\title{
Regional Groundwater Flow Model for C, K. L. and P Reactor Areas, Savannah River Site, Aiken, SC
}

by

G. P. Flach

Westinghouse Savannah River Company

Savannah River Site

Aiken, South Carolina 29808

M. K. Harris

R. A. Hiergesell

A. D. Smits

K. L. Hankins

This paper was prepared in connection with work done under the above contract number with the U. S.

Department of Energy. By acceptance of this paper, the publisher and/or recipient acknowledges the U. S.

Government's right to retain a nonexclusive, royalty-free license in and to any copyright covering this paper, along with the right to reproduce and to authorize others to reproduce all or part of the copyrighted paper. 
WSRC-TR-99-00248

Revision 0

\section{Regional Groundwater Flow Model for C, K, L, and P Reactor Areas, Savannah River Site, Aiken, South Carolina (U)}

Gregory P. Flach, Mary K. Harris, Robert A. Hiergesell, Andrew D. Smits, and Kelley L. Hawkins

September 1999

Prepared by:

Westinghouse Savannah River Company

Savannah River Site

Aiken, SC 29808

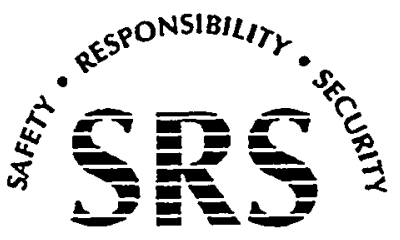

SAVANNAH RIVER SITE

Prepared for the U.S. Department of Energy Under

Contract Number DE-AC09-96SR18500 


\section{DISCLAIMER}

This report was prepared as an account of work sponsored by an agency of the United States Government. Neither the United States Government nor any agency thereof, nor any of their employees, makes any warranty, express or implied, or assumes any legal liability or responsibility for the accuracy, completeness, or usefulness of any information, apparatus, product or process disclosed, or represents that its use would not infringe privately owned rights. Reference herein to any specific commercial product, process or service by trade name, trademark, manufacturer, or otherwise does not necessarily constitute or imply its endorsement, recommendation, or favoring by the United States Government or any agency thereof. The views and opinions of authors expressed herein do not necessarily state or reflect those of the United States Government or any agency thereof.

This report has been reproduced directly from the best available copy.

Available for sale to the public, in paper, from: U.S. Department of Commerce, National Technical Information Service, 5285 Port Royal Road, Springfield, VA 22161, phone: (800) 553-6847

fax: (703) 605-6900

email: orders@ntis.fedworld.gov

online ordering: http://www.ntis.gov/ordering.htm

Available electronically at http://www.doe.gov/bridge

Available for a processing fee to U.S. Department of Energy and its contractors, in paper, from: U.S. Department of Energy, Office of Scientific and Technical Information, P.O. Box 62, Oak Ridge, TN 37831-0062, phone: (865) 576-8401

fax: (865) 576.5728

email: reports@adonis.osti.gov 


\section{DISCLAIMER}

Portions of this document may be illegible in electronic image products. Images are produced from the best available original document. 
WSRC-TR-99-00248

Revision 0

\section{Regional Groundwater Flow Model for C, K, L, and P Reactor Areas, Savannah River Site, Aiken, South Carolina (U)}

Gregory P. Flach, Mary K. Harris, Robert A. Hiergesell, Andrew D. Smits, and Kelley L. Hawkins

September 1999

Prepared by:

Westinghouse Savannah River Company Savannah River Site Aiken, SC 29808

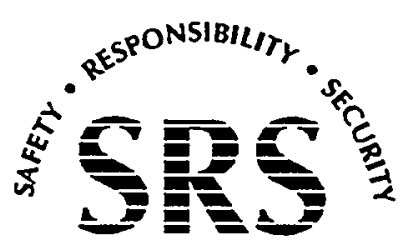

Prepared for the U.S. Department of Energy Under Contract Number DE-AC09-96SR18500 
11

WSRC-TR-99-00248. Rev. 0, Regional Groundwater Flow Model for C, K, L, and P Reactor Areas

Regional Groundwater Flow Model for C, K, L, and P Reactor Areas, Savannah River Site, Aiken, South Carolina (U)

Authentication and Approvals:
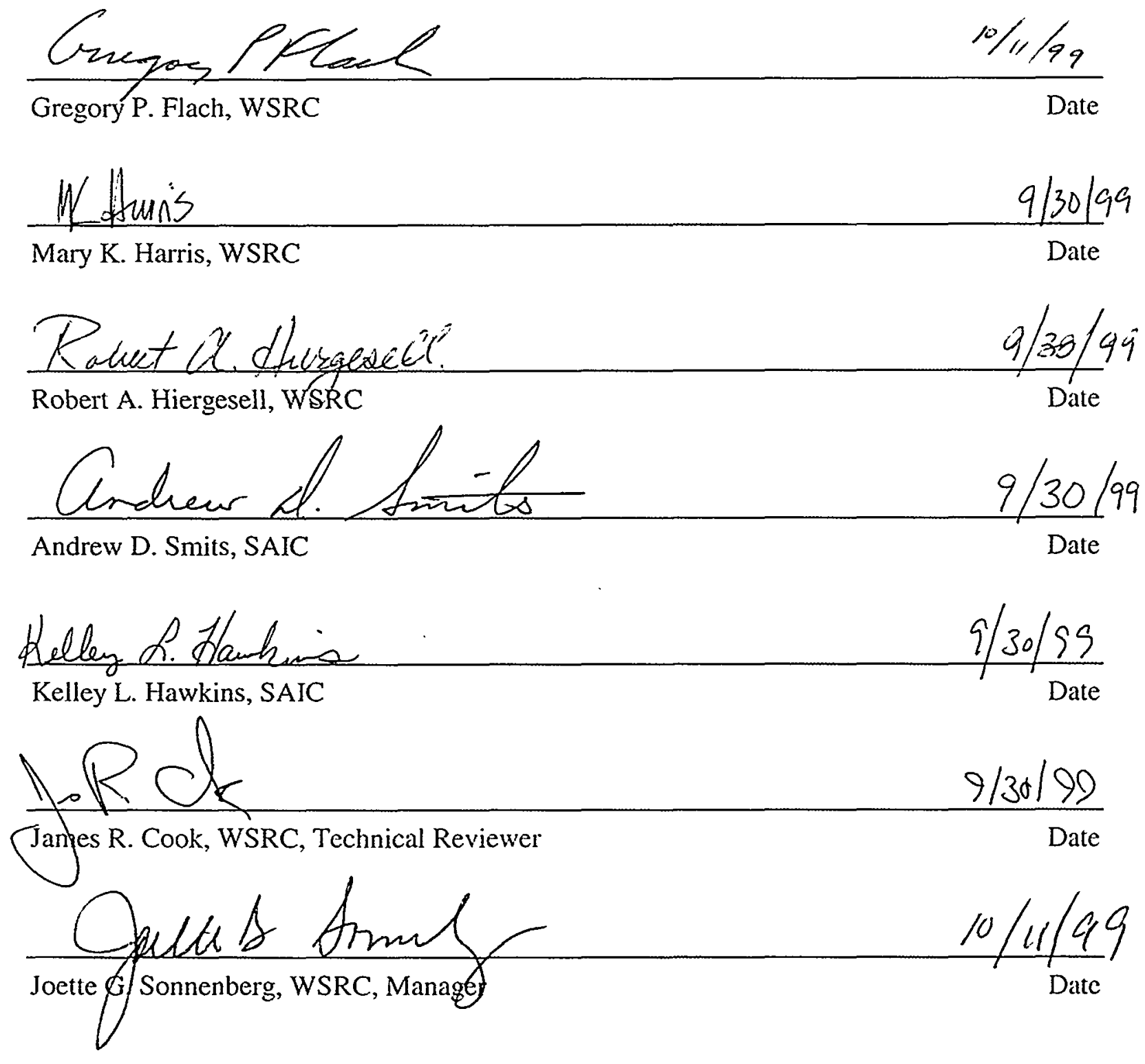


\section{ABSTRACT}

A regional groundwater flow model encompassing approximately $100 \mathrm{mi}^{2}$ surrounding the $\mathrm{C}$, $\mathrm{K}, \mathrm{L}$, and $\mathrm{P}$ reactor areas has been developed. The reactor flow model is designed to meet the planning objectives outlined in the General Groundwater Strategy for Reactor Area Projects by providing a common framework for analyzing groundwater flow, contaminant migration and remedial alternatives within the Reactor Projects team of the Environmental Restoration Department. The model provides a quantitative understanding of groundwater flow on a regional scale within the near surface aquifers and deeper semi-confined to confined aquifers. The model incorporates historical and current field characterization data up through Spring 1999. Model preprocessing is automated so that future updates and modifications can be performed quickly and efficiently. The CKLP regional reactor model can be used to guide characterization, perform scoping analyses of contaminant transport, and serve as a common base for subsequent finer-scale transport and remedial/feasibility models for each reactor area.

\section{MODEL SUMMARY}

The current groundwater flow model for $\mathrm{C}, \mathrm{K}, \mathrm{L}$, and $\mathrm{P}$ reactor areas simulates groundwater flow within the area bounded to the north by Upper Three Runs, to the west by the Savannah River, to the south by Steel Creek and Meyers Branch, and to the east by a line between McQueen Branch and Par Pond. Vertically the model extends from ground surface to the top of the Meyers Branch confining system. The model confirms that groundwater flow in upper aquifers at the Savannah River Site is recharge driven, with streams intercepting flow from higher elevations. The underlying Gordon aquifer is strongly influenced by and discharges to the Savannah River and Upper Three Runs. Nearly all recharge within the CKLP reactor region discharges to streams within or bounding the same area, usually the nearest stream, with the balance entering the Gordon aquifer. Simulated flow directions agree with the conceptual model of groundwater flow. Model calibration targets include groundwater recharge estimates, stream baseflow data and estimates, and water level measurements from more than 1000 wells. Model conductivity values in the Gordon aquifer and confining units are set directly to prior estimates based on field data. For the Upper Three Runs aquifer unit, conductivity values are defined through calibration to the groundwater flow and hydraulic head targets.

The chosen areal grid is 70,000 feet on a side, with a horizontal resolution of 500 square feet. The grid consists of 140 elements along each horizontal axis. The vertical resolution varies depending on hydrogeologic unit and terrain/hydrostratigraphic surface variations. The top 
surface of the mesh conforms to the ground surface. The bottom surface of the mesh coincides with the bottom of the Gordon aquifer unit. Interior node layers conform to the other stratigraphic surfaces. The "upper" aquifer zone of the Upper Three Runs aquifer includes the vadose zone and is represented by 3 finite-elements in the vertical direction. The "lower" aquifer zone of the Upper Three Runs aquifer contains 2 finite-elements, while the "tan clay" confining zone of the Upper Three Runs aquifer is represented by a single model element. The Gordon confining unit and Gordon aquifer unit are each assigned to one element, for a total of 8 vertical elements from ground surface to the bottom of the Gordon aquifer. The three-dimensional mesh is therefore $140 \times 140 \times 8$ with 156,800 elements or $141 \times 141 \times 9$ with 178,929 nodes. The finer vertical resolution in the "upper" zone of the Upper Three Runs aquifer is designed to support subsequent, finer-scale contaminant transport analyses.

Horizontal conductivity in the Gordon aquifer is set to $35 \mathrm{ft} /$ day based on the extensive field data from wells at the SRS and in the region surrounding the site. The vertical conductivity of the Gordon confining unit is set to $10^{-4} \mathrm{ft} /$ day in accordance with field measurements. Conductivity values within Upper Three Runs aquifer zones are set through model calibration to measured water levels. Horizontal conductivity in the "lower" aquifer zone is nominally $5.9 \mathrm{ft} /$ day, and varies from 4 to $20 \mathrm{ft} /$ day. Horizontal conductivity in the "upper" aquifer zone is nominally $8.3 \mathrm{ft} / \mathrm{day}$, and varies from 0.25 to $40 \mathrm{ft} /$ day. Vertical conductivity for the "tan clay" confining zone is nominally $3 \times 10^{-3} \mathrm{ft} / \mathrm{day}$, and varies between $1 \times 10^{-4}$ and $4 \times 10^{-3}$ $\mathrm{ft} /$ day. A typical ratio of horizontal to vertical conductivity is assumed to be 100 to 1 . Approximate soil characteristic curves are adopted for the vadose zone in the numerical model. An effective porosity value of $25 \%$ is assumed when computing the pore velocity field. 


\section{TABLE OF CONTENTS}

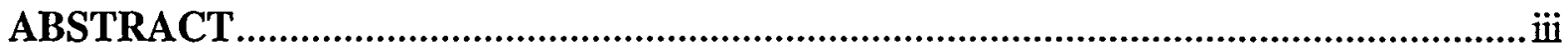

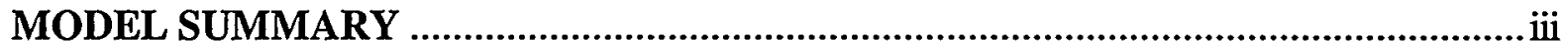

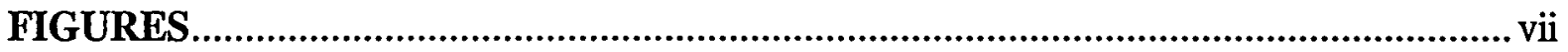

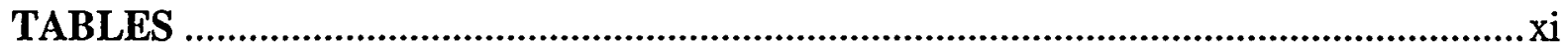

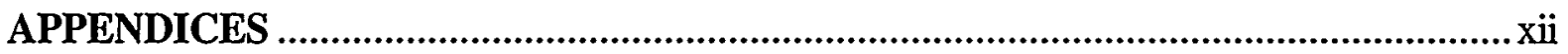

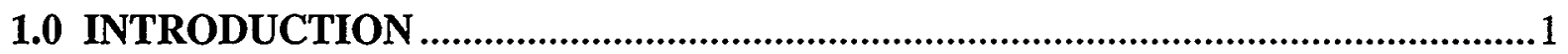

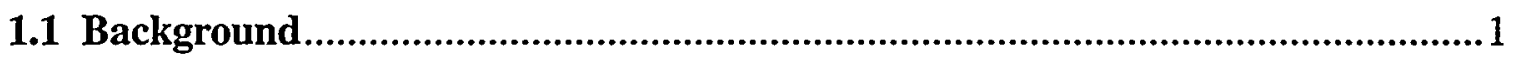

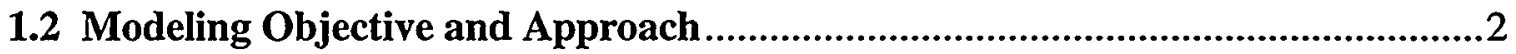

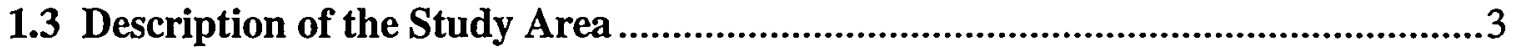

2.0 HYDROGEOLOGIC DATA AND CONCEPTUAL MODEL .....................................

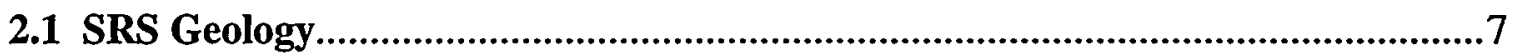

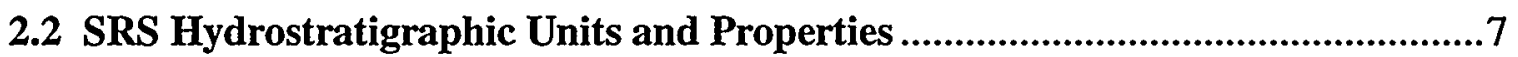

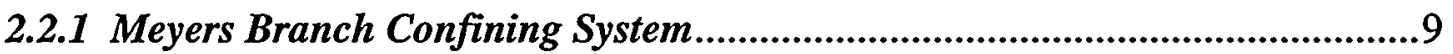

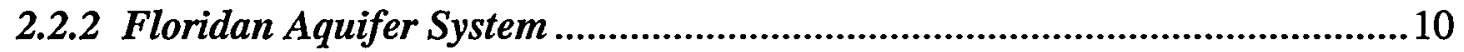

2.2.2.1 Gordon Aquifer ..............................................................................10

2.2.2.2 Gordon Confining Unit .....................................................................11

2.2.2.3 Upper Three Runs Aquifer .............................................................12

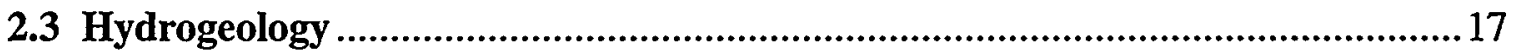

2.3.I Water Table

2.3.2 Gordon Aquifer Potentiometric Surface …................................................18

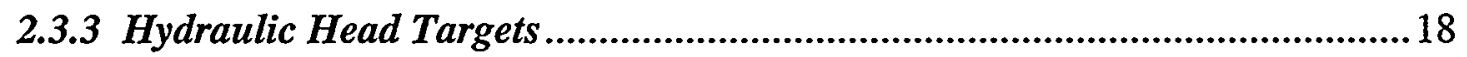


TABLE OF CONTENTS (Continued)

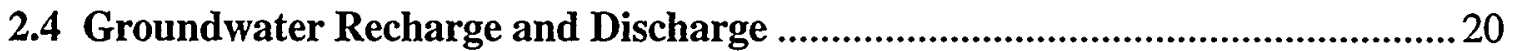

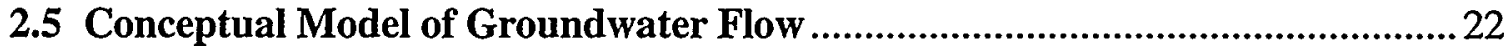

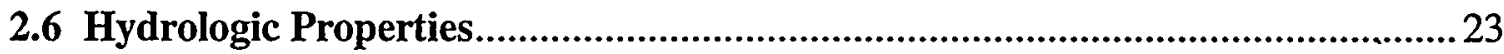

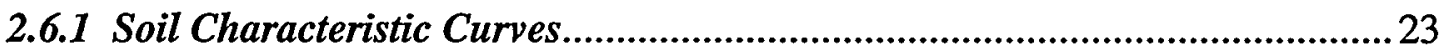

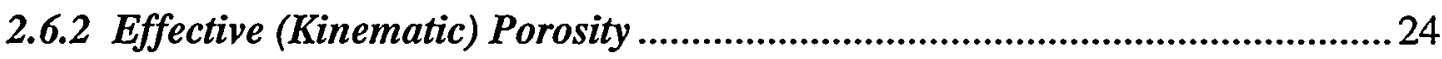

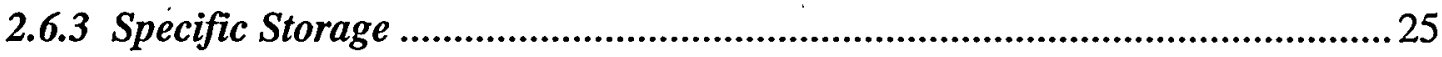

3.0 GROUNDWATER FLOW MODEL DEVELOPMENT ..........................................63

3.1 Code Selection and Description ............................................................................. 63

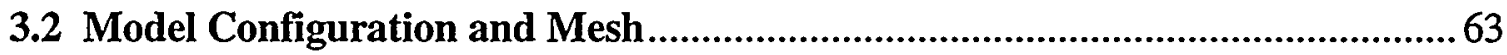

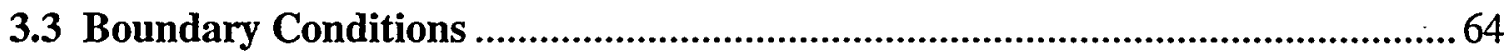

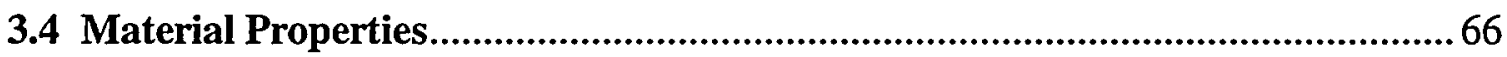

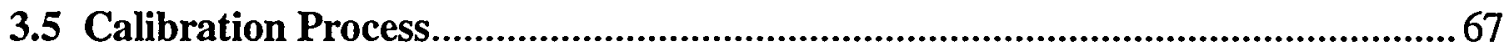

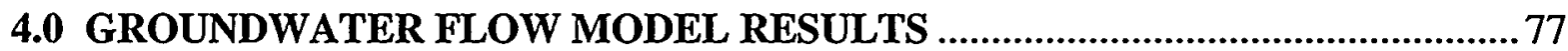

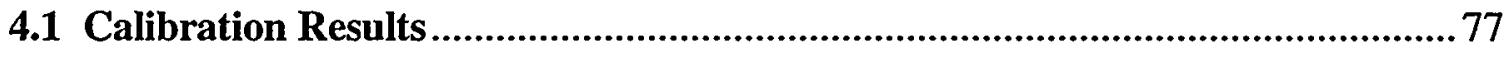

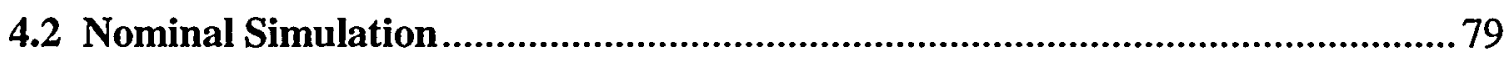

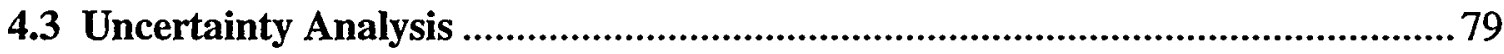

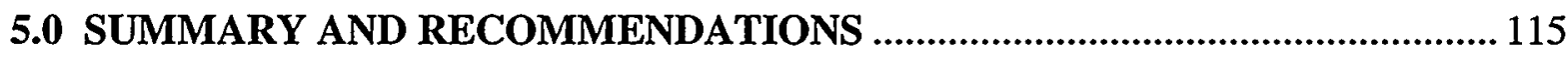

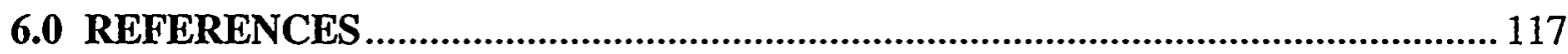




\section{FIGURES}

1-1 Location of the Savannah River Site and Model Areas............................................... 4

1-2 Location of Groundwater Basins at the Savannah River Site ...................................... 5

1-3 Location of Major Streams and Rivers in Model Area. Model Boundary

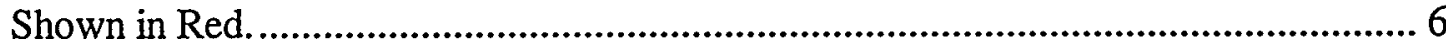

2-1 Comparison of Lithostratigraphic and Hydrostratigraphic Units at SRS ....................26

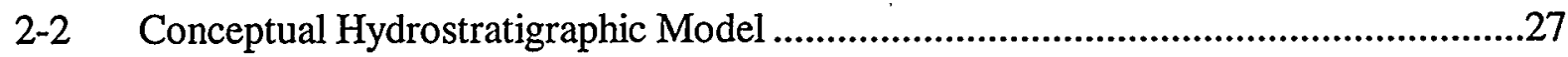

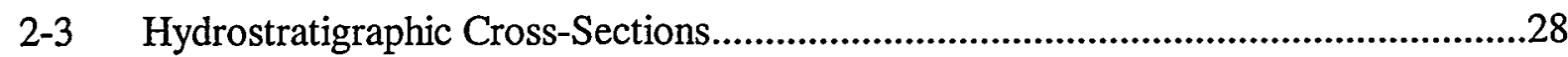

2-4 Base of Savannah River Alluvial Deposits..................................................................29

2-5 Isopach of Savannah River Valley Alluvial Fill .............................................................30

2-6 Altitude-Contour Map of the Top of the Meyers Branch Confining System.................31

2-7 Altitude-Contour Map of the Top of the Gordon Aquifer ..........................................32

2-8 Isopach Map of the Gordon Aquifer ........................................................................33

2-9 Altitude-Contour Map of the Top of the Gordon Confining Unit ................................34

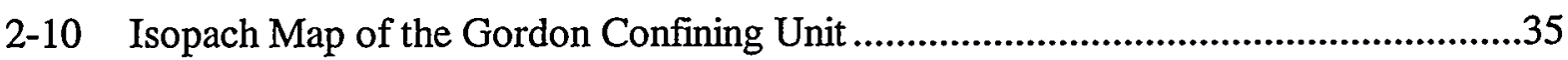

2-11 Altitude-Contour Map of the Top of the "Lower" Aquifer Zone ..................................36

2-12 Isopach Map of the "Lower" Aquifer Zone .................................................................37

2-13 Altitude-Contour Map of the Top of the "Tan Clay" Confining Zone...........................38

2-14 Isopach Map of the "Tan Clay" Confining Zone..........................................................39

2-15 Altitude-Contour Map of the Top of the "Transmissive Zone"....................................40

2-16 Isopach Map of the "Transmissive Zone"....................................................................41

2-17 Altitude-Contour Map of the Top of the "AA" Interval ..............................................42 


\section{FIGURES (Continued)}

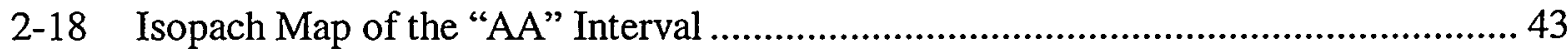

2-19 Altitude-Contour Map of the Top of the "A" Interval ........................................ 44

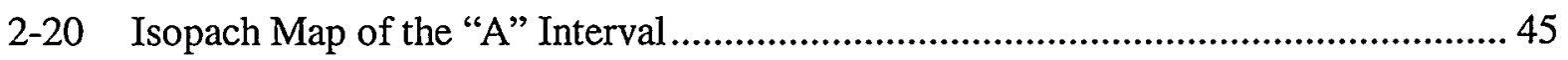

2-21 Isopach Map of the "UU" Interval .......................................................... 46

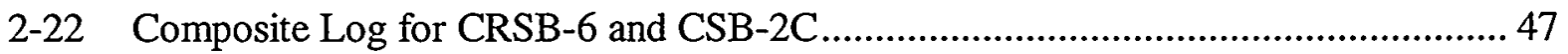

2-23 Water Table Map of CKLP Model Area .......................................................... 48

2-24 Location of Stream Baseflow Measurements for 1998 Field Study ........................ 49

2-25 Water Table Map for C Reactor Area .......................................................... 50

2-26 Water Table Map for K Reactor Area ...................................................... 51

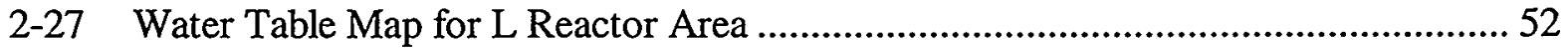

2-28 Water Table Map for P Reactor Area.......................................................... 53

2-29 Gordon Potentiometric Surface in the CKLP Model Area ................................... 54

2-30 Locations of Reactor Area Piezometers Installed in 1998 .................................... 55

2-31 Map of Live (Perennial) Stream Reaches as Determined by Field Observations ........ 56

2-32 Approximate Soil Characteristic Curves....................................................... 57

3-1 Plan View of Model Domain and Finite-Element Mesh ...................................... 70

3-2 Typical Cross-Sectional Slice through Finite-Element Mesh.............................. 71

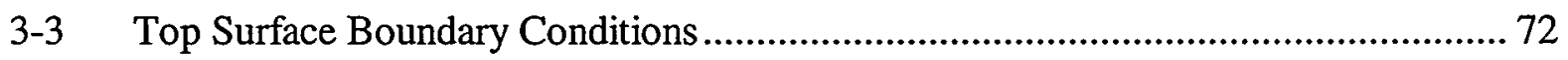

3-4 Boundary Conditions for Gordon Aquifer between the Top and Bottom Nodal

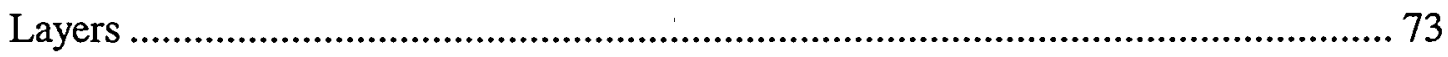

3-5 Boundary Conditions for Upper Three Runs Aquifer between the Top and Bottom Nodal Layers 


\section{FIGURES (Continued)}

3-6 Predicted Groundwater Flow Paths Prior to Refinement of "Tan Clay" Model Layer in C-Area near Caster Creek ............................................................................75

3-7 Predicted Groundwater Flow Paths after Refinement of "Tan Clay" Model Layer in C-Area near Caster Creek ........................................................................76

4-1 Simulated versus Measured Head for Each Aquifer Zone..........................................82

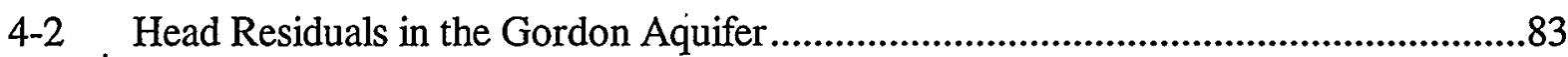

4-3 Head Residuals in the "Lower" UTR Aquifer Zone....................................................84

4-4 Head Residuals in the Transmissive Zone ("Upper" UTR Aquifer Zone).....................85

4-5 Head Residuals in the AA Horizon ("Upper" UTR Aquifer Zone)..............................86

4-6 Head Residuals in the A/UU Horizon ("Upper" UTR Aquifer Zone).........................87

4-7 Horizontal Conductivity in Element Layer 1 (Gordon Aquifer Except in

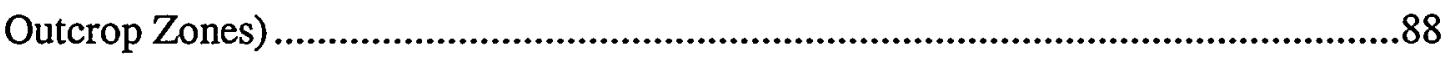

4-8 Vertical Conductivity in Element Layer 2 (Gordon Confining Unit Except in Outcrop Zones)

4-9 Horizontal Conductivity in Element Layer 3 (Lower Half of the "Lower" UTR Aquifer Zone Except in Outcrop Zones) ...................................................................90

4-10 Horizontal Conductivity in Element Layer 4 (Upper Half of the "Lower" UTR Aquifer Zone Except in Outcrop Zones) ......................................................................91

4-11 Vertical Conductivity in Element Layer 5 ("Tan Clay" Confining Zone Except in Outcrop Zones)

4-12 Horizontal Conductivity in Element Layer 6 (Transmissive Zone Except in Outcrop Zones)

4-13 Horizontal Conductivity in Element Layer 7 (AA Horizon Except in Outcrop Zones) 


\section{FIGURES (Continued)}

4-14 Horizontal Conductivity in Element Layer 8 (A/UU Horizons Except in

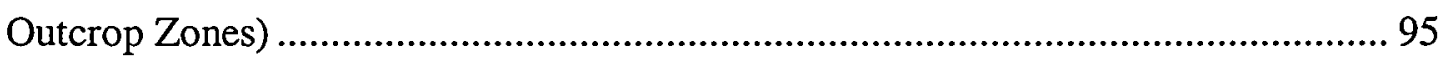

4-15 Horizontal Conductivity along a Cross-Section Through K-Area ............................. 96

4-16 Simulated Hydraulic Head in the Gordon Aquifer ................................................. 97

4-17 Simulated Hydraulic Head in the "Lower" UTR Aquifer ........................................... 98

4-18 Simulated Hydraulic Head in the "Upper" UTR Aquifer........................................... 99

4-19 Simulated Hydraulic Head in the Aquifer Zone Containing the Water Table ........... 100

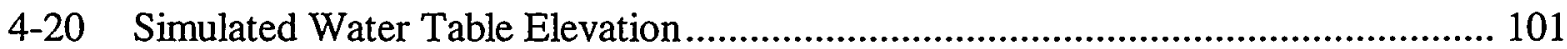

4-21 Simulated Flow Directions in the Gordon Aquifer ................................................ 102

4-22 Simulated Flow Directions in the "Lower" UTR Aquifer........................................... 103

4-23 Simulated Flow Directions in the "Upper" UTR Aquifer ......................................... 104

4-24 Simulated Seepage Faces.................................................................................... 105

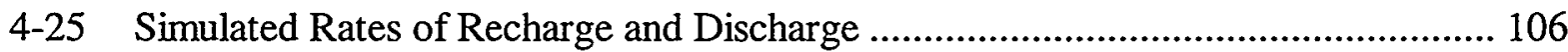

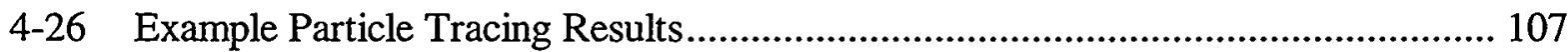

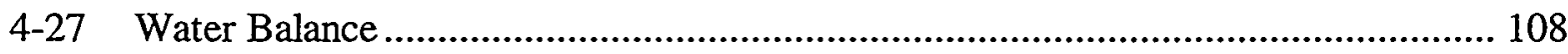




\section{TABLES}

2-1 Summary of Permeability Measurements .58

2-2 Summary of Permeability Measurements from Intervals within the "Upper"

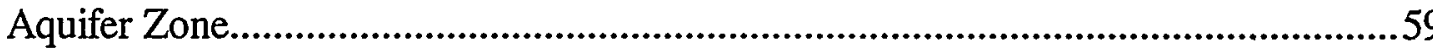

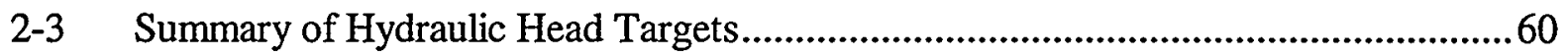

2-4 Base Flow Estimates Based on Hydrograph Separation of USGS Gauging

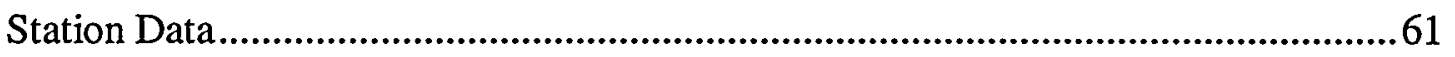

2-5 Base Flow Estimates Based on a Single Field Measurement under Low-Flow

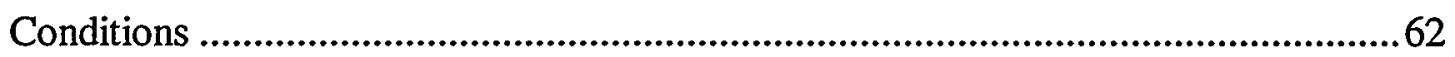

4-1 Calibration Summary for Groundwater Flow Targets .......................................... 109

4-2 Calibration Summary for Hydraulic Head Targets........................................... 110

4-3 Calibration Summary for Hydraulic Conductivity....................................... 111

4-4 Summary of Uncertainty Cases................................................................... 112

4-5 Calibration Summary for Uncertainty Cases............................................... 113 


\section{APPENDICES}

\section{APPENDIX A DATA COLLECTION AND HYDROGEOLOGIC MODEL METHODOLOGY}

APPENDIX B HYDROSTRATIGRAPHIC DATA

B-1 Locations of Sites within the Model Area

B-2 Hydrostratigraphic Boundaries

APPENDIX C PERMEABILITY DATA

C-1 Permeability Values Recorded from Pumping Tests

C-2 Permeability Values Recorded from Slug Tests

C-3 Permeability Values Recorded from Laboratory Tests

APPENDIX D DATA REFERENCES

APPENDIX E STREAM BASEFLOW DATA AND ESTIMATES

E-1 Stream Base Flow Estimates Based on USGS Gauging Station Data

E-2 Summary of Stream Baseflow and Water Table Work Conducted in Support of the R-Reactor and K-Reactor Groundwater Modeling Efforts (SRT-EST-98-110)

APPENDIX F HYDAULIC HEAD TARGET AND RESIDUAL DATA

APPENDIX G MODIFICATIONS TO C-AREA HYDROSTRATIGRAPHY BASED ON CPTU CHARACTERIZATION

APPENDIX H UNCERTAINTY ANALYSIS 


\subsection{INTRODUCTION}

\subsection{Background}

The Savannah River Site (SRS) is a U.S. Department of Energy (DOE) facility that occupies 300 square miles within Aiken, Barnwell, and Allendale counties in southwestern South Carolina (Figure 1-1). The SRS was set aside in 1950 as a controlled area to produce nuclear materials for national defense. The DOE and its contractors are responsible for the operation of the SRS. Westinghouse Savannah River Company (WSRC) is currently contracted to manage and operate the site.

The SRS operated five reactors to produce special radioactive materials during the Cold War Period. R Reactor was the first production reactor to go on-line, achieving criticality in December 1953. P Reactor achieved criticality in February of 1954, followed by L Reactor in August 1954, K Reactor in October 1954, and C Reactor in March 1955. The reactors produced plutonium-238, plutonium-239, and tritium for uses related to national defense, and also generated special isotopes for non-defense research, medical uses, and space programs. These special isotopes included cobalt- 60 , polonium-210, uranium-233, curium-244, and californium-252.

The past disposal practices associated with SRS reactor operations created waste units within and adjacent to the five reactor areas. Reactor area waste units include seepage basins, Bingham pump outage pits, burning/rubble pits, rubble piles, acid/caustic basins, coal pile runoff basins, and coal ash basins. WSRC (1997) provides a detailed discussion of these waste units.

The reactor areas lie within five major drainage systems (basins). These include the Fourmile Branch, Pen Branch, Steel Creek, Lower Three Runs and Upper Three Runs basins (Figure 1-2). SRS facilities are normally situated on well-drained, topographically high areas (divides) which separate the basins. This arrangement commonly places the waste units associated with a reactor within both of the adjacent groundwater basins. For example, L-Reactor waste units lie within the Pen Branch and Steel Creek basins and P-Reactor waste units lie within the Steel Creek and Lower Three Runs basins. 


\subsection{Modeling Objective and Approach}

The primary objective of this modeling effort is to establish a regional groundwater flow model to encompass the waste units associated with $\mathrm{C}, \mathrm{K}, \mathrm{L}$, and $\mathrm{P}$ reactor areas. The R-Reactor waste units are addressed in previous modeling efforts (HydroGeoLogic, 1997; 1998) and are not included in this report.

This model provides a basic understanding of the groundwater flow for these areas on a regional scale. This capability is important because of the various groundwater flow directions in the near surface aquifers and deep semi-confined to confined aquifers, and because it enables tracking of contaminant plumes from their source to discharge at the surface, potentially as far as the Savannah River and Upper Three Runs. The model for the reactor areas has been constructed to assist in scoping characterization and remedial activities by providing a common base for smaller-scale models of contaminant transport and remediation scenarios for each of these areas. In addition, the model allows waste units that are in close proximity to one another to be addressed comprehensively. This capability facilitates evaluation of the possibility of commingled plumes and assessment of the effects of one waste unit upon the other.

The model is designed to meet the planning objectives described in Section 4.2 of the General Groundwater Strategy for Reactor Area Projects (WSRC, 1997). The model incorporates all available data from geological and hydrological field characterizations into a project database that can be easily updated as additional field data are collected. This is consistent with the interactive approach described in WSRC (1997). The model will be able to incorporate new data as it is collected, allowing quick and cost-effective updates. The model can be evaluated to determine whether the available data are adequate to address a remediation issue. If more data are required, the model can assist in determining the types of data needed and from where they should be collected.

The reactors groundwater flow model uses EarthVision ${ }^{\circledR}$ proprietary software to calculate two-dimensional grids, maps, and cross-sections of the hydrogeology. The groundwater modeling is performed using the Flow And Contaminant Transport (FACT) code. The FACT code is a finite-element code developed by the Savannah River Technology Center (SRTC) (Hamm and others, 1997). 


\subsection{Description of the Study Area}

The SRS is centered 22.5 miles southeast of Augusta, Georgia, approximately 100 miles from the Atlantic Coast within the Upper Atlantic Coastal Plain Physiographic Province. The Savannah River forms the southwest boundary of the SRS (Figure 1-1). The SRS is situated on the Aiken Plateau of the Atlantic Coastal Plain at an approximate elevation of 300 feet above mean sea level ( $\mathrm{ft} \mathrm{msl}$ ). Overall, the plateau has a highly dissected surface and is characterized by broad inter-fluvial areas with narrow, steep-sided valleys. Local relief can attain 280 feet (Siple, 1967). The Aiken plateau is generally well drained, although many poorly drained sinks and depressions exist.

The model area, herein referred to as the C, K, L, and P Groundwater Model Area (CKLP GWMA) comprises approximately 100 square miles within the central and southern SRS. The CKLP GWMA has low to moderate topographic relief and drains to the west via perennial and intermittent streams (Figure 1-3). The CKLP GWMA is bounded to the north by Upper Three Runs, to the west by the Savannah River, to the south by Steel Creek and Meyers Branch, and to the east by a line between McQueen Branch and Par Pond (Figure 1-3). Upper Three Runs forms the northern boundary of the study area with an average elevation of $150 \mathrm{ft}$ msl, the Savannah River forms the western boundary with an average elevation between 85 and $90 \mathrm{ft} \mathrm{msl}$, and Steel Creek and Meyers Branch forms the south-southeastern boundary with elevations ranging from 100 to $105 \mathrm{ft}$ msl. Beyond the headwaters of Meyers Branch, the southern boundary extends to Par Pond in the area south of P Area. There is no single natural drainage at the eastern margin of the CKLP GWMA. A line running southeast from McQueen Branch, through the headwaters of Fourmile Branch, to Par Pond (Figure 1-3) defines an eastern boundary. 


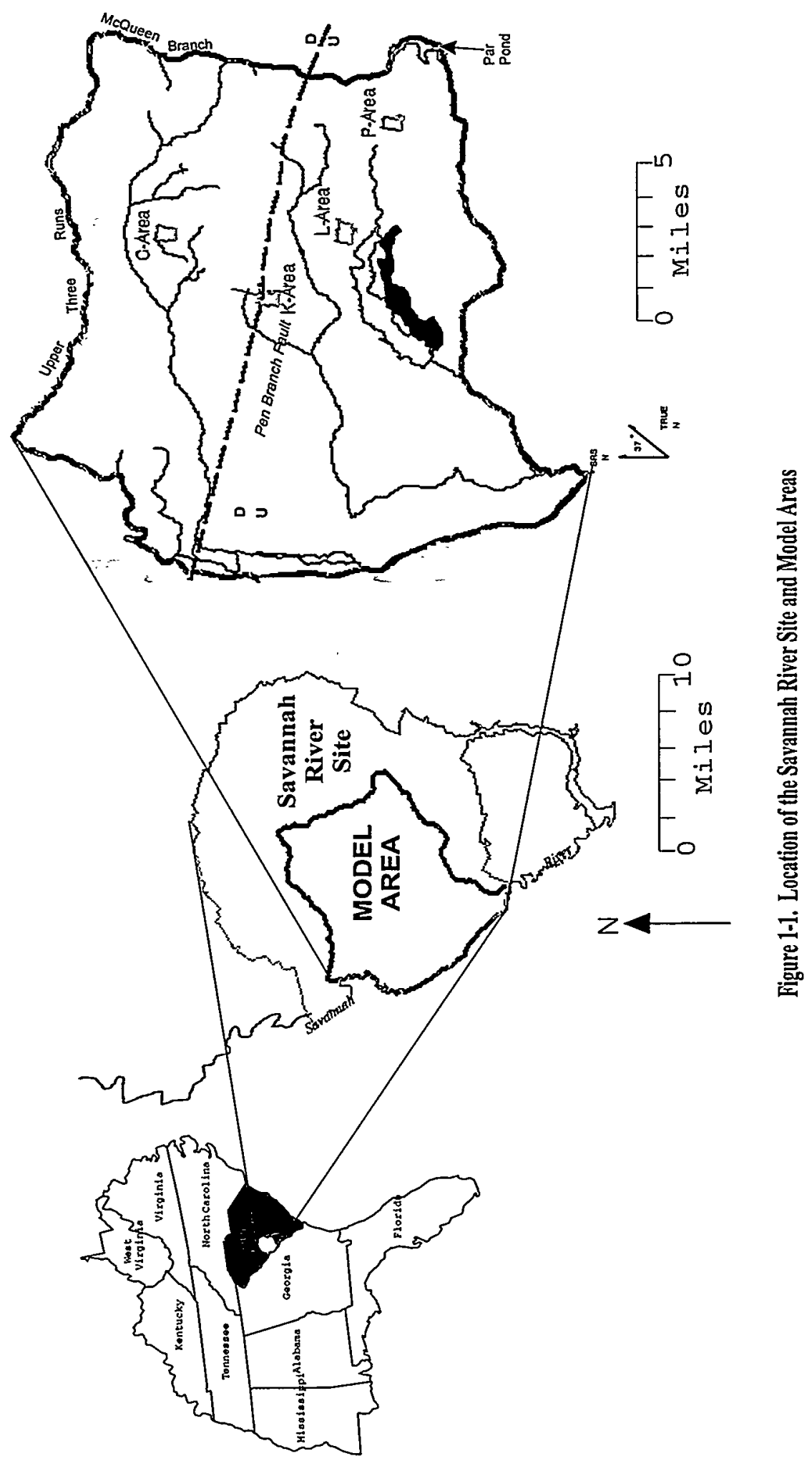




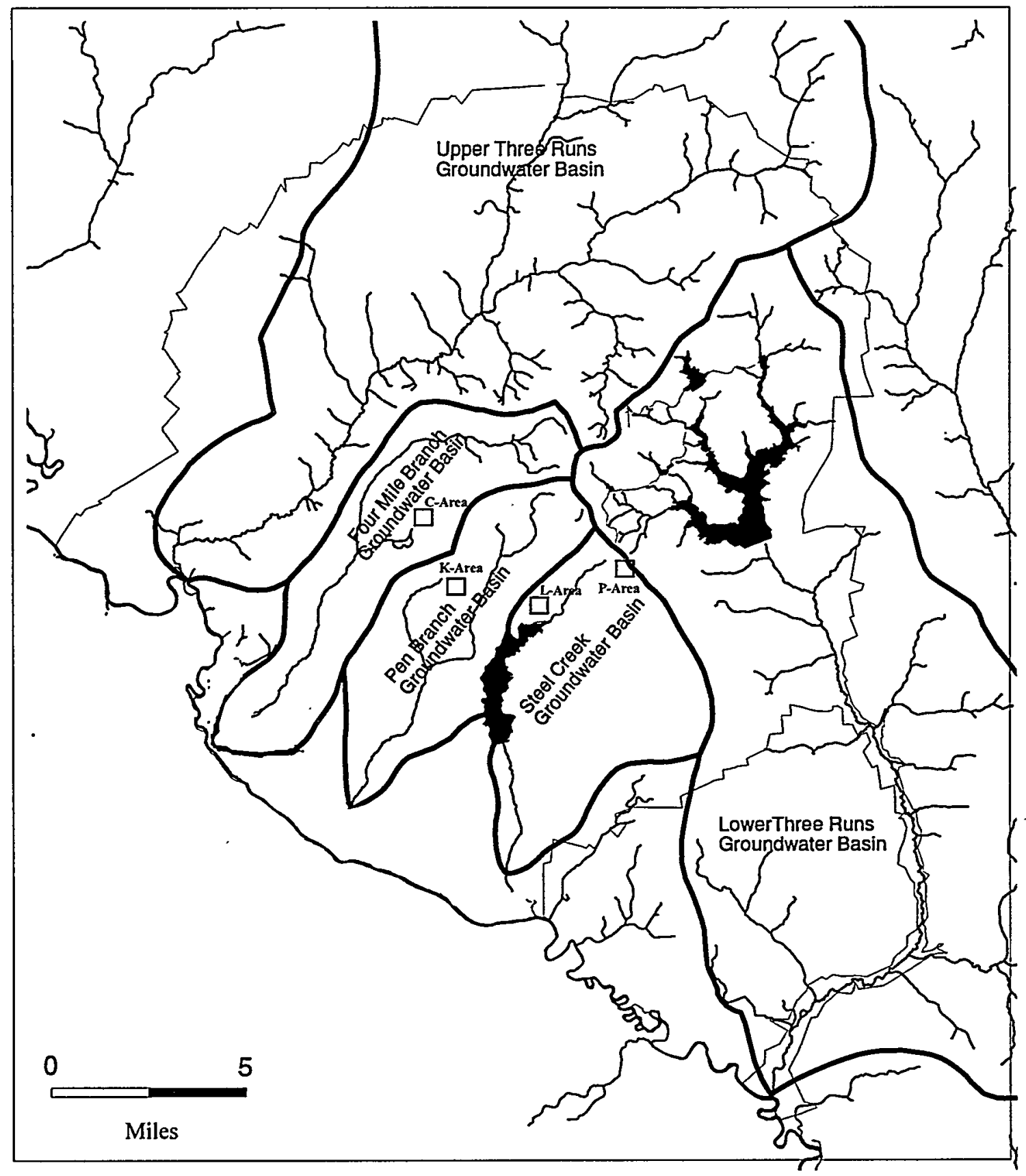

Figure 1-2. Location of Groundwater Basins at the Savannah River Site 


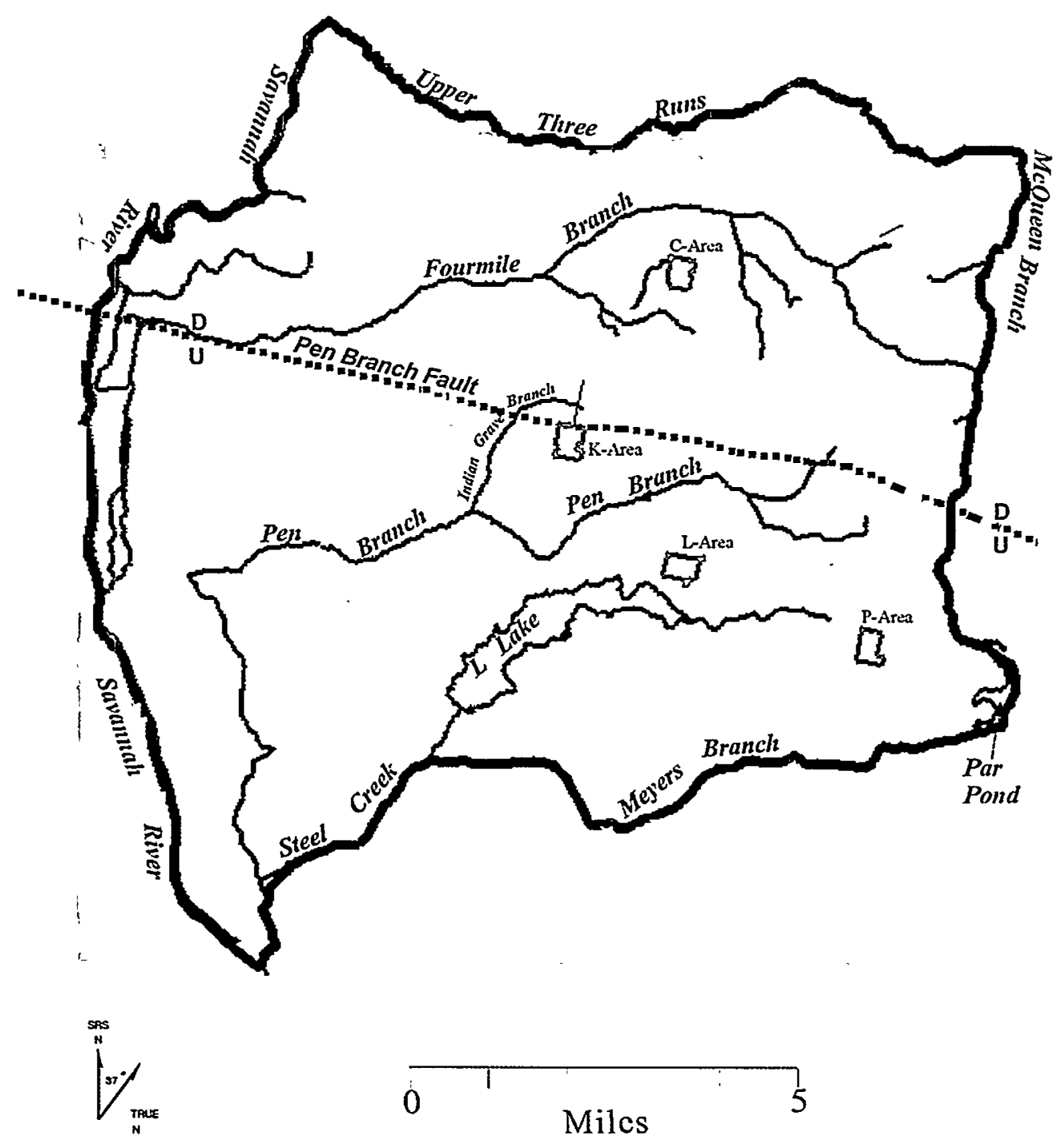

Figure 1-3. Location of Major Streams and Rivers in Model Area. Model Boundary Shown in Red 


\subsection{HYDROGEOLOGIC DATA AND CONCEPTUAL MODEL}

\subsection{SRS Geology}

The SRS lies within the Atlantic Coastal Plain, a southeast-dipping wedge of unconsolidated and semi-consolidated sediment that extends from its contact with the Piedmont Province at the Fall Line to the edge of the continental shelf. The sediment ranges from Late Cretaceous to Miocene in age and comprises layers of sand, muddy sand, and mud with minor amounts of calcareous sediment (Fallaw and Price, 1995). The Coastal Plain sediment rests unconformably on Triassic-aged sedimentary rock of the Dunbarton Basin and Paleozoic-aged crystalline rock of the Appalachian orogen.

The Pen Branch Fault (PBF) offsets basement rock and Late Cretaceous to Tertiary-aged sediment beneath the CKLP GWMA (Figure 1-1). Seismic studies and stratigraphic correlation indicate that the Pen Branch Fault is a sub-vertical growth fault with down-to-thenorthwest movement sense. The PBF probably represents reactivation of a border fault in the basement rock along the north margin of the Dunbarton Basin (Snipes and others, 1993; Stieve, 1994; Stieve and Stephenson, 1995). The part of the CKLP GWMA that is south of the PBF is underlain by Triassic-aged sedimentary rock.

\subsection{SRS Hydrostratigraphic Units and Properties}

The hydrostratigraphy of the SRS has been the subject of several different classification schemes. This report incorporates the hydrostratigraphic nomenclature currently established for the SRS region by Aadland and others (1995), who present a thorough review and description of the units. Figure 2-1 correlates the hydrostratigraphic nomenclature with the local lithostratigraphy as defined by Fallaw and Price (1995). This report addresses the up-dip part of the Floridan aquifer system and the top of the Meyers Branch confining system as defined by Aadland and others (1995).

The diagram in Figure 2-2 and the cross-sections in Figure 2-3 present the conceptual model used for this study and illustrate the relationship between the hydrostratigraphic units, the topography, and the recent alluvial material deposited in the Savannah River valley. The conceptual model accounts for movement along the PBF and its effect on the hydrostratigraphic units above the Meyers Branch confining system. The model assumes that strain accumulation within thees units is limited to non-brittle deformation of the sediment across the fault zone. The grid calculations made using EarthVision ${ }^{\circledR}$ software use a vertical fault to represent this zone which appears as a discontinuity in Figures 2-2 and 2-3. This 
method best approximates the flexure of strata across the fault zone in terms of the EarthVision ${ }^{\circledR}$ grids, which are used as input for the groundwater model. The groundwater model assumes that this flexure does not affect the hydrologic properties of the units.

The lateral and vertical extent of the hydrostratigraphic units and the recent alluvium are very important hydrologically. The topography is a major factor in controlling the distribution of surface water and the configuration of the water table. Major tributaries of the Savannah River incise the hydrostratigraphic units down to the "tan clay" confining zone and, to a lesser extent, to the "lower" aquifer zone of the Upper Three Runs aquifer. The Savannah River and Upper Three Runs cut down into the Gordon aquifer. The depth to which the streams and river incise the underlying hydrostratigraphic units is an important factor in the localized and regional flow systems in the study area. Leeth and Nagle (1996) installed a series of borings along the Savannah River in the vicinity of SRS to determine the shallow subsurface geology of the flood plain and river terraces. Their results were used as a guide in determining the lateral and vertical extent of the alluvium in the CKLP GWMA. The thickness of the recent alluvial material in the river valley varies, and attains a maximum thickness of approximately $50 \mathrm{ft}$ (Leeth and Nagle, 1996). Figures 2-2 and 2-3 depict the conceptual hydrostratigraphic model used for the CKLP GWMA and illustrate the extent to which the Savannah River has incised the hydrostratigraphic units. The Savannah River has cut down into the Gordon aquifer at the northern and southern ends of the valley but does not incise the Meyers Branch confining system within the model area. Figure 2-4 illustrates the base of the Savannah River alluvial valley as an altitude-contour map. The contours in Figure 2-4 define three smaller "valleys" that trend east-west across this surface. These structures represent input from the Upper Three Runs, Fourmile Branch, and Pen Branch tributaries into the main alluvial valley formed by the Savannah River. The isopach map in Figure 2-5 illustrates the thickness of the recent alluvium as used in the CKLP model. The alluvium attains a maximum thickness of between 40 and 50 feet in areas of confluence between major tributaries and the main river channel, and along the west bank of the present-day channel of the Savannah River. The asymmetry in the base of the alluvial valley and the variations in thickness of the alluvium is based on the findings of Leeth and Nagle (1996).

The following sections describe the lithologic characteristics along with the configuration of the tops and thickness of the hydrostratigraphic units mapped for this study. It should be noted that the tops of the hydrostratigraphic units correspond closely with unconformities in the SRS region recognized by Fallaw and Price (1995). All of the altitude contour maps have patterns that are consistent with the south-southeast dip of the Coastal Plain strata in this 
region (Fallaw and Price, 1995). Isopach contours indicate variability in thickness, which is related to the varying degrees of erosion at the unconformable surfaces and structural relations with the PBF. Both the altitude contour maps and isopach maps have been constructed to depict down cutting and deposition of recent alluvial material by the Savannah River and its major tributaries. All of the maps exhibit contour patterns that reflect the variability in data density across the area. Directional references given in the following discussion are made with respect to the SRS grid system.

The project database includes permeability data from aquifer pumping tests, borehole permeability tests (slug tests), and laboratory tests of core samples from locations within the CKLP GWMA. Table 2-1 presents a summary of the permeability data collected for this study. The summary incorporates only data from locations for which boundaries of the major hydrostratigraphic units have been established as part of this study. Statistical calculations were made using averaged values from multi-well pumping tests and the average values from wells with results from both rising and falling-head slug tests.

Appendix A presents a summary of the data collection and modeling methods that were utilized for this investigation. Appendix $B$ presents locations of data points, hydrostratigraphic boundaries, and a summary of the two-dimensional grids calculated from the boundaries. Appendix $C$ presents permeability data from locations within the model area. Appendix D lists source documents for the data presented in Appendices B and C and summarized in Table 2-1.

\subsubsection{Meyers Branch Confining System}

The Meyers Branch confining system (MBCS) defines the base of the Floridan aquifer system beneath the study area. In the CKLP GWMA, the top of the MBCS is delineated by laterally continuous layers of dense, gray to black, clay and sandy clay of the Lang Syne Formation of the Black Mingo Group (Figure 2-1) (Aadland and others, 1991 and 1995).

The configuration of the top of the MBCS is illustrated with altitude contours in Figure 2-6. The MBCS exhibits a relatively gentle dip in a south-southwest direction. The top of this unit shows vertical offset of approximately $40 \mathrm{ft}$ along the PBF. The Savannah River has not incised the MBCS within the CKLP GWMA.

Laboratory tests of 29 undisturbed samples taken from the MBCS indicate that vertical permeability ranges from 4.26E-06 to 3.40E-01 feet per day (ft/day)(Table 2-1). Tests of 27 undisturbed samples yield horizontal permeability values that range from $1.1 \mathrm{E}-05$ to 
$1.5 \mathrm{E}+00 \mathrm{ft} /$ day within this unit. These data show an arithmetic mean of $1.36 \mathrm{E}-02 \mathrm{ft} /$ day for vertical permeability and $8.42 \mathrm{E}-02 \mathrm{ft} /$ day for horizontal permeability, and a geometric mean of $2.43 \mathrm{E}-04 \mathrm{ft} /$ day for vertical permeability and $6.64 \mathrm{E}-04 \mathrm{ft} /$ day for horizontal permeability. The standard deviation is $5.58 \mathrm{E}-02$ for vertical permeability and $3.01 \mathrm{E}-01$ for horizontal permeability.

\subsubsection{Floridan Aquifer System}

The Floridan aquifer system overlies the MBCS and includes the Gordon aquifer, Gordon confining unit, and Upper Three Runs aquifer within the CKLP GWMA (Figure 2-1). The Upper Three Runs aquifer is recharged primarily by precipitation (Hiergesell, 1998a). Groundwater flow maintains a primarily vertical component from the Upper Three Runs aquifer down into the Gordon aquifer.

\subsubsection{Gordon Aquifer}

The Gordon aquifer constitutes the basal unit of the Floridan aquifer system beneath the CKLP GWMA and is the lowermost unit characterized in this report (Figure 2-1). Within the study area, the Gordon aquifer includes loose sand and clayey sand of the Congaree Formation and, where present, the sandy parts of the underlying Fourmile Branch and Snapp Formations (Figure 2-1), (Harris and others, 1990; Aadland and others, 1991 and 1995). The sand within the Gordon aquifer is yellowish to grayish orange and is sub- to well-rounded, moderately to poorly sorted, and medium- to coarse-grained. Pebbly layers and zones of sand cemented with iron and silica are common. The Gordon aquifer includes rare interbeds of light tan to gray clay that range up to three feet in thickness. Lenses of clay less than 6 inches in thickness are common near the base of this unit. The Gordon aquifer contains a small amount of sporadically distributed calcareous sediment.

The configuration of the top of the Gordon aquifer is illustrated in Figure 2-7. An isopach map is presented in Figure 2-8. The Gordon aquifer exhibits the same structural pattern as the MBCS with a regional dip to the south-southeast. The Gordon aquifer shows vertical offset approximately 40 feet along the PBF. The thickness of this unit is variable, ranging from approximately 60 feet to 160 feet. This variability is believed to be related to structural relations with overlying and underlying units and the presence of unconformities above and below the unit. In addition, the Gordon aquifer is incised along the Savannah River primarily in the vicinity of the PBF and along Upper Three Runs (Figure 2-7). 
Laboratory tests of 21 undisturbed samples taken from the Gordon aquifer indicate that vertical permeability within this unit ranges from 3.12E-06 to 3.62E+01 ft/day (Table 2-1). Horizontal permeability values range from $2.44 \mathrm{E}-05$ to $3.26 \mathrm{E}+01 \mathrm{ft} /$ day within this unit. These data show an arithmetic mean of $1.87 \mathrm{E}+00 \mathrm{ft} / \mathrm{day}$ for vertical permeability and $6.07 \mathrm{E}+00 \mathrm{ft} /$ day for horizontal permeability and a geometric mean of $2.09 \mathrm{E}-03 \mathrm{ft} /$ day for vertical permeability and 4.18E-02 ft/day for horizontal permeability. The standard deviation calculated from these data is $7.88 \mathrm{E}+00 \mathrm{ft} /$ day for vertical permeability and $1.18 \mathrm{E}+01 \mathrm{ft} /$ day for horizontal permeability.

Results from 50 slug tests conducted on wells screened within the Gordon aquifer indicate permeability ranges from $5.00 \mathrm{E}-03$ to $3.31 \mathrm{E}+01 \mathrm{ft} /$ day (Table $2-1$ ). The arithmetic mean from these data is $3.75 \mathrm{E}+00 \mathrm{ft} / \mathrm{day}$ and the geometric mean is $9.79 \mathrm{E}-01 \mathrm{ft} /$ day. The standard deviation calculated from these results is $6.47 \mathrm{E}+00 \mathrm{ft} /$ day. The permeability results were averaged for wells with both rising and falling-head tests.

Three multi-well pumping tests performed on wells screened within the Gordon aquifer give permeability values that range from $1.86 \mathrm{E}-04$ to $4.50 \mathrm{E}+01 \mathrm{ft} / \mathrm{day}$ (Table 2-1). The arithmetic mean is $2.57 \mathrm{E}+01 \mathrm{ft} /$ day and the geometric mean is $6.45 \mathrm{E}-01 \mathrm{ft} /$ day. The standard deviation calculated from these results is $2.32 \mathrm{E}+01 \mathrm{ft} / \mathrm{day}$.

Ten single-well pumping tests performed on wells screened within the Gordon aquifer give permeability values that range from $8.20 \mathrm{E}-01$ to $1.43 \mathrm{E}+02 \mathrm{ft} / \mathrm{day}$ (Table 2-1). The arithmetic mean is $2.25 \mathrm{E}+01 \mathrm{ft} /$ day and the geometric mean is $5.00 \mathrm{E}+00 \mathrm{ft} /$ day. The standard deviation calculated from these results is $4.50 \mathrm{E}+01 \mathrm{ft} /$ day.

Aadland and others (1995) present additional information on pumping tests conducted within the Gordon aquifer.

\subsubsection{Gordon Confining Unit}

The Gordon confining unit (GCU) separates the Gordon aquifer from the Upper Three Runs aquifer. This unit is commonly referred to as the "green clay" in previous SRS literature and includes sediment of the Warley Hill Formation (Figure 2-1). The unit comprises interbedded silty and clayey sand, sandy clay and clay. The clay is stiff to hard and is commonly fissile. Glauconite is a common constituent and imparts a distinctive greenish cast to the sediment, hence the informal name of "green clay" given to this unit. Zones of silica-cemented sand and clay are present within the GCU in some cores taken from the GSA. Beneath the CKLP GWMA, the GCU includes some calcareous sediment and limestone, primarily calcarenaceous 
sand and clayey sand with subordinate calcarenaceous clay, micritic clay, and sandy micrite and limestone.

The GCU dips toward the south-southeast, increasing from approximately 10 feet to 80 feet in thickness (Figures 2-9 and 2-10). The southeastward thickening is primarily due to an increase in the quantity of fine-grained calcareous material within this unit beneath the southern half of the study area. The GCU is incised along the Savannah River to the north and south of the PBF and also incised along the southern boundary of Upper Three Runs (Figure 2-9).

Laboratory tests of 47 undisturbed samples taken from the GCU indicate vertical permeability ranges from 1.14E-06 to 4.27E-01 ft/day (Table 2-1). Tests of 30 undisturbed samples yield horizontal permeability values that range from $5.40 \mathrm{E}-06$ to $1.22 \mathrm{E}-01 \mathrm{ft} / \mathrm{day}$ within this unit. These data show an arithmetic mean of 1.13E-02 ft/day for vertical permeability and 8.97E-03 $\mathrm{ft} /$ day for horizontal permeability and a geometric mean of $1.28 \mathrm{E}-04 \mathrm{ft} /$ day for vertical permeability and $1.65 \mathrm{E}-04 \mathrm{ft} /$ day for horizontal permeability. The standard deviation calculated from these data is $6.25 \mathrm{E}-02 \mathrm{ft} /$ day for vertical permeability and $2.83 \mathrm{E}-02 \mathrm{ft} /$ day for horizontal permeability.

Aadland and others (1995) discuss leakance estimates derived from multiple well pumping tests.

\subsubsection{Upper Three Runs Aquifer}

The Upper Three Runs aquifer (UTRA), as defined in this report, includes all strata from the ground surface to the top of the GCU. The UTRA includes the informally named "upland" unit, Tobacco Road Sand, Dry Branch Formation, Clinchfield Formation, and Santee Limestone (Figure 2-1). For the purposes of hydrostratigraphic analysis, the UTRA is often locally divided into informal "lower" and "upper" aquifer zones which are separated by the "tan clay" confining zone (Figure 2-1). The informal zones are further differentiated into intervals where local hydrologic conditions and model resolution requirements warrant additional hydrogeologic detail.

"Lower" Aquifer Zone. The "lower" aquifer zone (LAZ) of the UTRA beneath the CKLP GWMA consists of dominantly fine-grained, well-sorted sand and clayey sand of the Santee Formation and parts of the Dry Branch Formation which are beneath the "tan clay" confining zone (Figure 2-1). The bulk of the carbonate sediment beneath the CKLP GWMA is contained within the Santee Limestone and lower part of the Dry Branch Formation and is 
included in the LAZ. Descriptions of drill core indicate that the carbonate sediment in this vicinity has a significant siliciclastic component, and consists primarily of calcarenaceous sand, micritic sand, shelly sand, with minor amounts of sandy calcarenite and shelly limestone.

Altitude-contour and isopach maps of the LAZ are presented in Figures 2-11 and 2-12. The configuration of the top of the LAZ is similar to that of the GCU. The thickness of the LAZ ranges from approximately 30 feet to 110 feet. The wide range in thickness is attributed primarily to erosion of this unit on the overlying unconformity. The LAZ is deeply incised by Upper Three Runs and the Savannah River within the model area (Figure 2-11).

Laboratory tests of 30 undisturbed samples taken from the LAZ indicate that vertical permeability ranges from $4.54 \mathrm{E}-06$ to $3.42 \mathrm{E}+00 \mathrm{ft} /$ day (Table 2-1). Tests of 26 undisturbed samples yield horizontal permeability values that range from $1.59 \mathrm{E}-05$ to $1.11 \mathrm{E}+01 \mathrm{ft} / \mathrm{day}$ within this unit. These data show an arithmetic mean of $1.58 \mathrm{E}-01 \mathrm{ft} /$ day for vertical permeability and 7.07E-01 ft/day for horizontāl permeability and a geometric mean of $3.26 \mathrm{E}-03 \mathrm{ft} /$ day for vertical permeability and $1.31 \mathrm{E}-02 \mathrm{ft} /$ day for horizontal. The standard deviation calculated from these data is $6.25 \mathrm{E}-01 \mathrm{ft} /$ day for vertical permeability and $2.20 \mathrm{E}+00$ $\mathrm{ft} /$ day for horizontal permeability.

Results from 31 slug tests conducted within the LAZ indicate permeability ranges from 1.30E01 to $2.44 \mathrm{E}+01 \mathrm{ft} / \mathrm{day}$ (Table 2-1). The arithmetic mean from these data is $3.28 \mathrm{E}+00 \mathrm{ft} / \mathrm{day}$ and the geometric mean is $1.38 \mathrm{E}+00 \mathrm{ft} / \mathrm{day}$. The standard deviation calculated from these results is $5.60+00 \mathrm{ft} /$ day.

Two multi-well pumping tests of wells screened within the LAZ indicate permeability ranges from $1.23 \mathrm{E}+0.0$ to $2.10 \mathrm{E}+00 \mathrm{ft} /$ day (Table 1). The arithmetic mean is $1.67 \mathrm{E}+00 \mathrm{ft} /$ day and the geometric mean is $1.63 \mathrm{E}+00 \mathrm{ft} /$ day (Table 1). The standard deviation calculated from these results is $6.13 \mathrm{E}-01 \mathrm{ft} /$ day.

"Tan Clay" Confining Zone. The "tan clay" confining zone (TCCZ) of the UTRA is equivalent to the "tan clay" zone referred to in previous SRS reports. The includes sediment assigned to the Twiggs Clay and Irwinton Sand Members of the Dry Branch Formation (Figure 2-1). The zone contains light-yellowish tan to orange clay and sandy clay interbedded with clayey sand and sand. Clay layers are dispersed vertically and horizontally throughout the zone and are probably not laterally continuous over distances greater than 100 to 200 feet (Harris and others, 1990; Aadland and others, 1991). Beneath the CKLP GWMA, the TCCZ consists of two sequences of interbedded mud and sand with some calcareous sediment. The 
lower sequence contains light green to tan, slightly fissile clay and silty sand that is commonly interbedded with sand, silty sand, and coarse-grained, carbonate gravels. The carbonate gravels consist of oysters and other shell debris that are mixed with mud, sand, and gravel. The lower sequence commonly includes interbeds of relatively dense, well-indurated layers of a matrix-supported, shelly, sandy carbonate mudstone. This material consists of siliciclastic sand, mud, and gravel, shell debris, and other carbonate fragments contained in a matrix of mud-sized carbonate material (micrite).

The upper sequence within the TCCZ consists of interbedded siliciclastic sand and mud. The mud is waxy and commonly fissile, suggesting a very high clay content. The interbedded sand is generally well-sorted and medium-grained, with good interstitial porosity. The interbeds within this sequence vary from less than one to five feet in thickness. The individual layers of sand and mud cannot normally be correlated from core to core, but characteristic geophysical and CPT signatures can be readily identified at most data points, indicating that this interval is laterally continuous.

The configuration of the top of the TCCZ is illustrated in Figure 2-13 and an isopach map of the unit is presented in Figure 2-14. The configuration of the top of the TCCZ is very similar to that of the underlying LAZ. The measured thickness of the TCCZ ranges from approximately 10 feet to 20 feet. The TCCZ is deeply incised by the Savannah River, Upper Three Runs, Fourmile Branch, and Steel Creek and Meyers Branch within the model area (Figure 2-13).

Laboratory tests of 43 undisturbed samples taken from the TCCZ indicate vertical permeability ranges from $3.70 \mathrm{E}-08$ to $2.39 \mathrm{E}-01 \mathrm{ft} /$ day (Table 2-1). Tests of 29 undisturbed samples yield horizontal permeability values that range from $1.45 \mathrm{E}-05$ to $2.04 \mathrm{E}-01 \mathrm{ft} / \mathrm{day}$ within this unit. These data show an arithmetic mean of $9.96 \mathrm{E}-03 \mathrm{ft} /$ day for vertical permeability and 1.51E-02 ft/day for horizontal permeability and a geometric mean of $7.68 \mathrm{E}-05 \mathrm{ft} /$ day for vertical permeability and $2.68 \mathrm{E}-04 \mathrm{ft} /$ day for horizontal permeability. The standard deviation calculated from these data is $4.00 \mathrm{E}-02 \mathrm{ft} /$ day for vertical permeability and 4.83E-02 ft/day for horizontal permeability.

"Upper" Aquifer Zone. The "upper" aquifer zone (UAZ) of the UTRA includes all strata from the ground surface to the top of the TCCZ. The UAZ includes the "upland" unit, Tobacco Road Sand, and part of the Dry Branch Formation (Figure 2-1). Massive beds of sand and clayey sand with minor interbeds of clay characterize the UAZ. The sediment within the "upland" unit is commonly very dense and clayey and often contains gravely sand. 
The top of the UAZ is defined by the present-day topographic surface. The UAZ may be subdivided into four hydrostratigraphic intervals which are delineated by characteristic log signatures of tip, sleeve, and pore pressure data from the CPT tool. From the bottom up, these intervals include the "transmissive zone", "AA" interval, "A" interval, and an undifferentiated soils interval ("uu" interval) (Figure 2-1). Altitude-contour and isopach maps of these intervals are presented as Figures 2-15 through 2-21. Each interval bears distinctive CPT log signatures, which are indicative of their overall hydrogeology. These intervals have only been identified on a local basis prior to this report. This study presents the first attempt at correlating these intervals on a regional scale at the SRS.

The "transmissive zone" (TZ) corresponds with the interval of relatively clean sand that is correlative with the upper parts of the Dry Branch Formation (Figure 2-1). Logs of CPT data from this interval typically show very high tip and sleeve values with relatively low tip/sleeve ratios (Figure 2-22). The tip/sleeve ratios from the TZ normally produce very smooth patterns on CPT logs. This is probably indicative of the relatively massive sand layers that constitute this unit. The base of the TZ is commonly found at elevations between 170 and $190 \mathrm{ft} \mathrm{msl}$ and is picked where the relatively high friction ratio and pore pressure values of the TCCZ drop to values that are generally less than 2.0 , and the tip and sleeve values both demonstrate abrupt increases which are sustained with decreasing depth (Figure 2-22).

Figure 2-15 Presents an altitude contour map for the top of this unit. The map indicates that the TZ follows the regional dip toward the south-southeast. Local high and low areas on this surface generally correlate with highs and lows on the top of the TCCZ (Figure 2-13) The isopach map for the TZ is shown in Figure 2-16. The map indicates a prominent thick area in the eastern half of the central part of the study area. This area corresponds with an isolated low area on the top of the TCCZ (Figure 2-13). The thickness of the TZ varies locally, but generally increases in a southeast direction.

The base of the "AA" interval is delineated from CPT logs where relatively small and constant friction ratio values in the TZ increase abruptly, and the tip and sleeve values both show a significant decrease. The base of the "AA" interval is commonly found at elevations between 220 and 200 ( $\mathrm{ft} \mathrm{msl}$ ). Within the "AA" interval, logs of tip and sleeve readings indicate relatively low and generally consistent values which are accompanied by an erratic pattern of highly variable friction ratio readings (Figure 2-22). Logs of tip and sleeve readings from the "AA" interval generally have a "blocky" appearance when compared with tip and sleeve data from the TZ (Figure 2-22). The generally blocky pattern of the tip and sleeve curves and the highly variable and erratic friction ratio curve indicate the "AA" interval consists of a 
sequence of interbedded sand, and silty sand, probably of much lower permeability than the underlying TZ. The sediment contained within the " $A A$ " interval is generally correlative with the lower parts of the Tobacco Road Sand (Figure 2-1). A comparison of CPT logs .with geologic and geophysical data from the same location indicates that the base of the "AA" interval corresponds with the base of the Tobacco Road Sand.

Figure 2-17 Presents an altitude-contour map of the top of this unit. The contour patterns suggest two ridges and a trough that trend east-west across the area. The axis of the trough trends across the approximate center of the study area. The isopach map (Figure 2-18) suggests the ridges correspond with thicker parts of the unit, and the troughs with areas where the unit is thinner. The "grain" of the isopach contours in Figure 2-18 Follows the trends of the axes of the trough and ridges in Figure 2-17.

The " $\mathrm{A}$ " interval is correlative with the upper parts of the Tobacco Road Sand. The base of the " $\mathrm{A}$ " interval is delineated on CPT logs where the friction ratio curve changes abruptly from the highly variable values and erratic pattern of the "AA" interval to much smaller values with a "stable" curve pattern (Figure 2-22). This pattern is indicative of a more massively bedded unit with a permeability that is probably somewhat higher than that of the "AA" interval. The tip and sleeve values near the base of the "A" interval are often relatively large, and commonly show a gradual decrease up-section with a corresponding gradual increase in friction ratio values. These log-pattern changes correlate with the fining-upward sequence that is commonly observed in continuous core samples taken from the upper part of the Tobacco Road Sand. Figure 2-22 illustrates this relationship between the CPT log patterns and the lithology changes within this unit. Figures 2-19 and 2-20 present altitude-contour and isopach maps for this interval.

The uppermost UAZ consists of the fluvial sediments of the "upland" unit, recent alluvial material deposited by active stream reaches (outside of the Savannah River) and any local soil horizons which have formed in-situ from any of the lithostratigraphic units. For the purposes of this study, all of these have been grouped into a single "undifferentiated upper soils" ("uu") interval. Figure 2-1 illustrates the relative position of the "uu" interval with respect to the underlying units.

The "uu" interval can usually be identified on CPT logs where the sleeve stress values show a significant increase (up-section). This change accompanied by a drastic and abrupt increase in the friction ratio commonly to sustained values in excess of five (Figure 2-22). Friction ratio values within the "uu" interval generally exhibit an irregular pattern of drastic changes that 
often define groups, giving the curve a rough, "blocky" pattern (Figure 2-22). The top of the "uu" interval commonly only contains material that displays very low tip and sleeve values. These readings represent material that is interpreted to be the near-surface soil horizon. Because the "uu" interval includes the sediments assigned to the "upland" unit and surficial soil horizons, it typically appears as a "draping" over the top of the other units, when viewed in three dimensions or in cross-section (Figures 2-2 and 2-3). This is also suggested by the isopach contour patterns for this unit (Figure 2-21).

Laboratory tests of 17 undisturbed samples taken from the UAZ indicate vertical permeability ranges from 5.680E-06 to $2.77 \mathrm{E}-01 \mathrm{ft} /$ day (Table 2-1). Tests of 14 undisturbed samples yield horizontal permeability values that range from $3.12 \mathrm{E}-05$ to $6.04 \mathrm{E}+00 \mathrm{ft} /$ day within this unit. These data show an arithmetic mean of $1.90 \mathrm{E}+00 \mathrm{ft} /$ day for vertical permeability and $1.06 \mathrm{E}+00 \mathrm{ft} /$ day for horizontal permeability, and a geometric mean of $1.19 \mathrm{E}-02 \mathrm{ft} /$ day for vertical permeability and 4.02E-02 ft/day for horizontal permeability. The standard deviation calculated from these data is $6.67 \mathrm{E}+00 \mathrm{ft} /$ day for vertical permeability and $2.10 \mathrm{E}+00 \mathrm{ft} /$ day for horizontal permeability.

Results from 11 slug tests conducted within the UAZ give permeability values ranging from 6.32E-02 to $1.22 \mathrm{E}+01 \mathrm{ft} / \mathrm{day}$ (Table $2-1$ ). The arithmetic mean from these data is $2.09 \mathrm{E}+00$ $\mathrm{ft} /$ day and the geometric mean is $6.76 \mathrm{E}+00 \mathrm{ft} / \mathrm{day}$. The standard deviation calculated from these results is $3.62 \mathrm{E}+00 \mathrm{ft} /$ day.

Table 2-2 presents a summary of permeability measurements made on samples taken from locations where the informal intervals within the UAZ have been delineated.

\subsection{Hydrogeology}

\subsubsection{Water Table}

The water table aquifer is contained within the UTRA and includes all saturated material from the water table to the top of the GCU. The water table aquifer is commonly divided into the informal UAZ and LAZ, separated by the TCCZ. For this report, no distinction is made for the upper and lower zones because the majority of well data is from the upper zone as there are very few wells screened in the lower part of the water table aquifer within the model area at this time. A water table map of the reactor areas model domain shown in Figure 2-23.

The configuration of the water table is tightly controlled by the local topography and drainage system. Wells are scarce in the reactors area with the majority of the wells located around the 
reactor facilities. Therefore, for this project a study was conducted to characterize stream baseflow and supplement water table configuration along Indian Grave Branch and the upper part of Pen Branch within the model domain (Figure 2-24; Appendix E), (Hiergesell, 1998b,c). Water level measurements were obtained from selected wells along with careful examination of flowing reaches of the headwater segments of the streams. The water table map (Figure 2-23) was further refined with this data.

In addition to the regional water table map for the area, Figures 2-25 through 2-28 illustrate the water table configuration in $\mathrm{C}, \mathrm{K}, \mathrm{L}$, and $\mathrm{P}$ reactor areas. For further discussion of the water table in the reactor areas the reader is referred to Hiergesell (1988a).

\subsubsection{Gordon Aquifer Potentiometric Surface}

The Gordon aquifer is the lowermost aquifer addressed in this study and represents the basal unit of the Floridan aquifer system in the CKLP GWMA (Figure 2-1). Figure 2-29 illustrates the potentiometric surface of the Gordon aquifer in the model domain. Groundwater data from this unit are somewhat limited in the CKLP GWMA. The surface shown in Figure 2-29 incorporates mean water levels from wells screened within the Gordon aquifer and surface elevations from Upper Three Runs and Hollow Creek in areas where the Gordon aquifer meets the land surface (Hiergesell, 1999). The Gordon aquifer discharges to the Upper Three Runs valley to the north-northwest and to the Savannah River valley to the west-southwest.

\subsubsection{Hydraulic Head Targets}

In addition to construction of potentiometric maps for conceptual understanding of groundwater flow and boundary condition specification (e.g. Figures 2-23 and 2-29), hydraulic head data are valuable model calibration targets. Because steady-state groundwater flow is the focus of this effort, long-term, time-averaged head data are of the greatest interest as targets for model calibration. The primary source of uncertainty in mean water level is the transient fluctuation in individual readings that are on the order of a few feet. Errors in surveys, water-level readings, and other similar measurements are generally very small by comparison.

Water level data for most wells at the SRS are available from the Geochemical Information Management System (GIMS), which can be accessed through the Savannah River Information Network Environment (ShRINE). The data are also published in periodic well inventory and monitoring reports; see Environmental Protection Department and Exploration Resources, Inc. (1996a, b) for example. GIMS archives data obtained through a groundwater monitoring 
program administered by the Environmental Monitoring Section (EMS) of the Environmental Protection Department (EPD). The GIMS database is known to contain erroneous entries. Outliers were identified as single readings that deviated from the average value by more than $20 \mathrm{ft}$ and eliminated. With the remaining data, the sample standard deviation of the mean value was computed as (Walpole and Myers, 1978, section 5.5)

$$
s_{m}=\frac{s}{\sqrt{n}}=\frac{1}{\sqrt{n}} \times\left[\frac{1}{n-1} \sum_{i=1}^{n}\left(h_{i}-\bar{h}\right)^{2}\right]^{1 / 2}
$$

Mean values with an uncertainty exceeding $3 \mathrm{ft}$ at $95 \%$ confidence $\left(2 \mathrm{~s}_{\mathrm{m}}>3 \mathrm{ft}\right)$ were eliminated, with the idea that uncertainty in a hydraulic head target should not exceed the calibration goal. Previous models covering relatively small areas of the SRS have generally achieved a root-mean-square residual of $3 \mathrm{ft}$ (e.g. Camp Dresser \& McKee, 1989; GeoTrans, 1992; Flach and Harris, 1997). Given the large scale and coarse resolution anticipated for CKLP model, a calibration goal of $3 \mathrm{ft}$ may be too low. Sample standard deviations could not be computed for wells with a single reading, and the single reading was accepted the target for steady-state flow calibration.

Valuable data from wells not included in the EMS monitoring program are also available. The Environmental Science and Technology Department (ES\&TD) has monitored the P-series wells and other SRS wells for several years (Hiergesell, 1998). Water level data are also available from Environmental Restoration Department (ERD) docúments, such as the RFI/RI/BRA for the CMP Pits (WSRC, 1996). In 1998, piezometer clusters were installed at 17 locations across the reactors area for the purpose of defining water levels in remote areas (Figure 2-30) (WSRC, 1999). The two-piezometer clusters targeted the "upper" and "lower" UTRA. These data supplement the head targets derived from the GIMS database.

Appendix F contains the resulting list of hydraulic head targets. Each well was assigned to the appropriate hydrostratigraphic unit, as defined by the picks and grids presented in Section 2.2. The results are summarized in Table 2-3. Wells assigned to the Gordon aquifer are screened completely within the Gordon aquifer. Wells and piezometers above the Gordon confining unit are assigned to an aquifer zone if at least half the screen lies within that zone. Otherwise, the water level is ignored as model calibration target. There are a total of 1137 targets within the model domain. 


\subsection{Groundwater Recharge and Discharge}

Groundwater flow in upper aquifers at the Savannah River Site is driven by recharge, with streams intercepting flow from areas of higher groundwater elevations (Figures 2-23 and 2-29). Nearly all recharge within the CKLP model area discharges to streams within or bounding the same area, usually the nearest stream. For this type of groundwater flow system, recharge and discharge estimates, coupled with head measurements and confining unit leakance estimates, define the overall horizontal conductivity values of upper aquifers required to calibrate a numerical flow model. Because conductivity data at the model scale are typically non-existent, groundwater flow estimates are important model calibration targets.

At least three independent investigations of surface groundwater recharge have been performed in or near the SRS. Parizek and Root (1986) conducted a detailed hydrologic budget study of the McQueen Branch basin. They estimated average recharge for the basin at $15.6 \mathrm{in} / \mathrm{yr}$. Parizek and Root (1986) computed this value by dividing the total volumetric rate of recharge by the total basin area. The average recharge rate excluding seepage/wetland areas would therefore be somewhat larger. Hubbard $(1984,1986)$ conducted a multi-year lysimeter study at the SRS burial grounds in the General Separations Area and measured an average recharge of about $16 \mathrm{in} / \mathrm{yr}$ for grass cover. Based on lysimeters with small pine trees growing within them, Hubbard (1986) estimated recharge to be $6 \mathrm{in} / \mathrm{yr}$ for forested areas. Hubbard (1986) also reported that Denehy and McMahon (1985) measured $15 \mathrm{in} / \mathrm{yr}$ of recharge at the Chem-Nuclear site in Barnwell, South Carolina. Parizek and Root (1986) and Looney and others (1987) report that Cahill (1982) estimated recharge to be about $15 \mathrm{in} / \mathrm{yr}$ at the Low Level Radioactive Solid Waste Burial Site near Barnwell, South Carolina (ChemNuclear). It is unclear from the literature cited here whether the Denehy and McMahon (1985), and Cahill (1982) studies are related, apart from being conducted at the same location.

From these studies, the average recharge over the Savannah River Site is estimated to be about $15 \mathrm{in} / \mathrm{yr}$. The average rate excluding groundwater discharge areas would be somewhat higher. This estimate may be high due to a bias toward analysis of developed areas that tend to be less forested and flatter. The data for forested conditions are difficult to reconcile. Hubbard (1985) estimated recharge at $6 \mathrm{in} / \mathrm{yr}$ for forested areas. On the other hand, the vegetation of McQueen Branch basin studied by Parizek and Root (1986) study was 85\% evergreen and deciduous forest, and produced an estimate of nearly $16 \mathrm{in} / \mathrm{yr}$. The average of these two estimates is $10 \mathrm{in} / \mathrm{yr}$. Considering that the area of interest in this study is relatively undeveloped and heavily forested, perhaps a reasonable range to consider for groundwater flow modeling sensitivity studies is 10 to $16 \mathrm{in} / \mathrm{yr}$. 
To support this and subsequent modeling efforts in the $\mathrm{C}, \mathrm{K}, \mathrm{L}$ and $\mathrm{P}$ areas, stream base flow was estimated by analyzing U. S. Geological Survey (USGS) stream gauging station data (Cooney and others, 1998, for example) and measuring stream flow rate under low flow conditions (Hiergesell, 1998b, 1998c). The USGS data provide large-scale estimates of base flow. Complementing these estimates, Hiergesell $(1998 \mathrm{~b}, 1998 \mathrm{c})$ measured base flow for small streams. Appendix E-1 describes the simple hydrograph separation techniques that were used to estimate the long-term average rate of groundwater discharge to large-scale stream reaches within the CKLP reactor area. Appendix E-2 presents the data of Hiergesell (1998). Selected results from these studies are summarized in Tables 2-4 and 2-5. Table 2-5 emphasizes the larger stream flows around $\mathrm{C}$-area and $\mathrm{K}$-area because these reactors areas are currently of most interest. Hiergesell $(1998 \mathrm{~b}, 1998 \mathrm{c})$ also estimated the point of effluence along small streams and refined an ARC/INFO USGS coverage of live stream reaches, as illustrated by Figure 2-31.

The Pen Branch and Fourmile Branch base flow estimates are the most reliable calibration targets. For Meyers Branch and Upper Three Runs, there is added uncertainty in the fraction of base flow that can be attributed to the modeled area. The Steel Creek and Upper Three Runs estimates are based on the difference of large numbers compared to the baseflow estimate. Base flow estimates for these reaches have higher uncertainty as well. The more reliable base flow calibration targets may have an uncertainty of 15 to 25\% (Appendix E). The Steel Creek base flow estimate is negative and indicates a losing reach, perhaps reflecting artificial flow to L-Lake to maintain a historic level of $190 \mathrm{ft}$ msl. The Indian Grave Branch and Pen Branch field measurements in Table 2-5 are not consistent with the baseflow estimate for all of Pen Branch in Table 2-4. The measured flow in Pen Branch below its confluence with Indian Grave Branch ( $15.8 \mathrm{cfs}$ ) exceeds the estimated base flow for the entire stream $(13,3 \mathrm{cfs})$. The discrepancy is likely due to uncertainties and measurement errors in both analyses, or that base flow conditions were not present on December 18, 1997. 


\subsection{Conceptual Model of Groundwater Flow}

From Figure 2-23, groundwater flow in the Upper Three Runs aquifer is seen to be driven by recharge, with nearby streams intercepting flow from higher elevations. The underlying Gordon aquifer is strongly influenced by the Savannah River and Upper Three Runs, which appear to completely drain the aquifer and function as no-flow lines (Figure 2-29). Except for reactor area outfalls and the lower portion of $\mathrm{L}$ Lake, surface water bodies gain from groundwater discharge. Aadland and others (1995, Plate 17) gives the leakance of the Crouch Branch confining unit (of the Meyers Branch confining system) as roughly $3 \times 10^{-6}$ day $^{-1}$, which corresponds to $0.13 \mathrm{in} / \mathrm{yr}$ for every $10 \mathrm{ft}$ of head difference. The head difference across the Crouch Branch confining unit is centered near zero (Aadland and others, 1995, Figure 30). Flow across the unit is therefore a small fraction of total recharge, and could probably be neglected. A representative leakance coefficient for the Gordon confining unit in the study area appears to be roughly $10^{-5}$ day $^{-1}$ (Aadland and others, 1995, Plate 13). The head difference across the Gordon confining is highly variable due to large variation in the water table. Supposing a head difference of $50 \mathrm{ft}$ for example, the Darcy velocity through the unit would be $2.2 \mathrm{in} / \mathrm{yr}$ or $15 \%$ of surface recharge. Therefore, groundwater flow in the Gordon aquifer appears to be influenced significantly by recharge from the overlying UTR aquifer, and lateral flow into the model domain, mainly from the east. L-Lake and Par Pond are major lakes that have an important influence on nearby groundwater flow. The Site Utilities Department well database on ShRINE indicates that no more than three producing wells are screened in the Gordon aquifer (905-136G, 905-126G, and 905-103G). These wells serve small facilities and have a maximum capacity of $25 \mathrm{gpm}$ or less. Considering that actual usage would be much lower, the impact of these wells is insignificant at the regional scale. The impact of the Pen Branch fault on confining unit leakance is uncertain.

Solute groundwater contamination originating in the $\mathrm{C}, \mathrm{K}, \mathrm{L}$ or $\mathrm{P}$ areas is expected to be confined to the Upper Three Runs and Gordon aquifers. Most surface recharge discharges to the nearest stream, with the balance entering the Gordon aquifer. As groundwater in the Gordon aquifer flows toward the Savannah River or Upper Three Runs, the gradient between the Crouch Branch and Gordon aquifers becomes upward ensuring ultimate discharge to the Savannah River or Upper Three Runs. Contamination is not expected to enter the Crouch Branch aquifer. 


\subsection{Hydrologic Properties}

In addition to the unit-specific hydraulic conductivity data discussed above, soil characteristic curves, effective porosity, and specific storage data are needed for model development. The steady-state hydraulic head and Darcy velocity fields in the saturated zone are affected only by horizontal and vertical hydraulic conductivity, making these remaining properties less critical to model development. Soil characteristic curves (capillary suction and relative permeability as a function of water saturation) affect the flow solution in unsaturated regions. Effective porosity affects groundwater "particle" tracing results, which rely on the pore velocity field. Specific storage affects transient flow only, and then only in confined aquifer systems for practical purposes. Characterization data available for defining these hydraulic properties in the model are identified below. Given the general scarcity and uncertainty in the data, generic estimates to be applied model-wide are appropriate.

\subsubsection{Soil Characteristic Curves}

Relative permeability and capillary suction head as a function of water saturation are referred to as soil characteristic curves. These relationships are difficult to measure accurately, and testing is expensive. Very little data are available for SRS unconsolidated sediments. O'Brien \& Gere (1991) obtained a small set of water retention (capillary suction versus saturation) data for M-Area sediment samples. The data have been plotted by Flach and others (1996, Figures 11 and 12). Yu and others (1993) obtained both relative permeability and water retention data for remolded GSA sediments to be used for Environmental Restoration construction projects. Recently, Amidon (1996) obtained water retention data from 3 undisturbed soil samples collected from the vadose zone around the Burial Grounds Complex. According to Looney and others (1987), Gruber $(1981,1983)$ and Parizek and Root (1986) measured soil water content in the vadose zone and suggested the average water content is approximately $30 \%$ (water volume/total volume). Given the scarcity of the data and lacking a specific need for accurate vadose zone modeling in a regional scale model, a simplified approach for defining soil characteristic curves is taken as shown in Figure 2-32. The curves are chosen to align with data for sandy sediments as opposed to clayey sediments (see Flach and others (1996), Figures 11 and 12). The relative permeability at residual saturation is set to 0.1 instead of zero to avoid slow convergence. These "pseudo-soil" characteristic curves are adequate for transporting water and contaminants through the vadose zone to the water table, provided detailed, accurate information about the unsaturated zone is not needed. The most important aspect of these curves is the assumed residual saturation value (40\%), which 
has the strongest effect on average vadose zone saturation. Groundwater travel times through the vadose zone are affected by saturation through pore velocity.

\subsubsection{Effective (Kinematic) Porosity}

Aadland and others (1995, p. 44) analyzed laboratory data from 83 selected sediment samples taken from various low permeability beds within the Upper Three Runs aquifer. For 28 "clayey to very clayey, often silty, sand" samples the total porosity averaged $40 \%$. For 55 "sandy, often silty clay, and clay" samples, the average total porosity is $41 \%$. Aadland and others (1995, Table 3) also calculated the total porosity of the sandy portions of the Upper Three Runs aquifer using the Beard and Weyl (1973) method, and arrived at an average total porosity of 35\%. For the Gordon aquifer, the result is 34\% (Aadland and others, 1995, Table

7). More recently, Smits and others (1997) compiled a database of porosity measurements for the General Separations Area. The arithmetic average of these values, mostly from low permeability samples, is $45 \%$. From these data and analyses, total porosity in aquifer zones appears to average about $40 \%$.

An "effective" porosity value, smaller than the total porosity, is commonly used for transport simulations and particle tracing related to contaminant migration. As discussed by De Marsily (1986, Chapter 2), two types of porosity are commonly and unfortunately referred to as "effective porosity". The first is specific yield or drainage porosity of an unsaturated soil, $\omega_{\mathrm{d}}$, and the second is kinematic porosity of a saturated medium, $\omega_{\mathrm{c}}$. Section 2.3.3 of De Marsily (1986) summarizes which porosity (total included) to use for which application. For saturated-zone particle tracing and transport simulations, the kinematic porosity is appropriate and the focus of effective porosity discussions in this report.

An effective porosity can be used to account for regions of relatively immobile water, ranging from grain-sized "dead-end" pores to macro-scale clay intervals, which do not effectively participate in contaminant transport. The presence of immobile water does not necessarily dictate the use of an effective porosity (De Marsily, 1986, p. 259). If the solute contaminant perfectly penetrates the immobile water $\left(K^{\prime}=1, C^{\prime}=C\right.$ in De Marsily (1986)) (or there is no immobile water), then total porosity is appropriate $(\omega)$. On the other hand, if a model block contains sub-regions of immobile water that a solute will not penetrate $\left(K^{\prime}=0, C^{\prime}=0\right)$, then a lower, "effective" porosity is appropriate (kinematic, $\omega_{\mathcal{C}}$ ).

Effective porosity can be estimated by assuming that only the largest scale regions of relatively immobile water are not effectively penetrated by contaminant. At smaller scales, contaminant 
is able to effectively diffuse into regions of immobile water. Macro-scale regions of immobile water can reasonably be defined as sediment intervals with more than $25 \%$ mud. For the General Separations Area, 32\% of the nearly $40,000 \mathrm{ft}$ of sediment core contains greater than $25 \%$ mud, based on analysis of the lithologic data compiled by Smits and others (1997). This suggests that as low as $68 \%$ of a typical aquifer is effectively available for contaminant transport, and that effective porosity is approximately $25 \%$ (68\% of $40 \%$ total porosity). This estimate may be a conservative (low) estimate for effective conductivity, because in reality some contamination would penetrate the lower conductivity intervals. This value is consistent with the recommendations of Looney and others (1987, p. 39), who recommend assuming an effective porosity of 0.2 for risk calculations. Transport sensitivity studies should consider an effective porosity range of approximately $20 \%$ to $40 \%$.

\subsubsection{Specific Storage}

Specific storage is relevant only to transient flow simulations, and therefore has no effect on the steady-state results presented in later sections. Specific storage is defined by (Freeze and Cherry, 1979, p. 59)

$$
S_{s}=\rho g(\alpha+\eta \beta)
$$

where

$\mathrm{S}_{\mathrm{S}} \quad$ specific storage

$\rho$ density of water $\left(\sim 1000 \mathrm{~kg} / \mathrm{m}^{3}\right)$

g gravitational acceleration $\left(9.8 \mathrm{~m} / \mathrm{s}^{2}\right)$

$\alpha \quad$ compressibility of porous medium

$\eta \quad$ total porosity

$\beta \quad$ compressibility of water $\left(4.4 \times 10^{-10} \mathrm{~m}^{2} / \mathrm{N}\right)$.

Compressibility ranges from $10^{-6}$ to $10^{-8} \mathrm{~m}^{2} / \mathrm{N}$ for clay and from $10^{-7}$ to $10^{-9} \mathrm{~m}^{2} / \mathrm{N}$ for sand (Freeze and Cherry, 1979, Table 2.5). Assuming a nominal compressibility value of $5 \times 10^{-8} \mathrm{~m}^{2} / \mathrm{N}$ and a total porosity of $40 \%$ yields $1.5 \times 10^{-4} \mathrm{ft}^{-1}$ for specific storage. 


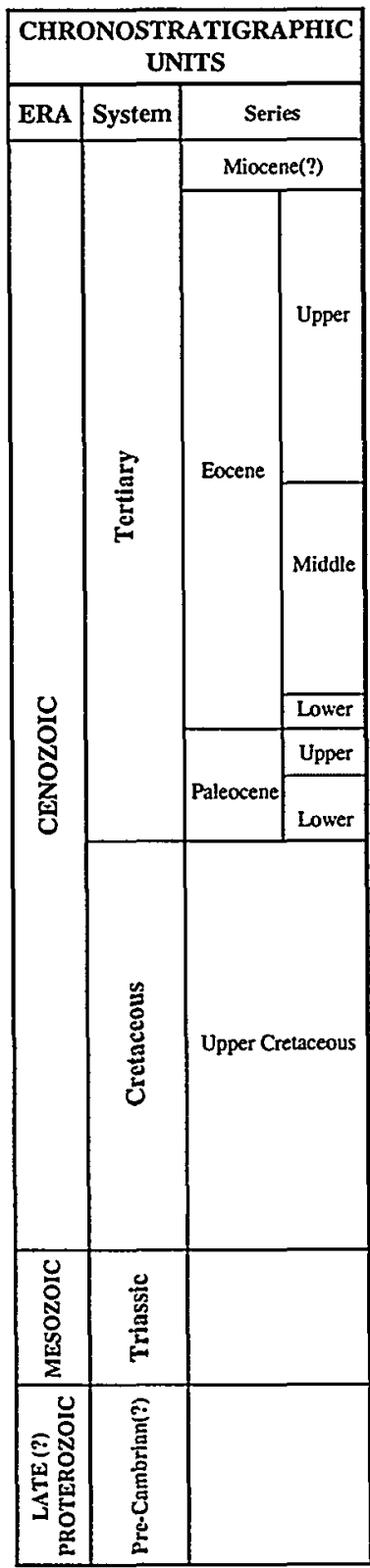

\begin{tabular}{|c|c|c|}
\hline \multicolumn{3}{|c|}{$\begin{array}{l}\text { LITHOSTRATIGRAPHIC UNITS } \\
\text { (Modified from Fallaw and Price, 1995) }\end{array}$} \\
\hline Group & \multicolumn{2}{|c|}{ Formation } \\
\hline & \multicolumn{2}{|c|}{ "upland" unit } \\
\hline \multirow{5}{*}{$\begin{array}{l}\text { Barnwell } \\
\text { Group }\end{array}$} & \multicolumn{2}{|c|}{ Tobacco Road Sand } \\
\hline & \multirow{3}{*}{$\begin{array}{l}\text { Dry Branch } \\
\text { Formation }\end{array}$} & Twiggs Clay Mbr. \\
\hline & & Grinins Landing Mbr. \\
\hline & & Irwinton Sand Mbr. \\
\hline & \multicolumn{2}{|c|}{$\begin{array}{l}\text { Clinchfield } \\
\text { Formation }\end{array}$} \\
\hline \multirow{3}{*}{$\begin{array}{l}\text { Orangeburg } \\
\text { Group }\end{array}$} & \multicolumn{2}{|c|}{ Santee } \\
\hline & \multicolumn{2}{|c|}{ Warley Hill Formation } \\
\hline & \multicolumn{2}{|c|}{ Congaree Formation } \\
\hline \multirow{5}{*}{$\begin{array}{l}\text { Black } \\
\text { Mingo } \\
\text { Group }\end{array}$} & \multicolumn{2}{|c|}{ Fourmile Branch Formation } \\
\hline & \multicolumn{2}{|c|}{ Snapp Formation } \\
\hline & \multicolumn{2}{|c|}{ Lang Syne Formation } \\
\hline & \multicolumn{2}{|c|}{ Sawdust Landing Formation } \\
\hline & \multicolumn{2}{|c|}{ Steel Creek Formation } \\
\hline \multicolumn{3}{|l|}{ Black Creek } \\
\hline & \multicolumn{2}{|c|}{ Middendorf Formation } \\
\hline & \multicolumn{2}{|c|}{ Cape Fear Formation } \\
\hline $\begin{array}{c}\text { Newark } \\
\text { Supergroup }\end{array}$ & \multicolumn{2}{|c|}{ Sedimentary Rock (Dunbarton Basin) } \\
\hline & \multicolumn{2}{|c|}{ Crystalline Basement Rock } \\
\hline & & \\
\hline
\end{tabular}

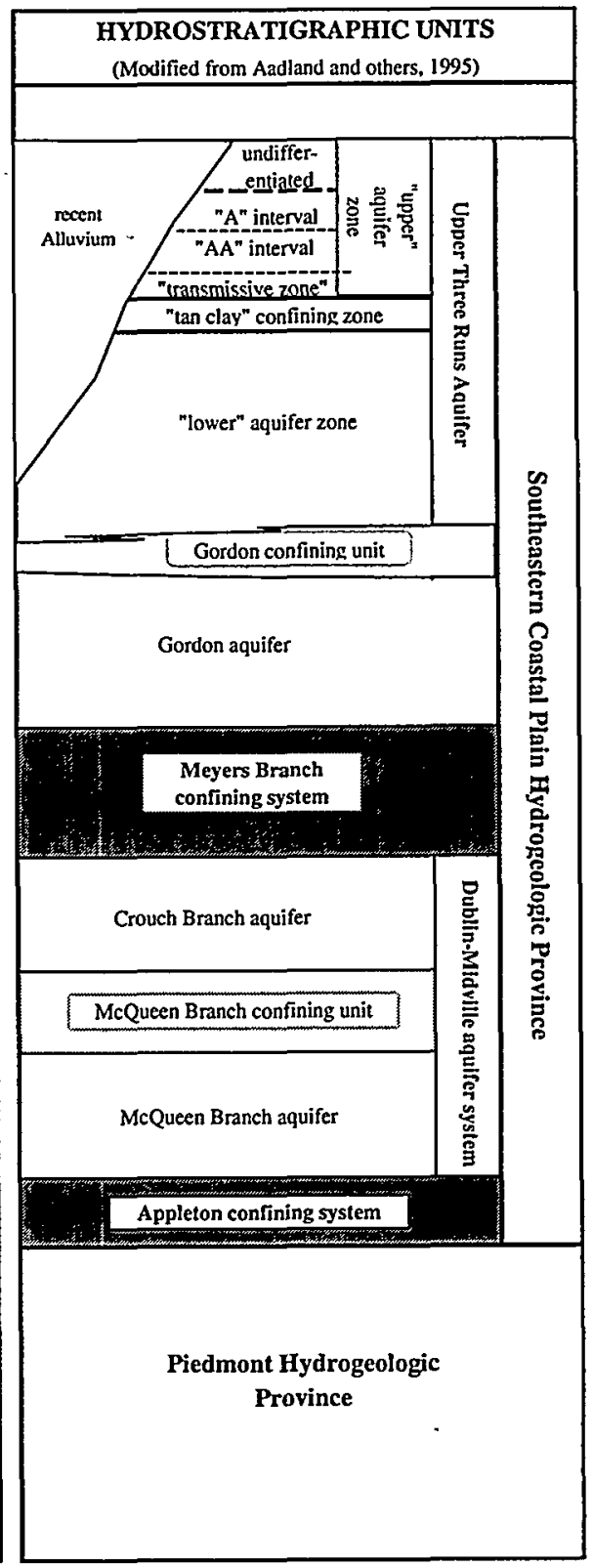

Figure 2-1. Comparison of Lithostratigraphic and Hydrostratigraphic Units at SRS 


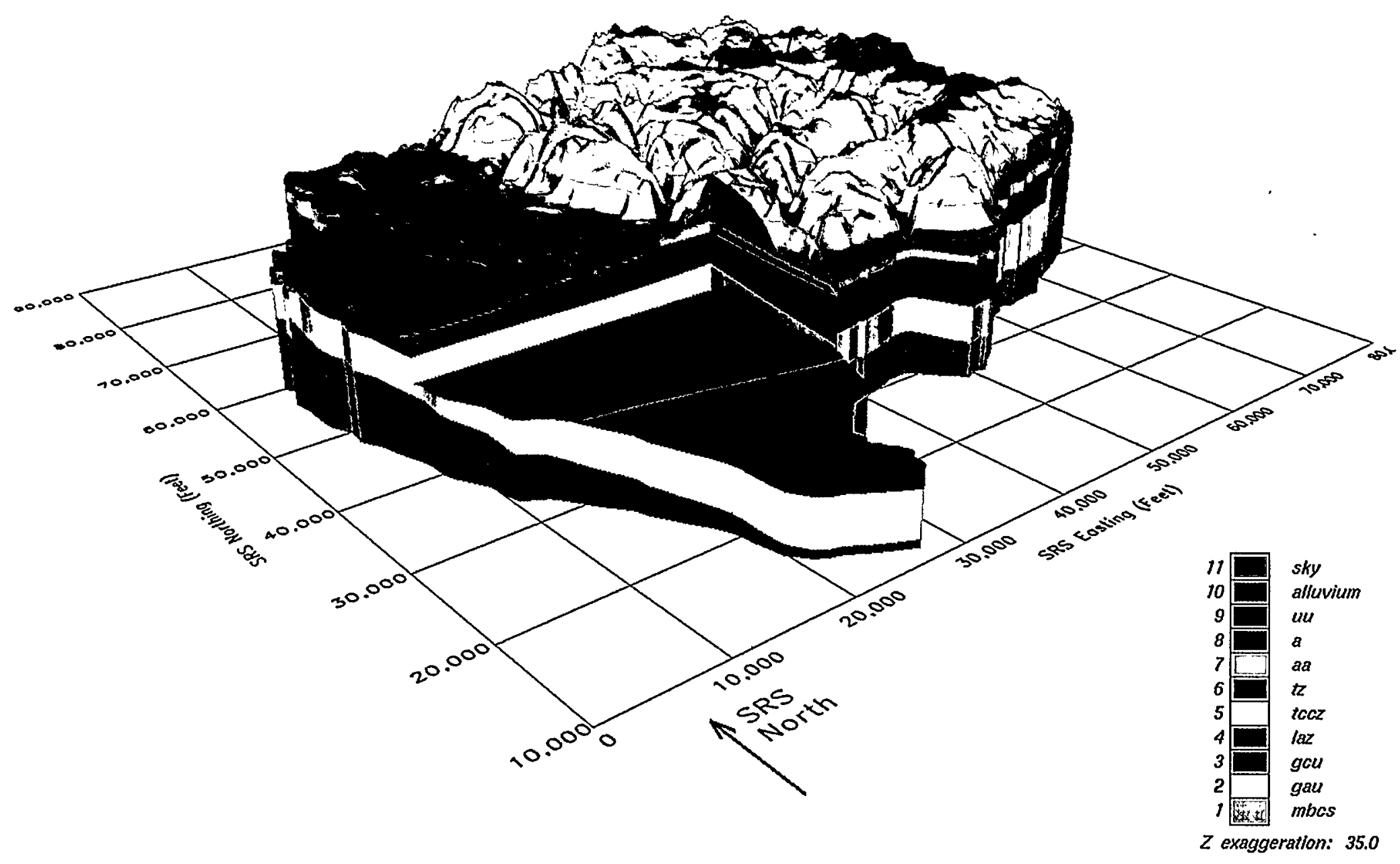

Figure 2-2. Conceptual Hydrostratigraphic Model 


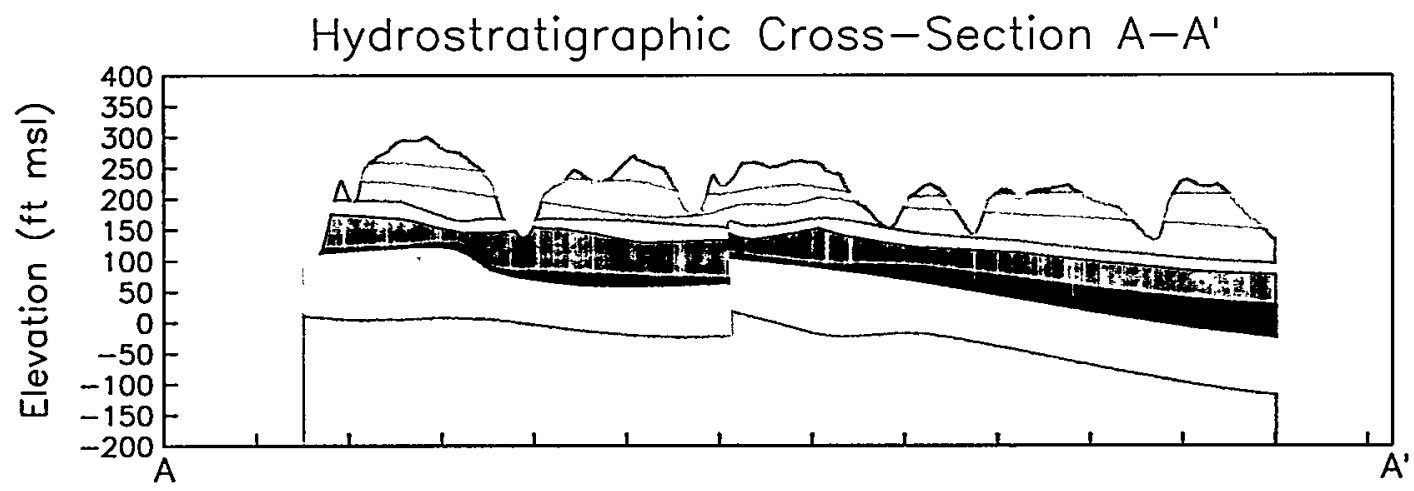

Hydrostratigraphic Cross-Section B-B'

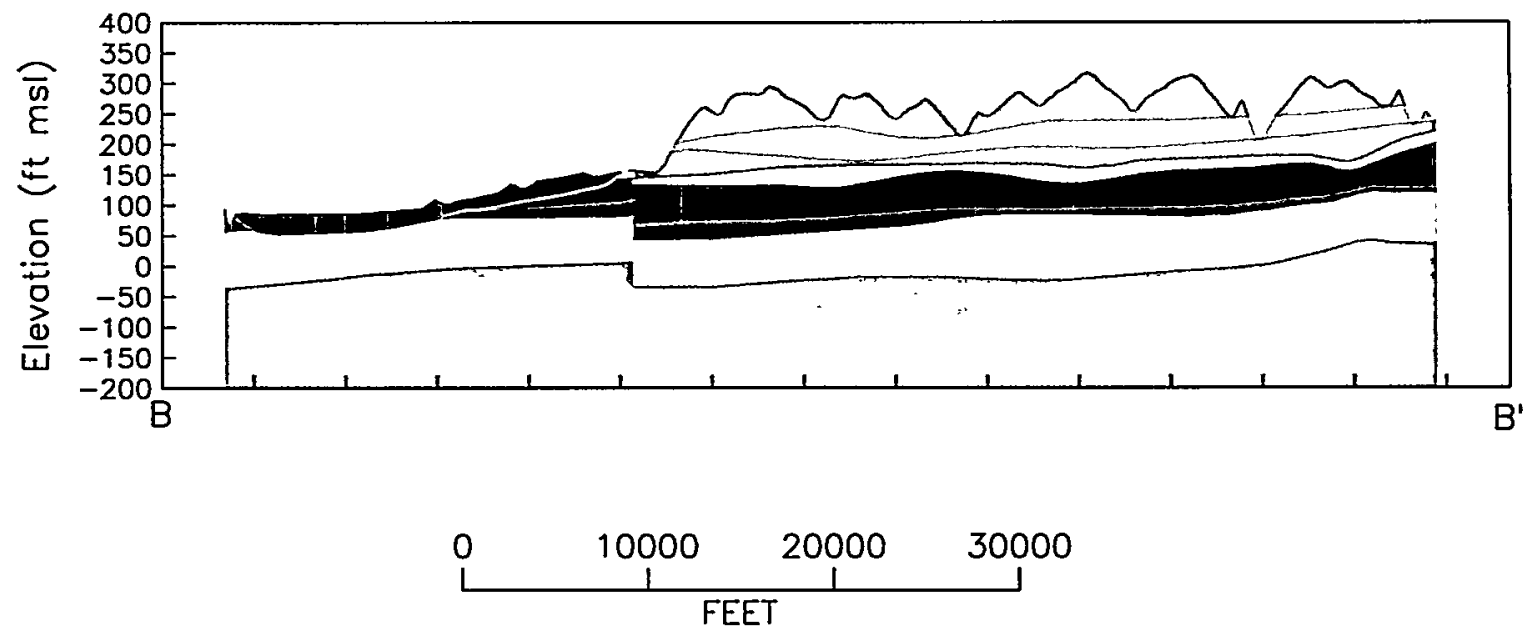

Vertical exaggeration $=25$

Location Map

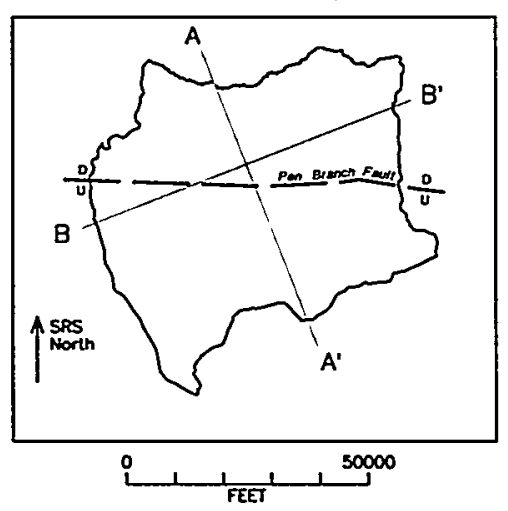

LEGEND

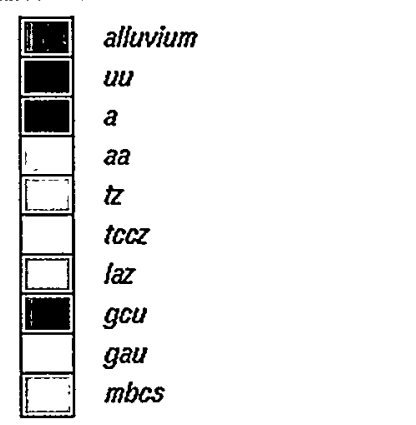

Figure 2-3. Hydrostratigraphic Cross-Sections 
Base of Savannah River Alluvial Deposits

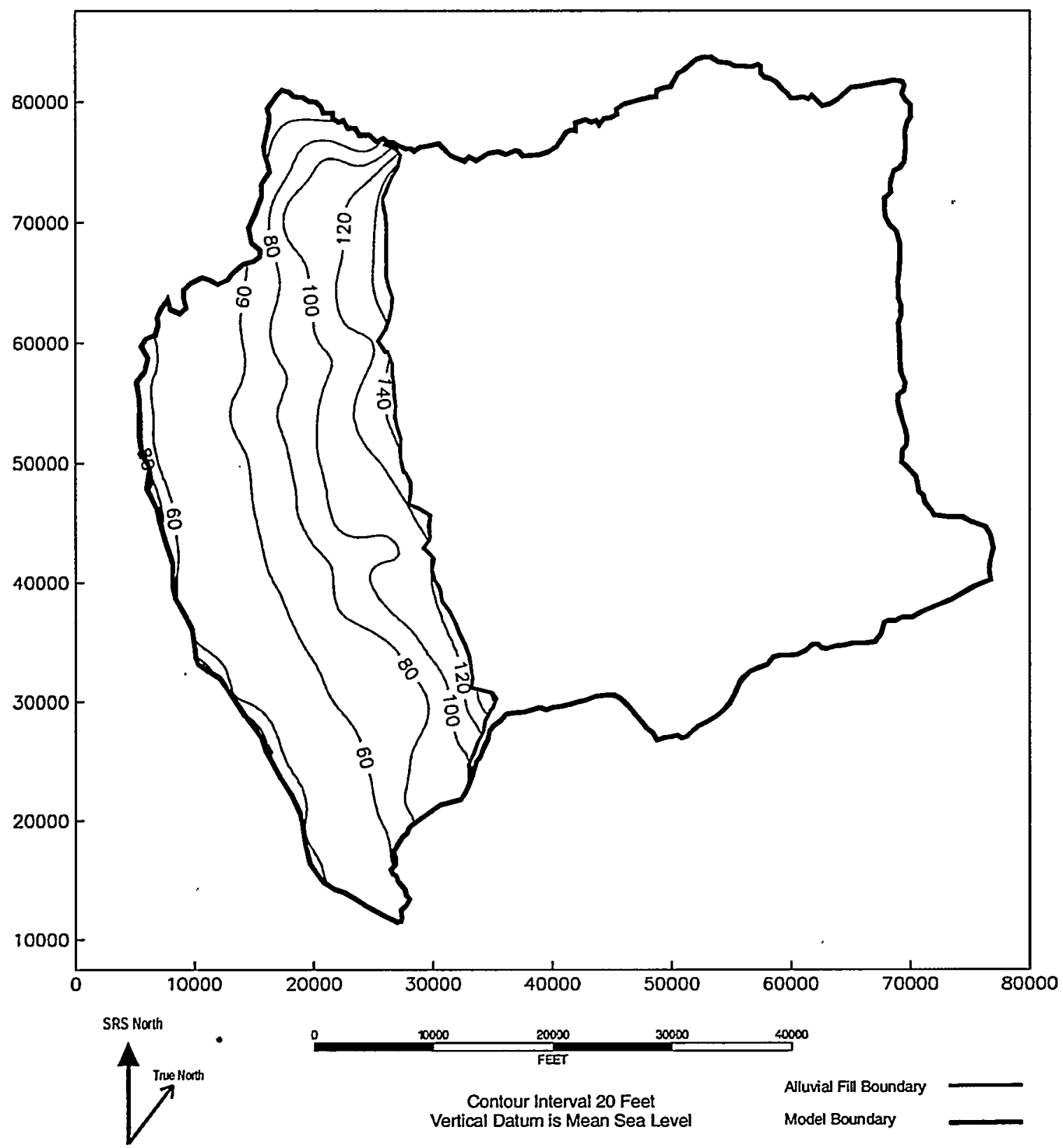

Figure 2-4. Base of Savannah River Alluvial Deposits 
Isopach of the Savannah River Valley Alluvial Fill

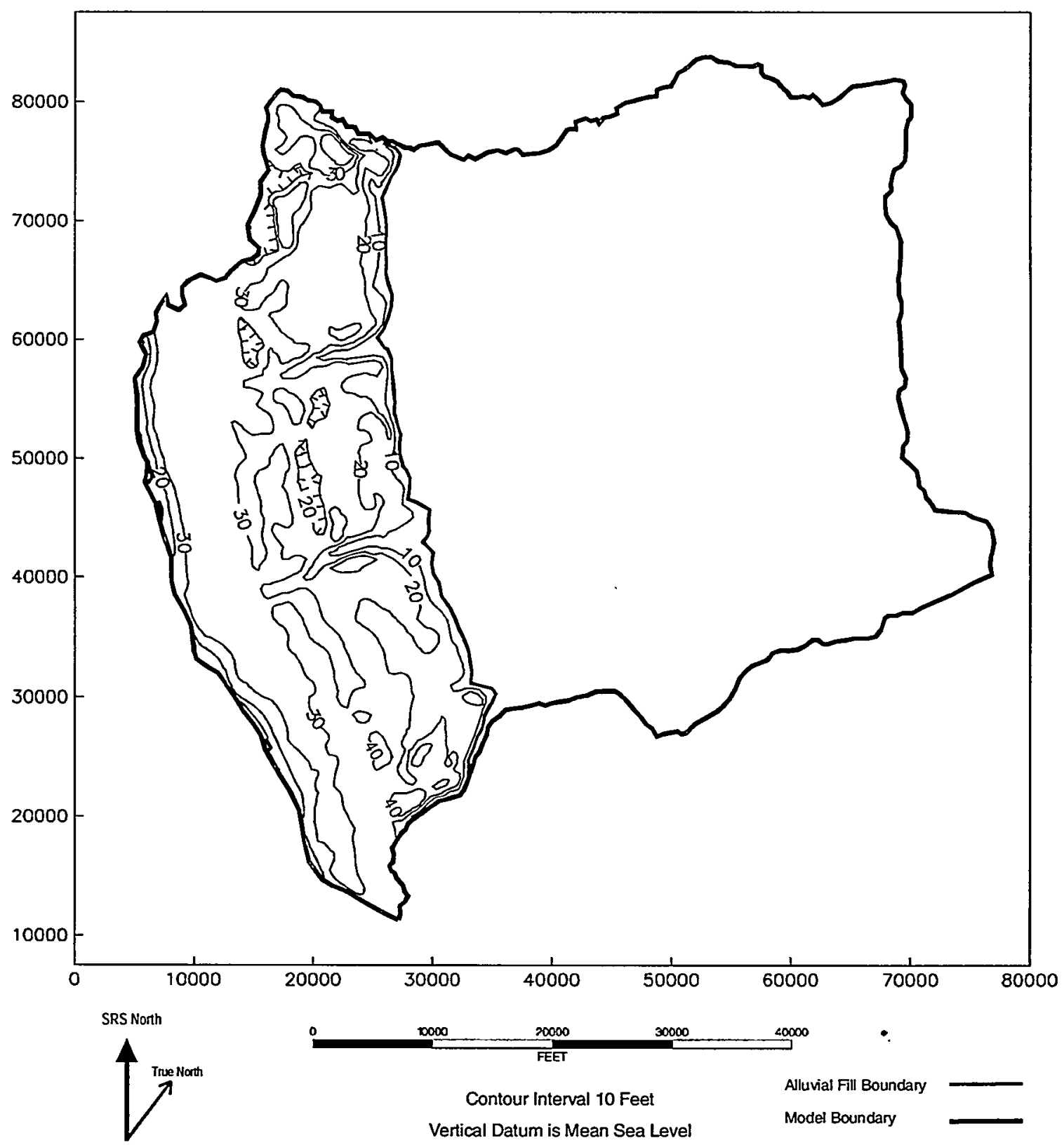

Figure 2-5. Isopach of the Savannah River Valley Alluvial Fill 
Top of the Meyers Branch Confining System

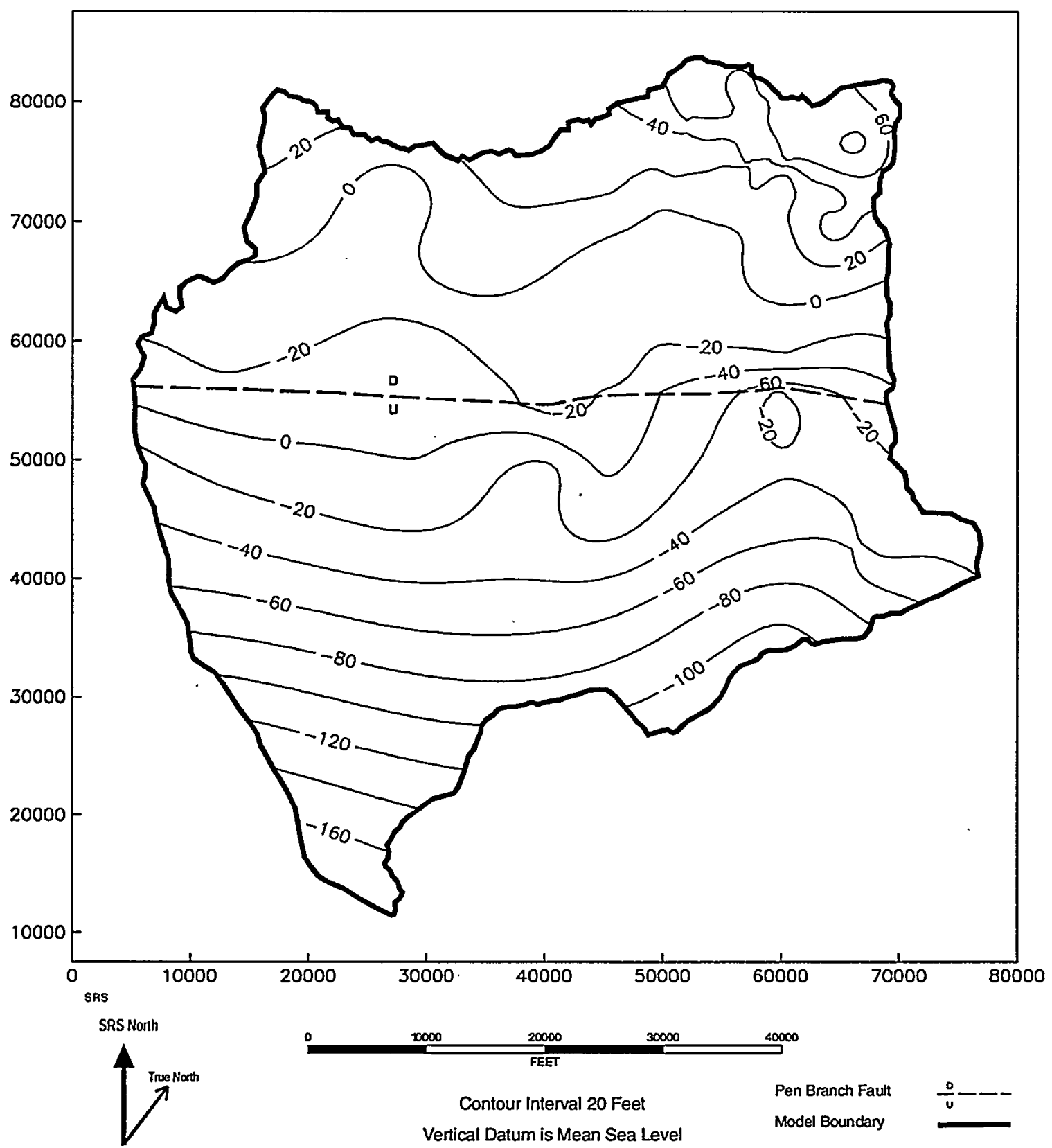

Figure 2-6. Altitude-Contour Map of the Top of the Meyers Branch Confining System 
Top of the Gordon Aquifer

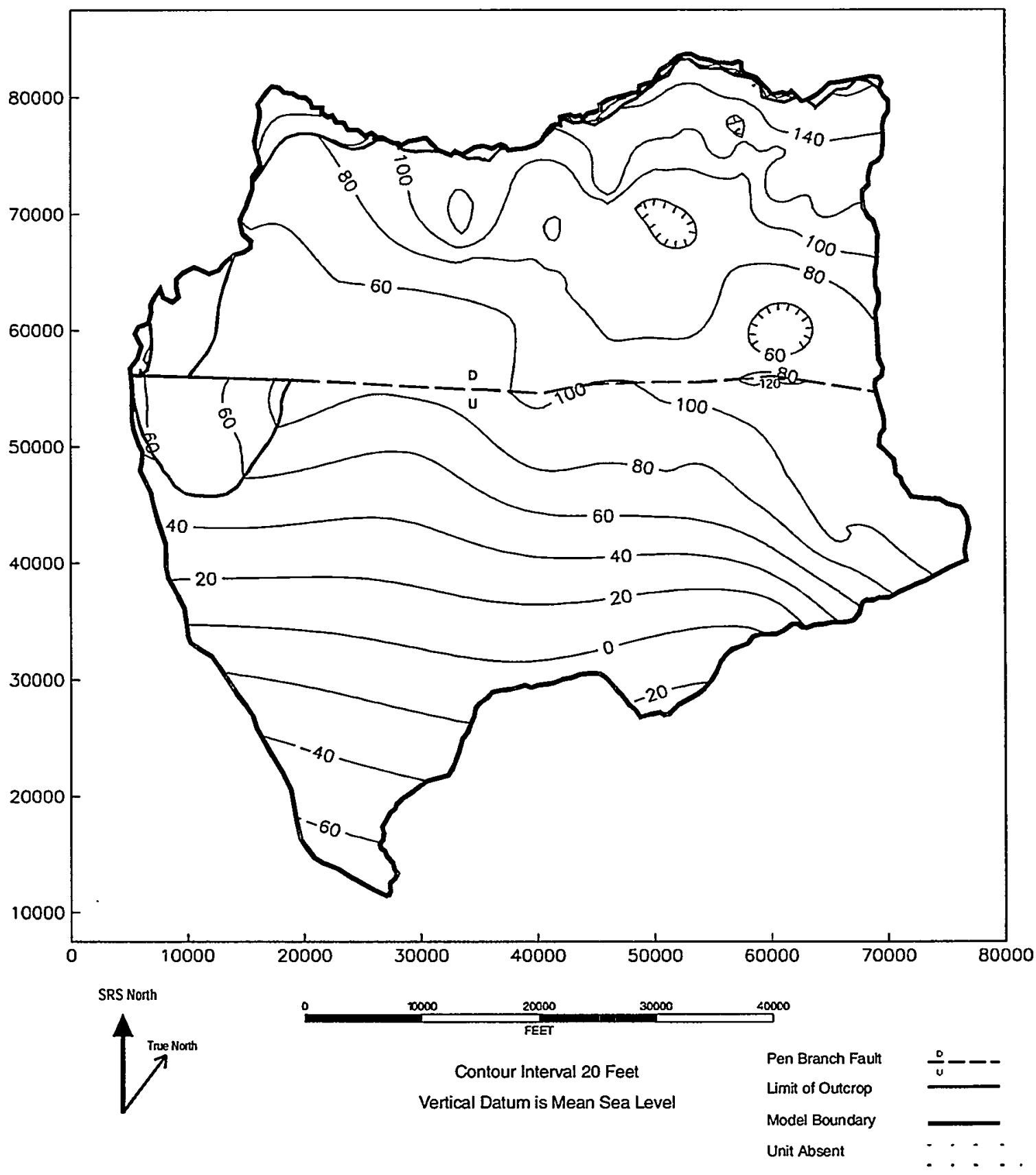

Figure 2-7. Altitude-Contour Map of the Top of the Gordon Aquifer 


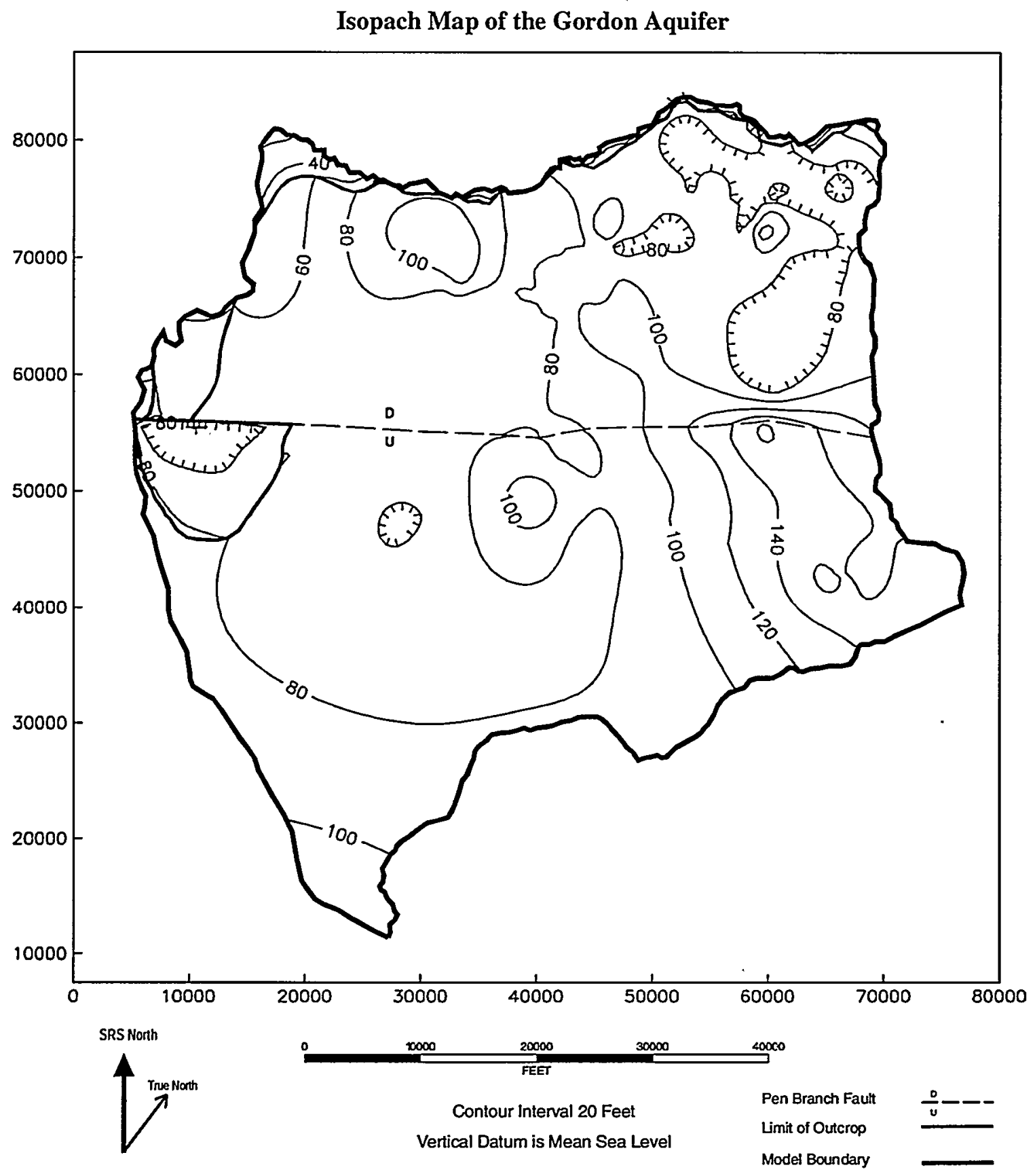

Figure 2-8. Isopach Map of the Gordon Aquifer 
Top of the Gordon Confining Unit

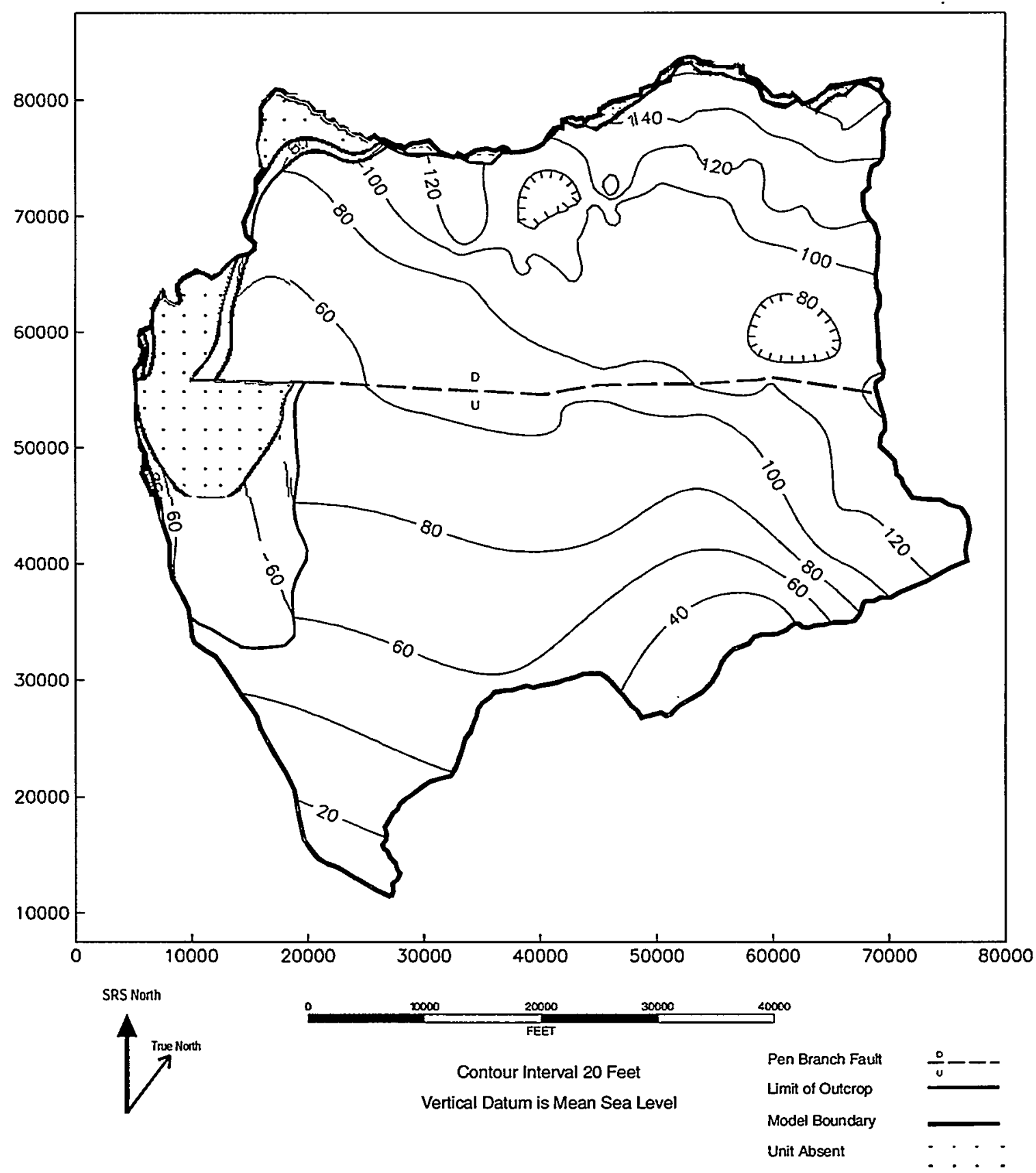

Figure 2-9. Altitude-Contour Map of the Top of the Gordon Confining Unit 


\section{Isopach Map of the Gordon Confining Unit}

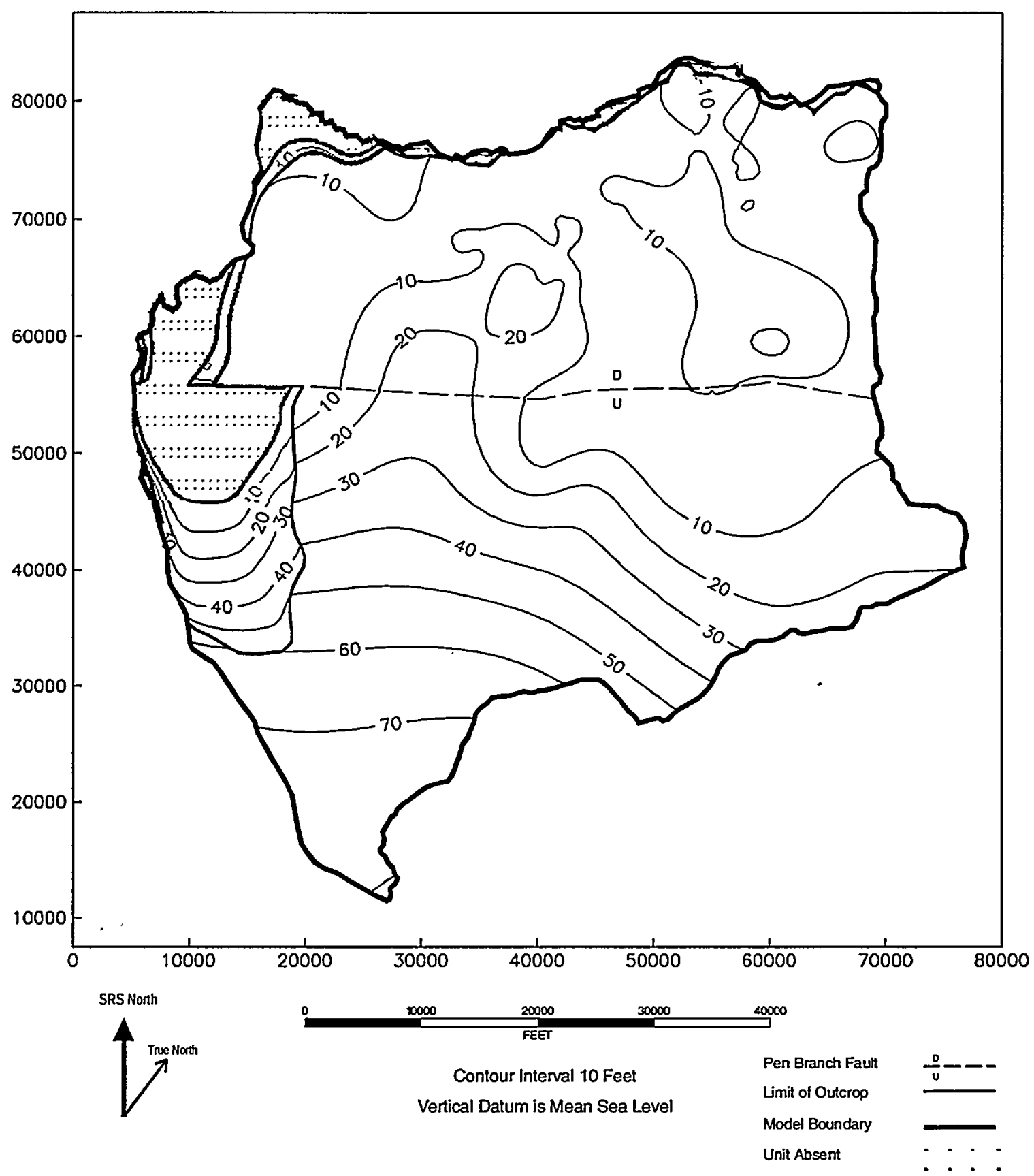

Figure 2-10. Isopach Map of the Gordon Confining Unit 
Top of the "LAZ"

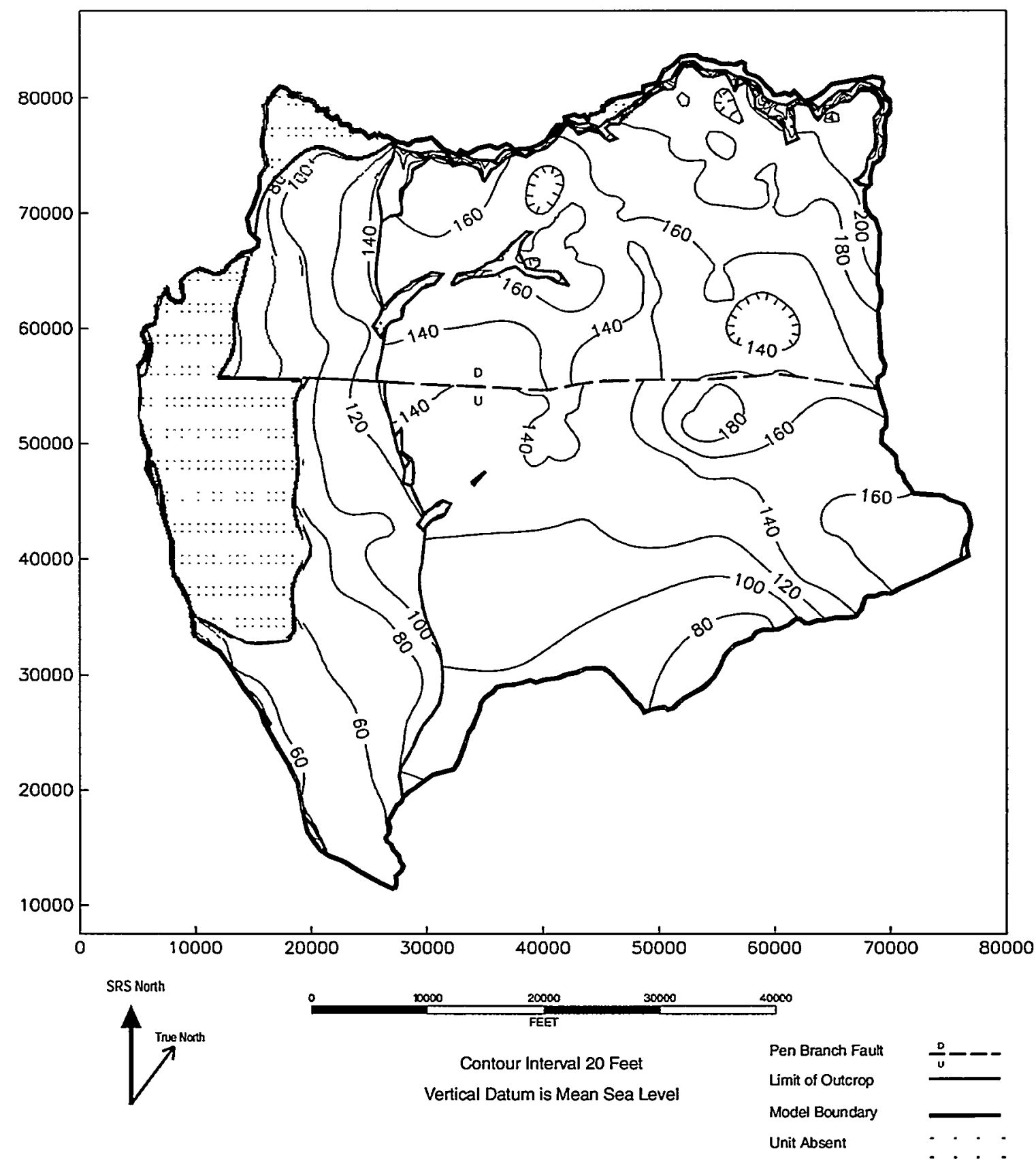

Figure 2-11. Altitude-Contour Map of the Top of the "Lower" Aquifer Zone 
Isopach Map of the "LAZ"

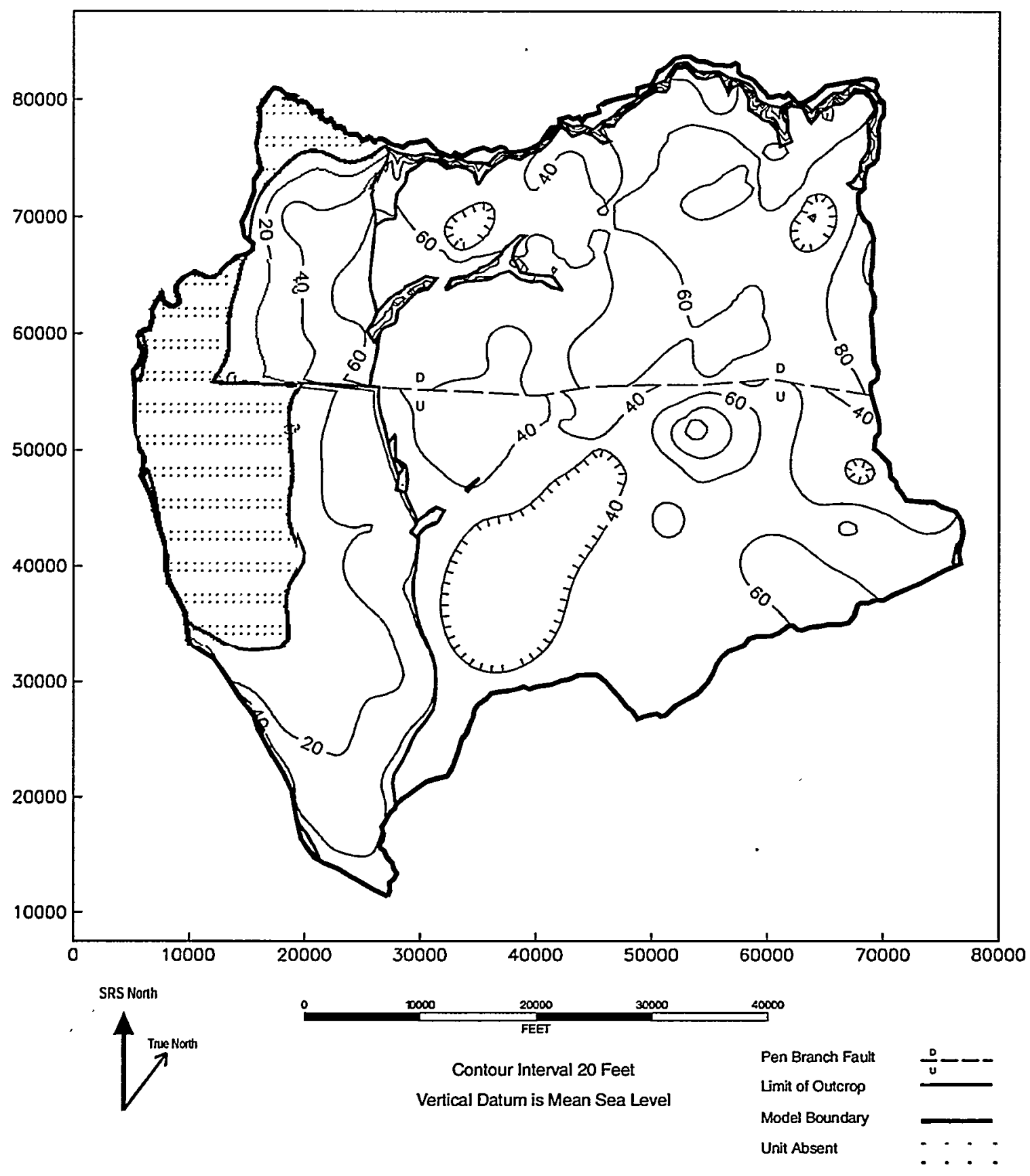

Figure 2-12. Isopach Map of the "Lower" Aquifer Zone 


\section{Top of the "Tan Clay" Confining Zone}

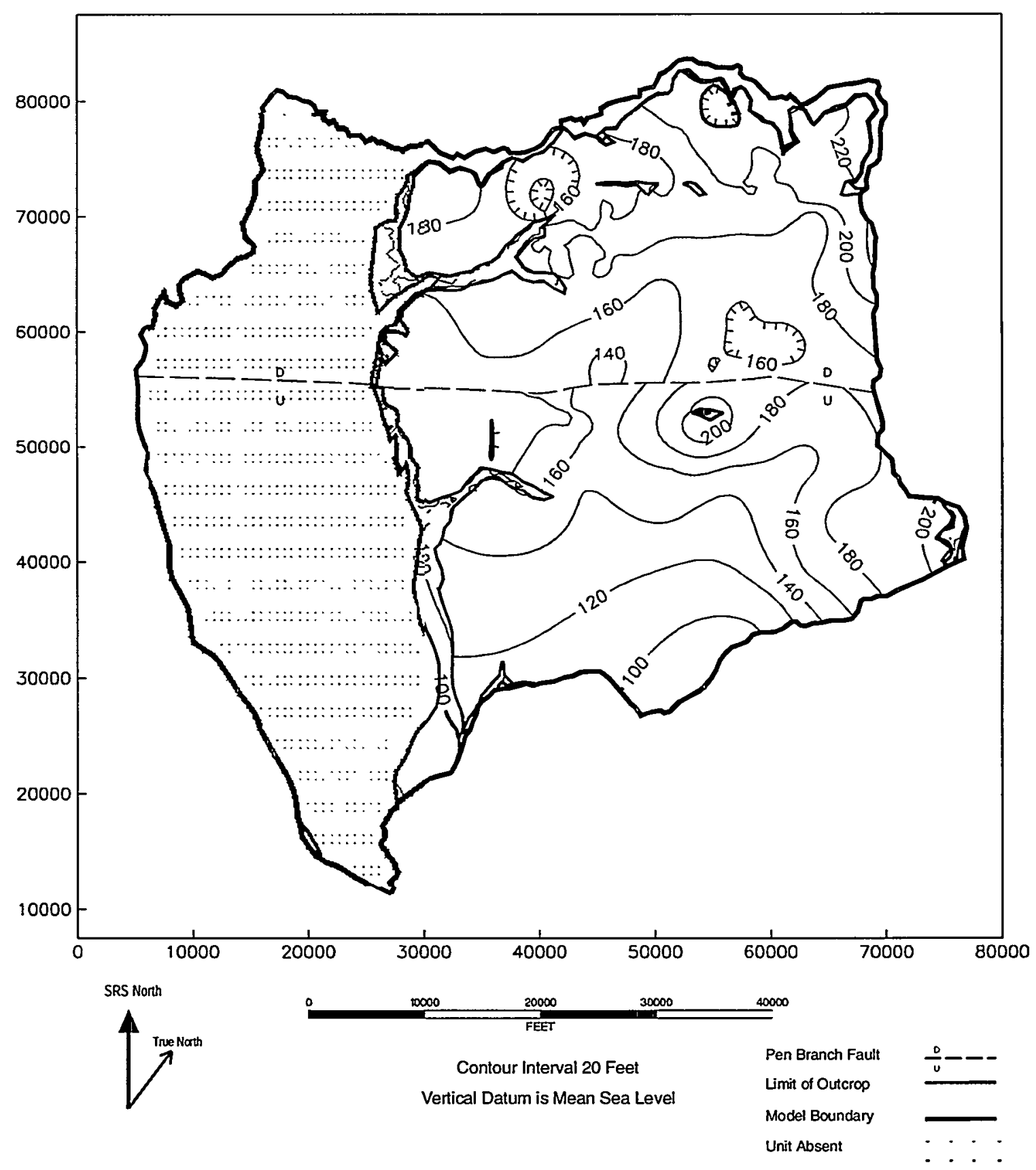

Figure 2-13. Altitude-Contour Map of the Top of the "Tan Clay" Confining Zone 
Isopach Map of the "Tan Clay" Confining Zone

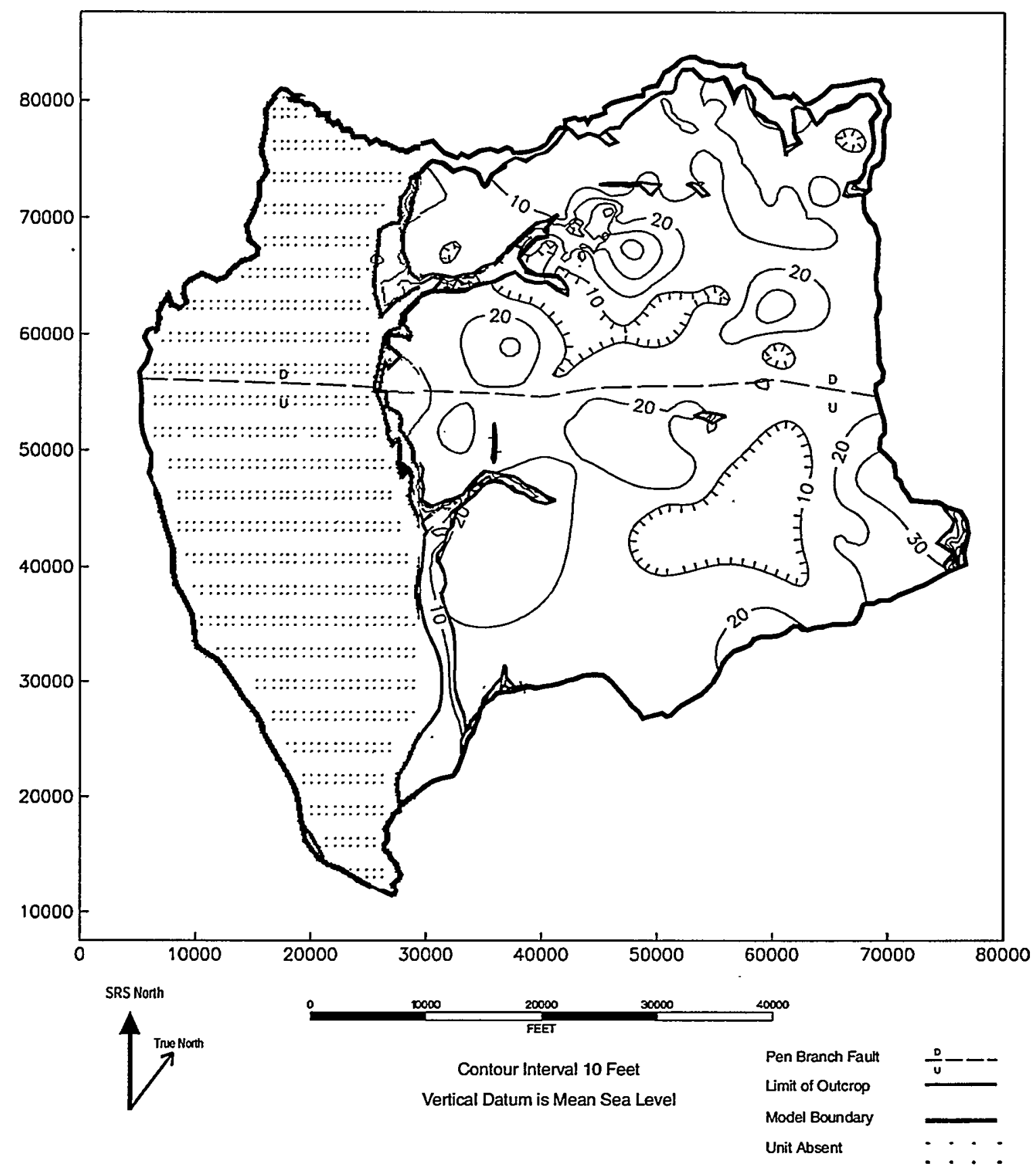

Figure 2-14. Isopach Map of the "Tan Clay" Confining Zone 
Top of the Transmissive Zone

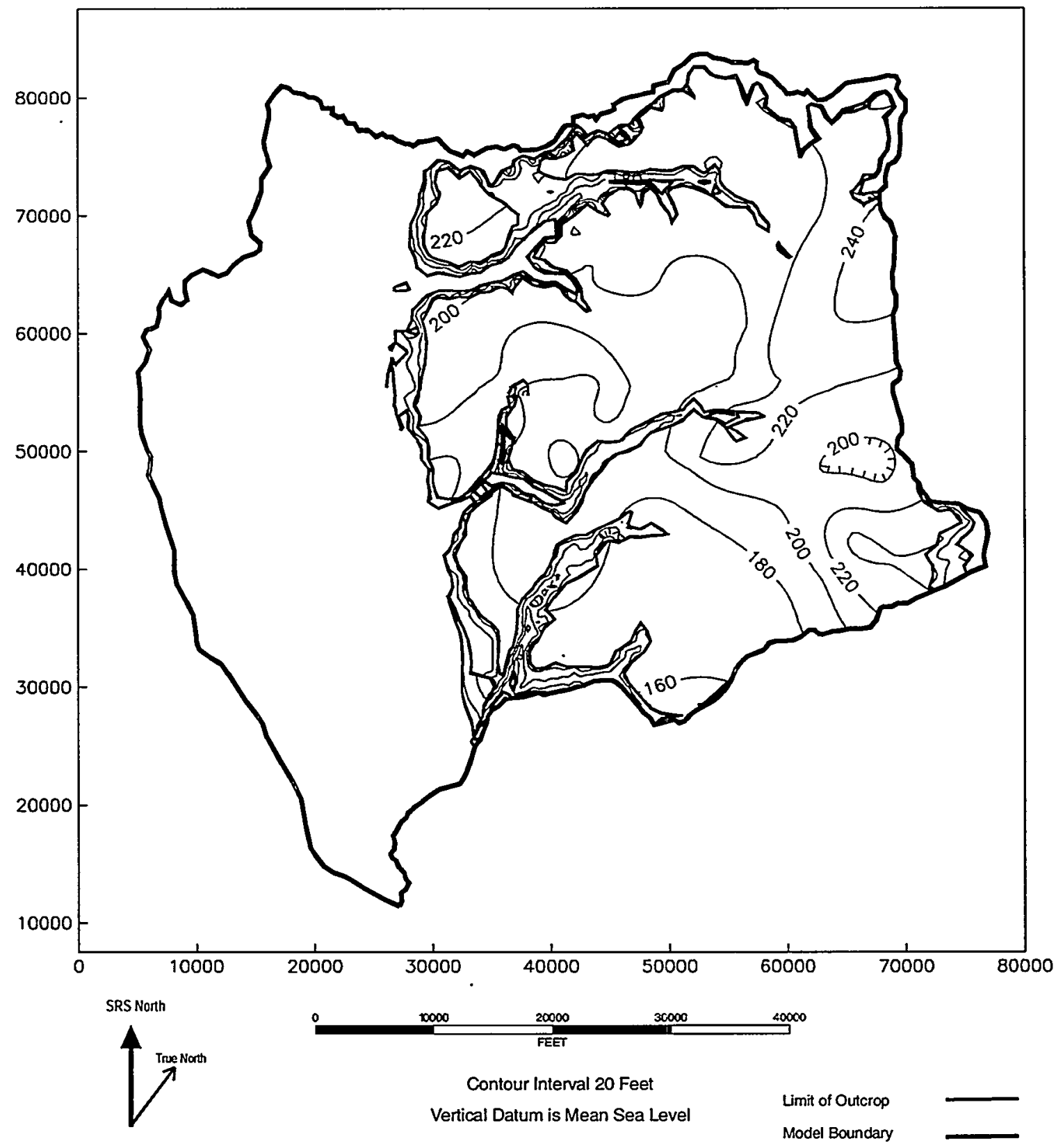

Figure 2-15. Altitude-Contour Map of the Top of the "Transmissive Zone" 
Isopach Map of the Transmissive Zone

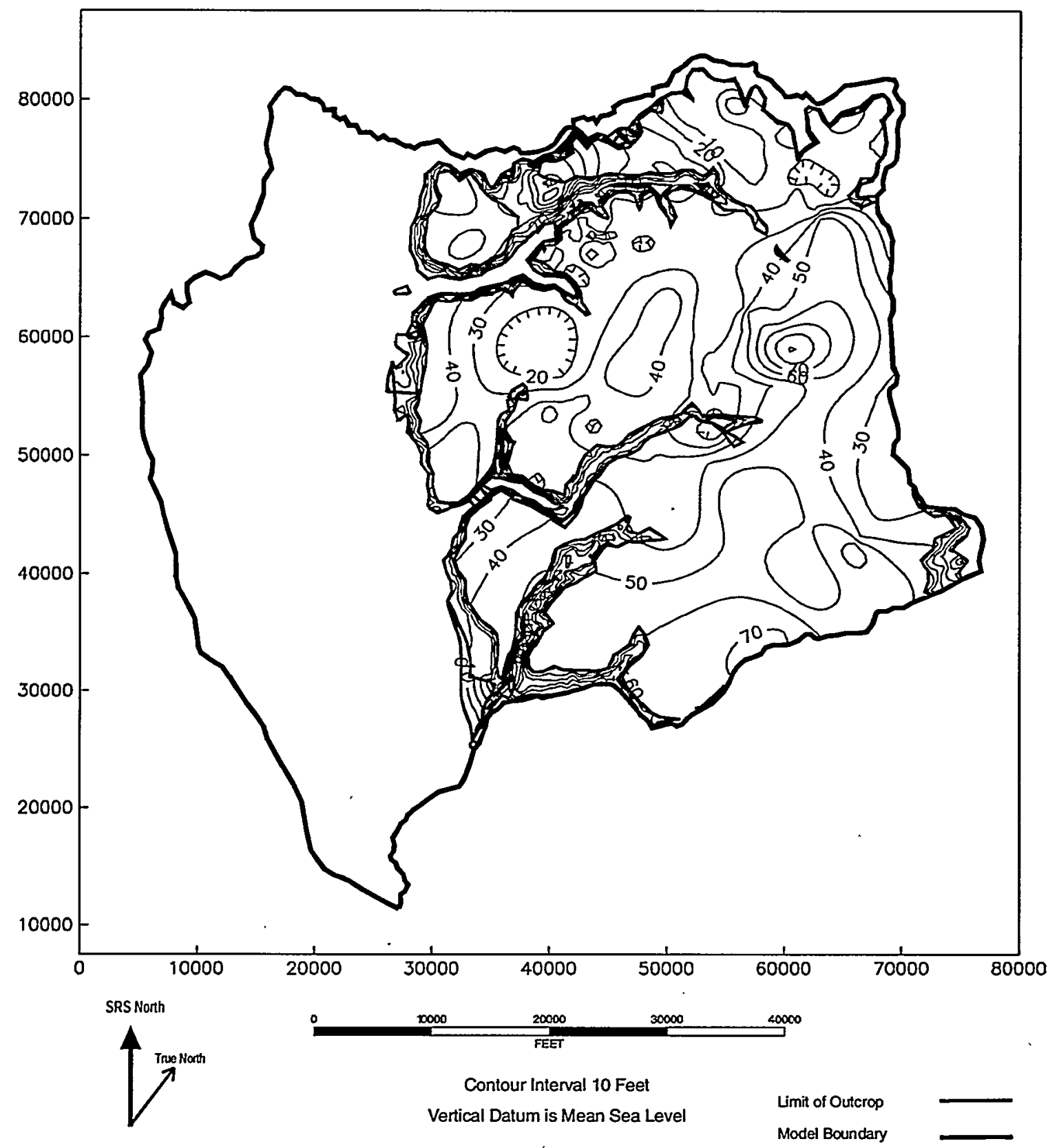

Figure 2-16. Isopach Map of the "Transmissive Zone" 


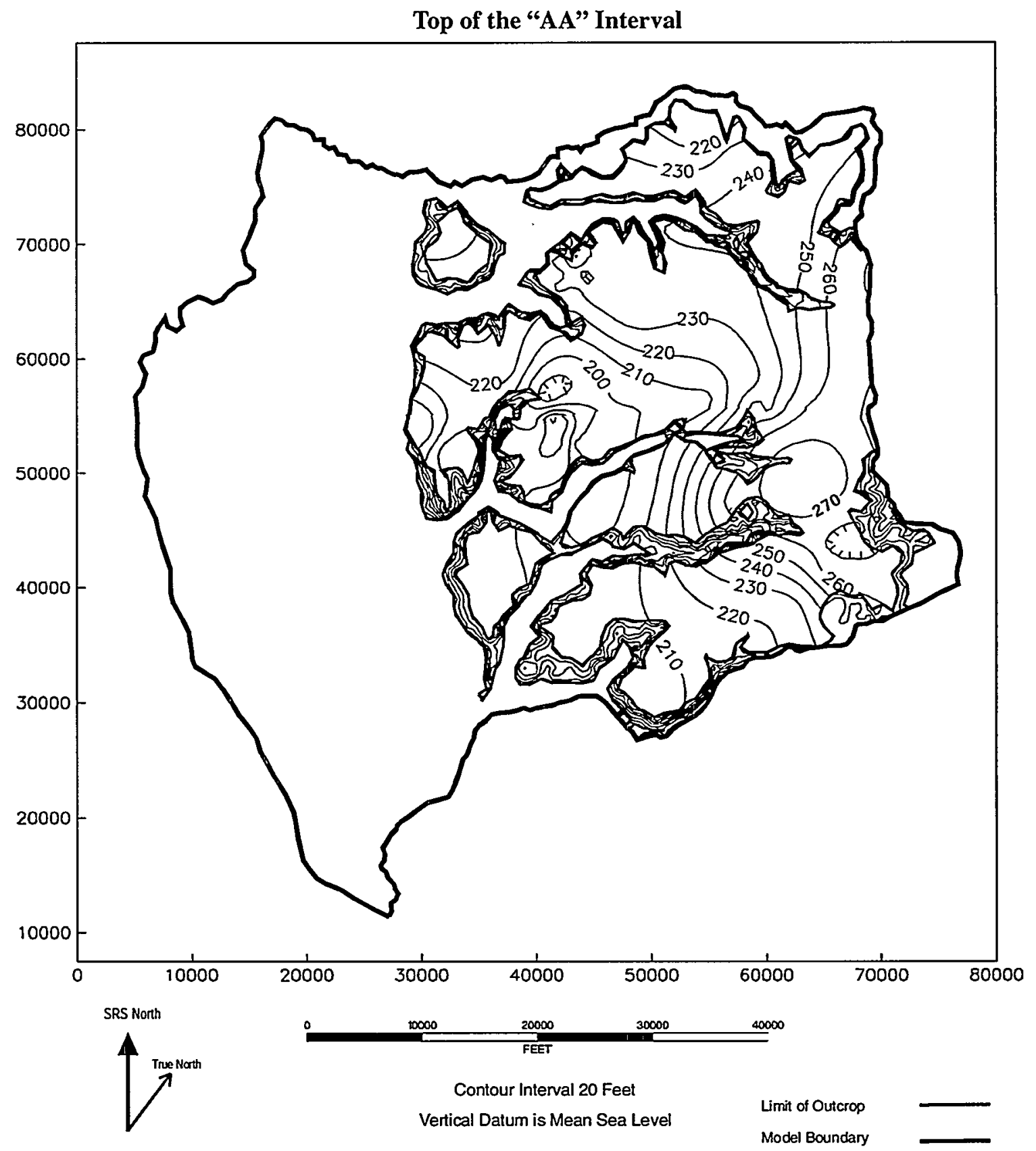

Figure 2-17. Altitude-Contour Map of the Top of the "AA" Interval 


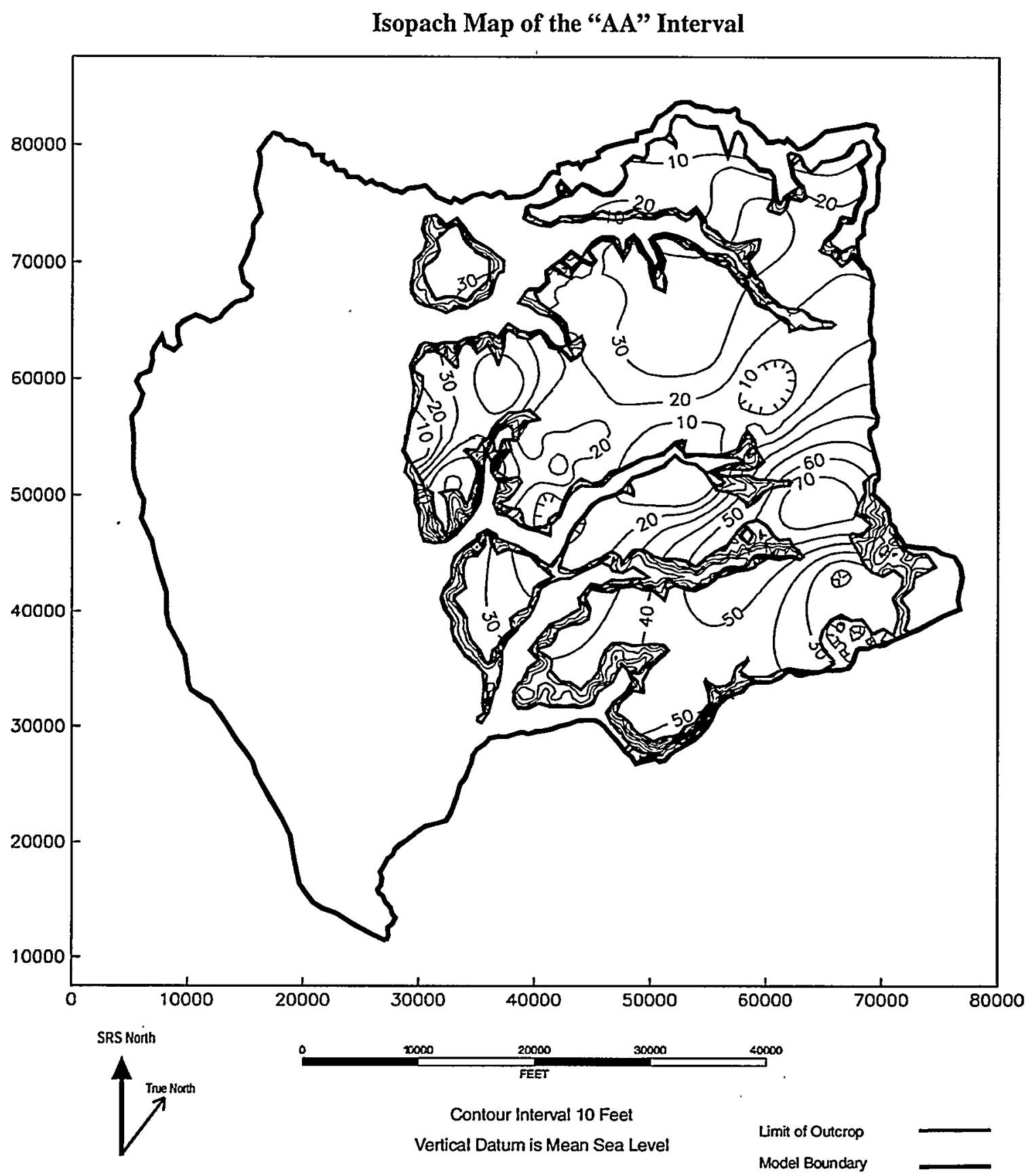

Figure 2-18. Isopach Map of the "AA" Interval 
Top of the "A" Interval

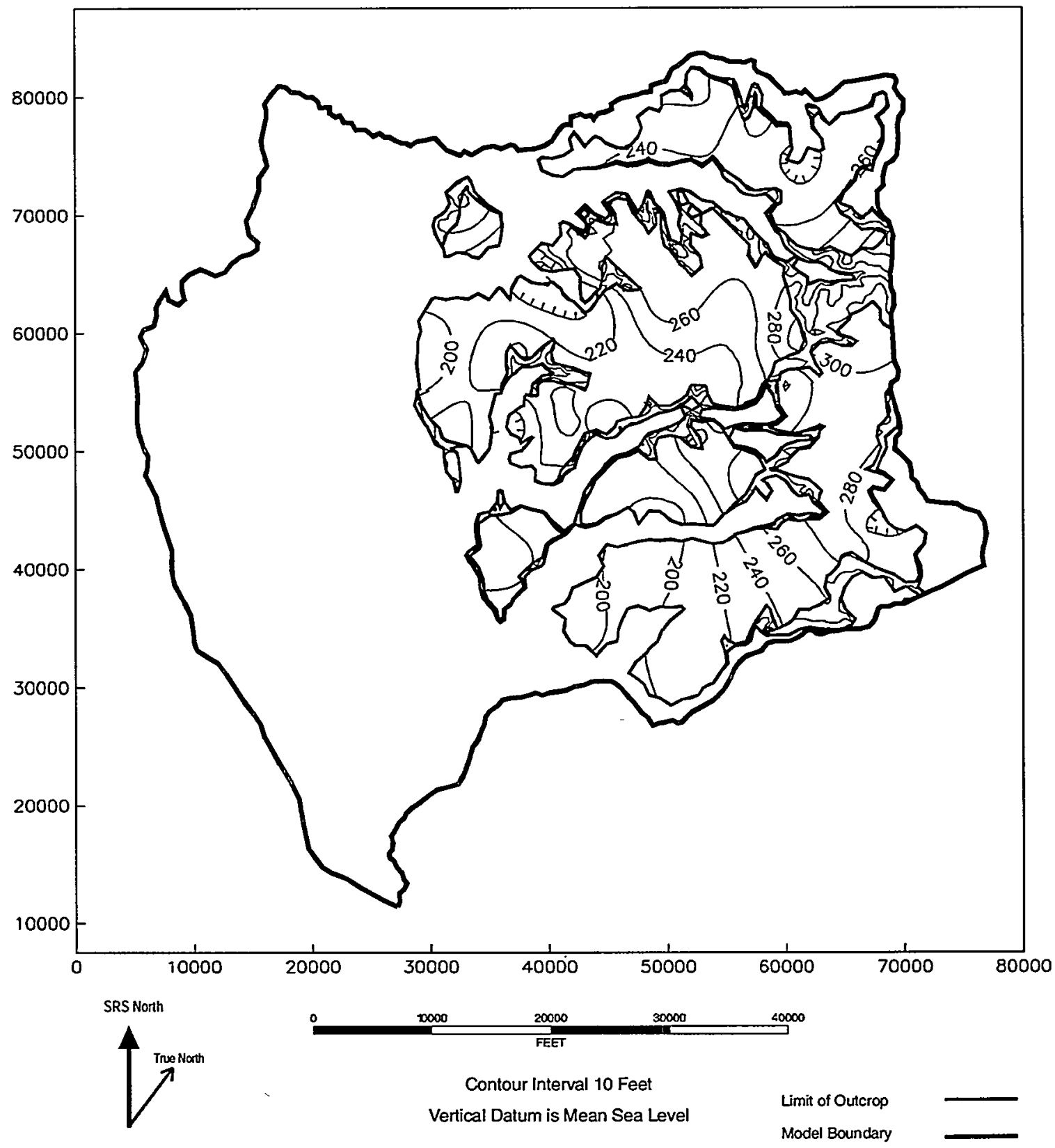

Figure 2-19. Altitude-Contour Map of the Top of the "A" Interval 


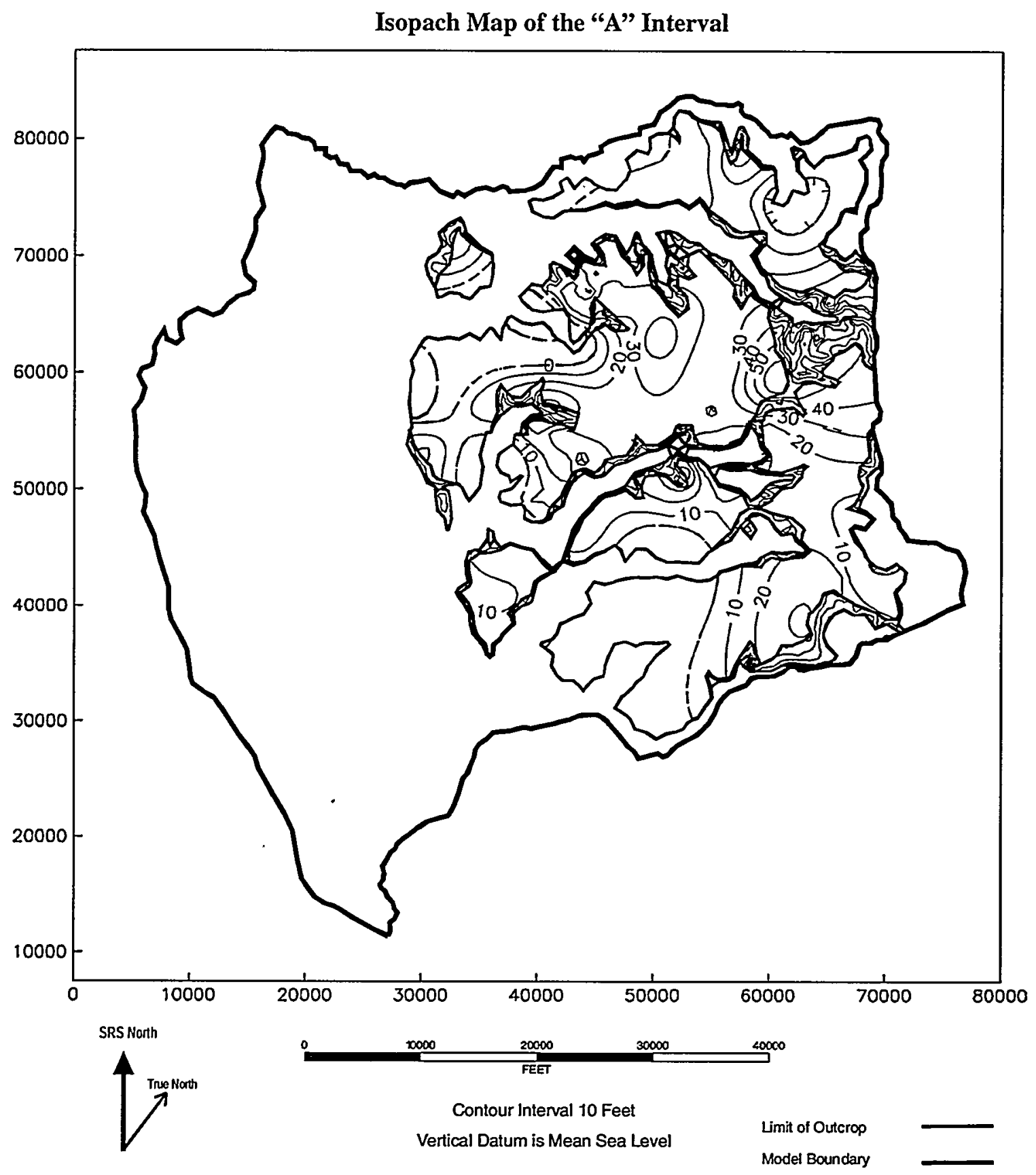

Figure 2-20. Isopach Map of the "A" Interval 


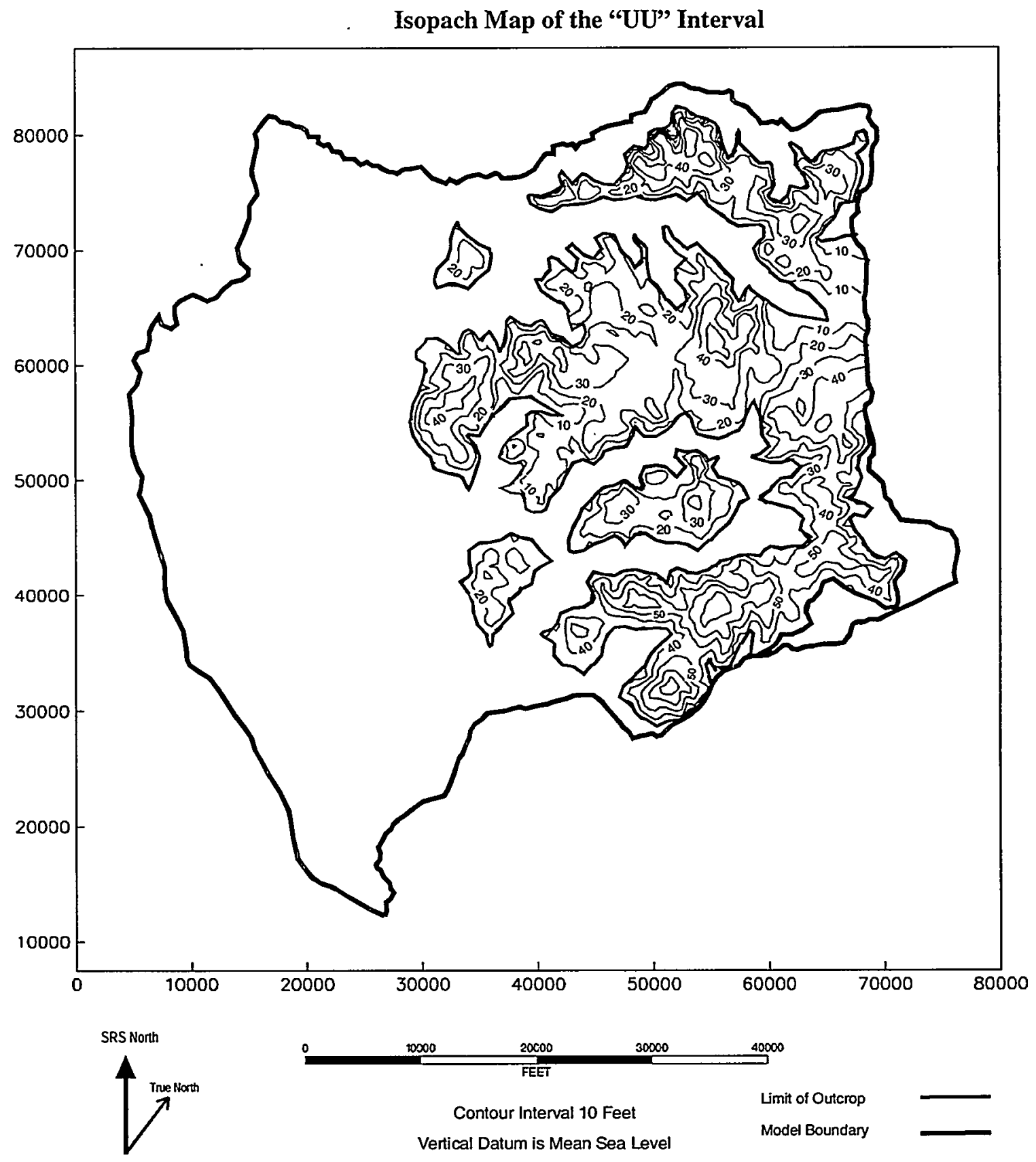

Figure 2-21. Isopach Map of the "UU" Interval 


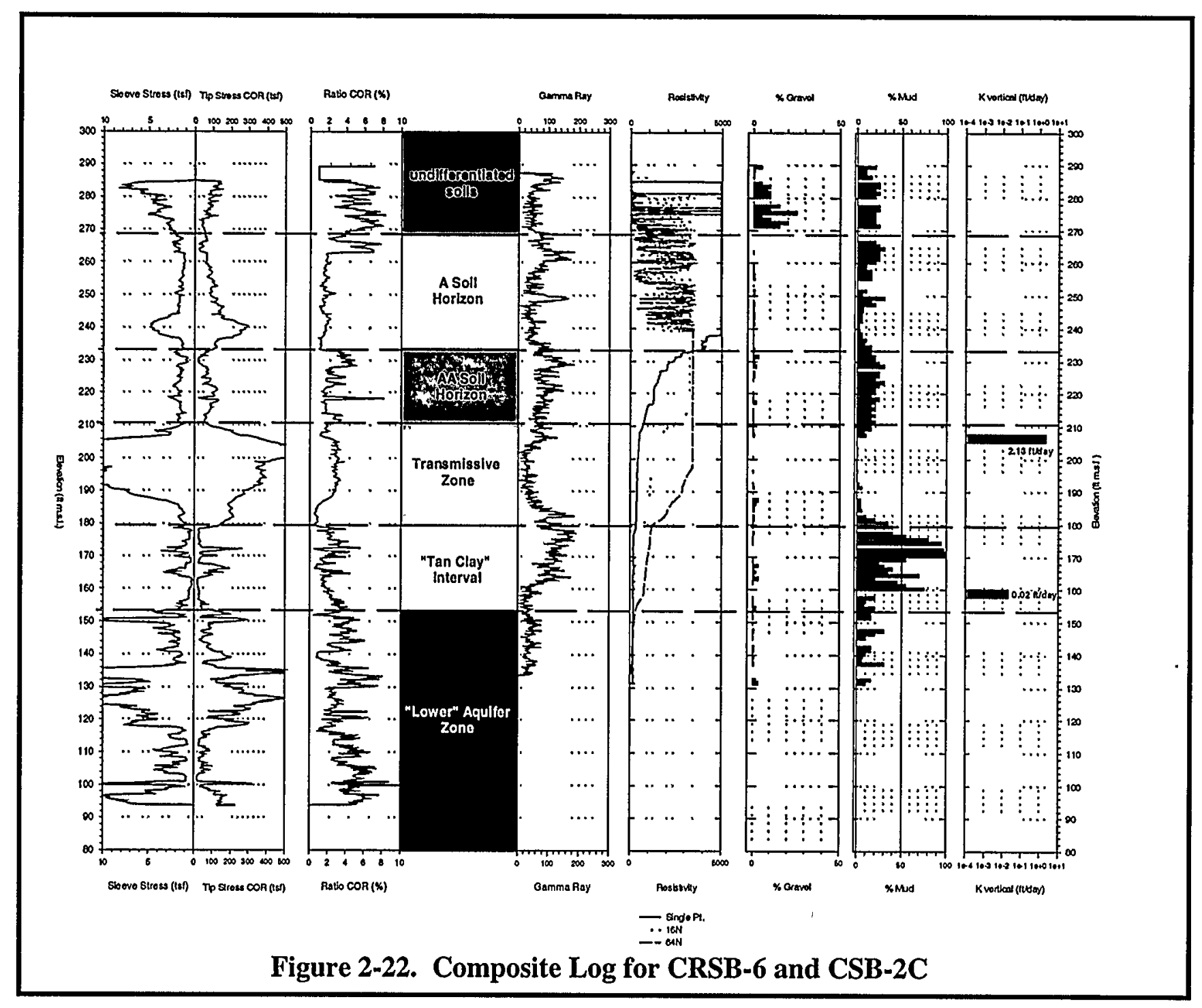




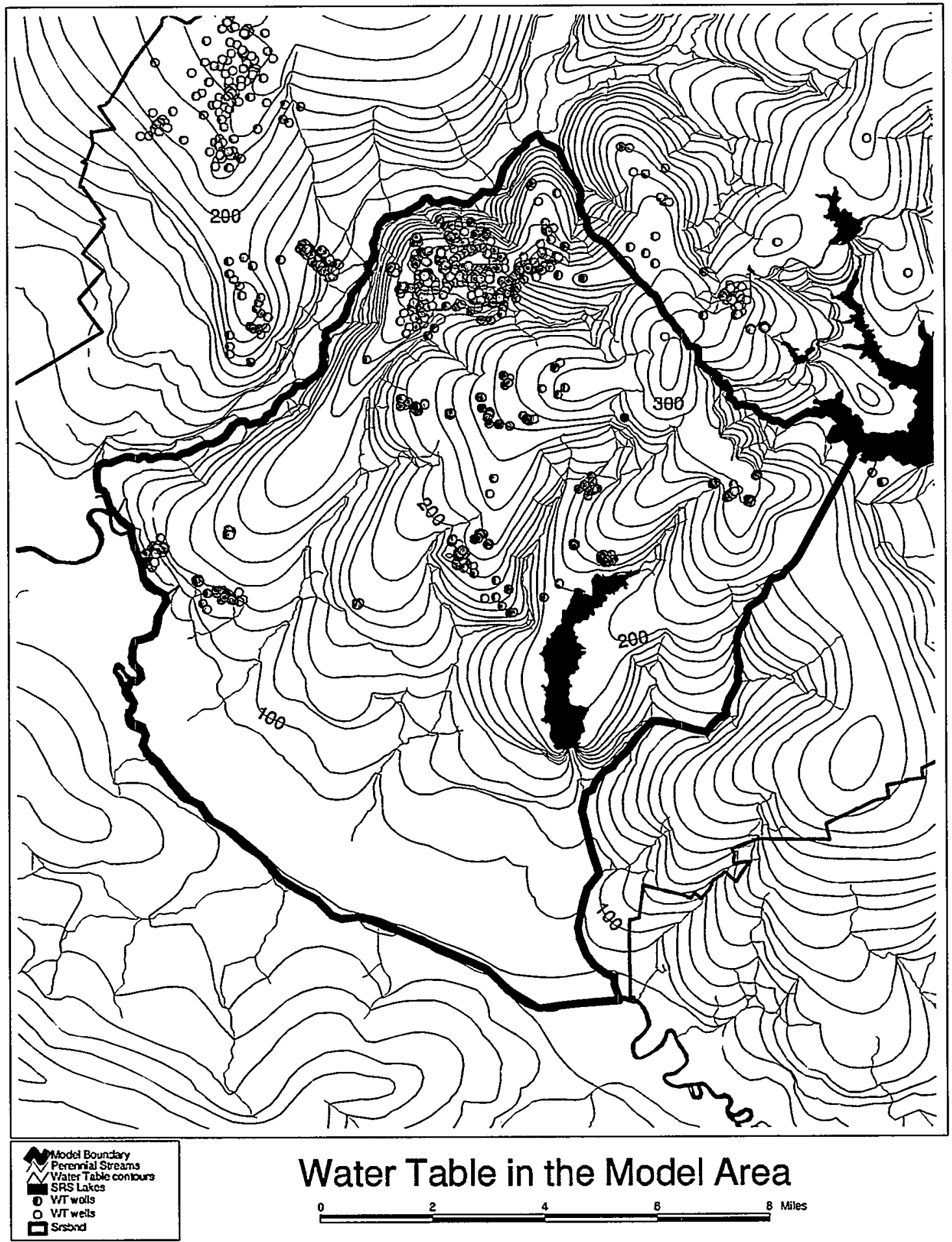

Figure 2-23. Water Table Map of CKLP Model Area 

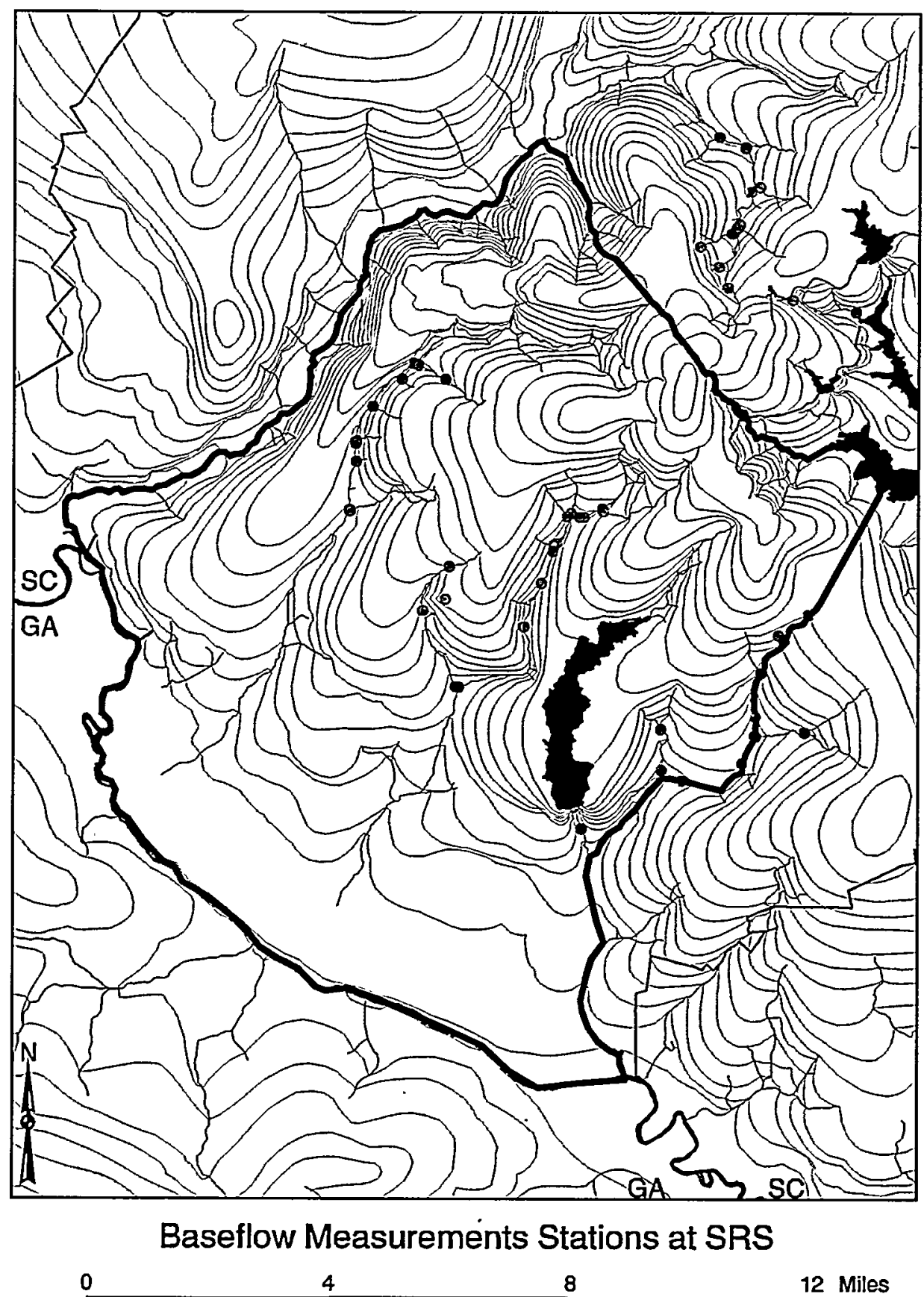

8 12 Miles

Figure 2-24. Location of Stream Baseflow Measurements for 1998 Field Study 

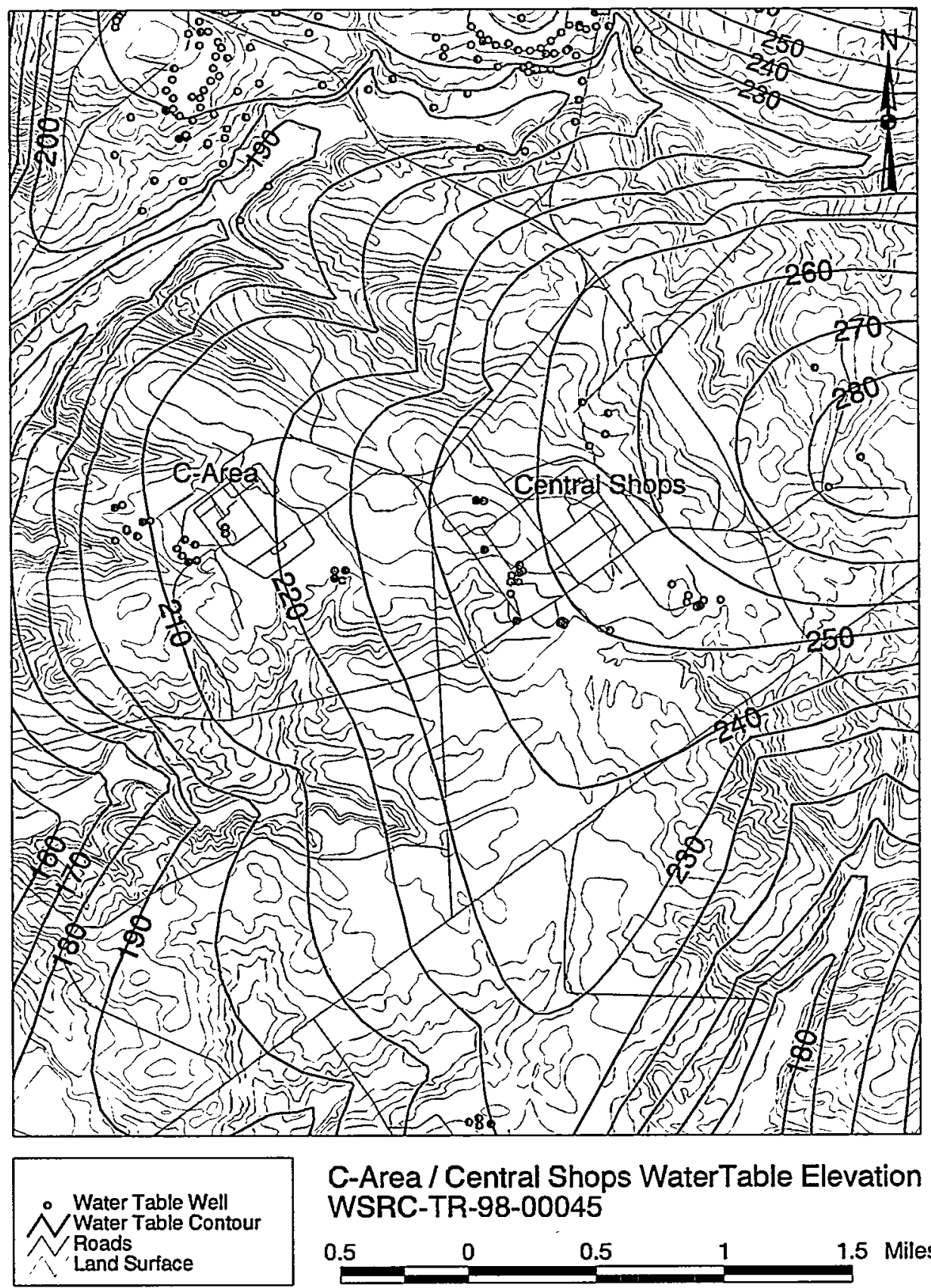

C-Area / Central Shops WaterTable Elevation WSRC-TR-98-00045

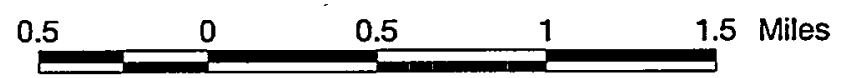

Figure 2-25. Water Table Map for C Reactor Area 

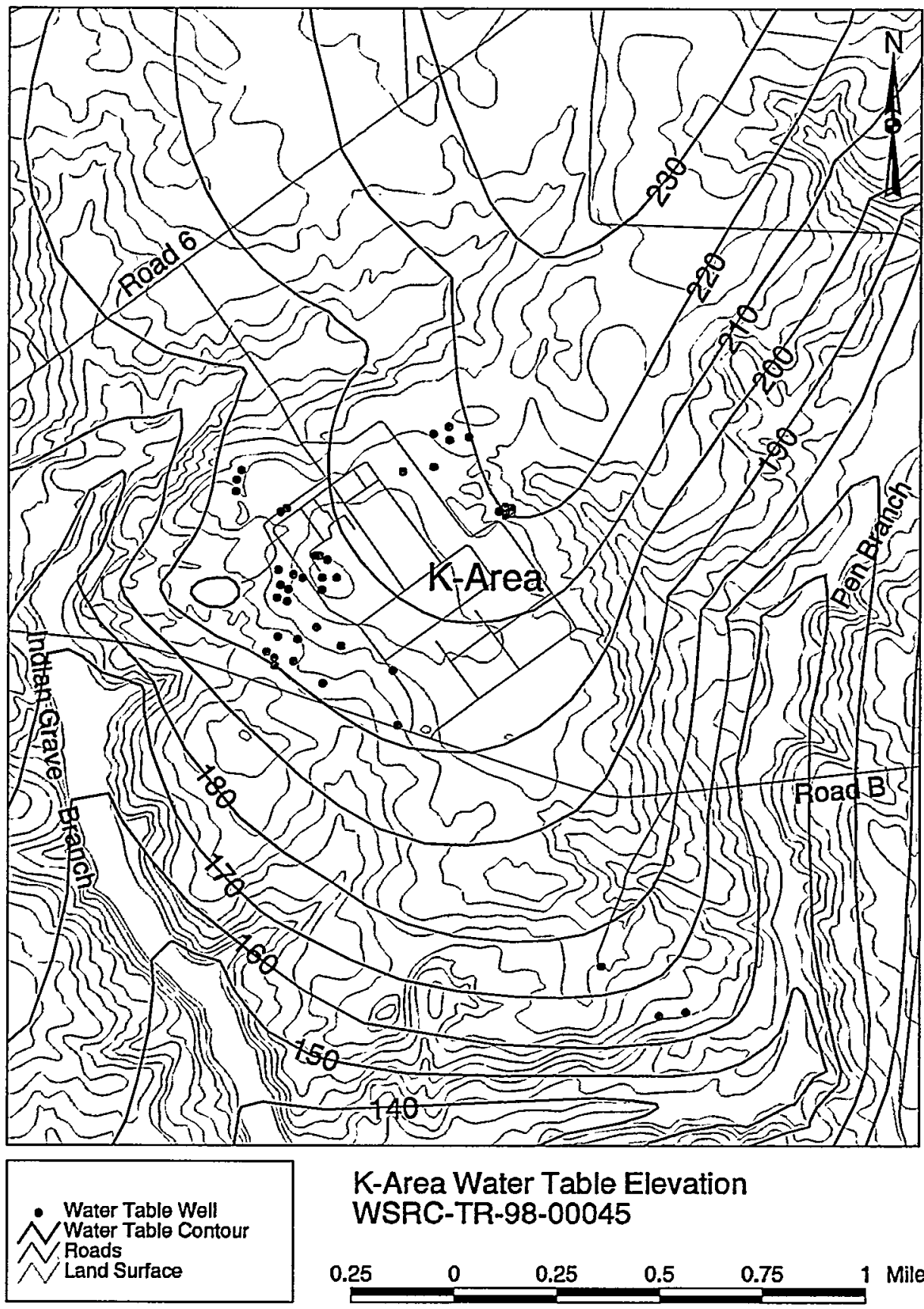

\section{K-Area Water Table Elevation WSRC-TR-98-00045}

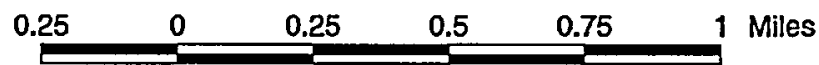

Figure 2-26. Water Table Map for K Reactor Area 

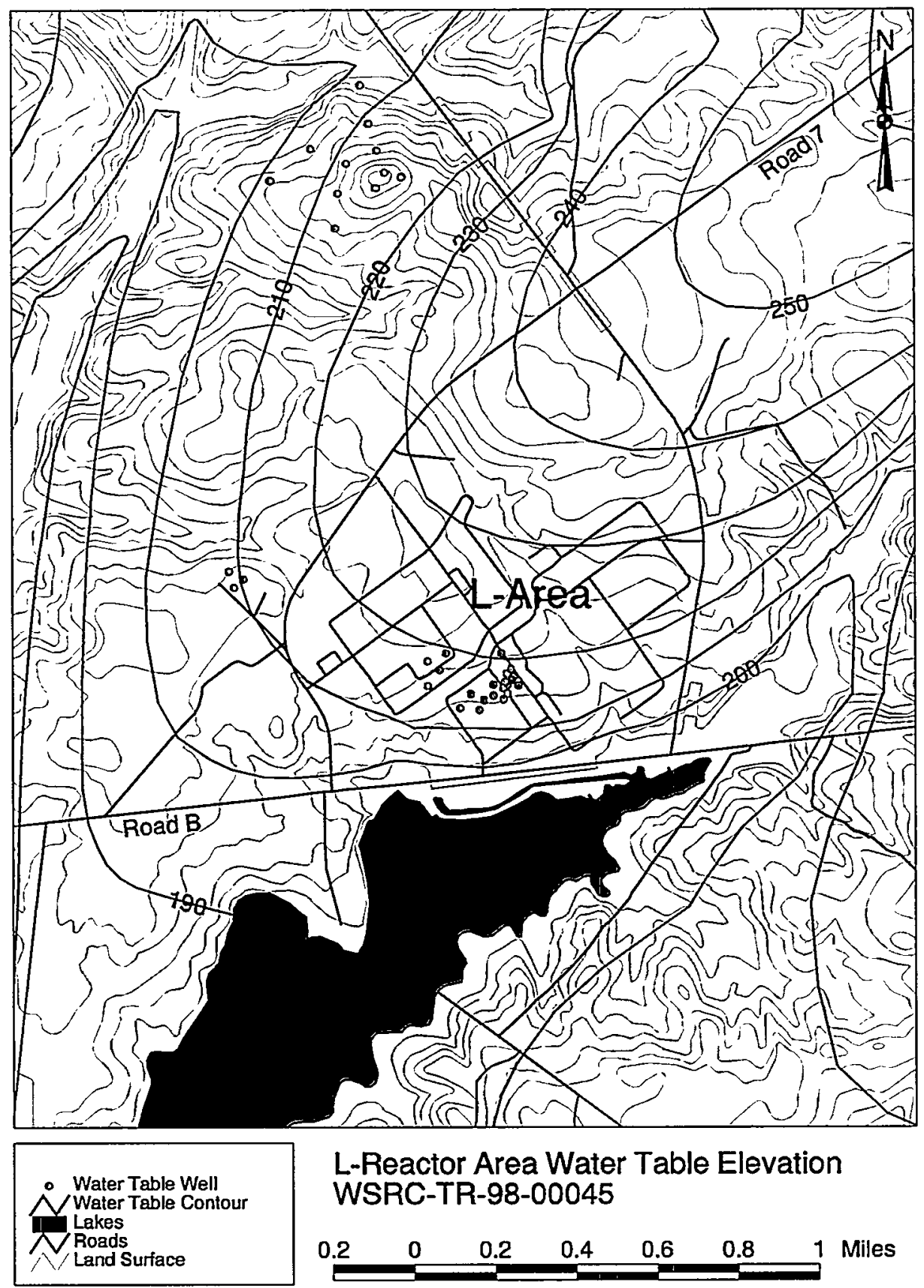

L-Reactor Area Water Table Elevation WSRC-TR-98-00045

$\begin{array}{lllllll}0.2 & 0 & 0.2 & 0.4 & 0.6 & 0.8 & 1\end{array}$

Figure 2-27. Water Table Map for L Reactor Area 


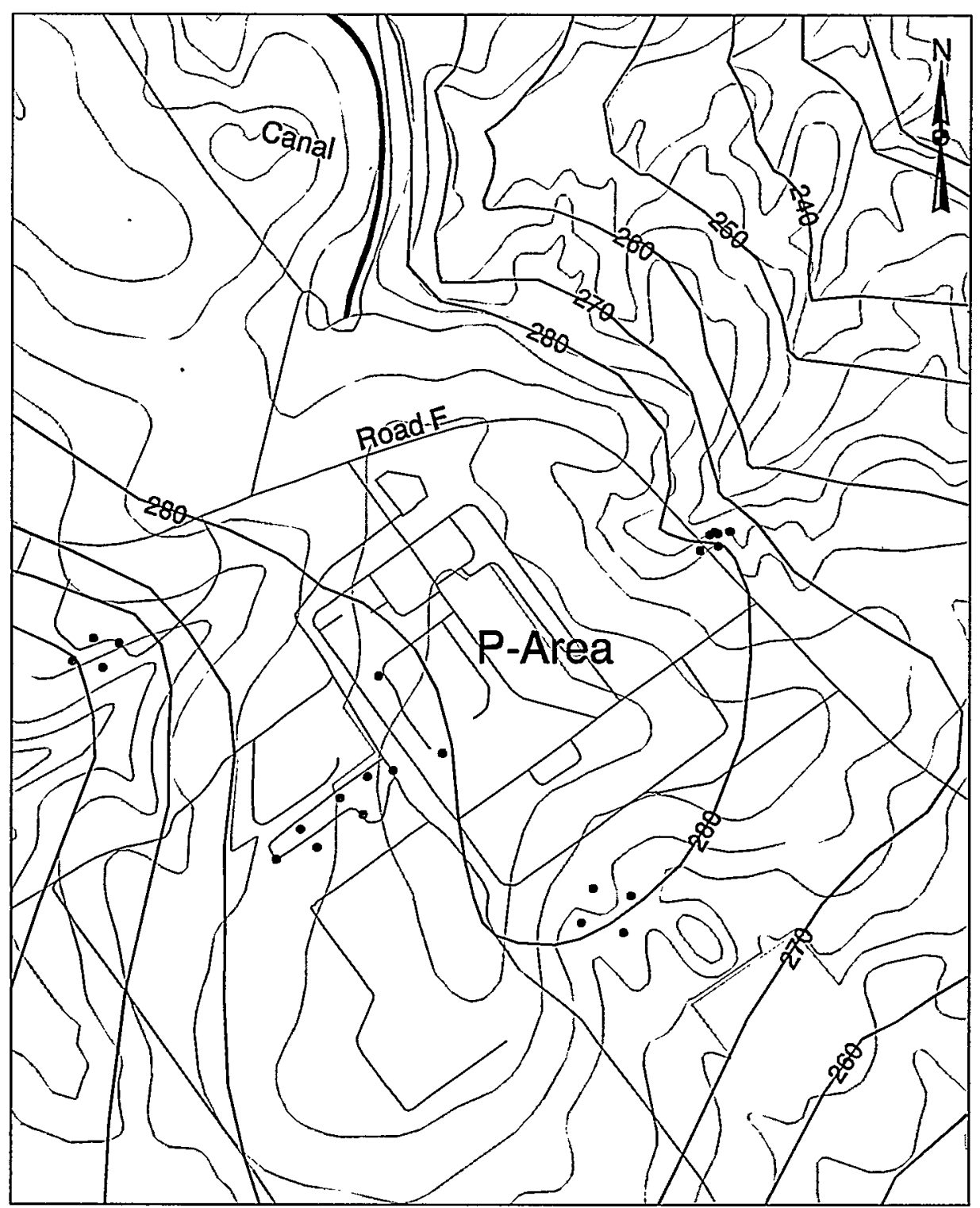

Water Table Well Water Table Contour P-Canal

P.Reactor Area Water Table Configuration WSRC-TR-98-00045

0.2

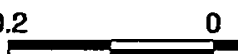

0.2 0.4 Miles

Figure 2-28. Water Table Map for P Reactor Area 


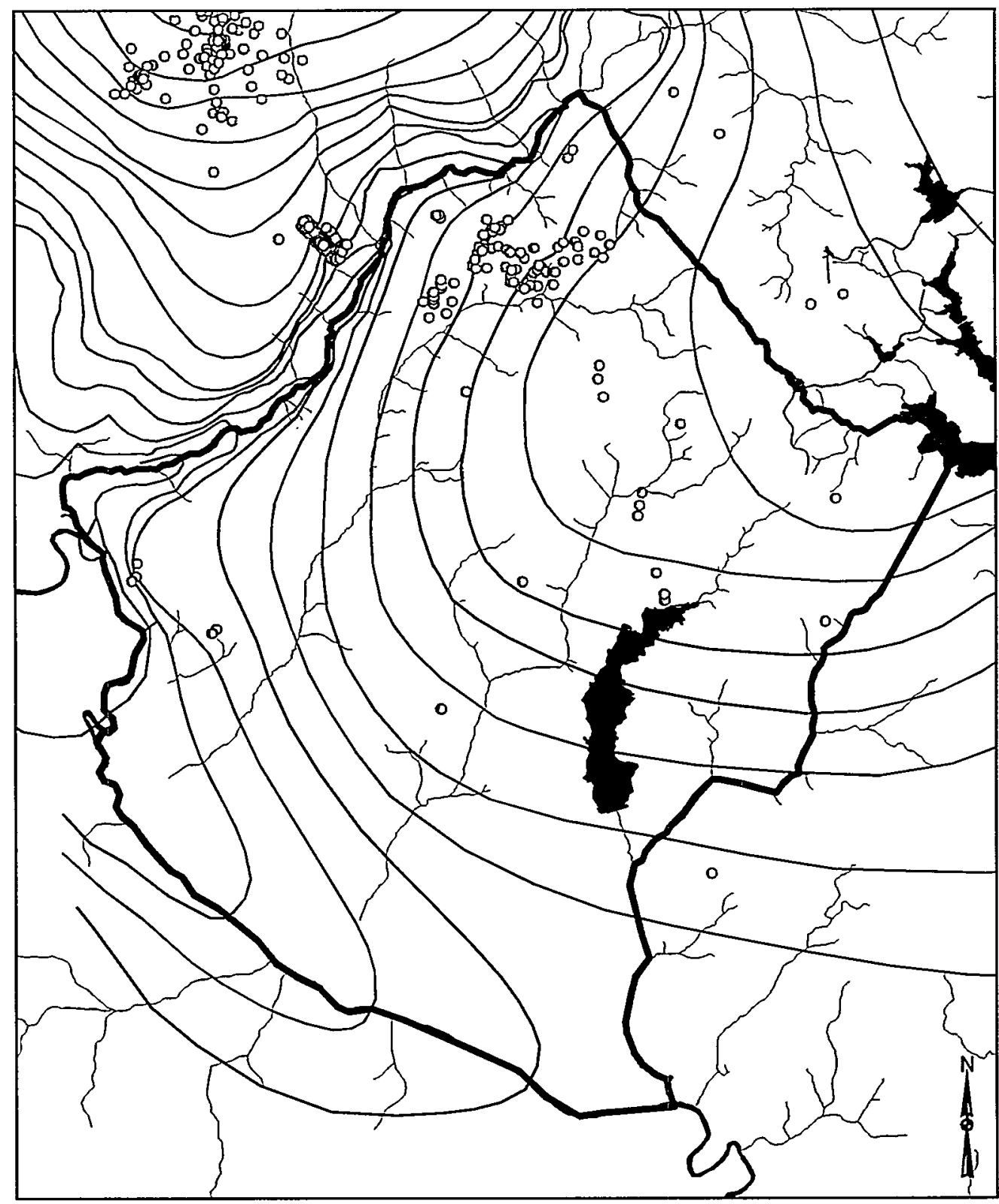

$\mathcal{N}_{\text {Perennial streams }}^{\text {Mol }}$ Gordon Potentiometric Level in the Model Area Gordon Aq. Wells

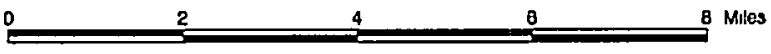

Figure 2-29. Gordon Potentiometric Surface in the CKLP Model Area 


\section{Reactor Area (RGW) Piezometers}

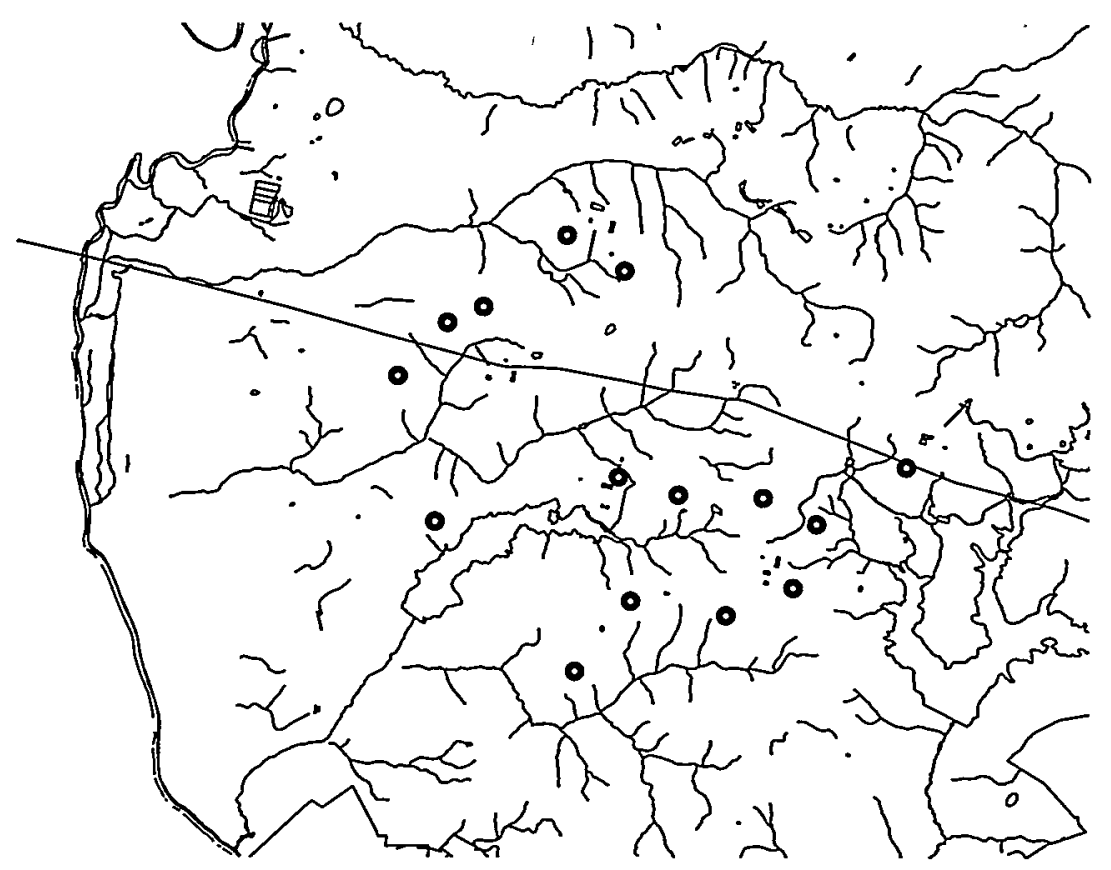

Figure 2-30. Locations of Reactor Area Piezometers Installed in 1998 


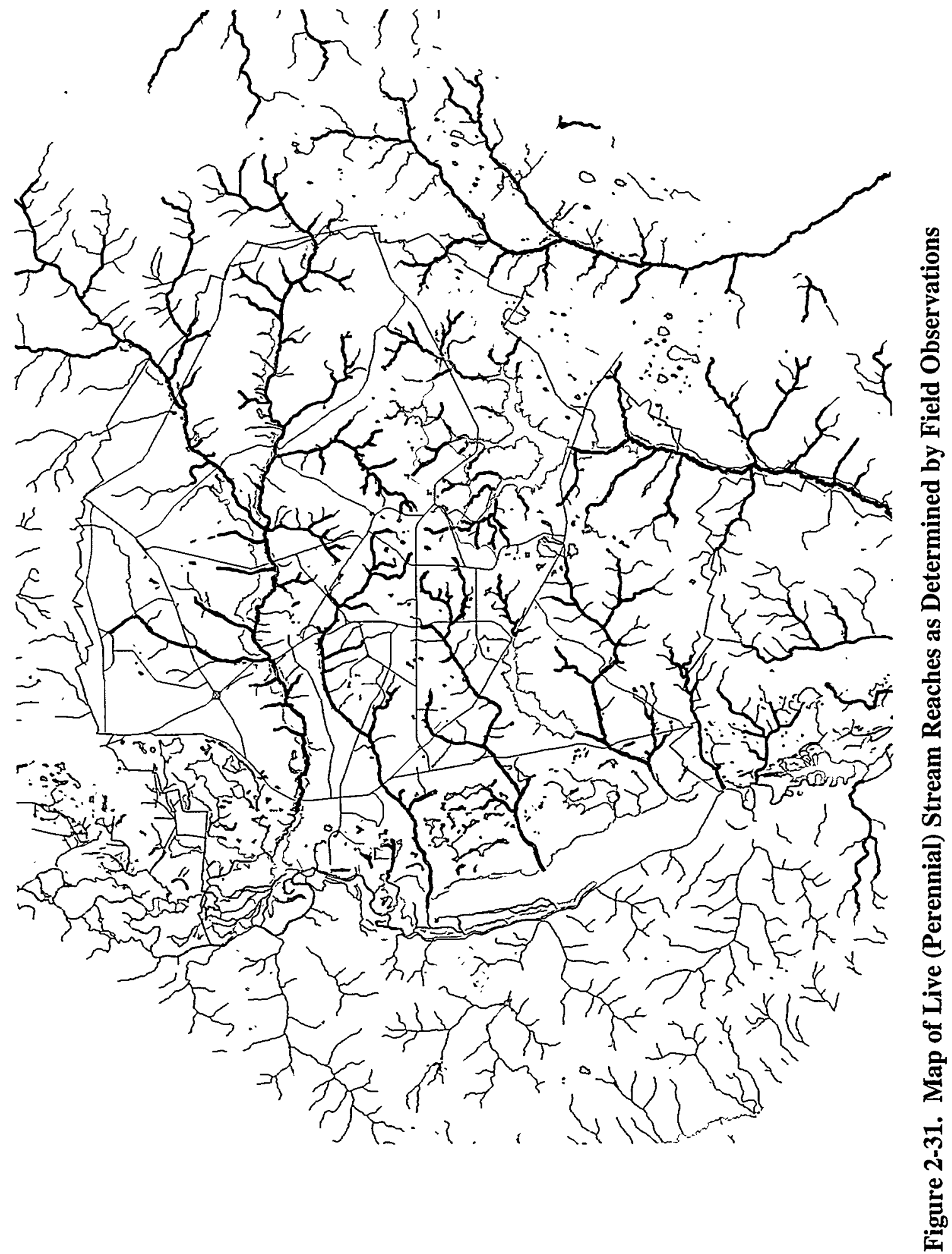




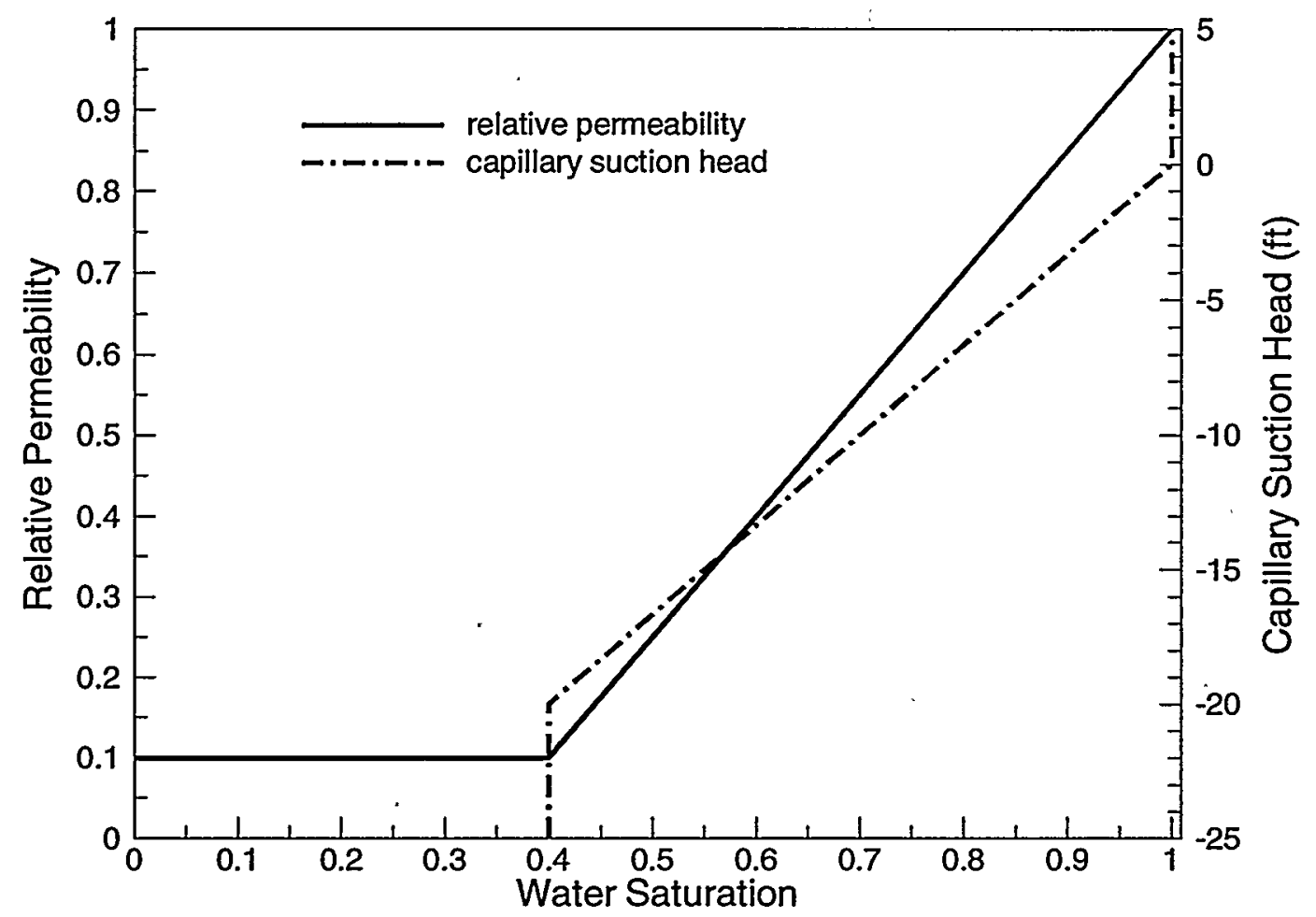

Figure 2-32. Approximate Soil Characteristic Curves 
Table 2-1. Summary of Permeability Measurements

\begin{tabular}{|c|c|c|c|c|c|}
\hline \multirow[b]{2}{*}{ Hydrostratigraphic Unit } & \multicolumn{2}{|c|}{ Laboratory Tests (feet/day) } & \multirow{2}{*}{$\begin{array}{l}\text { Slug Tests } \\
\text { (feet/day) }\end{array}$} & \multicolumn{2}{|c|}{ Pumping Tests (feet/day) } \\
\hline & Vertical & Horizontal & & Multi-Well & Single-Well \\
\hline \multicolumn{6}{|l|}{ "upper" aquifer zone } \\
\hline Number of Results & 17 & 14 & 11 & 0 & 0 \\
\hline Minimum & $5.68 \mathrm{E}-06$ & $3.12 \mathrm{E}-05$ & $6.32 \mathrm{E}-02$ & - & - \\
\hline Maximum & $2.77 \mathrm{E}+01$ & $6.04 \mathrm{E}+00$ & $1.22 \mathrm{E}+01$ & - & - \\
\hline Arithmetic Mean & $1.90 \mathrm{E}+00$ & $1.06 \mathrm{E}+00$ & $2.09 \mathrm{E}+00$ & - & - \\
\hline Geometric Mean & $1.19 \mathrm{E}-02$ & $4.02 \mathrm{E}-02$ & $6.76 \mathrm{E}-01$ & - & - \\
\hline Standard Deviation & $6.67 \mathrm{E}+00$ & $2.10 E+00$ & $3.62 \mathrm{E}+00$ & - & - \\
\hline \multicolumn{6}{|l|}{ "tan clay" confining zone } \\
\hline Number of Results & 43 & 29 & 2 & 0 & 0 \\
\hline Minimum & $3.70 \mathrm{E}-08$ & $1.45 \mathrm{E}-05$ & $3.60 \mathrm{E}-01$ & - & - \\
\hline Maximum & $2.39 \mathrm{E}-01$ & $2.04 \mathrm{E}-01$ & $5.27 \mathrm{E}+00$ & - & - \\
\hline Arithmetic Mean & $9.96 \mathrm{E}-03$ & $1.51 \mathrm{E}-02$ & $2.82 \mathrm{E}+00$ & - & - \\
\hline Geometric Mean & $7.68 \mathrm{E}-05$ & $2.68 \mathrm{E}-04$ & $1.38 \mathrm{E}+00$ & - & - \\
\hline Standard Deviation & 4.00E-02 & 4.83E-02 & $3.47 \mathrm{E}+00$ & - & - \\
\hline \multicolumn{6}{|l|}{ "lower" aquifer zone } \\
\hline Number of Results & 30 & 26 & 31 & 2 & 1 \\
\hline Minimum & $4.54 \mathrm{E}-06$ & $1.59 \mathrm{E}-05$ & $1.30 \mathrm{E}-01$ & $1.23 E+00$ & $1.68 \mathrm{E}+00$ \\
\hline Maximum & $3.42 \mathrm{E}+00$ & $1.11 E+01$ & $2.44 \mathrm{E}+01$ & $2.10 \mathrm{E}+00$ & - \\
\hline Arithmetic Mean & $1.58 \mathrm{E}-01$ & $7.07 \mathrm{E}-01$ & $3.28 \mathrm{E}+00$ & $1.67 \mathrm{E}+00$ & - \\
\hline Geometric Mean & $3.26 \mathrm{E}-03$ & $1.31 \mathrm{E}-02$ & $1.38 \mathrm{E}+00$ & $1.63 \mathrm{E}+00$ & - \\
\hline Standard Deviation & $6.25 \mathrm{E}-01$ & $2.20 \mathrm{E}+00$ & $5.60 \mathrm{E}+00$ & $6.13 \mathrm{E}-01$ & - \\
\hline \multicolumn{6}{|l|}{ Gordon Confining Unit } \\
\hline Number of Results & 47 & 30 & 1 & 0 & 0 \\
\hline Minimum & $1.14 \mathrm{E}-06$ & $5.40 \mathrm{E}-06$ & $2.04 \mathrm{E}+01$ & - & - \\
\hline Maximum & $4.27 \mathrm{E}-01$ & $1.22 \mathrm{E}-01$ & - & - & - \\
\hline Arithmetic Mean & $1.13 \mathrm{E}-02$ & 8.97E-03 & - & - & - \\
\hline Geometric Mean & $1.28 \mathrm{E}-04$ & $1.65 \mathrm{E}-04$ & - & - & - \\
\hline Standard Deviation & $6.25 \mathrm{E}-02$ & $2.83 \mathrm{E}-02$ & - & - & - \\
\hline \multicolumn{6}{|l|}{ Gordon Aquifer } \\
\hline Number of Results & 21 & 21 & 50 & 3 & 10 \\
\hline Minimum & $3.12 \mathrm{E}-06$ & $2.44 \mathrm{E}-05$ & $5.00 \mathrm{E}-03$ & $1.86 \mathrm{E}-04$ & $8.20 \mathrm{E}-01$ \\
\hline Maximum & $3.62 E+01$ & $3.26 \mathrm{E}+01$ & $3.31 \mathrm{E}+01$ & $4.50 \mathrm{E}+01$ & $1.43 E+02$ \\
\hline Arithmetic Mean & $1.87 \mathrm{E}+00$ & $6.07 \mathrm{E}+00$ & $3.75 E+00$ & $2.57 \mathrm{E}+01$ & $2.25 E+01$ \\
\hline Geometric Mean & $2.09 \mathrm{E}-03$ & $4.18 \mathrm{E}-02$ & $9.79 \mathrm{E}-01$ & $6.45 \mathrm{E}-01$ & $5.00 \mathrm{E}+00$ \\
\hline Standard Deviation & $7.88 \mathrm{E}+00$ & $1.18 \mathrm{E}+01$ & $6.47 \mathrm{E}+00$ & $2.32 E+01$ & $4.50 \mathrm{E}+01$ \\
\hline \multicolumn{6}{|c|}{ Meyers Branch Confining System } \\
\hline Number of Results & 29 & 29 & 0 & 0 & 0 \\
\hline Minimum & $4.26 \mathrm{E}-06$ & $1.11 \mathrm{E}-05$ & - & - & - \\
\hline Maximum & $3.40 \mathrm{E}-01$ & $1.50 \mathrm{E}+00$ & - & - & - \\
\hline Arithmetic Mean & $1.36 \mathrm{E}-02$ & $8.42 \mathrm{E}-02$ & - & - & - \\
\hline Geometric Mean & 2.43E-04 & $6.64 \mathrm{E}-04$ & - & - & - \\
\hline Standard Deviation & $5.58 \mathrm{E}-02$ & $3.01 \mathrm{E}-01$ & - & - & - \\
\hline
\end{tabular}


Table 2-2. Summary of Permeability Measurements from Intervals within the "Upper" Aquifer Zone

\begin{tabular}{|c|c|c|c|c|c|}
\hline \multirow[b]{2}{*}{ Hydrostratigraphic Unit } & \multicolumn{2}{|c|}{ Laboratory Tests (feet/day) } & \multirow{2}{*}{$\begin{array}{l}\text { Slug Tests } \\
\text { (feet/day) } \\
\end{array}$} & \multicolumn{2}{|c|}{ Pumping Tests (feet/day) } \\
\hline & Vertical & Horizontal & & Multi-Well & Single-Well \\
\hline \multicolumn{6}{|l|}{ "uu" interval } \\
\hline Number of Results & 0 & 0 & 0 & 0 & 0 \\
\hline Minimum & - & - & - & - & - \\
\hline Maximum & - & - & - & - & - \\
\hline Arithmetic Mean & - & - & - & - & - \\
\hline Geometric Mean & - & - & - & - & - \\
\hline Standard Deviation & - & - & - & - & - \\
\hline \multicolumn{6}{|l|}{ "A" interval } \\
\hline Number of Results & 1 & 1 & 0 & 0 & 0 \\
\hline Minimum & $1.85 \mathrm{E}-03$ & $2.04 \mathrm{E}-02$ & - & - & - \\
\hline Maximum & - & - & - & - & - \\
\hline Arithmetic Mean & - & - & - & - & - \\
\hline Geometric Mean & - & - & - & - & - \\
\hline Standard Deviation & - & - & - & - & - \\
\hline \multicolumn{6}{|l|}{ "AA" interval } \\
\hline Number of Results & 3 & 2 & 0 & 0 & 0 \\
\hline Minimum & 5.68E-06 & $3.12 \mathrm{E}-05$ & - & - & - \\
\hline Maximum & $2.13 \mathrm{E}+00$ & $2.69 \mathrm{E}-04$ & - & - & - \\
\hline Arithmetic Mean & $7.10 \mathrm{E}-01$ & $1.50 \mathrm{E}-04$ & - & - & - \\
\hline Geometric Mean & $9.48 \mathrm{E}-04$ & $9.16 \mathrm{E}-05$ & - & - & - \\
\hline Standard Deviation & $1.23 E+00$ & $1.68 \mathrm{E}-04$ & - & - & - \\
\hline \multicolumn{6}{|l|}{ Transmissive Zone } \\
\hline Number of Results & 3 & 1 & 0 & 0 & 0 \\
\hline Minimum & $4.80 \mathrm{E}-04$ & $9.60 \mathrm{E}-04$ & - & - & - \\
\hline Maximum & $3.98 \mathrm{E}-01$ & - & - & - & - \\
\hline Arithmetic Mean & $1.58 \mathrm{E}-01$ & - & - & - & - \\
\hline Geometric Mean & $2.45 \mathrm{E}-02$ & - & - & - & - \\
\hline Standard Deviation & $2.11 \mathrm{E}-01$ & - & - & - & - \\
\hline \multicolumn{6}{|l|}{ undifferentiated } \\
\hline Number of Results & 10 & 10 & 0 & 0 & 0 \\
\hline Minimum & $5.40 \mathrm{E}-04$ & $3.41 \mathrm{E}-03$ & - & - & - \\
\hline Maximum & $2.77 \mathrm{E}+01$ & $6.04 \mathrm{E}+00$ & - & - & - \\
\hline Arithmetic Mean & $2.97 \mathrm{E}+00$ & $1.49 E+00$ & - & - & - \\
\hline Geometric Mean & 2.47E-02 & $2.11 \mathrm{E}-01$ & - & - & - \\
\hline Standard Deviation & $8.70 \mathrm{E}+00$ & $2.38 E+00$ & - & - & - \\
\hline
\end{tabular}


Table 2-3. Summary of Hydraulic Head Targets

\begin{tabular}{lcc} 
Aquifer Unit or Zone & Category & Number of Hydraulic Head Targets \\
Gordon & 1 & 126 \\
"lower" UTRA & 2 & 344 \\
transmissive & 3 & 180 \\
"AA" & 4 & 308 \\
"A" and "uu" & 5 & 179 \\
\hline
\end{tabular}


Table 2-4. Base Flow Estimates Based on Hydrograph Separation of USGS Gauging Station Data

\begin{tabular}{|c|c|c|c|}
\hline Stream Reach & $\begin{array}{c}\text { Estimated } \\
\text { Base Flow } \\
\text { (cfs) }\end{array}$ & $\begin{array}{c}\text { Estimated Fraction } \\
\text { of Reach within } \\
\text { CKLP Model }\end{array}$ & $\begin{array}{c}\text { Base flow } \\
\text { Target } \\
\text { (cfs) }\end{array}$ \\
\hline $\begin{array}{l}\text { Meyers Branch } \\
\text { (headwaters to Road 9) }\end{array}$ & 9.5 & $1 / 3$ & 3.2 \\
\hline $\begin{array}{l}\text { Steel Creek } \\
\text { (above Road B to Road A; } \\
\text { includes L-Lake) }\end{array}$ & -2.2 & 1 & -2.2 \\
\hline $\begin{array}{l}\text { Pen Branch } \\
\text { (headwaters to Road A13; } \\
\text { includes Indian Grave } \\
\text { Branch) }\end{array}$ & 13.3 & 1 & 13.3 \\
\hline $\begin{array}{l}\text { Fourmile Branch } \\
\text { (headwaters to Road A12) }\end{array}$ & 14.1 & 1 & 14.1 \\
\hline $\begin{array}{l}\text { Upper Three Runs } \\
(\operatorname{Road} C \text { to } \operatorname{Road} A)\end{array}$ & 8.9 & $1 / 2$ & 4.5 \\
\hline
\end{tabular}

Source: Appendix E-1 
Table 2-5. Base Flow Estimates Based on a Single Field Measurement under Low-Flow Conditions

\begin{tabular}{lcc} 
Stream Reach & Field Measurement & Date \\
\hline Caster Creek & 2.9 & 7Dec98 \\
Central Shops outfall creek & 0.76 & 9 Dec98 \\
$\begin{array}{l}\text { Indian Grave Branch } \\
\text { (excluding K-18 outfall flow) }\end{array}$ & $5.5-0.7=4.8$ & 18 Dec97 \\
$\begin{array}{l}\text { Indian Grave above Road B } \\
\text { (excluding K-18 outfall flow) }\end{array}$ & $3.0-0.7=2.3$ & 18 Dec97 \\
$\begin{array}{l}\text { Pen Branch above Indian Grave Branch } \\
\text { Pen Branch above Road B }\end{array}$ & 11 & 18 Dec97 \\
\hline
\end{tabular}

Source: Appendix E-2 


\subsection{Groundwater Flow Model Development}

The process used to transform the hydrogeologic data and conceptual model into a numerical groundwater flow model is presented in this Section.

\subsection{Code Selection and Description}

The subsurface Flow and Contaminant Transport (FACT) code was selected for numerical flow simulations. FACT is a variably saturated, three-dimensional, finite-element groundwater flow and solute contaminant transport code developed by the Savannah River Technology Center (SRTC) (Hamm and Aleman, 1999). FACT is an outgrowth of the SAFT3D code developed jointly by HydroGeoLogic, Inc. and SRTC (Huyakorn and others, 1991). Version 2.0 of FACT was selected for the study so that more accurate head and particle tracking solutions could be obtained by replacing the influence coefficient algorithm used in Version 1.1 with more accurate Gaussian quadrature available in Version 2.0. Other distinguishing features of FACT include efficient memory management and numerical algorithms that make large grids feasible, and user-friendly boundary conditions. For example, the combination recharge/drain boundary condition automatically determines whether a surface node should receive recharge or be discharging groundwater, based on the head solution. The software has undergone extensive verification and validation (V\&V) testing, and has been used successfully to model other areas of the SRS. The reader is referred to the FACT User's Manual for a more thorough description of the code (Hamm and Aleman, 1999). FACT was selected primarily because

1) the variably saturated formulation enables explicit modeling of the vadose zone, which may be important for subsequent. modeling of contaminant transport or remedial actions using the present model or a derivative

2) the code meets the software Quality Assurance requirements of 1Q, 20-1

3) the authors have a strong working knowledge of the code

4) the source code is available.

\subsection{Model Configuration and Mesh}

As described in Section 2.5, groundwater recharge over the greater CKLP GWMA is thought to potentially travel as deep as the Gordon aquifer before discharging to the Savannah River, Upper Three Runs, or tributaries. Therefore contamination originating from $C, K, L$ and $P$ 
reactor facilities is expected to be confined to the Upper Three Runs and Gordon aquifer units between Upper Three Runs on the north, Steel Creek/Meyers Branch on the south, the Savannah River on the west, and an eastern line running from McQueen Branch to Par Pond. As shown in Figures 3-1 and 3-2, these are the boundaries chosen for the CKLP model. The rivers and streams bordering the selected domain also provide natural no-flow boundary conditions according to the conceptual model, and further motivation for choosing model boundaries as shown in Figure 3-1.

The chosen areal grid is $70,000 \mathrm{ft}$ on each side, with a resolution of 500 square feet(Figure 3-1). The mesh resolution is a compromise between the need to resolve topographic features that drive groundwater flow in the UTR aquifer, and computer memory, run-time, and storage limitations. There are 140 elements along the east-west and north-south model coordinate axes. The vertical resolution varies depending on hydrogeologic unit and stratigraphic variations (Figure 3-2). The top surface of the mesh conforms to the ground surface. The bottom surface of the mesh coincides with the bottom of the Gordon aquifer unit. Interior node layers conform to the other stratigraphic surfaces. The Pen Branch Fault is represented in the finite-element mesh as a gradual "bend" in hydrostratigraphic units, rather than the discontinuity shown in Figure ???, to be more consistent with the conceptual model of the fault. The Pen Branch Fault appears in Figure 3-2 between Indian Grave Branch and Pen Branch. The "upper" aquifer zone of the UTR aquifer unit is represented with 3 finiteelements in the vertical direction that conform to the transmissive, AA and A/uu horizons. The vadose zone is included in the model. The "tan clay" confining zone is modeled with a single element. The "lower" aquifer zone is subdivided into 2 finite-elements of equal size. The Gordon confining and aquifer units each contain one element, for a total of 8 vertical elements from ground surface to the bottom of the Gordon aquifer. The three-dimensional mesh size is therefore $140 \times 140 \times 8=156,800$ elements or $141 \times 141 \times 9=178,929$ nodes. The transmissive, AA and A/uu horizons within the "upper" UTR aquifer zone were explicitly modeled to improve model calibration and better support subsequent contaminant transport analyses compared to the previous model of Flach and others (1998). The prior effort only modeled these zones in K-area.

\subsection{Boundary Conditions}

The entire top surface of the mesh is assigned a combination recharge/drain boundary condition, except for the area covered by L Lake and Par Pond (Figure 3-3). This FACT code option automatically specifies a recharge boundary condition for nodes with a computed head below ground elevation, and a drain boundary condition for nodes with a computed head 
above ground surface, which is physically correct. The reader is referred to the FACT code manual for detailed information on how this boundary condition is numerically implemented in FACT (Hamm and Aleman, 1999). Surface drain coefficients are set to 1.0 day $^{-1}$ model wide. The selected drain coefficient is sufficiently large to ensure that computed head will be only slightly greater than ground elevation in discharge areas. Streams and rivers can be represented with the FACT recharge/drain boundary condition, instead of general head or river boundary condition, because they are gaining according to the conceptual model. For gaining surface bodies, the FACT recharge/drain, general head, river, and drain boundaries all function as drains and are equivalent. The maximum local recharge rate is generally specified as $12.5 \mathrm{in} / \mathrm{yr}$ based on model calibration (to be discussed), which is consistent with the estimated range of 10 to $16 \mathrm{in} / \mathrm{yr}$ for recharge developed in Section 2.4. Over the General Separations Area, recharge is set to $15 \mathrm{in} / \mathrm{yr}$ to reflect site-specific estimates of $15 \mathrm{in} / \mathrm{yr}$ recharge, less than average forest cover, and to be consistent with Flach and Harris (1997). However, recharge is set to $1.5 \mathrm{in} / \mathrm{yr}$ for capped areas within the Burial Ground Complex (E area).

The entire bottom surface of the mesh is assigned a general head boundary condition to account for flow into or out of the model domain across the Crouch Branch confining unit (Meyers Branch confining system). A leakance coefficient of $3 \times 10^{-6} \mathrm{~d}^{-1}$ is assumed based on Plate 17 of Aadland and others (1995). This value is supported by a scoping SRTC regional flow model for which model calibration indicates the leakance should be about $5 \times 10^{-6} d^{-1}$. Head distribution in the Crouch Branch aquifer is also taken from Plate 45 of Aadland and others (1995). General head boundary conditions are also specified for L Lake and Par Pond. L Lake is assumed to have a constant pool of $190 \mathrm{ft}$ and a drain coefficient of $1000 \mathrm{~d}^{-1}$. The drain coefficient is large enough that the lake and underlying aquifer have the same head along their boundary. Par Pond is similarly modeled as having a constant pool of $200 \mathrm{ft}$ and drain coefficient of $1000 \mathrm{~d}^{-1}$. Process water outfalls are not modeled because these features are too small to effectively resolve with a 500-ft finite-element size.

Boundary nodes between the top and bottom surfaces of the mesh are assigned either a no-flow or prescribed head boundary condition. Consistent with the conceptual model, boundary nodes underlying major streams and rivers are assigned no-flow boundary conditions because no groundwater is assumed to cross beneath these features. No-flow boundary conditions are also specified in the vadose zone. Where no-flow boundary conditions are inappropriate in the saturated zone, head is prescribed consistent with the potentiometric maps presented in Figures 2-14 and 2-20. For the Gordon aquifer, the result is 
no flow conditions along the west (Savannah River) and north (Upper Three Runs) boundaries, and prescribed head along the east and south boundaries as shown in Figure 3-4. For the Upper Three Runs aquifer, head is prescribed from the headwaters of McQueen Branch south to Par Pond, and from the headwaters of Meyers Branch east to Par Pond (Figure 3-5). Elsewhere, no flow boundary conditions are specified for this unit.

\subsection{Material Properties}

Horizontal conductivity in the Gordon aquifer is set to $35 \mathrm{ft} /$ day based on the extensive field data from both on and off the Savannah River Site reviewed in section 2.2.2.1 and by Aadland and others (1995). The vertical conductivity of the Gordon confining unit is set to $10^{-4} \mathrm{ft} /$ day in accordance with the field data summarized in section 2.2.2.2 and by Aadland and others (1995). Conductivity values in the Upper Three Runs aquifer unit are set through model calibration to well water level data, according to the procedure described in the next section. The ratio of horizontal to vertical conductivity is typically assumed to be 100:1. Because the Pen Branch Fault is assumed to exist in the model domain as a gradual bending of hydrostatigraphic units, the Gordon confining unit and "tan clay" confining zone are assumed to have the same leakance along the Pen Branch Fault as surrounding sediment. Model calibration to measured heads provided no evidence to the contrary. The approximate soil characteristic curves shown in Figure 2-23 are adopted for the numerical model. An effective porosity value of $25 \%$ is assumed for the purpose of computing a pore velocity field that may be used later for particle tracing. The assumed porosity value is consistent with the general recommendation of Looney and others $(1987$, p. 39). However, the value does not affect the steady-state head and Darcy velocity solutions, or set precedence for subsequent transport simulations. For specific storage a nominal value of $10^{-4} \mathrm{ft}^{-1}$ is input to the FACT code, and would only be important for transient flow simulations within a confined aquifer.

In C-area, initial groundwater pathlines originating from the C-Reactor Seepage Basin (CRSB) did not agree with the tritium plume movement observed from Cone Penetration Test (CPTu) concentration data, for any reasonable variation in horizontal and vertical conductivities. Subsequently, CPTu lithologic data were used to identify dominant confining intervals and zones of high horizontal conductivity within the Upper Three Runs aquifer, as described in Appendix G. In summary, the CPTu data indicate that, on the north side of Caster Creek, the "tan clay" confining zone largely disappears as a confining zone and a deeper confining zone appears within the lower zone of the Upper Three Runs aquifer. The underlying confining zone is interpreted to be a calcareous wackestone/mudstone based on the CPTu signature of low tip and sleeve resistance with high pore pressure. Also, the 
"transmissive zone" is significantly more conductive south of the CRSB. To best represent these features given the coarse vertical resolution of the numerical flow model, element layer 5 was redefined to coincide with the lower calcareous zone instead the tan clay near Caster Creek in C-area. Simulated groundwater flow paths were then in much better agreement with observed plume migration from the CRSB and C-area Burning/Rubble Pit (CBRP). Figures 3-6 and 3-7 illustrate the changes to the simulated flow paths that result from these refinements.

\subsection{Calibration Process}

Groundwater recharge and discharge estimates, monitoring well water level data, large-scale measurements of hydraulic conductivity, previous modeling efforts, and a general knowledge of groundwater flow directions and timing were used as targets for calibrating the CKLP flow model. The main parameters selected for calibration adjustment are recharge, horizontal conductivity in aquifer zones and vertical conductivity in confining zones within the Upper Three Runs aquifer, because the model is sensitive to these parameters, and each has significant uncertainty. Other input parameters have less impact on the steady-state flow results and/or lower uncertainty, and were set to their initial best-estimate value throughout calibration. For example, horizontal conductivity in the Gordon aquifer is relatively well known from extensive field-scale tests conducted both on and off the SRS. Therefore, the horizontal conductivity of the Gordon aquifer can be set to $35 \mathrm{ft} / \mathrm{day}$, and held fixed during model calibration. The overall calibration procedure involves 4 sequential, steps:

1) Set model recharge to a value consistent with the prior estimate, and such that simulated discharge agrees with prior baseflow estimates.

2) Adjust Gordon confining unit vertical conductivity to achieve agreement with measured head in the Gordon aquifer, while still agreeing with prior estimates.

3) For the Upper Three Runs aquifer, simultaneously adjust horizontal conductivity in the "lower" and "upper" aquifer zones and vertical conductivity in the "tan clay" confining zone to achieve agreement with head data in these zones.

4) Add zonal variation to unit conductivity values within the Upper Three Runs aquifer as needed to achieve better agreement with head targets.

In practice, the above procedure is iterated during calibration. The model is most sensitive to recharge, as this parameter drives groundwater flow according to the conceptual model 
(Section 2.5). As discussed in Section 2.4, average recharge is thought to lie within the range of 10 to $16 \mathrm{in} / \mathrm{yr}$. Taking the best-estimate value as the mid-point of the range, $13 \mathrm{in} / \mathrm{yr}$, the uncertainty would be plus or minus $25 \%$. The uncertainty of stream base-flow targets ranges from $\pm 15 \%$ to $25 \%$ (Appendix E). In step 1, equal weight is given to satisfying recharge and discharge targets because these data have similar reliability.

Next in importance is leakance through the Gordon confining unit, which is adjusted in step 2. Groundwater flow in the Gordon aquifer is controlled by recharge through the Gordon confining unit, flow across the east and south model boundaries, and horizontal conductivity. Because the head boundary conditions and horizontal conductivity are relatively well known for this unit, Gordon confining unit vertical conductivity is adjusted to achieve agreement with head targets.

With recharge and Gordon confining unit vertical conductivity fixed through steps 1 and 2, horizontal conductivity in the "upper" and "lower" aquifer zones and vertical conductivity in the "tan clay" become the next calibration parameters. The model is sensitive to these parameters, which are highly uncertain relative to other factors (e.g. boundary conditions). Zonal variation in conductivity is invoked as a last resort to achieving adequate agreement with head targets. In C-area CPTu lithologic data are used to guide specification of conductivity zones as described in Appendix G.

The goal of the calibration process is to achieve as good of agreement with prior targets as possible, without resorting to unjustifiable zonal variation in conductivity or other parameters. A lower estimate for achievable calibration accuracy is the uncertainty level in the target data. That is, one should not expect to match calibration targets better than the "noise" level in the data. As discussed in Section 2.3.3, head targets that are a result of time averaging have a "2sigma" uncertainty less than or equal to $3 \mathrm{ft}$, with most being well below $3 \mathrm{ft}$ (Appendix F). However, there are also a significant number of one-time head readings that have much larger uncertainty, typically $\pm 5 \mathrm{ft}$, that inflate average uncertainty. The recharge and stream base flow targets have an uncertainty of roughly $\pm 25 \%$ (Section 2.4 ; Appendix E). Previous models covering portions of the SRS have generally achieved a root-mean-square head residual of $3 \mathrm{ft}$ or so (e.g. Camp Dresser \& McKee, 1989; GeoTrans, 1992; Flach and Harris, 1997). Given the large scale, coarse mesh resolution, and relative uniformity of the conductivity field desired in the present model, a calibration goal of $3 \mathrm{ft}$ is too low, especially for the more heterogeneous aquifer zones. A more reasonable calibration goal for root-meansquare residual or mean-absolute residual is $5 \mathrm{ft}$. A reasonable calibration goal for the largest head residual is sometimes defined as $5-10 \%$ of the total head variation in the modeled system. 
For the Gordon aquifer, the total variation is about $120 \mathrm{ft}$ (Figure 2-20) suggesting a calibration goal of 6 to $12 \mathrm{ft}$ for the maximum residual. For the Upper Three Runs aquifer, the total variation is about $330 \mathrm{ft}$ (Figure 2-14) for a calibration goal of 16 to $33 \mathrm{ft}$. 


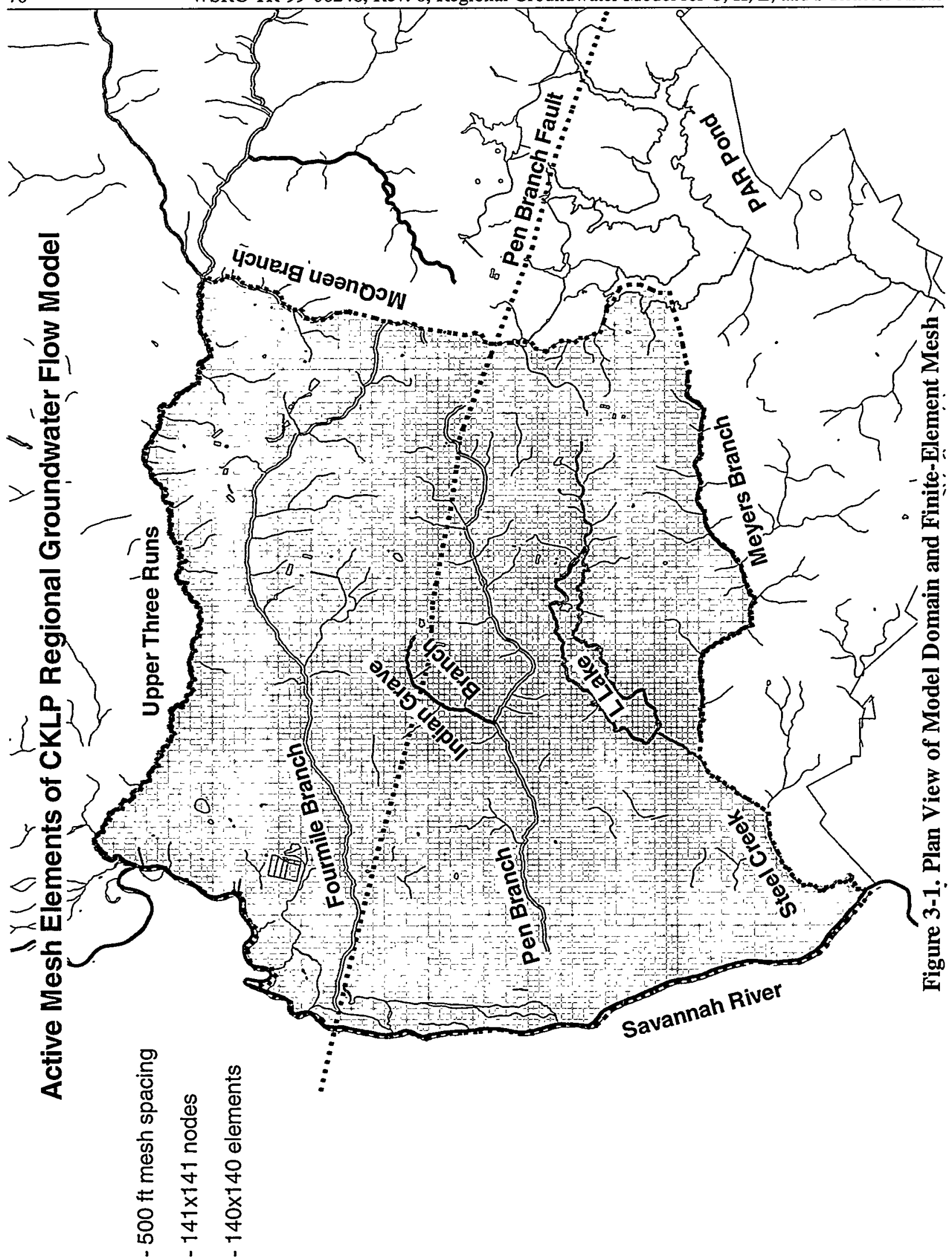




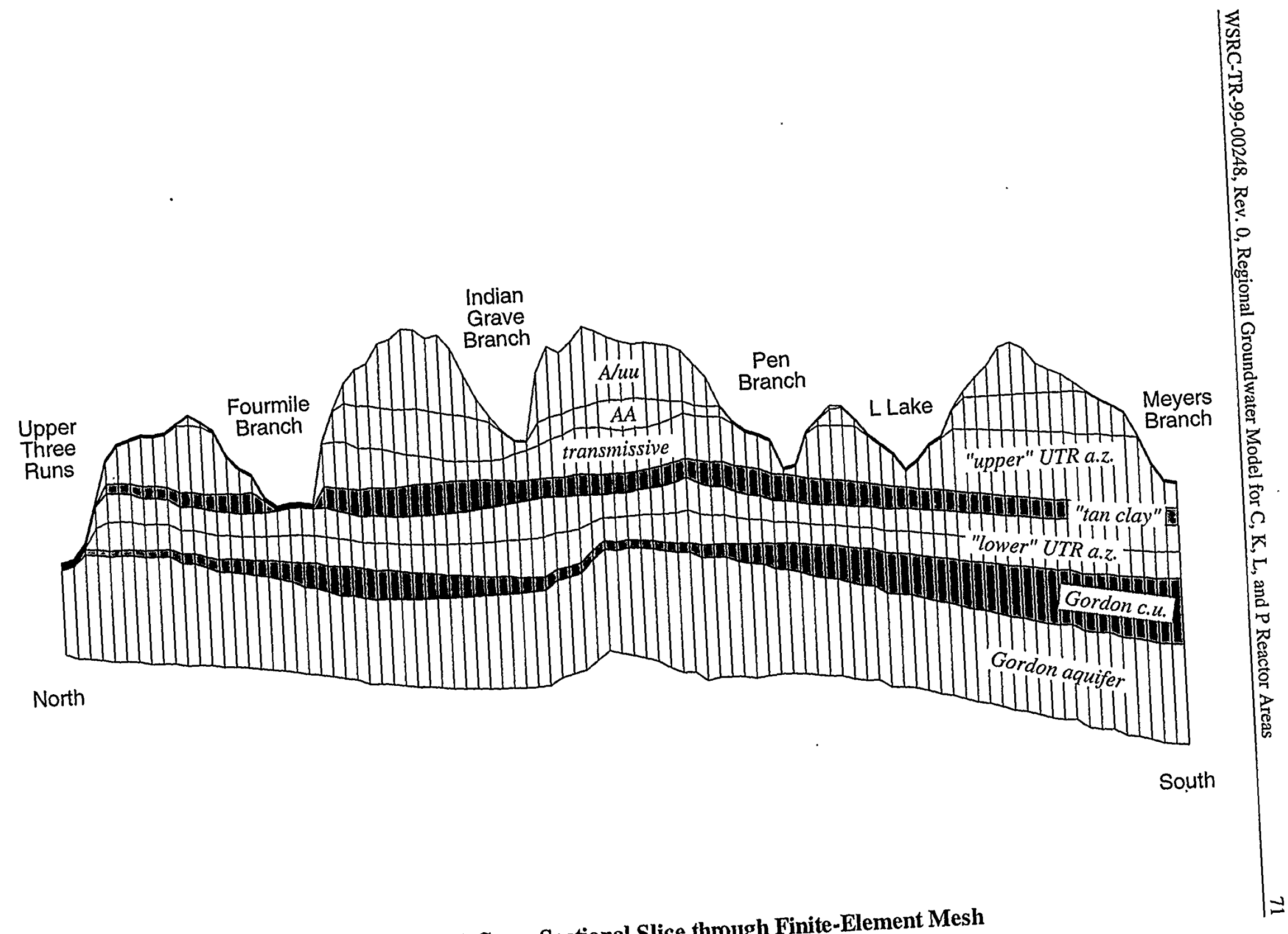

Figure 3-2. Typical Cross-Sectional Slice through Finite-Element Mesh 


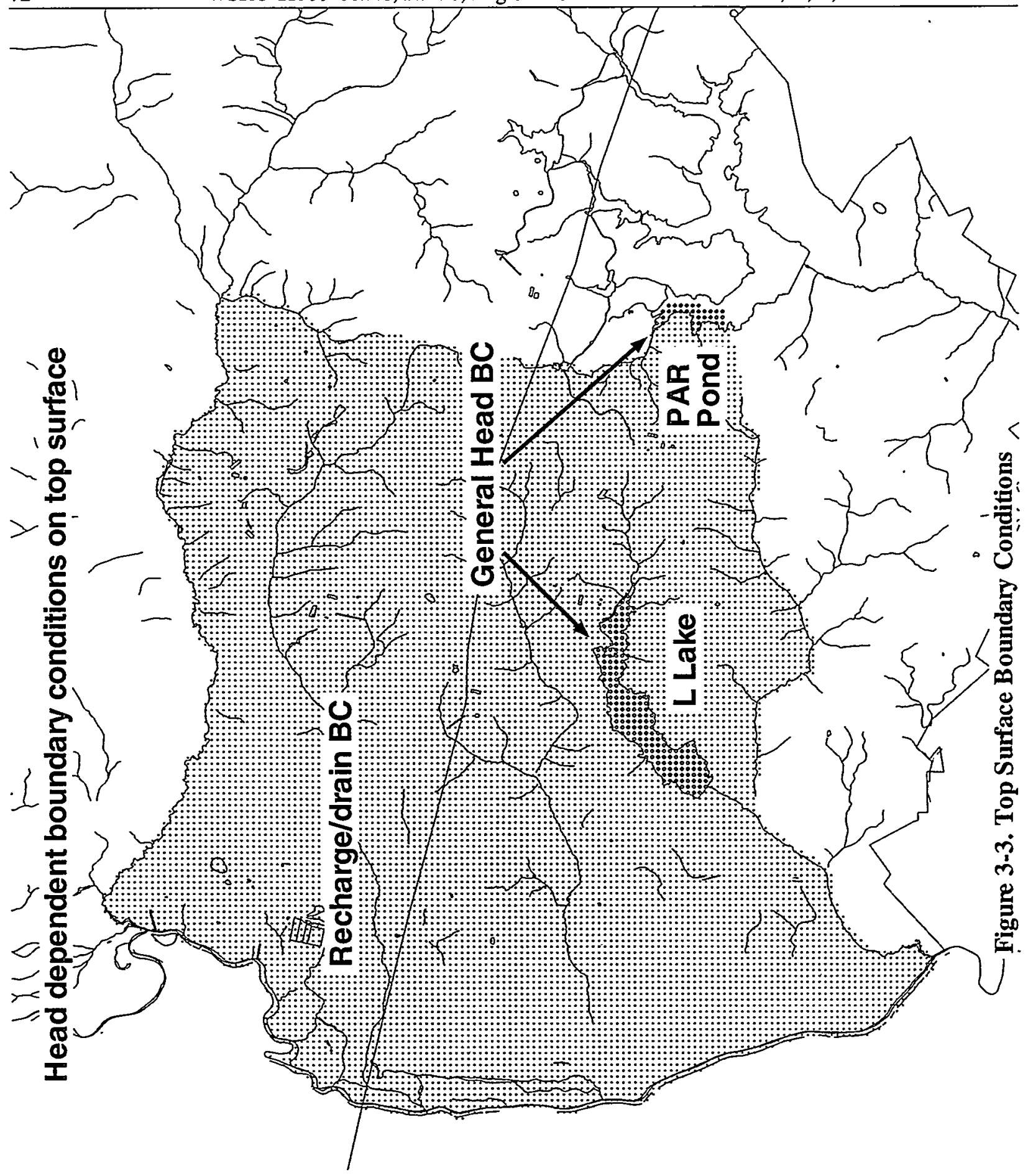




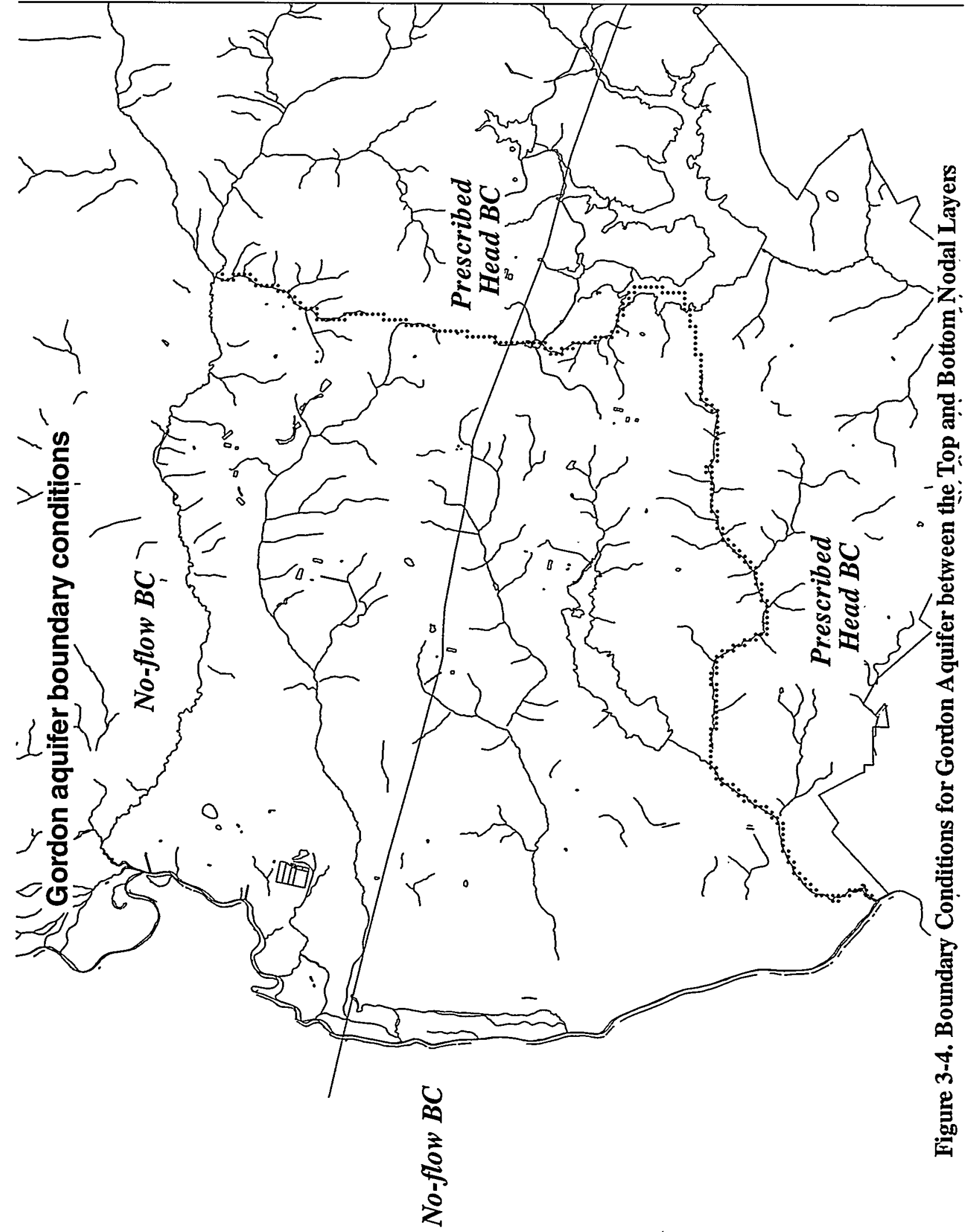




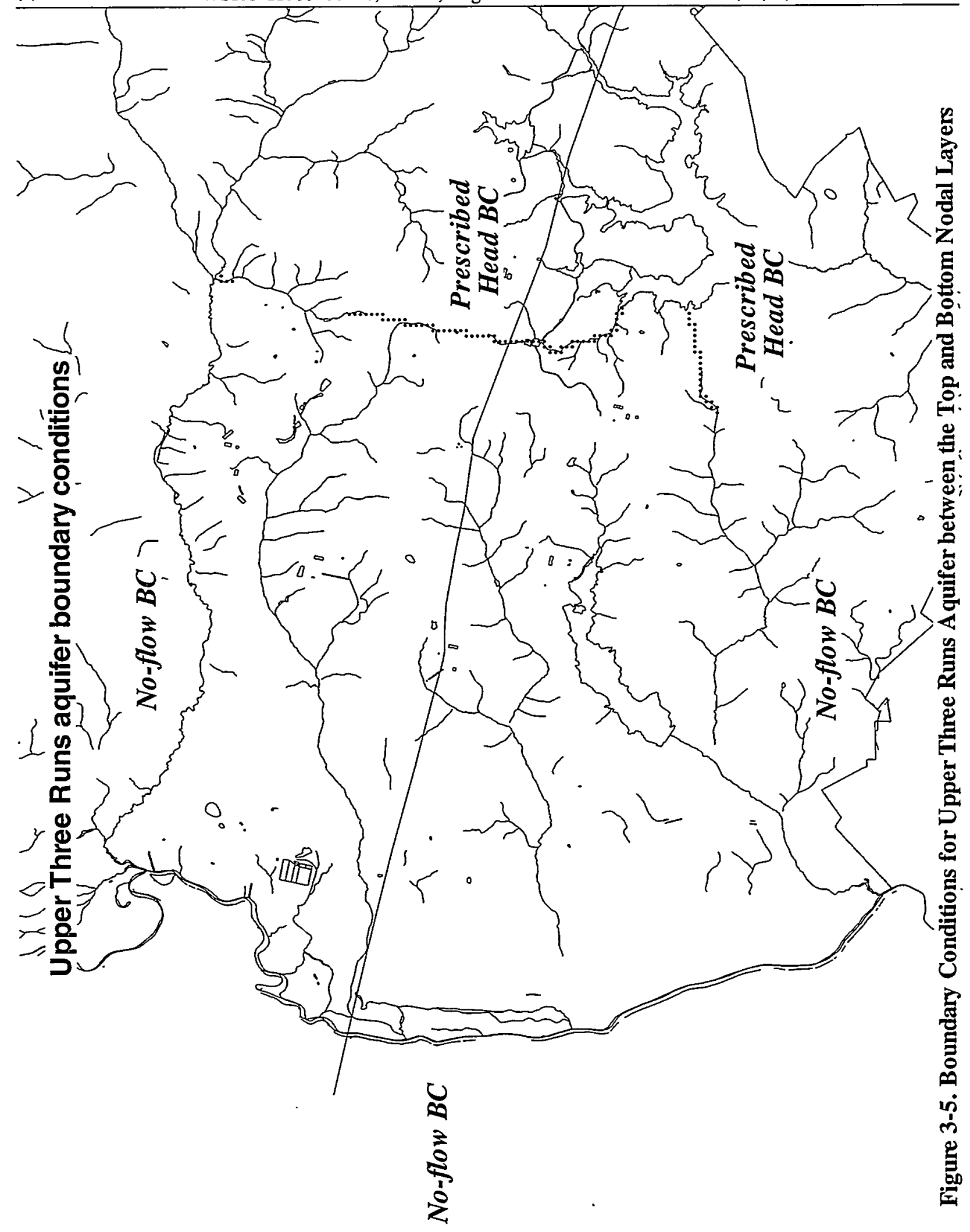




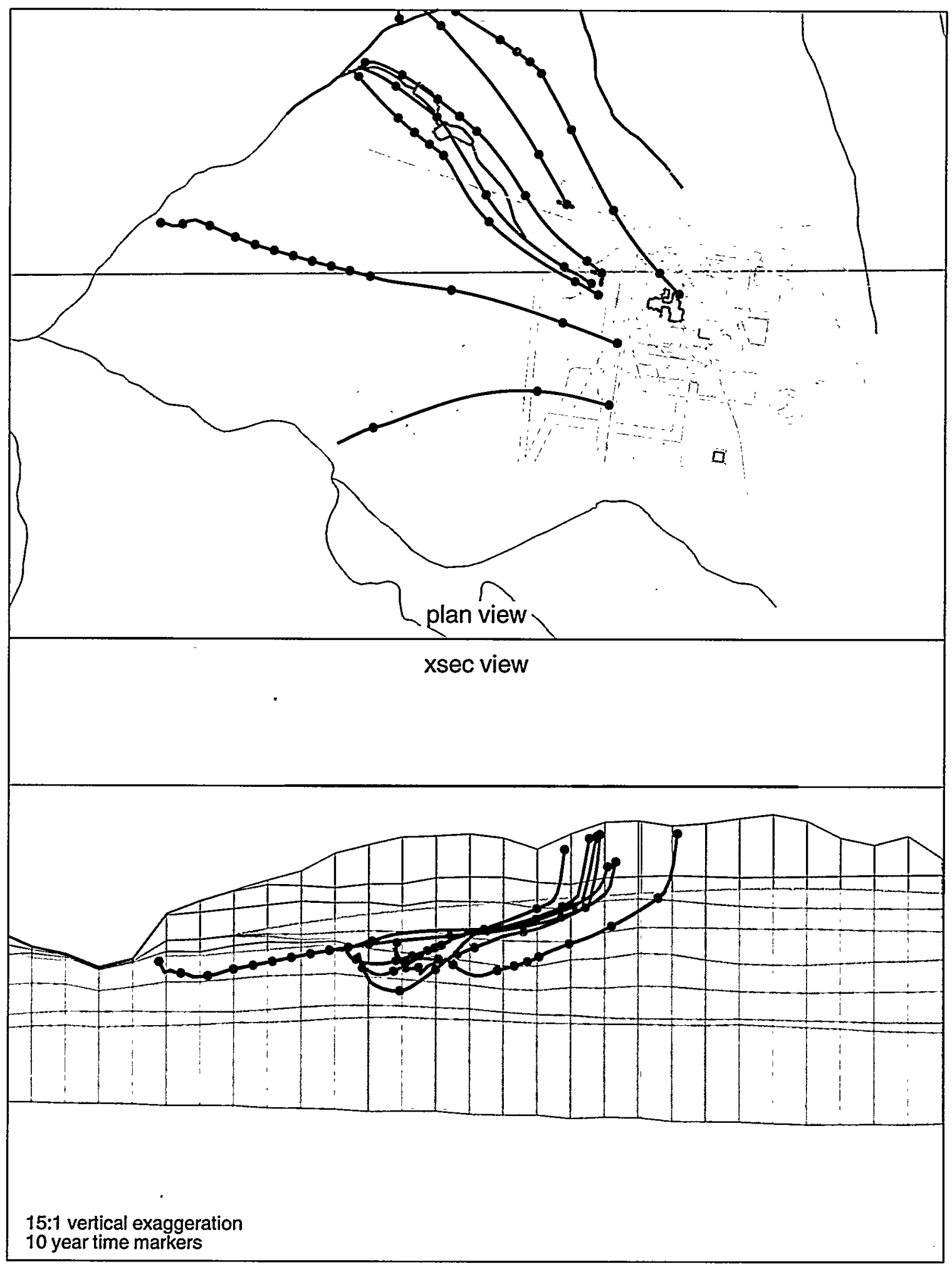

Figure 3-6. Predicted Groundwater Flow Paths Prior to Refinement of "Tan Clay" Model Layer in C-Area near Caster Creek 


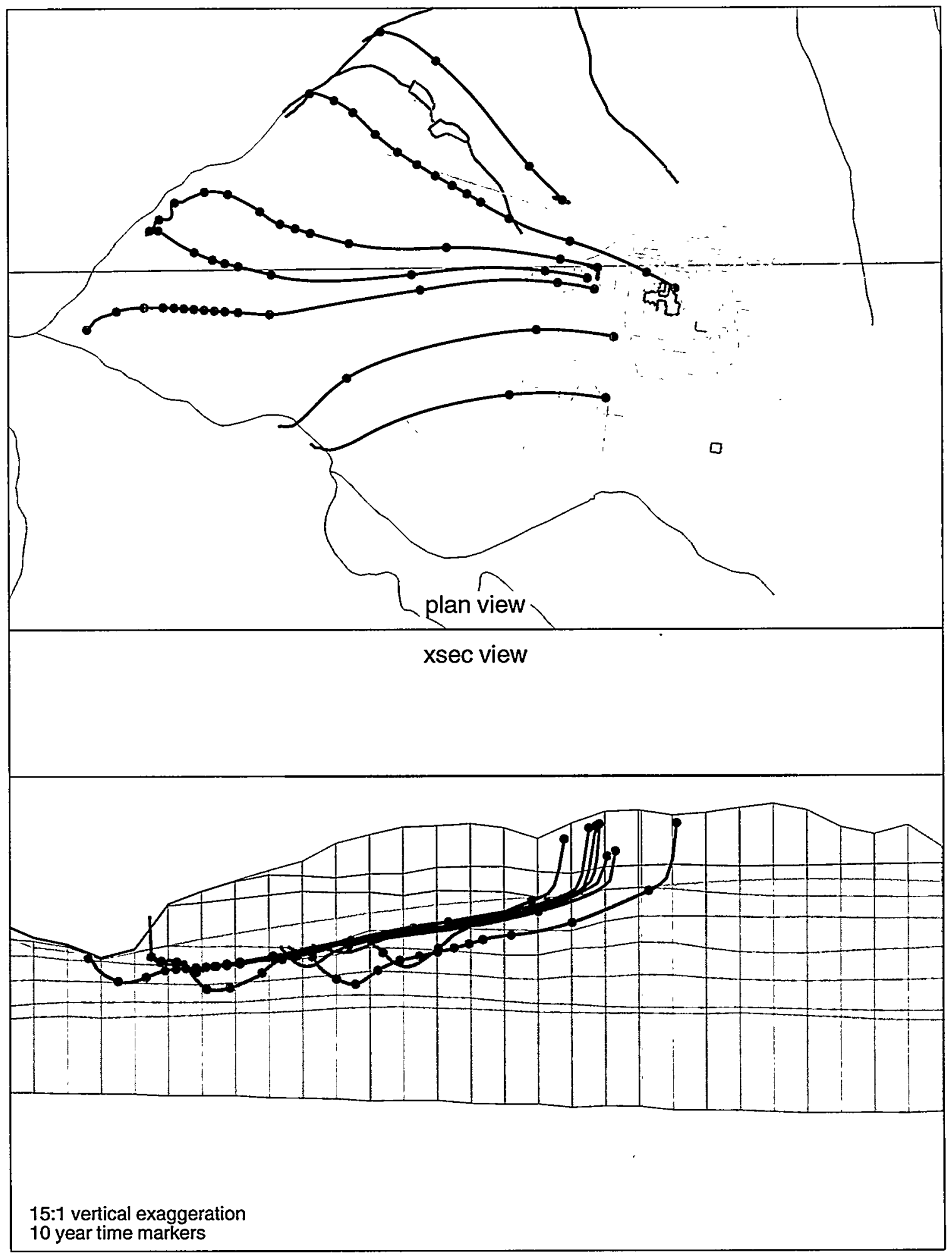

Figure 3-7. Predicted Groundwater Flow Paths after Refinement of "Tan Clay" Model Layer in C-Area near Caster Creek 


\subsection{GROUNDWATER FLOW MODEL RESULTS}

\subsection{Calibration Results}

Table 4-1 summarizes the calibration results for groundwater flow targets. The maximum rate of local recharge is set to $12.5 \mathrm{in} / \mathrm{yr}$ in the FACT recharge/drain boundary condition, except for the General Separations Area (Section 3.3). The modeled rate is $17 \%$ lower than the prior estimate of $15 \mathrm{in} / \mathrm{yr}$, but very close to the midpoint of the uncertainty range ( $13 \mathrm{in} / \mathrm{yr})$. Based on total area, which includes the Savannah River flood plain and other wetland areas, the average recharge rate is $9.0 \mathrm{in} / \mathrm{yr}$.

Excellent agreement is observed for Pen Branch and Fourmile Branch base flow, the most reliable targets. For Meyers Branch, the agreement is acceptable, being within the estimated confidence interval. Simulated base flow to Upper Three Runs between Road C and Road A is $40 \%$ higher than the prior estimate, but still within the uncertainty interval. A possible explanation is that Upper Three Runs receives significantly more base flow from the south side (model side), due to significantly steeper terrain and aquifer head gradients compared to the north side. The prior estimate is based on the assumption that base flow should be partitioned equally to each side. The model predicts L-Lake to be losing overall, in qualitative agreement with the fact that make-up water is required to maintain a historic level of $190 \mathrm{ft}$. However, a large discrepancy is noted for the combined baseflow for Steel Creek and L Lake. The data suggests a large net loss of $2.2 \mathrm{ft}^{3} / \mathrm{s}$ for this reach. While the model predicts L Lake to be losing at a rate of $0.3 \mathrm{ft}^{3} / \mathrm{s}$, Steel Creek gains at a rate of $4.1 \mathrm{ft}^{3} / \mathrm{s}$ for a net gain of 3.8 $\mathrm{ft}^{3} / \mathrm{s}$. A likely explanation is large uncertainty in the hydrograph estimate due to taking the difference of several numbers that are large compared to the balance. The small portion of Par Pond within the model domain is simulated to be gaining, as would be expected for an area away from the dam. Overall, the calibration goals for large-scale groundwater flow are met.

Also shown in Table 4-1 is a comparison to selected single-time field measurements. Excellent agreement is observed in C-area for Caster Creek and a creek receiving outfall from Central Shops. For K-area, the agreement between predicted and measured stream flow is poor. As noted in Section 2.4, the measured flow in Pen Branch below its confluence with Indian Grave Branch (15.8 cfs, Table 2-4) exceeds the estimated base flow for the entire stream (13.3 cfs, Table 2-3). Possibly the stream flow measurements in K-area are biased high. Conversely, the large gain along Pen Branch between Road B and Indian Grave Branch 
may be due to groundwater discharge induced by nearby L Lake. The model may be underestimating the flow from L Lake to Pen Branch.

Table 4-2 summarizes the calibration results for hydraulic head targets. Figure 4-1 graphically compares simulated head with measured head for each aquifer zone. Figures 4-2 through 4-6 illustrate the spatial distribution of head residuals. Appendix F contains a detailed listing of head residual information. The root-mean-square residual is $5.45 \mathrm{ft}$, which is acceptable compared to the calibration goal of $5 \mathrm{ft}$. The mean-absolute residual, which gives lower weight to outliers, is $4.0 \mathrm{ft}$ and well within the calibration goal. The residuals are largely unbiased, although the lower aquifer zone of the Upper Three Runs aquifer is biased high by more than $1 \mathrm{ft}$.

Agreement is excellent in the Gordon aquifer, except for 5 double-digit outliers. At TNX Area, the simulated heads for TBG-5B and P-26A are 13 to $16 \mathrm{ft}$ low, while the residual at nearby XSB-1A is only $-0.1 \mathrm{ft}$. The scale of the model appears to be too large to reproduce the sudden change in head in this area. In $\mathrm{K}$ Area, simulated head at P-25B is $14 \mathrm{ft}$ low. A possible explanation is that the Gordon aquifer is experiencing high recharge through the Pen Branch Fault. Northeast of P-25B, the residual for PW- $83 \mathrm{~N}$ is $+10 \mathrm{ft}$. Here the large discrepancy may be due to the target value being a single reading that is not reflective of the long-term average water level. In the General Separations Area, the measured water level at $\mathrm{BGO}-10 \mathrm{~A}$ is $13 \mathrm{ft}$ higher than the model prediction. However at BGO-10AA which is screened deeper, the agreement is excellent. The average Gordon aquifer residual is biased slightly low by $0.7 \mathrm{ft}$.

Large residuals are observed within the "lower" aquifer zone of the Upper Three Runs aquifer in the C-Area vicinity. In this area, the "lower" aquifer zone is interpreted to contain zones of calcareous sediment with low-permeability. These zones may serve to elevate the hydraulic head beneath the "tan clay" confining zone. These zones are largely unaccounted for in the model and may explain the large residuals. Other areas, such as the Chemicals, Metals and Pesticides (CMP) Pits, also contain double digit residuals. The discrepancies are likely caused by the heterogeneity in the "lower" aquifer zone that is not represented in the model. This heterogeneity is due to the fact that data points within the "lower" aquifer zone are relatively scarce and very widespread in areas outside of the General Separations Area. The overall mean-absolute residual is $5.1 \mathrm{ft}$, and acceptable compared to the calibration goal of $5 \mathrm{ft}$.

Head residuals are generally lower in the "upper" aquifer zone of the Upper Three Runs aquifer containing the "transmissive" zone, "AA horizon" and "A/uu horizon" (Table 4-2). 
Excellent agreement is observed in $\mathrm{C}$-area and $\mathrm{K}$-area, where additional characterization data has been obtained over the last year. As with the lower zone, the larger residuals are a result of uncertainty in the geology of specific areas due to observed areas of heterogeneity in the UTRA. The average residuals for the "transmissive" zone and "AA horizon" are well within the calibration goal. The average " $\mathrm{A} / \mathrm{uu}$ horizon: residual is slightly higher than desired.

Table 4-3 summarizes the calibration results for hydraulic conductivity. Figures 4-7 through 4-14 show variation in conductivity for each model layer in plan view. Horizontal conductivity is shown for the transmissive zones, and vertical conductivity for confining zones. Figure 4-15 illustrates a typical north-south vertical slice, in this case passing through $\mathrm{K}$ Area. The calibrated values are consistent with field data (Section 2.2) and previous groundwater flow models (e.g. HSI GeoTrans, 1998, Figure 4-6; HydroGeoLogic, 1998, Table 6.5; Flach, 1998, Table 4; Flach and Harris, 1997; GeoTrans, 1993, Table 4.1; GeoTrans, 1992, Table 3.6; Camp Dresser \& McKee, 1989, Table 3-3).

\subsection{Nominal Simulation}

Figures 4-16 through 4-18 illustrate simulated hydraulic head averaged over the entire thickness of the Gordon aquifer and "lower" and "upper" aquifer zones. Simulated head in the aquifer zone containing the water table is shown in Figure 4-19, and Figure 4-20 illustrates simulated water table elevation. For comparison to Figure 4-15, see Figure 2-20 which shows the Gordon potentiometric surface as based on measured water levels. The estimated water table based directly on head data is shown in Figure 2-14, and can be compared to Figures 417 through 4-20. Figures 4-21 through 4-23 illustrate flow directions that are vertically averaged over the entire thickness of the aquifer zones. Figure 4-24 shows simulated seepage faces, and Figure 4-25 illustrates rates of recharge and discharge. Figures 4-24 and 4-25 can be compared to Figure 2-21, which is based on field observations. Example particle tracing results are shown in Figure 4-26. A water balance for the model is depicted in Figure 4-27.

\subsection{Uncertainty Analysis}

Uncertainty in the nominal model can be estimated by varying the input parameters within their uncertainty range, and in a correlated manner such that agreement with calibration targets is preserved as much as possible. The nominal model is sensitive to recharge, which drives overall groundwater flow in this system, and Gordon confining unit (GCU) vertical conductivity $\left(\mathrm{K}_{\mathrm{V}}\right)$, which controls recharge to the Gordon aquifer (equal to leakance from the Upper Three Runs aquifer). Both of these input parameters have significant uncertainty. 
Table 4-4 summarizes four variations of these two parameters within their estimated range of uncertainty. For each uncertainty case, the model is recalibrated to maintain agreement with the prior head targets by adjusting conductivity values in the Upper Three Runs aquifer, and Gordon aquifer if necessary. Table 4-5 summarizes the calibration results for each sensitivity case.

Uncertainty cases 1 and 2 involve perturbations to the maximum local recharge rate of $\pm 20 \%$. As seen in Table 4-5, the results for cases 1 and 2 show equivalent agreement to hydraulic head targets compared to the nominal or base case. For higher recharge (case 1), predicted base flows are biased high for Pen Branch and Fourmile Branch, the most reliable targets. For lower recharge (case 2), simulated base flows are low for these streams. Horizontal conductivities in the "upper" and "lower" UTRA aquifer zones were adjusted by $+30 \%$ and $-26 \%$ to compensate for the recharge variations in cases 1 and 2, respectively. The resulting $\mathrm{K}_{\mathrm{h}}$ values for the uncertainty cases remain well within the data uncertainty range. No changes were made to the Gordon aquifer unit horizontal conductivity, or vertical conductivity in confining units/zones.

Uncertainty cases 3 and 4 involve increases and decreases to Gordon vertical conductivity by a factor of 5. For these cases, adjustment to Gordon aquifer unit horizontal conductivity was also required to maintain agreement with head targets, to the extent possible. Despite model recalibration, uncertainty cases 3 and 4 show significantly poorer agreement to calibration targets compared to the nominal case. For higher Gordon confining unit leakance, head residuals are large, and Pen Branch and Fourmile Branch base flows are significantly biased low. Horizontal conductivities for both the Gordon and UTR aquifers are barely credible. For lower Gordon confining unit leakance, head residuals are similar to the base case, and uncertainty cases 1 and 2. Simulated base flows for Pen Branch and Fourmile Branch are biased high. Reasonable horizontal conductivities are obtained for the UTR aquifer. However, the Gordon aquifer horizontal conductivity is significantly low compared to field data.

More detailed information about each uncertainty analysis case is presented in Appendix $\mathrm{H}$. In the appendix, model results in various forms are reproduced for each uncertainty case for comparison to the nominal results (Figures 4-1 through 4-14, 4-16 through 4-20, 4-25 and 4-26).

The uncertainty results presented here are generic. For specific applications of the model, additional uncertainty analysis should be performed, tailored to the sub-region and output 
parameter(s) of interest. For example, uncertainty cases 5 and 6 shown parenthetically in Table 4-4 would be useful for investigating uncertainty in plume migration, because they effectively provide upper and lower bounds on horizontal flow rates. Similarly, effective porosity should be considered for groundwater travel time and transport uncertainty analysis, because pore velocity is inversely proportional to this parameter. Specifically, transport sensitivity runs should include total porosity for an upper estimate $(\sim 40 \%)$, and a conservative (low) estimate for effective porosity $(\sim 25 \%)$. 

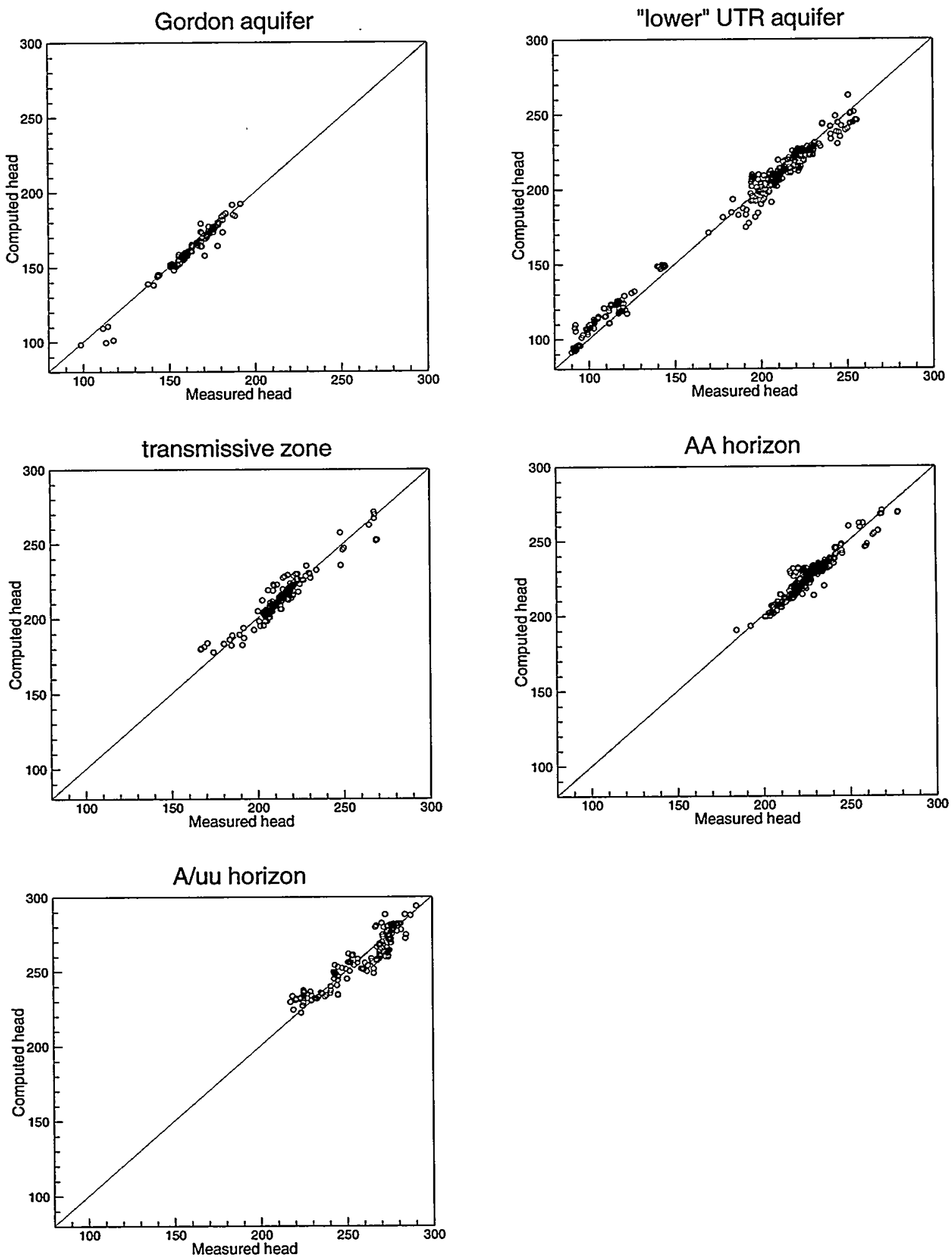

Figure 4-1. Simulated versus Measured Head for Each Aquifer Zone 


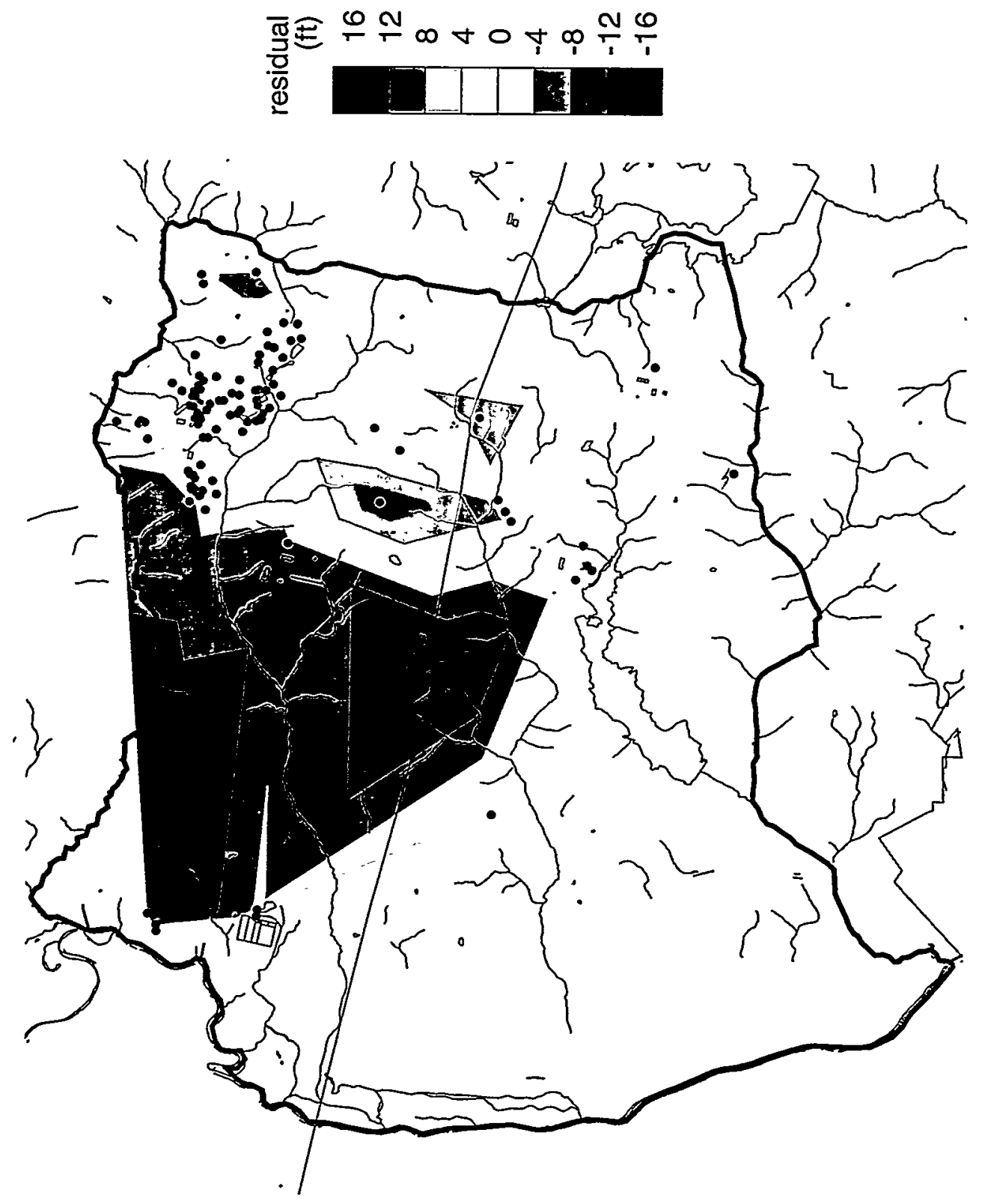



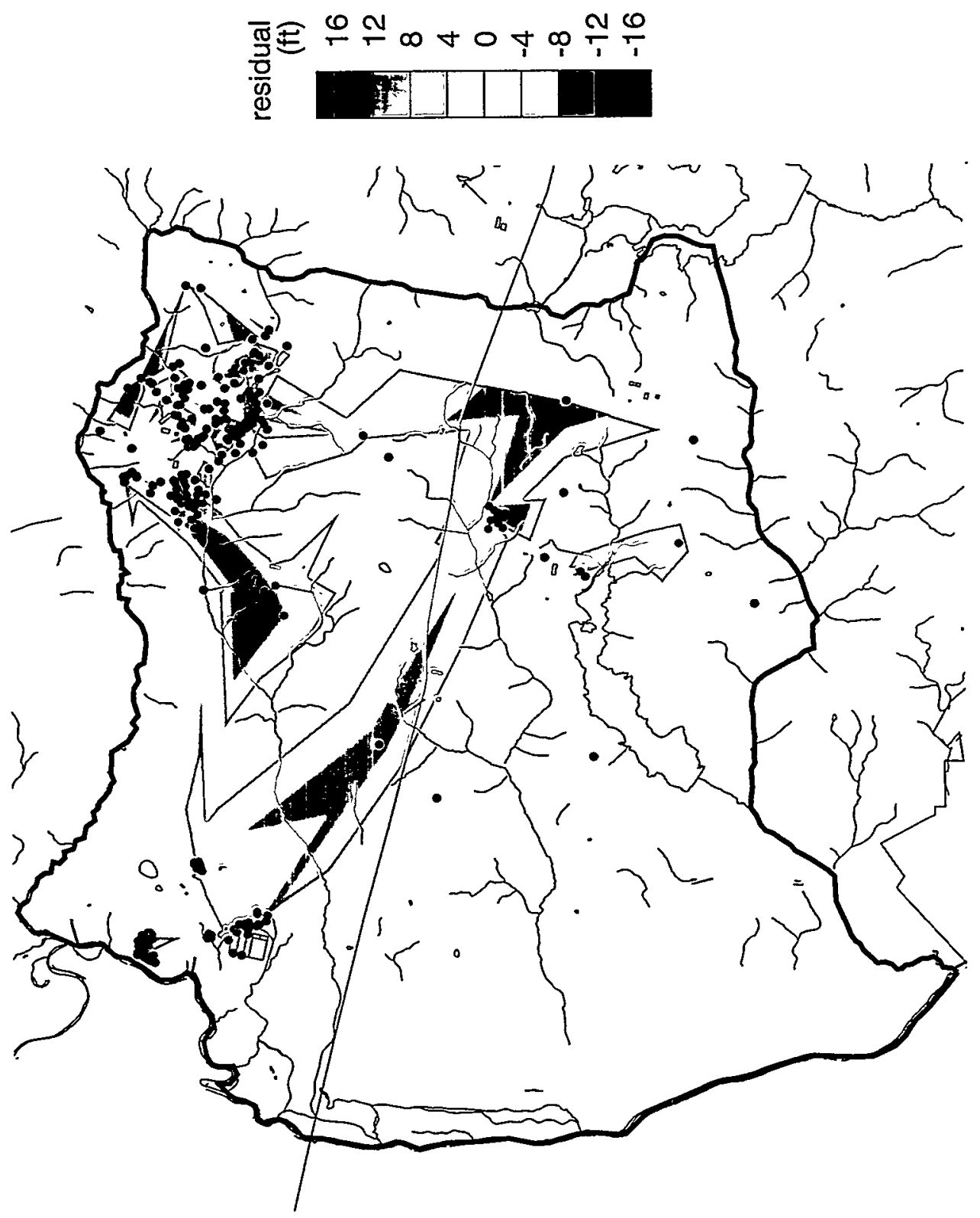

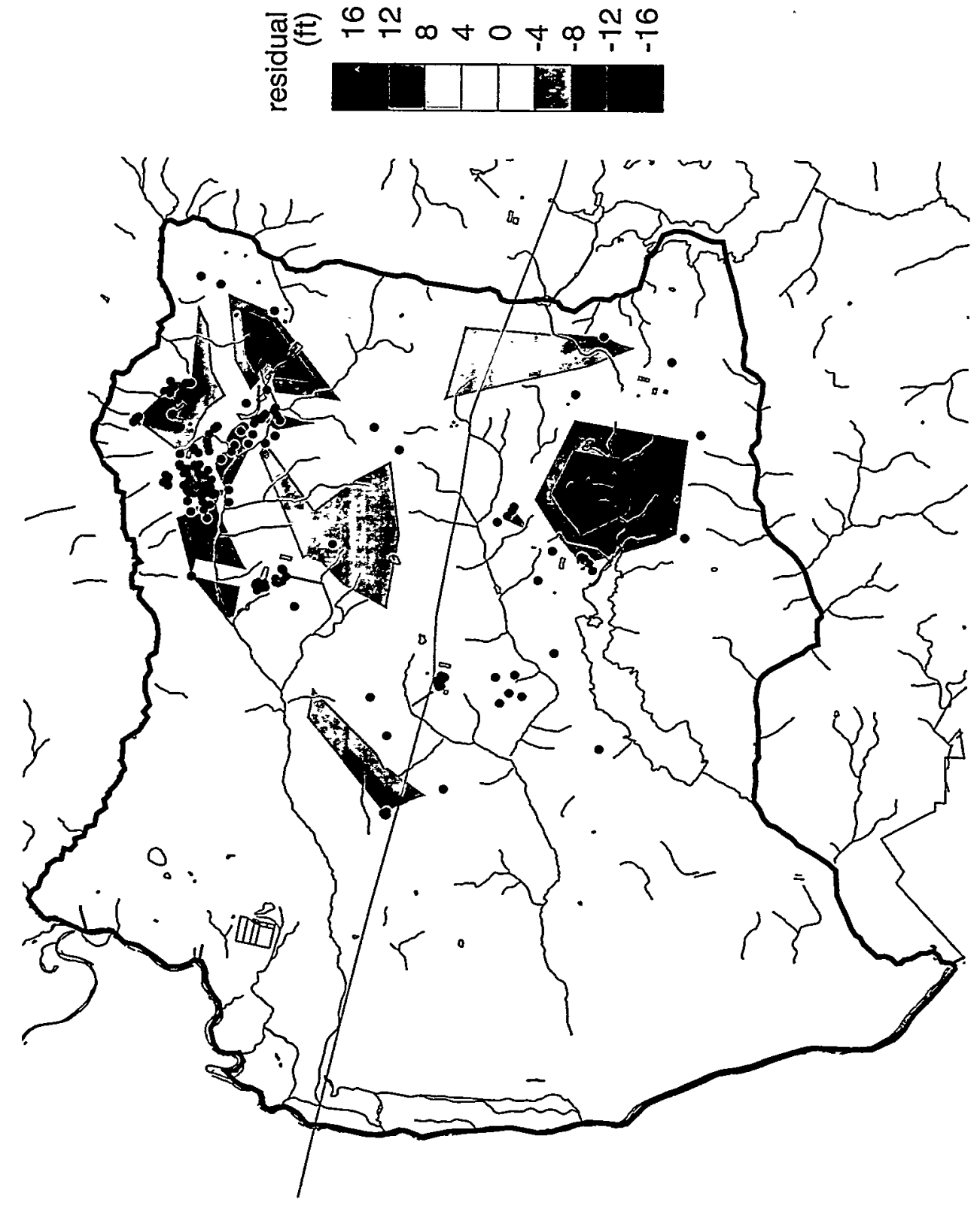

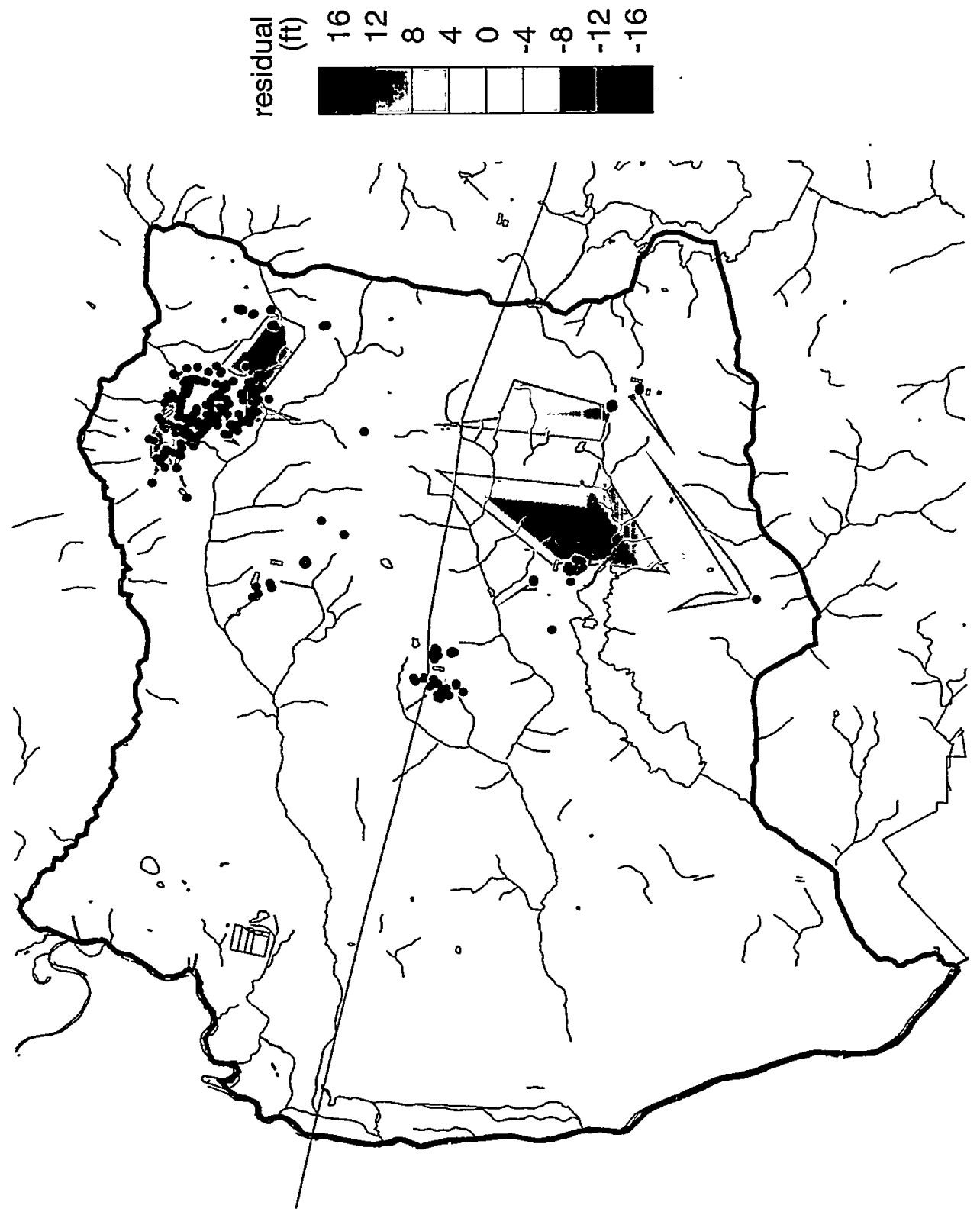


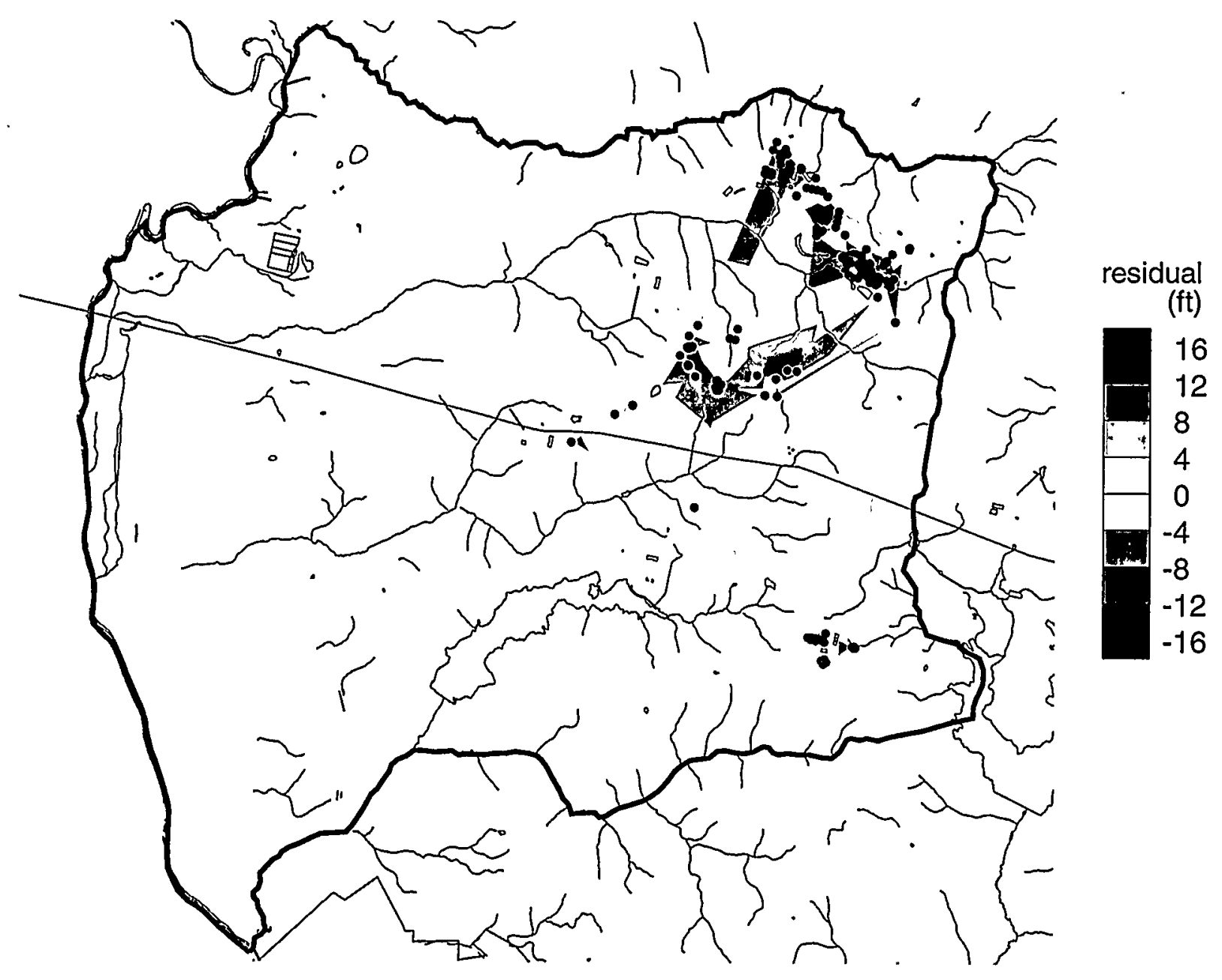




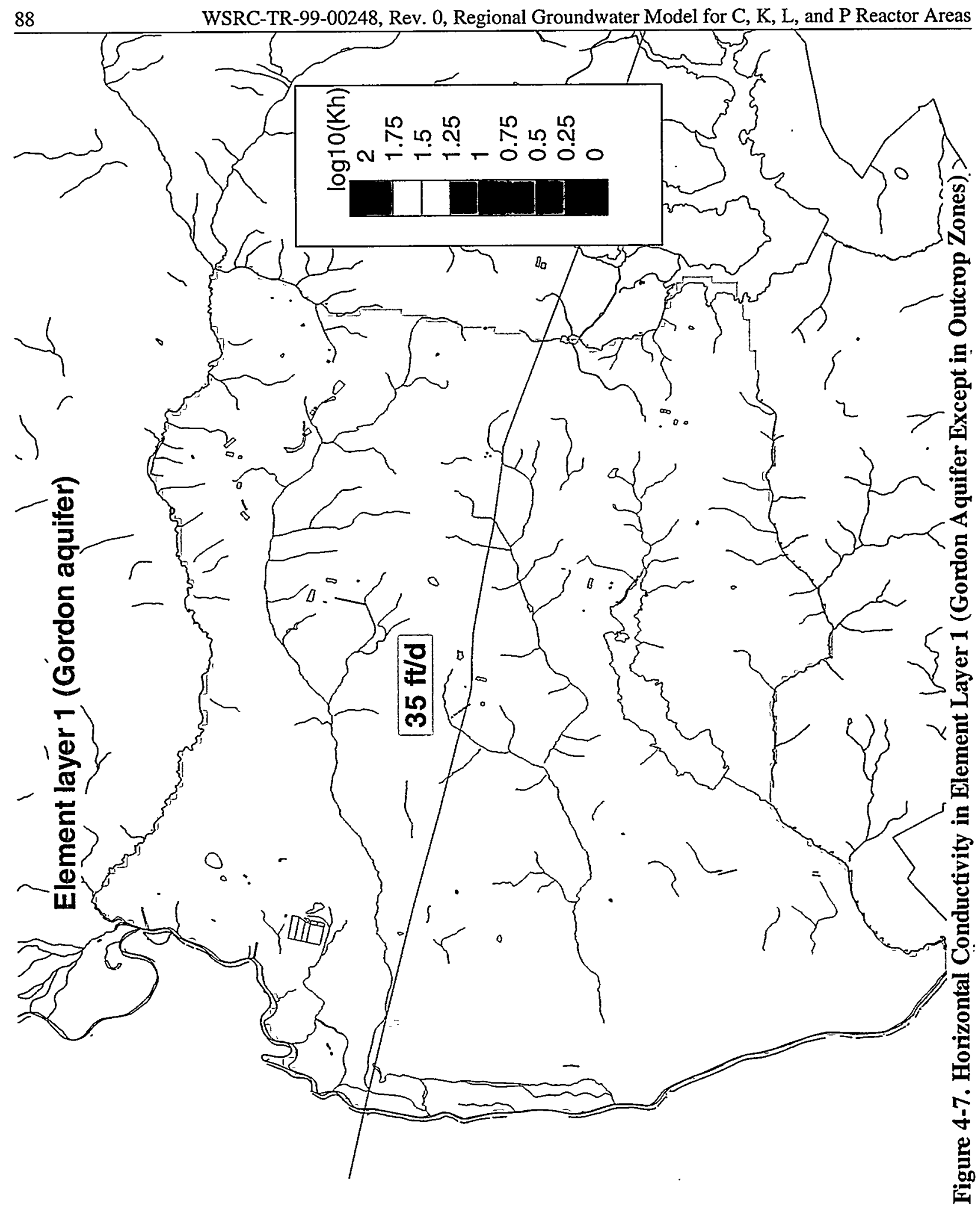




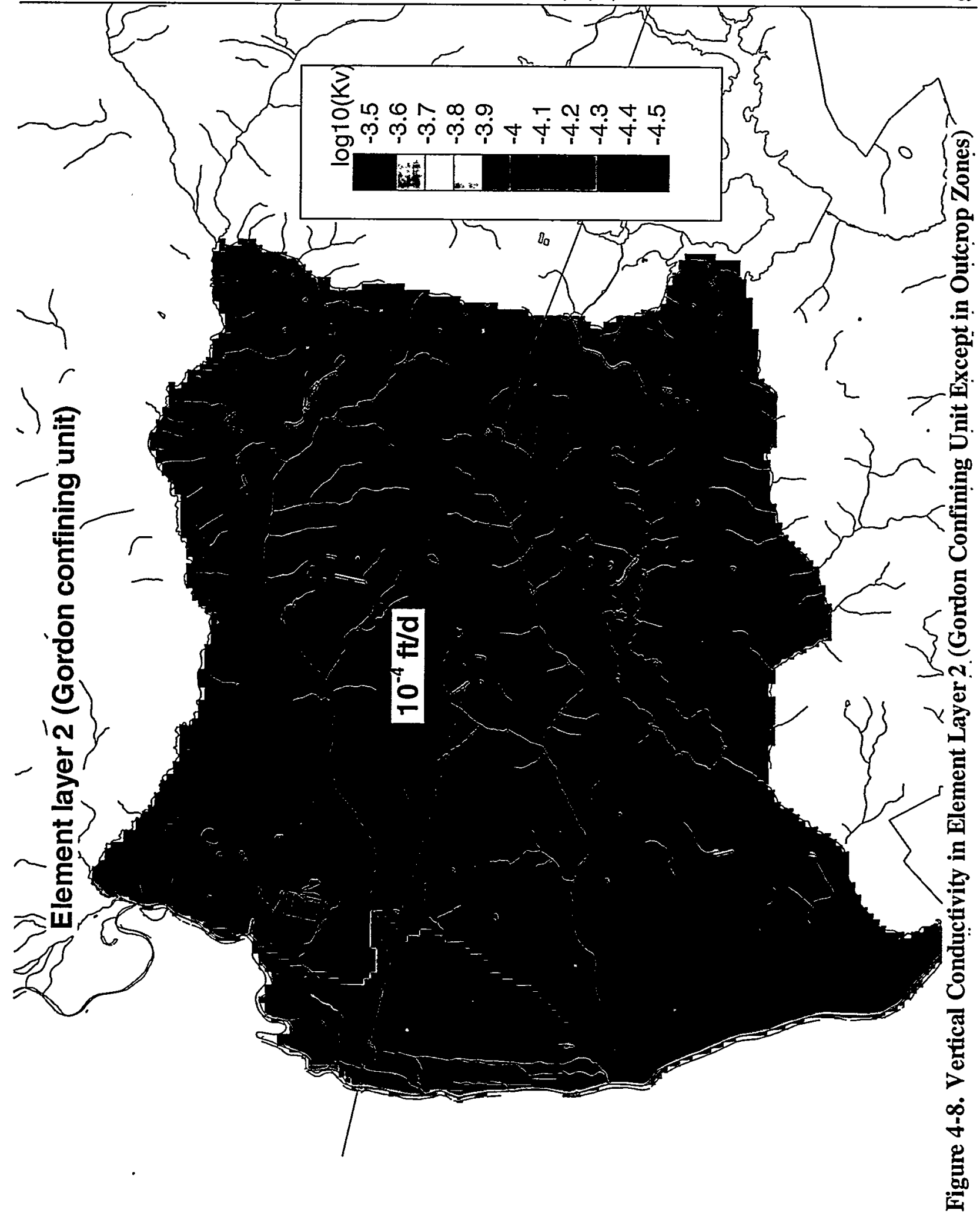




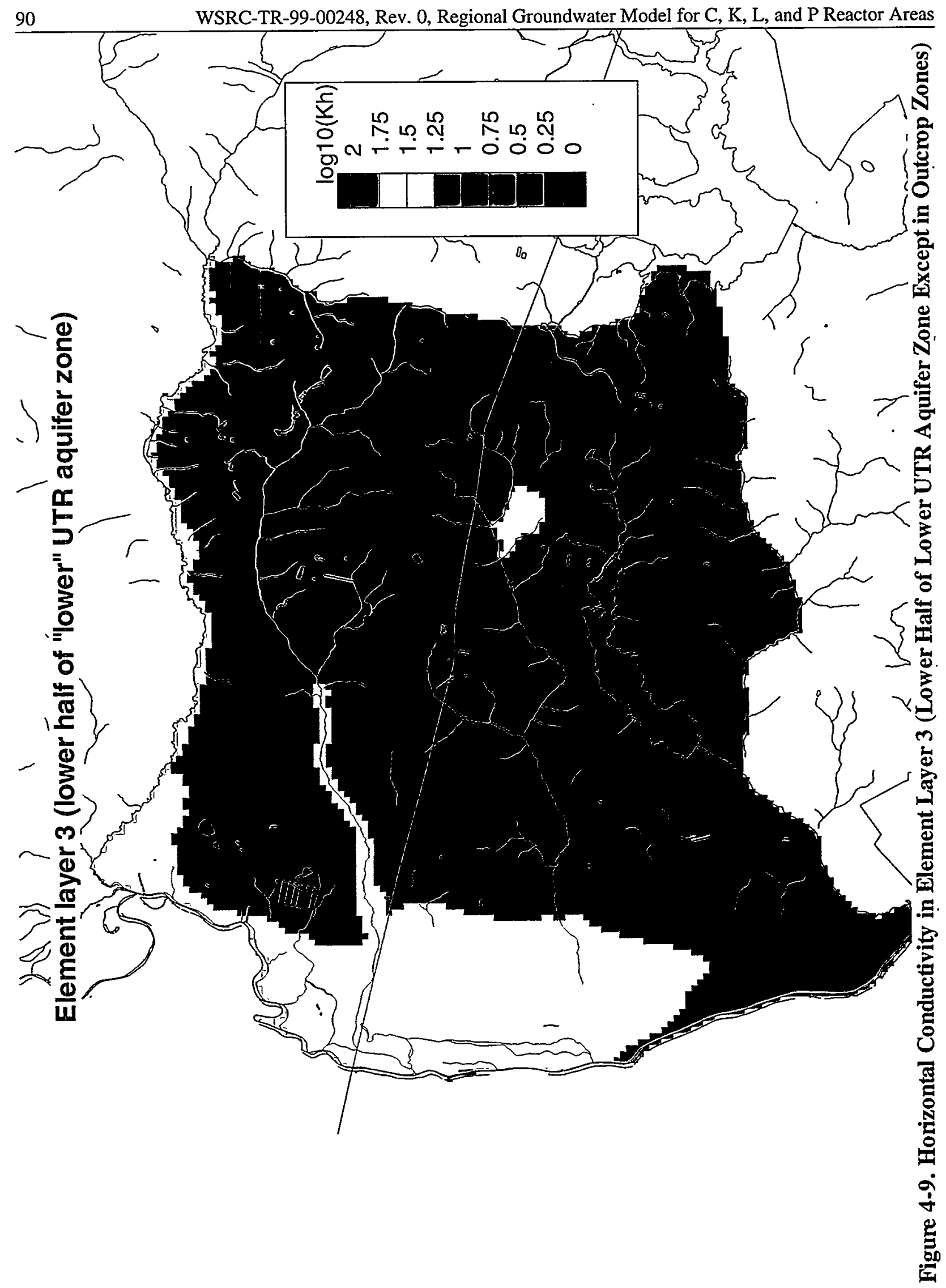




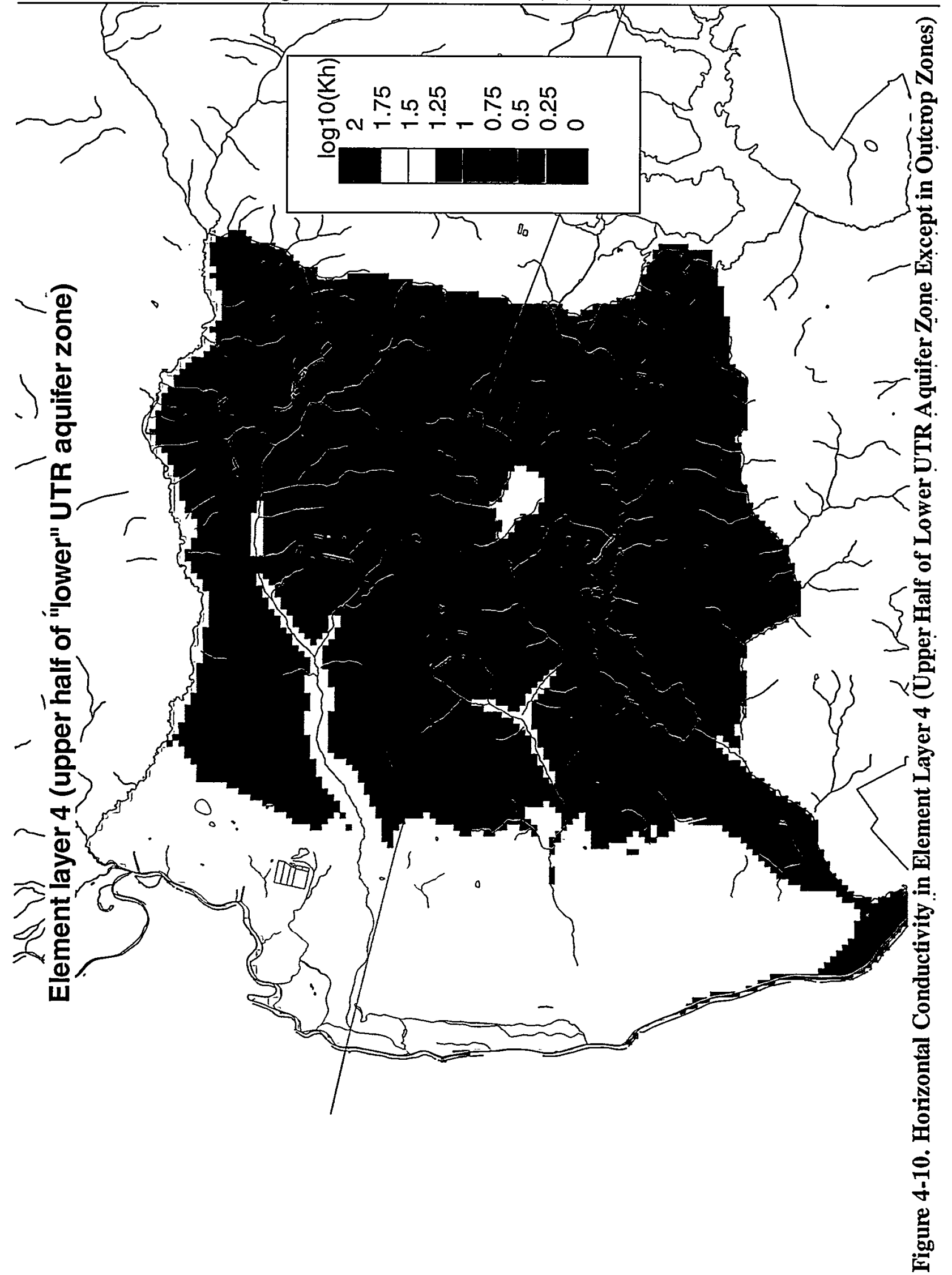




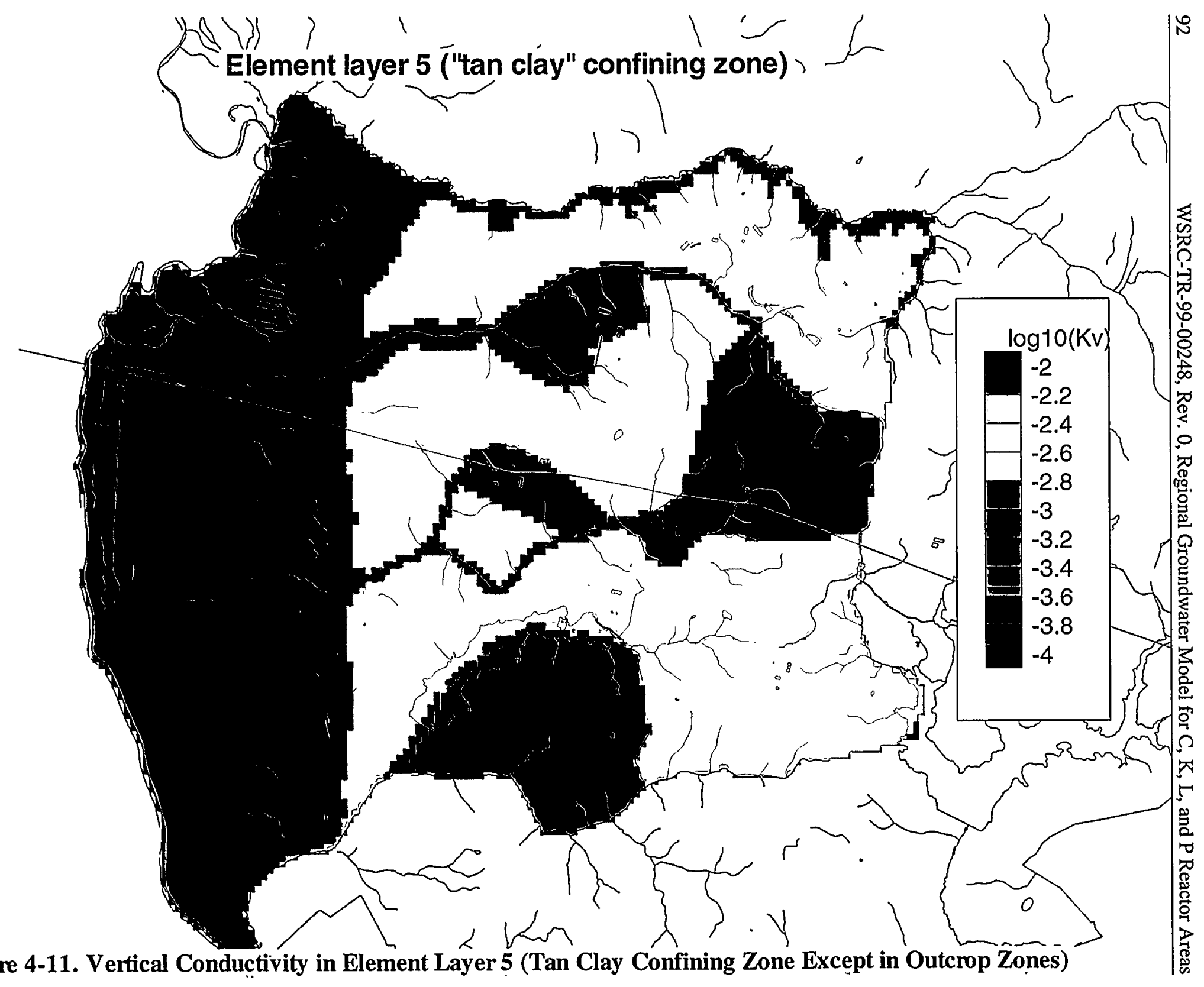




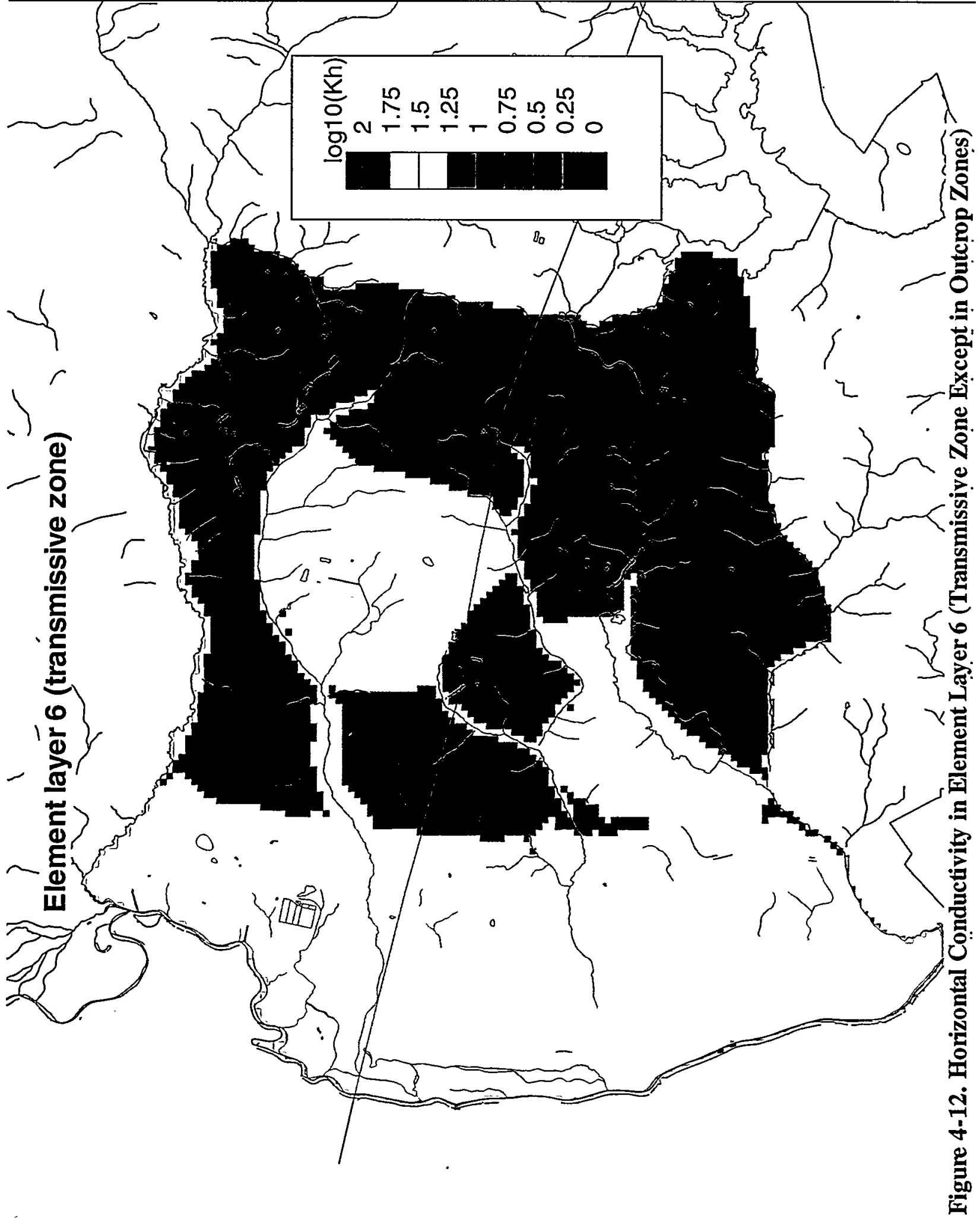




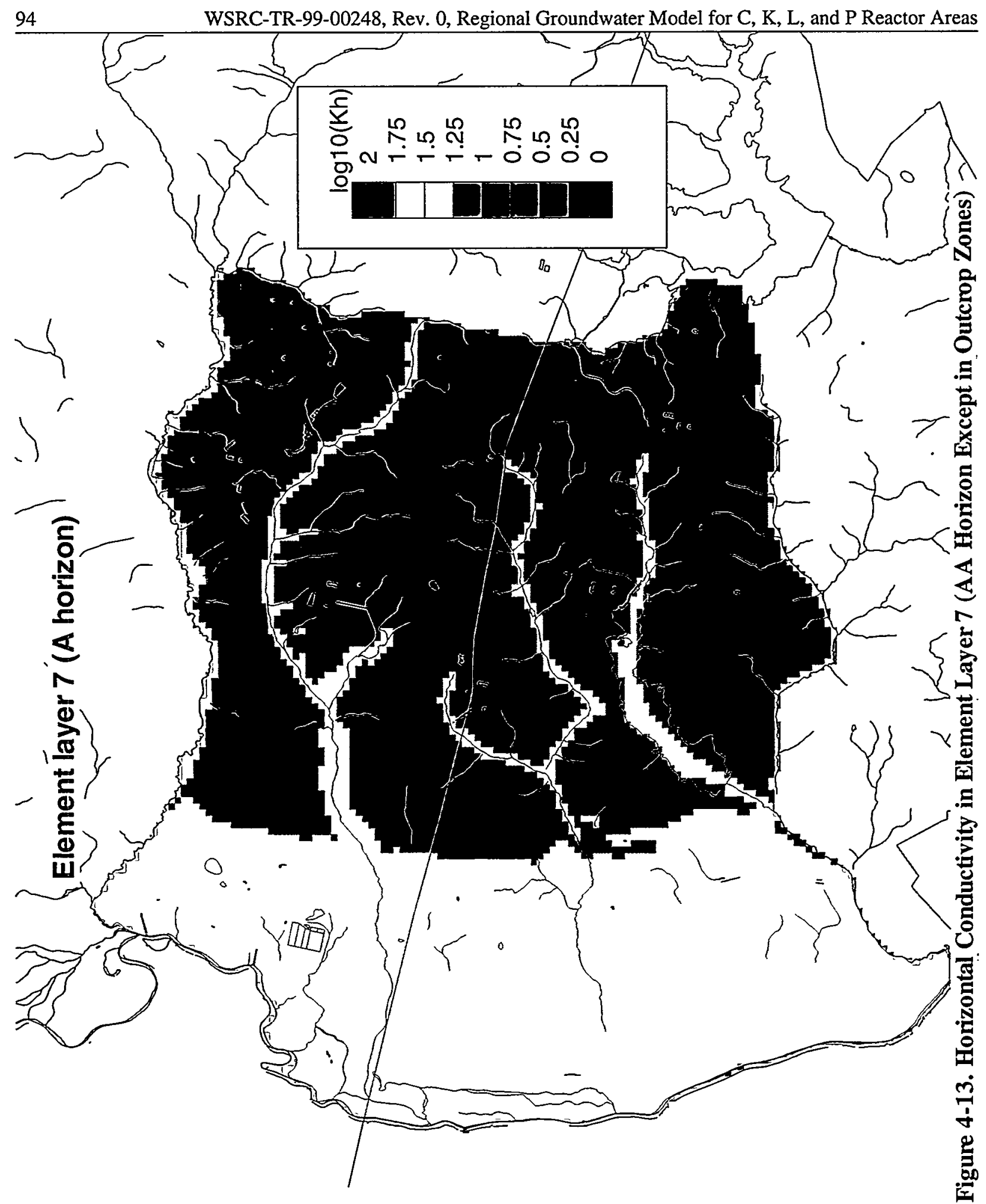




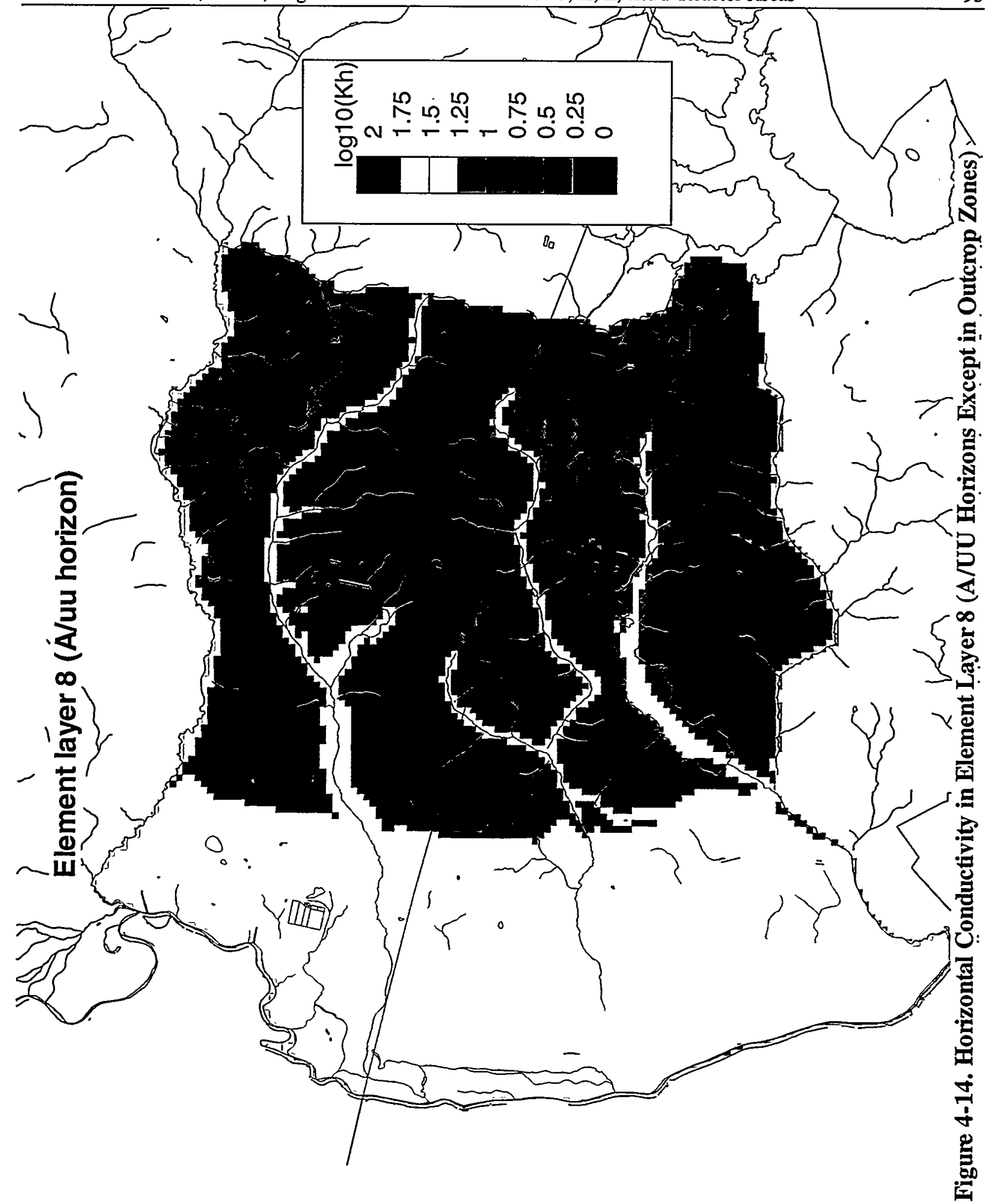



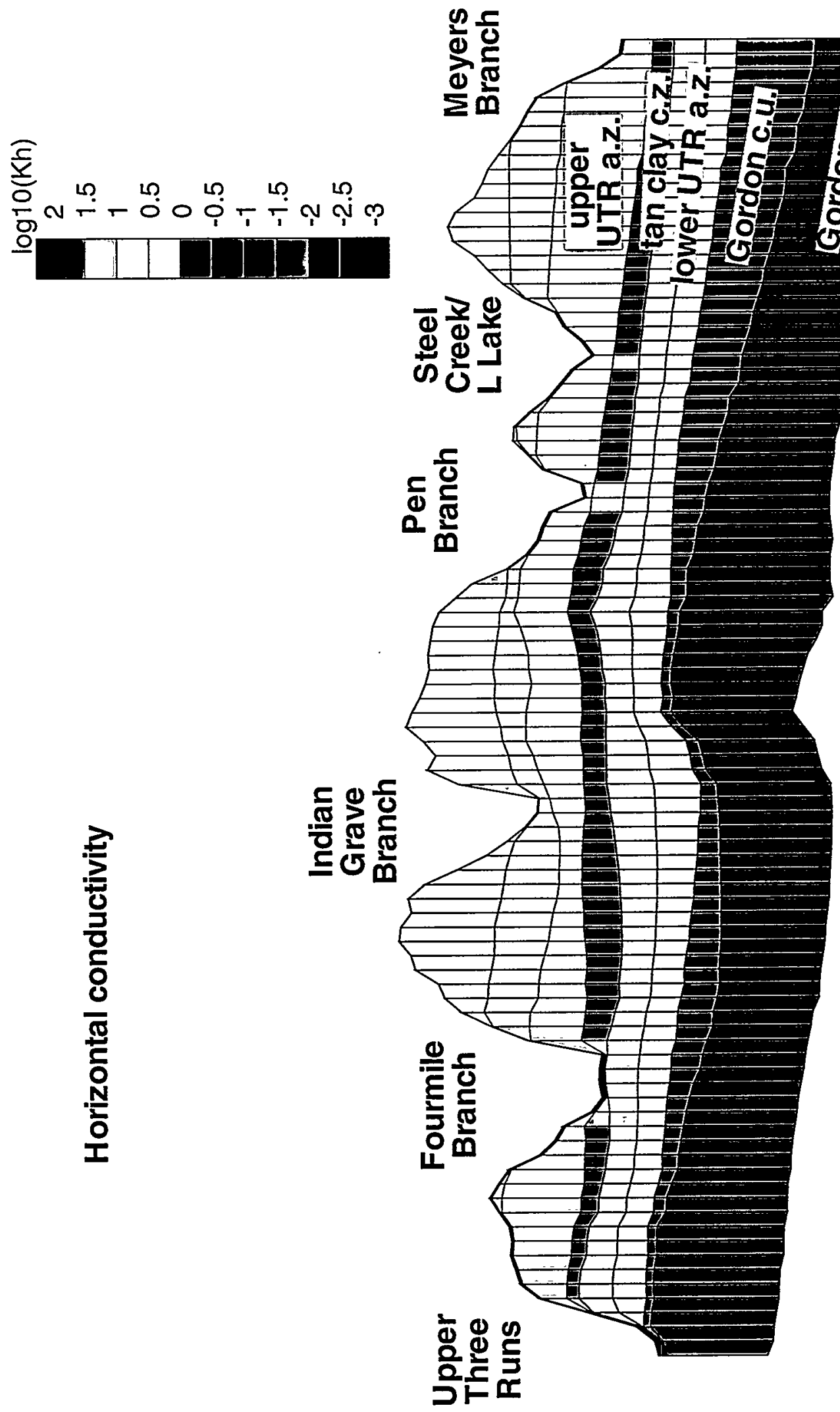

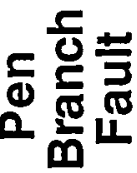

홍

옹

(5)

㿣

퐁

동

15

$\stackrel{8}{8}$

禺 
Simulated hydraulic head in Gordon aquifer

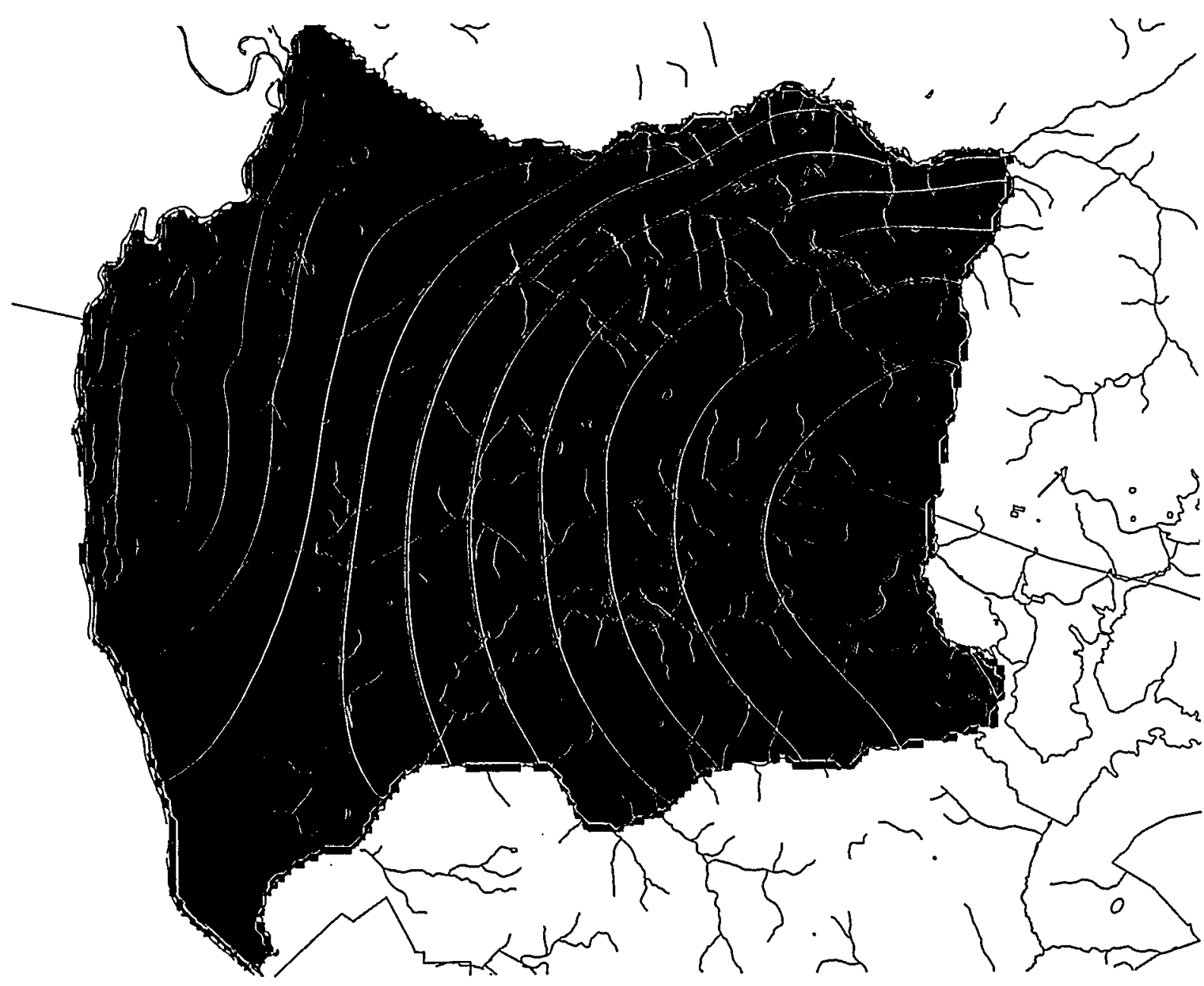

head

300 
Simulated hydraulic head in "lower" UTR aquifer zone

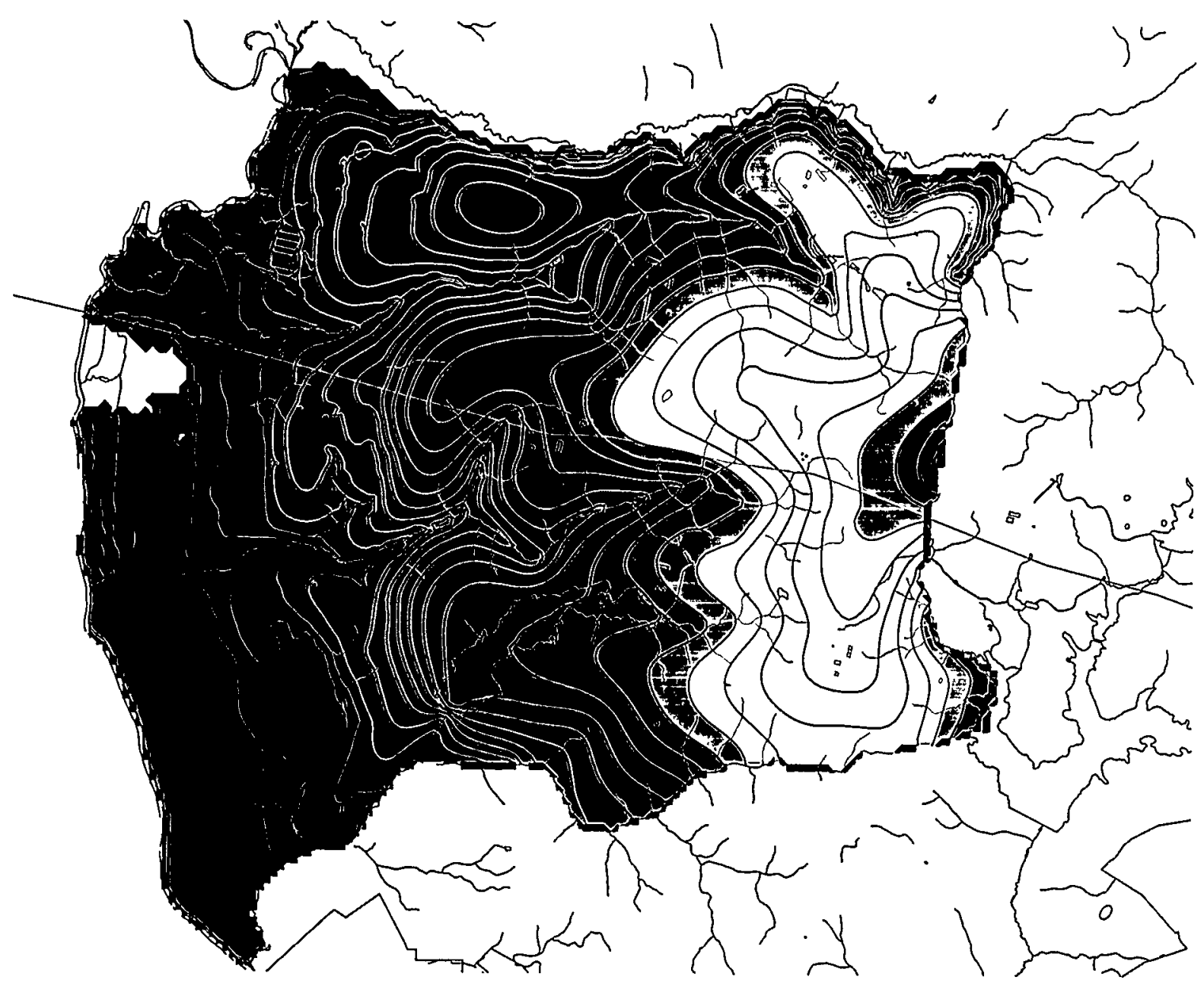

Figure 4-17. Simulated Hydraulic Head in the Lower UTR Aquifer 


\section{Simulated hydraulic head in "upper" UTR aquifer zone}

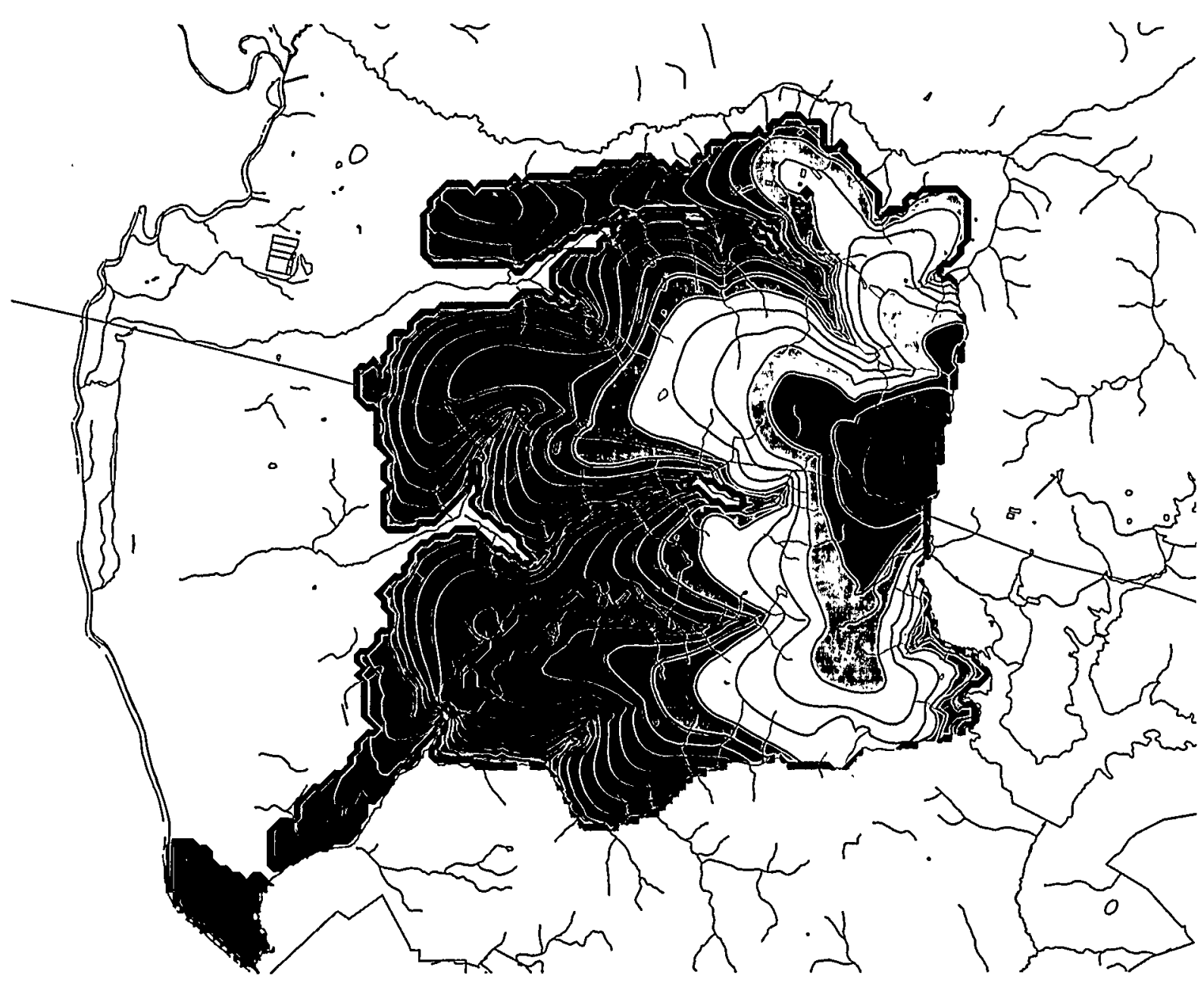




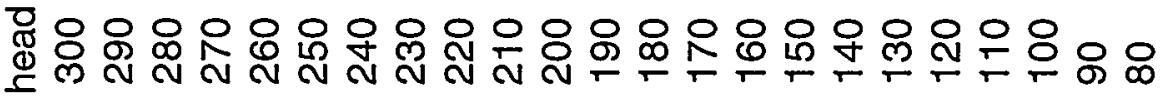

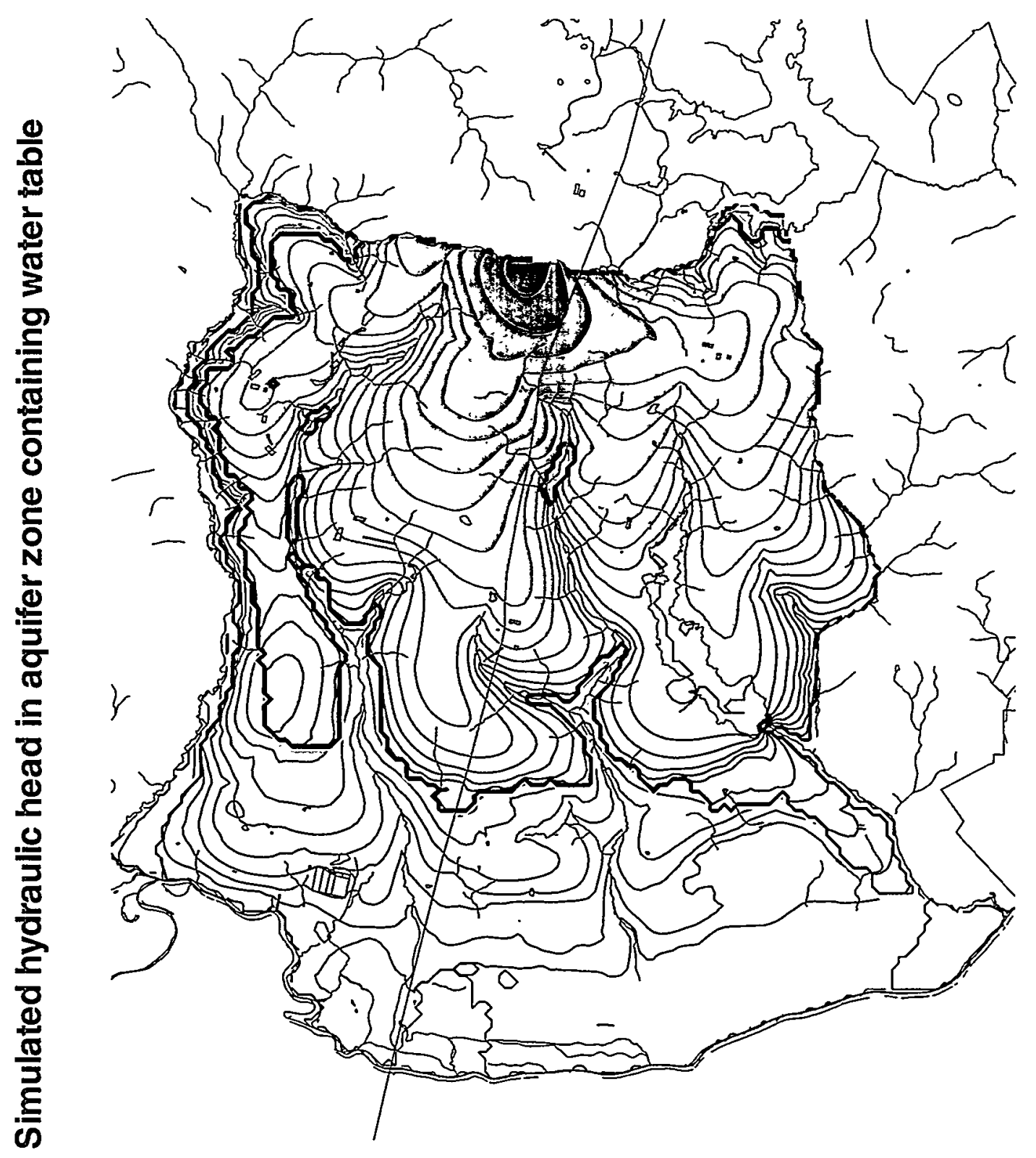

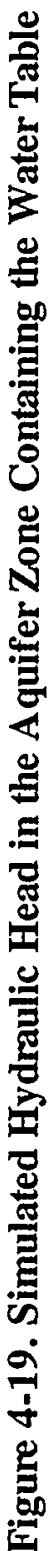




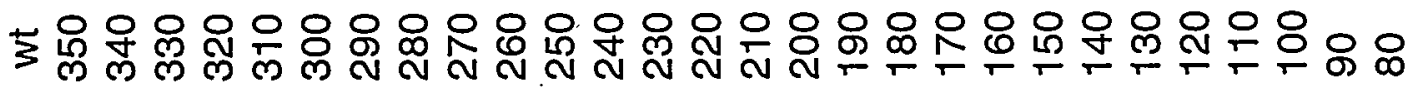

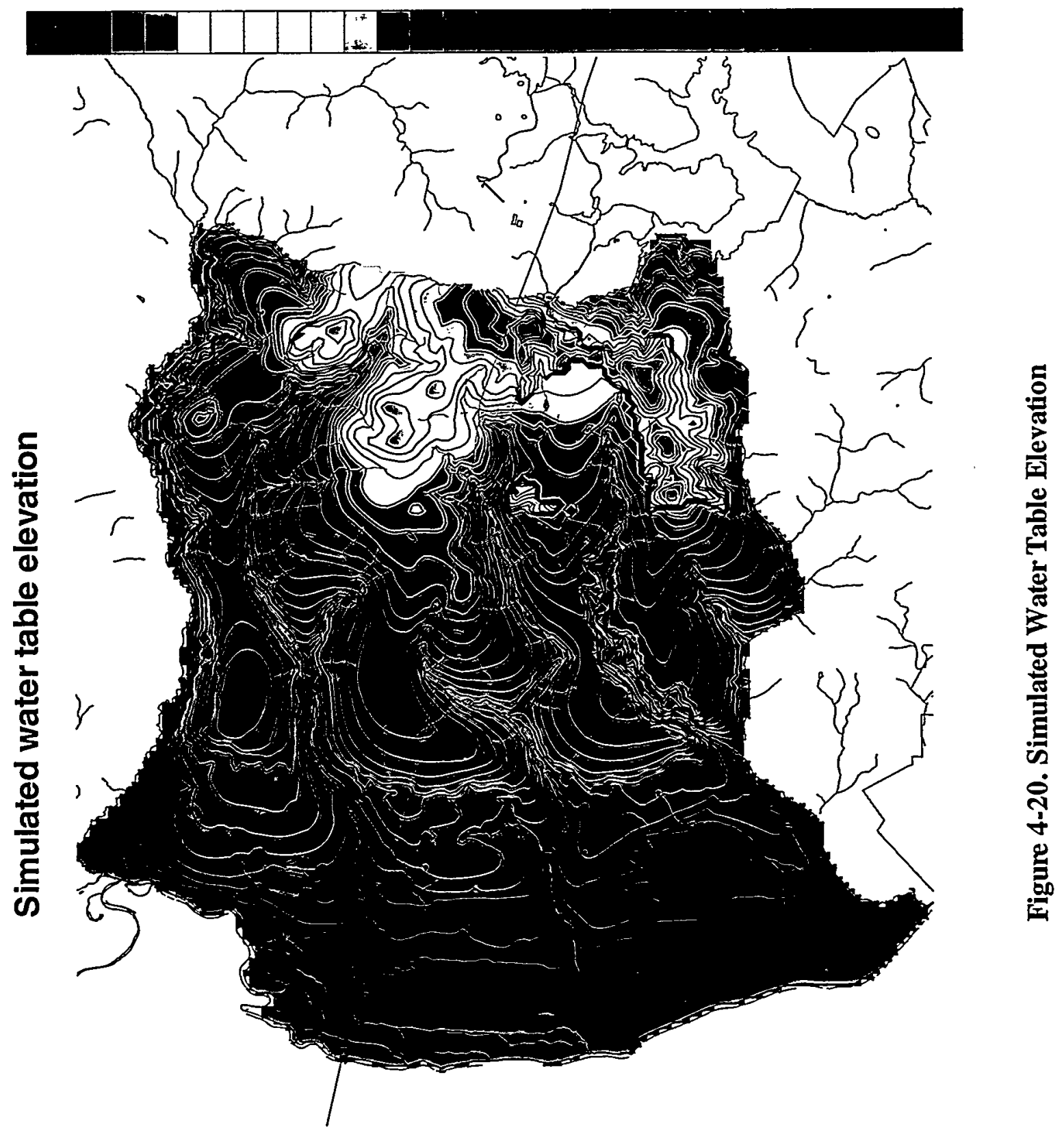




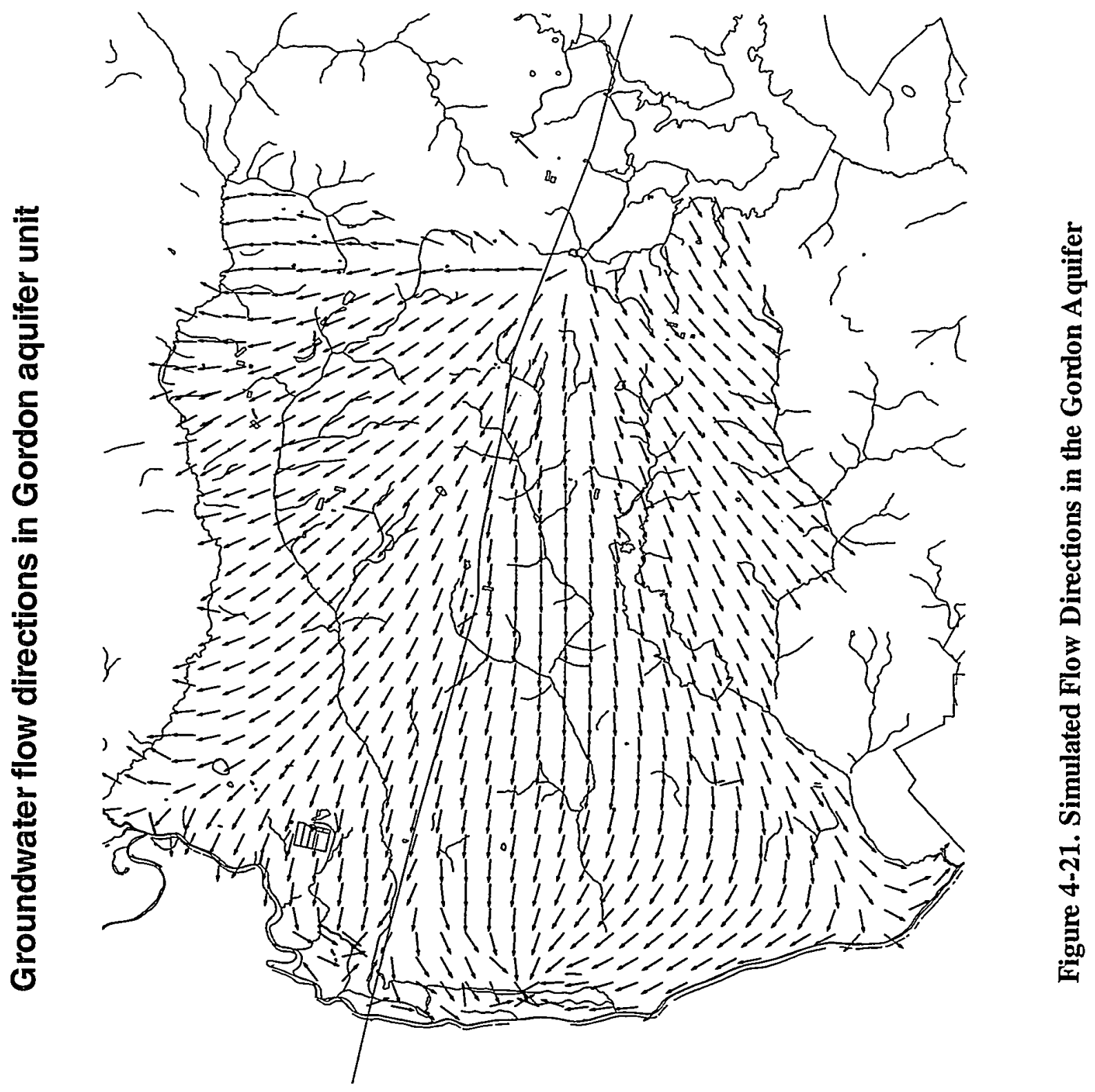




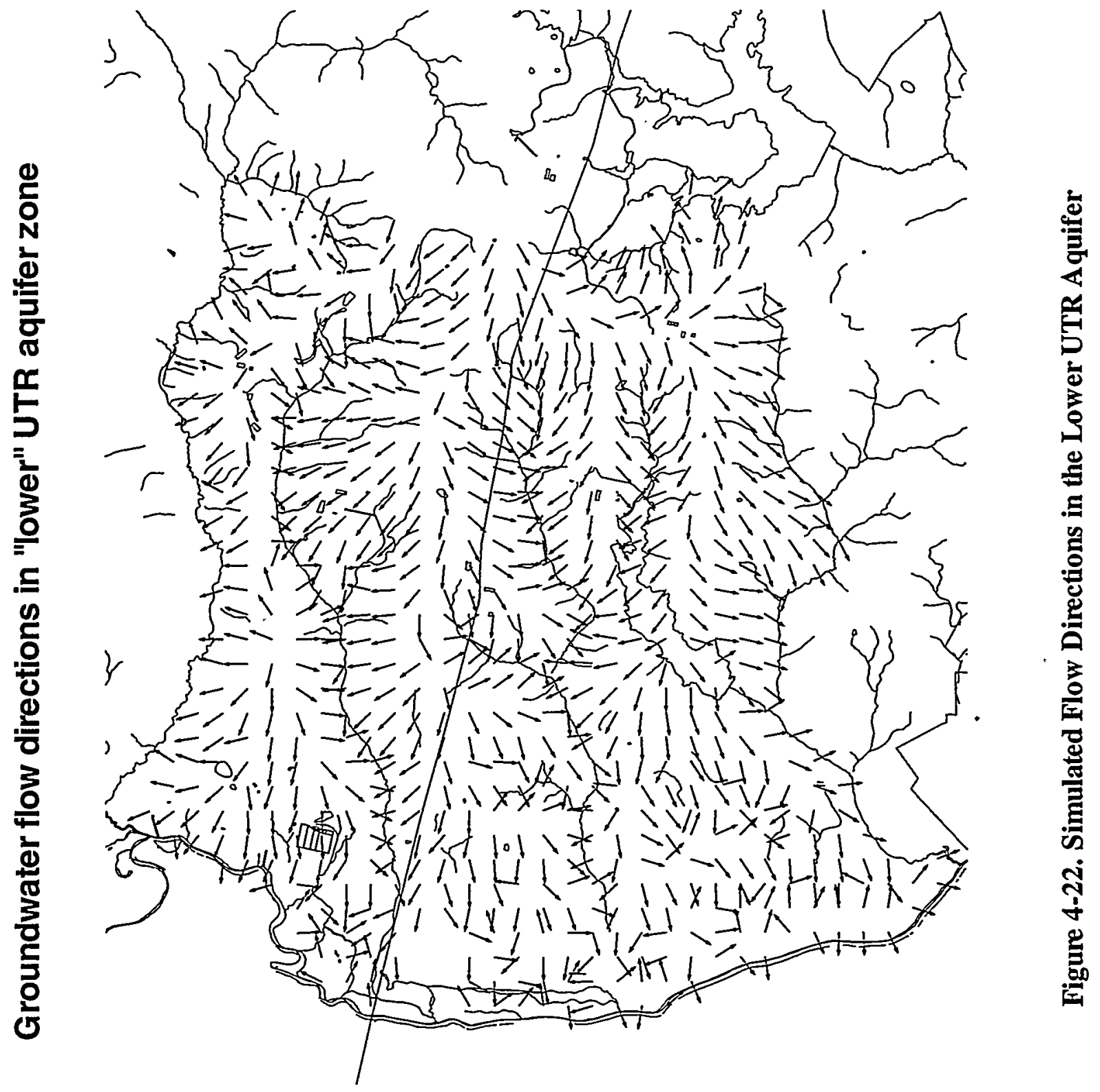




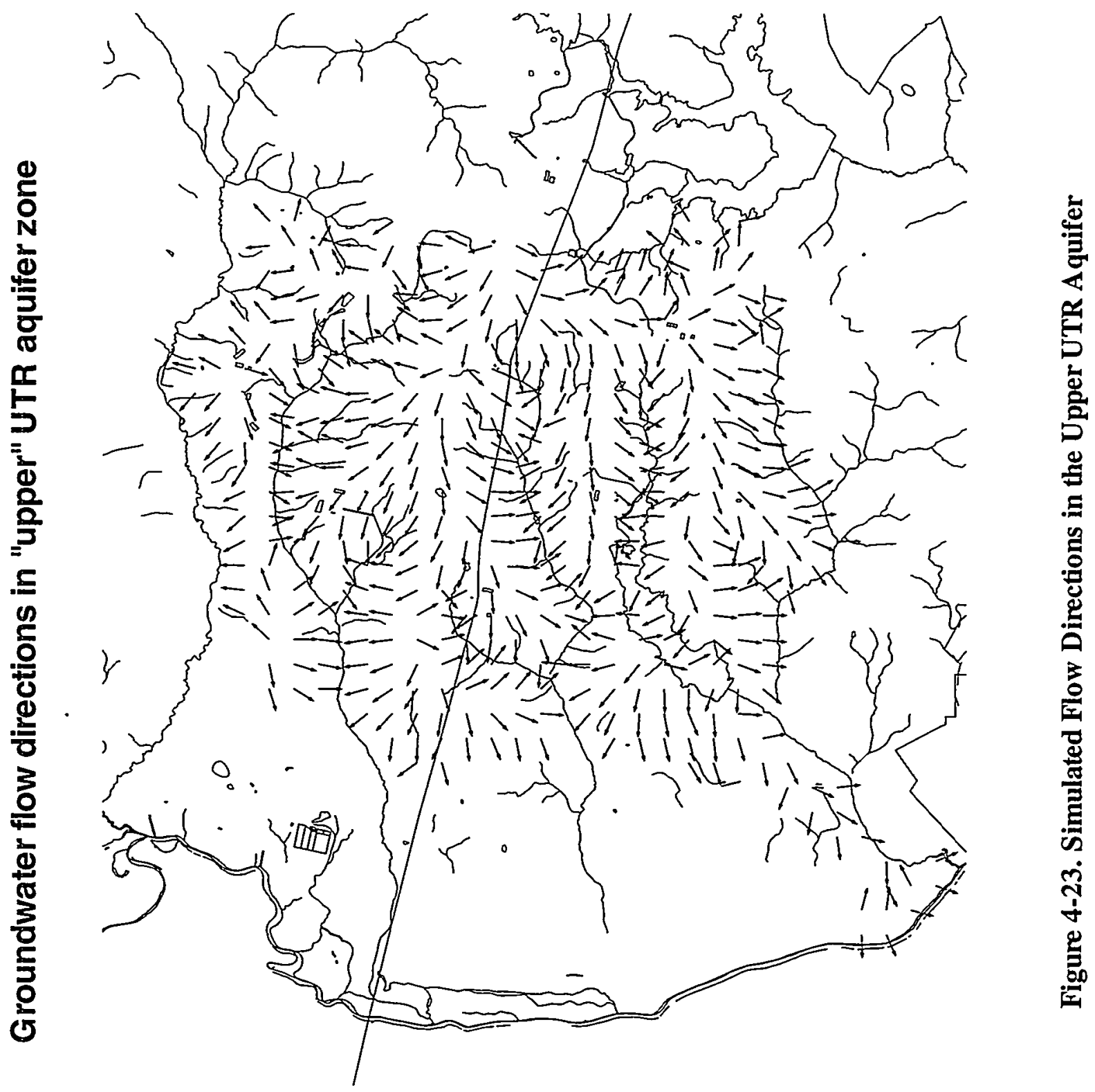




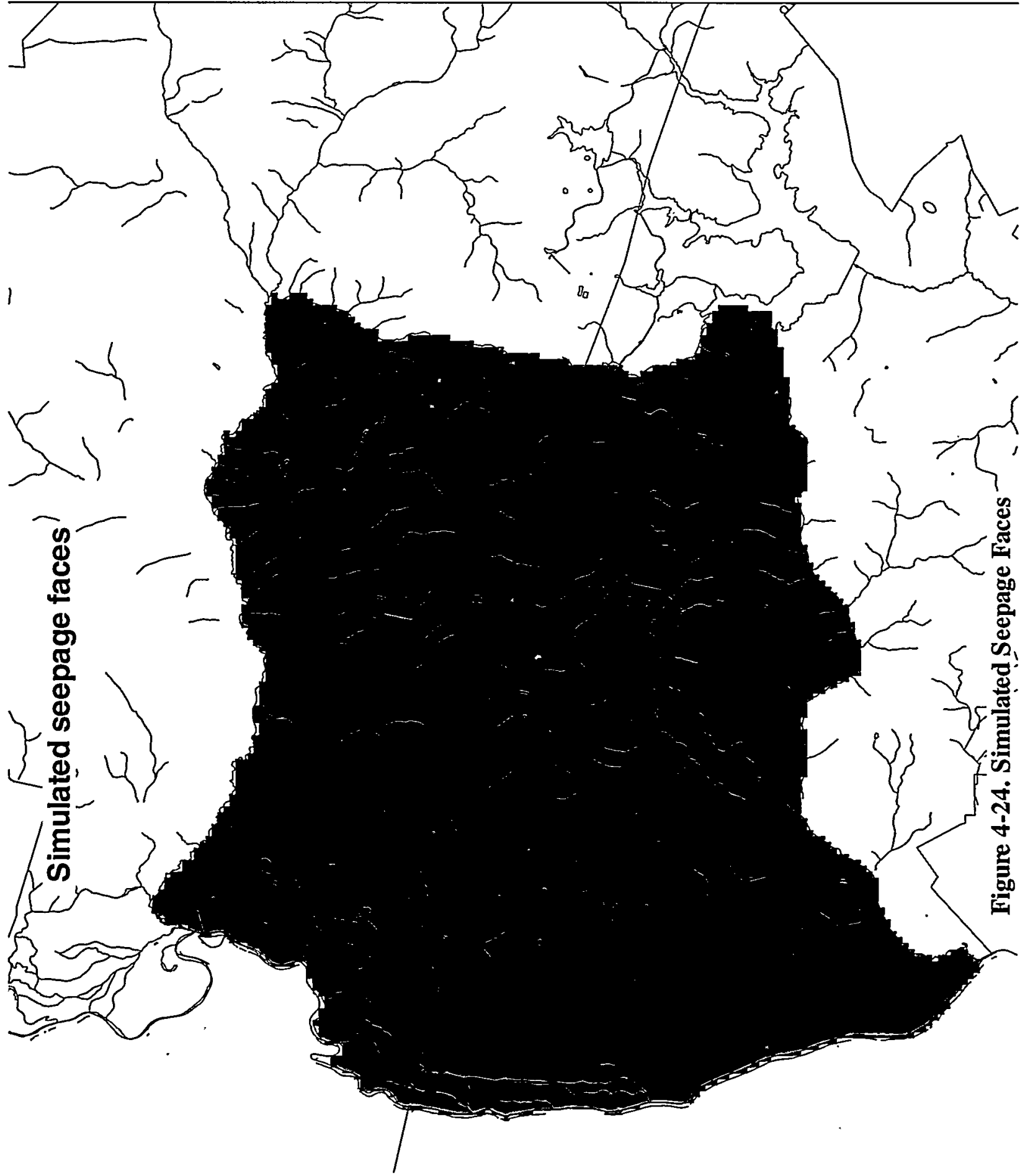




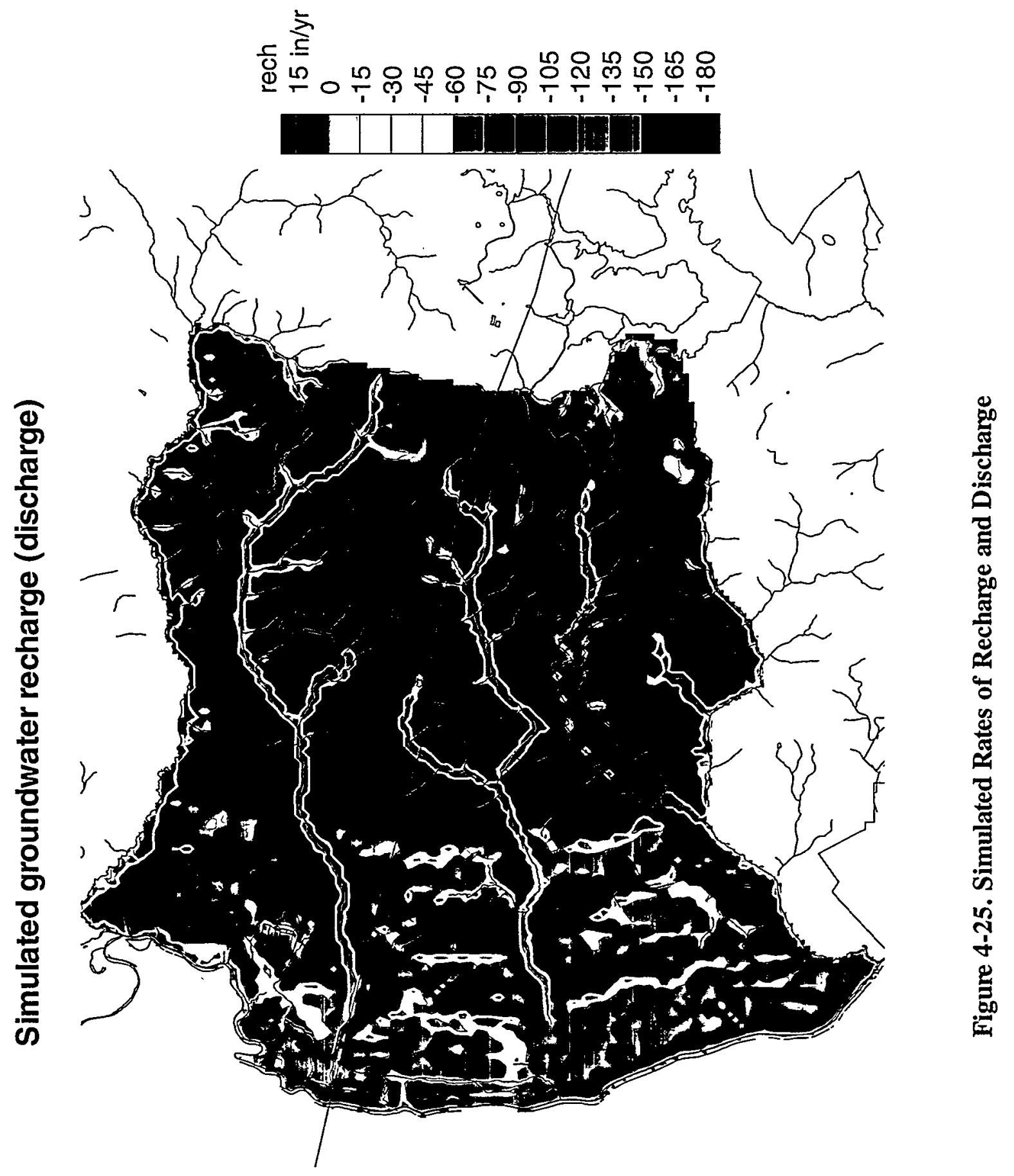




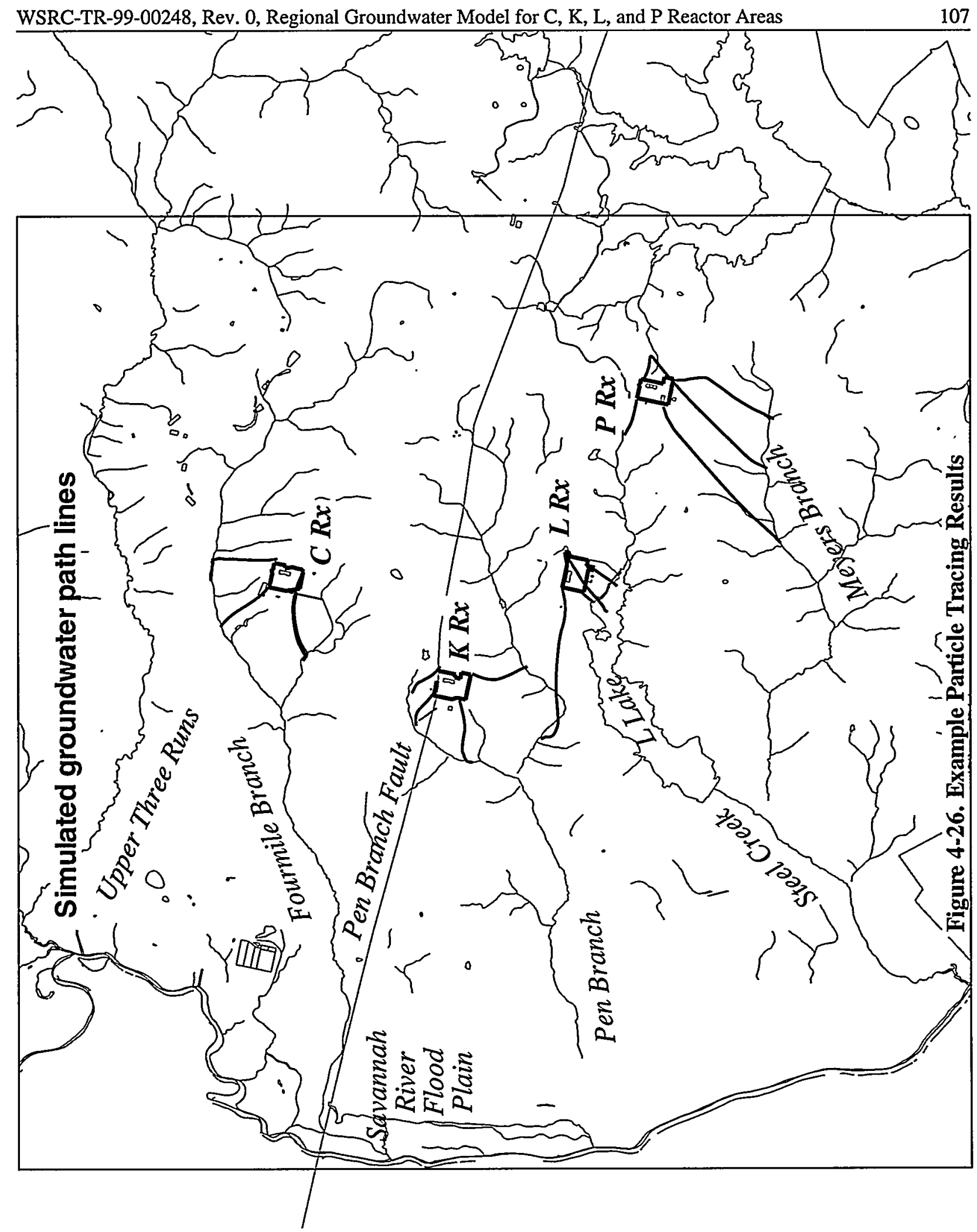




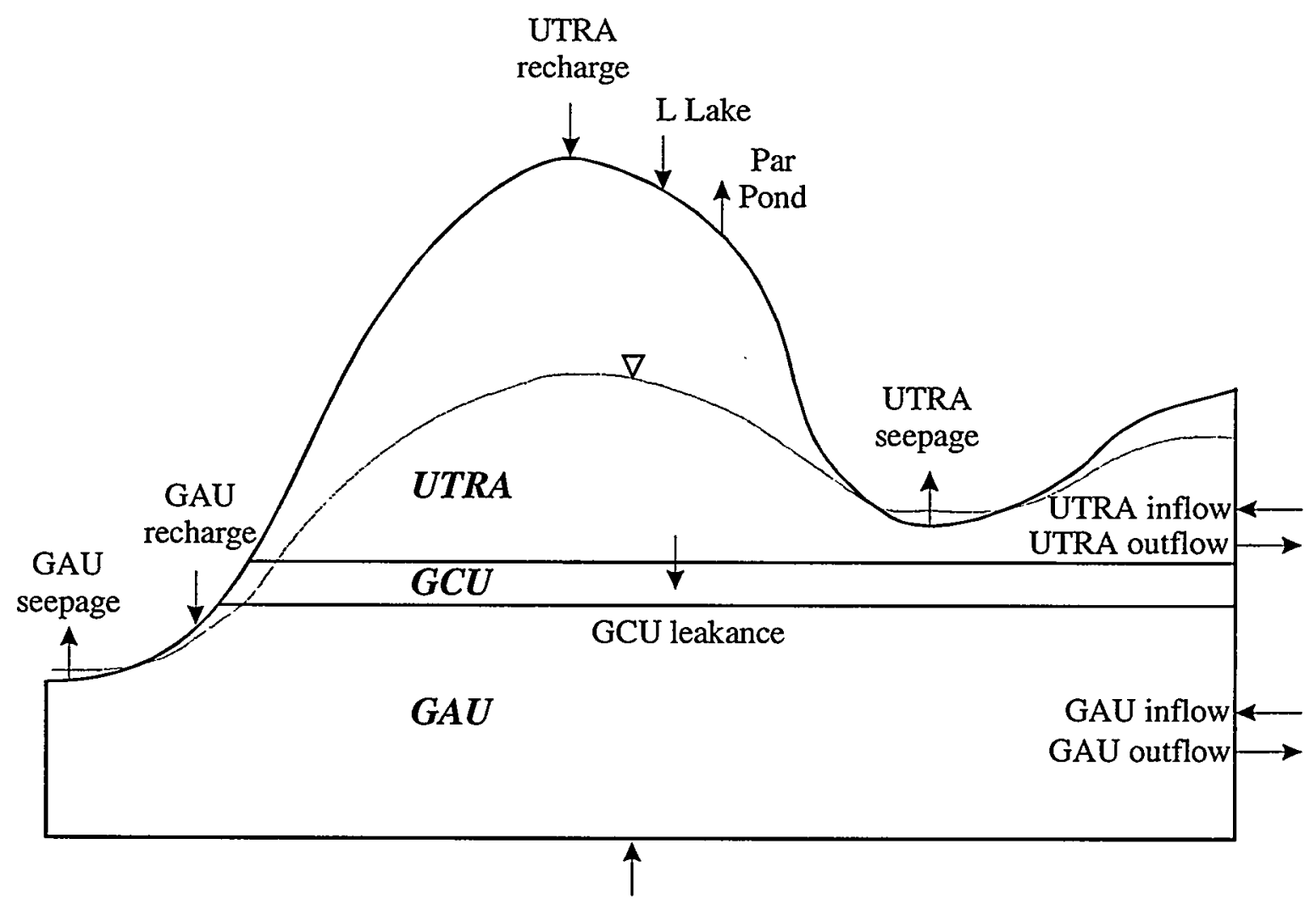

MBCS leakance

\begin{tabular}{lrrr}
\hline \multicolumn{1}{c}{ Flow component } & Entire model & UTR aquifer & Gordon aquifer \\
\hline Recharge & +79.9694 & +78.3983 & +1.5711 \\
Seepage & -80.7454 & -64.3827 & -16.3627 \\
In flows (head BCs) & +104.9055 & +0.5450 & +104.3605 \\
Out flows (head BCs) & -106.6514 & -2.2367 & -104.4148 \\
L Lake & +0.2734 & +0.2734 & N/A \\
Par Pond & -0.1225 & -0.1225 & N/A \\
Gordon c.u. leakage & N/A & -12.4745 & +12.4745 \\
Meyers Br. c.s. leakage & $\mathbf{+ 2 . 3 7 1 7}$ & $\mathrm{N} / \mathrm{A}$ & +2.3717 \\
\cline { 2 - 4 } Net total flow & $\mathbf{+ 0 . 0 0 0 7}$ & $\mathbf{+ 0 . 0 0 0 3}$ & $\mathbf{+ 0 . 0 0 0 3}$ \\
\hline
\end{tabular}

Figure 4-27. Water Balance 
Table 4-1. Calibration Summary for Groundwater Flow Targets

\begin{tabular}{|c|c|c|c|c|}
\hline Flow target & Prior estimate & $\begin{array}{l}\text { Range or } \\
\text { Uncertainty }\end{array}$ & $\begin{array}{l}\text { Model value } \\
\text { (in/yr for } \\
\text { recharge; } \\
\mathrm{ft}^{3} / \mathrm{s} \text { otherwise) } \\
\end{array}$ & Difference \\
\hline Surface recharge & $15 \mathrm{in} / \mathrm{yr}$ & $10-16 \mathrm{in} / \mathrm{yr}$ & $\begin{array}{c}12.5 \text { max. local } \\
(9.0 \text { based on total } \\
\text { area })\end{array}$ & $-17 \%$ \\
\hline $\begin{array}{l}\text { Meyers Branch base flow } \\
\text { (headwaters to Road 9) }\end{array}$ & 3.2 & $\pm 20-25 \%$ & 2.4 & $-25 \%$ \\
\hline $\begin{array}{l}\text { Steel Creek base flow } \\
\text { (above Road B to Road A; } \\
\text { includes L-Lake) }\end{array}$ & $\begin{array}{c}-2.2 \\
\text { (losing reach) }\end{array}$ & $\begin{array}{l} \pm 40-45 \% \\
\text { or more }\end{array}$ & $\begin{array}{l}3.1 \\
\text { (drain BCs: }+4.0 \\
\text { gen. head BCs: } \\
-0.9 \text { ) } \\
\end{array}$ & $+5.3 \mathrm{ft}^{3} / \mathrm{s}$ \\
\hline $\begin{array}{l}\text { Pen Branch base flow } \\
\text { (headwaters to Road A13; } \\
\text { includes Indian Grave } \\
\text { Branch) }\end{array}$ & 13.3 & $\pm 15-20 \%$ & 13.5 & $+2 \%$ \\
\hline $\begin{array}{l}\text { Fourmile Branch base flow } \\
\text { (headwaters to Road A12) }\end{array}$ & 14.1 & $\pm 15-20 \%$ & 14.7 & $+4 \%$ \\
\hline $\begin{array}{l}\text { Upper Three Runs base } \\
\text { flow } \\
\text { (Road C to Road A) }\end{array}$ & 4.5 & $\begin{array}{c} \pm 35-40 \% \\
\text { or more }\end{array}$ & 6.0 & $+33 \%$ \\
\hline L Lake & - & - & $\begin{array}{c}-0.9 \\
\text { (losing lake) }\end{array}$ & - \\
\hline $\begin{array}{l}\text { Par Pond } \\
\text { (portion within model) }\end{array}$ & - & - & 0.1 & - \\
\hline Caster Creek & 2.9 & - & 2.7 & $-7 \%$ \\
\hline $\begin{array}{l}\text { Central Shops outfall } \\
\text { creek }\end{array}$ & 0.76 & - & 0.72 & $-5 \%$ \\
\hline $\begin{array}{l}\text { Indian Grave Branch } \\
\text { (excluding K-18 outfall) }\end{array}$ & 4.8 & - & 2.6 & $-45 \%$ \\
\hline $\begin{array}{l}\text { Indian Grave above Road } \\
\text { B } \\
\text { (excluding K-18 outfall) }\end{array}$ & 2.3 & - & 1.0 & $-56 \%$ \\
\hline $\begin{array}{l}\text { Pen Branch above Indian } \\
\text { Grave Branch }\end{array}$ & 11 & - & 7.3 & $-34 \%$ \\
\hline Pen Branch above Road B & 1.9 & - & 5.3 & $+3.4 \mathrm{ft}^{3} / \mathrm{s}$ \\
\hline
\end{tabular}


Table 4-2. Calibration Summary for Hydraulic Head Targets

\begin{tabular}{|c|c|c|c|c|c|c|}
\hline $\begin{array}{c}\text { Measure } \\
\text { (ft) }\end{array}$ & $\begin{array}{c}\text { Gordon } \\
\text { aquifer }\end{array}$ & $\begin{array}{c}\text { "lower" } \\
\text { UTR } \\
\text { aquifer }\end{array}$ & $\begin{array}{c}\text { trans- } \\
\text { missive } \\
\text { zone }\end{array}$ & $\begin{array}{c}\text { AA } \\
\text { horizon }\end{array}$ & $\begin{array}{c}\text { A/uu } \\
\text { horizons }\end{array}$ & Overall \\
\hline $\begin{array}{c}\text { RMS } \\
\text { difference }\end{array}$ & 3.4 & 6.3 & 5.2 & 4.0 & 7.0 & 5.45 \\
\hline $\begin{array}{c}\text { Average } \\
\text { difference }\end{array}$ & -0.7 & +1.4 & -0.2 & -0.3 & -0.0 & - \\
\hline $\begin{array}{c}\text { Median } \\
\text { difference }\end{array}$ & -0.2 & +1.3 & -0.40 & -0.70 & +0.40 & - \\
\hline $\begin{array}{c}\text { Average } \\
\text { difference }\end{array}$ & 2.0 & 5.1 & 3.6 & 2.9 & 5.8 & - \\
\hline $\begin{array}{c}\text { Maximum } \\
\text { difference }\end{array}$ & -16.2 & +17.7 & -16.8 & -15.6 & -17.1 & - \\
\hline
\end{tabular}


Table 4-3. Calibration Summary for Hydraulic Conductivity

\begin{tabular}{|c|c|c|c|c|}
\hline Hydrostratigraphic Unit & $\begin{array}{c}\mathbf{K}_{\mathbf{h}} \\
\text { Average } \\
\text { (ft/day) }\end{array}$ & $\begin{array}{c}\mathrm{K}_{\mathbf{h}} \\
\text { Range } \\
\text { (ft/day) }\end{array}$ & $\begin{array}{c}\mathbf{K}_{\mathbf{V}} \\
\text { Average } \\
(\mathbf{f t} / \text { day })\end{array}$ & $\begin{array}{c}\mathbf{K}_{\mathbf{v}} \\
\text { Range } \\
\text { (ft/day) }\end{array}$ \\
\hline Gordon aquifer & 35 & - & 0.035 & - \\
\hline Gordon confining unit & 0.01 & - & $1 \times 10^{-4}$ & - \\
\hline "lower" UTR aquifer zone & 5.9 & $4-20$ & 0.058 & $0.004-0.1$ \\
\hline "tan clay" UTR confining zone & 0.3 & $0.01-0.4$ & $3 \times 10^{-3}$ & $\begin{array}{c}1 \times 10^{-4} \\
4 \times 10^{-3}\end{array}$ \\
\hline "upper" UTR aquifer zone: & 8.3 & - & 0.064 & - \\
\hline Transmissive zone & 13 & $3-40$ & 0.13 & $0.03-0.4$ \\
\hline AA horizon & 4.4 & $2-5$ & 0.044 & $0.02-0.05$ \\
\hline A horizon and above & 2.1 & $0.25-5$ & 0.021 & $\begin{array}{c}0.0025- \\
0.05\end{array}$ \\
\hline Alluvium & 20 & - & 0.2 & - \\
\hline
\end{tabular}




\section{Table 4-4. Summary of Uncertainty Cases}

\section{GCU Kv}

\begin{tabular}{|c|c|c|c|}
\cline { 2 - 4 } \multicolumn{1}{c}{ Recharge } & $5 \times 10^{-4} \mathrm{ft} /$ day & $10^{-4} \mathrm{ft} / \mathrm{day}$ & $2 \times 10^{-5} \mathrm{ft} /$ day \\
\hline \hline $15 \mathrm{in} / \mathrm{yr}$ & - & Case 1 & (Case 5) \\
\hline $12.5 \mathrm{in} / \mathrm{yr}$ & Case 3 & Nominal & Case 4 \\
\hline $10 \mathrm{in} / \mathrm{yr}$ & (Case 6) & Case 2 & - \\
\hline
\end{tabular}


Table 4-5. Calibration Summary for Uncertainty Cases

\begin{tabular}{|l|c|c|c|c|c|}
\hline Calibration measure & Nominal & Case 1 & Case 2 & Case 3 & Case 4 \\
\hline Overall RMS head residual (ft) & 5.45 & 5.48 & 5.45 & 9.59 & 5.62 \\
\hline $\begin{array}{l}\text { Gordon aquifer RMS head residual } \\
\text { (ft) }\end{array}$ & 3.4 & 3.3 & 3.5 & 3.4 & 3.1 \\
\hline $\begin{array}{l}\text { "lower" UTRA RMS head residual } \\
\text { (ft) }\end{array}$ & 6.3 & 6.6 & 6.1 & 11.6 & 6.8 \\
\hline $\begin{array}{l}\text { "upper" UTRA RMS head residual } \\
\text { (ft) }\end{array}$ & 4.0 & 4.0 & 4.1 & 7.5 & 4.4 \\
\hline \multicolumn{1}{|c|}{ Aluu } & 7.0 & 6.8 & 7.2 & 10.0 & 6.4 \\
\hline $\begin{array}{l}\text { Meyers Branch base flow residual } \\
\text { (cfs) }\end{array}$ & -0.8 & -0.2 & -1.3 & -2.1 & -0.4 \\
\hline Steel Creek base flow residual (cfs) & +5.3 & +6.0 & +4.7 & +4.2 & +5.3 \\
\hline $\begin{array}{l}\text { Pen Branch base flow residual (cfs) } \\
\text { Fourmile Branch base flow residual } \\
\text { (cfs) }\end{array}$ & +0.2 & +3.2 & -2.8 & -5.3 & +2.2 \\
\hline $\begin{array}{l}\text { Upper Three Runs base flow residual } \\
\text { (cfs) }\end{array}$ & +1.5 & +3.9 & -2.8 & -6.9 & +3.1 \\
\hline $\begin{array}{l}\text { Nominal Gordon aquifer unit, } \\
\text { Kh (ft/d) }\end{array}$ & 35 & 35 & 35 & 96 & 8.8 \\
\hline $\begin{array}{l}\text { Nominal "lower" UTR aquifer zone, } \\
\text { Kh (ft/day) }\end{array}$ & 5.9 & 7.7 & 4.4 & 0.7 & 7.8 \\
\hline $\begin{array}{l}\text { Nominal "tan clay" UTR confining } \\
\text { zone, Kv (ft/day) }\end{array}$ & $3 \times 10^{-3}$ & $3 \times 10^{-3}$ & $3 \times 10^{-3}$ & $6 \times 10^{-2}$ & $1 \times 10^{-3}$ \\
\hline $\begin{array}{l}\text { Nominal "upper" UTR aquifer zone, } \\
\text { Kh (ft/day) }\end{array}$ & 8.3 & 11 & 6.2 & 1.6 & 11.1 \\
\hline
\end{tabular}


This page intentionally left blank 


\subsection{SUMMARY AND RECOMMENDATIONS}

Important attributes of the baseline CKLP model are:

- The present baseline model is current with available characterization data from the reactor areas through Spring 1999. All the characterization data has been incorporated into a project database that can be easily updated as additional field data is obtained.

- Both the vadose and saturated zones are simulated in the model

- The Upper Three Runs aquifer is sub-divided into several vertical mesh layers that include the "transmissive" zone, "A horizon" and "A/uu horizons".

- The alluvial valley has been included in the model to realistically illustrate the extent to which the Savannah River has incised the hydrostratigraphic units. The Savannah River has cut down to the Gordon aquifer at the northern and southern ends of the valley but does not incise the Meyers Branch confining system within the model area.

- The model is based on the FACT code

Important implications of the CKLP model:

- The model meets the planning objectives of the General Groundwater Strategy for Reactor Area Projects (WSRC, 1997) by providing a common framework for analyzing groundwater flow, contaminant migration and remedial alternatives across ERD programs

- The CKLP groundwater flow model provides a good understanding of the groundwater flow regime for these reactor areas on a regional scale. The model is suited to assist in scoping characterization and remedial activities by providing a common base for the subsequent finer scale transport and remedial/feasibility models for each of these areas.

- The model has been constructed to incorporate new data as it is collected, providing quick and cost-effective updates.

Recommendations for future refinements to the CKLP model are:

- Pump tests in non-contaminated areas are needed for each reactor area in order to provide direct, field scale, conductivity measurements. In addition, previous conductivity measurements derived from slug tests and pump tests should be reviewed to determine data quality and validity of the measurements. 
- Additional research on the baseflow data for Upper Three Runs between Road C and Road A, Steel Creek and L Lake, and Pen Branch are needed to understand the surface water hydrology on a regional basis.

- Further evaluation of the high and low head residuals for both the Upper Three Runs and Gordon aquifers is needed by looking at the hydrostratigraphy and well screen intervals in greater detail. Additional well data and further understanding of the heterogeneity of the "lower" aquifer zone of the Upper Three Runs aquifer would improve the model. 


\subsection{REFERENCES}

Aadland, R. K., Gellici, J. A., and Thayer, P. A., 1995. Hydrogeologic Framework of WestCentral South Carolina. Report 5, Water Resources Division, South Carolina Department of Natural Resources, Columbia, SC.

Aadland, R. K., Harris, M. K., Lewis, C. M., Gaughan, T. F., and Westbrook, T. M., 1991. Hydrostratigraphy of the General Separations Area, Savannah River Site (SRS), South Carolina. WSRC-RP-91-13, Westinghouse Savannah River Company, Aiken, SC 29808.

Beard, D. C. and Weyl, P. K., 1973. "Influence of Texture on Porosity and Permeability of Unconsolidated Sand", American Association of Petroleum Geologists Bulletin, V. 57, p. 349-369.

Cahill, J. M., 1982. Hydrology of the Low Level Radioactive Solid Waste Burial Site and Vicinity near Barnwell, South Carolina, United States Geological Survey Open File Report 82-863.

Camp Dresser \& McKee, 1989. Numerical Simulation of Groundwater Flow and Contaminant Transport at the $K, L$, and $P$ Areas of the Savannah River Site, Aiken, South Carolina, Final Report, prepared for Westinghouse Savannah River Company.

Cooney, T. W., Drewes, P. A., Jones, K. H., Gissendanner, J. W., and Church, B. W., 1998. Water Resources Data - South Carolina, Water year 1997, Volume 1, U. S. Geological Survey Water-Data Report SC-97-1.

De Marsily, G., 1986. Quantitative Hydrogeology, Groundwater Hydrology for Engineers, Academic Press, Inc., Harcourt Brace Jovanovich, Publishers.

Denehy, K. and McMahon, P., 1985. Hydrologic and Micrometeorological Data for an Unsaturated Zone Flow Study at a Low-Level Radioactive Waste Burial Site, Barnwell, South Carolina, U. S. Geological Survey Open File Report OF85-476.

Environmental Protection Department and Exploration Resources, Inc., 1996a. Environmental Protection Department's Well Inventory (U), ESH-EMS-960488. Westinghouse Savannah River Company, Aiken, SC 29808. 
Environmental Protection Department and Exploration Resources, Inc., 1996b. The Savannah River Site's Groundwater Monitoring Program; Second Quarter 1996 (U), ESH-EMS960057. Westinghouse Savannah River Company, Aiken, SC 29808.

Fallaw, W. C. and Price, V., 1995. "Stratigraphy of the Savannah River Site and Vicinity", Southeastern Geology, 35: 21-58.

Flach, G. P. and Harris, M. K., 1997. Integrated Hydrogeological Model of the General Separations Area (U), Volume 2: Groundwater Flow Model (U), WSRC-TR-96-0399, Rev. 0, Westinghouse Savannah River Company, Aiken, SC 29808.

Flach, G. P., 1998. Impact of F- and H-Area Pump-Treat-Reinject Remediation Systems on the Old Radioactive Waste Burial Ground (U), SRT-EST-98-154.

Flach, G. P., Harris, M. K., Hiergesell, R. A., Smits, A. D., and Hawkins, K. L., 1998, Hydrogeological and groundwater flow model for the $C, K, L$, and $P$ reactor areas, Savannah River Site, Aiken, South Carolina (U), WSRC-TR-98-0028, Westinghouse Savannah River Company, Aiken, SC 29808.

Freeze, R. A. and Cherry, J. A., 1979. Groundwater, Prentice-Hall, Inc.

GeoTrans, 1992. Groundwater flow model of the General Separations Area, Savannah River Site, prepared for Westinghouse Savannah River Company, Project no. 3017-003.

GeoTrans, 1993. Groundwater Model Calibration and Review of Remedial Alternatives at the F- and H-Area Seepage Basins, WSRC-TR-93-384, Westinghouse Savannah River Company, Aiken, SC 29808.

Gruber, P., 1981. A Hydrology Study of the Unsaturated Zone Adjacent to a RadioactiveWaste Disposal Site at the Savannah River Plant, Aiken, South Carolina, M.S. Thesis, Geology Department, University of Georgia, Athens, GA.

Gruber, P., 1983. "A Hydrology Study of the Unsaturated Zone Adjacent to a RadioactiveWaste Disposal Site at the Savannah River Plant, Aiken, South Carolina", Role of Unsaturated Zone in Radioactive and Hazardous Waste Disposal, J.W. Mercer, P.S.C. Rao, and I.W. Marine (editors), Ann Arbor Science Publishers, Ann Arbor, MI. 
Hamm, L. L. and Aleman, S. E., 1999. FACT (Version 2.0): Subsurface Flow and Contaminant Transport; Documentation and User's Guide (U), WSRC-TR-99-00282, Westinghouse Savannah River Company, Aiken, SC 29808.

Hamm, L. L., Aleman, S. E., Flach, G. P, and Jones, W. F., 1997. Subsurface Flow and Contaminant Transport Documentation and User's Guide (U), WSRC-TR-95-0223, Savannah River Technology Center, Westinghouse Savannah River Company, Aiken, SC 29808.

Harris, M. K., Aadland, R. K., and Westbrook, T. M., 1990. "Lithological and Hydrological Characteristics of the Tertiary Hydrostratigraphic Systems of the General Separations Area, Savannah River Site, South Carolina". Savannah River Region: Transition Between the Gulf and Atlantic Coastal Plains, Proceedings of the Second Bald Head Island Conference on Coastal Plains Geology, University of North Carolina at Wilmington, pp 68-73.

Hiergesell, R. A., 1999. Preparation of Gordon Aquifer Regional Potentiometric Surface, Westinghouse Savannah River Company Inter-Office Memorandum, SRT-EST-99-309.

Hiergesell, R. A., 1998a. Regional Water Table Coverage of the Savannah River Site. WSRC-TR-98-00045, Rev. 0, Westinghouse Savannah River Company, Aiken, SC 29808.

Hiergesell, R. A., 1998b. Summary of Stream Baseflow and Water Table Work Conducted in Support of the R-Reactor and K-Reactor Groundwater Modeling Efforts. Westinghouse Savannah River Company Inter-Office Memorandum, SRT-EST-98-110.

Hiergesell, R. A., 1998c. Transmittal of Stream Baseflow Measurement Data, Westinghouse Savannah River Company Inter-Office Memorandum, SRT-EST-98-197.

HSI GeoTrans, 1998. Groundwater Flow and Solute Transport Modeling Report; K-Area Burning/Rubble Pit and Rubble Pile, WSRC-TR-98-5052. Westinghouse Savannah River Company, Aiken, SC 29808.

Hubbard, J. E. and Emslie, R. E., 1984. Water Budget for the SRP Burial Ground Area, DPST-83-742.

Hubbard, J. E., 1986. An Update on the SRP Burial Ground Area Water Balance and Hydrology, DPST-85-958. 
Huyakorn, P. S., Panday, S., and Birdie, T., 1991. Subsurface Analysis Finite Element Model for Flow and Transport in 3 Dimensions, Version 1.3, Documentation and User's Guide, prepared for Westinghouse Savannah River Company.

HydroGeoLogic, Inc., 1997. Groundwater Flow Model for the R-Reactor Area (U). WSRCOS-97-00006, Revision 0, HydroGeoLogic, Inc., Herndon, VA.

HydroGeoLogic, Inc., 1998. Groundwater Flow and Contaminant Transport for the $R$ Reactor Seepage Basins $(U)$, HydroGeoLogic, Inc., Herndon, VA.

Leeth, D. C. and Nagle, D. D., 1996. "Shallow Subsurface Geology of Part of the Savannah River Alluvial Valley in the Upper Coastal Plain of Georgia and South Carolina", Southeastern Geology, v. 36, no. 1, pp. 1-14.

Looney, B. B., Grant, M. W., and King, C. M., 1987. Estimation of Geochemical Parameters for Assessing Subsurface Transport at the Savannah River Plant, DPST-85-904.

O'Brien \& Gere Engineers, Inc., 1991. M Area Post-Test Characterization Geotechnical Testing, File: 4998.007 \#2.

Parizek, R. R. and Root, R. W., Jr., 1986. Development of a Ground-Water Velocity Model for the Radioactive Waste Management Facility, Savannah River Plant, South Carolina, DPST-86-658.

Siple, G. E., 1967. Geology and Ground Water of the Savannah River Plant and Vicinity, South Carolina, U.S. Geological Survey Water Supply Paper 1841.

Smits, A. D., Harris, M. K., Hawkins, K. L., and Flach, G. P., 1997. Integrated Hydrogeological Model of the General Separations Area (U), Volume I: Hydrogeologic Framework (U), WSRC-TR-96-0399, Westinghouse Savannah River Company, Aiken, SC, $118 \mathrm{pp}$.

Snipes, D. S., Fallaw, W. C., Price, V., Jr., and Cumbest, R. J., 1993. "The Pen Branch Fault: Documentation of Late Cretaceous-Tertiary Faulting in the Coastal Plain of South Carolina", Southeastern Geology, v. 33, no. 4, pp 195-218.

Stieve, A. L., 1994. Confirmatory Drilling Project Final Report (U), WSRC-RP-94-0136, Westinghouse Savannah River Company, Aiken, SC 29808. 
Stieve, A. and Stephenson, D., 1995. "Geophysical Evidence for Post Late Cretaceous Reactivation of Basement Structures in the Central Savannah River Area", Southeastern Geology, v. 35, no. 1, pp 1-20.

Walpole, R. E. and R. H. Meyers, 1978. Probability and Statistics for Engineers and Scientists, $2^{\text {nd }}$ Edition, MacMillan, New York.

WSRC, 1996. RCRA Facility Investigation/Remedial Investigation Report with Baseline Risk Assessment for the Chemicals, Metals, and Pesticides Pits (U), WSRC-RP-96-0012, Revision 1, Westinghouse Savannah River Company, Aiken, SC 29808.

WSRC, 1997. General Groundwater Strategy for Reactor Area Projects, WSRC-RP-97435, Revision 0, Westinghouse Savannah River Company, Environmental Restoration Division, Aiken, SC, 29808.

WSRC, 1999. Technical Oversight to Install Reactor Piezometers (U), WSRC-RP-99-4021, Westinghouse Savannah River Company, Aiken, SC, 29808.

Yu, A. D., Langton, C. A., and Serrato, M. G., 1993. Physical Properties Measurement Program (U), WSRC-RP-93-894, Westinghouse Savannah River Company, Aiken, SC 29808. 
This page intentionally left blank 
APPENDIX A. DATA COLLECTION AND HYDROGEOLOGIC MODEL METHODOLOGY 


\section{This page intentionally left blank}




\section{APPENDIX A. DATA COLLECTION AND HYDROGEOLOGIC MODEL METHODOLOGY}

\section{Hydrogeologic Data Collection}

Data utilized in this analysis include local grid coordinates and elevations of SRS and off-site wells, geophysical logs, drill-core descriptions, and logs of soil properties taken using cone penetrometer technology (CPT). The study included collecting permeability data from the recent and historical literature. These data originate from the results of different types of tests. These include aquifer-pumping tests, borehole permeability ("slug") tests, and laboratory tests performed on undisturbed samples.

\section{Project Database}

The project database used for the $\mathrm{C}, \mathrm{K}, \mathrm{L}$, and $\mathrm{P}$ groundwater model area (CKLP GWMA) is designed to support groundwater modeling conducted by the Environmental Sciences Section (ESS) of the Savannah River Technology Center (SRTC) such as that described in Flach, and Harris (1997). The scope of the groundwater modeling project database (GWMPD) for the CKLP GWMA includes the area bounded by Savannah River Site (SRS) local grid coordinates 10,000 to 85,000 feet North and 0 to 100,000 feet East.

The primary function of GWMPD is to record and report hydrogeologic parameters within the context of their spatial position and hydrostratigaphic assignment. The database is designed to accommodate geologic, geotechnical, geophysical, and stratigraphic data from any type of sampling location (site type). Current site types include soil borings, wells, and cone-penetrometer test (CPT) sites. Hydrogeologic data fall within three main categories: 1) field data collected during drilling and sampling; 2) Field analyses of borehole and aquifer permeability; and 3) laboratory analyses of core and undisturbed samples. The GWMPD also records "subjective" data such as stratigraphic analyses ("picks" for unit boundaries) in addition to the "objective" measurements described above.

The database is compiled in Paradox ${ }^{\circledR}$ software and incorporates a relational structure, which defines unique data locations (sites) by their coordinates and elevations. The sites are used as the key field by which different types of data are related. The unique site identifiers allow multiple data types to be associated with a single data site. The database is constructed so that revisions made to the "subjective" data (hydrostratigraphic "picks") are documented. The database records and dates each revision to the picked boundaries, and automatically regenerates updated output files for re-loading into EarthVision ${ }^{\circledR}$. This aspect of the database 
facilitates data evaluation and revision, and provides a means by which to maintain a history of the "subjective" data set.

The GWMPD maintains a bibliographic record of all documents reviewed and summarized for data, which are incorporated into the database. The database also records whether the documents serve as original sources for the data they contain, or summarize data extracted from previous reports. For example, a report that lists permeability values for undisturbed samples and includes copies of the laboratory reports in an appendix would be considered a "source" document. Similarly, a report that tabulates slug test results from several wells as average hydraulic conductivity values, and does not provide the test parameters or details of the individual analyses would be considered a "summary" document for those slug test results.

\section{Data Qualification}

Boundaries or "picks" for hydrostratigraphic units beneath the CKLP GWMA were established by the same method used in WSRC-RP-96-0399 for the General Separations Area (Smits and others, 1997). Because the CKLP GWMA includes the GSA modeling area, the picks were used to correlate hydrostratigraphic boundaries from within the GSA to cores in the remainder of the model area. The GWMPD uses the hydrostratigraphic nomenclature described in Aadland and others (1995). A rigorous Quality Review of the data was performed, comparing the core descriptions and geophysical logs with the list of unit boundaries. Geologists made refinements to these boundaries to ensure internal consistency between the unit boundaries and the lithology of the hydrostratigraphic units.

Hydrostratigraphic horizons include tops of the Meyers Branch confining system (MBCS), Gordon aquifer (GAU), Gordon confining unit (GCU), "lower" aquifer zone (LAZ), "tan clay" confining zone (TCCZ), and the informal intervals identified within the "upper" aquifer zone of the Upper Three Runs aquifer. These informal intervals include the "transmissive zone" and the "AA", "A", and "uu" intervals as originally identified in (WSRC, 1997)

\section{Hydrogeologic Model}

\section{Hydrostratigraphic Methods}

Hydrostratigraphic unit boundaries for the CKLP GWMA are based on a recent hydrostratigraphic analysis of the GSA. The CKLP GWMA includes the model area that was used for the recent GSA hydrostratigraphic model (Smits and others, 1997). The current 
CKLP model correlates the hydrostratigraphic picks made in the GSA with the cores in the remainder of the CKLP GWMA. Boundaries are determined through evaluation of:

Geophysical data. Gamma-ray logs in combination with resistivity logs are used to evaluate the potential confining properties of the strata. In general, low resistivity and high gamma-ray values indicate clay-rich sediment that impedes the flow of ground water.

Core description data. Core descriptions are used (in conjunction with the geophysical logs) to select boundaries between confining and transmissive units. Percentage of mud and estimated porosity are the primary criteria used. If core recovery is good, the foot-by-foot description is an excellent tool for determining the vertical extent of a confining or transmissive lithology.

CPT data. Logs of CPT data were used to delineate boundaries between the informal intervals within the "upper" aquifer zone. The boundaries were picked from logs of the tip stress, sleeve stress, friction ratio, and pore pressure readings. The logs were used to identify curve patterns that are characteristic of each interval.

The GWMPD was used to prepare a hydrogeologic model of the CKLP GWMA. The model was constructed with EarthVision ${ }^{\circledR}$ software. EarthVision ${ }^{\circledR}$ processes sets of spatial and property data by calculating minimum-tension grids to contour a "best fit" of the data. The grids can contour data in 3 dimensions ( $\mathrm{x}, \mathrm{y}, \mathrm{z})$, such as the top of a geologic unit, as twodimensional grids, or contour data in 4-dimensions: $x, y, z$, and a "property." An example of a property might be the variation of the percentage of mud within a geologic unit.

\section{Two-Dimensional Grid Calculation}

Data for hydrostratigraphic unit tops were exported from the Paradox ${ }^{\circledR}$ database into EarthVision ${ }^{\circledast}$. After minor format changes, the data was processed by an algorithm which produces a two-dimensional grid of the unit top surface. The two-dimensional grids were calculated so as to incorporate effects of the Pen Branch Fault. The off-set is assumed to be a consistent, vertical displacement along the trace of the fault. The south side of the fault is displaced up relative to the north side. The Pen Branch Fault is assumed to only affect the units beneath the "upper" aquifer zone of the Upper Three Runs aquifer. The top of the TCCZ is the shallowest horizon in the model that is displaced by the fault.

The EarthVision ${ }^{\circledR}$ model utilizes digitized $\mathrm{x}, \mathrm{y}, \mathrm{z}$ data for all U.S. Geological Survey topographic coverage of the GSA. The data was processed in the same manner as the data 
for the unit boundaries to produce a grid representing the topography of the study area. The high density of data points in this data set produced a two-dimensional grid of exceptional accuracy and detail. This grid was then used in subsequent grid calculation to determine the extent of the hydrostratigraphic units that crop out in the study area.

\section{Geologic Structure Builder}

\section{Altitude-Contour Maps}

Altitude-contour maps were constructed for the top of each hydrostratigraphic using the two-dimensional grids calculated from the scattered data for the unit tops. The maps are plotted using the Contour and Basemap module of EarthVision ${ }^{\circledast}$. Contour intervals are chosen by individual data sets so as to convey the information clearly and concisely, but virtually any level of detail is possible. An effort was made to keep the contour interval to within one-tenth of the range of the $\mathrm{z}$-values. This serves to minimize the number of contour lines, yet generally maintains a level of detail suitable for interpretation of the map.

\section{Isopach Maps}

Two-dimensional grids of unit thickness (isopach grids) were calculated by first comparing the two-dimensional grids of the unit base and unit top with the two-dimensional grid of the topography. Isopach maps of vertical unit thickness were calculated from comparison of the two-dimensional grids of the unit base and unit top. A value was then written to the corresponding nodes of the resultant grid (the isopach grid) equal to the vertical distance between the base and upper surface of the unit.

The resultant two-dimensional isopach grids were contoured using EarthVision ${ }^{\boxplus}$ in the same fashion as the structure-contour maps.

\section{REFERENCES}

Aadland, R. K., J. A. Gellici, and P. A. Thayer, 1995. Hydrogeologic Framework of WestCentral South Carolina, State of South Carolina Department of Natural Resources, Water Resources Division Report 5.

Flach, G. P., and M. K. Harris, 1997. Integrated Hydrogeological Model of the General Separations Area, Volume 2: Groundwater Flow Model (U), WSRC-TR-96-0399, Rev. 0, Westinghouse Savannah River Company, Savannah River Site, Aiken, SC, 29808. 
Smits, A. D., M. K. Harris, K. L. Hawkins, and G. P. Flach, 1997. Integrated Hydrogeological Model of the General Separations Area, Volume 1: Hydrogeologic Framework (U), WSRC-TR-96-0399, Rev. 0, Westinghouse Savannah River Company, Savannah River Site, Aiken, SC, 29808.

WSRC, 1997, Preliminary Characterization Report Phase I RFI/RI for the R-Reactor Seepage Basins/108-4R Overflow Basin (U),.WSRC-RP-97-196, Westinghouse Savannah River Company, Aiken, SC 29808. 
This page intentionally left blank 


\section{APPENDIX B. HYDROSTRATIGRAPHIC DATA}


Appendix B-1: Locations of Sites within the Model Area

\begin{tabular}{|c|c|c|c|c|}
\hline Well ID & $\begin{array}{c}\text { SRS } \\
\text { Northing } \\
(\mathrm{ft})\end{array}$ & $\begin{array}{c}\text { SRS } \\
\text { Easting } \\
(\mathrm{ft})\end{array}$ & $\begin{array}{c}\text { Surface } \\
\text { Elevation } \\
(\mathrm{ft} \text { m.s.l.) }\end{array}$ & Reference $^{1}$ \\
\hline
\end{tabular}

\begin{tabular}{|c|c|c|c|c|}
\hline $131 \mathrm{C}-100$ & 68535.42 & 42328.12 & 261.62 & Bechtel, 1998 \\
\hline $131 \mathrm{C}-104$ & 68301.83 & 41995.84 & 276.91 & Bechtel, 1998 \\
\hline $131 \mathrm{C}-105$ & 70348.82 & 41250.71 & 169.83 & Bechtel, 1999 \\
\hline $131 \mathrm{C}-49$ & 69151.44 & 43419.4 & 254.29 & Bechtel, 1999 \\
\hline 131C-51 & 69313.87 & 43526.89 & 264.97 & Bechtel, 1999 \\
\hline $131 C-54$ & 69970.8 & 42952.3 & 247.06 & Kirr, 1998 \\
\hline $131 \mathrm{C}-55$ & 69923.3 & 43145.9 & 245.01 & Kirr, 1998 \\
\hline $131 \mathrm{C}-59$ & 69874.5 & 42563.4 & 227.7 & Kirr, 1998 \\
\hline $131 \mathrm{C}-60$ & 69691 & 42639.3 & 218.2 & Kirr, 1998 \\
\hline 131C-63 & 69158.7 & 42902.8 & 211.14 & Kirr, 1998 \\
\hline $131 \mathrm{C}-64$ & 68969.9 & 42859.4 & 211.86 & Kirr, 1998 \\
\hline $131 C-67$ & 68968.3 & 43829 & 265.82 & Kirr, 1998 \\
\hline 131C-68 & 69068.1 & 43824.3 & 267.66 & Kirr, 1998 \\
\hline $131 \mathrm{C}-80$ & 70125.53 & 42073.82 & 205.72 & Bechtel, 1998 \\
\hline $131 \mathrm{C}-81$ & 70172.54 & 42140.63 & 217.85 & Bechtel, 1998 \\
\hline $131 \mathrm{C}-82$ & 70261.44 & 42225.03 & 232.96 & Bechtel, 1998 \\
\hline $131 \mathrm{C}-83$ & 70325.16 & 42299.17 & 240.51 & Bechtel, 1998 \\
\hline $131 \mathrm{C}-84$ & 70387.3 & 42379.51 & 241.87 & Bechtel, 1998 \\
\hline $131 \mathrm{C}-85$ & 70466.62 & 42439.04 & 237.49 & Kirr, 1998 \\
\hline 131C-91 & 69542.49 & 41626.7 & 233.65 & Bechtel, 1998 \\
\hline $131 \mathrm{C}-93$ & 69366.69 & 41518.62 & 241.89 & Kirr, 1998 \\
\hline 131C-95 & 69199.12 & 41402.24 & 241.27 & Bechtel, 1998 \\
\hline $131 C-96$ & 68789.08 & 42627.2 & 243.22 & Bechtel, 1999 \\
\hline $131 \mathrm{C}-98$ & 68661.72 & 42487.58 & 250.98 & Bechtel, 1998 \\
\hline 131C-R1 & 68682.8 & 44316 & 271 & WSRC, 1997 \\
\hline 131C-R2 & 68734.7 & 44066.1 & 266.8 & WSRC, 1997 \\
\hline 131C-R3 & 68882.9 & 43994.5 & 268.8 & WSRC, 1997 \\
\hline 131C-R4 & 68863.3 & 44197.3 & 285.6 & WSRC, 1997 \\
\hline 131C-R5 & 68830.8 & 44359.6 & 289 & WSRC, 1997 \\
\hline 131C-R6 & 69031.6 & 44274 & 276 & WSRC, 1997 \\
\hline BGO-10A & 76805.18 & 57050.92 & 299.1 & WSRC, 1996d \\
\hline BGO-10AA & 76997.88 & 56990.54 & 298.8 & WSRC, 1996d \\
\hline BGO-12A & 76804.63 & 56250.68 & 311.4 & WSRC, 1996d \\
\hline BGO-14A & 76377.54 & 55838.32 & 300.2 & WSRC, 1996d \\
\hline BGO-16A & 75756.95 & 56194.15 & 302.8 & WSRC, 1996d \\
\hline BGO-18A & 75599.89 & 56699.67 & 292.9 & WSRC, 1996d \\
\hline BGO-20AA & 74953.76 & 57114.81 & 280.88 & Rust, 1996 \\
\hline BGO-25A & 76158.5 & 55668.08 & 294.7 & WSRC, 1996d \\
\hline BGO-26A & 76144.6 & 55014.2 & 285.1 & WSRC, 1996d \\
\hline BGO-27C & 75666.3 & 54671.4 & 273.9 & WSRC, 1996d \\
\hline BGO-29A & 75560 & 54103.5 & 262.1 & WSRC, 1996d \\
\hline
\end{tabular}


Appendix B-1: Locations of Sites within the Model Area (Continued)

\begin{tabular}{|c|c|c|c|c|} 
Well ID & $\begin{array}{c}\text { SRS } \\
\text { Northing } \\
(\mathbf{f t})\end{array}$ & $\begin{array}{c}\text { SRS } \\
\text { Easting } \\
(\mathbf{f t})\end{array}$ & $\begin{array}{c}\text { Surface } \\
\text { Elevation } \\
(\mathbf{f t} \text { m.s.l. })\end{array}$ & Reference $^{\mathrm{I}}$ \\
\hline
\end{tabular}

\begin{tabular}{|l|r|r|r|l|}
\hline BGO-31C & 74978 & 54816.2 & 271.1 & WSRC, 1996d \\
\hline BGO-33C & 74479.7 & 55681.4 & 277.4 & WSRC, 1996d \\
\hline BGO-35C & 73953.9 & 56545.7 & 271.4 & WSRC, 1996d \\
\hline BGO-37C & 73498.2 & 57279.2 & 284.3 & WSRC, 1996d \\
\hline BGO-39A & 73572.52 & 57821.93 & 293.7 & WSRC, 1996d \\
\hline BGO-3A & 75561.7 & 58806.8 & 288.7 & WSRC, 1996d \\
\hline BGO-3D & 75351.3 & 58809.2 & 290.8 & WSRC, 1996d \\
\hline BGO-41A & 76469.52 & 55403.69 & 298.3 & WSRC, 1996d \\
\hline BGO-42C & 76404.71 & 55522.27 & 295.9 & WSRC, 1996d \\
\hline BGO-43AA & 77066.01 & 56268.64 & 312.2 & WSRC, 1996d \\
\hline BGO-44AA & 76757.02 & 57880.51 & 283.3 & WSRC, 1996d \\
\hline BGO-45A & 75830.03 & 54550.14 & 276.9 & WSRC, 1996d \\
\hline BGO-46B & 75012.1 & 54444.65 & 263.4 & WSRC, 1996d \\
\hline BGO-47A & 74728.83 & 54914.04 & 264.8 & WSRC, 1996d \\
\hline BGO-48C & 74599.64 & 55124.38 & 274.7 & WSRC, 1996d \\
\hline BGO-49A & 73902.78 & 56205.08 & 269.1 & WSRC, 1996d \\
\hline BGO-50A & 75201.16 & 54179.77 & 253.5 & WSRC, 1996d \\
\hline BGO-51AA & 74113.1 & 57867 & 287.2 & WSRC, 1996d \\
\hline BGO-52AA & 74638 & 57178.1 & 281.6 & WSRC, 1996d \\
\hline BGO-53AA & 76065 & 55431.5 & 288.9 & WSRC, 1996d \\
\hline BGO-5C & 76476.9 & 58794.5 & 294.2 & WSRC, 1996d \\
\hline BGO-6A & 76487.2 & 58316.8 & 283.8 & WSRC, 1996d \\
\hline BGO-6B & 76553.24 & 58346.46 & 284.5 & WSRC, 1996d \\
\hline BGO-8A & 76569 & 57618.3 & 281.3 & WSRC, 1996d \\
\hline BGO-9AA & 76975.69 & 57371.94 & 282.8 & WSRC, 1996d \\
\hline BGT-1 & 76700.6 & 59178.4 & 282.9 & WSRC, unknown \\
\hline BGT-10 & 79104.6 & 59507.2 & 215.2 & WSRC, unknown \\
\hline BGT-11 & 79566.9 & 59697.7 & 222.5 & Rust, 1996 \\
\hline BGT-12 & 77291.2 & 58045.9 & 284.2 & WSRC, unknown \\
\hline BGT-13 & 77488.9 & 58074 & 287.8 & WSRC, unknown \\
\hline BGT-14 & 77984 & 58143.4 & 280.7 & WSRC, unknown \\
\hline BGT-15 & 78479.2 & 58212.8 & 277.5 & WSRC, unknown \\
\hline BGT-16 & 78974.1 & 58283.5 & 250.7 & WSRC, unknown \\
\hline BGT-17 & 79469.7 & 58350 & 240.7 & WSRC, unknown \\
\hline BGT-18 & 79965.3 & 58416.5 & 216.5 & Rust, 1996 \\
\hline BGT-2 & 76957.6 & 59607.2 & 276.4 & WSRC, unknown \\
\hline BGT-20 & 80956.4 & 58549.6 & 159.5 & Rust, 1996 \\
\hline BGT-21 & 77280.7 & 56952.5 & 294.2 & WSRC, unknown \\
\hline
\end{tabular}


Appendix B-1: Locations of Sites within the Model Area (Continued)

\begin{tabular}{|c|c|c|c|c|}
\hline Well ID & $\begin{array}{c}\text { SRS } \\
\text { Northing } \\
\text { (ft) }\end{array}$ & $\begin{array}{c}\text { SRS } \\
\text { Easting } \\
\text { (ft) }\end{array}$ & $\begin{array}{l}\text { Surface } \\
\text { Elevation } \\
\text { (ft m.s.l.) }\end{array}$ & Reference $^{1}$ \\
\hline$\because$ &. & . & & $y$ \\
\hline BGT-25 & $\begin{array}{l}79278.7 \\
\end{array}$ & 57041.4 & 264.8 & WSRC, unknown \\
\hline BGT-27 & 80277.7 & 57085.9 & 256.9 & WSRC, unknown \\
\hline BGT-28 & 80777.2 & 57108.1 & 258.3 & Rust, 1996 \\
\hline BGT-29 & 81276.7 & 57130.4 & 243 & WSRC, unknown \\
\hline BGT-3 & 77197.6 & 60045.9 & 275.7 & Rust, 1996 \\
\hline BGT-30 & 81726.3 & 57150.4 & 219 & WSRC, unknown \\
\hline BGT-31 & 77229 & 56189.8 & 308.76 & WSRC, unknown \\
\hline BGT-32 & 77791.4 & 56121.1 & 310.12 & WSRC, unknown \\
\hline BGT-33 & 78404.5 & 56037.2 & 290.42 & WSRC, unknown \\
\hline BGT-34 & 78803.9 & 56027.5 & 286.76 & WSRC, unknown \\
\hline BGT-35 & 79305.8 & 55929.9 & 267.73 & WSRC, unknown \\
\hline BGT-36 & 79801.9 & 55867.5 & 261.36 & WSRC, unknown \\
\hline BGT-37 & 80298 & 55805 & 251.6 & WSRC, unknown \\
\hline BGT-38 & 80870.5 & 55733 & 240.14 & WSRC, unknown \\
\hline BGT-39 & 81290.2 & 55680.3 & 241.88 & WSRC, unknown \\
\hline BGT-4 & 77437.6 & 60484.5 & 259.2 & WSRC, unknown \\
\hline BGT-40 & 77297.2 & 55644.4 & 332.32 & WSRC, unknown \\
\hline BGT-41 & 77734.8 & 55490.1 & 328.37 & WSRC, unknown \\
\hline BGT-42 & 78240.7 & 55313.1 & 310.92 & WSRC, unknown \\
\hline BGT-43 & 79655.9 & 54816 & 277.08 & WSRC, unknown \\
\hline BGT-44 & 80127.7 & 54650.4 & 276.2 & WSRC, unknown \\
\hline BGT-45 & 80461.7 & 54533.1 & 285.28 & WSRC, unknown \\
\hline BGT-46 & 76714.3 & 55355 & 310 & WSRC, unknown \\
\hline BGT-47 & 77051.85 & 54986.57 & 317.32 & Rust, 1996 \\
\hline BGT-48 & 77135.7 & 54895.1 & 314.33 & WSRC, unknown \\
\hline BGT-49 & 76203.9 & 54946.3 & 297.26 & WSRC, unknown \\
\hline BGT-5 & 77677.6 & 60924.1 & 225.7 & Rust, 1996 \\
\hline BGT-50 & 76359.3 & 54756.2 & 296.27 & WSRC, unknown \\
\hline BGT-51 & 75519.8 & 54505.7 & 272.64 & WSRC, unknown \\
\hline BGT-53 & 75837.68 & 53422.04 & 278.25 & Rust, 1996 \\
\hline BGT-54 & 75941.7 & 52889.1 & 279.96 & WSRC, unknown \\
\hline BGT-56 & 73521.2 & 56265.8 & 262.94 & WSRC, unknown \\
\hline BGT-57 & 73268.5 & 56104.2 & 259.35 & WSRC, unknown \\
\hline BGT-58 & 73406.9 & 57399.6 & 285.76 & WSRC, unknown \\
\hline BGT-59 & 72802.6 & 57123.2 & 281.88 & WSRC, unknown \\
\hline BGT-6 & 77254.8 & 58746.7 & 282.2 & WSRC, unknown \\
\hline BGT-60 & 73120.6 & 58057.2 & 291.42 & WSRC, unknown \\
\hline BGT-61 & 72911.77 & 58490.09 & 284.3 & Rust, 1996 \\
\hline BGT-62 & 72854.4 & 58608 & 282.03 & WSRC, unknown \\
\hline BGT-63 & 73319.4 & 59146.3 & 293.67 & WSRC, unknown \\
\hline BGT-63A & 73646.4 & 58768.1 & 290.79 & WSRC, unknown \\
\hline
\end{tabular}


Appendix B-1: Locations of Sites within the Model Area (Continued)

\begin{tabular}{|c|c|c|c|c|}
\hline Well D & $\begin{array}{c}\text { SRS } \\
\text { Northing } \\
(\mathbf{f t})\end{array}$ & $\begin{array}{c}\text { SRS } \\
\text { Easting } \\
(\mathrm{ft})\end{array}$ & $\begin{array}{c}\text { Surface } \\
\text { Elevation } \\
\text { (ft m.s.l.) }\end{array}$ & Reference $^{1}$ \\
\hline
\end{tabular}

\begin{tabular}{|c|c|c|c|c|}
\hline BGT-64 & 73013.7 & 59500 & 283.25 & WSRC, unknown \\
\hline BGT-66 & 74476.6 & 60033.7 & 244.04 & WSRC, unknown \\
\hline BGT-67 & 74443.06 & 60426.74 & 242.03 & Rust, 1996 \\
\hline BGT-7 & 77717.8 & 58935.7 & 276.4 & WSRC, unknown \\
\hline BGT-8 & 78161.5 & 59118.6 & 249.3 & WSRC, unknown \\
\hline BGT-9 & 78642.3 & 59316.7 & 226 & Rust, 1996 \\
\hline BGX-11D & 75300.7 & 59581.4 & 273.8 & WSRC, 1996d \\
\hline BGX-1A & 76831.89 & 58590.35 & 289.1 & WSRC, 1996d \\
\hline BGX-2B & 77203.4 & 58256.5 & 289.2 & WSRC, 1996d \\
\hline BGX-4A & 77879.2 & 57215.6 & 288.8 & WSRC, 1996d \\
\hline BGX-7D & 78349.3 & 58312.8 & 277.1 & WSRC, 1996d \\
\hline BGX-9D & 76936 & 59522.1 & 277.4 & WSRC, 1996d \\
\hline BRR-1D & 77365.2 & 50588.2 & 293.8 & WSRC, 1996d \\
\hline BRR-3D & 77398.3 & 50203.5 & 289.5 & WSRC, 1996d \\
\hline BRR-6B & 77054.6 & 51100 & 293.9 & WSRC, 1996d \\
\hline BRR-7B & 77575.4 & 50707.5 & 289.6 & WSRC, 1996d \\
\hline BRR-8B & 77634.7 & 50116.5 & 276.7 & WSRC, 1996d \\
\hline CCP-1A & 66659.2 & 46981.3 & 287.1 & WSRC, 1996d \\
\hline CFD-1 & 55486.64 & 54875.37 & 268.8 & WSRC, 1994 \\
\hline CFD-18 & 56297.09 & 54935.66 & 248.3 & WSRC, 1994 \\
\hline CFD-5 & 55769.51 & 54803.57 & 257.8 & WSRC, 1994 \\
\hline CMP-1-CP & 52470.6 & 53252.6 & 248.9 & WSRC, 1998 \\
\hline CMP-30B & 51729.8 & 53166.9 & 286.5 & WSRC, 1996d \\
\hline CMP-32B & 52220 & 54052.8 & 251.7 & WSRC, 1996d \\
\hline CMP-4B-CP & 52688.5 & 53280.3 & $\overline{241.8}$ & WSRC, 1998 \\
\hline CMP-6A-CP & 52853.3 & 53105.7 & 238.4 & WSRC, 1998 \\
\hline CPC-1 & 66855.77 & 47183.78 & 285.1 & WSRC, 1990b \\
\hline CRP-5D & 68549.46 & 44515.25 & 274.5 & WSRC, 1996d \\
\hline CRP-9D & 69156.7 & 44243.2 & 268.4 & WSRC, 1996d \\
\hline CRSB-1 & 66908.216 & 45003.778 & 278.2 & Bechtel, 1999 \\
\hline CRSB-101 & 68802.14 & 43999.56 & 275.6 & Gregg, 1998a \\
\hline CRSB-11 & 67613.71 & 44513.8 & 276.71 & Bechtel, 1998 \\
\hline CRSB-14 & 67305.06 & 44515.82 & 275.55 & Bechtel, 1998 \\
\hline CRSB-16 & 67308.39 & 44827.9 & 282.56 & Bechtel, 1998 \\
\hline CRSB-17 & 67809.74 & 44718.16 & 281.01 & Bechtel, 1999 \\
\hline CRSB-18 & 68199.61 & 44399.48 & 273.78 & Rucker, 1999a \\
\hline CRSB-20 & 67799.39 & 44399.56 & 274.21 & Rucker, 1999a \\
\hline CRSB-21 & 67599.5 & 44399.5 & 272.85 & Rucker, 1999a \\
\hline CRSB-24 & 68003.84 & 44792.46 & 277.2 & Bechtel, 1999. \\
\hline CRSB-26 & 67873.31 & 45225.52 & 291.63 & Bechtel, 1999 \\
\hline CRSB-28 & 67576.46 & 45225.76 & 289.26 & Bechtel, 1998 \\
\hline
\end{tabular}


Appendix B-1: Locations of Sites within the Model Area (Continued)

\begin{tabular}{|c|c|c|c|c|}
\hline Well ID & $\begin{array}{c}\text { SRS } \\
\text { Northing } \\
\text { (ft) }\end{array}$ & $\begin{array}{c}\text { SRS } \\
\text { Easting } \\
\text { (ft) }\end{array}$ & $\begin{array}{c}\text { Surface } \\
\text { Elevation } \\
\text { (ft m.s.l.) }\end{array}$ & Reference $^{1}$ \\
\hline \multicolumn{2}{|l|}{ a } & 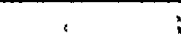 & & \\
\hline CRSB-30 & 67280.39 & 45223.19 & 285.4 & Bechtel, 1999 \\
\hline CRSB-31 & 67123.9 & 45211 & 284.43 & Bechtel, 1999 \\
\hline CRSB-32 & 67008.52 & 44392.01 & 274.13 & Bechtel, 1998 \\
\hline CRSB-35 & 67825.67 & 43793.81 & 257.4 & Bechtel, 1998 \\
\hline CRSB-36 & 67621.82 & 43785.52 & 265.62 & Bechtel, 1998 \\
\hline CRSB-37 & 67423.56 & 43790.49 & 270.47 & Bechtel, 1998 \\
\hline CRSB-4 & 67312.06 & 44995.71 & 282.76 & Bechtel, 1998 \\
\hline CRSB-40 & 68601.07 & 43409.99 & 227.33 & Bechtel, 1998 \\
\hline CRSB-43 & 67994.55 & 43410.39 & 258.5 & Gregg, 1998a \\
\hline CRSB-45 & 67594.3 & 43410.39 & 270.6 & Gregg, 1998a \\
\hline CRSB-5 & 67505.55 & 44990.65 & 288.3 & Bechtel, 1998 \\
\hline CRSB-50 & 69193.96 & 43418.21 & 255.61 & Bechtel, 1998 \\
\hline CRSB-52 & 68799.02 & 43405.07 & 233.14 & Bechtel, 1998 \\
\hline CRSB-54 & 68652.6 & 43191.69 & 224.72 & Bechtel, 1999 \\
\hline CRSB-55 & 68684.99 & 43004.6 & 229.14 & Bechtel, 1999 \\
\hline CRSB-56 & 68719.98 & 42804.2 & 232.54 & Bechtel, 1998 \\
\hline CRSB-57 & 68810.63 & 42405.28 & 248.17 & Bechtel, 1998 \\
\hline CRSB-58 & 68845.93 & 42209.69 & 253.86 & Bechtel, 1998 \\
\hline CRSB-6 & 67653.75 & 44996.22 & 289.386 & Bechtel, 1998 \\
\hline CRSB-60 & 68927.513 & 41797.987 & 257.035 & Bechtel, 1998; \\
\hline CRSB-61 & 68964.11 & 41602.18 & 257.28 & Bechtel, 1998 \\
\hline CRSB-63 & 69026.42 & 41190.06 & 240.38 & Bechtel, 1998 \\
\hline CRSB-64 & 69107.78 & 40798.67 & 229.92 & Bechtel, 1998 \\
\hline CRSB-65 & 69183.31 & 40519.91 & 214.8 & Bechtel, 1999 \\
\hline CRSB-86 & 69352.73 & 42845.6 & 206.55 & Bechtel, 1998 \\
\hline CRSB-88 & 68423.77 & 43795.59 & 253.2 & Bechtel, 1998 \\
\hline CRSB-89 & 68404.15 & 43986.53 & 260.61 & Bechtel, 1998 \\
\hline CRSB-90 & 68404.94 & 44200.19 & 268.39 & Bechtel, 1998 \\
\hline CRSB-91 & 68389.78 & 44400.57 & 273.1 & Bechtel, 1998 \\
\hline CRSB-92 & 67802.7 & 44995.83 & 287.5 & Kirr, 1998 \\
\hline CRSB-93 & 67950.27 & 44983.57 & 286.84 & Bechtel, 1998 \\
\hline CRSB-94 & 68103.96 & 44994.65 & 283.34 & Bechtel, 1999 \\
\hline CRSB-95 & 68252.96 & 44995.67 & 280.37 & Bechtel, 1998 \\
\hline CRSB-96 & 68401.76 & 44995.96 & 282.76 & Bechtel, 1998 \\
\hline CRSB-97 & 68718.65 & 44544.45 & 276.75 & Bechtel, 1998 \\
\hline CRSB-98 & 68851.71 & 44548.8 & 275.11 & Bechtel, 1998 \\
\hline CRSB-99 & 68994.32 & 44547.07 & 274.44 & Bechtel, 1998 \\
\hline CSB-101 & 66002.48 & 44868.43 & 281.2 & Rucker, 1999b \\
\hline CSB-102 & 65984.05 & 43109.96 & 283.5 & Rucker, 1999b \\
\hline CSB-103 & 64510.41 & 44149.64 & 257.6 & Rucker, 1999b \\
\hline CSB-104 & 63455.35 & 43787.85 & 224.9 & Rucker, 1999b \\
\hline
\end{tabular}


Appendix B-1: Locations of Sites within the Model Area (Continued)

\begin{tabular}{|c|c|c|c|c|}
\hline & $\begin{array}{c}\text { SRS } \\
\text { Northing } \\
(\mathrm{ft})\end{array}$ & $\begin{array}{c}\text { SRS } \\
\text { Easting } \\
(\mathrm{ft})\end{array}$ & $\begin{array}{c}\text { Surface } \\
\text { Elevation } \\
(\mathrm{ft} \text { m.s.l. })\end{array}$ & Reference $^{\mathbf{1}}$ \\
\hline
\end{tabular}

\begin{tabular}{|l|r|r|r|l|}
\hline CSB-105 & 63628.93 & 42549.54 & 212.1 & Rucker, 1999b \\
\hline CSB-106 & 64261.04 & 41634.09 & 183.8 & Rucker, 1999b \\
\hline CSB-107 & 64785.48 & 42446.08 & 220.7 & Rucker, 1999b \\
\hline CSB-108 & 65154.15 & 40531.22 & 196.7 & Rucker, 1999b \\
\hline CSB-109 & 65351.75 & 39098.64 & 172.9 & Rucker, 1999b \\
\hline CSB-110 & 66382.03 & 38102.37 & 184.6 & Rucker, 1999b \\
\hline CSB-111 & 66888.08 & 38022.32 & 172.1 & Rucker, 1999b \\
\hline CSB-112 & 67195.7 & 38892.52 & 195.3 & Rucker, 1999b \\
\hline CSB-113 & 67729.39 & 39311.45 & 210.4 & Rucker, 1999b \\
\hline CSB-114 & 68278.36 & 40206.45 & 196 & Rucker, 1999b \\
\hline CSB-116 & 67598.91 & 42794.74 & 274.7 & Rucker, 1999b \\
\hline CSB-117 & 67218.22 & 42698.32 & 276.5 & Rucker, 1999b \\
\hline CSB-118 & 66802.15 & 42707.65 & 277.8 & Rucker, 1999b \\
\hline CSB-119 & 67398.77 & 42193.79 & 268.3 & Rucker, 1999b \\
\hline CSB-120 & 66998.48 & 42197.24 & 282.8 & Rucker, 1999b \\
\hline CSB-121 & 66601.65 & 42197.24 & 274.2 & Rucker, 1999b \\
\hline CSB-122 & 65679.58 & 41120.9 & 222.9 & Rucker, 1999b \\
\hline CSB-123 & 65585.04 & 41352.9 & 224.2 & Rucker, 1999b \\
\hline CSB-2C & 67608.88 & 44973.31 & 289.9 & WSRC, 1996d \\
\hline CSB-3C & 67762.9 & 44617.79 & 280.9 & WSRC, 1996d \\
\hline CSB-47 & 67185.49 & 43419.51 & 282.7 & Bechtel, 1998 \\
\hline CSB-47A & 68006.2 & 44200.2 & 268.9 & ARA, 1999 \\
\hline CSB-48 & 69597.68 & 43418.61 & 259.8 & Bechtel, 1998 \\
\hline CSB-48A & 68205.2 & 44199.8 & 270.1 & ARA, 1999 \\
\hline FCHB-56 & 68602.13 & 43999.43 & 267 & Rucker, 1999a \\
\hline FSB-58 & 68028.13 & 41577.12 & 235.5 & Rucker, 1999a \\
\hline CSB-59 & 67751.28 & 41295.09 & 231.2 & Rucker, 1999a \\
\hline CSB-60 & 67502.28 & 40980.25 & 247 & Rucker, 1999a \\
\hline CSB-61 & 67245.08 & 40668.7 & 252.2 & Rucker, 1999a \\
\hline CSB-62 & 67014.1 & 40344.02 & 240.9 & Rucker, 1999a \\
\hline CSB-63 & 66792.95 & 40014.43 & 227.1 & Rucker, 1999a \\
\hline CSB-64 & 66530.84 & 39707.79 & 212.7 & Rucker, 1999a \\
\hline CSB-65 & 66173.72 & 39517.58 & 195.6 & Rucker, 1999a \\
\hline CSB-66A & 65783.83 & 39622.52 & 191.7 & Rucker, 1999a \\
\hline CSD-4D & 63143.8 & 50058.9 & 306.5 & WSRC, 1996d \\
\hline E-26 & 61550 & 59580 & 314.3 & D'Appolonia, \\
\hline E-4 & 58770 & 60440 & 315.4 & D'Appolonia, \\
\hline
\end{tabular}


Appendix B-1: Locations of Sites within the Model Area (Continued)

\begin{tabular}{|c|c|c|c|c|}
\hline Well W & $\begin{array}{c}\text { SRS } \\
\text { Northing } \\
\text { (ft) }\end{array}$ & $\begin{array}{c}\text { SRS } \\
\text { Easting } \\
\text { (ft) }\end{array}$ & $\begin{array}{c}\text { Surface } \\
\text { Elevation } \\
\text { (ft m.s.l.) }\end{array}$ & Reference $^{1}$ \\
\hline & & & & \\
\hline FCH-3 & 78059.22 & 52087.22 & 307.2 & WSRC, 1993a \\
\hline FCH-4 & 77514.56 & 52021.03 & 297.5 & WSRC, 1993a \\
\hline FCH-5 & 76992.12 & 51667.65 & 284.2 & WSRC, 1993a \\
\hline FCH-6 & 76410.33 & 51245.7 & 291.5 & WSRC, 1993a \\
\hline FIW-1MC & 76165.3 & 51354.4 & 293.3 & WSRC, 1996d \\
\hline FIW-2MA & 75930.8 & 51184.5 & 290.5 & WSRC, 1996d \\
\hline FNB-1A & 80154.5 & 54288.8 & 282.4 & WSRC, 1996d \\
\hline FNB-3A & 80557.2 & 54116.6 & 282.2 & WSRC, 1996d \\
\hline FSB-100A & 75534.4 & 50958.4 & 283.8 & WSRC, 1996d \\
\hline FSB-101A & 75719 & 51191.3 & 282.9 & WSRC, 1996d \\
\hline FSB-112A & 74231.4 & 48809.1 & 227 & WSRC, 1996d \\
\hline FSB-113A & 74167.5 & 51068.1 & 221.3 & WSRC, 1996d \\
\hline FSB-114A & 75297.4 & 52046.6 & 250 & WSRC, 1996d \\
\hline FSB-115C & 72515.5 & 49736 & 205.8 & WSRC, 1996d \\
\hline FSB-116C & 72725.5 & 50645.9 & 200.5 & WSRC, 1996d \\
\hline FSB-120A & 75538.9 & 49175.7 & 278 & WSRC, 1996d \\
\hline FSB-121C & 75155.7 & 48413.1 & 254.4 & WSRC, 1996d . \\
\hline FSB-122C & 73881.8 & 48195 & 216 & WSRC, 1996d \\
\hline FSB-123C & 74566.7 & 51750.5 & 236.3 & WSRC, 1996d \\
\hline FSB-1TA & 75649.1 & 51658.3 & 275.4 & WSRC, 1996d \\
\hline FSB-76A & 76131.9 & 51391.6 & 291.5 & WSRC, 1996d \\
\hline FSB-78A & 74757.7 & 50172.8 & 270.5 & WSRC, 1996d \\
\hline FSB-79A & 73664.5 & 50149.6 & 216.1 & WSRC, 1996d \\
\hline FSB-87A & 75601.7 & 50115.8 & 285.6 & WSRC, 1996d \\
\hline FSB-89C & 75553.2 & 51345.2 & 279.1 & WSRC, 1996d \\
\hline FSB-91C & 75213.3 & 50953.5 & 277 & WSRC, 1996d \\
\hline FSB-93C & 74897.3 & 50458.3 & 274 & WSRC, 1996d \\
\hline FSB-95C & 74971.7 & 50016.7 & 281.8 & WSRC, 1996d \\
\hline FSB-96A & 74882.2 & 49778.7 & 277.7 & WSRC, 1996d \\
\hline FSB-97A & 75171.2 & 49965.7 & 283.8 & WSRC, 1996d \\
\hline FSB-98A & 75389.8 & 50121.6 & 280.7 & WSRC, 1996d \\
\hline FSB-99A & 75675.6 & 50314.8 & 285.3 & WSRC, 1996d \\
\hline FSB-PC & 74090.2 & 50140 & 230.8 & WSRC, 1996d \\
\hline GAPWR-TW-1 & 51036.5 & 1544.1 & 219 & $\begin{array}{l}\text { Falls and others, } \\
1998\end{array}$ \\
\hline HAA-1TA & 69892.2 & 62953.3 & 290.2 & WSRC, 1996d \\
\hline HAA-2AA & 70925.4 & 61285.1 & 291.4 & WSRC, 1996d \\
\hline HAA-3AA & 71488 & 60201.9 & 274.5 & WSRC, 1996d \\
\hline HAA-4AA & 72223.2 & 61929.6 & 299.2 & WSRC, 1996d \\
\hline HAA-6AA & 71441 & 63860.2 & 279.8 & WSRC, 1996d \\
\hline $\mathrm{HC}-03 \mathrm{AA}$ & 54483.32 & 42333.62 & 263.8 & WSRC, 1996d \\
\hline $\mathrm{HC}-12 \mathrm{~A}$ & 73187 & 59504 & 287.3 & WSRC, 1996d \\
\hline
\end{tabular}


Appendix B-1: Locations of Sites within the Model Area (Continued)

\begin{tabular}{|c|c|c|c|c|}
\hline & $\begin{array}{c}\text { SRS } \\
\text { Northing } \\
\text { (ft) }\end{array}$ & $\begin{array}{c}\text { SRS } \\
\text { Easting } \\
\text { (ft) }\end{array}$ & $\begin{array}{c}\text { Surface } \\
\text { Elevation } \\
\text { (ft m.s.l.) }\end{array}$ & Reference $^{1}$ \\
\hline
\end{tabular}

\begin{tabular}{|c|c|c|c|c|}
\hline $\mathrm{HCA}-4 \mathrm{AA}$ & 72513.7 & 62942.5 & 308.6 & WSRC, 1996d \\
\hline $\mathrm{HCH}-1$ & 72796.38 & 60923.42 & 284 & WSRC, 1993a \\
\hline $\mathrm{HCH}-2$ & 72519.61 & 60091.79 & 270.9 & WSRC, 1993a \\
\hline $\mathrm{HCH}-3$ & 71998.82 & 59917.33 & 264 & WSRC, 1993a \\
\hline $\mathrm{HCH}-4$ & 72449.59 & 59139.93 & 269.9 & WSRC, 1993a \\
\hline $\mathrm{HCH}-5$ & 71810.36 & 59331.53 & 255 & WSRC, 1996d \\
\hline HIW-1BD & 72564.6 & 58342.2 & 275.8 & WSRC, 1996d \\
\hline HIW-1MC & 72500 & 58471.8 & 272.3 & WSRC, 1996d \\
\hline HIW-2A & 73249.7 & 56753 & 276.3 & WSRC, 1996d \\
\hline HIW-2MC & 73226.4 & 56698.4 & 269 & WSRC, 1996d \\
\hline HIW-4MC & 73160.1 & 56570.1 & 263.4 & WSRC, 1996d \\
\hline HIW-5MC & 73557.9 & 56498.9 & 266.1 & WSRC, 1996d \\
\hline HMD-1C & 78731.7 & 56973.3 & 262.7 & WSRC, 1996d \\
\hline HMD-2C & 79665.8 & 57269.7 & 259.3 & WSRC, 1996d \\
\hline HMD-3C & 79578.7 & 57745.2 & 257.2 & WSRC, 1996d \\
\hline HMD-4C & 79160.4 & 58188.5 & 248.5 & WSRC, 1996d \\
\hline HPC-1 & 70395.4 & 62493.6 & 293.5 & WSRC, 1996d \\
\hline HPT-1A & 74847.1 & 60587 & 232.9 & WSRC, 1996d \\
\hline HPT-2A & 75061.8 & 60200.5 & 257.8 & WSRC, 1996d \\
\hline HSB-101C & 72001.9 & 58604.4 & 256.3 & WSRC, 1996d \\
\hline HSB-103C & 71593.9 & 58323.6 & 245.2 & WSRC, 1996d \\
\hline HSB-104C & 71376.8 & 58082.6 & 245.5 & WSRC, 1996d \\
\hline $\mathrm{HSB}-105 \mathrm{C}$ & 71447.3 & 57883.8 & 247.2 & WSRC, 1996d \\
\hline HSB-106C & 71720.9 & 57651.5 & 250.7 & WSRC, 1996d \\
\hline HSB-107C & 71698.5 & 57432 & 259.3 & WSRC, 1996d \\
\hline HSB-109C & 71684.8 & 56895.6 & 259.4 & WSRC, 1996d \\
\hline HSB-110C & 71779.3 & 56680.7 & 253.4 & WSRC, 1996d \\
\hline HSB-111C & 71919.4 & 56501.9 & 253.7 & WSRC, 1996d \\
\hline HSB-112C & 72156.4 & 56417.4 & 252.6 & WSRC, 1996d \\
\hline HSB-113C & 72312.3 & 56160.4 & 258.7 & WSRC, 1996d \\
\hline HSB-115C & 72653.2 & 56043.2 & 266.8 & WSRC, 1996d \\
\hline HSB-117A & 72733.6 & 55170.1 & 234.8 & WSRC, 1996d \\
\hline HSB-118A & 72696.4 & 55775.6 & 245 & WSRC, 1996d \\
\hline HSB-119A & 73082.5 & 56100.2 & 254.8 & WSRC, 1996d \\
\hline HSB-120A & 73395.1 & 56431.9 & 266 & WSRC, 1996d \\
\hline HSB-121A & 72024.8 & 57389.6 & 272.3 & WSRC, 1996d \\
\hline HSB-122A & 72195.9 & 57747.4 & 269.4 & WSRC, 1996d \\
\hline HSB-123A & 72189.8 & 58124.8 & 263.6 & WSRC, 1996d \\
\hline HSB-124A & 72199.6 & 58514.6 & 263.9 & WSRC, 1996d \\
\hline HSB-132C & 71472.4 & 58787.7 & 238.3 & WSRC, 1996d \\
\hline HSB-139A & 71127.4 & 57365.4 & 231.5 & WSRC, 1996d \\
\hline
\end{tabular}


Appendix B-1: Locations of Sites within the Model Area (Continued)

\begin{tabular}{|c|c|c|c|c|}
\hline Well ID & $\begin{array}{c}\text { SRS } \\
\text { Northing } \\
\text { (ft) }\end{array}$ & $\begin{array}{c}\text { SRS } \\
\text { Easting } \\
\text { (ft) }\end{array}$ & $\begin{array}{l}\text { Surface } \\
\text { Elevation } \\
\text { (ft m.s.l.) }\end{array}$ & Reference $^{1}$ \\
\hline . & 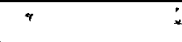 & - & & . \\
\hline HSB-140A & 70050.3 & 56535.4 & 234 & WSRC, 1996d \\
\hline HSB-141A & 71213.6 & 59168.7 & 252.6 & WSRC, 1996d \\
\hline HSB-142C & 73119 & 53505.3 & 201.6 & WSRC, 1996d \\
\hline HSB-143C & 73738.2 & 52773.2 & 220.1 & WSRC, 1996d \\
\hline HSB-144A & 71892.1 & 56200.5 & 233.6 & WSRC, 1996d \\
\hline HSB-145C & 71098.9 & 57769 & 233.7 & WSRC, 1996d \\
\hline HSB-146A & 70478.9 & 58454 & 249.5 & WSRC, 1996d \\
\hline HSB-148C & 70151.5 & 55344.2 & 248.9 & WSRC, 1996d \\
\hline HSB-151C & 72997.9 & 54014.9 & 211.6 & WSRC, 1996d \\
\hline HSB-152C & 72012 & 54346.7 & 212.1 & WSRC, 1996d \\
\hline HSB-65A & 72436.2 & 58436 & 270.7 & WSRC, 1996d \\
\hline HSB-68A & 71526.9 & 56892.1 & 247.4 & WSRC, 1996d \\
\hline HSB-69A & 71549.4 & 56465.1 & 234.1 & WSRC, 1996d \\
\hline HSB-83A & 71648.6 & 58606.1 & 234.9 & WSRC, 1996d \\
\hline HSB-84A & 71586.2 & 56359.1 & 226.7 & WSRC, 1996d \\
\hline HSB-85A & 73791.9 & 58943.4 & 292.1 & WSRC, 1996d \\
\hline HSB-86A & 72520.2 & 55985.9 & 260 & WSRC, 1996d \\
\hline HSB-PC & 72119.31 & 55650.03 & 227.8 & WSRC, 1996d \\
\hline HSB-TB & 72394 & 58696.1 & 267.1 & WSRC, 1996d \\
\hline HSL-6AA & 72692.6 & 60555.7| & 274.6 & WSRC, 1996d \\
\hline HSL-8AA & 72729.4 & 61113.8 & 286.7 & WSRC, 1996d \\
\hline IDB-2A & 77284.4 & 75391.1| & 302.4 & WSRC, 1996d \\
\hline IDP-3A & 85104.3 & 3778.11 & 282.2 & WSRC, 1996d \\
\hline IDQ-3A & 80553.7 & 35854 & 203.2 & WSRC, 1996d \\
\hline KAC-9D & 53197.8 & 42588.1 & 260.2 & WSRC, 1996d \\
\hline KPT-1 & 56460.7 & 40978.6 & 205.2 & Gregg, 1998b \\
\hline KPT-2 & 54110.9 & 43845.9 & 258.2 & Gregg, 1998b \\
\hline KPT-3 & 52641.2 & 43434 & 225.5 & Gregg, 1998b \\
\hline KPT-4 & 50028.8 & 41172.3 & 258.9 & Gregg, 1998b \\
\hline KPT-5 & 47731.6 & 39198.3 & 225.1 & Gregg, 1998b \\
\hline KРT-6 & 49191.9 & 39297.1 & 232.8 & Gregg, 1998b \\
\hline KРT-7 & 49340.5 & 37721.3 & 211.8 & Gregg, 1998b \\
\hline KPT-8 & 51888.2 & 39652.9 & 238.6 & Gregg, 1998b \\
\hline KPT-9 & 52456.1 & 36726.3 & 204.9 & Gregg, 1998b \\
\hline L3-CPT-PG-9 & 54369 & 41344 & 270 & ARA, 1992 \\
\hline LAC-5DL & 45365.4 & 51352 & 239.8 & WSRC, 1996d \\
\hline LAC-6DL & 45272.8 & 51188.1 & 239.8 & WSRC, 1996d \\
\hline LAC-7DL & 45097.1 & 51118.4 & 239.4 & WSRC, 1996d \\
\hline LAC-8DL & 45096.6 & 51300.9 & 234 & WSRC, 1996d \\
\hline LCO-5A & 44987 & 50866 & 230 & WSRC, 1996d \\
\hline LCO-5DL & 44974.5 & 50887.5 & 230.3 & WSRC, 1996d \\
\hline
\end{tabular}


Appendix B-1: Locations of Sites within the Model Area (Continued)

\begin{tabular}{|c|c|c|c|c|}
\hline Well ID & $\begin{array}{c}\text { SRS } \\
\text { Northing } \\
(\mathbf{f t})\end{array}$ & $\begin{array}{c}\text { SRS } \\
\text { Easting } \\
(\mathbf{f t})\end{array}$ & $\begin{array}{c}\text { Surface } \\
\text { Elevation } \\
(\text { ft m.s.l.) }\end{array}$ & Reference $^{1}$ \\
\hline
\end{tabular}

\begin{tabular}{|l|r|r|r|l|}
\hline LCO-8DL & 45586.1 & 51380.6 & 243.4 & WSRC, 1996d \\
\hline LFW-10SB & 83162.5 & 46137.5 & 168.4 & WSRC, 1991e \\
\hline LWN-1SB & 68131.9 & 33690.8 & 282.5 & WSRC, 1996d \\
\hline LWN-2SB & 66548.6 & 34739.1 & 231 & WSRC, 1996d \\
\hline LWN-3SB & 66900.2 & 32092.1 & 245.7 & WSRC, 1996d \\
\hline LWR-2SB & 71766 & 45998.8 & 248.1 & WSRC, 1996d \\
\hline LWR-3SB & 71243.3 & 47068.9 & 249.1 & WSRC, 1996d \\
\hline LWR-4SB & 70051.6 & 46749.6 & 293.8 & WSRC, 1996d \\
\hline LWR-8CC & 70409.4 & 45803 & 280.3 & WSRC, 1996d \\
\hline LWR-9SB & 71658.83 & 45406.62 & 238.2 & WSRC, 1996d \\
\hline M12-17 & 62294.6 & 56405.83 & 325.5 & LaW \\
& & & & Environmental, \\
\hline M121A & 62170.7 & 56819.9 & 303.7 & WSRC, 1996d \\
\hline M12A-29 & 60637.63 & 56403.41 & 321 & Law \\
\hline MW- & & & & Environmental, \\
\hline MWD-1A & 69592.8 & 75121.9 & 327.5 & WSRC, 1996d \\
\hline MWD-C3 & 69734.7 & 75249.03 & 322.5 & ARA, 1996 \\
\hline MWD-C5 & 69835.55 & 74766.97 & 328.7 & ARA, 1996 \\
\hline NPN-1A & 66632.1 & 70856.2 & 335.9 & WSRC, 1996d \\
\hline OFS-1SB & 74967.5 & 54032.6 & 261.6 & Amidon, 1995 \\
\hline OFS-2SB & 74671 & 53848 & 257.5 & Amidon, 1995 \\
\hline OFS-3SB & 74270 & 54579 & 258.1 & Amidon, 1995 \\
\hline OFS-4SB & 73874 & 55188 & 258.7 & Amidon, 1995 \\
\hline OFS-5SB & 73623 & 54298 & 228.7 & Amidon, 1995 \\
\hline P-13TA & 35600 & 60000 & 252.4 & WSRC, 1996d \\
\hline P-14TA & 72444.9 & 76439.6 & 294.4 & WSRC, 1996d \\
\hline P-18TA & 67578.5 & 47652.7 & 296.9 & WSRC, 1996d \\
\hline P-19TA & 55295.9 & 60034.6 & 297.4 & WSRC, 1996d \\
\hline P-20TA & 56094.1 & 76768.1 & 287.7 & WSRC, 1996d \\
\hline P-21TA & 24674.6 & 40739.2 & 207 & WSRC, 1996d \\
\hline P-22TA & 20593.4 & 73555.3 & 215.4 & WSRC, 1996d \\
\hline P-23TA & 48063.3 & 30931.3 & 181.5 & WSRC, 1996d \\
\hline P-24TA & 43096.2 & 66565.2 & 313.3 & WSRC, 1996d \\
\hline P-25TA & 52493.6 & 42261 & 265.1 & WSRC, 1996d \\
\hline P-26TA & 71958.6 & 18051.5 & 152.2 & WSRC, 1996d \\
\hline P-27TA & 70382 & 64022.9 & 274.1 & WSRC, 1996d \\
\hline P-28TA & 79284.3 & 55441.1 & 285.6 & WSRC, 1996d \\
\hline PBF-3 & 54221 & 40213 & 270 & ARA, 1992 \\
\hline
\end{tabular}


Appendix B-1: Locations of Sites within the Model Area (Continued)

\begin{tabular}{|c|c|c|c|c|}
\hline Well ID & $\begin{array}{c}\text { SRS } \\
\text { Northing } \\
\text { (ft) }\end{array}$ & $\begin{array}{c}\text { SRS } \\
\text { Easting } \\
\text { (ft) }\end{array}$ & $\begin{array}{c}\text { Surface } \\
\text { Elevation } \\
\text { (ft m.s.l.) }\end{array}$ & Reference $^{1}$ \\
\hline & 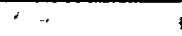 & 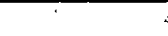 & \pm & . \\
\hline PBF-5 & 53591.29 & 30319.43 & 240.6 & Harris, 1997 \\
\hline PBF-6 & 55621.75 & 12814.48 & 92.5 & Harris, 1997 \\
\hline PBF-7 & 55420.69 & 59568.97 & 285.42 & Harris, 1997 \\
\hline PBF-8 & 55744.48 & 59812.89 & 292.01 & Harris, 1997 \\
\hline PCL-12 & 58948 & 37745 & 265.8 & Gregg, 1998b \\
\hline $\begin{array}{l}\text { PCL-13 } \\
\end{array}$ & 57115 & 35125 & 274.2 & Gregg, 1998b \\
\hline PCL-14 & 52068 & 32090 & 250.3 & Gregg, 1998b \\
\hline PCL-15 & 41222 & 37515 & 242.1 & Gregg, 1998b \\
\hline PCL-16 & 65993 & 43146 & 282.8 & Gregg, 1998b \\
\hline PCL-17 & 64174 & 48343 & 291 & Gregg, 1998b \\
\hline PCL-1A & 60442 & 80332 & 317.7 & Gregg, 1998b \\
\hline PCL-2 & 60238 & 76070 & 304.4 & Gregg, 1998b \\
\hline PCL-3A & 53512 & 74103 & 300 & Gregg, 1998b \\
\hline PCL-4 & 48651 & 63191 & 331.7 & Gregg, 1998b \\
\hline PCL-5 & 47475 & 67894 & 284.3 & Gregg, 1998b \\
\hline PCL-6 & 42040 & 67118 & 312.6 & Gregg, 1998b \\
\hline PCL-7B & 38735 & 62254 & 295.7 & Gregg, 1998b \\
\hline PCL-8 & 47839 & 51358 & 254.7 & Gregg, 1998b \\
\hline PCL-9 & 47441 & 56410 & 288.1 & Gregg, 1998b \\
\hline PPC-1 & 42727.22 & 66137.83 & 313.3 & WSRC, 1990b \\
\hline RCP-1A & 56968.1 & 74238.3 & 294.8 & WSRC, 1996d \\
\hline RSF-1 & 58505.3 & 74869.4 & 300.8 & WSRC, 1996d \\
\hline RSF-2 & 57670.4 & 74628.6 & 300.3 & WSRC, 1996d \\
\hline RSF-3 & 57621.4 & 75206.7 & 304.8 & WSRC, 1996d \\
\hline SDS-21 & 78951 & 67087 & 251 & WSRC, 1993b \\
\hline SDS-22 & 76887 & 66304 & 283 & WSRC, $1993 \mathrm{~b}$ \\
\hline SSW-1 & 71223.25 & 33206.83 & 311.3 & WSRC, 1989 \\
\hline SSW-2 & 72230.42 & 28236.98 & 167.3 & WSRC, 1996d \\
\hline SSW-3 & 70517.63 & 40532.31 & 178.7 & WSRC, 1989 \\
\hline SW-24CP & 74671 & 53848 & 257.5 & Amidon, 1995 \\
\hline T18N1A & 57015.7 & 45553.2 & 258.4 & WSRC, 1996d \\
\hline T18S1A & 46111.5 & 43897.6 & 233.5 & WSRC, 1996d \\
\hline T18W1A & 48773.1 & 40275.2 & 244.4 & WSRC, 1996d \\
\hline USGS-MP & 98367.1 & -8045.7 & 245 & $\begin{array}{l}\text { Falls and others, } \\
1998\end{array}$ \\
\hline VG-1 & 11543.5 & 17064.2 & 156.6 & Bechtel, 1982 \\
\hline VG-7 & 28828.3 & 5392.8 & 250.6 & Bechtel, 1982 \\
\hline VG-8 & -12412.6 & 22580.5 & 103.7 & Bechtel, 1982 \\
\hline YSC-1A & 78039.9 & 65438.93 & 268.9 & WSRC, 1996d \\
\hline YSC-1C & 78186.24 & 65855.46 & 272.5 & WSRC, 1996d \\
\hline YSC-2A & 78311.53 & 66100.08 & 281.7 & WSRC, 1996d \\
\hline YSC-3SB & 77680 & 65920 & 277 & WEGS, 1990 \\
\hline
\end{tabular}


Appendix B-1: Locations of Sites within the Model Area (Continued)

\begin{tabular}{|l|r|r|r|r|}
\hline Well ID & $\begin{array}{c}\text { SRS } \\
\text { Northing } \\
(\mathbf{f t})\end{array}$ & $\begin{array}{c}\text { SRS } \\
\text { Easting } \\
(\mathbf{f t})\end{array}$ & $\begin{array}{c}\text { Surface } \\
\text { Elevation } \\
(\mathbf{f t} \text { m.s.l.) }\end{array}$ & Reference $^{\mathbf{1}}$ \\
\hline
\end{tabular}

Notes:

ft.-feet

$\mathrm{ft}$ m.s.l.-feet above mean sea level

1-Detailed description of references in Appendix D 
Appendix B-2: Hydrostratigraphic Boundaries (continued)

\begin{tabular}{|c|c|c|c|c|c|c|c|}
\hline & & \multicolumn{2}{|c|}{ base of "uu" } & \multicolumn{2}{|c|}{ "AA" Interval } & \multicolumn{2}{|c|}{$\begin{array}{c}\text { Transmissive } \\
\text { Zone } \\
\end{array}$} \\
\hline Well ID & $\begin{array}{c}\text { Surface } \\
\text { Elevation (ft } \\
\text { m.s.l.) }\end{array}$ & $\begin{array}{c}\text { Elev. (ft } \\
\text { m.s.l.) }\end{array}$ & \begin{tabular}{|l} 
Depth (ft \\
b.g.l.)
\end{tabular} & $\begin{array}{l}\text { Elev. (ft } \\
\text { m.s.l.) }\end{array}$ & $\begin{array}{c}\text { Depth (ft } \\
\text { b.g.l. })\end{array}$ & $\begin{array}{l}\text { Elev. (ft } \\
\text { m.s.l.) }\end{array}$ & $\begin{array}{l}\text { Depth (ft } \\
\text { b.g.l.) }\end{array}$ \\
\hline
\end{tabular}

\begin{tabular}{|c|c|c|c|c|c|c|c|}
\hline $131 \mathrm{C}-100$ & 261.6 & 241.0 & 21 & & & 226 & 36 \\
\hline $131 \mathrm{C}-104$ & 276.9 & 258.0 & 19 & 237 & 40 & 220 & 57 \\
\hline $131 \mathrm{C}-105$ & 169.8 & & & & & & \\
\hline $131 \mathrm{C}-49$ & 254.3 & 240.0 & 14 & 224 & 30 & 212 & 42 \\
\hline $131 \mathrm{C}-51$ & 265.0 & 238.0 & 27 & 226 & 39 & 214 & $\overline{51}$ \\
\hline $131 \mathrm{C}-54$ & 247.1 & 232.0 & 15 & 219 & 28 & 208 & 39 \\
\hline $131 \mathrm{C}-55$ & 245.0 & 228.0 & 17 & 217 & 28 & 203 & $\overline{42}$ \\
\hline $131 \mathrm{C}-59$ & 227.7 & & & & & 203 & 25 \\
\hline $131 \mathrm{C}-60$ & 218.2 & & & & & 209 & 9 \\
\hline $131 \mathrm{C}-63$ & 211.1 & & & & & & \\
\hline $131 \mathrm{C}-64$ & 211.9 & & & & & & \\
\hline $131 \mathrm{C}-67$ & 265.8 & 248.0 & 18 & 220 & 46 & 209 & 57 \\
\hline $131 \mathrm{C}-68$ & 267.7 & 244.0 & 24 & 225 & 43 & 216 & 52 \\
\hline $131 \mathrm{C}-80$ & 205.7 & & & & & & \\
\hline $131 \mathrm{C}-81$ & 217.9 & & & & & 209 & 9 \\
\hline $131 \mathrm{C}-82$ & 233.0 & & & & & 217 & 16 \\
\hline $131 \mathrm{C}-83$ & 240.5 & & & & & 217 & 24 \\
\hline 131C-84 & 241.9 & & & & & 224 & 18 \\
\hline $131 \mathrm{C}-85$ & 237.5 & & & & & 222 & 15 \\
\hline $131 \mathrm{C}-91$ & 233.7 & & & & & 217 & 17 \\
\hline 131C-93 & 241.9 & & & & & 221 & 21 \\
\hline $131 \mathrm{C}-95$ & 241.3 & 226.0 & 15 & & & 222 & 19 \\
\hline $131 \mathrm{C}-96$ & 243.2 & 227.0 & 16 & & & 216 & 27 \\
\hline $131 \mathrm{C}-98$ & 251.0 & 230.0 & 21 & & & 216 & $\overline{35}$ \\
\hline 131C-R1 & 271.0 & 250.0 & 21 & 232 & 39 & 209 & 62 \\
\hline 131C-R2 & 266.8 & 251.0 & 16 & 224 & 43 & 201 & 66 \\
\hline 131C-R3 & 268.8 & 251.0 & 18 & 226 & 43 & 213 & 56 \\
\hline 131C-R4 & 285.6 & 250.0 & 36 & 228 & 58 & 211 & 75 \\
\hline 131C-R5 & 289.0 & 257.0 & 32 & 231 & 58 & 212 & 77 \\
\hline 131C-R6 & 276.0 & 251.0 & 25 & 219 & 57 & 205 & 71 \\
\hline BGO-10A & 299.1 & & & & & & \\
\hline BGO-10AA & 298.8 & & & & & & \\
\hline BGO-12A & 311.4 & & & & & & \\
\hline BGO-14A & 300.2 & & & & & & \\
\hline BGO-16A & 302.8 & & & & & & \\
\hline \begin{tabular}{|l|} 
BGO-18A \\
\end{tabular} & 292.9 & & & & & & \\
\hline BGO-20AA & 280.9 & & & & & & \\
\hline BGO-25A & 294.7 & & & & & & \\
\hline BGO-26A & 285.1 & & & & & & \\
\hline BGO-27C & 273.9 & & & & & & \\
\hline BGO-29A & 262.1 & & & & & & \\
\hline BGO-31C & 271.1 & & & & & & \\
\hline
\end{tabular}


B-14 WSRC-TR-99-00248, Rev. 0, Regional Groundwater Flow Model for C, K, L, and P Reactor Areas

Appendix B-2: Hydrostratigraphic Boundaries (continued)

\begin{tabular}{|c|c|c|c|c|c|c|c|}
\hline & & \multicolumn{2}{|c|}{ base of "uu" } & \multicolumn{2}{|c|}{ "AA" Interval } & \multicolumn{2}{|c|}{$\begin{array}{c}\text { Transmissive } \\
\text { Zone } \\
\end{array}$} \\
\hline Well ID & $\begin{array}{c}\text { Surface } \\
\text { Elevation (ft } \\
\text { m.s.l.) }\end{array}$ & $\begin{array}{l}\text { Elev. (ft } \\
\text { m.s.l.) }\end{array}$ & $\begin{array}{c}\text { Depth (ft } \\
\text { b.g.l.) }\end{array}$ & $\begin{array}{c}\text { Elev. (ft } \\
\text { m.s.l.) }\end{array}$ & $\begin{array}{l}\text { Depth (ft } \\
\text { b.g.I.) }\end{array}$ & $\begin{array}{c}\text { Elev. (ft } \\
\text { m.s.l.) }\end{array}$ & $\begin{array}{c}\text { Depth (ft } \\
\text { b.g.l.) }\end{array}$ \\
\hline
\end{tabular}

\begin{tabular}{|c|c|c|c|c|c|c|c|}
\hline BGO-33C & 277.4 & & & & & & \\
\hline BGO-35C & 271.4 & & & & & & \\
\hline BGO-37C & 284.3 & & & & & & \\
\hline BGO-39A & 293.7 & & & & & & \\
\hline BGO-3A & 288.7 & & & & & & \\
\hline BGO-41A & 298.3 & & & & & & \\
\hline BGO-42C & 295.9 & & & & & & \\
\hline $\mathrm{BGO}-43 \mathrm{AA}$ & 312.2 & & & & & & \\
\hline BGO-44AA & 283.3 & & & & & & \\
\hline BGO-45A & 276.9 & & & & & & \\
\hline BGO-46B & 263.4 & & & & & & \\
\hline BGO-47A & 264.8 & & & & & & \\
\hline BGO-48C & 274.7 & & & & & & \\
\hline BGO-49A & 269.1 & & & & & & \\
\hline BGO-50A & 253.5 & & & & & & \\
\hline BGO-51AA & 287.2 & & & & & & \\
\hline BGO-52AA & 281.6 & & & & & & \\
\hline BGO-53AA & 288.9 & & & & & & \\
\hline BGO-5C & 294.2 & & & & & & \\
\hline BGO-6A & 283.8 & & & & & & \\
\hline BGO-6B & 284.5 & & & & & & \\
\hline $\mathrm{BGO}-8 \mathrm{~A}$ & 281.3 & & & & & & \\
\hline BGO-9AA & 282.8 & & & & & & \\
\hline BGT-1 & 282.9 & & & & & & \\
\hline BGT-10 & 215.2 & & & & & & \\
\hline BGT-11 & 222.5 & & & & & & \\
\hline BGT-12 & 284.2 & & & & & & \\
\hline BGT-13 & 287.8 & & & & & & \\
\hline BGT-14 & 280.7 & & & & & & \\
\hline BGT-15 & 277.5 & & & & & & \\
\hline BGT-16 & 250.7 & & & & & & \\
\hline BGT-17 & 240.7 & & & & & & \\
\hline BGT-18 & 216.5 & & & & $\because$ & & \\
\hline BGT-2 & 276.4 & & & & & & \\
\hline BGT-20 & 159.5 & & & & & & \\
\hline BGT-21 & 294.2 & & & & & & \\
\hline BGT-22 & 281.0 & 265.0 & 16 & 234 & 47 & 212 & 69 \\
\hline BGT-23 & 270.0 & & & & & & \\
\hline BGT-24 & 265.8 & & & & & & \\
\hline BGT-25 & 264.8 & & & & & & \\
\hline BGT-27 & 256.9 & & & & & & \\
\hline BGT-28 & 258.3 & 250.0 & 8 & & & 230 & 28 \\
\hline
\end{tabular}


Appendix B-2: Hydrostratigraphic Boundaries (continued)

\begin{tabular}{|c|c|c|c|c|c|c|c|}
\hline & & \multicolumn{2}{|c|}{ base of "uu" } & \multicolumn{2}{|c|}{ "AA" Interval } & \multicolumn{2}{|c|}{$\begin{array}{c}\text { Transmissive } \\
\text { Zone }\end{array}$} \\
\hline Well ID & $\begin{array}{c}\text { Surface } \\
\text { Elevation (ft } \\
\text { m.s.l.) }\end{array}$ & $\begin{array}{l}\text { Elev. (ft } \\
\text { m.s.1.) }\end{array}$ & $\begin{array}{c}\text { Depth (ft } \\
\text { b.g.l.) }\end{array}$ & $\begin{array}{c}\text { Elev. (ft } \\
\text { m.s.l.) }\end{array}$ & $\begin{array}{c}\text { Depth (ft } \\
\text { b.g.l.). }\end{array}$ & $\begin{array}{l}\text { Elev. (ft } \\
\text { m.s.l.) }\end{array}$ & $\begin{array}{c}\text { Depth (ft } \\
\text { b.g.l.) }\end{array}$ \\
\hline
\end{tabular}

\begin{tabular}{|c|c|c|c|c|c|c|c|}
\hline BGT-29 & 243.0 & & & & & & \\
\hline BGT-3 & 275.7 & & & & & & \\
\hline BGT-30 & 219.0 & & & & & & \\
\hline BGT-31 & 308.8 & & & & & & \\
\hline BGT-32 & 310.1 & & & & & & \\
\hline BGT-33 & 290.4 & & & & & & \\
\hline BGT-34 & 286.8 & & & & & & \\
\hline BGT-35 & 267.7 & & & & & & \\
\hline BGT-36 & 261.4 & & & & & & \\
\hline BGT-37 & 251.6 & & & & & & \\
\hline BGT-38 & 240.1 & & & & & & \\
\hline BGT-39 & 241.9 & & & & & & \\
\hline$\overline{B G T-4}$ & 259.2 & & & & & & \\
\hline BGT-40 & 332.3 & & & & & & \\
\hline BGT-41 & 328.4 & & & & & & \\
\hline BGT-42 & 310.9 & & & & & & \\
\hline BGT-43 & 277.1 & & & & & & \\
\hline BGT-44 & 276.2 & & & & & & \\
\hline BGT-45 & 285.3 & & & & & & \\
\hline BGT-46 & 310.0 & & & & & & \\
\hline BGT-47 & 317.3 & & & & & & \\
\hline BGT-48 & 314.3 & & & & & & \\
\hline BGT-49 & 297.3 & & & & & & \\
\hline BGT-5 & 225.7 & & & & & & \\
\hline BGT-50 & 296.3 & & & & & & \\
\hline BGT-51 & 272.6 & & & & & & \\
\hline BGT-53 & 278.3 & & & & & & \\
\hline BGT-54 & 280.0 & & & & & & \\
\hline BGT-56 & 262.9 & & & & & & \\
\hline BGT-57 & 259.4 & & & & & & \\
\hline BGT-58 & 285.8 & & & & & & \\
\hline BGT-59 & 281.9 & & & & & & \\
\hline$\overline{\text { BGT-6 }}$ & 282.2 & & & & & & \\
\hline BGT-60 & 291.4 & & & & & & \\
\hline BGT-61 & 284.3 & 256.0 & 28 & 242 & 42 & 210 & 74 \\
\hline BGT-62 & 282.0 & & & & & & \\
\hline$\overline{B G T}_{-} 63$ & 293.7 & & & & & & \\
\hline BGT-63A & 290.8 & & & & & & \\
\hline BGT-64 & 283.3 & & & & & & \\
\hline BGT-66 & 244.0 & & & & & & \\
\hline BGT-67 & 242.0 & 232.0 & 10 & & & 209 & 33 \\
\hline BGT-7 & 276.4 & & & & & & \\
\hline
\end{tabular}


Appendix B-2: Hydrostratigraphic Boundaries (continued)

\begin{tabular}{|c|c||c|c|c|c|c|c|}
\hline & \multicolumn{3}{|c||}{ base of "uu" } & \multicolumn{2}{|c|}{ "AA" Interval } & \multicolumn{2}{c|}{$\begin{array}{c}\text { Transmissive } \\
\text { Zone }\end{array}$} \\
\hline Well ID & $\begin{array}{c}\text { Surface } \\
\text { Elevation (ft } \\
\text { m.s.l.) }\end{array}$ & $\begin{array}{c}\text { Elev. (ft } \\
\text { m.s.l.) }\end{array}$ & $\begin{array}{c}\text { Depth (ft } \\
\text { b.g.l.) }\end{array}$ & $\begin{array}{c}\text { Elev. (ft } \\
\text { m.s.l.) }\end{array}$ & $\begin{array}{c}\text { Depth (ft } \\
\text { b.g.l.) }\end{array}$ & $\begin{array}{c}\text { Elev. (ft } \\
\text { m.s.l.) }\end{array}$ & $\begin{array}{c}\text { Depth (ft } \\
\text { b.g.l.) }\end{array}$ \\
\hline
\end{tabular}

\begin{tabular}{|c|c|c|c|c|c|c|c|}
\hline BGT-8 & 249.3 & & & & & & \\
\hline BGT-9 & 226.0 & & & & & & \\
\hline$B G X-11 D$ & 273.8 & & & & & & \\
\hline BGX-1A & 289.1 & & & & & & \\
\hline BGX-2B & 289.2 & & & & & & \\
\hline BGX-4A & 288.8 & & & & & & \\
\hline BGX-7D & 277.1 & & & & & & \\
\hline BGX-9D & 277.4 & & & & & & \\
\hline BRR-1D & 293.8 & & & & & & \\
\hline BRR-3D & 289.5 & & & & & & \\
\hline BRR-6B & 293.9 & & & & & & \\
\hline BRR-7B & 289.6 & & & & & & \\
\hline BRR-8B & 276.7 & & & & & & \\
\hline CCP-1A & 287.1 & 267.0 & 20 & 232 & 55 & 205 & 82 \\
\hline CFD-1 & 268.8 & 237.0 & 32 & & & & \\
\hline CFD-18 & 248.3 & 228.0 & 20 & 208 & 40 & 198 & 50 \\
\hline CFD-5 & 257.8 & & & & & & \\
\hline CMP-1-CP & 248.9 & 238.0 & 11 & & & & \\
\hline CMP-30B & 286.5 & 262.0 & 25 & & & 245 & 42 \\
\hline CMP-32B & 251.7 & 230.0 & 22 & & & & \\
\hline CMP-4B-CP & 241.8 & 236.0 & 6 & & & & \\
\hline CMP-6A-CP & 238.4 & & & & & 222 & 16 \\
\hline CPC-1 & 285.1 & 267.0 & 18 & 230 & 55 & 187 & 98 \\
\hline CRP-5D & 274.5 & 264.0 & 11 & 232 & 43 & 192 & 83 \\
\hline CRP-9D & 268.4 & & & & & & \\
\hline CRSB-1 & 278.2 & 258.0 & 20 & 229 & 49 & 201 & 77 \\
\hline \begin{tabular}{|l|} 
CRSB-101 \\
\end{tabular} & 275.6 & 256.0 & 20 & 231 & 45 & 205 & 71 \\
\hline CRSB-11 & 276.7 & 254.0 & 23 & 227 & 50 & 204 & 73 \\
\hline \begin{tabular}{|l|} 
CRSB-14 \\
\end{tabular} & 275.6 & 252.0 & 24 & 235 & 41 & 207 & 69 \\
\hline CRSB-16 & 282.6 & 251.0 & 32 & 234 & 49 & 212 & 71 \\
\hline CRSB-17 & 281.0 & 261.0 & 20 & 231 & 50 & 207 & 74 \\
\hline CRSB-18 & 273.8 & 253.0 & 21 & 227 & 47 & 204 & 70 \\
\hline CRSB-20 & 274.2 & 257.0 & 17 & 228 & 46 & 203 & 71 \\
\hline CRSB-21 & 272.9 & 243.0 & 30 & 228 & 45 & 204 & 69 \\
\hline CRSB-24 & 277.2 & 264.0 & 13 & 238 & 39 & 217 & 60 \\
\hline CRSB-26 & 291.6 & 267.0 & 25 & 241 & 51 & 220 & 72 \\
\hline CRSB-28 & 289.3 & 256.0 & 33 & 228 & 61 & 205 & 84 \\
\hline CRSB-30 & 285.4 & 253.0 & 32 & 240 & 45 & 217 & 68 \\
\hline CRSB-31 & 284.4 & 248.0 & 36 & 235 & 49 & 214 & 70 \\
\hline CRSB-32 & 274.1 & 230.0 & 44. & 225 & 49 & 195 & 79 \\
\hline CRSB-35 & 257.4 & 237.0 & 20 & 221 & 36 & 196 & 61 \\
\hline CRSB-36 & 265.6 & 240.0 & 26 & 224 & 42 & 199 & 67 \\
\hline
\end{tabular}


Appendix B-2: Hydrostratigraphic Boundaries (continued)

\begin{tabular}{|c|c|c|c|c|c|c|c|}
\hline & & \multicolumn{2}{|c|}{ base of "uu" } & \multicolumn{2}{|c|}{ "AA" Interval } & \multicolumn{2}{|c|}{$\begin{array}{c}\text { Transmissive } \\
\text { Zone }\end{array}$} \\
\hline $\begin{array}{c}\text { Well ID } \\
\quad:\end{array}$ & $\begin{array}{c}\text { Surface } \\
\text { Elevation (ft } \\
\text { m.s.l.) }\end{array}$ & $\begin{array}{c}\text { Elev. (ft } \\
\text { m.s.l.). }\end{array}$ & $\begin{array}{c}\text { Depth (ft } \\
\text { b.g.l.) }\end{array}$ & $\begin{array}{l}\text { Elev. (ft } \\
\text {.m.s.l.) }\end{array}$ & $\begin{array}{l}\text { Depth (ft } \\
\text {.b.g.l.) }\end{array}$ & $\begin{array}{l}\text { Elev. (ft } \\
\text { m.s.l.) }\end{array}$ & $\begin{array}{c}\text { Depth (ft } \\
\text { b.g.l.) }\end{array}$ \\
\hline
\end{tabular}

\begin{tabular}{|c|c|c|c|c|c|c|c|}
\hline CRSB-37 & 270.5 & 251.0 & 19 & 236 & 34 & 209 & 61 \\
\hline CRSB-4 & 282.8 & 253.0 & 30 & 233 & 50 & 218 & 65 \\
\hline CRSB-40 & 227.3 & & & & & 196 & 31 \\
\hline CRSB-43 & 258.5 & 246.0 & 13 & 228 & 31 & 203 & 56 \\
\hline CRSB-45 & 270.6 & 257.0 & 14 & 238 & 33 & 216 & 55 \\
\hline CRSB-47 & 282.7 & 241.0 & 42 & 220 & 63 & 197 & 86 \\
\hline CRSB-48 & 259.8 & 247.0 & 13 & 236 & 24 & 226 & 34 \\
\hline CRSB-5 & 288.3 & 255.0 & 33 & 229 & 59 & 209 & 79 \\
\hline CRSB-50 & 255.6 & 231.0 & 25 & 223 & 33 & 213 & 43 \\
\hline CRSB-52 & 233.1 & & & 213 & 20 & 191 & 42 \\
\hline CRSB-54 & 224.7 & & & & & 204 & 21 \\
\hline CRSB-55 & 229.1 & & & & & 204 & 25 \\
\hline CRSB-56 & 232.5 & & & & & 212 & 21 \\
\hline CRSB-57 & 248.2 & 227.0 & 21 & & & 213 & 35 \\
\hline CRSB-58 & 253.9 & 237.0 & 17 & & & 219 & 35 \\
\hline CRSB-6 & 289.4 & 269.0 & 20 & 233 & 56 & 206 & 83 \\
\hline CRSB-60 & 257.0 & 240.0 & 17 & & & 218 & 39 \\
\hline CRSB-61 & 257.3 & 243.0 & 14 & 228 & 29 & 215 & 42 \\
\hline CRSB-63 & 240.4 & & & & & 221 & 19 \\
\hline CRSB-64 & 229.9 & & & & & 217 & 13 \\
\hline CRSB-65 & 214.8 & & & & & 209 & 6 \\
\hline CRSB-86 & 206.6 & & & & & & \\
\hline CRSB-88 & 253.2 & 233.0 & 20 & 214 & 39 & 189 & 64 \\
\hline CRSB-89 & 260.6 & 237.0 & 24 & 210 & 51 & 192 & 69 \\
\hline CRSB-90 & 268.4 & 243.0 & 25 & 219 & 49 & 201 & 67 \\
\hline CRSB-91 & 273.1 & 253.0 & 20 & 236 & 37 & 203 & 70 \\
\hline CRSB-92 & 287.5 & 269.0 & 19 & 236 & 52 & 215 & 73 \\
\hline CRSB-93 & 286.8 & 261.0 & 26 & 236 & 51 & 215 & 72 \\
\hline CRSB-94 & 283.3 & 268.0 & 15 & 237 & 46 & 214 & 69 \\
\hline CRSB-95 & 280.4 & 268.0 & 12 & 239 & 41 & 207 & 73 \\
\hline CRSB-96 & 282.8 & 248.0 & 35 & 234 & 49 & 203 & 80 \\
\hline CRSB-97 & 276.8 & 254.0 & 23 & 229 & 48 & 203 & 74 \\
\hline CRSB-98 & 275.1 & 253.0 & 22 & 229 & 46 & 209 & 66 \\
\hline CRSB-99 & 274.4 & 254.0 & 20 & 229 & 45 & 212 & 62 \\
\hline CSB-101 & 281.2 & 274.0 & 7 & 237 & 44 & 209 & 72 \\
\hline CSB-102 & 283.5 & 256.0 & 28 & 228 & 56 & 206 & 78 \\
\hline CSB-103 & 257.6 & 239.0 & 19 & 224 & 34 & 202 & 56 \\
\hline CSB-104 & 224.9 & 209.0 & 16 & & & 198 & 27 \\
\hline CSB-105 & 212.1 & & & & & 200 & $\overline{12}$ \\
\hline CSB-106 & 183.8 & & & & & & \\
\hline CSB-107 & 220.7 & & & & & 197 & 24 \\
\hline CSB-108 & 196.7 & & & & & & \\
\hline
\end{tabular}


Appendix B-2: Hydrostratigraphic Boundaries (continued)

\begin{tabular}{|c|c|c|c|c|c|c|c|}
\hline & & \multicolumn{2}{|c|}{ base of "uu" } & \multicolumn{2}{|c|}{ "AA" Interval } & \multicolumn{2}{|c|}{$\begin{array}{c}\text { Transmissive } \\
\text { Zone }\end{array}$} \\
\hline Well ID & $\begin{array}{c}\text { Surface } \\
\text { Elevation (ft } \\
\text { m.s.l.) }\end{array}$ & $\begin{array}{l}\text { Elev. (ft } \\
\text { m.s.l.) }\end{array}$ & $\begin{array}{l}\text { Depth (ft } \\
\text { b.g.l.) }\end{array}$ & $\begin{array}{c}\text { Elev. (ft } \\
\text { m.s.l.) }\end{array}$ & $\begin{array}{c}\text { Depth (ft } \\
\text { b.g.l.) }\end{array}$ & $\begin{array}{l}\text { Elev. (ft } \\
\text { m.s.l.) }\end{array}$ & \begin{tabular}{|c} 
Depth (ft \\
b.g.l.)
\end{tabular} \\
\hline
\end{tabular}

\begin{tabular}{|c|c|c|c|c|c|c|c|}
\hline CSB-109 & $172.9 \|$ & & & & & & \\
\hline CSB-110 & 184.6 & & & & & & \\
\hline CSB-111 & 172.1 & & & & & & \\
\hline CSB-112 & 195.3 & & & & & & \\
\hline CSB-113 & 210.4 & & & & & 200 & 10 \\
\hline CSB-114 & 196.0 & & & & & & \\
\hline CSB-116 & 274.7 & 255.0 & 20 & 232 & 43 & 207 & 68 \\
\hline CSB-117 & 276.5 & & & 244 & 33 & 221 & 56 \\
\hline CSB-118 & 277.8 & 258.0 & 20 & 224 & 54 & 202 & 76 \\
\hline CSB-119 & 268.3 & & & 228 & 40 & 202 & 66 \\
\hline CSB-120 & 282.8 & 248.0 & 35 & 225 & 58 & 202 & 81 \\
\hline CSB-121 & 274.2 & 254.0 & 20 & 234 & 40 & 213 & 61 \\
\hline CSB-122 & 222.9 & 197.0 & 26 & & & & \\
\hline CSB-123 & 224.2 & 210.0 & 14 & & & 204 & 20 \\
\hline CSB-2C & 289.9 & 269.0 & 21 & 235 & 55 & 206 & 84 \\
\hline CSB-3C & 280.9 & 250.0 & 31 & 233 & 48 & 202 & 79 \\
\hline CSB-47 & 282.7 & 253.0 & 30 & 238 & 45 & 210 & 73 \\
\hline CSB-47A & 268.9 & 263.0 & 6 & 244 & 25 & 220 & 49 \\
\hline CSB-48 & 259.8 & 247.0 & 13 & 236 & 24 & 216 & 44 \\
\hline CSB-48A & 270.1 & 252.0 & 18 & 236 & 34 & 210 & 60 \\
\hline CSB-56 & 267.0 & 246.0 & 21 & 242 & 25 & 212 & 55 \\
\hline CSB-58 & 235.5 & 222.0 & 14 & & & 214 & 22 \\
\hline CSB-59 & 231.2 & 221.0 & 10 & & & & \\
\hline CSB-60 & 247.0 & 234.0 & 13 & 221 & 26 & 209 & 38 \\
\hline CSB-61 & 252.2 & 238.0 & 14 & 224 & 28 & 210 & $\overline{42}$ \\
\hline CSB-62 & 240.9 & 231.0 & 10 & 228 & 13 & 217 & 24 \\
\hline CSB-63 & 227.1 & 213.0 & 14 & & & 204 & 23 \\
\hline CSB-64 & 212.7 & 192.0 & 21 & & & & \\
\hline CSB-65 & 195.6 & & & & & & \\
\hline CSB-66A & 191.7 & 179.0 & 13 & & & & \\
\hline CSD-4D & 306.5 & 279.0 & 28 & 232 & 75 & 194 & 113 \\
\hline E-26 & 314.3 & & & & & & \\
\hline $\mathrm{E}-4$ & 315.4 & & & & & & \\
\hline FAC-1SB & 312.2 & & & & & & \\
\hline FCH-1 & 316.8 & & & & & & \\
\hline $\mathrm{FCH}-2$ & 288.7 & & & & & & \\
\hline $\mathrm{FCH}-3$ & 307.2 & & & & & & \\
\hline $\mathrm{FCH}-4$ & 297.5 & & & & & & \\
\hline FCH-5 & 284.2 & & & & & & \\
\hline FCH-6 & 291.5 & & & & & & \\
\hline FIW-1MC & 293.3 & & & & & & \\
\hline FIW-2MA & 290.5 & & & & & & \\
\hline
\end{tabular}


Appendix B-2: Hydrostratigraphic Boundaries (continued)

\begin{tabular}{|c|c|c|c|c|c|c|c|}
\hline & & \multicolumn{2}{|c|}{ base of "uu" } & \multicolumn{2}{|c|}{ "AA" Interval } & \multicolumn{2}{|c|}{$\begin{array}{c}\text { Transmissive } \\
\text { Zone }\end{array}$} \\
\hline Well ID & $\begin{array}{c}\text { Surface } \\
\text { Elevation (ft } \\
\text { m.s.l.) }\end{array}$ & $\begin{array}{c}\text { Elev. (ft } \\
\text { m.s.1.) }\end{array}$ & $\begin{array}{c}\text { Depth (ft } \\
\text { b.g.1.) }\end{array}$ & $\begin{array}{c}\text { Elev. (ft } \\
\text { m.s.l.) }\end{array}$ & $\begin{array}{c}\text { Depth (ft } \\
\text {. b.g.l.) }\end{array}$ & \begin{tabular}{|c|} 
Elev. (ft \\
m.s.l.)
\end{tabular} & $\begin{array}{c}\text { Depth (ft } \\
\text { b.g.l.) }\end{array}$ \\
\hline
\end{tabular}

\begin{tabular}{|c|c|c|c|c|c|c|c|}
\hline FNB-1A & 282.4 & & & & & & \\
\hline FNB-3A & 282.2 & & & & & & \\
\hline FSB-100A & 283.8 & & & & & & \\
\hline FSB-101A & 282.9 & & & & & & \\
\hline FSB-112A & 227.0 & & & & & & \\
\hline FSB-113A & 221.3 & & & & & & \\
\hline FSB-114A & 250.0 & & & & & & \\
\hline FSB-115C & 205.8 & & & & & & \\
\hline FSB-116C & 200.5 & & & & & & \\
\hline FSB-120A & 278.0 & & & & & & \\
\hline FSB-121C & 254.4 & & & & & & \\
\hline FSB-122C & 216.0 & & & & & & \\
\hline FSB-123C & 236.3 & & & & & & \\
\hline FSB-1TA & 275.4 & & & & & & \\
\hline FSB-76A & 291.5 & & & & & & \\
\hline FSB-78A & 270.5 & & & & & & . \\
\hline FSB-79A & 216.1 & & & & & & \\
\hline FSB-87A & 285.6 & & & & & & \\
\hline FSB-89C & 279.1 & & & & & & \\
\hline FSB-91C & 277.0 & & & & & & \\
\hline FSB-93C & 274.0 & & & & & & \\
\hline FSB-95C & 281.8 & & & & & & \\
\hline FSB-96A & 277.7 & & & & & & \\
\hline FSB-97A & 283.8 & & & & & & \\
\hline FSB-98A & 280.7 & & & & & & \\
\hline FSB-99A & 285.3 & & & & & & \\
\hline FSB-PC & 230.8 & & & & & & \\
\hline $\begin{array}{l}\text { GAPWR-TW- } \\
1\end{array}$ & 219.0 & & & & & & \\
\hline HAA-1TA & 290.2 & 260.0 & 30 & 247 & 43 & 221 & 69 \\
\hline HAA-2AA & 291.4 & & & & & & \\
\hline HAA-3AA & 274.5 & & & & & & \\
\hline HAA-4AA & 299.2 & & & & & & \\
\hline HAA-6AA & 279.8 & & & & & & \\
\hline HC-03AA & 263.8 & 237.0 & 27 & 225 & 39 & 201 & 63 \\
\hline $\mathrm{HC}-12 \mathrm{~A}$ & 287.3 & & & & & & \\
\hline $\mathrm{HCA}-4 \mathrm{AA}$ & 308.6 & & & & & & \\
\hline $\mathrm{HCH}-1$ & 284.0 & & & & & & \\
\hline $\mathrm{HCH}-2$ & 270.9 & & & & & & \\
\hline $\mathrm{HCH}-3$ & 264.0 & & & & & & \\
\hline $\mathrm{HCH}-4$ & 269.9 & & & & & & \\
\hline HCH-5 & 255.0 & & & & & & \\
\hline
\end{tabular}


Appendix B-2: Hydrostratigraphic Boundaries (continued)

\begin{tabular}{|c|c|c|c|c|c|c|c|}
\hline & & \multicolumn{2}{|c|}{ base of "uu" } & \multicolumn{2}{|c|}{ "AA" Interval } & \multicolumn{2}{|c|}{$\begin{array}{c}\text { Transmissive } \\
\text { Zone }\end{array}$} \\
\hline Well ID & $\begin{array}{c}\text { Surface } \\
\text { Elevation (ft } \\
\text { m.s.l.) }\end{array}$ & $\begin{array}{l}\text { Elev. (ft } \\
\text { m.s.l.) }\end{array}$ & $\begin{array}{c}\text { Depth (ft } \\
\text { b.g.l.) }\end{array}$ & $\begin{array}{l}\text { Elev. (ft } \\
\text { m.s.l.) }\end{array}$ & $\begin{array}{c}\text { Depth (ft } \\
\text { b.g.l.) }\end{array}$ & $\begin{array}{l}\text { Elev. (ft } \\
\text { m.s.l.) }\end{array}$ & $\begin{array}{c}\text { Depth (ft } \\
\text { b.g.l.) }\end{array}$ \\
\hline
\end{tabular}

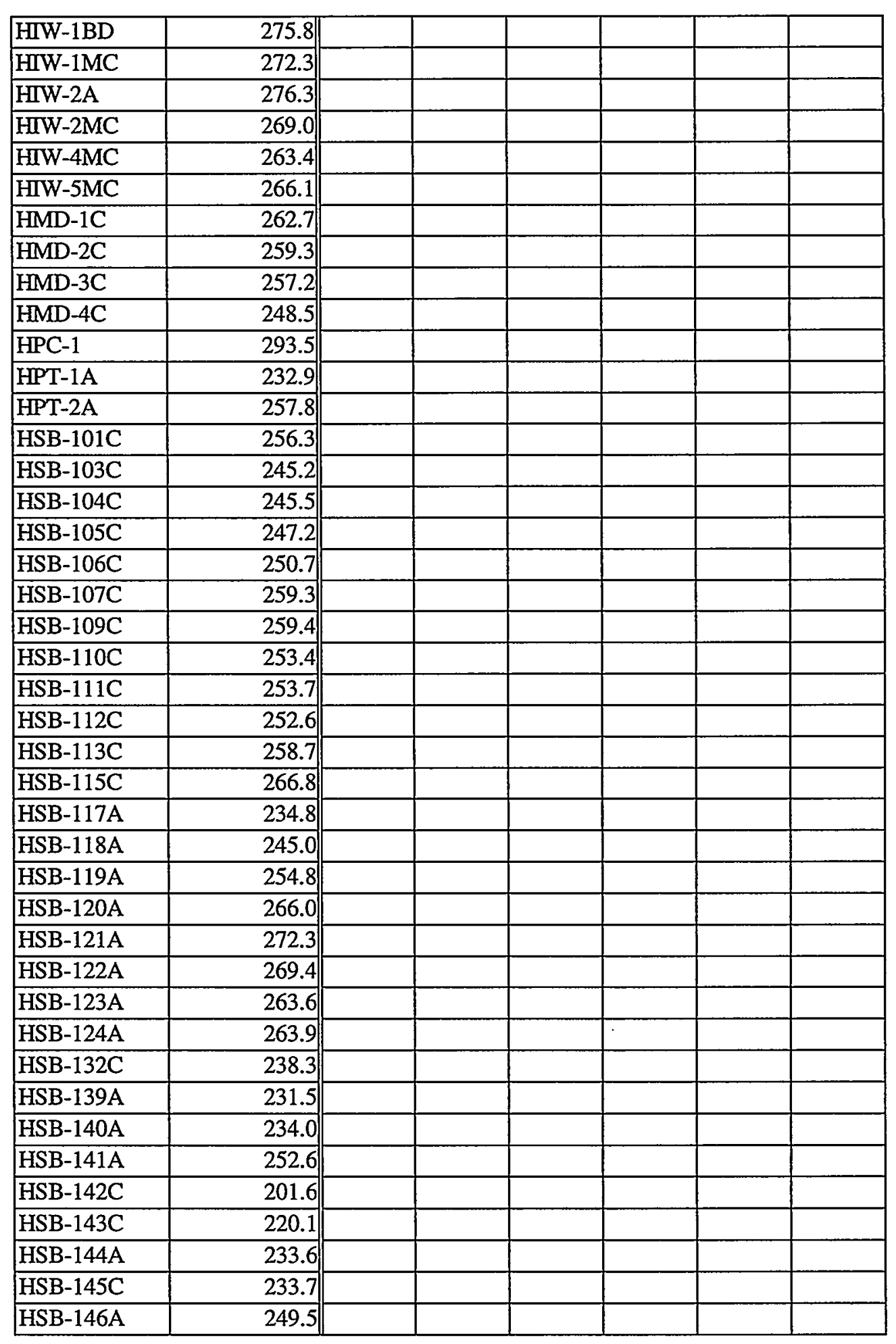


Appendix B-2: Hydrostratigraphic Boundaries (continued)

\begin{tabular}{|c|c|c|c|c|c|c|c|}
\hline & & \multicolumn{2}{|c|}{ base of "uu" } & \multicolumn{2}{|c|}{ "AA" Interval } & \multicolumn{2}{|c|}{$\begin{array}{c}\text { Transmissive } \\
\text { Zone }\end{array}$} \\
\hline Well ID & $\begin{array}{c}\text { Surface } \\
\text { Elevation (ft } \\
\text { m.s.l.) }\end{array}$ & $\begin{array}{l}\text { Elev. (ft } \\
\text { m.s.l.) }\end{array}$ & $\begin{array}{l}\text { Depth (ft } \\
\text { b.g.l.) }\end{array}$ & $\begin{array}{l}\text { Elev. (ft } \\
\text { m.s.l.) }\end{array}$ & $\begin{array}{l}\text { Depth (ft } \\
\text { b.g.l.) }\end{array}$ & $\begin{array}{l}\text { Elev. (ft } \\
\text { m.s.1.) }\end{array}$ & $\begin{array}{c}\text { Depth (ft } \\
\text { b.g.l.) }\end{array}$ \\
\hline
\end{tabular}

\begin{tabular}{|c|c|c|c|c|c|c|c|}
\hline HSB-148C & 248.9 & & & & & & \\
\hline HSB-151C & 211.6 & & & & & & \\
\hline HSB-152C & 212.1 & & & & & & \\
\hline HSB-65A & 270.7 & & & & & & \\
\hline HSB-68A & 247.4 & & & & & & \\
\hline HSB-69A & 234.1 & & & & & & \\
\hline HSB-83A & 234.9 & & & & & & \\
\hline HSB-84A & 226.7 & & & & & & \\
\hline HSB-85A & 292.1 & & & & & & \\
\hline HSB-86A & 260.0 & & & & & & \\
\hline HSB-PC & 227.8 & & & & & & \\
\hline HSB-TB & 267.1 & & & & & & \\
\hline HSL-6AA & 274.6 & & & & & & \\
\hline HSL-8AA & 286.7 & & & & & & \\
\hline IDB-2A & 302.4 & & & & & & \\
\hline IDP-3A & 282.2 & 245.0 & 37 & 232 & 50 & 204 & 78 \\
\hline IDQ-3A & 203.2 & 186.0 & 17 & & & & \\
\hline KAC-9D & 260.2 & 234.0 & 26 & 217 & 43 & 187 & 73 \\
\hline KPT-1 & 205.2 & & & 186 & 19 & 176 & 29 \\
\hline KPT-2 & 258.2 & 222.0 & 36 & 205 & 53 & 178 & 80 \\
\hline KPT-3 & 225.5 & 206.0 & 20 & 199 & 27 & 182 & 44 \\
\hline KPT-4 & 258.9 & 226.0 & 33 & & & 207 & 52 \\
\hline KPT-5 & 225.1 & 205.0 & 20 & & & & \\
\hline KPT-6 & 232.8 & 221.0 & 12 & 212 & 21 & 196 & 37 \\
\hline KPT-7 & 211.8 & & & & & 191 & 21 \\
\hline KPT-8 & 238.6 & & & & & & \\
\hline KPT-9 & 204.9 & 186.0 & 19 & & & 172 & 33 \\
\hline L3-CPT-PG-9 & 270.0 & 246.0 & 24 & 235 & 35 & 201 & 69 \\
\hline LAC-5DL & 239.8 & & & & & & \\
\hline LAC-6DL & 239.8 & & & & & & \\
\hline LAC-7DL & 239.4 & & & & & & \\
\hline LAC-8DL & 234.0 & & & & & & \\
\hline LCO-5A & 230.0 & 197.0 & 33 & & & & \\
\hline LCO-5DL & 230.3 & & & & & & \\
\hline LCO-8DL & 243.4 & & & & & & \\
\hline LFW-10SB & 168.4 & & & & & & \\
\hline LWN-1SB & 282.5 & & & & & & \\
\hline LWN-2SB & 231.0 & & & & & & \\
\hline LWN-3SB & 245.7 & . & & & & & \\
\hline LWR-2SB & 248.1 & . & & & & & \\
\hline LWR-3SB & 249.1 & & & & & & \\
\hline LWR-4SB & 293.8 & & & & & & \\
\hline
\end{tabular}


Appendix B-2: Hydrostratigraphic Boundaries (continued)

\begin{tabular}{|c|c|c|c|c|c|c|c|}
\hline & & \multicolumn{2}{|c|}{ base of "uu" } & \multicolumn{2}{|c|}{ "AA" Interval } & \multicolumn{2}{|c|}{$\begin{array}{c}\text { Transmissive } \\
\text { Zone }\end{array}$} \\
\hline Well ID & $\begin{array}{c}\text { Surface } \\
\text { Elevation (ft } \\
\text { m.s.l.) }\end{array}$ & $\begin{array}{c}\text { Elev. (ft } \\
\text { m.s.l.) }\end{array}$ & $\begin{array}{c}\text { Depth (ft } \\
\text { b.g.l.) }\end{array}$ & $\begin{array}{c}\text { Elev. (ft } \\
\text { m.s.l.) }\end{array}$ & $\begin{array}{c}\text { Depth (ft } \\
\text { b.g.l.) }\end{array}$ & $\begin{array}{c}\text { Elev. (ft } \\
\text { m.s.l.) }\end{array}$ & $\begin{array}{c}\text { Depth (ft } \\
\text { b.g.l.) }\end{array}$ \\
\hline
\end{tabular}

\begin{tabular}{|c|c|c|c|c|c|c|c|}
\hline LWR-8CC & 280.3 & 266.0 & 14 & 237 & 43 & 207 & 73 \\
\hline LWR-9SB & 238.2 & & & & & & \\
\hline M12-17 & 325.5 & & & & & & \\
\hline M121A & 303.7 & & & & & & \\
\hline M121A & 303.7 & & & & & & \\
\hline M12A-29 & 321.0 & 245.0 & 76 & 224 & 97 & 199 & 122 \\
\hline MWD-1A & 327.5 & 290.0 & 38 & 267 & 61 & 209 & 119 \\
\hline MWD-C3 & 322.5 & 289.0 & 34 & 264 & 59 & 210 & 113 \\
\hline MWD-C5 & 328.7 & 290.0 & 39 & 264 & 65 & 210 & 119 \\
\hline NPN-001 & 335.9 & 319.0 & 17 & 273 & 63 & 253 & 83 \\
\hline NPN-1A & 335.9 & & & & & & \\
\hline OFS-1SB & 261.6 & & & & & & \\
\hline OFS-2SB & 257.5 & & & & & & \\
\hline OFS-3SB & 258.1 & & & & & & \\
\hline OFS-4SB & 258.7 & & & & & & \\
\hline OFS-5SB & 228.7 & & & & & & \\
\hline P-13TA & 252.4 & & & & & & \\
\hline P-14TA & 294.4 & & & & & & \\
\hline P-18TA & 296.9 & 263.0 & 34 & 234 & 63 & 204 & 93 \\
\hline P-19TA & 297.4 & 255.0 & 42 & & & 225 & 72 \\
\hline P-20TA & 287.7 & 250.0 & 38 & 238 & 50 & 204 & 84 \\
\hline P-21TA & 207.0 & 188.0 & 19 & & & 151 & 56 \\
\hline P-22TA & 215.4 & & & & & 155 & 60 \\
\hline P-23TA & 181.5 & & & & & 156 & 26 \\
\hline P-24TA & 313.3 & 260.0 & 53 & 250 & 63 & 235 & 78 \\
\hline P-25TA & 265.1 & 251.0 & 14 & 221 & 44 & 183 & 82 \\
\hline P-26TA & 152.2 & & & & & & \\
\hline P-27TA & 274.1 & & & & & & \\
\hline P-28TA & 285.6 & 230.0 & 56 & 216 & 70 & 206 & 80 \\
\hline P4-СРТ-4 & 270.0 & 238.0 & 32 & 211 & 59 & 199 & 71 \\
\hline PBF-3 & 316.7 & 300.0 & 17 & & & 234 & 83 \\
\hline PBF-4 & 208.1 & 182.0 & 26 & & & & \\
\hline PBF-5 & 240.6 & 214.0 & 27 & 194 & 47 & 190 & 51 \\
\hline PBF-6 & 92.5 & & & & & & \\
\hline \begin{tabular}{|l|} 
PBF-7 \\
\end{tabular} & 285.4 & 269.0 & 16 & 250 & 35 & 221 & 64 \\
\hline PBF-8 & 292.0 & 258.0 & 34 & 237 & 55 & 216 & 76 \\
\hline PCL-12 & 265.8 & 241.0 & 25 & 223 & 43 & 174 & 92 \\
\hline PCL-13 & 274.2 & 235.0 & 39 & 219 & 55 & 182 & 92 \\
\hline PCL-14 & 250.3 & 232.0 & 18 & 224 & 26 & 168 & 82 \\
\hline PCL-15 & 242.1 & 223.0 & 19 & 212 & 30 & 188 & 54 \\
\hline PCL-16 & 282.8 & 258.0 & 25 & 228 & 55 & 194 & 89 \\
\hline PCL-17 & 291.0 & 265.0 & 26 & 236 & 55 & 202 & 89 \\
\hline
\end{tabular}


Appendix B-2: Hydrostratigraphic Boundaries (continued)

\begin{tabular}{|c|c|c|c|c|c|c|c|}
\hline & & \multicolumn{2}{|c|}{ base of "uu" } & \multicolumn{2}{|c|}{ "AA" Interval } & \multicolumn{2}{|c|}{$\begin{array}{c}\text { Transmissive } \\
\text { Zone }\end{array}$} \\
\hline Well ID & $\begin{array}{c}\text { Surface } \\
\text { Elevation (ft } \\
\text { m.s.l.) }\end{array}$ & $\begin{array}{l}\text { Elev.(ft } \\
\text { m.s.i.) }\end{array}$ & $\begin{array}{c}\text { Depth (ft } \\
\text { b.g.l.) }\end{array}$ & $\begin{array}{c}\text { Elev. (ft } \\
\text { m.s.l.) }\end{array}$ & $\begin{array}{l}\text { Depth (ft } \\
\text { b.g.l.) }\end{array}$ & $\begin{array}{l}\text { Elev. (ft } \\
\text { m.s.1.) }\end{array}$ & $\begin{array}{c}\text { Depth (ft } \\
\text { b.g.l.) }\end{array}$ \\
\hline
\end{tabular}

\begin{tabular}{|l|r||r|r|r|r|r|r|}
\hline PCL-1A & 317.7 & 292.0 & 26 & 257 & 61 & & \\
\hline PCL-2 & 304.4 & 250.0 & 54 & 230 & 74 & 206 & 98 \\
\hline PCL-3A & 300.0 & 250.0 & 50 & 228 & 72 & 208 & 92 \\
\hline PCL-4 & 331.7 & 290.0 & 42 & 278 & 54 & 199 & 133 \\
\hline PCL-5 & 284.3 & 270.0 & 14 & 266 & 18 & 199 & 85 \\
\hline PCL-6 & 312.6 & 275.0 & 38 & 266 & 47 & 242 & 71 \\
\hline PCL-7B & 295.7 & 259.0 & 37 & 228 & 68 & 193 & 103 \\
\hline PCL-8 & 254.7 & 228.0 & 27 & 217 & 38 & 192 & 63 \\
\hline PCL-9 & 288.1 & 271.0 & 17 & 260 & 28 & 208 & 80 \\
\hline PPC-1 & 313.3 & 285.0 & 28 & 263 & 50 & 245 & 68 \\
\hline RCP-1A & 294.8 & 273.0 & 22 & 243 & 52 & 208 & 87 \\
\hline RSF-1 & 300.8 & & & & & & \\
\hline RSF-2 & 300.3 & & & & & & \\
\hline RSF-3 & 304.8 & 251.0 & -54 & 223 & 82 & 210 & 95 \\
\hline SDS-21 & 251.0 & & & & & & \\
\hline SDS-22 & 283.0 & & & & & & \\
\hline SSW-1 & 311.3 & 281.0 & 30 & 255 & 56 & 230 & 81 \\
\hline SSW-2 & 167.3 & & & & & & \\
\hline SSW-3 & 178.7 & & & & & & \\
\hline SW-24CP & 257.5 & 242.0 & 16 & & & 221 & 37 \\
\hline T18N1A & 258.4 & & & & & & \\
\hline T18S1A & 233.5 & 213.0 & 21 & 197 & 37 & 186 & 48 \\
\hline T18W1A & 244.4 & 208.0 & 36 & & & 197 & 47 \\
\hline USGS-MP & 245.0 & & & & & & \\
\hline VG-1 & 156.6 & & & & & & \\
\hline VG-7 & 250.6 & & & & & & \\
\hline VG-8 & 103.7 & & & & & & \\
\hline YSC-1A & 268.9 & 247.0 & 22 & & & 237 & 32 \\
\hline YSC-1C & 272.5 & & & & & & \\
\hline YSC-2A & 281.7 & & & & & & \\
\hline YSC-3SB & 277.0 & & & & & & \\
\hline YSC-4A & 287.5 & & & & & & \\
\hline YSC-5A & 273.0 & & & & & & \\
\hline & & & & & & & \\
\hline
\end{tabular}




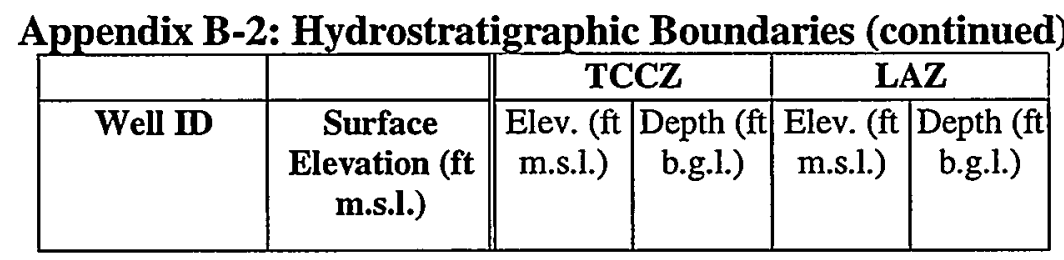

\begin{tabular}{|c|c|c|c|c|c|}
\hline $131 \mathrm{C}-100$ & 261.6 & 194 & 68 & 166 & 96 \\
\hline $131 \mathrm{C}-104$ & 276.9 & 187 & 90 & 161 & 116 \\
\hline $131 \mathrm{C}-105$ & 169.8 & & & 170 & 0 \\
\hline $131 \mathrm{C}-49$ & 254.3 & 186 & 68 & & \\
\hline $131 \mathrm{C}-51$ & 265.0 & 180 & 85 & & \\
\hline $131 \mathrm{C}-54$ & 247.1 & & & & \\
\hline $131 C-55$ & 245.0 & & & & \\
\hline $131 \mathrm{C}-59$ & 227.7 & & & & \\
\hline $131 \mathrm{C}-60$ & 218.2 & 181 & 37 & 147 & 71 \\
\hline $131 \mathrm{C}-63$ & 211.1 & 180 & 31 & & \\
\hline $131 \mathrm{C}-64$ & 211.9 & 182 & 30 & 166 & 46 \\
\hline $131 \mathrm{C}-67$ & 265.8 & 181 & 85 & & \\
\hline $131 \mathrm{C}-68$ & 267.7 & 184 & 84 & & \\
\hline $131 \mathrm{C}-80$ & 205.7 & 174 & 32 & 149 & 57 \\
\hline \begin{tabular}{|l|}
$131 C-81$ \\
\end{tabular} & 217.9 & 174 & 44 & 151 & 67 \\
\hline $131 \mathrm{C}-82$ & 233.0 & 174 & 59 & & \\
\hline $131 \mathrm{C}-83$ & 240.5 & 171 & 70 & 142 & 99 \\
\hline $131 \mathrm{C}-84$ & 241.9 & 170 & 72 & $\overline{152}$ & 90 \\
\hline $131 \mathrm{C}-85$ & 237.5 & 170 & 67 & 151 & 86 \\
\hline $131 \mathrm{C}-91$ & 233.7 & 178 & 56 & $\overline{144}$ & 90 \\
\hline 131C-93 & 241.9 & 180 & 62 & 143 & 99 \\
\hline $131 \mathrm{C}-95$ & 241.3 & 187 & 54 & 147 & 94 \\
\hline $131 \mathrm{C}-96$ & 243.2 & 183 & 60 & 166 & 77 \\
\hline 131C-98 & 251.0 & 179 & 72 & 162 & 89 \\
\hline 131C-R1 & 271.0 & 181 & 90 & & \\
\hline 131C-R2 & 266.8 & & & & \\
\hline 131C-R3 & 268.8 & 183 & 86 & & \\
\hline 131C-R4 & 285.6 & 181 & 105 & & \\
\hline 131C-R5 & 289.0 & 184 & 105 & & \\
\hline 131C-R6 & 276.0 & 168 & 108 & & \\
\hline BGO-10A & 299.1 & 209 & 90 & 207 & 92 \\
\hline BGO-10AA & 298.8 & 219 & 80 & 207 & 92 \\
\hline BGO-12A & 311.4 & 199 & 112 & 186 & 125 \\
\hline BGO-14A & 300.2 & 220 & 80 & 212 & 88 \\
\hline BGO-16A & 302.8 & 196 & 107 & 183 & 120 \\
\hline BGO-18A & 292.9 & 194 & 99 & 199 & 94 \\
\hline BGO-20AA & 280.9 & 206 & 75 & 194 & 87 \\
\hline BGO-25A & 294.7 & 212 & 83 & 201 & 94 \\
\hline BGO-26A & 285.1 & 219 & 66 & 205 & 80 \\
\hline BGO-27C & 273.9 & 199 & 75 & 192 & 82 \\
\hline BGO-29A & 262.1 & 196 & 66 & 185 & 77 \\
\hline
\end{tabular}


Appendix B-2: Hydrostratigraphic Boundaries (continued)

\begin{tabular}{|c|c||c|c|c|c|}
\hline & \multicolumn{2}{|c||}{ TCCZ } & \multicolumn{2}{c|}{ LAZ } \\
\hline Well ID & $\begin{array}{c}\text { Surface } \\
\text { Elevation (ft } \\
\text { m.s.l.) ? }\end{array}$ & $\begin{array}{c}\text { Elev. (ft } \\
\text { m.s.l.) }\end{array}$ & $\begin{array}{c}\text { Depth (ft } \\
\text { b.g.l.) }\end{array}$ & $\begin{array}{c}\text { Elev. (ft } \\
\text { m.s.l.) }\end{array}$ & Depth (ft \\
& & & $\vdots$ & \\
\hline
\end{tabular}

\begin{tabular}{|l|r|r|r|r|r|}
\hline BGO-31C & 271.1 & 198 & 73 & 188 & 83 \\
\hline BGO-33C & 277.4 & 200 & 77 & 191 & 86 \\
\hline BGO-35C & 271.4 & 204 & 67 & 197 & 74 \\
\hline BGO-37C & 284.3 & 199 & 85 & 191 & 93 \\
\hline BGO-39A & 293.7 & 204 & 90 & 202 & 92 \\
\hline BGO-3A & 288.7 & 198 & 91 & 189 & 100 \\
\hline BGO-41A & 298.3 & 217 & 81 & 208 & 90 \\
\hline BGO-42C & 295.9 & 216 & 80 & 209 & 87 \\
\hline BGO-43AA & 312.2 & 195 & 117 & 187 & 125 \\
\hline BGO-44AA & 283.3 & 222 & 61 & 199 & 84 \\
\hline BGO-45A & 276.9 & 207 & 70 & 201 & 76 \\
\hline BGO-46B & 263.4 & 199 & 64 & 193 & 70 \\
\hline BGO-47A & 264.8 & 198 & 67 & 190 & 75 \\
\hline BGO-48C & 274.7 & 198 & 77 & 192 & 83 \\
\hline BGO-49A & 269.1 & 201 & 68 & 192 & 77 \\
\hline BGO-50A & 253.5 & 194 & 60 & 184 & 70 \\
\hline BGO-51AA & 287.2 & 205 & 82 & 194 & 93 \\
\hline BGO-52AA & 281.6 & 207 & 75 & 197 & 85 \\
\hline BGO-53AA & 288.9 & 223 & 66 & 216 & 73 \\
\hline BGO-5C & 294.2 & 218 & 76 & 201 & 93 \\
\hline BGO-6A & 283.8 & 210 & 74 & 195 & 89 \\
\hline BGO-6B & 284.5 & 203 & 82 & 192 & 93 \\
\hline BGO-8A & 281.3 & 213 & 68 & 199 & 82 \\
\hline BGO-9AA & 282.8 & 224 & 59 & 211 & 72 \\
\hline BGT-1 & 282.9 & 222 & 61 & 208 & 75 \\
\hline BGT-10 & 215.2 & 201 & 14 & 194 & 21 \\
\hline BGT-11 & 222.5 & 228 & -5 & 223 & 0 \\
\hline BGT-12 & 284.2 & 225 & 59 & 214 & 70 \\
\hline BGT-13 & 287.8 & 224 & 64 & 216 & 72 \\
\hline BGT-14 & 280.7 & 215 & 66 & 209 & 72 \\
\hline BGT-15 & 277.5 & 209 & 69 & 201 & 77 \\
\hline BGT-16 & 250.7 & & & & \\
\hline BGT-17 & 240.7 & & & & \\
\hline BGT-18 & 216.5 & 222 & -5 & 217 & 0 \\
\hline BGT-2 & 276.4 & 213 & 63 & 198 & 78 \\
\hline BGT-20 & 159.5 & & & & \\
\hline BGT-21 & 294.2 & 223 & 71 & 216 & 78 \\
\hline BGT-22 & 281.0 & 200 & 81 & 178 & 103 \\
\hline BGT-23 & 270.0 & 216 & 54 & 210 & 60 \\
\hline BGT-24 & 227 & 39 & 220 & 46 \\
\hline BGT-25 & 229 & 36 & 224 & 41 \\
\hline BGT-27 & 217 & 40 & 207 & 50 \\
\hline
\end{tabular}


Appendix B-2: Hydrostratigraphic Boundaries (continued)

\begin{tabular}{|c|c||c|c|c|c|}
\hline & \multicolumn{2}{|c||}{ TCCZ } & \multicolumn{2}{c|}{ LAZ } \\
\hline Well ID & $\begin{array}{c}\text { Surface } \\
\text { Elevation (ft } \\
\text { m.s.l.) }\end{array}$ & $\begin{array}{c}\text { Elev. (ft } \\
\text { m.s.l.) }\end{array}$ & $\begin{array}{c}\text { Depth (ft } \\
\text { b.g.l.) }\end{array}$ & $\begin{array}{c}\text { Elev. (ft } \\
\text { m.s.l.) }\end{array}$ & $\begin{array}{c}\text { Depth (ft } \\
\text { b.g.l.) }\end{array}$ \\
\hline
\end{tabular}

\begin{tabular}{|l|r||r|r|r|r|}
\hline BGT-28 & 258.3 & 212 & 46 & 190 & 68 \\
\hline BGT-29 & 243.0 & 219 & 24 & 215 & 28 \\
\hline BGT-3 & 275.7 & 212 & 64 & 198 & 78 \\
\hline BGT-30 & 219.0 & & & & \\
\hline BGT-31 & 308.8 & 220 & 89 & 215 & 94 \\
\hline BGT-32 & 310.1 & 237 & 73 & 234 & 76 \\
\hline BGT-33 & 290.4 & 239 & 51 & 223 & 67 \\
\hline BGT-34 & 286.8 & 228 & 59 & 218 & 69 \\
\hline BGT-35 & 267.7 & 217 & 51 & 212 & 56 \\
\hline BGT-36 & 261.4 & 226 & 35 & 215 & 46 \\
\hline BGT-37 & 251.6 & 222 & 30 & 215 & 37 \\
\hline BGT-38 & 240.1 & & & & \\
\hline BGT-39 & 241.9 & & & & \\
\hline BGT-4 & 259.2 & 213 & 46 & 204 & 55 \\
\hline BGT-40 & 332.3 & 209 & 123 & 203 & 129 \\
\hline BGT-41 & 328.4 & 224 & 104 & 219 & 109 \\
\hline BGT-42 & 310.9 & 224 & 87 & 219 & 92 \\
\hline BGT-43 & 277.1 & 205 & 72 & 201 & 76 \\
\hline BGT-44 & 276.2 & 214 & 62 & 209 & 67 \\
\hline BGT-45 & 285.3 & 218 & 67 & 209 & 76 \\
\hline BGT-46 & 310.0 & 213 & 97 & 205 & 105 \\
\hline BGT-47 & 317.3 & 214 & 103 & 210 & 107 \\
\hline BGT-48 & 314.3 & 217 & 97 & 209 & 105 \\
\hline BGT-49 & 297.3 & 222 & 75 & 214 & 83 \\
\hline BGT-5 & 225.7 & 214 & 12 & 206 & 20 \\
\hline BGT-50 & 296.3 & 221 & 75 & 214 & 82 \\
\hline BGT-51 & 272.6 & 193 & 80 & 186 & 87 \\
\hline BGT-53 & 278.3 & 200 & 78 & 191 & 87 \\
\hline BGT-54 & 280.0 & 205 & 75 & 196 & 84 \\
\hline BGT-56 & 262.9 & 182 & 81 & 175 & 88 \\
\hline BGT-57 & 259.4 & 179 & 80 & 169 & 90 \\
\hline BGT-58 & 285.8 & 192 & 94 & 190 & 96 \\
\hline BGT-59 & 281.9 & 182 & 100 & 173 & 109 \\
\hline BGT-6 & 282.2 & 218 & 64 & & \\
\hline BGT-60 & 291.4 & 186 & 105 & 176 & 115 \\
\hline BGT-61 & 284.3 & 186 & 98 & 176 & 108 \\
\hline BGT-62 & 282.0 & 190 & 92 & 176 & 106 \\
\hline BGT-63 & 195 & 99 & 189 & 105 \\
\hline BGT-63A & 193 & 93 & 194 & 97 \\
\hline BGT-64 & 290.8 & 49 & 188 & 95 \\
\hline BGT-66 & & 188 & 56 \\
\hline BGT-67 & & & 180 & 62 \\
\hline
\end{tabular}


Appendix B-2: Hydrostratigraphic Boundaries (continued)

\begin{tabular}{|c|c||c|c|c|c|}
\hline & \multicolumn{2}{|c||}{} & \multicolumn{2}{c|}{ TCCZ } & \multicolumn{2}{c|}{ LAZ } \\
\hline Well ID & Surface & Elev. (ft & Depth (ft & Elev. (ft & Depth (ft \\
& $\begin{array}{c}\text { Elevation (ft } \\
\text { m.s.l.) }\end{array}$ & m.s.l.) & b.g.l.) & m.s.l.) & b.g.l.) \\
& & & & $\vdots$ & \\
\hline
\end{tabular}

\begin{tabular}{|l|r||r|r|r|r|}
\hline BGT-7 & 276.4 & 212 & 64 & 199 & 77 \\
\hline BGT-8 & 249.3 & 221 & 28 & 217 & 32 \\
\hline BGT-9 & 226.0 & 210 & 16 & 205 & 21 \\
\hline BGX-11D & 273.8 & 193 & 81 & 177 & 97 \\
\hline BGX-1A & 289.1 & 21.1 & 78 & 198 & 91 \\
\hline BGX-2B & 289.2 & 216 & 73 & 198 & 91 \\
\hline BGX-4A & 288.8 & 225 & 64 & 213 & 76 \\
\hline BGX-7D & 277.1 & 225 & 52 & 220 & 57 \\
\hline BGX-9D & 277.4 & 207 & 70 & 202 & 75 \\
\hline BRR-1D & 293.8 & 195 & 99 & & \\
\hline BRR-3D & 289.5 & 208 & 82 & 194 & 96 \\
\hline BRR-6B & 293.9 & 178 & 116 & 166 & 128 \\
\hline BRR-7B & 289.6 & 202 & 88 & 190 & 100 \\
\hline BRR-8B & 276.7 & 205 & 72 & 200 & 77 \\
\hline CCP-1A & 287.1 & 176 & 112 & 162 & 125 \\
\hline CFD-1 & 268.8 & 188 & 81 & 178 & 91 \\
\hline CFD-18 & 248.3 & 164 & 84 & 150 & 98 \\
\hline CFD-5 & 257.8 & 180 & 78 & 173 & 85 \\
\hline CMP-1-CP & 248.9 & 214 & 35 & & \\
\hline CMP-30B & 286.5 & 209 & 78 & 197 & 90 \\
\hline CMP-32B & 251.7 & 220 & 32 & 198 & 54 \\
\hline CMP-4B-CP & 241.8 & 212 & 30 & 194 & 48 \\
\hline CMP-6A-CP & 238.4 & 202 & 36 & 180 & 58 \\
\hline CPC-1 & 285.1 & 164 & 121 & 154 & 131 \\
\hline CRP-5D & 274.5 & 187 & 88 & 139 & 136 \\
\hline CRP-9D & 268.4 & 163 & 105 & & \\
\hline CRSB-1 & 278.2 & 172 & 106 & 153 & 125 \\
\hline CRSB-101 & 275.6 & 179 & 97 & 145 & 131 \\
\hline CRSB-11 & 276.7 & 170 & 107 & 155 & 122 \\
\hline CRSB-14 & 275.6 & 170 & 106 & 151 & 125 \\
\hline CRSB-16 & 282.6 & 181 & 102 & & \\
\hline CRSB-17 & 281.0 & 174 & 107 & 156 & 125 \\
\hline CRSB-18 & 273.8 & 173 & 101 & 137 & 137 \\
\hline CRSB-20 & 274.2 & 172 & 102 & 153 & 121 \\
\hline CRSB-21 & 272.9 & 170 & 103 & 152 & 121 \\
\hline CRSB-24 & 277.2 & 186 & 91 & 162 & 115 \\
\hline CRSB-26 & 291.6 & 190 & 102 & 170 & 122 \\
\hline CRSB-28 & 289.3 & 179 & 110 & 151 & 138 \\
\hline CRSB-30 & 285.4 & 184 & 101 & 165 & 120 \\
\hline CRSB-31 & 284.4 & 175 & 109 & 156 & 128 \\
\hline CRSB-32 & 161 & 113 & 130 & 144 \\
\hline CRSB-35 & & 93 & 148 & 109 \\
\hline & 257.4 & & & \\
\hline
\end{tabular}


Appendix B-2: Hydrostratigraphic Boundaries (continued)

\begin{tabular}{|c|c||c|c|c|c|}
\hline & \multicolumn{2}{|c||}{ TCCZ } & \multicolumn{2}{|c|}{ LAZ } \\
\hline Well ID & $\begin{array}{c}\text { Surface } \\
\text { Elevation (ft } \\
\text { m.s.l.) }\end{array}$ & $\begin{array}{c}\text { Elev. (ft } \\
\text { m.s.l.) }\end{array}$ & $\begin{array}{c}\text { Depth (ft } \\
\text { b.g.l.) }\end{array}$ & $\begin{array}{c}\text { Elev. (ft } \\
\text { m.s.l.) }\end{array}$ & $\begin{array}{c}\text { Depth (ft } \\
\text { b.g.l.) }\end{array}$ \\
\hline
\end{tabular}

\begin{tabular}{|l|r||r|r|r|r|}
\hline CRSB-36 & 265.6 & 164 & 102 & 135 & 131 \\
\hline CRSB-37 & 270.5 & 175 & 95 & & \\
\hline CRSB-4 & 282.8 & 179 & 104 & 148 & 135 \\
\hline CRSB-40 & 227.3 & 186 & 41 & 152 & 75 \\
\hline CRSB-43 & 258.5 & 169 & 90 & 143 & 116 \\
\hline CRSB-45 & 270.6 & 180 & 91 & 143 & 128 \\
\hline CRSB-47 & 282.7 & 163 & 120 & 135 & 148 \\
\hline CRSB-48 & 259.8 & 177 & 83 & 144 & 116 \\
\hline CRSB-5 & 288.3 & 175 & 113 & 135 & 153 \\
\hline CRSB-50 & 255.6 & 185 & 71 & 139 & 117 \\
\hline CRSB-52 & 233.1 & 181 & 52 & 156 & 77 \\
\hline CRSB-54 & 224.7 & 186 & 39 & 166 & 59 \\
\hline CRSB-55 & 229.1 & 182 & 47 & 169 & 60 \\
\hline CRSB-56 & 232.5 & 183 & 50 & 162 & 71 \\
\hline CRSB-57 & 248.2 & 182 & 66 & 170 & 78 \\
\hline CRSB-58 & 253.9 & 186 & 68 & 173 & 81 \\
\hline CRSB-6 & 289.4 & 178 & 111 & 160 & 129 \\
\hline CRSB-60 & 257.0 & 189 & 68 & 159 & 98 \\
\hline CRSB-61 & 257.3 & 185 & 72 & 160 & 97 \\
\hline CRSB-63 & 240.4 & 184 & 56 & 148 & 92 \\
\hline CRSB-64 & 229.9 & 189 & 41 & 147 & 83 \\
\hline CRSB-65 & 214.8 & 182 & 33 & 141 & 74 \\
\hline CRSB-86 & 206.6 & 182 & 25 & 165 & 42 \\
\hline CRSB-88 & 253.2 & 176 & 77 & 153 & 100 \\
\hline CRSB-89 & 260.6 & 182 & 79 & 161 & 100 \\
\hline CRSB-90 & 268.4 & 183 & 85 & 151 & 117 \\
\hline CRSB-91 & 273.1 & 185 & 88 & & \\
\hline CRSB-92 & 287.5 & 181 & 107 & 157 & 131 \\
\hline CRSB-93 & 286.8 & 181 & 106 & 163 & 124 \\
\hline CRSB-94 & 283.3 & 187 & 96 & 154 & 129 \\
\hline CRSB-95 & 280.4 & 191 & 89 & 165 & 115 \\
\hline CRSB-96 & 282.8 & 188 & 95 & 158 & 125 \\
\hline CRSB-97 & 276.8 & 181 & 96 & 156 & 121 \\
\hline CRSB-98 & 275.1 & 181 & 94 & 154 & 121 \\
\hline CRSB-99 & 274.4 & 174 & 100 & 142 & 132 \\
\hline CSB-101 & 281.2 & 181 & 100 & 156 & 125 \\
\hline CSB-102 & 283.5 & 186 & 98 & 182 & 102 \\
\hline CSB-103 & 257.6 & 179 & 79 & 168 & 90 \\
\hline CSB-104 & 224.9 & 175 & 50 & 168 & 57 \\
\hline CSB-105 & 212.1 & 177 & 35 & 175 & 37 \\
\hline CSB-106 & 18.8 & 169 & 15 \\
\hline CSB-107 & & & & \\
\hline & & & & \\
\hline
\end{tabular}


Appendix B-2: Hydrostratigraphic Boundaries (continued)

\begin{tabular}{|c|c||c|c|c|c|}
\hline & \multicolumn{2}{|c||}{ TCCZ } & \multicolumn{2}{c|}{ LAZ } \\
\hline Well ID & $\begin{array}{c}\text { Surface } \\
\text { Elevation (ft } \\
\text { m.s.l.) }\end{array}$ & $\begin{array}{c}\text { Elev. (ft } \\
\text { m.s.l.) }\end{array}$ & $\begin{array}{c}\text { Depth (ft } \\
\text { b.g.l.) }\end{array}$ & $\begin{array}{c}\text { Elev. (ft } \\
\text { m.s.l.) }\end{array}$ & Depth (ft \\
& b.g.l.) \\
\hline
\end{tabular}

\begin{tabular}{|l|r||r|r|r|r|}
\hline CSB-108 & 196.7 & 178 & 19 & 165 & 32 \\
\hline CSB-109 & 172.9 & & & & \\
\hline CSB-110 & 184.6 & & & 171 & 14 \\
\hline CSB-111 & 172.1 & & & 160 & 12 \\
\hline CSB-112 & 195.3 & 172 & 23 & 153 & 42 \\
\hline CSB-113 & 210.4 & 180 & 30 & 161 & 49 \\
\hline CSB-114 & 196.0 & 178 & 18 & 168 & 28 \\
\hline CSB-116 & 274.7 & 175 & 100 & 151 & 124 \\
\hline CSB-117 & 276.5 & 180 & 97 & 150 & 127 \\
\hline CSB-118 & 277.8 & 178 & 100 & 171 & 107 \\
\hline CSB-119 & 268.3 & 180 & 88 & 156 & 112 \\
\hline CSB-120 & 282.8 & 178 & 105 & 159 & 124 \\
\hline CSB-121 & 274.2 & 188 & 86 & 163 & 111 \\
\hline CSB-122 & 222.9 & 181 & 42 & 165 & 58 \\
\hline CSB-123 & 224.2 & 188 & 36 & 182 & 42 \\
\hline CSB-2C & 289.9 & 181 & 109 & 141 & 149 \\
\hline CSB-3C & 280.9 & 177 & 104 & & \\
\hline CSB-47. & 282.7 & 162 & 121 & 160 & 123 \\
\hline CSB-47A & 268.9 & 185 & 84 & 161 & 108 \\
\hline CSB-48 & 259.8 & 178 & 82 & 160 & 100 \\
\hline CSB-48A & 270.1 & 178 & 92 & 154 & 116 \\
\hline CSB-56 & 267.0 & 184 & 83 & 160 & 107 \\
\hline CSB-58 & 235.5 & 187 & 49 & 174 & 62 \\
\hline CSB-59 & 231.2 & 181 & 50 & 158 & 73 \\
\hline CSB-60 & 247.0 & 181 & 66 & 179 & 68 \\
\hline CSB-61 & 252.2 & 175 & 77 & 173 & 79 \\
\hline CSB-62 & 240.9 & 179 & 62 & 169 & 72 \\
\hline CSB-63 & 227.1 & 180 & 47 & 178 & 49 \\
\hline CSB-64 & 212.7 & 178 & 35 & 176 & 37 \\
\hline CSB-65 & 195.6 & 165 & 31 & 162 & 34 \\
\hline CSB-66A & 191.7 & & & & \\
\hline CSD-4D & 306.5 & 147 & 160 & 139 & 168 \\
\hline E-26 & 314.3 & 170 & 144 & 135 & 179 \\
\hline E-4 & 315.4 & 140 & 175 & 130 & 185 \\
\hline FAC-1SB & 312.2 & 224 & 88 & 217 & 95 \\
\hline FCH-1 & 316.8 & 214 & 103 & 202 & 115 \\
\hline FCH-2 & 288.7 & 213 & 76 & 197 & 92 \\
\hline FCH-3 & 307.2 & 207 & 100 & 196 & 111 \\
\hline FCH-4 & 297.5 & 197 & 101 & 187 & 111 \\
\hline FCH-5 & 284.2 & 196 & 88 & 191 & 93 \\
\hline FCH-6 & 189 & 103 & 182 & 110 \\
\hline FIW-1MC & & 103 & 185 & 108 \\
\hline
\end{tabular}


Appendix B-2: Hydrostratigraphic Boundaries (continued)

\begin{tabular}{|c|c||c|c|c|c|}
\hline & \multicolumn{2}{|c||}{ TCCZ } & \multicolumn{2}{c|}{ LAZ } \\
\hline Well ID & $\begin{array}{c}\text { Surface } \\
\text { Elevation (ft } \\
\text { m.s.l.) }\end{array}$ & $\begin{array}{c}\text { Elev. (ft } \\
\text { m.s.l.) }\end{array}$ & $\begin{array}{c}\text { Depth (ft } \\
\text { b.g.l.) }\end{array}$ & $\begin{array}{c}\text { Elev. (ft } \\
\text { m.s.l.) }\end{array}$ & $\begin{array}{c}\text { Depth (ft } \\
\text { b.g.l.) }\end{array}$ \\
\hline
\end{tabular}

\begin{tabular}{|c|c|c|c|c|c|}
\hline FIW-2MA & 290.5 & 189 & 102 & 180 & 111 \\
\hline FNB-1A & 282.4 & 208 & 74 & 202 & 80 \\
\hline FNB-3A & 282.2 & 211 & 71 & 208 & 75 \\
\hline FSB-100A & 283.8 & 185 & 99 & 183 & 101 \\
\hline FSB-101A & 282.9 & 191 & 92 & 183 & 100 \\
\hline FSB-112A & 227.0 & 164 & 63 & 144 & 83 \\
\hline FSB-113A & 221.3 & 178 & 43 & 171 & 50 \\
\hline FSB-114A & 250.0 & 178 & 72 & 173 & 77 \\
\hline FSB-115C & 205.8 & 181 & 25 & 165 & 41 \\
\hline FSB-116C & 200.5 & 176 & 25 & 171 & 30 \\
\hline FSB-120A & 278.0 & 181 & 97 & 165 & 113 \\
\hline FSB-121C & 254.4 & 173 & 81 & 162 & 92 \\
\hline FSB-122C & 216.0 & 164 & 52 & 148 & 68 \\
\hline FSB-123C & 236.3 & 183 & 53 & 172 & 64 \\
\hline FSB-1TA & 275.4 & 191 & 84 & 187 & $\overline{88}$ \\
\hline FSB-76A & 291.5 & abs. & abs. & \begin{tabular}{|l|} 
abs. \\
\end{tabular} & abs. \\
\hline FSB-78A & 270.5 & 163 & 108 & 147 & 124 \\
\hline FSB-79A & 216.1 & 173 & 43 & 164 & 52 \\
\hline FSB-87A & 285.6 & 176 & 110 & 173 & 113 \\
\hline FSB-89C & 279.1 & 186 & 93 & 180 & 99 \\
\hline FSB-91C & $\overline{277.0}$ & 168 & 109 & 161 & 116 \\
\hline FSB-93C & 274.0 & 166 & 108 & 151 & 123 \\
\hline FSB-95C & 281.8 & 174 & 108 & 158 & 124 \\
\hline FSB-96A & 277.7 & 167 & 111 & 154 & 124 \\
\hline FSB-97A & 283.8 & 163 & 121 & 152 & 132 \\
\hline FSB-98A & 280.7 & 172 & 109 & 160 & 121 \\
\hline FSB-99A & 285.3 & 178 & 107 & 173 & 112 \\
\hline FSB-PC & 230.8 & 161 & 70 & 157 & 74 \\
\hline $\begin{array}{l}\text { GAPWR-TW- } \\
1\end{array}$ & 219.0 & & & & \\
\hline HAA-1TA & 290.2 & 170 & 120 & 160 & 130 \\
\hline HAA-2AA & 291.4 & 190 & 102 & 186 & 106 \\
\hline HAA-3AA & 274.5 & 191 & 84 & 179 & 96 \\
\hline HAA-4AA & 299.2 & 202 & 97 & 194 & 106 \\
\hline HAA-6AA & 279.8 & 210 & 70 & 183 & 97 \\
\hline HC-03AA & 263.8 & & & & \\
\hline $\mathrm{HC}-12 \mathrm{~A}$ & 287.3 & 195 & 92 & 190 & 97 \\
\hline HCA-4AA & 308.6 & 234 & 75 & 230 & $\overline{79}$ \\
\hline HCH-1 & 284.0 & 202 & 82 & 187 & 97 \\
\hline $\mathrm{HCH}-2$ & 270.9 & 196 & 75 & 180 & 91 \\
\hline $\mathrm{HCH}-3$ & 264.0 & 197 & 67 & 179 & 85 \\
\hline $\mathrm{HCH}-4$ & 269.9 & 193 & 77 & 183 & 87 \\
\hline $\mathrm{HCH}-5$ & 255.0 & 192 & 63 & 180 & 75 \\
\hline
\end{tabular}


Appendix B-2: Hydrostratigraphic Boundaries (continued)

\begin{tabular}{|c|c|c|c|c|c|}
\hline & & \multicolumn{2}{|c|}{ TCCZ } & \multicolumn{2}{|c|}{ LAZ } \\
\hline Well ID & $\begin{array}{c}\text { Surface } \\
\text { Elevation (ft } \\
\vdots \text { m.s.l.) }\end{array}$ & $\begin{array}{c}\text { Elev. (ft } \\
\text { m.s.l.). }\end{array}$ & $\begin{array}{c}\text { Depth (f } \\
\text { b.g.l.) }\end{array}$ & $\begin{array}{c}\text { Elev. (ft } \\
\text { m.s.l.) }\end{array}$ & $\begin{array}{c}\text { Depth (ft } \\
\text { b.g.l.) }\end{array}$ \\
\hline
\end{tabular}

\begin{tabular}{|c|c|c|c|c|c|c|}
\hline HIW-1BD & 275.8 & 205 & & 71 & & \\
\hline HIW-1MC & 272.3 & 187 & & 86 & 80 & 93 \\
\hline HIW-2A & 276.3 & 202 & & 75 & 95 & 8 \\
\hline HIW-2MC & 269.0 & 199 & & 70 & 94 & 7 \\
\hline HIW-4MC & 263.4 & 197 & & 66 & 90 & 7 \\
\hline HIW-5MC & 266.1 & 184 & & 82 & 78 & 88 \\
\hline HMD-1C, & 262.7 & 229 & & 34 & 26 & 3 \\
\hline HMD-2C & 259.3 & 222 & & 37 & 16 & 4 \\
\hline HMD-3C & 257.2 & 223 & & 34 & 218 & 35 \\
\hline HMD-4C & 248.5 & 224 & & 25 & 20 & 2 \\
\hline HPC-1 & 293.5 & 195 & & 99 & 88 & 10 \\
\hline HPT-1A & 232.9 & & & & & \\
\hline HPT-2A & 257.8 & & & & & \\
\hline HSB-101C & 256.3 & 195 & & 61 & 89 & 6 \\
\hline HSB-103C & 245.2 & 195 & & 50 & 81 & 6 \\
\hline HSB-104C & 245.5 & 194 & & 52 & 85 & 6 \\
\hline HSB-105C & 247.2 & 190 & & 57 & 83 & 6 \\
\hline HSB-106C & 250.7 & 192 & & 59 & 84 & 6 \\
\hline HSB-107C & 259.3 & 199 & & 60 & 91 & 68 \\
\hline HSB-109C & 259.4 & 203 & & 56 & 89 & 7 \\
\hline HSB-110C & 253.4 & 192 & & 61 & 88 & 6 \\
\hline HSB-111C & 253.7 & 188 & & 66 & 72 & 8 \\
\hline HSB-112C & 252.6 & 191 & & 62 & 86 & 6 \\
\hline HSB-113C & 258.7 & 188 & & 71 & 74 & 8 \\
\hline HSB-115C & 266.8 & 209 & & 58 & 97 & 7 \\
\hline HSB-117A & 234.8 & 216 & & 19 & 92 & 4 \\
\hline HSB-118A & 245.0 & 183 & & 62 & 73 & 72 \\
\hline HSB-119A & 254.8 & 213 & & 42 & 95 & 6 \\
\hline HSB-120A & 266.0 & 203 & & 63 & 96 & 7 \\
\hline HSB-121A & 272.3 & 197 & & 75 & 84 & 88 \\
\hline HSB-122A & 269.4 & 188 & & 81 & 77 & 92 \\
\hline HSB-123A & 263.6 & 196 & & 68 & 86 & 78 \\
\hline HSB-124A & 263.9 & abs. & abs. & abs. & & abs. \\
\hline HSB-132C & 238.3 & 163 & & \begin{tabular}{l|l}
75 & \\
\end{tabular} & 58 & 8 \\
\hline HSB-139A & 231.5 & 190 & & 42 & 79 & 5 \\
\hline HSB-140A & 234.0 & 194 & & 40 & 81 & 5 \\
\hline HSB-141A & 252.6 & 181 & & 72 & 67 & 8 \\
\hline HSB-142C & 201.6 & abs. & abs. & & & \\
\hline HSB-143C & 220.1 & 198 & & 22 & 79 & 4 \\
\hline HSB-144A & 233.6 & 186 & & 48 & 79 & 5 \\
\hline HSB-145C & 233.7 & 184 & & 50 & 175 & $5 !$ \\
\hline HSB-146A & 249.5 & 174 & & 76 & 163 & 8 \\
\hline
\end{tabular}


Appendix B-2: Hydrostratigraphic Boundaries (continued)

\begin{tabular}{|c|c||c|c|c|c|}
\hline & & \multicolumn{2}{|c||}{ TCCZ } & \multicolumn{2}{c|}{ LAZ } \\
\hline Well W & $\begin{array}{c}\text { Surface } \\
\text { Elevation (ft } \\
\text { m.s.l.) }\end{array}$ & $\begin{array}{c}\text { Elev. (ft } \\
\text { m.s.l.) }\end{array}$ & $\begin{array}{c}\text { Depth (ft } \\
\text { b.g.l.) }\end{array}$ & $\begin{array}{c}\text { Elev. (ft } \\
\text { m.s.l.) }\end{array}$ & Depth (ft \\
& b.g.l.) \\
\hline
\end{tabular}

\begin{tabular}{|l|r||r|r|r|r|}
\hline HSB-148C & 248.9 & 187 & 62 & 171 & 78 \\
\hline HSB-151C & 211.6 & 193 & 19 & 183 & 29 \\
\hline HSB-152C & 212.1 & 198 & 14 & 186 & 26 \\
\hline HSB-65A & 270.7 & 204 & 67 & 199 & 72 \\
\hline HSB-68A & 247.4 & 198 & 49 & 193 & 54 \\
\hline HSB-69A & 234.1 & 187 & 47 & 181 & 53 \\
\hline HSB-83A & 234.9 & 195 & 40 & 188 & 47 \\
\hline HSB-84A & 226.7 & 205 & 22 & 181 & 46 \\
\hline HSB-85A & 292.1 & 204 & 88 & 200 & 92 \\
\hline HSB-86A & 260.0 & 185 & 75 & 178 & 82 \\
\hline HSB-PC & 227.8 & 188 & 40 & 178 & 50 \\
\hline HSB-TB & 267.1 & 207 & 60 & 199 & 68 \\
\hline HSL-6AA & 274.6 & 174 & 101 & 169 & 106 \\
\hline HSL-8AA & 286.7 & 193 & 94 & 186 & 101 \\
\hline IDB-2A & 302.4 & 245 & 57 & 228 & 74 \\
\hline IDP-3A & 282.2 & 191 & 91 & 183 & 99 \\
\hline IDQ-3A & 203.2 & & & 203 & 0 \\
\hline KAC-9D & 260.2 & 161 & 99 & 149 & 111 \\
\hline KPT-1 & 205.2 & 155 & 50 & 145 & 60 \\
\hline KPT-2 & 258.2 & 152 & 106 & 132 & 126 \\
\hline KPT-3 & 225.5 & 164 & 62 & 134 & 92 \\
\hline KPT-4 & 258.9 & 169 & 90 & 154 & 105 \\
\hline KPT-5 & 225.1 & 168 & 57 & 139 & 86 \\
\hline KPT-6 & 232.8 & 165 & 68 & 145 & 88 \\
\hline KPT-7 & 211.8 & 152 & 60 & 136 & 76 \\
\hline KPT-8 & 238.6 & & & & \\
\hline KPT-9 & 204.9 & 141 & 64 & 130 & 75 \\
\hline L3-CPT-PG-9 & 270.0 & 167 & 103 & 151 & 119 \\
\hline LAC-5DL & 239.8 & & & & \\
\hline LAC-6DL & 239.8 & & & & \\
\hline LAC-7DL & 239.4 & & & & \\
\hline LAC-8DL & 234.0 & 207 & 27 & 196 & 38 \\
\hline LCO-5A & 230.0 & 149 & 81 & 141 & 89 \\
\hline LCO-5DL & 230.3 & & & & \\
\hline LCO-8DL & 243.4 & & & & \\
\hline LFW-10SB & 168.4 & & & & \\
\hline LWN-1SB & 282.5 & 162 & 121 & 151 & 132 \\
\hline LWN-2SB & 231.0 & 168 & 63 & 151 & 80 \\
\hline LWN-3SB & 245.7 & 165 & 81 & 156 & 90 \\
\hline LWR-2SB & 248.1 & 177 & 71 & 167 & 81 \\
\hline LWR-3SB & 178 & 72 & 162 & 88 \\
\hline LWR-4SB & 178 & 116 & 165 & 129 \\
\hline & & & & \\
\hline
\end{tabular}


Appendix B-2: Hydrostratigraphic Boundaries (continued)

\begin{tabular}{|c|c|c|c|c|c|}
\hline & & \multicolumn{2}{|c|}{ TCCZ } & \multicolumn{2}{|c|}{ LAZ } \\
\hline Well ID & $\begin{array}{c}\text { Surface } \\
\text { Elevation (ft } \\
\text { m.s.l.) }\end{array}$ & $\begin{array}{c}\text { Elev. (ft } \\
\text { m.s.l.) }\end{array}$ & \begin{tabular}{|c|} 
Depth (ft \\
b.g.l.) \\
$t ;$
\end{tabular} & $\begin{array}{l}\text { Elev. (ft } \\
\text { m.s.l.) }\end{array}$ & $\begin{array}{c}\text { Depth (ft } \\
\text { b.g.l.) } \\
. .4\end{array}$ \\
\hline
\end{tabular}

\begin{tabular}{|l|r||r|r|r|r|}
\hline LWR-8CC & 280.3 & 182 & 98 & 132 & 148 \\
\hline LWR-9SB & 238.2 & 179 & 59 & 161 & 77 \\
\hline M12-17 & 325.5 & 169 & 157 & 158 & 168 \\
\hline M121A & 303.7 & 155 & 149 & 139 & 165 \\
\hline M121A & 303.7 & 155 & 149 & 139 & 165 \\
\hline M12A-29 & 321.0 & 159 & 162 & 135 & 186 \\
\hline MWD-1A & 327.5 & 171 & 157 & 165 & 163 \\
\hline MWD-C3 & 322.5 & 192 & 131 & 182 & 141 \\
\hline MWD-C5 & 328.7 & 190 & 139 & 180 & 149 \\
\hline NPN-001 & 335.9 & & & & \\
\hline NPN-1A & 335.9 & 235 & 101 & 224 & 112 \\
\hline OFS-1SB & 261.6 & 196 & 66 & 186 & 76 \\
\hline OFS-2SB & 257.5 & 198 & 60 & 188 & 70 \\
\hline OFS-3SB & 258.1 & 196 & 62 & 185 & 73 \\
\hline OFS-4SB & 258.7 & 196 & 63 & 192 & 67 \\
\hline OFS-5SB & 228.7 & 189 & 40 & 178 & 51 \\
\hline P-13TA & 252.4 & 108 & 144 & 86 & 167 \\
\hline P-14TA & 294.4 & 213 & 81 & 203 & 92 \\
\hline P-18TA & 296.9 & 186 & 111 & 167 & 130 \\
\hline P-19TA & 297.4 & 186 & 111 & 175 & 122 \\
\hline P-20TA & 287.7 & 166 & 122 & 152 & 136 \\
\hline P-21TA & 207.0 & 104 & 103 & 93 & 114 \\
\hline P-22TA & 215.4 & 128 & 87 & 120 & 95 \\
\hline P-23TA & 181.5 & 145 & 37 & 131 & 51 \\
\hline P-24TA & 313.3 & 196 & 117 & 183 & 130 \\
\hline P-25TA & 265.1 & 157 & 108 & 136 & 129 \\
\hline P-26TA & 152.2 & & & 152 & 0 \\
\hline P-27TA & 274.1 & 180 & 94 & 169 & 105 \\
\hline P-28TA & 285.6 & 181 & 105 & 174 & 112 \\
\hline P4-CPT-4 & 270.0 & 160 & 110 & 141 & 129 \\
\hline PBF-3 & 316.7 & 139 & 178 & 136 & 181 \\
\hline PBF-4 & 208.1 & 150 & 58 & 138 & 70 \\
\hline PBF-5 & 240.6 & 150 & 91 & 140 & 101 \\
\hline PBF-6 & 92.5 & & & & \\
\hline PBF-7 & 285.4 & 187 & 98 & 164 & 121 \\
\hline PBF-8 & 292.0 & 193 & 99 & 173 & 119 \\
\hline PCL-12 & 265.8 & 163 & 103 & 131 & 135 \\
\hline PCL-13 & 274.2 & 158 & 116 & 135 & 139 \\
\hline PCL-14 & 250.3 & 151 & 99 & 126 & 124 \\
\hline PCL-15 & 242.1 & 142 & 100 & 117 & 125 \\
\hline PCL-16 & 162 & 121 & 130 & 153 \\
\hline PCL-17 & 168 & 123 & & \\
\hline & & & & \\
\hline & 291.0 & & & & \\
\hline
\end{tabular}


Appendix B-2: Hydrostratigraphic Boundaries (continued)

\begin{tabular}{|c|c||c|c|c|c|}
\hline & & \multicolumn{2}{|c|}{ TCCZ } & \multicolumn{2}{c|}{ LAZ } \\
\hline Well ID & $\begin{array}{c}\text { Surface } \\
\text { Elevation (ft } \\
\text { m.s.l.) }\end{array}$ & $\begin{array}{c}\text { Elev. (ft } \\
\text { m.s.l.) }\end{array}$ & $\begin{array}{c}\text { Depth (ft } \\
\text { b.g.1.) }\end{array}$ & $\begin{array}{c}\text { Elev. (ft } \\
\text { m.s.l.) }\end{array}$ & $\begin{array}{c}\text { Depth (ft } \\
\text { b.g.l.) }\end{array}$ \\
\hline
\end{tabular}

\begin{tabular}{|l|r||r|r|r|r|}
\hline PCL-1A & 317.7 & & & & \\
\hline PCL-2 & 304.4 & 186 & 118 & 162 & 142 \\
\hline PCL-3A & 300.0 & 196 & 104 & 174 & 126 \\
\hline PCL-4 & 331.7 & 157 & 175 & 148 & 184 \\
\hline PCL-5 & 284.3 & 175 & 109 & 142 & 142 \\
\hline PCL-6 & 312.6 & 186 & 127 & 166 & 147 \\
\hline PCL-7B & 295.7 & 153 & 143 & 144 & 152 \\
\hline PCL-8 & 254.7 & 158 & 97 & 130 & 125 \\
\hline PCL-9 & 288.1 & 150 & 138 & 143 & 145 \\
\hline PPC-1 & 313.3 & 188 & 125 & 168 & 145 \\
\hline RCP-1A & 294.8 & 179 & 116 & 175 & 120 \\
\hline RSF-1 & 300.8 & 153 & 148 & 144 & 157 \\
\hline RSF-2 & 300.3 & 172 & 128 & 163 & 137 \\
\hline RSF-3 & 304.8 & 152 & 153 & 148 & 157 \\
\hline SDS-21 & 251.0 & 205 & 47 & 199 & 53 \\
\hline SDS-22 & 283.0 & 191 & 93 & 188 & 96 \\
\hline SSW-1 & 311.3 & 193 & 118 & 174 & 137 \\
\hline SSW-2 & 167.3 & & & 167 & 0 \\
\hline SSW-3 & 178.7 & & & & \\
\hline SW-24CP & 257.5 & $\cdot 195$ & 63 & 180 & 78 \\
\hline T18N1A & 258.4 & 135 & 123 & 121 & 138 \\
\hline T18S1A & 233.5 & 140 & 94 & 123 & 111 \\
\hline T18W1A & 244.4 & 163 & 81 & 153 & 91 \\
\hline USGS-MP & 245.0 & 178 & 67 & 172 & 73 \\
\hline VG-1 & 156.6 & & & & \\
\hline VG-7 & 250.6 & & & & \\
\hline VG-8 & 103.7 & & & & \\
\hline YSC-1A & 268.9 & 211 & 58 & 199 & 70 \\
\hline YSC-1C & 272.5 & 215 & 58 & 213 & 60 \\
\hline YSC-2A & 281.7 & 220 & 62 & 215 & 67 \\
\hline YSC-3SB & 277.0 & 211 & 66 & 205 & 72 \\
\hline YSC-4A & 287.5 & 223 & 65 & 214 & 74 \\
\hline YSC-5A & 273.0 & 221 & 52 & 209 & 64 \\
\hline & & & & & \\
\hline
\end{tabular}


Appendix B-2: Hydrostratigraphic Boundaries (continued)

\begin{tabular}{|c|c|c|c|c|c|c|c|}
\hline & & \multicolumn{2}{|c|}{ GCU } & \multicolumn{2}{|c|}{ GAU } & \multicolumn{2}{|c|}{ MBCS } \\
\hline Well ID & $\begin{array}{l}\text { Surface } \\
\text { Elevation (ft } \\
\text { m.s.l.) }\end{array}$ & $\begin{array}{c}\text { Elev. (ft } \\
\text { m.s.l.) }\end{array}$ & $\begin{array}{c}\text { Depth (ft } \\
\text { b.g.1.) }\end{array}$ & $\begin{array}{c}\text { Elev. (ft } \\
\text { m.s.l.) }\end{array}$ & \begin{tabular}{|} 
Depth (ft \\
b.g.l.)
\end{tabular} & $\begin{array}{l}\text { Elev. (ft } \\
\text { m.s.l.) }\end{array}$ & $\begin{array}{c}\text { Depth (ft } \\
\text { b.g.l.) }\end{array}$ \\
\hline
\end{tabular}

\begin{tabular}{|l|r||l|l|l|l|l|l|}
\hline $131 \mathrm{C}-100$ & 261.6 & & & & & & \\
\hline $131 \mathrm{C}-104$ & 276.9 & & & & & & \\
\hline $131 \mathrm{C}-105$ & 169.8 & & & & & & \\
\hline $131 \mathrm{C}-49$ & 254.3 & & & & & & \\
\hline $131 \mathrm{C}-51$ & 265.0 & & & & & & \\
\hline $131 \mathrm{C}-54$ & 247.1 & & & & & & \\
\hline $131 \mathrm{C}-55$ & 245.0 & & & & & & \\
\hline $131 \mathrm{C}-59$ & 227.7 & & & & & & \\
\hline $131 \mathrm{C}-60$ & 218.2 & & & & & & \\
\hline $131 \mathrm{C}-63$ & 211.1 & & & & & & \\
\hline $131 \mathrm{C}-64$ & 211.9 & & & & & & \\
\hline $131 \mathrm{C}-67$ & 265.8 & & & & & & \\
\hline $131 \mathrm{C}-68$ & 267.7 & & & & & & \\
\hline $131 \mathrm{C}-80$ & 205.7 & & & & & & \\
\hline $131 \mathrm{C}-81$ & 217.9 & & & & & & \\
\hline $131 \mathrm{C}-82$ & 233.0 & & & & & & \\
\hline $131 \mathrm{C}-83$ & 240.5 & & & & & & \\
\hline $131 \mathrm{C}-84$ & 241.9 & & & & & & \\
\hline $131 \mathrm{C}-85$ & 237.5 & & & & & & \\
\hline $131 \mathrm{C}-91$ & 233.7 & & & & & & \\
\hline $131 \mathrm{C}-93$ & 241.9 & & & & & & \\
\hline $131 \mathrm{C}-95$ & 241.3 & & & & & & \\
\hline $131 \mathrm{C}-96$ & 243.2 & & & & & & \\
\hline $131 \mathrm{C}-98$ & 251.0 & & & & & & \\
\hline $131 \mathrm{C}-\mathrm{R} 1$ & 271.0 & & & & & & \\
\hline $131 \mathrm{C}-\mathrm{R} 2$ & 266.8 & & & & & & \\
\hline $131 \mathrm{C}-\mathrm{R} 3$ & 268.8 & & & & & & \\
\hline $131 \mathrm{C}-\mathrm{R} 4$ & 285.6 & & & & & & \\
\hline $131 \mathrm{C}-\mathrm{R} 5$ & 289.0 & & & & & & \\
\hline $131 \mathrm{C}-\mathrm{R} 6$ & 276.0 & & & & & & \\
\hline BGO-10A & 299.1 & 131 & 168 & 124 & 175 & & \\
\hline BGO-10AA & 298.8 & 130 & 169 & 126 & 173 & & \\
\hline BGO-12A & 311.4 & 137 & 174 & 132 & 179 & & \\
\hline BGO-14A & 300.2 & 133 & 167 & 127 & 173 & & \\
\hline BGO-16A & 302.8 & 131 & 172 & 126 & 177 & & \\
\hline BGO-18A & 292.9 & 131 & 162 & 126 & 167 & & \\
\hline BGO-20AA & 280.9 & 125 & 156 & 114 & 167 & & \\
\hline BGO-25A & 294.7 & 138 & 157 & 128 & 167 & & \\
\hline BGO-26A & 285.1 & 133 & 152 & 129 & 156 & & \\
\hline BGO-27C & 273.9 & & & & & & \\
\hline BGO-29A & 262.1 & 124 & 138 & 113 & 149 & & \\
\hline BGO-31C & 271.1 & & & & & & \\
\hline
\end{tabular}


Appendix B-2: Hydrostratigraphic Boundaries (continued)

\begin{tabular}{|c|c|c|c|c|c|c|c|}
\hline & & \multicolumn{2}{|c|}{ GCU } & \multicolumn{2}{|c|}{ GAU } & \multicolumn{2}{|c|}{ MBCS } \\
\hline Well ID & $\begin{array}{l}\text { Surface } \\
\text { Elevation (ft } \\
\text { m.s.l.) }\end{array}$ & \begin{tabular}{|c|} 
Elev. (ft \\
m.s.l.)
\end{tabular} & $\begin{array}{c}\text { Depth (f } \\
\text { b.g.l.) }\end{array}$ & $\begin{array}{c}\text { Elev. }(\mathrm{ft} \\
\text { m.s.l.) }\end{array}$ & $\begin{array}{c}\text { Depth (ft } \\
\text { b.g.l.) }\end{array}$ & $\begin{array}{c}\text { Elev. (ft } \\
\text { m.s.l.) }\end{array}$ & $\begin{array}{c}\text { Depth (ft } \\
\text { b.g.l.) }\end{array}$ \\
\hline
\end{tabular}

\begin{tabular}{|c|c|c|c|c|c|c|c|}
\hline BGO-33C & 277.4 & & & & & & \\
\hline BGO-35C & 271.4 & & & & & & \\
\hline BGO-37C & 284.3 & & & & & & \\
\hline BGO-39A & 293.7 & 113 & 181 & 102 & 192 & 29 & 265 \\
\hline BGO-3A & 288.7 & 131 & 158 & 122 & 167 & 41 & 248 \\
\hline BGO-41A & 298.3 & 138 & 160 & 131 & 167 & & \\
\hline BGO-42C & 295.9 & & & & & & \\
\hline BGO-43AA & 312.2 & 135 & 177 & 127 & 185 & & \\
\hline BGO-44AA & 283.3 & 131 & 152 & 120 & 163 & & \\
\hline BGO-45A & 276.9 & 134 & 143 & 130 & 147 & & \\
\hline BGO-46B & 263.4 & 128 & 135 & 126 & 137 & & \\
\hline BGO-47A & 264.8 & 131 & 134 & 125 & 140 & & \\
\hline $\mathrm{BGO}-48 \mathrm{C}$ & 274.7 & & & & & & \\
\hline BGO-49A & 269.1 & 119 & 150 & 115 & 154 & & \\
\hline BGO-50A & 253.5 & 133 & 121 & 129 & 125 & & \\
\hline BGO-51AA & 287.2 & 107 & 180 & 93 & 194 & 32 & 255 \\
\hline BGO-52AA & 281.6 & 125 & 157 & 116 & 166 & 18 & 264 \\
\hline BGO-53AA & 288.9 & 138 & 151 & 132 & 157 & 29 & 260 \\
\hline BGO-5C & 294.2 & & & & & & \\
\hline BGO-6A & 283.8 & 121 & 163 & 120 & 164 & & \\
\hline BGO-6B & 284.5 & 137 & 148 & 123 & 162 & & \\
\hline BGO-8A & 281.3 & 130 & 151 & 120 & 161 & & \\
\hline BGO-9AA & 282.8 & 135 & 148 & 125 & 158 & & \\
\hline BGT-1 & 282.9 & & & & & & \\
\hline BGT-10 & 215.2 & 156 & 59 & 147 & 68 & & \\
\hline BGT-11 & 222.5 & 151 & 72 & 146 & 77 & 68 & 155 \\
\hline BGT-12 & 284.2 & & & & & & \\
\hline BGT-13 & 287.8 & & & & & & \\
\hline BGT-14 & 280.7 & & & & & & \\
\hline BGT-15 & 277.5 & .150 & 128 & & & & \\
\hline BGT-16 & 250.7 & 151 & 100 & & & & \\
\hline BGT-17 & 240.7 & 150 & 91 & & & & \\
\hline BGT-18 & 216.5 & 162 & 55 & 147 & 70 & 55 & 162 \\
\hline BGT-2 & 276.4 & & & & & & \\
\hline BGT-20 & 159.5 & 151 & 9 & 140 & 20 & 70 & 90 \\
\hline BGT-21 & 294.2 & & & & & & \\
\hline BGT-22 & 281.0 & 126 & 155 & 114 & 167 & 53 & 228 \\
\hline BGT-23 & 270.0 & & & & & & \\
\hline BGT-24 & 265.8 & & & & & & \\
\hline BGT-25 & 264.8 & & & & & & \\
\hline BGT-27 & 256.9 & 152 & 105 & & & & \\
\hline BGT-28 & 258.3 & 156 & 102 & 150 & 108 & 48 & 210 \\
\hline
\end{tabular}


Appendix B-2: Hydrostratigraphic Boundaries (continued)

\begin{tabular}{|c|c|c|c|c|c|c|c|}
\hline & & \multicolumn{2}{|c|}{$\mathbf{G C U}$} & \multicolumn{2}{|c|}{ GAU } & \multicolumn{2}{|c|}{ MBCS } \\
\hline Well ID & $\begin{array}{c}\text { Surface } \\
\text { Elevation (ft } \\
\text { m.s.l.) }\end{array}$ & $\begin{array}{c}\text { Elev. (ft } \\
\text { m.s.l.) } \\
t .\end{array}$ & $\begin{array}{c}\text { Depth (ft } \\
\text { b.g.l.) }\end{array}$ & $\begin{array}{c}\text { Elev. (ft } \\
\text { m.s.l.) }\end{array}$ & $\begin{array}{c}\text { Depth (ft } \\
\text { b.g.l.) }\end{array}$ & \begin{tabular}{c|} 
Elev. (ft \\
m.s.l.)
\end{tabular} & $\begin{array}{c}\text { Depth (ft } \\
\text { b.g.l.) }\end{array}$ \\
\hline
\end{tabular}

\begin{tabular}{|c|c|c|c|c|c|c|c|}
\hline BGT-29 & 243.0 & & & & & & \\
\hline BGT-3 & 275.7 & 143 & 133 & 141 & 135 & 65 & 211 \\
\hline BGT-30 & 219.0 & 147 & 72 & 141 & 78 & & \\
\hline BGT-31 & 308.8 & & & & & & \\
\hline BGT-32 & 310.1 & & & & & & \\
\hline BGT-33 & 290.4 & & & & & & \\
\hline BGT-34 & 286.8 & & & & & & \\
\hline BGT-35 & 267.7 & & & & & & \\
\hline BGT-36 & 261.4 & 148 & 113 & & & & \\
\hline BGT-37 & 251.6 & 133 & 119 & & & & \\
\hline BGT-38 & 240.1 & & & & & & \\
\hline BGT-39 & 241.9 & & & & & & \\
\hline BGT-4 & 259.2 & 149 & 110 & & & & \\
\hline BGT-40 & 332.3 & & & & & & \\
\hline BGT-41 & 328.4 & 149 & 179 & 142 & 186 & & \\
\hline BGT-42 & 310.9 & & & & & & \\
\hline BGT-43 & 277.1 & & & & & & \\
\hline BGT-44 & 276.2 & & & & & & \\
\hline BGT-45 & 285.3 & 150 & 135 & & & & \\
\hline BGT-46 & 310.0 & 134 & 176 & 125 & 8185 & & \\
\hline BGT-47 & 317.3 & 137 & 180 & 128 & 189 & & \\
\hline BGT-48 & 314.3 & & & & & & \\
\hline BGT-49 & 297.3 & 135 & 162 & 126 & 171 & & \\
\hline BGT-5 & 225.7 & 154 & 72 & 146 & 80 & 72 & 154 \\
\hline BGT-50 & 296.3 & 132 & 164 & 124 & 172 & & \\
\hline BGT-51 & 272.6 & & & & & & \\
\hline BGT-53 & 278.3 & 120 & 158 & 109 & 169 & 32 & 246 \\
\hline BGT-54 & 280.0 & & & & & & \\
\hline BGT-56 & 262.9 & & & & & & \\
\hline BGT-57 & 259.4 & & & & & & \\
\hline BGT-58 & 285.8 & 112 & 174 & 103 & 183 & & \\
\hline BGT-59 & 281.9 & & & & & & \\
\hline BGT-6 & 282.2 & & & & & & \\
\hline BGT-60 & 291.4 & & & & & & \\
\hline BGT-61 & 284.3 & 108 & 176 & 101 & 183 & & \\
\hline BGT-62 & 282.0 & & & & & & \\
\hline BGT-63 & 293.7 & & & & & & \\
\hline BGT-63A & 290.8 & & & & & & \\
\hline BGT-64 & 283.3 & 123 & 160 & & & & \\
\hline BGT-66 & 244.0 & & & & & & \\
\hline BGT-67 & 242.0 & 135 & 107| & 128 & 115 & 27 & 215 \\
\hline BGT-7 & 276.4 & & & & & & \\
\hline
\end{tabular}


Appendix B-2: Hydrostratigraphic Boundaries (continued)

\begin{tabular}{|c|c|c|c|c|c|c|c|}
\hline & & \multicolumn{2}{|c|}{ GCU } & \multicolumn{2}{|c|}{ GAU } & \multicolumn{2}{|c|}{ MBCS } \\
\hline Well ID & $\begin{array}{c}\text { Surface } \\
\text { Elevation (ft }\end{array}$ & \begin{tabular}{|c|} 
Elev. (ft \\
m.s.l.)
\end{tabular} & $\begin{array}{l}\text { Depth (ft } \\
\text { b.g.l.) }\end{array}$ & $\begin{array}{c}\text { Elev. (ft } \\
\text { m.s.l.) }\end{array}$ & \begin{tabular}{|c} 
Depth (ft \\
b.g.l.)
\end{tabular} & $\begin{array}{c}\text { Elev. (ft } \\
\text { m.s.l.) }\end{array}$ & \begin{tabular}{|c} 
Depth (f. \\
b.g.l.)
\end{tabular} \\
\hline
\end{tabular}

\begin{tabular}{|l|r||r|r|r|r|r|r|}
\hline BGT-8 & 249.3 & 149 & 100 & & & & \\
\hline BGT-9 & 226.0 & 149 & 77 & 141 & 85 & 64 & 162 \\
\hline BGX-11D & 273.8 & 126 & 148 & 117 & 157 & & \\
\hline BGX-1A & 289.1 & 132 & 157 & 127 & 162 & & \\
\hline BGX-2B & 289.2 & 140 & 149 & 127 & 162 & & \\
\hline BGX-4A & 288.8 & 130 & 159 & 124 & 165 & & \\
\hline BGX-7D & 277.1 & 157 & 120 & 144 & 133 & & \\
\hline BGX-9D & 277.4 & 139 & 138 & 131 & 146 & & \\
\hline BRR-1D & 293.8 & & & & & & \\
\hline BRR-3D & 289.5 & & & & & & \\
\hline BRR-6B & 293.9 & 123 & 171 & 108 & 186 & & \\
\hline BRR-7B & 289.6 & 135 & 155 & 122 & 168 & & \\
\hline BRR-8B & 276.7 & 131 & 146 & 126 & 151 & & \\
\hline CCP-1A & 287.1 & & & & & & \\
\hline CFD-1 & 268.8 & 112 & 157 & & & & \\
\hline CFD-18 & 248.3 & 94 & 154 & & & & \\
\hline CFD-5 & 257.8 & 93 & 165 & 89 & 169 & 28 & 230 \\
\hline CMP-1-CP & 248.9 & & & & & & \\
\hline CMP-30B & 286.5 & 95 & 192 & & & & \\
\hline CMP-32B & 251.7 & 95 & 157 & & & & \\
\hline CMP-4B-CP & 241.8 & & & & & & \\
\hline CMP-6A-CP & 238.4 & & & & & & \\
\hline CPC-1 & 285.1 & 96 & 189 & 87 & 198 & & -8 \\
\hline CRP-5D & 274.5 & & & & & & \\
\hline CRP-9D & 268.4 & 94 & 175 & 92 & 177 & & \\
\hline CRSB-1 & 278.2 & & & & & & \\
\hline CRSB-101 & 275.6 & & & & & & \\
\hline CRSB-11 & 276.7 & & & & & & \\
\hline CRSB-14 & 275.6 & & & & & & \\
\hline CRSB-16 & 282.6 & & & & & & \\
\hline CRSB-17 & 281.0 & & & & & & \\
\hline CRSB-18 & 273.8 & & & & & & \\
\hline CRSB-20 & 274.2 & & & & & & \\
\hline CRSB-21 & 272.9 & & & & & & \\
\hline CRSB-24 & 277.2 & & & & & & \\
\hline CRSB-26 & 291.6 & & & & & & \\
\hline CRSB-28 & 289.3 & & & & & & \\
\hline CRSB-30 & 285.4 & & & & & & \\
\hline CRSB-31 & 284.4 & & & & & & \\
\hline CRSB-32 & 274.1 & & & & & & \\
\hline CRSB-35 & 257.4 & & & & & & \\
\hline CRSB-36 & 265.6 & & & & & & \\
\hline
\end{tabular}


Appendix B-2: Hydrostratigraphic Boundaries (continued)

\begin{tabular}{|c|c|c|c|c|c|c|c|}
\hline & & \multicolumn{2}{|c|}{$\overline{\mathbf{G C U}}$} & \multicolumn{2}{|c|}{ GAU } & \multicolumn{2}{|c|}{ MBCS } \\
\hline Well ID & $\begin{array}{c}\text { Surface } \\
\text { Elevation (ft }\end{array}$ & $\begin{array}{l}\text { Elev. (ft } \\
\text { m.s.l.) }\end{array}$ & $\begin{array}{c}\text { Depth (ft } \\
\text { b.g.l.) }\end{array}$ & $\begin{array}{l}\text { Elev. (ft } \\
\text { m.s.l.) }\end{array}$ & $\begin{array}{c}\text { Depth (ft } \\
\text { b.g.l.) }\end{array}$ & $\begin{array}{c}\text { Elev. (ft } \\
\text { m.s.l.) }\end{array}$ & $\begin{array}{l}\text { Depth (ft } \\
\text { b.g.l.) }\end{array}$ \\
\hline
\end{tabular}

\begin{tabular}{|c|c|c|c|c|c|c|c|}
\hline CRSB-37 & 270.5 & & & & & & \\
\hline CRSB-4 & 282.8 & & & & & & \\
\hline CRSB-40 & 227.3 & & & & & & \\
\hline CRSB-43 & 258.5 & & & & & & \\
\hline CRSB-45 & 270.6 & & & & & & \\
\hline CRSB-47 & 282.7 & & & & & & \\
\hline CRSB-48 & 259.8 & & & & & & \\
\hline CRSB-5 & 288.3 & & & & & & \\
\hline CRSB-50 & 255.6 & & & & & & \\
\hline CRSB-52 & 233.1 & & & & & & \\
\hline CRSB-54 & 224.7 & & & & & & \\
\hline CRSB-55 & 229.1 & 103 & 126 & & & & \\
\hline CRSB-56 & 232.5 & & & & & & \\
\hline CRSB-57 & 248.2 & & & & & & \\
\hline CRSB-58 & 253.9 & & & & & & \\
\hline CRSB-6 6 & 289.4 & & & & & & \\
\hline CRSB-60 & 257.0 & & & & & & \\
\hline CRSB-61 & 257.3 & & & & & & \\
\hline CRSB-63 & 240.4 & & & & & & \\
\hline CRSB-64 & 229.9 & & & & & & \\
\hline CRSB-65 & 214.8 & & & & & & \\
\hline CRSB-86 & 206.6 & & & & & & \\
\hline CRSB-88 & 253.2 & & & & & & \\
\hline CRSB-89 & 260.6 & & & & & & \\
\hline CRSB-90 & 268.4 & & & & & & \\
\hline CRSB-91 & 273.1 & & & & & & \\
\hline CRSB-92 & 287.5 & & & & & & \\
\hline CRSB-93 & 286.8 & & & & & & \\
\hline CRSB-94 & 283.3 & & & & & & \\
\hline CRSB-95 & 280.4 & & & & & & \\
\hline CRSB-96 & 282.8 & & & & & & \\
\hline CRSB-97 & 276.8 & . & & & & & \\
\hline CRSB-98 & 275.1 & & & & & & \\
\hline CRSB-99 & 274.4 & & & & & & \\
\hline CSB-101 & 281.2 & & & & & & \\
\hline CSB-102 & 283.5 & & & & & & \\
\hline CSB-103 & 257.6 & & & & & & \\
\hline CSB-104 & 224.9 & 94 & 131 & & & & \\
\hline CSB-105 & 212.1 & 98 & 114 & 81 & 131 & & \\
\hline CSB-106 & 183.8 & 93 & 91 & 69 & 115 & & \\
\hline CSB-107 & 220.7 & 102 & 119 & 83 & 138 & & \\
\hline CSB-108 & 196.7 & 90 & 107 & 77 & 120 & & \\
\hline
\end{tabular}


B-40 WSRC-TR-99-00248, Rev. 0, Regional Groundwater Flow Model for C, K, L, and P Reactor Areas

Appendix B-2: Hydrostratigraphic Boundaries (continued)

\begin{tabular}{|c|c|c|c|c|c|c|c|}
\hline & & \multicolumn{2}{|c|}{ GCU } & \multicolumn{2}{|c|}{ GAU } & \multicolumn{2}{|c|}{ MBCS } \\
\hline Well ID & $\begin{array}{c}\text { Surface } \\
\text { Elevation ( } \mathrm{ft}\end{array}$ & \begin{tabular}{|c|} 
Elev. (ft \\
m.s.l.)
\end{tabular} & $\begin{array}{l}\text { Depth (ft } \\
\text { b.g.l.) }\end{array}$ & \begin{tabular}{|l} 
Elev. (ft \\
m.s.l.)
\end{tabular} & $\begin{array}{c}\text { Depth (ft } \\
\text { b.g.l.) }\end{array}$ & $\begin{array}{l}\text { Elev. }(\mathrm{ft} \\
\text { m.s.l.) }\end{array}$ & $\begin{array}{c}\text { Depth (ft } \\
\text { b.g.l.) }\end{array}$ \\
\hline
\end{tabular}

\begin{tabular}{|c|c|c|c|c|c|c|c|}
\hline CSB-109 & 172.9 & 102 & 71 & & & & \\
\hline CSB-110 & 184.6 & & & & & & \\
\hline CSB-111 & 172.1 & 101 & 71 & 87 & 85 & & \\
\hline CSB-112 & 195.3 & 103 & 92 & 91 & 104 & & \\
\hline CSB-113 & 210.4 & 96 & 114 & 81 & 129 & & \\
\hline CSB-114 & 196.0 & & & & & & \\
\hline CSB-116 & 274.7 & & & & & & \\
\hline CSB-117 & 276.5 & & & & & & \\
\hline CSB-118 & 277.8 & & & & & & \\
\hline CSB-119 & 268.3 & & & & & & \\
\hline CSB-120 & 282.8 & & & & & & \\
\hline$\overline{C S B}-121$ & 274.2 & & & & & & \\
\hline CSB-122 & 222.9 & 100 & 123 & 88 & 135 & & \\
\hline $\begin{array}{l}\text { CSB-123 } \\
\end{array}$ & 224.2 & & & & & & \\
\hline CSB-2C & 289.9 & & & & & & \\
\hline CSB-3C & 280.9 & & & & & & \\
\hline CSB-47 & 282.7 & & & & & & \\
\hline CSB-47A & 268.9 & & & & & & \\
\hline CSB-48 & 259.8 & & & & & & \\
\hline CSB-48A & 270.1 & & & & & & \\
\hline CSB-56 & 267.0 & & & & & & \\
\hline CSB-58 & 235.5 & & & & & & \\
\hline CSB-59 & 231.2 & & & & & & \\
\hline CSB-60 & 247.0 & 109 & 138 & & & & \\
\hline CSB-61 & 252.2 & 108 & 144 & & & & \\
\hline CSB-62 & 240.9 & & & & & & \\
\hline CSB-63 & 227.1 & 103 & 124 & & & & \\
\hline CSB-64 & 212.7 & 100 & 113 & 96 & 117 & & \\
\hline CSB-65 & 195.6 & 90 & 106 & 78 & 118 & & \\
\hline CSB-66A & 191.7 & 92 & 100 & & & & \\
\hline CSD-4D & 306.5 & & & & & & \\
\hline E-26 & 314.3 & 75 & 239 & & & & \\
\hline E-4 & 315.4 & 65 & 250 & & & & \\
\hline FAC-1SB & 312.2 & $\overline{149}$ & 163 & & & & \\
\hline $\mathrm{FCH}-1$ & 316.8 & 142 & 175 & 126 & 191 & 67 & 250 \\
\hline FCH-2 & 288.7 & 142 & 147 & 130 & 159 & 58 & 231 \\
\hline FCH-3 & 307.2 & 140 & 167 & 131 & 176 & 59 & 248 \\
\hline FCH-4 & 297.5 & 128 & 170 & 121 & 177 & 42 & 256 \\
\hline FCH-5 & 284.2 & 129 & 155 & 128 & 156 & 36 & 248 \\
\hline FCH-6 & 291.5 & 124 & 168 & 121 & 171 & 25 & 267 \\
\hline FIW-1MC & 293.3 & 121 & 172 & & & & \\
\hline FIW-2MA & 290.5 & 121 & 170 & 117 & 174 & & \\
\hline
\end{tabular}


Appendix B-2: Hydrostratigraphic Boundaries (continued)

\begin{tabular}{|c|c|c|c|c|c|c|c|}
\hline & & \multicolumn{2}{|c|}{ GCU } & \multicolumn{2}{|c|}{ GAU } & \multicolumn{2}{|c|}{ MBCS } \\
\hline Well ID & $\begin{array}{c}\text { Surface } \\
\text { Elevation (ft }\end{array}$ & $\begin{array}{c}\text { Elev. (ft } \\
\text { m.s.l.) }\end{array}$ & $\begin{array}{c}\text { Depth (ft } \\
\text { b.g.l.) }\end{array}$ & $\begin{array}{c}\text { Elev. (ft } \\
\text { m.s.l.) }\end{array}$ & $\begin{array}{c}\text { Depth (ff } \\
\text { b.g.l.) }\end{array}$ & $\begin{array}{c}\text { Elev. (ft } \\
\text { m.s.l.) }\end{array}$ & $\begin{array}{c}\text { Depth (ft } \\
\text { b.g.l.) }\end{array}$ \\
\hline
\end{tabular}

\begin{tabular}{|c|c|c|c|c|c|c|c|}
\hline FNB-1A & 282.4 & 151 & 131 & 138 & 145 & & \\
\hline FNB-3A & 282.2 & 146 & 136 & 140 & 142 & & \\
\hline FSB-100A & 283.8 & 118 & 166 & 114 & 170 & & \\
\hline$\overline{\text { FSB-101A }}$ & 282.9 & 119 & 164 & 116 & 167 & & \\
\hline FSB-112A & 227.0 & 103 & 124 & 98 & 129 & & \\
\hline FSB-113A & 221.3 & 109 & 112 & 104 & 117 & 22 & 199 \\
\hline FSB-114A & 250.0 & 114 & 136 & 110 & 140 & & \\
\hline FSB-115C & 205.8 & 101 & 105 & 86 & 120 & 6 & 200 \\
\hline FSB-116C & 200.5 & & & & & & \\
\hline FSB-120A & 278.0 & 112 & 166 & 110 & 168 & & \\
\hline FSB-121C & 254.4 & & & & & & \\
\hline FSB-122C & 216.0 & 104 & 112 & & & & \\
\hline FSB-123C & 236.3 & & & & & & \\
\hline FSB-1TA & 275.4 & 117 & 158 & 115 & 160 & 24 & 251 \\
\hline FSB-76A & 291.5 & 121 & 171 & 117 & 175 & & \\
\hline FSB-78A & 270.5 & 105 & 166 & 100 & 171 & & \\
\hline FSB-79A & 216.1 & 103 & 113 & 100 & 116 & 18 & 198 \\
\hline FSB-87A & 285.6 & 115 & 171 & 109 & 177 & & \\
\hline FSB-89C & 279.1 & & & & & & \\
\hline FSB-91C & 277.0 & & & & & & \\
\hline FSB-93C & 274.0 & & & & & & \\
\hline FSB-95C & 281.8 & & & & & & \\
\hline FSB-96A & 277.7 & 109 & 169 & 101 & 177 & & \\
\hline FSB-97A & 283.8 & 111 & 173 & 107 & 177 & & \\
\hline FSB-98A & 280.7 & 109 & 172 & 107 & 174 & & \\
\hline FSB-99A & 285.3 & 115 & 170 & 112 & 173 & & \\
\hline FSB-PC & 230.8 & & . & & & & \\
\hline $\begin{array}{l}\text { GAPWR-TW- } \\
1\end{array}$ & 219.0 & 87 & 132 & 55 & 164 & -31 & 250 \\
\hline HAA-1TA & 290.2 & 117 & 173 & 110 & 181 & & \\
\hline HAA-2AA & 291.4 & 125 & 167 & 119 & 173 & 30 & 262 \\
\hline HAA-3AA & 274.5 & 128 & 147 & 123 & 152 & 10 & 265 \\
\hline HAA-4AA & 299.2 & 125 & 175 & 119 & 181 & & \\
\hline HAA-6AA & 279.8 & 125 & 155 & 120 & 160 & 23 & 257 \\
\hline HC-03AA & 263.8 & & & & & & \\
\hline $\mathrm{HC}-12 \mathrm{~A}$ & 287.3 & & & & & & \\
\hline HCA-4AA & 308.6 & 124 & 185 & 117 & 192 & & \\
\hline $\mathrm{HCH}-1$ & 284.0 & 135 & 149 & 126 & 158 & 18 & 266 \\
\hline $\mathrm{HCH}-2$ & 270.9 & 131 & 140 & 123 & 148 & 0 & 271 \\
\hline $\mathrm{HCH}-3$ & 264.0 & 130 & 134 & 123 & 141 & & \\
\hline $\mathrm{HCH}-4$ & 269.9 & 123 & 147 & 114 & 156 & & \\
\hline $\mathrm{HCH}-5$ & 255.0 & 123 & 132 & 119 & 136 & -10 & 265 \\
\hline HIW-1BD & 275.8 & & & & & & \\
\hline
\end{tabular}


B-42 WSRC-TR-99-00248, Rev. 0, Regional Groundwater Flow Model for C, K, L, and P Reactor Areas

Appendix B-2: Hydrostratigraphic Boundaries (continued)

\begin{tabular}{|c|c|c|c|c|c|c|c|}
\hline & & \multicolumn{2}{|c|}{ GCU } & \multicolumn{2}{|c|}{ GAU } & \multicolumn{2}{|c|}{ MBCS } \\
\hline Well $\mathbf{~ D}$ & $\begin{array}{c}\text { Surface } \\
\text { Elevation (ft } \\
\text { m.s.l.) }\end{array}$ & $\begin{array}{l}\text { Elev. (ft } \\
\text { m.s.l.) }\end{array}$ & \begin{tabular}{|c} 
Depth (ft \\
b.g.l.)
\end{tabular} & $\begin{array}{c}\text { Elev. }(\mathrm{ft} \\
\text { m.s.l.) }\end{array}$ & $\begin{array}{c}\text { Depth (ft } \\
\text { b.g.l.) }\end{array}$ & $\begin{array}{c}\text { Elev. (ft } \\
\text { m.s.l.) }\end{array}$ & $\begin{array}{c}\text { Depth (ft } \\
\text { b.g.l.) }\end{array}$ \\
\hline
\end{tabular}

\begin{tabular}{|c|c|c|c|c|c|c|c|}
\hline HIW-1MC & 272.3 & & & & & & \\
\hline $\mathrm{HIW}-2 \mathrm{~A}$ & 276.3 & 116 & 160 & 110 & 166 & & \\
\hline HIW-2MC & 269.0 & & & & & & \\
\hline HIW-4MC & 263.4 & 112 & 151 & & & & \\
\hline HIW-5MC & 266.1 & & & & & & \\
\hline HMD-1C & 262.7 & 139 & 124 & 127 & 136 & & \\
\hline HMD-2C & 259.3 & 143 & 116 & 138 & 121 & & \\
\hline HMD-3C & 257.2 & 154 & 103 & 149 & 108 & & \\
\hline HMD-4C & 248.5 & 153 & 96 & 141 & 108 & & \\
\hline HPC-1 & 293.5 & 116 & 178 & 110 & 184 & 28 & 266 \\
\hline HPT-1A & 232.9 & 119 & 114 & 115 & 118 & 53 & 180 \\
\hline HPT-2A & 257.8 & 121 & 137 & 118 & 140 & 57 & 201 \\
\hline HSB-101C & 256.3 & & & & & & \\
\hline HSB-103C & 245.2 & & & & & & \\
\hline HSB-104C & 245.5 & & & & & & \\
\hline HSB-105C & 247.2 & & & & & & \\
\hline HSB-106C & 250.7 & & & & & & \\
\hline HSB-107C & 259.3 & & & & & & \\
\hline HSB-109C & 259.4 & & & & & & \\
\hline HSB-110C & 253.4 & & & & & & \\
\hline HSB-111C & 253.7 & & & & & & \\
\hline HSB-112C & 252.6 & & & & & & \\
\hline HSB-113C & 258.7 & & & & & & \\
\hline HSB-115C & 266.8 & & & & & & \\
\hline HSB-117A & 234.8 & 123 & 112 & 117 & 118 & & \\
\hline HSB-118A & 245.0 & 119 & 126 & 114 & 131 & & \\
\hline HSB-119A & 254.8 & 115 & 140 & 111 & 144 & & \\
\hline HSB-120A & 266.0 & 112 & 154 & 110 & 156 & & \\
\hline HSB-121A & 272.3 & 113 & 159 & 109 & 163 & & \\
\hline HSB-122A & 269.4 & 110 & 159 & 108 & 161 & & \\
\hline HSB-123A & 263.6 & 114 & 150 & 108 & 156 & & \\
\hline HSB-124A & 263.9 & 118 & 146 & & & & \\
\hline HSB-132C & 238.3 & & & & & & \\
\hline HSB-139A & 231.5 & 119 & 113 & 115 & 117 & & \\
\hline HSB-140A & 234.0 & 111 & 123 & 105 & 129 & & \\
\hline HSB-141A & 252.6 & 119 & 134 & 113 & 140 & & \\
\hline HSB-142C & 201.6 & & & & & & \\
\hline HSB-143C & 220.1 & & & & & & \\
\hline HSB-144A & 233.6 & 109 & 125 & 104 & 130 & & \\
\hline HSB-145C & 233.7 & & & & & & \\
\hline HSB-146A & 249.5 & 119 & 131 & 112 & 138 & & \\
\hline HSB-148C & 248.9 & & & & & & \\
\hline
\end{tabular}


Appendix B-2: Hydrostratigraphic Boundaries (continued)

\begin{tabular}{|c|c|c|c|c|c|c|c|}
\hline- & 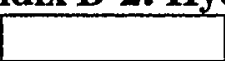 & \multicolumn{2}{|c|}{$\mathbf{G C U}$} & \multicolumn{2}{|c|}{ GAU } & \multicolumn{2}{|c|}{ MBCS } \\
\hline Well $\mathbf{m}$ & $\begin{array}{c}\text { Surface } \\
\text { Elevation (ft } \\
\text { m.s.l.) }\end{array}$ & $\begin{array}{l}\text { Elev. (ft } \\
\text { m.s.l.) }\end{array}$ & $\begin{array}{c}\text { Depth (ft } \\
\text { b.g.l.) }\end{array}$ & $\begin{array}{c}\text { Elev. (ft } \\
\text { m.s.l.) }\end{array}$ & $\begin{array}{c}\text { Depth (fi } \\
\text { b.g.1.) }\end{array}$ & \begin{tabular}{|c|} 
Elev. (ft \\
m.s.l.)
\end{tabular} & $\begin{array}{c}\text { Depth (ft } \\
\text { b.g.l. }\end{array}$ \\
\hline
\end{tabular}

\begin{tabular}{|l|r|r|r|r|r|r|r|}
\hline HSB-151C & 211.6 & & & & & & \\
\hline HSB-152C & 212.1 & & & & & & \\
\hline HSB-65A & 270.7 & 119 & 152 & 113 & 158 & & \\
\hline HSB-68A & 247.4 & 116 & 131 & 110 & 137 & & \\
\hline HSB-69A & 234.1 & 115 & 119 & 112 & 122 & & \\
\hline HSB-83A & 234.9 & 114 & 121 & 104 & 131 & 12 & 223 \\
\hline HSB-84A & 226.7 & 119 & 108 & 111 & 116 & & \\
\hline HSB-85A & 292.1 & 126 & 166 & 119 & 173 & & \\
\hline HSB-86A & 260.0 & 112 & 148 & 109 & 151 & & \\
\hline HSB-PC & 227.8 & & & & & & \\
\hline HSB-TB & 267.1 & 110 & 157 & 106 & 161 & 9 & 258 \\
\hline HSL-6AA & 274.6 & 126 & 149 & 121 & 154 & 9 & 266 \\
\hline HSL-8AA & 286.7 & 137 & 150 & 129 & 158 & & \\
\hline IDB-2A & 302.4 & 142 & 160 & 138 & 164 & -13 & 316 \\
\hline IDP-3A & 282.2 & 161 & 121 & & & & \\
\hline IDQ-3A & 203.2 & 132 & 71 & 124 & 79 & 55 & 148 \\
\hline KAC-9D & 260.2 & 95 & 165 & 89 & 171 & & \\
\hline KPT-1 & 205.2 & & & & & & \\
\hline KPTT-2 & 258.2 & & & & & & \\
\hline KPT-3 & 225.5 & & & & & & \\
\hline KPT-4 & 258.9 & & & & & & \\
\hline KPT-5 & 225.1 & & & & & & \\
\hline KPT-6 & 232.8 & & & & & & \\
\hline KPT-7 & 211.8 & & & & & & \\
\hline KPT-8 & 238.6 & & & & & & \\
\hline KPT-9 & 204.9 & & & & & & \\
\hline L3-CPT-PG-9 & 270.0 & & & & & & \\
\hline LAC-5DL & 239.8 & & & & & & \\
\hline LAC-6DL & 239.8 & & & & & & \\
\hline LAC-7DL & 239.4 & & & & & & \\
\hline LAC-8DL & 234.0 & & & & & & \\
\hline LCO-5A & 230.0 & 78 & 152 & 66 & 164 & & \\
\hline LCO-5DL & 230.3 & & & & & & \\
\hline LCO-8DL & 243.4 & & & & & & \\
\hline LFW-10SB & 168.4 & 170 & -2 & 168 & 0 & 44 & 124 \\
\hline LWN-1SB & 282.5 & 132 & 151 & 119 & 164 & & \\
\hline LWN-2SB & 231.0 & 90 & 141 & 82 & 149 & 11 & 220 \\
\hline LWN-3SB & 245.7 & 96 & 150 & 89 & 157 & 12 & 234 \\
\hline LWR-2SB & 248.1 & 146 & 102 & 131 & 117 & 17 & 231 \\
\hline LWR-3SB & 249.1 & 95 & 154 & 86 & 163 & 12 & 238 \\
\hline LWR-4SB & 293.8 & 104 & 190 & 99 & 198 & 7 & 287 \\
\hline LWR-8CC & 280.3 & & & & & & \\
\hline
\end{tabular}


B-44 WSRC-TR-99-00248, Rev. 0, Regional Groundwater Flow Model for C, K, L, and P Reactor Areas

Appendix B-2: Hydrostratigraphic Boundaries (continued)

\begin{tabular}{|c|c|c|c|c|c|c|c|}
\hline & & \multicolumn{2}{|c|}{ GCU } & \multicolumn{2}{|c|}{ GAU } & \multicolumn{2}{|c|}{ MBCS } \\
\hline Well ID & $\begin{array}{l}\text { Surface } \\
\text { Elevation (ft } \\
\text { m.s.l.) }\end{array}$ & $\begin{array}{c}\text { Elev. }(\mathrm{ft} \\
\text { m.s.l.) }\end{array}$ & \begin{tabular}{|c} 
Depth (ft \\
b.g.1.)
\end{tabular} & $\begin{array}{l}\text { Elev. (ft } \\
\text { m.s.l.) }\end{array}$ & $\begin{array}{c}\text { Depth (ft } \\
\text { b.g.l.) }\end{array}$ & $\begin{array}{c}\text { Elev. (ft } \\
\text { m.s.l. }\end{array}$ & $\begin{array}{c}\text { Depth (ft } \\
\text { b.g.l.) }\end{array}$ \\
\hline
\end{tabular}

\begin{tabular}{|l|r||r|r|r|r|r|r|}
\hline LWR-9SB & 238.2 & 110 & 128 & 108 & 130 & & \\
\hline M12-17 & 325.5 & & & & & & \\
\hline M121A & 303.7 & 89 & 215 & 74 & 230 & -5 & 309 \\
\hline M121A & 303.7 & 89 & 215 & 74 & 230 & -5 & 309 \\
\hline M12A-29 & 321.0 & & & & & & \\
\hline MWD-1A & 327.5 & 133 & 195 & 130 & 198 & 22 & 306 \\
\hline MWD-C3 & 322.5 & & & & & & \\
\hline MWD-C5 & 328.7 & & & & & & \\
\hline NPN-001 & 335.9 & & & & & & \\
\hline NPN-1A & 335.9 & 110 & 226 & 105 & 231 & 2 & 334 \\
\hline OFS-1SB & 261.6 & 129 & 133 & 126 & 136 & & \\
\hline OFS-2SB & 257.5 & 125 & 133 & 121 & 137 & & \\
\hline OFS-3SB & 258.1 & 125 & 133 & 120 & 138 & & \\
\hline OFS-4SB & 258.7 & 127 & 132 & 122 & 137 & & \\
\hline OFS-5SB & 228.7 & 122 & 107 & 117 & 112 & & \\
\hline P-13TA & 252.4 & 32 & 220 & 10 & 243 & -103 & 355 \\
\hline P-14TA & 294.4 & 139 & 155 & 131 & 163 & 26 & 269 \\
\hline P-18TA & 296.9 & 91 & 206 & 86 & 211 & -17 & 314 \\
\hline P-19TA & 297.4 & 120 & 178 & 114 & 184 & -56 & 353 \\
\hline P-20TA & 287.7 & 84 & 204 & 75 & 213 & -10 & 298 \\
\hline P-21TA & 207.0 & 48 & 160 & -24 & 231 & -115 & 322 \\
\hline P-22TA & 215.4 & 37 & 178 & -19 & 234 & -91 & 306 \\
\hline P-23TA & 181.5 & 89 & 93 & 58 & 124 & -4 & 186 \\
\hline P-24TA & 313.3 & 115 & 198 & 99 & 214 & -40 & 354 \\
\hline P-25TA & 265.1 & 100 & 165 & 95 & 171 & 13 & 253 \\
\hline P-26TA & 152.2 & 71 & 81 & 65 & 87 & 14 & 138 \\
\hline P-27TA & 274.1 & 129 & 145 & 127 & 147 & 49 & 225 \\
\hline P-28TA & 285.6 & 141 & 145 & 133 & 153 & 64 & 222 \\
\hline P4-CPT-4 & 270.0 & & & & & & \\
\hline PBF-3 & 316.7 & 79 & 238 & 50 & 267 & -23 & 340 \\
\hline PBF-4 & 208.1 & 66 & 142 & 41 & 167 & -32 & 240 \\
\hline PBF-5 & 240.6 & 103 & 138 & 78 & 163 & 6 & 235 \\
\hline PBF-6 & 92.5 & 95 & -2 & 93 & 0 & -2 & 95 \\
\hline PBF-7 & 285.4 & 118 & 168 & 114 & 172 & -55 & 340 \\
\hline PBF-8 & 292.0 & 114 & 178 & 110 & 182 & -44 & 336 \\
\hline PCL-12 & 265.8 & & & & & & \\
\hline PCL-13 & 274.2 & & & & & & \\
\hline PCL-14 & 250.3 & & & & & & \\
\hline PCL-15 & 242.1 & & & & & & \\
\hline PCL-16 & 282.8 & & & & & & \\
\hline PCL-17 & & & & & & \\
\hline PCL-1A & & & & & & \\
\hline & & & & & & \\
\hline
\end{tabular}


Appendix B-2: Hydrostratigraphic Boundaries (continued)

\begin{tabular}{|c|c|c|c|c|c|c|c|}
\hline & & \multicolumn{2}{|c|}{ GCU } & \multicolumn{2}{|c|}{ GAU } & \multicolumn{2}{|c|}{ MBCS } \\
\hline Well ID & $\begin{array}{c}\text { Surface } \\
\text { Elevation (ft } \\
\text { m.s.l.) }\end{array}$ & $\begin{array}{l}\text { Elev. (ft } \\
\text { m.s.l.) }\end{array}$ & $\begin{array}{c}\text { Depth (ft } \\
\text { b.g.l.) }\end{array}$ & $\begin{array}{c}\text { Elev. (ft } \\
\text { m.s.l.) }\end{array}$ & $\begin{array}{c}\text { Depth (ft } \\
\text { b.g.l.) }\end{array}$ & $\begin{array}{c}\text { Elev. (ft } \\
\text { m.s.l.) }\end{array}$ & \begin{tabular}{|c|} 
Depth (ft \\
b.g.l.)
\end{tabular} \\
\hline
\end{tabular}

\begin{tabular}{|l|r||r|r|r|r|r|r|}
\hline PCL-2 & 304.4 & $!$ & & & & & \\
\hline PCL-3A & 300.0 & & & & & & \\
\hline PCL-4 & 331.7 & $\cdot$ & & & & & \\
\hline PCL-5 & 284.3 & & & & & & \\
\hline PCL-6 & 312.6 & & & & & & \\
\hline PCL-7B & 295.7 & & & & & & \\
\hline PCL-8 & 254.7 & & & & & & \\
\hline PCL-9 & 288.1 & & & & & & \\
\hline PPC-1 & 313.3 & 120 & 193 & 106 & 208 & -56 & 370 \\
\hline RCP-1A & 294.8 & 83 & 212 & 78 & 217 & & \\
\hline RSF-1 & 300.8 & & & & & & \\
\hline RSF-2 & 300.3 & & & & & & \\
\hline RSF-3 & 304.8 & & & & & & \\
\hline SDS-21 & 251.0 & 163 & 89 & 148 & 104 & & \\
\hline SDS-22 & 283.0 & 149 & 134 & 138 & 145 & & \\
\hline SSW-1 & 311.3 & 126 & 185 & 123 & 188 & 12 & 299 \\
\hline SSW-2 & 167.3 & 109 & 59 & 96 & 72 & -10 & 177 \\
\hline SSW-3 & 178.7 & 89 & 90 & 84 & 95 & -7 & 186 \\
\hline SW-24CP & 257.5 & 120 & 138 & & & & \\
\hline T18N1A & 258.4 & 82 & 177 & 74 & 185 & -12 & 270 \\
\hline T18S1A & 233.5 & 96 & 138 & 71 & 163 & -4 & 238 \\
\hline T18W1A & 244.4 & 94 & 150 & 84 & 160 & -26 & 270 \\
\hline USGS-MP & 245.0 & 133 & 112 & 113 & 132 & 80 & 165 \\
\hline VG-1 & 156.6 & -1 & 158 & -80 & 237 & -198 & 355 \\
\hline VG-7 & 250.6 & 39 & 212 & -32 & 283 & -123 & 374 \\
\hline VG-8 & 103.7 & -38 & 142 & -117 & 221 & -239 & 343 \\
\hline YSC-1A & 268.9 & 159 & 110 & 154 & 115 & 69 & 200 \\
\hline YSC-1C & 272.5 & 164 & 109 & 157 & 116 & & \\
\hline YSC-2A & 281.7 & 162 & 120 & 151 & 131 & & \\
\hline YSC-3SB & 277.0 & 149 & 128 & 140 & 137 & & \\
\hline YSC-4A & 287.5 & 160 & 128 & 145 & 143 & 87 & 201 \\
\hline YSC-5A & 273.0 & 136 & 137 & 128 & 145 & & \\
\hline & & & & & & & \\
\hline & & & & & & & \\
\hline
\end{tabular}

Notes:

TCCZ - Tan Clay Confining Zone

LAZ - Lower Aquifer Zone

GCU - Gordon Confining Unit

GAU - Gordon Aquifer Unit

MBCS - Meyers Branch Confining System

ft m.s.l. - feet above mean sea level

ft b.g.l. - feet below ground level

Blank field indicates unit not penetrated

ND - Unit boundary not delineated

abs. - Unit absent 


\section{This page intentionally left blank}




\section{APPENDIX C. PERMEABILITY DATA}


This page intentionally left blank 
WSRC-TR-99-00248, Rev. 0, Regional Groundwater Flow Model for C, K, L, and P Reactor Areas

C-1

\section{Appendix C-1. Permeability Values Recorded from Pumping Tests}

\begin{tabular}{|c|c|c|c|c|c|c|}
\hline $\begin{array}{c}\text { Pumped } \\
\text { Well }\end{array}$ & $\begin{array}{c}\text { Observation } \\
\text { Well }\end{array}$ & $\begin{array}{c}\text { Test } \\
\text { Interval } \\
\text { Top } \\
\text { (ft b.g.l.) }\end{array}$ & $\begin{array}{c}\text { Test } \\
\text { Interval } \\
\text { Bottom } \\
\text { (ft b.g.l.) }\end{array}$ & $\begin{array}{c}\text { Permeability } \\
\text { (ft/day) }\end{array}$ & Analysis Method & Reference \\
\hline
\end{tabular}

\begin{tabular}{|c|c|c|c|c|c|c|}
\hline FSB-PC & FSB-25PC & 75.1 & - 125.0 & 0.80 & $\begin{array}{l}\text { Aqetsolv (Hantush } \\
\text { leaky) }\end{array}$ & WSRC, 1995b \\
\hline FSB-PC & FSB-50PC & 75.1 & 125.0 & 1.40 & $\begin{array}{l}\text { Aqetsolv (Hantush } \\
\text { leaky) }\end{array}$ & WSRC, $1995 \mathrm{~b}$ \\
\hline FSB-PC & FSB-79C & 75.1 & 125.0 & 3.00 & $\begin{array}{l}\text { Aqetsolv (Hantush } \\
\text { leaky) }\end{array}$ & WSRC, $1995 \mathrm{~b}$ \\
\hline FSB-PC & FSB-100PC & 75.1 & 125.0 & 2.10 & $\begin{array}{l}\text { Aqetsolv (Hantush } \\
\text { leaky) }\end{array}$ & WSRC, $1995 \mathrm{~b}$ \\
\hline FSB-PC & FSB-103C & 75.1 & 125.0 & 3.60 & $\begin{array}{l}\text { Aqetsolv (Hantush } \\
\text { leaky) }\end{array}$ & WSRC, $1995 \mathrm{~b}$ \\
\hline$\overline{\text { FSB-PC }}$ & FSB-106C & 75.1 & 125.0 & 3.40 & $\begin{array}{l}\text { Aqetsolv (Hantush } \\
\text { leaky) }\end{array}$ & WSRC, $1995 b$ \\
\hline FSB-PC & FSB-110C & 75.1 & 125.0 & 1.20 & $\begin{array}{l}\text { Aqetsolv (Hantush } \\
\text { leaky) }\end{array}$ & WSRC, $1995 \mathrm{~b}$ \\
\hline FSB-PC & FSB-150PC & 75.1 & 125.0 & 1.30 & $\begin{array}{l}\text { Aqetsolv (Hantush } \\
\text { leaky) }\end{array}$ & WSRC, 1995b \\
\hline FSB-PD & FSB-25PD & 37.3 & 81.0 & 46.40 & $\begin{array}{l}\text { Aqetsolv (Neuman } \\
\text { method) }\end{array}$ & WSRC, $1995 \mathrm{~b}$ \\
\hline FSB-PD & FSB-50PD & 37.3 & 81.0 & 62.50 & $\begin{array}{l}\text { Aqetsolv (Neuman } \\
\text { method) }\end{array}$ & WSRC, 1995b \\
\hline FSB-PD & FSB-100PD & 37.3 & 81.0 & 48.30 & $\begin{array}{l}\text { Aqetsolv (Neuman } \\
\text { method) }\end{array}$ & WSRC, $1995 \mathrm{~b}$ \\
\hline FSB-PD & FSB-150PD & 37.3 & 81.0 & 49.20 & $\begin{array}{l}\text { Aqetsolv (Neuman } \\
\text { method) }\end{array}$ & WSRC, 1995b \\
\hline FSB-76A & FSB-76A & 244.1 & 254.6 & 1.29 & $\begin{array}{l}\text { Jacob Semi- } \\
\text { Logarithmic }\end{array}$ & $\begin{array}{l}\text { Woodward-Clyde, } \\
\text { 1985a }\end{array}$ \\
\hline FSB-78A & FSB-78A & 233.0 & 243.4 & 0.82 & $\begin{array}{l}\text { Jacob Semi- } \\
\text { Logarithmic }\end{array}$ & $\begin{array}{l}\text { Woodward-Clyde, } \\
\text { 1985a }\end{array}$ \\
\hline FSB-79A & FSB-79A & 181.6 & 192.0 & 142.90 & $\begin{array}{l}\text { Jacob Semi- } \\
\text { Logarithmic }\end{array}$ & $\begin{array}{l}\text { Woodward-Clyde, } \\
\text { 1985a }\end{array}$ \\
\hline FSB-87A & FSB-87A & 242.0 & 252.5 & 51.02 & $\begin{array}{l}\text { Jacob Semi- } \\
\text { Logarithmic }\end{array}$ & $\begin{array}{l}\text { Woodward-Clyde, } \\
\text { 1985a }\end{array}$ \\
\hline HPT-1A & DRB-6WW & 127.0 & 178.0 & $1.47 \mathrm{E}-04$ & Hantush-Jacob & CH2M Hill, 1989 \\
\hline HPT-1A & HC-10A & 127.0 & 178.0 & $1.26 \mathrm{E}-03$ & Hantush-Jacob & CH2M Hill, 1989 \\
\hline HPT-1A & HPT-2A & 127.0 & 178.0 & $2.86 \mathrm{E}-04$ & Hantush-Jacob & CH2M Hill, 1989 \\
\hline HSB-PC & HSB-25PC & 57.0 & 110.6 & 0.90 & Hantush-Jacob & WSRC, 1995b \\
\hline HSB-PC & HSB-50PC & 57.0 & 110.6 & 1.30 & Hantush-Jacob & WSRC, $1995 b$ \\
\hline HSB-PC & HSB-100PC & 57.0 & 110.6 & 1.30 & Hantush-Jacob & WSRC, 1995b \\
\hline HSB-PC & HSB-136C & 57.0 & 110.6 & 1.60 & Hantush-Jacob & WSRC, 1995b \\
\hline HSB-PC & HSB-137C & 57.0 & 110.6 & 1.10 & Hantush-Jacob & WSRC, $1995 \mathrm{~b}$ \\
\hline HSB-PC & HSB-150PC & 57.0 & 110.6 & 1.20 & Hantush-Jacob & WSRC, 1995b \\
\hline HSB-65A & HSB-65A & \begin{tabular}{|c|}
197.5 \\
\end{tabular} & 208.2 & 1.74 & $\begin{array}{l}\text { Jacob Semi- } \\
\text { Logarithmic }\end{array}$ & $\begin{array}{l}\text { Woodward-Clyde, } \\
\text { 1985b }\end{array}$ \\
\hline HSB-68A & HSB-68A & 189.4 & 199.9 & 1.13 & $\begin{array}{l}\text { Jacob Semi- } \\
\text { Logarithmic }\end{array}$ & $\begin{array}{l}\text { Woodward-Clyde, } \\
\text { 1985b }\end{array}$ \\
\hline HSB-83A & HSB-83A & 158.9 & 169.7 & 10.48 & $\begin{array}{l}\text { Jacob Semi- } \\
\text { Logarithmic }\end{array}$ & $\begin{array}{l}\text { Woodward-Clyde, } \\
\text { 1985b }\end{array}$ \\
\hline HSB-84A & HSB-68A & 150.8 & 162.0 & 33.80 & $\begin{array}{l}\text { Aqtesolv (Non-Leaky } \\
\text { Theis) }\end{array}$ & Albenesius, 1990 \\
\hline
\end{tabular}


WSRC-TR-99-00248, Rev. 0, Regional Groundwater Flow Model for C, K, L, and P Reactor Areas

Appendix C-1. Permeability Values Recorded from Pumping Tests (Continued)

\begin{tabular}{|c|c|c|c|c|c|c|}
\hline $\begin{array}{c}\text { Pumped } \\
\text { Well }\end{array}$ & $\begin{array}{c}\text { Observation } \\
\text { Well }\end{array}$ & $\begin{array}{c}\text { Test } \\
\text { Interval } \\
\text { Top } \\
\text { (ft b.g.l.) }\end{array}$ & $\begin{array}{c}\text { Test } \\
\text { Interval } \\
\text { Bottom } \\
\text { (ft b.g.l.) }\end{array}$ & $\begin{array}{c}\text { Permeability } \\
\text { (ft/day) }\end{array}$ & Analysis Method & Reference \\
\hline
\end{tabular}

\begin{tabular}{|c|c|c|c|c|c|c|}
\hline HSB-84A & HSB-69A & 150.8 & 162.0 & 17.29 & $\begin{array}{l}\text { Aqtesolv (Non-Leaky } \\
\text { Theis) }\end{array}$ & Albenesius, 1990 \\
\hline HSB-84A & HSB-83A & 150.8 & 162.0 & 39.72 & $\begin{array}{l}\text { Aqtesolv (Non-Leaky } \\
\text { Theis) }\end{array}$ & Albenesius, 1990 \\
\hline HSB-84A & HSB-86A & 150.8 & 162.0 & 34.25 & $\begin{array}{l}\text { Aqtesolv (Non-Leaky } \\
\text { Theis) }\end{array}$ & Albenesius, 1990 \\
\hline HSB-84A & HSB-118A & 150.8 & 162.0 & 39.12 & $\begin{array}{l}\text { Aqtesolv (Non-Leaky } \\
\text { Theis) }\end{array}$ & Albenesius, 1990 \\
\hline HSB-84A & HSB-139A & 150.8 & 162.0 & 27.88 & $\begin{array}{l}\text { Aqtesolv (Non-Leaky } \\
\text { Theis) }\end{array}$ & Albenesius, 1990 \\
\hline HSB-85A & HSB-85A & 221.0 & 231.0 & 8.72 & $\begin{array}{l}\text { Aqtesolv (Non-Leaky } \\
\text { Theis) }\end{array}$ & $\begin{array}{l}\text { Woodward-Clyde, } \\
1985 \mathrm{~b}\end{array}$ \\
\hline HSB-86A & HSB-86A & 186.1 & 196.9 & 5.46 & $\begin{array}{l}\text { Aqtesolv (Non-Leaky } \\
\text { Theis) }\end{array}$ & $\begin{array}{l}\text { Woodward-Clyde, } \\
1985 \mathrm{~b}\end{array}$ \\
\hline $\begin{array}{l}\text { HSB- } \\
101 C\end{array}$ & HSB-101C & 80.0 & 90.0 & 1.68 & $\begin{array}{l}\text { Hantush and Jacob } \\
\text { (1955) Leaky Artesian } \\
\text { Solution }\end{array}$ & Evans, 1991 \\
\hline YSC-1A & YSC-1A & 132.0 & 192.1 & 62.00 & Cooper-Jacob & WEGS, 1990 \\
\hline YSC-1A & YSC-1A & 132.0 & 192.1 & 37.00 & Recovery & WEGS, 1990 \\
\hline YSC-1A & YSC-1A & 132.0 & 192.1 & 22.00 & Theis & WEGS, 1990 \\
\hline YSC-1A & YSC-1A & 132.0 & 192.1 & 52.00 & WHIP(Recovery) & WEGS, 1990 \\
\hline YSC-1A & YSC-1A & 132.0 & 192.1 & 57.00 & WHIP(Theis) & WEGS, 1990 \\
\hline YSC-1A & YSC-4A & 132.0 & 192.1 & 40.00 & Theis Non-Eq. & WEGS, 1990 \\
\hline YSC-1A & YSC-4A & 132.0 & 192.1 & 43.00 & WHIP(Theis) & WEGS, 1990 \\
\hline $\begin{array}{l}\mathrm{YSC}-1 \mathrm{~A} \\
\end{array}$ & YSC-4A & 132.0 & 192.1 & 47.00 & WHIP(Recovery) & WEGS, 1990 \\
\hline YSC-1A & YSC-4A & 132.0 & 192.1 & 52.00 & Recovery & WEGS, 1990 \\
\hline
\end{tabular}

Notes:

$\mathrm{ft}$ b.g.l. - feet below ground level

$\mathrm{ft} /$ day - feet per day

1 - Detailed description of references in Appendix D 
Appendix C-2. Permeability Values Recorded from Slug Tests

\begin{tabular}{|c|c|c|c|c|c|c|}
\hline Well ID & $\begin{array}{c}\text { Screen } \\
\text { Top } \\
\text { (ft b.g.l.) }\end{array}$ & $\begin{array}{c}\text { Screen } \\
\text { Bottom } \\
\text { (ft b.g.l.) }\end{array}$ & $\begin{array}{c}\text { Permeability } \\
\text { (ft/day) }\end{array}$ & Solution Method & Test Type & Reference \\
\hline
\end{tabular}

\begin{tabular}{|c|c|c|c|c|c|c|}
\hline BGO-1D & . 48 & 68 & 0.31 & Bouwer-Rice 1976 & Falling Head & \begin{tabular}{|l} 
S\&ME, \\
1988 \\
\end{tabular} \\
\hline BGO-3A & 175 & 185 & 3.25142 & Bouwer-Rice & Rising Head & $\begin{array}{l}\text { Amidon, } \\
1995\end{array}$ \\
\hline BGO-3A & 175 & 185 & 5.191 & Bouwer-Rice & Falling Head & $\begin{array}{l}\text { Amidon, } \\
1995\end{array}$ \\
\hline BGO-3D & 51.7 & 71.8 & 0.14 & Bouwer-Rice 1976 & Falling Head & $\begin{array}{l}\text { S\&ME, } \\
1988\end{array}$ \\
\hline BGO-5C & 101 & 111 & 0.13 & Hvorslev & Falling Head & $\begin{array}{l}\text { S\&ME, } \\
1988\end{array}$ \\
\hline BGO-6A & 166.3 & 176.3 & 0.77 & Hvorslev & Falling Head & \begin{tabular}{|l} 
S\&ME, \\
1988 \\
\end{tabular} \\
\hline BGO-8A & 166 & 176 & 0.21 & Hvorslev & Falling Head & $\begin{array}{l}\text { S\&ME, } \\
1988 \\
\end{array}$ \\
\hline BGO-10A & 178 & 188 & 0.16 & Hvorslev & Falling Head & \begin{tabular}{|l} 
S\&ME, \\
1988 \\
\end{tabular} \\
\hline $\begin{array}{l}\text { BGO- } \\
\text { 10AA }\end{array}$ & 208 & 218 & 0.43 & Bouwer-Rice 1976 & Falling Head & $\begin{array}{l}\text { WSRC, } \\
\text { 1992a }\end{array}$ \\
\hline BGO-12A & 195 & 205 & 0.005 & Hvorslev & Falling Head & \begin{tabular}{|l} 
S\&ME, \\
1988 \\
\end{tabular} \\
\hline BGO-14A & 180.6 & 190.6 & 0.04 & Hvorslev & Falling Head & $\begin{array}{l}\text { S\&ME, } \\
1988 \\
\end{array}$ \\
\hline BGO-16A & 190.3 & 200.3 & 0.15 & Hvorslev & Falling Head & $\begin{array}{l}\text { S\&ME, } \\
1988\end{array}$ \\
\hline BGO-18A & 183.4 & 193.4 & 11.98 & Hvorslev & Falling Head & $\begin{array}{l}\text { S\&ME, } \\
1988 \\
\end{array}$ \\
\hline BGO-21D & 45.3 & 65.3 & 0.79 & Bouwer-Rice 1976 & Falling Head & $\begin{array}{l}\text { S\&ME, } \\
1988 \\
\end{array}$ \\
\hline BGO-23D & 45 & 65 & 1.11 & Bouwer-Rice 1976 & Falling Head & $\begin{array}{l}\text { S\&ME, } \\
1988 \\
\end{array}$ \\
\hline BGO-25A & 180.6 & 190.6 & 0.5 & Hvorslev & Falling Head & $\begin{array}{l}\text { S\&ME, } \\
1988\end{array}$ \\
\hline $\mathrm{BGO}-41 \mathrm{~A}$ & 185 & 195 & 0.13 & Bouwer-Rice 1976 & Falling Head & $\begin{array}{l}\text { WSRC, } \\
1992 a\end{array}$ \\
\hline $\mathrm{BGO}-42 \mathrm{C}$ & 100 & 110 & 0.45 & Bouwer-Rice 1976 & Falling Head & $\begin{array}{l}\text { WSRC, } \\
\text { 1992a }\end{array}$ \\
\hline $\begin{array}{l}\text { BGO- } \\
43 \mathrm{AA}\end{array}$ & 240 & 250 & 0.86 & Bouwer-Rice 1976 & Falling Head & $\begin{array}{l}\text { WSRC, } \\
\text { 1992a }\end{array}$ \\
\hline $\begin{array}{l}\text { BGO- } \\
44 \mathrm{AA} \\
\end{array}$ & 212 & 222.1 & 4.36 & Bouwer-Rice 1976 & Falling Head & $\begin{array}{l}\text { WSRC, } \\
1992 \mathrm{a}\end{array}$ \\
\hline BGO-45A & 150 & 160 & 2.45 & Bouwer-Rice 1976 & Falling Head & $\begin{array}{l}\text { WSRC, } \\
1992 a\end{array}$ \\
\hline BGO-46B & 113 & 123 & 2.33 & Bouwer-Rice 1976 & Falling Head & $\begin{array}{l}\text { WSRC, } \\
1992 a\end{array}$ \\
\hline BGO-47A & 168 & 178 & 3.07 & Bouwer-Rice 1976 & Falling Head & $\begin{array}{l}\text { WSRC, } \\
1992 a\end{array}$ \\
\hline BGO-48C & 88 & 98 & 2.15 & Bouwer-Rice 1976 & Falling Head & $\begin{array}{l}\text { WSRC, } \\
\text { 1992a } \\
\end{array}$ \\
\hline BGO-49A & 184 & 194 & 0.49 & Bouwer-Rice 1976 & Falling Head & $\begin{array}{l}\text { WSRC, } \\
\text { 1992a }\end{array}$ \\
\hline
\end{tabular}


Appendix C-2. Permeability Values Recorded from Slug Tests (Continued)

\begin{tabular}{|c|c|c|c|c|c|c|}
\hline Well ID & $\begin{array}{c}\text { Screen } \\
\text { Top } \\
\text { (ft b.g.l.) }\end{array}$ & $\begin{array}{c}\text { Screen } \\
\text { Bottom } \\
\text { (ft b.g.l.) }\end{array}$ & $\begin{array}{l}\text { Permeability } \\
\text { (ft/day) }\end{array}$ & Solution Method & Test Type & Reference $^{1}$ \\
\hline
\end{tabular}

\begin{tabular}{|c|c|c|c|c|c|c|}
\hline BGO-50A & 153 & 163 & 0.4 & Bouwer-Rice 1976 & Falling Head & $\begin{array}{l}\text { WSRC, } \\
\text { 1992a }\end{array}$ \\
\hline $\begin{array}{l}\text { BGO- } \\
\text { 51AA }\end{array}$ & 248 & 263.6 & 0.7762 & Bouwer-Rice 1976 & Rising Head & $\begin{array}{l}\text { Amidon, } \\
1995\end{array}$ \\
\hline $\begin{array}{l}\text { BGO- } \\
51 \mathrm{AA}\end{array}$ & 248 & 263.6 & 1.188 & Bouwer-Rice 1976 & Falling Head & \begin{tabular}{|l} 
Amidon, \\
1995 \\
\end{tabular} \\
\hline $\begin{array}{l}\text { BGO- } \\
52 \mathrm{AA}\end{array}$ & 235 & 247.8 & 0.8996 & Böuwer-Rice 1976 & Rising Head & \begin{tabular}{|l} 
Amidon, \\
1995 \\
\end{tabular} \\
\hline $\begin{array}{l}\text { BGO- } \\
52 A A\end{array}$ & 235 & 247.8 & 8.15 & Bouwer-Rice 1976 & Falling Head & $\begin{array}{l}\text { Amidon, } \\
1995\end{array}$ \\
\hline $\begin{array}{l}\text { BGO- } \\
53 \mathrm{AA}\end{array}$ & 240 & 250 & 1.11744 & Bouwer-Rice 1976 & Falling Head & Rust, 1996 \\
\hline BGX-1A & 165 & 175.02 & 0.01 & Bouwer-Rice 1976 & Falling Head & $\begin{array}{l}\text { WSRC, } \\
\text { 1991a } \\
\end{array}$ \\
\hline BGX-2B & 142 & 151.95 & 0.21 & Bouwer-Rice 1976 & Falling Head & $\begin{array}{l}\text { WSRC, } \\
1991 \mathrm{a}\end{array}$ \\
\hline $\mathrm{BGX}-4 \mathrm{~A}$ & 172 & 182 & 1.83 & Bouwer-Rice 1976 & Falling Head & \begin{tabular}{|l} 
WSRC, \\
1991a
\end{tabular} \\
\hline BGX-7D & 63 & 83.03 & 20.38 & Bouwer-Rice 1976 & Rising Head & \begin{tabular}{|l} 
WSRC, \\
1991a
\end{tabular} \\
\hline BGX-9D & 45 & 65.01 & 0.36 & Bouwer-Rice 1976 & Rising Head & $\begin{array}{l}\text { WSRC, } \\
1991 \mathrm{a}\end{array}$ \\
\hline CMP-30B & 172.2 & 195.5 & 1.1 & Bouwer Rice & Rising Head & $\begin{array}{l}\text { WSRC, } \\
1996 c\end{array}$ \\
\hline CMP-30B & 172.2 & 195.5 & 1.4 & Bouwer Rice & Falling Head & $\begin{array}{l}\text { WSRC, } \\
1996 c\end{array}$ \\
\hline FSB-89C & 113 & 123 & 0.524 & Hvorslev & Falling Head & $\begin{array}{l}\text { WSRC, } \\
\text { 1991d }\end{array}$ \\
\hline FSB-91C & $\cdot 117.9$ & 127.9 & 0.141 & Hvorslev & Falling Head & $\begin{array}{l}\text { WSRC, } \\
\text { 1991d }\end{array}$ \\
\hline FSB-93C & 122 & 132 & 5.27 & Hvorslev & Falling Head & $\begin{array}{l}\text { WSRC, } \\
\text { 1991d }\end{array}$ \\
\hline FSB-97A & 188 & 198 & 0.852 & Hvorslev & Falling Head & $\begin{array}{l}\text { WSRC, } \\
\text { 1991d }\end{array}$ \\
\hline FSB-101A & 180 & 190 & 33.2 & Hvorslev & Falling Head & \begin{tabular}{|l|} 
Sirrine, \\
1987
\end{tabular} \\
\hline FSB-112A & 136 & 146 & 1.7 & Bouwer-Rice 1976 & Rising Head & $\begin{array}{l}\text { WEGS, } \\
1991\end{array}$ \\
\hline FSB-113A & 130 & 140 & 0.62 & Bouwer-Rice 1976 & Rising Head & \begin{tabular}{|l} 
WEGS, \\
1991
\end{tabular} \\
\hline FSB-114A & 145 & 155 & 0.44 & Bouwer-Rice 1976 & Rising Head & \begin{tabular}{|l} 
WEGS, \\
1991
\end{tabular} \\
\hline FSB-115C & 32 & 42 & 0.36 & Bouwer-Rice 1976 & Rising Head & \begin{tabular}{|l} 
WEGS, \\
1991
\end{tabular} \\
\hline FSB-116C & 30 & 40 & 0.69 & Bouwer-Rice 1976 & Rising Head & \begin{tabular}{|l} 
WEGS, \\
1991
\end{tabular} \\
\hline FSB-120A & 169 & 179 & 0.65 & Bouwer-Rice 1976 & Rising Head & \begin{tabular}{|l} 
WEGS, \\
1991
\end{tabular} \\
\hline FSB-121C & 96 & 106 & 11 & Bouwer-Rice 1976 & Rising Head & \begin{tabular}{|l} 
WEGS, \\
1991
\end{tabular} \\
\hline
\end{tabular}


WSRC-TR-99-00248, Rev. 0, Regional Groundwater Flow Model for C, K, L, and P Reactor Areas C-5

\section{Appendix C-2. Permeability Values Recorded from Slug Tests (Continued)}

\begin{tabular}{|c|c|c|c|c|c|c|}
\hline Well ID & $\begin{array}{c}\text { Screen } \\
\text { Top } \\
\text { (ft b.g.l.) }\end{array}$ & $\begin{array}{c}\text { Screen } \\
\text { Bottom } \\
\text { (ft b.g.l.) }\end{array}$ & $\begin{array}{c}\text { Permeability } \\
\text { (ft/day) }\end{array}$ & Solution Method & Test Type & Reference \\
\hline
\end{tabular}

\begin{tabular}{|c|c|c|c|c|c|c|}
\hline FSB $122 \mathrm{C}$ & $\because . .46$ & $\cdots 56$ & 2.6 & Bouwer-Rice 1976 & Rising Head & \begin{tabular}{|l} 
WEGS, \\
1991
\end{tabular} \\
\hline FSB-123C & 71 & 81 & 6.7 & Bouwer-Rice 1976 & Rising Head & \begin{tabular}{|l} 
WEGS, \\
1991 \\
\end{tabular} \\
\hline HAA-1TA & 310 & 320 & 0.548 & Bouwer-Rice 1976 & Rising Head & $\begin{array}{l}\text { WSRC, } \\
1995 \mathrm{a}\end{array}$ \\
\hline HAA-1TA & 310 & 320 & 0.786 & Bouwer-Rice 1976 & Falling Head & \begin{tabular}{|l|} 
WSRC, \\
$1995 \mathrm{a}$ \\
\end{tabular} \\
\hline HAA-2AA & 252 & 262 & 19.858 & Bouwer-Rice 1976 & Rising Head & $\begin{array}{l}\text { WSRC, } \\
1995 \mathrm{a} \\
\end{array}$ \\
\hline HAA-2AA & 252 & 262 & 30.6552 & Bouwer-Rice 1976 & Falling Head & \begin{tabular}{|l} 
WSRC, \\
$1995 a$
\end{tabular} \\
\hline HAA-3AA & 258 & 268 & 0.3229 & Bouwer-Rice 1976 & Rising Head & $\begin{array}{l}\text { WSRC, } \\
1995 \mathrm{a}\end{array}$ \\
\hline $\mathrm{HAA}-3 \mathrm{AA}$ & 258 & 268 & 0.504 & Bouwer-Rice 1976 & Falling Head & $\begin{array}{l}\text { WSRC, } \\
1995 \mathrm{a}\end{array}$ \\
\hline HAA-6AA & 244 & 254 & 0.224 & Bouwer-Rice 1976 & Rising Head & $\begin{array}{l}\text { WSRC, } \\
1995 a\end{array}$ \\
\hline HAA-6AA & 244 & 254 & 0.2587 & Bouwer-Rice 1976 & Falling Head & $\begin{array}{l}\text { WSRC, } \\
\text { 1'995a }\end{array}$ \\
\hline $\mathrm{HCA}-4 \mathrm{AA}$ & $: 265$ & 275 & 13.1717 & Bouwer-Rice 1976 & Rising Head & $\begin{array}{l}\text { WSRC, } \\
1995 a\end{array}$ \\
\hline $\mathrm{HCA}-4 \mathrm{AA}$ & $i^{265}$ & 275 & 13.78 & Bouwer-Rice 1976 & Falling Head & $\begin{array}{l}\text { WSRC, } \\
1995 \mathrm{a}\end{array}$ \\
\hline HSB-69A & 141 & 151 & 8.79 & Hvorslev & Rising Head & $\begin{array}{l}\text { WSRC, } \\
\text { 1991c }\end{array}$ \\
\hline HSB-101C & 80 & 90 & 4 & Hvorslev & Falling Head & $\begin{array}{l}\text { WSRC, } \\
1991 \mathrm{c}\end{array}$ \\
\hline HSB-103C & 76 & 86 & 3.1 & Hvorslev & Falling Head & $\begin{array}{l}\text { WSRC, } \\
1991 c\end{array}$ \\
\hline HSB-105C & 85 & 95 & 4.3 & Hvorslev & Falling Head & $\begin{array}{l}\text { WSRC, } \\
1991 \mathrm{c}\end{array}$ \\
\hline HSB-106C & 82 & 92 & 24.4 & Hvorslev & Falling Head & $\begin{array}{l}\text { WSRC, } \\
1991 \mathrm{c}\end{array}$ \\
\hline HSB-109C & 81 & 91 & 0.952 & Hvorslev & Falling Head & $\begin{array}{l}\text { Sirrine, } \\
1988 \\
\end{array}$ \\
\hline HSB-110C & 72 & 82 & 0.709 & Hvorslev & Falling Head & \begin{tabular}{|l} 
Sirrine, \\
1988 \\
\end{tabular} \\
\hline HSB-111C & 103 & 113 & 1.7 & Hvorslev & Falling Head & \begin{tabular}{|l} 
WSRC, \\
$1991 \mathrm{c}$ \\
\end{tabular} \\
\hline HSB-112C & 102 & 112 & 4.2 & Hvorslev & Falling Head & \begin{tabular}{|l|} 
WSRC, \\
1991c \\
\end{tabular} \\
\hline HSB-113C & 97 & 107 & 0.992 & Hvorslev & Falling Head & \begin{tabular}{|l} 
Sirrine, \\
1988 \\
\end{tabular} \\
\hline HSB-117A & 140 & 150 & 0.16 & Hvorslev & Falling Head & $\begin{array}{l}\text { WSRC, } \\
\text { 1991c } \\
\end{array}$ \\
\hline HSB-118A & 144 & 154 & 12 & Hvorslev & Falling Head & $\begin{array}{l}\text { WSRC, } \\
1991 c\end{array}$ \\
\hline HSB-122A & 85.8 & 94.8 & 6.8 & Hvorslev & Falling Head & \begin{tabular}{|l|} 
Sirrine, \\
1988 \\
\end{tabular} \\
\hline
\end{tabular}


C-6 WSRC-TR-99-00248, Rev. 0, Regional Groundwater Flow Model for C, K, L, and P Reactor Areas Appendix C-2. Permeability Values Recorded from Slug Tests (Continued)

\begin{tabular}{|c|c|c|c|c|c|c|}
\hline Well ID & $\begin{array}{c}\text { Screen } \\
\text { Top } \\
\text { (ft b.g.l.) }\end{array}$ & $\begin{array}{c}\text { Screen } \\
\text { Bottom } \\
\text { (ft b.g.l.) }\end{array}$ & $\begin{array}{l}\text { Permeability } \\
\text { (ft/day) }\end{array}$ & Solution Method & Test Type & Reference $^{1}$ \\
\hline
\end{tabular}

\begin{tabular}{|c|c|c|c|c|c|c|}
\hline HSB-132C & 60.2 & 69.2 & 0.22 & Hvorslev & Rising Head & $\begin{array}{l}\text { Sirrine, } \\
1988\end{array}$ \\
\hline HSB-132C & 60.2 & 69.2 & 0.28 & Hvorslev & Falling Head & \begin{tabular}{|l} 
Sirrine, \\
1988
\end{tabular} \\
\hline HSB-139A & 134.4 & 143.4 & 3.82 & Hvorslev & Falling Head & \begin{tabular}{|l|} 
Sirrine, \\
1988
\end{tabular} \\
\hline HSB-140A & 143 & 153 & 12 & Bouwer-Rice 1976 & Rising Head & \begin{tabular}{|l} 
WEGS, \\
1991 \\
\end{tabular} \\
\hline HSB-141A & 162 & 172 & 1.9 & Bouwer-Rice 1976 & Rising Head & \begin{tabular}{|l} 
WEGS, \\
1991 \\
\end{tabular} \\
\hline HSB-142C & 30 & 40 & 0.6 & Bouwer-Rice 1976 & Rising Head & \begin{tabular}{|l} 
WEGS, \\
1991
\end{tabular} \\
\hline HSB-143C & 41 & 51 & 2.4 & Bouwer-Rice 1976 & Rising Head & \begin{tabular}{|l} 
WEGS, \\
1991 \\
\end{tabular} \\
\hline HSB-144A & 145 & 155 & 0.22 & Bouwer-Rice 1976 & Rising Head & \begin{tabular}{|l} 
WEGS, \\
1991 \\
\end{tabular} \\
\hline HSB-145C & 59 & 69 & 0.38 & Bouwer-Rice 1976 & Rising Head & \begin{tabular}{|l} 
WEGS, \\
1991 \\
\end{tabular} \\
\hline HSB-146A & 154 & 164 & 9.4 & Bouwer-Rice 1976 & Rising Head & \begin{tabular}{|l} 
WEGS, \\
1991 \\
\end{tabular} \\
\hline HSB-148C & 80 & 90 & 1.8 & Bouwer-Rice 1976 & Rising Head & $\begin{array}{l}\text { WEGS, } \\
1991 \\
\end{array}$ \\
\hline HSB-151C & 31 & 41 & 0.8 & Bouwer-Rice 1976 & Rising Head & \begin{tabular}{|l} 
WEGS, \\
1991 \\
\end{tabular} \\
\hline HSB-152C & 29 & 59 & 0.8 & Bouwer-Rice 1976 & Rising Head & \begin{tabular}{|l} 
WEGS, \\
1991 \\
\end{tabular} \\
\hline HSL-6AA & 246 & 266 & 4.2 & Bouwer-Rice 1976 & Falling Head & $\begin{array}{l}\text { WSRC, } \\
1995 \mathrm{a} \\
\end{array}$ \\
\hline HSL-6AA & 246 & 266 & 6.7602 & Bouwer-Rice 1976 & Rising Head & $\begin{array}{l}\text { WSRC, } \\
1995 \mathrm{a} \\
\end{array}$ \\
\hline LAC-5DL & 53.6 & 63.6 & 0.61 & Bouwer-Rice 1976 & Rising Head & $\begin{array}{l}\text { WSRC, } \\
1996 a \\
\end{array}$ \\
\hline LAC-6DL & 52 & 62 & 1.33 & Bouwer-Rice 1976 & Rising Head & \begin{tabular}{|l|} 
WSRC, \\
$1996 a$
\end{tabular} \\
\hline LAC-7DL & 52 & 62 & 0.27 & Bouwer-Rice 1976 & Rising Head & $\begin{array}{l}\text { WSRC, } \\
1996 \mathrm{a} \\
\end{array}$ \\
\hline LAC-8DL & 43.6 & 53.6 & 0.74 & Bouwer-Rice 1976 & Rising Head & $\begin{array}{l}\text { WSRC, } \\
1996 \mathrm{a} \\
\end{array}$ \\
\hline LCO-5A & 190 & 200 & 0.1 & Bouwer-Rice 1976 & Rising Head & $\begin{array}{l}\text { WSRC, } \\
1996 a \\
\end{array}$ \\
\hline LCO-5DL & 45.4 & 55.4 & 4.62 & Bouwer-Rice 1976 & Rising Head & \begin{tabular}{|l} 
WSRC, \\
$1996 \mathrm{a}$ \\
\end{tabular} \\
\hline LCO-8DL & 55 & 65 & 12.17 & Bouwer-Rice 1976 & Rising Head & $\begin{array}{l}\text { WSRC, } \\
1996 a \\
\end{array}$ \\
\hline YSC-1C & 65 & 75 & 2.4 & Bouwer-Rice & Rising Head & $\begin{array}{l}\text { WEGS, } \\
1990 \\
\end{array}$ \\
\hline
\end{tabular}

Notes:

ft b.g.l. - feet below ground level

$\mathrm{ft} /$ day - feet per day

1 - Detailed description of references in Appendix D 
WSRC-TR-99-00248, Rev. 0, Regional Groundwater Flow Model for C, K, L, and P Reactor Areas C-7

Appendix C-3. Permeability Values Recorded from Laboratory Tests

\begin{tabular}{|c|c|c|c|c|c|}
\hline Well ID & $\begin{array}{c}\text { Interval Top } \\
\text { (ft b.g.l.) }\end{array}$ & $\begin{array}{c}\text { Interval } \\
\text { Bottom } \\
\text { (ft b.g.l.) } \\
\end{array}$ & $\begin{array}{c}\text { Permeability- } \\
\text { vertical } \\
\text { (ft/day) } \\
\end{array}$ & $\begin{array}{c}\text { Permeability- } \\
\text { horizontal } \\
\text { (ft/day) }\end{array}$ & Reference $^{l}$ \\
\hline BGO-3A & 162 & 164 & $1.14 \mathrm{E}-05$ & $2.10 \mathrm{E}-05$ & Amidon, 1995 \\
\hline BGO-3A & 266.1 & 267 & $1.40 \mathrm{E}-04$ & $2.30 \mathrm{E}-04$ & Amidon, 1995 \\
\hline BGO-9AA & 62 & 63.5 & $5.68 \mathrm{E}-05$ & $2.07 \mathrm{E}-04$ & WSRC, 1992 \\
\hline BGO-9AA & 137.4 & 137.7 & $1.38 \mathrm{E}-01$ & $1.00 \mathrm{E}-01$ & Core Laboratories, 1995 \\
\hline BGO-9AA & 142 & 142.3 & $2.27 \mathrm{E}-02$ & $2.69 \mathrm{E}-02$ & Core Laboratories, 1995 \\
\hline BGO-9AA & 158.7 & 158.8 & $3.49 \mathrm{E}-01$ & $2.08 \mathrm{E}-02$ & Core Laboratories, 1995 \\
\hline BGO-9AA & 222 & 223.5 & 7.67E-05 & $1.76 \mathrm{E}-03$ & WSRC, $1992 \mathrm{a}$ \\
\hline BGO-10A & 220 & 221.5 & $3.12 \mathrm{E}-06$ & $5.96 \mathrm{E}-05$ & WSRC, $1992 a$ \\
\hline BGO-20AA & 268 & 270 & $4.26 \mathrm{E}-06$ & $1.22 \mathrm{E}-02$ & RUST, 1996 \\
\hline BGO-39A & 280.5 & 282.5 & $1.36 \mathrm{E}-03$ & $6.53 \mathrm{E}-03$ & RUST, 1996 \\
\hline BGO-41A & 88 & 90 & $9.66 \mathrm{E}-02$ & $8.52 \mathrm{E}-04$ & WSRC, 1992a \\
\hline BGO-41A & 164 & 166 & $2.84 \mathrm{E}-05$ & $3.69 \mathrm{E}-04$ & WSRC, 1992a \\
\hline BGO-43AA & 185 & 187 & $8.80 \mathrm{E}-06$ & $2.44 \mathrm{E}-05$ & WSRC, 1992a \\
\hline BGO-44AA & 226 & 227.5 & $3.41 \mathrm{E}-02$ & $2.36 \mathrm{E}-01$ & WSRC, 1992a \\
\hline BGO-45A & 75 & 76.8 & $5.40 \mathrm{E}-05$ & $7.10 \mathrm{E}-05$ & WSRC, 1992a \\
\hline BGO-45A & 144 & 145 & $2.04 \mathrm{E}-04$ & $1.28 \mathrm{E}-04$ & WSRC, 1992a \\
\hline BGO-47A & 145.5 & 147 & $3.41 \mathrm{E}-03$ & $5.68 \mathrm{E}-03$ & WSRC, 1992a \\
\hline BGO-49A & 75 & 77 & $3.70 \mathrm{E}-08$ & $5.20 \mathrm{E}-05$ & WSRC, 1992a \\
\hline BGO-51AA & 298 & 299.75 & 2.27E-05 & $1.11 \mathrm{E}-05$ & Amidon, 1995 \\
\hline BGO-53AA & 265 & 267 & 2.27E-05 & $2.84 \mathrm{E}-05$ & RUST, 1996 \\
\hline BGT-9 & 72 & 74 & $8.24 \mathrm{E}-04$ & $1.70 \mathrm{E}-03$ & RUST, 1996 \\
\hline BGT-9 & 80 & 82 & $8.24 \mathrm{E}-04$ & $3.12 \mathrm{E}-03$ & RUST, 1996 \\
\hline BGT-11 & 70 & 71 & $3.12 \mathrm{E}-04$ & $9.90 \mathrm{E}-04$ & RUST, 1996 \\
\hline BGT-11 & 179.8 & 179.8 & $1.16 \mathrm{E}-04$ & 1.22E-04 & RUST, 1996 \\
\hline BGT-18 & 175 & 177 & $5.96 \mathrm{E}-05$ & $1.56 \mathrm{E}-04$ & RUST, 1996 \\
\hline BGT-18 & 192 & 194 & $1.99 \mathrm{E}-03$ & $4.54 \mathrm{E}-03$ & RUST, 1996 \\
\hline BGT-22 & 55 & 57 & $5.68 \mathrm{E}-06$ & $3.12 \mathrm{E}-05$ & RUST, 1996 \\
\hline BGT-22 & 165 & 167 & $8.52 \mathrm{E}-04$ & $1.85 \mathrm{E}-02$ & RUST, 1996 \\
\hline BGT-22 & 275 & 276 & $3.41 \mathrm{E}-05$ & & RUST, 1996 \\
\hline BGT-28 & 207.3 & 208.7 & $7.10 \mathrm{E}-05$ & $7.10 \mathrm{E}-04$ & RUST, 1996 \\
\hline BGT-47 & 108 & 109 & 2.84E-03 & & RUST, 1996 \\
\hline BGT-47 & 178 & 179 & $5.68 \mathrm{E}-03$ & $1.68 \mathrm{E}-02$ & RUST, 1996 \\
\hline BGT-53 & 88 & 90 & 5.11E-05 & $1.70 \mathrm{E}-05$ & RUST, 1996 \\
\hline BGT-53 & 314 & 315.6 & $1.42 \mathrm{E}-05$ & 1.33E-05 & RUST, 1996 \\
\hline BGT-61 & 102 & 104 & $1.42 \mathrm{E}-05$ & $4.26 \mathrm{E}-05$ & RUST, 1996 \\
\hline BGT-61 & 178 & 179.1 & 7.67E-06 & $8.52 \mathrm{E}-06$ & RUST, 1996 \\
\hline BGT-67 & 57 & 59 & $6.82 \mathrm{E}-02$ & $3.41 \mathrm{E}-03$ & RUST, 1996 \\
\hline BGT-67 & 112 & 113 & 7.67E-05 & $1.16 \mathrm{E}-05$ & RUST, 1996 \\
\hline BGX-1A & 80 & 82 & $3.41 \mathrm{E}-06$ & $2.53 \mathrm{E}-05$ & WSRC, 1991a \\
\hline BGX-2B & 75 & 77 & $4.26 \mathrm{E}-06$ & $1.99 \mathrm{E}-05$ & WSRC, 1991a \\
\hline BGX-2B & 156 & 156.8 & $1.85 \mathrm{E}-05$ & $3.98 \mathrm{E}-05$ & WSRC, 1991a \\
\hline BGX-4A & 65 & 67 & $8.24 \mathrm{E}-06$ & 1.70E-05 & WSRC, 1991a \\
\hline BGX-7D & 123 & 124.5 & $6.53 \mathrm{E}-05$ & & WSRC, 1992a \\
\hline BGX-9D & 70 & 70.8 & $9.66 \mathrm{E}-05$ & $3.41 \mathrm{E}-04$ & WSRC, 1991a \\
\hline
\end{tabular}


C-8 WSRC-TR-99-00248, Rev. 0, Regional Groundwater Flow Model for C, K, L, and P Reactor Areas

Appendix C-3. Permeability Values Recorded from Laboratory Tests (Continued)

\begin{tabular}{|c|c|c|c|c|c|}
\hline Well ID & $\begin{array}{c}\text { Interval Top } \\
\text { (ft b.g.l.) }\end{array}$ & $\begin{array}{c}\text { Interval } \\
\text { Bottom } \\
\text { (ft b.g.l.) }\end{array}$ & $\begin{array}{c}\text { Permeability- } \\
\text { vertical } \\
\text { (ft/day) }\end{array}$ & $\begin{array}{c}\text { Permeability- } \\
\text { horizontal } \\
\text { (ft/day) }\end{array}$ & Reference $^{\mathbf{1}}$ \\
\hline
\end{tabular}

\begin{tabular}{|c|c|c|c|c|c|}
\hline BGX-9D & 102.5 & 104.5 & $1.25 \mathrm{E}-04$ & 4.54E-04 & WSRC, 1992a \\
\hline $\mathrm{BGX}-11 \mathrm{D}$ & 94 & 96 & $1.14 \mathrm{E}-03$ & & WSRC, 1992a \\
\hline BGX-11D & 154 & 156 & 1.87E-05 & 9.09E-05 & WSRC, 1992a \\
\hline CPC-1 & 137.3 & 137.6 & $3.42 \mathrm{E}+00$ & $1.48 \mathrm{E}+00$ & Core Laboratories, 1995 \\
\hline $\mathrm{CSB} 002 \mathrm{C}$ & 83 & 84 & $2.13 \mathrm{E}+00$ & & Law, 1998 \\
\hline CSB002C & 130 & 132 & $1.85 \mathrm{E}-02$ & 2.04E-01 & Law, 1998 \\
\hline CSB003C & 130 & 132 & $2.39 \mathrm{E}-01$ & & Law, 1998 \\
\hline CSB003C & 160 & 162 & $3.69 \mathrm{E}-05$ & $3.12 \mathrm{E}-02$ & Law, 1998 \\
\hline CSB003C & 83 & 84 & $7.67 \mathrm{E}-02$ & & Law, 1998 \\
\hline CSB003C & 95 & 96 & $3.98 \mathrm{E}-01$ & & Law, 1998 \\
\hline CSB003C & 10 & 12.5 & $1.33 \mathrm{E}-03$ & & Law, 1998 \\
\hline CSB003C & 20 & 22.5 & $2.10 \mathrm{E}-01$ & $3.98 \mathrm{E}-01$ & Law, 1998 \\
\hline CSB003C & 35 & 37 & $1.85 \mathrm{E}-03$ & $2.04 \mathrm{E}-02$ & Law, 1998 \\
\hline FAC-1SB & 170 & 172 & $3.12 \mathrm{E}-05$ & $2.64 \mathrm{E}-04$ & WSRC, 1992b \\
\hline FCH-1 & 54.8 & 56.3 & $3.69 \mathrm{E}-02$ & $1.09 \mathrm{E}-01$ & WSRC, 1993a \\
\hline FCH-1 & 104.8 & 107.8 & $2.06 \mathrm{E}-05$ & & WSRC, 1993a \\
\hline FCH-1 & 145.8 & 146.8 & $8.78 \mathrm{E}-04$ & $1.17 \mathrm{E}-03$ & WSRC, 1993a \\
\hline FCH-1 & 169.8 & 170.8 & $1.02 \mathrm{E}-03$ & & WSRC, 1993a \\
\hline FCH-1 & 208.8 & 211.8 & $2.07 \mathrm{E}-05$ & $2.06 \mathrm{E}-05$ & WSRC, 1993a \\
\hline $\mathrm{FCH}-1$ & 260.3 & 261.8 & $7.29 \mathrm{E}-02$ & & WSRC, 1993a \\
\hline FCH-2 & 57.9 & 59.4 & $9.46 \mathrm{E}-05$ & $2.25 \mathrm{E}-04$ & WSRC, 1993a \\
\hline FCH-2 & 80.1 & 83.1 & $2.26 \mathrm{E}-05$ & & WSRC, 1993a \\
\hline FCH-2 & 135.7 & 138.7 & $1.94 \mathrm{E}-05$ & $2.55 \mathrm{E}-05$ & WSRC, 1993a \\
\hline FCH-2 & 150.1 & 153.1 & $2.24 \mathrm{E}-05$ & & WSRC, 1993a \\
\hline FCH-2 & 205.7 & 208.7 & $2.38 \mathrm{E}-05$ & $2.16 \mathrm{E}-05$ & WSRC, 1993a \\
\hline $\mathrm{FCH}-2$ & 229.4 & 230.9 & $1.60 \mathrm{E}-04$ & & WSRC, 1993a \\
\hline FCH-3 & 40.8 & 41.9 & $1.46 \mathrm{E}-04$ & $2.90 \mathrm{E}-04$ & WSRC, 1993a \\
\hline FCH-3 & 104.8 & 107.8 & $6.64 \mathrm{E}-05$ & & WSRC, 1993a \\
\hline FCH-3 & 130.9 & 132.2 & 1.52E-03 & $1.60 \mathrm{E}-03$ & WSRC, 1993a \\
\hline FCH-3 & 174.8 & 176 & $1.55 \mathrm{E}-03$ & & WSRC, 1993a \\
\hline FCH-3 & 199.2 & 202.2 & $6.36 \mathrm{E}-05$ & $6.90 \mathrm{E}-05$ & WSRC, 1993a \\
\hline FCH-3 & 265.1 & 266.2 & $2.05 \mathrm{E}-04$ & & WSRC, 1993a \\
\hline FCH-4 & 33.1 & 34.7 & $9.20 \mathrm{E}-05$ & $2.24 \mathrm{E}-04$ & WSRC, 1993a \\
\hline $\mathrm{FCH}-4$ & 103.9 & 106.9 & $2.98 \mathrm{E}-05$ & & WSRC, 1993a \\
\hline FCH-4 & 120 & 122.5 & $1.62 \mathrm{E}-05$ & $2.11 \mathrm{E}-05$ & WSRC, 1993a \\
\hline FCH-4 & 174.9 & 177.4 & $1.86 \mathrm{E}-05$ & & WSRC, 1993a \\
\hline $\mathrm{FCH}-4$ & 190.5 & 193.5 & $2.67 \mathrm{E}-05$ & $3.27 \mathrm{E}-05$ & WSRC, 1993a \\
\hline $\mathrm{FCH}-4$ & 262.7 & 264.3 & $1.58 \mathrm{E}-04$ & & WSRC, 1993a \\
\hline FCH-5 & 90 & 93 & $3.01 \mathrm{E}-05$ & & WSRC, 1993a \\
\hline FCH-5 & 122.2 & 124.2 & $1.10 \mathrm{E}+00$ & $1.52 \mathrm{E}+00$ & WSRC, 1993a \\
\hline FCH-5 & 160 & 162 & $1.31 \mathrm{E}+00$ & & WSRC, 1993a \\
\hline FCH-5 & 191.2 & 194.2 & $2.76 \mathrm{E}-05$ & $3.24 \mathrm{E}-05$ & WSRC, 1993a \\
\hline FCH-5 & 259 & 259.5 & $1.15 \mathrm{E}-04$ & & WSRC, 1993a \\
\hline FCH-6 & 105.7 & 108.2 & $1.86 \mathrm{E}-04$ & & WSRC, 1993a \\
\hline
\end{tabular}


WSRC-TR-99-00248, Rev. 0, Regional Groundwater Flow Model for C, K, L, and P Reactor Areas C-9

Appendix C-3. Permeability Values Recorded from Laboratory Tests (Continued)

\begin{tabular}{|c|c|c|c|c|c|}
\hline Well ID & $\begin{array}{c}\text { Interval Top } \\
\text { (ft b.g.l.) }\end{array}$ & $\begin{array}{c}\text { Interval } \\
\text { Bottom } \\
\text { (ft b.g.l.) }\end{array}$ & $\begin{array}{c}\text { Permeability- } \\
\text { vertical } \\
\text { (ft/day) }\end{array}$ & $\begin{array}{c}\text { Permeability- } \\
\text { horizontal } \\
\text { (ft/day) }\end{array}$ & Reference $^{1}$ \\
\hline
\end{tabular}

\begin{tabular}{|c|c|c|c|c|c|}
\hline FCH-6 & $\therefore \times 170.7$ & 171.7 & $1.11 \mathrm{E}-03$ & $\therefore$ & : WSRC, 1993a \\
\hline FCH-6 & 268.2 & 269.7 & $9.40 \mathrm{E}-04$ & & WSRC, 1993a \\
\hline FIW-1MC & 104 & 106 & $4.30 \mathrm{E}-05$ & 1.50E-03 & AT\&E, 1992 \\
\hline FIW-2MA & 103.5 & 105.5 & $3.70 \mathrm{E}-05$ & $2.30 \mathrm{E}-02$ & AT\&E, 1992 \\
\hline FIW-2MA & 171.2 & 172 & $2.10 \mathrm{E}-05$ & $4.30 \mathrm{E}-05$ & AT\&E, 1992 \\
\hline FSB-89C & 97 & 97.7 & $8.83 \mathrm{E}-05$ & & AT\&E, 1987 \\
\hline FSB-91C & 110 & 110.7 & $5.40 \mathrm{E}-05$ & & AT\&E, 1987 \\
\hline FSB-96A & 117 & 119.5 & & $8.43 \mathrm{E}-04$ & RUST, 1994 \\
\hline FSB-96A & 172 & 173 & & $1.05 \mathrm{E}-05$ & RUST, 1994 \\
\hline FSB-97A & 126.33 & 127.08 & 1.15E-04 & & AT\&E, 1987 \\
\hline FSB-97A & 127.5 & 128.25 & $1.87 \mathrm{E}-05$ & & AT\&E, 1987 \\
\hline FSB-101A & 166.5 & 167 & $3.81 \mathrm{E}-06$ & & AT\&E, 1987 \\
\hline FSB-114A & 138.5 & 140 & & $5.96 \mathrm{E}-05$ & WEGS, 1991 \\
\hline FSB-120A & 168 & 168.4 & & $8.50 \mathrm{E}-03$ & WEGS, 1991 \\
\hline FSB-122C & 70 & 72 & 9.00E-04 & & WEGS, 1991 \\
\hline $\mathrm{HCH}-1$ & 55 & 58 & $2.28 \mathrm{E}-01$ & $2.58 \mathrm{E}-01$ & WSRC, 1993a \\
\hline $\mathrm{HCH}-1$ & 155 & 156.5 & $4.32 \mathrm{E}-02$ & $1.01 \mathrm{E}-01$ & WSRC, 1993a \\
\hline $\mathrm{HCH}-1$ & 270 & 273 & 4.54E-05 & 5.42E-05 & WSRC, 1993a \\
\hline $\mathrm{HCH}-2$ & 65 & 68 & $1.37 \mathrm{E}-01$ & $1.79 \mathrm{E}-01$ & WSRC, 1993a \\
\hline $\mathrm{HCH}-2$ & 145 & 148 & 1.47E-04 & $1.76 \mathrm{E}-04$ & WSRC, 1993a \\
\hline $\mathrm{HCH}-2$ & 276.3 & 278 & $3.12 \mathrm{E}-04$ & $4.63 \mathrm{E}-04$ & WSRC, 1993a \\
\hline $\mathrm{HCH}-3$ & 85 & 88 & $1.54 \mathrm{E}-03$ & $2.09 \mathrm{E}-03$ & WSRC, 1993a \\
\hline $\mathrm{HCH}-3$ & 140 & 141.5 & $1.10 \mathrm{E}-03$ & $1.16 \mathrm{E}-03$ & WSRC, 1993a \\
\hline $\mathrm{HCH}-3$ & 255 & 258 & $4.80 \mathrm{E}-03$ & $1.10 \mathrm{E}-02$ & WSRC, 1993a \\
\hline $\mathrm{HCH}-4$ & 80 & 83 & $7.10 \mathrm{E}-05$ & $8.80 \mathrm{E}-05$ & WSRC, 1993a \\
\hline HCH-4 & 230 & 231.6 & $2.52 \mathrm{E}-04$ & $3.46 \mathrm{E}-04$ & WSRC, 1993a \\
\hline $\mathrm{HCH}-5$ & 140 & 142.5 & $1.23 \mathrm{E}-01$ & $1.58 \mathrm{E}-01$ & WSRC, 1993a \\
\hline $\mathrm{HCH}-5$ & 271.6 & 273.4 & $2.75 \mathrm{E}-04$ & $3.24 \mathrm{E}-04$ & WSRC, 1993a \\
\hline HIW-1MC & 79 & 81 & $5.40 \mathrm{E}-04$ & $1.40 \mathrm{E}-02$ & AT\&E, 1992 \\
\hline HIW-2A & 78 & 80 & $1.02 \mathrm{E}-05$ & $1.14 \mathrm{E}-03$ & RUST, 1994 \\
\hline HIW-2A & 165 & 165.4 & $2.84 \mathrm{E}-04$ & $3.41 \mathrm{E}-04$ & RUST, 1994 \\
\hline HMD-1C & 132 & 134 & $1.33 \mathrm{E}-06$ & $5.96 \mathrm{E}-06$ & WSRC, 1991b \\
\hline HMD-2C & 117 & 118.6 & $1.14 \mathrm{E}-06$ & 5.40E-06 & WSRC, 1991b \\
\hline HMD-3C & 107.3 & 108.6 & $1.90 \mathrm{E}-03$ & $3.41 \mathrm{E}-03$ & WSRC, 1991b \\
\hline HMD-4C & 29 & 31 & $9.09 \mathrm{E}-03$ & $7.67 \mathrm{E}-02$ & WSRC, 1991b \\
\hline HSB-TB & 112 & 112.6 & $5.30 \mathrm{E}-01$ & 1.97E-01 & Core Laboratories, 1995 \\
\hline HSB-TB & 127 & 127.3 & $1.40 \mathrm{E}-01$ & 5.29E-01 & Core Laboratories, 1995 \\
\hline HSB-TB & 151 & 151.3 & $4.00 \mathrm{E}-02$ & $1.00 \mathrm{E}-01$ & Core Laboratories, 1995 \\
\hline HSB-TB & 154.4 & 154.6 & & $1.73 E+00$ & Core Laboratories, 1995 \\
\hline HSB-69A & 120 & 120.8 & 1.52E-04 & & AT\&E, 1988 \\
\hline HSB-107C & 60.9 & 62 & $1.75 \mathrm{E}-04$ & & WSRC, 1990a \\
\hline HSB-117A & 111.67 & 112.34 & $1.81 \mathrm{E}-03$ & & AT\&E, 1988 \\
\hline HSB-118A & 128 & 129 & $1.57 \mathrm{E}-05$ & & WSRC, 1991c \\
\hline HSB-119A & 141.1 & 141.5 & $9.34 \mathrm{E}-04$ & & WSRC, 1991c \\
\hline
\end{tabular}


C-10 WSRC-TR-99-00248, Rev. 0, Regional Groundwater Flow Model for C, K, L, and P Reactor Areas

Appendix C-3. Permeability Values Recorded from Laboratory Tests (Continued)

\begin{tabular}{|c|c|c|c|c|c|}
\hline Well I & $\begin{array}{c}\text { Interval Top } \\
\text { (ft b.g.l.) }\end{array}$ & $\begin{array}{c}\text { Interval } \\
\text { Bottom } \\
\text { (ft b.g.l.) }\end{array}$ & $\begin{array}{c}\text { Permeability- } \\
\text { vertical } \\
\text { (ft/day) }\end{array}$ & $\begin{array}{c}\text { Permeability- } \\
\text { horizontal } \\
\text { (ft/day) }\end{array}$ & Reference $^{\mathbf{1}}$ \\
\hline
\end{tabular}

\begin{tabular}{|c|c|c|c|c|c|}
\hline HSB-120A & 155 & 155.5 & $2.72 \mathrm{E}-03$ & & WSRC, 1991c \\
\hline HSB-121A & 164 & 165 & $7.51 \mathrm{E}-05$ & & WSRC, 1991c \\
\hline HSB-122A & 65 & 65.5 & $7.40 \mathrm{E}-04$ & & WSRC, 1991c \\
\hline HSB-122A & 161 & 161.5 & $1.79 \mathrm{E}-05$ & & WSRC, 1991c \\
\hline HSB-123A & 150.5 & 151.5 & $6.69 \mathrm{E}-04$ & & WSRC, 1991c \\
\hline HSB-139A & 115 & 115.6 & $2.20 \mathrm{E}-04$ & & AT\&E, 1988 \\
\hline HSB-140A & 124 & 126 & $1.64 \mathrm{E}-04$ & & WEGS, 1991 \\
\hline HSB-146A & 131 & 132 & $3.40 \mathrm{E}-04$ & & WEGS, 1991 \\
\hline HSB-148C & 66 & 68 & $1.09 \mathrm{E}-03$ & & WEGS, 1991 \\
\hline IDP-3A & 261.25 & 262.75 & $3.41 \mathrm{E}-02$ & & Law Engineering, 1988 \\
\hline IDQ-3A & 49 & 51 & 2.04E-04 & & Law Engineering, 1988 \\
\hline IDQ-3A & 75 & 77 & $7.83 \mathrm{E}-03$ & & Law Engineering, 1988 \\
\hline IDQ-3A & 172 & 174 & $4.83 \mathrm{E}-05$ & & Law Engineering, 1988 \\
\hline LCO-5A & 18.1 & 19.9 & $9.94 \mathrm{E}-04$ & $3.41 \mathrm{E}-03$ & WSRC, 1996a \\
\hline MWD-1A & 115.5 & 116 & 7.04E-05 & 2.69E-04 & AT\&E, 1988 \\
\hline OFS-1SB & 67 & 68.65 & $3.41 \mathrm{E}-05$ & & Amidon, 1995 \\
\hline OFS-2SB & 108 & 108.3 & & $3.60 \mathrm{E}-02$ & Core Laboratories, 1995 \\
\hline OFS-2SB & 114 & 114.2 & $7.60 \mathrm{E}-02$ & $1.32 \mathrm{E}-03$ & Core Laboratories, 1995 \\
\hline OFS-3SB & 63 & 65 & $6.82 \mathrm{E}-05$ & $1.70 \mathrm{E}-01$ & Amidon, 1995 \\
\hline OFS-3SB & 135 & 137 & 4.80E-06 & $8.00 \mathrm{E}-06$ & Amidon, 1995 \\
\hline OFS-4SB & 25.2 & 25.5 & & $6.04 \mathrm{E}+00$ & Core Laboratories, 1995 \\
\hline OFS-4SB & 45 & 47 & $6.25 \mathrm{E}-04$ & $5.40 \mathrm{E}-03$ & Amidon, 1995 \\
\hline OFS-4SB & 47.5 & 47.8 & & $7.50 \mathrm{E}-01$ & Core Laboratories, 1995 \\
\hline OFS-4SB & 56 & 56.3 & $2.77 \mathrm{E}+01$ & $5.80 \mathrm{E}+00$ & Core Laboratories, 1995 \\
\hline OFS-4SB & 78 & 78.4 & & $2.17 \mathrm{E}-01$ & Core Laboratories, 1995 \\
\hline OFS-4SB & 85.6 & 86 & $1.60 \mathrm{E}-03$ & & Core Laboratories, 1995 \\
\hline OFS-4SB & 98.4 & 98.75 & 5.37E-03 & $1.80 \mathrm{E}-01$ & Core Laboratories, 1995 \\
\hline OFS-4SB & 109 & 109.35 & $1.89 \mathrm{E}-01$ & & Core Laboratories, 1995 \\
\hline OFS-4SB & 114.5 & 115 & $1.93 \mathrm{E}-03$ & & Core Laboratories, 1995 \\
\hline OFS-4SB & 126.4 & 126.7 & & $2.49 \mathrm{E}+00$ & Core Laboratories, 1995 \\
\hline OFS-4SB & 135 & 136.5 & $1.14 \mathrm{E}-05$ & $6.53 \mathrm{E}-05$ & Amidon, 1995 \\
\hline OFS-4SB & 143 & 143.3 & & $7.72 \mathrm{E}+00$ & Core Laboratories, 1995 \\
\hline OFS-4SB & 167 & 167.3 & & $2.41 \mathrm{E}+01$ & Core Laboratories, 1995 \\
\hline OFS-4SB & 175.7 & 176 & & $3.18 \mathrm{E}+01$ & Core Laboratories, 1995 \\
\hline OFS-5SB & 27 & 28.9 & $1.42 \mathrm{E}+00$ & $1.42 \mathrm{E}+00$ & Amidon, 1995 \\
\hline OFS-5SB & 56.5 & 56.8 & & $1.11 \mathrm{E}+01$ & Core Laboratories, 1995 \\
\hline OFS-5SB & 108 & 108.3 & 4.27E-01 & $1.22 \mathrm{E}-01$ & Core Laboratories, 1995 \\
\hline OFS-5SB & 108.6 & 109.6 & $4.50 \mathrm{E}-06$ & $1.79 \mathrm{E}-05$ & Amidon, 1995 \\
\hline OFS-5SB & 129.5 & 129.8 & $3.62 \mathrm{E}+01$ & $2.92 \mathrm{E}+01$ & Core Laboratories, 1995 \\
\hline OFS-5SB & 158.2 & 159.2 & & $3.26 \mathrm{E}+01$ & Core Laboratories, 1995 \\
\hline $\mathrm{P}-18 \mathrm{TA}$ & 180 & 182 & $7.60 \mathrm{E}-05$ & $5.60 \mathrm{E}-05$ & Bledsoe, 1987 \\
\hline P-18TA & 261 & 263 & $3.90 \mathrm{E}-02$ & $8.80 \mathrm{E}-02$ & Bledsoe, 1987 \\
\hline P-18TA & 410 & 412 & $9.00 \mathrm{E}-05$ & $1.00 \mathrm{E}-04$ & Bledsoe, 1987 \\
\hline P-18TA & 643 & 645 & $6.80 \mathrm{E}-05$ & & Bledsoe, 1987 \\
\hline
\end{tabular}


WSRC-TR-99-00248, Rev. 0, Regional Groundwater Flow Model for C, K, L, and P Reactor Areas

C-11

Appendix C-3. Permeability Values Recorded from Laboratory Tests (Continued)

\begin{tabular}{|c|c|c|c|c|c|}
\hline Well ID & $\begin{array}{c}\text { Interval Top } \\
\text { (ft b.g.I.) }\end{array}$ & $\begin{array}{c}\text { Interval } \\
\text { Bottom } \\
\text { (ft b.g.l.) }\end{array}$ & $\begin{array}{c}\text { Permeability- } \\
\text { vertical } \\
\text { (ft/day) }\end{array}$ & $\begin{array}{c}\text { Permeability- } \\
\text { horizontal } \\
\text { (ft/day) }\end{array}$ & Reference \\
\hline
\end{tabular}

\begin{tabular}{|c|c|c|c|c|c|}
\hline P-19TA & 190 & 192.9 & $3.40 \mathrm{E}-05$ & $7.90 \mathrm{E}-03$ & Bledsoe, 1987 \\
\hline P-19TA & 282 & 283 & $9.60 \mathrm{E}-03$ & $9.90 \mathrm{E}-02$ & Bledsoe, 1987 \\
\hline$\overline{\text { P-19TA }}$ & 355 & 358 & $3.90 \mathrm{E}-05$ & 5.90E-05 & Bledsoe, 1987 \\
\hline P-19TA & 495 & 497 & $8.50 \mathrm{E}-05$ & $6.50 \mathrm{E}-03$ & Bledsoe, 1987 \\
\hline P-19TA & 548 & 550 & 1.90E-02 & $7.90 \mathrm{E}-03$ & Bledsoe, 1987 \\
\hline P-21TA & 160 & 162 & $1.90 \mathrm{E}-03$ & 1.70E-02 & PSI, 1986 \\
\hline P-21TA & 325 & 327 & $3.40 \mathrm{E}-01$ & $1.50 \mathrm{E}+00$ & PSI, 1986 \\
\hline P-21TA & 380 & 382 & $5.40 \mathrm{E}-02$ & 6.80E-01 & PSI, 1986 \\
\hline P-21TA & 495 & 497 & $6.30 \mathrm{E}-04$ & $7.70 \mathrm{E}-04$ & PSI, 1986 \\
\hline P-21TA & 522 & 524 & $1.80 \mathrm{E}-05$ & $2.80 \mathrm{E}-05$ & PSI, 1986 \\
\hline P-21TA & 560 & 562 & $9.40 \mathrm{E}-05$ & $8.50 \mathrm{E}-05$ & PSI, 1986 \\
\hline P-22TA & 61 & 63 & $4.80 \mathrm{E}-04$ & 9.70E-04 & PSI, 1986 \\
\hline P-22TA & 140 & 142 & $3.70 \mathrm{E}-04$ & $1.90 \mathrm{E}-04$ & PSI, 1986 \\
\hline P-22TA & 331 & 333 & $1.40 \mathrm{E}-04$ & $1.20 \mathrm{E}-04$ & Bledsoe, 1987 \\
\hline P-22TA & 390 & 392 & $1.02 \mathrm{E}-03$ & & PSI, 1986 \\
\hline P-22TA & 612 & 614 & $1.20 \mathrm{E}-04$ & $2.80 \mathrm{E}-04$ & PSI, 1986 \\
\hline P-23TA & 97 & 99 & $3.60 \mathrm{E}-04$ & & Bledsoe, 1987 \\
\hline P-23TA & 185 & 187 & $9.60 \mathrm{E}-05$ & $1.10 \mathrm{E}-04$ & Bledsoe, 1987 \\
\hline P-23TA & 224 & 226 & $4.20 \mathrm{E}-05$ & $3.60 \mathrm{E}-05$ & Bledsoe, 1987 \\
\hline P-23TA & 301 & 303 & $3.40 \mathrm{E}-05$ & 1.10E-01 & Bledsoe, 1987 \\
\hline P-23TA & 361 & 363 & $9.60 \mathrm{E}-04$ & & Bledsoe, 1987 \\
\hline P-23TA & 401 & 403 & $1.10 \mathrm{E}-04$ & $2.40 \mathrm{E}-04$ & Bledsoe, 1987 \\
\hline YSC-1A & 65 & 67 & $7.38 \mathrm{E}-05$ & $8.24 \mathrm{E}-05$ & WEGS, 1990 \\
\hline YSC-1A & 113.1 & 113.7 & $1.48 \mathrm{E}-04$ & $4.54 \mathrm{E}-05$ & WEGS, 1990 \\
\hline YSC-1C & 59 & 60.9 & $1.25 \mathrm{E}-05$ & $5.96 \mathrm{E}-04$ & WEGS, 1990 \\
\hline YSC-1C & 113 & 114.5 & $2.58 \mathrm{E}-04$ & $3.98 \mathrm{E}-05$ & WEGS, 1990 \\
\hline YSC-2A & 121.8 & 122.6 & 2.61E-06 & & WEGS, 1990 \\
\hline YSC-3SB & 69 & 71 & $1.28 \mathrm{E}-05$ & $1.45 \mathrm{E}-05$ & WEGS, 1990 \\
\hline YSC-3SB & 127 & 128 & $4.54 \mathrm{E}-06$ & 5.11E-05 & WEGS, 1990 \\
\hline YSC-4A & 71.3 & 72 & $1.73 \mathrm{E}-03$ & $8.80 \mathrm{E}-05$ & WEGS, 1990 \\
\hline YSC-4A & 130.3 & 130.8 & $1.82 \mathrm{E}-03$ & & WEGS, 1990 \\
\hline YSC-4A & 140 & 141.1 & $2.67 \mathrm{E}-06$ & & WEGS, 1990 \\
\hline YSC-5A & 54 & 55 & $1.62 \mathrm{E}-05$ & $4.26 \mathrm{E}-05$ & WEGS, 1990 \\
\hline YSC-5A & 108.3 & 108.6 & $1.16 \mathrm{E}-04$ & $5.34 \mathrm{E}-02$ & Core Laboratories, 1995 \\
\hline YSC-5A & 110.3 & 110.6 & $1.43 \mathrm{E}-01$ & $1.88 \mathrm{E}-01$ & Core Laboratories, 1995 \\
\hline YSC-5A & 136 & 137 & $1.39 \mathrm{E}-03$ & $1.59 \mathrm{E}-05$ & WEGS, 1990 \\
\hline
\end{tabular}

Notes:

ft b.g.l. - feet below ground level

$\mathrm{ft} /$ day - feet per day

1 - Detailed description of references in Appendix D 
This page intentionally left blank 


\section{APPENDIX D. DATA SOURCES}




\section{This page intentionally left blank}




\section{APPENDIX D-1. DATA SOURCES}

Albenesius, P. W., Amidon, M. A., and Gaughan, T. F., 1990, HSB 84 A Pumping Test, WSRC-TR-90-0313, Westinghouse Savannah River Company, Aiken, SC 29808.

Amidon, M. A., 1995, Burial Ground Complex Field Investigation Preliminary Data Report \#1, WSRC-RP-94-1286, Westinghouse Savannah River Company, Aiken, SC 29808.

AT\&E, 1987, Laboratory Permeability Test Results “F”' Area Seepage Basins Job No. 7781, Report No. 1721, Atlanta Testing and Engineering, Duluth, GA.

AT\&E, 1988, Report of Laboratory Testing, Savannah River Plant, Job No. 7778-C, Report No. 3473, Atlanta Testing and Engineering, Duluth, GA.

AT\&E, 1992, F\&H-Area Seepage Basins Injection Wells/G2049, Soil Laboratory Testing, Job Number 11360, Report Number 63535, Atlanta Testing and Engineering, Duluth, GA.

Bechtel, 1982, Vogtle Electric Generating Plant, Studies of Postulated Millett Fault, Bechtel.

Bledsoe, H.W., 1987, Savannah River Plant Baseline Hydrogeologic Investigation - Phase II, DPST-86-674, E.I. DuPont de Nemours and Company, Savannah River Laboratory, Aiken, SC.

CH2M Hill, 1989, Congaree Aquifer Test Savannah River Site, CH2M Hill, Reston, VA.

Core Laboratories, 1995, Core Analysis Report, Savannah River Site, Core Laboratories, Carrollton, TX.

D'Appolonia, 1980, Data Report Away from Reactor Spent Fuel Storage Facility, D'Appolonoia.

Evans, E. K. and Parizek, R. R., 1991, Characterization of Hydraulic Conductivity Heterogeneity in Tertiary Sediments within the General Separations Area, Pennsylvania State University, Department of Geosciences.

Falls, W. F., Baum, J. S., Harrelson, L. G., Brown, L. H., and Jerden, J. L., 1997, Geology and Hydrogeology of Cretaceous and Tertiary Strata, and Confinement in the Vicinity of the U. S. Department of Energy Savannah River Site, South Carolina and Georgia: U. S. Geological Survey Water-Resources Investigations Report 97-4245, U. S. Geological Survey, Columbia, SC. 


\section{APPENDIX D-1. DATA SOURCES (Continued)}

Gregg, 1998a, Presentation of Cone Penetrometer Test Data, 131-C Reactor Seepage Basin Characterization, Task \#15, Subcontract AA8227N, submitted by Gregg In Situ, Inc. on September 14, 1998, Gregg In Situ, Inc., Aiken, SC

Gregg, 1998b, Presentation of Cone Penetrometer Test Data, Reactor Areas Characterization, Task \#14, Subcontract AA8227N, submitted by Gregg In Situ, Inc. on September 14, 1998, Gregg In Situ, Inc., Aiken, SC

Law Engineering, 1988, Results of Soil Testing, Purchase Order Number: AX0798832, Law Engineering, Atlanta, GA.

Law Engineering, 1998, Transmittal of Test Results: C Reactor Seepage Basins Geotechnical Testing Services WSRC Site Wide - Task Release No. 13, Law Engineering, Atlanta, GA.

Law Environmental, 1991, Savannah River Site Heavy Water Reactor Facility Phase I Geotechnical Study, Law Project No. 11-0674, submitted by Law Environmental on May 17, 1991.

PSI, 1986, Monitor Wells Hydrogeologic Study, Phase II, Savannah River Plant, Aiken, South Carolina, Service Subcontract AX-0681729, Professional Service Industries, Inc., Oak Brook, IL.

Rust, 1994, Central Shops Burning/Rubble Pit 631-6G Soil Sampling Report (U), WSRCRP-94-0382, Rust Environment and Infrastructure Inc., Aiken, SC.

Rust, 1996, Burial Ground Complex Field Investigation Preliminary Data Report \#2, WSRC-RP-96-060, Rust Environment and Infrastructure Inc., Aiken, SC.

S\&ME, 1988, Borehole Permeability Analyses Burial Ground Perimeter Wells, Soil and Materials Engineering, Inc., Columbia, SC 29203.

Sirrine, 1987, F-Area Compliance Monitoring Program Field Permeability Tests, Sirrine Environmental Consultants, Greenville, SC.

Sirrine, 1988, Field Permeability Tests, H-Area Seepage Basins, Sirrine Environmental Consultants, Greenville, SC.

WEGS, 1990, Hydrogeologic Characterization of the Proposed Waste Solidification and Disposal Facility Site, Y-Area, Westinghouse Environmental and Geotechnical Services, Inc., Columbia, SC 29203. 


\section{APPENDIX D-1. DATA SOURCES (Continued)}

WEGS, 1991, Summary of Well Installations and Aquifer Test Results $F$ and $H$ Seepage Basins Assessment (U), Westinghouse Environmental and Geotechnical Services, Inc., Columbia, SC 29203.

Woodward-Clyde Consultants, 1985a, F-Area Seepage Basins Site Assessment Report Part 1: Groundwater, Woodward-Clyde Consultants, Plymouth Meeting, Pennsylvania.

Woodward-Clyde Consultants, 1985b, H-Area Seepage Basins Site Assessment Report Part 1: Groundwater, Woodward-Clyde Consultants, Plymouth Meeting, Pennsylvania.

WSRC, 1989, Deep Corehole Project, Basic Data Report, Westinghouse Savannah River Company, Aiken, SC 29808.

WSRC, 1990a, Application for a Hazardous Waste Part B Post Closure Care Permit Volume V, Book 2, Westinghouse Savannah River Company, Aiken, SC 29808.

WSRC, 1990b, Design of Three Production Wells for P-Area, H-Area, and Central Shops, WSRC-RP-90-786, Westinghouse Savannah River Company, Aiken, SC 29808.

WSRC, 1991a, Burial Ground Expansion(U) Hydrogeologic Characterization, WSRC-RP91-0866, Westinghouse Savannah River Company, Aiken, SC 29808.

WSRC, 1991b, Hydrogeologic Characterization Hazardous Waste/Mixed Waste Disposal Facility, WSRC-RP-91-0423, Westinghouse Savannah River Company, Aiken, SC 29808.

WSRC, 1991c, RCRA/CERCLA Integration Document for the H-Area Seepage Basins Groundwater Unit, WSRC-RP-91-1019, Westinghouse Savannah River Company, Aiken, SC 29808.

WSRC, 1991d, RCRA/CERCLA Integration Document for the F-Area Seepage Basins Groundwater Unit, WSRC-RP-91-1018, Westinghouse Savannah River Company, Aiken, SC 29808.

WSRC, 1991e, Sanitary Landfill Hydrogeologic Characterization, WSRC-TR-91-0565, Westinghouse Savannah River Company, Aiken, SC 29808.

WSRC, 1992a, Mixed Waste Management Facility and Old Burial Groundwater Monitoring Assessment Well Installations, WSRC-RP-92-0195, Westinghouse Savannah River Company, Aiken, SC 29808.

WSRC, 1992b, Hydrogeology of the F-Area and K-Area Acid Caustic Basins, WSRC-RP91504, Westinghouse Savannah River Company, Aiken, SC 29808. 
APPENDIX D-1. DATA SOURCES (Continued)

WSRC, 1993, F- and H-Areas Inactive Process Sewer Lines Groundwater Assessment (U), WSRC-RP-93-0532, Westinghouse Savannah River Company, Aiken, SC 29808.

WSRC, 1993b, F- and H-Area Tank Farms Preliminary Characterization Part A: Phase 1 Report, WSRC-TR-93-197, Westinghouse Savannah River Company, Aiken, SC 29808.

WSRC, 1994, Confirmatory Drilling Project Final Report (U), WSRC-RP-94-0136, Westinghouse Savannah River Company, Aiken, SC 29808.

WSRC, 1995a, H-Area Tank 16 Monitoring Well Installation Field Report, WSRC-RP-950296, Westinghouse Savannah River Company, Aiken, SC 29808.

WSRC, 1995b, Summary Report for Aquifer Extraction (Pumping) Test Analyses Aquifer IIB, F\&H Area Seepage Basins, Westinghouse Savannah River Company, Aiken, SC 29808.

WSRC, 1996a, RFI/RI Report L-Area Oil and Chemical Basin and L-Area Acid/Caustic Basin, WSRC-RP-95-0305, Westinghouse Savannah River Company, Aiken, SC 29808.

WSRC, 1996b, $L$ - $P$ - and R-Areas Bingham Pump Outage Pits Addendum to the Work Plan for K-Area Bingham Pump Outage Pit, WSRC-RP-96-0173, Westinghouse Savannah River Company, Aiken, SC 29808.

WSRC, 1996c, Field Activities Report, Chemicals, Metals, and Pesticides Pits, Monitoring Well Installation $(U)$, WSRC-RP-96-00121, Westinghouse Savannah River Company, Aiken, SC 29808.

WSRC, 1996d, Environmental Protection Department's Well Inventory (U), ESH-EMS960488, Westinghouse Savannah River Company, Aiken, SC 29808. 


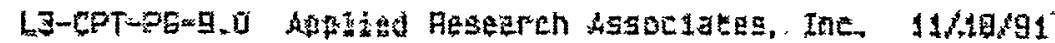

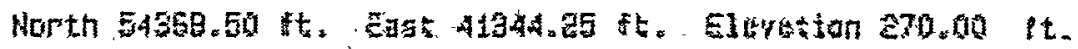
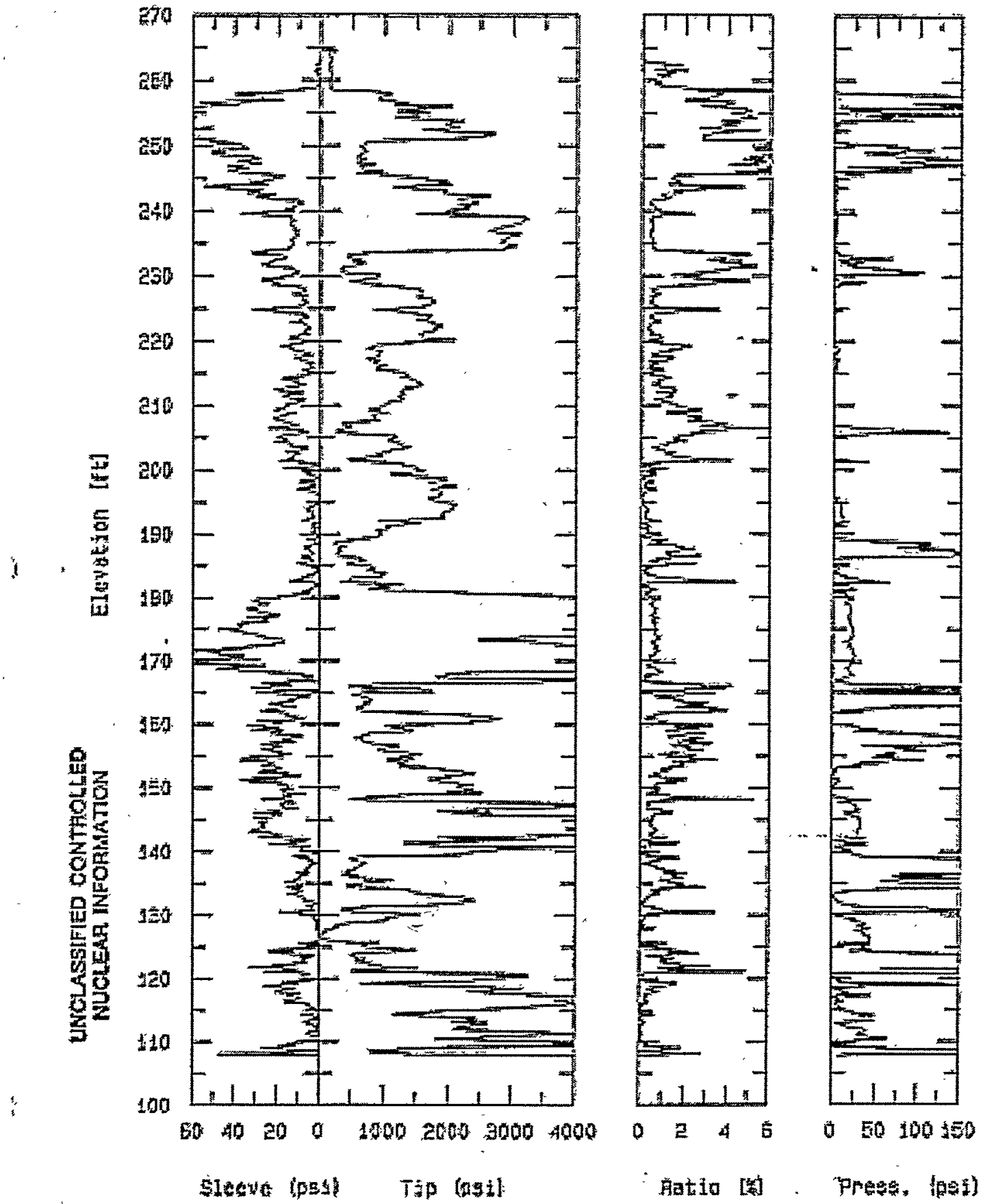

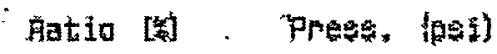


APPENDIX D-2. ARA, 1992, Paper Copies of CPT Data

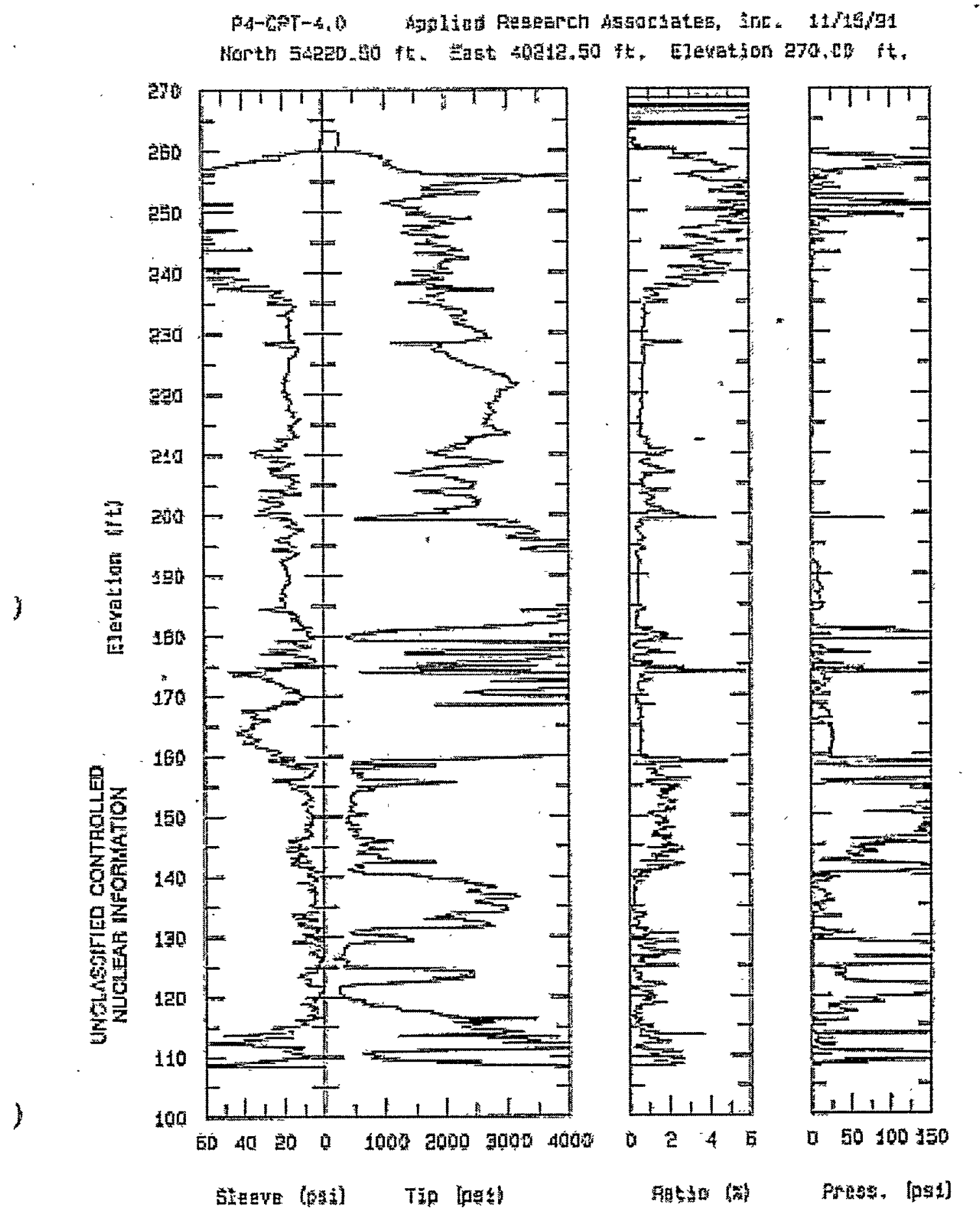




\title{
APPENDIX D-3. ARA, 1996, MWD-C3 and MWD-C5 Raw CPT Data
}

Applied Research Associates, Inc.

Electric Cone Penetrometer Data Cone_TAP v 2.23

\begin{abstract}
Test Id: MWD-C3
Site: SRS "F" AREA S.C.

Date: 02/29/96

Location: EAST OF F AREA IN

Cone Id: 108.013

Project: 5302

OODS

GWT (ft): 60.0

Surface Elev.: 322.5

Soil Density(pcf): 120.0

Northing: 69734.70 Easting: 75249.03
\end{abstract}

Sleeve Tip Stress Tip Stress Ratio Pore

Over- Eff. Wet Class. Class. Blow Depth Elevation Stress UNC COR COR Pressure Excitation burden Overburden Density FR PP Count

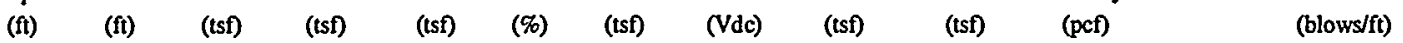

\begin{tabular}{|c|c|c|c|c|c|c|c|c|c|c|c|c|c|}
\hline 0 & 322.5 & 0 & 0 & 0 & 0 & 0 & 4 & 0 & 0 & 120 & -99 & -99 & -99 \\
\hline 0.19 & 322.31 & 0 & 18.9 & 18.9 & 0 & 0.02 & 4.002 & 0.01 & 0.01 & 120 & -99 & -99 & -99 \\
\hline 0.27 & 322.23 & 0 & 23.58 & 23.59 & 0 & 0.02 & 4.002 & 0.02 & 0.02 & 120 & -99 & -99 & -99 \\
\hline 0.35 & 322.15 & 0 & 28.27 & 28.27 & 0.01 & 0.01 & 4.002 & 0.02 & 0.02 & 120 & -99 & -99 & -99 \\
\hline 0.43 & 322.07 & 0.01 & 32.6 & 32.61 & 0.02 & 0.01 & 4.002 & 0.03 & 0.03 & 120 & -99 & -99 & -99 \\
\hline 0.51 & 321,99 & 0.02 & 35.73 & 35.73 & 0.05 & 0.01 & 4.002 & 0.03 & 0.03 & 120 & .99 & -99 & -99 \\
\hline 0.59 & 321.91 & 0.04 & 37.81 & 37.81 & 0.1 & 0.02 & 4.002 & 0.04 & 0.04 & 120 & -99 & -99 & 99 \\
\hline 0.67 & 321,83 & 0.06 & 36.85 & 36.85 & 0.17 & 0.01 & 4.003 & 0.04 & 0.04 & 120 & 7 & 7 & 8 \\
\hline 0.75 & 321.75 & 0.09 & 37.37 & 37.38 & 0.23 & 0.01 & 4.002 & 0.05 & 0.05 & 120 & 7 & 7 & 8 \\
\hline 0.83 & 321.67 & 0.09 & 38.15 & 38.16 & 0.24 & 0.01 & 4.002 & 0.05 & 0.05 & 120 & 7 & 7 & 8 \\
\hline 0.91 & 321.59 & 0.1 & 37.72 & 37.72 & 0.27 & 0.01 & 4.003 & 0.05 & 0.05 & 120 & 7 & 7 & 8 \\
\hline 0.99 & 321.51 & 0.09 & 36.42 & 36.42 & 0.26 & 0.01 & 4.002 & 0.06 & 0.06 & 120 & 7 & 7 & 8 \\
\hline 1.07 & 321.43 & 0.08 & 34.94 & 34.95 & 0.24 & 0.01 & 4.003 & 0.06 & 0.06 & 120 & 7 & 7 & 7 \\
\hline 1.15 & 321.35 & 0.07 & 33.12 & 33.12 & 0.21 & 0.01 & 4.003 & 0.07 & 0.07 & 120 & 7 & 7 & 7 \\
\hline 1.23 & 321.27 & 0.06 & 31.47 & 31.48 & 0.19 & 0.01 & 4.003 & 0.07 & 0.07 & 120 & 7 & 7 & 7 \\
\hline 1.31 & 321.19 & 0.04 & 30.18 & 30.18 & 0.14 & 0.02 & 4.002 & 0.08 & 0.08 & 120 & 7 & 7 & 6 \\
\hline 1.39 & 321.11 & 0.03 & 29.95 & 29.96 & 0.1 & 0.01 & 3.999 & 0.08 & 0.08 & 120 & 7 & 7 & 6 \\
\hline 1.47 & 321.03 & 0.02 & 29.35 & 29.35 & 0.07 & 0.01 & 3.999 & 0.09 & 0.09 & 120 & -99 & 7 & -99 \\
\hline 1.55 & 320.95 & 0.02 & 28 & 28.01 & 0.06 & 0.02 & 4.003 & 0.09 & 0.09 & 120 & -99 & 7 & -99 \\
\hline 1.63 & 320.87 & 0.01 & 27.83 & 27.83 & 0.04 & 0.02 & 4.003 & 0.1 & 0.1 & 120 & -99 & 7 & -99 \\
\hline 1.71 & 320.79 & 0 & 27.74 & 27.75 & 0.02 & 0.02 & 4.003 & 0.1 & 0.1 & 120 & -99 & 7 & -99 \\
\hline 1.79 & 320.71 & 0 & 27.83 & 27.83 & 0.01 & 0.02 & 4.003 & 0.11 & 0.11 & 120 & -99 & 7 & -99 \\
\hline 1.87 & 320.63 & 0 & 27.66 & 27.66 & 0.01 & 0.03 & 4.003 & 0.11 & 0.11 & 120 & -99 & 7 & -99 \\
\hline 1.95 & 320.55 & 0 & 27.57 & 27.57 & 0.01 & 0.02 & 4.003 & 0.12 & 0.12 & 120 & -99 & 7 & -99 \\
\hline 2.03 & 320.47 & 0.01 & 27.39 & 27.4 & 0.02 & 0.01 & 4.003 & 0.12 & 0.12 & 120 & -99 & 7 & -99 \\
\hline 2.11 & 320.39 & 0.01 & 27.48 & 27.48 & 0.04 & 0.01 & 4.003 & 0.13 & 0.13 & 120 & -99 & 7 & -99 \\
\hline 2.19 & 320.31 & 0.02 & 27.65 & 27.66 & 0.06 & 0.01 & 4.003 & 0.13 & 0.13 & 120 & -99 & 7 & -99 \\
\hline 2.27 & 320.23 & 0.02 & 28 & 28 & 0.08 & 0.01 & 4.003 & 0.14 & 0.14 & 120 & -99 & 7 & -99 \\
\hline 2.35 & 320.15 & 0.03 & 28.35 & 28.35 & 0.1 & 0.01 & 4.003 & 0.14 & 0.14 & 120 & 7 & 7 & 6 \\
\hline 2.42 & 320.08 & 0.03 & 29.04 & 29.04 & 0.12 & 0.01 & 4.003 & 0.15 & 0.15 & 120 & 7 & 7 & 6 \\
\hline 2.5 & 320 & 0.04 & 30.17 & 30.17 & 0.14 & 0.01 & 4.003 & 0.15 & 0.15 & 120 & 7 & $\because 7$ & 6 \\
\hline 2.57 & 319.93 & 0.05 & 31.64 & 31.64 & 0.15 & 0.01 & 4.003 & 0.15 & 0.15 & 120 & 7 & 7 & 7 \\
\hline 2.65 & 319.85 & 0.06 & 33.12 & 33.12 & 0.17 & 0.01 & 4.003 & 0.16 & 0.16 & 120 & 7 & 7 & 7 \\
\hline 2.72 & 319.78 & 0.06 & 34.85 & 34.85 & 0.18 & 0.01 & 4.003 & 0.16 & 0.16 & 120 & 7 & 7 & 7 \\
\hline 2,8 & 319.7 & 0.07 & 36.67 & 36.67 & 0.19 & 0.01 & 4.003 & 0.17 & 0.17 & 120 & 7 & 7 & 8 \\
\hline 2.87 & 319.63 & 0.08 & 38.23 & 38.23 & 0.2 & 0.01 & 4.003 & 0.17 & 0.17 & 120 & 7 & 7 & 8 \\
\hline 2.95 & 319.55 & 0.08 & 39.44 & 39.45 & 0.21 & 0.02 & 4.003 & 0.18 & 0.18 & 120 & 7 & 7 & 8 \\
\hline 3.02 & 319.48 & 0.1 & 40.23 & 40.23 & 0.25 & 0.01 & 4.003 & 0.18 & 0.18 & 120 & 7 & 7 & 9 \\
\hline 3.1 & 319.4 & 0.15 & 40.4 & 40.4 & 0.38 & 0.01 & 4.003 & 0.19 & 0.19 & 120 & 6 & 7 & 10 \\
\hline 3.34 & 319.16 & 0.51 & 36.42 & 36.42 & 1.4 & 0.02 & 4.003 & 0.2 & 0.2 & 120 & 6 & 7 & 9 \\
\hline 3.42 & 319.08 & 0.62 & 31.65 & 31.66 & 1.97 & 0.04 & 4.003 & 0.2 & 0.2 & 120 & 6 & 6 & 8 \\
\hline 3.49 & 319.01 & 0.75 & 28.78 & 28.8 & 2.61 & 0.06 & 4.003 & 0.21 & 0.21 & 120 & 5 & 6 & 9 \\
\hline 3.57 & 318.93 & 0.89 & 26.96 & 26.98 & 3.29 & 0.06 & 4.003 & 0.21 & 0.21 & 120 & 5 & 6 & 8 \\
\hline 3.64 & 318.86 & 1 & 26.44 & 26.47 & 3.79 & 0.12 & 4.003 & 0.22 & 0.22 & 120 & 8 & 6 & 18 \\
\hline 3.72 & 318.78 & 1.08 & 23.67 & 23.68 & 4.56 & 0.06 & 4.003 & 0.22 & 0.22 & 120 & 9 & 6 & 16 \\
\hline 3.8 & 318.7 & 1.11 & 21.76 & 21.78 & 5.1 & 0.11 & 4.003 & 0.23 & 0.23 & 120 & 9 & 6 & 15 \\
\hline 3.87 & 318.63 & 1.12 & 20.54 & 20.58 & 5.42 & 0.17 & 4.003 & 0.23 & 0.23 & 120 & 9 & 6 & 14 \\
\hline 3.94 & 318.56 & 1.09 & 18.55 & 18.63 & 5.85 & 0.39 & 4.003 & 0.24 & 0.24 & 120 & 9 & 6 & 12 \\
\hline 4.02 & 318.48 & 1.05 & 18.29 & 18.39 & 5.7 & 0.48 & 4.003 & 0.24 & 0.24 & 120 & 9 & 6 & 12 \\
\hline 4.09 & 318.41 & 1.04 & 18.67 & 18.83 & 5.52 & 0.79 & 3.999 & 0.25 & 0.25 & 120 & 9 & 6 & 12 \\
\hline 4.16 & 318.34 & 1.01 & 18.32 & 18.55 & 5.46 & 1.14 & 3.999 & 0.25 & 0.25 & 120 & 9 & 6 & 12 \\
\hline 4.24 & 318.26 & 0.98 & 17.42 & 17.71 & 5.55 & 1.42 & 4.003 & 0.25 & 0.25 & 120 & 9 & 6 & 12 \\
\hline
\end{tabular}


Applied Research Associates, Inc.

Electric Cone Penetrometer Data

Cone_TAP v 2.23

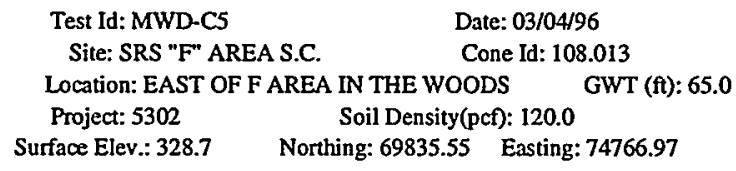

Sleeve Tip Stress Tip Stress Ratio Pore Overburde Eff. Wet Class. Class. Blow

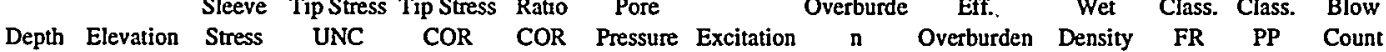

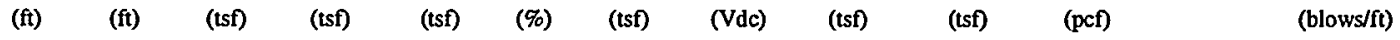

\begin{tabular}{|c|c|c|c|c|c|c|c|c|c|c|c|c|c|}
\hline 0 & 328.7 & 0 & 0 & 0 & 0 & 0 & 4.002 & 0 & 0 & 120 & -99 & -99 & -99 \\
\hline 0.15 & 328.55 & 0 & 9.91 & 9.91 & 0 & 0 & 4.002 & 0.01 & 0.01 & 120 & -99 & .99 & .99 \\
\hline 0.17 & 328.53 & 0 & 10.17 & 10.17 & 0 & 0 & 4.002 & 0.01 & 0.01 & 120 & -99 & -99 & -99 \\
\hline 0.21 & 328.49 & 0 & 10.61 & 10.6 & 0 & 0 & 4.001 & 0.01 & 0.01 & 120 & -99 & 7 & -99 \\
\hline 0.25 & 328.45 & 0 & 13.21 & 13.21 & 0 & 0 & 4.002 & 0.01 & 0.01 & 120 & -99 & 7 & -99 \\
\hline 0.29 & 328.41 & 0 & 16.51 & 16.51 & 0 & 0 & 4.002 & 0.02 & 0.02 & 120 & -99 & 7 & -99 \\
\hline 0.33 & 328.37 & 0 & 20.77 & 20.77 & 0 & 0 & 4.002 & 0.02 & 0.02 & 120 & -99 & -99 & -99 \\
\hline 0.38 & 328.32 & 0 & 25.81 & 25.81 & 0 & 0 & 4.002 & 0.02 & 0.02 & 120 & -99 & -99 & -99 \\
\hline 0.42 & 328.28 & 0 & 30.16 & 30.16 & 0.01 & 0 & 4.002 & 0.03 & 0.03 & 120 & -99 & -99 & -99 \\
\hline 0.46 & 328.24 & 0 & 33.2 & 33.2 & 0.01 & 0 & 4.002 & 0.03 & 0.03 & 120 & -99 & -99 & -99 \\
\hline 0.51 & 328.19 & 0 & 35.81 & 35.81 & 0 & 0 & 4.002 & 0.03 & 0.03 & 120 & -99 & -99 & -99 \\
\hline 0.56 & 328.14 & 0 & 38.33 & 38.33 & 0 & 0 & 4.002 & 0.03 & 0.03 & 120 & -99 & -99 & -99 \\
\hline 0.61 & 328.09 & 0 & 41.02 & 41.02 & 0 & 0 & 4.002 & 0.04 & 0.04 & 120 & -99 & -99 & -99 \\
\hline 0.65 & 328.05 & 0.01 & 44.31 & 44.31 & 0.01 & 0 & 3.997 & 0.04 & 0.04 & 120 & -99 & -99 & -99 \\
\hline 0.7 & 328 & 0.01 & 45.78 & 45.78 & 0.03 & 0 & 3.997 & 0.04 & 0.04 & 120 & -99 & -99 & -99 \\
\hline 0.75 & 327.95 & 0.02 & 46.5 & 46.5 & 0.04 & 0 & 4.002 & 0.05 & 0.05 & 120 & -99 & -99 & -99 \\
\hline 0.8 & 327.9 & 0.02 & 48.06 & 48.06 & 0.05 & 0 & 4.002 & 0.05 & 0.05 & 120 & -99 & 7 & -99 \\
\hline 0.85 & 327.85 & 0.03 & 49.19 & 49.19 & 0.06 & 0 & 4.002 & 0.05 & 0.05 & 120 & -99 & 7 & -99 \\
\hline 0.9 & 327.8 & 0.03 & 49.98 & 49.98 & 0.07 & 0 & 4.002 & 0.05 & 0.05 & 120 & -99 & 7 & -99 \\
\hline 0.95 & 327.75 & 0.04 & 50.32 & 50.32 & 0.07 & 0 & 4.002 & 0.06 & 0.06 & 120 & -99 & 7 & -99 \\
\hline 1.01 & 327.69 & 0.04 & 50.24 & 50.24 & 0.09 & 0 & 4.002 & 0.06 & 0.06 & 120 & -99 & 7 & -99 \\
\hline 1.06 & 327.64 & 0.05 & 50.15 & 50.15 & 0.1 & 0 & 4.002 & 0.06 & 0.06 & 120 & -99 & 7 & -99 \\
\hline 1.12 & 327.58 & 0.05 & 49.8 & 49.8 & 0.11 & 0 & 4.002 & 0.07 & 0.07 & 120 & 7 & 7 & 11 \\
\hline 1.17 & 327.53 & 0.05 & 49.28 & 49.28 & 0.11 & 0 & 4.002 & 0.07 & 0.07 & 120 & 7 & 7 & 11 \\
\hline 1.23 & 327.47 & 0.06 & 48.58 & 48.58 & 0.11 & 0 & 4.002 & 0.07 & 0.07 & 120 & 7 & 7 & 10 \\
\hline 1.28 & 327.42 & 0.06 & 47.8 & 47.8 & 0.12 & 0 & 4.002 & 0.08 & 0.08 & 120 & 7 & 7 & 10 \\
\hline 1.33 & 327.37 & 0.06 & 46.93 & 46.93 & 0.12 & 0 & 4.002 & 0.08 & 0.08 & 120 & 7 & 7 & 10 \\
\hline 1.39 & 327.31 & 0.06 & 45.8 & 45.8 & 0.12 & 0 & 4.002 & 0.08 & 0.08 & 120 & 7 & 7 & 10 \\
\hline 1.44 & 327.26 & 0.06 & 44.85 & 44.85 & 0.13 & 0 & 4.002 & 0.09 & 0.09 & 120 & 7 & 7 & 10 \\
\hline 1.5 & 327.2 & 0.06 & 43.89 & 43.89 & 0.13 & 0 & 4.002 & 0.09 & 0.09 & 120 & 7 & 7 & 9 \\
\hline 1.55 & 327.15 & 0.06 & 43.11 & 43.11 & 0.13 & 0 & 4.002 & 0.09 & 0.09 & 120 & 7 & 7 & 9 \\
\hline 1.61 & 327.09 & 0.05 & 42.15 & 42.15 & 0.13 & 0 & 4.002 & 0.1 & 0.1 & 120 & 7 & 7 & 9 \\
\hline 1.66 & 327.04 & 0.05 & 41.11 & 41.11 & 0.13 & 0 & 4.002 & 0.1 & 0.1 & 120 & 7 & 7 & 9 \\
\hline 1.72 & 326.98 & 0.05 & 40.15 & 40.15 & 0.12 & 0 & 4.002 & 0.1 & 0.1 & 120 & 7 & 7 & 9 \\
\hline 1.78 & 326.92 & 0.04 & 39.11 & 39.11 & 0.11 & 0 & 4.002 & 0.11 & 0.11 & 120 & 7 & 7 & 8 \\
\hline 1.84 & 326.86 & 0.04 & 38.33 & 38.33 & 0.11 & 0 & 4.002 & 0.11 & 0.11 & 120 & 7 & 7 & 8 \\
\hline 1.89 & 326.81 & 0.04 & 37.89 & 37.89 & 0.1 & 0 & 4.002 & 0.11 & 0.11 & 120 & -99 & 7 & .99 \\
\hline 1.95 & 326.75 & 0.04 & 37.28 & 37.28 & 0.09 & 0 & 4.002 & 0.12 & 0.12 & 120 & . -99 & 7 & .99 \\
\hline 2.01 & 326.69 & 0.03 & 37.02 & 37.02 & 0.09 & 0 & 4.002 & 0.12 & 0.12 & 120 & -99 & 7 & -99 \\
\hline 2.07 & 326.63 & 0.03 & 36.93 & 36.94 & 0.09 & 0.01 & 4.002 & 0.12 & 0.12 & 120 & -99 & 7 & -99 \\
\hline 2.13 & 326.57 & 0.03 & 37.11 & 37.11 & 0.09 & 0 & 4.002 & 0.13 & 0.13 & 120 & -99 & 7 & -99 \\
\hline 2.19 & 326.51 & 0.03 & 37.37 & 37.37 & 0.09 & 0 & 4.002 & 0.13 & 0.13 & 120 & -99 & 7 & -99 \\
\hline 2.25 & 326.45 & 0.04 & 37.8 & 37.8 & 0.1 & 0.01 & 4.002 & 0.14 & 0.14 & 120 & -99 & 7 & -99 \\
\hline 2.32 & 326.38 & 0.04 & 38.32 & 38.33 & 0.1 & 0 & 4.002 & 0.14 & 0.14 & 120 & 7 & 7 & 8 \\
\hline 2.38 & 326.32 & 0.04 & 40.94 & 40.94 & 0.1 & 0 & 3.995 & 0.14 & 0.14 & 120 & 7 & 7 & 9 \\
\hline 2.45 & 326.25 & 0.05 & 41.08 & 41.08 & 0.11 & 0 & 3.998 & 0.15 & 0.15 & 120 & 7 & 7 & 9 \\
\hline 2.51 & 326.19 & 0.05 & 40.85 & 40.85 & 0.12 & 0.01 & 4.002 & 0.15 & 0.15 & 120 & 7 & 7 & 9 \\
\hline 2.57 & 326.13 & 0.06 & 42.06 & 42.06 & 0.13 & 0.01 & 4.002 & 0.15 & 0.15 & 120 & 7 & 7 & 9 \\
\hline 2.64 & 326.06 & 0.06 & 43.19 & 43.19 & 0.15 & 0.01 & 4.002 & 0.16 & 0.16 & 120 & 7 & 7 & 9 \\
\hline 2.71 & 325.99 & 0.05 & 44.41 & 44.41 & 0.12 & 0.01 & 4.002 & 0.16 & 0.16 & 120 & 7 & 7 & 10 \\
\hline 2.77 & 325.93 & 0.04 & 45.28 & 45.28 & 0.08 & 0.01 & 4.002 & 0.17 & 0.17 & 120 & -99 & 7 & -99 \\
\hline 2.84 & 325.86 & 0.03 & 45.71 & 45.71 & 0.07 & 0.01 & 4.002 & 0.17 & 0.17 & 120 & -99 & 7 & -99 \\
\hline
\end{tabular}




\author{
Applied Research Associates \\ Electric Cone Penetrometer Data \\ Cone_TAP v 2.51
}

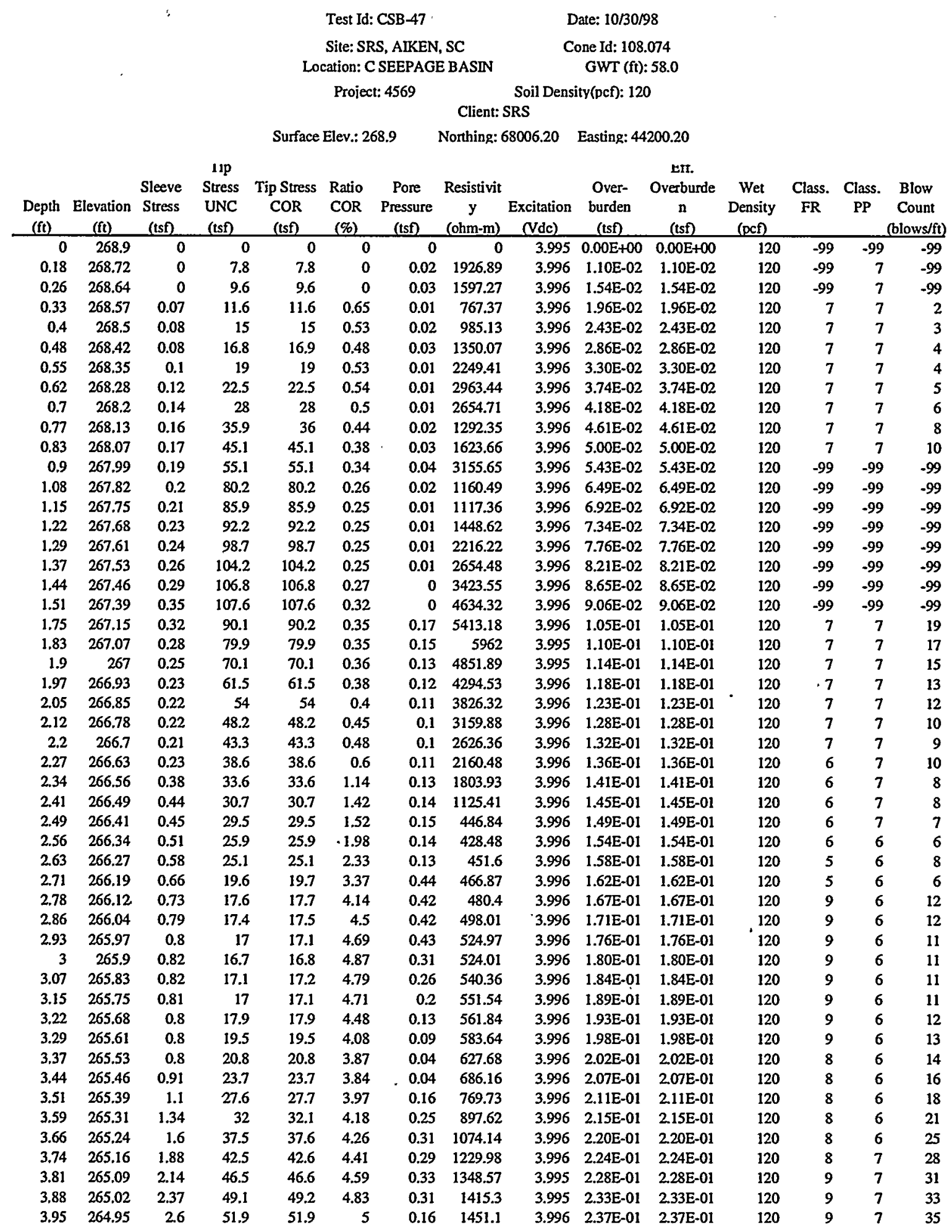


Applied Research Associates

Electric Cone Penetrometer Data Cone_TAP v 2.51

Test Id: CSB-48

Date: $10 / 30 / 98$

Site: SRS, AIKEN, SC

Cone Id: 108.074

Location: C SEEPAGE BASIN

GWT (ft): 55.8

Project: 4569

Soil Density(pcf): 120

Client: SRS

Surface Elev.: $270.1 \quad$ Northing: 68205.20 Easting: 44199.80

\begin{tabular}{|c|c|c|c|c|c|c|c|c|c|c|c|c|c|c|}
\hline $\begin{array}{l}\text { Depth } \\
\text { (ft) }\end{array}$ & $\begin{array}{c}\text { Elevation } \\
\text { (ft) }\end{array}$ & $\begin{array}{c}\text { Sleeve } \\
\text { Stress } \\
\text { (tsf) }\end{array}$ & $\begin{array}{l}\text { l1p } \\
\text { Stress } \\
\text { UNC } \\
\text { (tsf) }\end{array}$ & $\begin{array}{c}\text { Tip Stress } \\
\text { COR } \\
\text { (tsf) }\end{array}$ & $\begin{array}{l}\text { Ratio } \\
\text { COR } \\
(\%) \\
\end{array}$ & $\begin{array}{c}\text { Pore } \\
\text { Pressure } \\
\text { (tsf) }\end{array}$ & $\begin{array}{c}\text { Resistivit } \\
\text { y } \\
\text { (ohm-m) }\end{array}$ & $\begin{array}{c}\text { Excitation } \\
\text { (Vdc) }\end{array}$ & $\begin{array}{c}\text { Over- } \\
\text { burden } \\
\text { (tsf) }\end{array}$ & $\begin{array}{c}\text { EIr. } \\
\text { Overburde } \\
\mathbf{n} \\
\text { (tsf) } \\
\end{array}$ & $\begin{array}{c}\text { Wet } \\
\text { Density } \\
\text { (pcf) }\end{array}$ & $\begin{array}{l}\text { Class. } \\
\text { FR }\end{array}$ & $\begin{array}{c}\text { Class. } \\
\text { PP } \\
\end{array}$ & $\begin{array}{c}\text { Blow } \\
\text { Count } \\
\text { (blows/ft) }\end{array}$ \\
\hline 0 & 270.1 & 0 & 0 & 0 & 0 & 0 & 0 & 4.004 & $0.00 \mathrm{E}+00$ & $0.00 \mathrm{E}+00$ & 120 & -99 & -99 & -99 \\
\hline 0.17 & 269.93 & 0 & 14.2 & 14.2 & 0 & 0 & 0 & 4.004 & $1.01 \mathrm{E}-02$ & $1.01 E-02$ & 120 & -99 & -99 & -99 \\
\hline 0.24 & 269.86 & 0 & 22.1 & 22.1 & 0 & 0.01 & 1109.09 & 4.004 & $1.46 \mathrm{E}-02$ & $1.46 \mathrm{E}-02$ & 120 & -99 & -99 & .99 \\
\hline 0.31 & 269.79 & 0.09 & 28.2 & 28.2 & 0.33 & 0.02 & 1616.47 & 4.004 & $1.89 \mathrm{E}-02$ & $1.89 \mathrm{E}-02$ & 120 & -99 & -99 & -99 \\
\hline 0.39 & 269.71 & 0.11 & 33.3 & 33.3 & 0.33 & 0.01 & 3422.7 & 4.003 & 2.35E-02 & $2.35 \mathrm{E}-02$ & 120 & -99 & -99 & -99 \\
\hline 0.47 & 269.63 & 0.12 & 35.9 & 35.9 & 0.34 & 0.02 & 4918.34 & 4.003 & $2.80 \mathrm{E}-02$ & $2.80 \mathrm{E}-02$ & 120 & -99 & -99 & -99 \\
\hline 0.54 & 269.56 & 0.13 & 37.3 & 37.3 & 0.36 & 0.01 & 4854.76 & 4.004 & $3.24 \mathrm{E}-02$ & $3.24 \mathrm{E}-02$ & 120 & -99 & -99 & -99 \\
\hline 0.62 & 269.48 & 0.14 & 38.3 & 38.3 & 0.36 & 0.01 & 3728.25 & 4.004 & $3.71 \mathrm{E}-02$ & $3.71 \mathrm{E}-02$ & 120 & -99 & -99 & -99 \\
\hline 0.69 & 269.41 & 0.14 & 38.1 & 38.1 & 0.36 & 0 & 3975.84 & 4.004 & $4.15 \mathrm{E}-02$ & 4.15E-02 & 120 & 7 & 7 & 8 \\
\hline 0.76 & 269.34 & 0.13 & 37 & 37 & 0.36 & 0 & 4723.97 & 4.004 & 4.59E-02 & $4.59 \mathrm{E}-02$ & 120 & 7 & 7 & 8 \\
\hline 0.84 & 269.26 & 0.13 & 34.9 & 34.9 & 0.37 & 0.01 & 5951.98 & 4.004 & $5.04 \mathrm{E}-02$ & $5.04 \mathrm{E}-02$ & 120 & 7 & 7 & 7 \\
\hline 0.92 & 269.18 & 0.12 & 32.5 & 32.5 & 0.37 & 0 & 6285.92 & 4.004 & $5.49 \mathrm{E}-02$ & $5.49 \mathrm{E}-02$ & 120 & 7 & 7 & 7 \\
\hline 0.99 & 269.11 & 0.11 & 30.2 & 30.2 & 0.36 & 0.01 & 6114.31 & 4.004 & 5.95E-02 & 5.95E-02 & 120 & 7 & 7 & 6 \\
\hline 1.07 & 269.03 & 0.1 & 28.1 & 28.1 & 0.35 & 0 & 6022.94 & 4.004 & $6.40 \mathrm{E}-02$ & $6.40 \mathrm{E}-02$ & 120 & 7 & 7 & 6 \\
\hline 1.14 & 268.96 & 0.09 & 26.9 & 26.9 & 0.35 & 0 & 5827.38 & 4.004 & $6.83 \mathrm{E}-02$ & $6.83 \mathrm{E}-02$ & 120 & 7 & 7 & 6 \\
\hline 1.21 & 268.89 & 0.1 & 26.3 & 26.3 & 0.39 & 0 & 5956.58 & 4.004 & $7.28 \mathrm{E}-02$ & $7.28 \mathrm{E}-02$ & 120 & 7 & 7 & 6 \\
\hline 1.29 & 268.81 & 0.12 & 26.4 & 26.4 & 0.46 & 0 & 6658.11 & 4.004 & 7.72E-02 & $7.72 \mathrm{E}-02$ & 120 & 7 & 7 & 6 \\
\hline 1.36 & 268.74 & 0.14 & 26.6 & 26.6 & 0.52 & 0.01 & 2446.25 & 4.004 & $8.16 \mathrm{E}-02$ & $8.16 \mathrm{E}-02$ & 120 & 6 & 7 & 7 \\
\hline 1.43 & 268.67 & 0.17 & 26.3 & 26.3 & 0.64 & 0.01 & 1368.89 & 4.004 & 8.59E-02 & $8.59 \mathrm{E}-02$ & 120 & 6 & 7 & 7 \\
\hline 1.51 & 268.59 & 0.23 & 24 & 24 & 0.98 & 0.02 & 1008.66 & 4.004 & 9.04E-02 & $9.04 \mathrm{E}-02$ & 120 & 6 & 7 & 6 \\
\hline 1.75 & 268.35 & 0.53 & 21.3 & 21.3 & 2.5 & 0.01 & 531.4 & 4.004 & $1.05 \mathrm{E}-01$ & 1.05E-01 & 120 & 5 & 7 & 6 \\
\hline 1.82 & 268.28 & 0.61 & 21.1 & 21.1 & 2.88 & 0.02 & 541.5 & 4.004 & $1.09 \mathrm{E}-01$ & $1.09 \mathrm{E}-01$ & 120 & 8 & 7 & 14 \\
\hline 1.9 & 268.2 & 0.68 & 20.8 & 20.8 & 3.29 & 0.02 & 533.82 & 4.004 & 1.14E-01 & $1.14 \mathrm{E}-01$ & 120 & 8 & 7 & 14 \\
\hline 1.97 & 268.13 & 0.73 & 20.6 & 20.6 & 3.55 & 0 & 571.7 & 4.004 & 1.18E-01 & 1.18E-01 & 120 & 8 & 7 & 14 \\
\hline 2.05 & 268.05 & 0.75 & 20.8 & 20.8 & 3.59 & -0.01 & 622.57 & 4.003 & 1.23E-01 & $1.23 \mathrm{E}-01$ & 120 & 8 & 7 & 14 \\
\hline 2.12 & 267.98 & 0.76 & 21.5 & 21.5 & 3.53 & -0.02 & 617.5 & 4.003 & $1.27 \mathrm{E}-01$ & $1.27 \mathrm{E}-01$ & 120 & 8 & 7 & 14 \\
\hline 2.2 & 267.9 & 0.78 & 22.8 & 22.8 & 3.42 & -0.01 & 621.29 & 4.004 & $1.32 \mathrm{E}-01$ & $1.32 \mathrm{E}-01$ & 120 & 8 & 7 & 15 \\
\hline 2.27 & 267.83 & 0.8 & 25.7 & 25.7 & 3.12 & -0.07 & 640.19 & 4.004 & $1.36 \mathrm{E}-01$ & $1.36 \mathrm{E}-01$ & 120 & 8 & 7 & 17 \\
\hline 2.34 & 267.76 & 0.84 & 28.8 & 28.7 & 2.92 & -0.24 & 639.32 & 4.004 & 1.41E-0I & $1.41 \mathrm{E}-01$ & 120 & 8 & 7 & 19 \\
\hline 2.42 & 267.68 & 0.9 & 31.7 & 31.7 & 2.84 & -0.25 & 664.71 & 4.004 & 1.45E-01 & $1.45 \mathrm{E}-01$ & 120 & 8 & 7 & 21 \\
\hline 2.49 & 267.61 & 0.97 & 35.8 & 35.7 & 2.71 & -0.12 & 667.42 & 4.004 & $1.50 \mathrm{E}-01$ & $1.50 \mathrm{E}-01$ & 120 & 8 & 7 & 24 \\
\hline 2.57 & 267.53 & 1.07 & 40.1 & 40 & 2.66 & -0.1 & 709.21 & 4.004 & $1.54 \mathrm{E}-01$ & $1.54 \mathrm{E}-01$ & 120 & 8 & 7 & 27 \\
\hline 2.64 & 267.46 & 1.18 & 44.1 & 44 & 2.68 & -0.34 & 761.87 & 4.004 & $1.59 \mathrm{E}-01$ & $1.59 \mathrm{E}-01$ & 120 & 8 & 7 & 29 \\
\hline 2.71 & 267.39 & 1.31 & 49.5 & 49.4 & 2.66 & -0.32 & 862.69 & 4.004 & 1.63E-01 & $1.63 \mathrm{E}-01$ & 120 & 8 & 7 & 33 \\
\hline 2.79 & 267.31 & 1.49 & 54.9 & 54.8 & 2.71 & -0.4 & 1002.33 & 4.004 & $1.67 \mathrm{E}-01$ & $1.67 \mathrm{E}-01$ & 120 & 8 & 7 & 37 \\
\hline 2.87 & 267.23 & 1.69 & 58.1 & 58 & 2.92 & -0.43 & 1183.75 & 4.004 & $1.72 \mathrm{E}-01$ & 1.72E-01 & 120 & 8 & 7 & 39 \\
\hline 2.94 & 267.16 & 1.95 & 62.1 & 62 & 3.14 & -0.35 & 1398.46 & 4.004 & $1.77 \mathrm{E}-01$ & 1.77E-01 & 120 & 8 & 7 & 41 \\
\hline 3.02 & 267.08 & 2.22 & 64.8 & 64.8 & 3.43 & -0.42 & 1564.13 & 4.004 & $1.81 \mathrm{E}-01$ & $1.81 \mathrm{E}-01$ & 120 & 8 & 7 & 43 \\
\hline 3.09 & 267.01 & 2.45 & 65.8 & 65.7 & 3.72 & -0.47 & 1647.39 & 4.004 & $1.86 \mathrm{E}-01$ & $1.86 \mathrm{E}-01$ & 120 & 8 & 7 & 44 \\
\hline 3.16 & 266.94 & 2.61 & 68.1 & 68 & 3.84 & -0.5 & 1646.15 & 4.004 & $1.90 \mathrm{E}-01$ & $1.90 \mathrm{E}-01$ & 120 & 8 & 7 & 45 \\
\hline 3.24 & 266.86 & 2.74 & 70.3 & 70.2 & 3.9 & -0.52 & 1592.7 & 4.004 & $1.94 \mathrm{E}-01$ & $1.94 \mathrm{E}-01$ & 120 & 8 & 7 & 47 \\
\hline 3.32 & 266.78 & 2.85 & 72.6 & 72.5 & 3.93 & -0.53 & 1559.18 & 4.004 & 1.99E-01 & $1.99 \mathrm{E}-01$ & 120 & 8 & 7 & 48 \\
\hline 3.39 & 266.71 & 3 & 74 & 73.9 & 4.06 & -0.49 & 1540.74 & 4.004 & 2.03E-01 & 2.03E-01 & 120 & 8 & 7 & 49 \\
\hline 3.47 & 266.63 & 3.16 & 74.3 & 74.2 & 4.26 & -0.48 & 1556.21 & 4.004 & $2.08 \mathrm{E}-01$ & 2.08E-01 & 120 & 8 & 7 & 50 \\
\hline 3.54 & 266.56 & 3.26 & 74.9 & 74.8 & 4.36 & -0.51 & 1572.11 & 4.004 & $2.12 \mathrm{E}-01$ & 2.12E-01 & 120 & 8 & 7 & 50 \\
\hline 3.61 & 266.49 & 3.31 & 77.1 & 77 & 4.3 & -0.45 & 1572.66 & 4.004 & $2.17 \mathrm{E}-01$ & 2.17E-01 & 120 & 8 & 7 & 51 \\
\hline 3.69 & 266.41 & 3.31 & 81.3 & 81.4 & 4.07 & 0.29 & 1637.16 & 4.004 & $2.21 \mathrm{E}-01$ & $2.21 \mathrm{E}-01$ & 120 & 8 & 7 & 54 \\
\hline 3.76 & 266.34 & 3.36 & 85.2 & 85.4 & 3.94 & 0.88 & 1734.25 & 4.004 & $2.26 \mathrm{E}-01$ & $2.26 \mathrm{E}-01$ & 120 & 8 & 7 & 57 \\
\hline 3.84 & 266.26 & 3.43 & 88.4 & 88.8 & 3.87 & 2.05 & 1834.09 & 4.004 & 2.30E-01 & 2.30E-01 & 120 & 8 & 7 & 59 \\
\hline 3.91 & 266.19 & 3.49 & 90.3 & 90.7 & 3.85 & 1.9 & 1953.44 & 4.004 & 2.35E-01 & 2.35E-01 & 120 & 8 & 7 & 60 \\
\hline
\end{tabular}


APPENDIX D-5. Bechtel, 1998, As-built of CPT Locations 100-C Area

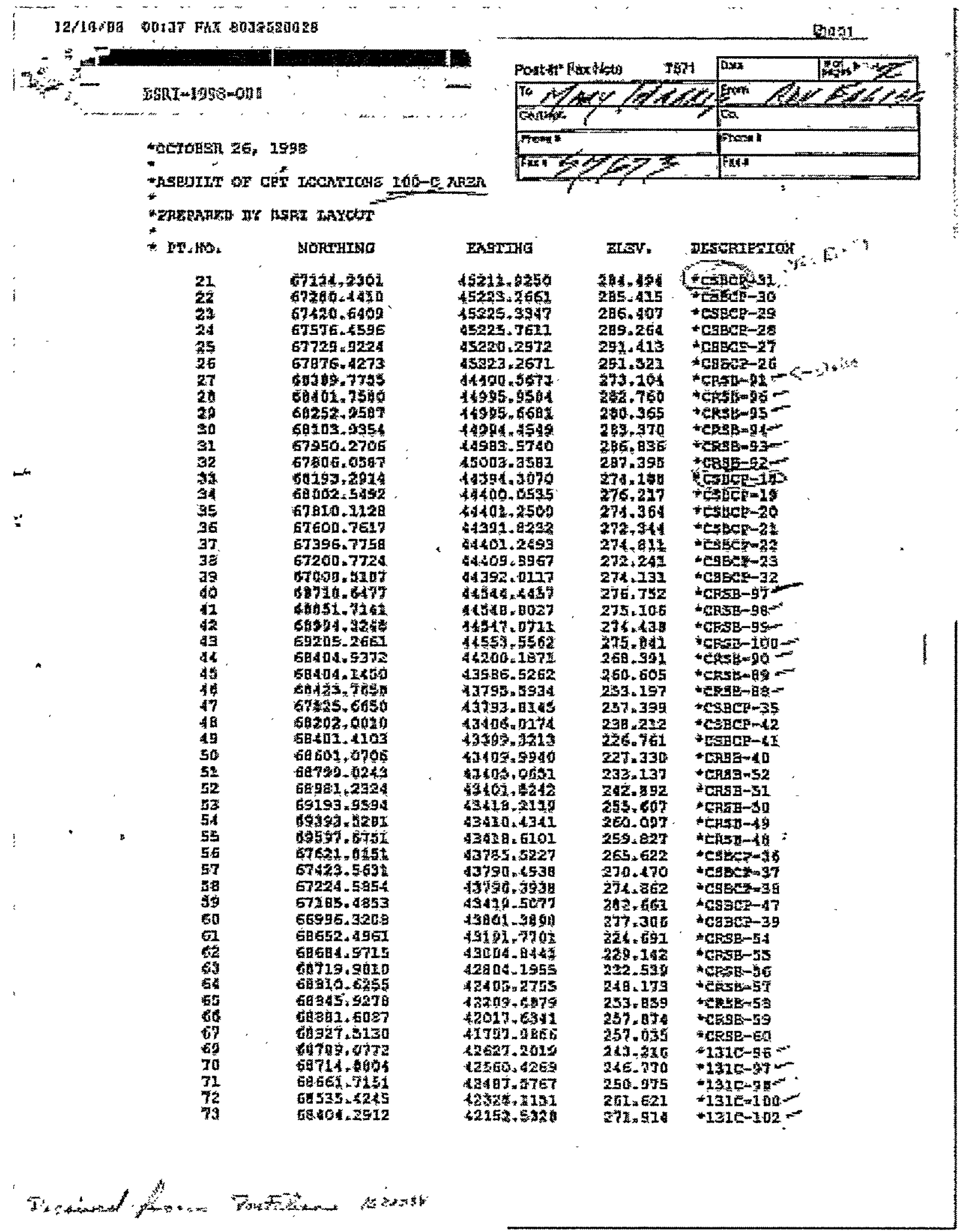


APPENDIX D-5. Bechtel, 1998, As-built of CPT Locations 100-C Area
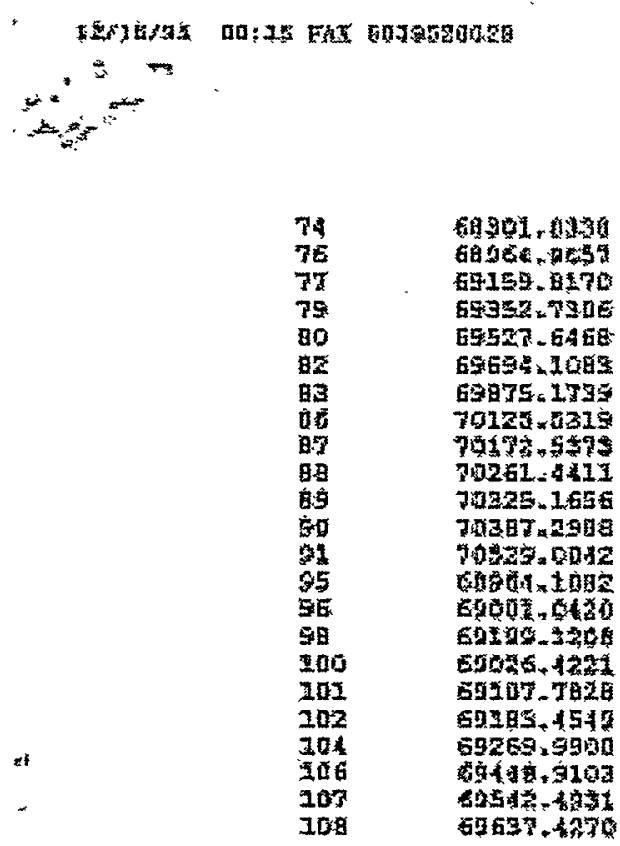

$4159 E^{4} 18485$

$42856 \times 9 \leq 01$

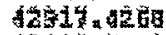

136 4 .

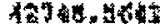

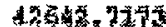

47574.1405

12073.6165

12145.63日4

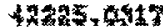

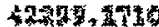

$4203 \times 5053$

20515. 0933

61602.3805

4255. àcs

4T615.2769

II.1Ep 9613

aby

16519.917

40023.5041

1571.5495

1E:5.704

11641.9085

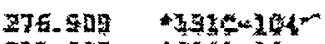

211.605 tenstum

ㄴ113.202 대표-67

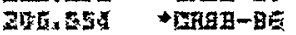

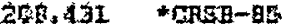

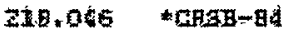

227. 200 HCFED-93

205. T30 MSte-jo-

1717.856 -

$232.353+1318-822$

40.

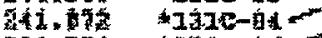

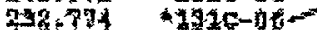

257.276 कFib-61

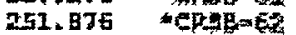

211.275 -1310 05 -

250.392 tassterso

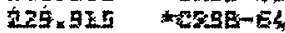

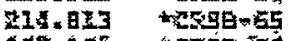

10\% It5

$235.4401414-22^{5}$

$23 x^{2} .5$

- ISIE- 130

002

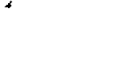


APPENDIX D-5. Bechtel, 1998, As-built of CPT Locations 100-C Area

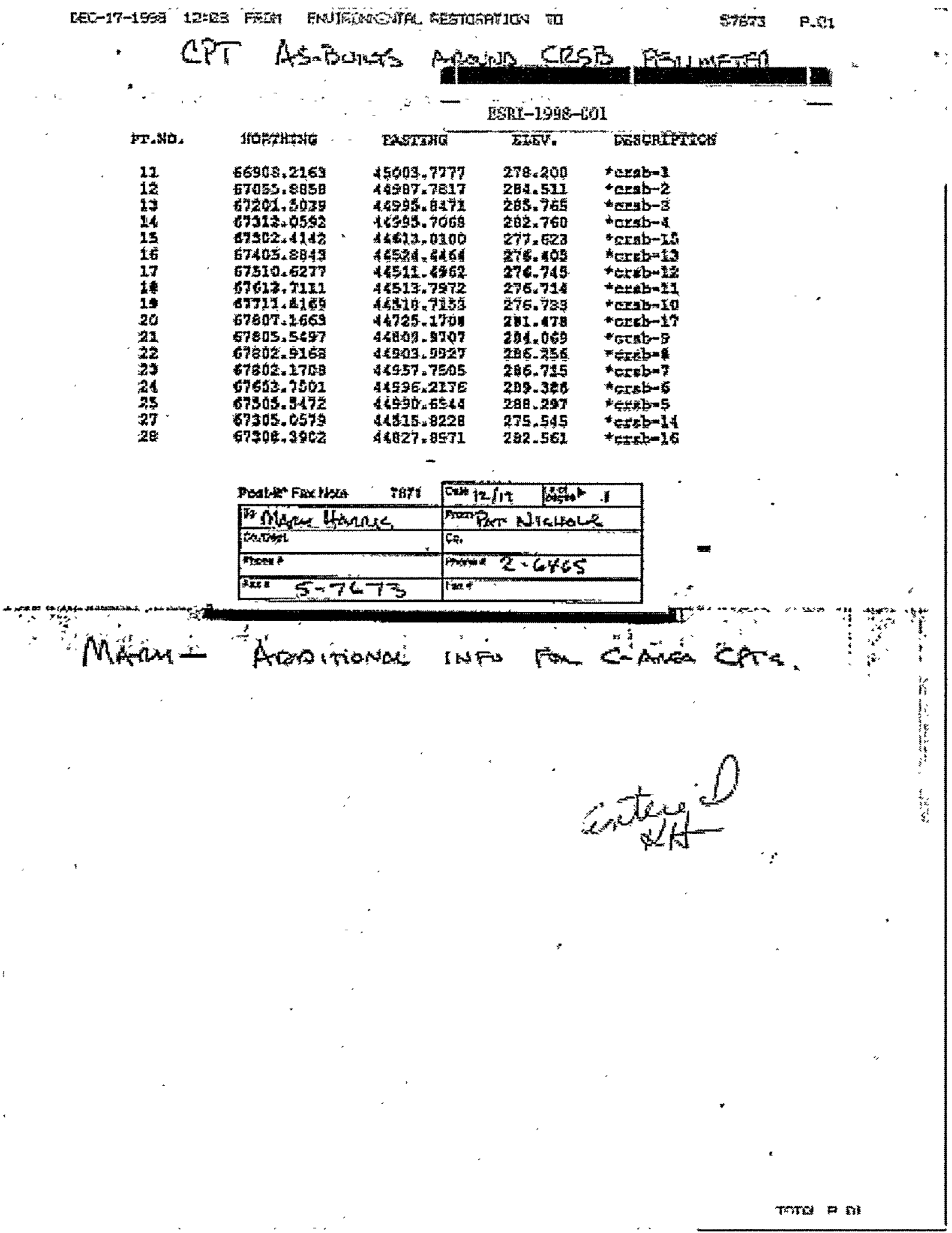


D-14 WSRC-TR-99-00248, Rev. 0, Regional Groundwater Flow Model for C, K, L, and P Reactor Areas APPENDIX D-6. Bechtel, 1999, As-built of CPT Locations at the 100-C Area Seepage Basins

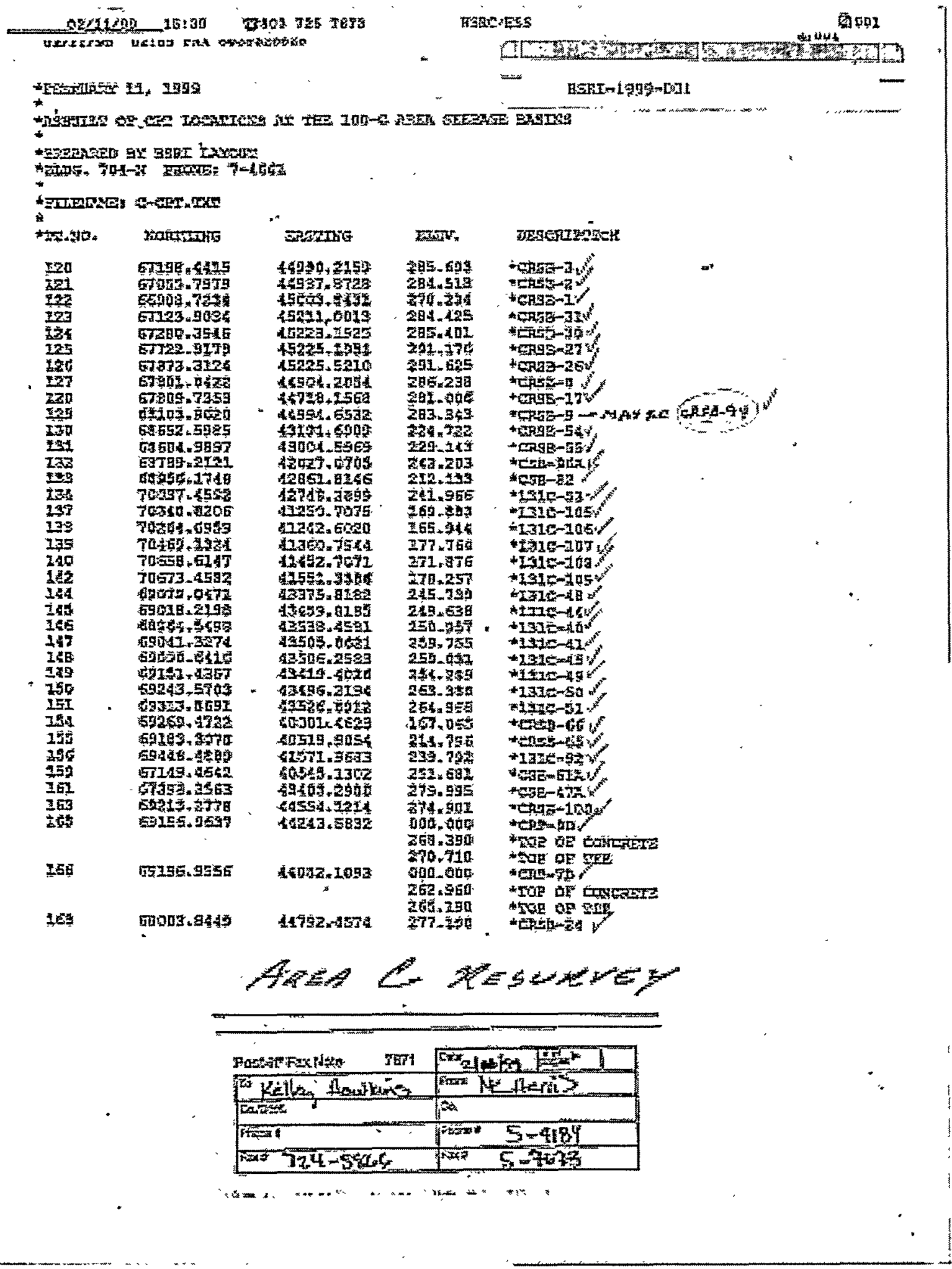


WSRC-TR-99-00248, Rev. 0, Regional Groundwater Flow Model for C, K, L, and P Reactor Areas D-15

APPENDIX D-7. Harris, 1997, borings.xls, electronic file received from M. K. Harris

\begin{tabular}{|ccccr|}
\hline WELL & $\begin{array}{c}\text { SRS } \\
\text { NORTHING }\end{array}$ & $\begin{array}{c}\text { SRS } \\
\text { EASTING }\end{array}$ & $\begin{array}{c}\text { ELEV. } \\
\text { CASIN } \\
\text { G }\end{array}$ & $\begin{array}{c}\text { GROUND } \\
\text { ELEV. }\end{array}$ \\
\hline & & & & \\
PBF 002 & $50,667.38$ & $91,082.89$ & 268.35 \\
PBF 002 & $50,667.38$ & $91,082.89$ & & 268.35 \\
PBF 003 & $58,766.62$ & $60,380.36$ & 316.65 \\
PBF 004 & $58,148.66$ & $29,985.13$ & 208.10 \\
PBF 005 & $53,591.29$ & $30,319.43$ & 240.60 \\
PBF 006 & $55,621.75$ & $12,814.48$ & 92.50 \\
PBF 006 & $55,621.75$ & $12,814.48$ & 285.42 \\
PBF 007 & $55,420.69$ & $59,568.97$ & \\
PBF 007 & $55,420.69$ & $59,568.97$ & \\
PBF 008 & $55,744.48$ & $59,812.89$ & & 285.42 \\
& & &
\end{tabular}


D-16 WSRC-TR-99-00248, Rev. 0, Regional Groundwater Flow Model for C, K, L, and P Reactor Areas APPENDIX D-8. Kirr, 1998, Well-Boring Locations.doc, electronic file received from J. Kirr

\begin{tabular}{|c|c|c|c|c|}
\hline Designation & Northing & Easting & $\frac{\text { Elevatio }}{\underline{\mathbf{n}}}$ & Description \\
\hline CRP-3C & 68697.04 & 44016.51 & 266.04 & Well \\
\hline CRP-3D & 68694.01 & 44012.93 & 265.31 & Well \\
\hline CRP-5C & 68535.99 & 44527.84 & 275.05 & Well \\
\hline CRP-5D & 68549.46 & 44515.25 & 274.50 & Well \\
\hline CRP-6DR & 68309.80 & 44007.90 & 261.50 & Well \\
\hline CRP-7D & 69197.00 & 44082.00 & 262.98 & Well \\
\hline CRP-8D & 68650.37 & 43681.94 & 246.08 & Well \\
\hline CRP-9D & 69156.95 & 44243.47 & 268.40 & Well \\
\hline CRP-10D & 68998.72 & 43741.10 & 264.84 & well \\
\hline CRP-11D & 68711.23 & 44168.20 & 268.93 & well-remedial action \\
\hline $131 \mathrm{C}-05$ & 68686.55 & 44092.00 & 286.40 & Soil Boring \\
\hline $131 \mathrm{C}-06$ & 68671.91 & 44181.38 & 270.30 & Soil Boring \\
\hline 131C-07 & 68659.91 & 44233.05 & 270.90 & Soil Boring \\
\hline $131 \mathrm{C}-08$ & 68649.28 & 44309.43 & 272.30 & Soil Boring \\
\hline 131C-09 & 68806.94 & 44545.51 & 275.81 & Soil Boring \\
\hline $131 \mathrm{C}-10$ & 68876.37 & 43991.49 & 286.80 & Soil Boring \\
\hline $131 \mathrm{C}-11$ & 68813.26 & 44479.47 & 289.70 & Soil Boring \\
\hline $131 \mathrm{C}-12$ & 68946.35 & 44462.48 & 284.10 & Soil Boring \\
\hline $131 \mathrm{C}-13$ & 68739.33 & 44193.46 & 270.10 & Soil Boring \\
\hline $131 \mathrm{C}-14$ & 68959.22 & 44302.84 & 283.10 & Soil Boring \\
\hline $131 \mathrm{C}-15$ & 68558.37 & 43893.00 & 256.58 & Surface Sample \\
\hline $131 C-16$ & 68481.21 & 44209.67 & 270.00 & Surface Sample \\
\hline $131 \mathrm{C}-19$ & 68825.41 & 44385.92 & 289.79 & Soil Boring \\
\hline $131 \mathrm{C}-20$ & 68848.61 & 44261.09 & 286.38 & Soil Boring \\
\hline $131 \mathrm{C}-21$ & 68871.79 & 44154.21 & 285.58 & Soil Boring \\
\hline 131C-16-CPT & 68489.63 & 44589.14 & & CPT \\
\hline 131C-17-CPT & 68323.35 & 44053.31 & & CPT \\
\hline 131C-18-CPT & 68719.30 & 44158.79 & & CPT \\
\hline 131C-19-CPT & 68250.65 & 43902.38 & & CPT \\
\hline 131C-20-CPT & 68387.70 & 43890.75 & & CPT \\
\hline 131C-21-CPT & 69047.72 & 44066.46 & & CPT \\
\hline 131C-22-CPT & 69178.44 & 43996.57 & & CPT \\
\hline 131C-23-CPT & 68469.44 & 43981.91 & & CPT \\
\hline 131C-24-CPT & 68420.34 & 44249.81 & & CPT \\
\hline 131C-25-CPT & 68272.82 & 44358.99 & & CPT \\
\hline 131C-26-CPT & 68099.70 & 44236.10 & & CPT \\
\hline 131C-27-CPT & 68868.40 & 43833.60 & 263.67 & CPT \\
\hline 131C-28-CPT & 68786.40 & 43776.30 & 256.28 & $\mathrm{CPT}$ \\
\hline 131C-29-CPT & 68968.30 & 43829.00 & 265.82 & $\mathrm{CPT}$ \\
\hline
\end{tabular}


APPENDIX D-8. Kirr, 1998, Well-Boring Locations.doc, electronic file received from J. Kirr

\begin{tabular}{|c|c|c|c|c|}
\hline Designation & Northing & Easting & $\frac{\text { Elevatio }}{\underline{\mathbf{n}}}$ & Description \\
\hline 131C-30-CPT & 68703.90 & 43720.20 & 248.98 & CPT (not used) \\
\hline 131C-31-CPT & 69068.10 & 43824.30 & 267.66 & $\mathrm{CPT}$ \\
\hline 131C-32-CPT & 69168.00 & 43819.50 & 267.32 & $\mathrm{CPT}$ \\
\hline 131C-33-CPT & 68760.10 & 43637.40 & 245.55 & CPT (not used) \\
\hline 131C-34-CPT & 68842.70 & 43693.80 & 252.60 & CPT \\
\hline 131C-35-CPT & 68923.20 & 43750.00 & 260.77 & $\mathrm{CPT}$ \\
\hline 131C-36-CPT & 68816.50 & 43554.90 & 242.54 & CPT (not used) \\
\hline 131C-37-CPT & 68899.10 & 43611.00 & 251.43 & CPT (not used) \\
\hline 131C-38-CPT & 68978.00 & 43666.40 & 259.84 & CPT (not used) \\
\hline 131C-39-CPT & 69024.70 & 43746.40 & 265.26 & CPT (not used) \\
\hline 131C-40-CPT & 68987.60 & 43564.20 & 249.50 & CPT (not used) \\
\hline 131C-41-CPT & 69034.30 & 43652.70 & 259.40 & CPT (not used) \\
\hline 131C-42-CPT & 69080.80 & 43741.30 & 266.30 & CPT (not used) \\
\hline 131C-43-CPT & 69127.70 & 43829.50 & 266.30 & CPT (not used) \\
\hline 131C-44-CPT & 69075.80 & 43517.40 & 248.50 & CPT (not used) \\
\hline 131C-45-CPT & 69122.60 & 43606.00 & 258.50 & CPT (not used) \\
\hline $131 \mathrm{C}-46-\mathrm{CPT}$ & 69169.30 & 43694.50 & 265.30 & CPT (not used) \\
\hline 131C-47-CPT & 69216.00 & 43782.80 & 265.40 & CPT (not used) \\
\hline 131C-48-CPT & 69164.30 & 43470.70 & 244.00 & + \\
\hline 131C-49-CPT & 69211.00 & 43559.00 & 254.20 & \\
\hline 131C-50-CPT & 69257.90 & 43648.00 & 261.90 & \\
\hline 131C-51-CPT & 69304.40 & 43736.00 & 265.30 & \\
\hline 131C-52-CPT & 70071.8 & 42566.50 & 230.34 & \\
\hline 131C-53-CPT & 70022.4 & 42759.40 & 243.34 & \\
\hline 131C-54-CPT & 69970.8 & 42952.30 & 247.06 & \\
\hline 131C-55-CPT & 69923.3 & 43145.90 & 245.01 & \\
\hline 131C-56-CPT & $69875: 7$ & 43339.80 & 248.84 & \\
\hline 131C-57-CPT & 69831.3 & 43533.90 & 249.36 & \\
\hline 131C-58-CPT & 69790.8 & 43728.20 & 243.11 & \\
\hline 131C-59-CPT & 69874.5 & 42563.40 & 227.70 & \\
\hline 131C-60-CPT & 69691.0 & 42639.30 & 218.20 & \\
\hline 131C-61-CPT & 69523.0 & 42745.40 & 208.54 & \\
\hline 131C-62-CPT & 69346.2 & 42836.60 & 207.16 & \\
\hline 131C-63-CPT & 69158.7 & 42902.80 & 211.14 & \\
\hline 131C-64-CPT & 68969.9 & 42859.40 & 211.86 & \\
\hline 131C-65-CPT & 68868.40 & 43833.60 & 263.67 & CPT-28 (dual location) \\
\hline 131C-66-CPT & 68868.40 & 43833.60 & 263.67 & CPT-27 (dual location) \\
\hline 131C-67-CPT & 68968.30 & 43829.00 & 265.82 & CPT-29 (dual location) \\
\hline 131C-68-CPT & 69068.10 & 43824.30 & 267.66 & CPT-31 (dual location) \\
\hline 131C-69-CPT & 69168.00 & 43819.50 & 267.32 & CPT-32 (dual location) \\
\hline
\end{tabular}


D-18 WSRC-TR-99-00248, Rev. 0, Regional Groundwater Flow Model for C, K, L, and P Reactor Areas

APPENDIX D-8. Kirr, 1998, Well-Boring Locations.doc, electronic file received from J. Kirr

\begin{tabular}{|c|c|c|c|c|}
\hline Designation & Northing & Easting & $\frac{\text { Elevatio }}{\underline{\mathbf{n}}}$ & Description \\
\hline CPT 70 thru 76 & $\begin{array}{l}\text { Contingent } \\
\text { Locations }\end{array}$ & $\begin{array}{l}\text { Not Used -only } \\
\text { designated }\end{array}$ & & \\
\hline 131C-80-CPT & 70122.76 & 42076.62 & 206.0 & \\
\hline 131C-81-CPT & 70172.7 & 42140.8 & 218.0 & \\
\hline 131C-82-CPT & 70264.36 & 42218.22 & 232.97 & \\
\hline 131C-83-CPT & 70328.34 & 42295.50 & 240.08 & \\
\hline 131C-84-CPT & 70405.96 & 42359.58 & 239.80 & \\
\hline 131C-85-CPT & 70466.62 & 42439.04 & 237.49 & \\
\hline 131C-86-CPT & 70547.24 & 42500.84 & 231.84 & \\
\hline 131C-87-CPT & 70616.97 & 42572.93 & 226.81 & \\
\hline 131C-88-CPT & 70688.59 & 42642.17 & 218.62 & \\
\hline 131C-89-CPT & 69733.61 & 41646.48 & 219.21 & \\
\hline 131C-90-CPT & 69635.71 & 41644.49 & 227.38 & \\
\hline 131C-91-CPT & 69534.62 & 41626.52 & 233.76 & \\
\hline 131C-92-CPT & 69449.63 & 41574.46 & 239.59 & \\
\hline 131C-93-CPT & 69366.69 & 41518.62 & 241.89 & \\
\hline 131C-94-CPT & 69282.99 & 41463.87 & 241.43 & \\
\hline 131C-95-CPT & 69203.51 & 41402.77 & 241.31 & \\
\hline 131C-96-CPT & 68800.66 & 42630.16 & 242.58 & \\
\hline 131C-97-CPT & 68720.26 & 42554.06 & 246.72 & \\
\hline 131C-98-CPT & 68655.72 & 42481.42 & 251.58 & \\
\hline 131C-99-CPT & 68597.42 & 42400.45 & 256.73 & \\
\hline 131C-100-CPT & 68535.41 & 42322.07 & 261.78 & \\
\hline 131C-101-CPT & 68474.57 & 42242.58 & 266.72 & \\
\hline 131C-102-CPT & 68415.79 & 42161.62 & 271.46 & \\
\hline 131C-103-CPT & 68363.00 & 42075.15 & 275.46 & \\
\hline 131C-104-CPT & 68303.94 & 41995.22 & 277.06 & \\
\hline 131C-105-CPT & 70356.80 & 41240.70 & 169.30 & \\
\hline 131C-106-CPT & 70265.80 & 41241.70 & 165.80 & \\
\hline 131C-107-CPT & 70472.20 & 41366.00 & 178.40 & \\
\hline 131C-108-CPT & 70658.70 & 41452.20 & 171.10 & \\
\hline 131C-109-CPT & 70703.60 & 41537.20 & 172.00 & \\
\hline 131C-R1-CPT & 68682.80 & 44316.00 & 271.00 & IAPP SAMPLES \\
\hline 131C-R2-CPT & 68734.70 & 44066.10 & 266.80 & IAPP SAMPLES \\
\hline 131C-R3-CPT & 68882.90 & 43994.50 & 268.80 & IAPP SAMPLES \\
\hline 131C-R4-CPT & 68863.30 & 44197.30 & 285.60 & IAPP SAMPLES \\
\hline 131C-R5-CPT & 68830.80 & 44359.60 & 289.00 & IAPP SAMPLES \\
\hline 131C-R6-CPT & 69031.60 & 44274.00 & 276.00 & IAPP SAMPLES \\
\hline
\end{tabular}


APPENDIX D-8. Kirr, 1998, Well-Boring Locations.doc, electronic file received from J. Kirr

\begin{tabular}{lllll} 
Designation & Northing & Easting & $\underline{\underline{\text { Elevatio }}}$ & Description \\
\cline { 3 - 4 } & & & $\underline{\underline{\mathbf{n}}}$ & \\
131C-R7-CPT & 68696.7 & 44230.0 & 269.58 & IAPP SAMPLES \\
131C-R8-CPT & 68724.1 & 44113.4 & 267.75 & IAPP SAMPLES \\
131C-R9-CPT & 68725.9 & 44025.0 & 266.20 & IAPP SAMPLES \\
131C-R10-CPT & 68693.3 & 44100.7 & 268.20 & IAPP SAMPLES \\
Orange Ball & 68713.98 & 44059.54 & & \\
Orange Ball & 68667.05 & 44054.39 & & Pit Area \\
Orange Ball & 68611.40 & 44335.05 & & Pit Area \\
Orange Ball & 68661.19 & 44349.12 & & Pit Area \\
Corner Marker & 68776.82 & 43994.46 & & Pit Area \\
Corner Marker & 68985.12 & 44022.36 & & Mounded Area \\
Corner Marker & 68678.88 & 44528.36 & & Mounded Area \\
Corner Marker & 69014.24 & 44532.13 & & Mounded Area \\
& & & & Mounded Area
\end{tabular}


D-20 WSRC-TR-99-00248, Rev. 0, Regional Groundwater Flow Model for C, K, L, and P Reactor Areas APPENDIX D-9. Rucker, 1999a, grucker.990113.csbvoc.xls, CRSB Analytical Data received from $\mathrm{G}$. Rucker

\begin{tabular}{|c|c|c|c|c|c|c|c|c|}
\hline SAMPLE ID & ANALYTE & EASTING & NORTHING & DEPMPI & DL & $\mathbf{R Q}$ & RESULT & UNITS \\
\hline CRSB-56-01 & 1,1,1-Trichloroethan & $43,999.43$ & $68,602.13$ & 67.0 & 1 & $\mathrm{u}$ & 1 & UGL \\
\hline CRSB-58-08 & Tritium & $41,577.12$ & $68,028.13$ & 118.0 & 1301 & & $17,300.0$ & $\mathrm{pCi} / \mathrm{L}$ \\
\hline CRSB-59-01 & $1,1,1$-Trichloroethan & $41,295.09$ & $67,751.28$ & 40.0 & 1.00 & $\mathbf{U}$ & 1 & UGL \\
\hline CRSB-60-08 & Tritium & $40,980.25$ & $67,502.28$ & 158.0 & 1301 & & $25,300.0$ & $\mathrm{pCi} / \mathrm{L}$ \\
\hline CRSB-61-01 & $1,1,1$-Trichloroethan & $40,668.70$ & $67,245.08$ & 69.0 & 1 & $\mathrm{U}$ & 1 & UGL \\
\hline CRSB- $62-08$ & Tritium & $40,344.02$ & $67,014.10$ & 159.0 & 1301 & & 187.0 & $\mathrm{pCi} / \mathrm{L}$ \\
\hline CRSB-63-01 & $1,1,1$-Trichloroethan & $40,014.43$ & $66,792.95$ & 53.0 & 1 & $\mathbf{U}$ & 1 & UGL \\
\hline CRSB- $64-08$ & 1,2-Dichloroethylene & $39,707.79$ & $66,530.84$ & 140.0 & 1 & $\mathrm{U}$ & 1 & UGL \\
\hline CRSB-65-08 & Tritium & $39,517.58$ & $66,173.72$ & 148.0 & 1301 & & 219.0 & $\mathrm{pCi} / \mathrm{L}$ \\
\hline CRSB-66A-01 & $1,1,1$-Trichloroethan & $39,622.52$ & $65,783.83$ & 28.0 & 1 & $\mathrm{U}$ & 1 & UGL \\
\hline
\end{tabular}




\begin{tabular}{|l|r|r|r|}
\hline Borehole & \multicolumn{1}{|c|}{ Northing } & \multicolumn{1}{l|}{ Easting } & \multicolumn{1}{l|}{ Eleve } \\
\hline CSB-101 & 66002.48 & 44868.43 & 281.20 \\
\hline CSB-102 & 65984.05 & 43109.96 & 283.50 \\
\hline CSB-103 & 64510.41 & 44149.64 & 257.60 \\
\hline CSB-104 & 63455.35 & 43787.85 & 224.90 \\
\hline CSB-105 & 63628.93 & 42549.54 & 212.10 \\
\hline CSB-106 & 64261.04 & 41634.09 & 183.80 \\
\hline CSB-107 & 64785.48 & 42446.08 & 220.70 \\
\hline CSB-108 & 65154.15 & 40531.22 & 196.70 \\
\hline CSB-109 & 65351.75 & 39098.64 & 172.93 \\
\hline CSB-110 & 66382.03 & 38102.37 & 184.60 \\
\hline CSB-111 & 66888.08 & 38022.32 & 172.10 \\
\hline CSB-112 & 67195.7 & 38892.52 & 195.30 \\
\hline CSB-113 & 67729.39 & 39311.45 & 210.40 \\
\hline CSB-114 & 68278.36 & 40206.45 & 196.00 \\
\hline CSB-115 & 69107.78 & 40798.67 & 229.92 \\
\hline CSB-116 & 67598.91 & 42794.74 & 274.74 \\
\hline CSB-117 & 67218.22 & 42698.32 & 276.50 \\
\hline CSB-118 & 66802.15 & 42707.65 & 277.80 \\
\hline CSB-119 & 67398.77 & 42193.79 & 268.30 \\
\hline CSB-120 & 66998.48 & 42197.24 & 282.80 \\
\hline CSB-121 & 66601.65 & 42197.24 & 274.24 \\
\hline CSB-122 & 65679.58 & 41120.9 & 222.9 \\
\hline CSB-123 & 65585.04 & 41352.9 & 224.2 \\
\hline
\end{tabular}


APPENDIX D-11. WSRC, 1998, CMP Pits Sample Locations

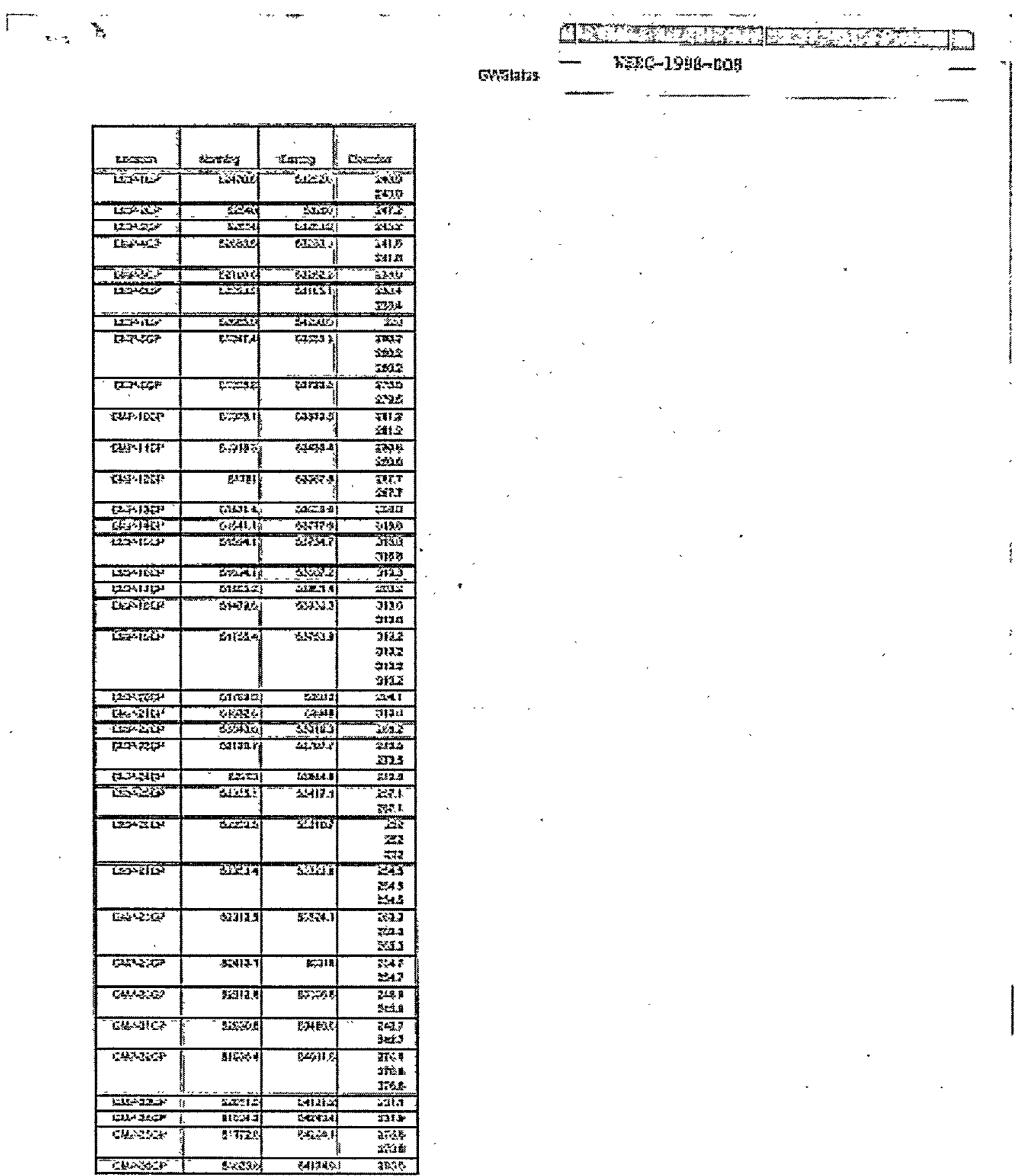


APPENDIX D-11. WSRC, 1998, CMP Pits Sample Locations

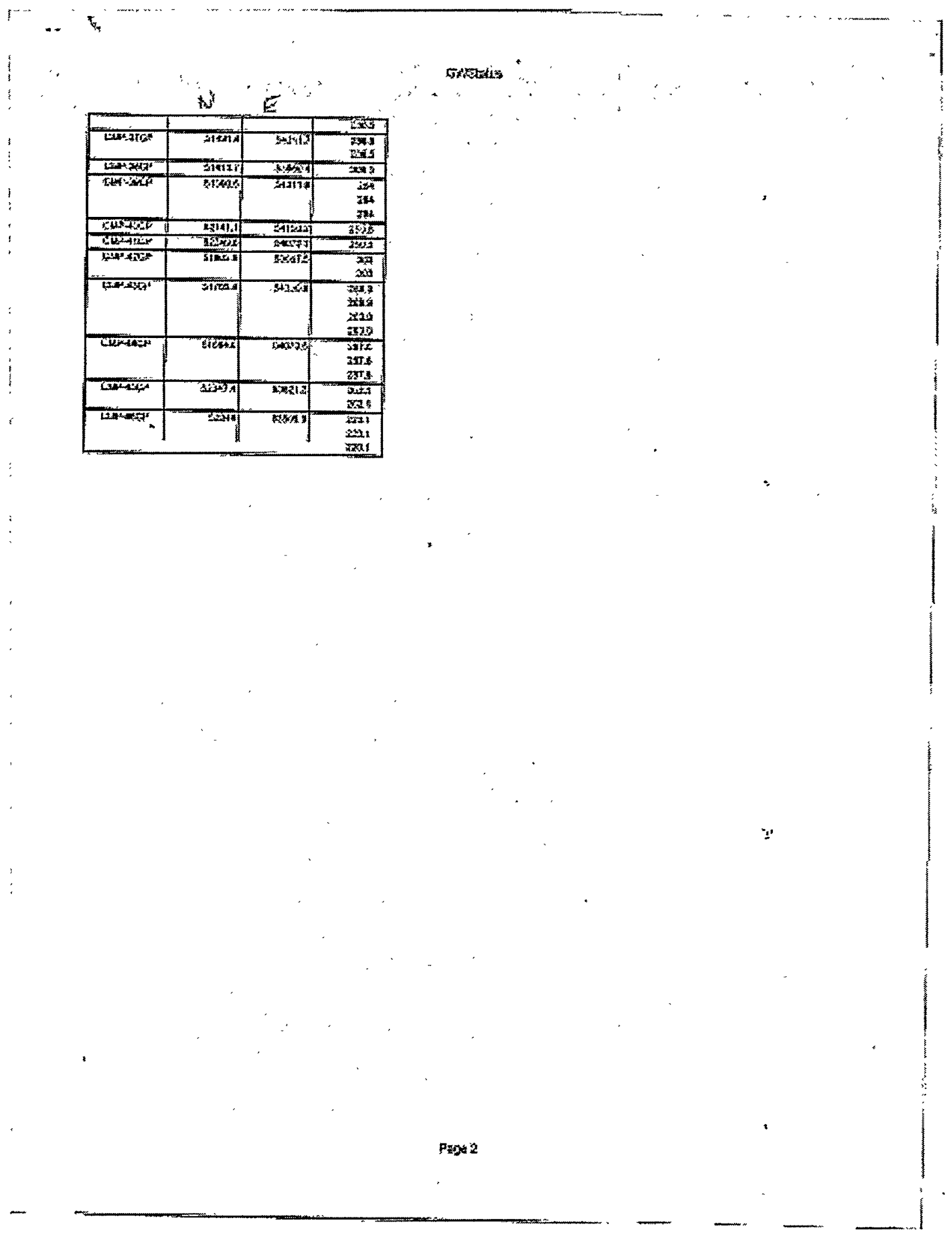


D-24 WSRC-TR-99-00248, Rev. 0, Regional Groundwater Flow Model for C, K, L, and P Reactor Areas APPENDIX D-12. WSRC, unknown, Location of BGT Cores, Data received from $\mathrm{M}$. Amidon

\begin{tabular}{|l|c|c|c|}
\hline Site ID & Northing & Easting & $\begin{array}{c}\text { Grnd } \\
\text { Elevation } \\
\text { (ft.) }\end{array}$ \\
\hline BGT001 & 76700.6 & 59178.4 & 282.9 \\
\hline BGT002 & 76957.6 & 59607.2 & 276.4 \\
\hline BGT003 & 77197.6 & 60045.9 & 275.7 \\
\hline BGT004 & 77437.6 & 60484.5 & 259.2 \\
\hline BGT005 & 77677.6 & 60924.1 & 225.7 \\
\hline BGT006 & 77254.8 & 58746.7 & 282.2 \\
\hline BGT007 & 77717.8 & 58935.7 & 276.4 \\
\hline BGT008 & 78161.5 & 59118.6 & 249.3 \\
\hline BGT009 & 78642.3 & 59316.7 & 226.0 \\
\hline BGT010 & 79104.6 & 59507.2 & 215.2 \\
\hline BGT011 & 79566.9 & 59697.7 & 222.5 \\
\hline BGT012 & 77291.2 & 58045.9 & 284.2 \\
\hline BGT013 & 77488.9 & 58074.0 & 287.8 \\
\hline BGT014 & 77984.0 & 58143.4 & 280.7 \\
\hline BGT015 & 78479.2 & 58212.8 & 277.5 \\
\hline BGT016 & 78974.1 & 58283.5 & 250.7 \\
\hline BGT017 & 79469.7 & 58350.0 & 240.7 \\
\hline BGT018 & 79965.3 & 58416.5 & 216.5 \\
\hline BGT019 & 80460.8 & 58483.0 & 236.3 \\
\hline BGT020 & 80956.4 & 58549.6 & 159.3 \\
\hline BGT021 & 77280.7 & 56952.5 & 294.2 \\
\hline BGT022 & 77680.3 & 56970.3 & 281.0 \\
\hline BGT023 & 78279.7 & 56997.0 & 270.0 \\
\hline BGT024 & 78779.2 & 57019.2 & 265.8 \\
\hline BGT025 & 79278.7 & 57041.4 & 264.8 \\
\hline BGT026 & 79778.2 & 57063.7 & 250.2 \\
\hline BGT027 & 80277.7 & 57085.9 & 256.9 \\
\hline BGT028 & 80777.2 & 57108.1 & 258.3 \\
\hline BGT029 & 81276.7 & 57130.4 & 243.0 \\
\hline BGT030 & 81726.3 & 57150.4 & 219.0 \\
\hline BGT031 & 77229.0 & 56189.8 & 308.76 \\
\hline BGT032 & 77791.4 & 56121.1 & 310.12 \\
\hline BGT033 & 78404.5 & 56037.2 & 290.42 \\
\hline BGT034 & 78803.9 & 56027.5 & 286.76 \\
\hline BGT035 & 79305.8 & 55929.9 & 267.73 \\
\hline BGT036 & 79801.9 & 55867.5 & 261.36 \\
\hline BGT037 & 80298.0 & 55805.0 & 251.60 \\
\hline BGT038 & 80870.5 & 55733.0 & 240.14 \\
\hline BGT039 & 81290.2 & 55680.3 & 241.88 \\
\hline BGT040 & 77297.2 & 55644.4 & 332.32 \\
\hline BGT041 & 77734.8 & 55490.1 & 328.37 \\
\hline BGT042 & 78240.7 & 55313.1 & 310.92 \\
\hline BGT043 & 79655.9 & 54816.0 & 277.08 \\
\hline BGT044 & 80127.7 & 54650.4 & 276.20 \\
\hline BGT045 & 80461.7 & 54533.1 & 285.28 \\
\hline
\end{tabular}


APPENDIX D-12. WSRC, unknown, Location of BGT Cores, Data received from $\mathrm{M}$. Amidon

\begin{tabular}{|l|l|l|l|}
\hline BGT046 & 76714.3 & 55355.0 & 310.00 \\
\hline BGT047 & 77051.9 & 54986.6 & 317.32 \\
\hline BGT048 & 77135.7 & 54895.1 & 314.33 \\
\hline BGT049 & 76203.9 & 54946.3 & 297.26 \\
\hline BGT050 & 76359.3 & 54756.2 & 296.27 \\
\hline BGT051 & 75519.8 & 54505.7 & 272.64 \\
\hline BGT052 & 75640.6 & 54093.8 & 264.26 \\
\hline BGT053 & 75837.7 & 53422.0 & 278.25 \\
\hline BGT054 & 75941.7 & 52889.1 & 279.96 \\
\hline BGT055 & 76009.4 & 52382.3 & 270.68 \\
\hline BGT056 & 73521.2 & 56265.8 & 262.94 \\
\hline BGT057 & 73268.5 & 56104.2 & 259.35 \\
\hline BGT058 & 73406.9 & 57399.6 & 285.76 \\
\hline BGT059 & 72802.6 & 57123.2 & 281.88 \\
\hline BGT060 & 73120.6 & 58057.2 & 291.42 \\
\hline BGT061 & 72911.8 & 58490.1 & 284.30 \\
\hline BGT062 & 72854.4 & 58609.0 & 282.03 \\
\hline BGT063A & 73646.4 & 58768.1 & 290.79 \\
\hline BGT063 & 73319.4 & 59146.3 & 293.67 \\
\hline BGT064 & 73013.7 & 59500.0 & 283.25 \\
\hline BGT065 & 72734.8 & 59822.7 & 276.29 \\
\hline BGT066 & 74476.6 & 60033.7 & 244.04 \\
\hline BGT067 & 74443.1 & 60426.7 & 242.03 \\
\hline & & & \\
\hline
\end{tabular}


This page intentionally left blank 


\section{APPENDIX E-1. STREAM BASE FLOW ESTIMATES BASED ON USGS GAUGING STATION DATA}


(This page intentionally left blank) 


\section{Appendix E-1. Stream Base Flow Estimates Based on USGS Gauging Station Data}

Groundwater flow in upper aquifers at the Savannah River Site is recharge driven, with streams intercepting flow from higher elevations. Nearly all recharge within the CKLP reactor region discharges to streams within or bounding the same area, usually the nearest stream. For this type of groundwater flow system, recharge and discharge estimates, coupled with head measurements and confining unit leakance estimates, define the overall horizontal conductivities of upper aquifers required to calibrate a numerical flow model. Because conductivity data at the model scale are typically non-existent, stream base flow estimates are important model calibration targets. In this appendix, simple hydrograph separation techniques are used to estimate the long-term average rate of groundwater discharge to certain stream reaches within the CKLP reactor area.

The U. S. Geological Survey has monitored stream flows at numerous locations across the Savannah River Site for decades. The data are published annually for the preceding water year (Cooney and others, 1998, for example), and made available electronically from the United States NWIS-W data retrieval web site (http://h2o-nwisw.er.usgs.gov/nwis-w/US/). Figure E-1 illustrates the location and identification number of each USGS gauging station. Industrial discharges from SRS operations are monitored near outfalls by the USGS, separate from NPDES outfall monitoring conducted by SRS. Figures E-2, E-3 and E-4 show the relationship between USGS and NPDES gauging stations for the General Separations Area/C-Area, K-Area , and L- and P-Areas, respectively.

Given the locations of the USGS gauging stations, regional scale base flows are most easily estimated for the stream reaches and wetland areas enclosed by the polygons depicted in Figure E-5. For example, base flow between the headwaters of Meyers Branch and Road 9 is more conveniently estimated than base flow over the entire reach, because there is not a gauging station on Meyers Branch just above its confluence with Steel Creek. Similarly, base flow will be estimated for portions of Upper Three Runs and Steel Creek. On the other hand, gauging stations are located where Pen Branch and Fourmile Branch enter the Savannah River Swamp, so base flow for the entire drainage can be conveniently estimated.

Figure E-6 is an example hydrograph produced from USGS data for the two gauging stations located on Meyers Branch for water years 1993 through 1996. Station 021973561 is located at Road 9 and station 02197354 monitors the P007 outfall. Discharges to P007 are small relative to the total flow at Road 9. Not surprisingly, the downstream data exhibits a seasonal variation with elevated average flows occurring from late fall through early spring. Over 
shorter periods, individual rainfall events are readily observed as a step increase in daily flow followed by an exponential decline. Presumably these peaks are due to direct precipitation, surface runoff and subsurface stormflow, and not reflective of base flow.

Because downstream USGS gauging stations measure total stream flow, the base flow component must be separated from other contributors to a hydrograph of total flow. These include the direct precipitation, surface runoff and subsurface stormflow components mentioned above, as well as process water discharges to outfalls. Shirmohammadi and others (1984) observed that "daily values of precipitation and streamflow are not sufficient for detailed hydrograph analysis using traditional hydrograph separation techniques" and developed an approximate method for partitioning daily total streamflow data, such as that available from the USGS for SRS streams. Hydrograph separation for this project is accomplished with a simplified version of the approach of Shirmohammadi and others (1984). The following steps are applied to the time series of daily total stream flow:

1) Compute the average, $F_{a v g}$, of the downstream flow, $F$

2) Subtract outfall flows from the downstream flow leaving "natural" flow components,

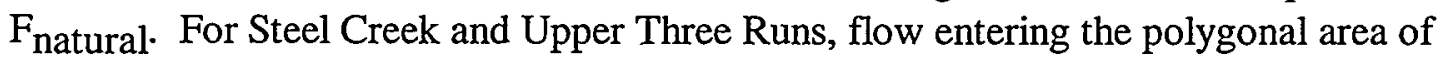
interest from upstream is also subtracted.

3) Remove the remaining direct precipitation, surface runoff and subsurface stormflow components by creating a "clipped" time series, $\mathrm{F}_{\text {base flow, }}$ according to

$F_{\text {base flow }}=\min \left[F_{\text {natural }}, 1.05 \times \max \left(F_{\text {natural, previous }}, F_{\text {avg }}\right)\right]$

4) Smooth the base flow component, Fbase flow, over 4 water years, 1993 to 1996, for easier visualization using a running digital filter:

$\mathrm{F}_{\text {smooth }}=\left(\mathrm{F}_{\text {base flow, } \mathrm{i}-1}+\mathrm{F}_{\text {base flow, } \mathrm{i}}+\mathrm{F}_{\text {base flow, } \mathrm{i}+1}\right) / 3$

5) Average the smoothed base flow component, $F_{\text {smooth }}$, over the 4 water years from 1993 to 1996 , producing $\mathrm{F}_{\text {smthavg. }}$

The third step is based on the assumption that the base flow component responds slowly to rainfall events, and therefore cannot increase very rapidly from one day to the next (5\% or less). No restriction on the rate of decrease is imposed. The fourth step does not affect the average computed in the fifth step. 
The maximum rate of base flow increase, specified in step 3, was selected according to the recommendations of Linsley and others (1982, chapter 7). As a rule of thumb, the duration of direct runoff following the end of a rainfall is approximately $\mathrm{A}^{0.2}$ days, where $\mathrm{A}$ is the drainage area in square miles (Linsley and others, 1982, equation 7-4). Pen Branch has a drainage area of 21 square miles resulting in runoff terminating after about 2 days. The drainage area of Fourmile Branch is 22 square miles yielding essentially the same duration. Taking these streams as representative, base flow should typically depart from total flow at the start of a rainfall event and rejoin the total flow after 2 days plus the duration of the rain. The total time of departure would be roughly 2 to 3 days. However, inspection of several individual rainfall events in comparison to the qualitative guidelines of Linsley and others (1982, Figure 7-5) suggests that runoff often lasts longer, sometimes up to roughly 6 days following a heavy rain. The maximum rate of base flow increase was set to 1.05 to yield a 2 to 6 day duration. Figure E- 6 shows a sample segment of total and estimated base flow for Meyers Branch.

Applying the above procedure to the appropriate data for each drainage basin yields the results illustrated in Figures E-7 through E-12. In these figures, the upper plots (a) show the reference downstream flow and any outfall or upstream flows. Additional detail on the more significant outfall flow rates is provided by Figures E-13 through E-20. Note that generally outfall and upstream flows form a large component of the downstream flow. The curves (b) at the bottom of Figures E-6 through E-11 show the "base flow", "smooth" and "smthavg" components as defined in steps 3) to 5) above.

Table E-1 summarizes the bottom line results. The appropriate base flow target for CKLP model calibration is gotten by multiplying the base flow estimate for the stream reach by the fraction of the reach contained within the model domain. For Upper Three Runs between Road C and Road A, which lies on the CKLP model boundary, a reasonable assumption is that each side contributes equally. The main branch of Meyers Branch forms a boundary of the model, but a major tributary just south of Dunbarton Road is totally outside the model domain. Overall, perhaps $1 / 3$ of this reach lies within the model. Far more uncertain is the fraction of base flow to the larger Upper Three Runs reach that should be attributed to groundwater from within the model; $1 / 4$ is suggested in Table E-1. The Steel Creek estimate is negative and indicates a losing reach, presumably reflecting artificial flows to L-Lake to maintain the current lake level.

The stated accuracy of the various raw USGS gauging station data is typically "good" $(<10 \%$ error $95 \%$ of the time), "fair" ( $<15 \%$ error $95 \%$ of the time) or "poor" (less than "fair" 
accuracy) (Cooney and others, 1998, p. 16). Uncertainty in the long-term average flows ultimately used in this analysis are much smaller than uncertainty in a daily flow. Larger contributors to overall uncertainty are biases in the hydrograph separation procedure, and the estimated fraction of the analyzed reach that lies within the model domain. The uncertainty of the results summarized in Table E-1 can be estimated by considering different values for the assumed maximum rate of base flow increase. As shown by Table E-2, the base flow estimates appear to have an uncertainty around plus or minus $10 \%$ due to uncertainty in the chosen rate. Biases in hydrograph separation technique might add another 5 to $10 \%$. For example, base flow continues to decrease during flood conditions before ascending. The hydrograph separation technique used here allows baseflow to increase immediately, and might produce slightly high estimates. Some of the model calibration targets contain added uncertainty in the amount that should be partitioned to the model domain. Overall, the baseflow targets may have an uncertainty of 15 to $25 \%$.

Exceptions include the base flow estimates for Steel Creek and Upper Three Runs. These base flow estimates are derived by taking the difference of large, nearly equal, flows. Uncertainty in daily measurements can not be neglected for these reaches. The additional uncertainty can be estimated as follows. Assume the $2 \square$ confidence interval on a daily flow measurement is $15 \%$ of the mean, $\square$ :

$$
2 \sigma=0.15 \mu
$$

For independent errors in daily flow, the uncertainty in the long-term average is

$$
2 \sigma_{\mu}=\frac{2 \sigma}{\sqrt{\mathrm{n}}}=\frac{0.15 \mu}{\sqrt{\mathrm{n}}}
$$

where $\mathrm{n}$ is the number of individual measurements, approximately $4 \times 365=1460$ for 4 Water Years of data. For a base flow estimate that is a linear combination of long-term flow station estimates, the uncertainty is

$$
2 \sigma_{\sum \mu}=\sqrt{\sum\left(2 \sigma_{\mu}\right)^{2}}=\sqrt{\sum\left(\frac{0.15 \mu}{\sqrt{\mathrm{n}}}\right)^{2}}
$$

where the errors are again assumed to be independent. For Steel Creek, the result is 


\begin{tabular}{|c|c|}
\hline Station & Mean (cfs) \\
\hline Steel Creek at Road A & 78 \\
\hline L007 outfall & 69 \\
\hline Steel Creek above Road B & 7.5 \\
\hline Meyers Branch at Road 9 & 10.5 \\
\hline Base flow estimate & 2.2 \\
\hline
\end{tabular}

For Upper Three Runs between Roads C and A, the result is

\begin{tabular}{|c|c|}
\hline Station & Mean (cfs) \\
\hline Upper Three Runs at Road A & 244 \\
\hline Upper Three Runs at Road A & 228 \\
\hline Base flow estimate & 8.9 \\
\hline $\begin{array}{l}\text { Absolute uncertainty } \\
\text { Relative uncertainty }\end{array}$ & $\begin{array}{c}1.3 \\
15 \%\end{array}$ \\
\hline
\end{tabular}

These additional uncertainties are reflected in Table E-1. For biased measurements, the uncertainty would be higher. The above analysis is based on the independent, unbiased errors and therefore underestimates the actual uncertainty.

\section{References}

Cooney, T: W., P. A. Drewes, K. H. Jones, J. W. Gissendanner and B. W. Church, 1998, Water resources data - South Carolina, Water year 1997, Volume 1, U. S. Geological Survey Water-Data Report SC-97-1.

Linsley, R. K. Jr., M. A. Kohler and J. L. H. Paulhus, 1982, Hydrology for engineers, $3^{\text {rd }}$ edition, McGraw-Hill, New York.

Shirmohammadi, A., W. G. Knisel and J. M. Sheridan, 1984, An approximate method for partitioning daily streamflow data, Journal of Hydrology, v74, p. 335-354. 


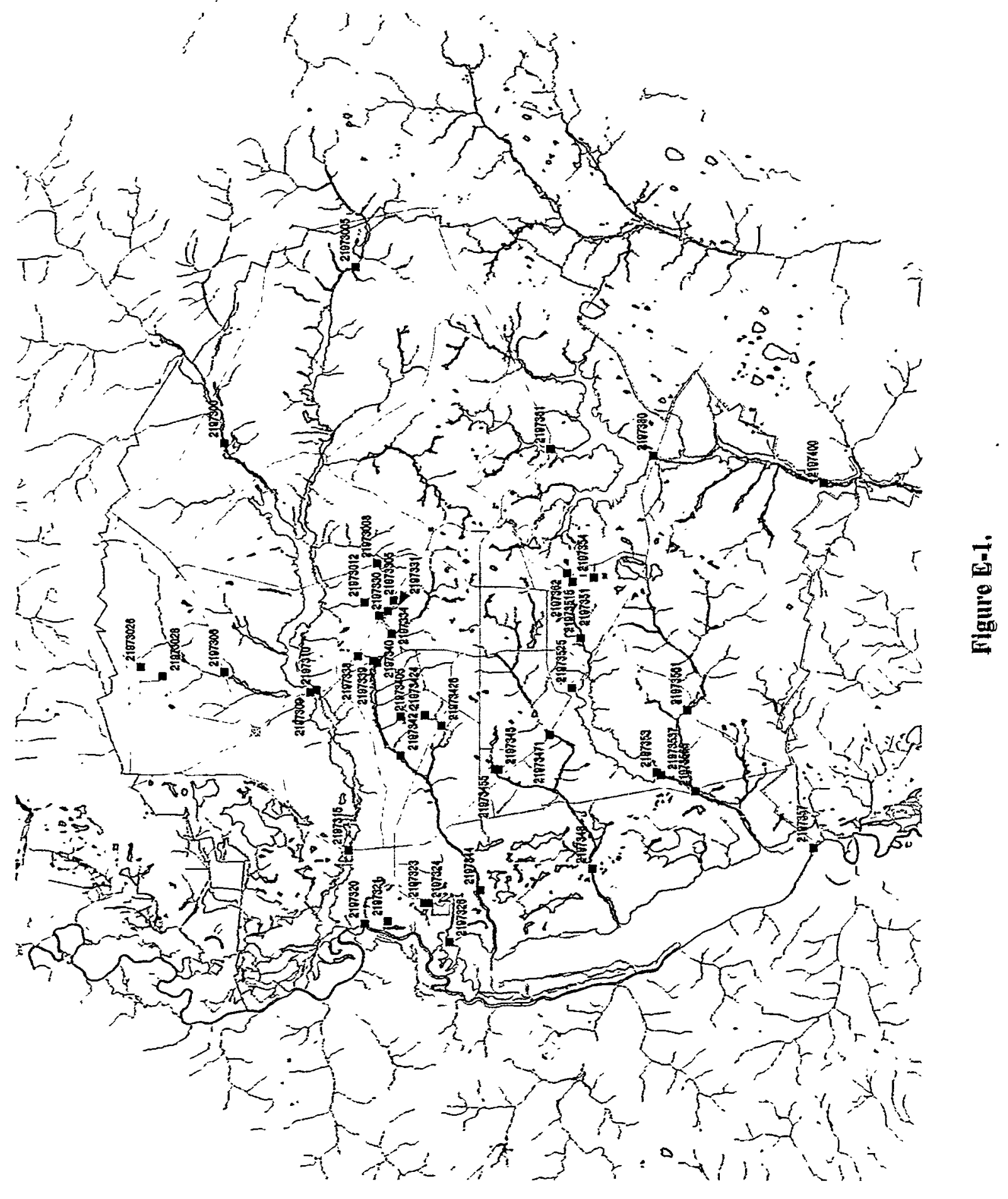




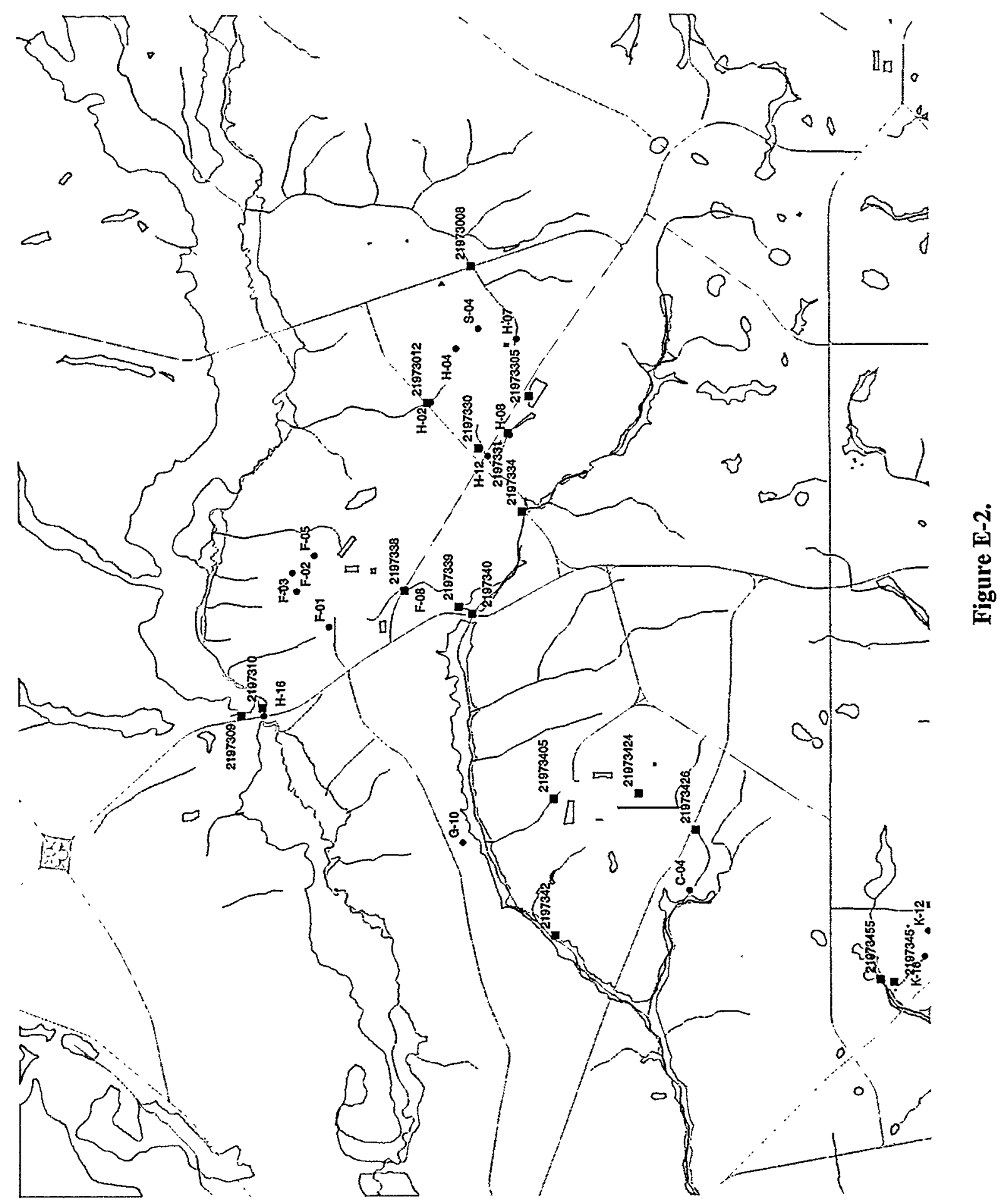




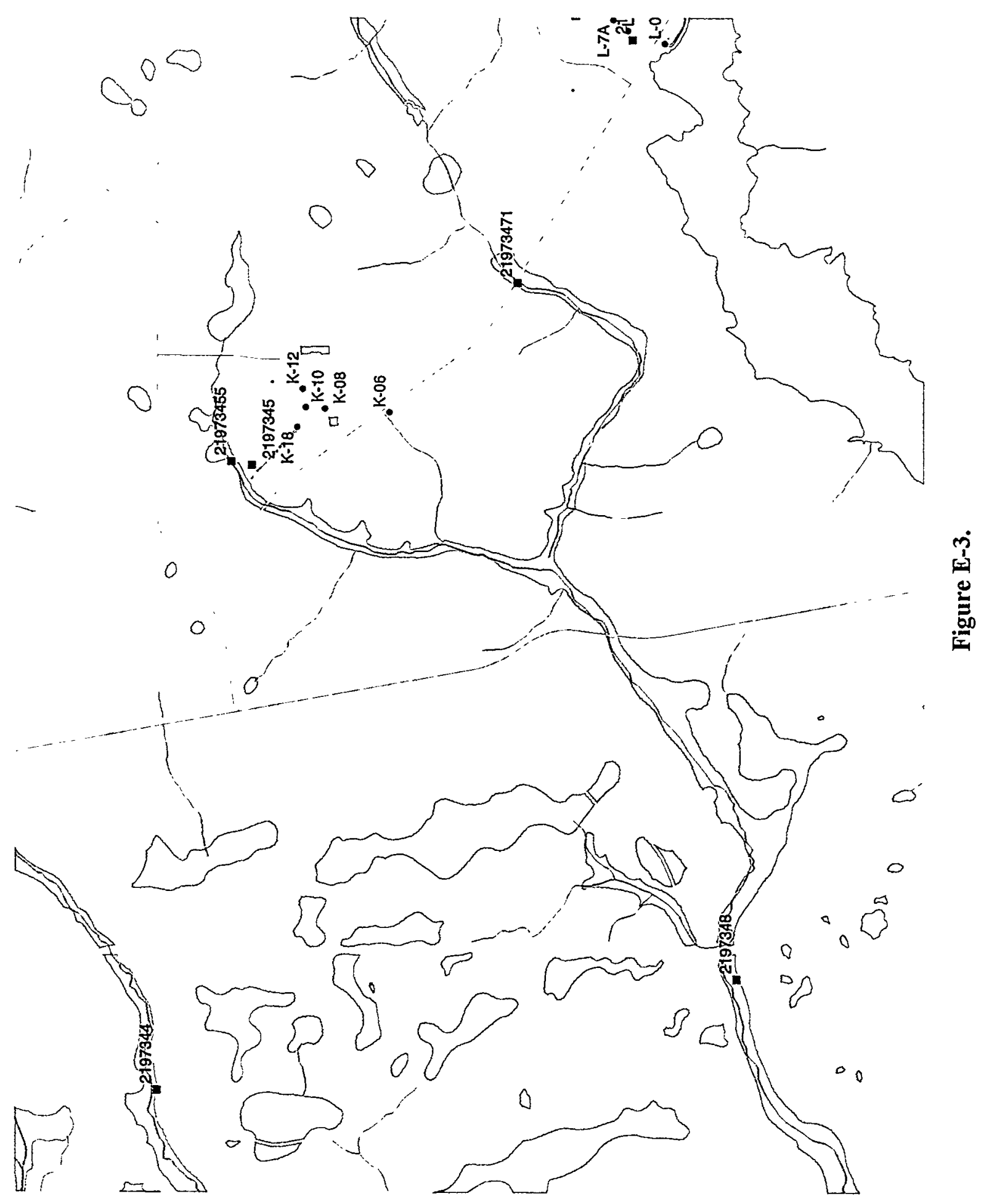




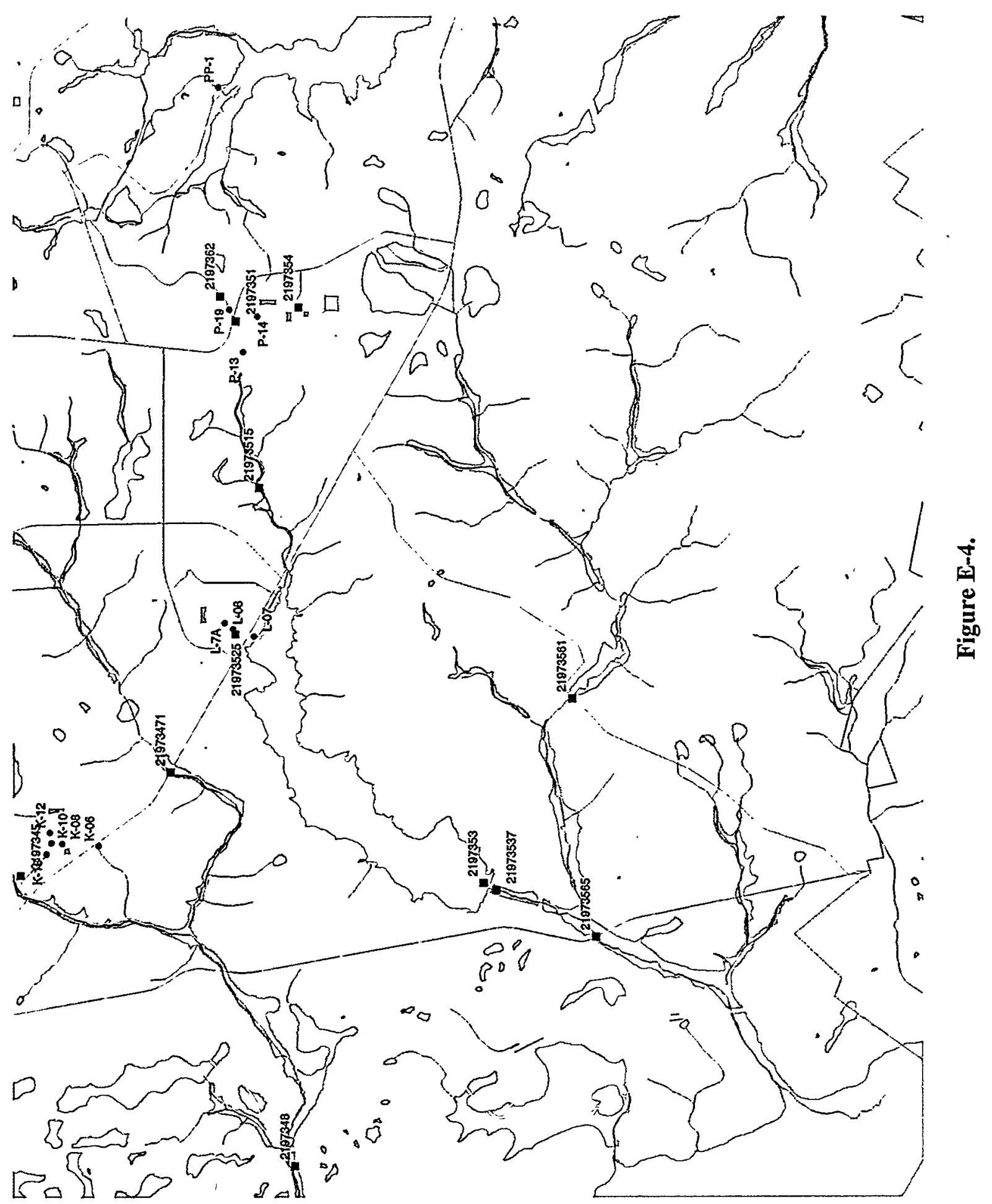




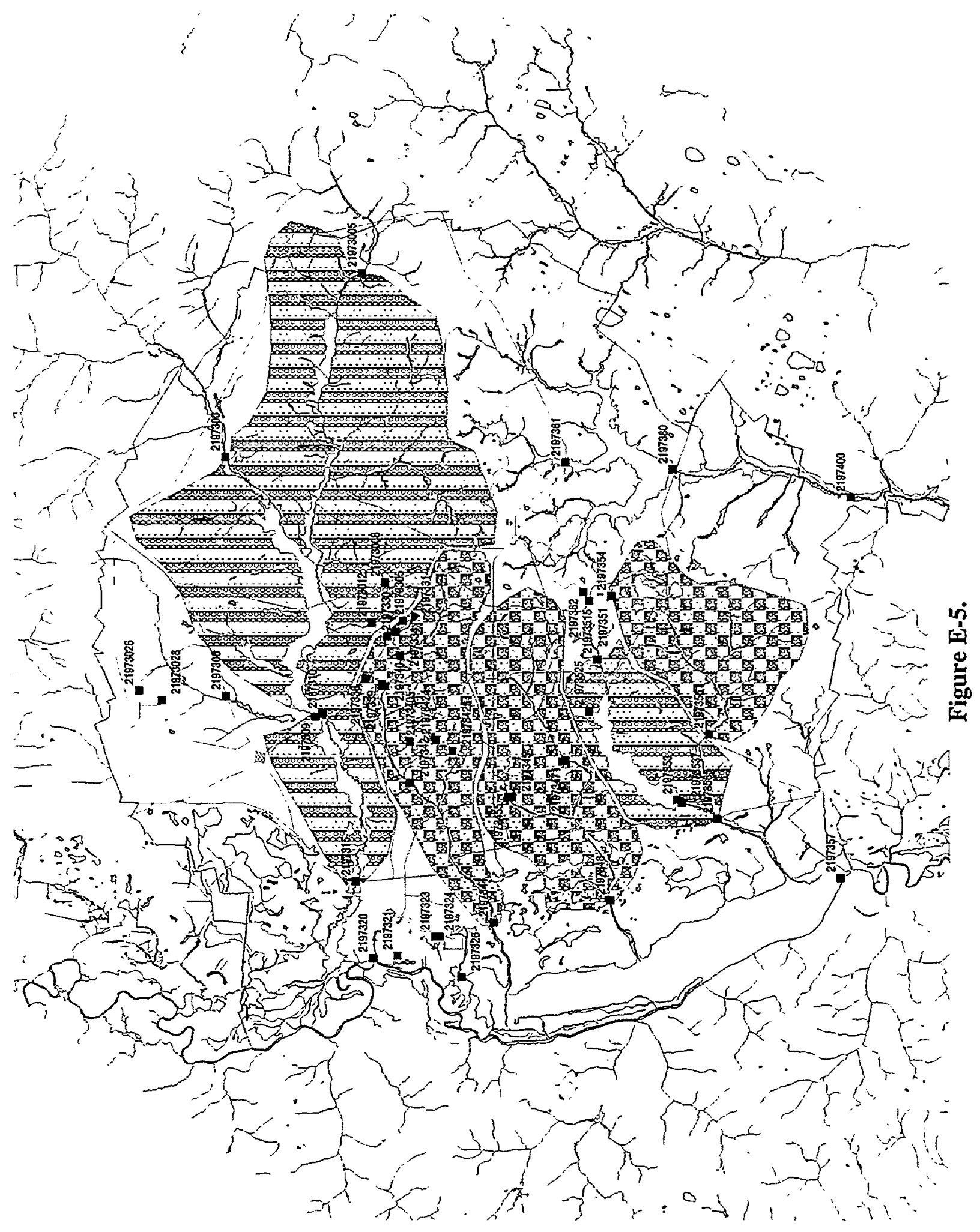




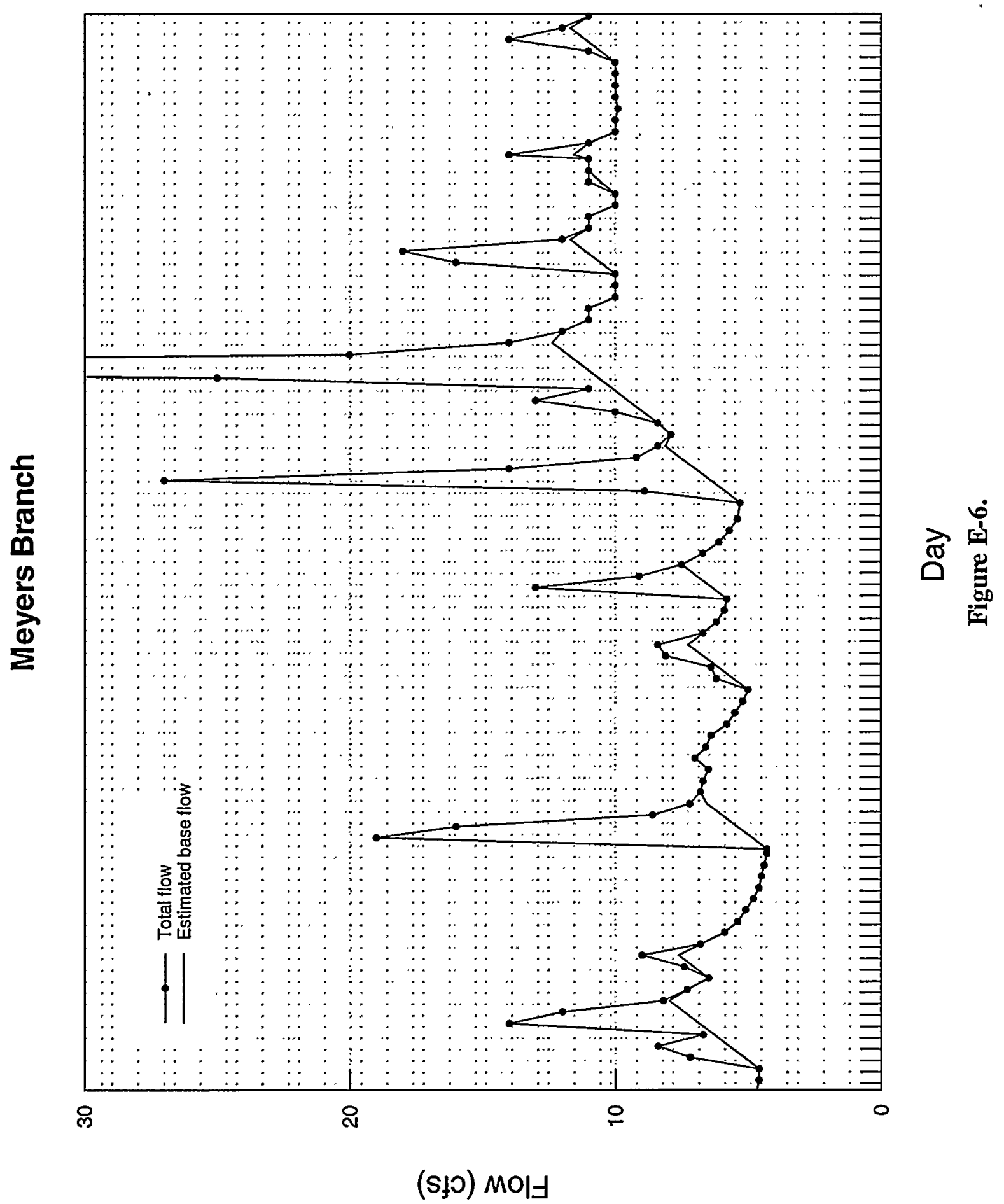




\section{Meyers Branch}

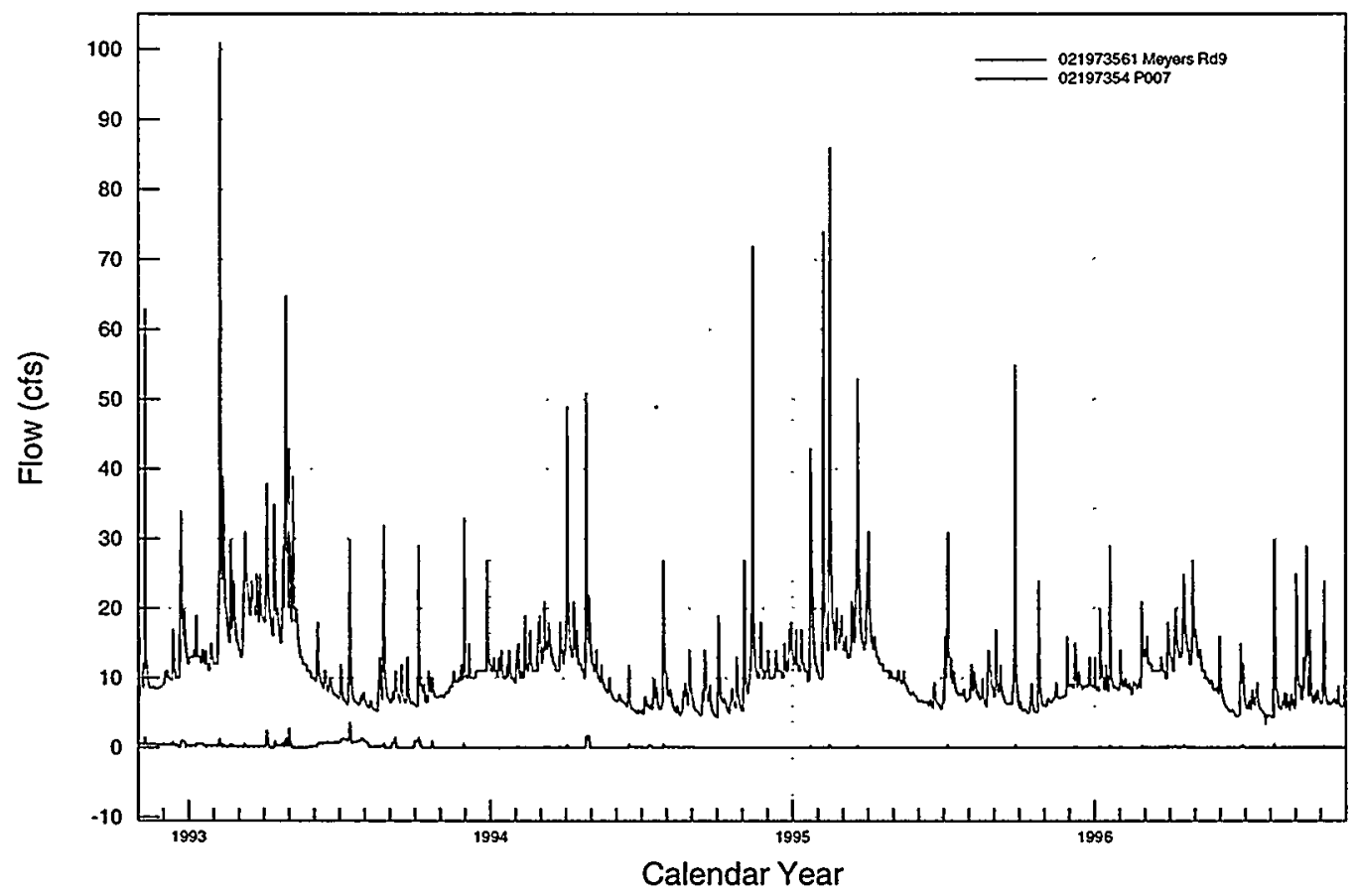

Meyers Branch

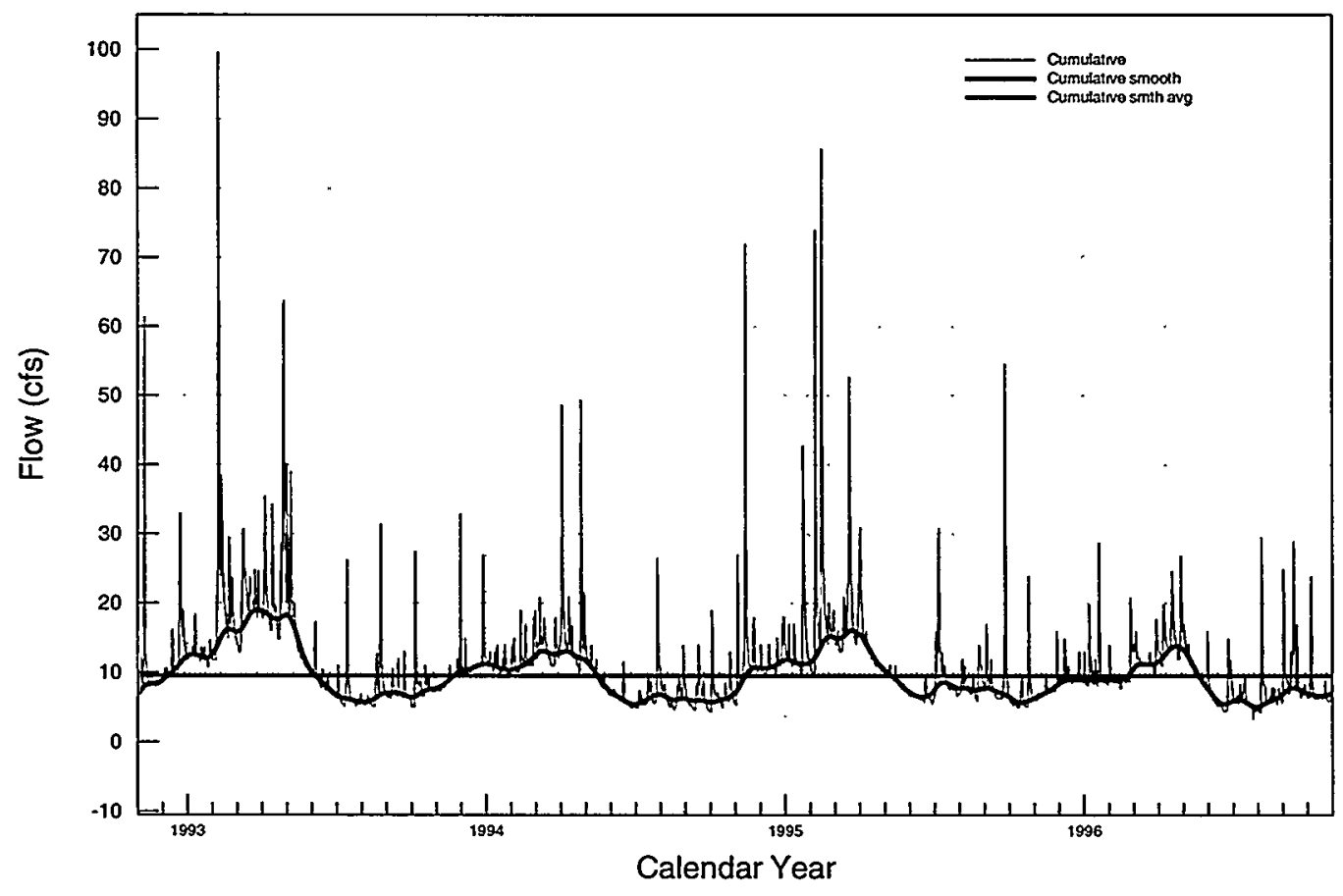

Figure E-7. 


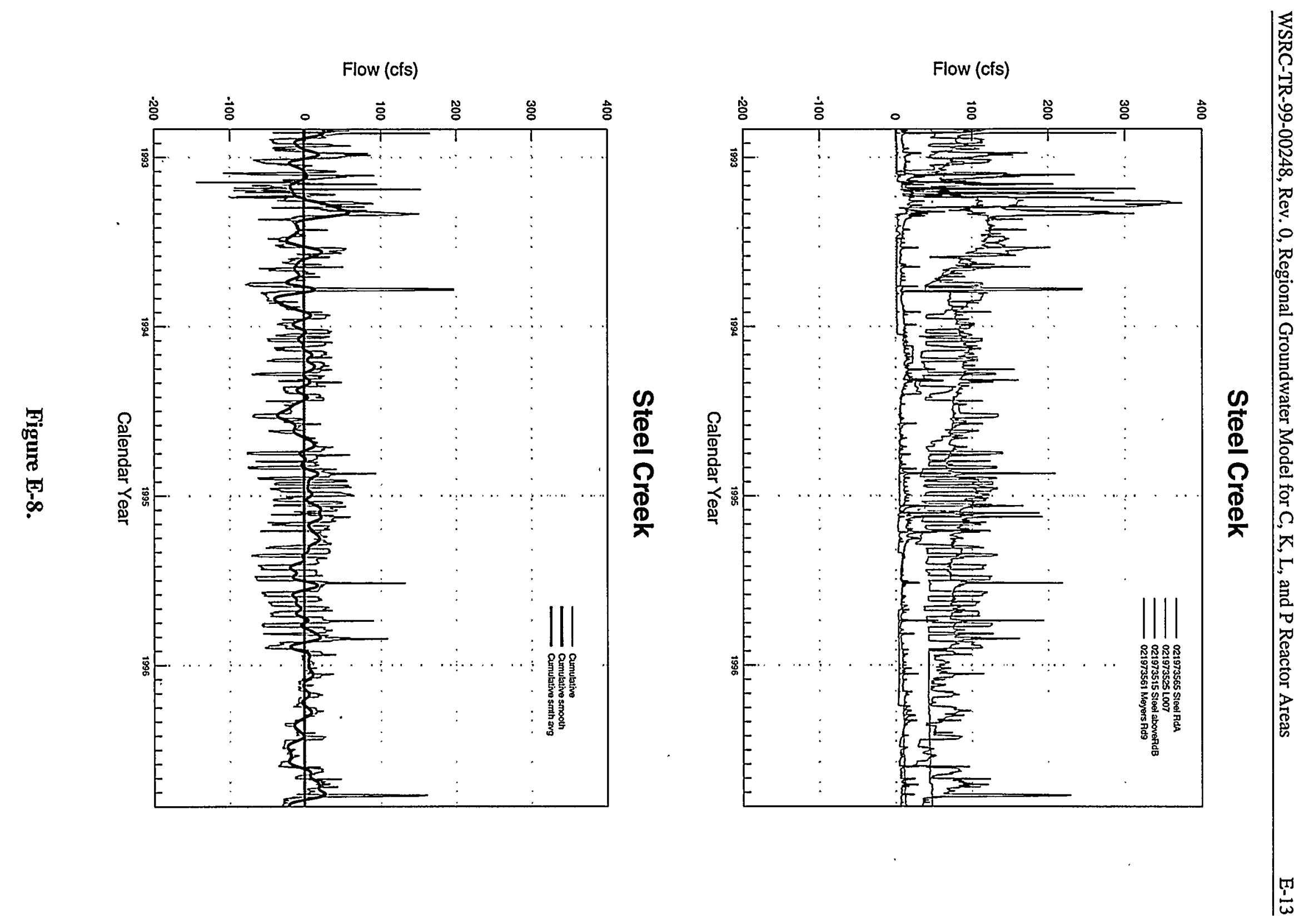




\section{Pen Branch}

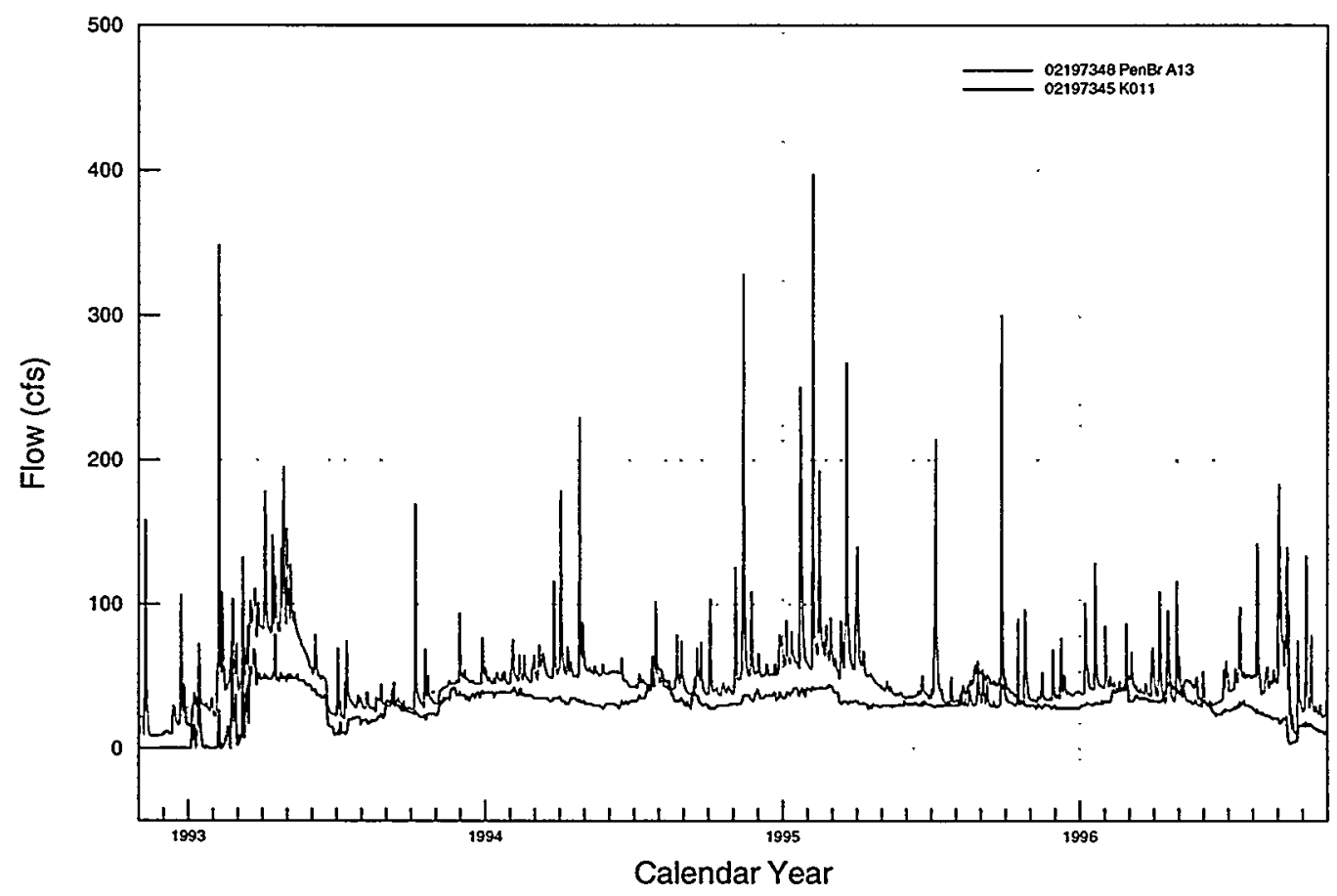

Pen Branch

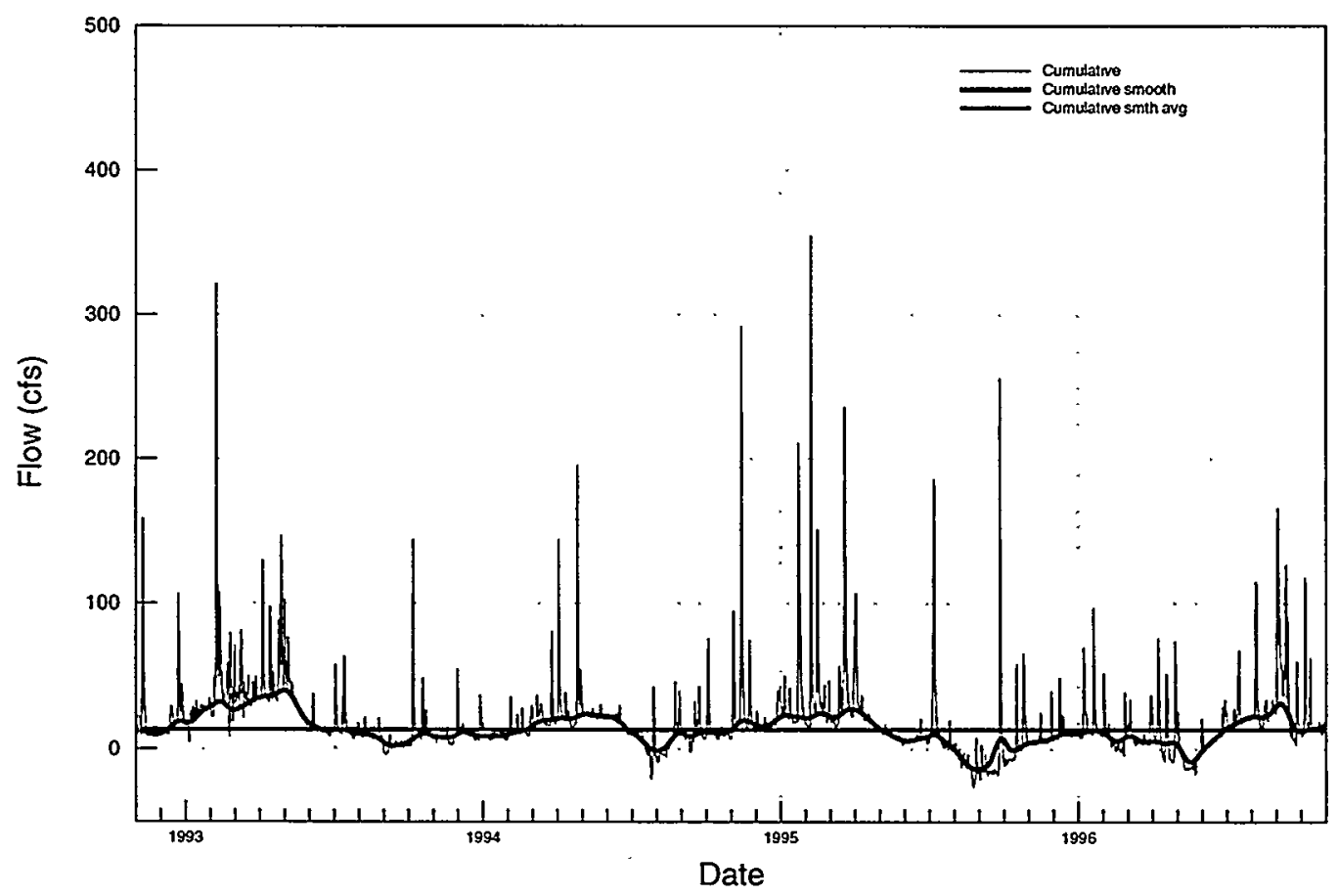

Figure E-9. 
Fourmile Branch

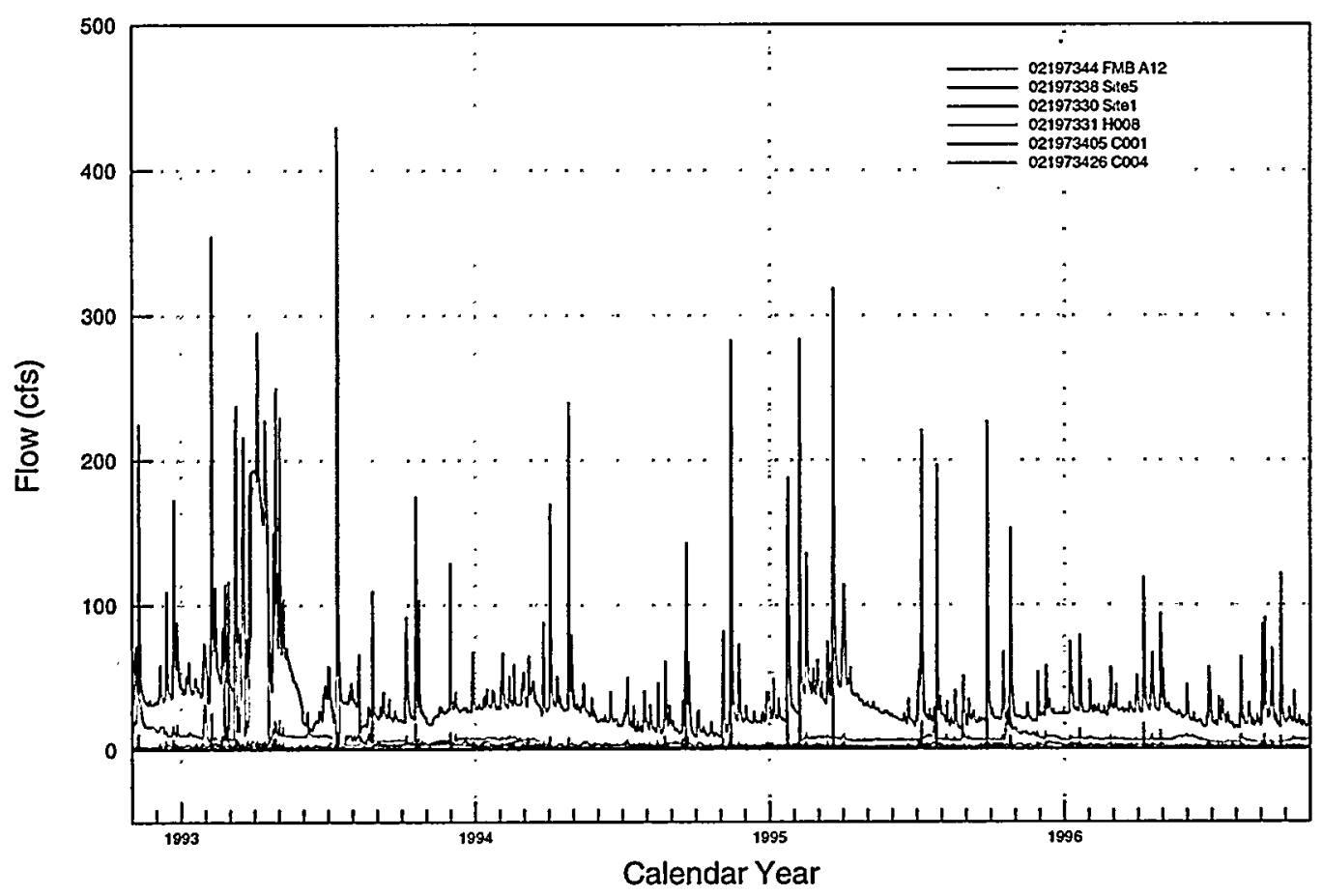

Fourmile Branch

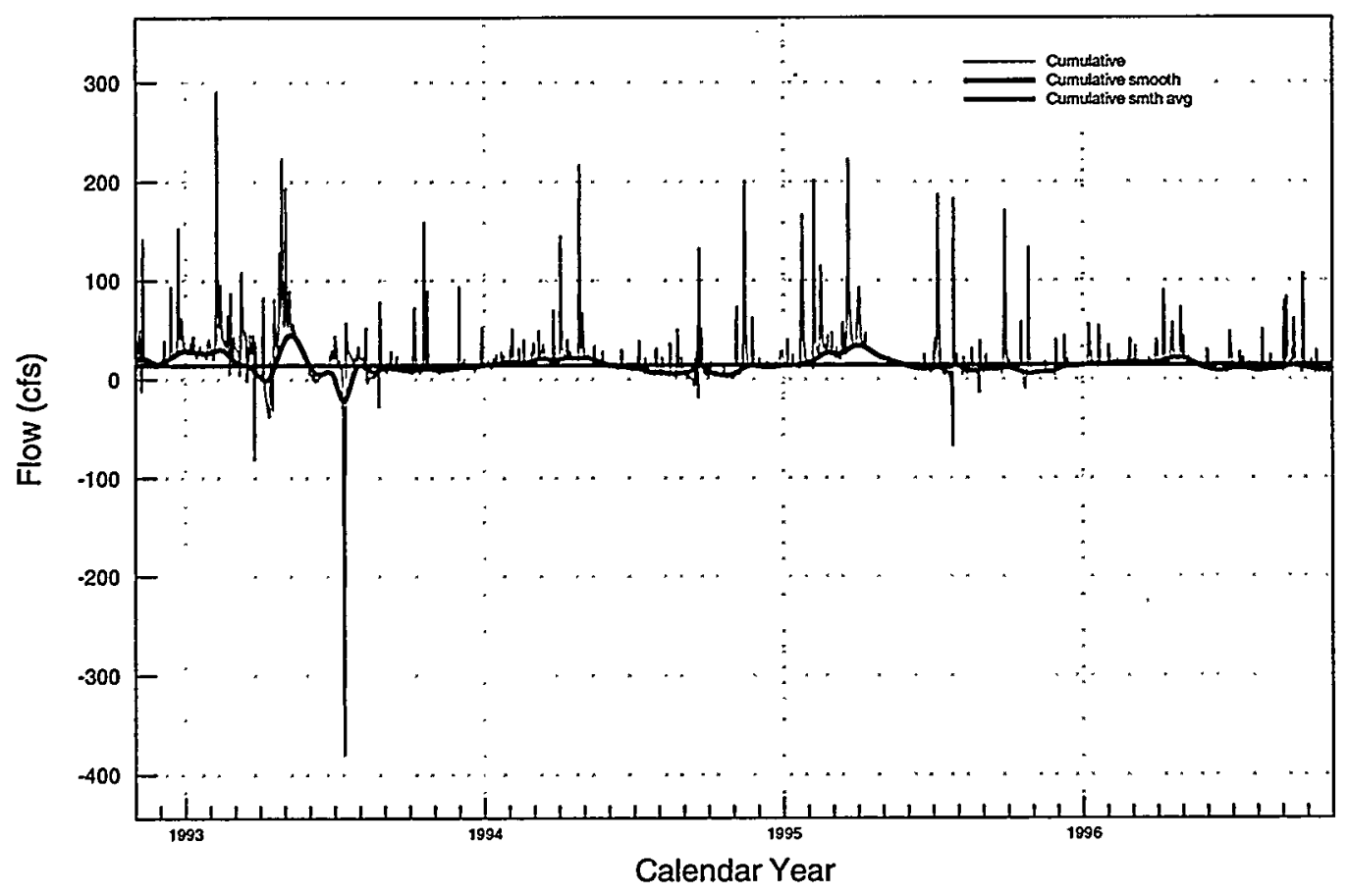

Figure E-10. 


\section{Upper Three Runs}

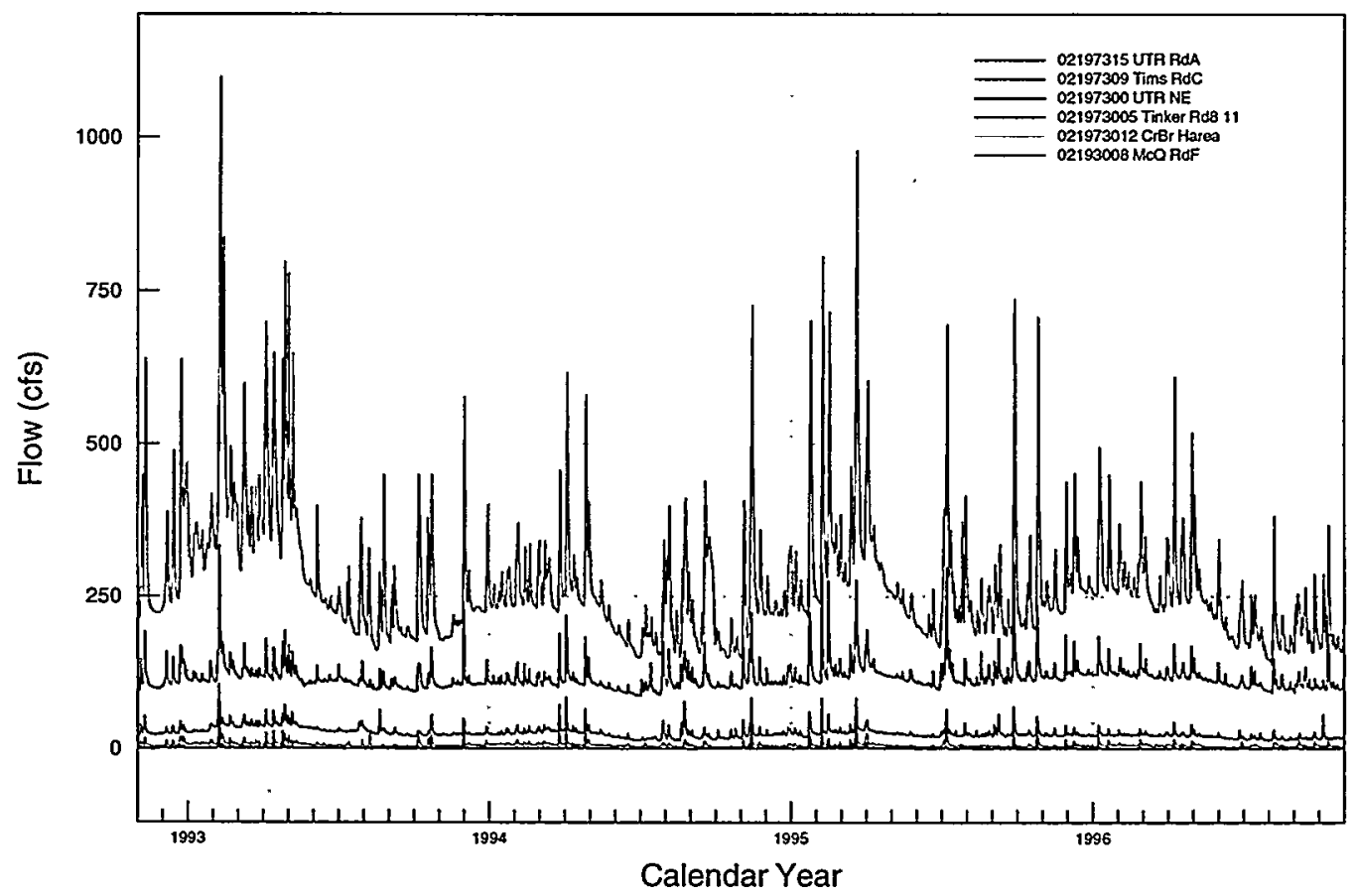

\section{Upper Three Runs}

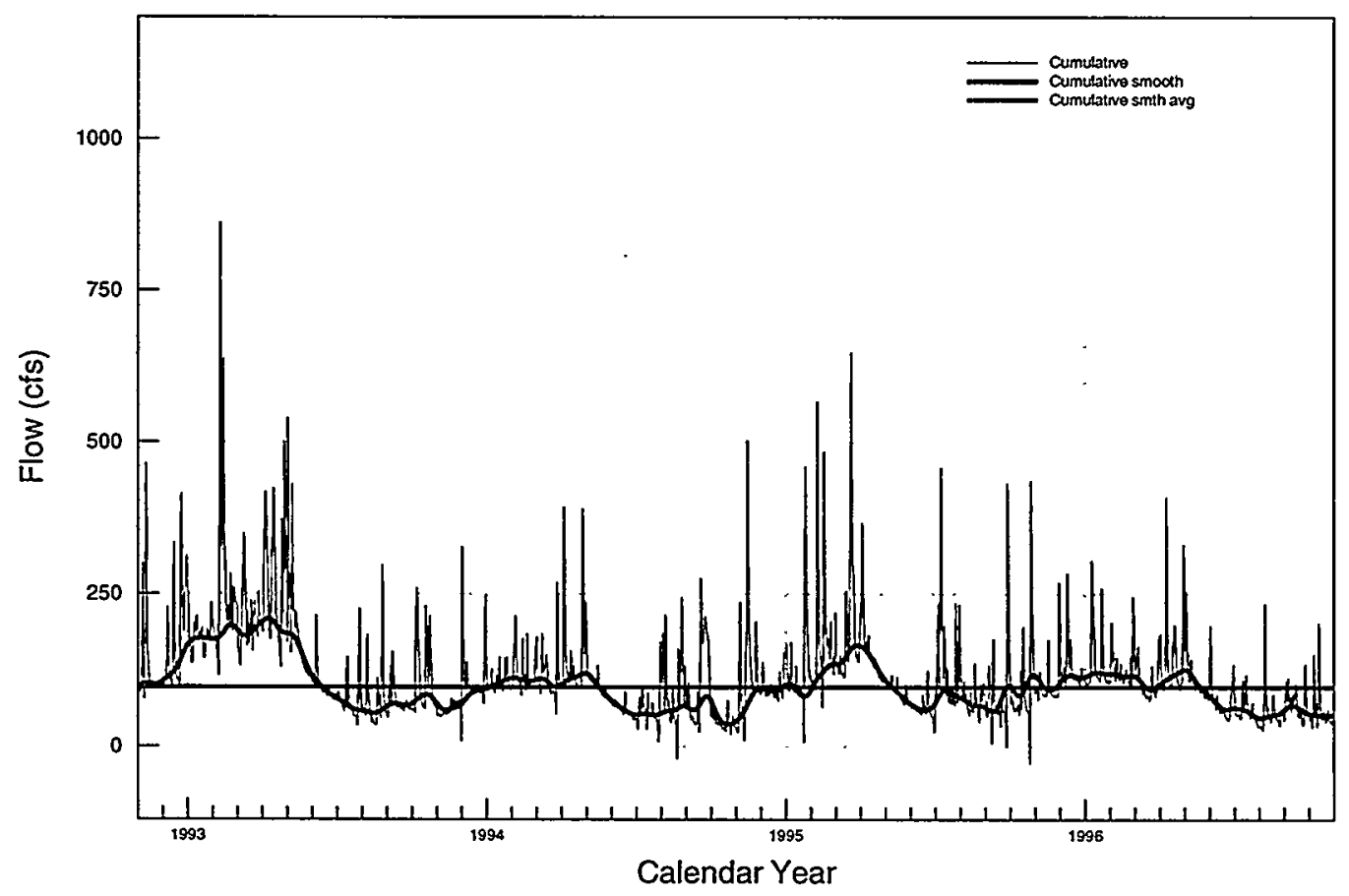

Figure E-11. 


\section{Upper Three Runs}

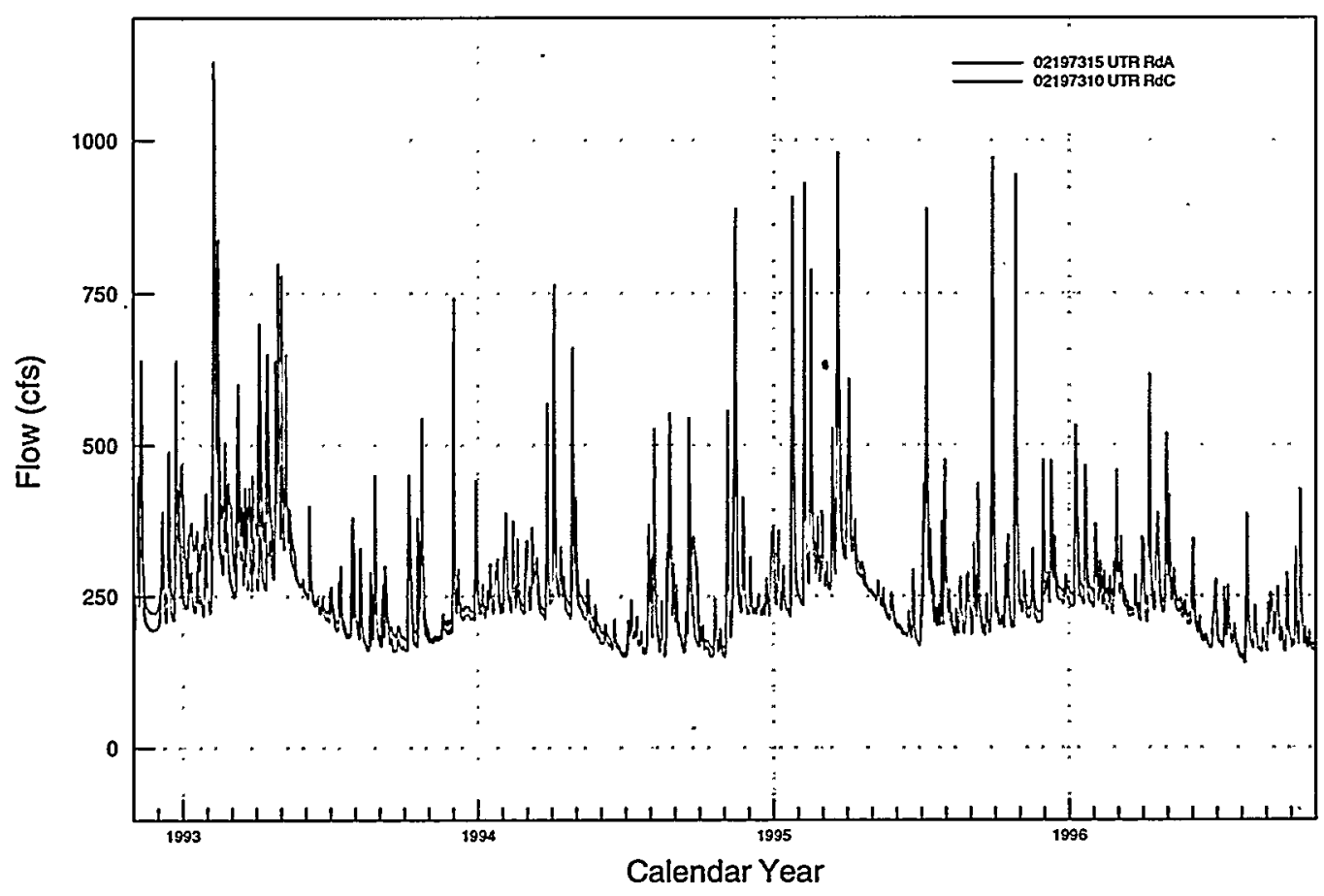

Upper Three Runs

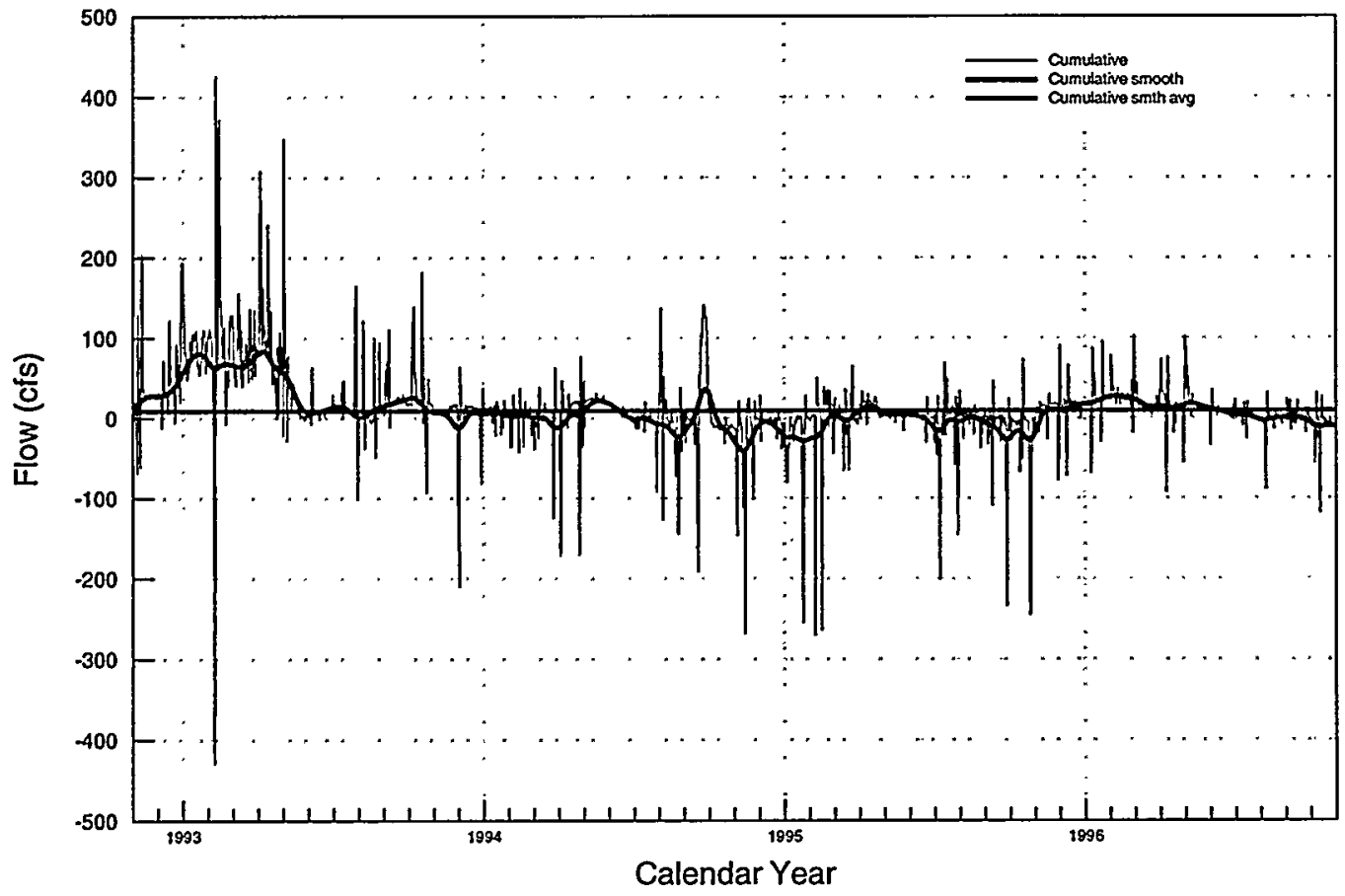

Figure E-12. 


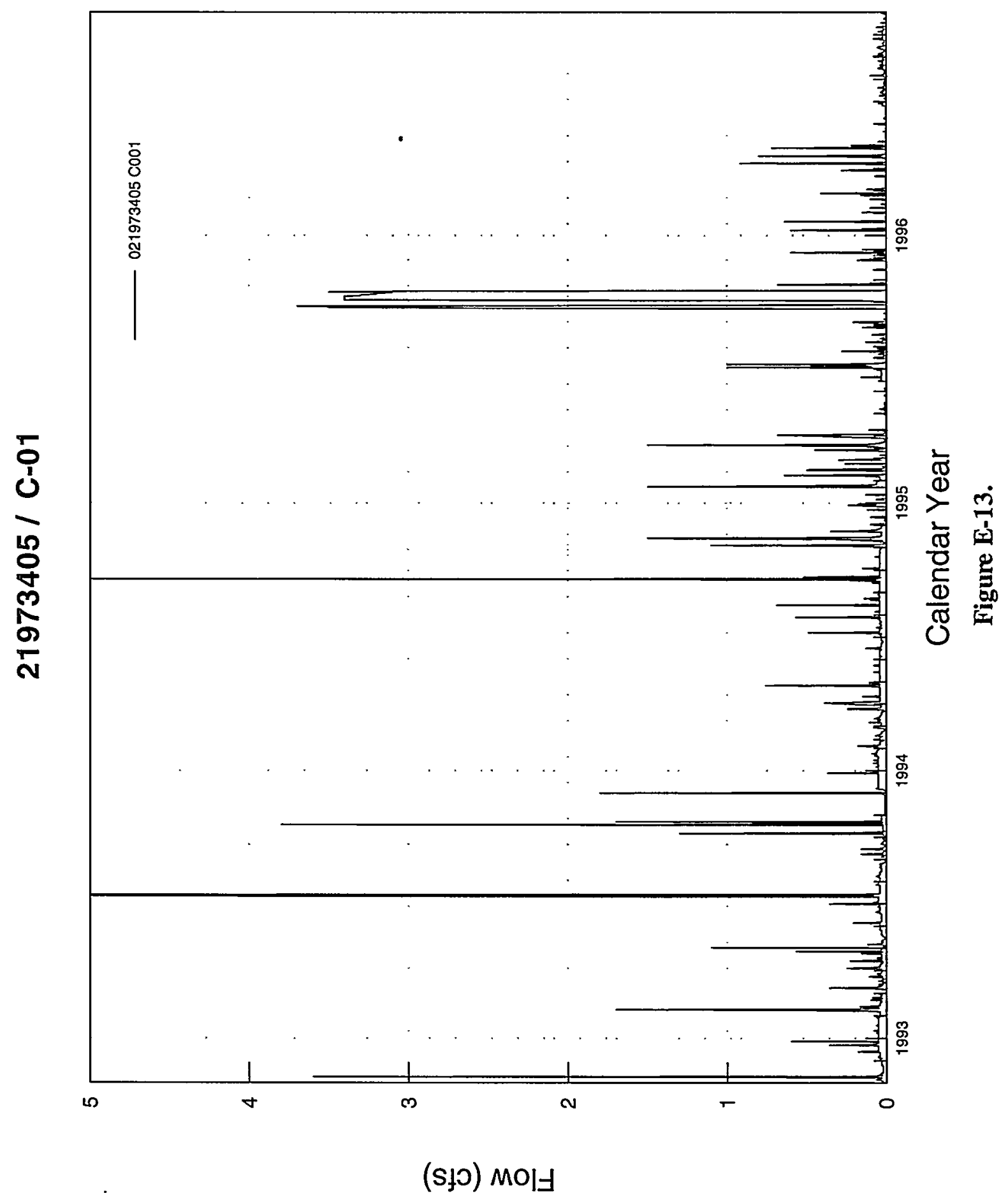




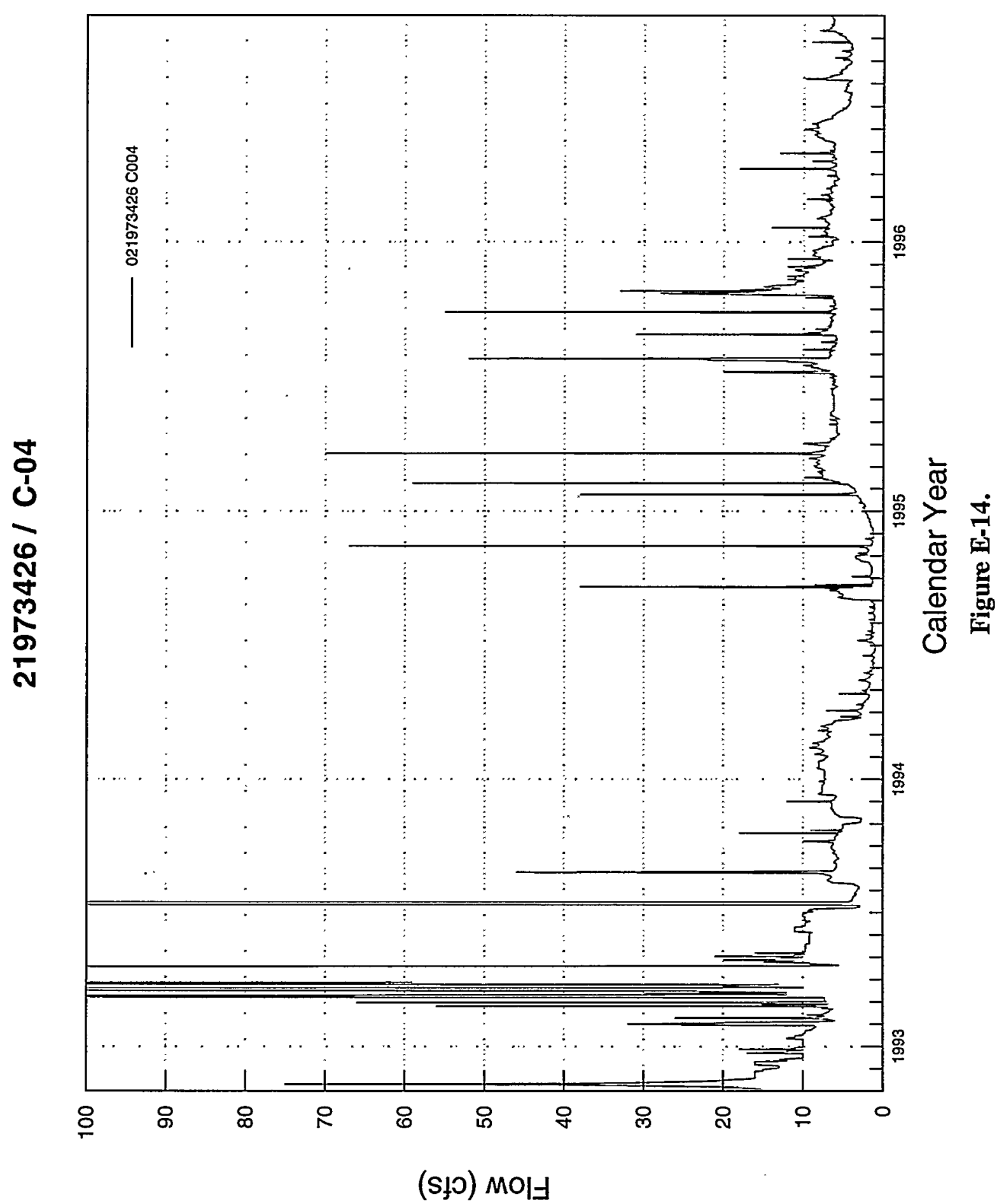




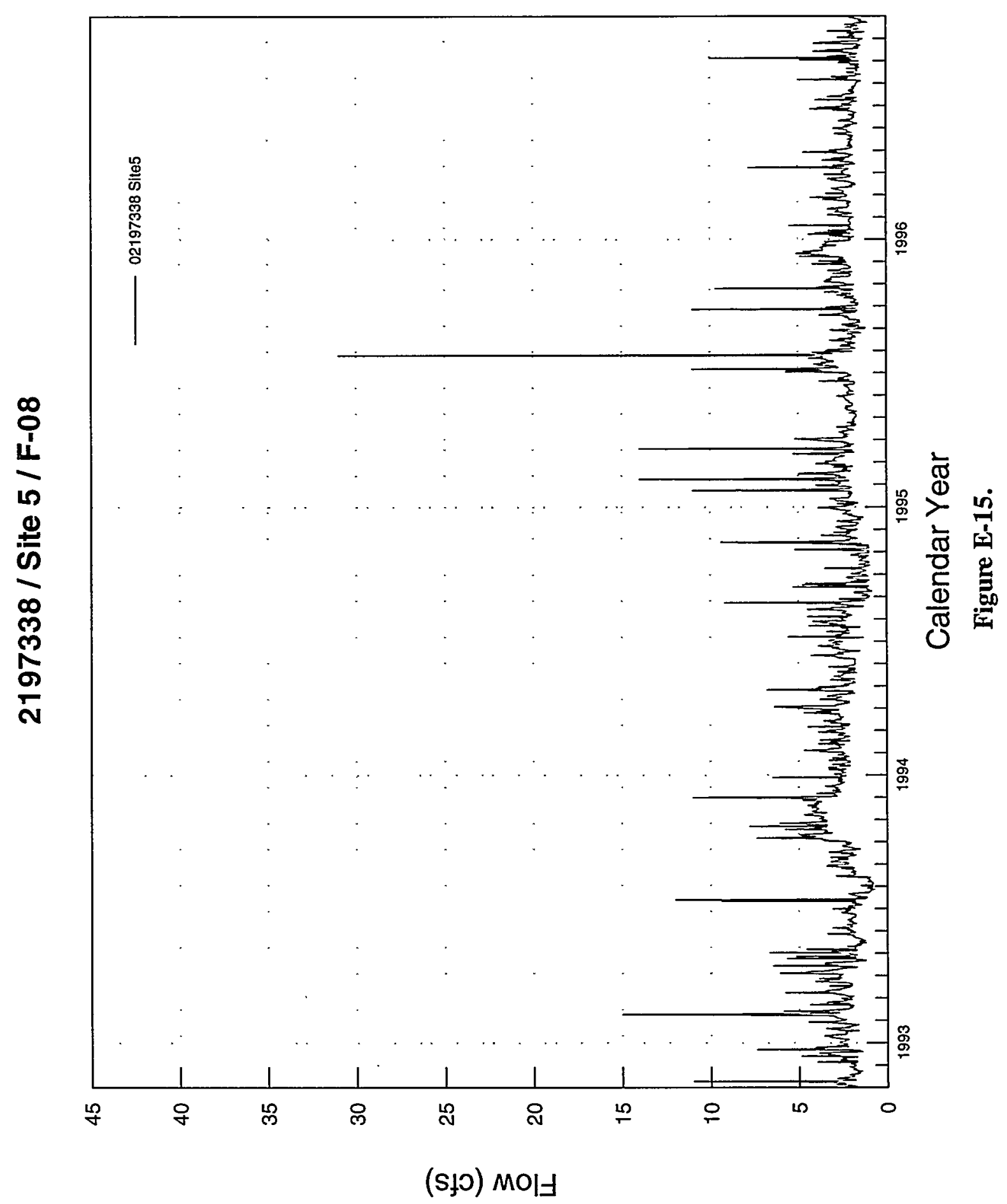




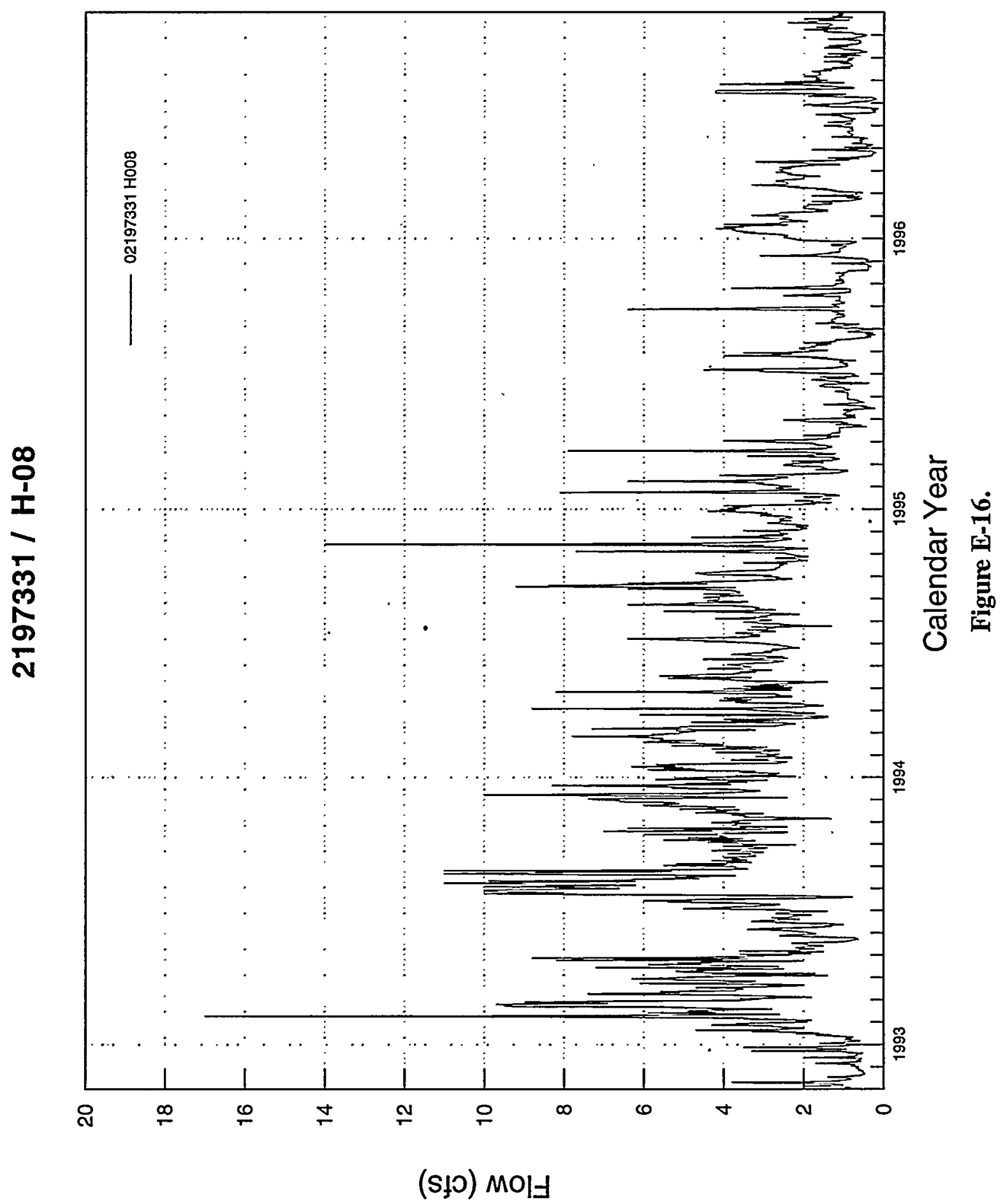




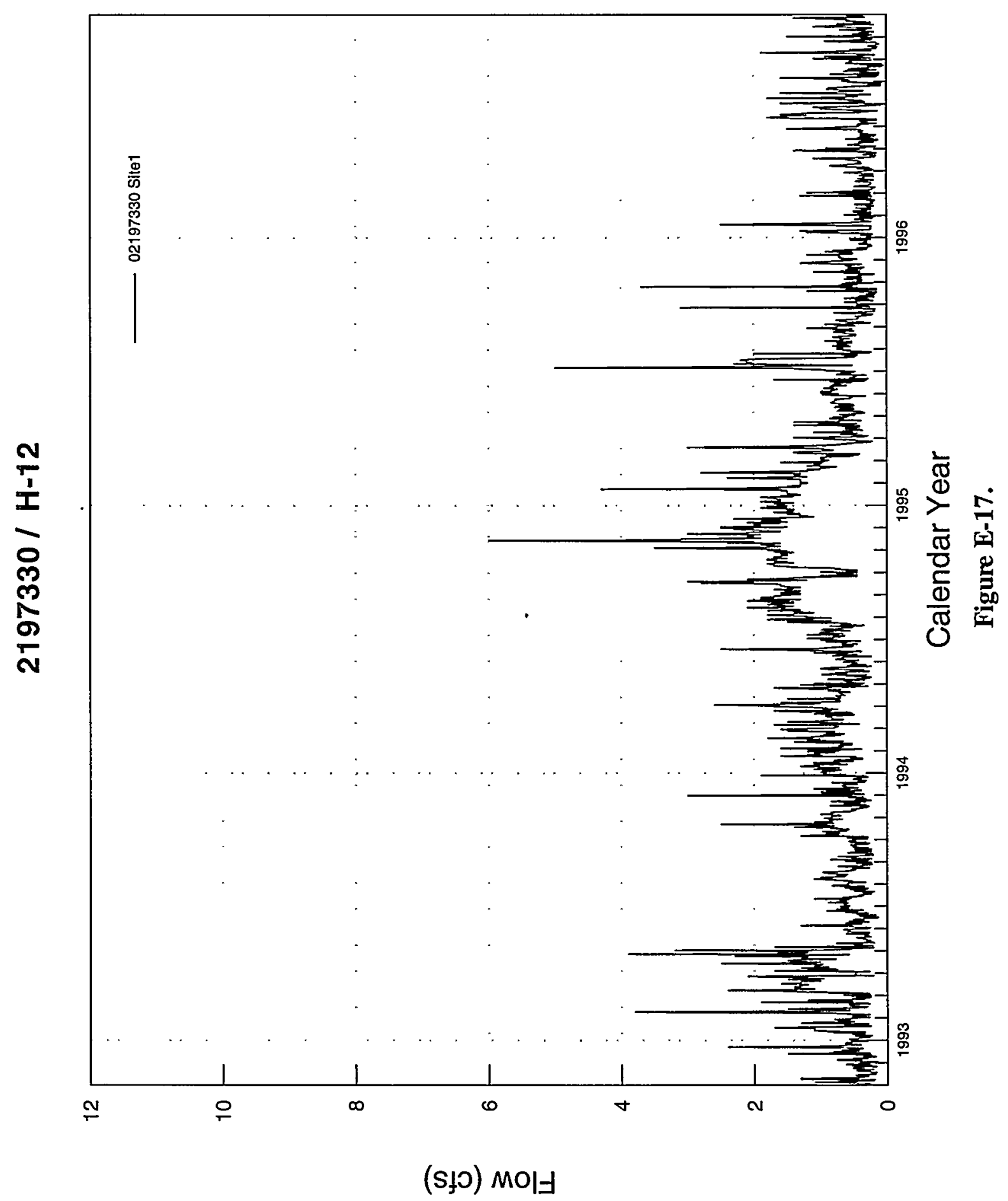




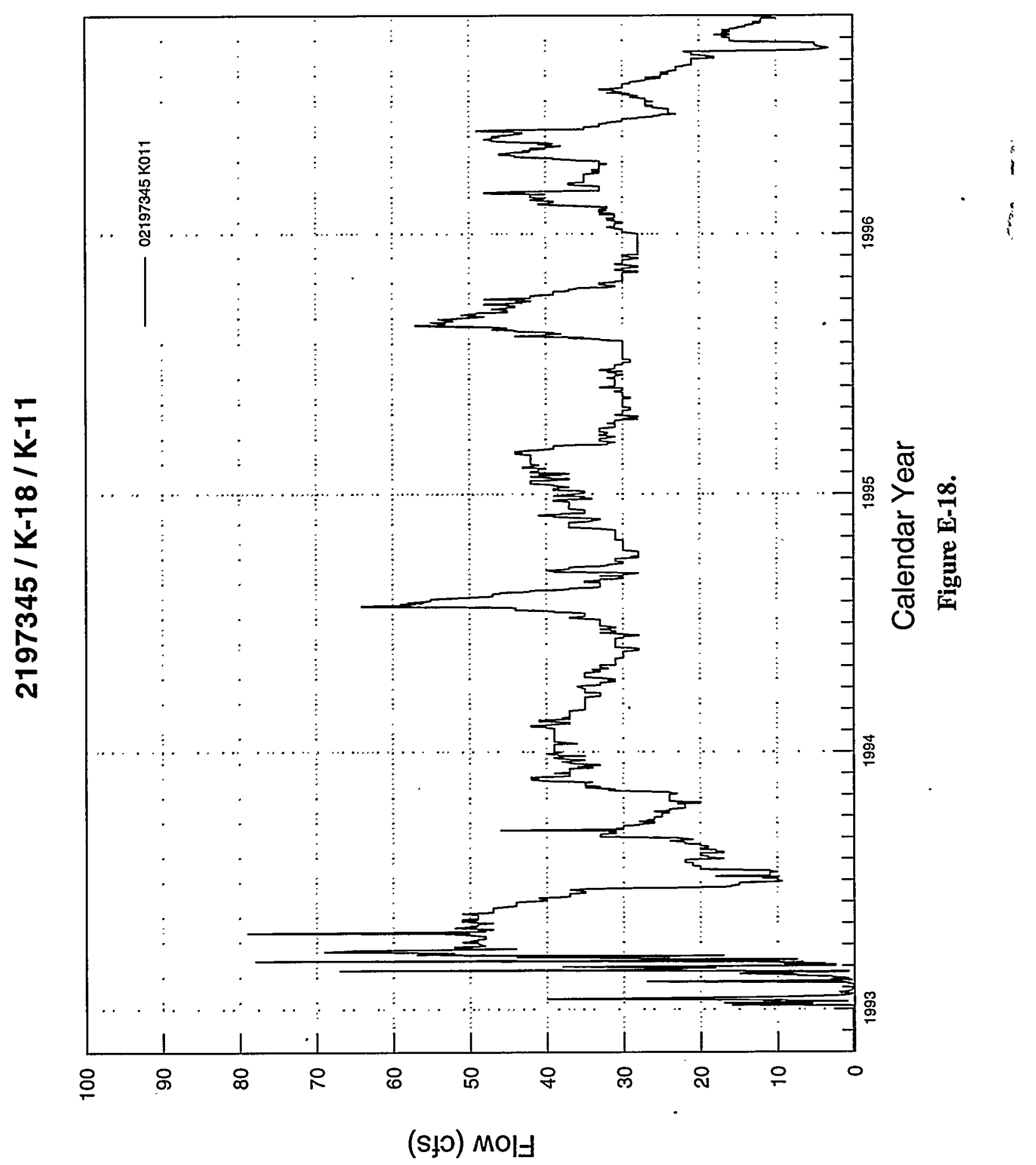




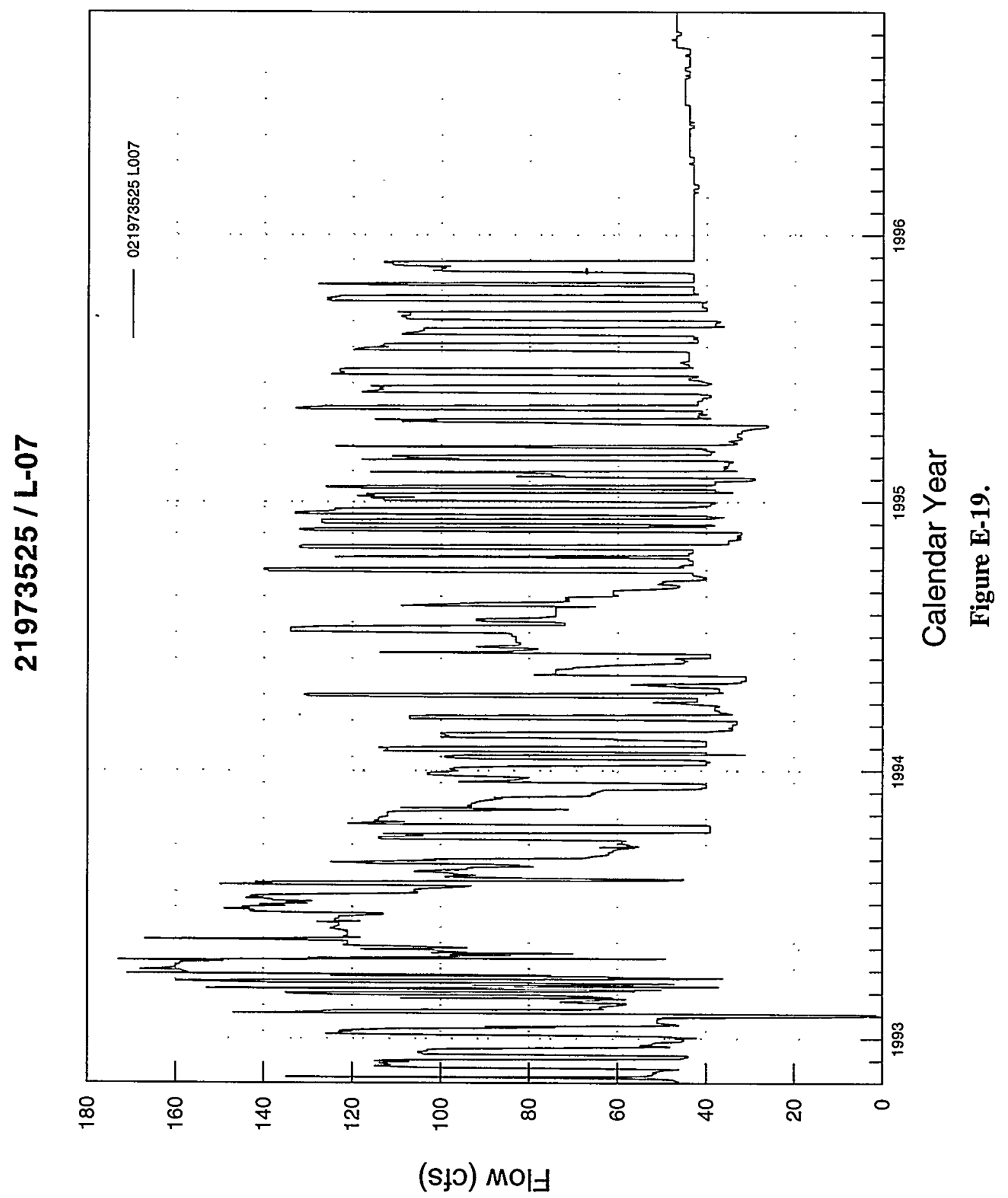




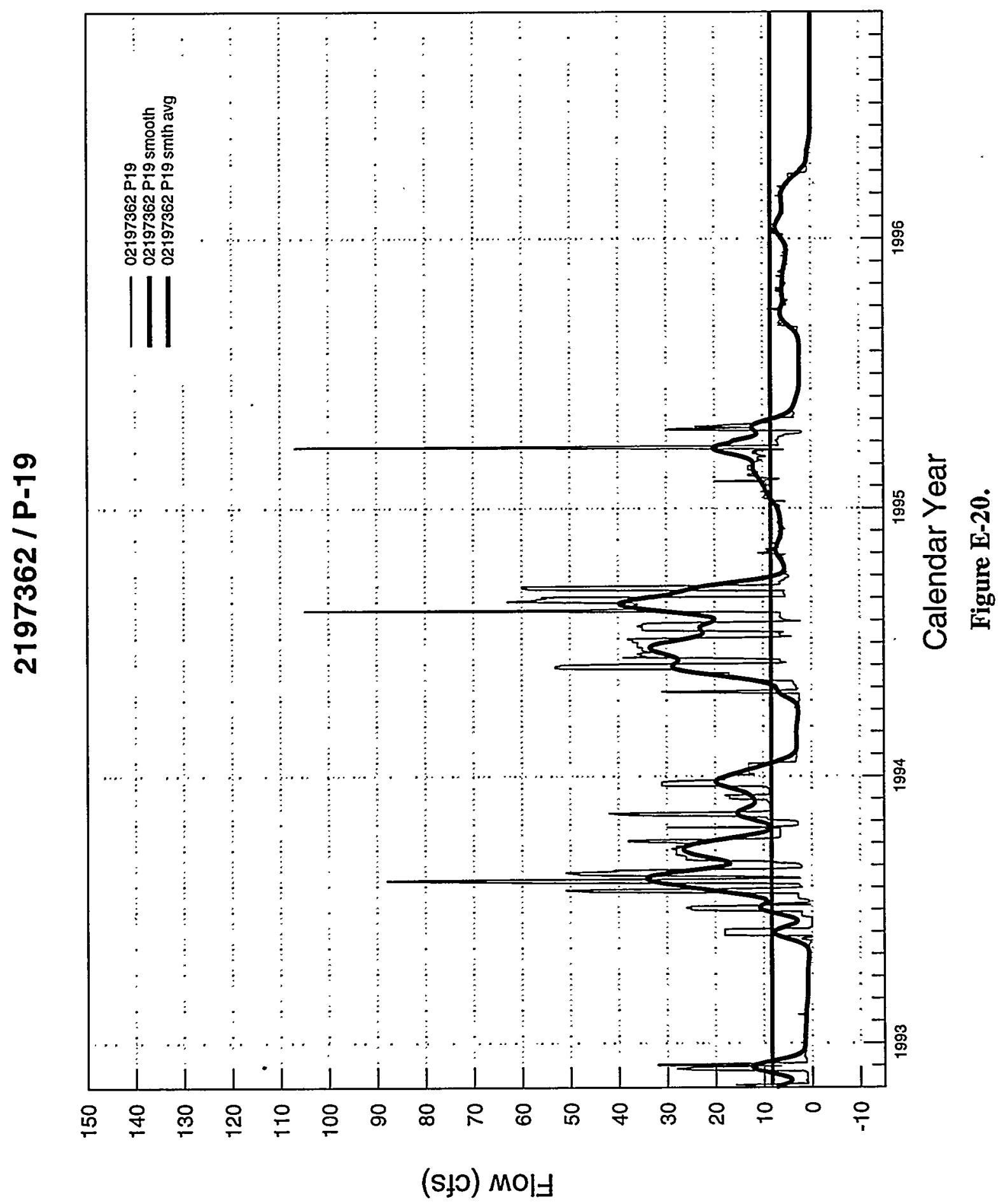


Table E-1. Base flow estimates based on hydrograph separation of USGS gauging station data

\begin{tabular}{|c|c|c|c|c|}
\hline Stream reach & $\begin{array}{l}\text { Estimated } \\
\text { base flow } \\
\text { (cfs) }\end{array}$ & $\begin{array}{c}\text { Fraction of } \\
\text { reach } \\
\text { within } \\
\text { CKLP } \\
\text { model }\end{array}$ & $\begin{array}{c}\text { Base flow } \\
\text { target } \\
\text { (cfs) }\end{array}$ & $\begin{array}{c}\text { Estimated } \\
\text { uncertainty }\end{array}$ \\
\hline $\begin{array}{l}\text { Meyers Branch } \\
\text { (headwaters to Road 9) }\end{array}$ & 9.5 & $1 / 3 ?$ & $3.2 ?$ & $\pm 20-25 \%$ \\
\hline $\begin{array}{l}\text { Steel Creek } \\
\text { (above Road B to Road A; } \\
\text { includes L-Lake) }\end{array}$ & -2.2 & 1 & -2.2 & $\begin{array}{l} \pm 40-45 \% \\
\text { or more }\end{array}$ \\
\hline $\begin{array}{l}\text { Pen Branch } \\
\text { (headwaters to Road A13; } \\
\text { includes Indian Grave Branch) }\end{array}$ & 13.3 & 1 & 13.3 & $\pm 15-20 \%$ \\
\hline $\begin{array}{l}\text { Fourmile Branch } \\
\text { (headwaters to Road A12) }\end{array}$ & 14.1 & 1 & 14.1 & $\pm 15-20 \%$ \\
\hline $\begin{array}{l}\text { Upper Three Runs } \\
\text { (Tims Branch at Road C/UTR near } \\
\text { site boundary/Tinker Creek at } \\
\text { Road 8-11 to UTR at Road A) }\end{array}$ & 97 & $1 / 4 ? ?$ & $24 ? ?$ & - \\
\hline $\begin{array}{l}\text { Upper Three Runs } \\
\text { (Road C to Road A) }\end{array}$ & 8.9 & $1 / 2$ & 4.5 & $\begin{array}{l} \pm 35-40 \% \\
\text { or more }\end{array}$ \\
\hline
\end{tabular}


Table E-2. Sensitivity of base flow estimates to the assumed maximum rate of daily base flow increase.

\begin{tabular}{|l|c|c|c|}
\hline Stream reach & $\begin{array}{c}\text { Estimated } \\
\text { base flow } \\
\text { for } \\
\mathbf{r}=\mathbf{1 . 0 2 5} \\
\text { (cfs) }\end{array}$ & $\begin{array}{c}\text { Estimated } \\
\text { base flow } \\
\text { for } \\
\mathbf{r}=\mathbf{1 . 0 5} \\
\text { (cfs) }\end{array}$ & $\begin{array}{c}\text { Estimated } \\
\text { base flow } \\
\text { for } \\
\text { r=1.10 } \\
\text { (cfs) }\end{array}$ \\
\hline $\begin{array}{l}\text { Meyers Branch } \\
\text { (headwaters to Road 9) }\end{array}$ & $\begin{array}{c}9.2 \\
(-3 \%)\end{array}$ & 9.5 & $\begin{array}{c}9.8 \\
(+3 \%)\end{array}$ \\
\hline $\begin{array}{l}\text { Steel Creek } \\
\text { (above Road B to Road A; } \\
\text { includes L-Lake) }\end{array}$ & $\begin{array}{c}-2.2 \\
(0 \%)\end{array}$ & -2.2 & $\begin{array}{c}-2.2 \\
(0 \%)\end{array}$ \\
\hline $\begin{array}{l}\text { Pen Branch } \\
\text { (headwaters to Road A13; } \\
\text { includes Indian Grave Branch) }\end{array}$ & $\begin{array}{c}12.3 \\
(-8 \%)\end{array}$ & 13.3 & $\begin{array}{c}14.3 \\
(+8 \%)\end{array}$ \\
\hline $\begin{array}{l}\text { Fourmile Branch } \\
\text { (headwaters to Road A12) }\end{array}$ & $\begin{array}{c}(-8 \%) \\
\text { site boundary/Tinker Creek at } \\
\text { Road 8-11 to UTR at Road A) } \\
\text { (Tims Branch at Road C/UTR near }\end{array}$ & $\begin{array}{c}(-6 \%) \\
\text { (Road C to Road A) }\end{array}$ & $\begin{array}{c}14.1 \\
(+7 \%)\end{array}$ \\
\hline $\begin{array}{l}\text { Upper Three Runs } \\
(+13 \%)\end{array}$ & $\begin{array}{c}104 \\
(+7 \%)\end{array}$ \\
\hline
\end{tabular}


This page intentionally left blank 
(This page intentionally left blank) 


\section{APPENDIX E-2. STREAM BASEFLOW MEASUREMENTS AT THE} SAVANNAH RIVER SITE

A program was initiated in the mid 1990's to acquire information on the extent of perennial streams on the SRS and in a band approximately 5 miles wide surrounding the SRS on the South Carolina side of the Savannah River. The purpose of the program was to determine the full extent of flowing stream reaches in their headwater areas and obtain numerous measurements of flow rates along individual SRS streams at times that reflected baseflow conditions.

Acquisition of baseflow measurements in SRS streams began in the fall of 1997 and has continued as necessary for calibration of regional groundwater flow models. Baseflow measurement acquisition is listed below, by stream reach.

\section{STREAM REACH}

Upper Pen Branch

Mill Creek

Indian Grave Branch

R-Canal Turnout Drainageway

Meyers Branch

Four Mile Branch

and tributaries near C-Reactor
MEASUREMENT PERIOD

September 1997

December 1997

December 1997

February 1998

February 1998 and April 1998

It was assumed that baseflow conditions are reached relatively quickly following rainfall events, after approximately 8-10 days without rainfall following a significant rainfall event. Examination of SRS stream hydrographs tends to support this assumption. Most of the measurements, however, were obtained after much longer periods without rainfall.

Two types of instruments were used depending on the magnitude of flow at a selected station. A cutthroat flume for stream reaches with flow rates less than 0.02 to 0.15 cubic feet/second (cfs). An instream flow velocity indicator (Marsh-McBirney Portable Water Flow Meter Model 201) was utilized to acquire stream flow rates greater than approximately $0.15 \mathrm{cfs}$. The following procedures contained in WSRC-L-14.1 Rev. 43 were utilized to operate the Marsh McBirney Flow Meter and to perform the stream flow rate measurements: EESOP-2-102, Stream Velocity and Discharge Measurements and EESOP-2-103, MarshMcBirney Portable Current Meter Operation and Maintenance. There is no formal SRS procedure for the use of the cutthroat flume, however the manufacturer's guidelines were utilized to obtain these measurements.

The strategy was to start at the headwaters of a stream and obtain measurements at different stations while working in the downstream direction. If possible, measurements were obtained just upstream of the point of entry of significant tributary branches. Measurements were also obtained on the tributaries themselves so that their relative contribution could be quantified. 
All measurements along a single stream reach were obtained within a short a period of time, generally over a period of 1 to 2 days, to minimize the possibility that baseflow conditions might be impacted by rainfall events. In the case of Meyers Branch, where measurements were obtained on different days separated by a two-month period, one measurement station was re-measured on the second measurement date to provide a means to compare measurements obtained on the two days.

For each of the streams a map is presented illustrating its perennial reaches and the perennial reaches of its primary tributaries. Also shown are the ground water basin boundaries associated with each stream reach, the position of measurement stations along the stream reaches and a posting of the baseflow measurement at each station. Data used to calculate baseflow of each station is provided on the pages following each illustration. 


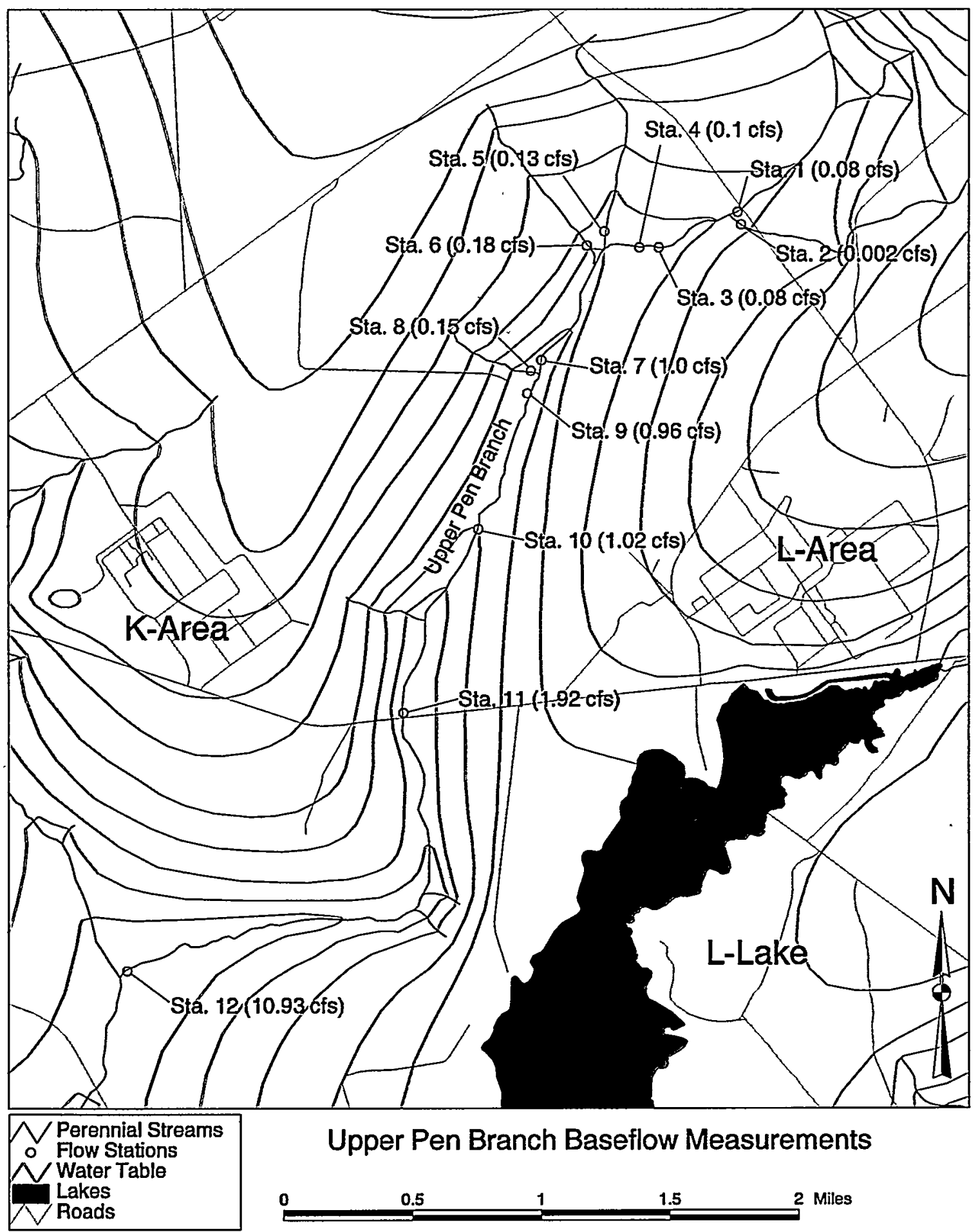




\section{UPPER PEN BRANCH BASEFLOW}

Station 1 Just downstream from Road C, Near confluence of easternmost two headwater tributaries

Approx UTM Coord. 441732E, 3676976N

$(10 / 14 / 97)$

Flume $\quad 4$ " throat width, fluid height $=0.2, Q=0.082 \mathrm{cfs}$ or Measurement $\quad 37.3 \mathrm{gpm}$

Station 2 Just downstream from Road C, Near confluence of easternmost two headwater tributaries

Approx UTM Coord. 441740E, 3676937N

$(10 / 14 / 97)$

Flume Measurement, 4" throat width, fluid height $=0.03$ ', $Q=0.0019 \mathrm{cfs}$ or $0.84 \mathrm{gpm}$

Station 3 Just downstream from powerline road, approximately $150 \mathrm{ft}$.

Approx UTM coordinates 441114E, 3676771N

$(10 / 14 / 97)$

Flume Measurement; 4" throat width, height $=0.192, \mathrm{Q}=0.078 \mathrm{cfs}$ or $35 \mathrm{gpm}$

Station 4 Just downstream from powerline road, approximately $200 \mathrm{ft}$.

Approx UTM coordinates 441044E, 36767791N

$(10 / 14 / 97)$

Flume Measurement; 4" throat width, height $=0.22, \mathrm{Q}=0.101 \mathrm{cfs}$ or 45.1gpm 
Station 5 On central headwater tributary of Pen Branch, just upstream of confluence with the headwater branch containing the eastern two headwater tributaries.

Approx UTM coordinates 440930E, 36768281N

$(10 / 14 / 97)$

Flume Measurement; 4" throat width, height $=0.25, \mathrm{Q}=0.13 \mathrm{cfs}$ or $58.3 \mathrm{gpm}$

Station 6 On westernmost headwater tributary of Pen Branch, just upstream from confluence with the main branch (containing the three easternmost headwater tributaries.)

Approx UTM coordinates 440850E, 36767511N

$(10 / 14 / 97)$

Flume Measurement; 4" throat width, height $=0.29, \mathrm{Q}=0.175 \mathrm{cfs}$ or 78.4gpm

Station 7 On main segment Pen Branch, approx $780 \mathrm{ft}$ downstream from Sta. 6 Approx UTM coordinates 440523E, 36760281N

$(10 / 15 / 97)$

Flume Measurement; 4 " throat width, height $=0.3$

$8, \mathrm{Q}=0.3 \mathrm{cfs}$ or $135 \mathrm{gpm}$

Flowmeter Measurement

Segment depth(cm) depth(ft) Width(ft) velocity(ft/sec) Q (cfs)

$\begin{array}{llllll}1 & 8 & 0.26 & 1 & 0.96 & 0.25 \\ 2 & 10 & 0.33 & 1 & 0.96 & 0.31 \\ 3 & 9 & 0.30 & 1 & 0.69 & 0.20 \\ 4 & 9 & 0.30 & 1 & 0.79 & 0.23\end{array}$

Tot Flow $=1.00$

Station 8 On lateral tributary to main branch of Pen Branch, close to sta. 7.

Approx UTM coordinates 440487E, 36759941N

$(10 / 15 / 97)$

Flume Measurement; 4" throat width, height $=0.29, \mathrm{Q}=0.175 \mathrm{cfs}$ or 78.4gpm 
Station 9 On main fork of Pen Branch, close to stations 7 and 8, downstream of their confluence

Approx UTM coordinates 440479E, 36758991N

$(10 / 15 / 97)$

Flowmeter Measurement

Segment depth(cm) depth(ft) width(ft) velocity(ft/sec) Q $\quad$ (cfs)

$\begin{array}{llllll}1 & 9 & 0.30 & 1 & 0.08 & 0.02\end{array}$

$\begin{array}{llllll}2 & 10 & 0.33 & 1 & 0.31 & 0.10\end{array}$

$\begin{array}{llllll}3 & 13 & 0.43 & 1 & 0.52 & 0.22\end{array}$

$\begin{array}{llllll}4 & 12 & 0.39 & 1 & 0.39 & 0.15\end{array}$

$\begin{array}{llllll}5 & 12 & 0.39 & 1 & 0.5 & 0.20\end{array}$

$\begin{array}{llllll}6 & 13 & 0.43 & 1 & 0.52 & 0.22\end{array}$

$\begin{array}{llllll}7 & 12 & 0.39 & 1 & 0.21 & 0.08\end{array}$

$\begin{array}{llllll}8 & 9 & 0.30 & 1 & -0.13 & -0.04\end{array}$

Tot Flow $=0.96$

Station 10 On Pen Branch at Road 6.2, approximately $100 \mathrm{ft}$ downstream from bridge.

Approx UTM coordinates 440122E, 3674981N

$(10 / 15 / 97)$

Flowmeter Measurement

$\begin{array}{lllllll}\begin{array}{l}\text { Tape } \\ \text { msmt }\end{array} & \text { Segment } & \text { depth(cm) } & \text { depth(ft) } & \text { width(ft) } \begin{array}{l}\text { Velocity } \\ \text { (ft/sec) }\end{array} & \text { Q (cfs) } \\ 1.5 & 1 & 8 & 0.26 & 1 & 0.22 & 0.06 \\ 2.5 & 2 & 10 & 0.33 & 1 & 0.2 & 0.07 \\ 3.5 & 3 & 9 & 0.30 & 1 & 0.49 & 0.14 \\ 4.5 & 4 & 10 & 0.33 & 1 & 0.55 & 0.18 \\ 5.5 & 5 & 8 & 0.26 & 1 & 0.63 & 0.17 \\ 6.5 & 6 & 10 & 0.33 & 1 & 0.52 & 0.17 \\ 7.5 & 7 & 7 & 0.23 & 1 & 0.41 & 0.09 \\ 8.5 & 8 & 7 & 0.23 & 1 & 0.32 & 0.07 \\ 9.5 & 9 & 8 & 0.26 & 1 & 0.21 & 0.06 \\ 10.5 & 10 & 6 & 0.20 & 1 & 0.06 & 0.01\end{array}$

Tot Flow $=1.02$ 
Station 11 On Pen Branch at B Road, approximately $75 \mathrm{ft}$ upstream from bridge.

Approx UTM coordinates 439666E, 3673894N

$(10 / 15 / 97)$

Flowmeter Measurement

$\begin{array}{lllll}\text { Tape } & \text { Segment } \operatorname{depth}(\mathrm{cm}) & \operatorname{depth}(\mathrm{ft}) & \text { width(ft) } & \text { velocity(ft/sec) }\end{array}$ msmt

$\begin{array}{lllllll}1.5 & 1 & 9 & 0.30 & 1 & 0.11 & 0.03 \\ 2.5 & 2 & 15 & 0.49 & 1 & 0.26 & 0.13 \\ 3.5 & 3 & 14 & 0.46 & 1 & 0.28 & 0.13 \\ 4.5 & 4 & 14 & 0.46 & 1 & 0.84 & 0.39 \\ 5.5 & 5 & 10 & 0.33 & 1 & 0.87 & 0.29 \\ 6.5 & 6 & 10 & 0.33 & 1 & 0.9 & 0.30 \\ 7.5 & 7 & 10 & 0.33 & 1 & 0.99 & 0.32 \\ 8.5 & 8 . & 10 & 0.33 & 1 & 0.82 & 0.27 \\ 9.5 & 9 & 9 & 0.30 & 1 & 0.18 & 0.05 \\ 10.5 & 10 & 6 & 0.20 & 1 & 0.07 & 0.01 \\ & & & & & & \text { Tot Flow }=1.92\end{array}$


Station 12 On Pen Branch just upstream from confluence with Indian Grave Branch Approx UTM coordinates 437922E, 3672243N

$12 / 18 / 97$

Flowmeter Measurements

Segment Tape $\operatorname{depth}(\mathrm{cm}) \operatorname{depth}(\mathrm{ft}) \quad \begin{array}{r}\text { velocity } \\ \text { width(f }(\mathrm{ft} / \mathrm{sec})\end{array}$

corr.

$\begin{array}{llllllll}1 & 0.5 & 24 & 0.79 & 1 & -0.05 & 0 & 0.00 \\ 2 & 1.5 & 26 & 0.85 & 1 & 0.22 & 0.27 & 0.23 \\ 3 & 2.5 & 39 & 1.28 & 1 & 0.9 & 0.95 & 1.22 \\ 4 & 3.5 & 35 & 1.15 & 1 & 0.8 & 0.85 & 0.98 \\ 5 & 4.5 & 33 & 1.08 & 1 & 0.91 & 0.96 & 1.04 \\ 6 & 5.5 & 29 & 0.95 & 1 & 0.88 & 0.93 & 0.88 \\ 7 & 6.5 & 27 & 0.89 & 1 & 0.94 & 0.99 & 0.88 \\ 8 & 7.5 & 26 & 0.85 & 1 & 0.98 & 1.03 & 0.88 \\ 9 & 8.5 & 27 & 0.89 & 1 & 0.87 & 0.92 & 0.82 \\ 10 & 9.5 & 26 & 0.85 & 1 & 0.97 & 1.02 & 0.87 \\ 11 & 10.5 & 26 & 0.85 & 1 & 0.79 & 0.84 & 0.72 \\ 12 & 11.5 & 24 & 0.79 & 1 & 0.59 & 0.64 & 0.50 \\ 13 & 12.5 & 25 & 0.82 & 1 & 0.48 & 0.53 & 0.43 \\ 14 & 13.5 & 30 & 0.98 & 1 & 0.62 & 0.67 & 0.66 \\ 15 & 14.5 & 26 & 0.85 & 1 & 0.66 & 0.71 & 0.61 \\ 16 & 15.5 & 16 & 0.52 & 1 & 0.38 & 0.43 & 0.23 \\ & & & & & & \text { Tot Flow }=10.93\end{array}$




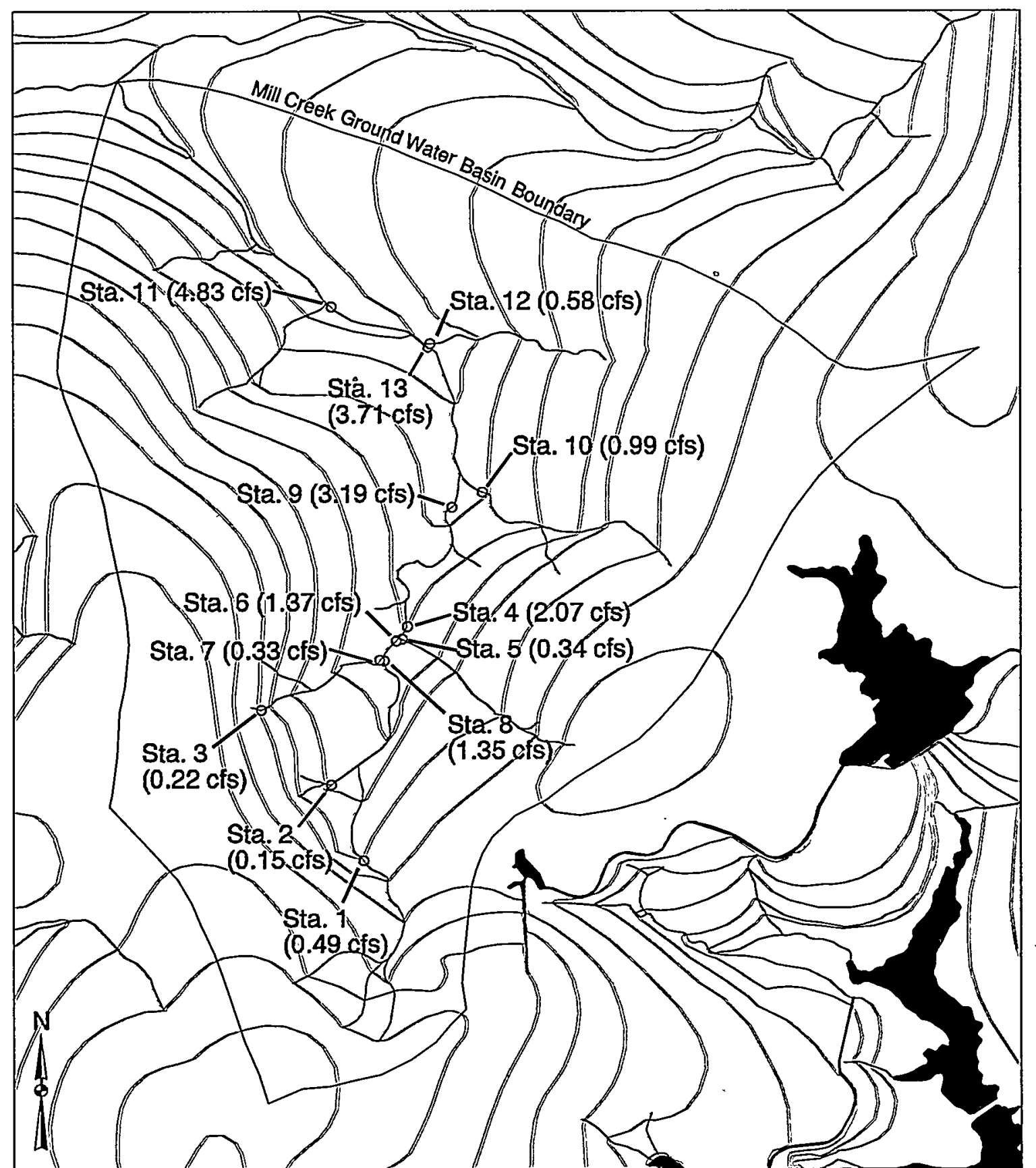

$\sim$ Perennial Streams - Measurement Station $\checkmark$ Mill Creek Basin Water Table Lakes
Mill Creek Baseflow Measurements and Groundwater Basin

0.5
0.5 1.5 Miles 


\section{MILL CREEK BASEFLOW}

Station 1 Mill Creek where Woodward Rd. crosses, upstream side of road.

Approximate UTM Coordinates:

$445115 \mathrm{E}, 3682835 \mathrm{~N}$

$(12 / 4 / 97)$

Flume Measurement: $8^{\prime \prime}$ throat, fluid height $=0.5 \mathrm{ft} ., \mathrm{Q}=1.06 \mathrm{cfs}$ or 475gmp

Flowmeter Measurement

Segment Tape width depth depth (ft) (ft/sec) (ft/sec) Q (cfs)

$\begin{array}{llllllll} & & (\mathrm{ft} .) & (\mathrm{cm}) & & & & \\ & 0.5 & 1 & 9 & 0.30 & 0.01 & 0.06 & 0.018\end{array}$

$\begin{array}{llllllll}2 & 1.5 & 1 & 12 & 0.39 & 0.03 & 0.08 & 0.031\end{array}$

$\begin{array}{llllllll}3 & 2.5 & 1 & 16 & 0.52 & 0.18 & 0.23 & 0.121\end{array}$

$\begin{array}{llllllll}4 & 3.5 & 1 & 18 & 0.59 & 0.40 & 0.45 & 0.266\end{array}$

$\begin{array}{llllllll}5 & 4.5 & 1 & 11 & 0.36 & 0.11 & 0.16 & 0.058\end{array}$

Station 2 Along Monroe-Owens Rd. First tributary north of main channel of Mill Creek.

Approximate UTM Coordinates: 444846E, 3683390N

(12/4/97)

Flume Measurement: 8 " throat, fluid height $=0.19 \mathrm{ft}$., $\mathrm{Q}=0.152 \mathrm{cfs}$ or 69gmp

Station 3 Along Monroe-Owens Rd. Second tributary north of main channel of Mill Creek.

Approximate UTM Coordinates: 444344E, 3683928N

$(12 / 4 / 97)$

Flume Measurement: 8 " throat, fluid height $=0.23 \mathrm{ft} ., \mathrm{Q}=0.22 \mathrm{cfs}$ or 101 gmp 
Station 4 Main branch of Mill Creek, downstream of first major tributary entering from south.

Approximate UTM Coordinates: 445355E, 3684472N

(12/4/97)

Flowmeter Measurement

Segment Tape width (ft.) dept

$1 \quad 0.5 \quad 1$

$2 \quad 1.5 \quad 1$

$\begin{array}{lll}3 & 2.5 & 1\end{array}$

$\begin{array}{lll}4 & 3.5 & 1\end{array}$

$\begin{array}{lll}5 & 4.5 & 1\end{array}$

$\begin{array}{lll}6 & 5.5 & 1\end{array}$

$\begin{array}{lll}7 & 6.5 & 1\end{array}$

$\begin{array}{lll}8 & 7.5 & 1\end{array}$

$\begin{array}{lll}9 & 8.5 & 1\end{array}$

10

$$
\text { (cm) }
$$

9.25

0.5

14
15
14
24
22
25
30
26
25
20

$\begin{array}{llll}\text { depth } & \begin{array}{l}\text { flow vel. } \\ \text { (ft/sec) }\end{array} & \begin{array}{c}\text { corrected } \\ \text { flow vel. } \\ \text { (ft/sec) }\end{array} & \text { Q (cfs) } \\ 0.46 & 0.16 & 0.21 & 0.096 \\ 0.49 & 0.35 & 0.40 & 0.197 \\ 0.46 & 0.26 & 0.31 & 0.142 \\ 0.79 & 0.34 & 0.39 & 0.307 \\ 0.72 & 0.24 & 0.29 & 0.209 \\ 0.82 & 0.36 & 0.41 & 0.336 \\ 0.98 & 0.40 & 0.45 & 0.443 \\ 0.85 & 0.22 & 0.27 & 0.230 \\ 0.82 & 0.07 & 0.12 & 0.098 \\ 0.66 & -0.02 & 0.03 & 0.010 \\ & & \text { Tot. flow }= & 2.07\end{array}$

Station 5 Tributary to Mill Creek, entering from south, just upstream of confluence with Mill Ck.

Approximate UTM Coordinates:

445348E, 3684437N

(12/4/97)

Flowmeter Measurement

Segment Tape width

(ft.)

1

2

3

4

1.5

1

3.5

1

1

(cm)

$0.5 \quad 1 \quad 4$

10

17

8

depth $\mathrm{cm})$

0.13

0.13

0.33

0.56

0.26

corrected

flow vel. flow vel.

$\operatorname{depth}(\mathrm{ft})(\mathrm{ft} / \mathrm{sec}) \quad(\mathrm{ft} / \mathrm{sec}) \quad \mathrm{Q}(\mathrm{cfs})$

$0.06 \quad 0.11$

0.014

$\begin{array}{ll}0.15 & 0.20\end{array}$

0.066

0.37

0.42

0.234

$0.09 \quad 0.024$

Tot. flow $=0.34$ 
Station 6 Main branch of Mill Creek, upstream of first major tributary entering from south.

Approximate UTM Coordinates:

$445318 \mathrm{E}, 3684317 \mathrm{~N}$

$(12 / 4 / 97)$

Flowmeter Measurement

corrected

Segment Tape width

flow vel. flow vel.

$\begin{array}{llllllll}\text { Segment } & \text { Tape } & \begin{array}{l}\text { width } \\ \text { (ft.) }\end{array} & \begin{array}{l}\text { depth } \\ \text { (cm) }\end{array} & \text { depth }(\mathrm{ft}) & (\mathrm{ft} / \mathrm{sec}) & (\mathrm{ft} / \mathrm{sec}) & \begin{array}{l}\mathrm{Q} \\ \text { (cfs) }\end{array} \\ 1 & 0.5 & 1 & 9 & 0.30 & 0.38 & 0.43 & 0.127 \\ 2 & 1.5 & 1 & 20 & 0.66 & 0.10 & 0.15 & 0.098 \\ 3 & 2.5 & 1 & 20 & 0.66 & 0.51 & 0.56 & 0.367 \\ 4 & 3.5 & 1 & 20 & 0.66 & 0.79 & 0.84 & 0.551 \\ 5 & 4.5 & 1 & 15 & 0.49 & 0.05 & 0.10 & 0.049 \\ 6 & 5.5 & 1 & 17 & 0.56 & 0.04 & 0.09 & 0.050 \\ 7 & 6.5 & 1 & 10 & 0.33 & 0.35 & 0.40 & 0.131 \\ & & & & & & \text { Tot. flow }= & 1.37\end{array}$

Station 7 Northernmost trib. to Mill $\mathrm{Ck}$ that crosses powerline road, just above entry into Mill Ck.

Approximate UTM Coordinates:

$445200 \mathrm{E}, 3684304 \mathrm{~N}$

(12/4/97)

Flowmeter Measurement

Segment Tape width

1

(ft.)

0.5

1

depth

(cm)

corrected

flow vel. flow vel.

depth (ft) $(\mathrm{ft} / \mathrm{sec}) \quad(\mathrm{ft} / \mathrm{sec}) \quad \mathrm{Q}(\mathrm{cfs})$

$\begin{array}{llll}0.79 & 0.37 & 0.42 & 0.331\end{array}$

Tot. flow $=0.33$ 
Station 8 Main branch of Mill Creek, upstream of confluence with northernmost powerline tirbutary

Approximate UTM Coordinates:

$445216 \mathrm{E}, 3684298 \mathrm{~N}$

(12/4/97)

Flowmeter Measurement

Segment Tape width

$\begin{array}{lll} & & (\mathrm{ft} .) \\ 1 & 0.5 & 1 \\ 2 & 1.5 & 1 \\ 3 & 2.5 & 1 \\ 4 & 3.5 & 1 \\ 5 & 4.5 & 1 \\ 6 & 5.5 & 1 \\ 7 & 6.5 & 1\end{array}$

corrected

flow vel. flow vel.

\begin{tabular}{|c|c|c|c|c|}
\hline $\begin{array}{l}\text { depth } \\
(\mathrm{cm})\end{array}$ & depth $(\mathrm{ft})$ & $(\mathrm{ft} / \mathrm{sec})$ & $(\mathrm{ft} / \mathrm{sec})$ & $\begin{array}{l}Q \\
\text { (cfs) }\end{array}$ \\
\hline 12 & 0.39 & 0.04 & 0.09 & 0.035 \\
\hline 13 & 0.43 & 0.26 & 0.31 & 0.132 \\
\hline 13 & 0.43 & 0.38 & 0.43 & 0.183 \\
\hline 14 & 0.46 & 0.44 & 0.49 & 0.225 \\
\hline 18 & 0.59 & 0.48 & 0.53 & 0.313 \\
\hline 20 & 0.66 & 0.35 & 0.40 & 0.262 \\
\hline 20 & 0.66 & 0.25 & 0.30 & 0.197 \\
\hline & & & Tot. flow $=$ & 135 \\
\hline
\end{tabular}

Station 9 Main branch of Mill Creek, upstream of confluence with 2'nd major trib. from south

Approximate UTM Coordinates:

445744E, 3685452N

(12/4/97)

Flowmeter Measurement

Segment Tape widt
2

3

4

5

6

7

8

9

10

11

12

13 (ft.)

0.5

1.5

2.5

3.5

4.5

5.5

6.5

7.5

8.5

9.5

10.5

11.5

12.5

\section{1}

1

1

1

1

1

1

1

1

1

1

1

1

depth
(cm)
22
30
40
50
48
48
45
43
48
48
56
54
46

corrected

flow vel. flow vel.

$\operatorname{depth}(\mathrm{ft})(\mathrm{ft} / \mathrm{sec}) \quad(\mathrm{ft} / \mathrm{sec}) \quad \mathrm{Q}(\mathrm{cfs})$

$\begin{array}{llll}0.72 & 0.02 & 0.07 & 0.051 \\ 0.98 & 0.06 & 0.11 & 0.108 \\ 1.31 & 0.04 & 0.09 & 0.118 \\ 1.64 & 0.12 & 0.17 & 0.279 \\ 1.57 & 0.16 & 0.21 & 0.331 \\ 1.57 & 0.20 & 0.25 & 0.394 \\ 1.48 & 0.22 & 0.27 & 0.399 \\ 1.41 & 0.20 & 0.25 & 0.353 \\ 1.57 & 0.13 & 0.18 & 0.283 \\ 1.57 & 0.11 & 0.16 & 0.252 \\ 1.84 & 0.07 & 0.12 & 0.220 \\ 1.77 & 0.10 & 0.15 & 0.266 \\ 1.51 & 0.04 & 0.09 & 0.136 \\ & & \text { Tot. flow }= & 3.19\end{array}$


Station 10 2'nd major tributary to Mill Ck. from south, just upstream from confluence.

Approximate UTM Coordinates:

445899E, 3685493N

$(12 / 4 / 97)$

Flowmeter Measurement

Segment Tape widt

$\begin{array}{lll} & & \text { (ft.) } \\ 1 & 0.5 & 1 \\ 2 & 1.5 & 1 \\ 3 & 2.5 & 1 \\ 4 & 3.5 & 1 \\ 5 & 4.5 & 1 \\ 6 & 5.5 & 1 \\ 7 & 6.5 & 1 \\ 8 & 7.5 & 1\end{array}$

corrected

flow vel. flow vel.

$\begin{array}{lllll}\begin{array}{l}\text { depth } \\ (\mathrm{cm})\end{array} & \text { depth }(\mathrm{ft}) & (\mathrm{ft} / \mathrm{sec}) & (\mathrm{ft} / \mathrm{sec}) & \begin{array}{l}\mathrm{Q} \\ (\mathrm{cfs})\end{array} \\ 6 & 0.20 & 0.24 & 0.29 & 0.057 \\ 6 & 0.20 & 0.58 & 0.63 & 0.124 \\ 5 & 0.16 & 0.61 & 0.66 & 0.108 \\ 6 & 0.20 & 0.78 & 0.83 & 0.163 \\ 7 & 0.23 & 0.64 & 0.69 & 0.158 \\ 7 & 0.23 & 0.65 & 0.70 & 0.161 \\ 8 & 0.26 & 0.55 & 0.60 & 0.157 \\ 8 & 0.26 & 0.17 & 0.22 & 0.058 \\ & & & \text { Tot. flow }= & 0.99\end{array}$

Station 11 Main branch of Mill Creek, at USGS station site where "buried cable road" crosses.

Approximate UTM Coordinates:

$444821 \mathrm{E}, 3686806 \mathrm{~N}$

$(12 / 8 / 97)$

Flowmeter Measurement

Segment Tape width

$\begin{array}{lll} & & \text { (ft.) } \\ 1 & 0.5 & 1 \\ 2 & 1.5 & 1 \\ 3 & 2.5 & 1 \\ 4 & 3.5 & 1 \\ 5 & 4.5 & 1 \\ 6 & 5.5 & 1 \\ 7 & 6.5 & 1 \\ 8 & 7.5 & 1 \\ 9 & 8.5 & 1 \\ 10 & 9.5 & 1 \\ 11 & 10.5 & 1 \\ 12 & 11.5 & 1\end{array}$

corrected

flow vel. flow vel.

\begin{tabular}{|c|c|c|c|c|}
\hline $\begin{array}{l}\text { depth } \\
\text { (cm) }\end{array}$ & depth (f & $(\mathrm{ft} / \mathrm{sec})$ & $(\mathrm{ft} / \mathrm{sec})$ & $Q(\mathrm{cfs})$ \\
\hline 19 & 0.62 & 0.04 & 0.09 & 0.056 \\
\hline 22 & 0.72 & 0.43 & 0.48 & 0.346 \\
\hline 22 & 0.72 & 0.54 & 0.59 & 0.426 \\
\hline 22 & 0.72 & 0.58 & 0.63 & 0.455 \\
\hline 22 & 0.72 & 0.63 & 0.68 & 0.491 \\
\hline 22 & 0.72 & 0.70 & 0.75 & 0.541 \\
\hline 22 & 0.72 & 0.67 & 0.72 & 0.520 \\
\hline 24 & 0.79 & 0.65 & 0.70 & 0.551 \\
\hline 24 & 0.79 & 0.58 & 0.63 & 0.496 \\
\hline 25 & 0.82 & 0.41 & 0.46 & 0.377 \\
\hline 22 & 0.72 & 0.34 & 0.39 & 0.282 \\
\hline \multirow[t]{2}{*}{19} & 0.62 & 0.41 & 0.46 & 0.287 \\
\hline & & & Tot. flow $=$ & 4.83 \\
\hline
\end{tabular}


Station 12 1'st major tributary from east as you proceed upstream from Tinker Ck. Measured very close to confluence with Mill Creek.

Approximate UTM Coordinates:

$445559 \mathrm{E}, 3686545 \mathrm{~N}$

$(12 / 8 / 97)$

Flowmeter Measurement

Segment Tape width

$\begin{array}{lll} & & \text { (ft.) } \\ 1 & 0.5 & 1 \\ 2 & 1.5 & 1 \\ 3 & 2.5 & 1 \\ 4 & 3.5 & 1\end{array}$

corrected

flow vel. flow vel.

$\begin{array}{llll}\text { depth } & \text { depth }(\mathrm{ft})(\mathrm{ft} / \mathrm{sec}) & (\mathrm{ft} / \mathrm{sec}) & \mathrm{Q}(\mathrm{cfs})\end{array}$ (cm) 9

12

12

8

$$
0.30
$$

0.39

0.39

0.26
$0.23 \quad 0.28$

$0.50 \quad 0.55$

$0.53 \quad 0.58$

0.16

0.21
0.083

0.217

0.228

Tot. flow $=0.58$

Station 13 Main branch of Mill Creek, just upstream of confluence with 1'st major tributary to enter Mill Creek from east, as you proceed upstream from Tinker Creek.

Approximate UTM Coordinates:

445534E, 3686510N

$(12 / 8 / 97)$

Flowmeter Measurement

Segment Tape width

$\begin{array}{lll} & & \text { (ft.) } \\ 1 & 0.5 & 1 \\ 2 & 1.5 & 1 \\ 3 & 2.5 & 1 \\ 4 & 3.5 & 1 \\ 5 & 4.5 & 1 \\ 6 & 5.5 & 1 \\ 7 & 6.5 & 1 \\ 8 & 7.5 & 1 \\ 9 & 8.5 & 1 \\ 10 & 9.5 & 1\end{array}$

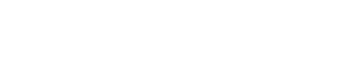

\begin{tabular}{lllll} 
& \multicolumn{5}{c}{ flow vel. flow vel. } \\
depth & depth (ft) $(\mathrm{ft} / \mathrm{sec})$ & $(\mathrm{ft} / \mathrm{sec})$ & $\mathrm{Q}(\mathrm{cfs})$ \\
(cm) & & & & \\
8 & 0.26 & 0.25 & 0.30 & 0.079 \\
16 & 0.52 & 0.34 & 0.39 & 0.205 \\
19 & 0.62 & 0.67 & 0.72 & 0.449 \\
22 & 0.72 & 0.79 & 0.84 & 0.606 \\
21 & 0.69 & 0.85 & 0.90 & 0.620 \\
19 & 0.62 & 0.71 & 0.76 & 0.474 \\
20 & 0.66 & 0.71 & 0.76 & 0.499 \\
20 & 0.66 & 0.65 & 0.70 & 0.459 \\
12 & 0.39 & 0.56 & 0.61 & 0.240 \\
8 & 0.26 & 0.27 & 0.32 & 0.084 \\
& & & Tot. flow $=3.71$
\end{tabular}



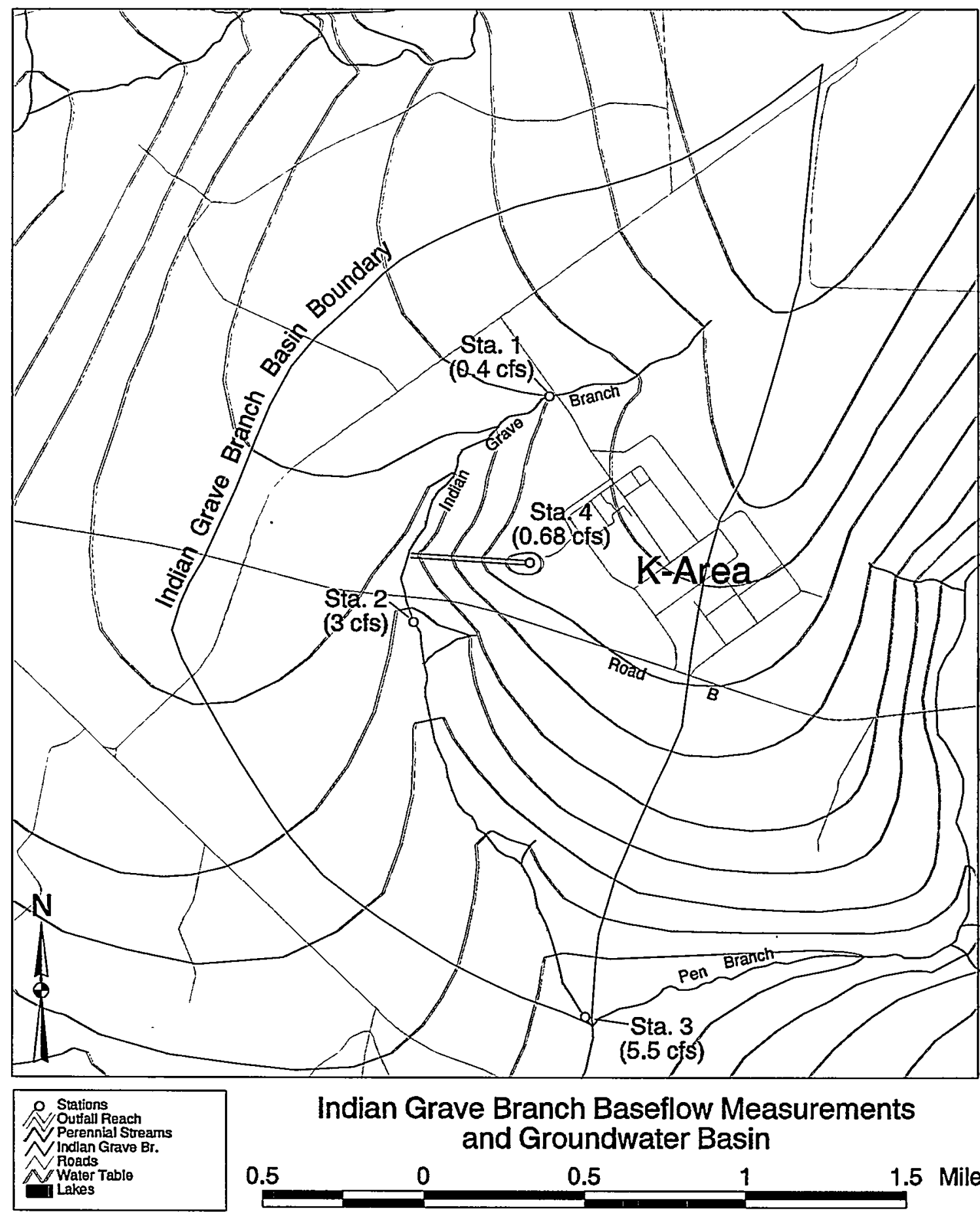
Indian Grave Branch Baseflow Measurements and Groundwater Basin

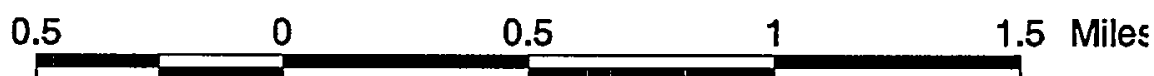




\section{INDIAN GRAVE BRANCH BASEFLOW}

Station 1 Indian Grave Branch, approximately $100 \mathrm{ft}$. downstream from road 6.4

Approximate UTM Coordinates: 437677E, 3675456N

$(12 / 18 / 97)$

Flowmeter Measurement

Segment Tape widt

$\begin{array}{lll} & & \text { (ft.) } \\ 1 & 0.5 & 1 \\ 3 & 1.5 & 1 \\ 3 & 2.5 & 1 \\ 4 & 3.5 & 1\end{array}$

corrected

flow vel. flow vel.

$\begin{array}{llll}\text { depth } & \text { depth }(\mathrm{ft})(\mathrm{ft} / \mathrm{sec}) & \text { (ft/sec) } & \mathrm{Q}(\mathrm{cfs})\end{array}$

(cm)

6

0.20

$-0.03$

0.02

0.004

12

0.39

$-0.04$

0.01

0.004

12

0.39

0.45

0.50

0.197

12

$0.40 \quad 0.45$

0.177

Tot. flow $=0.38$

Station 2 Indian Grave Branch about 150 yards downstream from Road B Approximate UTM Coordinates: 436979E, 3674290N

$(12 / 18 / 97)$

Flowmeter Measurement Segment Tape width (ft.)

1

2

3

4

5

6

6

7

8

9

10

11

12

1

0.5

\section{5}

2.5

3.5

4.5

5.5

6.5

7.5

8.5

9.5

10.5

11.5
1

1

1

1

1

1 .

1

1

1

1

1 depth

$$
\text { (cm) }
$$

7

14

17

20

22

26

30

24

24

21

16

10 flow vel. flow vel.

$$
\operatorname{depth}(\mathrm{ft})(\mathrm{ft} / \mathrm{sec}) \quad(\mathrm{ft} / \mathrm{sec}) \quad \mathrm{Q}(\mathrm{cfs})
$$

0.23

0.02

0.07

0.016

0.46

0.27

0.32

0.61

0.53

0.52

0.41

0.61

0.43

0.28

0.23

0.12

0.07

Tot. flow $=2.98$ 
Station 3 Indian Grave Branch just above confluence with Pen Branch

Approximate UTM Coordinates: 437845E, 3672268N

\section{$(12 / 18 / 97)$}

Flowmeter Measurement

Segment Tape width

(ft.)

1

2

3

$3-2.5$

$4 \quad 3.5$

$5 \quad 4.5$

$6 \quad 5.5$

$7 \quad 6.5$

$8 \quad 7.5$

$9 \quad 8.5$

10

$0.5 \quad 1$

$1.5 \quad 1$

$2.5 \quad 1$

5.51

5.51

$.5 \quad 1$

$6.5-1$

7.51

$8.5 \quad 1$

$9.5 \quad 1$

$\begin{aligned} & \text { depth } \\ & \text { (cm) }\end{aligned}$
6
9
12
16
16
20
20
17
14
12

flow vel. flow vel.

$\operatorname{depth}(\mathrm{ft})(\mathrm{ft} / \mathrm{sec}) \quad(\mathrm{ft} / \mathrm{sec}) \quad \mathrm{Q}(\mathrm{cfs})$

$\begin{array}{llll}0.20 & 0.20 & 0.25 & 0.049\end{array}$

$\begin{array}{llll}0.30 & 0.64 & 0.69 & 0.204\end{array}$

$\begin{array}{llll}0.39 & 1.13 & 1.18 & 0.465\end{array}$

$\begin{array}{llll}0.52 & 1.33 & 1.38 & 0.724\end{array}$

$\begin{array}{llll}0.52 & 1.39 & 1.44 & 0.756\end{array}$

$\begin{array}{llll}0.66 & 1.51 & 1.56 & 1.024\end{array}$

$\begin{array}{llll}0.66 & 1.63 & 1.68 & 1.102\end{array}$

$\begin{array}{llll}0.56 & 1.02 & 1.07 & 0.597\end{array}$

$\begin{array}{llll}0.46 & 0.87 & 0.92 & 0.423\end{array}$

12

0.39

$0.37 \quad 0.42$

0.165

Tot. flow $=5.51$

Station 4 Discharge at K-18 Outfall 


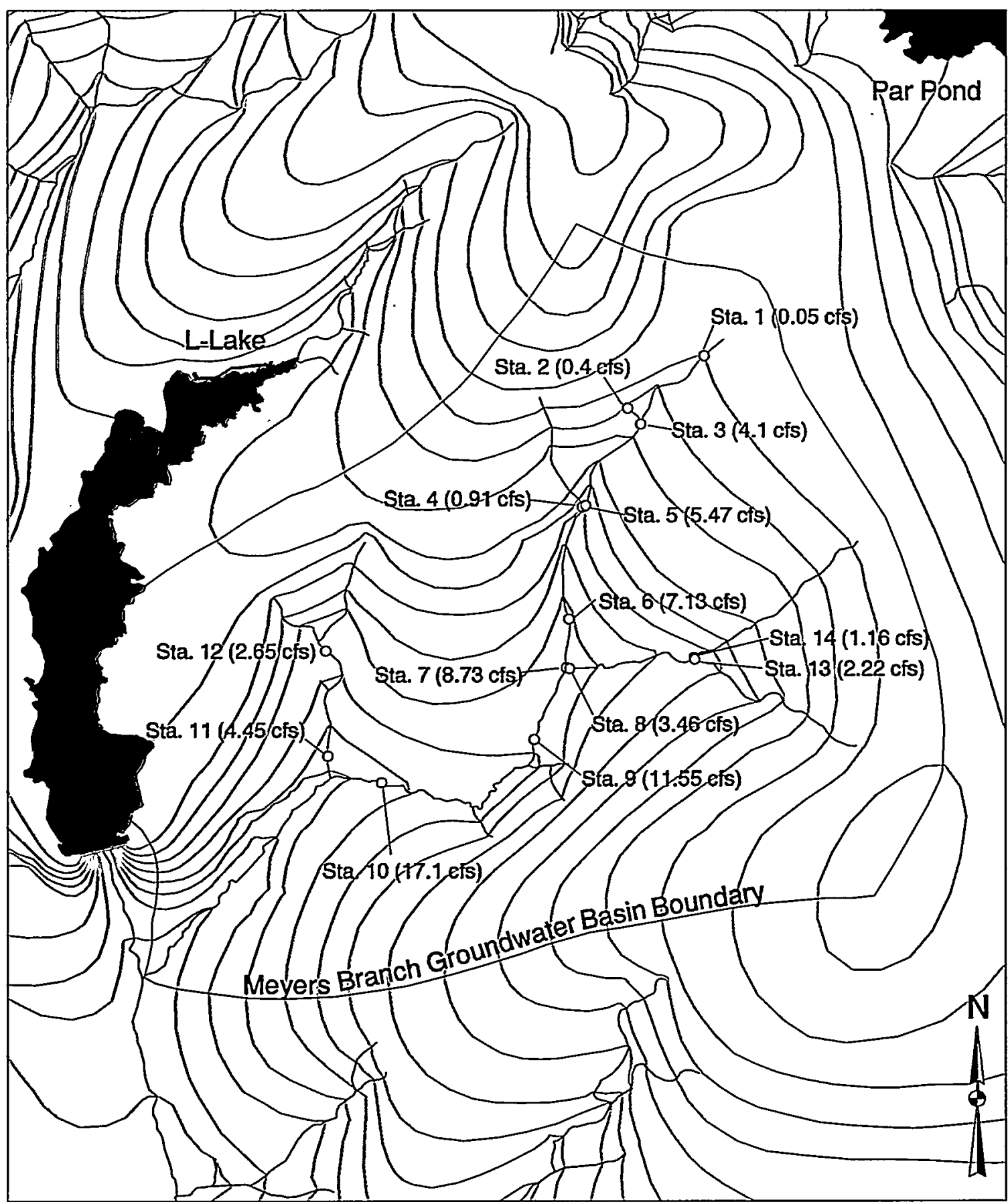

o Baseflow Stations Perennial Streams Meyer Branch GW Basin Water Tablo

Lakes
Meyers Branch Baseflow Measurements and Groundwater Basin

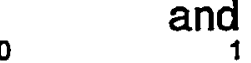




\section{MEYERS BRANCH BASEFLOW}

Station 1 Meyers Branch headwaters of main branch, $0.3 \mathrm{mi}$ S. of Rd. B on an abandoned road.

Approximate UTM Coordinates:

$447,249 \mathrm{E}, 3,674,190 \mathrm{~N}$

(2/2/98) Flume Measurement: 8 " throat, fluid height=0.11 ft., $\mathrm{Q}=0.05 \mathrm{cfs}$ or $23 \mathrm{gmp}$

Station 2 Tributary to Meyers Branch, entering from northwest.

Approximate UTM Coordinates:

$446,457 \mathrm{E}, 3673647 \mathrm{~N}$

$(2 / 2 / 98)$

Flowmeter

Measurement

Segment Tape

$\begin{array}{lll}1 & 0.5 & 1 \\ 2 & 1.5 & 1 \\ 3 & 2.5 & 1 \\ 4 & 3.5 & 1\end{array}$

corrected flow vel. flow vel.

width depth depth (ft) (ft/sec) (ft/sec) Q (cfs) (ft.) (cm)

$\begin{array}{lllll}5 & 0.16 & 0.12 & 0.12 & 0.020\end{array}$

$\begin{array}{lllll}8 & 0.26 & 0.37 & 0.37 & 0.097\end{array}$

$\begin{array}{lllll}6 & 0.20 & 0.71 & 0.71 & 0.140\end{array}$

$\begin{array}{lllll}8 & 0.26 & 0.75 & 0.75 & 0.197\end{array}$

Tot. flow $=0.45$ 
Station 3 Main channel of Meyers Branch, downstream of confluence with tributary entering from northwest.

Approximate UTM Coordinates:

$446591 \mathrm{E}, 36734822 \mathrm{~N}$

$(2 / 2 / 98)$

Flowmeter Measurement

Segment Tape width

$\begin{array}{lll} & & \text { (ft.) } \\ 1 & 0.5 & 1 \\ 2 & 1.5 & 1 \\ 3 & 2.5 & 1 \\ 4 & 3.5 & 1 \\ 5 & 4.5 & 1 \\ 6 & 5.5 & 1 \\ 7 & 6.5 & 1 \\ 8 & 7.5 & 1 \\ 9 & 8.5 & 1 \\ 10 & 9.25 & 0.5\end{array}$

flow vel. flow vel.

$\begin{array}{llll}\text { depth } & \text { depth }(\mathrm{ft})(\mathrm{ft} / \mathrm{sec}) & (\mathrm{ft} / \mathrm{sec}) & \mathrm{Q}(\mathrm{cfs})\end{array}$

(cm)

7

14

18

16

14

16

14

18

16

10

0.30

0.30

0.069

0.23

1.12

1.12

0.514

0.59

0.95

0.95

0.561

0.52

0.46

1.14

1.14

0.598

1.51

1.51

0.694

0.52

1.32

1.32

0.693

0.46

0.94

0.94

0.432

0.59

0.56

0.56

0.331

0.52

0.30

0.30

0.157

0.33

0.31

0.31

0.051

Station 4 Tributary to Meyers Branch, entering from northwest, about $150 \mathrm{ft}$ from confluence with Meyers Br.

Approximate UTM Coordinates:

$445985 \mathrm{E}, 3672628 \mathrm{~N}$

$(2 / 2 / 98)$

Flowmeter Measurement

Segment Tape width

$\begin{array}{llll} & & \text { (ft.) } & \text { (cm) } \\ 1 & 0.5 & 1 & 7 \\ 2 & 1.5 & 1 & 12 \\ 3 & 2.5 & 1 & 11 \\ 4 & 3.5 & 1 & 12 \\ 5 & 4.5 & 1 & 14 \\ 6 & 6.5 & 1 & 9 \\ 7 & 6.25 & 0.5 & 2\end{array}$

corrected

flow vel. flow vel.

$\operatorname{depth}(\mathrm{ft})(\mathrm{ft} / \mathrm{sec}) \quad(\mathrm{ft} / \mathrm{sec}) \quad \mathrm{Q}(\mathrm{cfs})$

$\begin{array}{llll}0.23 & 0.12 & 0.12 & 0.028\end{array}$

$\begin{array}{llll}0.39 & 0.22 & 0.22 & 0.087\end{array}$

$\begin{array}{llll}0.36 & 0.74 & 0.74 & 0.267\end{array}$

0.39

0.67

0.67

0.264

0.46

0.37

0.37

0.170

0.30

0.33

0.33

0.097

0.07

0.04

0.04

0.001

Tot. flow $=0.91$ 
Station 5 Main channel of Meyers Branch, upstream of confluence with tributary entering from northwest.

Approximate UTM Coordinates:

$446020 \mathrm{E}, 3672643 \mathrm{~N}$

$(2 / 2 / 98)$

Flowmeter Measurement

Segment Tape width

$\begin{array}{lll}1 & 0.5 & \text { (ft. }\end{array}$

$2 \quad 1.5 \quad 1$

$3 \quad 2.5 \quad 1$

$4 \quad 3.5 \quad 1$

$5 \quad 4.5 \quad 1$

$\begin{array}{lll}6 & 5.5 & 1\end{array}$

$\begin{array}{lll}7 & 6.5 & 1\end{array}$

$\begin{array}{lll}8 & 7.5 & 1\end{array}$

$\begin{array}{lll}9 & 8.5 & 1\end{array}$

$\begin{array}{lll}10 & 9.25 & 0.5\end{array}$ (ft.)

depth

(cm)

4

13

17

17

15

15

15

11

10

6

\section{corrected}

flow vel. flow vel.

$\operatorname{depth}(\mathrm{ft})(\mathrm{ft} / \mathrm{sec}) \quad(\mathrm{ft} / \mathrm{sec}) \quad \mathrm{Q}(\mathrm{cfs})$

$\begin{array}{llll}0.13 & 0.02 & 0.02 & 0.003\end{array}$

$\begin{array}{llll}0.43 & 0.78 & 0.78 & 0.333\end{array}$

$\begin{array}{llll}0.56 & 1.62 & 1.62 & 0.904\end{array}$

$\begin{array}{llll}0.56 & 2.52 & 2.52 & 1.406\end{array}$

$\begin{array}{llll}0.49 & 2.35 & 2.35 & 1.157\end{array}$

$\begin{array}{llll}0.49 & 1.67 & 1.67 & 0.822\end{array}$

$\begin{array}{llll}0.49 & 1.31 & 1.31 & 0.645\end{array}$

$\begin{array}{llll}0.36 & 0.28 & 0.28 & 0.101\end{array}$

$\begin{array}{llll}0.33 & 0.31 & 0.31 & 0.102\end{array}$

$\begin{array}{llll}0.20 & 0.02 & 0.02 & 0.002\end{array}$

Tot. flow $=5.47$ 
Station 6 Meyers Branch, approx. 150 yds downstream from Old Dunbarton Rd.

Approximate UTM Coordinates:

$445846 \mathrm{E}, 3671467 \mathrm{~N}$

$(2 / 2 / 98)$

Flowmeter

Measurement

Segment Tape

$\begin{array}{lll}1 & 0.5 & \text { (ft) } \\ 2 & 1.5 & 1 \\ 3 & 2.5 & 1 \\ 4 & 3.5 & 1 \\ 5 & 4.5 & 1 \\ 6 & 5.5 & 1 \\ 7 & 6.5 & 1 \\ 8 & 7.5 & 1 \\ 9 & 8.5 & 1 \\ 10 & 9.5 & 1 \\ 11 & 10.5 & 1 \\ 12 & 11.5 & 1 \\ 13 & 12.5 & 1 \\ 14 & 13.5 & 1\end{array}$

width depth depth (ft) $(\mathrm{ft} / \mathrm{sec}) \quad(\mathrm{ft} / \mathrm{sec}) \quad \mathrm{Q}(\mathrm{cfs})$ (ft.) (cm)

20

$38: 125$

$37 \quad 1.21$

$35 \quad 1.15$

36

38

42

43

43

42

32

20

4

3

$\begin{array}{ll}1.45 & 1.45-1.665\end{array}$

$\begin{array}{llll}1.18 & 1.35 & 1.35 & 1.595\end{array}$

$\begin{array}{llll}1.25 & 1.05 & 1.05 & 1.309\end{array}$

$\begin{array}{llll}1.38 & 0.60 & 0.60 & 0.827\end{array}$

$\begin{array}{llll}1.41 & 0.20 & 0.20 & 0.282\end{array}$

$\begin{array}{llll}1.41 & 0.05 & 0.05 & 0.071\end{array}$

$\begin{array}{llll}1.38 & -0.09 & -0.09 & -0.124\end{array}$

$\begin{array}{llll}1.05 & -0.12 & -0.12 & -0.126\end{array}$

$\begin{array}{llll}0.66 & -0.23 & -0.23 & -0.151\end{array}$

$\begin{array}{llll}0.13 & -0.19 & -0.19 & -0.025\end{array}$

$\begin{array}{llll}0.10 & -0.11 & -0.11 & -0.011\end{array}$ corrected

flow vel. flow vel.

Tot. flow $=7.13$ 
Station 7 Main channel of Meyers Branch, upstream of confluence with trib. entering from east.

Approximate UTM Coordinates:

$445821 \mathrm{E}, 3670958 \mathrm{~N}$

$(2 / 2 / 98)$

Flowmeter Measurement

Segment Tape width

$\begin{array}{lll} & & \text { (ft.) } \\ 1 & 0.5 & 1 \\ 2 & 1.5 & 1 \\ 3 & 2.5 & 1 \\ 4 & 3.5 & 1 \\ 5 & 4.5 & 1 \\ 6 & 5.5 & 1 \\ 7 & 6.5 & 1 \\ 8 & 7.5 & 1 \\ 9 & 8.5 & 1 \\ 10 & 9.5 & 1 \\ 11 & 10.5 & 1 \\ 12 & 11.5 & 1 \\ 13 & 12.5 & 1\end{array}$

corrected

flow vel. flow vel.

\begin{tabular}{|c|c|c|c|c|}
\hline depth & depth (ft) & $(\mathrm{ft} / \mathrm{sec})$ & $(\mathrm{ft} / \mathrm{sec})$ & $Q$ (cfs) \\
\hline 17 & 0.56 & 0.83 & 0.83 & 0.463 \\
\hline 18 & 0.59 & 0.65 & 0.65 & 0.384 \\
\hline 18 & 0.59 & 1.02 & 1.02 & 0.602 \\
\hline 12 & 0.39 & 1.51 & 1.51 & 0.595 \\
\hline 14 & 0.46 & 1.48 & 1.48 & 0.680 \\
\hline 15 & 0.49 & 1.42 & 1.42 & 0.699 \\
\hline 16 & 0.52 & 1.52 & 1.52 & 0.798 \\
\hline 16 & 0.52 & 1.85 & 1.85 & 0.971 \\
\hline 18 & 0.59 & 1.70 & 1.70 & 1.004 \\
\hline 18 & 0.59 & 1.88 & 1.88 & 1.110 \\
\hline 14 & 0.46 & 1.78 & 1.78 & 0.818 \\
\hline 14 & 0.46 & 1.39 & 1.39 & 0.638 \\
\hline & 0.30 & -0.10 & -0.10 & -0.030 \\
\hline & & & Tot. flow $=$ & 8.73 \\
\hline
\end{tabular}

Station 8 Tributary to Meyers Branch. entering main channel from east, just upstream from confluence.

Approximate UTM Coordinates:

445899E, 3685493N

$(2 / 2 / 98)$

Flowmeter Measurement

Segment Tape width (ft.)

1

2

3

4

5

6

$\begin{array}{llllllll} & & \text { (ft.) } & \text { (cm) } & & & & \\ 1 & 0.5 & 1 & 16 & 0.52 & 0.35 & 0.35 & 0.184 \\ 2 & 1.5 & 1 & 24 & 0.79 & 1.46 & 1.46 & 1.150 \\ 3 & 2.5 & 1 & 27 & 0.89 & 1.70 & 1.70 & 1.506 \\ 4 & 3.5 & 1 & 28 & 0.92 & 0.59 & 0.59 & 0.542 \\ 5 & 4.5 & 1 & 16 & 0.52 & 0.18 & 0.18 & 0.094 \\ 6 & 5.5 & 1 & 4 & 0.13 & -0.11 & -0.11 & -0.014 \\ & & & & & & \text { Tot. flow }= & 3.46\end{array}$

$\begin{array}{llllllll} & & \text { (ft.) } & \text { (cm) } & & & & \\ 1 & 0.5 & 1 & 16 & 0.52 & 0.35 & 0.35 & 0.184 \\ 2 & 1.5 & 1 & 24 & 0.79 & 1.46 & 1.46 & 1.150 \\ 3 & 2.5 & 1 & 27 & 0.89 & 1.70 & 1.70 & 1.506 \\ 4 & 3.5 & 1 & 28 & 0.92 & 0.59 & 0.59 & 0.542 \\ 5 & 4.5 & 1 & 16 & 0.52 & 0.18 & 0.18 & 0.094 \\ 6 & 5.5 & 1 & 4 & 0.13 & -0.11 & -0.11 & -0.014 \\ & & & & & & \text { Tot. flow }= & 3.46\end{array}$

depth depth (ft) (ft/sec)

corrected

flow vel. flow vel. 
Station 9 Meyers Branch, just upstream from first point it passes under Seaboard Coast RR Line.

Approximate UTM Coordinates:

445482E, 3670216N

$(2 / 2 / 98)$

Flowmeter Measurement

Segment Tape width

$\begin{array}{llll} & & \text { (ft.) } & (\mathrm{cm}) \\ 1 & 0.5 & 1 & 14 \\ 2 & 1.5 & 1 & 24 \\ 3 & 2.5 & 1 & 36 \\ 4 & 3.5 & 1 & 36 \\ 5 & 4.5 & 1 & 34 \\ 6 & 5.5 & 1 & 29 \\ 7 & 6.5 & 1 & 26 \\ 8 & 7.5 & 1 & 22 \\ 9 & 8.5 & 1 & 24 \\ 10 & 9.5 & 1 & 24 \\ 11 & 10.5 & 1 & 28 \\ 12 & 11.5 & 1 & 20 \\ 13 & 12.5 & 1 & 18 \\ 14 & 13.5 & 1 & 14 \\ 15 & 14.25 & 0.5 & 4\end{array}$

corrected

flow vel. flow vel.

$\operatorname{depth}(\mathrm{ft})(\mathrm{ft} / \mathrm{sec}) \quad(\mathrm{ft} / \mathrm{sec}) \quad \mathrm{Q}(\mathrm{cfs})$

$\begin{array}{lll}0.46 & 0.33 \quad 0.33\end{array}$

$\begin{array}{lll}0.79 & 0.48 & 0.48\end{array}$

$\begin{array}{lll}1.18 & 1.10 & 1.10\end{array}$

1.18

1.12

0.95

0.85

0.72

0.79

0.79

0.92

0.66

0.59

0.46

0.13
1.28

1.32

1.18

1.27

1.40

1.03

1.38

1.12

0.91

1.00

0.76

0.46

\subsection{8}

1.32

1.18

1.27

1.40

1.03

1.38

1.12

0.91

1.00

0.76

0.46

Tot. flow=
0.152

0.378

1.299

1.512

1.473

1.123

1.083

1.011

0.811

1.087

1.029

0.597

0.591

0.349

0.030 
Station 10 Meyers Branch, downstream approx. 150 yards from bridge on Rd 9.

Approximate UTM Coordinates:

$443897 \mathrm{E}, 3669769 \mathrm{~N}$

$(2 / 2 / 98)$

Flowmeter Measurement

Segment Tape width

$\begin{array}{lll} & & \text { (ft.) } \\ 1 & 0.5 & 1 \\ 2 & 1.5 & 1 \\ 3 & 2.5 & 1 \\ 4 & 3.5 & 1 \\ 5 & 4.5 & 1 \\ 6 & 5.5 & 1 \\ 7 & 6.5 & 1 \\ 8 & 7.5 & 1 \\ 9 & 8.5 & 1 \\ 10 & 9.5 & 1 \\ 11 & 10.5 & 1 \\ 12 & 11.5 & 1 \\ 13 & 12.5 & 1 \\ 14 & 13.5 & 1 \\ 15 & 14.5 & 1\end{array}$

\begin{tabular}{lllll} 
& \multicolumn{5}{c}{$\begin{array}{c}\text { corrected } \\
\text { depth } \\
\text { (cm) }\end{array}$} & depth (ft) & (ft/sec) & $\begin{array}{c}\text { flow vel. } \\
\text { (ft/sec) }\end{array}$ & Q (cfs) \\
20 & 0.66 & 0.37 & 0.37 & 0.243 \\
22 & 0.72 & 0.55 & 0.55 & 0.397 \\
27 & 0.89 & 0.85 & 0.85 & 0.753 \\
28 & 0.92 & 0.90 & 0.90 & 0.827 \\
28 & 0.92 & 1.20 & 1.20 & 1.102 \\
32 & 1.05 & 2.10 & 2.10 & 2.205 \\
30 & 0.98 & 2.35 & 2.35 & 2.313 \\
30 & 0.98 & 2.15 & 2.15 & 2.116 \\
26 & 0.85 & 1.85 & 1.85 & 1.578 \\
26 & 0.85 & 2.00 & 2.00 & 1.706 \\
24 & 0.79 & 2.10 & 2.10 & 1.654 \\
22 & 0.72 & 1.65 & 1.65 & 1.191 \\
20 & 0.66 & 1.35 & 1.35 & 0.886 \\
10 & 0.33 & 0.42 & 0.42 & 0.138 \\
6 & 0.20 & -0.05 & -0.05 & -0.010 \\
& & & Tot. flow= & 17.10
\end{tabular}


Station 11 First major trib. to Meyer Branch east of L-Lake, enters from north, just N. of steep road grade.

Approximate UTM Coordinates:

$443342 \mathrm{E}, 3670046 \mathrm{~N}$

$(2 / 2 / 98)$

Flowmeter Measurement

Segment Tape width

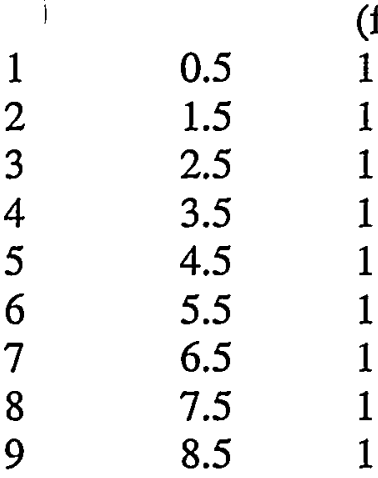

(ft.) $\quad(\mathrm{cm})$

12

28

32

32

42

43

36

30

15 corrected flow vel. flow vel.

$\begin{array}{lllll}\text { depth } & \text { depth (ft) } & (\mathrm{ft} / \mathrm{sec}) & (\mathrm{ft} / \mathrm{sec}) & \mathrm{Q}(\mathrm{cfs}) \\ \mathrm{cm}) & & & & \\ 2 & 0.39 & -0.05 & -0.05 & -0.020 \\ 28 & 0.92 & 0.22 & 0.22 & 0.202 \\ 32 & 1.05 & 0.43 & 0.43 & 0.451 \\ 32 & 1.05 & 0.70 & 0.70 & 0.735 \\ 42 & 1.38 & 0.90 & 0.90 & 1.240 \\ 43 & 1.41 & 1.08 & 1.08 & 1.524 \\ 36 & 1.18 & 0.43 & 0.43 & 0.508 \\ 30 & 0.98 & -0.13 & -0.13 & -0.128 \\ 5 & 0.49 & -0.12 & -0.12 & -0.059 \\ & & & \text { Tot. flow }= & 4.45\end{array}$

Station 12 Tributary to Meyers Br. Located east of L-Lake, enters Meyers Br. from the north.

Approximate UTM Coordinates: $443320 \mathrm{E}, 3671135 \mathrm{~N}$

$(4 / 2 / 98)$

Flowmeter Measurement Segment Tape width

$\begin{array}{lll} & & \text { (ft.) } \\ 1 & 0.5 & 1 \\ 2 & 1.5 & 1 \\ 3 & 2.5 & 1 \\ 4 & 3.5 & 1 \\ 5 & 4.5 & 1 \\ 6 & 5.5 & 1\end{array}$

corrected flow vel. flow vel. $\operatorname{depth}(\mathrm{ft})(\mathrm{ft} / \mathrm{sec}) \quad(\mathrm{ft} / \mathrm{sec}) \quad \mathrm{Q}(\mathrm{cfs})$ (cm) 30 36 0.98

0.10

0.10

0.098

37

37

34

29
1.18

1.21

1.21

1.12

0.95
0.55

0.50

0.50

0.40

0.25
0.55

0.50

0.50

0.40

0.25

Tot. flow $=2.65$ 
Station 13 Confluence of 2 tribs to MeyersBr. Just S. of RR line. Southernmost of the 2 tributaries.

Approx UTM coordinates:

$447153 \mathrm{E} ; 3671048 \mathrm{~N}$

$(4 / 2 / 98)$

Flowmeter Measurement

Segment Tape width

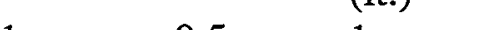

$\begin{array}{llll}1 & 0.5 & 1 & 10\end{array}$

$\begin{array}{llll}2 & 1.5 & 1 & 12\end{array}$

$\begin{array}{llll}3 & 2.5 & 1 & 10\end{array}$

$\begin{array}{llll}4 & 3.5 & 1 & 10\end{array}$

$\begin{array}{llll}5 & 4.5 & 1 & 12\end{array}$

$\begin{array}{lll}6 & 5.5 & 1\end{array}$

$\begin{array}{lll}7 & 6.5 & 1\end{array}$

$\begin{array}{lll}8 & 7.5 & 1\end{array}$

$1 \quad 16$

12
10
10
12
16
12
12

12

10
12
10
0
12
16
12
12

corrected

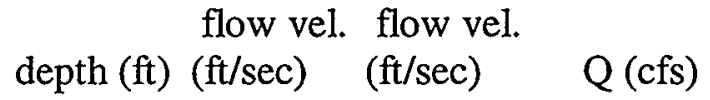

$\begin{array}{llll}0.33 & 0.20 & 0.20 & 0.066\end{array}$

$\begin{array}{llll}0.39 & 0.90 & 0.90 & 0.354\end{array}$

$\begin{array}{llll}0.33 & 0.85 & 0.85 & 0.279\end{array}$

$\begin{array}{llll}0.33 & 1.05 & 1.05 & 0.345\end{array}$

$\begin{array}{llll}0.39 & 0.90 & 0.90 & 0.354\end{array}$

$\begin{array}{llll}0.52 & 1.15 & 1.15 & 0.604\end{array}$

$\begin{array}{llll}0.39 & 0.50 & 0.50 & 0.197\end{array}$

$\begin{array}{llll}0.39 & 0.05 & 0.05 & 0.020\end{array}$

Tot. flow $=2.22$

Station 14 Confluence of 2 tribs to MeyersBr. Just S. of RR line. Northernmost of the 2 tributaries.

Approx UTM coordinates: 447149E; 3671064N

$(4 / 2 / 98)$

Flowmeter measurement

Segment Tape width

(ft.) (cm)

$1-0.5-120$

$\begin{array}{llll}2 & 1.5 & 1 & 14\end{array}$

$\begin{array}{llll}3 & 2.5 & 1 & 14\end{array}$

$\begin{array}{llll}4 & 3.5 & 1 & 9\end{array}$ corrected

flow vel. flow vel.

depth (ft) $(\mathrm{ft} / \mathrm{sec}) \quad(\mathrm{ft} / \mathrm{sec}) \quad \mathrm{Q}(\mathrm{cfs})$

$\begin{array}{llll}0.39 & 0.05 & 0.05 & 0.020\end{array}$

$\begin{array}{llll}0.46 & 0.80 & 0.80 & 0.367\end{array}$

$\begin{array}{llll}0.46 & 1.30 & 1.30 & 0.597\end{array}$

$\begin{array}{llll}0.30 & 0.60 & 0.60 & 0.177\end{array}$

Tot. flow $=1.16$ 


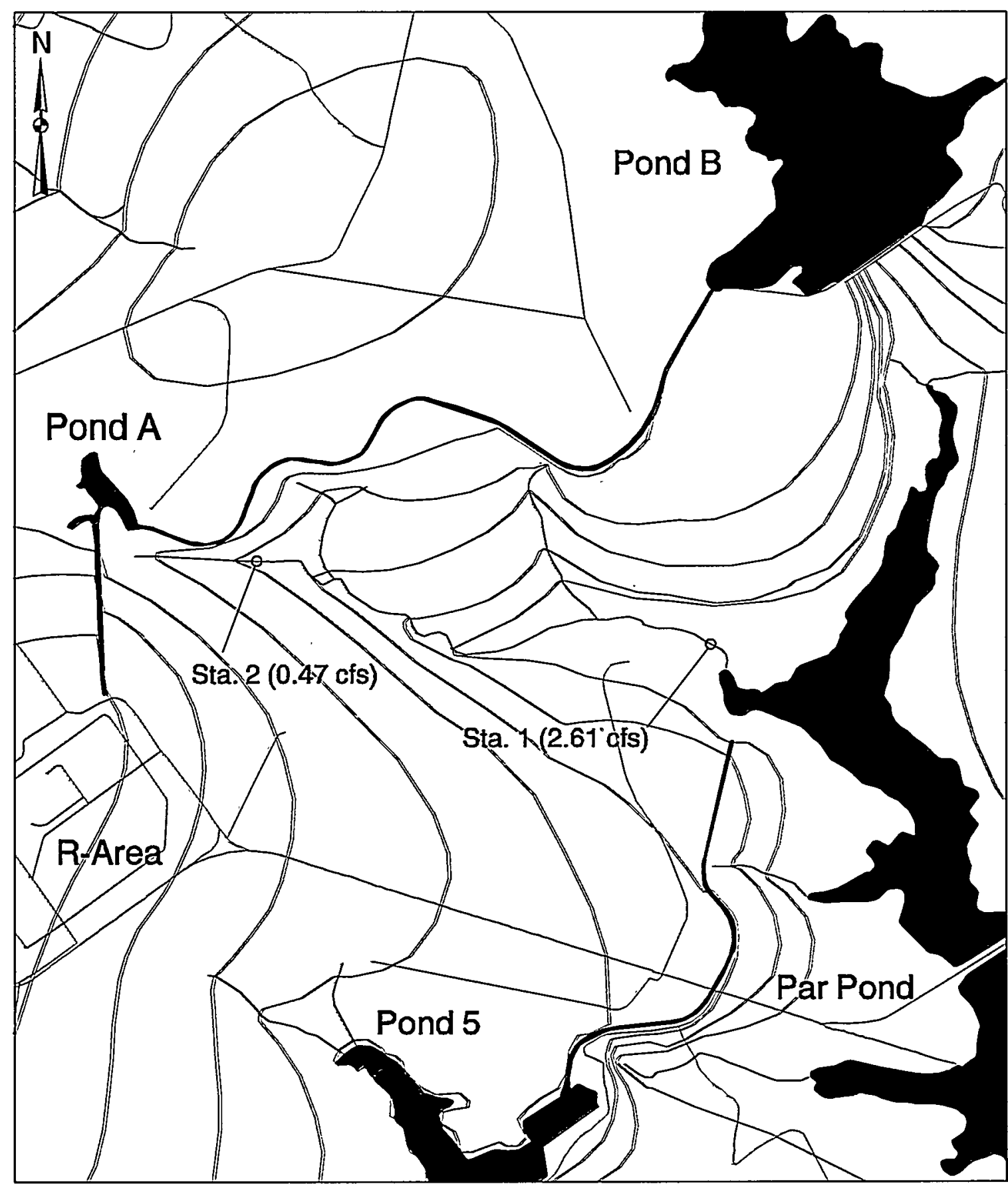

Perennial Streams O RCanal Ste Roads

\section{OLD R-CANAL DISCHARGE TURNOUT} BASEFLOW MEASUREMENTS

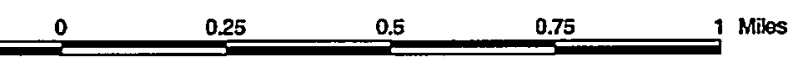




\section{OLD R-CANAL DISCHARGE TURNOUT BASEFLOW}

Station 1 R-Canal discharge turnout drainage, near entrance to Par Pond

Approximate UTM Coordinates:

$447931 \mathrm{E}, 3682189 \mathrm{~N}$

$(2 / 2 / 98)$

Flowmeter Measurement

Segment Tape width

(ft.)

$\begin{array}{lll}1 & 0.5 & 1\end{array}$

$\begin{array}{lll}2 & 1.5 & 1\end{array}$

$\begin{array}{lll}3 & 2.5 & 1\end{array}$

$\begin{array}{lll}4 & 3.5 & 1\end{array}$

$\begin{array}{lll}5 & 4.5 & 1\end{array}$

$\begin{array}{lll}6 & 5.5 & 1\end{array}$

$\begin{array}{lll}7 & 6.5 & 1\end{array}$

$\begin{array}{lll}8 & 7.5 & 1\end{array}$

$\begin{array}{lll}9 & 8.5 & 1\end{array}$ (ft.) (cm)

10

28

30

27

26

34

34

24

8 corrected

flow vel. flow vel. depth (ft) $(\mathrm{ft} / \mathrm{sec}) \quad(\mathrm{ft} / \mathrm{sec}) \quad \mathrm{Q}(\mathrm{cfs})$

0.33

0.92

0.98

0.89

0.85

1.12

1.12

0.79

0.26
0.14

0.38

0.41

0.53

0.52

0.42

0.30

0.13

$-0.02$
0.14

0.38

0.41

0.53

0.52

0.42

0.30

0.13

$-0.02$

Tot. flow $=2.61$

Station 2 R-Canal discharge turnout drainage, $0.3 \mathrm{mi}$ downstream from diversion box.

Approximate UTM Coordinates:

$446804 \mathrm{E}, 3682517 \mathrm{~N}$

$(2 / 2 / 98)$

Flowmeter Measurement

Segment Tape width

$\begin{array}{lll} & & \text { (ft.) } \\ 1 & 0.5 & 1 \\ 2 & 1.5 & 1 \\ 3 & 2.5 & 1 \\ 4 & 3.5 & 1\end{array}$

\begin{tabular}{|c|c|c|c|c|}
\hline $\begin{array}{l}\text { depth } \\
\text { (cm) }\end{array}$ & $\operatorname{depth}(\mathrm{ft})$ & $\begin{array}{l}\text { flow vel. } \\
(\mathrm{ft} / \mathrm{sec})\end{array}$ & $\begin{array}{l}\text { corrected } \\
\text { flow vel. } \\
(\mathrm{ft} / \mathrm{sec})\end{array}$ & $\mathrm{Q}(\mathrm{cfs})$ \\
\hline 5 & 0.16 & 0.12 & 0.12 & 0.020 \\
\hline 8 & 0.26 & 0.37 & 0.37 & 0.097 \\
\hline 6 & 0.20 & 0.71 & 0.71 & 0.140 \\
\hline 8 & 0.26 & 0.75 & 0.80 & 0.210 \\
\hline
\end{tabular}




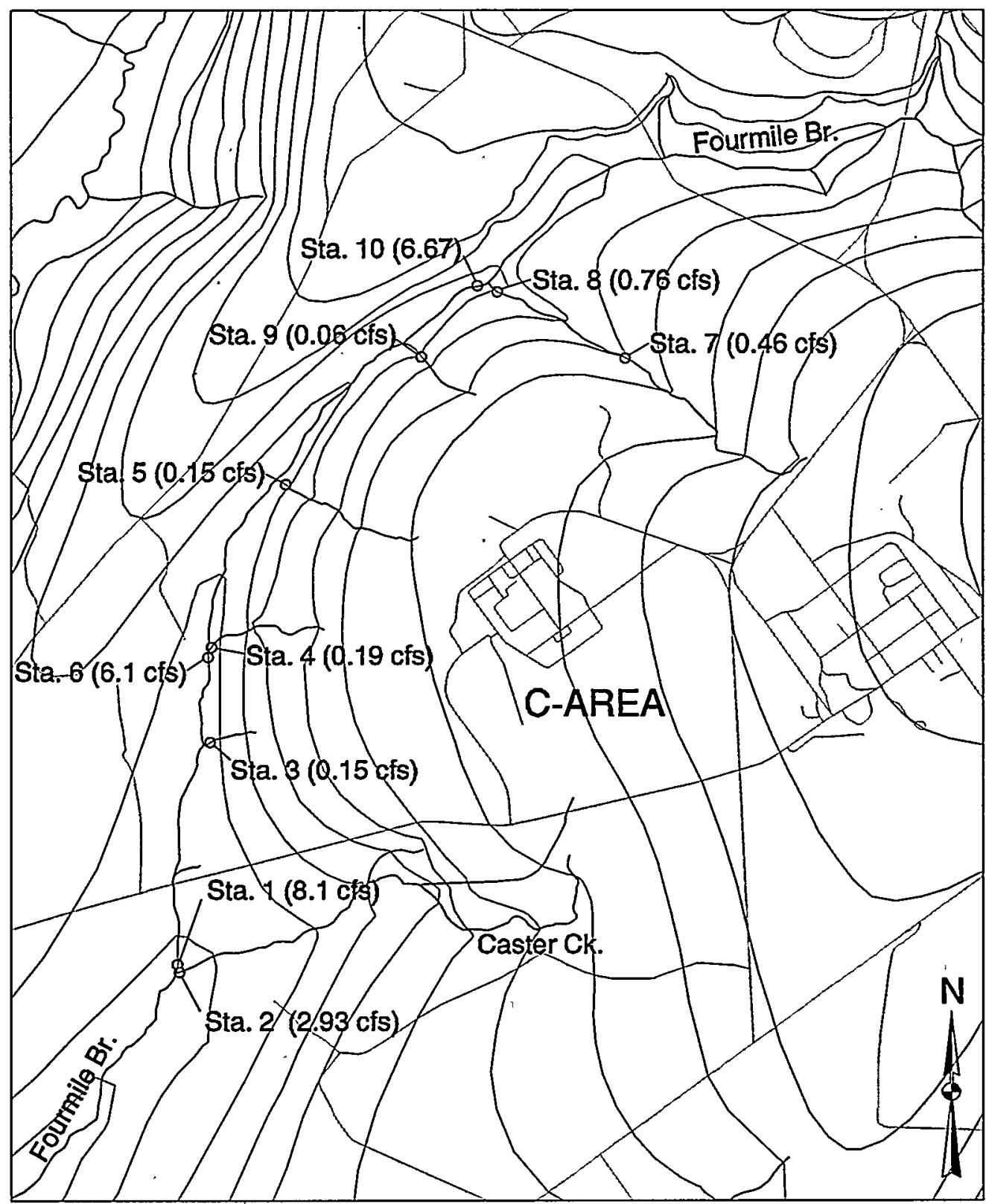

$\checkmark$ Perennial Streams $\checkmark$ Roads Flow Stations Water Table
Baseflow Measurements near C-Reactor

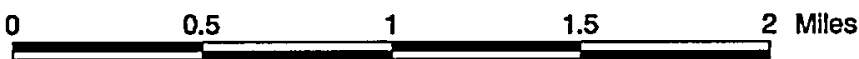




\section{C-AREA STREAM BASEFLOW MEASUREMENTS IN FOURMILE BRANCH AND ITS MAIN TRUBUTARIES FROM THE C-REACTOR SIDE}

Station 1 Main branch of Fourmile Br., 100' upstream of confluence with Caster Ck.

$$
\text { Approximate UTM Coordinates: } \quad 3676961 \text { N, } 434965 \text { E }
$$

12/7/98-1

Flowmeter Measurement

Segment Tape width

$\begin{array}{lll}1 & \text { (ft.) }\end{array}$

$2 \quad 1.5 \quad 1$

$3 \quad 2.5 \quad 1$

$4 \quad 3.5 \quad 1$

$5 \quad 4.5 \quad 1$

$\begin{array}{lll}6 & 5.5 & 1\end{array}$

$\begin{array}{lll}7 & 6.5 & 1\end{array}$

$8 \quad 7.5 \quad 1$

$\begin{array}{lll}9 & 8.5 & 1\end{array}$

$9.5 \quad 1$

$10.5 \quad 1$

$11.5 \quad 1$

$12.5 \quad 1$

$13.5 \quad 1$

$14.5 \quad 1$

$15.5 \quad 1$

$16.5 \quad 1$

$17.5 \quad 1$

$18.5 \quad 1$

$19.5 \quad 1$

flow vel. flow vel.

$\begin{array}{llll}\text { ft.) } & \text { depth (ft) }(\mathrm{ft} / \mathrm{sec}) & (\mathrm{ft} / \mathrm{sec}) & \mathrm{Q} \\ & & & (\mathrm{cfs})\end{array}$

$\begin{array}{lllll}1 & 0.50 & 0.39 & 0.38 & 0.190 \\ 1 & 0.80 & 0.48 & 0.47 & 0.376 \\ 1 & 0.85 & 0.73 & 0.72 & 0.612 \\ 1 & 0.90 & 0.46 & 0.45 & 0.405 \\ 1 & 0.85 & 0.05 & 0.04 & 0.034 \\ 1 & 1.10 & 0.23 & 0.22 & 0.242 \\ 1 & 1.10 & 0.22 & 0.21 & 0.231 \\ 1 & 1.20 & 0.52 & 0.51 & 0.612 \\ 1 & 1.10 & 0.80 & 0.79 & 0.869 \\ 1 & 1.20 & 0.70 & 0.69 & 0.828 \\ 1 & 1.10 & 0.65 & 0.64 & 0.704 \\ 1 & 1.20 & 0.32 & 0.31 & 0.372 \\ 1 & 1.20 & 0.02 & 0.01 & 0.012 \\ 1 & 1.20 & 0.08 & 0.07 & 0.084 \\ 1 & 1.10 & 0.32 & 0.31 & 0.341 \\ 1 & 1.30 & 0.30 & 0.29 & 0.377 \\ 1 & 1.30 & 0.15 & 0.14 & 0.182 \\ 1 & 1.10 & 1.00 & 0.99 & 1.089 \\ 1 & 1.20 & 0.38 & 0.37 & 0.444 \\ 1 & 1.10 & 0.04 & 0.03 & 0.033 \\ 1 & 1.00 & 0.08 & 0.07 & 0.070 \\ 0.5 & 0.80 & 0.01 & 0.00 & 0.000 \\ & 0.80 & -0.01 & -0.02 & -0.008 \\ & & & \text { Tot. flow= } & 8.10\end{array}$


Station 2 Caster Ck. About 20 feet upstream of confluence with Fourmile Br.

$$
\text { Approximate UTM Coordinates: } \quad 3676914 \mathrm{~N}, 435026 \mathrm{E}
$$

12/7/98_2

\begin{tabular}{|c|c|c|c|c|c|c|}
\hline \multirow{2}{*}{\multicolumn{3}{|c|}{ Flowmeter Measurement }} & \multicolumn{4}{|c|}{ corrected } \\
\hline & & & & flow vel & flow vel. & \\
\hline Segment & Tape & $\begin{array}{l}\text { width } \\
\text { (ft.) }\end{array}$ & $\operatorname{depth}(\mathrm{ft})$ & $(\mathrm{ft} / \mathrm{sec})$ & $(\mathrm{ft} / \mathrm{sec})$ & $\begin{array}{l}Q \\
\text { (cfs) }\end{array}$ \\
\hline 1 & 0.5 & 1 & 0.70 & 0.03 & 0.02 & 0.014 \\
\hline 2 & 1.5 & 1 & 0.70 & 0.07 & 0.06 & 0.042 \\
\hline 3 & 2.5 & 1 & 0.70 & 0.31 & 0.30 & 0.210 \\
\hline 4 & 3.5 & 1 & 0.70 & 0.45 & 0.44 & 0.308 \\
\hline 5 & 4.5 & 1 & 0.70 & 0.50 & 0.49 & 0.343 \\
\hline 6 & 5.5 & 1 & 0.80 & 0.35 & 0.34 & 0.272 \\
\hline 7 & 6.5 & 1 & 0.90 & 0.15 & 0.14 & 0.126 \\
\hline 8 & 7.5 & 1 & 0.80 & 0.10 & 0.09 & 0.072 \\
\hline 9 & 8.5 & 1 & 0.90 & 0.22 & 0.21 & 0.189 \\
\hline 10 & 9.5 & 1 & 1.10 & 0.32 & 0.31 & 0.341 \\
\hline 11 & 10.5 & 1 & 1.10 & 0.42 & 0.41 & 0.451 \\
\hline 12 & 11.5 & 1 & 1.10 & 0.26 & 0.25 & 0.275 \\
\hline 13 & 12.5 & 1 & 1.10 & 0.08 & 0.07 & 0.077 \\
\hline 14 & 13.5 & 1 & 1.00 & 0.09 & 0.08 & 0.080 \\
\hline 15 & 14.5 & 1 & 0.90 & 0.07 & 0.06 & 0.054 \\
\hline 16 & 15.5 & 1 & 0.70 & 0.09 & 0.08 & 0.056 \\
\hline 17 & 16.5 & 1 & 0.30 & 0.08 & 0.07 & 0.021 \\
\hline & & & & & Tot. flow= & 2.93 \\
\hline
\end{tabular}

Station 3 First tributary to $4 \mathrm{MB}$ south of the "Twin Lakes" tributary, just above mouth

Approx. UTM Coordinates: $\quad 3678228$ N, $435196 \mathrm{E}$

12/8/98_1

. Flowmeter Measurement

Segment Tape width

(ft.)

$\begin{array}{lll}1 & 0.75 & 1.5\end{array}$ corrected

flow vel. flow vel.

$\operatorname{depth}(\mathrm{ft})(\mathrm{ft} / \mathrm{sec}) \quad(\mathrm{ft} / \mathrm{sec}) \quad \mathrm{Q}$

(cfs)

$\begin{array}{llll}1.00 & 0.11 & 0.10 & 0.150\end{array}$

Tot. flow $=0.15$ 
Station 4 "Twin Lakes" tributary to $4 \mathrm{MB}$, just above the mouth.

Approx. UTM Coordinates: 3678776 N, $435211 \mathrm{E}$

12/8/98_2

Flowmeter Measurement

Segment Tape width

$\begin{array}{lllll}1 & & \text { (ft.) } & & \\ 2 & 0.5 & 1 & 0.20 & 0.50 \\ 3 & 1.5 & 1 & 0.30 & 0.31 \\ & 2.25 & 0.5 & 0.20 & -0.01\end{array}$

corrected

flow vel. flow vel.

$\operatorname{depth}(\mathrm{ft})(\mathrm{ft} / \mathrm{sec}) \quad(\mathrm{ft} / \mathrm{sec}) \quad \mathrm{Q}(\mathrm{cfs})$

$0.49 \quad 0.098$

$0.30 \quad 0.090$

$-0.02-0.002$

Station 5 First drainage north of "Twin Lakes" drainage, near confluence with 4MB

Approx. UTM Coordinates: 3679757 N, $435631 \mathrm{E}$

12/8/98_3

Flowmeter Measurement

Segment Tape width

$\begin{array}{lll}1 & 0.5 & 1 \\ 2 & 1.5 & 1 \\ 3 & 2.5 & 1\end{array}$

corrected

flow vel. flow vel.

$\operatorname{depth}(\mathrm{ft})(\mathrm{ft} / \mathrm{sec}) \quad(\mathrm{ft} / \mathrm{sec}) \quad \mathrm{Q}(\mathrm{cfs})$

$\begin{array}{llll}0.20 & 0.21 & 0.20 & 0.040\end{array}$

$\begin{array}{llll}0.35 & 0.32 & 0.31 & 0.109\end{array}$

$\begin{array}{llll}0.10 & 0.01 & 0.00 & 0.000\end{array}$

Tot. flow $=0.15$ 
Station 6 Fourmile Branch, just below confluence with Twin Lakes drainage and about $200 \mathrm{ft}$ upstream from Sewage Plant discharge line.

Approx. UTM Coordinates: 3678674 N, $435160 \mathrm{E}$

12/8/98_4

Flowmeter Measurement

Segment Tape width

$$
\text { (ft.) }
$$

2

3

4

5

6

7

8

9

10

11

12

13

14

15

16

$\begin{array}{ll}0.5 & 1 \\ 1.5 & 1 \\ 2.5 & 1 \\ 3.5 & 1 \\ 4.5 & 1 \\ 5.5 & 1 \\ 6.5 & 1 \\ 7.5 & 1 \\ 8.5 & 1 \\ 9.5 & 1 \\ 10.5 & 1 \\ 11.5 & 1 \\ 12.5 & 1 \\ 13.5 & 1 \\ 14.5 & 1 \\ 15.5 & 1\end{array}$

0.10

0.30

0.40

0.50

0.60

0.55

0.70

0.70

0.80

0.85

0.75

0.65

0.60

0.50

0.30

0.25

corrected

flow vel. flow vel.
(ft/sec)

$0.26 \quad 0.25$

$\begin{array}{ll}0.63 & 0.62\end{array}$

$\begin{array}{ll}0.89 & 0.88\end{array}$

$0.93 \quad 0.92$

$0.91 \quad 0.90$

Q

(cfs)

0.025

0.186

0.352

0.460

0.540

$1.04 \quad 1.03$

0.567

$0.96 \cdot 0.95$

0.665

$0.93 \quad 0.92$

0.644

$\begin{array}{ll}0.78 & 0.77\end{array}$

0.616

$\begin{array}{ll}0.84 & 0.83\end{array}$

0.706

$0.53 \quad 0.52$

0.390

$0.22 \quad 0.21$

0.137

0.66

0.65

0.390

0.42

0.41

0.205

0.56

0.55

0.165

0.23

0.22

0.055

Tot. flow $=6.10$

Station 7 "Construction Outfall" tributary to $4 \mathrm{MB}$, near asbestos pits, near fork in drainage.

Approx. UTM Coordinates: 3680419 N, 437588 E

12/9/98_1

Flowmeter Measurement

Segment Tape width

(ft.)

$\begin{array}{lll}1 & 0.5 & 1 \\ 2 & 1.5 & 1 \\ 3 & 2.5 & 1 \\ 4 & 3.5 & 1 \\ 5 & 4.25 & 0.5\end{array}$

corrected

flow vel. flow vel.

$\operatorname{depth}(\mathrm{ft})(\mathrm{ft} / \mathrm{sec}) \quad(\mathrm{ft} / \mathrm{sec}) \quad \mathrm{Q}(\mathrm{cfs})$

$\begin{array}{llll}0.20 & 0.24 & 0.23 & 0.046\end{array}$

$\begin{array}{llll}0.30 & 0.71 & 0.70 & 0.210\end{array}$

0.25

0.75

0.74

0.185

0.10

0.22

0.21

0.021

0.05

0.05

0.04

0.001

Tot. flow $=0.46$ 
Station 8 "Construction Outfall" tributary to 4MB, near asbestos pits, 250' above mouth.

Approx. UTM Coordinates: 3680843 N, 436833 E

12/9/98_2

Flowmeter

Measurement

Segment Tape

$\begin{array}{lll} & & \text { (ft.) } \\ 1 & 0.5 & 1 \\ 2 & 1.5 & 1 \\ 3 & 2.5 & 1 \\ 4 & 3.5 & 1 \\ 5 & 4.5 & 1 \\ 6 & 5.5 & 1 \\ 7 & 6.25 & 0.5\end{array}$

corrected

flow vel. flow vel.

Station 9 First trib. to $4 \mathrm{MB}$ south of "construction outfall" tributary. About 200 yds upstream from mouth.

Approx. UTM Coordinates: 3680423 N, $436400 \mathrm{E}$

12/9/98_3

Flowmeter Measurement

Segment Tape width

(ft.)

1

2

3 widt

(t.)

0.50

0.50

0.30

0.30

0.20

0.15

0.15 (ft/sec) Q

(cfs)

0.030

0.345

0.192

0.105

0.056

0.032

$-0.002$

Tot. flow $=0.76$

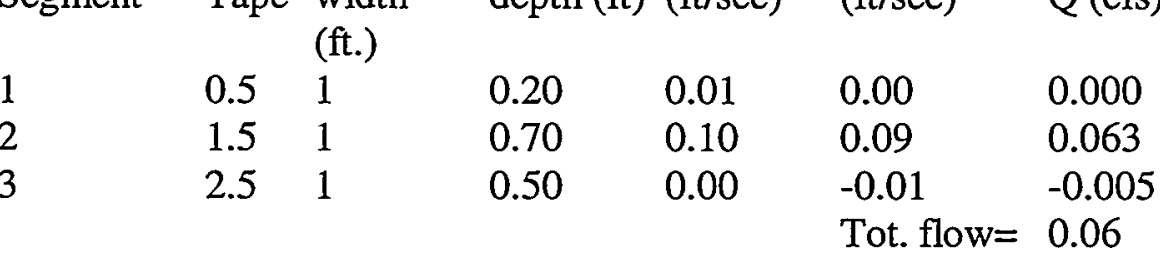


Station 10 Fourmile Branch about 150 feet downstream of steamline road, north of C-Area.

Approx. UTM Coordinates: 3680839 N, $436684 \mathrm{E}$

$(12 / 8 / 97)$

FlowmeterMeasurement

Segment Tape width

1
2
3
4
5
6
7
8
9

10

11

12

13

14

15

16

17

18

19

20 (ft.)

$0.5 \quad 1$

1.51

$2.5 \quad 1$

$3.5 \quad 1$

4.51

$5.5 \quad 1$

$6.5,1$

$7.5 \quad 1$

8.51

$9.5 \quad 1$

$10.5 \quad 1$

$11.5 \quad 1$

$12.5 \quad 1$

$13.5 \quad 1$

$14.5 \quad 1$

$15.5 \quad 1$

$16.5 \quad 1$

$17.5 \quad 1$

$18.5 \quad 1$

19.251 corrected

flow vel. flow vel.

$\operatorname{depth}(\mathrm{ft})(\mathrm{ft} / \mathrm{sec}) \quad(\mathrm{ft} / \mathrm{sec}) \quad \mathrm{Q}$

0.70

0.70

0.70

0.60

0.60

0.50

0.40

0.40

0.45

0.60

0.50

0.60

0.70

0.60

0.50

0.40

0.45

0.65

0.65

0.40
0.38

0.70

0.72

0.65

0.50

0.40

0.46

0.60

0.70

0.76

0.88

0.98

0.88

0.75

0.75

0.58

0.40

0.52

0.31

0.05
0.37

0.69

0.71

0.64

0.49

0.39

0.45

0.59

0.69

0.75

0.87

0.97

0.87

0.74

0.74

0.57

0.39

0.51

0.30

0.04

Tot. flow $=6.67$ (cfs)

0.259

0.483

0.497

0.384

0.294

0.195

0.180

0.236

0.311

0.450

0.435

0.582

0.609

0.444

0.370

0.228

0.176

0.332

0.195

0.016 
This page intentionally left blank 
APPENDIX F. HYDRAULIC HEAD TARGET AND RESIDUAL DATA 


\section{(This page intentionally left blank)}




\section{APPENDIX F. HYDRAULIC HEAD TARGET AND RESIDUAL DATA}

\section{Hydraulic Head Targets}

Table F-1 summarizes the hydraulic head data available for model calibration. When multiple measurements are available for a given well, the mean water level is shown. Otherwise, the single reading is given. The sample standard deviation of the mean, sample standard deviation of the population, and number of readings follow the target head, where applicable. The "category" column refers to the aquifer zone: $1=$ Gordon, $2=$ "lower" UTRA, $3=$ transmissive zone, $4=\mathrm{AA}$ horizon, $5=\mathrm{A} / \mathrm{uu}$ horizon and $6=$ mixed or other. The average head target has a "2 sigma" uncertainty of $\pm 0.8 \mathrm{ft}$, not counting one-time readings which inflate the overall average uncertainty.

Table F-1. Hydraulic Head Targets for Model Calibration

\begin{tabular}{|c|c|c|c|c|c|c|c|c|c|}
\hline 'BG & 26 & ' & 58809.70 & 73958.40 & 210.7 & 230.7 & 239.35 & 0.85 & 1.202 \\
\hline - BG & 27 & - & 58810.00 & 74356.70 & 234.4 & 254.4 & 240.95 & 0.85 & 1.202 \\
\hline 'BG & 28 & - & 58810.20 & 74752.00 & 239.7 & 259.7 & 247.10 & 0.60 & 0.852 \\
\hline ' $\mathrm{BG}$ & 29 & - & 58809.90 & 75151.60 & 231.6 & 251.6 & 245.00 & 0.60 & 0.852 \\
\hline 'BG & 30 & ' & 58809.10 & 75550.10 & 231.7 & 251.7 & 237.55 & 0.05 & 0.072 \\
\hline 'BG & 31 & ' & 58803.70 & 75949.90 & 223.3 & 243.3 & 233.70 & 0.50 & 0.712 \\
\hline 'BG & 32 & ' & 58803.50 & 76349.90 & 226.9 & 246.9 & 233.40 & 0.30 & 0.422 \\
\hline 'BG & 33 & . & 58526.00 & 76479.90 & 221.2 & 241.2 & 232.90 & 0.30 & 0.422 \\
\hline 'BG & 34 & ' & 58107.40 & 76493.60 & 217.4 & 237.4 & 232.85 & 0.45 & 0.642 \\
\hline 'BG & 35 & ' & 57726.40 & 76495.30 & 228.0 & 248.0 & 232.90 & 0.20 & 0.282 \\
\hline - BG & 36 & ' & 57620.30 & 76747.60 & 223.3 & 243.3 & 232.50 & 0.60 & 0.852 \\
\hline - BG & 37 & - & 57251.00 & 76804.90 & 227.8 & 247.8 & 232.85 & 0.55 & 0.782 \\
\hline 'BG & 38 & ' & 56851.10 & 76805.00 & 225.9 & 245.9 & 232.30 & 0.40 & 0.572 \\
\hline 'BG & 39 & ' & 56451.30 & 76804.90 & 226.0 & 246.0 & 231.70 & 0.50 & 0.712 \\
\hline 'BG & 40 & ' & 56051.00 & 76805.10 & 221.9 & 241.9 & 231.40 & 0.50 & 0.712 \\
\hline 'BG & 41 & ' & 55868.80 & 76576.30 & 221.0 & 241.0 & 230.75 & 0.25 & 0.352 \\
\hline 'BG & 42 & ' & 55869.50 & 76178.80 & 217.1 & 237.1 & 230.70 & 0.60 & 0.852 \\
\hline 'BG & 43 & ' & 56039.40 & 75852.50 & 222.9 & 242.9 & 230.50 & 0.40 & 0.572 \\
\hline 'BG & 51 & ' & 58599.30 & 73864.30 & 221.2 & 241.2 & 240.70 & -1.00 & -1.001 \\
\hline 'BG & 52 & ' & 55524.30 & 75910.40 & 223.8 & 243.8 & 229.32 & 0.28 & 1.4827 \\
\hline 'BG & 53 & - & 55073.90 & 76157.30 & 214.7 & 234.7 & 228.04 & 0.31 & 0.949 \\
\hline 'BG & 54 & - & 54830.30 & 75837.90 & $215.2^{\circ}$ & 235.2 & 228.61 & 0.32 & 1.7129 \\
\hline 'BG & 55 & ' & 54590.50 & 75525.30 & 214.9 & 234.9 & 226.56 & 0.61 & 3.2829 \\
\hline 'BG & 56 & , & 54481.90 & 75206.50 & 210.9 & 230.9 & 225.05 & 0.27 & 0.768 \\
\hline 'BG & 57 & ' & 54820.00 & 75000.40 & 214.6 & 234.6 & 225.27 & 0.15 & 0.459 \\
\hline 'BG & 58 & ' & 55162.30 & 74790.90 & 218.2 & 238.2 & 226.78 & 0.27 & 0.819 \\
\hline 'BG & 59 & - & 55508.30 & 74593.40 & 217.7 & 237.7 & 229.85 & 0.40 & 2.0426 \\
\hline 'BG & 60 & . & 55850.30 & 74386.30 & 215.5 & 235.5 & 230.80 & 0.39 & 2.0126 \\
\hline 'BG & 61 & ' & 56360.80 & 74075.40 & 225.0 & 245.0 & 232.83 & 0.48 & 2.4226 \\
\hline 'BG & 62 & ' & 56530.90 & 73971.60 & 222.5 & 242.5 & 233.41 & 0.44 & 1.4711 \\
\hline 'BG & 63 & ' & 56870.50 & 73754.50 & 224.2 & 244.2 & 235.24 & 0.43 & 1.3510 \\
\hline 'BG & 64 & ' & 57212.40 & 73547.20 & 227.3 & 247.3 & 238.13 & 0.38 & 1.2110 \\
\hline 'BG & 65 & ' & 57552.70 & 73340.60 & 230.9 & 250.9 & 235.74 & 0.42 & 1.3110 \\
\hline 'BG & 66 & ' & 57805.00 & 73585.00 & 231.0 & 251.0 & 235.20 & 0.60 & 1.819 \\
\hline 'BG & 67 & . & 57902.60 & 73954.10 & 224.7 & 244.7 & 236.49 & 0.55 & 2.9028 \\
\hline 'BG & 68 & ' & 58251.50 & 76553.60 & 216.5 & 242.9 & 232.22 & -1.00 & -1.001 \\
\hline 'BG & 69 & ' & 58226.20 & 76553.80 & 222.2 & 242.2 & 232.48 & -1.00 & -1.001 \\
\hline 'BG & 80 & ' & 57962.60 & 76596.50 & 226.2 & 248.6 & 232.73 & -1.00 & -1.001 \\
\hline 'BG & 81 & - & 57983.00 & 76621.90 & 222.9 & 246.9 & 227.35 & -1.00 & -1.001 \\
\hline 'BG & 84 & ' & 57955.40 & 76695.90 & 227.2 & 247.2 & 232.58 & -1.00 & -1.001 \\
\hline 'BG & 85 & ' & 57928.90 & 76712.00 & 228.0 & 248.0 & 232.55 & -1.00 & -1.001 \\
\hline 'BG & 86 & , & 57979.40 & 76721.40 & 228.0 & 248.0 & 232.48 & -1.00 & -1.001 \\
\hline
\end{tabular}


Table F-1. Hydraulic Head Targets for Model Calibration (Continued)

\begin{tabular}{|c|c|c|c|c|c|c|c|c|c|c|c|}
\hline 'BG & 87 & , & 57951.90 & 76748.90 & 226.2 & 245.8 & 232.30 & -1.00 & -1.001 & 4 & \\
\hline 'BG & 91 & ' & 56649.40 & 78031.30 & 205.4 & 235.4 & 218.82 & 0.51 & 2.2419 & 4 & \\
\hline 'BG & 92 & ' & 56828.00 & 79019.60 & 197.2 & 227.2 & 208.97 & 0.69 & 3.0119 & 3 & \\
\hline 'BG & 93 & , & 57160.80 & 79930.80 & 180.5 & 210.5 & 199.16 & 1.00 & 4.3619 & 6 & ? \\
\hline 'BG & 94 & - & 57494.00 & 80867.20 & 152.8 & 182.8 & 191.19 & 0.27 & 1.2020 & 2 & \\
\hline 'BG & 95 & , & 58407.00 & 80059.90 & 152.5 & 182.5 & 192.86 & 0.26 & 1.1720 & 2 & \\
\hline ' BG & 96 & , & 58297.80 & 79396.30 & 177.2 & 207.2 & 197.82 & 0.64 & 2.7719 & 2 & \\
\hline 'BG & 98 & , & 57398.70 & 77597.90 & 212.5 & 242.5 & 224.46 & -1.00 & -1.001 & 4 & \\
\hline 'BG & 99 & , & 58404.10 & 76904.60 & 215.9 & 245.9 & 232.53 & -1.00 & -1.001 & 4 & \\
\hline 'BG & 100 & - & 58899.10 & 77815.60 & 203.3 & 233.3 & 224.80 & -1.00 & -1.001 & 4 & \\
\hline 'BG & 103 & ' & 59752.10 & 77883.60 & 169.5 & 199.5 & 199.95 & 0.34 & 1.4217 & 2 & \\
\hline 'BG & 107 & ' & 60120.10 & 74803.60 & 208.3 & 228.3 & 234.59 & 0.91 & 4.1521 & 4 & \\
\hline 'BG & 108 & . & 59827.90 & 74383.00 & 217.3 & 247.3 & 238.99 & 0.35 & 1.5620 & 4 & \\
\hline 'BG & 109 & . & 59626.10 & 73926.20 & 228.4 & 258.4 & 240.79 & 0.54 & 2.4020 & 5 & \\
\hline 'BG & 110 & ' & 59277.20 & 73354.70 & 224.3 & 254.3 & 241.51 & 0.50 & 2.1819 & 4 & \\
\hline 'BG & 113 & - & 59386.00 & 77410.20 & 196.4 & 216.4 & 217.10 & -1.00 & -1.001 & 6 & ? \\
\hline 'BG & 115 & . & 57884.50 & 77207.20 & 198.9 & 218.9 & 215.80 & -1.00 & -1.001 & 3 & \\
\hline 'BG & 119 & - & 57004.90 & 77743.70 & 209.2 & 229.2 & 215.37 & -1.00 & -1.001 & 4 & \\
\hline 'BG & 122 & ' & 56789.70 & 78581.10 & 189.9 & 209.9 & 211.34 & 0.35 & 1.5219 & 3 & \\
\hline 'BG & 124 & - & 57095.00 & 77254.00 & 214.8 & 234.8 & 231.82 & -1.00 & -1.001 & 4 & \\
\hline 'BGO & 1D & ' & 58779.30 & 73737.90 & 225.0 & 245.0 & 238.52 & 0.34 & 2.8469 & 4 & \\
\hline ' $\mathrm{BGO}$ & $2 \mathrm{D}$ & - & 58809.70 & 74552.90 & 218.9 & 238.9 & 238.19 & 0.19 & 1.4359 & 4 & \\
\hline 'BGO & $3 \mathrm{~A}$ & ' & 58806.80 & 75561.70 & 103.7 & 113.7 & 163.04 & 0.09 & 0.4624 & 1 & \\
\hline 'BGO & $3 \mathrm{C}$ & - & 58806.40 & 75550.40 & 178.7 & 188.7 & 225.92 & 0.19 & 1.0027 & 2 & \\
\hline ' BGO & $3 \mathrm{D}$ & ' & 58809.20 & 75351.30 & 227.6 & 247.6 & 235.57 & 0.18 & 1.1238 & 4 & \\
\hline 'BGO & $3 D R$ & & 58820.00 & 75512.30 & 217.5 & 237.6 & 231.91 & 0.13 & 0.5921 & 4 & \\
\hline 'BGO & $4 \mathrm{D}$ & ' & 58803.70 & 76150.10 & 220.6 & 240.6 & 232.59 & 0.61 & 3.4732 & 4 & \\
\hline 'BGO & $5 \mathrm{C}$ & - & 58794.50 & 76476.90 & 183.2 & 193.2 & 216.25 & 0.33 & 2.5057 & 2 & \\
\hline 'BGO & 50 & ' & 58784.80 & 76477.50 & 219.3 & 239.3 & 230.66 & 0.35 & 2.7260 & 4 & \\
\hline 'BGO & $6 \mathrm{~A}$ & - & 58316.80 & 76487.20 & 107.5 & 117.5 & 159.33 & 0.07 & 0.5861 & 1 & \\
\hline 'BGO & $6 \mathrm{~B}$ & - & 58346.50 & 76553.20 & 139.7 & 149.7 & 219.05 & 0.15 & 1.0145 & 2 & \\
\hline ' $\mathrm{BGO}$ & $6 \mathrm{C}$ & ' & 58307.00 & 76487.10 & 158.0 & 168.0 & 220.32 & 0.23 & 1.8564 & 2 & \\
\hline ' BGO & $6 \mathrm{D}$ & ' & 58297.10 & 76487.30 & 217.2 & 237.2 & 231.38 & 0.09 & 0.7465 & 4 & \\
\hline 'BGO & 7D & - & 57917.20 & 76494.50 & 220.2 & 240.2 & 232.64 & 0.29 & 2.2461 & 4 & \\
\hline 'BGO & $8 \mathrm{~A}$ & - & 57618.30 & 76569.00 & 105.3 & 115.3 & 160.96 & 0.77 & 2.4310 & 1 & \\
\hline ' BGO & 8AR & & 57617.50 & 76598.80 & 94.6 & 104.6 & 160.90 & 0.21 & 1.4751 & 1 & \\
\hline 'BGO & $8 \mathrm{C}$ & - & 57618.70 & 76579.20 & 174.3 & 184.3 & 224.31 & 0.50 & 3.9061 & 2 & \\
\hline 'BGO & 8D & - & 57617.80 & 76588.80 & 220.6 & 240.6 & 232.73 & 0.40 & 3.1462 & 4 & \\
\hline 'BGO & $9 A A$ & & 57371.90 & 76975.70 & 73.8 & 83.8 & 158.01 & 0.08 & 0.5043 & 1 & \\
\hline 'BGO & $9 \mathrm{D}$ & ' & 57478.90 & 76811.60 & 209.2 & 229.2 & 230.13 & 0.43 & 3.4264 & 4 & \\
\hline ' BGO & $10 A$ & - & 57050.90 & 76805.20 & 111.1 & 121.1 & 170.74 & 1.35 & 5.8719 & 1 & \\
\hline 'BGO & $10 A A$ & & 56990.50 & 76997.90 & 80.8 & 90.8 & 157.61 & 0.49 & 3.0639 & 1 & \\
\hline - BGO & 10AR & & 57063.80 & 76806.00 & 96.5 & 106.5 & 158.49 & 0.08 & 0.5545 & 1 & \\
\hline 'BGO & $10 B$ & ' & 56978.80 & 76982.10 & 139.0 & 149.0 & 219.85 & 0.21 & 1.3039 & 2 & \\
\hline 'BGO & $10 \mathrm{C}$ & - & 57041.10 & 76805.20 & 157.3 & 167.3 & 220.45 & 0.14 & 1.1765 & 2 & \\
\hline 'BGO & $10 \mathrm{DR}$ & & 57073.70 & 76804.80 & 218.3 & 238.3 & 231.68 & 0.23 & 1.5546 & 4 & \\
\hline 'BGO & $11 D$ & ' & 56651.30 & 76805.10 & 216.3 & 236.3 & 230.91 & 0.36 & 2.3643 & 4 & \\
\hline - BGO & $11 \mathrm{DR}$ & & 56650.40 & 76849.30 & 213.1 & 233.0 & 230.54 & 0.25 & 1.1120 & 4 & \\
\hline ' BGO & $12 \mathrm{AR}$ & & 56259.90 & 76803.80 & 99.3 & 109.3 & 157.82 & 0.09 & 0.4627 & 1 & \\
\hline 'BGO & $12 A X$ & & 56258.00 & 76834.80 & 99.5 & 109.5 & 157.35 & 0.14 & 0.6521 & 1 & \\
\hline 'BGO & $12 \mathrm{C}$ & ' & 56241.10 & 76805.20 & 153.6 & 163.6 & 220.05 & 0.24 & 0.7710 & 2 & \\
\hline 'BGO & D $12 \mathrm{CR}$ & & 56215.20 & 76806.00 & 144.0 & 154.0 & 221.95 & 0.19 & 1.0228 & 2 & \\
\hline ' BGO & $12 \mathrm{CX}$ & & 56230.40 & 76834.50 & 141.2 & 151.2 & 230.39 & 0.25 & 1.1421 & 2 & \\
\hline ' BGO & $12 D$ & ' & 56231.10 & 76805.20 & 217.8 & 237.8 & 231.43 & 0.20 & 1.3043 & 4 & \\
\hline ' $\mathrm{BGO}$ & $12 \mathrm{DR}$ & & 56214.70 & 76834.60 & 212.7 & 232.8 & 220.13 & 0.28 & 1.2621 & 4 & \\
\hline 'BGO & I3DR & & 55840.40 & 76824.70 & 210.3 & 220.3 & 230.99 & 0.19 & 1.3651 & 4 & \\
\hline 'BGO & $14 \mathrm{~A}$ & - & 55838.30 & 76377.50 & 109.6 & 119.6 & 157.25 & 0.72 & 2.7715 & 1 & \\
\hline 'BGO & D I4AR & & 55788.90 & 76351.80 & 96.8 & 106.8 & 159.14 & 0.23 & 1.6047 & 1 & \\
\hline 'BGO & $14 \mathrm{C}$ & ' & 55839.00 & 76367.70 & 192.1 & 202.1 & 221.36 & 0.95 & 3.5514 & 2 & \\
\hline 'BGO & $14 \mathrm{CR}$ & & 55789.00 & 76337.80 & 190.1 & 200.1 & 223.75 & 0.19 & 1.3450 & 2 & \\
\hline ' BGO & 14DR & ? & 55789.40 & 76322.10 & 218.1 & 238.1 & 230.64 & 0.22 & 1.5248 & 4 & \\
\hline 'BGO & 15D & , & 55859.10 & 75973.50 & 218.7 & 238.7 & 230.02 & 0.20 & 1.6062 & 4 & \\
\hline 'BGO & $16 A$ & ' & 56194.20 & 75757.00 & 102.5 & 112.5 & 160.99 & 0.22 & 1.0423 & 1 & \\
\hline 'BGO & $16 \mathrm{AR}$ & & 56217.10 & 75743.20 & 103.7 & 113.7 & 161.03 & 0.08 & 0.4841 & 1 & \\
\hline ' BGO & $16 \mathrm{~B}$ & - & 56183.80 & 75767.50 & 136.0 & 146.0 & 218.39 & 0.19 & 1.2444 & 2 & \\
\hline 'BGO & 16D & ' & 56202.10 & 75751.40 & 217.3 & 237.3 & 230.98 & 0.15 & 1.1663 & 4 & \\
\hline 'BGO & D 17D & - & 56399.40 & 75599.60 & 204.0 & 224.0 & 232.40 & 1.06 & 3.6712 & 4 & \\
\hline 'BGO & 17DR & & 56407.20 & 75604.00 & 216.9 & 236.9 & 231.62 & 0.55 & 3.5943 & 4 & \\
\hline
\end{tabular}


Table F-1. Hydraulic Head Targets for Model Calibration (Continued)

\begin{tabular}{|c|c|c|c|c|c|c|c|c|c|c|}
\hline 'BGO & $18 \mathrm{~A}$ & $56699: 70$ & 75599.90 & 99.5 & 109.5 & 161.16 & 0.09 & 0.7563 & 1 & \\
\hline 'BGO & $18 D$ & 56711.20 & 75600.00 & 219.6 & 239.6 & 232.04 & $0.15^{\circ}$ & 1.1659 & 4 & \\
\hline ' BGO & 19D' & 56997.30 & 75350.00 & 196.8 & 216.8 & 234.13 & 0.23 & 1.2329 & 6 & ? \\
\hline BGO & 19DR' & 56800.70 & 75520.00 & 196.7 & 216.7 & 231.49 & 0.19 & 0.8720 & 6 & ? \\
\hline 'BGO & $20 A^{\prime}$ & 57100.40 & 74966.40 & 86.3 & 96.3 & 163.48 & 0.39 & 1.7921 & 1 & \\
\hline 'BGO & $20 A^{\prime}$ & 57089.50 & 74949.80 & 18.3 & 28.3 & 161.56 & 0.10 & 0.4622 & 6 & ? \\
\hline BGO & $20 \mathrm{~B}$ & 57119.80 & 74951.50 & 131.0 & 141.0 & 227.59 & 0.21 & 1.2032 & 2 & \\
\hline 'BGO & $20 C^{\prime}$ & 57106.00 & 74937.60 & 174.0 & 184.0 & 228.80 & 0.21 & 1.1529 & 2 & \\
\hline 'BGO & $20 D^{\prime}$ & 57113.80 & 74962.20 & 216.3 & 236.3 & 233.83 & 0.19 & 1.6069 & 4 & \\
\hline BGO & 21D, & 57470.70 & 74688.50 & 217.7 & 237.7 & 234.95 & 0.21 & 1.6966 & 4 & \\
\hline 'BGO & 22D & 57817.30 & 74482.20 & 194.2 & 214.2 & 232.63 & 0.20 & 0.9222 & 6 & ? \\
\hline BGO & 222DR' & 57831.50 & 74471.50 & 219.2 & 239.2 & 236.43 & 0.76 & 3.8025 & 4 & \\
\hline 'BGO & 22DX' & 57770.74 & 74560.48 & 217.9 & 237.9 & 234.35 & 0.24 & 0.9315 & 4 & \\
\hline 'BGO & 23D & 58133.00 & 74238.10 & 222.0 & 242.0 & 236.03 & 0.14 & 1.1063 & 4 & \\
\hline 'BGO & 24D' & 58438.80 & 74012.40 & 221.0 & 241.0 & 237.09 & 0.15 & 1.2264 & 4 & \\
\hline 'BGO & $25 A^{\prime}$ & 55668.10 & 76158.50 & 104.1 & 114.1 & 160.78 & 0.14 & 1.1159 & 1 & \\
\hline 'BGO & $26 \mathrm{~A}$ & 55014.20 & 76144.60 & 81.0 & 91.0 & 160.71 & 0.59 & 4.0347 & 1 & \\
\hline 'BGO & $26 \mathrm{D}$ & 55015.20 & 76128.00 & 213.4 & 233.5 & 227.68 & 0.29 & 2.2157 & 4 & \\
\hline 'BGO & $27 c^{\prime}$ & 54671.40 & 75666.30 & 154.9 & 163.9 & 220.88 & 0.20 & 1.4752 & 2 & \\
\hline 'BGO & 27D & 54680.20 & 75677.30 & 209.3 & 229.3 & 227.64 & 0.19 & 1.4759 & 4 & \\
\hline 'BGO & $28 \mathrm{D}$ & 54457.90 & 75348.30 & 210.1 & 230.1 & 226.34 & 0.20 & 1.5057 & 4 & \\
\hline 'BGO & $29 A^{\prime}$ & 54103.50 & 75560.00 & 102.5 & 112.5 & 159.70 & 0.13 & 0.9253 & 1 & \\
\hline 'BGO & $29 \mathrm{C}^{\prime}$ & 54099.10 & 75577.80 & 176.8 & 186.8 & 223.08 & 0.20 & 1.3544 & 2 & \\
\hline 'BGO & $29 D^{\prime}$ & 54099.40 & 75592.50 & 208.5 & 228.5 & 226.38 & 0.21 & 1.4546 & 4 & \\
\hline 'BGO & $30 C^{\prime}$ & 54512.30 & 75181.00 & 178.4 & 188.4 & 219.09 & 0.25 & 1.8555 & 2 & \\
\hline 'BGO & $30 D^{\prime}$ & 54499.20 & 75187.70 & 207.8 & 227.8 & 225.74 & 0.20 & 1.5357 & 4 & \\
\hline 'BGO & $31 \mathrm{C}$ & 54816.20 & 74978.00 & 176.4 & 186.4 & 225.57 & 0.20 & 1.5157 & 2 & \\
\hline 'BGO & $31 D^{\prime}$ & 54841.70 & 74985.30 & 211.1 & 231.1 & 226.69 & 0.21 & 1.5957 & 4 & \\
\hline 'BGO & 32D & 55250.20 & 74727.00 & 214.5 & 234.5 & 227.73 & 0.20 & 1.5559 & 4 & \\
\hline 'BGO & $33 C^{\prime}$ & 55681.40 & 74479.70 & 177.8 & 187.8 & 225.18 & 0.18 & 1.3959 & 2 & \\
\hline 'BGO & $33 \mathrm{D}$ & 55695.40 & 74468.70 & 213.1 & 233.1 & 230.25 & 0.25 & 2.1170 & 4 & \\
\hline 'BGO & $34 \mathrm{D}$ & 56082.60 & 74228.80 & 212.7 & 232.7 & 232.82 & 0.21 & 1.7069 & 4 & \\
\hline 'BGO & $35 \mathrm{C}$ & 56545.70 & 73953.90 & 161.9 & 171.9 & 228.87 & 0.19 & 1.4661 & 2 & \\
\hline 'BGO & $35 D^{\prime}$ & 56556.50 & 73946.00 & 219.4 & 239.4 & 234.68 & 0.25 & 2.1370 & 4 & \\
\hline 'BGO & $36 \mathrm{D}$ & 56888.10 & 73743.80 & 223.3 & 243.3 & 237.08 & 0.26 & 2.2171 & 4 & . \\
\hline 'BGO & $37 C^{\prime}$ & 57279.20 & 73498.20 & 168.8 & 178.8 & 230.95 & 0.29 & 2.0450 & 2 & \\
\hline 'BGO & 37D & 57292.90 & 73490.80 & 226.1 & 246.1 & 238.64 & 0.29 & 2.45 .72 & 4 & \\
\hline ' BGO & $38 D^{\prime}$ & 57557.50 & 73329.30 & 222.3 & 242.3 & 236.46 & 0.25 & 2.1574 & 4 & \\
\hline ' BGO & $39 \mathrm{~A}$ & 57821.90 & 73573.20 & 84.8 & 94.8 & 167.67 & 0.09 & 0.4422 & 1 & \\
\hline 'BGO & $39 C^{\prime}$ & 57816.10 & 73563.30 & 174.9 & 184.9 & 231.08 & 0.61 & 3.4432 & 2 & \\
\hline 'BGO & $39 D^{\prime}$ & 57831.00 & 73583.50 & 224.7 & 244.7 & 235.64 & 0.18 & 1.4567 & 4 & \\
\hline ' BGO & $40 D^{\prime}$ & 54638.60 & 76125.80 & 216.6 & 226.5 & 222.46 & 0.21 & 1.4548 & 4 & \\
\hline ' BGO & $41 \mathrm{~A}$ & 55403.70 & 76469.50 & 103.3 & 113.3 & 158.55 & 0.11 & 0.7139 & 1 & \\
\hline 'BGO & $42 \mathrm{C}^{\prime}$ & 55522.30 & 76404.70 & 185.9 & 195.9 & 223.40 & 0.21 & 1.3842 & 2 & \\
\hline 'BGO & $43 \mathrm{~A}$ & 56253.40 & 77061.40 & 105.9 & 115.9 & 158.98 & 0.33 & 2.0137 & 1 & \\
\hline 'BGO & $43 \mathrm{AA}^{\prime}$ & 56268.60 & 77066.00 & 62.2 & 72.2 & 156.69 & 0.13 & 0.8442 & 1 & \\
\hline ' BGO & $43 \mathrm{CR}^{\prime}$ & 56237.20 & 77035.20 & 178.4 & 188.4 & 225.40 & 0.23 & 1.4540 & 2 & \\
\hline 'BGO & $43 D^{\prime}$ & 56238.80 & 77056.70 & 198.2 & 208.2 & 231.49 & 0.20 & 1.3243 & 6 & ? \\
\hline 'BGO & $44 \mathrm{~A}$ & 57851.20 & 76755.20 & 98.0 & 108.0 & 158.06 & 0.46 & 2.9943 & 1 & \\
\hline ' BGO & $44 A A^{\prime}$ & 57880.50 & 76757.00 & 61.2 & 71.3 & 158.70 & 0.06 & 0.4141 & 1 & \\
\hline 'BGO & $44 B^{\prime}$ & 57865.80 & 76756.00 & 148.1 & 158.1 & 221.00 & 0.50 & 3.3445 & 2 & \\
\hline ' BGO & $44 C^{\prime}$ & 57894.90 & 76757.80 & 190.6 & 200.6 & 220.63 & 0.44 & 2.9545 & 6 & ? \\
\hline ' BGO & $44 \mathrm{D}^{\prime}$ & 57910.00 & 76759.50 & 223.4 & 233.4 & 232.54 & 0.17 & 1.1342 & 4 & \\
\hline ' BGO & $45 A^{\prime}$ & 54550.10 & 75830.00 & 116.9 & 126.9 & 160.81 & 0.07 & 0.4845 & 6 & ? \\
\hline 'BGO & $45 B^{\prime}$ & 54563.60 & 75840.30 & 137.0 & 147.0 & 219.06 & 0.35 & 2.3145 & 2 & \\
\hline 'BGO & $45 \mathrm{C}$ & 54577.40 & 75835.00 & 190.5 & 200.5 & 222.90 & 0.32 & 2.1244 & 2 & \\
\hline ' BGO & $45 D^{\prime}$ & 54585.60 & 75854.30 & 209.6 & 229.6 & 227.50 & 0.43 & 2.8844 & 4 & . \\
\hline ' BGO & $46 B^{\prime}$ & 54444.70 & 75012.10 & 140.4 & 150.4 & 218.15 & 0.17 & 1.1342 & 2 & \\
\hline ' BGO & $46 C^{\prime}$ & 54433.90 & 75022.20 & 178.0 & 188.0 & 219.60 & 0.24 & 1.6145 & 2 & \\
\hline ' BGO & $46 \mathrm{D}$ & 54420.00 & 75033.80 & 202.1 & 212.1 & 225.26 & 0.27 & 1.7844 & 3 & \\
\hline 'BGO & $47 A^{\prime}$ & 54914.00 & 74728.80 & 86.8 & 96.8 & 162.44 & 0.07 & 0.4643 & 1 & \\
\hline ' BGO & $47 C^{\prime}$ & 54933.40 & 74752.00 & 178.6 & 188.6 & 222.92 & 0.19 & 1.2644 & 2 & \\
\hline 'BGO & 47D & 54922.90 & 74739.70 & 203.4 & 213.4 & 226.24 & 0.30 & 1.9543 & 3 & \\
\hline ' BGO & $48 C^{\prime}$ & 55124.40 & 74599.60 & 176.7 & 186.7 & 223.56 & 0.20 & 1.2944 & 2 & \\
\hline ' BGO & $48 \mathrm{D}$ & 55121.00 & 74586.40 & 202.0 & 212.0 & 226.75 & 0.22 & 1.4344 & 3 & \\
\hline ' BGO & $49 A^{\circ}$ & 56205.10 & 73902.80 & 75.1 & 85.1 & 166.76 & 1.02 & 4.0916 & 1 & \\
\hline ' BGO & $49 C^{\prime}$ & 56202.20 & 73917.20 & 166.0 & 176.0 & 228.15 & 0.21 & 1.5353 & 2 & \\
\hline
\end{tabular}


Table F-1. Hydraulic Head Targets for Model Calibration (Continued)

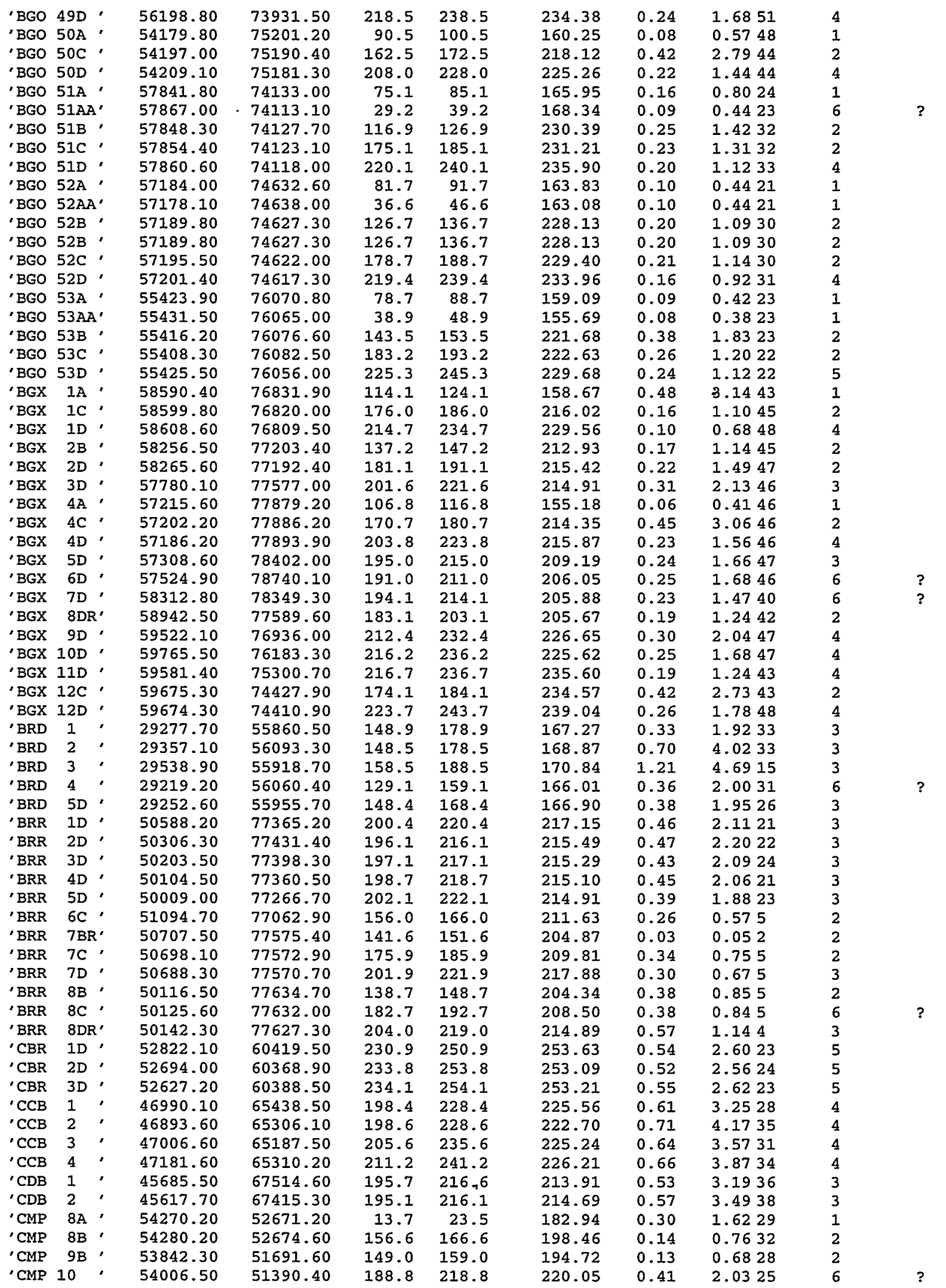


Table F-1. Hydraulic Head Targets for Model Calibration (Continued)

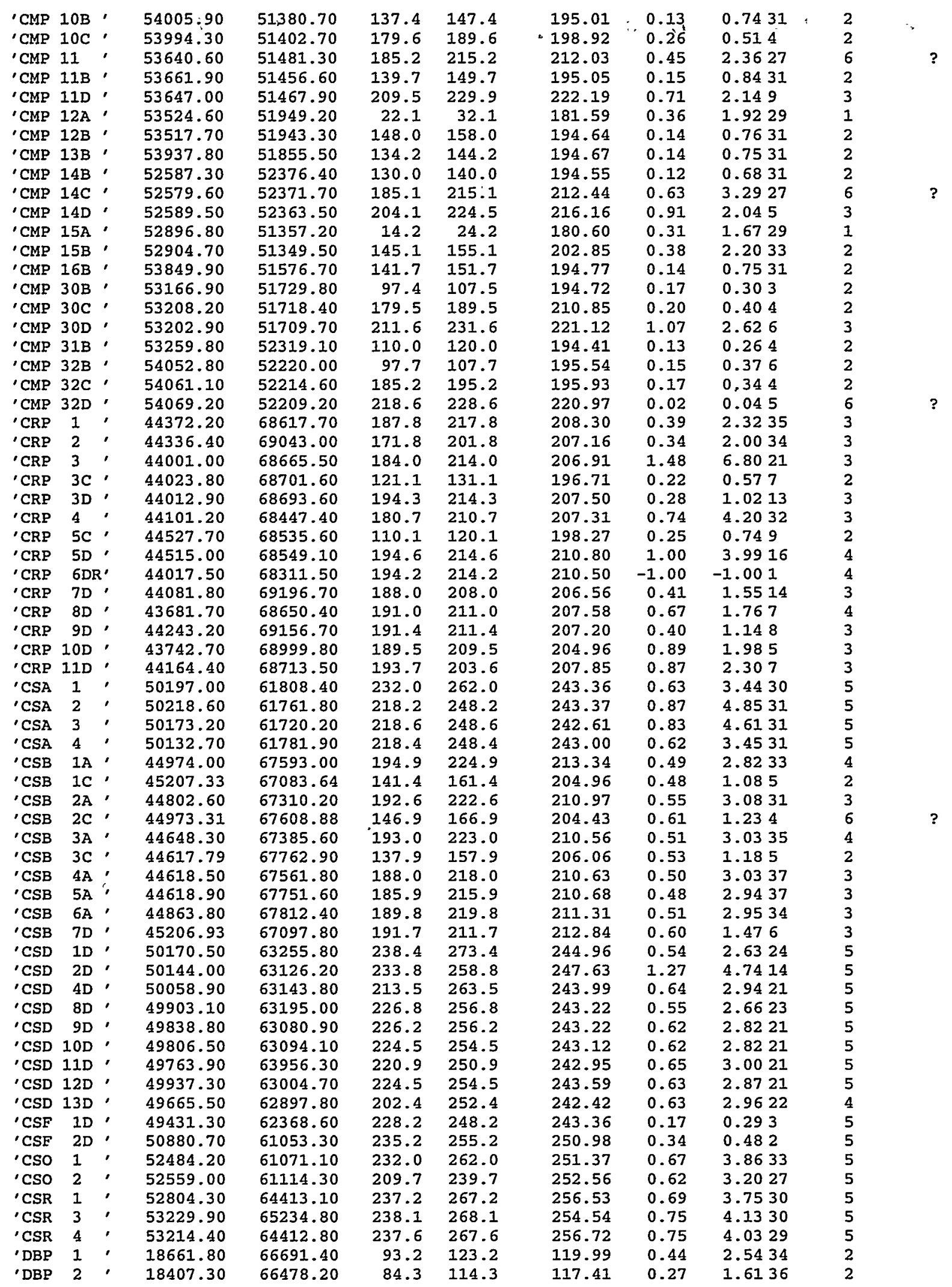


Table F-1. Hydraulic Head Targets for Model Calibration (Continued)

\begin{tabular}{|c|c|c|c|c|c|c|c|c|c|}
\hline 'DBP & 3 & ' & 18427.50 & 66775.50 & 86.4 & 116.4 & 121.06 & 0.36 & 2.2137 \\
\hline DBP & 4 & ' & 18342.10 & 66679.60 & 84.2 & 114.2 & 118.97 & 0.33 & 1.9133 \\
\hline 'DBP & 5 & - & 18605.20 & 66485.60 & 96.1 & 116.1 & 117.52 & 0.49 & 1.8915 \\
\hline 'DCB & $1 \mathrm{~A}$ & - & 19856.30 & 64028.50 & 90.1 & 120.1 & 115.37 & 0.17 & 0.8728 \\
\hline 'DCB & $2 \mathrm{~A}$ & ' & 20895.20 & 63436.10 & 97.4 & 127.4 & 124.85 & 0.24 & 1.3230 \\
\hline 'DCB & $3 \mathrm{~A}$ & - & 20899.90 & 62674.90 & 96.2 & 126.2 & 120.65 & 0.21 & 1.1329 \\
\hline 'DCB & $4 \mathrm{~A}$ & . & 20493.80 & 62678.80 & 92.5 & 122.5 & 119.15 & 0.15 & 0.8833 \\
\hline 'DCB & $5 A$ & - & 20139.80 & 63126.10 & 85.9 & 115.9 & 118.85 & 0.15 & 0.8232 \\
\hline 'DCB & 6 & - & 19979.30 & 64167.90 & 109.5 & 129.5 & 116.77 & 0.16 & 0.8932 \\
\hline 'DCB & 7 & - & 20036.30 & 64001.40 & 108.9 & 128.9 & 118.01 & 0.15 & 0.8632 \\
\hline 'DCB & 8 & ، & 21014.10 & 63473.90 & 110.3 & 130.3 & 126.45 & 0.26 & 1.5033 \\
\hline 'DCB & 9 & , & 19807.40 & 64190.60 & 97.3 & 117.3 & 114.90 & 0.17 & 0.9430 \\
\hline 'DCB & 10 & . & 19852.30 & 63803.10 & 99.8 & 119.8 & 116.64 & 0.36 & 2.0432 \\
\hline 'DCB & 11 & ' & 19248.60 & 64638.30 & 106.8 & 126.8 & 122.09 & 0.29 & 1.6031 \\
\hline 'DCB & 12 & , & 18529.80 & 65150.00 & 92.0 & 112.0 & 109.75 & 0.13 & 0.7432 \\
\hline 'DCB & 13 & ' & 19235.40 & 63842.50 & 102.0 & 122.1 & 117.17 & 0.76 & 4.3533 \\
\hline 'DCB & 14 & . & 19392.40 & 64909.80 & 94.6 & 114.6 & 111.57 & 1.41 & 4.229 \\
\hline 'DCB & 15 & , & 17635.90 & 64607.40 & 99.8 & 119.9 & 111.61 & 0.46 & 2.2524 \\
\hline 'DCB & 16 & ' & 17611.20 & 63956.00 & 100.1 & 120.1 & 111.99 & 0.24 & 1.3129 \\
\hline 'DCB & $17 \mathrm{~A}$ & ' & 19841.80 & 64583.20 & 109.4 & 119.4 & 116.52 & 0.15 & 0.304 \\
\hline 'DCB & $17 \mathrm{~B}$ & - & 19844.70 & 64588.80 & 99.2 & 101.7 & 116.85 & 0.14 & 0.274 \\
\hline 'DCB & $17 \mathrm{C}$ & ' & 19846.50 & 64593.70 & 87.4 & 89.9 & 116.03 & 0.13 & 0.254 \\
\hline 'DCB & $18 \mathrm{~A}$ & ' & 19881.30 & 64051.80 & 110.1 & 120.1 & 116.16 & 0.34 & 0.765 \\
\hline DCB & $18 \mathrm{~B}$ & ' & 19874.50 & 64046.10 & 100.5 & 103.0 & 113.23 & 0.32 & 0.654 \\
\hline DCB & $18 \mathrm{C}$ & ' & 19869.40 & 64041.40 & 87.7 & 90.2 & 112.63 & 0.29 & 0.574 \\
\hline 'DCB & $19 \mathrm{~A}$ & . & 19890.30 & 64022.10 & 111.9 & 121.9 & 120.05 & 0.64 & 1.284 \\
\hline 'DCB & $19 B$ & , & 19885.30 & 64016.50 & 101.9 & 104.4 & 117.27 & 0.31 & 0.624 \\
\hline 'DCB & $19 \mathrm{C}$ & - & 19879.70 & 64010.90 & 89.1 & 91.6 & 116.33 & 0.33 & 0.664 \\
\hline DCB & $20 \mathrm{~A}$ & ' & 20106.50 & 63931.00 & 110.9 & 120.9 & 117.07 & 0.15 & 0.304 \\
\hline 'DCB & $20 B$ & ' & 20102.60 & 63935.30 & 100.3 & 102.8 & 116.36 & 0.16 & 0.324 \\
\hline 'DCB & $20 \mathrm{C}$ & ' & 20098.10 & 63940.40 & 89.4 & 91.9 & 116.29 & 0.17 & 0.354 \\
\hline 'DCB & $20 D$ & ' & 20087.90 & 63953.30 & 46.2 & 48.7 & 114.29 & 0.30 & 0.594 \\
\hline 'DCB & $21 \mathrm{~A}$ & - & 19854.70 & 63914.80 & 110.1 & 120.1 & 116.75 & 0.34 & 0.684 \\
\hline 'DCB & $21 B$ & - & 19851.50 & 63920.00 & 102.2 & 104.7 & 113.26 & 0.23 & 0.464 \\
\hline 'DCB & $21 C$ & ' & 19849.20 & 63925.30 & 88.3 & 90.8 & 112.85 & 0.22 & 0.444 \\
\hline DCB & $22 \mathrm{~A}$ & ' & 19794.20 & 63907.60 & 109.8 & 119.8 & 112.73 & 0.12 & 0.265 \\
\hline DCB & $22 \mathrm{~B}$ & . & 19790.70 & 63913.10 & 100.9 & 103.4 & 112.66 & 0.19 & 0.374 \\
\hline 'DCB & $22 \mathrm{C}$ & - & 19788.70 & 63919.10 & 88.1 & 90.6 & 112.74 & 0.39 & 0.784 \\
\hline 'DCB & $23 \mathrm{~A}$ & ' & 19608.30 & 63870.40 & 105.7 & 115.7 & 111.77 & 0.12 & 0.286 \\
\hline 'DCB & $23 B$ & ' & 19607.00 & 63876.30 & 94.1 & 96.6 & 108.72 & 0.08 & 0.164 \\
\hline DCB & $23 c$ & - & 19605.70 & 63882.50 & 86.6 & 89.1 & 108.95 & 0.15 & 0.294 \\
\hline 'DCB & 23D & - & 19602.20 & 63900.00 & 49.1 & 51.6 & 111.44 & 0.27 & 0.544 \\
\hline 'DCB & $24 \mathrm{~A}$ & ' & 19983.10 & 63321.60 & 109.2 & 119.2 & 115.39 & 0.13 & 0.254 \\
\hline 'DCB & $24 B$ & ' & 19972.40 & 63318.20 & 100.6 & 103.1 & 115.15 & 0.06 & 0.103 \\
\hline 'DCB & $24 \mathrm{C}$ & - & 19966.40 & 63315.70 & 87.6 & 90.1 & 116.21 & 0.12 & 0.234 \\
\hline 'DOB & 1 & , & 23567.80 & 68438.10 & 114.7 & 144.7 & 143.23 & 0.40 & 2.7347 \\
\hline DOB & 2 & , & 23340.80 & 68568.00 & 115.3 & 145.3 & 143.31 & 0.43 & 2.9647 \\
\hline 'DOB & 3 & ' & 23633.30 & 68693.50 & 115.9 & 145.9 & 143.13 & 0.53 & 3.1435 \\
\hline 'DOB & 4 & ' & 23815.60 & 68514.40 & 109.2 & 139.2 & 142.39 & 0.51 & 3.0235 \\
\hline 'DOB & 7 & ' & 23485.70 & 68315.80 & 125.7 & 145.7 & 143.18 & 0.46 & 1.8316 \\
\hline 'DOB & 8 & , & 23710.40 & 68429.20 & 128.3 & 148.3 & 143.78 & 0.48 & 1.9116 \\
\hline DOB & 9 & ' & 23690.70 & 68811.60 & 128.5 & 148.5 & 144.10 & 0.42 & 0.733 \\
\hline 'DOB & 10 & ' & 23291.40 & 68490.90 & 128.3 & 148.3 & 143.30 & 0.42 & 1.6716 \\
\hline 'DOB & 11 & ' & 23400.80 & 68445.20 & 126.7 & 131.7 & 140.92 & 0.32 & 0.563 \\
\hline DOB & 12 & , & 23398.78 & 68450.08 & 133.1 & 138.1 & 140.56 & 0.36 & 1.5418 \\
\hline 'DOB & 14 & , & 23527.50 & 68335.00 & 132.6 & 137.6 & 139.68 & 0.33 & 0.583 \\
\hline 'DOB & 15 & ' & 23189.90 & 68139.60 & 110.9 & 116.0 & 141.92 & 0.20 & 0.343 \\
\hline DOB & 16 & , & 23190.80 & 68133.90 & 103.5 & 108.6 & 141.39 & 0.19 & 0.323 \\
\hline 'DOL & 1 & ' & 23586.10 & 68794.40 & 109.2 & 119.2 & 144.27 & 0.40 & 0.693 \\
\hline ' $F$ & 2 & ' & 51484.30 & 75677.40 & 207.0 & 217.0 & 218.37 & 1.40 & 2.423 \\
\hline $\mathrm{F}$ & $18 \mathrm{~A}$ & , & 50108.00 & 74170.20 & 194.4 & 204.4 & 203.79 & 1.24 & 3.719 \\
\hline 'FAB & 1 & ' & 54915.50 & 77798.80 & 215.4 & 235.4 & 228.36 & 0.22 & 0.7813 \\
\hline 'FAB & 2 & ' & 55137.50 & 77470.10 & 216.5 & 236.5 & 229.12 & 0.21 & 0.7714 \\
\hline ' $\mathrm{FAB}$ & 3 & ' & 55030.80 & 77151.20 & 211.8 & 231.8 & 228.85 & 0.21 & 0.7212 \\
\hline ' $F A B$ & 4 & ' & 54759.70 & 77584.60 & 214.2 & 234.2 & 228.54 & 0.20 & 0.6811 \\
\hline 'FAC & 3 & ' & 55322.70 & 78018.30 & 224.8 & 254.8 & 229.11 & 0.28 & 1.5831 \\
\hline 'FAC & 4 & ' & 55472.90 & 78223.80 & 207.8 & 237.8 & 228.52 & 0.26 & 1.4531 \\
\hline 'FAC & 5 & ' & 55241.30 & 77960.30 & 214.0 & 234.0 & 224.91 & 0.60 & 3.1828 \\
\hline
\end{tabular}


Table F-1. Hydraulic Head Targets for Model Calibration (Continued)

\begin{tabular}{|c|c|c|c|c|c|c|c|c|c|c|c|}
\hline 'FAC & $5 \mathrm{P}$ & ' & $55314 \div 80$ & 78175.70 & 225.7 & 235.7 & 229.73 & 0.38 & 0.86 .5 & 5 & \\
\hline 'FAC & 6 & ' & 55335.50 & 78129.00 & 216.2 & 236.2 & 220.78 & 0.80 & 4.1026 & 5 & \\
\hline 'FAC & 7 & ' & 55356.20 & 78123.40 & 215.7 & 235.7 & 223.18 & 1.03 & 5.5729 & 5 & \\
\hline 'FAC & 8 & ' & 55366.00 & 78090.90 & 216.0 & 236.0 & 227.17 & 0.73 & 3.9129 & 5 & \\
\hline ' FAC & $9 \mathrm{C}$ & ' & 55339.30 & 78030.50 & 197.4 & 207.4 & 217.42 & 0.28 & 0.574 & 3 & \\
\hline 'FAC & $10 \mathrm{C}$ & ' & 55298.40 & 78119.70 & 200.2 & 210.2 & 217.42 & 0.28 & 0.635 & 3 & \\
\hline 'FAC & $11 C$ & ' & 55231.90 & 78100.30 & 201.4 & 211.4 & 217.49 & 0.28 & 0.635 & 3 & \\
\hline 'FAC & $12 \mathrm{C}$ & ' & 55226.40 & 78047.20 & 198.0 & 208.0 & 217.55 & 0.28 & 0.635 & 3 & \\
\hline 'FAL & 1 & ' & 53756.40 & 78115.90 & 207.0 & 238.5 & 218.73 & 0.29 & 1.7435 & 5 & \\
\hline 'FAL & 2 & ' & 53757.40 & 78231.90 & 206.6 & 238.0 & 217.15 & 0.29 & 1.7134 & 6 & ? \\
\hline 'FBP & $1 \mathrm{~A}$ & ' & 51080.70 & 78893.00 & 161.8 & 191.8 & 206.72 & 0.37 & 2.1333 & 2 & \\
\hline 'FBP & $2 A$ & ' & 50534.10 & 79711.40 & 137.1 & 167.1 & 191.51 & 0.47 & 2.7033 & 2 & \\
\hline 'FBP & $3 A$ & ' & 50913.40 & 79838.90 & 141.0 & 171.0 & 194.31 & 0.46 & 2.7837 & 2 & \\
\hline 'FBP & 4 & ' & 51368.20 & 79320.00 & 165.2 & 195.2 & 212.39 & 0.33 & 1.9233 & 2 & \\
\hline 'FBP & 5D & ' & 51073.90 & 79193.80 & 192.6 & 212.6 & 205.23 & 0.41 & 1.5314 & 6 & ? \\
\hline ' FBP & 6D & ' & 50547.10 & 79672.90 & 178.3 & 198.3 & 194.77 & 0.55 & 2.2016 & 2 & \\
\hline 'FBP & 7D & ' & 50878.90 & 79805.70 & 183.2 & 203.2 & 194.09 & 0.28 & 0.686 & 2 & \\
\hline ' $\mathrm{FBP}$ & 8D & ' & 51386.40 & 79291.80 & 172.8 & 192.8 & 207.08 & 0.49 & 1.8214 & 2 & \\
\hline ' $\mathrm{FBP}$ & $9 D$ & ' & 51074.00 & 79565.10 & 177.9 & 197.9 & 200.29 & 0.71 & 2.5413 & 2 & \\
\hline 'FBP & 11D & ' & 50767.90 & 79099.30 & 192.0 & 212.1 & 203.03 & 0.15 & 0.417 & 6 & ? \\
\hline 'FBP & $12 \mathrm{D}$ & ' & 51165.70 & 78932.30 & 182.1 & 202.1 & 208.42 & 0.45 & 1.5812 & 2 & \\
\hline 'FBP & $13 \mathrm{D}$ & ' & 50694.10 & 79748.90 & 172.7 & 192.7 & 194.94 & 0.91 & 3.5115 & 2 & \\
\hline 'FC & $1 \mathrm{~A}$ & ' & 53115.10 & 79664.50 & 96.7 & 101.7 & 143.51 & -1.00 & -1.001 & 1 & \\
\hline 'FC & 1B & ' & 53115.00 & 79672.40 & $151: 8$ & 156.8 & 210.78 & -1.00 & -1.001 & 2 & \\
\hline 'FC & $1 \mathrm{C}$ & ' & 53115.10 & 79680.10 & 183.9 & 188.9 & 214.00 & -1.00 & -1.001 & 2 & \\
\hline 'FC & ID & ' & 53114.50 & 79688.30 & 217.2 & 222.2 & 223.63 & -1.00 & -1.001 & 5 & \\
\hline 'FC & $3 A$ & ' & 57620.00 & 78726.60 & 21.5 & 26.5 & 175.47 & -1.00 & -1.001 & 6 & ? \\
\hline 'FC & $3 \mathrm{~B}$ & ' & 57629.90 & 78727.70 & 61.2 & 66.2 & 150.63 & -1.00 & -1.001 & 1 & \\
\hline 'FC & $3 C$ & ' & 57639.00 & 78728.00 & 121.0 & 126.0 & 151.80 & -1.00 & -1.001 & 1 & \\
\hline 'FC & 3D & ' & 57647.90 & 78728.40 & 165.9 & 170.9 & 206.43 & -1.00 & -1.001 & 2 & \\
\hline 'FC & $3 E$ & ' & 57655.50 & 78728.80 & 185.7 & 190.7 & 205.33 & -1.00 & -1.001 & 2 & \\
\hline 'FC & $3 F$ & ' & 57663.20 & 78729.10 & 205.1 & 210.1 & 206.25 & -1.00 & -1.001 & 3 & \\
\hline 'FC & $4 \mathrm{~A}$ & ' & 53896.50 & 82242.50 & -28.0 & -23.0 & 173.31 & -1.00 & -1.001 & 6 & ? \\
\hline 'FC & $4 \mathrm{~B}$ & ' & 53901.30 & 82249.00 & 76.1 & 81.1 & 140.96 & -1.00 & -1.001 & 1 & \\
\hline 'FC & $4 \mathrm{C}$ & ' & 53905.90 & 82255.40 & 116.3 & 121.3 & 137.64 & -1.00 & -1.001 & 1 & \\
\hline 'FC & 4D & ' & 53910.70 & 82262.20 & 146.4 & 151.4 & 151.03 & -1.00 & -1.001 & 6 & ? \\
\hline 'FC & $4 \mathrm{E}$ & ' & 53915.30 & 82268.90 & 176.4 & 181.4 & 185.19 & -1.00 & -1.001 & 2 & \\
\hline ' $\mathrm{ECA}$ & $2 \mathrm{C}$ & ' & 53712.20 & 78296.00 & 295.3 & 299.3 & 297.45 & 0.56 & 2.6322 & 5 & \\
\hline ' $\mathrm{FCA}$ & 2D & ' & 53715.20 & 78295.80 & 219.0 & 239.0 & 225.03 & 0.35 & 2.2842 & 5 & \\
\hline ' $\mathrm{FCA}$ & 9D & ' & 53733.10 & 78600.50 & 221.9 & 241.9 & 225.20 & 0.23 & 1.3132 & 5 & \\
\hline 'FCA & 9DR & & 53734.50 & 78608.80 & 207.7 & 227.7 & 224.00 & 0.41 & 1.5214 & 4 & \\
\hline ' $F C A$ & $10 \mathrm{~A}$ & ' & 53571.90 & 78640.40 & 221.0 & 241.0 & 225.27 & 0.22 & 1.4042 & 5 & \\
\hline 'FCA & 10D & ' & 53732.00 & 78640.00 & 219.5 & 239.5 & 225.76 & 0.38 & 2.0328 & 5 & \\
\hline ' FCA & $16 \mathrm{~A}$ & ' & 53568.80 & 78899.50 & 215.1 & 235.1 & 225.18 & 0.20 & 1.2842 & 5 & \\
\hline 'FCA & $16 \mathrm{~B}$ & ' & 53571.00 & 78898.00 & 295.3 & 299.3 & 298.00 & 0.41 & 1.7318 & 5 & \\
\hline 'FCA & $16 \mathrm{D}$ & ' & 53719.50 & 78898.50 & 221.1 & 241.1 & 224.96 & 0.26 & 1.3728 & 5 & \\
\hline ' $\mathrm{FCA}$ & 19D & ' & 53719.10 & 78271.90 & 209.7 & 229.7 & 217.22 & 0.34 & 1.8529 & 4 & \\
\hline ' FCB & 1 & ' & 54871.80 & 76835.40 & 205.6 & 235.6 & 230.23 & 0.24 & 0.342 & 4 & \\
\hline ' $\mathrm{FCB}$ & 2 & ' & 55046.70 & 76679.70 & 205.2 & 235.2 & 228.60 & 0.79 & 4.4432 & 4 & \\
\hline 'FCB & 3 & ' & 54874.40 & 76427.80 & 195.3 & 225.3 & 223.94 & 0.31 & 1.7230 & 6 & ? \\
\hline ' $\mathrm{FCB}$ & 4 & ' & 54605.90 & 76780.40 & 204.5 & 234.5 & 228.18 & 0.60 & 3.4232 & 4 & \\
\hline 'FCB & 5 & ' & 54773.00 & 76492.60 & 217.1 & 237.1 & 228.84 & 0.32 & 1.7330 & 4 & \\
\hline 'FCB & 6 & ' & 54733.40 & 76582.10 & 215.1 & 235.1 & 229.08 & 0.27 & 1.4026 & 4 & \\
\hline 'FET & 1D & ' & 53299.90 & 76165.60 & 206.9 & 226.9 & 223.75 & 0.27 & 1.5030 & 4 & \\
\hline 'FET & $2 \mathrm{D}$ & ' & 52981.20 & 76045.80 & 209.5 & 229.5 & 222.40 & 0.29 & 1.5730 & 4 & \\
\hline 'FET & 3D & ' & 53025.70 & 75961.00 & 203.0 & 223.0 & 222.60 & 0.29 & 1.5730 & 3 & \\
\hline 'FET & $4 \mathrm{D}$ & ' & 53149.00 & 75959.30 & 205.1 & 225.1 & 222.95 & 0.28 & 1.5932 & 3 & \\
\hline 'FIW & 1D & ' & 51420.00 & 76114.90 & 198.9 & 218.9 & 214.73 & -1.00 & -1.001 & 3 & \\
\hline 'FIW & IID & & 51362.50 & 76171.60 & 194.0 & 214.0 & 219.04 & 0.86 & 2.106 & 3 & \\
\hline 'FIW & $2 I C$ & & 51202.60 & 75924.50 & 125.3 & 175.2 & 212.61 & 0.78 & 2.208 & 2 & \\
\hline 'FIW & 2MA & & 51184.50 & $75930.80^{\circ}$ & 100.5 & 110.5 & 151.83 & 0.23 & 0.648 & 1 & \\
\hline 'FIW & $2 \mathrm{MC}$ & & 51263.50 & 75757.90 & 127.9 & 167.9 & 210.05 & 1.30 & 3.899 & 2 & \\
\hline 'FIW & $2 M D$ & & 51202.40 & 75934.90 & 190.9 & 220.8 & 217.09 & 0.81 & 2.288 & 3 & \\
\hline 'FNB & 1 & ' & 54271.60 & 80151.50 & 177.2 & 207.2 & 210.94 & 0.40 & 2.2230 & 6 & ? \\
\hline ' FNB & $1 \mathrm{~A}$ & ' & 54288.80 & 80154.50 & 107.9 & 117.9 & 144.29 & 0.10 & 0.257 & 1 & \\
\hline ' FNB & 2 & ' & 54362.10 & 80442.30 & 180.8 & 210.8 & 207.10 & 0.42 & 2.3331 & 6 & ? \\
\hline 'FNB & $2 A$ & ' & 54355.80 & 80454.70 & 111.1 & 121.1 & 143.59 & 0.15 & 0.407 & 1 & \\
\hline
\end{tabular}


Table F-1. Hydraulic Head Targets for Model Calibration (Continued)

\begin{tabular}{|c|c|c|c|c|c|c|c|c|c|c|c|}
\hline 'FNB & 3 & ' & 54105.80 & 80553.10 & 182.1 & 212.1 & 209.27 & 0.43 & 2.3731 & 6 & ? \\
\hline ‘ FNB & $3 \mathrm{~A}$ & ' & 54116.60 & 80557.20 & 109.2 & 119.2 & 143.14 & 0.22 & 0.597 & 1 & \\
\hline 'FNB & 4 & , & 53843.50 & 80409.80 & 179.6 & 209.6 & 213.61 & 0.48 & 2.7031 & 6 & ? \\
\hline ' FNB & 5 & - & 54295.20 & 80556.10 & 193.5 & 203.5 & 206.66 & 0.40 & 1.128 & 6 & ? \\
\hline 'FNB & 6 & ' & 54096.28 & 80822.49 & 200.6 & 210.6 & 208.73 & 0.21 & 0.434 & 3 & \\
\hline ' FNB & 7 & ' & 54398.46 & 80649.18 & 192.4 & 202.4 & 203.95 & 0.22 & 0.444 & 6 & ? \\
\hline 'FNB & 8 & ' & 54550.33 & 80521.45 & 195.4 & 205.4 & 202.85 & 0.15 & 0.335 & 3 & \\
\hline 'FOB & $1 \mathrm{D}$ & ' & 50026.60 & 73812.76 & 175.4 & 195.4 & 204.05 & 0.81 & 1.976 & 3 & \\
\hline 'FOB & $4 D$ & . & 49338.12 & 74430.27 & 174.0 & 194.1 & 206.94 & 0.93 & 2.286 & 3 & \\
\hline 'FOB & $5 C$ & - & 49730.31 & 74607.04 & 129.3 & 149.3 & 202.57 & 0.97 & 2.748 & 2 & \\
\hline 'FOB & $7 \mathrm{C}$ & ' & 50235.60 & 76074.12 & 148.9 & 168.9 & 209.84 & 0.73 & 2.088 & 2 & \\
\hline FOB & $7 D$ & - & 50244.28 & 76085.38 & 193.9 & 213.9 & 212.71 & 1.10 & 2.465 & 3 & \\
\hline ' $F O B$ & $8 \mathrm{D}$ & ' & 49940.18 & 75772.14 & 191.4 & 211.4 & 212.33 & 1.17 & 2.866 & 3 & \\
\hline 'FOB & $9 \mathrm{C}$ & ' & 50797.38 & 75773.51 & 155.5 & 175.5 & 211.99 & 0.73 & 2.068 & 2 & \\
\hline ' $F O B$ & 9D & - & 50782.52 & 75774.99 & 192.6 & 212.6 & 214.82 & 1.16 & 2.846 & 3 & \\
\hline 'FOB & $11 \mathrm{C}$ & - & 51920.55 & 75613.91 & 156.2 & 176.2 & 214.27 & 0.80 & 2.268 & 2 & \\
\hline ' $F O B$ & $11 D$ & ' & 51909.29 & 75602.78 & 199.0 & 219.0 & 217.69 & 0.91 & 2.407 & 3 & \\
\hline 'FOB & $12 \mathrm{D}$ & ' & 49785.91 & 75596.56 & 179.3 & 199.3 & 211.49 & 1.13 & 2.766 & 3 & \\
\hline 'FRB & 1 & ' & 53914.94 & 76229.52 & 212.2 & 232.2 & 227.65 & 0.90 & 2.025 & 4 & \\
\hline 'FRB & 3 & , & 53588.10 & 76117.53 & 216.2 & 231.2 & 224.91 & 0.87 & 1.945 & 4 & \\
\hline ' FRB & 4 & ' & 53653.31 & 76076.19 & 214.6 & 229.6 & 224.30 & 0.67 & 1.787 & 4 & \\
\hline 'FSB & OPD & & 49849.80 & 74549.20 & 171.6 & 215.3 & 207.62 & 0.38 & 0.926 & 3 & \\
\hline 'FSB & $50 \mathrm{PD}$ & & 49874.60 & 74600.90 & 174.7 & 219.8 & 207.23 & 1.04 & 2.948 & 3 & \\
\hline 'FSB & 76 & ' & 51388.80 & 76141.60 & 197.0 & 227.0 & 218.17 & 0.26 & 2.1869 & 3 & \\
\hline 'FSB & $76 \mathrm{~A}$ & ' & 51391.60 & 76131.90 & 36.9 & 47.4 & 155.27 & 0.11 & 0.9272 & 1 & \\
\hline 'FSB & $76 \mathrm{~B}$ & - & 51394.00 & 76122.40 & 99.2 & 109.7 & 151.77 & 0.08 & 0.6672 & 1 & \\
\hline 'FSB & $76 \mathrm{C}$ & - & 51396.40 & 76112.40 & 154.8 & 165.3 & 213.00 & 0.17 & 1.5182 & 2 & \\
\hline 'FSB & 77 & ' & 50713.10 & 75129.40 & 186.4 & 216.4 & 212.49 & 0.20 & 1.7478 & 3 & \\
\hline FSB & 78 & ' & 50164.70 & 74764.00 & 187.7 & 217.7 & 208.84 & 0.24 & 2.1783 & 3 & \\
\hline 'FSB & $78 \mathrm{~A}$ & ' & 50172.80 & 74757.70 & 27.0 & 37.5 & 156.26 & 0.09 & 0.7874 & 1 & \\
\hline 'FSB & $78 \mathrm{~B}$ & ' & 50178.80 & 74765.90 & 82.4 & 92.8 & 154.65 & 0.09 & 0.7671 & 1 & \\
\hline FSB & $78 \mathrm{C}$ & ' & 50170.20 & 74772.50 & 141.6 & 151.4 & 208.00 & 0.16 & 1.4181 & 2 & \\
\hline 'FSB & 79 & ' & 50139.70 & 73663.10 & 174.1 & 204.1 & 201.63 & 0.32 & 2.8481 & 3 & \\
\hline 'FSB & $79 A$ & ' & 50149.60 & 73664.50 & 24.0 & 34.4 & 158.19 & 0.17 & 1.4373 & 1 & \\
\hline 'FSB & $79 B$ & ' & 50159.20 & 73666.10 & 80.7 & 91.2 & 158.28 & 0.10 & 0.8872 & 1 & \\
\hline 'FSB & $79 \mathrm{C}$ & ' & 50171.30 & 73668.00 & 149.8 & 159.6 & 196.88 & 0.12 & 1.0780 & 2 & \\
\hline 'FSB & $87 \mathrm{~A}$ & ' & 50115.80 & 75601.70 & 33.1 & 43.6 & 153.99 & 0.09 & 0.7472 & 1 & \\
\hline 'FSB & $87 \mathrm{~B}$ & ' & 50104.90 & 75597.00 & 90.0 & 100.5 & 150.76 & 0.08 & 0.6573 & 1 & \\
\hline 'FSB & $87 \mathrm{C}$ & ' & 50093.40 & 75591.90 & 148.8 & 159.3 & 208.78 & 0.22 & 1.9375 & 2 & \\
\hline 'FSB & 87D & ' & 50081.10 & 75586.30 & 187.4 & 216.8 & 213.95 & 0.48 & 2.5428 & 3 & \\
\hline 'FSB & $88 \mathrm{C}$ & . & 51518.00 & 75619.40 & 158.4 & 168.4 & 212.72 & 0.21 & 1.7771 & 2 & \\
\hline 'FSB & $88 \mathrm{D}$ & ' & 51527.00 & 75621.80 & 202.1 & 222.1 & 216.26 & 0.20 & 1.7977 & 3 & \\
\hline 'FSB & $89 \mathrm{C}$ & ' & 51345.20 & 75553.20 & 156.1 & 166.1 & 212.07 & 0.19 & 1.6070 & 2 & \\
\hline 'FSB & 89D & ' & 51335.80 & 75548.30 & 201.9 & 221.9 & 215.66 & 0.21 & 1.7572 & 3 & \\
\hline 'FSB & $90 \mathrm{C}$ & ' & 51148.60 & 75382.90 & 158.1 & 168.1 & 211.09 & 0.20 & 1.7073 & 2 & \\
\hline 'FSB & $90 \mathrm{D}$ & ' & 51140.70 & 75376.90 & 205.1 & 225.1 & 215.39 & 0.53 & 3.6548 & 3 & \\
\hline 'FSB & $91 \mathrm{C}$ & ' & 50953.50 & 75213.30 & 149.1 & 159.1 & 210.87 & 0.16 & 1.4378 & 2 & \\
\hline 'FSB & 91D & ' & 50946.60 & 75207.60 & 200.9 & 220.9 & 213.86 & 0.21 & 1.8073 & 3 & \\
\hline 'FSB & $92 \mathrm{C}$ & ' & 50564.00 & 75053.20 & 147.6 & 157.6 & 209.42 & 0.47 & 3.2448 & 2 & \\
\hline 'FSB & $92 \mathrm{D}$ & ' & 50557.60 & 75045.80 & 201.7 & 221.7 & 212.07 & 0.19 & 1.6171 & 3 & \\
\hline 'FSB & $93 \mathrm{C}$ & ' & 50458.30 & 74897.30 & 142.0 & 152.0 & 208.90 & 0.16 & 1.3978 & 2 & \\
\hline 'FSB & 93D & ' & 50452.40 & 74888.50 & 197.9 & 217.9 & 210.85 & 0.20 & 1.7377 & 3 & \\
\hline 'FSB & $94 \mathrm{C}$ & ' & 50180.00 & 74869.00 & 139.8 & 149.8 & 207.96 & 0.25 & 2.2478 & 2 & \\
\hline 'FSB & 94DR & & 50162.90 & 74869.10 & 183.3 & 203.4 & 210.21 & 0.19 & 1.4858 & 3 & \\
\hline 'FSB & $95 \mathrm{C}$ & ' & 50016.70 & 74971.70 & 145.8 & 155.8 & 205.64 & 0.30 & 1.0512 & 2 & \\
\hline 'FSB & $95 \mathrm{CR}$ & $\mathrm{R}^{\prime}$ & 49987.80 & 75001.90 & 151.9 & 161.9 & 208.00 & 0.16 & 1.2963 & 2 & \\
\hline 'FSB & $95 \mathrm{D}$ & ' & 50008.90 & 74977.50 & 207.8 & 227.8 & 210.45 & 1.18 & 3.7210 & 4 & \\
\hline 'FSB & 95DR & $R^{\prime}$ & 49996.00 & 74991.70 & 187.0 & 207.0 & 210.47 & 0.19 & 1.4660 & 3 & \\
\hline 'FSB & $96 \mathrm{~A}$ & ' & 49778.70 & 74882.20 & 85.7 & 95.7 & 152.05 & 0.11 & 0.3712 & 1 & \\
\hline 'FSB & 96AR & & 49746.60 & 74914.90 & 79.0 & 89.0 & 153.47 & 0.07 & 0.5258 & 1 & \\
\hline 'FSB & $97 \mathrm{~A}$ & ' & 49965.70 & 75171.20 & 85.8 & 95.8 & 152.31 & 0.08 & 0.6671 & 1 & \\
\hline 'FSB & $97 \mathrm{C}$ & ' & 49970.60 & 75179.60 & 143.8 & 153.8 & 208.32 & 0.17 & 1.5076 & 2 & \\
\hline 'FSB & 97D & ' & 49975.50 & 75188.90 & 196.9 & 216.9 & 210.84 & 0.18 & 1.6279 & 3 & \\
\hline 'FSB & $98 \mathrm{~A}$ & ' & 50121.60 & 75389.80 & 84.7 & 94.7 & 150.61 & 0.14 & 0.5114 & 1 & \\
\hline 'FSB & $98 \mathrm{AR}$ & $R^{\prime}$ & 50105.80 & 75362.00 & 82.1 & 92.1 & 151.90 & 0.05 & 0.3853 & 1 & \\
\hline 'FSB & $98 \mathrm{C}$ & ' & 50116.50 & 75381.20 & 148.4 & 158.4 & 209.30 & 0.20 & 1.7473 & 2 & \\
\hline 'FSB & $98 \mathrm{D}$ & $'$ & 50111.60 & 75371.90 & 200.3 & 220.3 & 212.31 & 0.19 & 1.6776 & 3 & \\
\hline 'FSB & $99 \mathrm{~A}$ & ' & 50314.80 & 75675.60 & 92.9 & 102.9 & 150.78 & 0.08 & 0.6371 & 1 & \\
\hline
\end{tabular}


Table F-1. Hydraulic Head Targets for Model Calibration (Continued)

\begin{tabular}{|c|c|c|c|c|c|c|c|c|c|}
\hline 'FSB & $99 \mathrm{C}$ & ' & 50320.60 & 75683.70 & 157.2 & 167.2 & 209.91 & 0.21 & 1.7371 \\
\hline 'FSB & 99D & ' & 50326.90 & 75691.70 & 198.1 & 218.1 & 212.38 & 0.24 & 2.1177 \\
\hline 'ESB1 & $.00 \mathrm{~A}$ & ' & 50958.40 & 75534.40 & 95.8 & 105.8 & 151.62 & 0.08 & 0.6870 \\
\hline 'FSB1 & $01 \mathrm{~A}$ & ' & 51191.30 & 75719.00 & 92.9 & 102.9 & 151.84 & 0.09 & 0.7770 \\
\hline 'ESB1 & $.02 \mathrm{C}$ & ' & 50834.80 & 73582.90 & 145.9 & 155.9 & 195.37 & 0.06 & 0.4771 \\
\hline 'FSB1 & $.03 C$ & ' & 49651.30 & 74210.00 & 147.1 & 157.1 & 202.62 & 0.14 & 1.2780 \\
\hline 'FSB1 & $.04 \mathrm{C}$ & ' & 49248.60 & 73872.60 & 150.7 & 160.7 & 201.08 & 0.15 & 1.2670 \\
\hline 'FSB1 & $.04 \mathrm{D}$ & ' & 49255.40 & 73865.20 & 190.4 & 210.4 & 204.58 & 0.17 & 1.4873 \\
\hline 'FSB1 & $.05 \mathrm{C}$ & ' & 49828.00 & 75234.20 & 141.5 & 151.5 & 207.89 & 0.31 & 2.7480 \\
\hline 'FSB1 & .05D & ' & 49833.30 & 75244.30 & 203.7 & 223.7 & 208.26 & 0.31 & 1.0211 \\
\hline 'FSB1 & $.05 \mathrm{DR}$ & $z^{\prime}$ & 49841.00 & 75258.10 & 188.5 & 208.6 & 210.97 & 0.21 & 1.6158 \\
\hline 'FSB1 & $.06 \mathrm{C}$ & ' & 50651.30 & 74190.10 & 156.0 & 166.0 & 201.45 & 0.13 & 1.1075 \\
\hline 'FSB1 & $.06 \mathrm{D}$ & ' & 50636.80 & 74193.00 & 202.9 & 222.9 & 207.12 & 0.28 & 1.8342 \\
\hline 'FSB1 & $.07 \mathrm{C}$ & ' & 51158.10 & 75184.00 & 150.8 & 160.8 & 210.33 & 0.34 & 2.8671 \\
\hline 'FSB1 & .07D & ' & 51149.80 & 75177.20 & 200.9 & 220.9 & 213.85 & 0.24 & 2.0878 \\
\hline 'FSB1 & $.08 \mathrm{D}$ & ' & 51142.30 & 76260.70 & 203.8 & 223.8 & 217.70 & 0.25 & 2.4897 \\
\hline 'FSB1 & $.09 \mathrm{D}$ & ' & 50488.60 & 75855.90 & 205.8 & 225.8 & 213.69 & 0.25 & 2.1574 \\
\hline 'FSB1 & $10 \mathrm{C}$ & ' & 50150.60 & 74190.70 & 137.2 & 147.2 & 201.26 & 0.20 & 1.7276 \\
\hline 'FSB1 & $10 \mathrm{D}$ & ' & 50141.60 & 74193.30 & 191.1 & 211.1 & 205.64 & 0.15 & 1.3278 \\
\hline 'FSB1 & $11 \mathrm{C}$ & ' & 51526.30 & 75383.30 & 159.0 & 169.0 & 212.18 & 0.20 & 1.6572 \\
\hline 'ESBI & IID & ' & 51515.90 & 75382.90 & 201.7 & 221.7 & 215.55 & 0.25 & 2.1374 \\
\hline 'FSBI & $12 \mathrm{~A}$ & ' & 48809.10 & 74231.40 & 81.0 & 91.0 & 153.58 & 0.06 & 0.4559 \\
\hline 'FSB1 & $12 \mathrm{C}$ & ' & 48794.80 & 74227.50 & 129.1 & 139.1 & 201.90 & 0.21 & 1.6261 \\
\hline 'FSBI & $12 \mathrm{D}$ & ' & 48780.00 & 74223.70 & 188.9 & 208.9 & 206.15 & 0.18 & 1.3658 \\
\hline 'FSBI & $13 \mathrm{~A}$ & ' & 51068.10 & 74167.50 & 81.0 & 91.3 & 159.00 & 0.36 & 2.7859 \\
\hline 'FSBI & $13 \mathrm{C}$ & ' & 51084.20 & 74160.70 & 154.0 & 164.0 & 202.64 & 0.18 & 1.3860 \\
\hline 'FSBI & $13 \mathrm{D}$ & ' & 51098.40 & 74154.80 & 189.6 & 209.6 & 207.42 & 0.17 & 1.3359 \\
\hline 'FSBI & $14 \mathrm{~A}$ & ' & 52046.60 & 75297.40 & 95.2 & 105.0 & 155.76 & 0.06 & 0.4859 \\
\hline 'FSBI & $14 \mathrm{C}$ & ' & 52033.80 & 75288.50 & 158.0 & 168.0 & 213.68 & 0.17 & 1.3061 \\
\hline 'FSB1 & $14 \mathrm{D}$ & ' & 52018.60 & 75278.60 & 197.7 & 217.8 & 217.17 & 0.19 & 1.4759 \\
\hline 'FSBI & $15 \mathrm{C}$ & ' & 49736.00 & 72515.50 & 163.8 & 173.8 & 184.41 & 0.11 & 0.8864 \\
\hline 'FSB1 & $15 \mathrm{D}$ & ' & 49728.30 & 72504.30 & 182.5 & 192.5 & 191.38 & 0.16 & 1.2663 \\
\hline 'ESB1 & $16 \mathrm{C}$ & ' & 50645.90 & 72725.50 & 160.5 & 170.5 & 189.59 & 0.23 & 1.7258 \\
\hline 'FSB1 & $16 \mathrm{D}$ & ' & 50629.70 & 72727.40 & 186.4 & 196.4 & 191.96 & 0.16 & 1.2866 \\
\hline 'FSB1 & $17 \mathrm{D}$ & ' & 50486.80 & 74070.40 & 189.7 & 209.7 & 205.18 & 0.14 & 1.0658 \\
\hline 'FSB1 & $18 \mathrm{D}$ & ' & 51276.30 & 74697.90 & 191.3 & 211.3 & 211.68 & 0.21 & 1.5957 \\
\hline 'FSB1 & $19 \mathrm{D}$ & ' & 50600.60 & 74599.70 & 193.1 & 213.1 & 208.38 & 0.16 & 1.3769 \\
\hline 'FSB1 & $20 \mathrm{~A}$ & ' & 49175.70 & 75538.90 & $99: 0$ & 109.0 & 152.77 & 1.14 & 8.3954 \\
\hline 'FSB1 & $20 \mathrm{C}$ & ' & 49171.10 & 75549.80 & 150.7 & 160.7 & 206.31 & 0.17 & 1.3358 \\
\hline 'FSB1 & $20 \mathrm{D}$ & ' & 49163.70 & 75568.70 & 196.5 & 216.5 & 209.68 & 0.24 & 1.9065 \\
\hline 'FSB1 & $21 \mathrm{C}$ & ' & 48413.10 & 75155.70 & 148.4 & 158.4 & 204.39 & 0.18 & 1.3758 \\
\hline 'FSB1 & 21DR & & 48429.70 & 75151.90 & 191.3 & 211.3 & 207.24 & 0.20 & 1.5054 \\
\hline 'FSB1 & $22 \mathrm{C}$ & $'$ & 48195.00 & 73881.80 & 160.0 & 170.0 & 200.11 & 0.25 & 1.8959 \\
\hline 'FSB1 & $22 \mathrm{D}$ & ' & 48201.70 & 73865.50 & 186.6 & 206.6 & 203.45 & 0.27 & 2.0758 \\
\hline 'FSB1 & $23 \mathrm{C}$ & ' & 51750.50 & 74566.70 & 155.3 & 165.3 & 210.45 & 0.19 & 1.4757 \\
\hline 'FSB1 & $23 \mathrm{D}$ & ' & 51734.80 & 74562.70 & 194.1 & 214.1 & 212.29 & 0.17 & 1.3058 \\
\hline 'FSB1 & $50 P C$ & & 49990.10 & 74090.00 & 107.6 & 160.1 & 198.57 & 0.46 & 1.308 \\
\hline 'FSB1 & .50PD & & 49717.90 & 74615.80 & 176.2 & 221.3 & 207.99 & 0.70 & 1.998 \\
\hline 'FSL & 1D & ' & 52992.50 & 79063.10 & 208.5 & 228.6 & 224.68 & 0.16 & 1.1250 \\
\hline 'FSL & 2D & ' & 52790.60 & 78636.50 & 208.7 & 228.8 & 225.10 & 0.17 & 1.3057 \\
\hline 'FSL & 3D & ' & 52465.20 & 77765.20 & 205.9 & 226.0 & 222.76 & 0.25 & 1.8555 \\
\hline 'FSL & $4 \mathrm{D}$ & ' & 52230.40 & 77452.40 & 204.0 & 224.1 & 217.51 & 0.19 & 1.3850 \\
\hline 'FSL & 5D & ' & 51903.30 & 77047.70 & 203.5 & 223.7 & 220.79 & 0.21 & 1.4848 \\
\hline 'FSL & 6D & ' & 51727.90 & 76733.10 & 202.1 & 222.1 & 220.15 & 0.20 & 1.4148 \\
\hline 'FSL & 7D & ' & 51485.60 & 76327.80 & 199.5 & 219.6 & 218.24 & 0.28 & 1.9650 \\
\hline 'FSL & 8D & ' & 51513.50 & 76054.70 & 202.7 & 222.8 & 217.62 & 0.22 & 1.4946 \\
\hline 'FSL & 9D & ' & 51543.90 & 75768.40 & 201.4 & 221.5 & 216.80 & 0.48 & 3.3247 \\
\hline 'FSS & 1D & ' & 53897.60 & 75257.60 & 209.9 & 229.9 & 223.69 & 0.21 & 1.5555 \\
\hline 'FSS & $2 \mathrm{D}$ & ' & 53918.90 & 75103.50 & 204.4 & 224.4 & 222.88 & 0.23 & 1.7053 \\
\hline 'FSS & 3D & ' & 53548.00 & 74960.50 & 205.8 & 225.8 & 220.71 & 0.23 & 1.7054 \\
\hline 'FSS & $4 \mathrm{D}$ & ' & 52876.10 & 75537.80 & 202.6 & 222.6 & 218.96 & 0.26 & 1.8753 \\
\hline 'FST & ID & ' & 49102.00 & 81242.60 & 119.5 & 129.5 & 125.73 & 0.36 & $1.038^{\circ}$ \\
\hline 'FTF & 1 & ' & 53179.80 & 77413.30 & 221.2 & 241.2 & 227.43 & 1.45 & 5.4414 \\
\hline 'FTF & 2 & ' & 53275.10 & 77336.00 & 219.4 & 239.4 & 225.16 & 0.71 & 3.5525 \\
\hline 'FTF & 3 & ' & 53244.80 & 77235.30 & 218.2 & 221.2 & 223.85 & 0.66 & 3.6230 \\
\hline 'FTF & 4 & ' & 53268.20 & 77132.90 & 216.6 & 236.6 & 223.81 & 0.37 & 2.0731 \\
\hline 'FTF & 5 & ' & 53168.30 & 77035.60 & 215.3 & 235.3 & 223.45 & 0.50 & 2.8432 \\
\hline
\end{tabular}


Table F-1. Hydraulic Head Targets for Model Calibration (Continued)

\begin{tabular}{|c|c|c|c|c|c|c|c|c|c|c|c|}
\hline ‘FTF & 6 & - & 53062.00 & 77151.40 & 216.9 & 236.9 & 223.41 & 0.42 & 2.2629 & 5 & \\
\hline ‘FTF & 7 & , & 53089.70 & 77235.90 & 222.1 & 226.1 & 223.74 & 0.36 & 2.0533 & 4 & \\
\hline 'FTF & 8 & ' & 53059.90 & 77336.20 & 219.6 & 239.6 & 225.07 & 0.74 & 3.1318 & 5 & \\
\hline ‘FTF & 9 & ' & 52769.50 & 77482.80 & 216.4 & 236.4 & 221.13 & 0.90 & 4.7828 & 5 & \\
\hline 'FTF & 10 & ' & 52905.00 & 77336.00 & 215.1 & 235.1 & 220.09 & 1.23 & 5.6421 & 4 & \\
\hline ' FTF & 11 & ' & 52748.80 & 77180.70 & 215.8 & 235.8 & 220.49 & 1.31 & 5.9921 & 5 & \\
\hline 'FTF & 12 & - & 52648.50 & 77321.40 & 215.0 & 235.0 & 226.76 & 0.28 & 1.5431 & 4 & \\
\hline 'FTF & 13 & ' & 53098.40 & 76637.80 & 216.1 & 236.1 & 228.19 & 1.20 & 6.8032 & 4 & \\
\hline 'FTF & 15 & - & 53230.00 & 76732.00 & 197.5 & 227.5 & 225.11 & 0.58 & 3.3433 & 6 & ? \\
\hline 'FTF & 16 & ' & 52879.80 & 76758.60 & 203.8 & 233.8 & 223.33 & 0.47 & 2.7233 & 6 & ? \\
\hline 'FTF & 17 & ' & 52884.00 & 76872.00 & 200.6 & 230.6 & 222.94 & 0.35 & 2.0434 & 6 & ? \\
\hline 'FTF & 18 & ' & 52879.20 & 76955.80 & 202.3 & 232.3 & 223.26 & 0.51 & 2.9032 & 6 & ? \\
\hline 'FTF & 19 & ' & 52670.40 & 77139.10 & 198.3 & 228.3 & 222.37 & 0.36 & 2.0533 & 6 & ? \\
\hline 'FTF & 20 & ' & 52500.00 & 77015.00 & 198.3 & 228.3 & 221.81 & 0.31 & 1.8234 & 6 & ? \\
\hline 'FTF & 21 & ' & 52498.60 & 76866.70 & 198.7 & 228.7 & 223.06 & 0.27 & 1.5934 & 3 & \\
\hline 'FTF & 22 & . & 52494.70 & 76751.30 & 212.6 & 242.6 & 221.71 & 0.35 & 2.0234 & 6 & ? \\
\hline 'FTF & 23 & ' & 52660.30 & 76611.80 & 201.2 & 231.2 & 222.18 & 0.36 & 2.0833 & 6 & ? \\
\hline 'FTF & $24 \mathrm{~A}$ & . & 52780.80 & 77256.60 & 212.7 & 232.7 & 222.00 & 0.69 & 3.9032 & 4 & \\
\hline 'FTF & $25 \mathrm{~A}$ & ' & 52868.70 & 77308.40 & 212.8 & 232.8 & 223.18 & 0.33 & 1.9233 & 4 & \\
\hline 'FTF & 26 & ' & 52875.40 & 77250.00 & 206.3 & 226.3 & 223.26 & 0.33 & 1.8832 & 4 & \\
\hline 'FTF & 27 & ' & 52823.50 & 77227.20 & 213.5 & 243.5 & 223.33 & 0.39 & 2.2332 & 5 & \\
\hline${ }^{\prime} \mathrm{H}$ & 6 & , & 58335.40 & 72009.10 & 225.2 & 235.2 & 231.04 & 0.86 & 1.915 & 4 & \\
\hline ' $\mathrm{H}$ & 7 & ' & 58336.10 & 71949.20 & 224.9 & 234.9 & 228.96 & 0.48 & 1.075 & 4 & \\
\hline${ }^{\prime} \mathrm{H}$ & 8 & ' & 58233.90 & 71615.40 & 218.4 & 228.4 & 227.01 & 0.18 & 0.487 & 4 & \\
\hline${ }^{\prime} \mathrm{H}$ & 9 & ' & 58275.30 & 71572.60 & 207.4 & 217.4 & 226.76 & 0.33 & 0.745 & 4 & \\
\hline${ }^{\prime} \mathrm{H}$ & 10 & ' & 57822.80 & 71607.20 & 222.5 & 232.5 & 227.27 & 0.39 & 1.047 & 4 & \\
\hline${ }^{\prime} \mathrm{H}$ & 11 & ' & 57779.40 & 71565.90 & 212.0 & 222.0 & 227.70 & 0.41 & 0.925 & 4 & \\
\hline ' $\mathrm{H}$ & $18 \mathrm{~A}$ & ' & 57337.70 & 71339.60 & 217.5 & 227.5 & 224.08 & 0.25 & 1.0919 & 4 & \\
\hline ' $\mathrm{H}$ & 19 & ' & 57041.70 & 71434.20 & 219.6 & 221.1 & 227.63 & 1.02 & 3.5212 & 4 & \\
\hline 'HAA & $1 A$ & ' & 62967.90 & 69879.10 & 94.9 & 104.9 & 181.25 & 0.22 & 0.669 & 1 & \\
\hline 'HAA & $1 \mathrm{AA}$ & & 62960.40 & 69885.70 & 13.6 & 23.6 & 181.15 & 0.30 & 0.909 & 6 & ? \\
\hline 'HAA & $1 \mathrm{~B}$ & ' & 62976.00 & 69872.20 & 119.3 & 129.3 & 251.76 & 0.61 & 1.839 & 2 & \\
\hline ' $\mathrm{HAA}$ & $1 \mathrm{C}$ & ' & 62983.00 & 69866.20 & 147.4 & 157.4 & 252.36 & 0.63 & 1.889 & 2 & \\
\hline 'HAA & 1D & ' & 62991.00 & 69859.10 & 261.8 & 281.8 & 276.83 & 0.63 & 1.899 & 5 & \\
\hline ' HAA & 1TA & & 62953.30 & 69892.20 & -29.8 & -19.8 & 180.96 & 0.48 & 1.449 & 6 & ? \\
\hline ' HAA & $2 A$ & ' & 61276.00 & 70930.40 & 107.3 & 117.3 & 177.19 & 0.19 & 0.579 & 1 & \\
\hline 'HAA & $2 A A$ & & 61285.10 & 70925.40 & 29.4 & 39.4 & 177.77 & 0.23 & 0.709 & 6 & ? \\
\hline 'HAA & $2 \mathrm{~B}$ & ' & 61267.50 & 70935.40 & 127.2 & 137.2 & 253.24 & 0.42 & 1.269 & 2 & \\
\hline ' HAA & $2 \mathrm{C}$ & ' & 61258.90 & 70940.40 & 171.9 & 181.9 & 254.82 & 0.35 & 1.049 & 2 & \\
\hline 'HAA & $2 \mathrm{D}$ & ' & 61250.60 & 70945.40 & 260.3 & 280.4 & 276.61 & 0.39 & 1.3412 & 5 & \\
\hline 'HAA & $3 A$ & ' & 60190.40 & 71470.90 & 96.8 & 106.8 & 175.78 & 0.19 & 0.579 & 1 & \\
\hline 'HAA & $3 A A$ & & 60201.90 & 71488.00 & 6.5 & 16.5 & 175.03 & 0.19 & 0.589 & 6 & ? \\
\hline ' HAA & $3 B$ & ' & 60178.40 & 71453.20 & 125.9 & 135.9 & 240.59 & 0.39 & 1.3011 & 2 & \\
\hline ' HAA & $3 C$ & ' & 60167.40 & 71436.90 & 163.3 & 173.3 & 243.95 & 0.49 & 1.489 & 2 & \\
\hline 'HAA & $3 \mathrm{D}$ & ' & 60154.30 & 71418.40 & 246.7 & 266.7 & 264.58 & 0.82 & 3.9123 & 5 & \\
\hline ' HAA & $4 \mathrm{~A}$ & ' & 61920.00 & 72223.00 & 105.4 & 115.3 & 174.85 & 0.21 & 0.639 & 1 & \\
\hline 'HAA & $4 A A$ & $a^{\prime}$ & 61929.60 & 72223.20 & 32.2 & 42.2 & 175.13 & 0.17 & 0.5912 & 1 & \\
\hline 'HAA & $4 \mathrm{~B}$ & ' & 61909.90 & 72222.90 & 124.5 & 135.0 & 250.41 & 0.38 & 1.149 & 6 & ? \\
\hline ' HAA & $4 \mathrm{C}$ & ' & 61899.90 & 72223.10 & 158.3 & 168.3 & 251.83 & 0.39 & 1.118 & 2 & \\
\hline ' $\mathrm{HAA}$ & $4 D$ & ' & 61890.00 & 72223.30 & 255.7 & 275.7 & 270.14 & 0.43 & 1.289 & 5 & \\
\hline 'HAA & $5 \mathrm{~A}$ & ' & 62657.40 & 70601.10 & 100.7 & 110.7 & 179.23 & 1.29 & 2.243 & 1 & \\
\hline ' HAA & $6 \mathrm{~A}$ & ' & 63870.00 & 71440.90 & 95.6 & 105.6 & 178.92 & 0.19 & 0.579 & 1 & \\
\hline 'HAA & $6 \mathrm{AA}$ & & 63860.20 & 71441.00 & 25.8 & 35.8 & 178.60 & 0.18 & 0.549 & 1 & \\
\hline 'HAA & $6 \mathrm{~B}$ & ' & 63879.80 & 71440.40 & 131.3 & 141.4 & 235.67 & 0.27 & 0.829 & 2 & \\
\hline 'HAA & $6 C$ & ' & 63889.90 & 71440.60 & 161.1 & 171.1 & 235.86 & 0.28 & 0.849 & 2 & \\
\hline ' HAA & $6 \mathrm{D}$ & ' & 63900.20 & 71440.30 & 247.1 & 267.2 & 264.83 & 0.37 & 1.3012 & 5 & \\
\hline 'HAC & 1 & ' & 61415.20 & 72171.00 & 258.8 & 278.8 & 269.40 & 0.31 & 1.6428 & 5 & \\
\hline ' $\mathrm{HAC}$ & 2 & ' & 61366.90 & 72220.20 & 258.8 & 278.8 & 268.99 & 0.34 & 1.7928 & 5 & \\
\hline ' HAC & 3 & ' & 61313.60 & 72183.40 & 255.0 & 275.0 & 269.11 & 0.29 & 1.5629 & 5 & \\
\hline ' HAC & 4 & ' & 61372.00 & 72120.30 & 254.1 & 274.1 & 269.63 & 0.31 & 1.6628 & 5 & \\
\hline ' HAP & 1 & ' & 63398.80 & 71209.80 & 256.3 & 276.3 & 270.90 & 0.30 & 1.4824 & 5 & \\
\hline 'HAP & 2 & ' & 63519.80 & 71122.90 & 243.8 & 263.8 & 270.35 & 0.24 & 1.2729 & 5 & \\
\hline ' $\mathrm{HC}$ & $1 \mathrm{~A}$ & ' & 61867.00 & 71755.00 & 89.5 & 94.5 & 175.80 & 0.00 & 0.002 & 1 & \\
\hline 'HC & 1D & ' & 61867.00 & 71746.00 & 206.5 & 211.5 & 268.95 & 0.75 & 1.062 & 3 & \\
\hline ' $\mathrm{HC}$ & $1 E$ & ' & 61864.00 & 71746.00 & 251.5 & 256.5 & 275.00 & 0.50 & 0.712 & 5 & \\
\hline ' $\mathrm{HC}$ & $2 \mathrm{~A}$ & ' & 61866.00 & 71794.00 & 72.2 & 77.2 & 175.80 & 0.50 & 0.712 & 1 & \\
\hline ' HC & $2 B$ & ' & 61876.00 & 71785.00 & 85.7 & 90.7 & 175.00 & 1.00 & 1.412 & 1 & \\
\hline 'HC & $2 \mathrm{C}$ & ' & 61872.00 & 71784.00 & 135.7 & 140.7 & 253.70 & 0.50 & 0.712 & 2 & \\
\hline
\end{tabular}


Table F-1. Hydraulic Head Targets for Model Calibration (Continued)

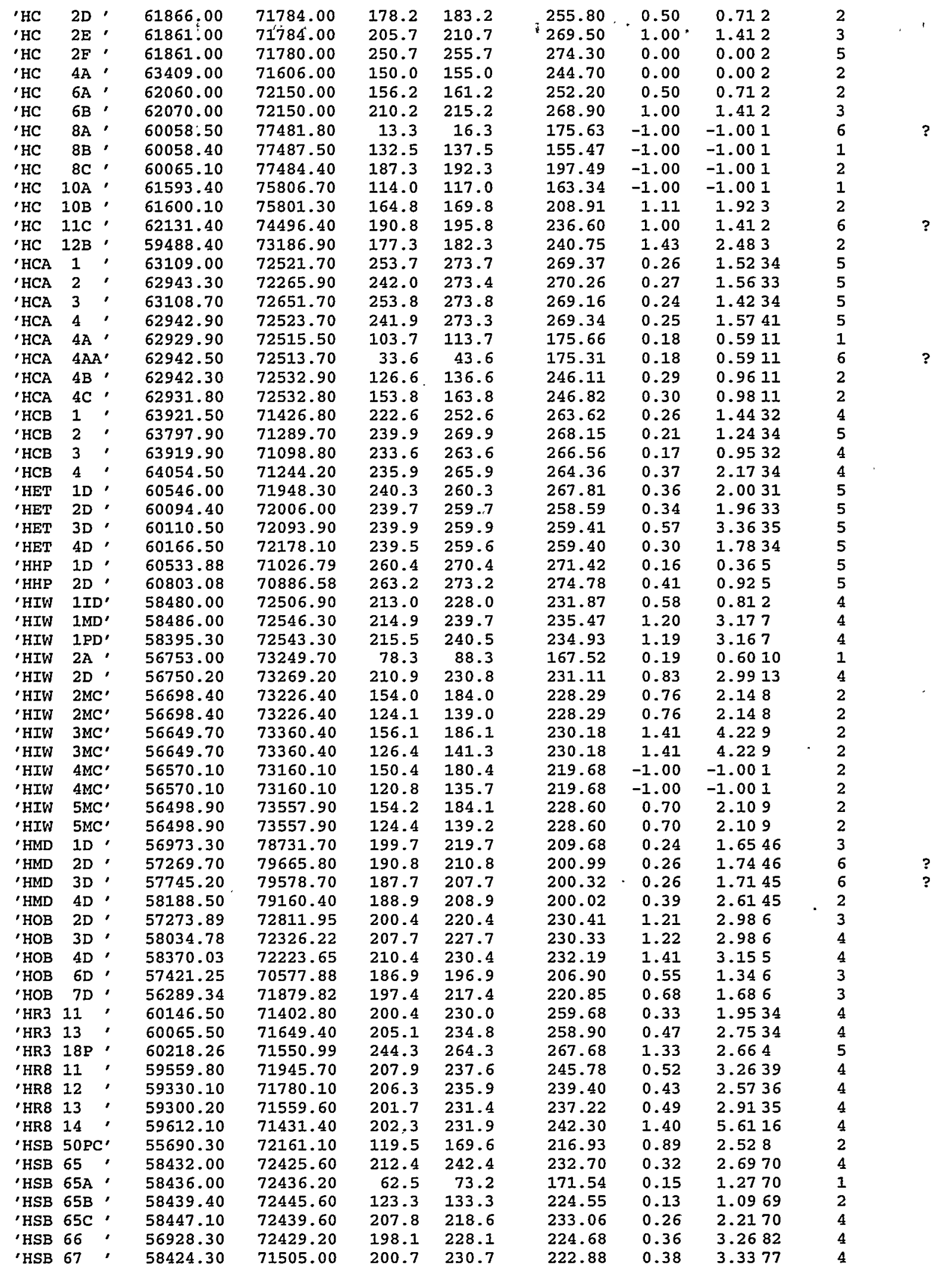


Table F-1. Hydraulic Head Targets for Model Calibration (Continued)

\begin{tabular}{|c|c|c|c|c|c|c|c|c|c|c|c|}
\hline 'HSB & 68 & ' & 56901.00 & 71528.00 & 213.3 & 243.3 & 221.72 & 0.23 & 1.7859 & 4 & \\
\hline HSB & $68 \mathrm{~A}$ & ' & 56892.10 & 71526.90 & 47.5 & 58.0 & 171.95 & 0.12 & 1.0070 & 1 & \\
\hline 'HSB & $68 \mathrm{~B}$ & ' & 56882.10 & 71525.50 & 123.5 & 134.5 & 216.75 & 0.25 & 2.0972 & 2 & \\
\hline 'HSB & $68 \mathrm{C}$ & ' & 56872.70 & 71524.10 & 168.4 & 179.5 & 217.68 & 0.18 & 1.5472 & 2 & \\
\hline 'HSB & 69 & ' & 56475.10 & 71546.90 & 199.0 & 229.0 & 219.38 & 0.11 & 0.9477 & 4 & \\
\hline 'HSB & $69 \mathrm{~A}$ & ' & 56465.10 & 71549.40 & 83.1 & 93.1 & 171.67 & 0.42 & 3.4468 & 1 & \\
\hline 'HSB & 70 & ' & 55758.90 & 72606.90 & 205.7 & 235.7 & 223.94 & 0.39 & 3.2268 & 4 & \\
\hline 'HSB & $70 \mathrm{C}$ & ' & 55757.10 & 72597.30 & 164.9 & 174.9 & 223.06 & 0.23 & 1.9071 & 2 & \\
\hline ' HSB & 71 & ' & 55279.20 & 72875.90 & 204.8 & 234.8 & 223.96 & 0.41 & 3.3568 & 4 & \\
\hline ' HSB & $71 \mathrm{C}$ & ' & 55281.50 & 72866.60 & 171.9 & 181.9 & 222.56 & 0.25 & 2.1370 & 2 & \\
\hline 'HSB & $83 \mathrm{~A}$ & ' & 58606.10 & 71648.60 & 65.2 & 76.0 & 173.05 & 0.26 & 2.3782 & 1 & \\
\hline 'HSB & $83 \mathrm{~B}$ & ' & 58594.90 & 71639.60 & 121.2 & 132.1 & 223.00 & 0.15 & 1.2472 & 2 & \\
\hline ' HSB & $83 \mathrm{C}$ & ' & 58614.80 & 71636.90 & 160.2 & 171.2 & 224.85 & 0.14 & 1.1269 & 2 & \\
\hline ' HSB & 83D & ' & 58601.70 & 71628.10 & 198.7 & 228.7 & 224.88 & 0.14 & 1.1572 & 4 & \\
\hline ' HSB & $84 \mathrm{~A}$ & ' & 56359.10 & 71586.20 & 64.7 & 75.9 & 171.98 & 0.11 & 0.9570 & 1 & \\
\hline ' HSB & $84 \mathrm{~B}$ & ' & 56352.40 & 71603.30 & 121.8 & 132.9 & 210.78 & 0.10 & 0.8171 & 2 & \\
\hline ' HSB & $84 \mathrm{C}$ & ' & 56360.10 & 71597.10 & 170.9 & 181.8 & 213.51 & 0.22 & 1.8773 & 2 & \\
\hline 'HSB & $84 \mathrm{D}$ & - & 56349.90 & 71583.90 & 199.5 & 219.5 & 218.86 & 0.11 & 0.9170 & 3 & \\
\hline ' HSB & $85 \mathrm{~A}$ & ' & 58943.40 & 73791.90 & 61.1 & 71.1 & 168.87 & 0.09 & 0.7983 & 1 & \\
\hline ' HSB & $85 \mathrm{~B}$ & ' & 58953.30 & 73789.30 & 133.2 & 143.2 & 233.85 & 0.18 & 1.7287 & 2 & \\
\hline ' HSB & $85 \mathrm{C}$ & ' & 58947.40 & 73802.30 & 214.2 & 224.2 & 239.19 & 0.23 & 2.0174 & 4 & \\
\hline 'HSB & $86 \mathrm{~A}$ & ' & 55985.90 & 72520.20 & 63.1 & 73.9 & 168.77 & 0.11 & 0.8971 & 1 & \\
\hline ' HSB & $86 B$ & ' & 55976.90 & 72519.00 & 113.8 & 124.0 & 221.56 & 0.17 & 1.4172 & 2 & \\
\hline ' HSB & $86 \mathrm{C}$ & - & 55984.60 & 72529.70 & 189.4 & 199.4 & 223.45 & 0.21 & 1.7973 & 6 & ? \\
\hline ' HSB & $86 \mathrm{D}$ & ' & 55996.50 & 72522.10 & 206.6 & 236.6 & 223.42 & 0.21 & 1.8171 & 4 & \\
\hline ' HSBI & $00 \mathrm{C}$ & ' & 58806.50 & 72077.20 & 153.0 & 163.0 & 226.95 & 0.22 & 1.8271 & 2 & \\
\hline 'HSBI & 000 & ' & 58796.90 & 72073.80 & 216.9 & 236.9 & 233.86 & 0.21 & 1.8676 & 4 & \\
\hline ' HSBI & $100 \mathrm{PC}$ & & 55720.00 & 72058.30 & 117.6 & 167.7 & 215.72 & 1.04 & 2.958 & 2 & \\
\hline ' HSB] & $100 \mathrm{PD}$ & & 56379.50 & 71445.30 & 195.0 & 214.9 & 217.05 & 0.38 & 1.088 & 3 & \\
\hline 'HSB] & $101 \mathrm{C}$ & - & 58604.40 & 72001.90 & 166.3 & 176.3 & 226.25 & 0.25 & 2.1672 & 2 & \\
\hline ' HSB1 & 101D & ' & 58594.80 & 71997.50 & 216.1 & 236.1 & 230.69 & 0.22 & 1.9276 & 4 & \\
\hline 'HSB1 & $102 \mathrm{C}$ & ' & 58399.70 & 71960.10 & 166.7 & 176.7 & 224.74 & 0.17 & 1.4270 & 2 & \\
\hline ' HSB] & 102D & ' & 58393.40 & 71952.40 & 216.3 & 236.3 & 228.71 & 0.20 & 1.7173 & 4 & \\
\hline 'HSB] & $103 \mathrm{C}$ & ' & 58323.60 & 71593.90 & 159.2 & 169.2 & 223.51 & 0.15 & 1.2871 & 2 & \\
\hline ' HSB] & LO3D & ' & 58315.60 & 71588.10 & 213.7 & 233.7 & 225.70 & 0.15 & 1.2974 & 4 & \\
\hline ' HSB] & $104 \mathrm{C}$ & ' & 58082.60 & 71376.80 & 163.5 & 173.5 & 220.71 & 0.13 & 1.0671 & 2 & \\
\hline ' HSB] & $104 \mathrm{D}$ & ' & 58075.80 & 71370.20 & 210.6 & 230.6 & 224.80 & 0.23 & 1.9573 & 4 & \\
\hline ' HSB] & $105 \mathrm{C}$ & - & 57883.80 & 71447.30 & 152.2 & 162.2 & 219.69 & 0.12 & 0.9769 & 2 & \\
\hline ' HSB] & $105 \mathrm{D}$ & - & 57877.40 & 71454.80 & 211.8 & 231.8 & 225.31 & 0.16 & 1.4377 & 4 & \\
\hline ' $\mathrm{HSB}]$ & $106 \mathrm{C}$ & - & 57651.50 & 71720.90 & 158.7 & 168.7 & 221.87 & 0.13 & 1.0570 & 2 & \\
\hline ' HSB] & $106 \mathrm{D}$ & - & 57644.80 & 71727.80 & 210.7 & 230.7 & 226.04 & 0.16 & 1.3772 & 4 & \\
\hline 'HSB] & $107 \mathrm{C}$ & - & 57432.00 & 71698.50 & 159.3 & 169.3 & 219.49 & 0.14 & 1.2171 & 2 & \\
\hline ' HSB] & 107D & ' & 57412.20 & 71696.60 & 215.1 & 235.1 & 224.74 & 0.15 & 1.2874 & 4 & \\
\hline 'HSB] & $108 \mathrm{C}$ & - & 57155.50 & 71688.70 & 186.0 & 196.0 & 218.61 & 0.16 & 1.3171 & 6 & ? \\
\hline ‘HSB1 & $108 \mathrm{D}$ & - & 57145.60 & 71688.00 & 212.0 & 232.0 & 223.53 & 0.15 & 1.2366 & 4 & \\
\hline ‘ HSB] & $109 \mathrm{C}$ & ' & 56895.60 & 71684.80 & 168.4 & 178.4 & 218.83 & 0.12 & 0.9972 & 2 & \\
\hline 'HSB] & 109D & ' & 56885.50 & 71685.60 & 213.0 & 233.0 & 222.67 & 0.18 & 1.4663 & 4 & \\
\hline ' HSB] & $110 \mathrm{C}$ & ' & 56680.70 & 71779.30 & 171.4 & 181.4 & 219.14 & 0.12 & 0.9971 & 2 & \\
\hline ' HSB] & $110 \mathrm{D}$ & ' & 56672.10 & 71785.20 & 211.4 & 231.4 & 222.06 & 0.16 & 1.3170 & 4 & \\
\hline ' HSBI & $111 \mathrm{C}$ & $\cdot$ & 56501.90 & 71919.40 & 140.7 & 150.7 & 220.30 & 0.13 & 1.0871 & 2 & \\
\hline ‘HSBI & $111 D$ & ' & 56494.50 & 71926.20 & 185.7 & 195.7 & 221.90 & 0.16 & 1.3171 & 6 & ? \\
\hline 'HSB] & $111 E$ & ' & 56487.20 & 71932.80 & 211.7 & 231.7 & 221.89 & 0.17 & 1.4673 & 4 & \\
\hline 'HSB] & $112 \mathrm{C}$ & ' & 56417.40 & 72156.40 & 140.6 & 150.6 & 221.61 & 0.15 & 1.2971 & 2 & \\
\hline 'HSB] & $112 \mathrm{D}$ & ' & 56408.10 & 72161.60 & 188.3 & 198.3 & 222.81 & 0.23 & 1.9872 & 6 & ? \\
\hline 'HSBI & $112 \mathrm{E}$ & ' & 56399.50 & 72166.60 & 211.7 & 231.7 & 222.52 & 0.16 & 1.4579 & 4 & \\
\hline 'HSB1 & $113 \mathrm{C}$ & - & 56160.40 & 72312.30 & 154.7 & 164.7 & 221.86 & 0.16 & 1.3569 & 2 & \\
\hline ' HSB] & $113 \mathrm{C}$ & ' & 56160.40 & 72312.30 & 151.7 & 161.7 & 221.86 & 0.16 & 1.3569 & 2 & \\
\hline ‘HSB] & $113 \mathrm{D}$ & ' & 56164.30 & 72302.70 & 216.2 & 236.2 & 222.48 & 0.19 & 1.6776 & 4 & \\
\hline ' HSB] & $114 \mathrm{C}$ & ' & 56107.00 & 72464.60 & 185.6 & 195.6 & 223.33 & 0.20 & 1.6470 & 6 & ? \\
\hline 'HSB] & $114 \mathrm{D}$ & - & 56104.60 & 72474.20 & 212.8 & 232.8 & 223.19 & 0.21 & 1.8074 & 4 & \\
\hline 'HSB] & $115 \mathrm{C}$ & ' & 56043.20 & 72653.20 & 182.8 & 192.8 & 224.16 & 0.30 & 2.5472 & 2 & \\
\hline 'HSB1 & $115 D$ & - & 56039.80 & 72662.30 & 213.9 & 233.9 & 223.85 & 0.21 & 1.9685 & 4 & \\
\hline ' HSB] & $116 \mathrm{C}$ & ' & 55989.10 & 72888.10 & 180.5 & 190.5 & 225.02 & 0.21 & 1.7469 & 2 & \\
\hline 'HSB] & $116 D$ & ' & 55988.20 & 72898.10 & 214.5 & 234.5 & 225.88 & 0.42 & 3.5975 & 4 & \\
\hline 'HSB] & $117 \mathrm{~A}$ & ' & 55170.10 & 72733.60 & 84.8 & 94.8 & 166.76 & 0.09 & 0.7269 & 1 & \\
\hline 'HSB] & $117 \mathrm{C}$ & ' & 55162.90 & 72740.70 & 165.1 & 175.1 & 221.68 & 0.34 & 2.8571 & 2 & \\
\hline 'HSB] & 117D & ' & 55155.60 & 72747.60 & 200.3 & 220.3 & 223.72 & 0.32 & 2.7775 & 3 & \\
\hline 'HSB1 & $118 \mathrm{~A}$ & ' & 55775.60 & 72696.40 & 91.0 & 101.0 & 167.78 & 0.11 & 0.9270 & 6 & ? \\
\hline
\end{tabular}


Table F-1. Hydraulic Head Targets for Model Calibration (Continued)

\begin{tabular}{|c|c|c|c|c|c|c|c|c|c|c|}
\hline 'HSB119A & ' & 56100.20 & 73082.50 & 93.3 & 103.3 & 167.08 & 0.10 & 0.8570 & 6 & $?$ \\
\hline 'HSB120A & ' & 56431.90 & 73395.10 & 91.0 & 101.0 & 166.41 & $0.10^{4}$ & $0.83^{\prime} 69$ & 1 & $\therefore$ \\
\hline 'HSB121A & ' & 57389.60 & 72024.80 & 88.3 & 98.3 & 171.74 & 0.11 & 0.9168 & 1 & \\
\hline 'HSB122A & ' & 57747.40 & 72195.90 & 85.4 & 95.4 & 171.59 & 0.13 & 1.0669 & 1 & \\
\hline 'HSB123A & ' & 58124.80 & 72189.80 & 93.6 & 103.6 & 171.98 & 0.29 & 2.5375 & 6 & ? \\
\hline 'HSB124AR & & 58531.70 & 72202.70 & 94.6 & 104.6 & 172.19 & 0.11 & 0.8253 & 1 & \\
\hline 'HSB125C & ' & 58592.80 & 71503.60 & 145.6 & 155.6 & 223.51 & 0.14 & 1.1969 & 2 & \\
\hline 'HSB125D & ' & 58584.10 & 71498.20 & 199.4 & 219.4 & 221.27 & 0.13 & 1.0870 & 3 & \\
\hline 'HSB126C & ' & 57178.20 & 70627.70 & 176.3 & 181.3 & 203.99 & 0.06 & 0.5066 & 2 & \\
\hline 'HSB126D & ' & 57169.60 & 70633.40 & 190.5 & 200.5 & 205.17 & 0.06 & 0.5471 & 3 & \\
\hline 'HSB127C & ' & 56792.10 & 71210.10 & 148.4 & 158.4 & 210.39 & 0.07 & 0.6070 & 2 & \\
\hline 'HSB127D & ' & 56788.00 & 71218.90 & 197.8 & 217.8 & 218.03 & 0.11 & 0.9269 & 3 & \\
\hline 'HSB129C & ' & 55110.00 & 71830.40 & 147.8 & 157.8 & 205.74 & 0.15 & 1.2771 & 2 & \\
\hline 'HSB129D & ' & 55103.40 & 71837.10 & 185.2 & 205.2 & 208.44 & 0.11 & 0.9569 & 3 & \\
\hline 'HSB130C & ' & 54643.60 & 70762.40 & 159.9 & 169.9 & 199.98 & 0.06 & 0.5271 & 2 & \\
\hline 'HSB130D & ' & 54651.70 & 70757.20 & 182.1 & 202.1 & 200.24 & 0.09 & 0.7271 & 3 & \\
\hline 'HSB131C & ' & 56894.90 & 70374.70 & 148.5 & 158.5 & 203.93 & 0.07 & 0.5469 & 2 & \\
\hline 'HSB131D & ' & 56891.10 & 70365.00 & 195.7 & 205.7 & 205.10 & 0.14 & 1.2475 & 3 & \\
\hline 'HSB132C & ' & 58787.70 & 71472.40 & 168.6 & 178.6 & 221.57 & 0.10 & 0.8769 & 2 & \\
\hline 'HSB132D & ' & 58799.30 & 71469.50 & 206.5 & 226.5 & 221.18 & 0.16 & 1.3068 & 4 & \\
\hline 'HSB133C & ' & 59110.30 & 71949.50 & 173.3 & 183.3 & 230.77 & 0.17 & 1.4370 & 2 & \\
\hline 'HSB133D & ' & 59102.30 & 71943.50 & 208.5 & $228 \cdot 5$ & 235.37 & 0.21 & 1.7270 & 4 & \\
\hline 'HSB134C & ' & 58289.90 & 71210.30 & 149.1 & 159.1 & 220.97 & 0.12 & 1.0272 & 2 & \\
\hline 'HSB134D & ' & 58296.50 & 71217.30 & 205.8 & 225.8 & 222.09 & 0.21 & 1.7472 & 4 & \\
\hline 'HSB135C & ' & 56560.80 & 71390.20 & 147.3 & 157.3 & 206.79 & 0.07 & 0.5567 & 2 & \\
\hline 'HSB135D & ' & 56552.80 & 71396.70 & 199.9 & 219.9 & 218.26 & 0.11 & 0.8767 & 3 & \\
\hline 'HSB136C & ' & 55949.60 & 71900.30 & 160.5 & 170.5 & 217.28 & 0.16 & 1.3171 & 2 & \\
\hline 'HSB136D & $'$ & 55941.70 & 71906.00 & 200.2 & 220.2 & 220.72 & 0.16 & 1.3170 & 3 & \\
\hline 'HSB137C & ' & 55700.20 & 72269.90 & 163.8 & 173.8 & 220.04 & 0.19 & 1.6573 & 2 & \\
\hline 'HSB137D & ' & 55696.10 & 72278.90 & 205.3 & 225.3 & 221.96 & 0.21 & 1.7270 & 4 & \\
\hline 'HSB138D & 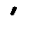 & 55260.70 & 73160.20 & 208.1 & 228.1 & 223.78 & 0.29 & 2.4670 & 4 & \\
\hline 'HSB139A & ' & 57365.40 & 71127.40 & 87.6 & 97.6 & 173.74 & 0.12 & 0.9867 & 1 & \\
\hline 'HSB139C & ' & 57374.50 & 71129.80 & 148.5 & 158.5 & 214.48 & 0.09 & 0.8073 & 2 & \\
\hline 'HSB139D & ' & 57384.40 & 71133.20 & 206.7 & 226.7 & 222.54 & 0.22 & 1.8470 & 4 & \\
\hline 'HSB140A & ' & 56535.40 & 70050.30 & 81.0 & 91.0 & 175.50 & 0.37 & 2.7155 & 6 & ? \\
\hline 'HSB140C & 1 & 56551.80 & 70049.20 & 161.6 & 171.6 & 205.64 & 0.28 & 2.1256 & 2 & \\
\hline 'HSB140D & $\cdot$ & 56560.60 & 70036.00 & 194.1 & 214.1 & 213.86 & 0.37 & 2.7656 & 3 & \\
\hline 'HSB141A & ' & 59168.70 & 71213.60 & 80.6 & 90.6 & 175.12 & 0.16 & 1.2260 & 1 & \\
\hline 'HSB141C & ' & 59170.20 & 71196.70 & 154.7 & 164.7 & 229.07 & 0.32 & 1.5825 & 2 & \\
\hline 'HSB141CR & 8 & 59167.20 & 71226.70 & 152.1 & 162.1 & 229.33 & 0.55 & 3.0631 & 2 & \\
\hline 'HSB141D & ' & 59170.90 & 71184.40 & 217.8 & 237.8 & 240.83 & 0.60 & 4.7162 & 4 & \\
\hline 'HSB142C & ' & 53505.30 & 73119.00 & 161.6 & 171.6 & 198.28 & 0.27 & 2.0458 & 2 & \\
\hline 'HSB142D & ' & 53493.10 & 73113.00 & 189.7 & 199.7 & 197.94 & 0.29 & 2.2159 & 3 & \\
\hline 'HSB143C & ' & 52773.20 & 73738.20 & 169.1 & 179.1 & 209.34 & 0.14 & 1.0857 & 2 & \\
\hline 'HSB143D & ' & 52774.50 & 73754.00 & 196.9 & 216.9 & 213.22 & 0.18 & 1.3557 & 3 & \\
\hline 'HSB144A & ' & 56200.50 & 71892.10 & 78.6 & 88.6 & 171.37 & 0.32 & 2.4959 & 1 & \\
\hline 'HSB145C & ' & 57769.00 & 71098.90 & 164.7 & 174.7 & 213.53 & 0.21 & 1.6459 & 2 & \\
\hline 'HSB145D & ' & 57753.90 & 71088.00 & 184.2 & 194.2 & 220.43 & 0.22 & 1.6760 & 6 & $?$ \\
\hline 'HSB146A & ' & 58454.00 & 70478.90 & 85.5 & 95.5 & 176.09 & 0.08 & 0.5756 & 1 & \\
\hline 'HSBI46C & ' & 58473.10 & 70471.60 & 152.3 & 162.3 & 210.01 & 0.15 & 1.1557 & 2 & \\
\hline 'HSB146D & ' & 58493.00 & 70469.70 & 204.0 & 224.1 & 222.57 & 0.43 & 3.2758 & 4 & \\
\hline 'HSB147D & ' & 55804.40 & 73827.90 & 215.2 & 235.2 & 231.53 & 0.32 & 2.4359 & 4 & \\
\hline 'HSB148C & ' & 55344.20 & 70151.50 & 158.9 & 168.9 & 201.78 & 0.08 & 0.6263 & 2 & \\
\hline 'HSB148D & ' & 55355.70 & 70160.90 & 198.1 & 218.1 & 213.46 & 0.20 & 1.6365 & 3 & \\
\hline 'HSB149D & ' & 57286.30 & 71338.80 & 207.0 & 227.0 & 222.61 & 0.41 & 3.1057 & 4 & \\
\hline 'HSBI5OD & ' & 58692.80 & 71692.60 & 206.9 & 226.9 & 226.80 & 0.35 & 2.7260 & 4 & \\
\hline 'HSB150PC & $\because$ & 55543.90 & 72236.40 & 119.5 & 169.6 & 217.37 & 0.85 & 2.428 & 2 & \\
\hline 'HSB151C & ' & 54014.90 & 72997.90 & 170.6 & 180.6 & 207.82 & 0.16 & 1.2159 & 2 & \\
\hline 'HSB151D & ' & 54026.40 & 72997.80 & 197.6 & 207.6 & 207.16 & 0.18 & 1.3757 & 3 & \\
\hline 'HSB152C & ' & 54346.70 & 72012.00 & 173.1 & 183.1 & 199.04 & 0.06 & 0.4558 & 2 & \\
\hline 'HSB152D & ' & 54362.10 & 72011.70 & 197.0 & 207.0 & 205.56 & 0.35 & 1.3515 & 3 & \\
\hline 'HSL 1D & ' & 58925.00 & 72179.60 & 219.8 & 239.8 & 235.58 & 0.33 & 2.2647 & 4 & \\
\hline 'HSL 2D & ' & 59423.50 & 72190.80 & 225.2 & 245.3 & 242.11 & 0.26 & 1.8048 & 4 & \\
\hline 'HSL & ' & 59770.60 & 72251.50 & 233.7 & 253.8 & 250.38 & 0.32 & 2.3252 & 5 & \\
\hline 'HSL $\quad 4 D$ & ' & 60171.90 & 72453.70 & 245.0 & 265.1 & 261.98 & 0.28 & 1.9850 & 5 & \\
\hline 'HSL 5D & ' & 60339.40 & 72562.20 & 247.8 & 267.7 & 265.94 & 0.46 & 3.1747 & 5 & \\
\hline 'HSL 5D & ' & 60339.40 & 72562.20 & 242.6 & 247.7 & 265.94 & 0.46 & 3.1747 & 5 & \\
\hline
\end{tabular}


Table F-1. Hydraulic Head Targets for Model Calibration (Continued)

\begin{tabular}{|c|c|c|c|c|c|c|c|c|c|c|c|}
\hline 'HSL & $6 \mathrm{~A}$ & ' & 60549.50 & 72684.50 & 104.7 & 114.7 & 168.42 & 0.14 & 0.5616 & 1 & \\
\hline 'HSL & $6 A A$ & & 60555.70 & 72692.60 & 18.6 & 28.6 & 169.13 & 0.14 & 0.5617 & 1 & \\
\hline 'HSL & $6 \mathrm{~B}$ & ' & 60543.60 & 72676.30 & 127.9 & 137.9 & 244.59 & 0.30 & 1.1916 & 2 & \\
\hline 'HSL & $6 \mathrm{C}$ & ' & 60537.60 & 72667.50 & 157.6 & 167.6 & 245.42 & 0.28 & 1.1617 & 2 & \\
\hline 'HSL & $6 \mathrm{D}$ & ' & 60531.10 & 72659.70 & 243.9 & 264.0 & 260.12 & 0.32 & 2.3452 & 5 & \\
\hline 'HSL & 6D & ' & 60531.10 & 72659.70 & 239.4 & 243.9 & 260.12 & 0.32 & 2.3452 & 4 & \\
\hline 'HSL & $7 \mathrm{D}$ & ' & 60723.00 & 72674.40 & 242.3 & 262.4 & 259.98 & 0.29 & 2.0750 & 5 & \\
\hline 'HSL & $8 \mathrm{~A}$ & , & 61113.90 & 72721.00 & 108.8 & 118.8 & 172.78 & 0.14 & 0.5717 & 1 & \\
\hline 'HSL & $8 \mathrm{AA}$ & & 61113.80 & 72729.40 & 28.7 & 38.7 & 175.72 & 0.47 & 2.1421 & 1 & \\
\hline 'HSL & $8 B$ & ' & 61115.00 & 72710.20 & 138.7 & 148.7 & 249.22 & 0.28 & 1.1517 & 2 & \\
\hline 'HSL & $8 \mathrm{C}$ & ' & 61115.90 & 72700.50 & 171.7 & 181.7 & 250.39 & 0.24 & 1.0519 & 2 & \\
\hline 'HSL & 8D & ' & 61117.10 & 72688.10 & 248.4 & 268.4 & 260.94 & 0.23 & 1.7456 & 5 & \\
\hline 'HSS & 1D & ' & 64675.60 & 67610.30 & 236.5 & 256.5 & 268.77 & 0.66 & 3.4227 & 4 & \\
\hline 'HSS & $2 \mathrm{D}$ & ' & 64785.90 & 67355.90 & 234.5 & 254.5 & 267.87 & 0.66 & 3.4227 & 4 & \\
\hline 'HSS & 3D & , & 64709.50 & 68257.50 & 262.6 & 282.6 & 281.85 & 0.72 & 3.7327 & 5 & \\
\hline ' $\mathrm{HTF}$ & 1 & ' & 62067.00 & 71745.00 & 236.9 & 256.9 & 272.90 & 0.30 & 1.7333 & 5 & \\
\hline ' HTF & 2 & ' & 62175.00 & 71610.00 & 237.0 & 257.0 & 274.33 & 0.28 & 1.6032 & 5 & \\
\hline ' $\mathrm{HTF}$ & 4 & ' & 61942.00 & 71630.00 & 235.2 & 255.2 & 274.34 & 0.27 & 1.5231 & 5 & \\
\hline ' HTF & 5 & ' & 62110.00 & 71390.00 & 264.3 & 284.3 & 279.58 & 1.35 & 6.8926 & 5 & \\
\hline ' $\mathrm{HTF}$ & 7 & ' & 62112.00 & 71130.00 & 263.5 & 283.5 & 275.95 & 0.35 & 1.8026 & 5 & \\
\hline ' $\mathrm{HTF}$ & 8 & ' & 61965.00 & 71270.00 & 263.6 & 283.6 & 273.92 & 0.55 & 2.6223 & 5 & \\
\hline 'HTF & 9 & ' & 61698.00 & 71652.00 & 245.8 & 265.8 & 273.81 & 0.35 & 2.0734 & 5 & \\
\hline ' HTF & 10 & ' & 61838.00 & 71520.00 & 245.2 & 265.2 & 273.32 & 0.28 & 1.5631 & 5 & \\
\hline ' HTF & 11 & ' & 61722.00 & 71398.00 & 238.9 & 258.9 & 274.03 & 0.33 & 1.8732 & 5 & \\
\hline ' HTF & 12 & ' & 61593.00 & 71520.00 & 242.9 & 262.9 & 273.47 & 0.35 & 2.0836 & 5 & \\
\hline 'HTF & 13 & ' & 61586.00 & 71856.00 & 262.6 & 282.6 & 274.19 & 0.30 & 1.6530 & 5 & \\
\hline 'HTF & 14 & ' & 61462.00 & 71858.00 & 261.9 & 281.9 & 273.23 & 0.41 & 2.1527 & 5 & \\
\hline ' HTF & 15 & ' & 61353.00 & 71700.00 & 260.7 & 280.7 & 273.55 & 0.23 & 1.3737 & 5 & \\
\hline 'HTF & 16 & ' & 61950.00 & 72150.00 & 248.3 & 268.3 & 269.67 & 0.41 & 2.0024 & 5 & \\
\hline ' HTF & 17 & ' & 61188.00 & 72600.00 & 238.4 & 258.4 & 262.53 & 0.53 & 3.1836 & 5 & \\
\hline ' HTF & 18 & ' & 61223.30 & 71771.80 & 251.7 & 271.7 & 271.64 & 0.28 & 1.7237 & 5 & \\
\hline ' HTF & 19 & ' & 61079.20 & 71902.50 & 245.7 & 265.7 & 269.17 & 0.26 & 1.6037 & 5 & \\
\hline ' HTF & 20 & ' & 61086.40 & 72073.30 & 251.9 & 271.9 & 267.89 & 0.35 & 2.0836 & 5 & \\
\hline ' HTF & 21 & ' & 61261.00 & 71998.20 & 242.6 & 262.6 & 269.60 & 0.28 & 1.6635 & 5 & \\
\hline ' HTF & 22 & - & 62553.60 & 71363.40 & 251.4 & 271.4 & 275.47 & 0.32 & 1.8332 & 5 & \\
\hline ' HTF & 23 & , & 62670.30 & 71363.10 & 256.8 & 276.8 & 274.55 & 0.38 & 2.2133 & 5 & \\
\hline ' HTF & 24 & ' & 62775.60 & 71362.60 & 257.8 & 277.8 & 274.11 & 0.31 & 1.7531 & 5 & \\
\hline ' $\mathrm{HTF}$ & 25 & ' & 62902.00 & 71224.30 & 252.5 & 272.5 & 274.67 & 0.51 & 2.9333 & 5 & \\
\hline ' HTF & 26 & ' & 62815.70 & 71090.70 & 255.5 & 275.5 & 275.44 & 0.47 & 2.7133 & 5 & \\
\hline ' HTF & 27 & ' & 62660.30 & 71057.90 & 259.1 & 279.1 & 276.90 & 0.68 & 3.9934 & 5 & \\
\hline ' HTF & 28 & ' & 62515.70 & 71080.10 & 251.9 & 271.9 & 275.99 & 0.26 & 1.4631 & 5 & \\
\hline ' HTF & 29 & ' & 62414.90 & 71229.90 & 259.9 & 289.9 & 275.79 & 0.32 & 1.7932 & 5 & \\
\hline ' $\mathrm{HTF}$ & 31 & ' & 62662.50 & 70747.00 & 246.7 & 266.7 & 275.61 & 0.31 & 1.6829 & 5 & \\
\hline ' HTF & 32 & ' & 62807.90 & 70880.60 & 251.1 & 271.1 & 274.74 & 0.28 & 1.6133 & 5 & \\
\hline ' HTF & 34 & ' & 61978.50 & 71144.10 & 251.7 & 271.7 & 274.33 & 1.27 & 6.3525 & 5 & \\
\hline 'HWP & $1 D$ & ' & 59852.50 & 72158.08 & 239.9 & 249.9 & 245.25 & 0.20 & 0.292 & 5 & \\
\hline 'HWP & $2 \mathrm{D}$ & ' & 59918.86 & 72368.22 & 253.0 & 263.0 & 262.96 & 0.52 & 1.034 & 5 & \\
\hline 'HWS & $1 \mathrm{~A}$ & ' & 50234.80 & 64885.10 & 225.2 & 255.2 & 244.89 & 0.34 & 1.8730 & 5 & \\
\hline 'HWS & 2 & ' & 50346.40 & 64786.30 & 215.3 & 245.3 & 245.48 & 0.40 & 2.1128 & 4 & \\
\hline 'HXB & 1 & ' & 52557.80 & 60549.70 & 214.2 & 244.2 & 251.71 & 0.57 & 3.0028 & 5 & \\
\hline ' $\mathrm{HXB}$ & 2 & ' & 52892.80 & 60866.50 & 212.1 & 242.1 & 252.90 & 0.63 & 3.2727 & 5 & \\
\hline ' $\mathrm{HXB}$ & 3 & , & 52707.30 & 60631.20 & 212.2 & 242.2 & 252.21 & 0.61 & 3.1727 & 5 & \\
\hline 'HXB & $4 D$ & ' & 52617.30 & 60685.70 & 234.9 & 254.9 & 253.85 & 0.46 & 2.3125 & 5 & \\
\hline ' HXB & $5 D$ & . & 52510.40 & 60587.70 & 234.2 & 254.2 & 253.00 & 0.46 & 2.3225 & 5 & \\
\hline 'IDP & $3 A$ & ' & 37781.10 & 85104.30 & -86.7 & -81.3 & 167.18 & 0.24 & 1.0920 & 6 & ? \\
\hline 'IDP & $3 B$ & ' & 37785.30 & 85119.50 & 95.7 & 100.7 & 157.42 & 0.47 & 2.1020 & 1 & \\
\hline 'IDP & $3 c$ & ' & 37790.10 & 85133.70 & 164.1 & 169.1 & 202.15 & 0.85 & 3.5918 & 2 & \\
\hline IDP & 4 & ' & 38615.40 & 82812.60 & 189.5 & 199.6 & 191.13 & 1.27 & 5.5419 & 2 & \\
\hline 'IDP & 5 & ' & 38284.50 & 83521.50 & 186.4 & 206.6 & 198.41 & 0.75 & 3.3420 & 2 & \\
\hline 'IDP & 6 & ' & 38248.50 & 84113.90 & 184.5 & 209.1 & 201.54 & 0.74 & 3.2920 & 2 & \\
\hline 'IDP & 7 & ' & 38713.90 & 84460.10 & 188.6 & 208.6 & 200.79 & 0.82 & 3.6720 & 2 & \\
\hline 'IDP & 8 & ' & 39174.30 & 84740.40 & 185.4 & 204.5 & 199.25 & 1.03 & 4.6320 & 2 & \\
\hline 'IDQ & $3 \mathrm{~A}$ & ' & 35854.00 & 80553.70 & -189.8 & -184.3 & 166.06 & 0.27 & 1.2020 & 6 & ? \\
\hline 'IDQ & $3 B$ & ' & 35858.80 & 80578.40 & 108.4 & 113.4 & 140.21 & 1.28 & 5.8721 & 1 & \\
\hline 'IDQ & $3 c$ & ' & 35863.50 & 80601.70 & 136.6 & 141.6 & 164.58 & 0.84 & 3.6419 & 2 & \\
\hline 'IDQ & 4 & ' & 36726.20 & 83125.10 & 185.6 & 205.6 & 198.39 & 0.71 & 3.1720 & 2 & \\
\hline 'IDQ & 5 & ' & 36851.80 & 82763.60 & 187.4 & 207.5 & 198.31 & 1.46 & 6.3519 & 2 & \\
\hline 'IDQ & 6 & ' & 37299.30 & 82414.40 & 181.9 & 202.1 & 193.81 & 0.66 & 3.0221 & 2 & \\
\hline
\end{tabular}


Table F-1. Hydraulic Head Targets for Model Calibration (Continued)

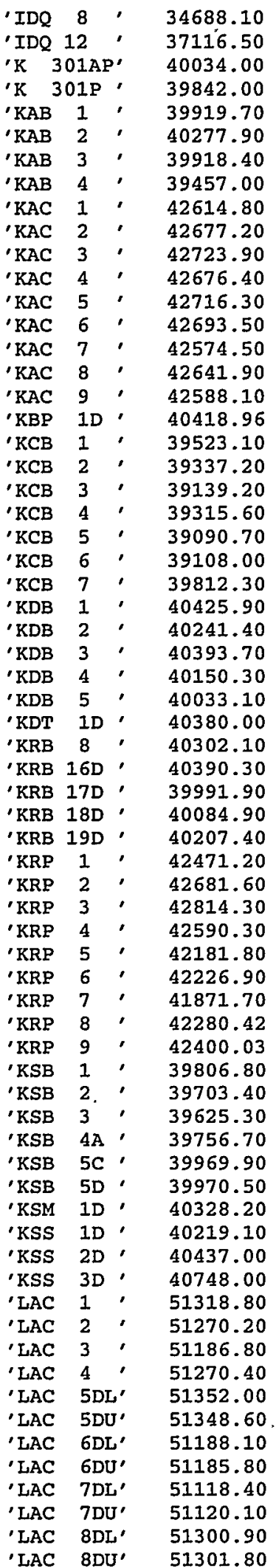

\subsection{0} 81913.70

54284.00

54320.00

53055.60

52410.80

51807.70

52807.10

53167.00

53255.50

53201.80

53053.50

53161.70

53139.90

53252.90

53136.00

53197.80

52439.60

53453.00

53634.40

53440.50

53256.10

53353.70

53559.20

53435.60

54050.50

53907.30

53794.60

53787.40

54052.20

54154.10

54893.60

54888.00

55446.40

55563.70

55620.90

54544.00

54503.60

54248.70

54362.90

54606.60

54206.70

54390.30

54470.74

54360.10

54044.40

53927.60

54040.20

54140.40

54165.60

54156.50

54188.00

47758.90

46803.80

46644.30

45238.80

45330.40

45201.90

45213.10

45365.40

45345.90

45272.80

45252.50

45097.10

45114.70

45096.60

45116.00
180.4

194.7

$194.0 \quad 224.0$

$198.6 \quad 228.6$

$193.0 \quad 223.0$

$187.0 \quad 217.0$

$199.0 \quad 229.0$

195.4225 .4

$195.8 \quad 225.8$

$178.0 \quad 208.0$

204.3224 .3

204.6224 .6

203.0223 .0

$192.3 \quad 212.3$

$\begin{array}{ll}195.7 & 215.7\end{array}$

$192.0 \quad 202.1$

$183.6 \quad 213.6$

$187.7 \quad 217.7$

184.1214 .1

$188.9 \quad 218.9$

$189.3 \quad 209.3$

188.7208 .7

$196.5 \quad 216.5$

$184.8 \quad 205.8$

$182.5 \quad 203.5$

$184.2 \quad 205.4$

$189.2 \quad 209.2$

188.5208 .5

$193.7 \quad 213.7$

$\begin{array}{ll}195.8 & 215.8\end{array}$

$191.5 \quad 211.5$

186.8206 .8

$185.8 \quad 205.8$

$186.8 \quad 206.8$

$207.0 \quad 237.0$

$199.2 \quad 229.2$

$207.5 \quad 237.5$

$188.7 \quad 218.7$

$200.8 \quad 210.8$

$203.1 \quad 213.1$

203.1213 .2

$200.1 \quad 210.1$

$200.8 \quad 210.8$

$\begin{array}{ll}175.6 & 205.6\end{array}$

$\begin{array}{ll}173.8 & 203.8\end{array}$

$169.7 \quad 199.7$

$169.6 \quad 199.6$

$172.9 \quad 182.9$

$194.5 \quad 214.5$

$193.7 \quad 213.7$

$157.4 \quad 177.5$

$144.6 \quad 164.7$

$139.3 \quad 159.3$

191.1221 .1

193.4223 .4

$\begin{array}{ll}190.7 & 220.7\end{array}$

$185.3 \quad 215.3$

$176.2 \quad 186.2$

$207.9 \quad 227.8$

$175.9 \quad 185.9$

$201.7 \quad 221.7$

$177.4 \quad 187.4$

204.9224 .8

$180.4 \quad 190.4$

$199.8 \quad 219.8$
189.90

187.30

208.77

205.10

205.85

209.37

203.60

202.90

219.27

221.51

221.96

218.07

222.40

222.34

219.45

221.18

220.84

208.15

204.68

202.83

202.32

205.48

200.65

201.15

205.30

208.48

206.99

207.75

207.05

205.51

208.10

208.55

209.30

206.03

204.58

203.82

218.59

219.46

219.16

218.52

216.28

217.85

215.92

217.10

218.30

204.19

203.86

203.13

203.45

204.88

204.48

208.29

174.31

164.65

163.82

216.48

216.09

216.52

216.07

219.74

219.48

217.89

218.97

215.11

218.06

217.45

218.07

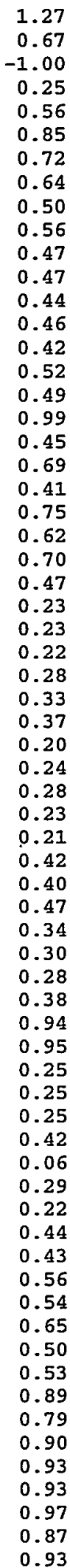

2.5014

$-1.001$

1.7248

3.0129

4.7231

3.7527

3.4529

2.8132

3.1231

2. 6832

2. 6532

2.3929

2.4528

2.2930

2.0215

1.8915

2.215

2. 6935

3.9232

2.4636

2.7013

1.869

1.988

1.4910

1.8366

1.9269

1.8570

1.5029

2.1944

1.6319

0.6511

1.1121

1.3021

1.0721

0.9421

2.5437

2.3234

2. 6932

2.0636

0.675

0.839

1.078

1.633

1.653

1.9664

2.0368

2.0267

3.3663

0.103

0.869

1.2934

2.3328

2.2828

2.9628

2.9630

3.6832

2.8533

2.8629

2.186

2.107

2.216

2.276

2.296

2.386

2.126

2.296
$?$

? 
Table F-1. Hydraulic Head Targets for Model Calibration (Continued)

\begin{tabular}{|c|c|c|c|c|c|c|c|c|c|}
\hline ' LAW & $1 \mathrm{C}$ & - & 50603.60 & 44562.40 & -34.0 & -29.0 & 176.21 & 0.29 & 0.645 \\
\hline ' LAW & $1 D$ & - & 50595.60 & 44562.00 & 6.6 & 11.6 & 176.50 & 0.17 & 0.5510 \\
\hline ' LAW & $1 E$ & ' & 50579.00 & 44561.20 & 90.1 & 95.1 & 205.04 & 0.59 & 1.335 \\
\hline ' LAW & $1 F$ & ' & 50567.10 & 44562.10 & 165.9 & 185.9 & 203.89 & 0.96 & 2.879 \\
\hline ' L.AW & $2 B$ & - & 49635.50 & 45641.00 & -9.8 & -4.8 & 176.23 & 0.17 & 0.5210 \\
\hline - LAW & $2 C$ & ' & 49638.70 & 45610.90 & 171.2 & 191.2 & 209.19 & 0.34 & 2.2444 \\
\hline ' LAW & $3 B$ & ' & 52269.50 & 45600.70 & -1.0 & 4.0 & 178.20 & 0.17 & 0.5510 \\
\hline ‘ LAW & $3 c$ & - & 52272.90 & 45616.10 & 194.9 & 214.9 & 235.20 & 0.58 & 2.0112 \\
\hline - LCO & 1 & ' & 50957.70 & 45198.20 & 195.8 & 225.8 & 214.76 & 0.60 & 3.3531 \\
\hline ' LCO & 2 & ' & 51043.40 & 45317.80 & 196.6 & 226.6 & 215.14 & 0.58 & 3.3032 \\
\hline - LCO & 3 & ' & 51113.20 & 45203.00 & 196.3 & 226.3 & 229.09 & 0.51 & 2.8130 \\
\hline ' LCO & 4 & ' & 51036.10 & 45087.40 & 192.3 & 222.3 & 212.57 & 0.61 & 3.5233 \\
\hline ' LCO & $5 A$ & ' & 50866.00 & 44987.00 & 30.0 & 40.0 & 177.24 & 0.28 & 0.686 \\
\hline 'LCO & $5 c$ & ' & 50881.80 & 44988.50 & 110.5 & 120.5 & 210.99 & 0.60 & 1.486 \\
\hline ' LCO & 5DL & & 50887.50 & 44974.50 & 174.9 & 184.9 & 212.95 & 0.86 & 2.106 \\
\hline ' LCO & 6DL & & 50921.20 & 45069.30 & 178.0 & 188.0 & 213.67 & 0.83 & 2.026 \\
\hline ' LCO & 7DL & & 51055.90 & 44946.90 & 170.2 & 180.2 & 213.31 & 0.83 & 2.036 \\
\hline ' LCO & $8 \mathrm{DL}$ & & 51380.60 & 45586.10 & 178.4 & 188.4 & 220.55 & 0.83 & 2.046 \\
\hline ' LCO & 8DU & & 51361.70 & 45586.10 & 211.1 & 226.1 & 220.60 & 0.84 & 2.056 \\
\hline ' LDB & 1 & ' & 50590.50 & 45886.50 & 185.0 & 215.0 & 217.40 & 0.38 & 3.0363 \\
\hline ' LDB & 2 & ' & 50784.60 & 46007.40 & 184.5 & 214.5 & 219.44 & 0.37 & 2.9765 \\
\hline ' LDB & 3 & - & 50525.80 & 46068.90 & 199.3 & 219.3 & 218.93 & 0.38 & 2.5544 \\
\hline ' LDB & 4 & - & 50339.50 & 45809.00 & 200.7 & 220.7 & 216.92 & 0.41 & 2.6943 \\
\hline ' LFW & 6 & - & 45241.20 & 84537.80 & 141.1 & 160.4 & 154.01 & 0.31 & 1.8635 \\
\hline 'LFW & $6 \mathrm{R}$ & ' & 45194.00 & 84413.90 & 134.3 & 154.3 & 153.81 & 0.35 & 1.2012 \\
\hline 'LFW & 7 & ' & 45318.90 & 84310.30 & 140.5 & 159.8 & 152.18 & 0.22 & 1.2934 \\
\hline ' LFW & 8 & • & 45415.30 & 84032.60 & 139.9 & 159.2 & 150.00 & 0.20 & 1.1936 \\
\hline ' LFW & $8 \mathrm{R}$ & ' & 45414.60 & 83949.00 & 134.9 & 154.9 & 150.75 & 0.24 & 0.8212 \\
\hline ' LFW & $10 \mathrm{~A}$ & ' & 45935.60 & 84369.60 & 134.4 & 164.4 & 157.05 & 0.42 & 2.7743 \\
\hline ' LFW & 16 & ' & 45852.60 & 84748.90 & 131.2 & 161.2 & 155.48 & 0.28 & 1.5933 \\
\hline ' LFW & 17 & ' & 45607.30 & 84602.80 & 128.5 & 158.5 & 153.68 & 0.51 & 2.9233 \\
\hline ' LFW & 18 & - & 45459.40 & 84577.30 & 137.7 & 167.7 & 161.41 & 0.59 & 3.8542 \\
\hline ' LFW & 19 & - & 45135.40 & 84817.20 & 130.0 & 160.0 & 156.16 & 0.29 & 1.6733 \\
\hline ' LFW & 20 & , & 45582.90 & 85262.60 & 135.0 & 165.0 & 159.04 & 0.31 & 1.8134 \\
\hline ' LFW & 21 & ' & 46149.40 & 84178.30 & 137.9 & 167.9 & 157.52 & 0.62 & 4.0443 \\
\hline · LFW & 22 & ' & 46325.20 & 84223.60 & 122.4 & 152.4 & 151.34 & 0.30 & 1.7534 \\
\hline ‘ LFW & 23 & ' & 46456.10 & 84251.30 & 125.1 & 155.1 & 151.36 & 0.63 & 3.7435 \\
\hline ‘ LFW & $23 \mathrm{R}$ & ' & 46512.90 & 84206.10 & 118.2 & 138.2 & 149.44 & 0.74 & 2.5612 \\
\hline ‘ LFW & 24 & ' & 46520.80 & 84544.20 & 124.5 & 154.5 & 154.38 & 0.36 & 2.0433 \\
\hline ' LFW & 25 & - & 46425.70 & 84967.20 & 123.2 & 153.2 & 156.78 & 0.35 & 2.0333 \\
\hline 'LFW & 26 & - & 45633.80 & 85654.60 & 143.2 & 164.2 & 161.19 & 0.33 & 1.8832 \\
\hline ' LFW & 27 & • & 45596.10 & 85839.10 & 142.9 & 163.9 & 162.11 & 0.33 & 1.9133 \\
\hline ' LFW & 28 & ' & 45555.30 & 86079.60 & 141.1 & 162.1 & 163.56 & 0.30 & 1.9441 \\
\hline 'LFW & 29 & ' & 45503.30 & 86372.70 & 143.9 & 164.9 & 164.72 & 0.36 & 2.0733 \\
\hline 'LFW & 30 & - & 45170.90 & 86318.40 & 141.7 & 162.7 & 164.97 & 0.34 & 2.1138 \\
\hline 'LFW & 31 & ' & 44869.00 & 86262.20 & 145.0 & 166.0 & 164.82 & 0.37 & 2.5447 \\
\hline 'LFW & 32 & ' & 44935.90 & 85836.80 & 144.3 & 165.3 & 162.39 & 0.27 & 1.7842 \\
\hline 'LFW & $32 \mathrm{C}$ & ' & 44923.00 & 85837.80 & 98.6 & 113.6 & 161.61 & 0.38 & 0.764 \\
\hline ‘ LFW & 33 & ' & 44973.00 & 85633.80 & 144.4 & 165.4 & 161.01 & 0.31 & 1.8435 \\
\hline ‘ LFW & 34 & - & 45016.90 & 85409.50 & 143.7 & 164.7 & 159.94 & 0.28 & 1.7339 \\
\hline 'LFW & 35 & ' & 45378.80 & 85237.40 & 143.4 & 164.4 & 158.79 & 0.31 & 1.7633 \\
\hline 'LFW & 36 & ' & 45582.30 & 83535.50 & 130.3 & 151.3 & 145.93 & 0.20 & 1.1935 \\
\hline ' LFW & $36 R$ & - & 45519.10 & 83537.30 & 121.8 & 141.8 & 146.10 & 0.23 & 0.8213 \\
\hline ' LFW & 37 & ' & 45667.70 & 83113.20 & 129.8 & 150.8 & 142.84 & 0.17 & 0.9835 \\
\hline ‘ LFW & 38 & ' & 46018.50 & 83172.30 & 130.5 & 151.5 & 143.36 & 0.32 & 1.7932 \\
\hline 'LFW & 39 & ' & 46218.50 & 83213.10 & 131.2 & 152.2 & 143.71 & 0.32 & 1.8132 \\
\hline 'LFW & 40 & ' & 46395.10 & 83248.80 & 131.2 & 152.2 & 143.54 & 0.32 & 1.6828 \\
\hline 'LFW & 41 & ' & 46626.90 & 83304.90 & 130.3 & 151.3 & 145.20 & 0.43 & 2.5435 \\
\hline ' LFW & $41 R$ & ' & 46635.30 & 83238.30 & 120.2 & 140.2 & 142.17 & 1.38 & 5.3315 \\
\hline ' LFW & 42 & ' & 46532.90 & 83776.20 & 130.2 & 151.2 & 147.42 & 0.43 & 2.5234 \\
\hline ' LFW & $43 B$ & - & 45240.50 & 86459.20 & 90.4 & 100.4 & 166.04 & 0.19 & 1.1736 \\
\hline ' LFW & $43 C$ & ' & 45234.90 & 86480.60 & 128.5 & 138.5 & 166.27 & 0.19 & 1.1937 \\
\hline ' LFW & $43 \mathrm{D}$ & - & 45244.50 & 86443.20 & 150.9 & 170.9 & 166.70 & 0.24 & 1.4336 \\
\hline 'LFW & $44 D$ & ' & 45022.60 & 84524.40 & 139.5 & 159.3 & 155.33 & 0.15 & 0.6719 \\
\hline ' LFW & 45D & - & 45142.00 & 84217.80 & 134.7 & 154.7 & 152.55 & 0.15 & 0.8332 \\
\hline 'LFW & $46 \mathrm{D}$ & - & 45162.80 & 84054.00 & 137.3 & 157.1 & 151.48 & 0.14 & 0.6219 \\
\hline ' LFW & $46 D$ & ' & 45162.80 & 84054.00 & 109.5 & 119.6 & 151.48 & 0.14 & 0.6219 \\
\hline 'LFW & $47 \mathrm{C}$ & ' & 45161.60 & 83823.30 & 105.7 & 115.8 & 148.91 & 0.11 & 0.4619 \\
\hline
\end{tabular}


Table F-1. Hydraulic Head Targets for Model Calibration (Continued)

\begin{tabular}{|c|c|c|}
\hline - LEW & 47D & 45150.80 \\
\hline ' LFW & $48 \mathrm{C}$ & 45413.30 \\
\hline LFW & $48 \mathrm{D}$ & 45426.70 \\
\hline ‘LFW & $55 \mathrm{C}$ & 45205.90 \\
\hline & 55D & 45189.30 \\
\hline EW & $56 \mathrm{D}$ & 45306.60 \\
\hline EW & $57 \mathrm{~B}$ & 45440.60 \\
\hline FW & $57 \mathrm{C}$ & 45411.10 \\
\hline 'LFW & 57D & 45417.40 \\
\hline 'LFW & $58 D$ & 45700.20 \\
\hline$F W$ & $59 B$ & 46047.40 \\
\hline JFW & $59 \mathrm{C}$ & 46052.00 \\
\hline IFW & 59D & 46056.10 \\
\hline ' LFW' & $60 \mathrm{~B}$ & 45710.20 \\
\hline 'LFW & $60 \mathrm{C}$ & 45711.90 \\
\hline ' LFW & $60 \mathrm{D}$ & 45722.30 \\
\hline 'LFW & $61 C$ & 46489.60 \\
\hline 'LFW & 61D & 46471.10 \\
\hline ' LFW & $62 B$ & 45915.50 \\
\hline 'LFW & $62 C$ & 45906.70 \\
\hline 'LFW & 62D & 45922.90 \\
\hline ' LFW & $63 B$ & 45550.70 \\
\hline 'LFW & $63 C$ & 45559.20 \\
\hline 'LFW & 63D & 45569.10 \\
\hline ' LFW & $64 \mathrm{~B}$ & 45268.80 \\
\hline 'LFW & $64 \mathrm{C}$ & 45271.30 \\
\hline 'LFW & $64 \mathrm{D}$ & 45280.70 \\
\hline 'LFW & $65 \mathrm{~B}$ & 46061.80 \\
\hline 'LFW & $65 C$ & 46064.40 \\
\hline 'LFW & 65D & 46071.80 \\
\hline 'LFW & $66 \mathrm{~B}$ & 46195.90 \\
\hline 'LFW & $66 \mathrm{C}$ & 46186.00 \\
\hline 'LFW & 66D & 46173.70 \\
\hline 'LFW & $67 \mathrm{~B}$ & 46517.10 \\
\hline 'LFW & $67 \mathrm{C}$ & 46527.50 \\
\hline 'LFW & 67D & 46529.90 \\
\hline 'LFW & $68 \mathrm{~B}$ & 46885.30 \\
\hline ' LFW & $68 C$ & 46876.20 \\
\hline 'LEW & 68D & 46868.00 \\
\hline ' LFW & $69 \mathrm{~B}$ & 45492.00 \\
\hline 'LFW & $69 \mathrm{C}$ & 45494.50 \\
\hline 'LFW & 69D & 45501.00 \\
\hline FW & $70 B$ & 45825.50 \\
\hline 'LFW & $70 \mathrm{C}$ & 45833.40 \\
\hline 'LFW & 700 & 45839.80 \\
\hline ' IFW & $71 B$ & 46340.40 \\
\hline IFW & $71 \mathrm{C}$ & 46329.80 \\
\hline 'LFW & $71 D$ & 46319.80 \\
\hline LFW & $72 B$ & 46944.30 \\
\hline$F W$ & $72 \mathrm{C}$ & 46937.10 \\
\hline LFW & $72 \mathrm{D}$ & 46943.00 \\
\hline 'LFW & $74 \mathrm{C}$ & 45097.80 \\
\hline LFW & $74 \mathrm{D}$ & 45098.00 \\
\hline LFW & $75 \mathrm{C}$ & 45357.00 \\
\hline 'LFW & $75 D$ & 45355.60 \\
\hline FW & 76 & 44758.60 \\
\hline LET & 77 & 44866.50 \\
\hline LFW & 78 & 44726.50 \\
\hline LRI & 1 & 49128.70 \\
\hline$R T$ & 2 & 49214.40 \\
\hline$R$ & 3 & 49057.70 \\
\hline & 4 & 48964.70 \\
\hline LSB & 1 & 50700.90 \\
\hline & 2 & 50824.50 \\
\hline & 3 & 50729.70 \\
\hline & 4 & 50513.00 \\
\hline & 36 & 57891.50 \\
\hline
\end{tabular}

\begin{tabular}{|c|c|c|}
\hline 3838.60 & 134.9 & \\
\hline 33856.40 & 108 & \\
\hline 33856.90 & 134.9 & \\
\hline 3613.20 & 94.1 & \\
\hline 3601.30 & 121.2 & \\
\hline 3398.00 & 131.3 & \\
\hline 3196.70 & 68.4 & \\
\hline 3200.10 & 107.8 & \\
\hline 3190.20 & 130.6 & \\
\hline 2940.60 & 127 & \\
\hline 3027.10 & 66.0 & \\
\hline 3011.00 & 100.3 & \\
\hline 83000.10 & 129.3 & \\
\hline 2517.50 & 67.7 & \\
\hline 82529.60 & 98.3 & \\
\hline 82531.50 & 123.8 & \\
\hline 8308 & 111 & \\
\hline 83089.10 & 130.3 & \\
\hline 83001.20 & 62.8 & \\
\hline 33012.70 & 108.4 & \\
\hline $\begin{array}{l}82991.60 \\
82740.80\end{array}$ & $\begin{array}{r}127.6 \\
66.1\end{array}$ & \\
\hline 82746.10 & 96.2 & \\
\hline 82751.80 & 126.4 & \\
\hline 82736.40 & 51.9 & \\
\hline 82744.80 & 83.0 & \\
\hline 82737.80 & 115.2 & \\
\hline 8258 & 53.5 & \\
\hline 82592.90 & 86.1 & \\
\hline 82598.40 & 111.5 & 131 \\
\hline 82838.30 & 70.3 & \\
\hline 82836.50 & 100.0 & \\
\hline 5.10 & 121.8 & \\
\hline 82847.10 & 55.6 & \\
\hline 82844.20 & 86.1 & \\
\hline 82855.00 & 120.6 & \\
\hline 83023.30 & 56.7 & \\
\hline 830 & 88.3 & \\
\hline 83031.60 & 124.6 & 144 \\
\hline 82451.20 & 52.0 & \\
\hline 82458.60 & 79.1 & \\
\hline 82452.00 & 119.0 & \\
\hline & 61.5 & \\
\hline 8230 & 78.8 & \\
\hline 82316.30 & 118.3 & $13 \varepsilon$ \\
\hline 82616.70 & 57.0 & \\
\hline 8261 & 80.4 & \\
\hline .10 & 115.5 & \\
\hline 82872.10 & 50.9 & \\
\hline 82875.80 & 87.8 & \\
\hline 82881.50 & 120.0 & \\
\hline 85813.80 & 101.0 & \\
\hline 85828.10 & 152.7 & \\
\hline 5.80 & 100.6 & \\
\hline 85868.00 & 151.0 & \\
\hline 85682.10 & 142.9 & \\
\hline 86461.70 & 144.2 & \\
\hline 86064.90 & 149.9 & \\
\hline 48548.60 & 185.8 & \\
\hline & 184.7 & \\
\hline 48333.60 & 191.4 & \\
\hline 48440.20 & 173.3 & \\
\hline 45153.10 & 192.7 & \\
\hline 45224.00 & 195.0 & \\
\hline 453 & 196.6 & \\
\hline 45321.60 & 191.5 & \\
\hline 73904.00 & 234.2 & \\
\hline
\end{tabular}

$\begin{array}{llll}149.44 & 0.10 & 0.5734 & 1 \\ 148.98 & 0.13 & 0.6322 & 1 \\ 149.40 & 0.13 & 0.6022 & 1 \\ 146.97 & 0.09 & 0.4019 & 1 \\ 147.10 & 0.09 & 0.4119 & 1 \\ 145.48 & 0.08 & 0.4534 & 1 \\ 143.76 & 0.09 & 0.4021 & 1 \\ 143.94 & 0.08 & 0.3821 & 1 \\ 143.96 & 0.08 & 0.3921 & 1 \\ 141.98 & 0.09 & 0.5134 & 1 \\ 142.81 & 0.12 & 0.5321 & 1 \\ 142.62 & 0.14 & 0.6018 & 1 \\ 142.83 & 0.36 & 2.1235 & 1 \\ 137.86 & 0.07 & 0.2110 & 1 \\ 138.27 & 0.29 & 1.4324 & 1 \\ 138.25 & 0.07 & 0.4537 & 1 \\ 142.09 & 0.17 & 0.8021 & 1 \\ 144.12 & 0.27 & 1.4228 & 1 \\ 142.30 & 0.10 & 0.4421 & 1 \\ 142.75 & 0.13 & 0.5921 & 1 \\ 143.49 & 0.18 & 1.0030 & 1 \\ 140.19 & 0.07 & 0.3324 & 1 \\ 140.21 & 0.08 & 0.3923 & 1 \\ 140.56 & 0.13 & 0.6224 & 1 \\ 140.04 & 0.07 & 0.2411 & 1 \\ 140.30 & 0.17 & 0.8224 & 1 \\ 140.35 & 0.05 & 0.2725 & 1 \\ 137.94 & 0.06 & 0.3022 & 1 \\ 137.93 & 0.09 & 0.4123 & 1 \\ 138.39 & 0.17 & 0.8425 & 1 \\ 140.84 & 0.12 & 0.3810 & 1 \\ 140.68 & 0.34 & 1.0910 & 1 \\ 141.90 & 0.26 & 1.0115 & 1 \\ 139.11 & 0.10 & 0.4923 & 1 \\ 138.75 & 0.15 & 0.6821 & 1 \\ 141.94 & 0.31 & 1.5024 & 1 \\ 140.21 & 0.15 & 0.4710 & 1 \\ 139.61 & 0.22 & 0.7110 & 1 \\ 142.66 & 0.33 & 1.6224 & 1 \\ 137.56 & 0.06 & 0.1810 & 1 \\ 137.78 & 0.06 & 0.2923 & 1 \\ 137.87 & 0.06 & 0.3124 & 1 \\ 136.20 & 0.07 & 0.2210 & 1 \\ 136.23 & 0.06 & 0.2010 & 1 \\ 135.67 & 0.12 & 0.3710 & 1 \\ 137.77 & 0.09 & 0.4424 & 1 \\ 137.92 & 0.08 & 0.3724 & 1 \\ 137.39 & 0.13 & 0.6323 & 1 \\ 138.08 & 0.16 & 0.5411 & 1 \\ 137.63 & 0.24 & 0.7610 & 1 \\ 138.74 & 0.27 & 0.8710 & 1 \\ 163.30 & 0.25 & 0.7810 & 1 \\ 163.16 & 0.22 & 0.8214 & 1 \\ 162.91 & 0.29 & 0.9912 & 1 \\ 163.24 & 0.31 & 1.0612 & 1 \\ 160.77 & 0.27 & 0.615 & 1 \\ 164.94 & 0.91 & 1.292 & 1 \\ 162.53 & 0.31 & 0.624 & 1 \\ 209.30 & 0.55 & 3.0731 & 6 \\ 210.15 & 1.00 & 5.6732 & 4 \\ 209.47 & 0.53 & 3.0132 & 4 \\ 208.61 & 0.53 & 2.9832 & 3 \\ 211.61 & 0.48 & 2.9137 & 4 \\ 212.39 & 0.51 & 3.0235 & 4 \\ 217.16 & 0.51 & 3.1538 & 4 \\ 216.84 & 0.74 & 4.5338 & 4 \\ 240.63 & 1.37 & 5.6617 & 5\end{array}$


Table F-1. Hydraulic Head Targets for Model Calibration (Continued)

\begin{tabular}{|c|c|c|c|c|c|c|c|c|c|c|}
\hline 'MGC & 9 & 55610.70 & 75372.10 & 217.3 & 237.3 & 229.55 & 0.29 & 1.5027 & 4 & \\
\hline 'MGC & 11 & 55770.70 & 75252.30 & 219.2 & 239.2 & 233.42 & 0.81 & 2.9413 & 4 & \\
\hline ' MGC & 19 & 56408.70 & 74770.10 & 230.6 & 234.6 & 232.22 & 0.39 & 2.0527 & 4 & \\
\hline 'MGC & 23 & 56726.60 & 74528.30 & 227.9 & 247.9 & 234.29 & 1.11 & 4.4616 & 4 & \\
\hline 'MGC & 32 & 57448.80 & 73982.10 & 232.0 & 252.0 & 245.08 & 0.37 & 1.8926 & 5 & \\
\hline 'MGC & 36 & 57776.00 & 73738.90 & 234.4 & 254.4 & 236.08 & 0.38 & 1.9326 & 5 & \\
\hline - MGE & 9 & 55489.40 & 75215.10 & 218.1 & 238.1 & 227.47 & 0.77 & 3.9126 & 4 & \\
\hline 'MGE & 21 & 56446.20 & 74487.80 & 227.9 & 247.9 & 230.59 & 1.16 & 5.4322 & 4 & \\
\hline 'MGE & 30 & 57175.40 & 73935.80 & 229.3 & 249.3 & 236.14 & 0.64 & 3.1825 & 4 & \\
\hline 'MGE & 34 & 57495.10 & 73695.00 & 237.2 & 257.2 & 241.03 & 1.23 & 5.3419 & 5 & \\
\hline 'MGG & 15 & 55851.50 & 74699.00 & 223.3 & 243.3 & 232.82 & 1.20 & 4.9717 & 4 & \\
\hline 'MGG & 19 & 56174.30 & 74456.00 & 226.0 & 246.0 & 231.38 & 1.03 & 4.8122 & 4 & \\
\hline 'MGG & 23 & 56491.80 & 74214.00 & 227.1 & 247.1 & 232.19 & 1.25 & 5.8622 & 4 & \\
\hline ' MGG & 36 & 57541.70 & 73413.00 & 232.5 & 252.5 & 238.45 & 0.82 & 4.1225 & 5 & \\
\hline 'NBG & 1 & 53879.30 & 79300.40 & 200.9 & 232.3 & 224.47 & 0.18 & 1.0635 & 5 & \\
\hline 'NBG & 2 & 53958.40 & 79099.80 & 203.6 & 233.6 & 224.96 & 0.18 & 1.0634 & 5 & \\
\hline 'NBG & 3 & 54068.10 & 78939.60 & 202.1 & 233.5 & 217.54 & 0.36 & 2.0833 & 5 & \\
\hline 'NBG & 4 & 54329.20 & 78942.10 & 196.1 & 227.5 & 217.05 & 0.31 & 1.8034 & 6 & ? \\
\hline 'NBG & 5 & 54515.60 & 78943.40 & 194.9 & 226.4 & 217.77 & 0.35 & 2.0634 & 6 & ? \\
\hline 'NPM & 1 & 56851.60 & 62153.40 & 257.1 & 277.1 & 287.37 & 0.67 & 2.2311 & 5 & \\
\hline 'NPM & 2 & 58252.00 & 63056.80 & 244.2 & 264.2 & 271.77 & 0.74 & 2.5812 & 5 & \\
\hline 'NPM & 3 & 55417.60 & 62109.20 & 247.2 & 267.2 & 274.61 & 0.63 & 2.1712 & 5 & \\
\hline 'NPM & 4 & 57215.00 & 60883.20 & 256.7 & 276.7 & 284.21 & 0.67 & 2.3312 & 5 & \\
\hline 'NPM & $4 D D$ & 57218.80 & 60893.10 & 296.4 & 306.4 & 305.60 & 1.40 & 4.6411 & 5 & \\
\hline 'NPM & $19 \mathrm{~A}$ & 57551.80 & 62970.70 & 248.2 & 268.2 & 270.74 & 0.74 & 2.5612 & 5 & \\
\hline 'NPM & $19 \mathrm{~B}$ & 57558.30 & 62981.80 & 217.7 & 227.7 & 268.88 & 0.73 & 2.5212 & 4 & \\
\hline 'NPM & $19 \mathrm{C}$ & 57575.40 & 62977.10 & 193.5 & 203.5 & 268.05 & 0.72 & 2.4812 & 3 & \\
\hline 'NPM & $19 D$ & 57567.90 & 62960.90 & 97.5 & 107.5 & 243.32 & 0.44 & 1.5312 & 2 & \\
\hline 'NPM & $19 E$ & 57582.60 & 62991.70 & 33.9 & 43.9 & 188.33 & 0.84 & 2.9212 & 1 & \\
\hline 'NPM & $34 \mathrm{~A}$ & 56301.20 & 60774.50 & 279.8 & 289.8 & 290.75 & 0.68 & 2.3512 & 5 & \\
\hline 'NPM & $34 \mathrm{~B}$ & 56315.10 & 60768.90 & 225.6 & 235.6 & 271.12 & 0.53 & 1.8512 & 5 & \\
\hline 'NPM & $34 \mathrm{C}$ & 56329.10 & 60764.20 & 181.8 & 191.8 & 267.71 & 0.53 & 1.8312 & 3 & \\
\hline 'NPM & $34 \mathrm{D}$ & 56354.90 & 60752.00 & 86.4 & 96.4 & 253.85 & 0.47 & 1.6212 & 2 & \\
\hline 'NPM & $34 \mathrm{E}$ & 56342.80 & 60758.80 & 33.1 & 43.1 & 187.16 & 0.19 & 0.6612 & 1 & \\
\hline ' PAC & 1 & 66753.40 & 43543.30 & 253.9 & 283.9 & 284.74 & 0.22 & 1.2732 & 5 & \\
\hline - PAC & 2 & 66980.90 & 43527.70 & 247.9 & 277.9 & 271.02 & 0.29 & 1.6431 & 5 & \\
\hline - PAC & 3 & 66861.40 & 43585.60 & 252.9 & 282.9 & 271.35 & 0.38 & 2.1532 & 5 & \\
\hline ' PAC & 4 & 66863.20 & 43495.40 & 250.6 & 280.6 & 284.42 & 0.17 & 0.9231 & 5 & \\
\hline ' PAC & 5 & 66907.10 & 43561.70 & 255.1 & 275.1 & 275.05 & 0.48 & 2.5629 & 5 & \\
\hline ' PAC & 6 & 66894.70 & 43580.10 & 255.2 & 275.2 & 274.58 & 0.38 & 2.0228 & 5 & \\
\hline ' $\mathrm{PCB}$ & $1 \mathrm{~A}$ & 65070.60 & 41988.20 & 263.5 & 293.5 & 280.71 & 0.50 & 2.7029 & 5 & \\
\hline - PCB & $2 \mathrm{~A}$ & 64891.40 & 41821,40 & 257.8 & 287.8 & 279.52 & 0.49 & 2.6529 & 5 & \\
\hline ' PCB & $3 A$ & 64706.30 & 42036.00 & 262.7 & 292.7 & 281.55 & 0.50 & 2.8232 & 5 & \\
\hline ' $\mathrm{PCB}$ & $4 \mathrm{~A}$ & 64901.40 & 42171.00 & 262.9 & 292.9 & 279.71 & 0.47 & 2.5630 & 5 & \\
\hline ' PDB & 2 & 64743.10 & 43513.10 & 247.7 & 268.7 & 278.00 & 0.38 & 2.3639 & 4 & \\
\hline ' PDB & 3 & 64938.20 & 43542.20 & 248.1 & 269.1 & 278.27 & 0.37 & 2.3039 & 4 & \\
\hline ' PDB & 4 & 64623.80 & 43455.10 & 266.2 & 286.2 & 279.16 & 0.40 & 1.4413 & 5 & \\
\hline - PDB & 5 & 64584.40 & 44106.60 & 264.2 & 284.2 & 277.79 & 0.33 & 1.1913 & 5 & \\
\hline 'PRP & $1 \mathrm{~A}$ & 63032.70 & 45349.80 & 232.9 & 262.9 & 249.50 & 0.47 & 2.7033 & 4 & \\
\hline ' PRP & 2 & 63229.00 & 45389.50 & 234.1 & 264.1 & 255.57 & 0.78 & 4.5834 & 4 & \\
\hline 'PRP & 3 & 63165.50 & 45200.70 & 228.6 & 258.6 & 255.97 & 0.66 & 3.8033 & 4 & \\
\hline 'PRP & 4 & 63345.90 & 45268.90 & 232.9 & 262.9 & 257.79 & 0.43 & 2.5435 & 4 & \\
\hline 'PSB & $1 \mathrm{~A}$ & 64141.40 & 43619.30 & 257.4 & 287.4 & 276.87 & 0.46 & 3.0042 & 5 & \\
\hline 'PSB & $2 \mathrm{~A}$ & 63916.50 & 43612.40 & 257.2 & 287.2 & 276.72 & 0.48 & 3.0842 & 5 & \\
\hline 'PSB & $3 A$ & 63590.40 & 43599.80 & 256.5 & 286.5 & 275.57 & 0.50 & 3.2242 & 5 & \\
\hline 'PSB & $4 \mathrm{~A}$ & 63347.00 & 43534.20 & 255.5 & 285.5 & 274.83 & 0.54 & 3.5743 & 5 & \\
\hline ' PSB & $5 \mathrm{~A}$ & 63606.50 & 43440.50 & 262.3 & 292.3 & 276.11 & 0.52 & 3.4043 & 5 & \\
\hline 'PSB & $6 \mathrm{~A}$ & 63975.70 & 43436.00 & 262.1 & 292.1 & 277.59 & 0.49 & 3.1542 & 5 & \\
\hline 'PSB & $7 \mathrm{~A}$ & 64301.00 & 43553.30 & 259.0 & 289.0 & 277.46 & 0.45 & $2.97 \quad 43$ & 5 & \\
\hline 'PSS & $1 D$ & 75773.30 & 37298.40 & 182.1 & 202.1 & 198.15 & 0.69 & 3.6929 & 6 & ? \\
\hline 'PSS & $2 \mathrm{D}$ & 75910.10 & 36037.90 & 177.1 & 197.1 & 195.25 & 0.67 & 3.5328 & 6 & ? \\
\hline ' RSS & $3 D$ & 76138.70 & 35974.10 & 178.5 & 198.5 & 198.73 & 1.43 & 5.7116 & 6 & ? \\
\hline ' $P W$ & $83 \mathrm{~N}$ & 52202.00 & 61394.00 & 4.0 & 9.0 & 168.43 & -1.00 & -1.001 & 1 & \\
\hline ' RAC & 1 & 74570.70 & 55107.30 & 247.3 & 277.3 & 273.98 & 0.33 & 1.8531 & 5 & \\
\hline ' RAC & 2 & 74555.50 & 55026.30 & 243.4 & 273.4 & 272.59 & 0.23 & 1.2430 & 5 & \\
\hline 'RAC & 3 & 74667.50 & 55015.30 & 242.3 & 272.3 & 272.37 & 0.26 & 1.4731 & 5 & \\
\hline ' RAC & 4 & 74588.80 & 54984.00 & 238.2 & 268.2 & 271.71 & 0.30 & 1.6530 & 5 & \\
\hline 'RBW & ICL & 74227.40 & 62038.50 & 105.5 & 115.5 & 255.93 & 0.37 & 0.643 & 2 & \\
\hline
\end{tabular}


Table F-1. Hydraulic Head Targets for Model Calibration (Continued)

\begin{tabular}{|c|c|c|c|}
\hline 'RBW & $1 \mathrm{CU}$ & & 74214.00 \\
\hline 'RBW & ID & & 74237.50 \\
\hline 'RBW & $2 \mathrm{CL}$ & & 71795.10 \\
\hline 'RBW & $2 \mathrm{CU}$ & & 71785.90 \\
\hline 'RBW & $2 \mathrm{D}$ & & 71776.70 \\
\hline ' RCP & $1 \mathrm{~A}$ & ' & 74238.30 \\
\hline ' $\mathrm{RCI}$ & ID & ' & 74223.50 \\
\hline ' RDB & ID & ' & 844.50 \\
\hline ' RDB & $2 \mathrm{D}$ & ' & 74782.20 \\
\hline 'RDB & $3 \mathrm{D}$ & ' & 74899.00 \\
\hline ' RPC & $1 \mathrm{CL}$ & & 74261.86 \\
\hline 'RPC & 1D & & 74215.65 \\
\hline 'RPC & 7DL & & 74726.38 \\
\hline ' RPC & $7 D U$ & & 4720.18 \\
\hline ' RPC & 8DL & & 4671.66 \\
\hline ' RPC & $8 \mathrm{DU}$ & & 74664.76 \\
\hline 'RPC & 9DL & & 74507.87 \\
\hline 'RPC & 9DU & & 74507.71 \\
\hline 'RPC & 10DL & & 74551.49 \\
\hline 'RPC & 10DU & & 74540.11 \\
\hline ' RPC & 11DL & & 75240.08 \\
\hline ' RPC & 11DU & & 75250.01 \\
\hline 'RRP & 1 & ' & 75634.60 \\
\hline 'RRP & 2 & ' & 75829.80 \\
\hline 'RRP & 3 & ' & 75853.00 \\
\hline 'RRP & 4 & ' & 75723.30 \\
\hline 'RSB & 7 & ' & 75044.30 \\
\hline 'RSB & 8 & 1 & 75178.20 \\
\hline ' RSC & 2 & - & 74378.60 \\
\hline 'RSC & 3 & ' & 74699.70 \\
\hline SSC & 9 & ' & 74565.30 \\
\hline 'RSD & 1 & ' & 75035.10 \\
\hline 'RSD & 3 & ' & 74702.30 \\
\hline 'RSD & 4 & ' & 75154.60 \\
\hline 'RSD & 5 & ' & 75207.00 \\
\hline 'RSD & 6 & ' & 75256.60 \\
\hline 'RSD & 7 & ' & 75178.40 \\
\hline 'RSD & 8 & ' & 75229.60 \\
\hline 'RSD & 9 & ' & 75185.90 \\
\hline RSE & $1 \mathrm{~A}$ & ' & 74712.70 \\
\hline 'RSE & 1B & ' & 74698.10 \\
\hline RSE & IC & ' & 74684.10 \\
\hline 'RSE & 2 & ' & 74743.50 \\
\hline 'RSE & $3 A$ & ' & 74931.20 \\
\hline 'RSE & $4 \mathrm{~A}$ & ' & 75101.10 \\
\hline RSE & 7 & ' & 3.70 \\
\hline RSE & 8 & ' & 74869.40 \\
\hline 'RSE & 9 & ' & 74971.10 \\
\hline 'RSE & 10 & ' & 74848.30 \\
\hline 'RSE & 11 & ' & 74787.70 \\
\hline ' RSE & 12 & , & 74842.30 \\
\hline RSE & 18 & ' & 74839.50 \\
\hline 'RSE & 19 & ' & 74791.20 \\
\hline 'RSE & 24 & ' & 74638.90 \\
\hline 'RSE & 25 & , & 74544.50 \\
\hline 'RSF & 1 & ' & 4869.40 \\
\hline RSF & 2 & ' & 74628.60 \\
\hline 'RSF & 3 & - & 75206.70 \\
\hline 'RSP & ID & ' & 74426.80 \\
\hline ' RSP & $2 \mathrm{D}$ & ' & 75568.60 \\
\hline 'SBG & 1 & ' & 63749.10 \\
\hline SBG & 2 & , & 64939.60 \\
\hline ' SBG & 3 & ' & 65265.60 \\
\hline ' SBG & 4 & ' & 65010.20 \\
\hline ' SBG & 5 & ' & 64499.00 \\
\hline 'SBG & 6 & ' & 63860.00 \\
\hline & 2 & ' & 64697.10 \\
\hline
\end{tabular}

\begin{tabular}{|c|c|c|}
\hline 2047.40 & 156.1 & 166 \\
\hline 62031.60 & 243.0 & 263.1 \\
\hline 58712.00 & 96.4 & 106.4 \\
\hline 58715.40 & 145.1 & 55 \\
\hline 58719.90 & 284.9 & 304 \\
\hline 56968.10 & 46.8 & \\
\hline 967.90 & 261.3 & 281.3 \\
\hline 57097.30 & 265.5 & 285 \\
\hline 56879.80 & 265.7 & 285.7 \\
\hline 56881.90 & 265.8 & 285.8 \\
\hline 57923.24 & 103.3 & 113.3 \\
\hline 57931.26 & 264.5 & 284.5 \\
\hline 3812.32 & 209.9 & 219.9 \\
\hline 303.87 & 240.8 & 277 \\
\hline 58276.90 & 204.1 & 21 \\
\hline 58279.13 & 273.0 & 288.0 \\
\hline 57908.37 & 216.4 & 226 \\
\hline 57898.35 & 268.3 & 283.3 \\
\hline 57380.20 & 200.5 & 210.5 \\
\hline 57380.29 & 272.5 & 287 \\
\hline $\begin{array}{l}57380.02 \\
57380.43\end{array}$ & $\begin{array}{l}180.2 \\
271.2\end{array}$ & \\
\hline 54563.50 & 242.4 & 272.4 \\
\hline 54468.30 & 242.5 & 272.5 \\
\hline 54303.00 & 238.1 & 268.1 \\
\hline 54294.50 & 238.3 & 268.3 \\
\hline 57692.80 & 272.7 & 292.6 \\
\hline 57612.90 & 274.3 & 294.3 \\
\hline 58543.00 & 261.9 & 281.9 \\
\hline 58724.70 & 258.6 & 278.6 \\
\hline 59241.20 & 251.6 & 271 \\
\hline 57440.80 & 267.9 & 287.7 \\
\hline 57451.60 & 269.3 & 289.1 \\
\hline 57441.40 & 270.6 & 290.6 \\
\hline 57439.90 & 269.6 & 289.6 \\
\hline 57441.30 & 270.1 & 290 \\
\hline 57394.30 & 267.3 & 287.3 \\
\hline 57394.00 & 267.3 & 287.3 \\
\hline 57245.60 & 251.7 & 271.7 \\
\hline 57734.50 & 274.8 & 294.8 \\
\hline 57731.40 & 275.7 & \\
\hline 57730.80 & 268.5 & 288.5 \\
\hline 57594.90 & 269.7 & 289.5 \\
\hline 57445.80 & 268.2 & 288.0 \\
\hline 57528.40 & 260.6 & 270.6 \\
\hline 58481.50 & 266.5 & \\
\hline 58538.80 & 271.2 & 291. \\
\hline 58463.30 & 266.7 & 286.7 \\
\hline 58420.70 & 270.7 & 290.5 \\
\hline 58357.60 & 262.1 & 272. \\
\hline 58318.20 & 259.1 & 269 \\
\hline 58247.20 & 268.1 & 288.1 \\
\hline 58318.40 & 262.5 & 282.5 \\
\hline 57370.40 & 237.6 & 257.6 \\
\hline 55824.50 & 237.5 & 257.5 \\
\hline 58505.30 & 228.8 & \\
\hline 57670.40 & 224.8 & 235.3 \\
\hline 57621.40 & 229.8 & 239.8 \\
\hline 56879.40 & 274.7 & 289.7 \\
\hline 55947.10 & 260.3 & 280.3 \\
\hline 74619.40 & 190.7 & 220.7 \\
\hline 74570.20 & 205.9 & \\
\hline 73699.90 & 206.6 & 236 \\
\hline 72399.80 & 185.6 & 215.6 \\
\hline 72208.30 & 199.4 & 219 \\
\hline 73599.30 & 208.1 & \\
\hline 73850. & 215.9 & \\
\hline
\end{tabular}

255.80

259.01

269.44

269.85

297.55

194.29

281.78

286.02

285.28

282.97

256.90

276.77

274.90

275.68

278.92

290.58

279.10

280.96

280.56

290.42

278.49

289.45

265.61

264.76

264.33

264.37

286.01

288.00

278.28

276.72

271.79

286.45

286.84

288.51

287.18

287.07

285.23

285.51

283.77

288.55

288.84

288.72

286.72

285.20

286.61

280.80

284.00

279.23

281.74

282.84

276.42

279.61

280.91

279.63

275.41

277.64

278.38

279.63

289.71

278.40

237.91

237.86

237.08

241.00

249.51

244.56

242.49
0.40

0.45

0.53

0.57

0.72

0.20

0.64

0.24

0.21

0.30

0.17

0.16

0.67

0.57

0.92

1.11

0.54

0.87

0.10

0.54

0.00

0.85

0.65

0.91

0.67

0.82
0.87

0.73

0.79

0.60

0.72

0.52

0.55

0.47

0.46

0.44
0.34

0.75

0.99

1.11

1.09

0.75

0.63

0.86

1.32

1.14

1.35

0.49

0.74

1.14

0.57

0.47

0.68

0.60

0.64

1.19

$-1.00$

0.29

0.33

0.47

0.27

0.31

0.32

0.36
0.693

0.783

0.913

0.993

1.253

0.6711

2.2012

1.2627

1.0727

1.5627

0.303

0.283

0.952

0.812

1.292

1.562

0.992

0.762

1.222

0.142

0.762

0.012

4.7631

3.4128

5.1432

3.6530

4.4129

4.2424

3.4222

4.2923

3.6321

3.3231

4.0632

2.7328

2.8928

2.5028

2.4629

2.3328

1.7526

4.4335

5.3229

5.8828

5.7628

4.0329

3.4129

4.7931

6.9928

4.1529

5.9327

6.9927

1.6912

3.8427

5.9227

3.0028

2.5629

3.6629

3.2529

3.3928

1.682

$-1.001$

1.5227

1.7629

2.6031

1.3927

1.6227

1.7429

1.7022

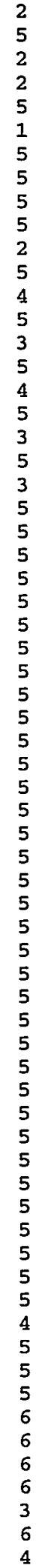


Table F-1. Hydraulic Head Targets for Model Calibration (Continued)

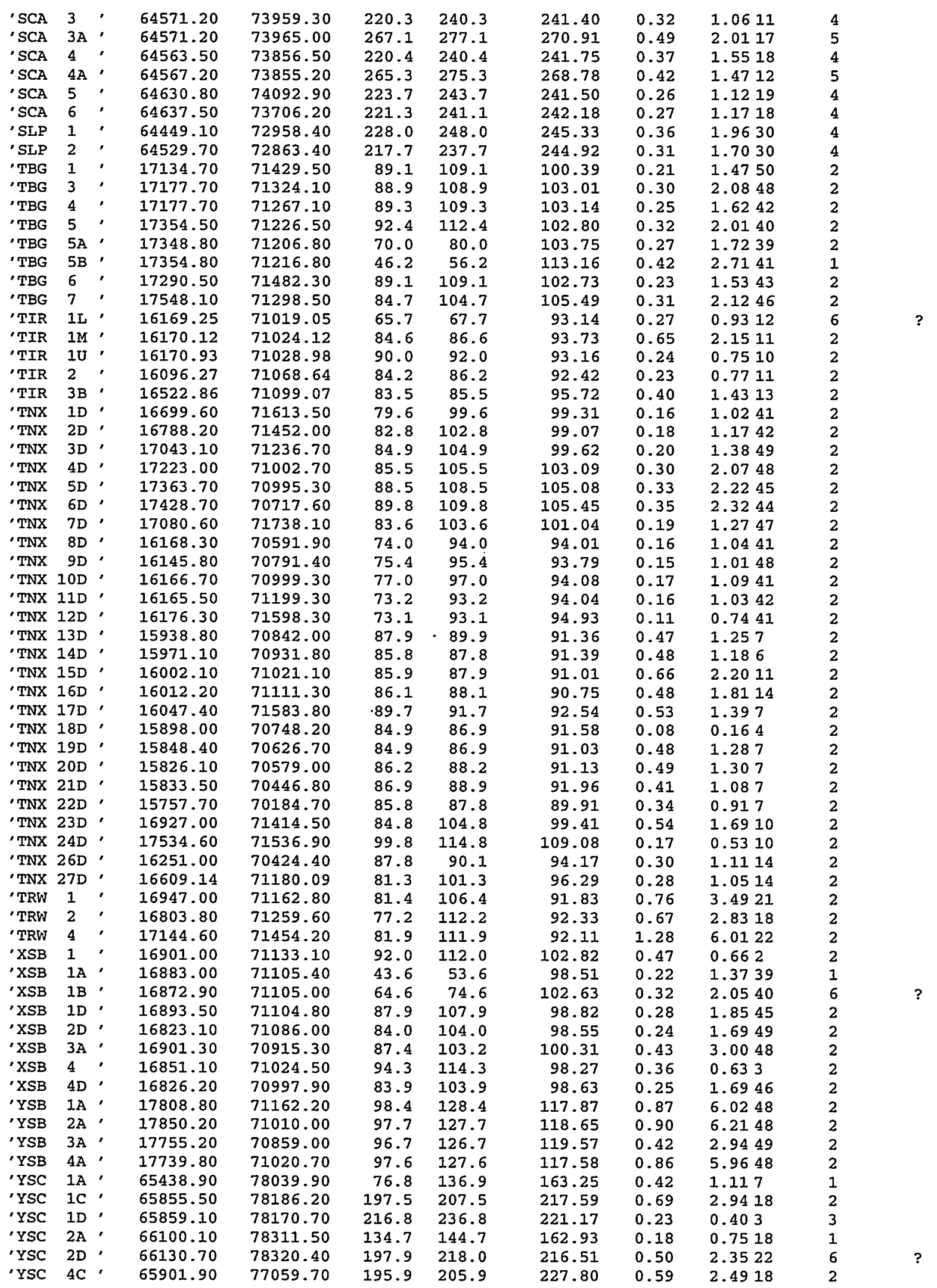


Table F-1. Hydraulic Head Targets for Model Calibration (Continued)

\begin{tabular}{|c|c|c|c|c|c|c|c|c|c|c|c|}
\hline 'YSC & $5 A$ & ' & 67134.90 & 74295.90 & 116.0 & 121.0 & 181.23 & 1.18 & 5.0218 & & 1 \\
\hline ' $\mathrm{z}$ & 2 & ' & 53181.60 & 74785.30 & 214.0 & 214.5 & 219.42 & 0.47 & 1.7614 & & 3 \\
\hline ' $\mathrm{z}$ & 3 & ' & 51328.30 & $75086: 20$ & 206.6 & 207.1 & 212.57 & 0.71 & 2.028 & & 3 \\
\hline ' & 8 & , & 51584.90 & 76640.50 & 213.6 & 214.1 & 219.33 & 0.55 & 2.1415 & & 3 \\
\hline ' & 9 & ' & 50570.50 & 77732.00 & 207.5 & 227.5 & 215.04 & 0.43 & 2.1926 & & 4 \\
\hline ' & 12 & ' & 61400.90 & 71198.90 & 251.3 & 251.8 & 274.33 & 0.22 & 0.536 & & 5 \\
\hline 'z & 13 & ' & 62203.60 & 70785.80 & 256.6 & 257.1 & 276.13 & 0.79 & 2.9514 & & 5 \\
\hline ' & 17 & , & 43797.80 & 72260.90 & 148.2 & 148.7 & 169.52 & 0.31 & 1.1514 & & 2 \\
\hline$' \mathrm{z}$ & 18 & ' & 43774.10 & 73077.20 & 159.9 & 160.4 & 184.80 & 0.58 & 2.1714 & & ? \\
\hline ' $z$ & 20 & ' & 43722.40 & 74080.70 & 173.4 & 193.4 & 184.74 & 0.36 & 0.805 & & 3 \\
\hline 2 & $20 B$ & , & 43721.00 & 74085.00 & 175.6 & 195.6 & 191.15 & 0.85 & 2.8311 & & 3 \\
\hline ' ZBG & 1 & , & 65584.10 & 76584.20 & 220.0 & 240.1 & 234.17 & 0.48 & 3.0239 & & 3 \\
\hline ' ZBG & 2 & ' & 67472.90 & 76170.50 & 210.9 & 230.9 & 222.03 & 0.41 & 2.6241 & & ? \\
\hline ' ZDT & 1 & ' & 65114.80 & 71644.40 & 227.0 & 247.0 & 239.91 & 0.15 & 0.8836 & & 4 \\
\hline 'ZDT & 2 & ' & 65059.90 & 71696.50 & 225.1 & 245.1 & 241.45 & 0.16 & 0.9736 & & 4 \\
\hline ' ZW & 2 & ' & 54388.70 & 80701.50 & 194.8 & 204.8 & 207.29 & 0.58 & 2.4518 & & 3 \\
\hline ' $\mathrm{ZW}$ & 3 & ' & 57078.20 & 80746.50 & 194.6 & 205.1 & 200.78 & 0.42 & 1.8419 & & ? \\
\hline$' \mathrm{ZW}$ & 4 & ' & 56556.90 & 77667.40 & 229.2 & 239.7 & 232.31 & 0.47 & 2.0319 & & 5 \\
\hline ' $\mathrm{ZW}$ & 5 & ' & 54708.60 & 75767.40 & 221.0 & 231.0 & 227.40 & 0.27 & 1.3023 & & 4 \\
\hline ' $2 W$ & 6 & ' & 52030.80 & 76166.00 & 216.7 & 227.2 & 220.13 & 0.78 & 3.2017 & & 4 \\
\hline ' $\mathrm{ZW}$ & 7 & ' & 60300.70 & 72399.50 & 254.5 & 264.8 & 265.84 & 0.36 & 1.5017 & & 5 \\
\hline ' $\mathrm{ZW}$ & 8 & ' & 63801.50 & 70800.80 & 254.1 & 264.1 & 270.85 & 0.21 & 0.9118 & & 5 \\
\hline ' $\mathrm{zW}$ & 9 & , & 61400.30 & 73198.40 & 242.4 & 252.4 & 251.97 & 0.45 & 1.9318 & & 5 \\
\hline ' $\mathrm{ZW}$ & 10 & ' & 63401.00 & 73212.40 & 242.2 & 252.2 & 249.72 & 0.86 & 4.1323 & & 5 \\
\hline avg & sigm: & & $399174 \mathrm{nu}$ & 1307 & & & & & & & \\
\hline ' CMP & $10 \mathrm{C}$ & ' & 53994.30 & 51402.70 & 179.6 & 189.6 & 198.53 & -1.00 & -1.001 & & 2 \\
\hline ' CMP & 1OD & - & 53994.30 & 51392.50 & 209.6 & 229.6 & 229.84 & -1.00 & -1.001 & & 3 \\
\hline ' CMP & 11D & ' & 53647.00 & 51467.90 & 209.5 & 229.9 & 223.34 & -1.00 & -1.001 & & 3 \\
\hline 'CMP & 14D & - & 52589.50 & 52363.50 & 204.1 & 224.5 & 217.43 & -1.00 & -1.001 & & 3 \\
\hline 'CMP & $15 \mathrm{C}$ & ' & 52907.80 & 51361.40 & 220.6 & 250.6 & 244.53 & -1.00 & -1.001 & & 5 \\
\hline 'CMP & $30 \mathrm{~B}$ & ' & 53166.90 & 51729.80 & 97.4 & 107.5 & 195.00 & $-1: 00$ & -1.001 & & 2 \\
\hline 'CMP & $30 \mathrm{c}$ & , & 53208.20 & 51718.40 & 179.5 & 189.5 & 210.55 & -1.00 & -1.001 & - & 2 \\
\hline 'CMP & $30 D$ & - & 53202.90 & 51709.70 & 211.6 & 231.6 & 227.97 & -1.00 & -1.001 & & 3 \\
\hline ' CMP & $31 \mathrm{C}$ & , & 53255.70 & 52389.70 & 197.9 & 207.9 & 210.78 & -1.00 & -1.001 & & 6 \\
\hline ' CMP & $32 \mathrm{~B}$ & , & 54052.80 & 52220.00 & 97.7 & 107.7 & 195.31 & -1.00 & -1.001 & & 2 \\
\hline 'CMP & $32 \mathrm{C}$ & ' & 54061.10 & 52214.60 & 185.2 & 195.2 & 195.44 & -1.00 & -1.001 & & 2 \\
\hline 'CMP & $32 \mathrm{D}$ & - & 54069.20 & 52209.20 & 218.6 & 228.6 & 220.77 & -1.00 & -1.001 & & 6 \\
\hline 'NPM & 2 & , & 58252.00 & 63056.80 & 244.2 & 264.2 & 267.00 & -1.00 & -1.001 & & 5 \\
\hline 'NPM & 3 & ' & 55417.60 & $62109: 20$ & 247.2 & 267.2 & 267.60 & -1.00 & -1.001 & & 5 \\
\hline 'NPM & 4 & ' & 57215.00 & 60883.20 & 256.7 & 276.7 & 272.70 & -1.00 & -1.001 & & 5 \\
\hline 'NPN & 1 & ' & 70879.60 & 66661.40 & 257.3 & 277.4 & 277.50 & -1.00 & -1.001 & & 4 \\
\hline 'NPN & 2 & ' & 72541.50 & 67394.10 & 257.9 & 278.0 & 273.50 & -1.00 & -1.001 & & 4 \\
\hline 'NPN & 3 & ' & 70029.20 & 67989.80 & 260.0 & 280.1 & 276.70 & -1.00 & -1.001 & & 4 \\
\hline 'NPN & 4 & ' & 71021.80 & 65357.20 & 265.4 & 285.5 & 278.50 & -1.00 & -1.001 & & 5 \\
\hline 'NTN & 1 & ' & 45562.30 & 56993.70 & 212.4 & 232.4 & 233.70 & -1.00 & -1.001 & & 5 \\
\hline 'NTN & 2 & ' & 46735.10 & 57935.50 & 207.2 & 227.2 & 235.20 & -1.00 & -1.001 & & 5 \\
\hline 'NTS & 1 & ' & 43893.90 & 46082.00 & 164.3 & 184.4 & 180.40 & -1.00 & -1.001 & & 3 \\
\hline 'NTS & 2 & ' & 45825.20 & 46262.60 & 174.7 & 194.8 & 192.30 & -1.00 & -1.001 & & 4 \\
\hline 'NTW & 1 & ' & 40257.70 & 48776.50 & 168.9 & 188.8 & 183.60 & -1.00 & -1.001 & & 3 \\
\hline 'NTW & 2 & ' & 39353.20 & 49309.30 & 171.5 & 191.5 & 183.90 & -1.00 & -1.001 & & 3 \\
\hline 'NTW & 3 & ' & 41208.70 & 50040.00 & 176.7 & 196.6 & 191.80 & -1.00 & -1.001 & & 3 \\
\hline 'NTW & 4 & ' & 41678.20 & 48636.30 & 166.0 & 185.8 & 180.40 & -1.00 & -1.001 & & 3 \\
\hline$p$ & $13 \mathrm{~A}$ & , & 60000.00 & 35600.00 & -67.3 & -57.4 & 173.07 & 0.17 & 1.2456 & & 1 \\
\hline 'P & $13 B$ & ' & 60000.00 & 35600.00 & -7.2 & 3.0 & 175.65 & 0.22 & 1.6456 & & 1 \\
\hline 'P & $15 \mathrm{~A}$ & - & 51376.30 & 46755.30 & -97.0 & -87.0 & 176.77 & 0.23 & 1.6351 & & 6 \\
\hline 'p & $18 \mathrm{~A}$ & , & 47688.10 & 67592.80 & 12.0 & 22.0 & 168.48 & 0.16 & 1.2764 & & 1 \\
\hline 'P & $18 \mathrm{~B}$ & ' & 47680.90 & 67578.90 & 67.0 & 77.0 & 169.01 & 0.16 & 1.3064 & & 1 \\
\hline 'P & $19 \mathrm{~A}$ & - & 60031.30 & 55347.10 & -36.7 & -26.7 & 186.83 & -1.00 & $1.60-1$ & & 1 \\
\hline 'P & $21 B$ & , & 40757.60 & 24641.80 & -82.2 & -72.2 & 134.01 & 0.16 & 1.2964 & & 1 \\
\hline 'P & $23 \mathrm{~A}$ & ' & 30914.50 & 48114.90 & -38.8 & -28.8 & 146.11 & 0.17 & 1.4469 & & 6 \\
\hline 'P & $23 B$ & - & 30925.30 & 48101.20 & 41.5 & 46.5 & 137.85 & 0.19 & 1.5869 & & 1 \\
\hline P & $24 \mathrm{~A}$ & - & 66569.70 & 43142.20 & -1.9 & 8.9 & 191.47 & -1.00 & $1.00-1$ & & 1 \\
\hline P & $25 B$ & ' & 42241.90 & 52521.40 & 80.6 & 90.6 & 178.24 & 0.65 & 5.1764 & & 1 \\
\hline 'P & $26 \mathrm{~A}$ & ' & 18055.90 & 72010.40 & 22.0 & 32.0 & 117.48 & 0.33 & 2.7369 & & 1 \\
\hline ' $P$ & $27 B$ & ' & 64000.30 & 70405.90 & 74.8 & 94.8 & 179.76 & 0.15 & 1.1862 & & 1 \\
\hline 'RGW & $3 c$ & , & 74059.23 & 53473.51 & 137.6 & 147.6 & 255.11 & -1.00 & -1.001 & & 2 \\
\hline 'RGW & $3 D$ & ' & 74067.32 & 53481.33 & 217.2 & 227.2 & 265.49 & -1.00 & -1.001 & & 4 \\
\hline
\end{tabular}


Table F-1. Hydraulic Head Targets for Model Calibration (Continued)

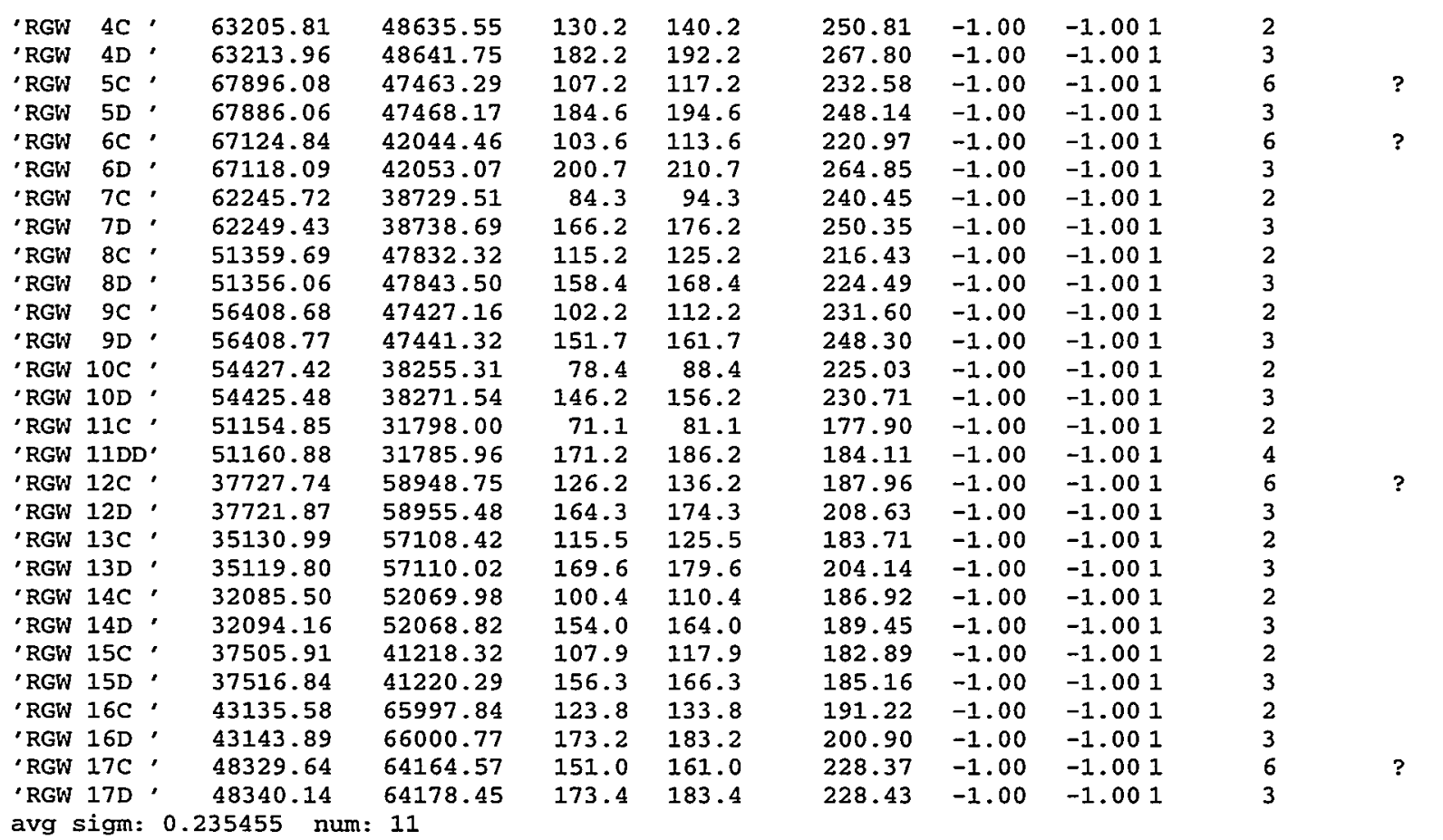




\section{Hydraulic Head Residuals}

The complete contents of the FACT code observation well file are listed in Table F-2. FACT does not compute a hydraulic head for wells outside of the saturated zone and model domain (model area). Wells outside the model area. are denoted by a simulated head of " 0.0 " in Table F-2. The associated (large negative) residual is meaningless, and ignored in the calculation of summary statistics.

Table F-2. Summary of Group Statistical Parameters

\begin{tabular}{|c|c|c|c|c|c|c|c|c|c|c|}
\hline \multicolumn{2}{|c|}{ Group St } & \multicolumn{6}{|c|}{ stati } & \multicolumn{2}{|c|}{5.45} & \\
\hline **G & GOUP & 1 & ** & \multirow{2}{*}{$\begin{array}{l}\text { rms of } \\
\text { avg of } \\
\text { avg of } \\
\text { max of } \\
566.62\end{array}$} & \multirow{2}{*}{$\begin{array}{c}\text { (FACT-data) } \\
\text { (FACT-data) } \\
\text { |FACT-data) } \\
\text { \{FACT-data\} } \\
55791.31\end{array}$} & \multicolumn{2}{|c|}{$\begin{array}{l}\text { differences: } \\
\text { differences: } \\
\text { differences: } \\
\text { differences: }\end{array}$} & \multicolumn{2}{|c|}{$\begin{array}{r}3.366 \\
-0.737 \\
1.983 \\
-16.208\end{array}$} & \\
\hline "BGO & $3 \mathrm{~A}$ & $"$ & 57 & & & 103.7 & 113.7 & 163.0 & 163.5 & 0.5 \\
\hline "BGO & $6 \mathrm{~A}$ & $"$ & 57 & 379.75 & 56798.46 & 107.5 & 117.5 & 159.3 & 159.9 & 0.6 \\
\hline " BGO & $8 \mathrm{~A}$ & $"$ & 56 & 713.52 & 57023.70 & 105.3 & 115.3 & 161.0 & 159.1 & -1.9 \\
\hline " BGO & $8 \mathrm{AR}$ & & 56 & 718.93 & 57053.02 & 94.6 & 104.6 & 160.9 & 159.1 & -1.8 \\
\hline "BGO & $9 A A$ & & & 557.06 & 57472.74 & 73.8 & 83.8 & 158.0 & 157.8 & -0.2 \\
\hline " BGO & $10 \mathrm{~A}$ & & & 207.63 & 57372.71 & 111.1 & 121.1 & 170.7 & 157.9 & -12.8 \\
\hline " BGO & $10 \mathrm{AA}$ & & & 188.61 & 57573.76 & 80.8 & 90.8 & 157.6 & 157.4 & -0.2 \\
\hline " BGO & $10 \mathrm{AR}$ & & 56 & 220.41 & 57370.81 & 96.5 & 106.5 & 158.5 & 158.0 & -0.5 \\
\hline "BGO & $12 \mathrm{AR}$ & & & 433.62 & 57535.80 & 99.3 & 109.3 & 157.8 & 157.3 & -0.5 \\
\hline "BGO & $12 \mathrm{AX}$ & & 55 & 438.21 & 57566.51 & 99.5 & 109.5 & 157.4 & 157.2 & -0.2 \\
\hline "BGO & $14 \mathrm{~A}$ & $"$ & & 932.60 & 57206.47 & 109.6 & 119.6 & 157.3 & 158.0 & 0.7 \\
\hline "BGO & $14 \mathrm{AR}$ & & 54 & 878.94 & 57191.60 & 96.8 & 106.8 & 159.1 & 158.1 & -1.0 \\
\hline "BGO & $16 \mathrm{~A}$ & $"$ & 55 & 151.72 & 56525.53 & 102.5 & 112.5 & 161.0 & 160.0 & -1.0 \\
\hline "BGO & $16 \mathrm{AR}$ & & 55 & 171.25 & 56507.27 & 103.7 & 113.7 & 161.0 & 160.1 & -0.9 \\
\hline " BGO & $18 \mathrm{~A}$ & " & 55 & 613.51 & 56266.77 & 99.5 & 109.5 & 161.2 & 161.0 & -0.2 \\
\hline "BGO & $20 \mathrm{~A}$ & $"$ & 55 & 873.74 & 55563.80 & 86.3 & 96.3 & 163.5 & 163.2 & -0.3 \\
\hline "BGO & $25 \mathrm{~A}$ & $"$ & & 720.59 & 57027.64 & 104.1 & 114.1 & 160.8 & 158.4 & -2.4 \\
\hline "BGO & $26 \mathrm{~A}$ & " & 54 & 078.09 & 57150.00 & 81.0 & 91.0 & 160.7 & 157.8 & -2.9 \\
\hline "BGO & $29 A$ & " & 53 & 065.74 & 56767.52 & 102.5 & 112.5 & 159.7 & 157.8 & -1.9 \\
\hline "BGO & $39 A$ & $"$ & 56 & 289.81 & 54051.04 & 84.8 & 94.8 & 167.7 & 8.1 & 0.4 \\
\hline "BGO & $41 \mathrm{~A}$ & " & 54 & 526.63 & 57386.82 & 103.3 & 113.3 & 158.6 & 157.3 & -1.3 \\
\hline "BGO & $43 \mathrm{~A}$ & $"$ & 55 & 480.82 & 57789.12 & 105.9 & 115.9 & 159.0 & 156.5 & -2.5 \\
\hline "BGO & $43 A A$ & & & 496.65 & 57790.46 & 62.2 & 72.2 & 156.7 & 156.7 & 0.0 \\
\hline "BGO & $44 \mathrm{~A}$ & " & 56 & 980.04 & 57157.41 & 98.0 & 108.0 & 158.1 & 158.7 & 0.6 \\
\hline "BGO & $44 \mathrm{AA}$ & & 57 & 009.08 & 57153.08 & 61.2 & 71.3 & 158.7 & 159.0 & 0.3 \\
\hline "BGO & $47 \mathrm{~A}$ & $"$ & & 685.72 & 55785.97 & 86.8 & 96.8 & 162.4 & 161.0 & -1.4 \\
\hline "BGO & $49 A$ & " & 54 & 776.87 & 54709.58 & 75.1 & 85.1 & 166.8 & 164.7 & -2.1 \\
\hline "BGO & $50 \mathrm{~A}$ & $"$ & & 065.78 & 56400.69 & 90.5 & 100.5 & 160.2 & 158.8 & -1.4 \\
\hline "BGO & $51 \mathrm{~A}$ & $"$ & & 425.66 & 54594.46 & 75.1 & 85.1 & 166.0 & 166.6 & 0.6 \\
\hline "BGO & $52 \mathrm{~A}$ & " & 55 & 886.11 & 55219.91 & 81.7 & 91.7 & 163.8 & 164.2 & 0.4 \\
\hline
\end{tabular}


Table F-2. Summary of Group Statistical Parameters (Continued)

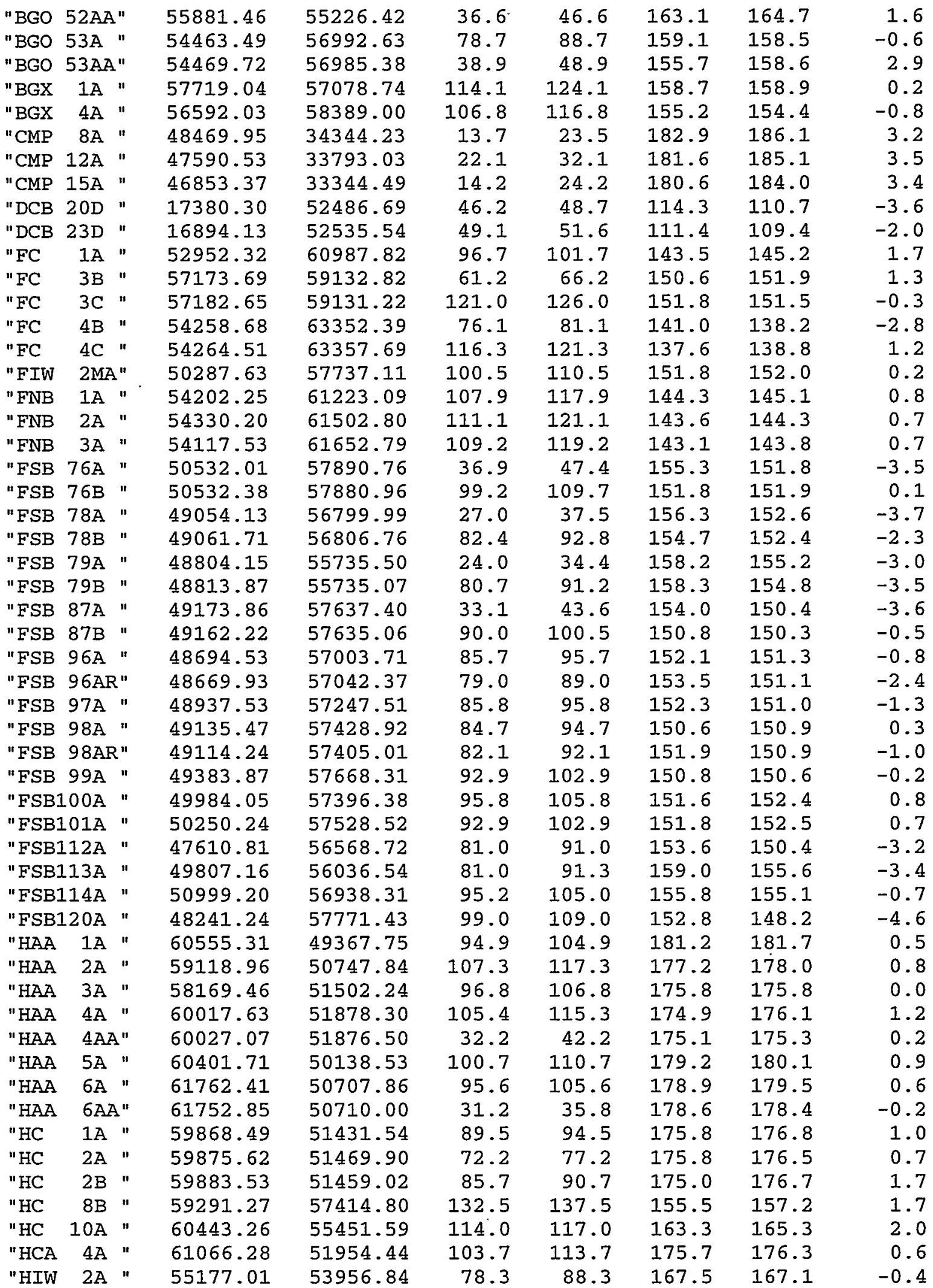


Table F-2. Summary of Group Statistical Parameters (Continued)

\begin{tabular}{|c|c|c|c|c|c|c|c|c|c|}
\hline "HSB & $65 \mathrm{~A}$ & & 56654.09 & 52811.20 & 62.5 & 73.2 & 171.5 & 171.6 & 0.1 \\
\hline "HSB & $68 \mathrm{~A}$ & 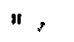 & 54954.88 & $52242: 77$ & 47.5 & $\therefore 58.0: 1$ & 171.9 & 171.0 & $-0: 9$ \\
\hline "HSB & $69 A$ & " & 54541.89 & 52353.55 & 83.1 & 93.1 & $17 i .7$ & 170.4 & -1.3 \\
\hline "HSB & $83 A$ & " & 56656.73 & 52005.45 & 65.2 & 76.0 & 173.1 & 173.3 & 0.2 \\
\hline "HSB & $84 \mathrm{~A}$ & $"$ & 54445.85 & 52411.59 & 64.7 & 75.9 & 172.0 & 170.2 & -1.8 \\
\hline "HSB & $85 A$ & " & 57432.27 & 54031.78 & 61.1 & 71.1 & 168.9 & 169.3 & 0.4 \\
\hline "HSB & $86 \mathrm{~A}$ & $"$ & 54275.00 & 53402.77 & 63.1 & 73.9 & 168.8 & 167.6 & -1.2 \\
\hline "HSBI & $.17 \mathrm{~A}$ & $"$ & 53521.40 & 53781.12 & 84.8 & 94.8 & 166.8 & 165.8 & -1.0 \\
\hline "HSB1 & $20 \mathrm{~A}$ & $"$ & 54893.16 & 54165.82 & 91.0 & 101.0 & 166.4 & 166.2 & -0.2 \\
\hline "HSB1 & $21 \mathrm{~A}$ & $"$ & 55545.03 & 52626.35 & 88.3 & 98.3 & 171.7 & 171.0 & -0.7 \\
\hline "HSB] & $22 \mathrm{~A}$ & $"$ & 55930.58 & 52719.32 & 85.4 & 95.4 & 171.6 & 171.2 & -0.4 \\
\hline "HSB1 & $24 A R$ & & 56699.16 & 52562.91 & 94.6 & 104.6 & 172.2 & 172.4 & 0.2 \\
\hline "HSB1 & $39 A$ & $"$ & 55334.77 & 51753.59 & 87.6 & 97.6 & 173.7 & 172.7 & -1.0 \\
\hline "HSBI & $-41 A$ & $"$ & 57116.59 & 51462.98 & 80.6 & 90.6 & 175.1 & 174.9 & -0.2 \\
\hline "HSB] & $-44 \mathrm{~A}$ & $"$ & 54354.32 & 52743.78 & 78.6 & 88.6 & 171.4 & 169.3 & -2.1 \\
\hline "HSB] & $-46 \AA$ & $"$ & 56264.76 & 50892.93 & 85.5 & 95.5 & 176.1 & 175.3 & -0.8 \\
\hline "HSL & $6 \mathrm{~A}$ & $"$ & 58773.03 & 52614.66 & 104.7 & 114.7 & 168.4 & 173.8 & 5.4 \\
\hline "HSL & $6 A A$ & & 58780.78 & 52621.29 & 18.6 & 28.6 & 169.1 & 173.0 & 3.9 \\
\hline "HSL & $8 \mathrm{~A}$ & $"$ & 59332.69 & 52533.01 & 108.8 & 118.8 & 172.8 & 174.3 & 1.5 \\
\hline "HSL & $8 A A$ & & 59334.34 & 52541.25 & 28.7 & 38.7 & 175.7 & 173.6 & -2.1 \\
\hline " LAW & 1D & $"$ & 43189.65 & 27176.23 & 6.6 & 11.6 & 176.5 & 176.3 & -0.2 \\
\hline " LAW & $2 \mathrm{~B}$ & " & 42474.87 & 28431.27 & -9.8 & -4.8 & 176.2 & 175.4 & -0.8 \\
\hline " LAW & $3 B$ & " & 45042.93 & 27844.21 & -1.0 & 4.0 & 178.2 & 179.9 & 1.7 \\
\hline " LCO & $5 \mathrm{~A}$ & " & 43542.51 & 27535.73 & 30.0 & 40.0 & 177.2 & 177.2 & 0.0 \\
\hline "NPM & $19 E$ & $"$ & 53855.72 & 43750.52 & 33.9 & 43.9 & 188.3 & 184.4 & -3.9 \\
\hline "NPM & $34 \mathrm{E}$ & " & 52178.77 & 41824.18 & 33.1 & 43.1 & 187.2 & 185.2 & -2.0 \\
\hline "PW & $83 N$ & $"$ & 48260.52 & 43306.42 & 4.0 & 9.0 & 168.4 & 179.3 & 10.9 \\
\hline "TBG & $5 B$ & $"$ & 16217.09 & 60159.71 & 46.2 & 56.2 & 113.2 & 99.8 & -13.4 \\
\hline "XSB & $1 \mathrm{~A}$ & " & 15732.44 & 60148.84 & 43.6 & 53.6 & 98.5 & 98.4 & -0.1 \\
\hline "YSC & $1 \mathrm{~A}$ & " & 64669.04 & 56836.46 & 76.8 & 136.9 & 163.3 & 160.8 & -2.5 \\
\hline "YSC & $2 \mathrm{~A}$ & " & 65372.26 & 56964.66 & 134.7 & 144.7 & 162.9 & 160.8 & -2.1 \\
\hline "YSC & $5 A$ & $"$ & 65549.56 & 52821.66 & 116.0 & 121.0 & 181.2 & 173.5 & -7.7 \\
\hline "P & $13 \mathrm{~A}$ & " & 50525.24 & 16454.79 & -67.3 & -57.4 & 173.1 & 177.3 & 4.2 \\
\hline "P & $13 B$ & " & 50525.24 & 16454.79 & -7.2 & 3.0 & 175.7 & 177.2 & 1.5 \\
\hline "P & $18 \mathrm{~A}$ & " & 45134.06 & 50308.26 & 12.0 & 22.0 & 168.5 & 164.1 & -4.4 \\
\hline " $P$ & $18 \mathrm{~B}$ & $"$ & 45124.13 & 50296.16 & 67.0 & 77.0 & 169.0 & 164.2 & -4.8 \\
\hline " P & $19 \mathrm{~A}$ & " & 54661.51 & 35763.86 & -36.7 & -26.7 & 186.8 & 191.8 & 5.0 \\
\hline " P & $23 \mathrm{~B}$ & $"$ & 24685.04 & 34727.78 & 41.5 & 46.5 & 137.8 & 139.1 & 1.3 \\
\hline "P & $24 \mathrm{~A}$ & $"$ & 58519.49 & 22466.26 & -1.9 & 8.9 & 191.5 & 192.4 & 0.9 \\
\hline " $P$ & $25 B$ & " & 36673.35 & 36698.53 & 80.6 & 90.6 & 178.2 & 164.4 & -13.8 \\
\hline "P & $26 \mathrm{~A}$ & " & 17067.87 & 60790.20 & 22.0 & 32.0 & 117.5 & 101.3 & -16.2 \\
\hline "P & $27 \mathrm{~B}$ & $"$ & 61674.68 & 49668.39 & 74.8 & 94.8 & 179.8 & 181.5 & 1.7 \\
\hline \multirow{4}{*}{\multicolumn{2}{|c|}{ ** GROUP }} & 2 & rms of & (FACT-data) & \multicolumn{2}{|c|}{ differences: } & \multicolumn{2}{|l|}{6.304} & \\
\hline & & & avg of & (FACT-data) & \multicolumn{2}{|c|}{ differences: } & \multicolumn{2}{|l|}{1.404} & \\
\hline & & & avg of & |FACT-data | & \multirow{2}{*}{\multicolumn{2}{|c|}{$\begin{array}{l}\text { differences: } \\
\text { differences: }\end{array}$}} & \multicolumn{2}{|l|}{5.068} & \\
\hline & & & $\max$ of & \{FACT-data\} & & & 17.737 & & \\
\hline "BG & 94 & " & 57485.58 & 61253.82 & 152.8 & 182.8 & 191.2 & 174.8 & -16.4 \\
\hline "BG & 95 & " & 58210.78 & 60274.34 & 152.5 & 182.5 & 192.9 & 177.4 & -15.5 \\
\hline "BG & 96 & $"$ & 57966.00 & 59647.94 & 177.2 & 207.2 & 197.8 & 196.6 & -1.2 \\
\hline "BG 1 & .03 & $"$ & 59074.01 & 57865.93 & 169.5 & 199.5 & 199.9 & 199.6 & -0.3 \\
\hline "BGO & $3 C$ & $"$ & 57663.88 & 55780.34 & 178.7 & 188.7 & 225.9 & 225.6 & -0.3 \\
\hline "BGO & $5 \mathrm{C}$ & $"$ & 57844.87 & 56689.07 & 183.2 & 193.2 & 216.3 & 221.1 & 4.8 \\
\hline
\end{tabular}


Table F-2. Summary of Group Statistical Parameters (Continued)

\begin{tabular}{|c|c|c|c|c|c|c|c|c|c|}
\hline " BGO & $6 \mathrm{~B}$ & " & 57422.52 & 56856.84 & 139.7 & 149.7 & 219.1 & 221.5 & 2.4 \\
\hline " BGO & $6 \mathrm{C}$ & " & 57370.14 & 56800.40 & 158.0 & 168.0 & 220.3 & 222.3 & 2.0 \\
\hline "BGO & $8 \mathrm{C}$ & " & 56716.03 & 57033.59 & 174.3 & 184.3 & 224.3 & 224.5 & 0.2 \\
\hline "BGO & $10 \mathrm{~B}$ & $"$ & 56173.88 & 57560.73 & 139.0 & 149.0 & 219.9 & 223.6 & 3.7 \\
\hline "BGO & $10 \mathrm{C}$ & $"$ & 56198.04 & 57374.75 & 157.3 & 167.3 & 220.4 & 224.4 & 4.0 \\
\hline "BGO & $12 \mathrm{C}$ & $"$ & 55415.52 & 57541.08 & 153.6 & 163.6 & 220.1 & 225.4 & 5.3 \\
\hline "BGO & $12 \mathrm{CR}$ & & 55390.36 & 57547.24 & 144.0 & 154.0 & 221.9 & 225.2 & 3.3 \\
\hline "BGO & $12 \mathrm{CX}$ & & 55411.15 & 57571.96 & 141.2 & 151.2 & 230.4 & 225.1 & -5.3 \\
\hline "BGO & $14 \mathrm{C}$ & $"$ & 54931.25 & 57196.74 & 192.1 & 202.1 & 221.4 & 227.1 & 5.7 \\
\hline " BGO & $14 \mathrm{CR}$ & & 54876.13 & 57177.89 & 190.1 & 200.1 & 223.8 & 227.0 & 3.2 \\
\hline "BGO & $16 \mathrm{~B}$ & $"$ & 55143.73 & 56537.96 & 136.0 & 146.0 & 218.4 & 225.6 & 7.2 \\
\hline " BGO & $20 \mathrm{~B}$ & $"$ & 55889.62 & 55545.19 & 131.0 & 141.0 & 227.6 & 226.7 & -0.9 \\
\hline "BGO & $20 \mathrm{C}$ & $"$ & 55873.23 & 55534.46 & 174.0 & 184.0 & 228.8 & 227.5 & -1.3 \\
\hline "BGO & $27 C$ & $"$ & 53643.34 & 56753.42 & 154.9 & 163.9 & 220.9 & 223.5 & 2.6 \\
\hline "BGO & $29 \mathrm{C}$ & $"$ & 53065.14 & 56785.84 & 176.8 & 186.8 & 223.1 & 222.6 & -0.5 \\
\hline "BGO & $30 \mathrm{C}$ & $"$ & 53386.81 & 56311.81 & 178.4 & 188.4 & 219.1 & 222.0 & 2.9 \\
\hline " BGO & $31 \mathrm{C}$ & $"$ & 53641.87 & 56050.06 & 176.4 & 186.4 & 225.6 & 222.0 & -3.6 \\
\hline "BGO & $33 c$ & " & 54384.56 & 55382.76 & 177.8 & 187.8 & 225.2 & 223.3 & -1.9 \\
\hline "BGO & $35 \mathrm{C}$ & $"$ & 55120.65 & 54688.75 & 161.9 & 171.9 & 228.9 & 224.8 & -4.1 \\
\hline "BGO & $37 \mathrm{C}$ & $"$ & 55743.38 & 54090.51 & 168.8 & 178.8 & 230.9 & 226.8 & -4.1 \\
\hline "BGO & $39 c$ & $"$ & 56282.08 & 54042.56 & 174.9 & 184.9 & 231.1 & 228.9 & -2.2 \\
\hline " BGO & $42 \mathrm{C}$ & $"$ & 54629.16 & 57298.77 & 185.9 & 195.9 & 223.4 & 226.9 & 3.5 \\
\hline "BGO & $43 \mathrm{CR}$ & ?" & 55459.53 & 57766.86 & 178.4 & 188.4 & 225.4 & 225.6 & 0.2 \\
\hline " BGO & $44 B$ & $"$ & 56994.49 & 57155.16 & 148.1 & 158.1 & 221.0 & 222.5 & 1.5 \\
\hline "BGO & $45 B$ & " & 53574.07 & 56946.03 & 137.0 & 147.0 & 219.1 & 223.5 & 4.4 \\
\hline "BGO & $45 C$ & " & 53586.46 & 56937.98 & 190.5 & 200.5 & 222.9 & 225.3 & 2.4 \\
\hline "BGO & $46 \mathrm{~B}$ & " & 53285.57 & 56160.65 & 140.4 & 150.4 & 218.2 & 219.8 & 1.6 \\
\hline "BGO & $46 \mathrm{C}$ & " & 53277.11 & 56172.78 & 178.0 & 188.0 & 219.6 & 220.9 & 1.3 \\
\hline " BGO & $47 \mathrm{C}$ & " & 53709.52 & 55804.63 & 178.6 & 188.6 & 222.9 & 221.4 & -1.5 \\
\hline "BGO & $48 \mathrm{C}$ & " & 53864.66 & 55615.85 & 176.7 & 186.7 & 223.6 & 221.5 & -2.1 \\
\hline "BGO & $49 \mathrm{C}$ & $"$ & 54777.03 & 54724.27 & 166.0 & 176.0 & 228.2 & 223.4 & -4.8 \\
\hline "BGO & $50 \mathrm{C}$ & $"$ & 53080.36 & 56386.55 & 162.5 & 172.5 & 218.1 & 220.5 & 2. \\
\hline "BGO & $51 B$ & " & 56430.92 & 54587.93 & 116.9 & 126.9 & 230.4 & 227.8 & -2.6 \\
\hline "BGO & $51 \mathrm{C}$ & " & 56435.93 & 54582.16 & 175.1 & 185.1 & 231.2 & 229.2 & -2.0 \\
\hline "BGO & $52 \mathrm{~B}$ & $"$ & 55890.68 & 55213.52 & 126.7 & 136.7 & 228.1 & 226.7 & -1 \\
\hline "BGO & $52 \mathrm{~B}$ & " & 55890.68 & 55213.52 & 126.7 & 136.7 & 228.1 & 226.7 & -1 \\
\hline "BGO & $52 \mathrm{C}$ & " & 55895.16 & 55207.15 & 178.7 & 188.7 & 229.4 & 227.9 & -1 \\
\hline "BGO & $53 \mathrm{~B}$ & " & 54457.17 & 57000.00 & 143.5 & 153.5 & 221.7 & 225.4 & 3. \\
\hline "BGO & $53 c$ & " & 54450.67 & 57007.32 & 183.2 & 193.2 & 222.6 & 226.6 & 4.0 \\
\hline "BGX & IC & $"$ & 57725.76 & 57065.15 & 176.0 & 186.0 & 216.0 & 219.7 & 3.7 \\
\hline "BGX & $2 \mathrm{~B}$ & $"$ & 57469.67 & 57511.55 & 137.2 & 147.2 & 212.9 & 218.2 & 5.3 \\
\hline "BGX & 2D & " & 57476.29 & 57498.90 & 181.1 & 191.1 & 215.4 & 219.4 & 4. \\
\hline "BGX & $4 \mathrm{C}$ & $"$ & 56580.37 & 58398.63 & 170.7 & 180.7 & 214.3 & 219.2 & 4 . \\
\hline "BGX & $8 \mathrm{DR}$ & & 58220.98 & 57746.68 & 183.1 & 203.1 & 205.7 & 212.2 & 6.5 \\
\hline "BGX & $12 \mathrm{C}$ & $"$ & 58280.41 & 54501.71 & 174.1 & 184.1 & 234.6 & 228.6 & -6. \\
\hline " BRR & $6 \mathrm{C}$ & $"$ & 50435.16 & 58863.14 & 156.0 & 166.0 & 211.6 & 214.1 & 2. \\
\hline "BRR & $7 \mathrm{BR}$ & $2 "$ & 50162.98 & 59444.94 & 141.6 & 151.6 & 204.9 & 211.0 & 6.1 \\
\hline " BRR & $7 \mathrm{C}$ & $"$ & 50153.27 & 59444.45 & 175.9 & 185.9 & 209.8 & 212.2 & 2.5 \\
\hline "BRR & $8 \mathrm{~B}$ & $"$ & 49597.22 & 59625.82 & 138.7 & 148.7 & 204.3 & 207.1 & 2. \\
\hline "CMP & $8 B$ & $"$ & 48480.44 & 34345.48 & 156.6 & 166.6 & 198.5 & 206.4 & 7. \\
\hline "CMP & $9 B$ & " & 47847.73 & 33475.01 & 149.0 & 159.0 & 194.7 & 208.4 & 13. \\
\hline " CMP & $10 B$ & " & 47943.12 & 33136.89 & 137.4 & 147.4 & 195.0 & 209.8 & 14. \\
\hline " CMP & $10 \mathrm{C}$ & " & 47936.35 & 33160.82 & 179.6 & 189.6 & 198.9 & 209.7 & \\
\hline "CMP & $11 B$ & $"$ & 47622.42 & 33282.65 & 139.7 & 149.7 & 195.1 & 208.6 & 13. \\
\hline
\end{tabular}


Table F-2. Summary of Group Statistical Parameters (Continued)

\begin{tabular}{|c|c|c|c|c|c|c|c|c|c|}
\hline " CMP & $12 \mathrm{~B}$ & $"$ & 47582.56 & 33788.69 & 148.0 & 158.0 & 194.6 & 206.7 & 12.1 \\
\hline "CMP & $13 \mathrm{~B}$ & $"$ & 47975.22 & 33615.47 & 134.2 & 144.2 & 194.7 & 208.2 & 13.5 \\
\hline "CMP & $14 \mathrm{~B}$ & " & $46762: 54$ & 34405.77 & $130: 0$ & 140.0 & 194.6 & 202.0 & 7.4 \\
\hline "CMP & $15 \mathrm{~B}$ & " & 46859.50 & 33335.32 & 145.1 & 155.1 & 202.9 & 206.7 & 3.8 \\
\hline "CMP & $16 \mathrm{~B}$ & " & 47831.28 & 33361.04 & 141.7 & 151.7 & 194.8 & 208.7 & 13.9 \\
\hline " CMP & $30 \mathrm{~B}$ & $"$ & 47195.03 & 33652.79 & 97.4 & 107.5 & 194.7 & 206.1 & 11.4 \\
\hline "CMP & $30 \mathrm{C}$ & " & 47233.06 & 33633.06 & 179.5 & 189.5 & 210.8 & 206.5 & -4.3 \\
\hline "CMP & $31 B$ & $"$ & 47408.43 & 34209.90 & 110.0 & 120.0 & 194.4 & 204.7 & 10.3 \\
\hline "CMP & $32 B$ & " & 48163.49 & 33948.09 & 97.7 & 107.7 & 195.5 & 207.6 & 12.1 \\
\hline "CMP & $32 \mathrm{C}$ & " & 48170.49 & 33941.09 & 185.2 & 195.2 & 195.9 & 207.4 & 11.5 \\
\hline "CRP & $3 c$ & " & 41780.37 & 52154.68 & 121.1 & 131.1 & 196.7 & 181.5 & -15.2 \\
\hline "CRP & $5 c$ & " & 42238.74 & 51887.54 & 110.1 & 120.1 & 198.3 & 184.2 & -14.1 \\
\hline "CSB & $1 \mathrm{C}$ & " & 42601.64 & 50326.00 & 141.4 & 161.4 & 205.0 & 198.8 & -6.2 \\
\hline "CSB & $3 c$ & " & 42166.21 & 51112.99 & 137.9 & 157.9 & 206.1 & 191.4 & -14.7 \\
\hline " DBP & 1 & $"$ & 16554.65 & 55461.46 & 93.2 & 121.4 & 120.0 & 121.2 & 1.2 \\
\hline "DBP & 2 & " & 16261.38 & 55305.83 & 84.3 & 114.3 & 117.4 & 118.7 & 1.3 \\
\hline "DBP & 3 & " & 16342.95 & 55592.44 & 86.4 & 116.4 & 121.1 & 119.8 & -1.3 \\
\hline "DBP & 4 & $"$ & 16239.48 & 55516.39 & 84.2 & 114.2 & 119.0 & 118.9 & -0.1 \\
\hline "DBP & 5 & $"$ & 16456.49 & 55271.93 & 96.1 & 116.1 & 117.5 & 120.3 & 2.8 \\
\hline " DCB & $1 \mathrm{~A}$ & " & 17169.39 & 52608.40 & 90.1 & 120.1 & 115.4 & 123.5 & 8.1 \\
\hline "DCB & $2 \mathrm{~A}$ & " & 18062.42 & 51812.95 & 97.4 & 127.4 & 124.8 & 131.1 & 6.3 \\
\hline "DCB & $3 \mathrm{~A}$ & " & 17908.76 & 51067.40 & 96.2 & 126.2 & 120.6 & 129.2 & 8.6 \\
\hline "DCB & $4 \mathrm{~A}$ & " & 17512.34 & 51155.65 & 92.5 & 122.5 & 119.1 & 126.5 & 7.4 \\
\hline "DCB & $5 A$ & " & 17259.08 & 51666.78 & 85.9 & 115.9 & 118.8 & 124.4 & 5.6 \\
\hline "DCB & 6 & $"$ & 17318.69 & 52719.18 & 109.5 & 128.2 & 116.8 & 125.0 & 8.2 \\
\hline "DCB & 7 & $"$ & 17339.83 & 52544.47 & 108.9 & 128.9 & 118.0 & 125.4 & 7.4 \\
\hline "DCB & 8 & $"$ & 18186.59 & 51825.20 & 110.3 & 130.3 & 126.4 & 132.1 & 5.7 \\
\hline " DCB & 9 & $"$ & 17155.27 & 52777.13 & 97.3 & 117.3 & 114.9 & 123.1 & 8.2 \\
\hline " DCB & 10 & $"$ & 17118.62 & 52388.76 & 99.8 & 119.8 & 116.6 & 123.3 & 6.7 \\
\hline "DCB & 11 & $"$ & 16701.76 & 53331.22 & 106.8 & 117.0 & 122.1 & 117.3 & -4.8 \\
\hline "DCB & 12 & $"$ & 16105.05 & 53981.19 & 92.0 & 112.0 & 109.8 & 115.7 & 5.9 \\
\hline "DCB & 13 & $"$ & 16523.39 & 52555.56 & 102.0 & 117.6 & 117.2 & 117.5 & 0.3 \\
\hline "DCB & 14 & $"$ & 16898.86 & 53566.89 & 94.6 & 114.6 & 111.6 & 119.3 & 7.7 \\
\hline "DCB & 15 & $"$ & 15117.88 & 53636.30 & 99.8 & 114.5 & 111.6 & 110.8 & -0.8 \\
\hline "DCB & 16 & " & 14958.28 & .27 & 100 & 4.7 & 112.0 & 111.2 & -0.8 \\
\hline "DCB & $17 \mathrm{~A}$ & $"$ & 17270.54 & 53154.00 & 109.4 & 119.4 & 116.5 & 123.8 & 7.3 \\
\hline "DCB & $17 \mathrm{~B}$ & " & 17274.54 & 53158.87 & 99.2 & 101.7 & 116.9 & 123.7 & 6.8 \\
\hline "DCB & $17 \mathrm{C}$ & " & 17277.32 & 53163.29 & 87.4 & 89.9 & 116.0 & 123.6 & 7.6 \\
\hline "DCB & $18 \mathrm{~A}$ & $"$ & 17198.69 & 52626.00 & 110.1 & 120.1 & 116.2 & 124.0 & 7.8 \\
\hline "DCB & $18 \mathrm{~B}$ & $"$ & 17190.86 & 52621.83 & 100.5 & 103.0 & 113.2 & 123.6 & 10.4 \\
\hline " DCB & $18 \mathrm{C}$ & " & 17184.89 & 52618.30 & 87.7 & 90.2 & 112.6 & 123.4 & 10.8 \\
\hline " DCB & $19 A$ & $"$ & 17201.32 & 52595.07 & 111.9 & 121.9 & 120.1 & 124.1 & 4.0 \\
\hline "DCB & $19 B$ & " & 17195.27 & 52590.63 & 101.9 & 104.4 & 117.3 & 123.8 & 6.5 \\
\hline "DCB & $19 \mathrm{C}$ & $"$ & 17188.62 & 52586.32 & 89.1 & 91.6 & 116.3 & 123.5 & 7.2 \\
\hline "DCB & $20 \mathrm{~A}$ & $"$ & 17393.86 & 52461.01 & 110.9 & 120.9 & 117.1 & 125.9 & 8.8 \\
\hline "DCB & $20 B$ & " & 17390.93 & 52466.03 & 100.3 & 102.8 & 116.4 & 125.7 & 9.3 \\
\hline "DCB & $20 \mathrm{C}$ & " & 17387.59 & 52471.95 & 89.4 & 91.9 & 116.3 & 125.4 & 9.1 \\
\hline " DCB & $21 \mathrm{~A}$ & " & 17144.19 & 52497.52 & 110.1 & 120.1 & 116.8 & 123.7 & 6.9 \\
\hline " DCB & $21 B$ & " & 17142.14 & 52503.27 & 102.2 & 104.7 & 113.3 & 123.4 & 10.1 \\
\hline " DCB & $21 C$ & " & 17140.99 & 52508.93 & 88.3 & 90.8 & 112.8 & 123.1 & 10.3 \\
\hline "DCB & $22 \mathrm{~A}$ & " & 17083.51 & 52503.06 & 109.8 & 119.8 & 112.7 & 123.2 & 10.5 \\
\hline "DCB & $22 B$ & $"$ & 17081.23 & 52509.16 & 100.9 & 103.4 & 112.7 & 122.8 & 10.1 \\
\hline "DCB & $22 \mathrm{C}$ & " & 17080.53 & 52515.45 & 88.1 & 90.6 & 112.7 & 122.5 & 9.8 \\
\hline "DCB & $23 A$ & " & 16893.94 & 52505.32 & 105.7 & 115.7 & 111.8 & 121.3 & 9.5 \\
\hline
\end{tabular}


Table F-2. Summary of Group Statistical Parameters (Continued)

\begin{tabular}{|c|c|c|c|c|c|c|c|c|}
\hline " DCB & $23 B$ & 16893.90 & 52511.36 & 94.1 & 96.6 & 108.7 & 121.0 & 12.3 \\
\hline "DCB & $23 \mathrm{C}$ & 16893.92 & 52517.70 & 86.6 & 89.1 & 108.9 & 120.9 & 12.0 \\
\hline " DCB & $24 \mathrm{~A}$ & 17146.45 & 51890.59 & 109.2 & 119.2 & 115.4 & 123.5 & 8.1 \\
\hline $\mathrm{DCB}$ & $24 B$ & 17135.28 & 51889.49 & 100.6 & 103.1 & 115.1 & 123.2 & 8.1 \\
\hline $\mathrm{DCB}$ & $24 \mathrm{C}$ & 17128.89 & 51888.29 & 87.6 & 90.1 & 116.2 & 123.0 & \\
\hline DOB & 1 & 21716.60 & 56149.98 & 114.7 & 144.7 & 143.2 & 148.9 & 5. \\
\hline DOB & 2 & 21521.56 & 56324.23 & 115.3 & 145.3 & 143.3 & 148.1 & 4. \\
\hline "DOB & 3 & 21833.77 & 56386.18 & 115.9 & 145.9 & 143.1 & 149.3 & 6.2 \\
\hline " DOB & 4 & 21974.85 & 56173.09 & 109.2 & 139.2 & 142.4 & 149.8 & 7.4 \\
\hline " DOB & 7 & 21610.86 & 56047.42 & 125.7 & 145.7 & 143.2 & 148.7 & 5.5 \\
\hline " DOB & 8 & 21854.23 & 56111.62 & 128.3 & 148.3 & 143.8 & 149.5 & 5.7 \\
\hline "DOB & 9 & 21914.47 & 56489.76 & 128.5 & 148.5 & 144.1 & 149.6 & 5. \\
\hline "DOB & 10 & 21457.21 & 56259.09 & 128.3 & 148.2 & 143.3 & 148.0 & 4. \\
\hline "DOB & 11 & 21554.72 & 56191.64 & 126.7 & 131.7 & 140.9 & 148.3 & 7. \\
\hline "DOB & 12 & 21553.76 & 56196.84 & 133.1 & 138.1 & 140.6 & 148.4 & 7. \\
\hline " DOB & 14 & 21655.74 & 56057.51 & 132.6 & 137.6 & 139.7 & 148.8 & 9.7 \\
\hline " DOB & 15 & 21284.89 & 55936.57 & 110.9 & 116.0 & 141.9 & 147.2 & 5.3 \\
\hline "DOB & 16 & 21284.59 & 55930.81 & 103.5 & 108.6 & 141.4 & 147.1 & 5. \\
\hline " DOL & 1 & 21808.58 & 56494.69 & 109.2 & 119.2 & 144.3 & 148.9 & 4. \\
\hline "FBP & $1 \mathrm{~A}$ & 50801.97 & 60656.16 & 161.8 & 191.8 & 206.7 & 208.1 & 1. \\
\hline " FBP & $2 \mathrm{~A}$ & 50437.47 & 61570.32 & 137.1 & 167.1 & 191.5 & 186.2 & -5 \\
\hline "FBP & $3 A$ & 50834.99 & 61616.17 & 141.0 & 171.0 & 194.3 & 192.3 & -2 \\
\hline "FBP & 4 & 51171.97 & 61014.05 & 165.2 & 195.2 & 212.4 & 207.0 & -5 \\
\hline "FBP & $6 \mathrm{D}$ & 50442.18 & 61529.96 & 178.3 & 198.3 & 194.8 & 195.4 & 0 \\
\hline "FBP & $7 D$ & 50794.34 & 61590.87 & 183.2 & 203.2 & 194.1 & 197.2 & 3. \\
\hline " FBP & $8 D$ & 51183.90 & 60982.69 & 172.8 & 192.8 & 207.1 & 207.5 & 0.4 \\
\hline "FBP & $9 D$ & 50935.15 & 61314.97 & 177.9 & 197.9 & 200.3 & 201.7 & 1. \\
\hline "FBP & $12 \mathrm{D}$ & 50893.28 & 60676.93 & 182.1 & 202.1 & 208.4 & 209.6 & 1.2 \\
\hline "FBP & 13D & 50601.77 & 61573.73 & 172.7 & 192.7 & 194.9 & 195.4 & 0 . \\
\hline "FC & $1 \mathrm{~B}$ & 52953.86 & 60995.57 & 151.8 & 156.8 & 210.8 & 213.8 & 3. \\
\hline "FC & $1 C$ & 52955.56 & 61003.08 & 183.9 & 188.9 & 214.0 & 214.5 & 0 . \\
\hline "FC & $3 D$ & 57191.44 & 59129.76 & 165.9 & 170.9 & 206.4 & 209.3 & 2. \\
\hline "FC & $3 E$ & 57198.96 & 59128.57 & 185.7 & 190.7 & 205.3 & 209.9 & 4. \\
\hline "FC & $4 \mathrm{E}^{\prime}$ & 54276.52 & 63368.94 & 176.4 & 166.4 & 185.2 & 0.0 & -185.2 \\
\hline "FIW & $2 I C^{\prime}$ & 50304.02 & 57727.18 & 125.3 & 175.2 & 212.6 & 212.2 & -0 \\
\hline "FIW & $2 \mathrm{MC}$ & 50328.95 & 57551.56 & 127.9 & 167.9 & 210.1 & 211.8 & 1. \\
\hline "FOB & $5 C^{\prime \prime}$ & 48589.99 & 56744.62 & 129.3 & 149.3 & 202.6 & 199.4 & -3 \\
\hline "FOB & $7 \mathrm{C}^{\prime \prime}$ & 49389.26 & 58074.58 & 148.9 & 168.9 & 209.8 & 208.8 & -1. \\
\hline "FOB & $9 \mathrm{C}$ & 49876.26 & 57663.74 & 155.5 & 175.5 & 212.0 & 210.6 & -1. \\
\hline "FOB & $11 \mathrm{C}$ & 1.71 & 57274.11 & 156.2 & 176.2 & 214.3 & 214.5 & 0.2 \\
\hline "FSB & $76 \mathrm{C}$ & 50532.65 & 57870.68 & 154.8 & 165.3 & 213.0 & 214.0 & 1. \\
\hline "FSB & $78 \mathrm{C}$ & 49054.67 & 56815.01 & 141.6 & 151.4 & 208.0 & 202.2 & -5 \\
\hline "FSB & $79 c$ & 48826.10 & 55734.41 & 149.8 & 159.6 & 196.9 & 192.2 & -4 \\
\hline "FSB & $87 \mathrm{C}$ & 49149.91 & 57632.47 & 148.8 & 159.3 & 208.8 & 206.5 & -2 \\
\hline "FSB & $88 \mathrm{C}$ & 50549.09 & 57363.18 & 158.4 & 168.4 & 212.7 & 212.8 & 0.1 \\
\hline "FSB & $89 \mathrm{C}$ & 50366.31 & 57334.35 & 156.1 & 166.1 & 212.1 & 211.7 & -0 \\
\hline "FSB & $90 \mathrm{C}$ & 50138.59 & 57208.65 & 158.1 & 168.1 & 211.1 & 210.2 & -0 \\
\hline "FSB & $91 \mathrm{C}^{\prime}$ & 49912.50 & 57083.32 & 149.1 & 159.1 & 210.9 & 208.1 & -2 \\
\hline "FSB & $92 \mathrm{C}$ & 49498.22 & 57007.70 & 147.6 & 157.6 & 209.4 & 205.7 & -3 \\
\hline "FSB & $93 C^{\prime}$ & 49362.42 & 56877.18 & 142.0 & 152.0 & 208.9 & 204.0 & -4 \\
\hline "FSB & $94 \mathrm{C}$ & 49084.32 & 56907.36 & 139.8 & 149.8 & 208.0 & 202.8 & -5 \\
\hline "FSB & $95 \mathrm{C}$ & 48945.94 & 57041.77 & 145.8 & 155.8 & 205.6 & 203.2 & -2 . \\
\hline "FSB & 95CR' & 48923.95 & 57077.32 & 151.9 & 161.9 & 208.0 & 203.5 & \\
\hline 'FSB & $97 C^{\prime}$ & 48944.07 & 57254.71 & 143.8 & 153.8 & 2.08 .3 & 204.0 & -4 \\
\hline
\end{tabular}


Table F-2. Summary of Group Statistical Parameters (Continued)

\begin{tabular}{|c|c|c|c|c|c|c|c|c|c|}
\hline "FSB & $98 \mathrm{C}$ & " & 49128.70 & 57421.57 & .148 .4 & 158.4 & 209.3 & 205.7 & -3.6 \\
\hline "FSB & $99 \mathrm{C}$ & " & $49391.23 \%$ & 57675.02 & .157 .2 & 167.2 & 209.9 & 208.1 & $-1: 8$ \\
\hline "FSB1 & $102 \mathrm{C}$ & $"$ & 49457.41 & 55513.22 & 145.9 & 155.9 & 195.4 & 192.5 & -2.9 \\
\hline "FSB1 & $103 c$ & $"$ & 48430.15 & 56372.68 & 147.1 & 157.1 & 202.6 & 196.5 & -6.1 \\
\hline "FSB1 & $104 \mathrm{C}$ & $"$ & 47966.11 & 56126.38 & 150.7 & 160.7 & 201.1 & 192.5 & -8.6 \\
\hline "FSB1 & $105 C$ & $"$ & 48815.94 & 57337.76 & 141.5 & 151.5 & 207.9 & 203.6 & -4.3 \\
\hline "FSBI & $106 \mathrm{C}$ & $"$ & 49404.17 & 56145.31 & 156.0 & 166.0 & 201.5 & 199.4 & -2.1 \\
\hline "FSB1 & $107 \mathrm{C}$ & $"$ & 50106.53 & 57012.12 & 150.8 & 160.8 & 210.3 & 208.8 & -1.5 \\
\hline "FSB1 & $110 \mathrm{C}$ & $"$ & 48914.53 & 56249.99 & 137.2 & 147.2 & 201.3 & 197.3 & -4.0 \\
\hline "FSB1 & $111 \mathrm{C}$ & " & 50508.12 & 57130.51 & 159.0 & 169.0 & 212.2 & 211.7 & -0.5 \\
\hline "FSB1 & $112 \mathrm{C}$ & " & 47596.01 & 56567.88 & 129.1 & 139.1 & 201.9 & 193.7 & -8.2 \\
\hline "FSB1 & $113 C$ & $"$ & 49821.49 & 56026.54 & 154.0 & 164.0 & 202.6 & 200.3 & -2.3 \\
\hline "FSB1 & $114 \mathrm{C}$ & " & 50984.82 & 56932.27 & 158.0 & 168.0 & 213.7 & 213.1 & -0.6 \\
\hline "FSB1 & $116 \mathrm{C}$ & $"$ & 49094.38 & 54713.83 & 160.5 & 170.5 & 189.6 & 187.2 & -2.4 \\
\hline "FSB1 & $120 \mathrm{C}$ & " & 48239.01 & 57783.04 & 150.7 & 160.7 & 206.3 & 202.4 & -3.9 \\
\hline "FSB1 & $121 \mathrm{C}$ & " & 47415.63 & 57555.15 & 148.4 & 158.4 & 204.4 & 197.8 & -6.6 \\
\hline "FSB1 & $122 \mathrm{C}$ & $"$ & 46937.44 & 56354.44 & 160.0 & 170.0 & 200.1 & 190.1 & -10.0 \\
\hline "FSB1 & $123 \mathrm{C}$ & $"$ & 50557.64 & 56285.14 & 155.3 & 165.3 & 210.5 & 206.5 & -4.0 \\
\hline "FSB1 & $150 P C$ & & 48736.60 & 56184.86 & 107.6 & 160.1 & 198.6 & 195.7 & -2.9 \\
\hline "HAA & $1 \mathrm{~B}$ & $"$ & 60561.80 & 49359.31 & 119.3 & 129.3 & 251.8 & 250.3 & -1.5 \\
\hline " HAA & $1 \mathrm{C}$ & $"$ & 60567.40 & 49351.99 & 147.4 & 157.4 & 252.4 & 251.1 & -1.3 \\
\hline "HAA & $2 \mathrm{~B}$ & $"$ & 59111.69 & 50754.50 & 127.2 & 137.2 & 253.2 & 244.4 & -8.8 \\
\hline " HAA & $2 \mathrm{C}$ & " & 59104.31 & 50761.18 & 171.9 & 181.9 & 254.8 & 246.1 & -8.7 \\
\hline "HAA & $3 B$ & $"$ & 58154.04 & 51487.42 & 125.9 & 135.9 & 240.6 & 236.6 & -4.0 \\
\hline "HAA & $3 C$ & $"$ & 58139.89 & 51473.76 & 163.3 & 173.3 & 244.0 & 238.2 & -5.8 \\
\hline "HAA & $4 \mathrm{C}$ & " & 59997.99 & 51882.57 & 158.3 & 168.3 & 251.8 & 243.8 & -8.0 \\
\hline "HAA & $6 \mathrm{~B}$ & $"$ & 61771.90 & 50705.33 & 131.3 & 141.4 & 235.7 & 243.2 & 7.5 \\
\hline "HAA & $6 \mathrm{C}$ & " & 61781.82 & 50703.43 & 161.1 & 171.1 & 235.9 & 243.9 & 8.0 \\
\hline " $\mathrm{HC}$ & $2 \mathrm{C}$ & " & 59879.41 & 51458.87 & 135.7 & 140.7 & 253.7 & 244.6 & -9.1 \\
\hline "HC & $2 \mathrm{D}$ & $"$ & 59873.54 & 51460.12 & 178.2 & 183.2 & 255.8 & 245.9 & -9.9 \\
\hline "HC & $4 \mathrm{~A}$ & $"$ & 61345.81 & 50965.20 & 150.0 & 155.0 & 244.7 & 244.4 & -0.3 \\
\hline "HC & $6 \mathrm{~A}$ & $"$ & 60139.40 & 51777.79 & 156.2 & 161.2 & 252.2 & 244.0 & -8.2 \\
\hline "HC & $8 \mathrm{C}$ & $"$ & 59297.17 & 57410.38 & 187.3 & 192.3 & 197.5 & 201.2 & 3.7 \\
\hline "HC & $10 B$ & $"$ & 60448.69 & 55444.91 & 164.8 & 169.8 & 208.9 & 207.4 & -1.5 \\
\hline "HC & $12 \mathrm{~B}$ & $"$ & 57839.58 & 53326.69 & 177.3 & 182.3 & 240.8 & 233.3 & -7.5 \\
\hline "HCA & $4 B$ & $"$ & 61082.03 & 51968.88 & 126.6 & 136.6 & 246.1 & 235.0 & -11.1 \\
\hline " $\mathrm{HCA}$ & $4 \mathrm{C}$ & $"$ & 61071.73 & 51970.96 & 153.8 & 163.8 & 246.8 & 242.2 & -4.6 \\
\hline "HIW & $2 \mathrm{MC}$ & & 55118.76 & 53945.40 & 154.0 & 184.0 & 228.3 & 223.5 & -4.8 \\
\hline "HIW & $2 \mathrm{MC}$ & & 55118.76 & 53945.40 & 124.1 & 139.0 & 228.3 & 222.5 & -5.8 \\
\hline "HIW & $3 M C$ & & 55098.98 & 54086.60 & 156.1 & 186.1 & 230.2 & 223.8 & -6.4 \\
\hline "HIW & $3 M C$ & & 55098.98 & 54086.60 & 126.4 & 141.3 & 230.2 & 222.8 & -7.4 \\
\hline "HIW & $4 \mathrm{MC}$ & & 54979.48 & 53907.23 & 150.4 & 180.4 & 219.7 & 222.5 & 2.8 \\
\hline "HIW & 4MC & & 54979.48 & 53907.23 & 120.8 & $1: 35.7$ & 219.7 & 221.6 & 1.9 \\
\hline "HIW & $5 \mathrm{MC}$ & & 54992.54 & 54311.14 & 154.2 & 184.1 & 228.6 & 223.7 & -4.9 \\
\hline "HIW & $5 M C$ & & 54992.54 & 54311.14 & 124.4 & 139.2 & 228.6 & 222.7 & -5.9 \\
\hline " HMD & $4 \mathrm{D}$ & $"$ & 57810.04 & 59439.92 & 188.9 & 208.9 & 200.0 & 203.0 & 3.0 \\
\hline " HSB & $50 P C$ & & 53911.20 & 53112.98 & 119.5 & 169.6 & 216.9 & 211.4 & -5.5 \\
\hline "HSB & $65 B$ & $"$ & 56659.37 & 52819.69 & 123.3 & 133.3 & 224.6 & 227.1 & 2.5 \\
\hline " HSB & $68 \mathrm{~B}$ & $"$ & 54944.81 & 52243.48 & 123.5 & 134.5 & 216.8 & .213 .4 & -3.4 \\
\hline "HSB & $68 \mathrm{C}$ & $"$ & 54935.32 & 52244.06 & 168.4 & 179.5 & 217.7 & 214.1 & -3.6 \\
\hline "HSB & $70 \mathrm{C}$ & $"$ & 54067.23 & 53525.76 & 164.9 & 174.9 & 223.1 & 215.4 & -7.7 \\
\hline "HSB & $71 \mathrm{C}$ & $"$ & 53658.01 & 53888.06 & 171.9 & 181.9 & 222.6 & 213.8 & -8.8 \\
\hline "HSB & $83 \mathrm{~B}$ & $"$ & 56643.90 & 51998.97 & 121.2 & 132.1 & 223.0 & 224.9 & 1.9 \\
\hline "HSB & $83 c$ & $"$ & 56662.80 & 51992.19 & 160.2 & 171.2 & 224.8 & 225.7 & 0.9 \\
\hline
\end{tabular}


Table F-2. Summary of Group Statistical Parameters (Continued)

\begin{tabular}{|c|c|c|c|c|c|c|c|c|}
\hline "HSB 84B & " & 54442.86 & 52429.71 & 121.8 & 132.9 & 210.8 & 211.1 & 0.3 \\
\hline "HSB 84C & $"$ & 54449.10 & 52422.04 & 170.9 & 181.8 & 213.5 & 211.7 & -1.8 \\
\hline "HSB 85B & $"$ & 57441.42 & 54027.18 & 133.2 & 143.2 & 233.8 & 230.1 & -3.7 \\
\hline "HSB 86B & $"$ & 54265.95 & 53403.47 & 113.8 & 124.0 & 221.6 & 214.8 & -6.8 \\
\hline "HSB100C & $"$ & 56941.86 & 52383.02 & 153.0 & 163.0 & 226.9 & 228.6 & 1.7 \\
\hline " HSB100PC & & 53918.88 & 53006.25 & 117.6 & 167.7 & 215.7 & 210.9 & -4 \\
\hline "HSB101C & $"$ & 56728.52 & 52351.38 & 166.3 & 176.3 & 226.2 & 227.4 & 1. \\
\hline "HSB102C & " & 56519.60 & 52353.05 & 166.7 & 176.7 & 224.7 & 226.0 & 1.3 \\
\hline "HSB103C & $"$ & 56369.03 & 52010.68 & 159.2 & 169.2 & 223.5 & 223.3 & -0 \\
\hline "HSB104C & " & 56088.16 & 51848.43 & 163.5 & 173.5 & 220.7 & 220.3 & -0 \\
\hline "HSB105C & " & 55908.36 & 51958.72 & 152.2 & 162.2 & 219.7 & 219.3 & -0. \\
\hline "HSB106C & " & 55738.02 & 52274.64 & 158.7 & 168.7 & 221.9 & 219.9 & -2 . \\
\hline "HSB107C & " & 55518.66 & 52298.37 & 159.3 & 169.3 & 219.5 & 218.5 & -1 \\
\hline "HSB109C & $"$ & 54991.13 & 52396.49 & 168.4 & 178.4 & 218.8 & 215.7 & -3 \\
\hline "HSB110C & " & 54800.57 & 52533.61 & 171.4 & 181.4 & 219.1 & 215.3 & -3 . \\
\hline "HSB111C & $"$ & 54654.81 & 52707.82 & 140.7 & 150.7 & 220.3 & 214.5 & -5 \\
\hline "HSB112C & $"$ & 54621.43 & 52957.21 & 140.6 & 150.6 & 221.6 & 215.7 & -5 . \\
\hline "HSB113C & $"$ & 54402.46 & 53163.13 & 154.7 & 164.7 & 221.9 & 215.7 & -6 . \\
\hline "HSB113C & $"$ & 54402.46 & 53163.13 & 151.7 & 161.7 & 221.9 & 215.6 & -6 \\
\hline "HSB115C & $"$ & 54358.70 & 53520.95 & 182.8 & 192.8 & 224.2 & 218.3 & -5 \\
\hline "HSB116C & $"$ & 54354.62 & 53761.97 & 180.5 & 190.5 & 225.0 & 218.9 & -6 \\
\hline "HSB117C & $"$ & 53515.83 & 53789.56 & 165.1 & 175.1 & 221.7 & 212.0 & -9 . \\
\hline "HSB125C & $"$ & 56613.57 & 51866.38 & 145.6 & 155.6 & 223.5 & 224.8 & 1. \\
\hline "HSB126C & $"$ & 55047.77 & 51303.73 & 176.3 & 181.3 & 204.0 & 205.8 & 1. \\
\hline "HSB127C & $"$ & 54791.20 & 51953.68 & 148.4 & 158.4 & 210.4 & 210.5 & \\
\hline "HSB129C & $"$ & 53274.82 & 52910.16 & 147.8 & 157.8 & 205.7 & 205.1 & -0 . \\
\hline "HSB130C & $"$ & 52596.56 & 51962.46 & 159.9 & 169.9 & 200.0 & 204.2 & 4. \\
\hline "HSB131C & $"$ & 54718.06 & 51115.16 & 148.5 & 158.5 & 203.9 & 206.4 & 2 . \\
\hline "HSB132C & " & 56797.72 & 51795.34 & 168.6 & 178.6 & 221.6 & 226.5 & 4. \\
\hline "HSB133C & $"$ & 57212.47 & 52194.94 & 173.3 & 183.3 & 230.8 & 230.7 & -0 . \\
\hline "HSB134C & $"$ & 56256.31 & 51642.47 & 149.1 & 159.1 & 221.0 & 220.9 & -0 . \\
\hline "HSB135C & $"$ & 54602.40 & 7.94 & 147.3 & 157.3 & 206.8 & 210.6 & 3 \\
\hline "HSB136C & $"$ & 54110.61 & 52803.97 & 160.5 & 170.5 & 217.3 & 211.7 & -5 . \\
\hline "HSB137C & $"$ & 53943.50 & 53217.34 & 163.8 & 173.8 & 220.0 & 212.9 & -7 \\
\hline "HSB139C & $"$ & 55344.17 & 51754.05 & 148.5 & 158.5 & 214.5 & 213.2 & -1 \\
\hline "HSB140C & $"$ & 54314.78 & 50868.11 & 161.6 & 171.6 & 205.6 & 206.9 & \\
\hline "HSB141C & $"$ & 57114.54 & 51446.14 & 154.7 & 164.7 & 229.1 & 229.0 & -0 . \\
\hline " $\mathrm{HSB} 141 \mathrm{CR}$ & & 5711 & 5.11 & 152.1 & 162.1 & 229.3 & 228.9 & -0 . \\
\hline "HSB142C & $"$ & 51973.10 & 54504.23 & 161.6 & 171.6 & 198.3 & 198.9 & \\
\hline "HSB143C & $"$ & 51385.74 & 55262.11 & 169.1 & 179.1 & 209.3 & 202.0 & -7 \\
\hline "HSB145C & $"$ & 55723.63 & 51641.80 & 164.7 & 174.7 & 213.5 & 215.5 & \\
\hline "HSB146C & $"$ & 56281.92 & 50881.82 & 152.3 & 162.3 & 210.0 & 219.5 & \\
\hline "HSB148C & $"$ & 53154.84 & 51219.25 & 158.9 & 168.9 & 201.8 & 209.1 & 7. \\
\hline " HSB150PC & :" & 53783.65 & 53217.07 & 119.5 & 169.6 & 217.4 & 210.9 & -6 . \\
\hline "HSB151C & $"$ & 52446.39 & 54279.83 & 170.6 & 180.6 & 207.8 & 202.7 & -5 \\
\hline "HSB152C & " & 52565.96 & 53246.49 & 173.1 & 183.1 & 199.0 & 198.0 & -1 \\
\hline "HSL $\quad 6 \mathrm{~B}$ & $"$ & 58765.56 & 52607.86 & 127.9 & 137.9 & 244.6 & 230.3 & -14 \\
\hline "HSL & $"$ & 58757.86 & 52600.50 & 157.6 & 167.6 & 245.4 & 237.9 & -7 \\
\hline "HSL & $"$ & 59331.52 & 52522.22 & 138.7 & 148.7 & 249.2 & 239.4 & -9 \\
\hline "HSL & $"$ & 59330.38 & 52512.54 & 171.7 & 181.7 & 250.4 & 240.4 & -10 \\
\hline " LAW & $"$ & 43173.25 & 27178.90 & 90.1 & 95.1 & 205.0 & 202.5 & -2 \\
\hline$" \mathrm{LCO} \quad 5 \mathrm{C}$ & $"$ & 43558.27 & 27533.91 & 110.5 & 120.5 & 211.0 & 205.9 & -5 \\
\hline "NPM 19D & $"$ & 53834.94 & 43723.45 & 97.5 & 107.5 & 243.3 & 248.7 & 5. \\
\hline "NPM 34D & $"$ & 52189.19 & 41815.02 & 86.4 & 96.4 & 253.9 & 251.5 & -2 . \\
\hline
\end{tabular}


Table F-2. Summary of Group Statistical Parameters (Continued)

\begin{tabular}{|c|c|c|c|c|c|c|c|c|c|}
\hline "TBG & 1 & " & 16046.02 & 60413.52 & 89.1 & 109.1 & 100.4 & 109.9 & 9.5 \\
\hline "TBG & 3 & & $16066.17 \%$ & 60301.49 & 88.9 & 108.9. & 103.0 & 110.8 & $7 . .8$ \\
\hline "TBG & 4 & " & 16054.32 & 60245.73 & 89.3 & 109.3 & 103.1 & 111.0 & 7.9 \\
\hline "TBG & 5 & " & 16218.81 & 60169.26 & 92.4 & 112.4 & 102.8 & 113.4 & 10.6 \\
\hline "TBG & $5 \mathrm{~A}$ & " & 16209.14 & 60151.18 & 70.0 & 80.0 & 103.7 & 112.7 & 9.0 \\
\hline "TBG & 6 & " & 16209.40 & 60432.78 & 89.1 & 109.1 & 102.7 & 111.7 & 9.0 \\
\hline "TBG & 7 & " & 16423.15 & 60199.44 & 84.7 & 104.7 & 105.5 & 115.3 & 9.8 \\
\hline "TIR & $I M$ & $"$ & 15018.24 & 60217.55 & 84.6 & 86.6 & 93.7 & 96.0 & 2.3 \\
\hline "TIR & 10 & $"$ & 15020.04 & 60222.14 & 90.0 & 92.0 & 93.2 & 96.0 & 2.8 \\
\hline "TIR & 2 & $"$ & 14955.26 & 60276.45 & 84.2 & 86.2 & 92.4 & 95.2 & 2.8 \\
\hline "TIR & $3 B$ & " & 15378.85 & 60217.52 & 83.5 & 85.5 & 95.7 & 101.3 & 5.6 \\
\hline "TNX & 1D & " & 15658.69 & 60683.97 & 79.6 & 99.6 & 99.3 & 103.5 & 4.2 \\
\hline "TNX & $2 \mathrm{D}$ & " & 15711.77 & 60507.57 & 82.8 & 102.8 & 99.1 & 105.0 & 5.9 \\
\hline "TNX & 3D & " & 15916.34 & 60243.98 & 84.9 & 104.9 & 99.6 & 109.0 & 9.4 \\
\hline "TNX & 4D & $"$ & 16043.66 & 59977.69 & 85.5 & 105.5 & 103.1 & 112.4 & 9.3 \\
\hline "TNX & 5D & " & 16179.74 & 59941.20 & 88.5 & 108.5 & 105.1 & 114.0 & 8.9 \\
\hline "TNX & 6D & $"$ & 16185.59 & 59656.06 & 89.8 & 109.8 & 105.4 & 115.1 & 9.7 \\
\hline "TNX & D & " & 16057.27 & 60726.63 & 83.6 & 103.6 & 101.0 & 108.1 & 7.1 \\
\hline "TNX & 8D & " & 14926.59 & 59795.15 & 74.0 & 94.0 & 94.0 & 95.7 & 1.7 \\
\hline "TNX & $9 D$ & $"$ & 14946.06 & 59994.97 & 75.4 & 95.1 & 93.8 & 95.5 & 1.7 \\
\hline "TNX & $10 \mathrm{D}$ & " & 15009.73 & 60193.98 & 77.0 & 95.9 & 94.1 & 95.9 & 1.8 \\
\hline "TNX & 11D & $"$ & 15050.14 & 60389.86 & 73.2 & 93.2 & 94.0 & 96.3 & 2.3 \\
\hline TNX & $12 \mathrm{D}$ & $"$ & 15143.66 & 60777.90 & 73.1 & 93.1 & 94.9 & 95.7 & 0.8 \\
\hline "TNX & $13 D$ & " & 14754.11 & 60087.50 & 87.9 & 89.9 & 91.4 & 93.6 & 2.2 \\
\hline "TNX & $14 \mathrm{D}$ & " & 14804.37 & 60168.63 & 85.8 & 87.8 & 91.4 & 94.0 & 2.6 \\
\hline "TNX & $15 \mathrm{D}$ & $"$ & 14853.26 & 60249.53 & 85.9 & 87.9 & 91.0 & 94.3 & 3.3 \\
\hline "TNX & $16 \mathrm{D}$ & $"$ & 14881.89 & 60335.66 & 86.1 & 88.1 & 90.8 & 94.5 & 3.7 \\
\hline "TNX & 17D & " & 15014.56 & 60790.52 & 89.7 & 91.7 & 92.5 & 92.9 & 0.4 \\
\hline "TNX & $18 \mathrm{D}$ & $"$ & 14694.70 & 60004.24 & 84.9 & 86.9 & 91.6 & 93.2 & 1.6 \\
\hline "TNX & $19 D$ & " & 14620.92 & 59895.70 & 84.9 & 86.9 & 91.0 & 92.7 & 1.7 \\
\hline "TNX & $20 \mathrm{D}$ & " & 14589.19 & 59853.68 & 86.2 & 88.2 & 91.1 & 92.4 & 1.3 \\
\hline "TNX & $21 D$ & " & 14568.94 & 59722.83 & 86.9 & 88.9 & 92.0 & 92.4 & 0.4 \\
\hline "TNX & $22 \mathrm{D}$ & " & 14440.30 & 59482.22 & 85.8 & 87.8 & 89.9 & 91.5 & 1.6 \\
\hline "TNX & $23 \mathrm{D}$ & $"$ & 15839.74 & 60442.04 & 84.8 & 104.8 & 99.4 & 106.9 & 7.5 \\
\hline "TNX & $24 \mathrm{D}$ & " & 16459.51 & 60435.43 & 99.8 & 114.8 & 109.1 & 115.2 & 6.1 \\
\hline "TNX & $26 \mathrm{D}$ & $"$ & 14972.66 & 59614.12 & 87.8 & 90.1 & 94.2 & 96.4 & 2.2 \\
\hline "TNX & $27 \mathrm{D}$ & " & 15480.09 & 60278.84 & 81.3 & 101.3 & 96.3 & 102.9 & 6.6 \\
\hline "TRW & 1 & $"$ & 15806.97 & 60191.68 & 81.4 & 106.4 & 91.8 & 107.8 & 16.0 \\
\hline "TRW & 2 & $"$ & 15687.03 & 60316.14 & 77.2 & 112.2 & 92.3 & 105.6 & 13.3 \\
\hline "TRW & 4 & $"$ & 16060.84 & 60435.63 & 81.9 & 111.9 & 92.1 & 109.8 & 17.7 \\
\hline "XSB & 1 & " & 15755.80 & 60172.19 & 92.0 & 112.0 & 102.8 & 107.5 & 4.7 \\
\hline "XSB & 1D & " & 15742.58 & 60146.07 & 87.9 & 107.9 & 98.8 & 107.4 & 8.6 \\
\hline "XSB & $2 \mathrm{D}$ & " & 15669.81 & 60142.32 & 84.0 & 104.0 & 98.5 & 106.1 & 7.6 \\
\hline "XSB & $3 A$ & " & 15710.81 & 59959.09 & 87.4 & 103.2 & 100.3 & 107.6 & 7.3 \\
\hline "XSB & 4 & $"$ & 15684.42 & 60076.34 & 94.3 & 114.3 & 98.3 & 107.1 & 8.8 \\
\hline "XSB & $4 \mathrm{D}$ & " & 15654.53 & 60055.50 & 83.9 & 103.9 & 98.6 & 106.3 & 7.7 \\
\hline$S B$ & $1 \mathrm{~A}$ & " & 16649.82 & 60011.91 & 98.4 & 128.4 & 117.9 & 118.9 & 1.0 \\
\hline "YSB & $2 \mathrm{~A}$ & " & 16658.67 & 59854.43 & 97.7 & 127.7 & 118.7 & 119.6 & 0.9 \\
\hline "YSB & $3 A$ & $"$ & 16534.35 & 59726.48 & 96.7 & 126.7 & 119.6 & 118.9 & -0.7 \\
\hline "YSB & $4 \mathrm{~A}$ & " & 16552.90 & 59887.85 & 97.6 & 127.6 & 117.6 & 118.4 & 0.8 \\
\hline "YSC & $1 \mathrm{C}$ & " & 65106.95 & 56892.95 & 197.5 & 207.5 & 217.6 & 213.8 & -3.8 \\
\hline SC & $4 \mathrm{C}$ & " & 64918.13 & 55781.42 & 195.9 & 205.9 & 227.8 & 222.8 & -5.0 \\
\hline 2 & 17 & $"$ & 42299.33 & 55683.19 & 148.2 & 148.7 & 169.5 & 171.2 & 1.7 \\
\hline "CMP & $10 \mathrm{C}$ & " & 47936.35 & 33160.82 & 179.6 & 189.6 & 198.5 & 209.7 & 11.2 \\
\hline
\end{tabular}


Table F-2. Summary of Group Statistical Parameters (Continued)

\begin{tabular}{|c|c|c|c|c|c|c|c|c|c|}
\hline "CMP & $30 \mathrm{~B}$ & " & 47195.03 & 33652.79 & 97.4 & 107.5 & 195.0 & 206.1 & 11.1 \\
\hline "CMP & $30 \mathrm{C}$ & $"$ & 47233.06 & 33633.06 & 179.5 & 189.5 & 210.6 & 206.5 & -4.1 \\
\hline "CMP & $32 \mathrm{~B}$ & $"$ & 48163.49 & 33948.09 & 97.7 & 107.7 & 195.3 & 207.6 & 12.3 \\
\hline "CMP & $32 \mathrm{C}$ & $"$ & 48170.49 & 33941.09 & 185.2 & 195.2 & 195.4 & 207.4 & 12.0 \\
\hline " RGW & J $4 \mathrm{C}$ & $"$ & 56371.24 & 28538.95 & 130.2 & 140.2 & 250.8 & 262.5 & 11.7 \\
\hline " RGW & $7 \mathrm{C}$ & $"$ & 53372.55 & 19049.00 & 84.3 & 94.3 & 240.4 & 241.7 & 1.3 \\
\hline "RGW & $8 \mathrm{C}$ & $"$ & 44616.98 & 30216.22 & 115.2 & 125.2 & 216.4 & 214.7 & -1.7 \\
\hline " RGW & $9 \mathrm{C}$ & $"$ & 49471.40 & 28770.17 & 102.2 & 112.2 & 231.6 & 231.3 & -0.3 \\
\hline "RGW & $10 \mathrm{C}$ & $"$ & 45626.50 & 20210.68 & 78.4 & 88.4 & 225.0 & 219.7 & -5.3 \\
\hline "RGW & V $11 \mathrm{C}$ & $"$ & 41082.90 & 14574.88 & 71.1 & 81.1 & 177.9 & 181.4 & 3.5 \\
\hline " RGW & J $13 C$ & $"$ & 30671.53 & 42663.76 & 115.5 & 125.5 & 183.7 & 193.4 & 9.7 \\
\hline "RGW & V $14 \mathrm{C}$ & $"$ & 26645.04 & 38368.61 & 100.4 & 110.4 & 186.9 & 182.9 & -4.0 \\
\hline " RGW & V $15 c$ & $"$ & 29690.81 & 26627.12 & 107.9 & 117.9 & 182.9 & 184.6 & 1.7 \\
\hline " RGW & V $16 \mathrm{C}$ & $"$ & 40349.41 & 49694.67 & 123.8 & 133.8 & 191.2 & 183.1 & -8.1 \\
\hline \multicolumn{2}{|c|}{ ** GROUP } & 3 & $\begin{array}{l}\text { * } \text { rms of } \\
\text { avg of } \\
\text { avg of } \\
\max \text { of }\end{array}$ & $\begin{array}{l}\text { (FACT-data) } \\
\text { (FACT-data) } \\
\text { |FACT-data) } \\
\text { \{FACT-data }\end{array}$ & \multicolumn{2}{|c|}{$\begin{array}{l}\text { differences: } \\
\text { differences: } \\
\text { differences: } \\
\text { differences: }\end{array}$} & $\begin{array}{r}5.23 \\
0.17 \\
3.60 \\
-16.83\end{array}$ & & \\
\hline "BG & 92 & $"$ & 56450.00 & 59585.06 & 197 & 227.2 & 209.0 & 218.7 & 9.7 \\
\hline "BG : & 115 & $"$ & 57106.59 & 57592.61 & 198.9 & 218.9 & 215.8 & 228.2 & 12.4 \\
\hline "BG : & 122 & $"$ & 56321.37 & 59164.11 & 189.9 & 209.9 & 211.3 & 222.6 & 11.3 \\
\hline "BGO & $46 D$ & $"$ & 53265.93 & 56187.01 & 202.1 & 212.1 & 225.3 & 225.7 & 0.4 \\
\hline " BGO & $47 D$ & $"$ & 53696.69 & 55794.78 & 203.4 & 213.4 & 226.2 & 225.9 & -0.3 \\
\hline "BGO & $48 D$ & $"$ & 53858.59 & 55603.64 & 202.0 & 212.0 & 226.8 & 225.8 & -1.0 \\
\hline "BGX & $3 \quad 3 D$ & $"$ & 57081.36 & 57976.03 & 201.6 & 221.6 & 214.9 & 227.3 & 12.4 \\
\hline "BGX & $5 D$ & $"$ & 56791.69 & 58881.04 & 195.0 & 215.0 & 209.2 & 222.7 & 13.5 \\
\hline " BRD & 1 & " & 24686.69 & 42660.07 & 148.9 & 178.9 & 167.3 & 180.4 & 13.1 \\
\hline " BRD & 2 & $"$ & 24812.76 & 42871.28 & 148.5 & 178.5 & 168.9 & 181.3 & 12.4 \\
\hline " BRD & 3 & $"$ & 24954.28 & 42662.69 & 158.5 & 188.5 & 170.8 & 183.8 & 13.0 \\
\hline "BRD & $5 \mathrm{D}$ & $"$ & 24681.93 & 42758.41 & 148.4 & 168.4 & 166.9 & 179.7 & 12.8 \\
\hline "BRR & $1 \mathrm{D}$ & $"$ & 50002.58 & 59264.14 & 200.4 & 220.4 & 217.1 & 216.9 & -0.2 \\
\hline " BRR & $2 \mathrm{D}$ & $"$ & 49740.61 & 59387.51 & 196.1 & 216.1 & 215.5 & 215.1 & -0.4 \\
\hline " BRR & $3 D$ & $"$ & 49633.17 & 59376.50 & 197.1 & 217.1 & 215.3 & 4.5 & -0.8 \\
\hline " BRR & $4 \mathrm{D}$ & $"$ & 49528.48 & 59360.11 & 198.7 & 218.7 & 215.1 & 213.9 & -1.2 \\
\hline " BRR & $5 \mathrm{D}$ & $"$ & 49415.56 & 59288.22 & 202.1 & 222.1 & 214.9 & 213.5 & -1.4 \\
\hline "BRR & $7 \mathrm{D}$ & $"$ & 50143.22 & 59444.34 & 201.9 & 221.9 & 217.9 & 217.5 & -0.4 \\
\hline "BRR & $8 D$ & ?" & 49620.92 & 59613.22 & 204.0 & 219.0 & 214.9 & 213.8 & -1.1 \\
\hline "CDB & 1 & $"$ & 43158.97 & 50648.13 & 195.7 & 216.6 & 213.9 & 216.0 & 2.1 \\
\hline "CDB & 2 & $"$ & 43072.00 & 50565.10 & 195.1 & 216.1 & 214.7 & 215.8 & 1.1 \\
\hline "CMP & $11 D$ & $"$ & 47610.19 & 33296.80 & 209.5 & 229.9 & 222.2 & 229.7 & 7.5 \\
\hline " CMP & $14 \mathrm{D}$ & $"$ & 46762.01 & 34392.70 & 204.1 & 224.5 & 216.2 & 216.0 & -0.2 \\
\hline "CMP & $30 \mathrm{D}$ & $"$ & 47226.07 & 33625.65 & 211.6 & 231.6 & 221.1 & 228.5 & 7.4 \\
\hline "CRP & 1 & $"$ & 42103.71 & 52000.00 & 187.8 & 217.8 & 208.3 & 206.6 & -1.7 \\
\hline "CRP & 2 & $"$ & 42157.12 & 52423.62 & 171.8 & 201.8 & 207.2 & 204.9 & -2.3 \\
\hline "CRP & 3 & $"$ & 41750.56 & 52124.11 & 184.0 & 214.0 & 206.9 & 204.7 & -2.2 \\
\hline "CRP & $3 D$ & $"$ & 41768.04 & 52149.12 & 194.3 & 214.3 & 207.5 & 204.7 & -2.8 \\
\hline "CRP & 4 & " & 41803.23 & 51889.94 & 180.7 & 210.7 & 207.3 & 205.6 & -1.7 \\
\hline "CRP & 7D & $"$ & 41940.04 & 52626.90 & 188.0 & 208.0 & 206.6 & 203.3 & -3.3 \\
\hline ' CRP & D & $"$ & 42089.59 & 52554.22 & 191.4 & 211.4 & 207.2 & 204.2 & -3.0 \\
\hline CRP & 10D & " & 41567.41 & 52504.81 & 189.5 & 209.5 & 205.0 & 202.5 & -2.5 \\
\hline "CRP & $11 D$ & $"$ & 41920.37 & 52137.09 & 193.7 & 203.6 & 207.9 & 205.3 & \\
\hline "CSB & $2 \mathrm{~A}$ & $"$ & 42252.86 & 50631.76 & 192.6 & 222.6 & 211.0 & 210.5 & -0.5 \\
\hline
\end{tabular}


Table F-2. Summary of Group Statistical Parameters (Continued)

\begin{tabular}{|c|c|c|c|c|c|c|c|c|c|}
\hline "CSB & $4 \mathrm{~A}$ & $"$ & 42125.10 & 50916.14 & 188.0 & 218.0 & 210.6 & 209.1 & -1.5 \\
\hline CSB & $5 \mathrm{~A}$ & $" \because$ & $42164.95 \ldots$ & 51101.71 & $\because 185.9$ & 215.9 & 210.7 & 209.0 & -1.7 \\
\hline " CSB & $6 \mathrm{~A}$ & $"$ & 42417.14 & 51110.26 & 189.8 & 219.8 & 211.3 & 210.6 & -0.7 \\
\hline "CSB & $7 \mathrm{D}$ & $"$ & 42604.20 & 50339.94 & 191.7 & 211.7 & 212.8 & 213.4 & 0.6 \\
\hline "F & 2 & $"$ & 50528.19 & 57426.91 & 207.0 & 217.0 & 218.4 & 218.3 & -0.1 \\
\hline " $F$ & $18 \mathrm{~A}$ & $"$ & 48868.60. & 56238.80 & 194.4 & 204.4 & 203.8 & 203.2 & -0.6 \\
\hline "FAC & $9 \mathrm{C}$ & $"$ & 54788.19 & 58927.09 & 197.4 & 207.4 & 217.4 & 229.1 & 11.7 \\
\hline "FAC & $10 \mathrm{C}$ & $"$ & 54766.72 & 59022.85 & 200.2 & 210.2 & 217.4 & 228.9 & 11.5 \\
\hline "FAC & $11 \mathrm{C}$ & " & 54697.64 & 59017.70 & 201.4 & 211.4 & 217.5 & 229.1 & 11.6 \\
\hline " FAC & $12 \mathrm{C}$ & " & 54681.22 & 58966.90 & 198.0 & 208.0 & 217.5 & 229.2 & 11.7 \\
\hline "FC & $3 F$ & $"$ & 57206.55 & 59127.26 & 205.1 & 210.1 & 206.2 & 219.0 & 12.8 \\
\hline "FET & 3D & $"$ & 52094.87 & 57383.84 & 203.0 & 223.0 & 222.6 & 226.2 & 3.6 \\
\hline " FET & $4 \mathrm{D}$ & $"$ & 52215.12 & 57356.54 & 205.1 & 225.1 & 223.0 & 226.8 & 3.8 \\
\hline "FIW & 1D & $"$ & 50556.26 & 57868.22 & 198.9 & 218.9 & 214.7 & 219.6 & 4.9 \\
\hline "FIW & 1ID & & 50511.80 & 57935.64 & 194.0 & 214.0 & 219.0 & 219.4 & 0.4 \\
\hline "FIW & $2 \mathrm{MD}$ & & 50305.99 & 57737.40 & 190.9 & 220.8 & 217.1 & 217.8 & 0.7 \\
\hline "FNB & 6 & " & 54152.82 & 61916.51 & 200.6 & 210.6 & 208.7 & 210.1 & 1.4 \\
\hline "FNB & 8 & $"$ & 54534.35 & 61527.65 & 195.4 & 205.4 & 202.8 & 212.2 & 9.4 \\
\hline "FOB & 1D & " & 48714.66 & 55906.09 & 175.4 & 195.4 & 204.0 & 198.0 & -6.0 \\
\hline " FOB & $4 \mathrm{D}$ & $"$ & 48169.62 & 56653.25 & 174.0 & 194.1 & 206.9 & 202.6 & -4.3 \\
\hline " FOB & $7 D$ & $"$ & 49400.09 & 58083.79 & 193.9 & 213.9 & 212.7 & 214.0 & 1.3 \\
\hline "FOB & 8D & $"$ & 49037.51 & 57840.62 & 191.4 & 211.4 & 212.3 & 211.7 & -0.6 \\
\hline "FOB & 9D & $"$ & 49862.03 & 57668.28 & 192.6 & 212.6 & 214.8 & 215.3 & 0.5 \\
\hline "FOB & 11D & $"$ & 50928.38 & 57265.56 & 199.0 & 219.0 & 217.7 & 219.6 & 1.9 \\
\hline "FOB & 12D & $"$ & 48850.11 & 57700.96 & 179.3 & 199.3 & 211.5 & 210.1 & -1.4 \\
\hline "FSB & OPD & & 48694.84 & 56663.20 & 171.6 & 215.3 & 207.6 & 205.0 & -2.6 \\
\hline "FSB & 50PD & & 48729.85 & 56708.61 & 174.7 & 219.8 & 207.2 & 205.6 & -1.6 \\
\hline "FSB & 76 & $"$ & 50531.29 & 57900.83 & 197.0 & 227.0 & 218.2 & 219.6 & 1.4 \\
\hline "FSB & 77 & " & 49659.91 & 57051.23 & 186.4 & 216.4 & 212.5 & 212.0 & -0.5 \\
\hline "FSB & 78 & " & 49047.52 & 56807.83 & 187.7 & 217.7 & 208.8 & 207.9 & -0.9 \\
\hline "FSB & 79 & " & 48794.18 & 55736.19 & 174.1 & 204.1 & 201.6 & 195.1 & -6.5 \\
\hline "FSB & 87D & " & 49136.71 & 57629.55 & 187.4 & 216.8 & 214.0 & 211.7 & -2.3 \\
\hline "FSB & $88 \mathrm{D}$ & $"$ & 50558.40 & 57363.65 & 202.1 & 222.1 & 216.3 & 218.1 & 1.8 \\
\hline "FSB & 89D & $"$ & 50356.09 & 57331.51 & 201.9 & 221.9 & 215.7 & 216.9 & 1.2 \\
\hline "FSB & $90 \mathrm{D}$ & " & 50129.62 & 57204.42 & 205.1 & $225.1^{\circ}$ & 215.4 & 215.3 & -0.1 \\
\hline "FSB & 91D & " & 49904.56 & 57079.18 & 200.9 & 220.9 & 213.9 & 213.6 & -0.3 \\
\hline "FSB & $92 D$ & " & 49490.42 & 57001.79 & 201.7 & 221.7 & 212.1 & 211.3 & -0.8 \\
\hline "FSB & 93D & $"$ & 49354.82 & 56869.80 & 197.9 & 217.9 & 210.8 & 209.9 & -0.9 \\
\hline "FSB & $94 \mathrm{DR}$ & & 49067.61 & 56911.01 & 183.3 & 203.4 & 210.2 & 208.4 & -1.8 \\
\hline "FSB & 95DR & R" & 48929.85 & 57065.63 & 187.0 & 207.0 & 210.5 & 208.7 & -1.8 \\
\hline "FSB & 97D & $"$ & 48950.80 & 57262.79 & 196.9 & 216.9 & 210.8 & 209.8 & -1.0 \\
\hline "FSB & 98D & " & 49121.97 & 57413.49 & 200.3 & 220.3 & 212.3 & 211.2 & -1.1 \\
\hline "FSB & 99D & " & 49399.05 & 57681.54 & 198.1 & 218.1 & 212.4 & 213.3 & 0.9 \\
\hline "FSB1 & $04 D$ & " & 47971.22 & 56117.73 & 190.4 & 210.4 & 204.6 & 198.5 & -6.1 \\
\hline "FSB1 & $.05 D$ & $"$ & 48823.22 & 57346.54 & 203.7 & 223.7 & 208.3 & 209.7 & 1.4 \\
\hline "FSB1 & $.05 \mathrm{DR}$ & & 48833.62 & 57358.44 & 188.5 & 208.6 & 211.0 & 209.4 & -1.6 \\
\hline "FSB1 & $106 \mathrm{D}$ & $"$ & 49390.58 & 56151.16 & 202.9 & 222.9 & 207.1 & 205.0 & -2.1 \\
\hline "FSB1 & $107 \mathrm{D}$ & $"$ & 50097.00 & 57007.19 & 200.9 & 220.9 & 213.8 & 214.2 & 0.4 \\
\hline "FSB1 & $.08 \mathrm{D}$ & " & 50314.94 & 58068.57 & 203.8 & 223.8 & 217.7 & 218.9 & 1.2 \\
\hline "FSB1 & $.09 \mathrm{D}$ & " & 49591.36 & 57808.53 & 205.8 & 225.8 & 213.7 & 214.6 & 0.9 \\
\hline "FSB1 & $10 \mathrm{D}$ & " & 48906.27 & 56254.41 & 191.1 & 211.1 & 205.6 & 203.4 & -2.2 \\
\hline "FSB1 & $11 \mathrm{D}$ & " & 50497.87 & 57132.28 & 201.7 & 221.7 & 215.6 & 216.8 & 1.2 \\
\hline "FSB1 & $12 \mathrm{D}$ & " & 47580.74 & 56567.24 & 188.9 & 208.9 & 206.2 & 200.4 & -5.8 \\
\hline "FSB1 & $13 \mathrm{D}$ & $"$ & 49834.16 & 56017.82 & 189.6 & 209.6 & 207.4 & 205.8 & -1.6 \\
\hline
\end{tabular}


Table F-2. Summary of Group Statistical Parameters (Continued)

\begin{tabular}{|c|c|c|c|c|c|c|c|c|}
\hline " FSB & $14 \mathrm{D}$ & 50967.90 & 56925.74 & 197.7 & 217.8 & 217.2 & 218.3 & 1.1 \\
\hline " FSB1 & $5 \mathrm{D}$ & 48150.84 & 54688.25 & 182.5 & 185.6 & 191.4 & 0.0 & -191.4 \\
\hline "FSB1 & $16 D$ & 49078.92 & 54719.06 & 186.4 & 194.7 & 192.0 & 187.1 & -4.9 \\
\hline "FSBI & $17 \mathrm{D}$ & 49218.37 & 56062.42 & 189.7 & 209.7 & 205.2 & 203.1 & -2.1 \\
\hline "FSBI & $8 \mathrm{D} "$ & 50121.08 & 56512.06 & 191.3 & 211.3 & 211.7 & 211.4 & -0.3 \\
\hline "FSBI & $19 \mathrm{D} "$ & 49439.73 & 56556.50 & 193.1 & 213.1 & 208.4 & 208.4 & 0 . \\
\hline "FSBI & 20D" & 48235.70 & 57803.07 & 196.5 & 216.5 & 209.7 & 207.8 & -1 \\
\hline "FSB1 & 21DR" & 47431.08 & 57547.99 & 191.3 & 211.3 & 207.2 & 203.1 & -4 \\
\hline "FSB1 & 22D" & 46940.61 & 56337.10 & 186.6 & 206.6 & 203.5 & 195.4 & -8.1 \\
\hline "FSB1 & 23D & 50541.46 & 56284.49 & 194.1 & 214.1 & 212.3 & 211.9 & -0. \\
\hline "FSB1 & 50PD" & 48579.67 & 56755.77 & 176.2 & 221.3 & 208.0 & 205.3 & -2 \\
\hline "FSL & 6D" & 50985.96 & 58408.90 & 202.1 & 222.1 & 220.2 & 222.9 & 2 \\
\hline "FSL & $7 D$ & 50664.69 & 58062.83 & 199.5 & 219.6 & 218.2 & 220.6 & 2 . \\
\hline "FSI & $8 D$ & 50635.20 & 57789.90 & 202.7 & 222.8 & 217.6 & 219.9 & 2 . \\
\hline "FSL & 9D & 50605.41 & 57503.53 & 201.4 & 221.5 & 216.8 & 219.0 & 2 . \\
\hline "FSS & $2 \mathrm{D}$ & 52790.27 & 56359.37 & 204.4 & 224.4 & 222.9 & 224.9 & 2 . \\
\hline "FSS & $3 \mathrm{D}$ & 52397.74 & 56296.61 & 205.8 & 225.8 & 220.7 & 222.5 & 1. \\
\hline "FSS & $4 \mathrm{D}$ & 51860.55 & 57000.99 & 202.6 & 222.6 & 219.0 & 223.5 & 4. \\
\hline "FTF & $21 "$ & 51767.59 & 58379.34 & 198.7 & 228.7 & 223.1 & 226.8 & 3. \\
\hline "HC & 1D" & 59866.62 & 51422.74 & 206.5 & 211.5 & 268.9 & 252.9 & -16. \\
\hline "HC & $2 \mathrm{E}$ & 59868.65 & 51461.16 & 205.7 & 210.7 & 269.5 & 252.7 & -16.8 \\
\hline " $\mathrm{HC}$ & $6 \mathrm{~B}$ & 60149.18 & 51775.71 & 210.2 & 215.2 & 268.9 & 252.1 & -16.8 \\
\hline "HMD & 1D & 56532.27 & 59273.24 & 199.7 & 219.7 & 209.7 & 221.8 & 12. \\
\hline " НОВ & $2 \mathrm{D}$ & 55595.50 & 53420.36 & 200.4 & 220.4 & 230.4 & 229.7 & -0 . \\
\hline " НОВ & $6 \mathrm{D}$ & 55275.15 & 51204.47 & 186.9 & 196.9 & 206.9 & 206.7 & -0 . \\
\hline "НОВ & $7 D$ & 54438.67 & 52713.30 & 197.4 & 217.4 & 220.9 & 218.2 & -2 \\
\hline "HSB & 84D" & 54436.38 & 52411.25 & 199.5 & 219.5 & 218.9 & 215.0 & -3 \\
\hline "HSB1 & 00PD" & 54436.51 & 52269.53 & 195.0 & 214.9 & 217.1 & 212.8 & -4 \\
\hline "HSB1 & 17D" & 53510.12 & 53797.83 & 200.3 & 220.3 & 223.7 & 217.9 & -5 \\
\hline "HSB1 & $25 \mathrm{D}$ & 56603.94 & 51862.91 & 199.4 & 219.4 & 221.3 & 224.4 & 3. \\
\hline "HSB1 & $26 \mathrm{D}$ & 55040.55 & 51311.10 & 190.5 & 200.5 & 205.2 & 204.8 & -0 \\
\hline " HSB1 & $27 \mathrm{D} "$ & 54789.02 & 51963.14 & 197.8 & 217.8 & 218.0 & 212.8 & -5. \\
\hline " HSBI & $29 \mathrm{D} "$ & 53269.76 & 52918.08 & 185.2 & 205.2 & 208.4 & 206.8 & -1 \\
\hline "HSB] & 30D & 52603.41 & 51955.69 & 182.1 & 202.1 & 200.2 & 205.0 & 4. \\
\hline "HSB1 & 31D & 54712.33 & 51106.47 & 195.7 & 201.2 & 205.1 & 201.8 & -3 \\
\hline "HSB1 & $35 \mathrm{D}$ & 54595.92 & 52185.96 & 199.9 & 219.9 & 218.3 & 213.8 & -4 \\
\hline " HSB1 & $36 \mathrm{D}$ & 54104.07 & 52811.18 & 200.2 & 220.2 & 220.7 & 216.4 & -4 \\
\hline "HSB1 & $40 \mathrm{D}$ & 54320.65 & 50853.37 & 194.1 & 214.1 & 213.9 & 206.3 & -7.6 \\
\hline "HSB1 & 42D" & 51959.92 & 54500.90 & 189 & 192.7 & 197.9 & 192.5 & -5 \\
\hline "HSB1 & 43D" & .30 & 77.30 & 19 & 216.9 & 213.2 & 206.4 & -6 \\
\hline "HSB1 & 48D" & 53168.04 & 51226.05 & 198.1 & 218.1 & 213.5 & 211.2 & -2 \\
\hline "HSB1 & $510 "$ & 52457.62 & 54277.34 & 197.6 & 200.9 & 207.2 & 200.9 & -6 \\
\hline "HSB1 & $52 \mathrm{D}$ & 52580.96 & 53242.99 & 197.0 & 199.2 & 205.6 & 198.4 & -7 \\
\hline "K 3 & $01 \mathrm{AP}$ & 34880.17 & 38881.66 & 193.3 & 197.7 & 208.8 & 206.5 & -2 \\
\hline " KDB & 1 & 35214.96 & 38571.78 & 184.8 & 205.8 & 208.5 & 209.6 & 1. \\
\hline " KDB & 2 & 35004.72 & 38470.07 & 182.5 & 203.5 & 207.0 & 208.4 & 1. \\
\hline "KDB & 3 & 35130.26 & 38328.17 & 184.2 & 205.4 & 207.8 & 209.3 & 1. \\
\hline "KSB & 1 & 34608.12 & 38694.54 & 175.6 & 205.6 & 204.2 & 205.1 & 0. \\
\hline " KSB & 2 & 34482.69 & 38601.79 & 173.8 & 203.8 & 203.9 & 204.4 & 0. \\
\hline "KSB & 3 & 34429.71 & 38728.16 & 169.7 & 199.7 & 203.1 & 203.5 & 0. \\
\hline "KSB & $4 \mathrm{~A}$ & 34579.07 & 38798.85 & 169.6 & 199.6 & 203.4 & 204.4 & 1. \\
\hline "KSB & $5 C$ & 34792.85 & 38779.18 & 172.9 & 182.9 & 204.9 & 205.9 & 1. \\
\hline "KSS & $1 D$ & 33704.58 & 32460.67 & 157.4 & 177.5 & 174.3 & 177.8 & 3. \\
\hline "LAC & 5DL" & 44096.56 & 27804.81 & 176.2 & 186.2 & 219.7 & 213.8 & -5 . \\
\hline
\end{tabular}


Table F-2. Summary of Group Statistical Parameters (Continued)

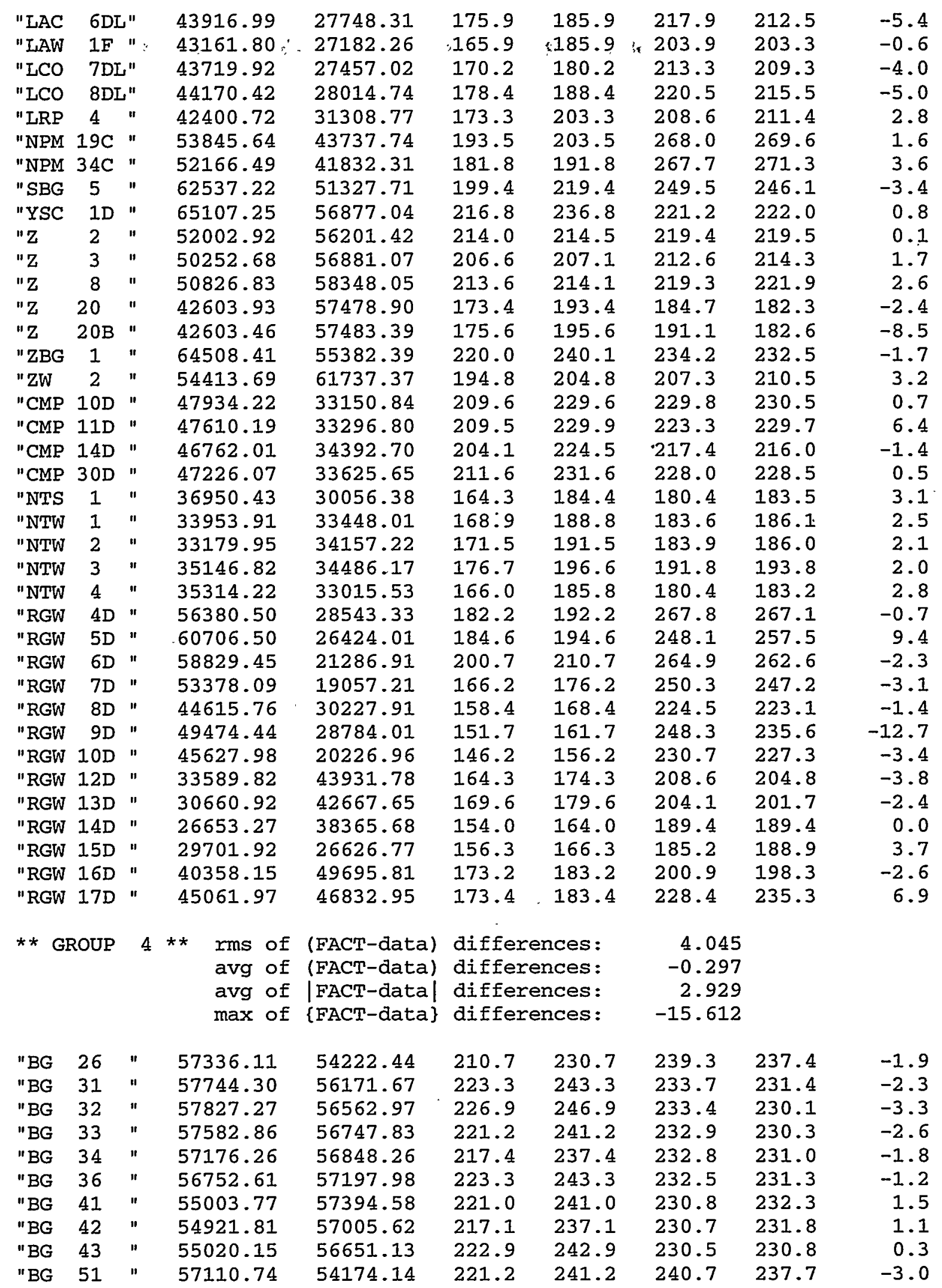


Table F-2. Summary of Group Statistical Parameters (Continued)

\begin{tabular}{|c|c|c|c|c|c|c|c|c|c|}
\hline "BG & 52 & $"$ & 54528.35 & 56814.86 & 223.8 & 243.8 & 229.3 & 230.5 & 1.2 \\
\hline "BG & 53 & " & 54139.12 & 57150.01 & 214.7 & 234.7 & 228.0 & 231.2 & 3.2 \\
\hline "BG & 54 & $"$ & 53834.44 & 56888.23 & 215.2 & 235.2 & 228.6 & 230.2 & 1.6 \\
\hline BG & 55 & " & 53534.89 & 56632.32 & 214.9 & 234.9 & 226.6 & 228.8 & 2.2 \\
\hline "BG & 56 & $"$ & 53362.38 & 56343.07 & 210.9 & 230.9 & 225.0 & 227.3 & 2.3 \\
\hline "BG & 57 & $"$ & 53650.24 & 56071.18 & 214.6 & 234.6 & 225.3 & 227.3 & 2.0 \\
\hline "BG & 58 & $"$ & 53941.50 & 55795.09 & 218.2 & 238.2 & 226.8 & 227.5 & 0.7 \\
\hline "BG & 59 & $"$ & 54238.88 & 55529.97 & 217.7 & 237.7 & 229.8 & 227.9 & -1.9 \\
\hline "BG & 60 & 18 & 54530.35 & 55256.29 & 215.5 & 235.5 & 230.8 & 228.7 & -2.1 \\
\hline "BG & 61 & $"$ & 54965.05 & 54846.04 & 225.0 & 245.0 & 232.8 & 230.3 & -2.5 \\
\hline "BG & 62 & $"$ & 55109.85 & 54709.14 & 222.5 & 242.5 & 233.4 & 230.7 & -2.7 \\
\hline "BG & 63 & $"$ & 55396.89 & 54426.18 & 224.2 & 244.2 & 235.2 & 231.7 & -3.5 \\
\hline "BG & 64 & " & 55688.22 & 54152.33 & 227.3 & 247.3 & 238.1 & 232.7 & -5.4 \\
\hline "BG & 65 & $"$ & 55978.13 & 53879.49 & 230.9 & 250.9 & 235.7 & 234.1 & -1.6 \\
\hline "BG & 66 & $"$ & 56275.73 & 54066.09 & 231.0 & 251.0 & 235.2 & 235.2 & 0.0 \\
\hline "BG & 67 & $"$ & 56447.94 & 54406.83 & 224.7 & 244.7 & 236.5 & 235.3 & -1.2 \\
\hline "BG & 68 & $"$ & 57329.68 & 56876.99 & 216.5 & 242.9 & 232.2 & 230.5 & -1.7 \\
\hline " BG & 69 & $"$ & 57304.98 & 56882.44 & 222.2 & 242.2 & 232.5 & 230.8 & -1.7 \\
\hline "BG & 80 & $"$ & 57056.01 & 56979.02 & 226.2 & 248.6 & 232.7 & 231.2 & -1.5 \\
\hline "BG & 81 & " & 57081.25 & 57000.00 & 222.9 & 246.9 & 227.3 & 231.0 & 3.7 \\
\hline "BG & 84 & $"$ & 57069.64 & 57077.74 & 227.2 & 247.2 & 232.6 & 231.0 & -1.6 \\
\hline "BG & 85 & $"$ & 57048.52 & 57105.85 & 228.0 & 248.0 & 232.6 & 231.1 & -1.5 \\
\hline "BG & 86 & $"$ & 57098.42 & 57097.69 & 228.0 & 248.0 & 232.5 & 231.0 & -1.5 \\
\hline "BG & 87 & $"$ & 57077.23 & 57130.31 & 226.2 & 245.8 & 232.3 & 230.9 & -1.4 \\
\hline "BG & 91 & $"$ & 56069.82 & 58655.49 & 205.4 & 235.4 & 218.8 & 229.1 & 10.3 \\
\hline "BG & 98 & $"$ & 56712.64 & 58075.77 & 212.5 & 242.5 & 224.5 & 229.2 & 4.7 \\
\hline "BG & 99 & $"$ & 57551.92 & 57188.59 & 215.9 & 245.9 & 232.5 & 228.8 & -3.7 \\
\hline "BG & 100 & $"$ & 58225.51 & 57976.77 & 203.3 & 233.3 & 224.8 & 218.1 & -6.7 \\
\hline "BG & 107 & $"$ & 58793.60 & 54776.73 & 208.3 & 228.3 & 234.6 & 231.4 & -3.2 \\
\hline "BG & 108 & $"$ & 58420.34 & 54426.07 & 217.3 & 247.3 & 239.0 & 237.3 & -1.7 \\
\hline "BG & 110 & $"$ & 57667.88 & 53534.74 & 224.3 & 254.3 & 241.5 & 240.6 & -0.9 \\
\hline "BG & 119 & $"$ & 56357.76 & 58300.26 & 209.2 & 229.2 & 215.4 & 229.6 & 14.2 \\
\hline "BG & 124 & $"$ & 56344.08 & 57802.53 & 214.8 & 234.8 & 231.8 & 231.0 & -0.8 \\
\hline " BGO & $1 D$ & $"$ & 57260.53 & 54013.08 & 225.0 & 245.0 & 238.5 & 238.6 & 0.1 \\
\hline " BGO & $2 \mathrm{D}$ & " & 57459.71 & 54803.95 & 218.9 & 238.9 & 238.2 & 236.1 & -2.1 \\
\hline "BGO & $3 \mathrm{D}$ & " & 57625.22 & 55585.01 & 227.6 & 247.6 & 235.6 & 233.8 & -1.8 \\
\hline "BGO & 3DR & & 57669.26 & 55740.24 & 217.5 & 237.6 & 231.9 & 232.8 & 0.9 \\
\hline " BGO & $4 \mathrm{D}$ & $"$ & 57785.92 & 56367.50 & 220.6 & 240.6 & 232.6 & 230.6 & -2.0 \\
\hline "BGO & $5 \mathrm{D}$ & " & 57835.51 & 56691.67 & 219.3 & 239.3 & 230.7 & 229.3 & -1.4 \\
\hline " BGO & $6 \mathrm{D}$ & " & 57360.50 & 56802.66 & 217.2 & 237.2 & 231.4 & 230.7 & -0.7 \\
\hline "BGO & 7D & $"$ & 56990.40 & 56888.68 & 220.2 & 240.2 & 232.6 & 231.4 & -1.2 \\
\hline "BGO & 8D & $"$ & 56717.15 & 57043.17 & 220.6 & 240.6 & 232.7 & 231.5 & -1.2 \\
\hline "BGO & $9 \mathrm{D}$ & $"$ & 56627.61 & 57289.98 & 209.2 & 229.2 & 230.1 & 230.9 & 0.8 \\
\hline " BGO & $10 \mathrm{DR}$ & & 56229.85 & 57367.58 & 218.3 & 238.3 & 231.7 & 231.6 & -0.1 \\
\hline "BGO & $11 D$ & $"$ & 55816.74 & 57455.69 & 216.3 & 236.3 & 230.9 & 231.8 & 0.9 \\
\hline "BGO & $11 \mathrm{DR}$ & & 55825.05 & 57499.11 & 213.1 & 233.0 & 230.5 & 231.7 & 1.2 \\
\hline "BGO & $12 \mathrm{D}$ & $"$ & 55405.74 & 57543.15 & 217.8 & 237.8 & 231.4 & 232.1 & 0.7 \\
\hline "BGO & $12 \mathrm{DR}$ & & 55395.81 & 57575.32 & 212.7 & 232.8 & 220.1 & 232.0 & 11.9 \\
\hline "BGO & $13 \mathrm{DR}$ & & 55027.63 & 57643.46 & 210.3 & 220.3 & 231.0 & 231.8 & 0.8 \\
\hline " BGO & $14 \mathrm{DR}$ & & 54873.25 & 57162.45 & 218.1 & 238.1 & 230.6 & 231.9 & 1.3 \\
\hline "BGO & $15 \mathrm{D}$ & $"$ & 54868.95 & 56806.97 & 218.7 & 238.7 & 230.0 & 231.1 & 1.1 \\
\hline "BGO & $16 \mathrm{D}$ & $"$ & 55158.28 & 56518.41 & 217.3 & 237.3 & 231.0 & 230.7 & -0.3 \\
\hline "BGO & $17 D$ & $"$ & 55319.71 & 56328.91 & 204.0 & 224.0 & 232.4 & 230.7 & -1.7 \\
\hline "BGO & $17 \mathrm{DR}$ & & 55328.25 & 56331.59 & 216.9 & 236.9 & 231.6 & 231.2 & -0.4 \\
\hline
\end{tabular}


Table F-2. Summary of Group Statistical Parameters (Continued)

\begin{tabular}{|c|c|c|c|c|c|c|c|c|c|}
\hline "BGO & $18 D$ & " & 55624.78 & 56264.47 & 219.6 & 239.6 & 232.0 & 231.8 & -0.2 \\
\hline "BGO & 20D & " & 5 & 55556.90 & $: 216: 3$ & 1236.3. & 233.8 & 232.9 & -0.9 \\
\hline "BGO & 21D & $"$ & 56178.17 & 55214.98 & 217.7 & 237.7 & 235.0 & 233.8 & -1.2 \\
\hline "BGO & $22 \mathrm{DR}$ & & 56485.97 & 54927.71 & 219.2 & 239.2 & 236.4 & 234.9 & -1.5 \\
\hline "BGO & $22 \mathrm{DX}$ & & 56445.03 & 55027.38 & 217.9 & 237.9 & 234.4 & 234.7 & 0.3 \\
\hline "BGO & 23D & $"$ & 56732.35 & 54636.72 & 222.0 & 242.0 & 236.0 & 235.9 & -0.1 \\
\hline "BGO & $24 \mathrm{D}$ & " & 56984.54 & 54352.38 & 221.0 & 241.0 & 237.1 & 237.0 & -0.1 \\
\hline "BGO & $26 \mathrm{D}$ & $"$ & 54075.62 & 57133.55 & 213.4 & 233.5 & 227.7 & 231.2 & 3.5 \\
\hline "BGO & 27D & " & 53654.23 & 56762.35 & 209.3 & 229.3 & 227.6 & 229.3 & 1.7 \\
\hline BGO & 28D & " & 53368.39 & 56486.76 & 210.1 & 230.1 & 226.3 & 227.8 & 1.5 \\
\hline "BGO & 29D & $"$ & 53068.49 & 56800.16 & 208.5 & 228.5 & 226.4 & 228.4 & 2.0 \\
\hline "BGO & 30D & " & 53375.39 & 56321.08 & 207.8 & 227.8 & 225.7 & 227.1 & 1.4 \\
\hline "BGO & $31 D$ & " & 53668.33 & 56051.90 & 211.1 & 231.1 & 226.7 & 227.2 & 0.5 \\
\hline "BGO & $32 \mathrm{D}$ & " & 54014.20 & 55714.31 & 214.5 & 234.5 & 227.7 & 227.4 & -0.3 \\
\hline "BGO & $33 D$ & " & 54395.96 & 55369.09 & 213.1 & 233.1 & 230.2 & 228.2 & -2.0 \\
\hline "BGO & $34 \mathrm{D}$ & " & 54724.82 & 55053.93 & 212.7 & 232.7 & 232.8 & 229.1 & -3.7 \\
\hline "BGO & $35 D$ & $"$ & 55129.57 & 54678.78 & 219.4 & 239.4 & 234.7 & 230.7 & -4.0 \\
\hline "BGO & $36 \mathrm{D}$ & " & 55411.89 & 54412.06 & 223.3 & 243.3 & 237.1 & 231.7 & -5.4 \\
\hline "BGO & $37 \mathrm{D}$ & " & 55755.24 & 54080.42 & 226.1 & 246.1 & 238.6 & 232.9 & -5.7 \\
\hline "BGO & $38 \mathrm{D}$ & $"$ & 55980.48 & 53867.44 & 222.3 & 242.3 & 236.5 & 233.7 & -2.8 \\
\hline "BGO & $39 D$ & " & 56300.85 & 54059.22 & 224.7 & 244.7 & 235.6 & 235.0 & -0.6 \\
\hline "BGO & $40 D$ & " & 53706.79 & 57209.70 & 216.6 & 226.5 & 222.5 & 231.0 & 8.5 \\
\hline "BGO & $44 \mathrm{D}$ & $"$ & 57038.45 & 57149.39 & 223.4 & 233.4 & 232.5 & 230.8 & -1.7 \\
\hline "BGO & $45 D$ & $"$ & 53598.50 & 56955.15 & 209.6 & 229.6 & 227.5 & 230.0 & 2.5 \\
\hline "BGO & $49 \mathrm{D}$ & " & 54776.67 & 54738.97 & 218.5 & 238.5 & 234.4 & 229.4 & -5.0 \\
\hline "BGO & $50 \mathrm{D}$ & " & 53090.30 & 56375.14 & 208.0 & 228.0 & 225.3 & 226.5 & 1.2 \\
\hline "BGO & $51 \mathrm{D}$ & " & 56440.93 & 54575.88 & 220.1 & 240.1 & 235.9 & 235.1 & -0.8 \\
\hline "BGO & $52 \mathrm{D}$ & " & 55899.95 & 55201.33 & 219.4 & 239.4 & 234.0 & 233.2 & -0.8 \\
\hline "BGX & $1 D$ & $"$ & 57732.18 & 57053.05 & 214.7 & 234.7 & 229.6 & 228.2 & -1.4 \\
\hline "BGX & $4 \mathrm{D}$ & $"$ & 56566.33 & 58409.49 & 203.8 & 223.8 & 215.9 & 228.1 & 12.2 \\
\hline "BGX & $9 D$ & $"$ & 58652.02 & 56986.86 & 212.4 & 232.4 & 226.7 & 221.7 & -5.0 \\
\hline "BGX & $10 \mathrm{D}$ & $"$ & 58733.61 & 56200.00 & 216.2 & 236.2 & 225.6 & 225.3 & -0.3 \\
\hline "BGX & $11 D$ & " & 58370.03 & 55374.96 & 216.7 & 236.7 & 235.6 & 231.6 & -4.0 \\
\hline "BGX & 12D & " & 58275.90 & 54485.29 & 223.7 & 243.7 & 239.0 & 237.5 & -1.5 \\
\hline " CCB & 1 & $"$ & 44003.41 & 48346.16 & 198.4 & 228.4 & 225.6 & 227.0 & 1.4 \\
\hline " CCB & 2 & $"$ & 43881.49 & 48236.71 & 198.6 & 228.6 & 222.7 & 226.6 & 3.9 \\
\hline "CCB & 3 & $"$ & 43967.36 & 48097.21 & 205.6 & 235.6 & 225.2 & 227.7 & 2.5 \\
\hline " CCB & 4 & $"$ & 44164.05 & 48180.84 & 211.2 & 241.2 & 226.2 & 228.6 & 2.4 \\
\hline "CRP & $5 \mathrm{D}$ & $"$ & 42229.13 & 51903.38 & 194.6 & 214.6 & 210.8 & 207.4 & -3.4 \\
\hline "CRP & $6 \mathrm{DR}$ & & 41693.10 & 51774.41 & 194.2 & 214.2 & 210.5 & 205.6 & -4.9 \\
\hline "CRP & 8D & $"$ & 41435.10 & 52175.72 & 191.0 & 211.0 & 207.6 & 203.2 & -4.4 \\
\hline "CSB & $1 \mathrm{~A}$ & " & 42479.31 & 50872.75 & 194.9 & 224.9 & 213.3 & 211.6 & -1.7 \\
\hline "CSB & $3 \mathrm{~A}$ & " & 42117.61 & 50737.59 & 193.0 & 223.0 & 210.6 & 209.4 & -1.2 \\
\hline "CSD & 13D & $"$ & 46092.11 & 45304.73 & 202.4 & 252.4 & 242.4 & 245.6 & 3.2 \\
\hline " FAB & 1 & $"$ & 54325.47 & 58788.57 & 215.4 & 235.4 & 228.4 & 233.0 & 4.6 \\
\hline "FAB & 2 & $"$ & 54474.28 & 58420.90 & 216.5 & 236.5 & 229.1 & 233.5 & 4.4 \\
\hline "FAB & 3 & $"$ & 54303.61 & 58131.15 & 211.8 & 231.8 & 228.9 & 232.4 & 3.5 \\
\hline "FAB & 4 & $"$ & 54128.54 & 58611.44 & 214.2 & 234.2 & 228.5 & 233.0 & 4.5 \\
\hline "FAC & 4 & $"$ & 54959.05 & 59088.39 & 207.8 & 237.8 & 228.5 & 231.0 & 2.5 \\
\hline "FAC & 5 & $"$ & 54677.73 & 58878.80 & 214.0 & 234.0 & 224.9 & 231.7 & 6.8 \\
\hline " FCA & 9DR & & 53338.69 & 59826.41 & 207.7 & 227.7 & 224.0 & 229.8 & 5.8 \\
\hline " FCA & 19D & $"$ & 53253.58 & 59500.00 & 209.7 & 229.7 & 217.2 & 231.4 & 14.2 \\
\hline $\mathrm{CB}$ & 1 & $"$ & 54082.43 & 57855.31 & 205.6 & 235.6 & 230.2 & 232.7 & 2.5 \\
\hline " FCB & 2 & $"$ & 54221.13 & 57666.65 & 205.2 & 235.2 & 228.6 & 232.3 & 3.7 \\
\hline
\end{tabular}


Table F-2. Summary of Group Statistical Parameters (Continued)

\begin{tabular}{|c|c|c|c|c|c|c|c|c|c|}
\hline " FCB & 4 & " & 53810.90 & 57856.79 & 204.5 & 234.5 & 228.2 & 232.4 & 4.2 \\
\hline "FCB & 5 & " & 53914.51 & 57540.54 & 217.1 & 237.1 & 228.8 & 232.3 & 3.5 \\
\hline " FCB & 6 & " & 53894.39 & 57636.32 & 215.1 & 235.1 & 229.1 & 232.5 & 3.4 \\
\hline "FET & 1D & " & 52405.62 & 57526.96 & 206.9 & 226.9 & 223.8 & 228.4 & 4.6 \\
\hline "FET & $2 \mathrm{D}$ & " & 52068.97 & 57476.04 & 209.5 & 229.5 & 222.4 & 226.7 & 4.3 \\
\hline "FRB & 1 & " & 53020.51 & 57461.61 & 212.2 & 232.2 & 227.7 & 231.0 & 3.3 \\
\hline "FRB & 3 & " & 52677.52 & 57420.02 & 216.2 & 231.2 & 224.9 & 229.7 & 4.8 \\
\hline "FRB & 4 & $"$ & 52732.71 & 57366.03 & 214.6 & 229.6 & 224.3 & 229.6 & 5.3 \\
\hline "FSB & $95 \mathrm{D}$ & $"$ & 48939.51 & 57049.06 & 207.8 & 227.8 & 210.5 & 209.1 & -1.4 \\
\hline "FSL & $3 \mathrm{D}$ & " & 51921.73 & 59265.15 & 205.9 & 226.0 & 222.8 & 227.5 & 4.7 \\
\hline "FSL & 4D & $"$ & 51627.03 & 59008.00 & 204.0 & 224.1 & 217.5 & 226.3 & 8.8 \\
\hline "FSL & 5D & " & 51222.93 & 58680.16 & 203.5 & 223.7 & 220.8 & 224.3 & 3.5 \\
\hline "FSS & 1D & $"$ & 52801.47 & 56514.53 & 209.9 & 229.9 & 223.7 & 226.0 & 2.3 \\
\hline "FTF & 3 & $"$ & 52574.12 & 58584.74 & 218.2 & 221.2 & 223.8 & 230.5 & 6.7 \\
\hline " FTF & 5 & $"$ & 52457.78 & 58405.31 & 215.3 & 235.3 & 223.5 & 231.7 & 8.2 \\
\hline "FTF & 7 & $"$ & 52422.54 & 58617.58 & 222.1 & 226.1 & 223.7 & 230.3 & 6.6 \\
\hline "FTF & 10 & $"$ & 52262.69 & 58753.89 & 215.1 & 235.1 & 220.1 & 231.2 & 11.1 \\
\hline " FTF & 12 & $"$ & 52008.76 & 58792.94 & 215.0 & 235.0 & 226.8 & 230.2 & 3.4 \\
\hline "FTF & 13 & $"$ & 52306.70 & 58030.74 & 216.1 & 236.1 & 228.2 & 229.6 & 1.4 \\
\hline " FTF & $24 \mathrm{~A}$ & $"$ & 52124.69 & 58702.05 & 212.7 & 232.7 & 222.0 & 229.8 & 7.8 \\
\hline " FTF & $25 \mathrm{~A}$ & " & 52221.44 & 58734.44 & 212.8 & 232.8 & 223.2 & 230.3 & 7.1 \\
\hline "FTF & 26 & $"$ & 52215.85 & 58675.92 & 206.3 & 226.3 & 223.3 & 229.0 & 5.7 \\
\hline "H & 6 & $"$ & 56466.89 & 52414.35 & 225.2 & 235.2 & 231.0 & 231.6 & 0.6 \\
\hline "H & 7 & " & 56455.12 & 52355.62 & 224.9 & 234.9 & 229.0 & 230.4 & 1.4 \\
\hline "H & 8 & $"$ & 56285.76 & 52050.36 & 218.4 & 228.4 & 227.0 & 224.6 & -2.4 \\
\hline "H & 9 & $"$ & 56317.35 & 52000.00 & 207.4 & 217.4 & 226.8 & 223.6 & -3.2 \\
\hline "H & 10 & $"$ & 55881.94 & 52127.81 & 222.5 & 232.5 & 227.3 & 225.5 & -1.8 \\
\hline "H & 11 & " & 55830.90 & 52096.44 & 212.0 & 222.0 & 227.7 & 223.6 & -4.1 \\
\hline "H & $18 \mathrm{~A}$ & $"$ & 55351.80 & 51966.92 & 217.5 & 227.5 & 224.1 & 219.0 & -5.1 \\
\hline "H & 19 & $"$ & 55081.94 & 52120.99 & 219.6 & 221.1 & 227.6 & 0.0 & -227.6 \\
\hline " $\mathrm{HCB}$ & 1 & $"$ & 61809.86 & 50683.36 & 222.6 & 252.6 & 263.6 & 254.1 & -9.5 \\
\hline " $\mathrm{HCB}$ & 3 & $"$ & 61740.10 & 50362.86 & 233.6 & 263.6 & 266.6 & 256.8 & -9.8 \\
\hline " $\mathrm{HCB}$ & 4 & $"$ & 61901.99 & 50477.10 & 235.9 & 265.9 & 264.4 & 255.1 & -9.3 \\
\hline "HIW & IID & & 56711.83 & 52871.21 & 213.0 & 228.0 & 231.9 & 235.1 & 3.2 \\
\hline "HIW & $1 \mathrm{MD}$ & & 56725.89 & 52908.50 & 214.9 & 239.7 & 235.5 & 235.8 & 0.3 \\
\hline "HIW & $1 P D$ & & 56636.55 & 52924.42 & 215.5 & 240.5 & 234.9 & 235.3 & 0.4 \\
\hline "HIW & $2 \mathrm{D}$ & $"$ & 55178.32 & 53976.50 & 210.9 & 230.8 & 231.1 & 230.0 & -1.1 \\
\hline " $\mathrm{HOB}$ & $3 \mathrm{D}$ & " & 56238.78 & 52787.04 & 207.7 & 227.7 & 230.3 & 231.4 & 1.1 \\
\hline " $\mathrm{HOB}$ & 4D & " & 56545.37 & 52617.01 & 210.4 & 230.4 & 232.2 & 233.0 & 0.8 \\
\hline "HR3 & 11 & $"$ & 58112.36 & 51444.75 & 200.4 & 230.0 & 259.7 & 246.5 & -13.2 \\
\hline "HR3 & 13 & $"$ & 58084.40 & 51702.80 & 205.1 & 234.8 & 258.9 & 246.0 & -12.9 \\
\hline " HR8 & 11 & $"$ & 57651.36 & 52097.77 & 207.9 & 237.6 & 245.8 & 241.6 & -4.2 \\
\hline " HR8 & 12 & $"$ & 57392.25 & 51983.55 & 206.3 & 235.9 & 239.4 & 238.3 & -1.1 \\
\hline "HR8 & 13 & " & 57317.15 & 51774.08 & 201.7 & 231.4 & 237.2 & 236.7 & -0.5 \\
\hline "HR8 & 14 & " & 57595.58 & 51583.84 & 202.3 & 231.9 & 242.3 & 241.4 & -0.9 \\
\hline "HSB & 65 & " & 56647.98 & 52801.67 & 212.4 & 242.4 & 232.7 & 234.8 & 2.1 \\
\hline "HSB & $65 C$ & " & 56665.66 & 52812.22 & 207.8 & 218.6 & 233.1 & 233.9 & 0.8 \\
\hline "HSB & 66 & $"$ & 55177.89 & 53117.82 & 198.1 & 228.1 & 224.7 & 226.5 & 1.8 \\
\hline "HSB & 67 & " & 56449.04 & 51902.78 & 200.7 & 221.1 & 222.9 & 222.8 & -0.1 \\
\hline "HSB & 68 & " & 54963.81 & 52241.99 & 213.3 & 238.5 & 221.7 & 218.7 & -3.0 \\
\hline "HSB & 69 & " & 54551.15 & 52349.03 & 199.0 & 227.7 & 219.4 & 215.4 & -4.0 \\
\hline "HSB & 70 & $"$ & 54070.99 & 53534.77 & 205.7 & 235.7 & 223.9 & 221.8 & -2.1 \\
\hline ISB & 71 & " & 53657.70 & 53897.63 & 204.8 & 234.8 & 224.0 & 219.7 & -4.3 \\
\hline HSB & $83 D$ & " & 56648.16 & 51986.31 & 198.7 & 223.3 & 224.9 & 225.5 & 0.6 \\
\hline
\end{tabular}


Table F-2. Summary of Group Statistical Parameters (Continued)

\begin{tabular}{|c|c|c|c|c|c|c|c|c|c|}
\hline "HSB & $85 \mathrm{C}$ & " & 57438.35 & 54041.12 & 214.2 & 224.2 & 239.2 & 238.0 & -1.2 \\
\hline "HSB & $86 \mathrm{D}$ & " & 54285.76 & 53402.43 & 206.6 & 236.6 & $223: 4$ & 222.6 & -0.8 \\
\hline "HSB1 & $.00 \mathrm{D}$ & " & 56931.76 & 52381.69 & 216.9 & 236.9 & 233.9 & 234.6 & 0.7 \\
\hline "HSB1 & .01D & " & 56718.21 & 52349.07 & 216.1 & 236.1 & 230.7 & 232.1 & 1.4 \\
\hline "HSB1 & D & " & 56511.84 & 52346.83 & 216.3 & 236.3 & 228.7 & 230.0 & 1.3 \\
\hline "HSB1 & $.03 D$ & " & 56360.00 & 52006.67 & 213.7 & 224.5 & 225.7 & 223.5 & -2.2 \\
\hline "HSB1 & .04D & $"$ & 56080.13 & 51843.39 & 210.6 & 224.8 & 224.8 & 221.2 & -3.6 \\
\hline "HSB1 & .05D & " & 55903.66 & 51967.39 & 211.8 & 231.6 & 225.3 & 222.9 & -2.4 \\
\hline "HSB1 & $.06 \mathrm{D}$ & " & 55732.90 & 52282.78 & 210.7 & 230.7 & 226.0 & 224.9 & -1.1 \\
\hline "HSBI & 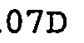 & " & 55498.90 & 52300.62 & $215.1^{\circ}$ & 235.1 & 224.7 & 223.7 & -1.0 \\
\hline "HSB1 & .08D & $"$ & 55236.33 & 52347.64 & 212.0 & 232.0 & 223.5 & 222.0 & -1.5 \\
\hline "HSBI & .09D & " & 54981.42 & 52399.37 & 213.0 & 233.0 & 222.7 & 220.7 & -2.0 \\
\hline "HSB1 & $10 \mathrm{D}$ & " & 54793.39 & 52541.16 & 211.4 & 231.4 & 222.1 & 220.2 & -1.9 \\
\hline "HSB1 & $11 \mathrm{E}$ & " & 54643.22 & 52723.98 & 211.7 & 231.7 & 221.9 & 220.6 & -1.3 \\
\hline "HSB1 & $12 \mathrm{E}$ & " & 54606.04 & 52970.91 & 211.7 & 231.7 & 222.5 & 222.6 & 0.1 \\
\hline "HSB1 & $13 \mathrm{D}$ & $"$ & 54404.28 & 53152.93 & 216.2 & 236.2 & 222.5 & 222.7 & 0.2 \\
\hline "HSB1 & $14 \mathrm{D}$ & " & 54381.54 & 53333.10 & 212.8 & 232.8 & 223.2 & 223.2 & 0.0 \\
\hline "HSB1 & $15 \mathrm{D}$ & $"$ & 54357.27 & 53530.56 & 213.9 & 233.9 & 223.9 & 224.2 & 0.3 \\
\hline "HSB1 & $16 \mathrm{D}$ & " & 54355.82 & 53771.94 & 214.5 & 234.5 & 225.9 & 225.1 & -0.8 \\
\hline "HSB1 & $32 \mathrm{D}$ & " & 56808.47 & 51790.09 & 206.5 & 226.5 & 221.2 & 227.2 & 6.0 \\
\hline "HSB1 & $33 \mathrm{D}$ & " & 57203.40 & 52190.74 & 208.5 & 228.5 & 235.4 & 235.6 & 0.2 \\
\hline "HSB1 & $34 \mathrm{D}$ & " & 56264.22 & 51647.94 & 205.8 & 218.4 & 222.1 & 219.0 & -3.1 \\
\hline "HSB1 & ס373. & " & 53941.36 & 53227.00 & 205.3 & 225.3 & 222.0 & 218.9 & -3.1 \\
\hline "HSB1 & $38 D$ & " & 53698.71 & 54179.56 & 208.1 & 228.1 & 223.8 & 221.1 & -2.7 \\
\hline "HSB1 & 390 & $"$ & 55354.57 & 51755.32 & 206.7 & 222.5 & 222.5 & 214.2 & -8.3 \\
\hline "HSB1 & 41D & " & 57112.67 & 51433.96 & 217.8 & 237.8 & 240.8 & 235.1 & -5.7 \\
\hline "HSB1 & D6 & " & 56300.99 & 50875.82 & 204.0 & 224.1 & 222.6 & 222.7 & 0.1 \\
\hline "HSB1 & D77 & " & 54369.35 & 54719.63 & 215.2 & 235.2 & 231.5 & 227.5 & -4.0 \\
\hline "HSB1 & 49D & ". & 55301.36 & 51976.82 & 207.0 & 227.0 & 222.6 & 218.0 & -4.6 \\
\hline "HSB1 & $50 \mathrm{D}$ & " & 56750.68 & 52030.46 & 206.9 & 226.9 & 226.8 & 227.1 & 0.3 \\
\hline "HSL & $1 D$ & " & 57079.06 & 52458.54 & 219.8 & 239.8 & 235.6 & 237.1 & 1.5 \\
\hline "HSL & $2 \mathrm{D}$ & $"$ & 57568.99 & 52365.85 & 225.2 & 245.3 & 242.1 & 241.8 & -0.3 \\
\hline "HSL & 6D & " & 58749.88 & 52594.22 & 239.4 & 243.9 & 260.1 & 247.9 & -12.2 \\
\hline "HSS & 1D & " & 61753.98 & 46793.47 & 236.5 & 256.5 & 268.8 & 268.1 & -0.7 \\
\hline "HSS & $2 D$ & " & 61808.98 & 46521.70 & 234.5 & 254.5 & 267.9 & 267.9 & 0.0 \\
\hline "HWS & 2 & " & 47150.77 & 47010.39 & 215.3 & 245.3 & 245.5 & 243.4 & -2.1 \\
\hline "K & $01 P$ & $"$ & 34699.85 & 38956.80 & 194.4 & 201.0 & 205.1 & 205.3 & 0.2 \\
\hline "KAB & 1 & $"$ & 34512.97 & 37703.87 & 194.0 & 224.0 & 205.9 & 205.6 & -0.3 \\
\hline "KAB & 2 & $"$ & 34729.28 & 36998.69 & 198.6 & 228.6 & 209.4 & 205.8 & -3.6 \\
\hline "KAB & 3 & $"$ & 34252.24 & 36483.51 & 193.0 & 223.0 & 203.6 & 199.7 & -3.9 \\
\hline "KAB & 4 & " & 34008.71 & 37557.00 & 187.0 & 217.0 & 202.9 & 201.4 & -1.5 \\
\hline "KAC & 1 & " & 37172.33 & 37252.49 & 199.0 & 229.0 & 219.3 & 219.3 & 0.0 \\
\hline " KAC & 2 & $"$ & 37251.77 & 37326.09 & 195.4 & 225.4 & 221.5 & 220.0 & -1.5 \\
\hline "KAC & 3 & $"$ & 37286.28 & 37263.85 & 195.8 & 225.8 & 222.0 & 220.1 & -1.9 \\
\hline "KAC & 4 & $"$ & 37208.99 & 37128.67 & 178.0 & 208.0 & 218.1 & 217.6 & -0.5 \\
\hline "KAC & 5 & $"$ & 37270.51 & 37226.21 & 204.3 & 224.3 & 222.4 & 220.4 & -2.0 \\
\hline "KAC & 6 & " & 37243.68 & 37209.62 & 204.6 & 224.6 & 222.3 & 220.1 & -2.2 \\
\hline " KAC & 7 & " & 37150.77 & 37344.90 & 203.0 & 223.0 & 219.5 & 219.7 & 0.2 \\
\hline " KAC & 8 & " & 37192.40 & 37216.54 & 192.3 & 212.3 & 221.2 & 218.3 & -2.9 \\
\hline "KAC & 9 & " & 37152.62 & 37288.17 & 195.7 & 215.7 & 220.8 & 218.5 & -2.3 \\
\hline " KBP & $1 \mathrm{D}$ & " & 34873.24 & 36997.53 & 192.0 & 202.1 & 208.2 & 206.2 & -2.0 \\
\hline " KCB & 1 & " & 34207.66 & 38175.04 & 183.6 & 213.6 & 204.7 & 202.9 & -1.8 \\
\hline " KCB & 2 & " & 34063.53 & 38391.13 & 187.7 & 217.7 & 202.8 & 201.6 & $-1 . .2$ \\
\hline "KCB & 3 & $"$ & 33829.55 & 38242.63 & 184.1 & 214.1 & 202.3 & 199.6 & -2.7 \\
\hline
\end{tabular}


F-40 WSRC-TR-99-00248, Rev. 0, Regional Groundwater Flow Model for C, K, L, and P Reactor Areas

Table F-2. Summary of Group Statistical Parameters (Continued)

\begin{tabular}{|c|c|c|c|c|c|c|c|c|c|}
\hline " KCB & 4 & $"$ & 33963.75 & 38025.59 & 188.9 & 218.9 & 205.5 & 201.2 & -4.3 \\
\hline " KCB & 5 & $"$ & 33764.06 & 38167.82 & 189.3 & 209.3 & 200.7 & 199.3 & -1.4 \\
\hline " KCB & 6 & $"$ & 33823.71 & 38365.23 & 188.7 & 208.7 & 201.2 & 199.5 & -1.7 \\
\hline " KCB & 7 & $"$ & 34486.92 & 38097.90 & 196.5 & 216.5 & 205.3 & 205.6 & 0.3 \\
\hline " KDB & 4 & $"$ & 34890.68 & 38371.73 & 189.2 & 209.2 & 207.0 & 207.9 & 0.9 \\
\hline " KDB & 5 & $"$ & 34831.09 & 38655.12 & 188.5 & 208.5 & 205.5 & 207.0 & 1.5 \\
\hline " KDT & 1D & $"$ & 35191.60 & 38682.66 & 193.7 & 213.7 & 208.1 & 209.5 & 1.4 \\
\hline " KRB & 8 & $"$ & 35269.15 & 39422.20 & 195.8 & 215.8 & 208.5 & 207.3 & -1.2 \\
\hline " KRB & $16 \mathrm{D}$ & $"$ & 35354.26 & 39398.39 & 191.5 & 211.5 & 209.3 & 207.8 & -1.5 \\
\hline " KRB & 17D & $"$ & 35080.66 & 40027.41 & 186.8 & 206.8 & 206.0 & 201.9 & -4.1 \\
\hline "KRB & $18 \mathrm{D}$ & $"$ & 35196.02 & 40122.82 & 185.8 & 205.8 & 204.6 & 201.7 & -2.9 \\
\hline " KRB & 19D & $"$ & 35327.74 & 40153.30 & 186.8 & 206.8 & 203.8 & 202.2 & -1.6 \\
\hline "KRP & 1 & $"$ & 37318.17 & 38629.26 & 207.0 & 237.0 & 218.6 & 221.2 & 2.6 \\
\hline " KRP & 2 & $"$ & 37515.57 & 38546.00 & 199.2 & 229.2 & 219.5 & 222.4 & 2.9 \\
\hline " KRP & 4 & " & 37397.01 & 38427.35 & 188.7 & 218.7 & 218.5 & 221.0 & 2.5 \\
\hline " KRP & 5 & $"$ & 37048.11 & 38750.66 & 200.8 & 210.8 & 216.3 & 219.1 & 2.8 \\
\hline "KRP & 6 & $"$ & 37009.08 & 38350.12 & 203.1 & 213.1 & 217.9 & 219.7 & 1.8 \\
\hline " KRP & 7 & $"$ & 36699.81 & 38603.56 & 203.1 & 213.2 & 215.9 & 218.1 & 2.2 \\
\hline " KRP & 8 & $"$ & 37116.32 & 38597.27 & 200.1 & 210.1 & 217.1 & 219.7 & 2.6 \\
\hline " KRP & 9 . & $"$ & 37210.32 & 38464.17 & 200.8 & 210.8 & 218.3 & 220.3 & 2.0 \\
\hline "KSB & $5 D$ & $"$ & 34791.55 & 38770.15 & 194.5 & 214.5 & 204.5 & 206.6 & 2.1 \\
\hline "KSM & 1D & $"$ & 35147.98 & 38726.59 & 193.7 & 213.7 & 208.3 & 209.1 & 0.8 \\
\hline "LAC & 1 & $"$ & 44037.76 & 27687.88 & 191.1 & 221.1 & 216.5 & 214.4 & -2.1 \\
\hline "LAC & 2 & $"$ & 44009.27 & 27787.58 & 193.4 & 223.4 & 216.1 & 214.9 & -1.2 \\
\hline "LAC & 3 & " & 43900.98 & 27679.23 & 190.7 & 220.7 & 216.5 & 213.6 & -2.9 \\
\hline "LAC & 4 & $"$ & 43985.08 & 27672.80 & 185.3 & 215.3 & 216.1 & 213.8 & -2.3 \\
\hline "LAC & $5 \mathrm{DU}$ & & 44089.18 & 27786.44 & 207.9 & 227.8 & 219.5 & 215.8 & -3.7 \\
\hline "LAC & $6 \mathrm{DU}$ & & 43910.52 & 27728.93 & 201.7 & 221.7 & 219.0 & 214.3 & -4.7 \\
\hline "ILAC & $7 \mathrm{DI}$ & & 43812.28 & 27590.94 & 177.4 & 187.4 & 215.1 & 211.0 & -4.1 \\
\hline "LAC & $7 D U$ & & 43817.61 & 27607.80 & 204.9 & 224.8 & 218.1 & 213.3 & -4.8 \\
\hline "LAC & 8DI & & 43990.69 & 27552.51 & 180.4 & 190.4 & 217.5 & 212.0 & -5.5 \\
\hline "LAC & $8 \mathrm{DU}$ & & 43995.60 & 27571.30 & 199.8 & 219.8 & 218.1 & 213.8 & -4.3 \\
\hline "LAW & $2 \mathrm{C}$ & $"$ & 42471.74 & 28401.16 & 171.2 & 191.2 & 209.2 & 207.8 & -1.4 \\
\hline "LAW & $3 c$ & $"$ & 45049.46 & 27858.57 & 194.9 & 214.9 & 235.2 & 219.9 & -15.3 \\
\hline "LCO & 1 & $"$ & 43676.11 & 27723.24 & 195.8 & 225.8 & 214.8 & 212.8 & -2.0 \\
\hline "LCO & 2 & $"$ & 43784.81 & 27822.41 & 196.6 & 226.6 & 215.1 & 214.0 & -1.1 \\
\hline "LCO & 3 & $"$ & 43829.21 & 27695.61 & 196.3 & 226.3 & 229.1 & 213.5 & -15.6 \\
\hline "LCO & 4 & $"$ & 43729.76 & 27598.57 & 192.3 & 222.3 & 212.6 & 212.2 & -0.4 \\
\hline "LCO & $5 D I$ & & 43560.94 & 27519.03 & 174.9 & 184.9 & 212.9 & 209.0 & -3.9 \\
\hline "LCO & 6DI & & 43613.61 & 27604.75 & 178.0 & 188.0 & 213.7 & 210.1 & -3.6 \\
\hline "LCO & $8 \mathrm{DU}$ & & 44151.94 & 28018.67 & 211.1 & 226.1 & 220.6 & 217.5 & -3.1 \\
\hline "LDB & 1 & $"$ & 43460.04 & 28472.85 & 185.0 & 215.0 & 217.4 & 215.3 & -2.1 \\
\hline "LDB & 2 & " & 43675.04 & 28550.75 & 184.5 & 214.5 & 219.4 & 216.6 & -2.8 \\
\hline "LDB & 3 & $"$ & 43434.68 & 28664.71 & 199.3 & 219.3 & 218.9 & 216.6 & -2.3 \\
\hline "LDB & 4 & " & 43198.42 & 28449.23 & 200.7 & 220.7 & 216.9 & 214.3 & -2.6 \\
\hline "LRP & 2 & " & 42626.81 & 31171.46 & 184.7 & 214.7 & 210.2 & 214.1 & 3.9 \\
\hline "LRP & 3 & " & 42469.52 & 31185.16 & 191.4 & 221.4 & 209.5 & 214.2 & 4.7 \\
\hline "LSB & 1 & $"$ & 43415.55 & 27732.52 & 192.7 & 222.7 & 211.6 & 211.2 & -0.4 \\
\hline "LSB & 2 & $"$ & 43551.19 & 27776.17 & 195.0 & 225.0 & 212.4 & 212.4 & 0.0 \\
\hline "LSB & 3 & $"$ & 43492.70 & 27956.99 & 196.6 & 226.6 & 217.2 & 213.2 & -4.0 \\
\hline "LSB & 4 & " & 43266.79 & 27936.41 & 191.5 & 221.5 & 216.8 & 211.5 & -5.3 \\
\hline "MGC & 9 & $"$ & 54500.94 & 56270.36 & 217.3 & 237.3 & 229.6 & 229.5 & -0.1 \\
\hline "MGC & 11 & $"$ & 54632.54 & 56119.91 & 219.2 & 239.2 & 233.4 & 229.5 & -3.9 \\
\hline "MGC & 19 & $"$ & 55156.34 & 55515.60 & 230.6 & 234.6 & 232.2 & 230.9 & -1.3 \\
\hline
\end{tabular}


Table F-2. Summary of Group Statistical Parameters (Continued)

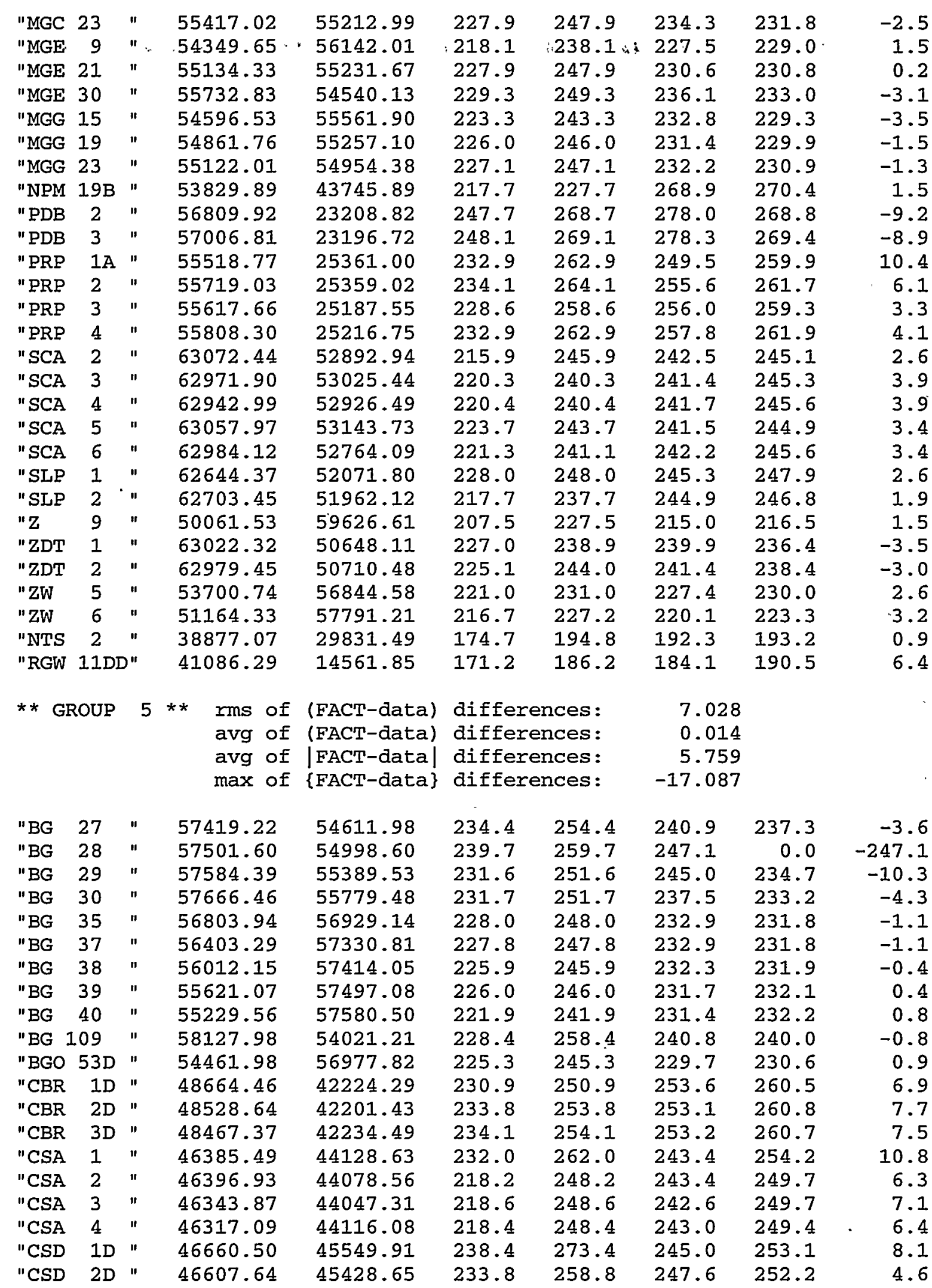


Table F-2. Summary of Group Statistical Parameters (Continued)

\begin{tabular}{|c|c|c|c|c|c|c|c|c|c|}
\hline "CSD & $4 \mathrm{D}$ & $"$ & 46528.06 & 45463.56 & 213.5 & 263.5 & 244.0 & 248.4 & 4.4 \\
\hline "CSD & 8D & $"$ & 46386.31 & 45546.03 & 226.8 & 256.8 & 243.2 & 249.3 & 6.1 \\
\hline "CSD & 9D & $"$ & 46299.69 & 45447.80 & 226.2 & 256.2 & 243.2 & 249.1 & 5.9 \\
\hline CSD & $10 D$ & $"$ & 46270.84 & 45467.42 & 224.5 & 254.5 & 243.1 & 248.3 & 5.2 \\
\hline ESD & $11 D$ & $"$ & 46408.43 & 46319.64 & 220.9 & 250.9 & 243.0 & 245.1 & 2 . \\
\hline ESD & $12 \mathrm{D}$ & " & 46380.19 & 45352.78 & 224.5 & 254.5 & 243.6 & 249.0 & \\
\hline CSF & ID & $"$ & 45753.00 & 44835.79 & 228.2 & 248.2 & 243.4 & 247.5 & \\
\hline "CSF & $2 \mathrm{D}$ & $"$ & 46897.26 & 43247.88 & 235.2 & 255.2 & 251.0 & 6.0 & \\
\hline "CSO & 1 & $"$ & 48469.42 & 42931.91 & 232.0 & 262.0 & 251.4 & 51.9 & 10. \\
\hline Cso & 2 & $"$ & 48551.57 & 42958.61 & 209.7 & 239.7 & 252.6 & 255.8 & 3.2 \\
\hline $\operatorname{CSR}$ & 1 & $"$ & 49477.36 & 46134.32 & 237.2 & 267.2 & 256.5 & 255.8 & -0 \\
\hline CSR & 3 & $"$ & 50064.50 & 46849.58 & 238.1 & 268.1 & 254.5 & 254.3 & -0 . \\
\hline CSR & 4 & $"$ & 49878.44 & 46048.77 & 237.6 & 267.6 & 256.7 & 258.3 & \\
\hline "FAC & 3 & $"$ & 54769.41 & 58918.61 & 224.8 & 254.8 & 229.1 & 236.5 & 7 . \\
\hline "FAC & $5 \mathrm{P}$ & $"$ & 54794.41 & 59074.22 & 225.7 & 235.7 & 229.7 & 233.9 & 4. \\
\hline "FAC & 6 & $"$ & 54804.95 & 59024.23 & 216.2 & 236.2 & 220.8 & 232.0 & 11. \\
\hline "FAC & 7 & $"$ & 54824.03 & 59014.45 & 215.7 & 235.7 & 223.2 & 231.8 & 8. \\
\hline "FAC & 8 & $"$ & 54826.86 & 58980.62 & 216.0 & 236.0 & 227.2 & 232.0 & \\
\hline "FAL & 1 & $"$ & 53257.63 & 59339.73 & 207.0 & 238.5 & 218.7 & 233.5 & \\
\hline "FC & $1 \mathrm{D}$ & $"$ & 52956.68 & 1.23 & 217.2 & 222.2 & 223.6 & 22.4 & \\
\hline "FCA & $2 C$ & $"$ & 53251.84 & 59525.09 & 295.3 & 299.3 & 297.4 & 0.0 & -297 \\
\hline "FCA & $2 \mathrm{D}$ & $"$ & 53254.73 & 59524.27 & 219.0 & 239.0 & 225.0 & 235.8 & 10 \\
\hline " FCA & $9 D$ & $"$ & 53335.59 & 59818.59 & 221.9 & 241.9 & 225.2 & 237.5 & 12 \\
\hline "FCA & $10 \mathrm{~A}$ & $"$ & 53186.21 & 59891.13 & 221.0 & 241.0 & 225.3 & 236.7 & 11. \\
\hline " FCA & $10 D$ & $"$ & 53342.73 & 59857.45 & 219.5 & 239.5 & 225.8 & 235.8 & \\
\hline "FCA & $16 \mathrm{~A}$ & $"$ & 53237.05 & 60145.21 & 215.1 & 235.1 & 225.2 & 232.1 & 6 \\
\hline "FCA & $16 \mathrm{~B}$ & $"$ & 53238.89 & 60143.29 & 295.3 & 299.3 & 298.0 & 0.0 & -298 . \\
\hline "FCA & $16 \mathrm{D}$ & $"$ & 53384.25 & 60112.90 & 221.1 & 241.1 & 225.0 & 236.3 & 11. \\
\hline "FSL & $1 D$ & $"$ & 52707.36 & 60425.06 & 208.5 & 228.6 & 224.7 & 227.0 & \\
\hline FTF & 1 & $"$ & 52547.55 & 58772.37 & 221.2 & 241.2 & 227.4 & 235.1 & \\
\hline FTF & 2 & $"$ & 52624.70 & 6.94 & 219.4 & 239.4 & 225.2 & 234.3 & \\
\hline "FTF & 4 & $"$ & 52575.72 & 9.71 & 216.6 & 36.6 & 223.8 & 232.7 & \\
\hline "FTF & 6 & $"$ & 52377.87 & 58540.68 & 216.9 & 236.9 & 223.4 & 232.2 & 8. \\
\hline "FTF & 8 & $"$ & 52414.24 & 58721.88 & 219.6 & 239.6 & 225.1 & 233.8 & 8. \\
\hline "FTF & 9 & $"$ & 52160.67 & 58925.65 & 216.4 & 236.4 & 221.1 & 231.4 & 10. \\
\hline TF & 11 & $"$ & 52077.61 & 58634.46 & 215.8 & 235.8 & 220.5 & 230.7 & 10. \\
\hline FTF & 27 & $"$ & .35 & & 3.5 & 3.5 & 223.3 & 231.4 & \\
\hline IAA & $1 D$ & $"$ & 60573.75 & 3.38 & 261.8 & 281.8 & 276.8 & 280.3 & \\
\hline " HAA & $2 \mathrm{D}$ & $"$ & 59097.23 & 50767.79 & 260.3 & 280.4 & 276.6 & 275.3 & -1 \\
\hline " HAA & 3D & $"$ & 58123.23 & 51458.39 & 246.7 & 264.3 & 264.6 & 258.6 & -6 . \\
\hline HAA & $4 \mathrm{D}$ & $"$ & 59988.35 & 51884.83 & 255.7 & 275.7 & 270.1 & 268.2 & -1 \\
\hline " HAA & 6D & $"$ & 61791.83 & 50700.99 & 247.1 & 267.2 & 264.8 & 256.8 & -8 . \\
\hline $\mathrm{HAC}$ & 1 & $"$ & 59513.05 & 51932.39 & 258.8 & 278.8 & 269.4 & 268.7 & -0 . \\
\hline $\mathrm{HAC}$ & 2 & $"$ & 6.04 & 55 & .8 & 278.8 & 9.0 & 7.5 & $\perp$ \\
\hline$A C$ & 3 & $"$ & 59416.25 & 51965.64 & 255.0 & 275.0 & 269.1 & 267.3 & \\
\hline $\mathrm{HAC}$ & 4 & $"$ & 59460.26 & 51891.78 & 254.1 & 274.1 & 269.6 & 267.7 & -1 \\
\hline HAP & 1 & $"$ & 61253.46 & 50579.78 & 256.3 & 276.3 & 270.9 & 269.7 & -1 \\
\hline HAP & 2 & $"$ & 61353.75 & 50469.62 & 243.8 & 263.8 & 270.4 & 261.4 & -9 \\
\hline$\theta C$ & $1 \mathrm{E}$ & $"$ & 59863.68 & 51423.36 & 251.5 & 256.5 & 275.0 & 264.3 & -10 \\
\hline${ }^{2}$ & $2 F$ & $"$ & 59867.82 & 51457.24 & 250.7 & 255.7 & 274.3 & 263.6 & -10 \\
\hline ICA & 1 & $"$ & 61242.75 & 51923.26 & 253.7 & 273.7 & 269.4 & 261.5 & \\
\hline$\sim A$ & 2 & $"$ & 61027.49 & 51707.50 & 242.0 & 273.4 & 270.3 & 260.2 & \\
\hline $\mathrm{CA}$ & 3 & $"$ & 61269.49 & 52050.48 & 253.8 & 273.8 & 269.2 & 260.7 & -8 \\
\hline $\mathrm{HCA}$ & 4 & $"$ & 61080.70 & 51959.75 & 241.9 & 273.3 & 269.3 & 258.7 & -10 \\
\hline
\end{tabular}


Table F-2. Summary of Group Statistical Parameters (Continued)

\begin{tabular}{|c|c|c|c|c|c|c|c|c|c|}
\hline " $\mathrm{HCB}$ & 2 & " & 61660.45 & 50574.96 & 239.9 & 269.9 & 268.1 & 257.4 & -10.7 \\
\hline "HET & ID & $"$ & 58616.55 & 51895.27 & 240.3 & 260.3 & 267.8 & 257.3 & -10.5 \\
\hline " HET & $2 \mathrm{D}$ & $"$ & 58186.81 & 52045.60 & 239.7 & 259.7 & 258.6 & 252.0 & -6.6 \\
\hline "HET & $3 D$ & $"$ & 58220.83 & 52128.23 & 239.9 & 259.9 & 259.4 & 251.7 & -7.7 \\
\hline HET & $4 \mathrm{D}$ & $"$ & 58293.12 & 52198.95 & 239.5 & 259.6 & 259.4 & 252.0 & -7.4 \\
\hline GHP & $1 D$ & " & 58413.10 & 50996.42 & 260.4 & 270.1 & 271.4 & 267.1 & -4.3 \\
\hline " HHP & $2 D$ & " & 58647.26 & 50803.30 & 263.2 & 273.2 & 274.8 & 270.8 & -4.0 \\
\hline " HR3 & $18 \mathrm{P}$ & $"$ & 58213.36 & 51574.78 & 244.3 & 264.3 & 267.7 & 258.2 & -9.5 \\
\hline "HSL & $3 D$ & " & 57921.13 & 52353.06 & 233.7 & 253.8 & 250.4 & 245.0 & -5.4 \\
\hline HSL & $4 \mathrm{D}$ & " & 58355.70 & 52467.41 & 245.0 & 265.1 & 262.0 & 250.1 & -11.9 \\
\hline HSL & $5 \mathrm{D}$ & " & 58542.10 & 52538.71 & 247.8 & 267.7 & 265.9 & 251.7 & -14.2 \\
\hline "HSL & $5 \mathrm{D}$ & " & 58542.10 & 52538.71 & 242.6 & 247.7 & 265.9 & 248.8 & -17.1 \\
\hline HSL & $6 \mathrm{D}$ & $"$ & 58749.88 & 52594.22 & 243.9 & 264.0 & 260.1 & 251.2 & -8.9 \\
\hline HSL & D & $"$ & 58940.64 & 52568.70 & 242.3 & 262.4 & 260.0 & 251.9 & -8.1 \\
\hline ASL & $8 D$ & " & 59328.98 & 52500.00 & 248.4 & 268.4 & 260.9 & 255.7 & -5. \\
\hline Ass & 3D & " & 61921.70 & 47419.48 & 262.6 & 282.6 & 281.9 & 277.9 & -4 \\
\hline HTF & 1 & $"$ & 60062.04 & 51380.18 & 236.9 & 256.9 & 272.9 & 259.9 & -13.0 \\
\hline ATF & 2 & $"$ & 60139.61 & 51225.68 & 237.0 & 257.0 & 274.3 & 260.6 & -13 \\
\hline "HTF & 4 & $"$ & 59915.86 & 51293.68 & 235.2 & 255.2 & 274.3 & 259.7 & -14.6 \\
\hline$A T F$ & 5 & $"$ & 60030.29 & 51024.00 & 264.3 & 284.3 & 279.6 & 280.6 & 1.0 \\
\hline " HTF & 7 & $"$ & 59978.19 & 50769.26 & 263.5 & 283.5 & 275.9 & 280.5 & 4.6 \\
\hline "HTF & 8 & $"$ & 59863.51 & 50936.77 & 263.6 & 283.6 & 273.9 & 280.1 & 6.2 \\
\hline " HTF & 9 & $"$ & 59681.77 & 51365.93 & 245.8 & 265.8 & 273.8 & 265.8 & -8 \\
\hline "HTF & 10 & $"$ & 59791.26 & 51207.71 & 245.2 & 265.2 & 273.3 & 266.0 & -7.3 \\
\hline "HTF & 11 & $"$ & 59652.43 & 51112.49 & 238.9 & 258.9 & 274.0 & 262.1 & -11.9 \\
\hline "HTF & 12 & $"$ & 59551.62 & 58.65 & 242.9 & 262.9 & 273.5 & 264.0 & -9.5 \\
\hline "HTF & 13 & $"$ & 59614.63 & 51588.76 & 262.6 & 282.6 & 274.2 & 276.9 & 2 \\
\hline "HTF & 14 & " & 59493.75 & 51616.50 & 261.9 & 281.9 & 273.2 & 276.0 & 2.8 \\
\hline " HTF & 15 & " & 59354.29 & 51484.61 & 260.7 & 280.7 & 273.6 & 275.9 & 2. \\
\hline "HTF & 16 & $"$ & 60031.80 & 51800.66 & 248.3 & 268.3 & 269.7 & 264.2 & -5 \\
\hline $\mathrm{ATF}$ & 17 & $"$ & 59380.01 & 52399.25 & 238.4 & 258.4 & 262.5 & 254.0 & -8.5 \\
\hline "HTF & 18 & $"$ & 59242.35 & 51581.81 & 251.7 & 271.7 & 271.6 & 268.4 & -3.2 \\
\hline "HTF & 19 & " & 59128.57 & 51739.61 & 245.7 & 265.7 & 269.2 & 263.0 & -6.2 \\
\hline "HTF & 20 & " & 59171.13 & 51905.18 & 251.9 & 271.9 & 267.9 & 266.5 & -1 \\
\hline "HTF & 21 & " & 59326.30 & 51795.42 & 242.6 & 262.6 & 269.6 & 260.6 & -9 \\
\hline "HTF & 22 & ". & 60458.67 & 50905.75 & 251.4 & 271.4 & 275.5 & 270.9 & -4 \\
\hline "HTF & 23 & " & 60572.75 & 50881.19 & 256.8 & 276.8 & 274.6 & 274.5 & -0. \\
\hline "HTF & 24 & $"$ & 60675.65 & 8.81 & 7.8 & 277.8 & 4.1 & 274.6 & 0.5 \\
\hline $\mathrm{ATF}$ & 25 & " & 60770.53 & .25 & .5 & 27 & 27 & 271.0 & -3.7 \\
\hline $\mathrm{ATF}$ & 26 & " & 60658.34 & 50584.51 & 255.5 & 275.5 & 275.4 & 274.1 & -1. \\
\hline " HTF & 27 & " & 60500.00 & 50584.74 & 259.1 & 279.1 & 276.9 & 277.3 & 0.8 \\
\hline "HTF & 28 & " & 60362.69 & 50636.52 & 251.9 & 271.9 & 276.0 & 272.2 & -3 \\
\hline " HTF & 29 & " & 60295.24 & 50804.00 & 259.9 & 289.9 & 275.8 & 281.4 & 5.6 \\
\hline " HTF & 31 & " & 60437.03 & 50280.18 & 246.7 & 266.7 & 275.6 & 269.2 & -6. \\
\hline "HTF & 32 & $"$ & 60607.03 & 50380.63 & 251.1 & 271.1 & 274.7 & 271.7 & -3 \\
\hline " HTF & 34 & " & 59850.54 & 50810.81 & 251.7 & 271.7 & 274.3 & 271.8 & -2 \\
\hline HWP & 1D & " & 57981.82 & .52244 .65 & 239.9 & 249.9 & 245.2 & 246.6 & 1 \\
\hline 'HWP & $2 \mathrm{D}$ & & 58090.42 & 52436.40 & 253.0 & 263.0 & 263.0 & 0.0 & -263.0 \\
\hline "HWS & $1 \mathrm{~A}$ & " & 47062.15 & 47130.24 & 225.2 & 255.2 & 244.9 & 244.3 & -0 \\
\hline "HXB & 1 & $"$ & 48433.00 & 42406.60 & 214.2 & 244.2 & 251.7 & 256.4 & 4.7 \\
\hline HXB & 2 & $"$ & 48826.55 & 42646.82 & 212.1 & 242.1 & 252.9 & 257.5 & 4. \\
\hline $\mathrm{HXB}$ & 3 & $"$ & 48596.18 & 42455.23 & 212.2 & 242.2 & 252.2 & 256.6 & 4. \\
\hline $\mathrm{AXB}$ & $4 \mathrm{D}$ & $"$ & 48519.48 & 42527.26 & 234.9 & 254.9 & 253.9 & 261.3 & 7. \\
\hline " HXB & $5 \mathrm{D}$ & & 48394.54 & 42453.62 & 234.2 & 254.2 & 253.0 & 260.6 & 7. \\
\hline
\end{tabular}


Table F-2. Summary of Group Statistical Parameters (Continued)

\begin{tabular}{|c|c|c|c|c|c|c|c|c|c|}
\hline " KRP & 3 & $"$ & 37592.37 & 38269.08 & 207.5 & 237.5 & 219.2 & 224.4 & 5.2 \\
\hline "MGA & 36 & $"$ & 56426.67 & 54360.14 & 234.2 & 254.2 & 240.6 & 235.6 & -5.0 \\
\hline "MGC & 32 & $"$ & 56009.88 & 54528.57 & 232.0 & 252.0 & 245.1 & 234.1 & -11.0 \\
\hline "MGC & 36 & $"$ & 56279.36 & 54222.66 & 234.4 & 254.4 & 236.1 & 235.2 & -0.9 \\
\hline "MGE & 34 & " & 55995.47 & 54238.12 & 237.2 & 257.2 & 241.0 & 0.0 & -241.0 \\
\hline "MGG & 36 & " & 55982.43 & 53952.59 & 232.5 & 252.5 & 238.4 & 234.2 & -4.2 \\
\hline "NBG & 1 & $"$ & 53624.12 & 60472.79 & 200.9 & 232.3 & 224.5 & 227.3 & 2.8 \\
\hline "NBG & 2 & " & 53659.78 & 60260.13 & 203.6 & 233.6 & 225.0 & 229.0 & 4.0 \\
\hline "NBG & 3 & $"$ & 53733.78 & 60080.63 & 202.1 & 233.5 & 217.5 & 229.6 & 12.1 \\
\hline "NPM & 1 & $"$ & 52966.40 & 43082.52 & 257.1 & 277.1 & 287.4 & 287.6 & 0.2 \\
\hline "NPM & 2 & $"$ & 54524.03 & 43675.02 & 244.2 & 264.2 & 271.8 & 279.7 & 7.9 \\
\hline "NPM & 3 & " & 51554.55 & 43337.43 & 247.2 & 267.2 & 274.6 & 280.7 & 6.1 \\
\hline "NPM & 4 & " & 53057.77 & 41764.52 & 256.7 & 276.7 & 284.2 & 288.2 & 4.0 \\
\hline " NPM & $4 \mathrm{DD}$ & & 53063.55 & 41773.42 & 296.4 & 306.4 & 305.6 & 297.4 & -8.2 \\
\hline "NPM & $19 A$ & $"$ & 53821.23 & 43736.38 & 248.2 & 268.2 & 270.7 & 282.4 & 11.7 \\
\hline "NPM & $34 \mathrm{~A}$ & " & 52141.34 & 41848.19 & 279.8 & 289.8 & 290.8 & 293.8 & 3.0 \\
\hline "NPM & $34 \mathrm{~B}$ & " & 52153.77 & 41839.82 & 225.6 & 235.6 & 271.1 & 274.5 & 3.4 \\
\hline " PAC & 1 & " & 58782.57 & 22820.40 & 253.9 & 283.9 & 284.7 & 274.6 & -10.1 \\
\hline " PAC & 2 & " & 59001.85 & 22757.84 & 247.9 & 277.9 & 271.0 & 269.9 & -1.1 \\
\hline "PAC & 3 & $"$ & 58897.00 & 22839.32 & 252.9 & 282.9 & 271.3 & 273.2 & 1.9 \\
\hline " PAC & 4 & $"$ & 58880.01 & 22750.72 & 250.6 & 280.6 & 284.4 & 272.2 & -12.2 \\
\hline " PAC & 5 & $"$ & 58936.73 & 22806.44 & 255.1 & 275.1 & 275.1 & 270.9 & -4.2 \\
\hline " PAC & 6 & " & 58928.43 & 22827.02 & 255.2 & 275.2 & 274.6 & 271.0 & -3.6 \\
\hline " PCB & $1 \mathrm{~A}$ & $"$ & 56813.22 & 21649.15 & 263.5 & 293.5 & 280.7 & 281.9 & 1.2 \\
\hline " PCB & $2 \mathrm{~A}$ & $"$ & 56603.25 & 21523.26 & 257.8 & 287.8 & 279.5 & 277.1 & -2.4 \\
\hline " PCB & $3 A$ & $"$ & 56466.82 & 21771.65 & 262.7 & 292.7 & 281.6 & 282.1 & 0.5 \\
\hline " PCB & $4 \mathrm{~A}$ & $"$ & 56685.72 & 21863.14 & 262.9 & 292.9 & 279.7 & 282.0 & 2.3 \\
\hline " PDB & 4 & " & 56681.17 & 23176.89 & 266.2 & 286.2 & 279.2 & 281.6 & 2.4 \\
\hline " PDB & 5 & " & 56778.08 & 23822.35 & 264.2 & 284.2 & 277.8 & 278.9 & 1.1 \\
\hline " PSB & $1 \mathrm{~A}$ & $"$ & 56243.45 & 23437.80 & 257.4 & 287.4 & 276.9 & 277.8 & 0.9 \\
\hline "PSB & $2 \mathrm{~A}$ & $"$ & 56022.03 & 23477.81 & 257.2 & 287.2 & 276.7 & 277.5 & 0.8 \\
\hline " PSB & $3 \mathrm{~A}$ & " & 55700.43 & 23533.29 & 256.5 & 286.5 & 275.6 & 276.1 & 0.5 \\
\hline " PSB & $4 \mathrm{~A}$ & " & 55448.71 & 23519.73 & 255.5 & 285.5 & 274.8 & 274.8 & 0.0 \\
\hline "PSB & $5 A$ & $"$ & 55683.06 & 23374.12 & 262.3 & 292.3 & 276.1 & 281.5 & 5.4 \\
\hline " PSB & $6 \mathrm{~A}$ & " & 56043.26 & 23292.96 & 262.1 & 292.1 & 277.6 & 281.9 & 4.3 \\
\hline " PSB & $7 \mathrm{~A}$ & " & 56385.84 & 23340.06 & 259.0 & 289.0 & 277.5 & 279.2 & 1.7 \\
\hline " SCA & $3 A$ & $"$ & 62973.08 & 53031.02 & 267.1 & 272.4 & 270.9 & 0.0 & -270.9 \\
\hline "SCA & $4 \mathrm{~A}$ & $"$ & 62946.34 & 52924.45 & 265.3 & 274.0 & 268.8 & 0.0 & -268.8 \\
\hline " z & 12 & $"$ & 59296.96 & 50984.50 & 251.3 & 251.8 & 274.3 & 263.2 & -11.1 \\
\hline "z & 13 & $"$ & 59996.23 & 50413.54 & 256.6 & 257.1 & 276.1 & 269.4 & -6.7 \\
\hline " ZW & 4 & " & 55903.68 & 58318.78 & 229.2 & 239.7 & 232.3 & 231.9 & -0.4 \\
\hline "ZW & 7 & $"$ & 58470.42 & 52387.61 & 254.5 & 264.8 & 265.8 & 255.7 & -10.1 \\
\hline " ZW & 8 & " & 61562.33 & 50095.99 & 254.1 & 264.1 & 270.8 & 262.9 & -7.9 \\
\hline " ZW & 9 & $"$ & 59712.09 & 52940.43 & 242.4 & 252.4 & 252.0 & 250.3 & -1.7 \\
\hline "ZW & 10 & " & 61671.98 & 52538.16 & 242.2 & 252.2 & 249.7 & 251.7 & 2.0 \\
\hline "CMP & $15 \mathrm{C}$ & $"$ & 46865.00 & 33346.32 & 220.6 & 250.6 & 244.5 & 240.7 & -3.8 \\
\hline "NPM & 2 & $"$ & 54524.03 & 43675.02 & 244.2 & 264.2 & 267.0 & 279.7 & 12.7 \\
\hline "NPM & 3 & $"$ & 51554.55 & 43337.43 & 247.2 & 267.2 & 267.6 & 280.7 & 13.1 \\
\hline "NPM & 4 & $"$ & 53057.77 & 41764.52 & 256.7 & 276.7 & 272.7 & 288.2 & 15.5 \\
\hline "NTN & 1 & $"$ & 40851.04 & 40382.75 & 212.4 & 232.4 & 233.7 & 232.9 & -0.8 \\
\hline "NTN & 2 & $"$ & 42194.02 & 41060.13 & 207.2 & 227.2 & 235.2 & 235.7 & 0.5 \\
\hline
\end{tabular}




\section{APPENDIX G. MODIFICATIONS TO C-AREA HYDROSTRATIGRAPHY BASED ON CPTU CHARACTERIZATION}




\section{This page intentionally left blank}




\section{APPENDIX G. MODIFICATIONS TO C-AREA HYDROSTRATIGRAPHY BASED ON CPTU CHARACTERIZATION}

\section{Overview}

In C-area, initial groundwater modeling pathlines originating from the C-Reactor Seepage Basins (CRSB) did not agree with the tritium and TCE plume movement observed from Cone Penetration Test concentration data, for any reasonable variation in horizontal and vertical conductivities. Figure G-1 illustrates the CPT tritium data associated with the CRSB, while Figure G-2 shows CPT TCE data associated with the CRSB and C-area Burning/Rubble Pit (CBRP). Figure G-3 shows a typical example of simulated groundwater flow. Note that predicted pathlines from the CRSB travel towards Twin Lakes while the plume data suggests groundwater is migrating toward the confluence of Fourmile Branch and Caster Creek. The lack of calibration success in matching pathlines with plume migration suggested a flaw in the conceptual model for $\mathrm{C}$-area and motivated a reexamination of the hydrostratigraphic framework.

Cone Penetration Tests (CPTu) were used almost exclusively at the $\mathrm{C}$-area Burning/Rubble Pit and C-Reactor Seepage Basins to define contaminant plumes and local hydrostratigraphy, rather than conventional borehole techniques (e.g. monitoring wells, cores, electric well logs, slug and pumping tests). In this study, CPTu lithologic data are further used to identify dominant confining intervals and zones of high horizontal conductivity within the Upper Three Runs aquifer in C-area. The approach is to correlate tip, sleeve and pore pressure measurements to percent fines and hydraulic conductivity. Then the small-scale conductivity data are upscaled and interpolated onto the regional-scale flow model grid using stochastic theory.

The CPTu predictions indicate that, on the north side of Caster Creek, the tan clay largely disappears as a confining unit and is simultaneously underlain by a deeper confining zone within the "lower" UTRA. The underlying confining zone is interpreted to be a calcareous wackestone/mudstone based on the CPTu signature of low tip and sleeve resistance with high pore pressure. Also, the transmissive zone is significantly more conductive south of the CRSB. These predicted hydraulic conductivity variations suggest element layer 5 in the model should be redefined to coincide with the lower calcareous zone instead the tan clay near Caster Creek in C-area. With this modification, simulated groundwater flow paths, as shown in Figure G-4, are in much better agreement with observed plume migration from the CRSB and CBRP (Figures G-1 and G-2). 


\section{CPTu correlations}

Several soil classification charts have been developed in the literature that correlate CPTu tip resistance, sleeve resistance, and pore pressure to soil categories (Syms and Others, 1999). Such charts define a discrete soil index, but do not predict gradation or percent fines content. Following Jefferies and Davies (1993), Robertson and Fear (1995) and Syms and Others (1999), concepts employed in soil classification were applied to prediction of percent fines. The predicted \%fines and normalized pore pressure measurement were then correlated to small-scale hydraulic conductivity.

\section{CPTu measurement and normalized parameters}

$\mathrm{CPTu}$ data consist of uncorrected tip resistance, $\mathrm{q}_{\mathrm{c}}$, sleeve friction, $\mathrm{f}_{\mathrm{s}}$, and pore pressure, $u$, typically in units of tons per square foot (tsf). These dimensional parameters are commonly normalized in the recent literature to produce their dimensionless counterparts $Q_{t n}, F_{s n}$ and $B_{q}$, respectively. Normalized tip resistance, $Q_{t n}$, is defined by

$$
\mathrm{Q}_{\mathrm{tn}}=\frac{\mathrm{q}_{\mathrm{t}}-\sigma}{\bar{\sigma}}
$$

where the corrected tip resistance, $\mathrm{q}_{\mathrm{t}}$, is

$$
q_{t}=q_{c}+u\left(1-\frac{A_{N}}{A_{T}}\right)
$$

and $\sigma$ and $\bar{\sigma}$ are the total and effective overburdens. In equation (G.2), the total tip stress, $q_{t}$, is equal to the mechanical rod stress (measured force/tip area), $q_{c}$, plus fluid pressure on the backside of the cone over the area $\left(\mathrm{A}_{\mathrm{T}}-\mathrm{A}_{\mathrm{N}}\right)$ divided by tip area. The numerator of equation (G.1) is the net stress required to advance the tip and deform the medium. The denominator is the grain-to-grain contact, or "effective", stress. Above the water table, the total overburden is

$$
\sigma=\left(\gamma_{\mathrm{d}}+\gamma \omega \mathrm{S}_{\mathrm{w}}\right) \mathrm{z}
$$

where $\mathrm{z}$ is depth below ground surface. The dry soil weight density per unit total volume (bulk density), $\gamma_{\mathrm{d}}$, is approximately $100 \mathrm{lbf} / \mathrm{ft}^{3}$. Water weight density per unit water volume, $\gamma$, is $62.4 \mathrm{lbf} / \mathrm{ft}^{3}$. A reasonable total porosity, $\omega$, is $45 \%$, and an average vadose zone saturation might be $70 \%$. Below the water table, the total overburden is

$$
\sigma=\left(\gamma_{\mathrm{d}}+\gamma \omega \mathrm{S}_{\mathrm{w}}\right) \mathrm{z}_{\mathrm{WT}}+\left(\gamma_{\mathrm{d}}+\gamma \omega\right)\left(\mathrm{z}-\mathrm{z}_{\mathrm{WT}}\right)
$$


Effective overburden is computed from the relation

$$
\sigma=\bar{\sigma}+p
$$

where $\mathrm{p}$ is assumed to be hydrostatic pressure (deMarsily, 1986, §5.2; Freeze and Cherry, §2.9). Above the water table, the usual assumption is $p=0$, or $\bar{\sigma}=\sigma$.

Normalized friction ratio (normalized sleeve friction) is defined by

$$
F_{s n}=\frac{f_{s}}{q_{t}-\sigma} \times 100 \%
$$

and represents sleeve friction relative to the net force required to advance the cone tip (deform the medium). Normalized pore pressure is similarly defined as

$$
B_{q}=\frac{u-p}{q_{t}-\sigma}
$$

The numerator is net (measured - hydrostatic) pore pressure.

\section{\%Fines correlation}

Syms and Others (1999) conducted an extensive literature review of soil classification charts based on CPTu measurements, and applied several to SRS sediments. They observed that the cited charts "do not work well for SRS soils". Syms and Others (1999) also considered the approach of Robertson and Fear (1995), who extended an approach introduced by Jefferies and Davies (1993) for correlating \%fines (\#200 sieve) to the CPTu parameters, $Q_{\text {tn }}$ and $F_{s n}$. They achieved marginal success for sediments of the Altamaha, Tobacco Road / Dry Branch, and Santee formations within the General Separations Area. Much better results were obtained by optimizing coefficients in the correlation for each formation, using paired CPTu and sieve data from 3 locations in F-area. This study investigates the extent to which \% fines are correlated to CPTu parameters, using data from $\mathrm{F}-, \mathrm{H}$ - and $\mathrm{R}$-areas.

An initial indication of the degree of correlation between \%fines and CPTu can be gained by computing the correlation coefficient, $\mathrm{r}$, for each $\mathrm{CPTu}$ parameter. Table G-1 summarizes the results. The parameter exhibiting the strongest (linear) correlation to \%fines is $\log _{10} F_{\text {sn }}$, followed by $B_{q}$ and then $\log _{10} Q_{\text {tn }}$. Table G-1 suggests choosing these parameters for the purpose of generating a \%fines correlation. The correlation is apparently weak as a linear variation in $\log _{10} \mathrm{~F}_{\mathrm{sn}}$ only explains $21.6 \%\left(\mathrm{r}^{2}\right)$ of the variation in \% FC, for example. 
Following Syms and Others (1999), the CPTu data are next segregated into $4 \% \mathrm{FC}$ categories based on sieve results: 0-15\% (group 1, blue), 15-30\% (group 2, green), 3050\% (group 3, red) and 50\%-100\% (group 4, yellow/black). Figures G-5 and G-6 present the color-coded CPTu data in terms of $\log _{10} Q_{\text {tn }}$ versus $\log _{10} F_{S n}$, and $B_{q}$ versus $\log _{10} \mathrm{~F}_{\mathrm{sn}}$. Also plotted in these figures are the average positions (centers of mass) of each \%FC group. Figures G-7 and G-8 show the same data, but with each \%FC group displayed separately. The $\% \mathrm{FC}$ groups significantly overlap, confirming the preliminary conclusion from Table G-1. The \%FC groups would have to be substantially separated in order to develop an accurate correlation. Nevertheless, on average, the data from each group are separated, particularly with respect to $\log _{10} F_{\mathrm{sn}}$ and $\mathrm{B}_{\mathrm{q}}$. This is most easily observed from the "center of mass" points depicted in each figure. The first three groups follow a definite, nearly linear, trend. Specifically, \%FC increases with friction ratio and pore pressure as expected. The data show little dependence on normalized tip resistance. The $50-100 \%$ fines group deviates from this trend. This suggests that a significantly better correlation can be achieved for sieve data in the 0-50\% range compared to the entire set. Table G-2 summarizes correlation statistics for the first 3 groups comprising the $0-50 \%$ fines data. Indeed, the degree of linear correlation is significantly improved compared to Table G-1. For example, $\mathrm{r}^{2}$ has increased from $21.6 \%$ to $37.9 \%$ for normalized friction ratio.

The ultimate objective of this study is to distinguish between high and low conductivity sediments. This breakpoint occurs for a relatively low \% fines content and lessens the need to correctly predict \%fines at the high end. For this reason, and that a much better correlation can be achieved by ignoring data with greater than $50 \%$ fines, regression analysis will be performed only on the first three \%FC groups. The following linear functional form is considered

$$
\% \mathrm{FC}=\mathrm{A}+\mathrm{B} * \log _{10} \mathrm{~F}_{\mathrm{sn}}+\mathrm{C} * \mathrm{~B}_{\mathrm{q}}+\mathrm{D} * \log _{10} \mathrm{Q}_{\mathrm{tn}}
$$

The regression is linear (in the regression coefficients) and produces the following result

$$
\% \mathrm{FC}=5.009+15.98 * \log _{10} \mathrm{~F}_{\mathrm{sn}}+24.14 * \mathrm{~B}_{\mathrm{q}}+6.869 * \log _{10} \mathrm{Q}_{\mathrm{tn}}
$$

Regression statistics from JMP (SAS Institute, 1995) are presented in Table G-3. In summary, the correlation explains about $45 \%\left(\mathrm{r}^{2}\right)$ of the variation in $\% \mathrm{FC}$. For the data used to develop the correlation (0-50\% fines), Figure G-9 shows the variation in residuals with respect to each CPTu parameter, and compares predicted to measured \%FC. Figure G-10 presents the same information for the entire paired data set. As expected, the correlation is a very poor predictor of high \% fines. 
Table G-1 Correlation measures for \%fines and CPTu parameters.

\begin{tabular}{|c|c|c|c|c|}
\hline & PAIR & $\mathrm{COV}$ & $r$ & $r^{\wedge} 2$ \\
\hline & $F C \& Q t n:$ & -19.418 & -0.023 & 0.001 \\
\hline & FC \& Fsn: & 13.216 & 0.348 & 0.121 \\
\hline & $F C \& B q:$ & 1.164 & 0.393 & 0.155 \\
\hline FC \& & $\log 10 Q \operatorname{tn}:$ & -1.346 & -0.169 & 0.029 \\
\hline FC \& & log10Fsn: & 2.948 & 0.465 & 0.216 \\
\hline$(1-\mathrm{Bq}$ & ) log10Qtn: & -2.247 & -0.250 & 0.062 \\
\hline
\end{tabular}

Table G-2 Correlation measures for \%fines and CPTu parameters; 0-50\% fines.

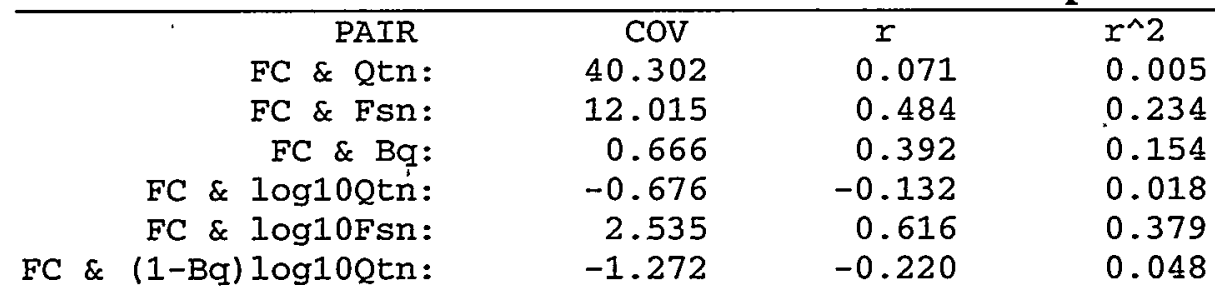

Table G-3 Regression statistics.

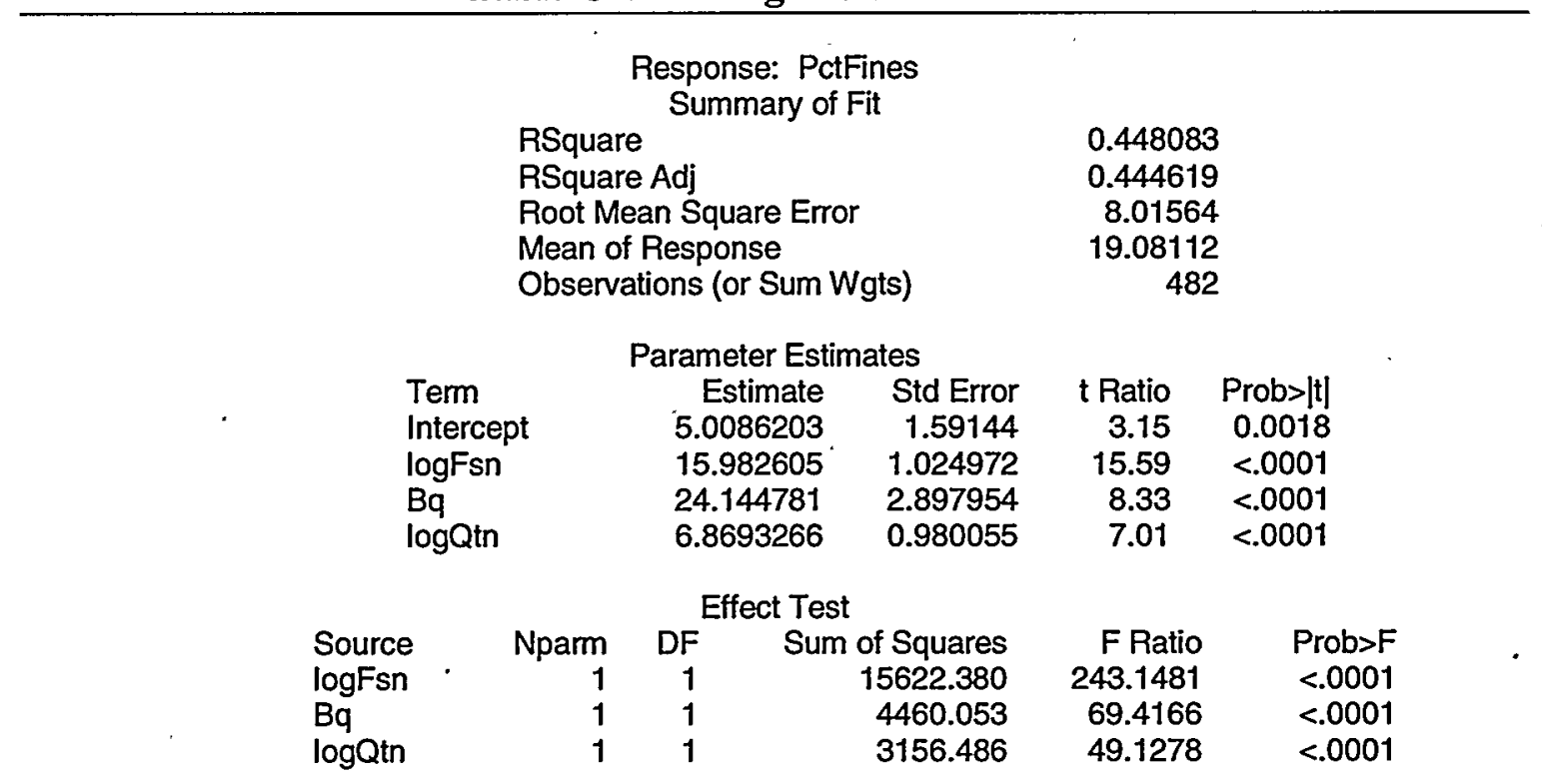

\section{Hydraulic conductivity correlation}

Hydraulic conductivity can be directly correlated to CPTu measurements, as Parsons and ARA (1997) and Celeste (1998) have done for SRS sediments. Alternatively, conductivity can be correlated to \%fines (e.g. Kegley, 1993; Parsons and ARA, 1997; Celeste, 1998; Flach and others, 1998; Flach and Harris, 1999), which may then be related to CPTu measurements using the correlation developed in the previous section. In this study we apply a hybrid approach which relates conductivity to CPTu measurements indirectly through predicted \%fines, and directly through the $\mathrm{B}_{\mathrm{q}}$ parameter. The approach is motivated-by the absence of small-scale conductivity measurements in $\mathrm{C}$-area that can be used to directly correlate conductivity to CPTu. 
The $\%$ FC ranges of $0-15 \%, 15-30 \%$ and $>30 \%$ are considered to correspond to "high", "medium" and "low" conductivity. Based on conductivity data taken at various scales, previous flow models, and preliminary model calibration, reasonable values for these conductivity categories appear to be 20,2 and $0.01 \mathrm{ft} / \mathrm{d}$. Recognizing that equation (G.8) is a poor predictor of fine-grained sediment while $B_{q}$ is good predictor of low conductivity intervals, we also use $B_{q}>0.1$ to define an "extra low" conductivity category. The "extra low" conductivity zone is assigned a value of $0.0001 \mathrm{ft} / \mathrm{d}$, irrespective of predicted \%fines. The chosen hydraulic conductivity relationship is summarized in Table G-4.

The conductivity settings listed in Table G-4 are based in part on qualitative knowledge of how conductivity varies with lithology and support scale. As with all models, the specific values of conductivity are ultimately based on model calibration. The settings in Table G-4 are typical values for small-scale conductivity measurements on sediments ranging from clean sand (high K) to clay (extra low K) (e.g. Kegley, 1993; Parsons and ARA, 1997; Celeste, 1998; Flach and others, 1998; Flach and Harris, 1999). Example small-scale tests include the laboratory falling head permeameter and minipermeameter. After the correlation is applied to the CPT data, the small-scale conductivity estimates are scaled up to the flow model grid (field scale) through a process described in the next section. The upscaled estimates for horizontal conductivity compare favorably with average field-scale measurement data from slug and pumping tests, and previous calibrated flow models (see Figures G-28 to G-59 presented in next section). The predicted conductivity fields based on Table G-4 are viewed as a starting point for final flow model calibration to additional targets such as water level and plume data.

Table G-4 Hydraulic conductivity correlation.

\begin{tabular}{|c|c|c|}
\hline Conductivity category & Definition & Value (ft/d) \\
\hline High & $0-15 \%$ fines predicted from equation (G.8) & 20 \\
\hline Medium & $15-30 \%$ fines predicted from equation (G.8) & 2 \\
\hline Low & $>30 \%$ fines predicted from equation (G.8) & 0.01 \\
\hline Extra Low & $\mathrm{Bq}>0.1$ irrespective of predicted \%fines & 0.0001 \\
\hline
\end{tabular}

\section{Upscaling}

CPTu measurements have a vertical resolution of $0.1 \mathrm{ft}$, and the radius of influence of the cone in the horizontal plane is equally small. Therefore, a method for upscaling 
CPTu measurements to the coarser resolution of the flow model mesh is required. Before developing an appropriate approach for $\mathrm{C}$-area, we first review upscaling approaches from the stochastic hydrology literature.

\section{Review of upscaling in the stochastic hydrology literature}

Upscaling refers to the process of replacing a heterogeneous conductivity field within a particular finite volume with a single, "equivalent", conductivity value. The equivalent conductivity is defined as the value that reproduces some average behavior of the block, such as mean flow for a given head difference. A closely related problem is that of determining the "effective" conductivity of a heterogeneous media. As stated by Sanchez-Vila and others (1995), "effective parameters are defined as representative values of the mean behavior through an ensemble of realizations, while equivalent parameters are associated with a certain geometry and defined as spatial averages computed on a single realization. These two definitions should converge to the same value for very large geometries and under the assumption of ergodicity." The problem of defining effective conductivity is considered first, as this topic is better developed in the literature.

Of principal importance to this study is the work of Gelhar and Axness (1983) who derived analytical expressions for the effective conductivity tensor of an infinite, ergodic, anisotropically-correlated medium subjected to a uniform mean flow. The threedimensional anisotropy of the heterogeneous medium is defined in terms an exponential autocovariance function with distinct correlation scales for each coordinate direction, $\lambda_{1}$, $\lambda_{2}$ and $\lambda_{3}$. When the mean flow is aligned with the bedding $\left(\lambda_{1}=\lambda_{2}>\lambda_{3}\right)$, the non-zero components of the conductivity tensor are

$$
\begin{gathered}
\overline{\mathrm{K}}_{11}=\overline{\mathrm{K}}_{22}=\overline{\mathrm{K}}_{\mathrm{h}}=\mathrm{K}_{\mathrm{g}}\left[1+\sigma^{2}\left(\frac{1}{2}-\mathrm{g}_{11}\right)\right] \\
\overline{\mathrm{K}}_{33}=\overline{\mathrm{K}}_{\mathrm{v}}=\mathrm{K}_{\mathrm{g}}\left[1+\sigma^{2}\left(\frac{1}{2}-\mathrm{g}_{33}\right)\right]
\end{gathered}
$$

where

$$
\begin{aligned}
& \overline{\mathrm{K}}_{\mathrm{h}} \equiv \text { effective horizontal conductivity } \\
& \overline{\mathrm{K}}_{\mathrm{v}} \equiv \text { effective vertical conductivity } \\
& \mathrm{Kg} \equiv \text { geometric mean of point conductivity field }
\end{aligned}
$$




$$
\sigma^{2} \equiv \text { variance of the natural logarithm of point conductivities }
$$

and $g_{11}$ and $g_{33}$ are functions of the correlation scales. For case being considered here, they are defined in terms of the ratio of horizontal to vertical correlation, $\rho=\lambda_{h} / \lambda_{v}>1$, as follows:

$$
\begin{aligned}
& g_{11}=\frac{1}{2} \frac{1}{\rho^{2}-1}\left[\frac{\rho^{2}}{\left(\rho^{2}-1\right)^{1 / 2}} \tan ^{-1}\left(\rho^{2}-1\right)^{1 / 2}-1\right] \\
& g_{33}=\frac{\rho^{2}}{\rho^{2}-1}\left[1-\frac{1}{\left(\rho^{2}-1\right)^{1 / 2}} \tan ^{-1}\left(\rho^{2}-1\right)^{1 / 2}\right]
\end{aligned}
$$

The above analytical results are based on a first-order perturbation analysis, and strictly speaking, only exact in the limit as the variance approaches zero. Accurate results can be expected for small variances. For large variances, the predictions may become increasingly inaccurate, or even nonphysical. For example, $\overline{\mathrm{K}}_{\mathrm{v}}$ is negative when $\lambda_{\mathrm{h}} / \lambda_{\mathrm{v}}$ $\rightarrow \infty$ and the variance of $\operatorname{lnK}$ exceeds 2 . To remedy such nonphysical results and hopefully extend the range of applicability of effective conductivity predictions, Gelhar and Axness (1983) proposed the following generalization of equations (G.9) and (G.10)

$$
\begin{aligned}
& \overline{\mathrm{K}}_{\mathrm{h}}=\mathrm{K}_{\mathrm{g}} \exp \left[\sigma^{2}\left(\frac{1}{2}-\mathrm{g}_{11}\right)\right] \\
& \overline{\mathrm{K}}_{\mathrm{v}}=\mathrm{K}_{\mathrm{g}} \exp \left[\sigma^{2}\left(\frac{1}{2}-\mathrm{g}_{33}\right)\right]
\end{aligned}
$$

The generalization is motivated by the observation that a Taylor series expansion of equations (G.13) and (G.14) contains equations (G.9) and (G.10), respectively, as the first two terms. Subsequent comparison of equation (G.9) to numerical simulations indicates that the exponential generalization is accurate for isotropic systems and variances up to 7 , but overpredicts effective conductivity for anisotropic systems (Gelhar, 1997, p. 161).

Ababou and Wood (1990) point out that equations such as (G.13) and (G.14) can alternatively be written in terms of a "p-norm" defined by

$$
\mathrm{K}_{\mathrm{p}} \equiv\left[\frac{1}{\mathrm{~N}} \sum_{\mathrm{i}}\left(\mathrm{K}_{\mathrm{i}}\right)^{\mathrm{p}}\right]^{1 / \mathrm{p}}=\left(\overrightarrow{\mathrm{K}^{\mathrm{p}}}\right)^{1 / \mathrm{p}}
$$

because 


$$
\mathrm{K}_{\mathrm{p}}=\mathrm{K}_{\mathrm{g}} \exp \left(\mathrm{p} \sigma^{2} / 2\right)
$$

Comparing equations (G.13) and (G.14) with (G.16), one finds

$$
\begin{aligned}
& \mathrm{p}_{\mathrm{h}}=1-2 \mathrm{~g}_{11} \\
& \mathrm{p}_{\mathrm{v}}=1-2 \mathrm{~g}_{33}
\end{aligned}
$$

where $p_{h}$ and $p_{v}$ are the averaging exponent associated with horizontal and vertical effective conductivity. That is, equations (G.13) and (G.14) are exactly equivalent to

$$
\overline{\mathrm{K}}_{\mathrm{h}}=\left(\overline{\mathrm{K}^{\mathrm{ph}_{\mathrm{h}}}}\right)^{1 / \mathrm{ph}}
$$

and

$$
\overline{\mathrm{K}}_{\mathrm{v}}=\left(\overline{\mathrm{K}^{\mathrm{p}_{\mathrm{v}}}}\right)^{1 / \mathrm{p}_{\mathrm{v}}}
$$

As explained by Ababou and Wood (1990), the p-norm encompasses the familiar averages of arithmetic $(p=1)$, geometric $(p \rightarrow 0)$, and harmonic $(p=-1)$ as well as any blend in between. In more recent years, numerous authors have developed expressions for effective conductivity based on less restrictive assumptions than those adopted by Gelhar and Axness (1995), such as bounded media with various boundary conditions, gradually varying mean flow, non-stationary conductivity, and radial flow. Frequently the effective conductivity is formulated as a power-average (G.15) with the power $p$ having been determined from numerical simulations or a combined numerical-analytical approach (Sanchez-Vila and others, 1995).

The related problem of determining "equivalent" block conductivities has received less attention in literature, but is of great practical importance because "effective" conductivity estimates are strictly valid only for regions that span at least 10 or 100 times the integral correlation scale (Kitanidis, 1997). Frequently model blocks, and even the entire model domain, are not significantly larger than the scale of heterogeneity. This observation is especially supported by recent research that suggests variability exists at all scales without bound and motivates the use of fractal models. Sanchez-Vila and others (1995) summarize and compare upscaling approaches proposed to-date. Two out of the four approaches reviewed by Sanchez-Vila and others (1995) are practical in that a mechanism for computing block conductivity from point values was provided by the author(s). Of these approaches, Desbarats (1992) is particularly appealing because of its simplicity. 
Desbarats (1992) conjectures that equivalent block conductivities can be formulated as a power-average, a reasonable hypothesis considering the successful use of $\mathrm{p}$-norms in defining effective conductivity. Desbarats (1992) empirically determined the appropriate power through numerical experimentation. For cubic blocks and an isotropic conductivity field, the optimal averaging exponent was determined to be $p=1 / 3$. Interestingly, this is the same power as is appropriate for the effective conductivity of an infinite domain, as can be seen from equations (G.11) and (G.17). As Desbarats (1992) notes, this observation further supports the empirical result. For an anisotropic media with $\lambda_{h}=10 \lambda_{v}$ and block dimensions of $L_{h} / \lambda_{h}=L_{v} / \lambda_{v}=3$, the optimal averaging exponents were found through numerical experimentation to be $\mathrm{p}_{\mathrm{h}}=0.59$ and $\mathrm{p}_{\mathrm{v}}=-0.33$. Unlike the isotropic case, these results differ from the averaging exponents for effective conductivity of an infinite medium with $\lambda_{h}=10 \lambda_{v}$. The latter results are $p_{h}=0.86$ and $p_{v}$ $=-0.72$ (see equations (G.11), (G.12), (G.17) and (G.18)). The discrepancy may be a reflection of equation (G.9) already overpredicting $\mathrm{K}_{\mathrm{h}}$ in infinite anisotropic media for large variances, as previously stated. Desbarats (1992) recommends that numerical calibration experiments be used to define the power exponents for the specific combination of block geometry and correlation scales of interest. However as Desbarats (1992) notes, equations such as (G.17) and (G.18) give the correct values for the limiting cases of an isotropic or perfectly stratified medium, and "provide a convenient alternative to tedious numerical experiments".

Practical upscaling procedures frequently involve vertical averaging of borehole/CPT data over a stratigraphic layer, followed by horizontal averaging or interpolation across the layer. Assuming equivalent block conductivities can be expressed as a known power average, then certain restrictions apply to such a two-stage averaging/interpolation process in order to avoid biased estimates. First consider vertical averaging of threedimensional point data, followed by horizontal averaging of the two-dimensional intermediate-scale estimates. The first stage can be written

$$
\overline{\mathrm{K}_{\mathrm{q}}}=\left[\frac{1}{\mathrm{~m}} \sum_{\mathrm{i}}\left(\mathrm{K}_{\mathrm{i}}\right)^{\mathrm{q}}\right]^{1 / \mathrm{q}}
$$

where $q$ is unspecified at the moment. The second stage involves averaging the $2 \mathrm{D}$ intermediate-scale data according to

$$
\overline{\mathrm{K}}=\left[\frac{1}{\mathrm{n}} \sum_{\mathrm{j}}\left(\overline{\mathrm{K}_{\mathrm{qj}}}\right)^{\mathrm{r}}\right]^{1 / \mathrm{r}}
$$

where $r$ is also unspecified. Combining (G.21) and (G.22) produces 


$$
\overline{\mathrm{K}}=\left\{\frac{1}{\mathrm{n}} \sum_{\mathrm{j}}\left[\frac{1}{\mathrm{~m}} \sum_{\mathrm{i}}\left(\mathrm{K}_{\mathrm{i}}\right)^{\mathrm{q}}\right]^{\mathrm{r} / \mathrm{q}}\right\}^{1 / \mathrm{r}}
$$

which is the effective conductivity of the layer. Alternatively, the effective conductivity can be computed directly from the known power average of the 3D point data:

$$
\overline{\mathrm{K}}=\left[\frac{1}{\mathrm{~N}} \sum_{\mathrm{i}}\left(\mathrm{K}_{\mathrm{i}}\right)^{\mathrm{p}}\right]^{1 / \mathrm{p}}
$$

where $\mathrm{N}=\mathrm{m} \times \mathrm{n}$. In order for the two-step averaging process (G.23) to be consistent (unbiased) with respect to (G.24) we require $q=r=p$ so that equation (G.23) becomes

$$
\overline{\mathrm{K}}=\left\{\frac{1}{\mathrm{n}} \sum_{\mathrm{j}}\left[\frac{1}{\mathrm{~m}} \sum_{\mathrm{i}}\left(\mathrm{K}_{\mathrm{i}}\right)^{\mathrm{p}}\right]\right\}^{1 / \mathrm{p}}
$$

or

$$
\overline{\mathrm{K}}^{\mathrm{p}}=\frac{1}{\mathrm{n}} \sum_{\mathrm{j}}\left[\frac{1}{\mathrm{~m}} \sum_{\mathrm{i}}\left(\mathrm{K}_{\mathrm{i}}\right)^{\mathrm{p}}\right]
$$

Equation (G.26) implies that upscaling should be conducted according to the following sequence in order to avoid biased estimation:

1) Transform the point data $K$ to $K^{p}$

2) Estimate $\mathrm{K}^{\mathrm{P}}$ through one or more arithmetic averaging and/or interpolation steps

3) Transform $\overline{K^{p}}$ back to $\bar{K}$ through the transform $\left(\bar{K}^{p}\right)^{1 / p}$

Step 2), for example, could involve vertical averaging followed by two-dimensional block kriging.

\section{C-area upscaling approach}

Having reviewed the relevant stochastic hydrology literature on upscaling, we now consider an appropriate upscaling approach for $\mathrm{C}$-area specifically. The conductivity estimates inferred from CPTu measurements have a vertical resolution of $0.1 \mathrm{ft}$ and are 
considered to be "point" measurements. Atlantic Coastal Plain sediments are clearly stratified and imply anisotropic correlation scales, $\lambda_{\mathrm{h}}$ and $\lambda_{v}$. Their ratio cannot be derived from the CPTu data because none of the 164 locations are close enough in the horizontal plane (i.e. within inches). However, judgement based on knowledge of the depositional environment and visual inspection of outcrops suggests a reasonable ratio is approximately $\lambda_{h} / \lambda_{v}=10$. Furthermore, the vertical and horizontal correlation scales within stratigraphic zones are probably on the order of a few inches and feet, respectively. Considering that the typical model block will be a few feet thick and span on the order of $100 \mathrm{ft}$ in the horizontal plane, the "equivalent" block conductivity should be close to the "effective" conductivity, in the terminology of Sanchez-Vila and others (1995). Therefore, power averaging parameters derived from Gelhar and Axness (1983) can reasonably be applied to upscaling of C-area CPTu data. For $\lambda_{h} / \lambda_{v}=10$, the $\mathrm{p}$-norm results are $\mathrm{p}_{\mathrm{h}}=0.86$ and $\mathrm{p}_{\mathrm{v}}=-0.72$ for horizontal and vertical conductivity, respectively (see equations (G.11), (G.12), (G.17) and (G.18)). As mentioned previously, Gelhar and Axness' (1983) predictions overpredict $p_{h}$ for anisotropic media. Considering this and many other uncertainties in the data and underlying assumptions, $p_{h}$ and $p_{v}$ are uncertain and may be viewed as calibration parameters.

To avoid introducing bias into the predicted conductivity fields, the following upscaling process is chosen for $\mathrm{K}_{\mathrm{h}}$ and $\mathrm{K}_{\mathrm{v}}$ :

1) Transform the point data $\mathrm{K}$ to $\mathrm{K}^{\mathrm{p}}$, where $\mathrm{p}=+0.86$ for horizontal conductivity and $p=-0.72$ for vertical conductivity

2) For each CPTu push, arithmetically average $\mathrm{K}^{\mathrm{P}}$ over the thickness of each model layer

3) Interpolate $\overline{\mathrm{K}^{\mathrm{p}}}$ within each layer using two-dimensional block kriging

4) Back transform $\mathrm{K}^{\mathrm{p}}$ block estimates by computing $\left(\mathrm{K}^{\mathrm{p}}\right)^{1 / \mathrm{p}}$, where $\mathrm{p}$ takes on the value used in step 1)

Vertical averaging in step 2) reduces the three-dimensional CPTu data to a sequence of two-dimensional data sets, one per model layer. Model layers conform to stratigraphic units, zones or sub-zones. Kriging is chosen in step 3) because it is an exact interpolator for point estimation and can be optimized for the characteristics of individual formations through the variogram model. An isotropic exponential semivariogram model is chosen. The functional form is

$$
\gamma(h)=c\left[1-\exp \left(-\frac{3 h}{a}\right)\right]
$$


where

$$
\begin{aligned}
& \gamma \equiv \text { semivariogram; } \frac{1}{2} \mathrm{E}\left\{[\mathrm{K}(\overrightarrow{\mathrm{x}}+\overrightarrow{\mathrm{h}})-\mathrm{K}(\overrightarrow{\mathrm{x}})]^{2}\right\} \\
& \mathrm{h} \equiv \text { lag distance, }|\overrightarrow{\mathrm{h}}| \\
& \mathrm{c} \equiv \text { contribution } \\
& \mathrm{a} \equiv \text { effective range }
\end{aligned}
$$

A variogram analysis of the synthetic data for each hydrostratigraphic unit was performed to estimate the effective range, a. The estimated ranges varied from 500 to $1000 \mathrm{ft}$. The average of $750 \mathrm{ft}$ was subsequently used in the block kriging performed for each unit. The contribution, $c$, does not affect the point or block estimates so its specification can be arbitrary. The contribution does affect the uncertainty of the estimates and an estimate would be needed if an uncertainty analysis were conducted.

\section{Predicted conductivity fields for $\mathrm{C}$-area}

There were 164 lithologic CPTu pushes in C-area, among a larger total number that included groundwater sampling. The $131 \mathrm{C}$ series of pushes are located at and downgradient of the C-area Burning/Rubble Pit. The CRSB series is located in the vicinity of the C-Reactor Seepage Basins and down-gradient. The CSB series are located further down-gradient of the CRSBs and also along Caster Creek, Fourmile Branch and the C-04 outfall. The 164 data locations are shown in Figure G-11.

\section{Initial hydrostratigraphy (unaltered tan clay horizon)}

Figures G-12 through G-27 show upscaled predicted horizontal and vertical hydraulic conductivity for model layers 3 through 8 . The correspondence between model layers and hydrostratigraphy is defined in Table G-5. The upper half of each figure shows the point kriging estimates of $\mathrm{K}^{\mathrm{p}}$ in units of $(\mathrm{ft} / \mathrm{d})^{\mathrm{p}}$, which are intermediate results prior to block kriging and back transformation. Of more interest is the lower half of each figure which shows the final (back-transformed) block kriging estimates $\bar{K}$ in units of $\mathrm{ft} / \mathrm{d}$.

Note in Figure G-21 that the predicted vertical conductivity of the "tan clay" confining zone abruptly increases by one to two orders of magnitude in the vicinity of Caster Creek. Simultaneously, the horizontal (Figure G-18) and vertical (Figure G-19) conductivity of the underlying "lower" UTRA aquifer zone decrease abruptly. Hence, the CPTu predictions indicate that, on the north side of Caster Creek, the tan clay largely disappears as a confining unit and is simultaneously underlain by a deeper, more 
significant confining zone within the "lower" UTRA. The underlying confining zone is interpreted to be a calcareous wackestone/mudstone based on the CPTu signature of low tip and sleeve resistance with high pore pressure.

The predicted hydraulic conductivity variation in the transmissive zone shown in Figure G-22 is also notable. The transmissive zone is significantly more conductive near Caster Creek in general, and especially so midway between the C-04 outfall and Fourmile Branch. This variation is significant enough to justify a corresponding high conductivity zone in regional flow model.

Table G-5 Correspondence between model layers and hydrostratigraphy.

\begin{tabular}{|c|c|c|}
\hline Model layer & Hydrostratigraphic zone & Abbreviation \\
\hline 8 & A and uu horizons & A/uu \\
\hline 7 & AA horizon & AA \\
\hline 6 & transmissive zone & TZ \\
\hline 5 & tan clay & TCCZ \\
\hline 4 & upper half of "lower" UTRA & uLAZ \\
\hline 3 & lower half of "lower" UTRA & ILAZ \\
\hline 2 & Gordon confining unit & GCU \\
\hline 1 & Gordon aquifer unit & GAU \\
\hline
\end{tabular}

\section{Modified hydrostratigraphy (modified tan clay horizon)}

Inspection of the CPTu logs and the predicted conductivity fields presented above indicates that additional hydrostratigraphic zones should be defined in C-area. Specifically, two additional confining zones should be delineated within the "lower" UTRA, as indicated in Table G-6. The vertical resolution of the regional flow model is insufficient to individually represent each hydrostratigraphic zone in the expanded framework. Given this limitation the best alternative is to redefine element layer 5 such that it coincides with the lower calcareous zone (CC1) instead the tan clay (TCCZ) near Caster Creek in $\mathrm{C}$-area. The specific modifications made to the "tan clay" model layer are provided in Table G-7 as revised picks for the top of the TCCZ and LAZ. Figures G-28 through G-43 illustrate the predicted hydraulic conductivity results based on CPTu data for the redefined model stratigraphy. These plots correspond to Figures G-12 through G-27 presented previously. Note from Figure G-37 that the model "tan clay" layer (element layer 5) now functions as a confining unit throughout $\mathrm{C}$-area. 
Table G-6 Expanded hydrostratigraphy in C-area.

\begin{tabular}{|c|c|}
\hline Hydrostratigraphic zone & Abbreviation \\
\hline A and uu horizons & $\mathrm{A} / \mathrm{uu}$ \\
\hline AA horizon & $\mathrm{AA}$ \\
\hline transmissive zone & $\mathrm{TZ}$ \\
\hline tan clay & $\mathrm{TCCZ}$ \\
\hline $\begin{array}{c}\text { upper aquifer interval } \\
\text { within "lower" UTRA }\end{array}$ & $\mathrm{uLAZ}$ \\
\hline $\begin{array}{c}\text { calcareous (confining) interval 1 } \\
\text { within "lower" UTRA }\end{array}$ & $\mathrm{CC1}$ \\
\hline $\begin{array}{c}\text { middle aquifer interval } \\
\text { within "lower" UTRA }\end{array}$ & $\mathrm{mLAZ}$ \\
\hline $\begin{array}{c}\text { calcareous (confining) interval 2 } \\
\text { within "lower" UTRA }\end{array}$ & $\mathrm{CC} 2$ \\
\hline $\begin{array}{c}\text { lower aquifer interval } \\
\text { within "lower" UTRA }\end{array}$ & lLAZ \\
\hline Gordon confining unit & $\mathrm{GCU}$ \\
\hline Gordon aquifer unit & $\mathrm{GAU}$ \\
\hline
\end{tabular}


Table G-7 Modified hydrostratigraphic picks for defining model layer 5.

\begin{tabular}{|l|l|l|l|}
\hline SiteID & TCCZ & LAZ & Comment \\
\hline CSB-102 & 186 & 182 & prior \\
CSB-102 & 158 & 127 & revised \\
\hline CSB-103 & 179 & 168 & prior \\
CSB-103 & 148 & 121 & revised \\
\hline CSB-104 & 175 & 168 & prior \\
CSB-104 & 144 & 130 & revised \\
\hline CSB-105 & 177 & 175 & prior \\
CSB-105 & 147 & 129 & revised \\
\hline CSB-106 & 173 & 169 & prior \\
CSB-106 & 152 & 127 & revised \\
\hline CSB-107 & $1.0 \mathrm{E}+20$ & $1.0 \mathrm{E}+20$ & prior \\
CSB-107 & 156 & 132 & revised \\
\hline CSB-108 & 178 & 165 & prior \\
CSB-108 & 156 & 128 & revised \\
\hline CSB-109 & $1.0 \mathrm{E}+20$ & $1.0 \mathrm{E}+20$ & prior \\
CSB-109 & 149 & 132 & revised \\
\hline CSB-110 & $1.0 \mathrm{E}+20$ & 171 & prior \\
CSB-110 & 164 & 148 & revised \\
\hline CSB-111 & $1.0 \mathrm{E}+20$ & 160 & prior \\
CSB-111 & 154 & 134 & revised \\
\hline CSB-112 & 172 & 153 & prior \\
CSB-112 & 151 & 129 & revised \\
\hline CSB-113 & 180 & 161 & prior \\
CSB-113 & 159 & 144 & revised \\
\hline CSB-114 & 178 & 168 & prior \\
CSB-114 & 160 & 148 & revised \\
\hline CSB-118 & 178 & 171 & prior \\
CSB-118 & 154 & 137 & revised \\
\hline CSB-120 & 178 & 159 & prior \\
CSB-120 & 157 & 145 & revised \\
\hline CSB-121 & 188 & 163 & prior \\
CSB-121 & 161 & 151 & revised \\
\hline CSB-122 & 181 & 165 & prior \\
CSB-122 & 150 & 129 & revised \\
\hline CSB-123 & 188 & 182 & prior \\
CSB-123 & 158 & 139 & revised \\
\hline CSB-60 & 181 & 179 & prior \\
CSB-60 & 162 & 149 & revised \\
\hline CSB-61 & 175 & 173 & prior \\
CSB-61 & 155 & 146 & revised \\
\hline CSB-62 & 179 & 169 & prior \\
CSB-62 & 146 & 140 & revised \\
\hline CSB-63 & 180 & 178 & prior \\
CSB-63 & 157 & 142 & revised \\
\hline CSB-64 & 178 & 176 & prior \\
CSB-64 & 156 & 140 & revised \\
\hline CSB-65 & 165 & 162 & prior \\
CSB-65 & 160 & 131 & revised \\
\hline CSB-66A & $1.0 \mathrm{E}+20$ & $1.0 \mathrm{E}+20$ & prior \\
revised \\
\hline
\end{tabular}




\section{- References}

Ababou, R. and E. F. Wood, 1990, Comment on "Effective groundwater model parameter values: influence of spatial variability of hydraulic conductivity, leakance, and recharge" by J. J. Gomez-Hernandez and S. M. Gorelick, Water Resources Research, v26, n8, 1843-1846.

Celeste, 1998, Permeability of R-area sediments, Savannah River Site, South Carolina: Minipermeameter vs. cone penetrometer technology, M. S. Thesis, Department of Earth Sciences, University of North Carolina at Wilmington.

de Marsily, G., 1986, Quantitative hydrogeology; Groundwater hydrology for engineers, Academic Press.

Desbarats, A. J., 1992, Spatial averaging of hydraulic conductivity in three-dimensional heterogeneous porous media, Mathematical Geology, v24, n3, 249-267.

Flach, G. P., L. L. Hamm, M. K. Harris, P. A. Thayer, J. S. Haselow and A. D. Smits, 1998, A method for characterizing hydrogeologic heterogeneity using lithologic data, in G. S. Fraser and J. M. Davis, Hydrogeologic models of sedimentary aquifers, SEPM Concepts in Hydrogeology and Environmental Geology No. 1.

Flach, G. P. and M. K. Harris, 1999, Integrated hydrogeological modeling of the General Separations Area, Volume 2, Groundwater flow model, WSRC-TR-96-0399, Rev. 1 .

Freeze, R. A. and J. A. Cherry, 1979, Groundwater, Prentice-Hall.

Gelhar, L. W., 1997, Perspectives on field-scale application of stochastic subsurface hydrology, in G. Dagan and S. P. Neuman, eds., Subsurface flow and transport: a stochastic approach, Cambridge University Press.

Gelhar, L. W. and C. L. Axness, 1983, Three-dimensional stochastic analysis of macrodispersion in aquifers, Water Resources Research, v19, n1, 161-180.

Jefferies, M. G. and M. P. Davies, 1993, Use of the CPTu to estimate equivalent SPT $\mathrm{N}_{60}$, Geotechnical Testing Journal, GTJODJ, v16, n4, December, 458-468.

Kegley, W. P., 1993, Distribution of permeability at the MWD well field, Savannah River Site, Aiken, South Carolina, M. S. Thesis, Clemson University. 
Kitanidis, P. K., 1997, Groundwater flow in heterogeneous formations, in G. Dagan and S. P. Neuman, eds., Subsurface flow and transport: a stochastic approach, Cambridge University Press.

Parsons Engineering Science, Inc. and Applied Research Associates, Inc., 1997, Evaluation of cone penetrometer data for permeability correlation at the Savannah River Site, WSRC-RP-97-63.

Robertson, P. K. and C. E. Fear, 1995, Liquefaction of sands and its evaluation, IS TOKYO 95, First International Conference on Earthquake Geotechnical Engineering, Keynote Lecture, November.

Sanchez-Vila, X., J. P. Giradi and J. Carrera, 1995, A synthesis of approaches to upscaling of hydraulic conductivities, Water Resources Research, v31, n4, 867882.

Syms and Others, 1999, in preparation. 


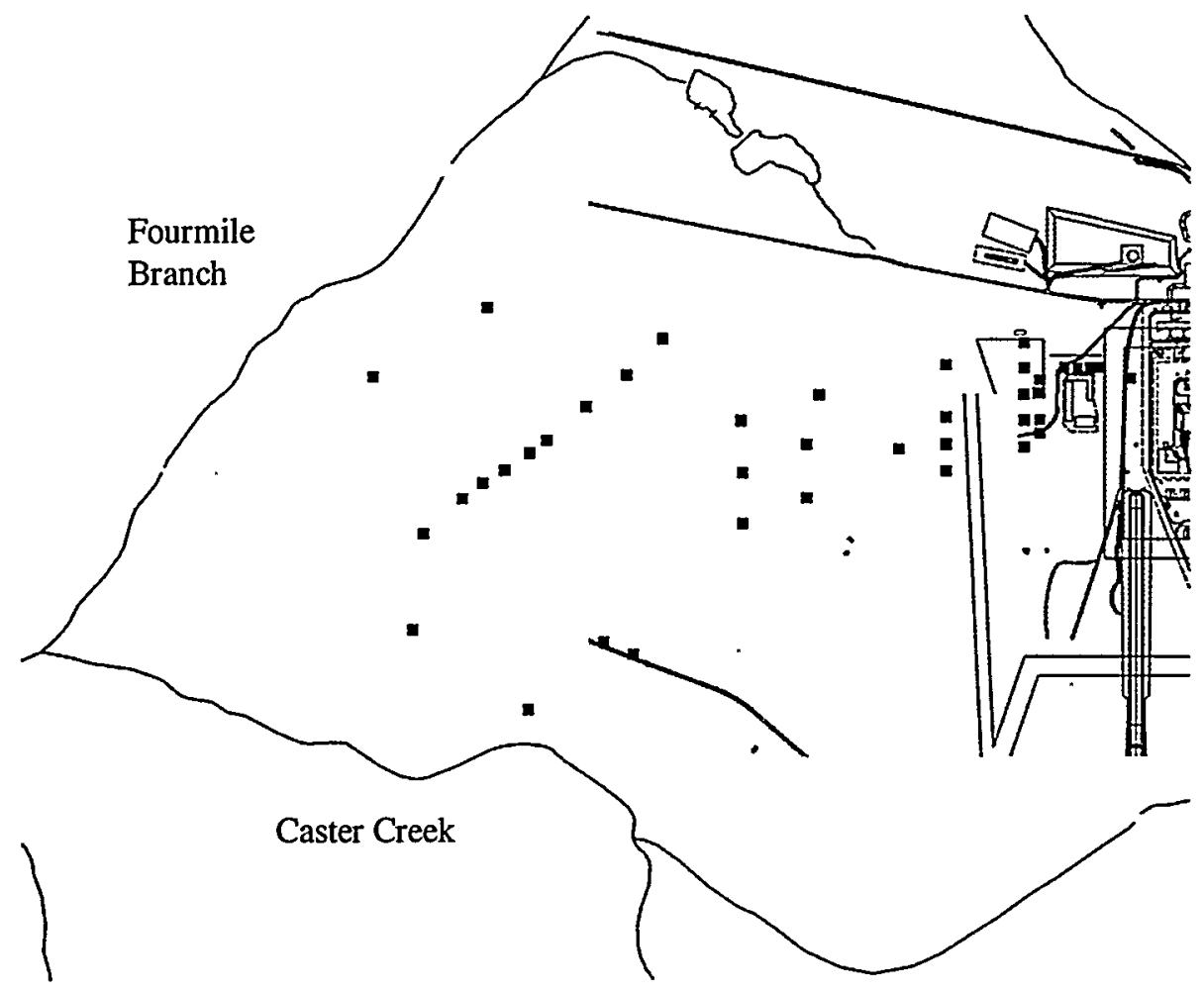

Figure G-1. Plan view of CPT tritium concentration data $\geq 400 \mathrm{pCi} / \mathrm{ml}$.

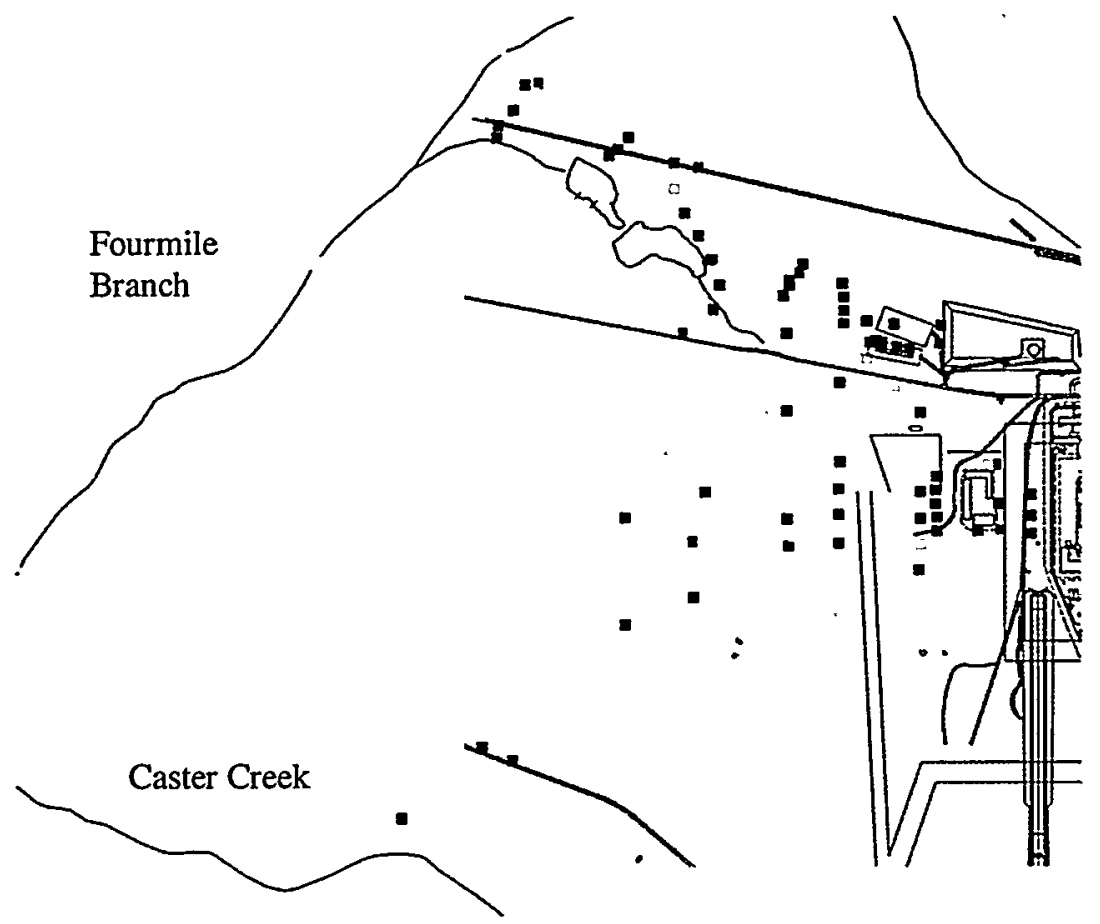

Figure G-2. Plan view of CPT TCE concentration data $\geq 5.2 \mathrm{ppb}$. 


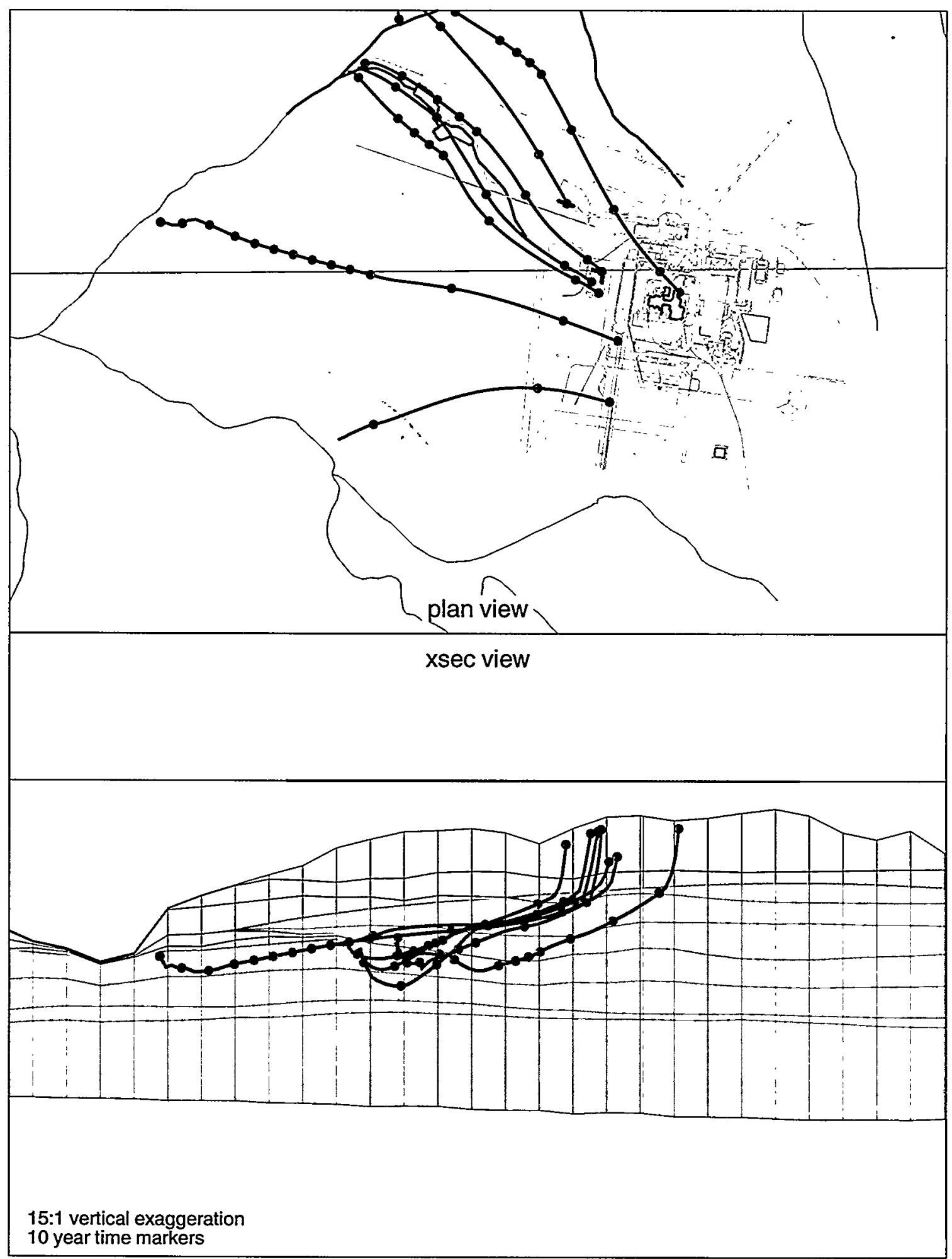

Figure G-3. Predicted groundwater flow paths before revising "tan clay" model layer in C-area near Caster Creek. 


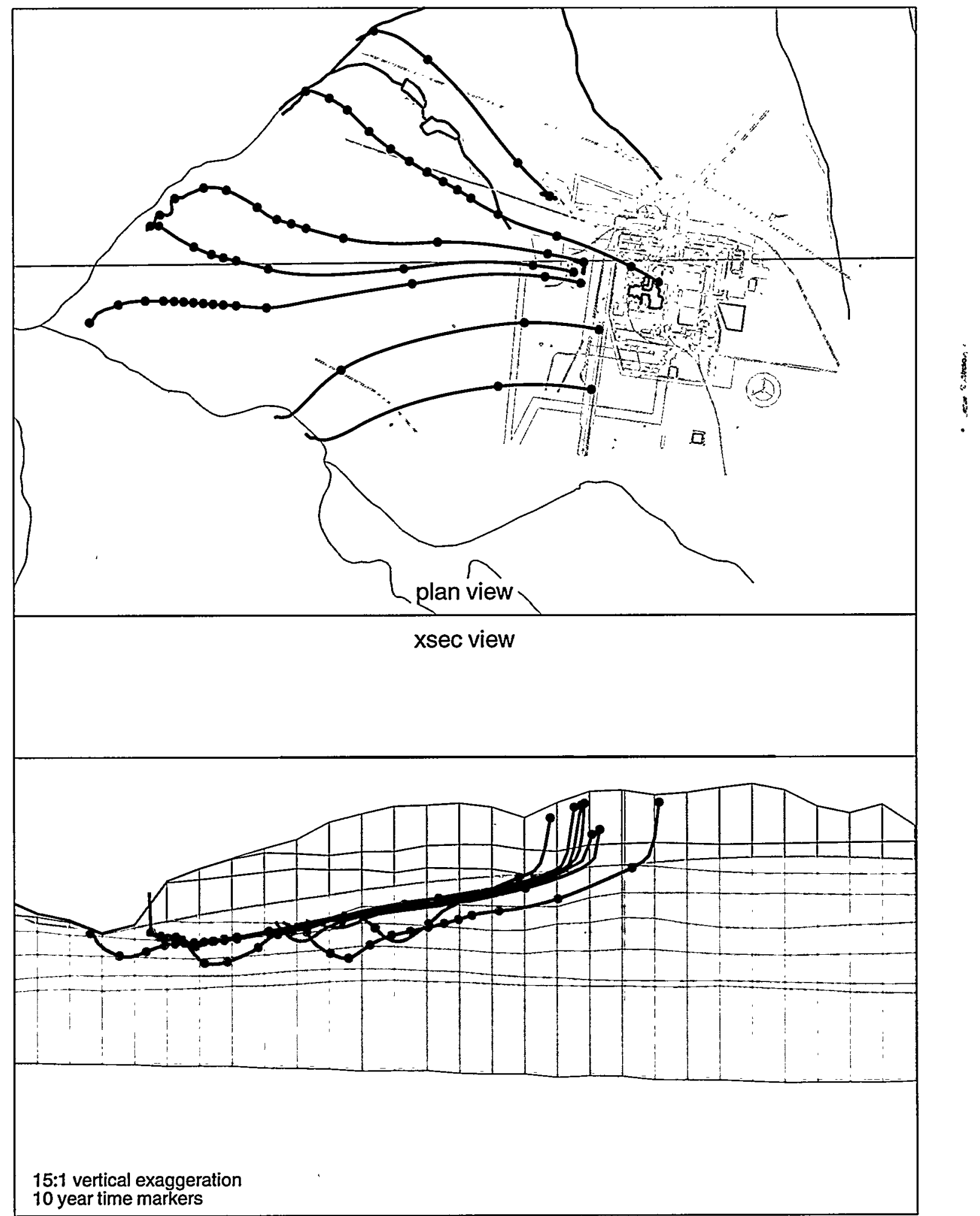

Figure G-4. Predicted groundwater flow paths after revising "tan clay" model layer in C-area near Caster Creek. 


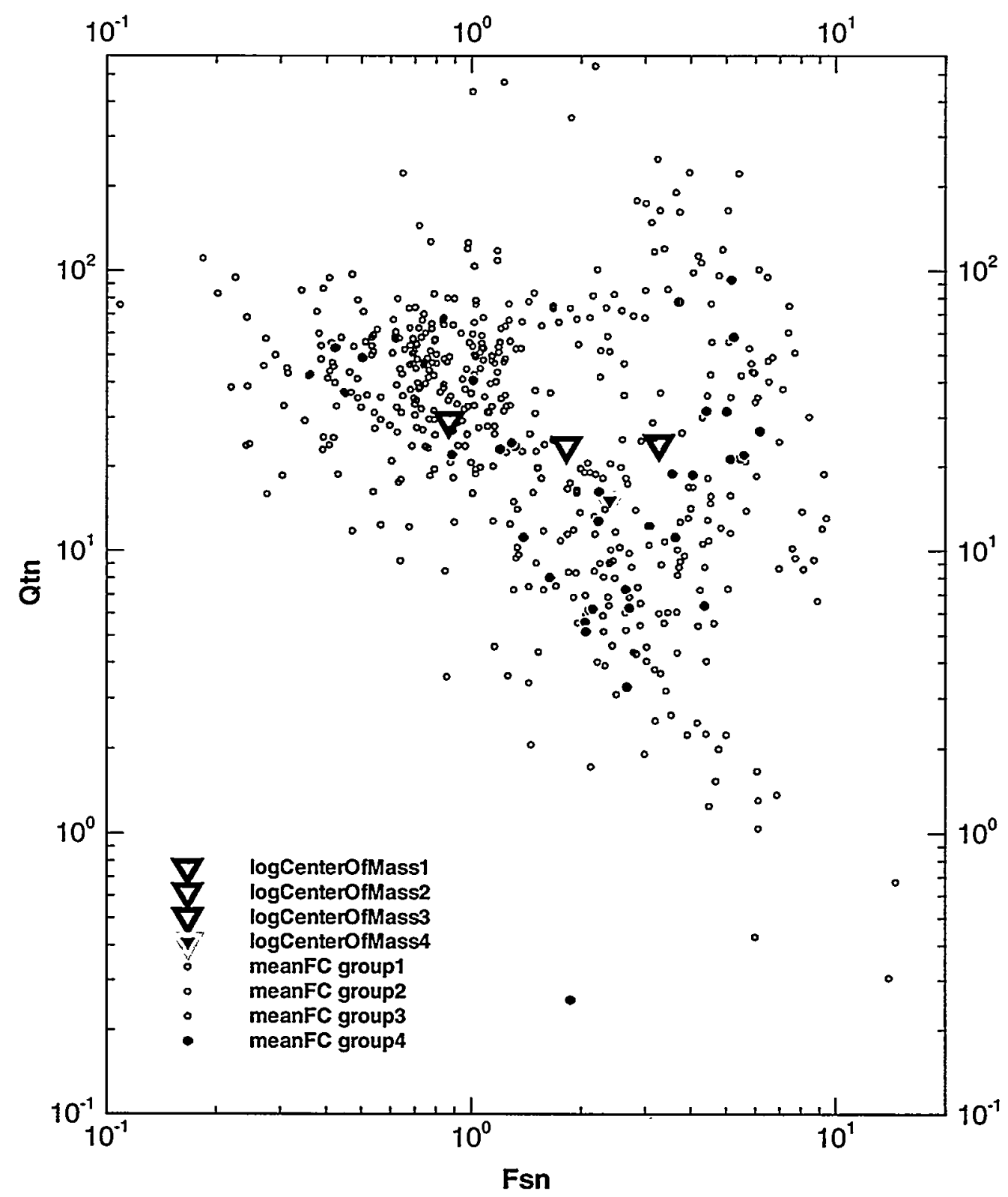

Figure G-5. Normalized tip resistance and friction ratio CPTu data, color-coded by \%fines content (group $1 /$ blue $=0$ to $15 \%$; group $2 /$ green $=15$ to $30 \%$; group $3 /$ red $=30$ to $50 \%$; group $4 /$ yellow $\&$ black $=50$ to $100 \%)$. 


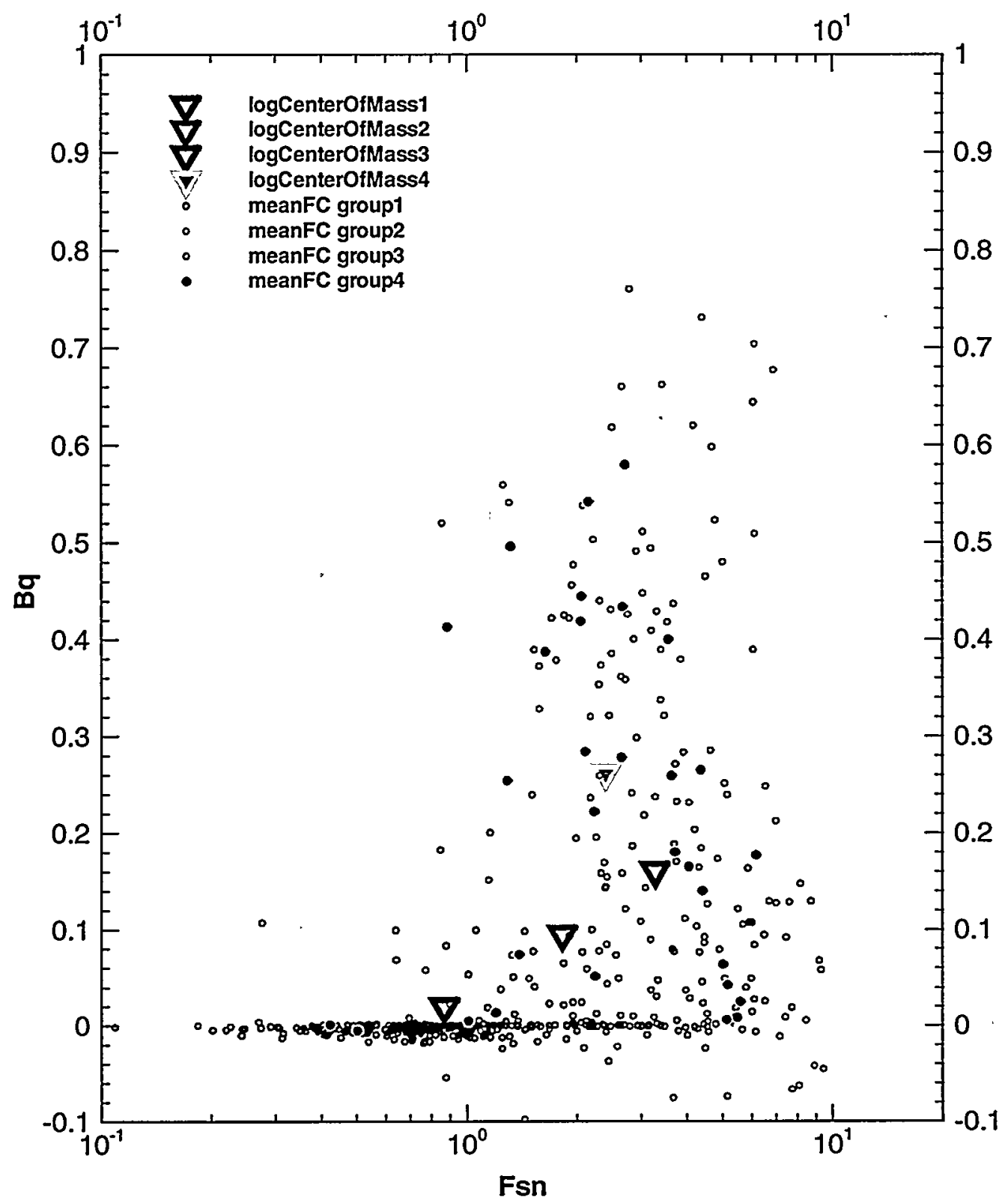

Figure G-6. Normalized pore pressure and friction ratio CPTu data, color-coded by \%fines content (group $1 /$ blue $=0$ to $15 \%$; group 2/green $=15$ to 30\%; group $3 /$ red $=30$ to 50\%; group $4 /$ yellow \& black $=50$ to $100 \%)$. 

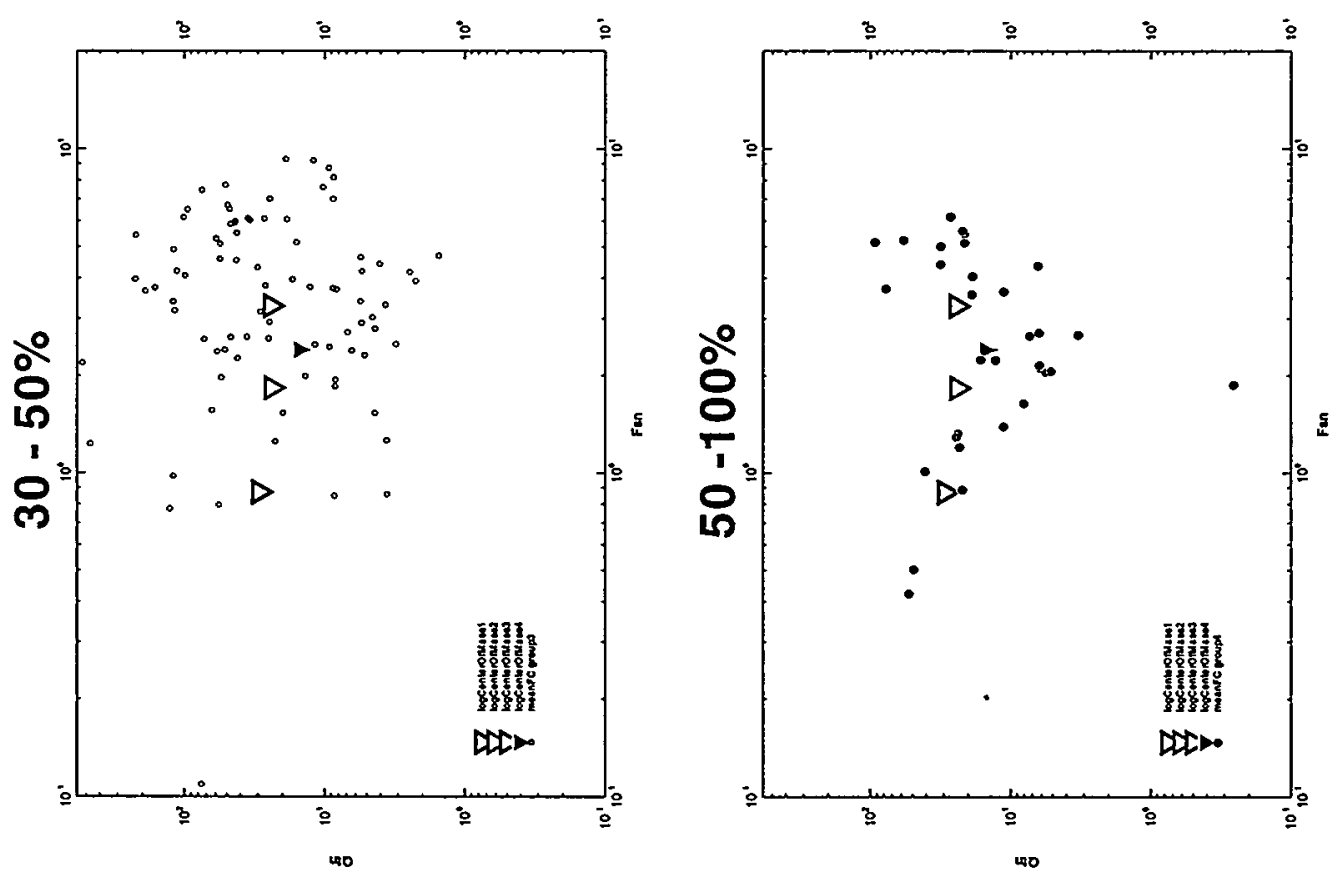

로요용
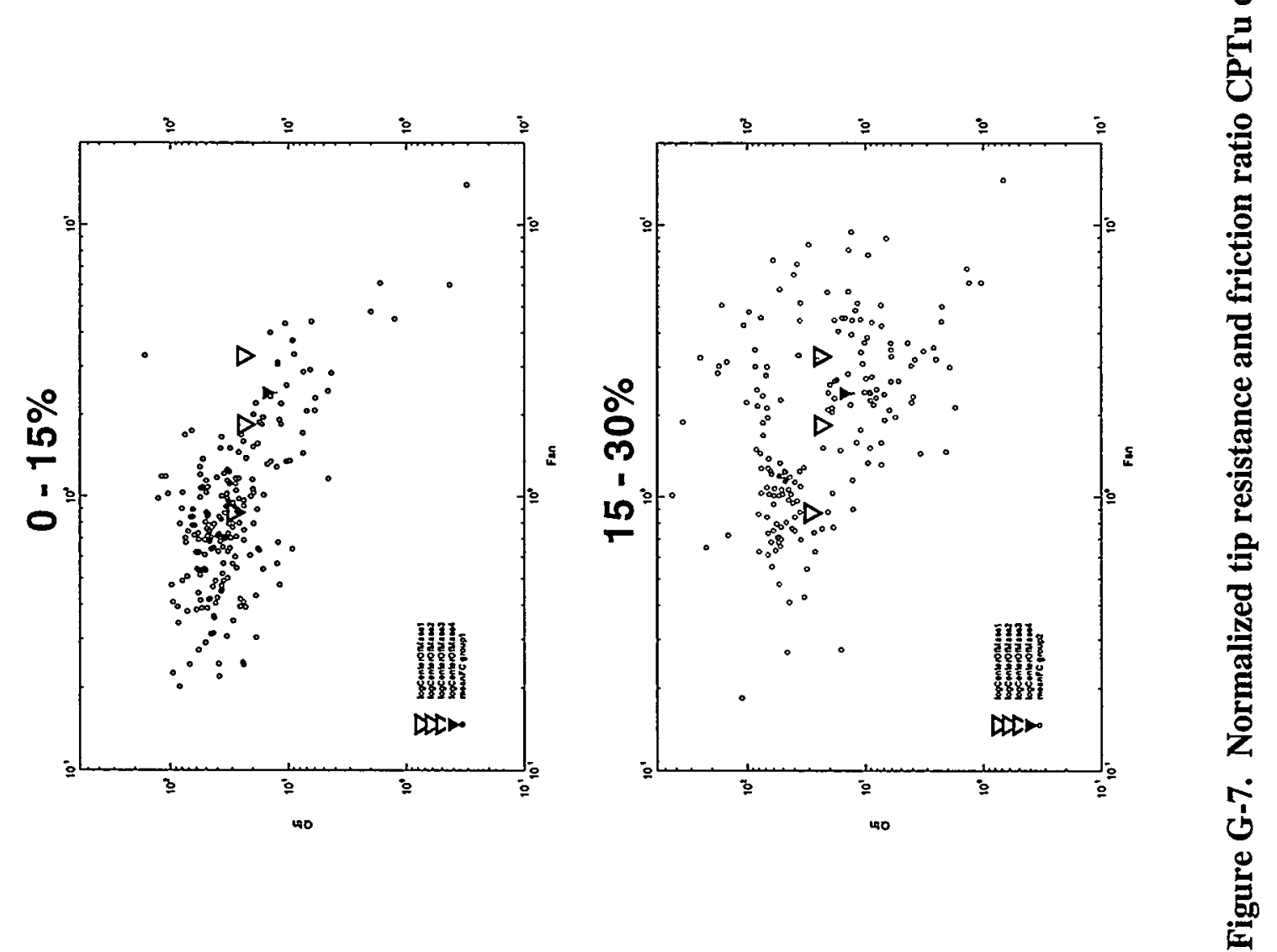

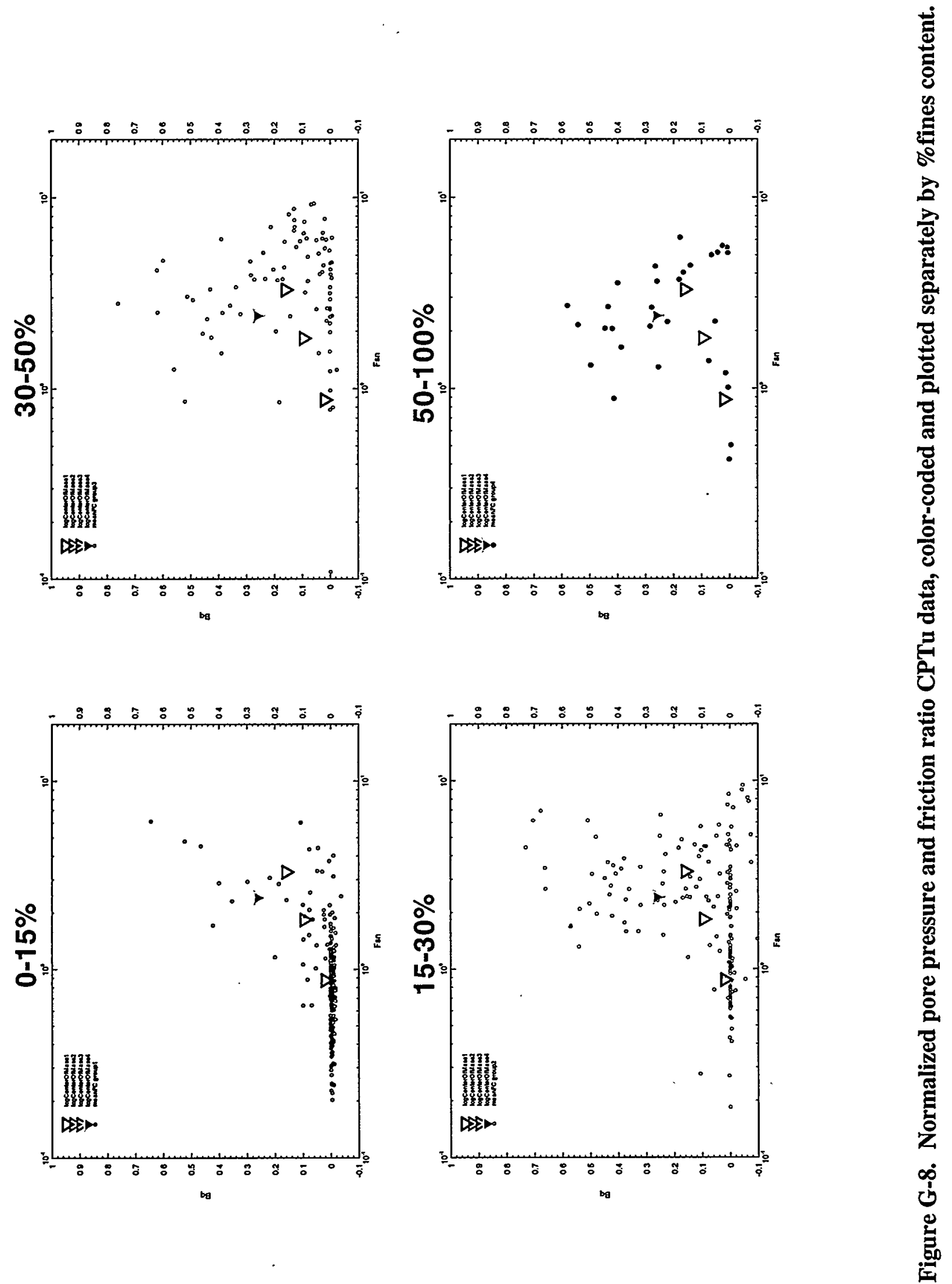

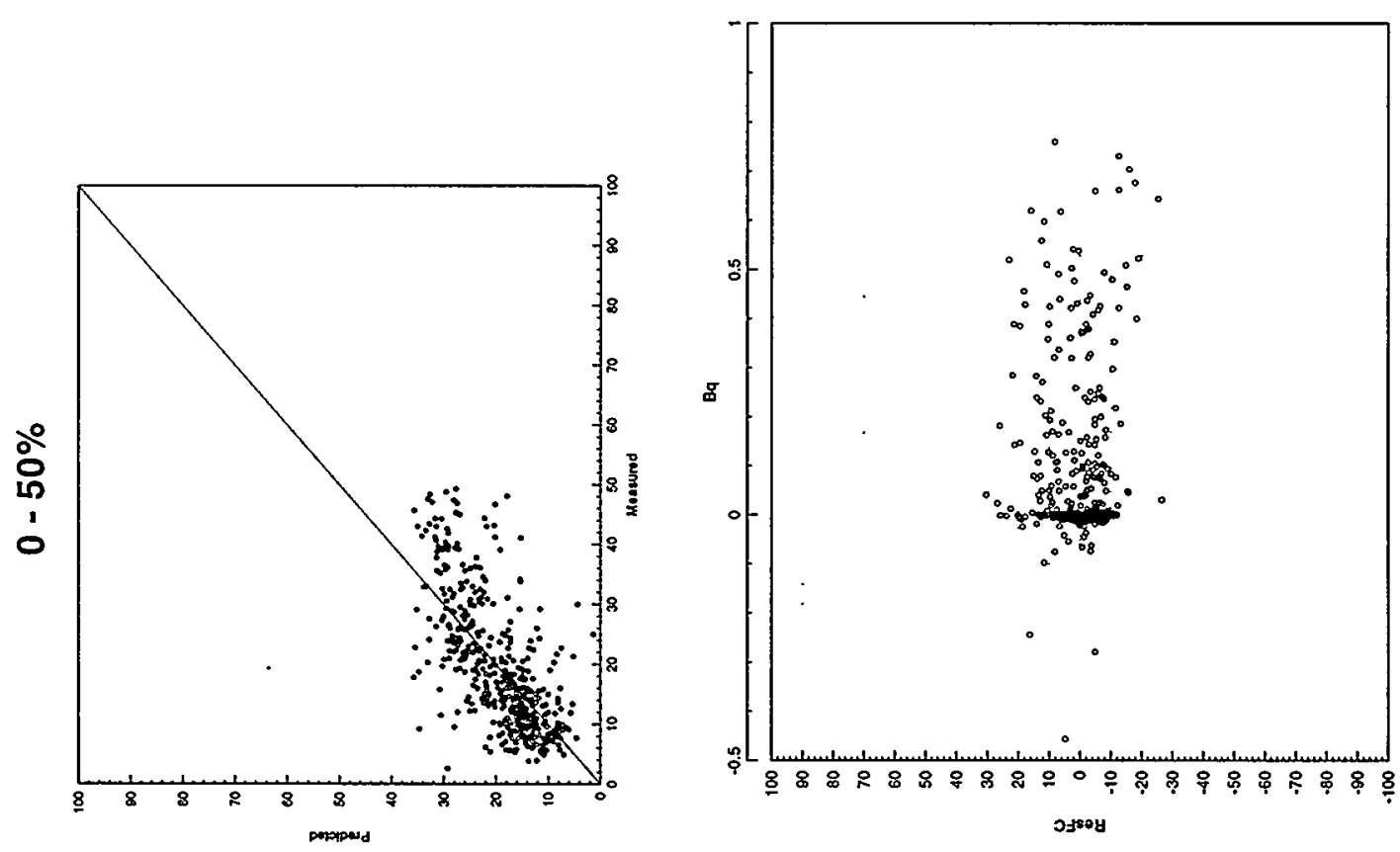

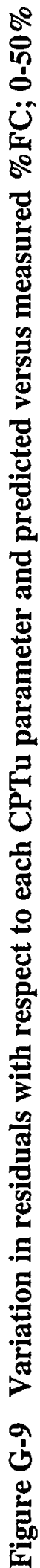



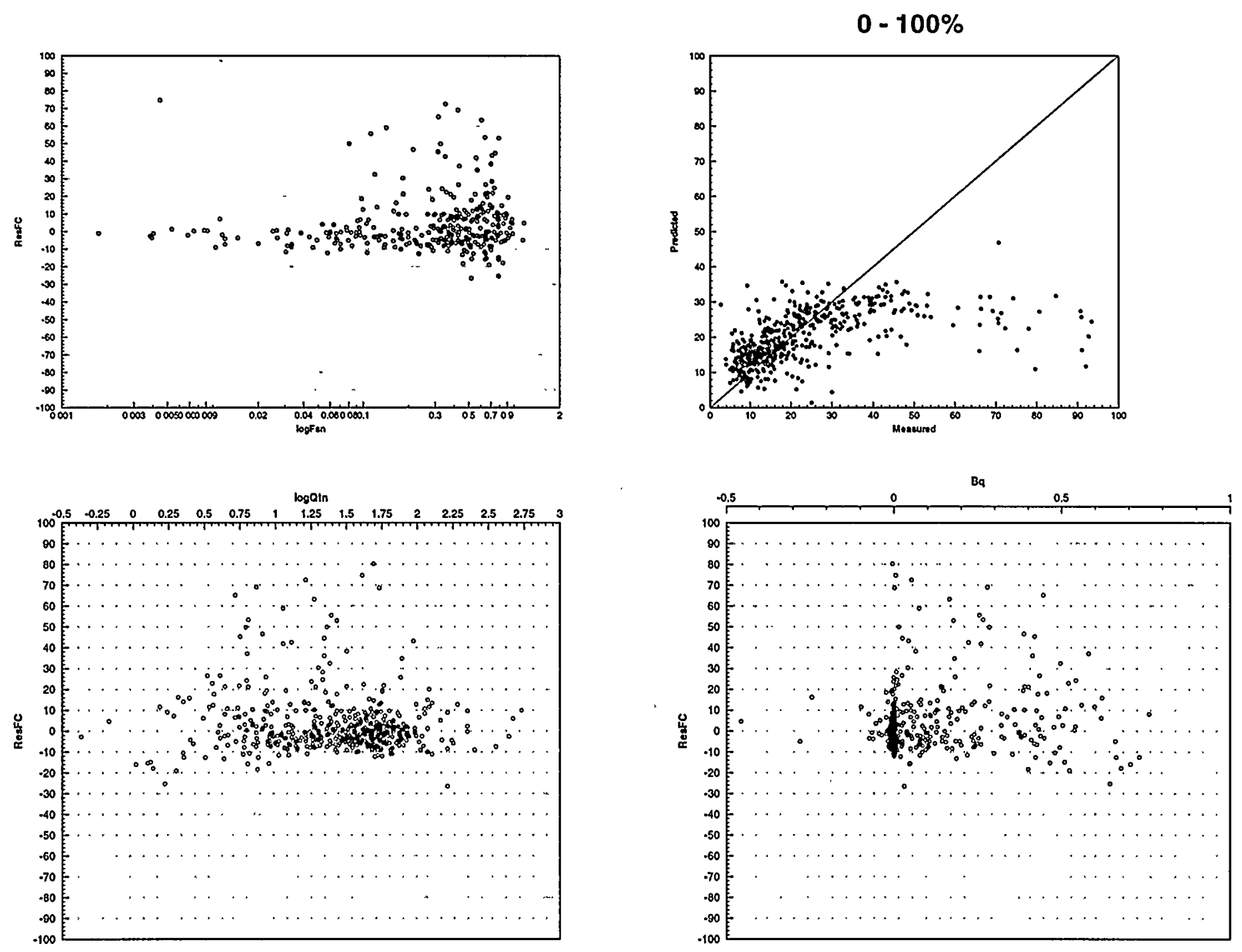

Figure G-10 Variation in residuals with respect to each CPTu parameter and predicted versus measured \%FC; 0-100\% 


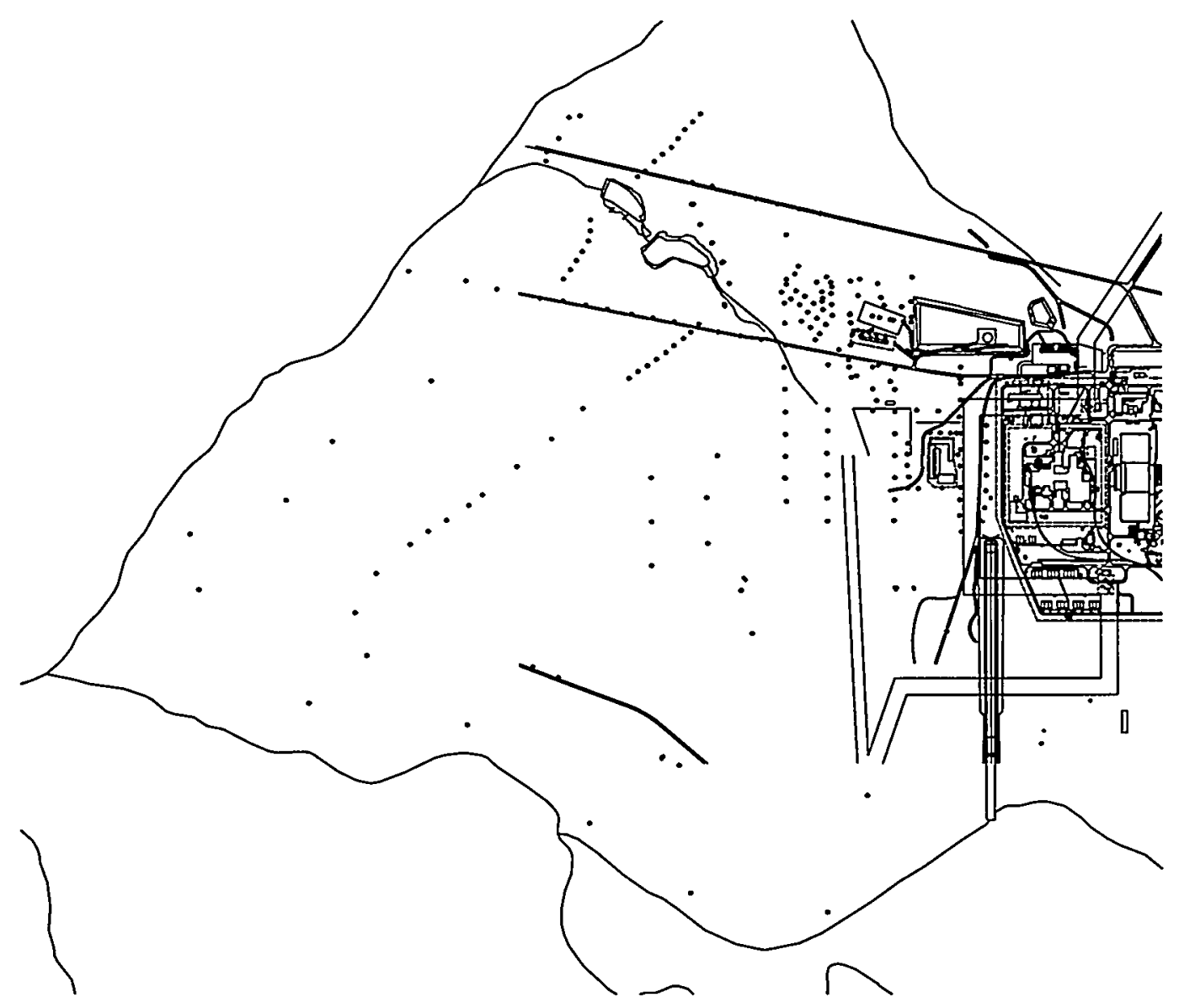

Figure G-11. Locations of C-area CPTu lithologic pushes. 


\section{Point Kriging Estimation}

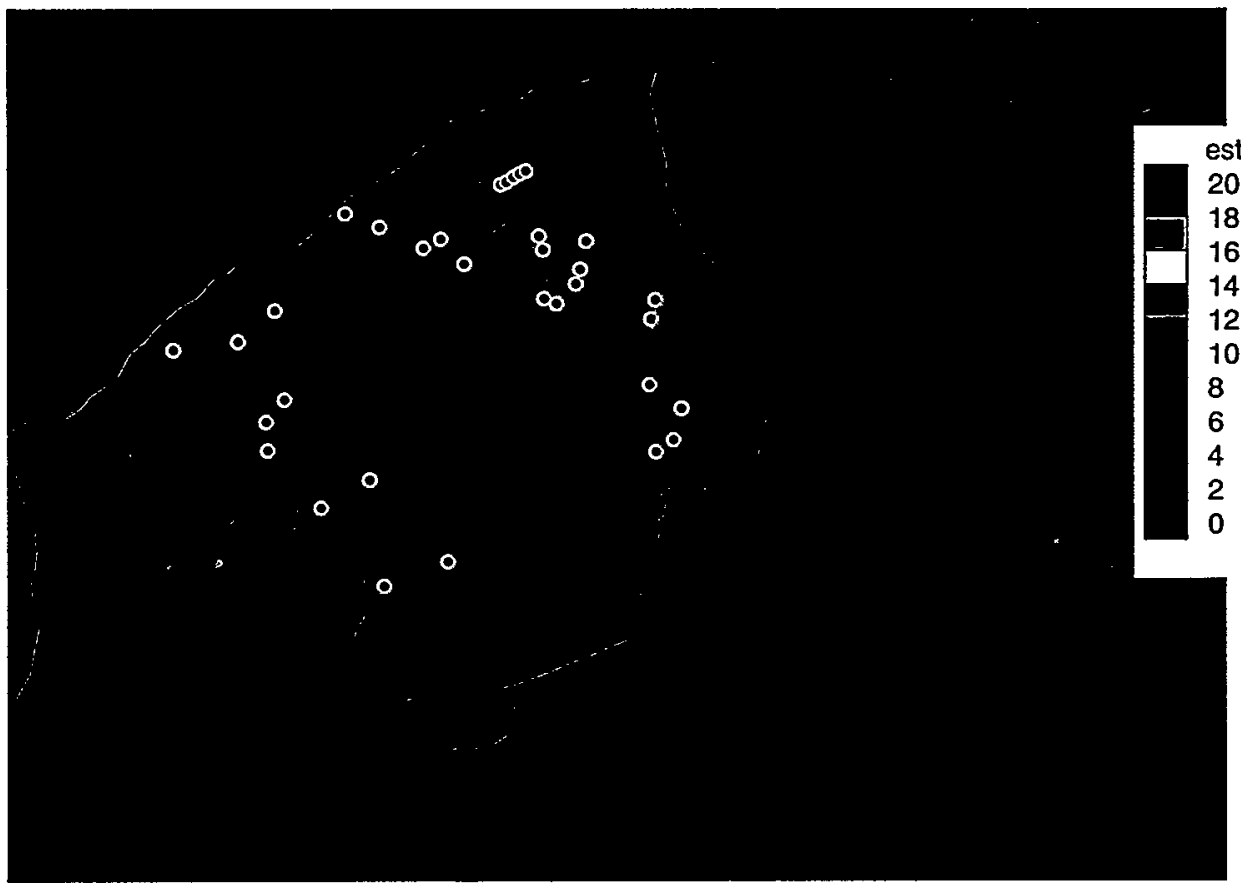

\section{Block Kriging Estimation}

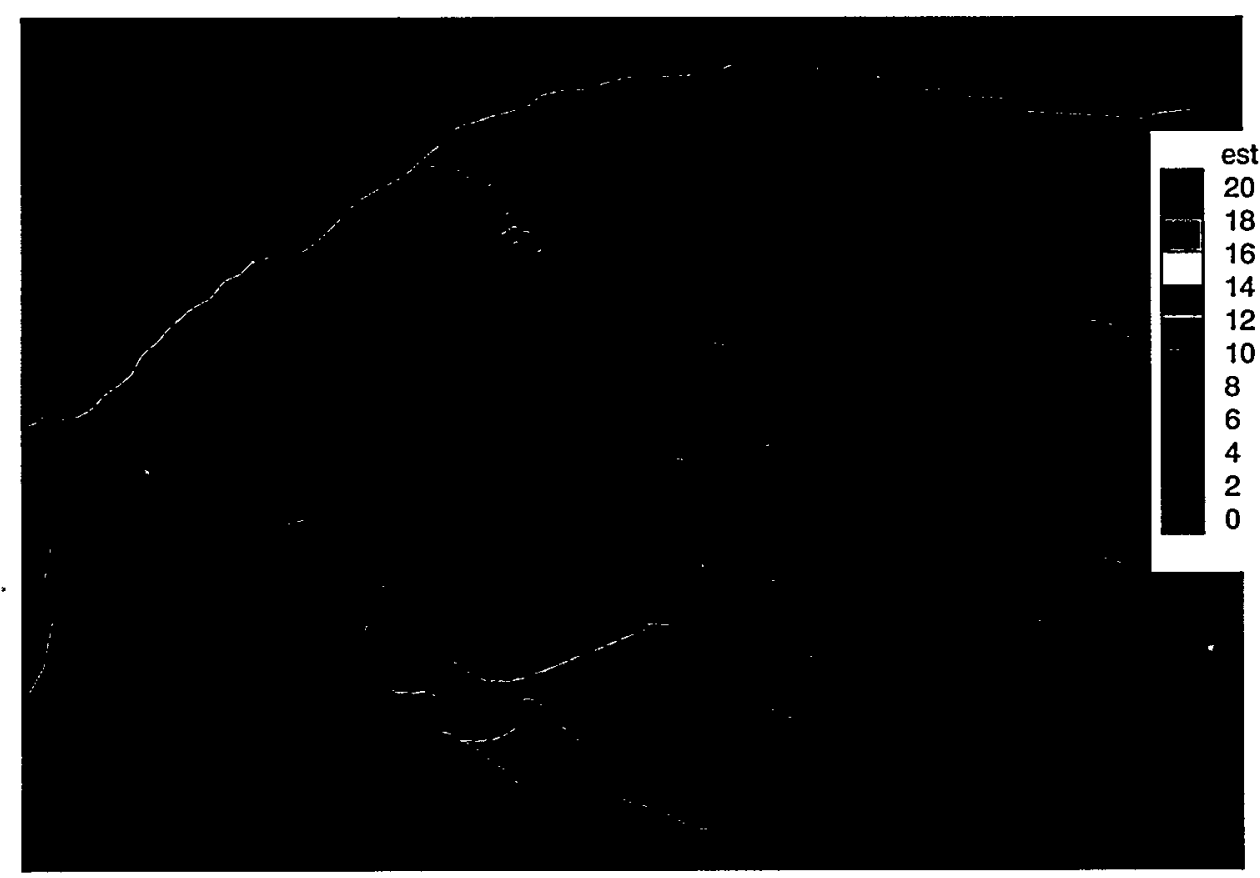

Figure G-12. Kriging estimates for Kh in model layer 1 ( GAU); before TCCZ mods. 


\section{Point Kriging Estimation}

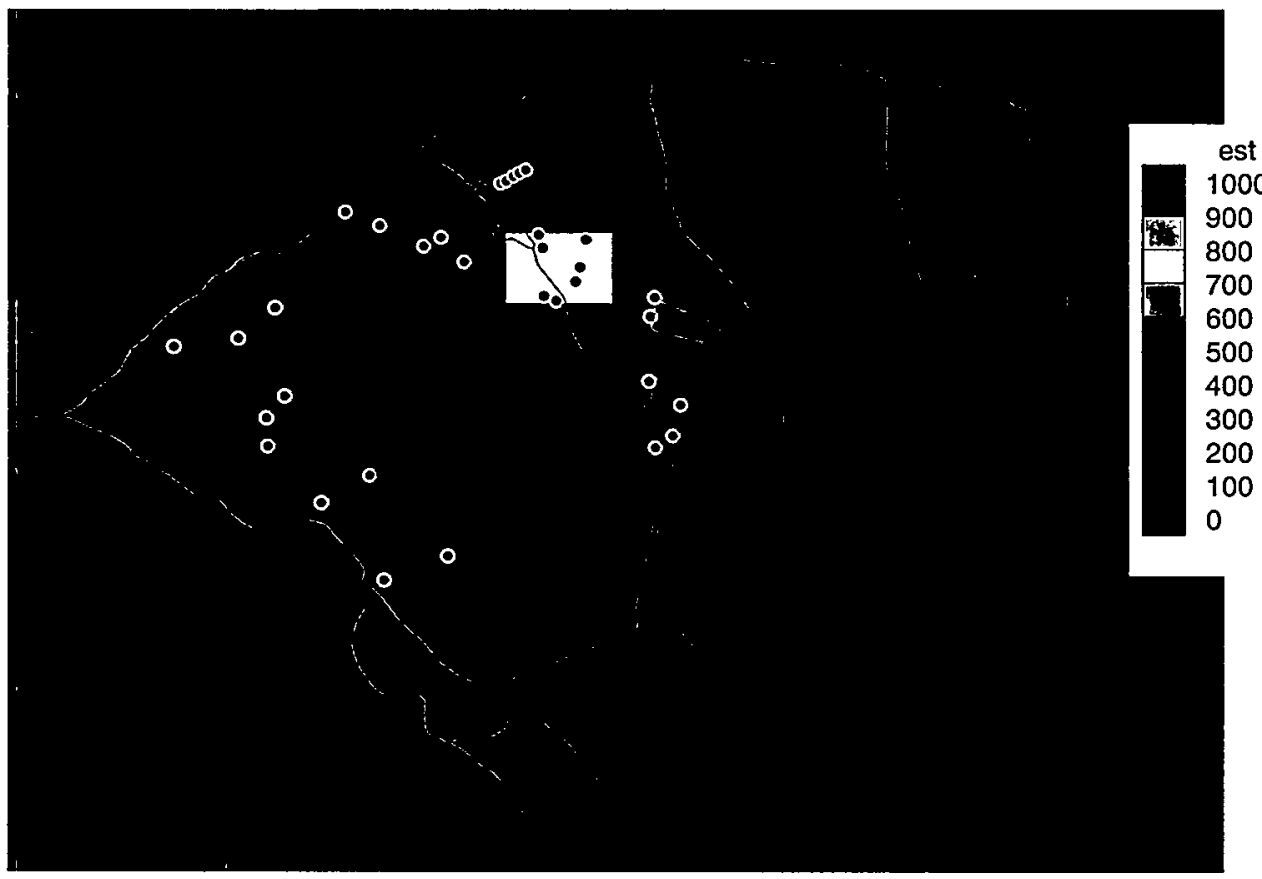

\section{Block Kriging Estimation}

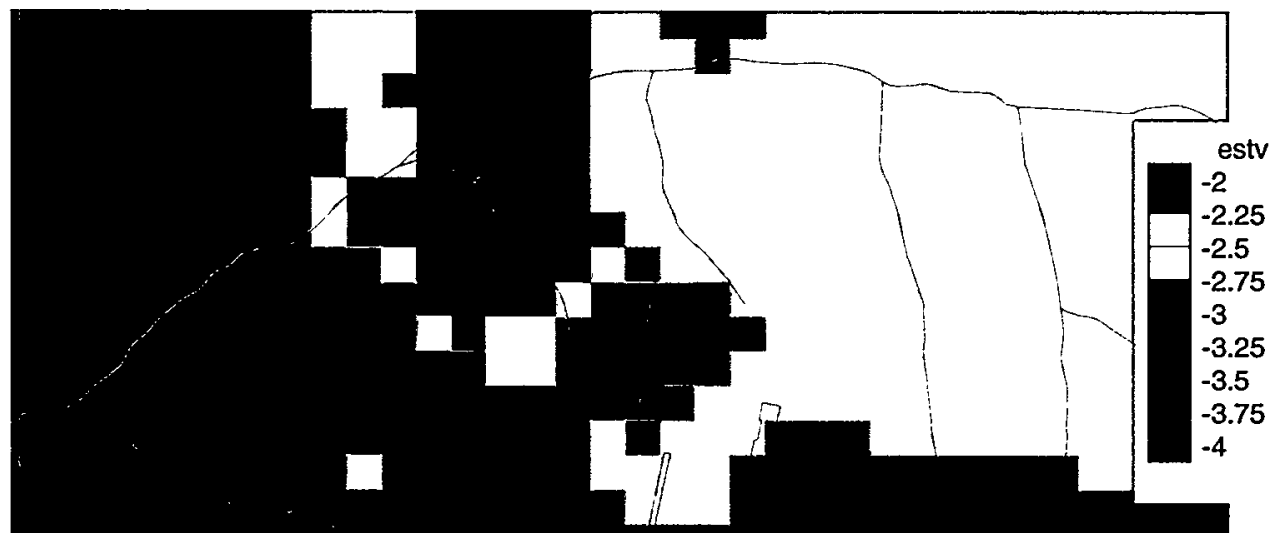

Figure G-13. Kriging estimates for Kv in model layer 1 ( GAU); before TCCZ mods. 


\section{Point Kriging Estimation}

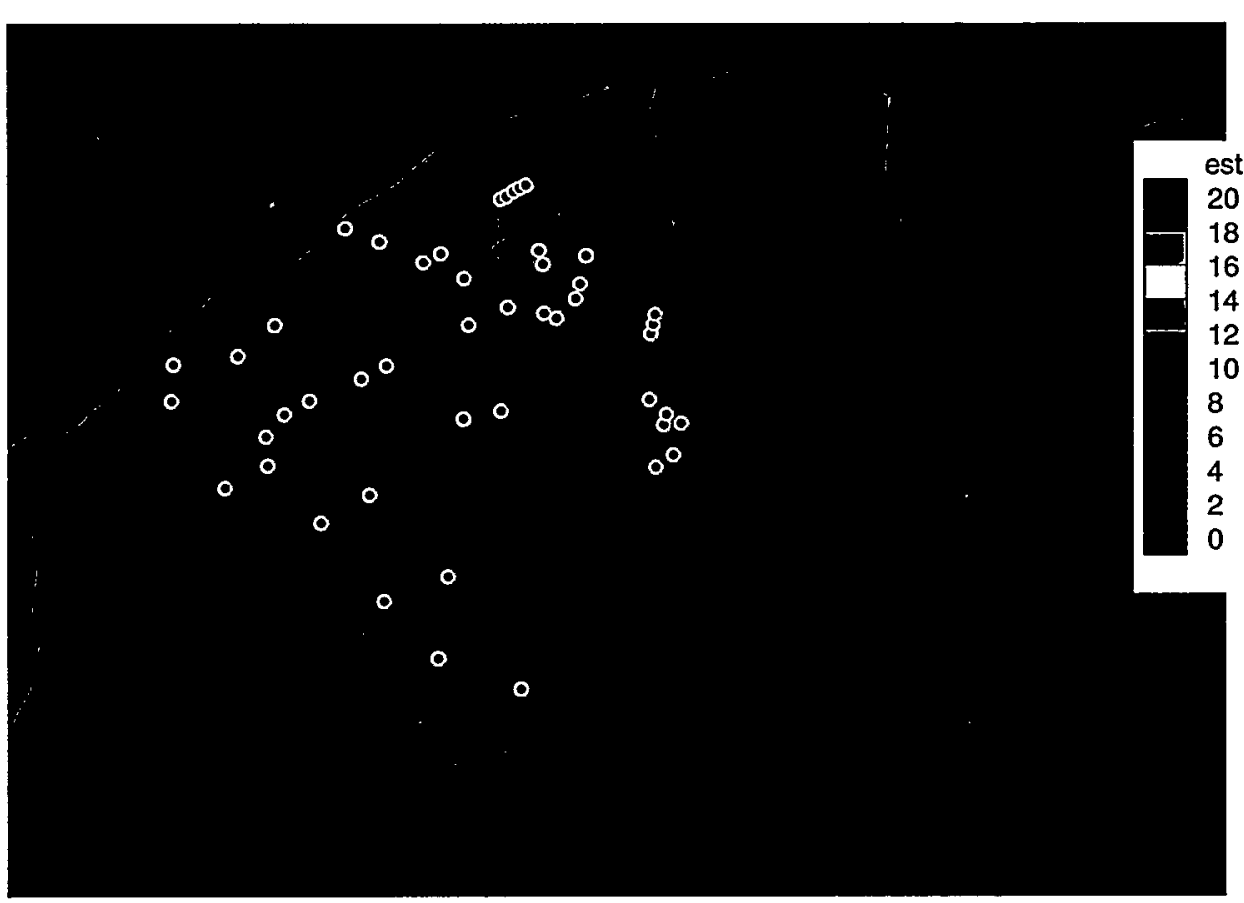

\section{Block Kriging Estimation}

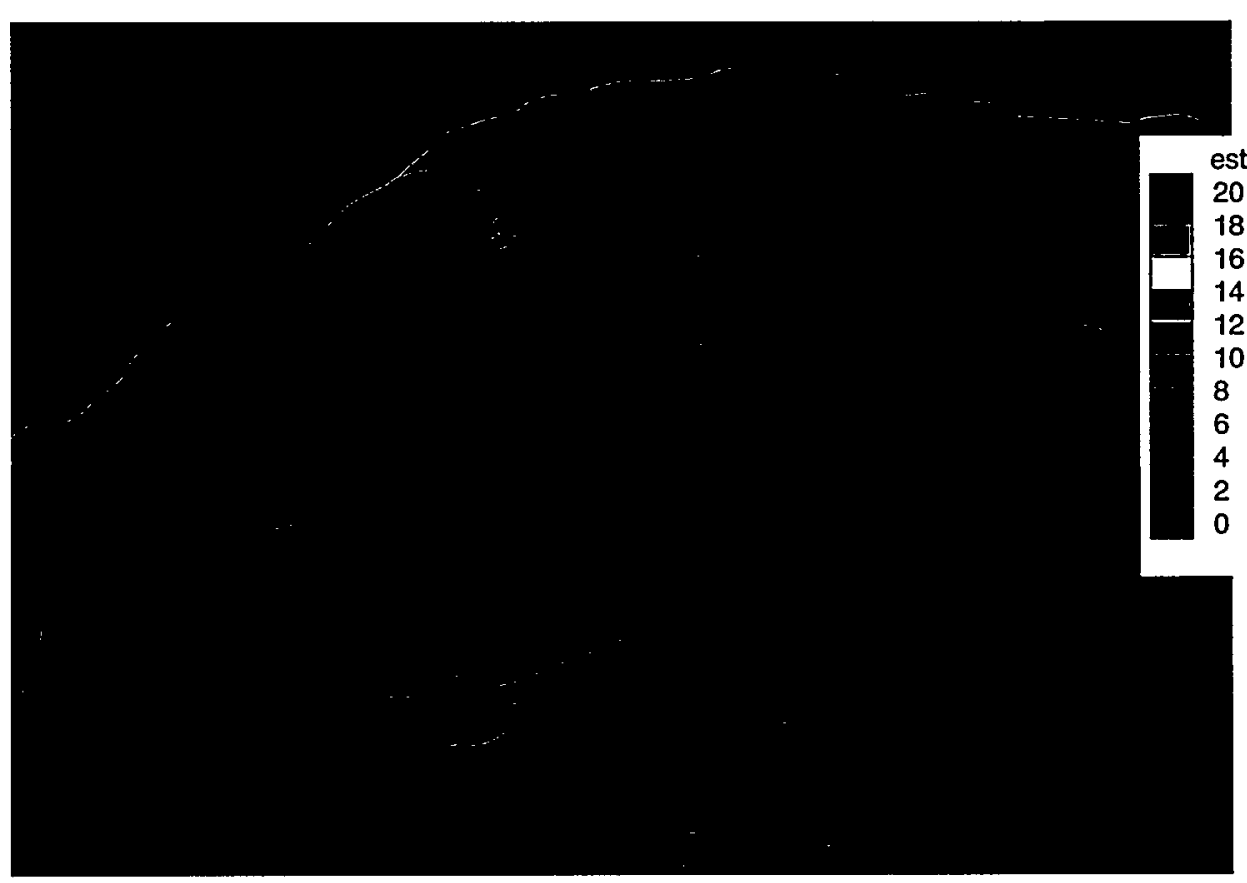

Figure G-14. Kriging estimates for Kh in model layer 2 ( GCU); before TCCZ mods. 


\section{Point Kriging Estimation}

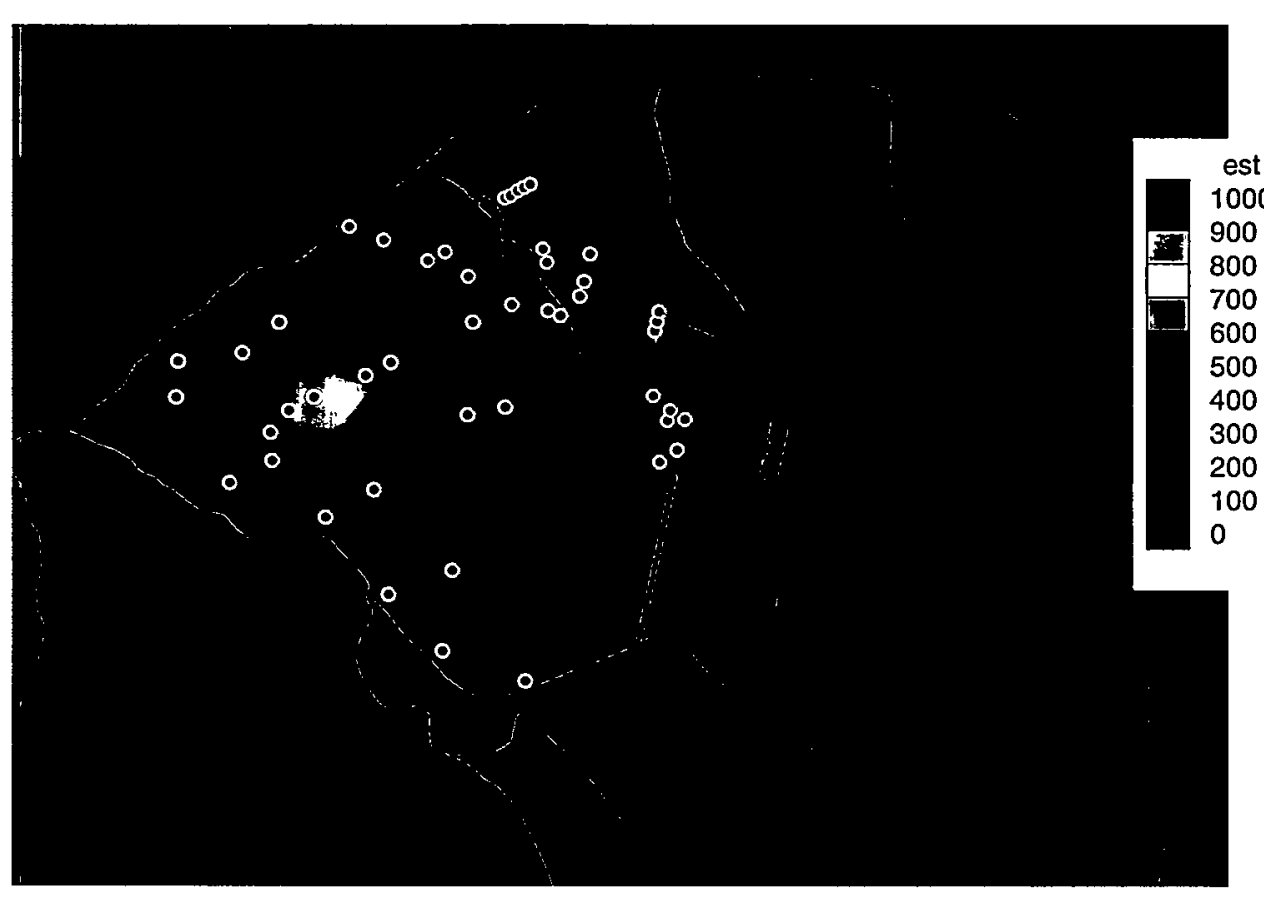

\section{Block Kriging Estimation}

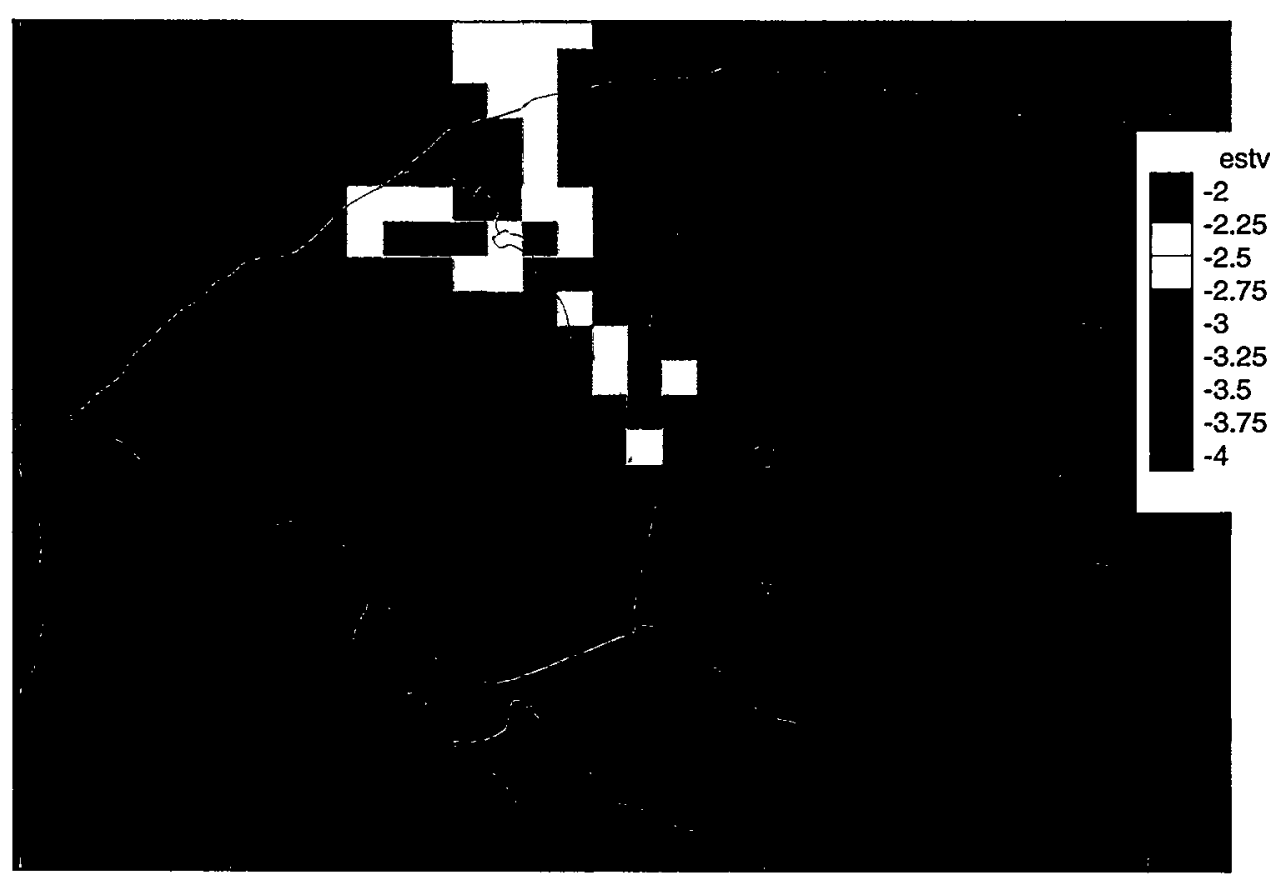

Figure G-15. Kriging estimates for Kv in model layer 2 ( GCU); before TCCZ mods. 


\section{Point Kriging Estimation}

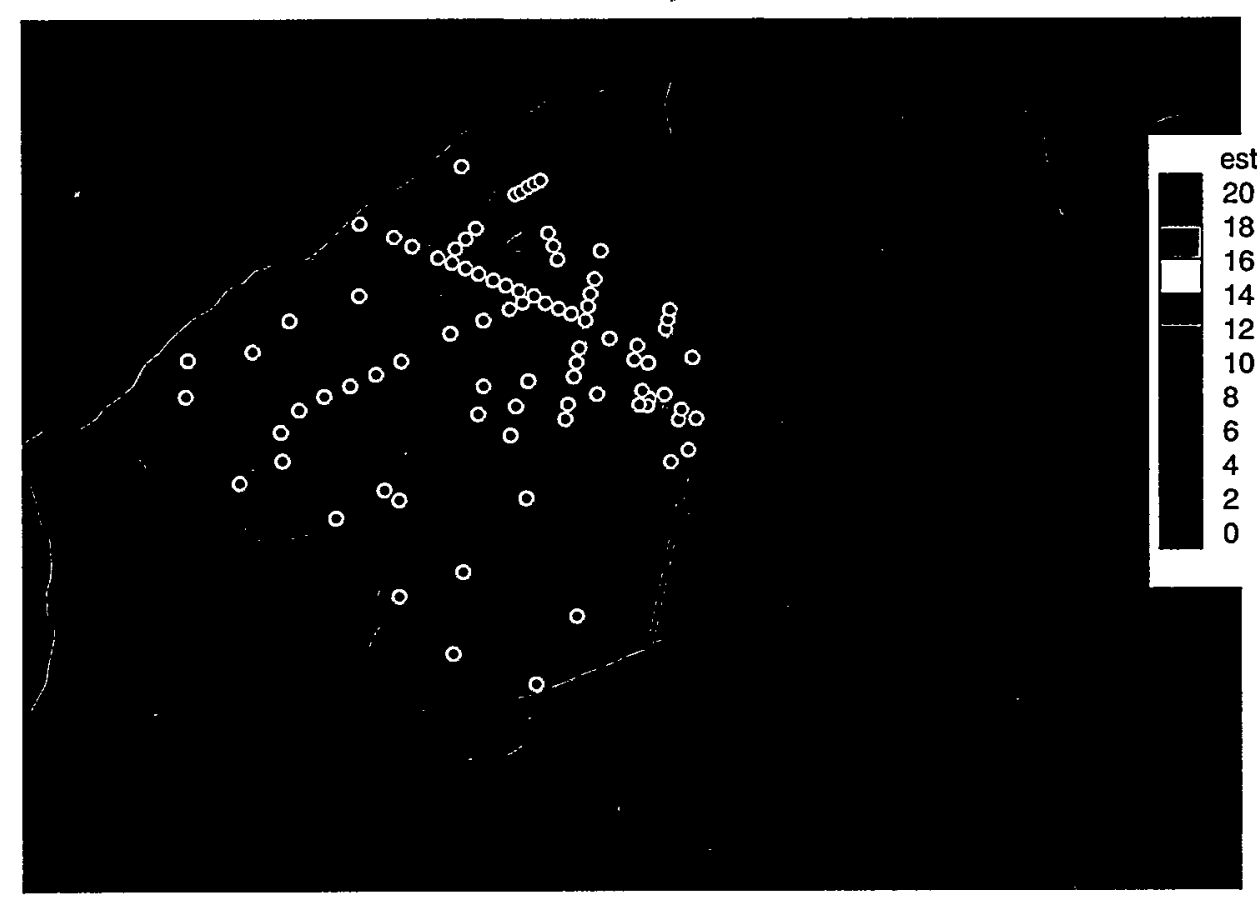

\section{Block Kriging Estimation}

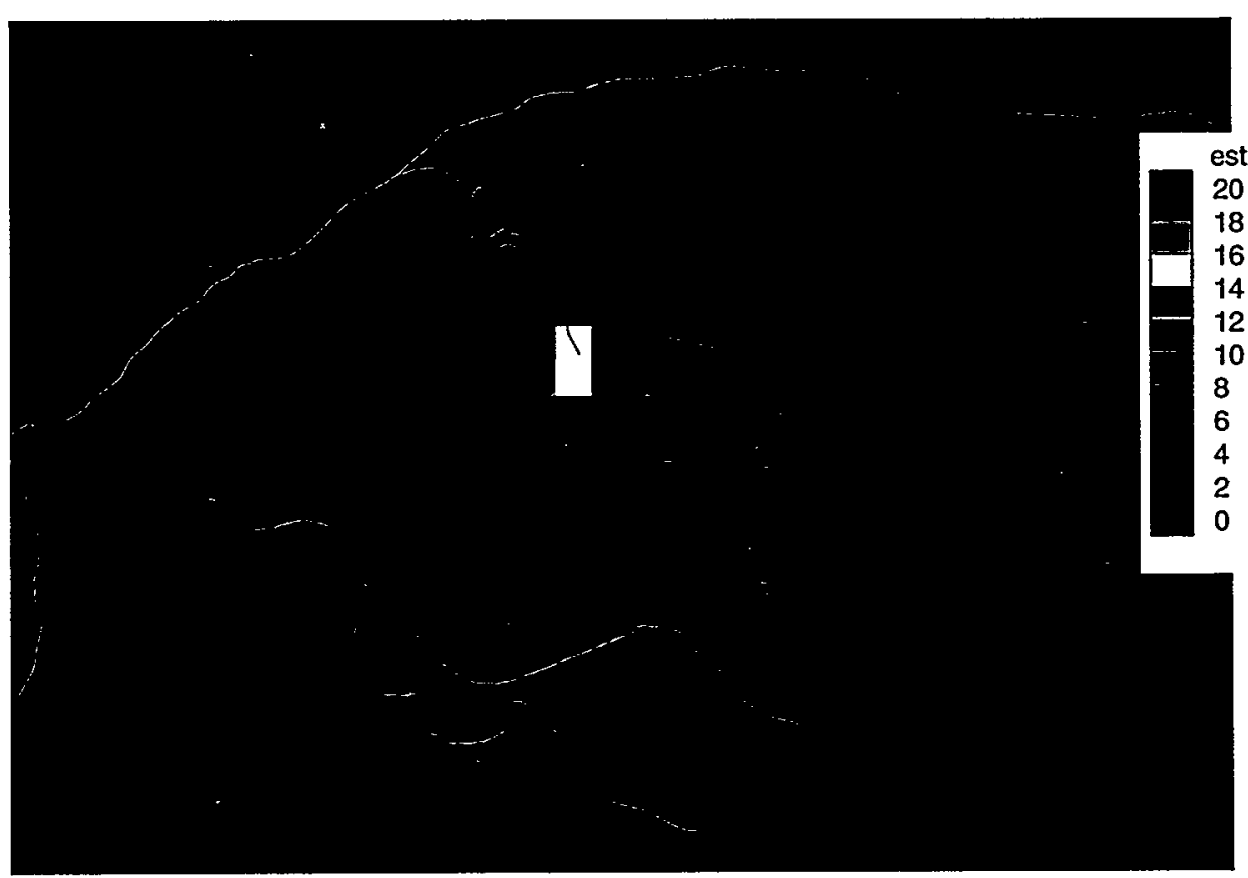

Figure G-16. Kriging estimates for $\mathrm{Kh}$ in model layer 3 ( $\mathrm{lLAZ}$ ); before TCCZ mods. 


\section{Point Kriging Estimation}

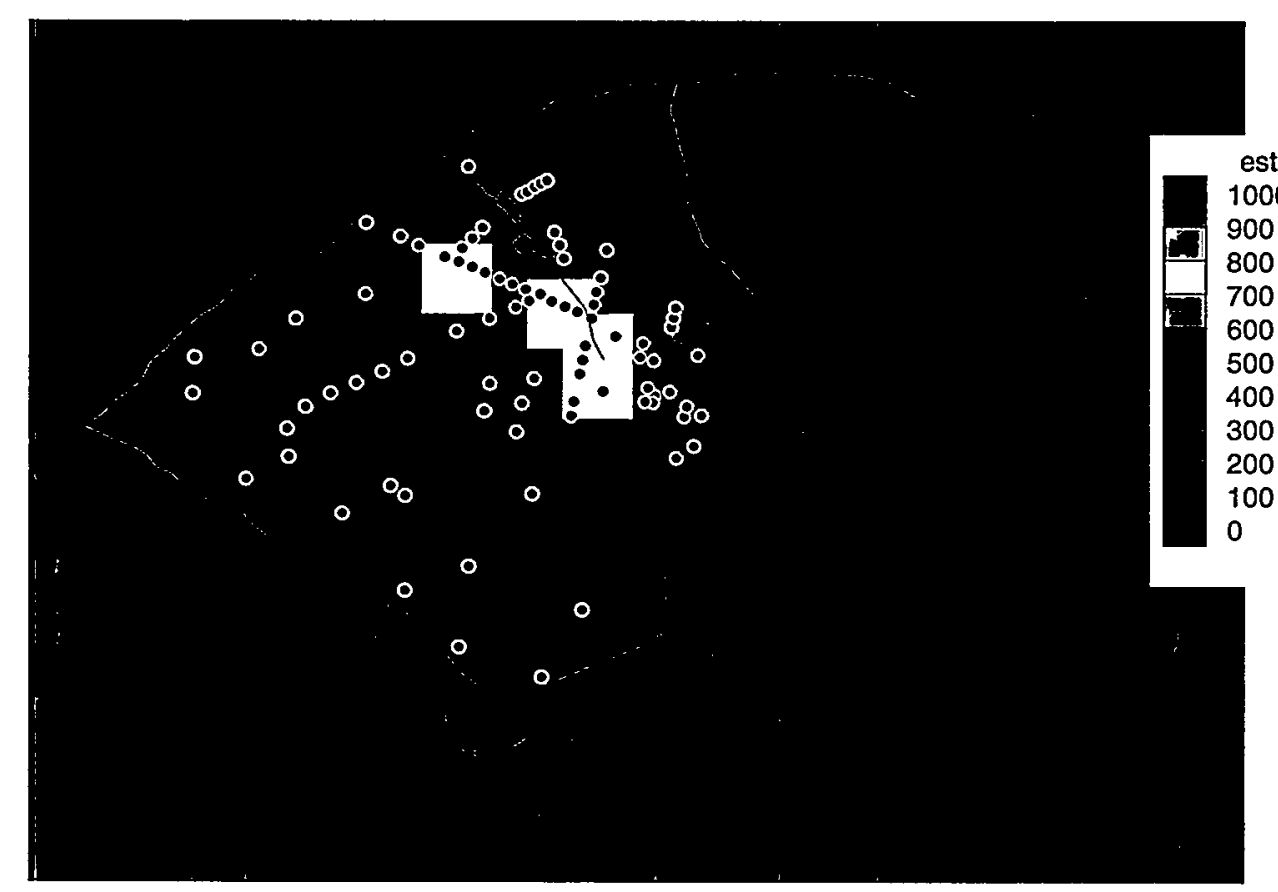

\section{Block Kriging Estimation}

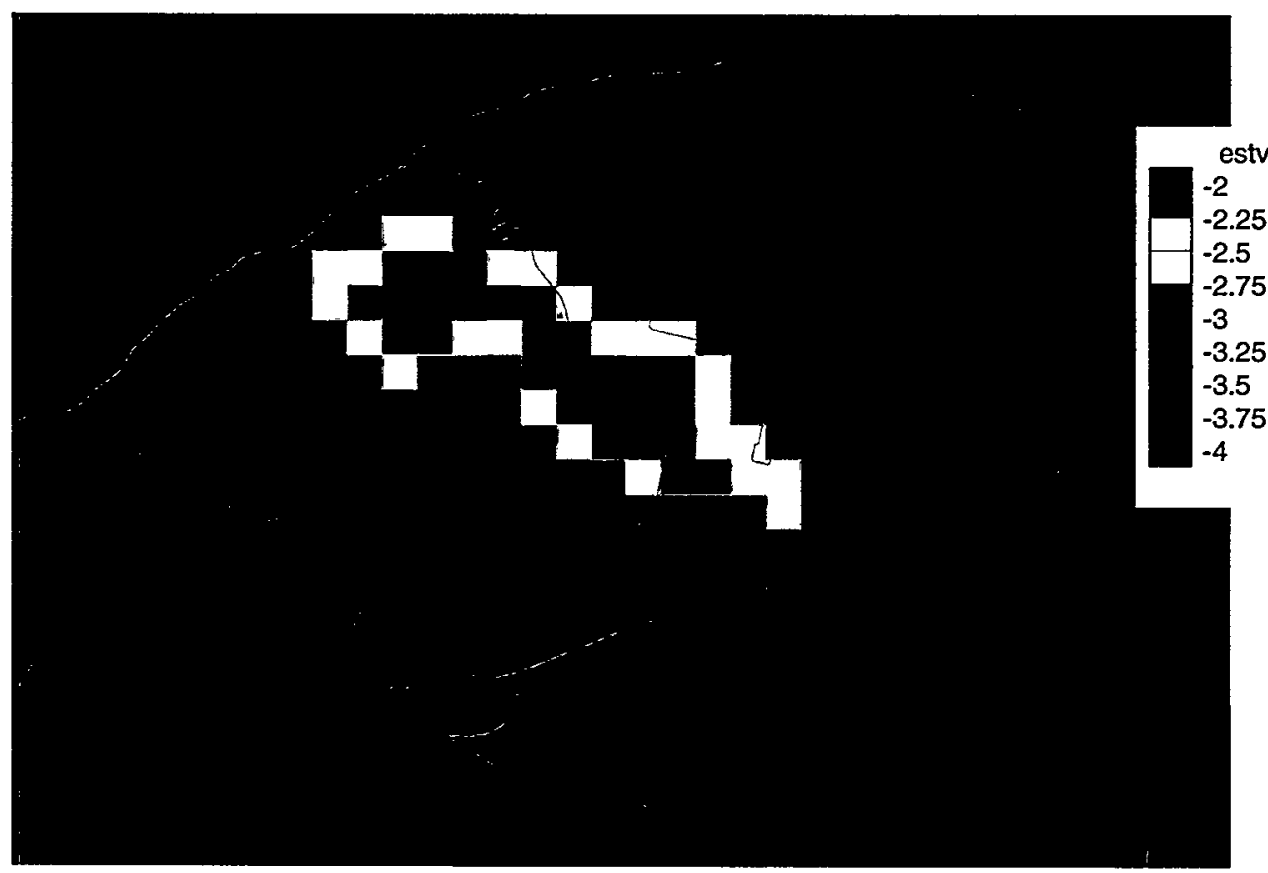

Figure G-17. Kriging estimates for Kv in model layer 3 ( $-\mathrm{LAZ})$; before TCCZ mods. 


\section{Point Kriging Estimation}

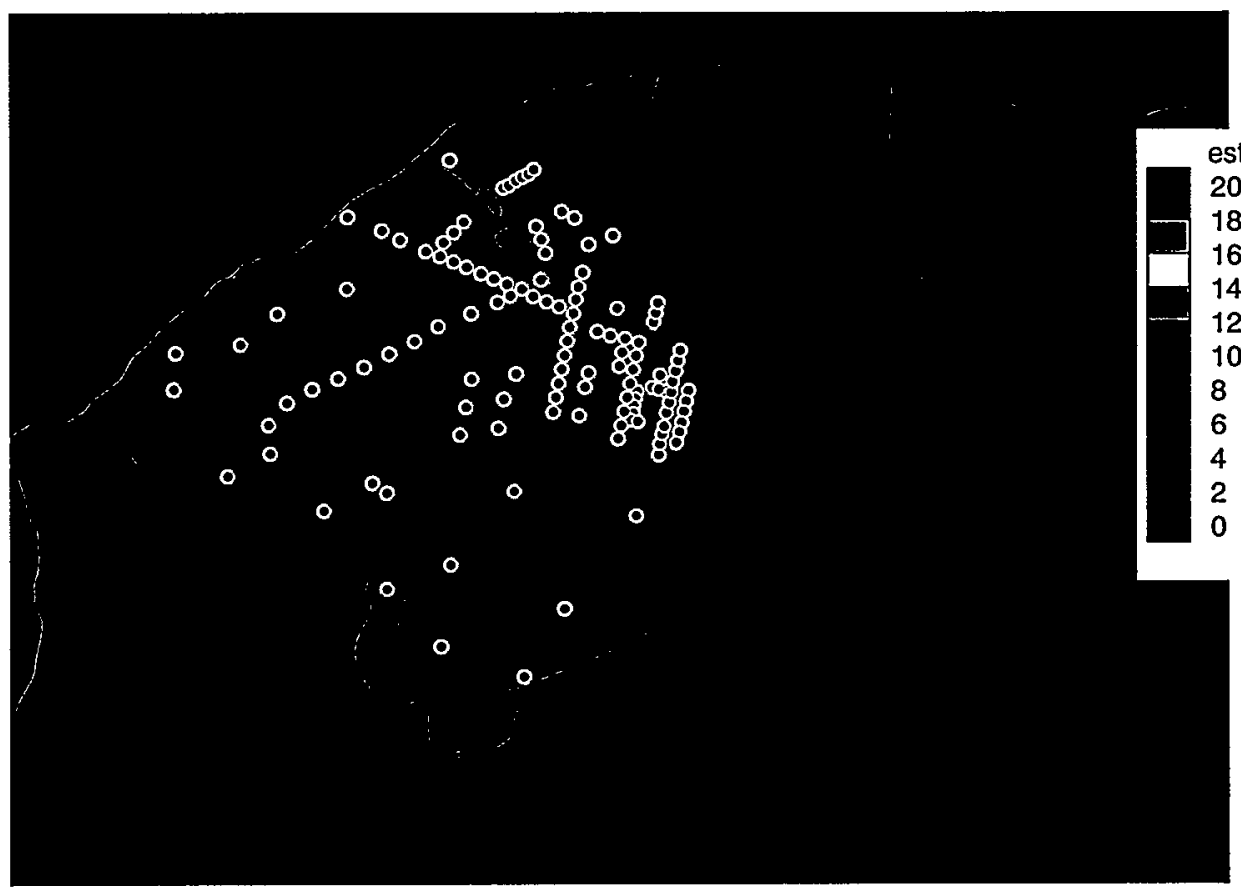

Block Kriging Estimation

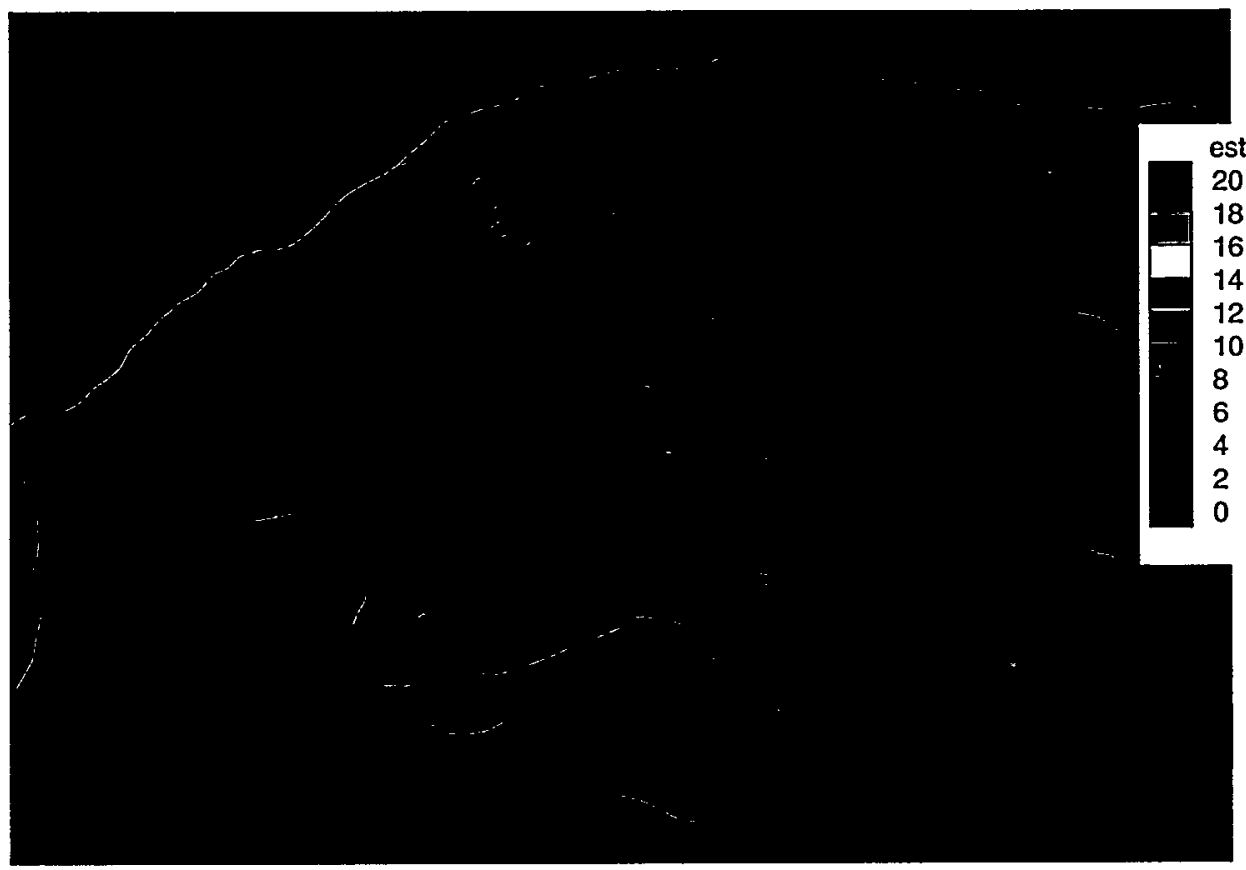

Figure G-18. Kriging estimates for Kh in model layer 4 ( uLAZ); before TCCZ mods. 


\section{Point Kriging Estimation}

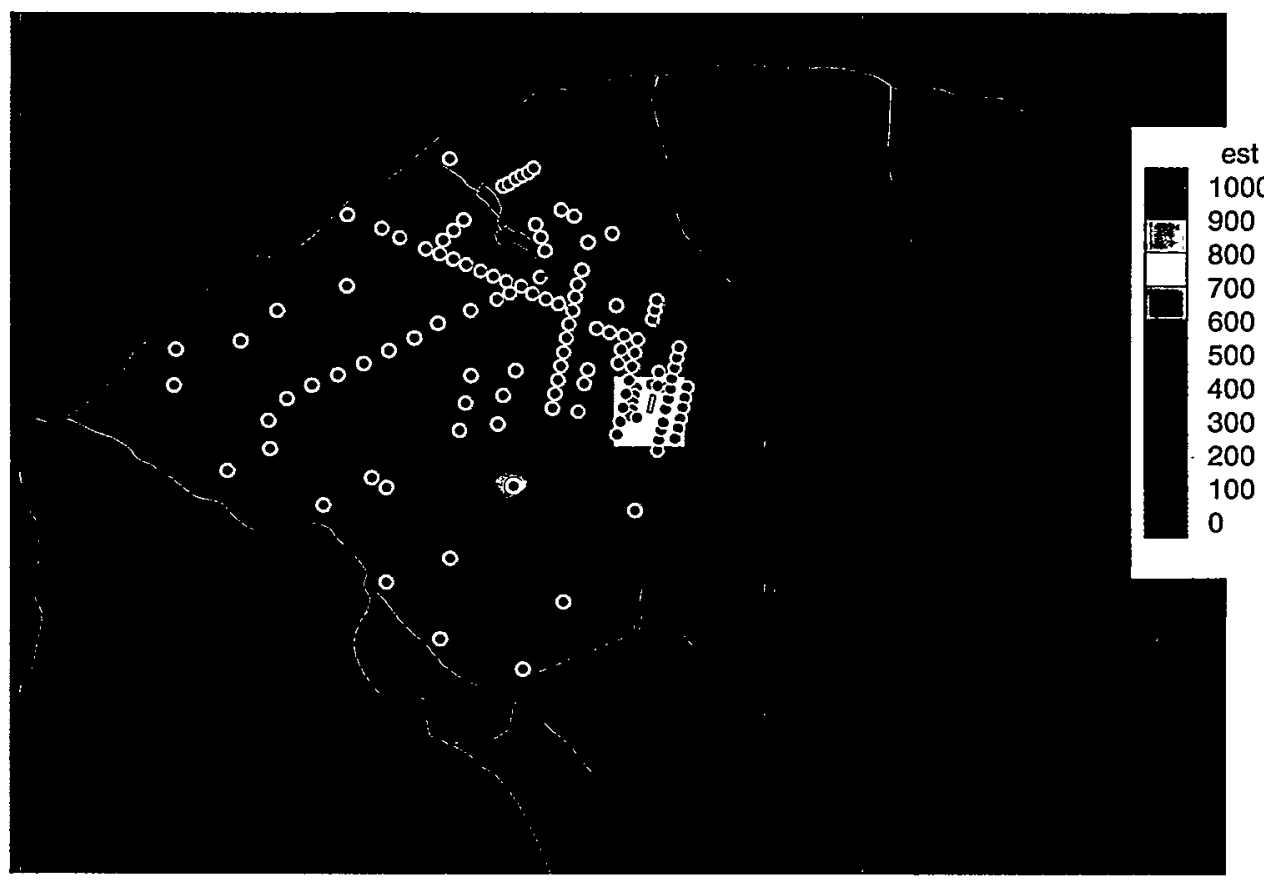

\section{Block Kriging Estimation}

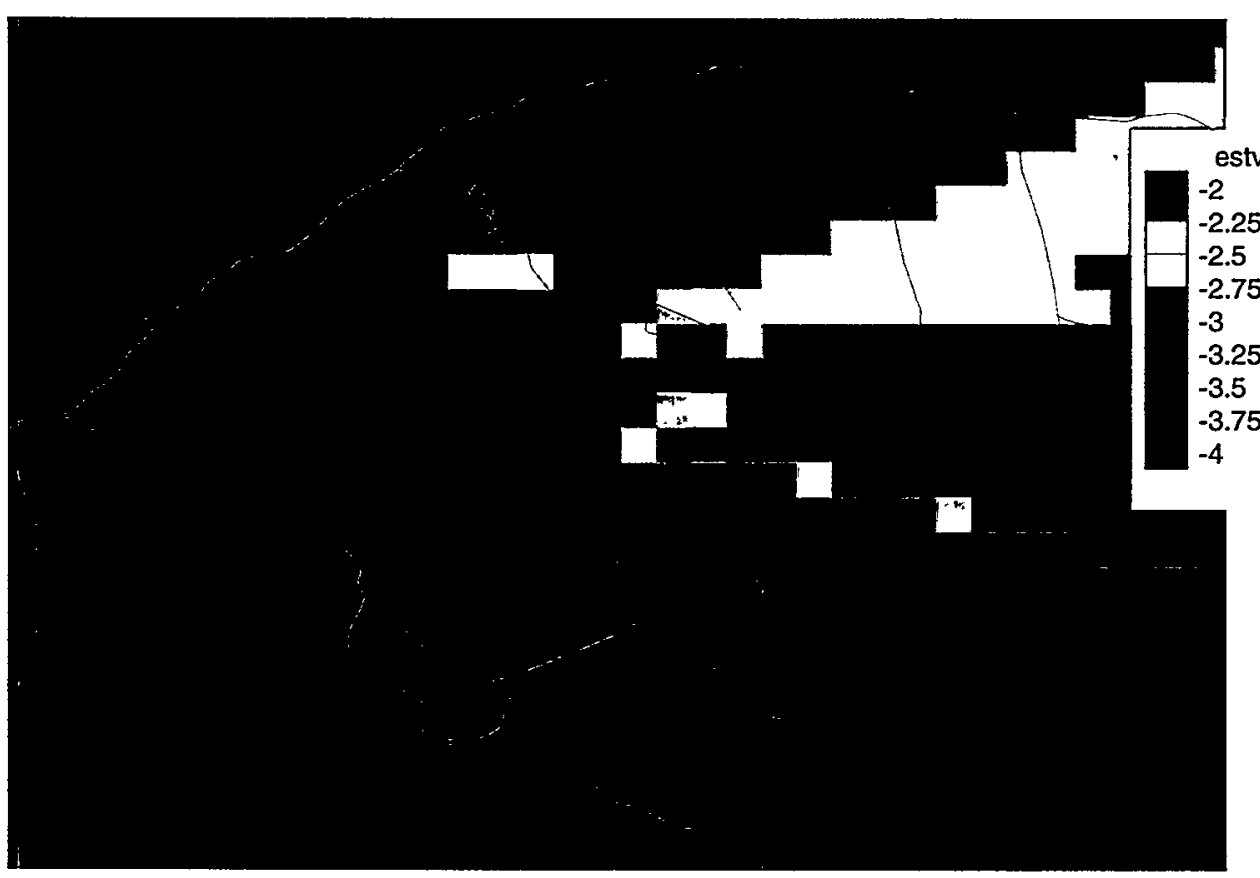

Figure G-19. Kriging estimates for Kv in model layer 4 ( uLAZ); before TCCZ mods. 


\section{Point Kriging Estimation}

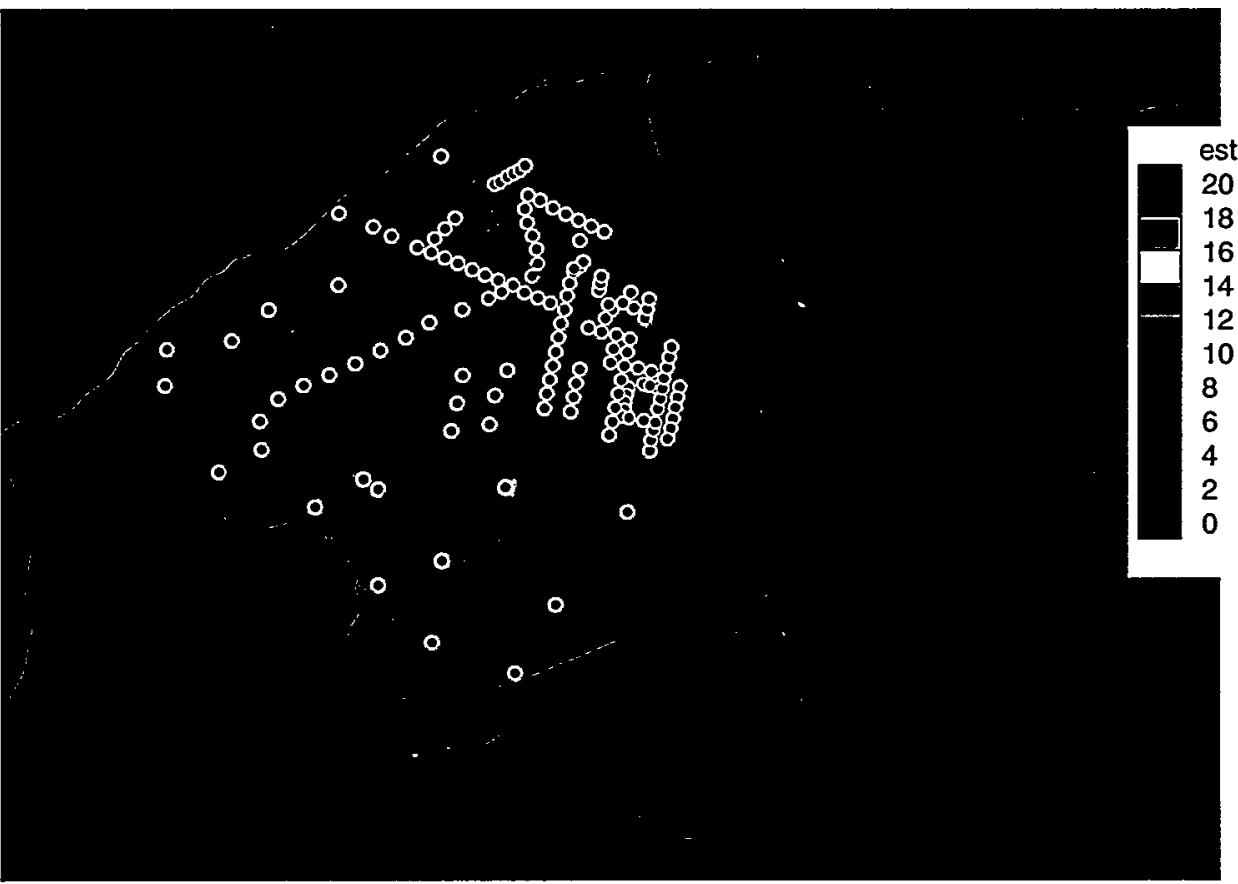

\section{Block Kriging Estimation}

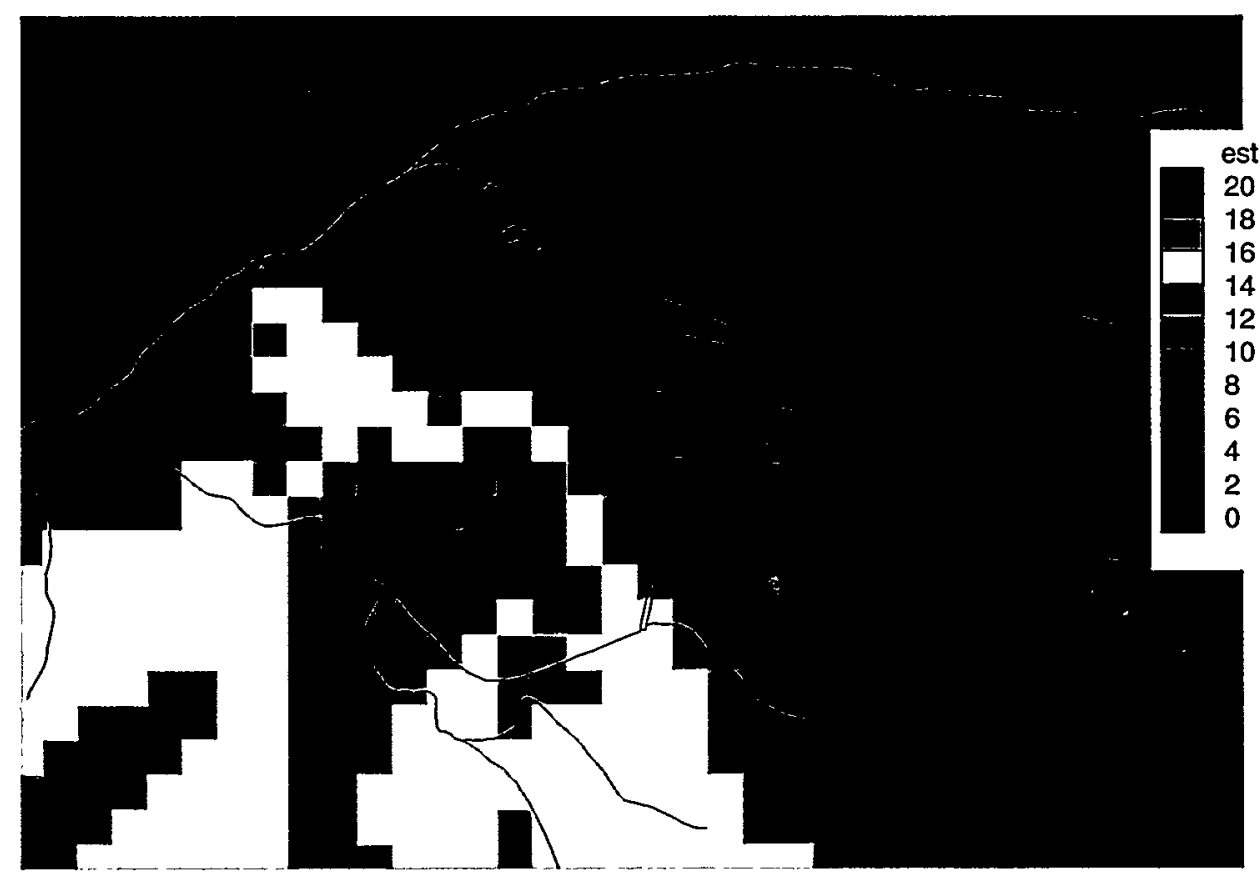

Figure G-20. Kriging estimates for Kh in model layer 5 ( $\sim \mathrm{TCCZ})$; before TCCZ mods. 


\section{Point Kriging Estimation}

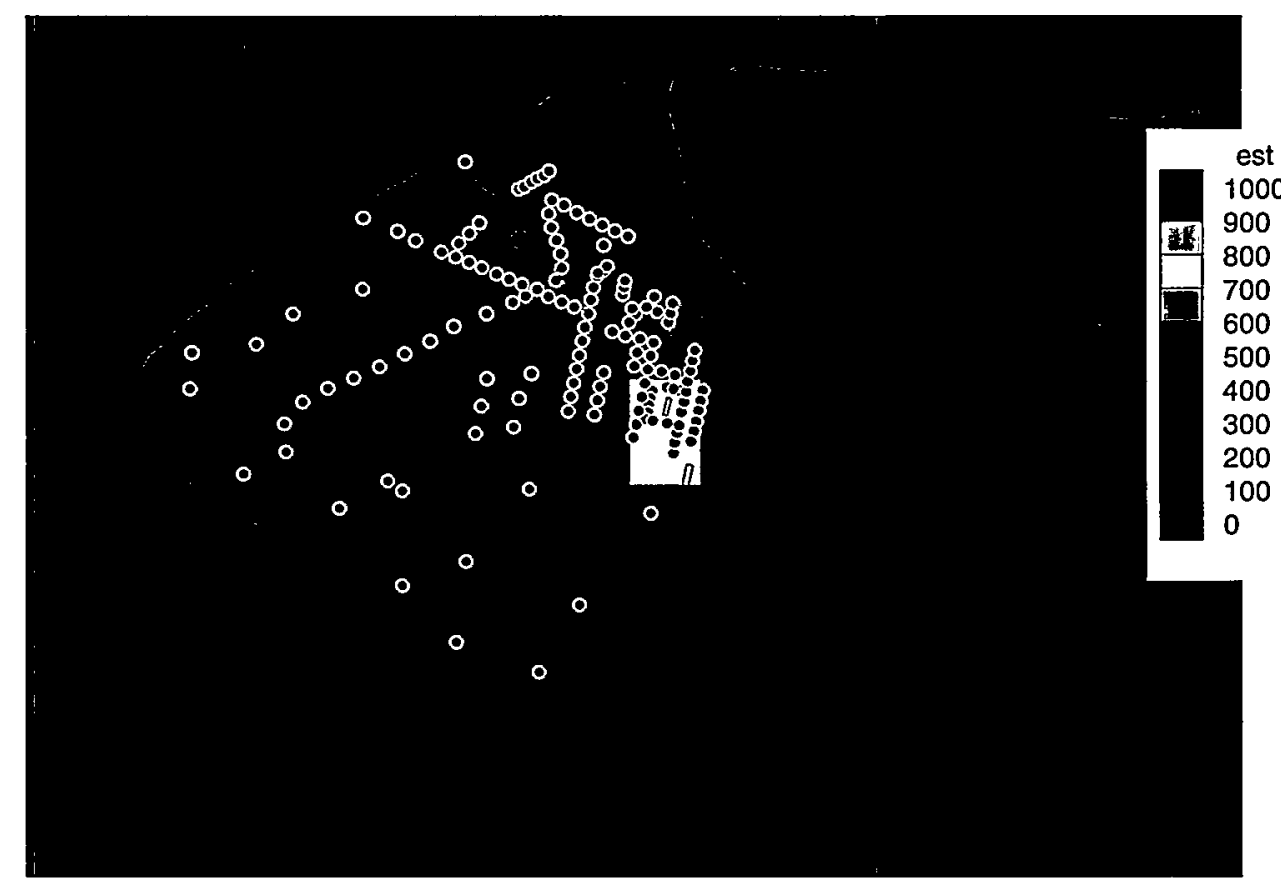

\section{Block Kriging Estimation}

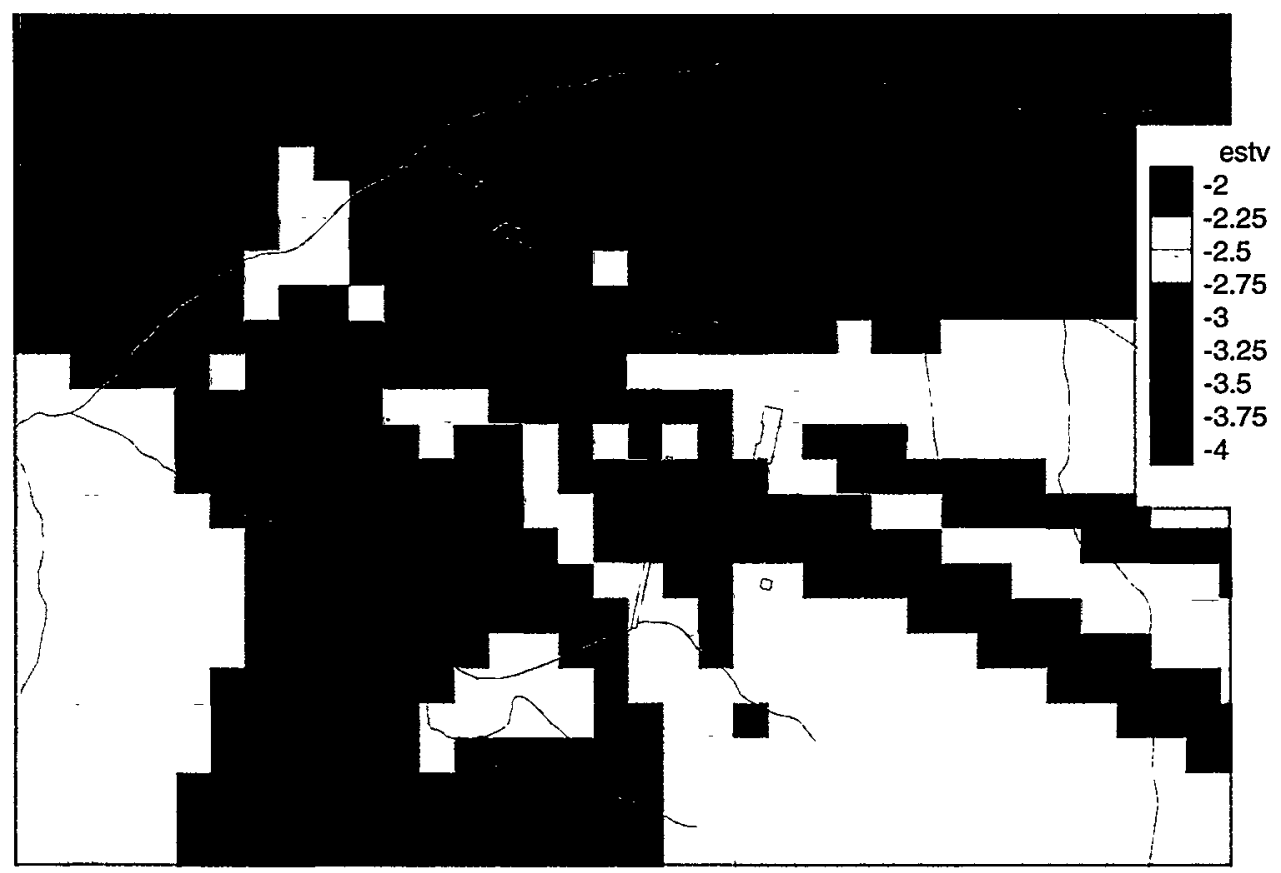

Figure G-21. Kriging estimates for Kv in model layer 5 ( TCCZ); before TCCZ mods. 


\section{Point Kriging Estimation}

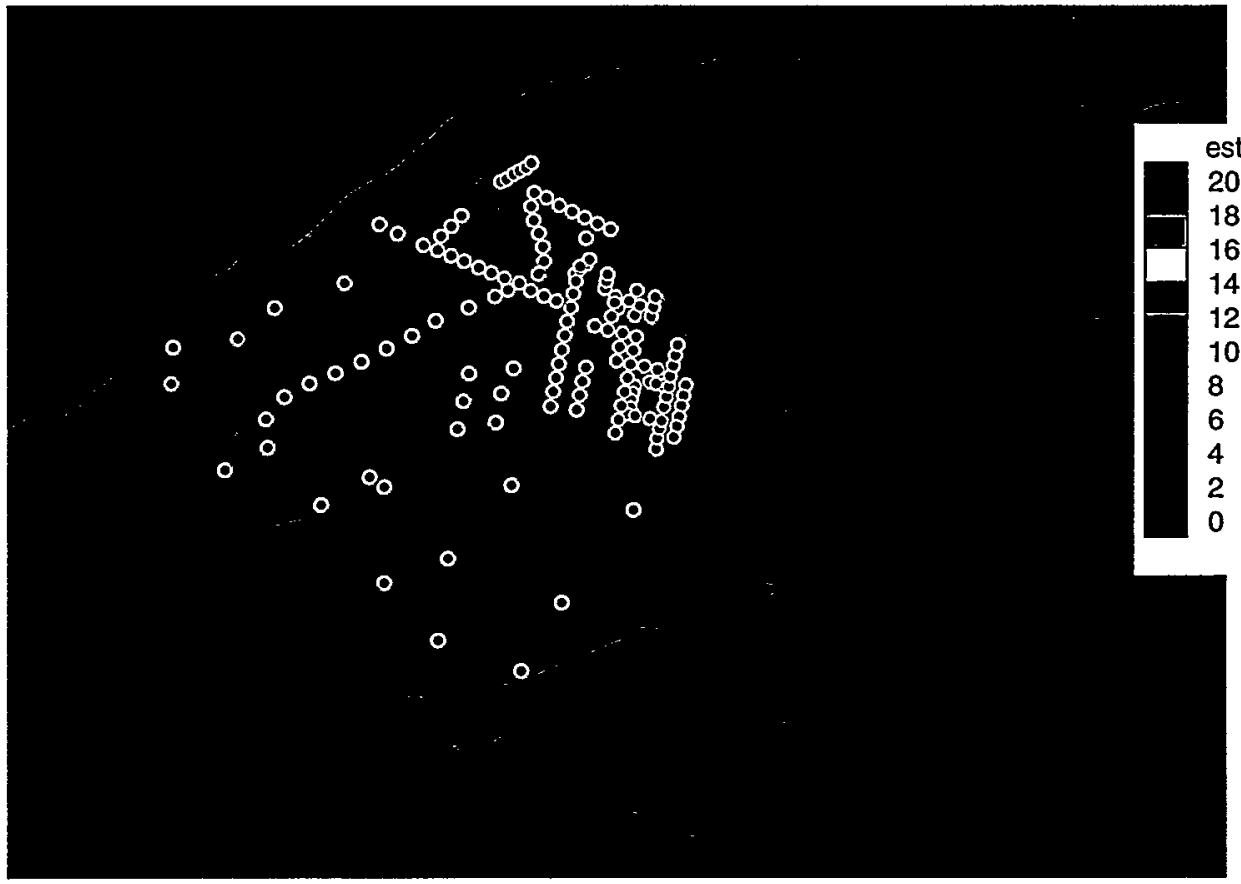

Block Kriging Estimation

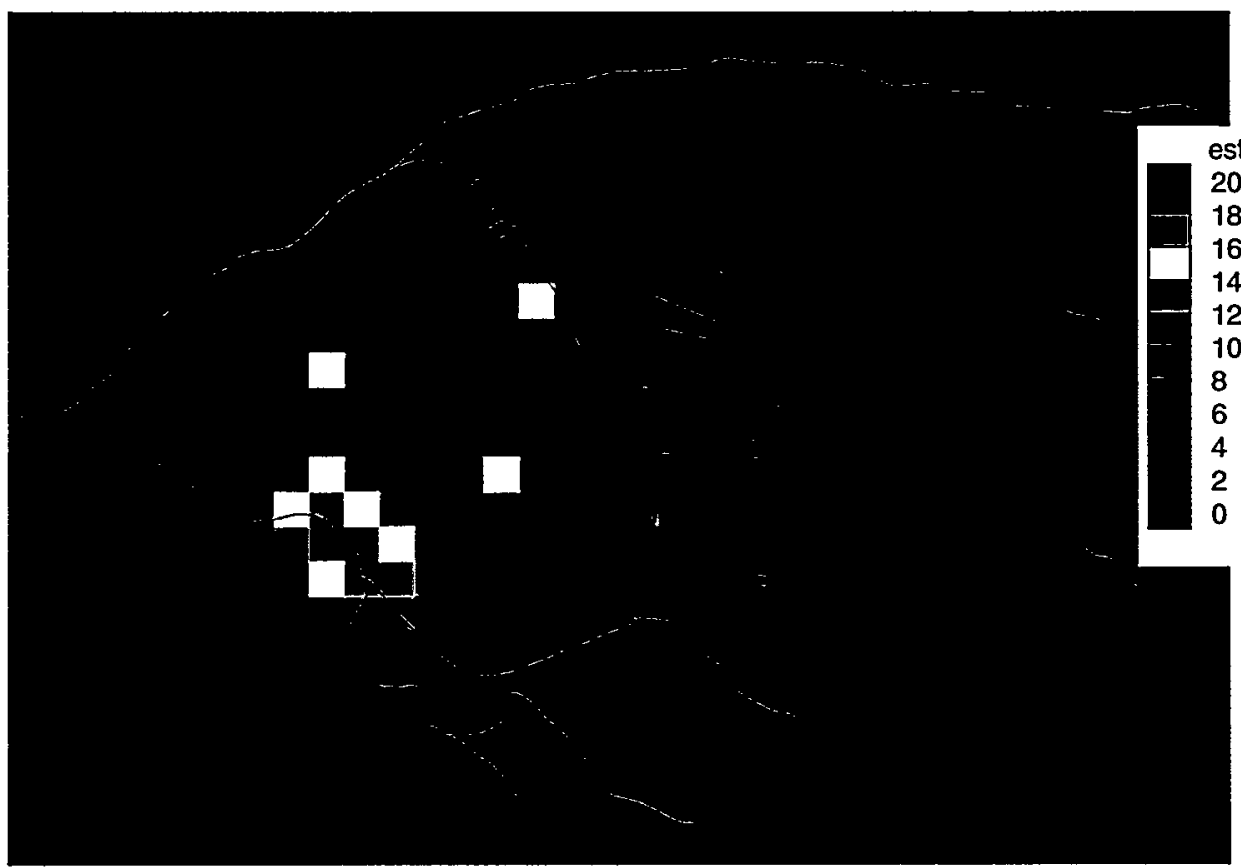

Figure G-22. Kriging estimates for Kh in model layer $6(\sim \mathrm{TZ})$; before TCCZ mods. 


\section{Point Kriging Estimation}

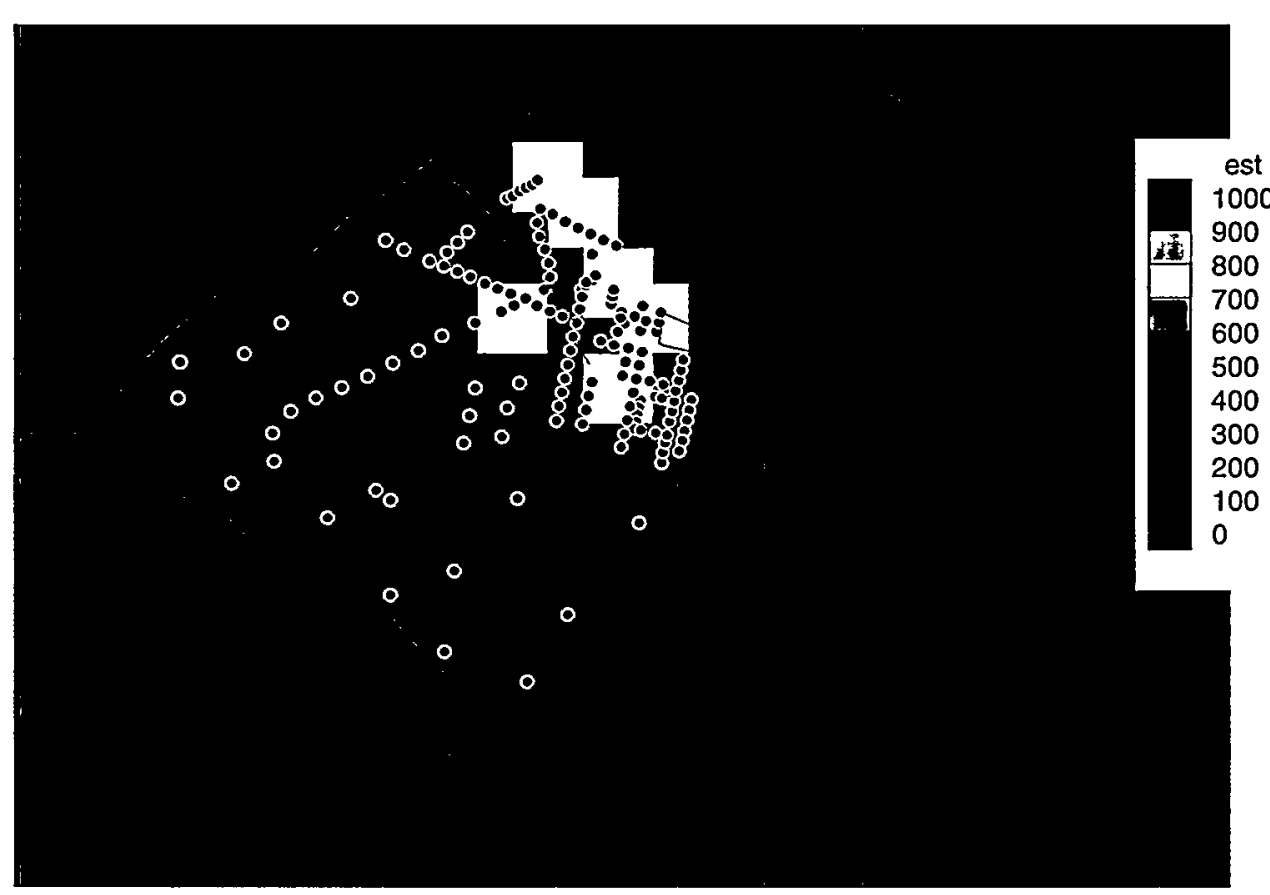

\section{Block Kriging Estimation}

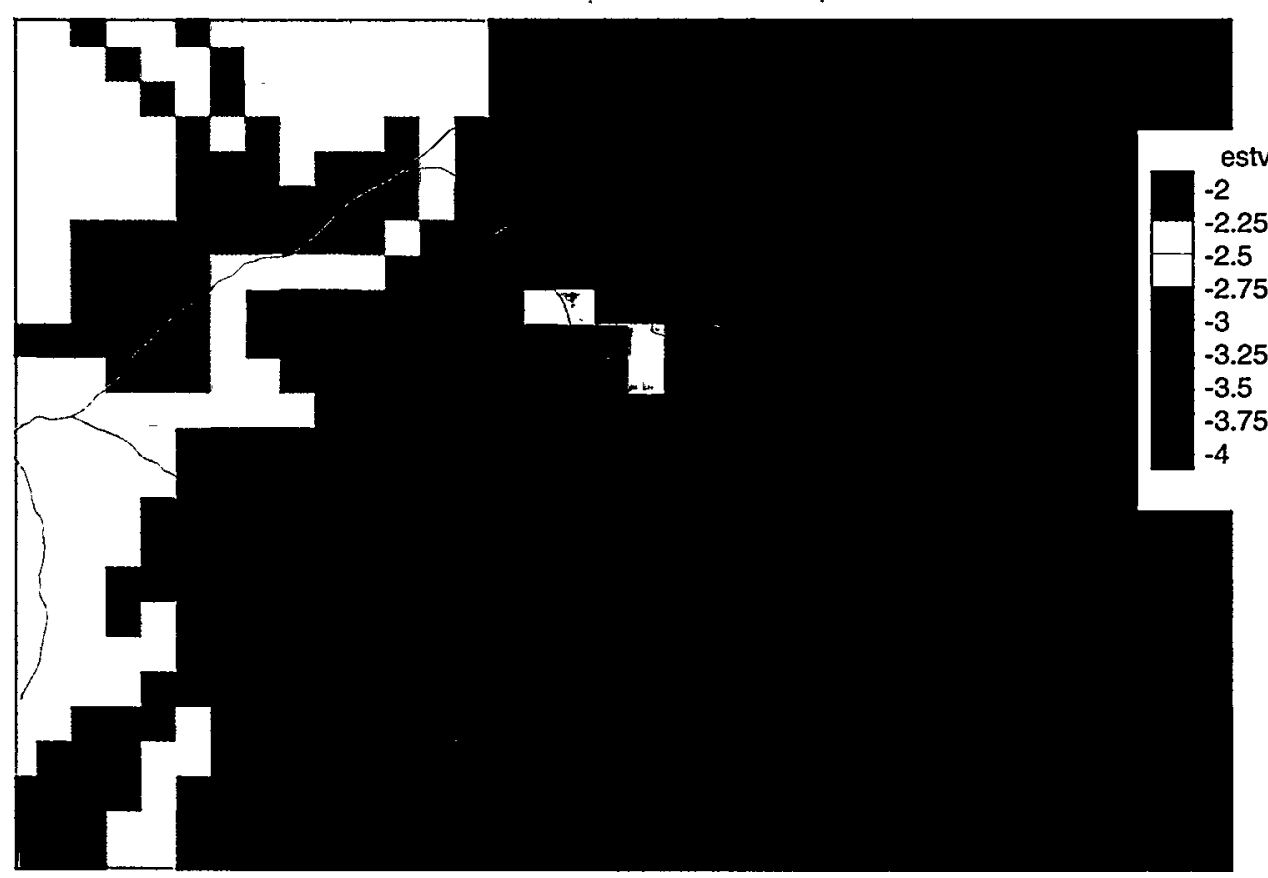

Figure G-23. Kriging estimates for $\mathrm{Kv}$ in model layer 6 ( TZ); before TCCZ mods. 


\section{Point Kriging Estimation}

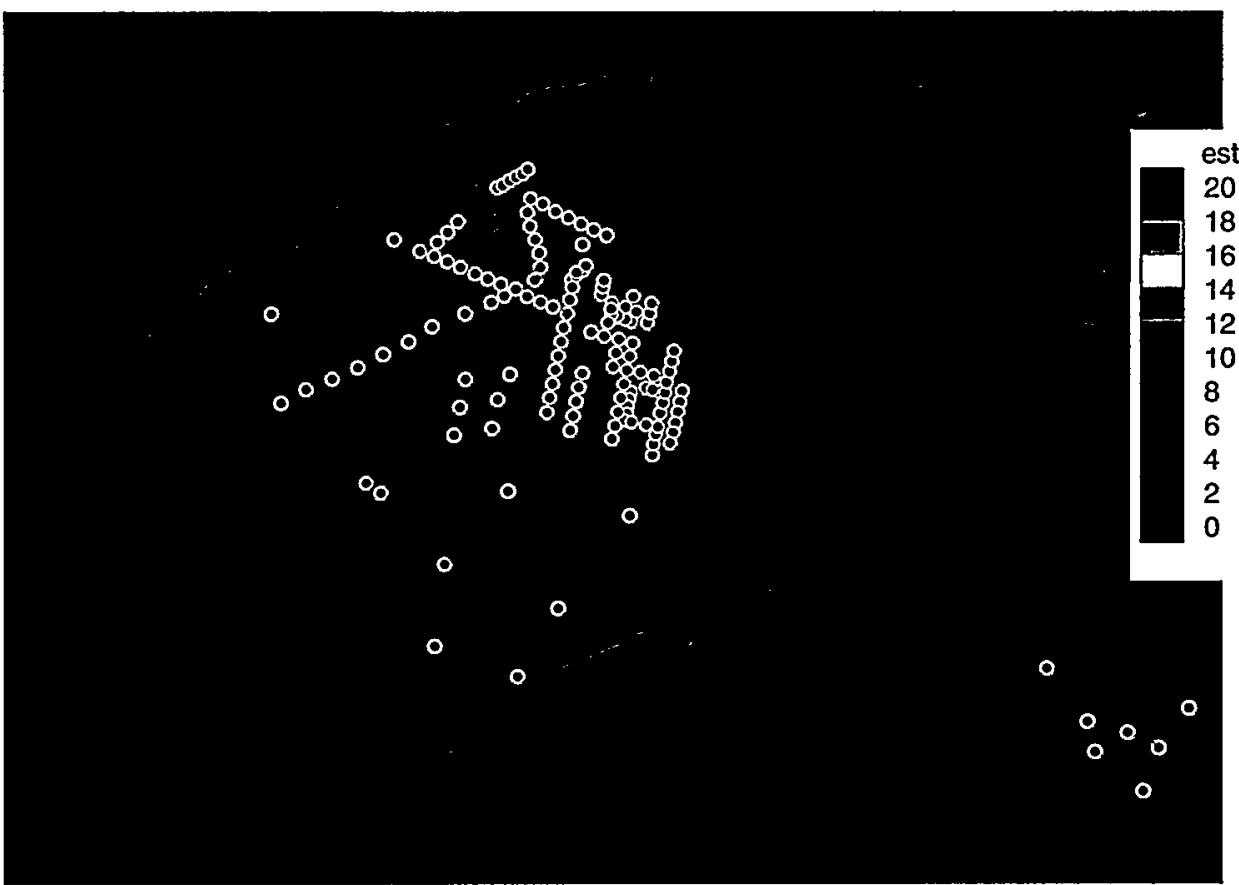

\section{Block Kriging Estimation}

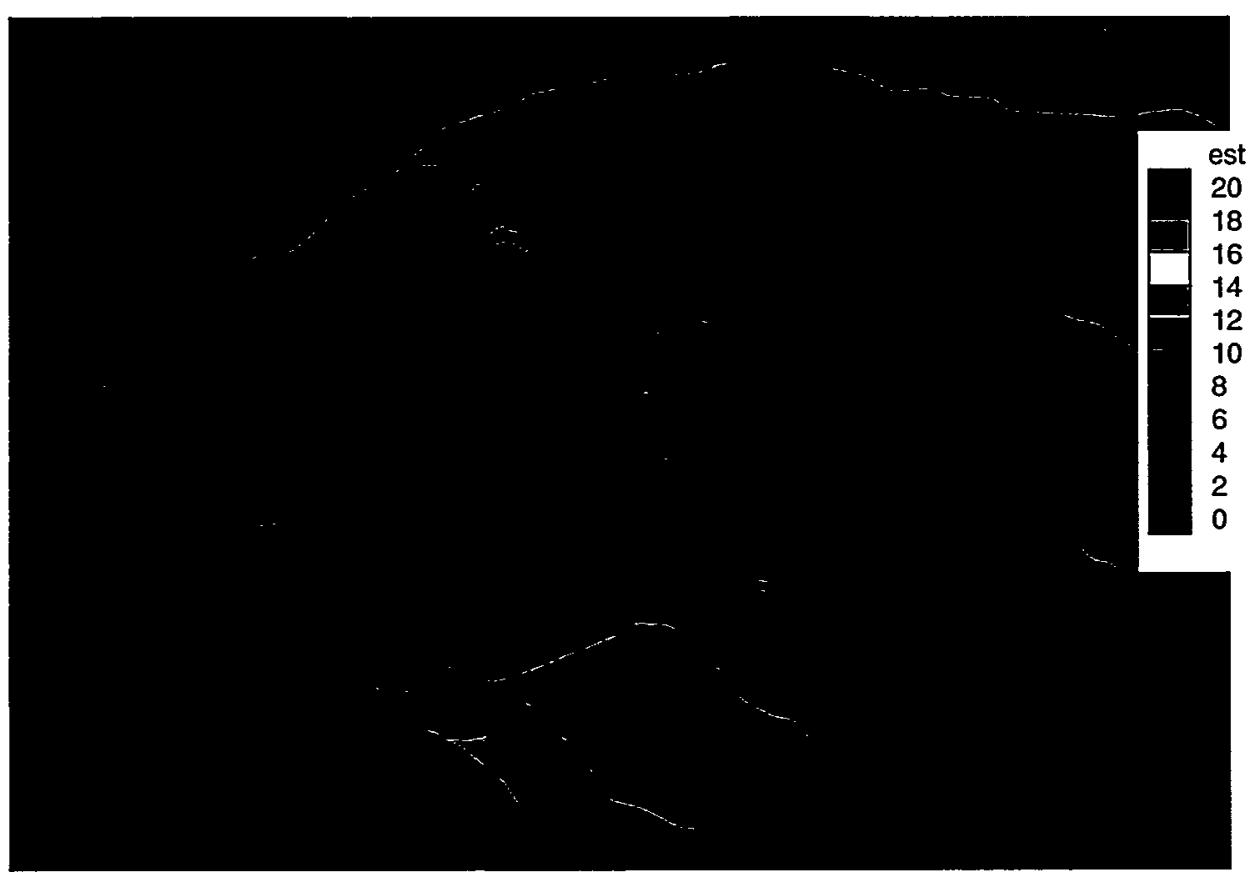

Figure G-24. Kriging estimates for Kh in model layer 7 ( AA); before TCCZ mods. 


\section{Point Kriging Estimation}

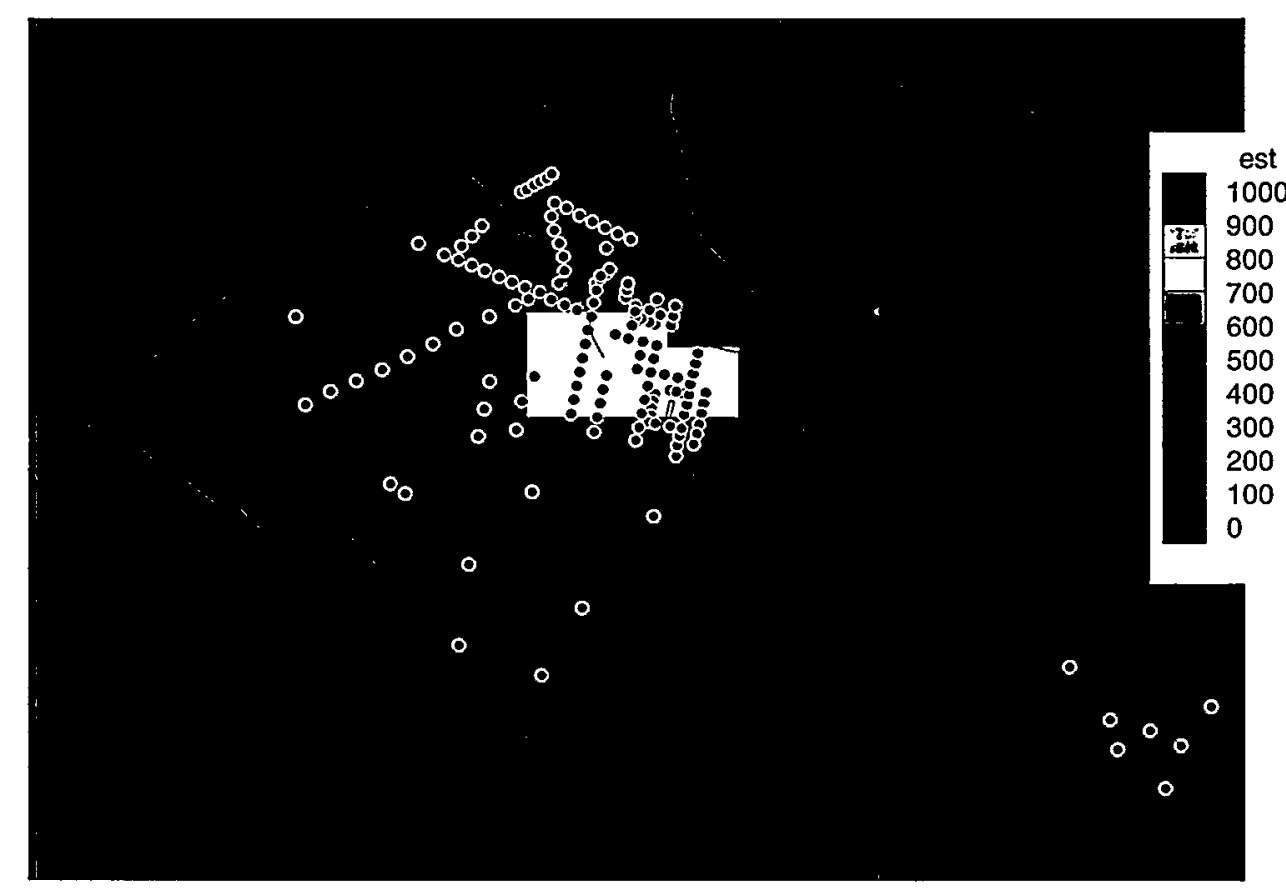

\section{Block Kriging Estimation}

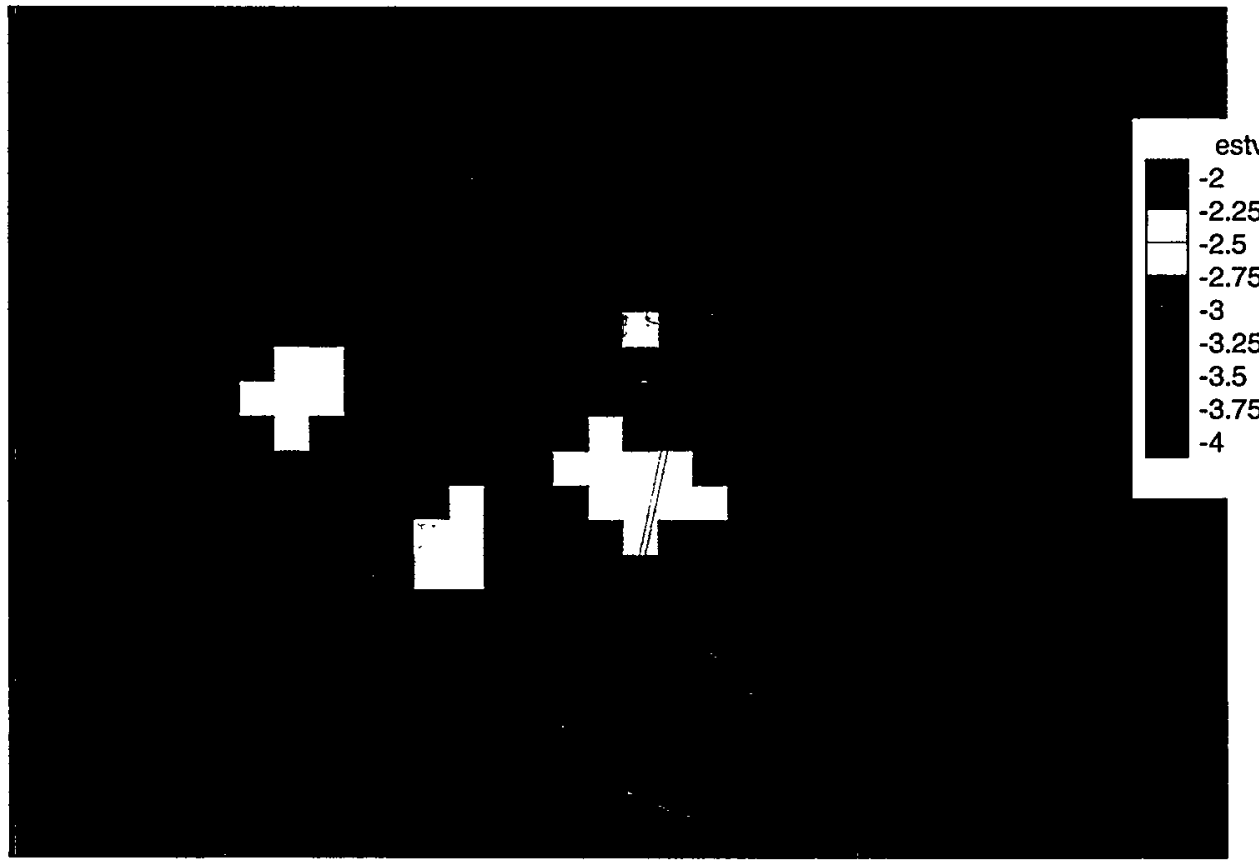

Figure G-25. Kriging estimates for Kv in model layer $7(\sim \mathrm{AA})$; before TCCZ mods. 


\section{Point Kriging Estimation}

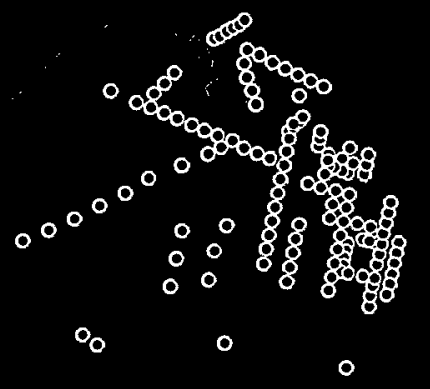

o

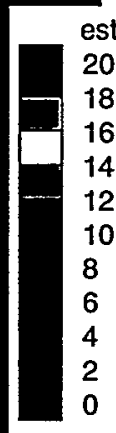

$\circ$

$\circ$ $0_{0}^{\circ} 0$

\section{Block Kriging Estimation}

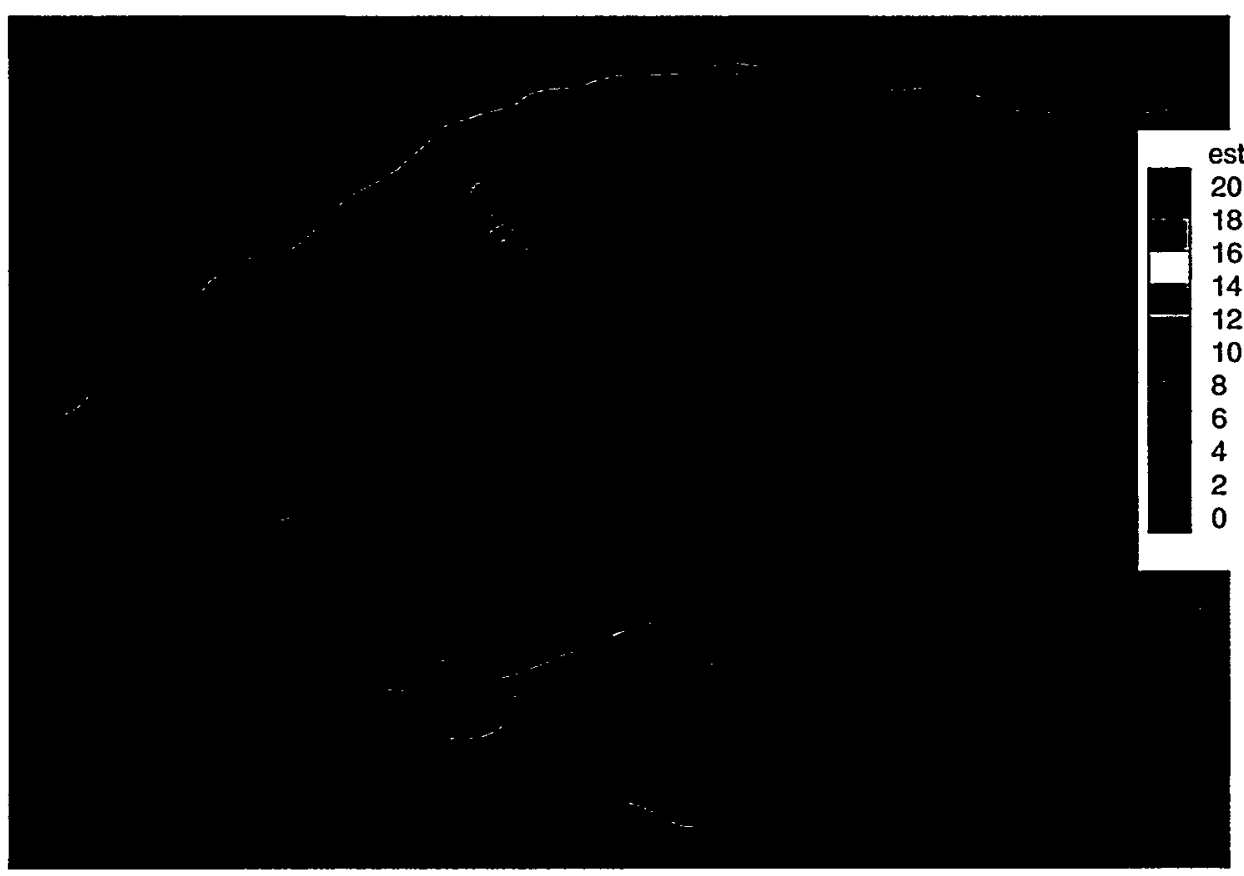

Figure G-26. Kriging estimates for Kh in model layer 8 ( $\sim \mathrm{A} / \mathrm{uu})$; before TCCZ mods. 


\section{Point Kriging Estimation}

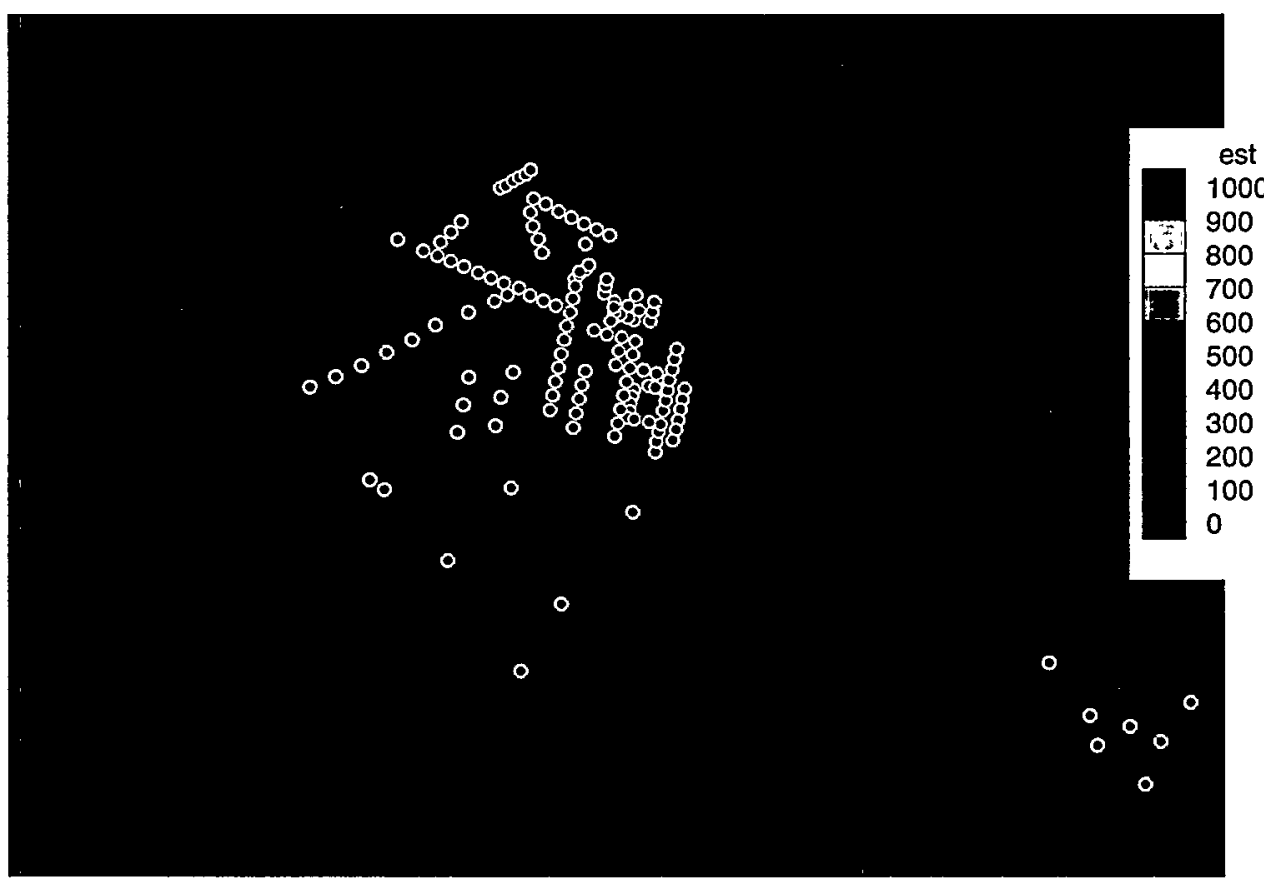

\section{Block Kriging Estimation}

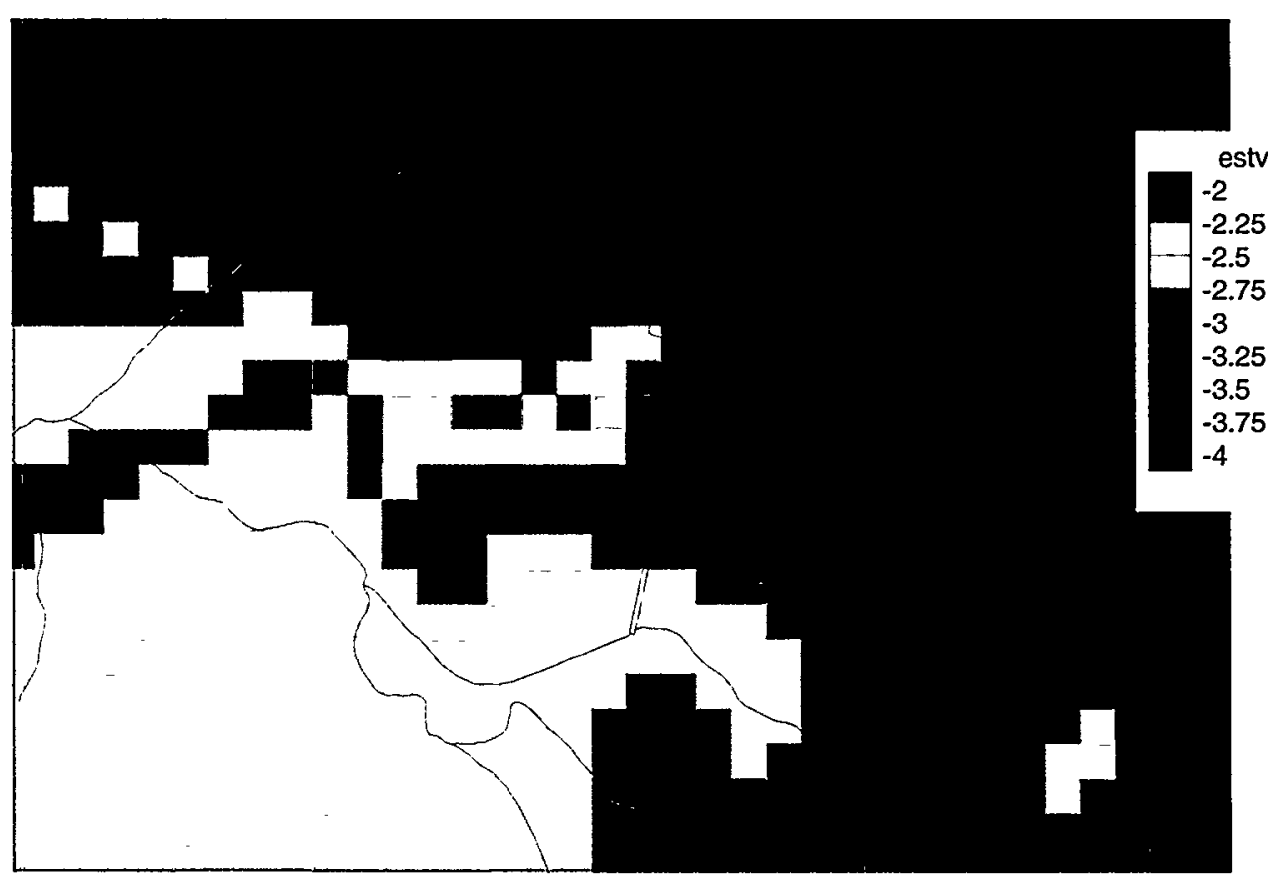

Figure G-27. Kriging estimates for Kv in model layer 8 ( A/uu); before TCCZ mods. 


\section{APPENDIX H. UNCERTAINTY ANALYSIS}


This page intentionally left blank 


\section{APPENDIX H. Uncertainty Analysis}

Four uncertainty cases were considered as summarized in the table below. For each case, numerous plots of the simulated groundwater flow results are provided for comparison to the nominal case. Discussion of the comparison is provided in the Section 4.3 of the main text.

GCU Kv

\begin{tabular}{|c|c|c|c|}
\cline { 2 - 4 } \multicolumn{1}{c}{ Recharge } & $5 \times 10^{-4} \mathrm{ft} / \mathrm{day}$ & $10^{-4} \mathrm{ft} /$ day & $2 \times 10^{-5} \mathrm{ft} / \mathrm{day}$ \\
\hline $15 \mathrm{in} / \mathrm{yr}$ & - & Case 1 & - \\
\hline $12.5 \mathrm{in} / \mathrm{yr}$ & Case 3 & Nominal & Case 4 \\
\hline $10 \mathrm{in} / \mathrm{yr}$ & - & Case 2 & - \\
\hline
\end{tabular}


This page intentionally left blank 


\section{Simulation results for uncertainty case 1}

Uncertainty case 1 involves an increase in recharge of $20 \%$ to $15 \mathrm{in} / \mathrm{yr}$ (Table 4-4). Summary calibration results are provided in Table 4-5. This appendix presents detailed simulation results for uncertainty case 1 for comparison to the nominal results shown in figures in the main text. The correspondence between figures for the nominal and uncertainty case 1 is as follows:

\begin{tabular}{|l|l|l|}
\hline Plot type & Nominal case & Uncertainty case 1 \\
\hline Head residual summary & Figure 4-1 & Figure H-1-1 \\
\hline Head residuals in Gordon aquifer & Figure 4-2 & Figure H-1-2 \\
\hline Head residuals in "lower" UTRA & Figure 4-3 & Figure H-1-3 \\
\hline Head residuals in transmissive zone & Figure 4-4 & Figure H-1-4 \\
\hline Head residuals in AA horizon & Figure 4-5 & Figure H-1-5 \\
\hline Head residuals in A/uu horizons & Figure 4-6 & Figure H-1-6 \\
\hline Kh in element layer 1 & Figure 4-7 & Figure H-1-7 \\
\hline Kv in element layer 2 & Figure 4-8 & Figure H-1-8 \\
\hline Kh in element layer 3 & Figure 4-9 & Figure H-1-9 \\
\hline Kh in element layer 4 & Figure 4-10 & Figure H-1-10 \\
\hline Kv in element layer 5 & Figure 4-11 & Figure H-1-11 \\
\hline Kh in element layer 6 & Figure 4-12 & Figure H-1-12 \\
\hline Kh in element layer 7 & Figure 4-13 & Figure H-1-13 \\
\hline Kh in element layer 8 & Figure 4-14 & Figure H-1-14 \\
\hline Gordon aquifer head & Figure 4-16 & Figure H-1-15 \\
\hline "Lower" UTRA head & Figure 4-17 & Figure H-1-16 \\
\hline "Upper" UTRA head & Figure 4-18 & Figure H-1-17 \\
\hline Head in aquifer containing water table & Figure 4-19 & Figure H-1-18 \\
\hline Water table & Figure 4-20 & Figure H-1-19 \\
\hline Recharge/discharge & Figure 4-25 & Figure H-1-20 \\
\hline Example particle tracing & Figure 4-26 & Figure H-1-21 \\
\hline
\end{tabular}



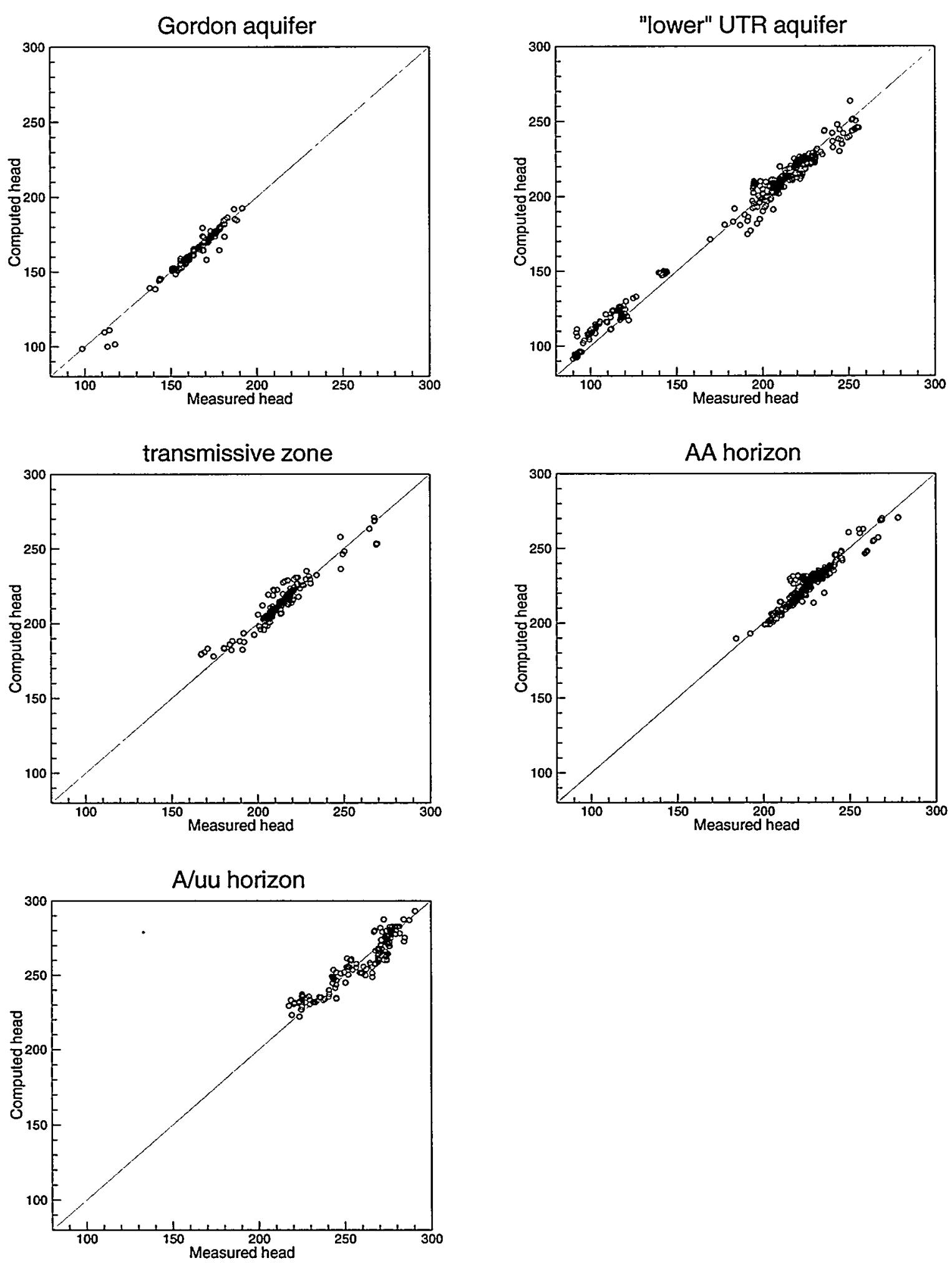

Figure H-1-1. (uncertainty case 1; compare to Figure 4-1) 


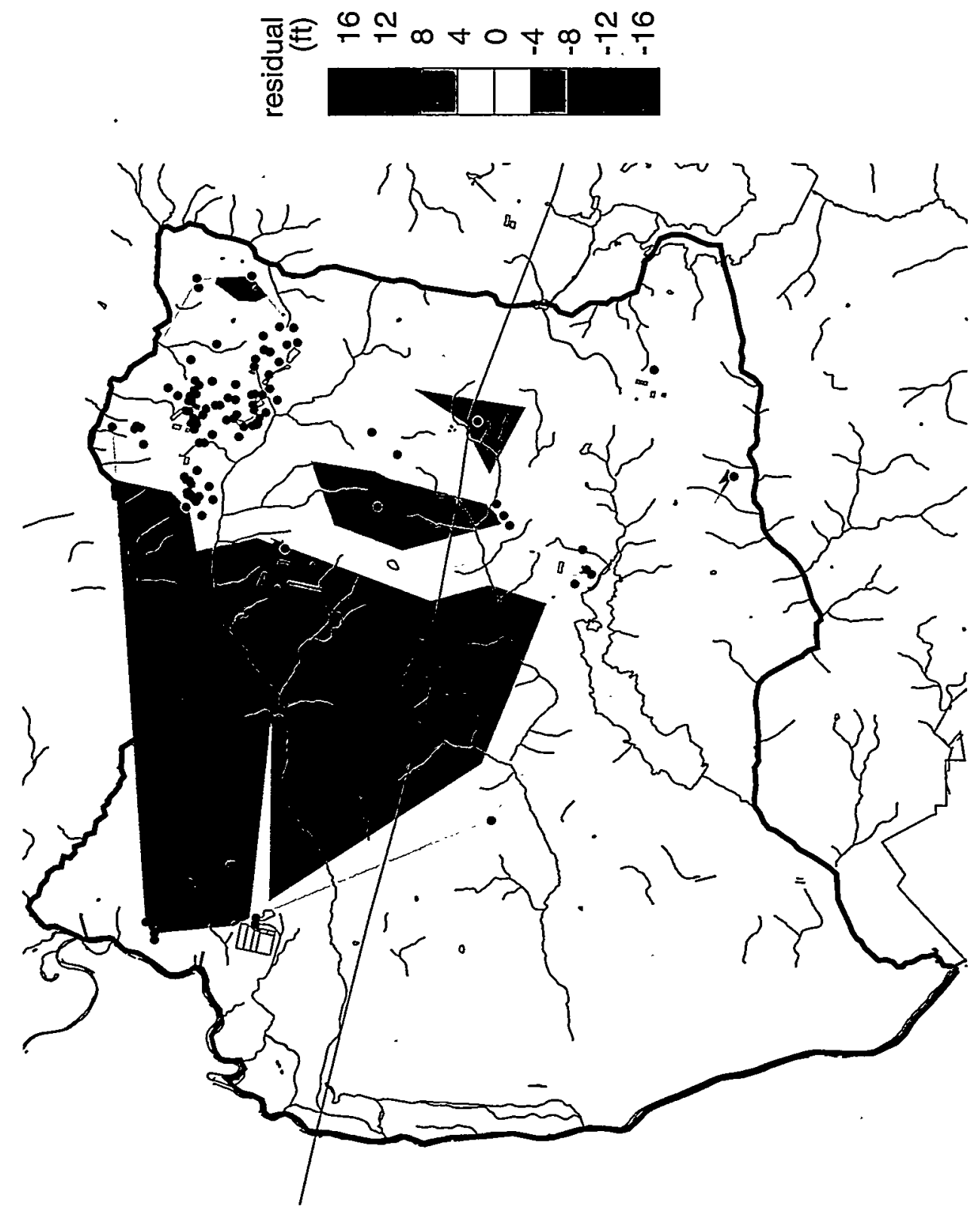




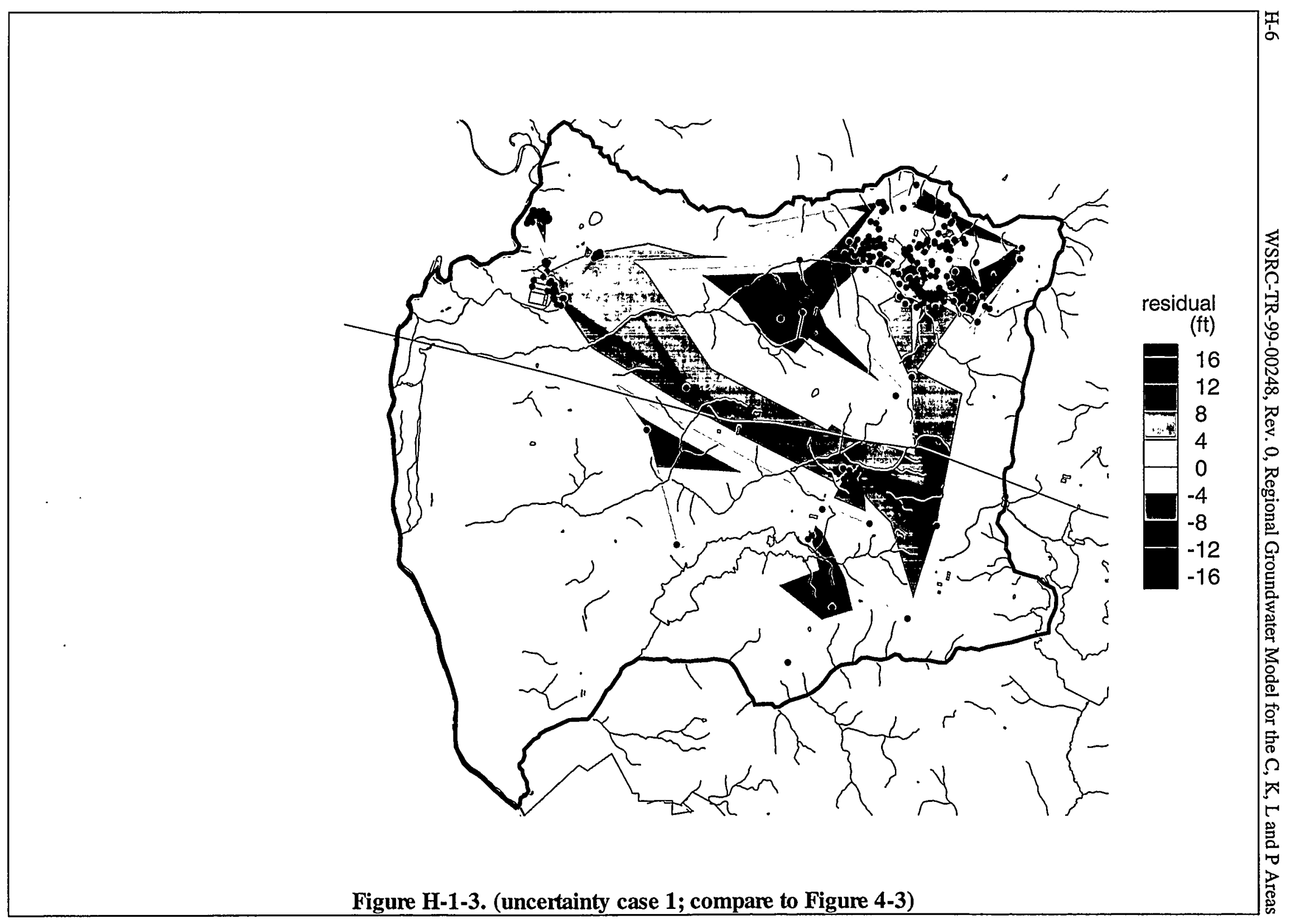




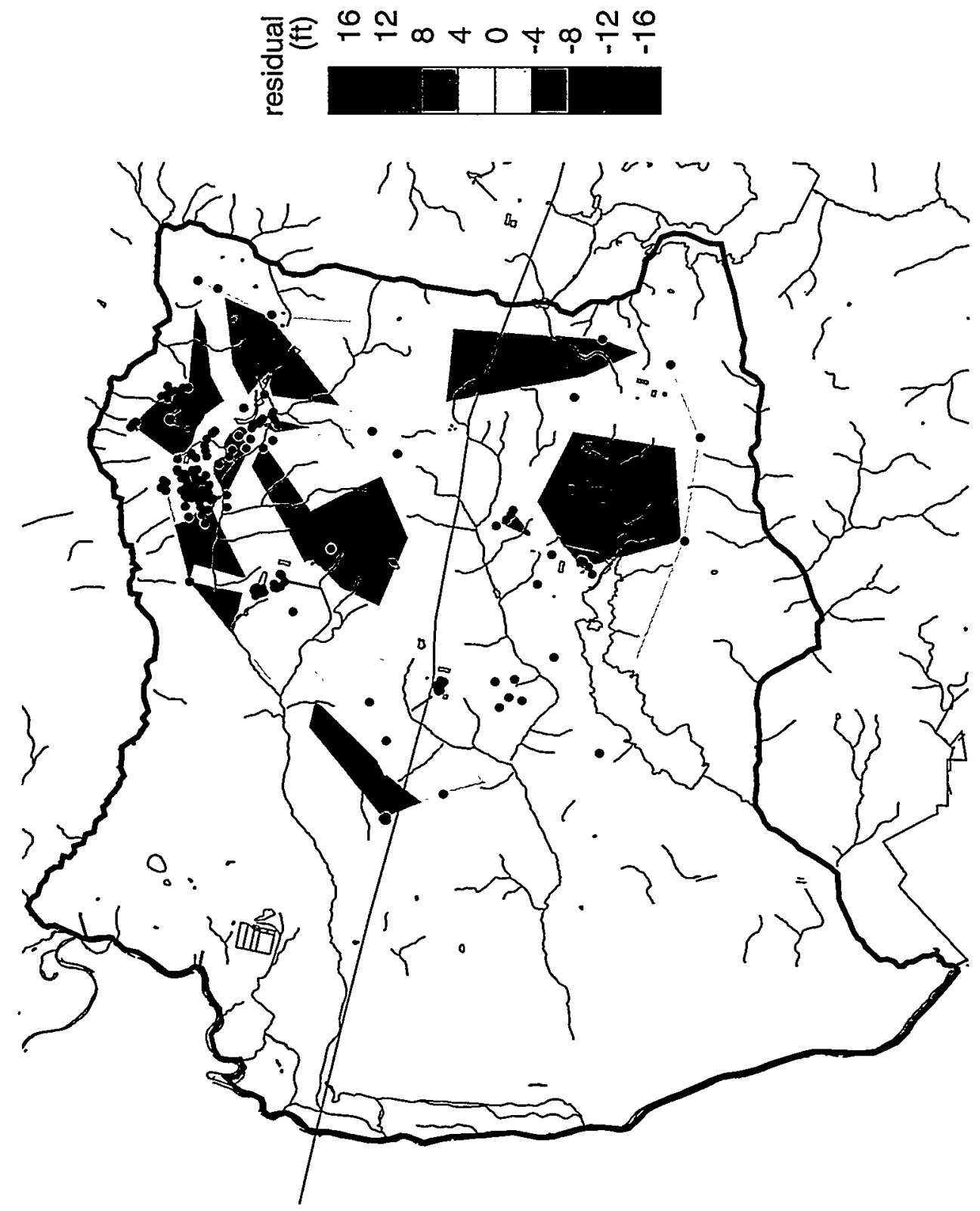




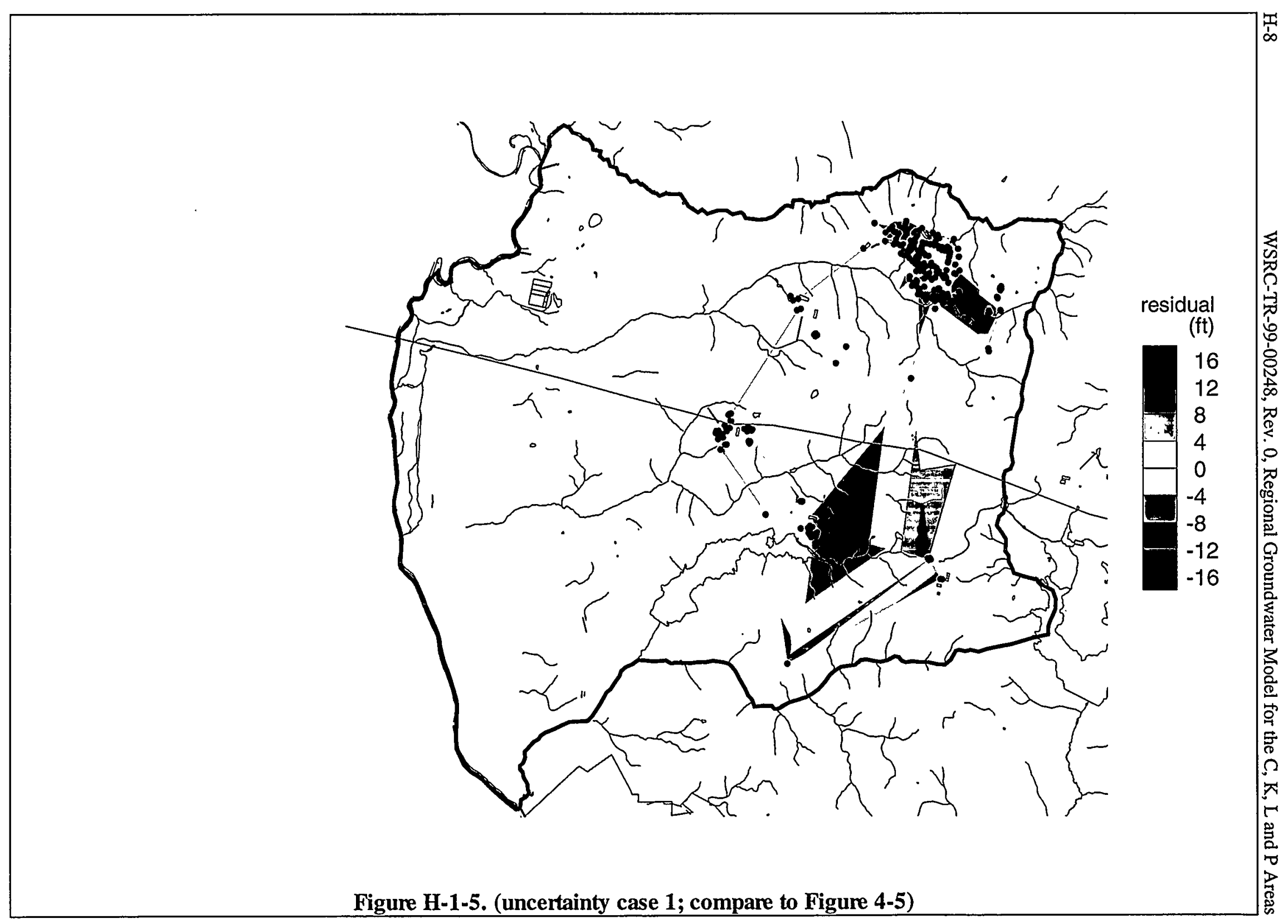


WSRC-TR-99-00248, Rev. 0, Regional Groundwater Model for the C, K, L and P Areas

H-9
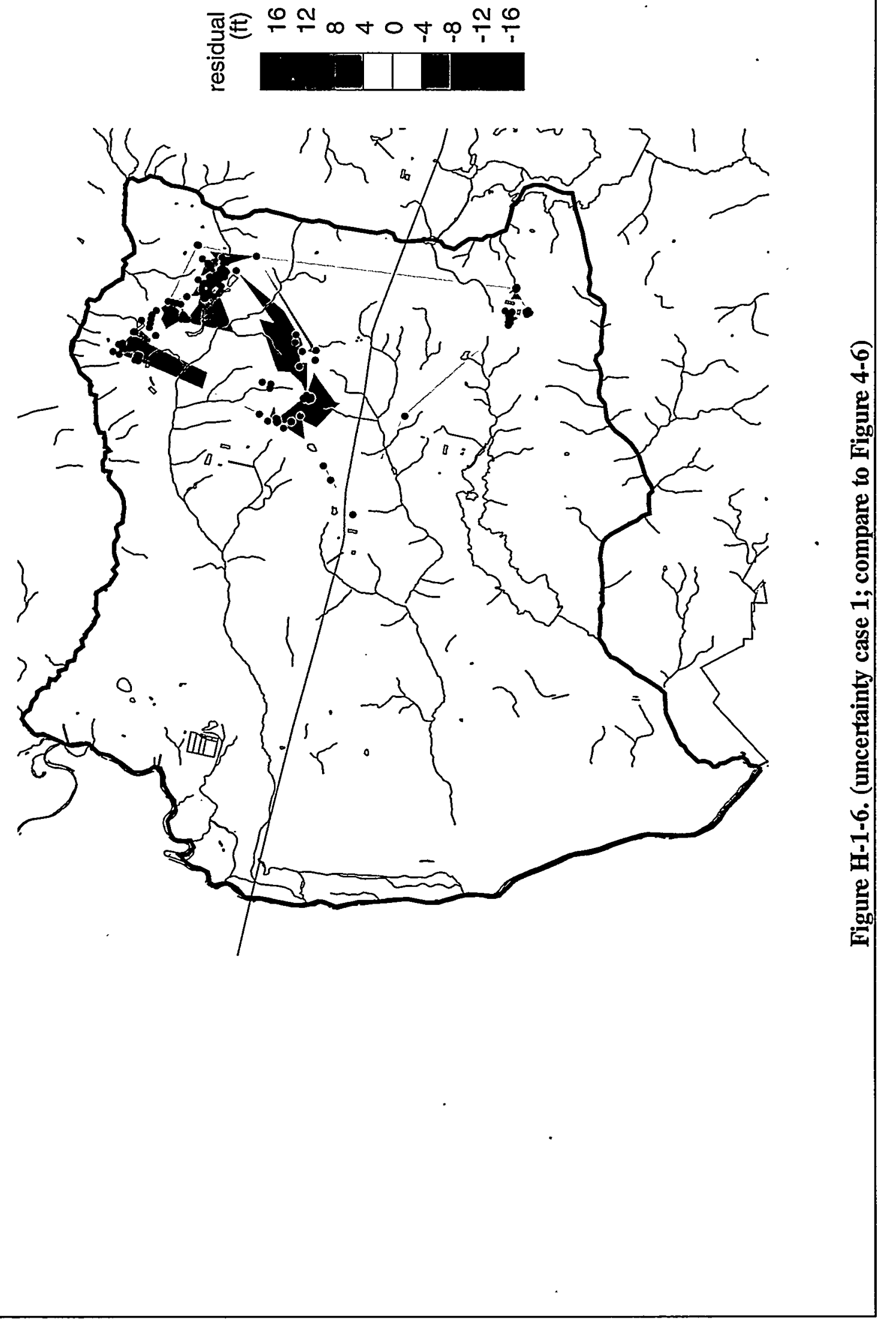


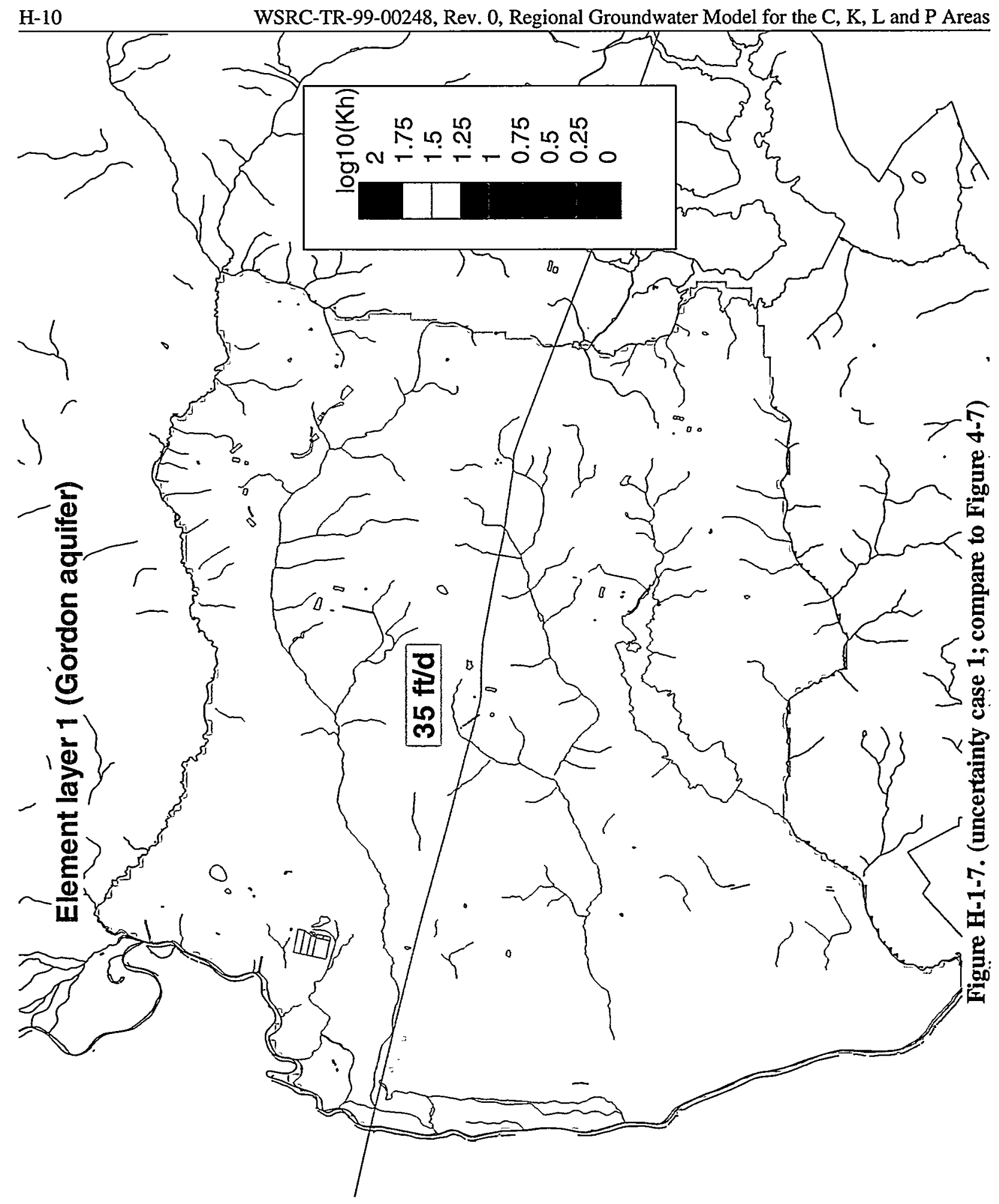




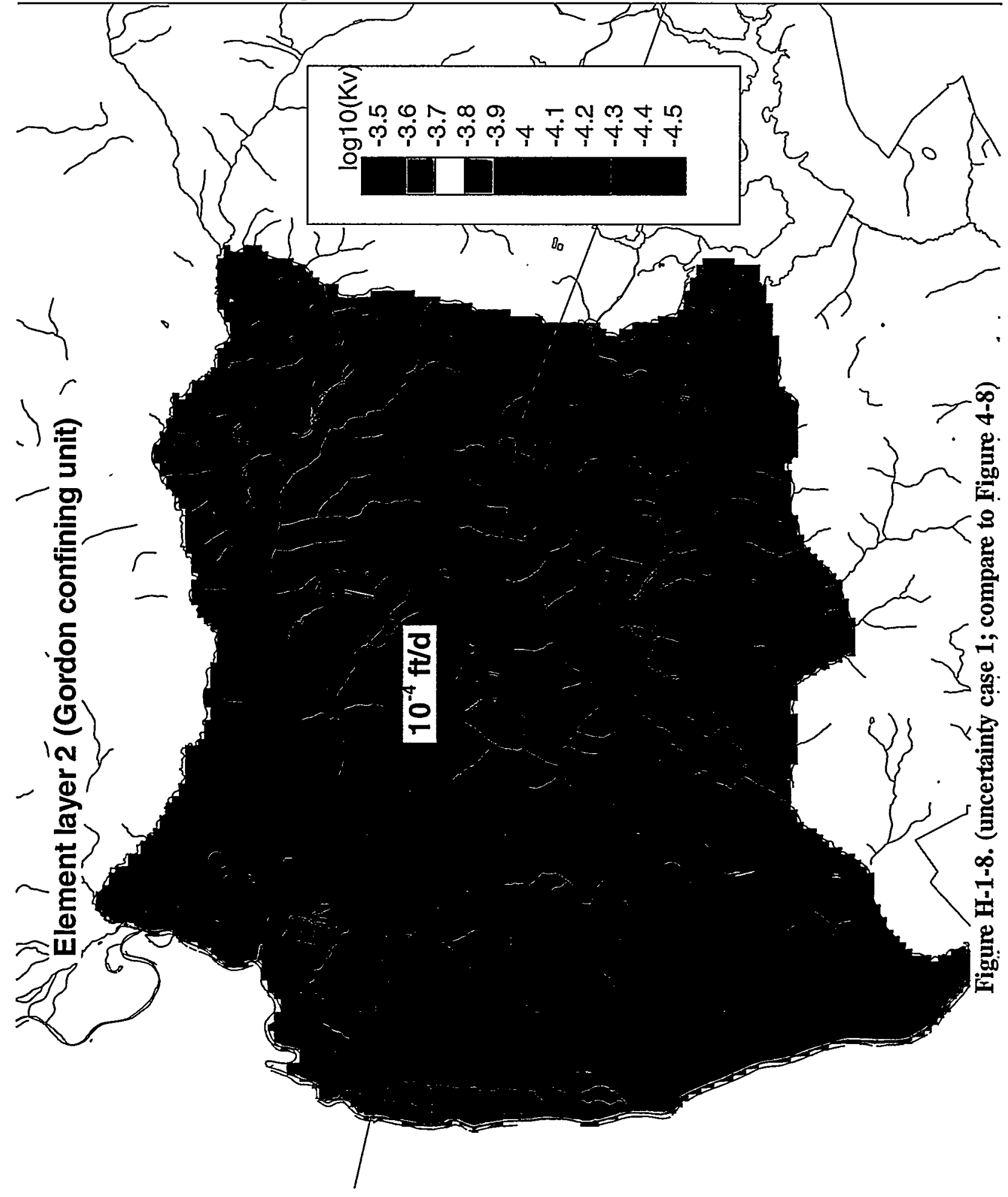




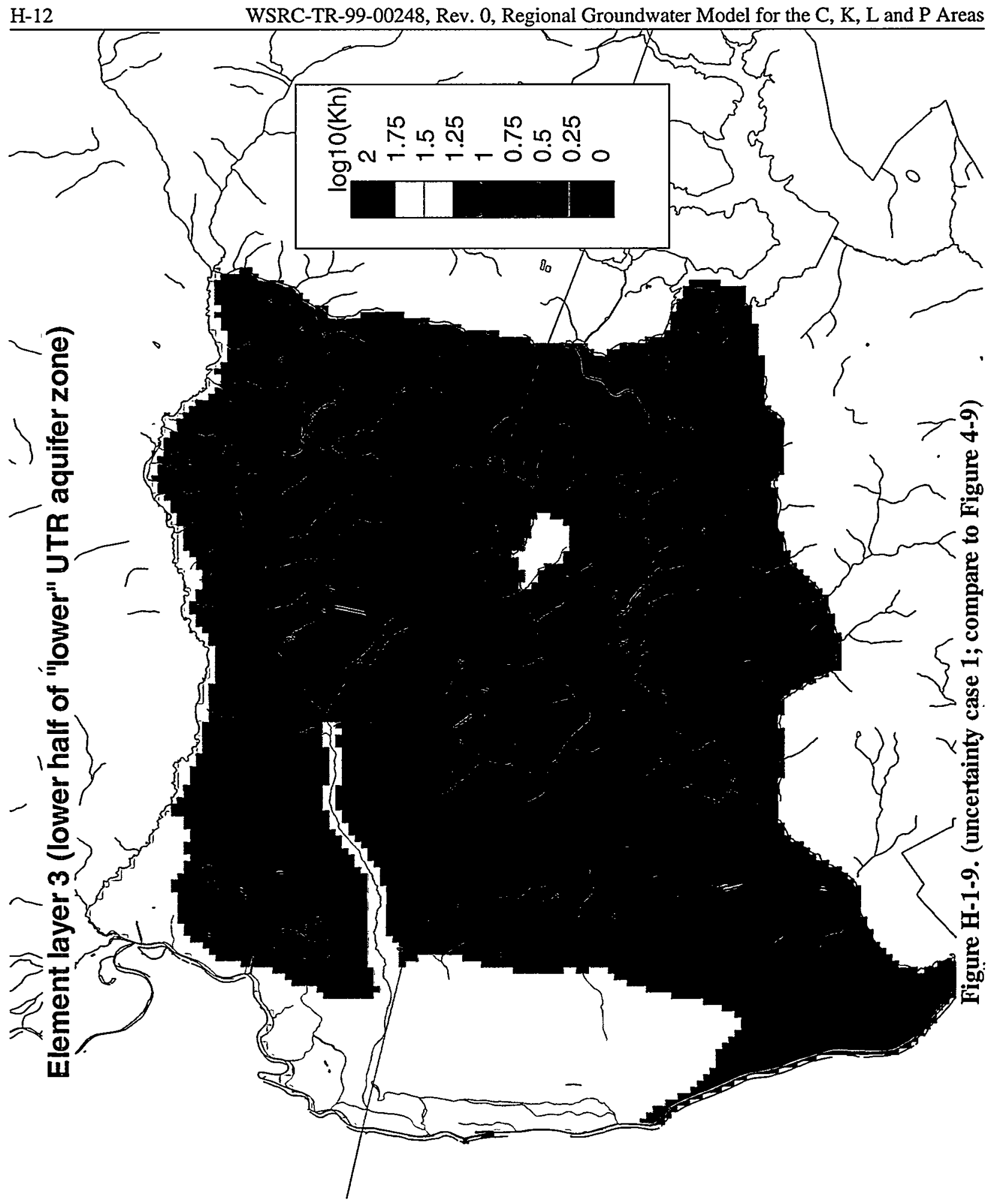




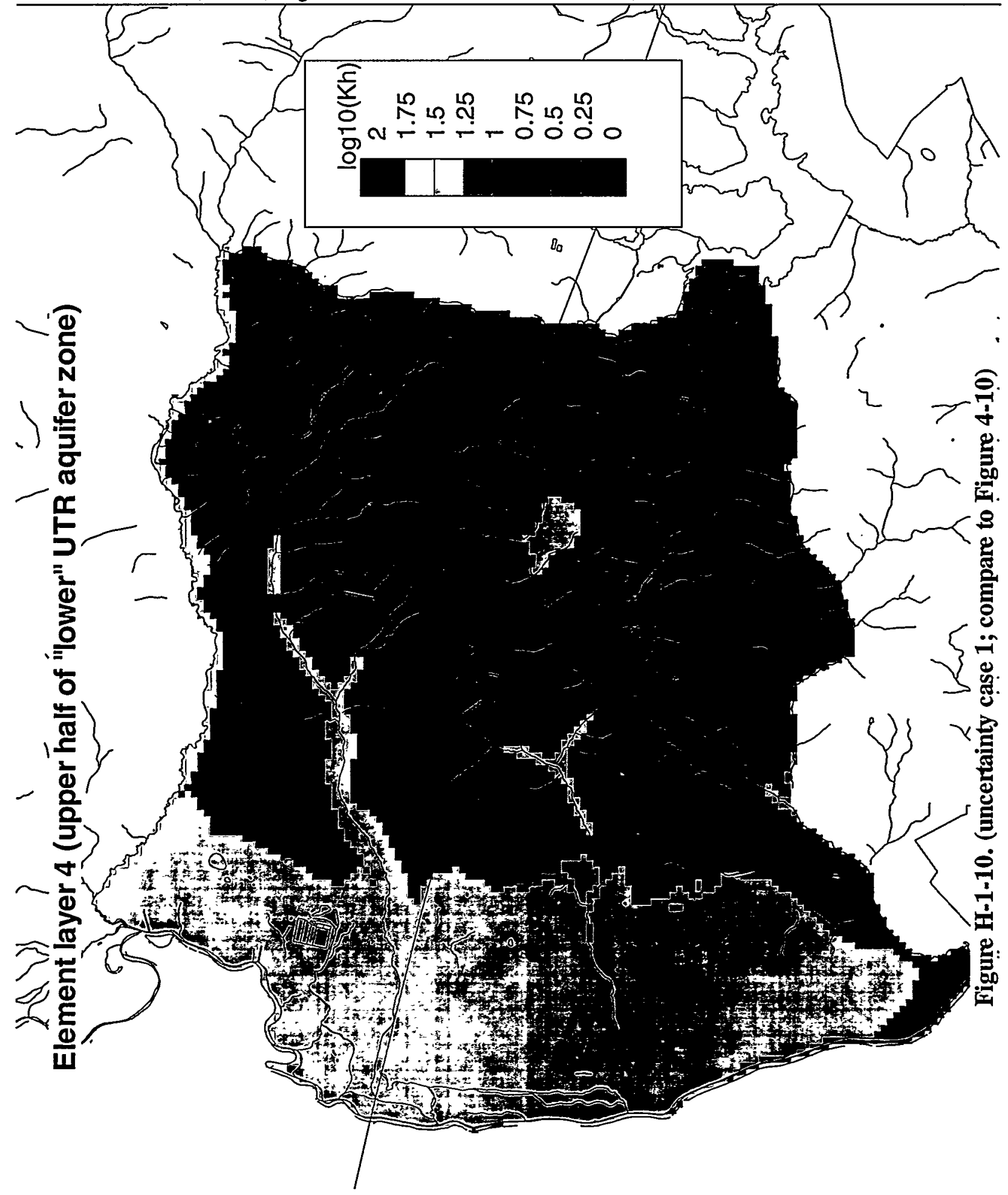




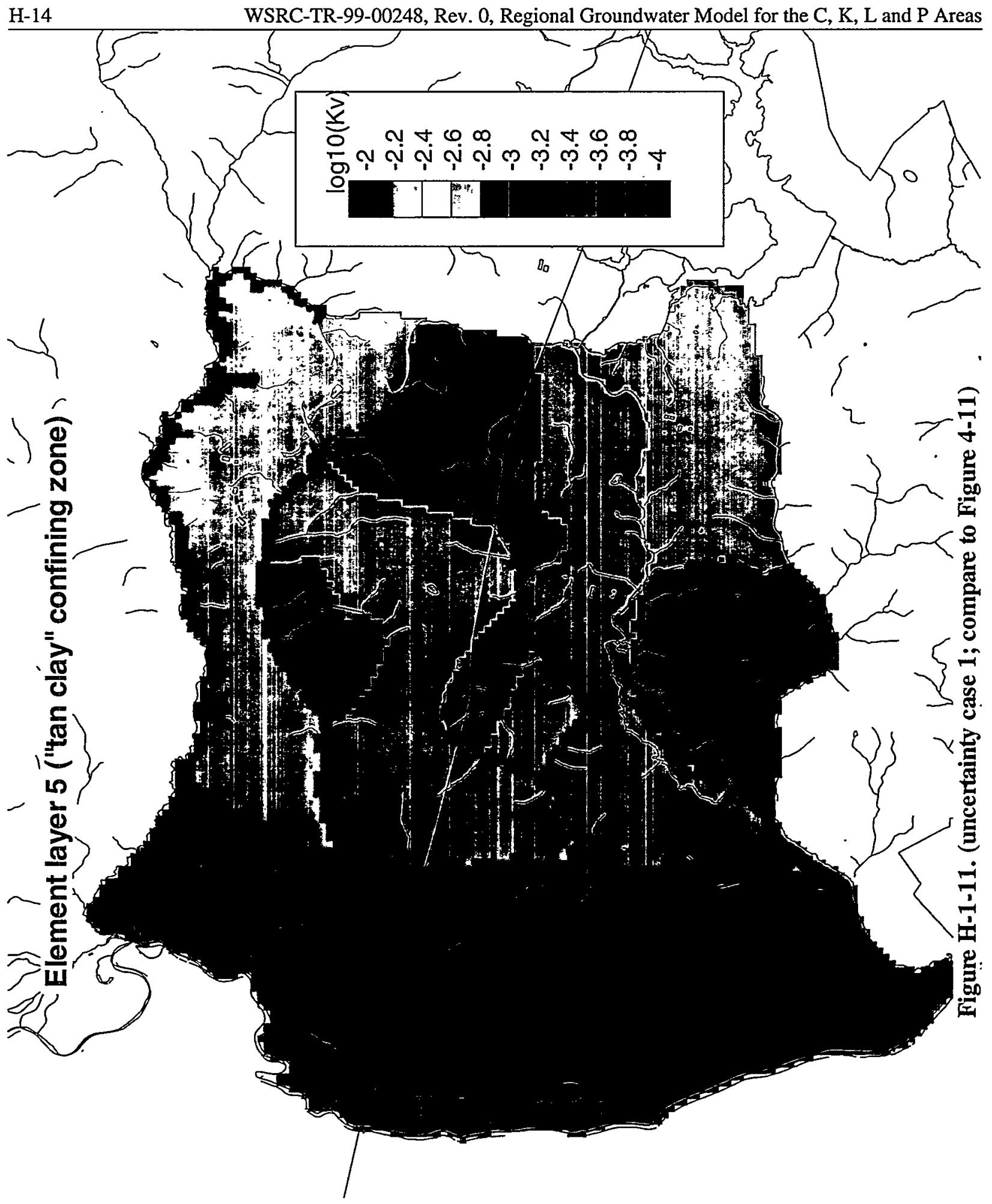




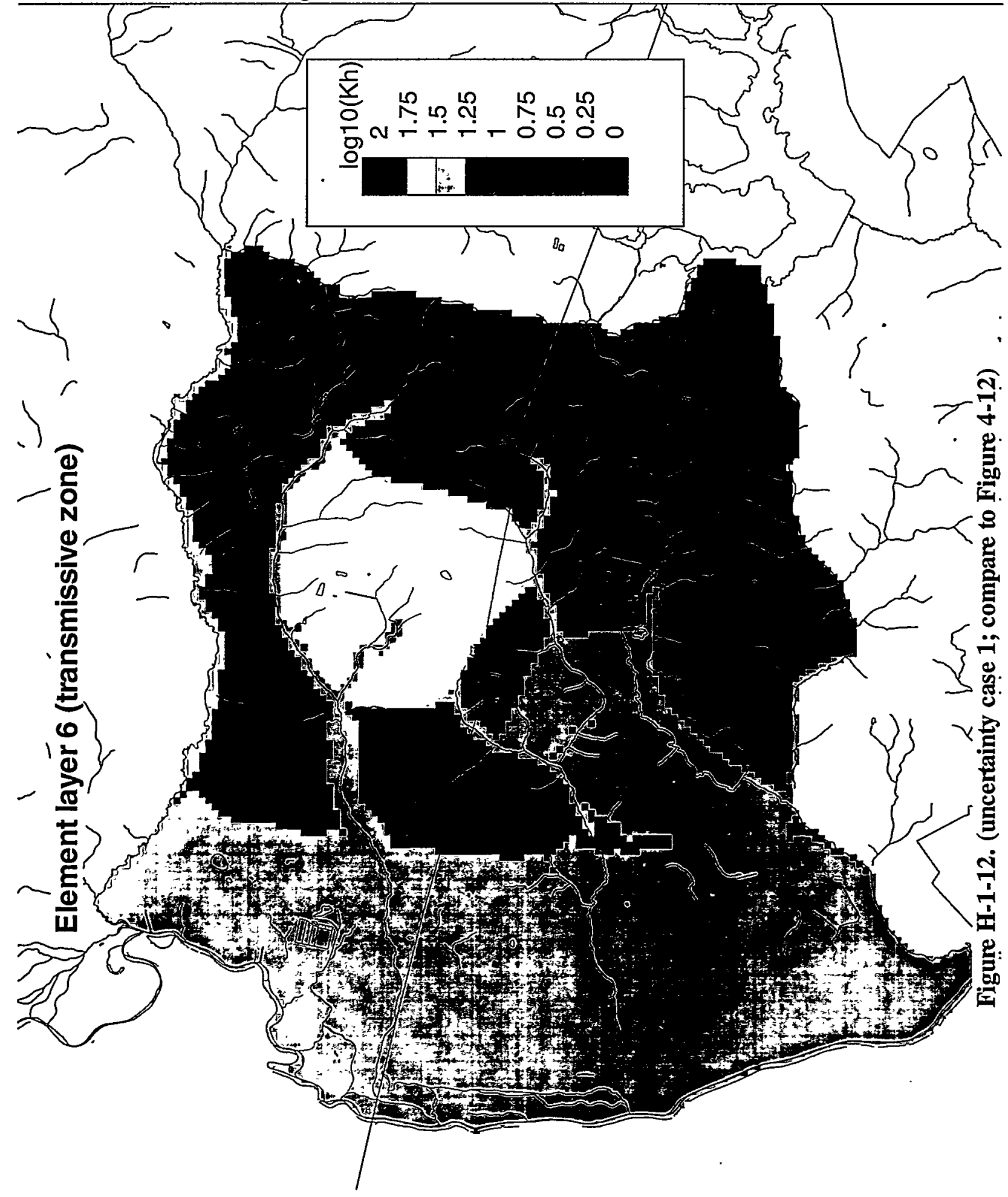




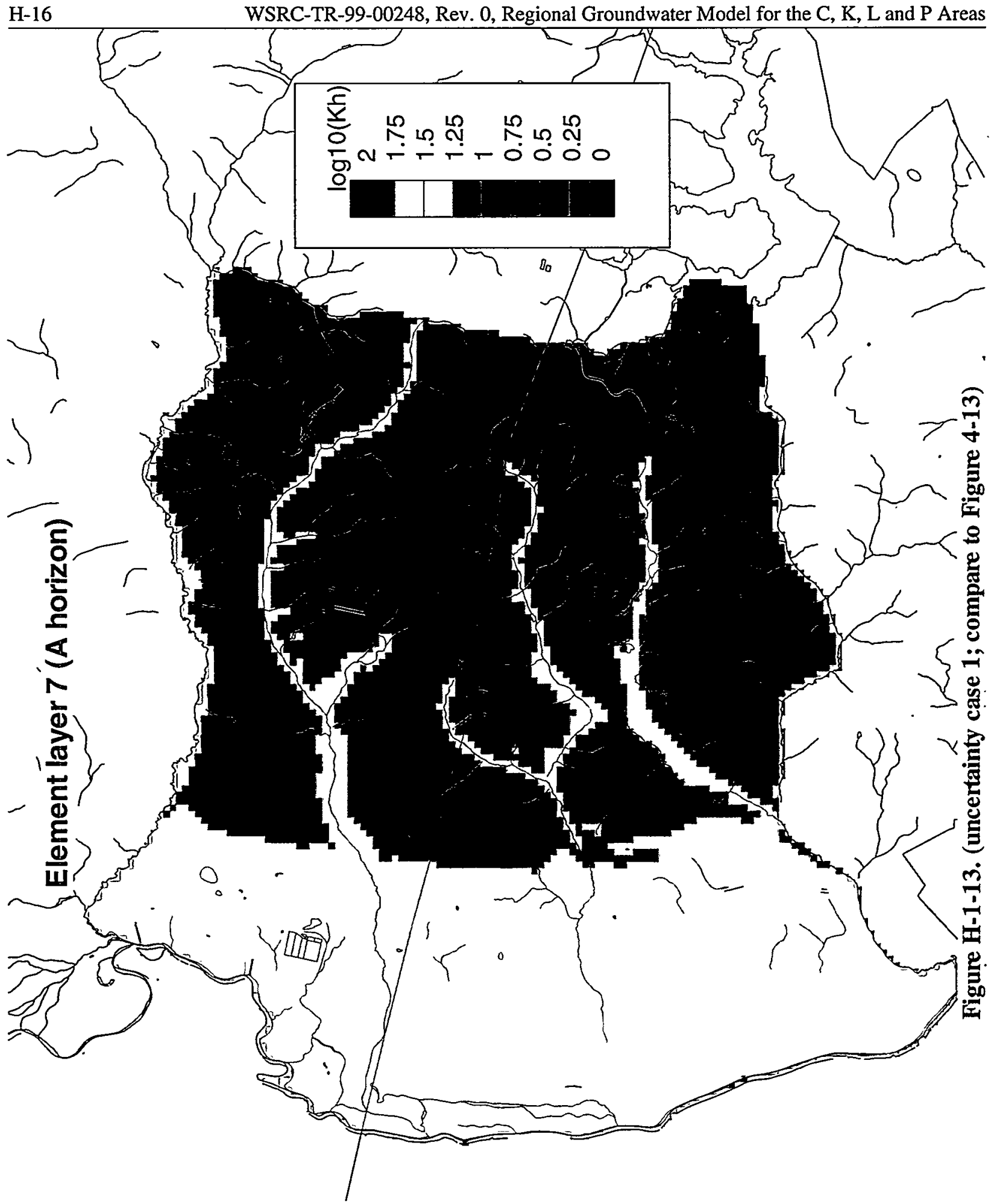


WSRC-TR-99-00248, Rev. 0, Regional Groundwater Model for the C, K, L and P Areas

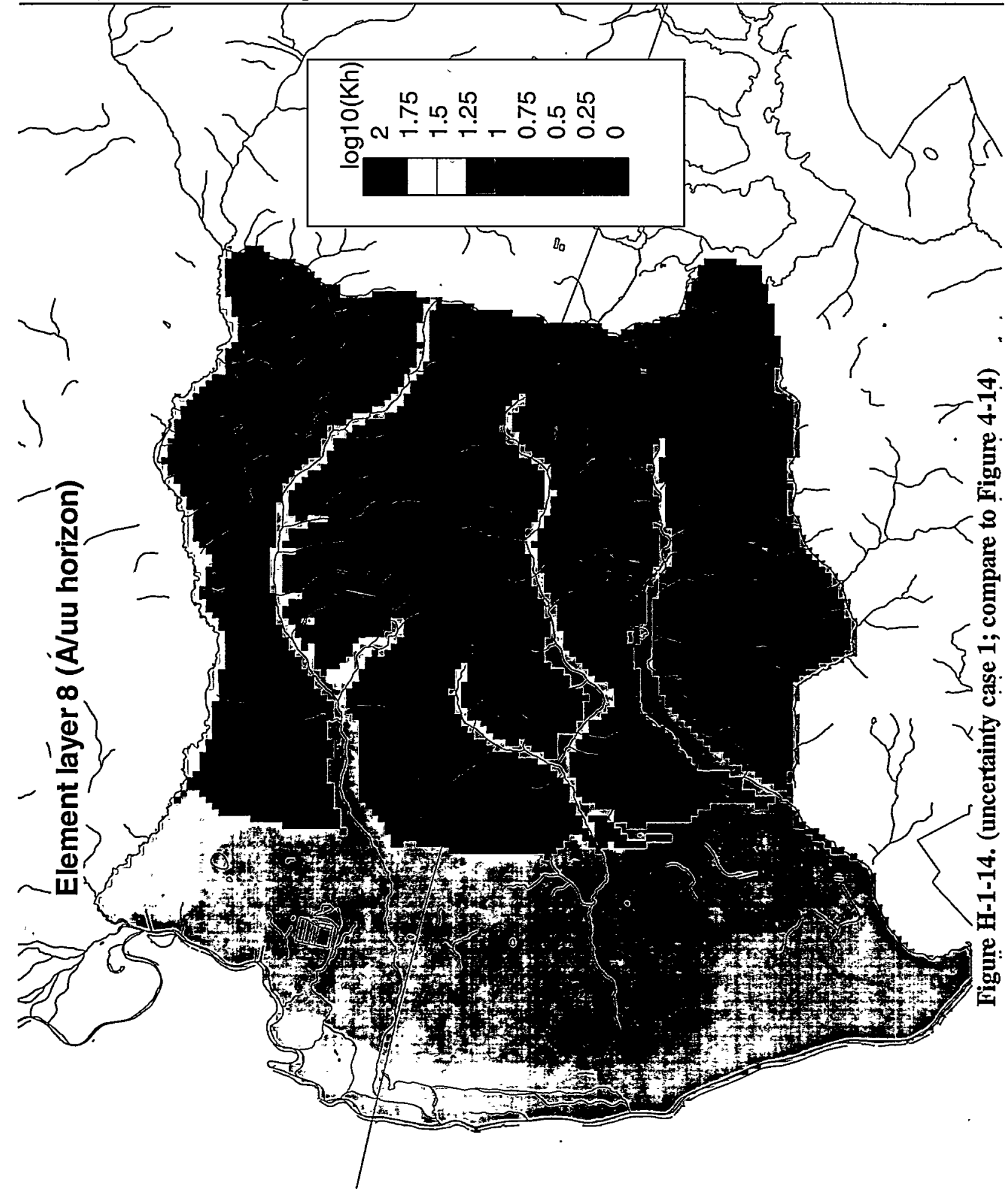




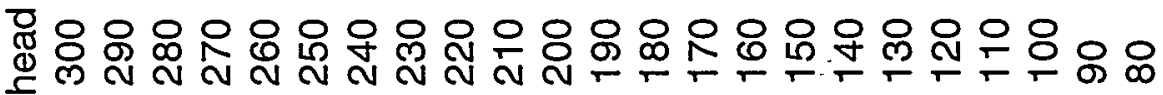
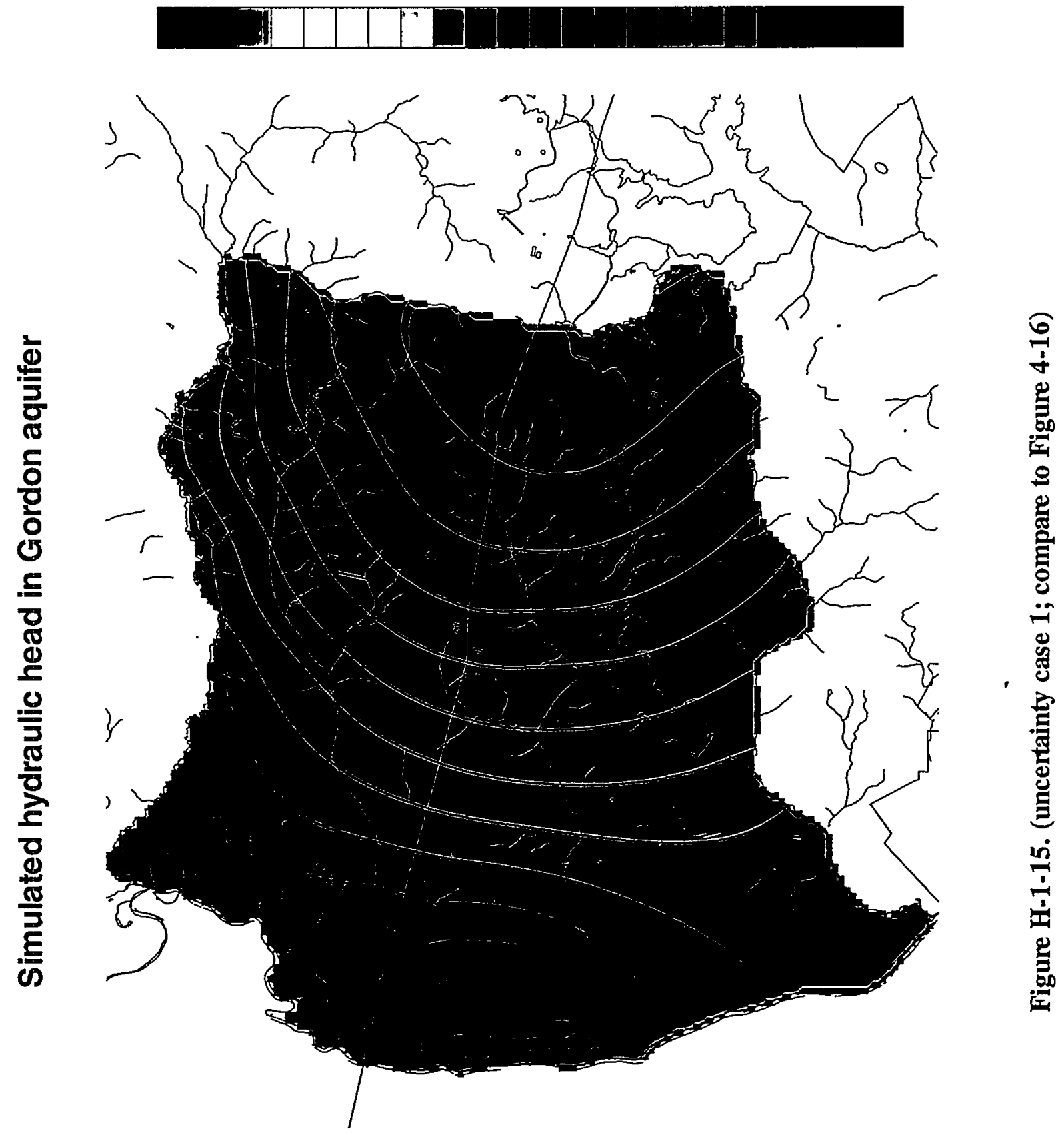
Simulated hydraulic head in "lower" UTR aquifer zone

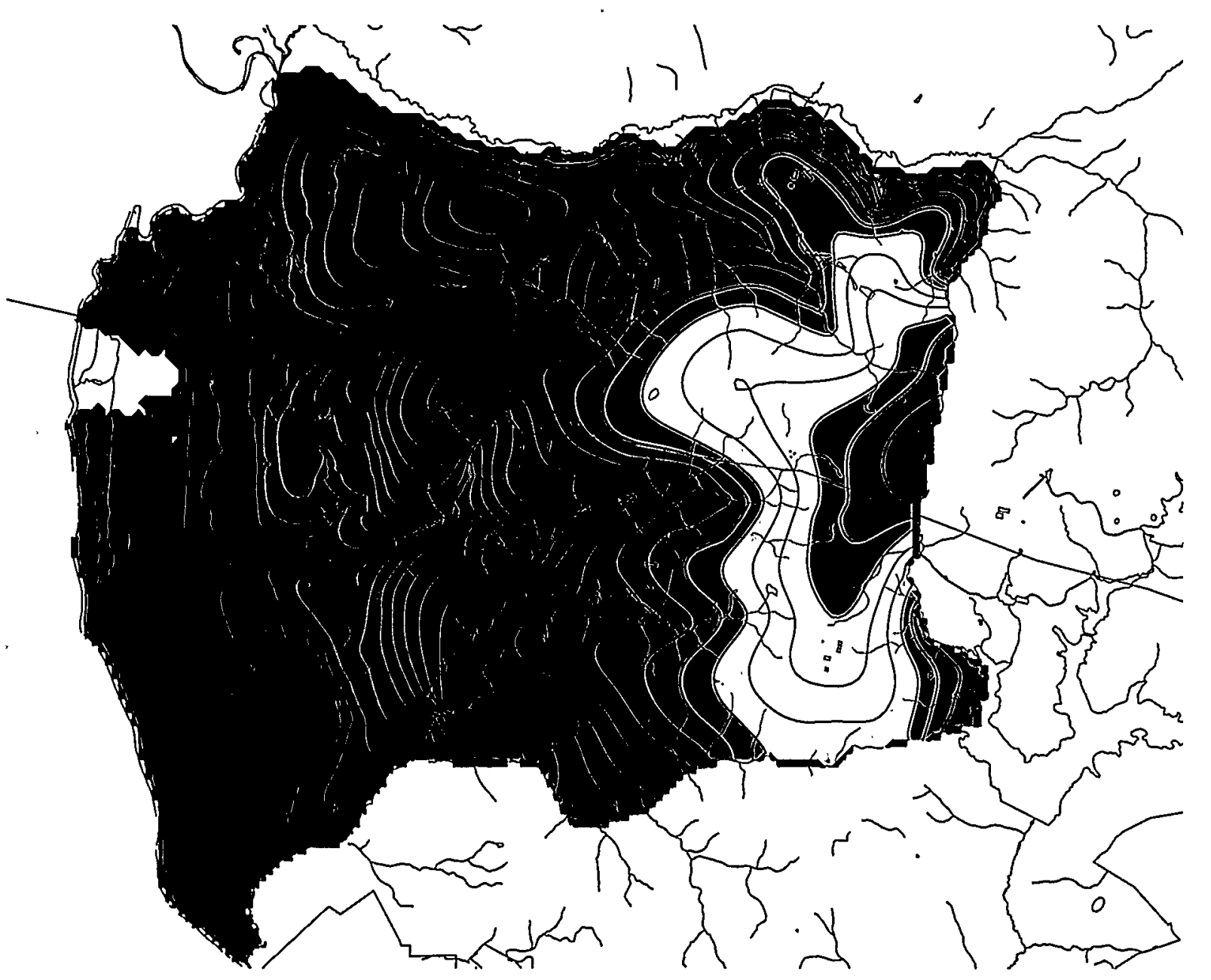

head

Figure H-1-16. (uncertainty case 1; compare to Figure 4-17) 


\section{Simulated hydraulic head in "upper" UTR aquifer zone}

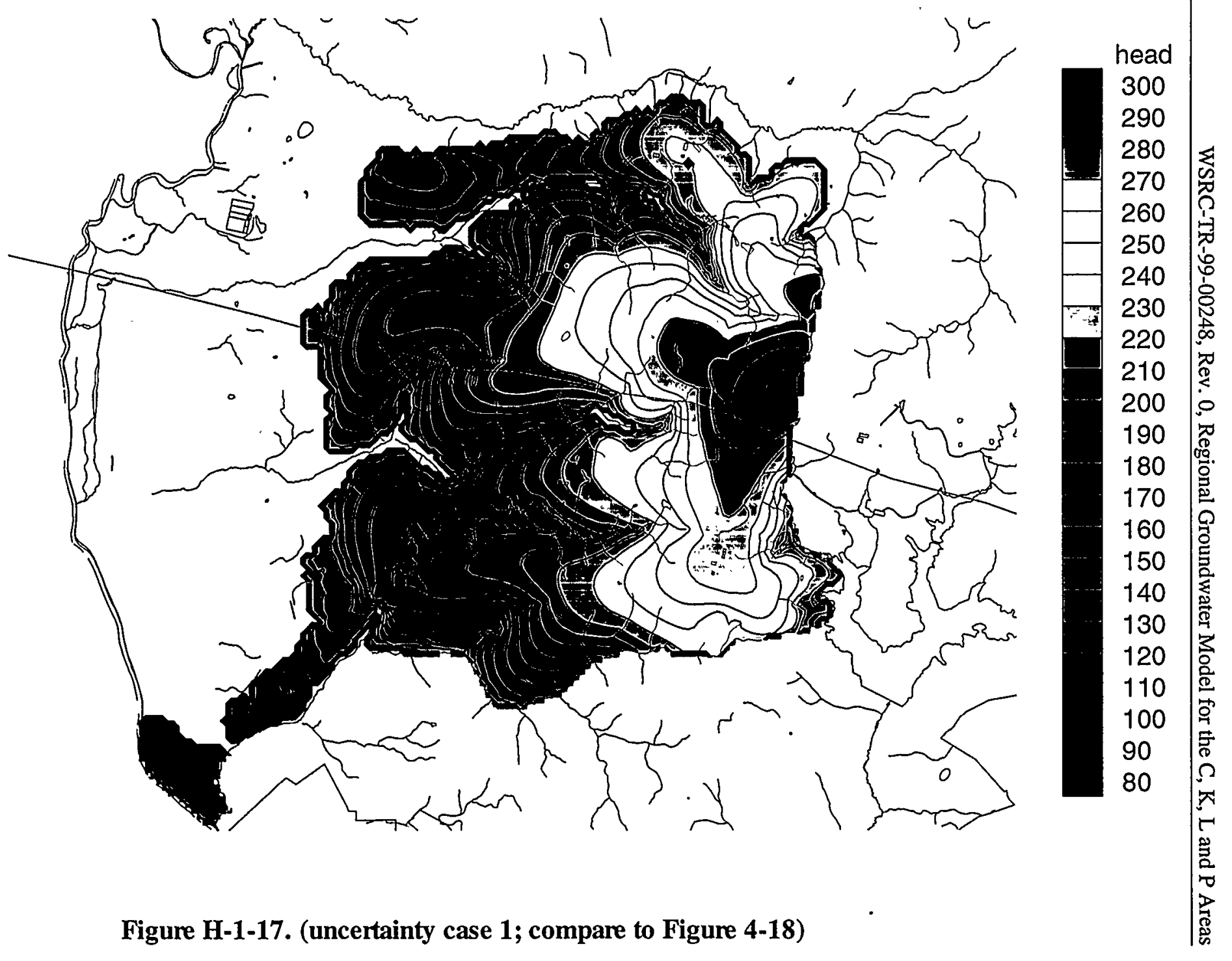


Simulated hydraulic head in aquifer zone containing water table

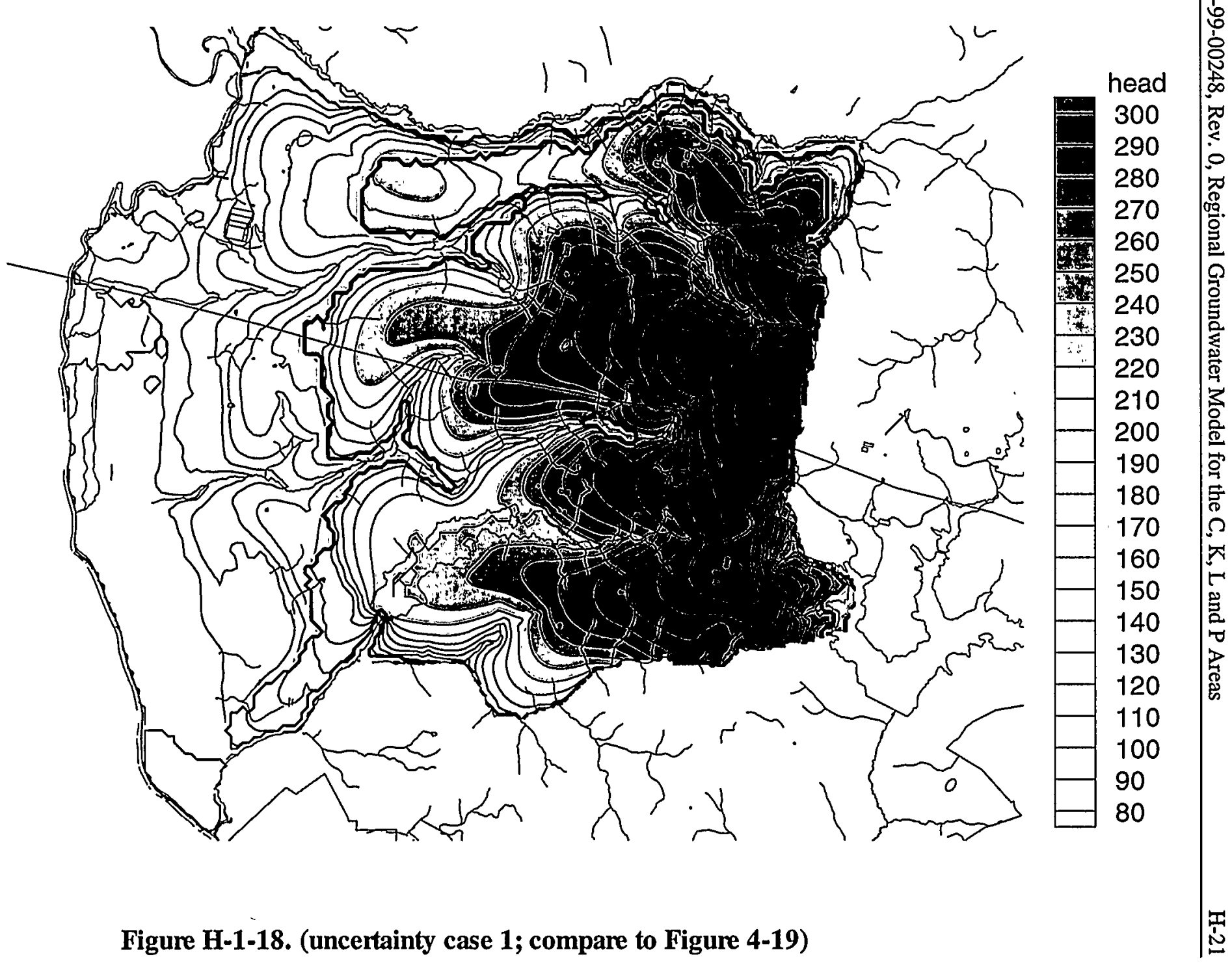




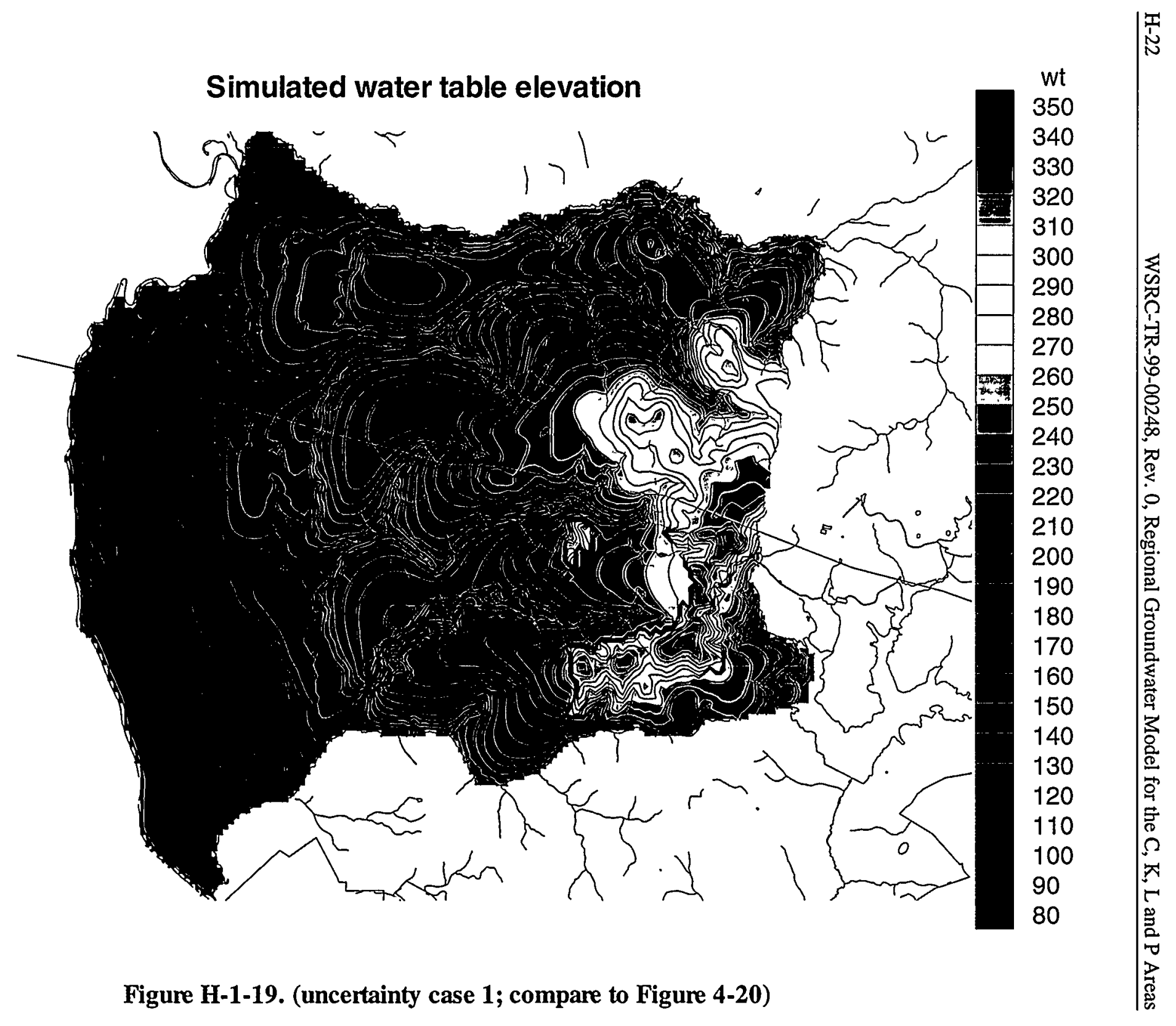




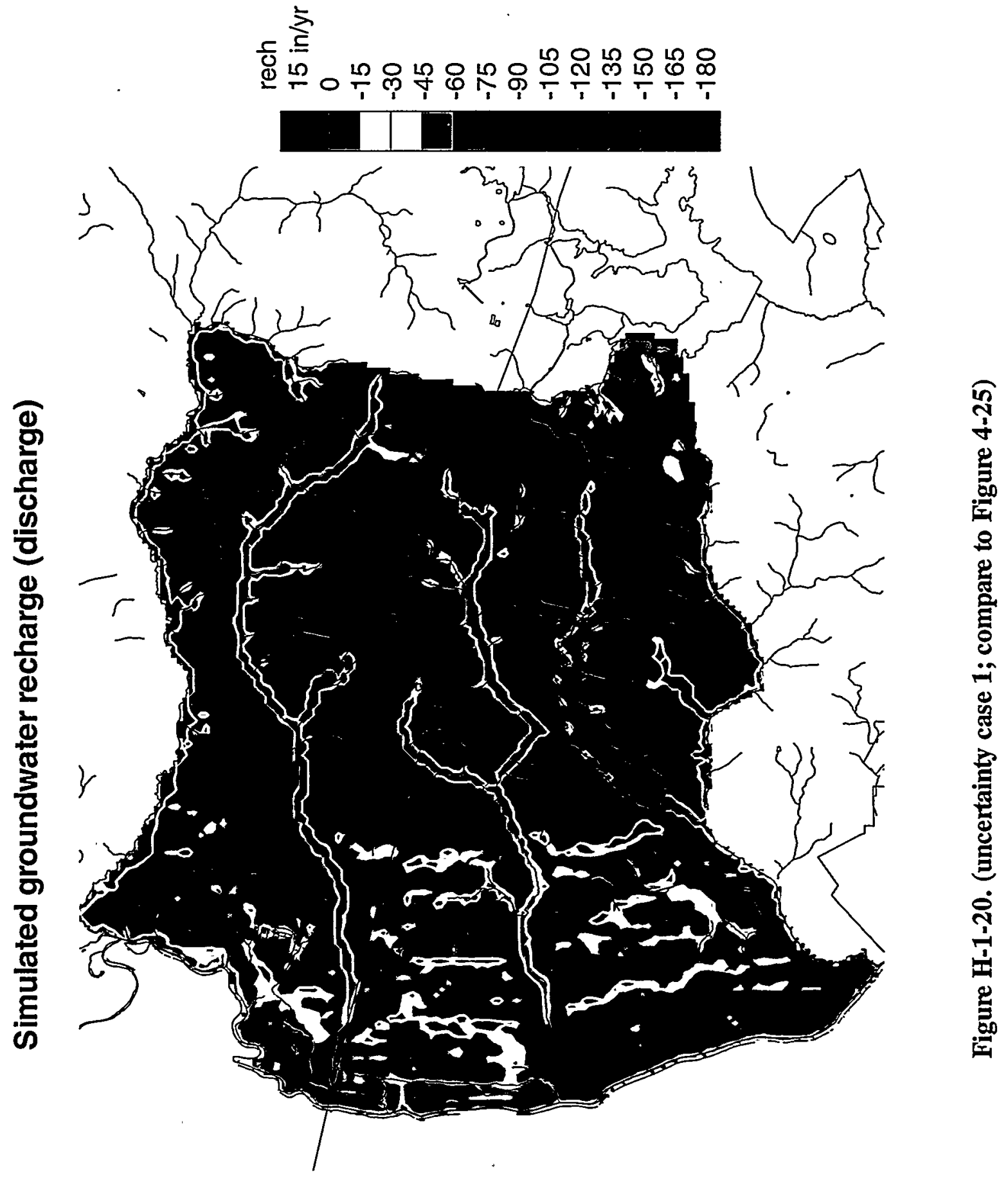




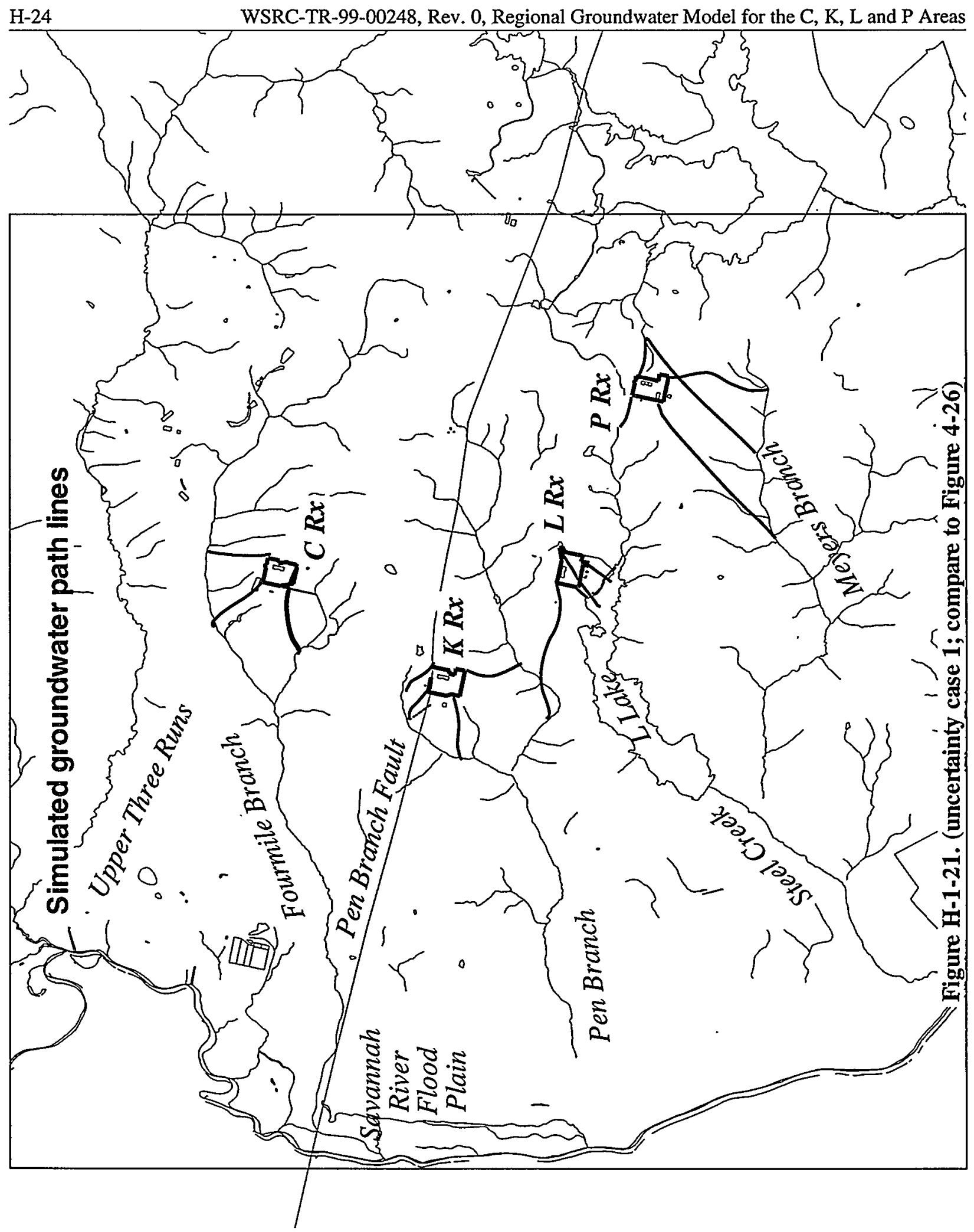




\section{Simulation results for uncertainty case 2}

Uncertainty case 1 involves a decrease in recharge of $20 \%$ to $10 \mathrm{in} / \mathrm{yr}$ (Table 4-4). Summary calibration results are provided in Table 4-5. This appendix presents detailed simulation results for uncertainty case 2 for comparison to the nominal results shown in figures in the main text. The correspondence between figures for the nominal and uncertainty case 2 is as follows:

\begin{tabular}{|l|l|l|}
\hline Plot type & Nominal case & Uncertainty case 2 \\
\hline Head residual summary & Figure 4-1 & Figure H-2-1 \\
\hline Head residuals in Gordon aquifer & Figure 4-2 & Figure H-2-2 \\
\hline Head residuals in "lower" UTRA & Figure 4-3 & Figure H-2-3 \\
\hline Head residuals in transmissive zone & Figure 4-4 & Figure H-2-4 \\
\hline Head residuals in AA horizon & Figure 4-5 & Figure H-2-5 \\
\hline Head residuals in A/uu horizons & Figure 4-6 & Figure H-2-6 \\
\hline Kh in element layer 1 & Figure 4-7 & Figure H-2-7 \\
\hline Kv in element layer 2 & Figure 4-8 & Figure H-2-8 \\
\hline Kh in element layer 3 & Figure 4-9 & Figure H-2-9 \\
\hline Kh in element layer 4 & Figure 4-10 & Figure H-2-10 \\
\hline Kv in element layer 5 & Figure 4-11 & Figure H-2-11 \\
\hline Kh in element layer 6 & Figure 4-12 & Figure H-2-12 \\
\hline Kh in element layer 7 & Figure 4-13 & Figure H-2-13 \\
\hline Kh in element layer 8 & Figure 4-14 & Figure H-2-14 \\
\hline Gordon aquifer head & Figure 4-16 & Figure H-2-15 \\
\hline "Lower" UTRA head & Figure 4-17 & Figure H-2-16 \\
\hline "Upper" UTRA head & Figure 4-18 & Figure H-2-17 \\
\hline Head in aquifer containing water table & Figure 4-19 & Figure H-2-18 \\
\hline Water table & Figure 4-20 & Figure H-2-19 \\
\hline Recharge/discharge & Figure 4-25 & Figure H-2-20 \\
\hline Example particle tracing & Figure 4-26 & Figure H-2-21 \\
\hline & &
\end{tabular}



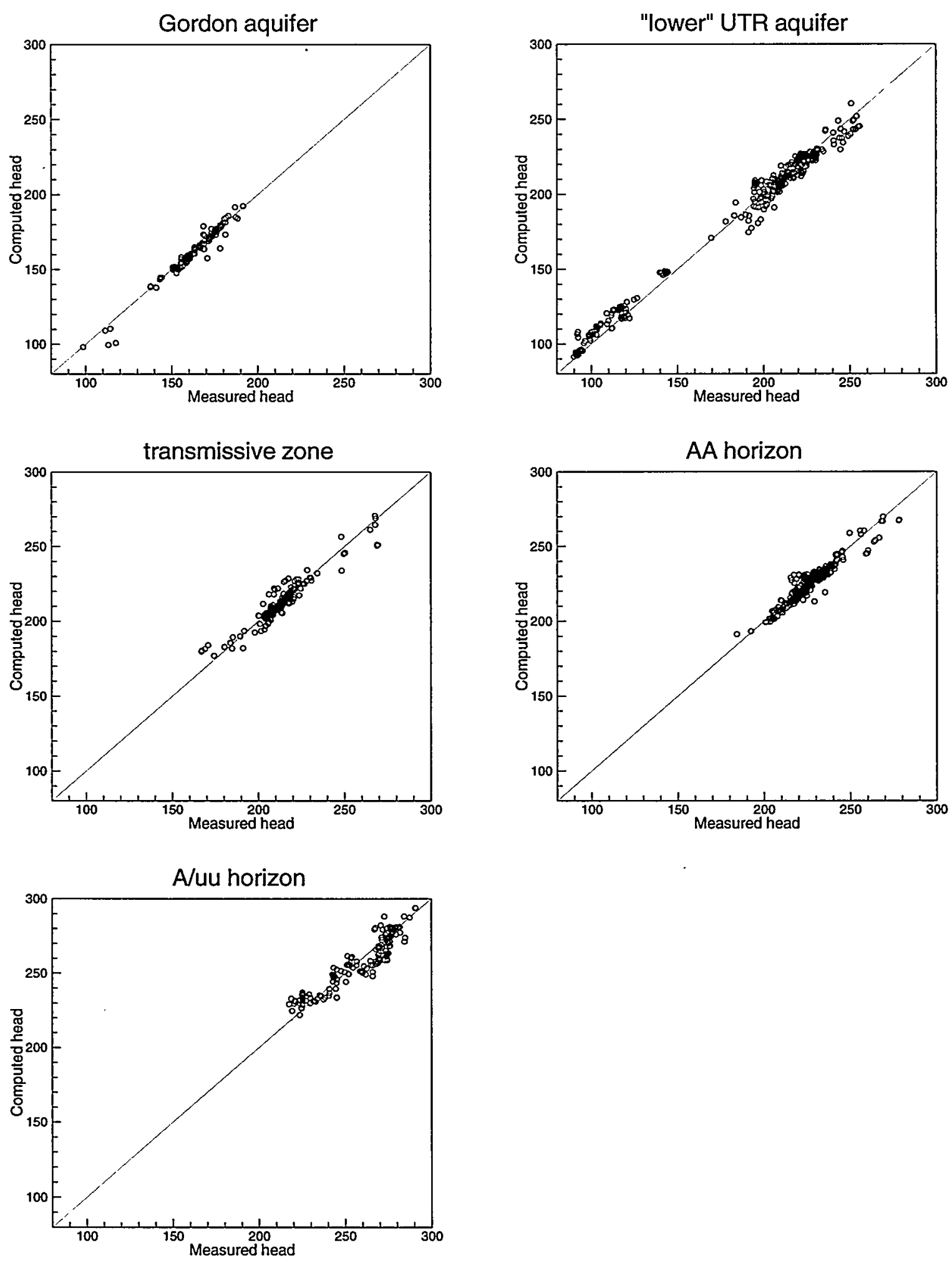

Figure H-2-1. (uncertainty case 2; compare to Figure 4-1) 


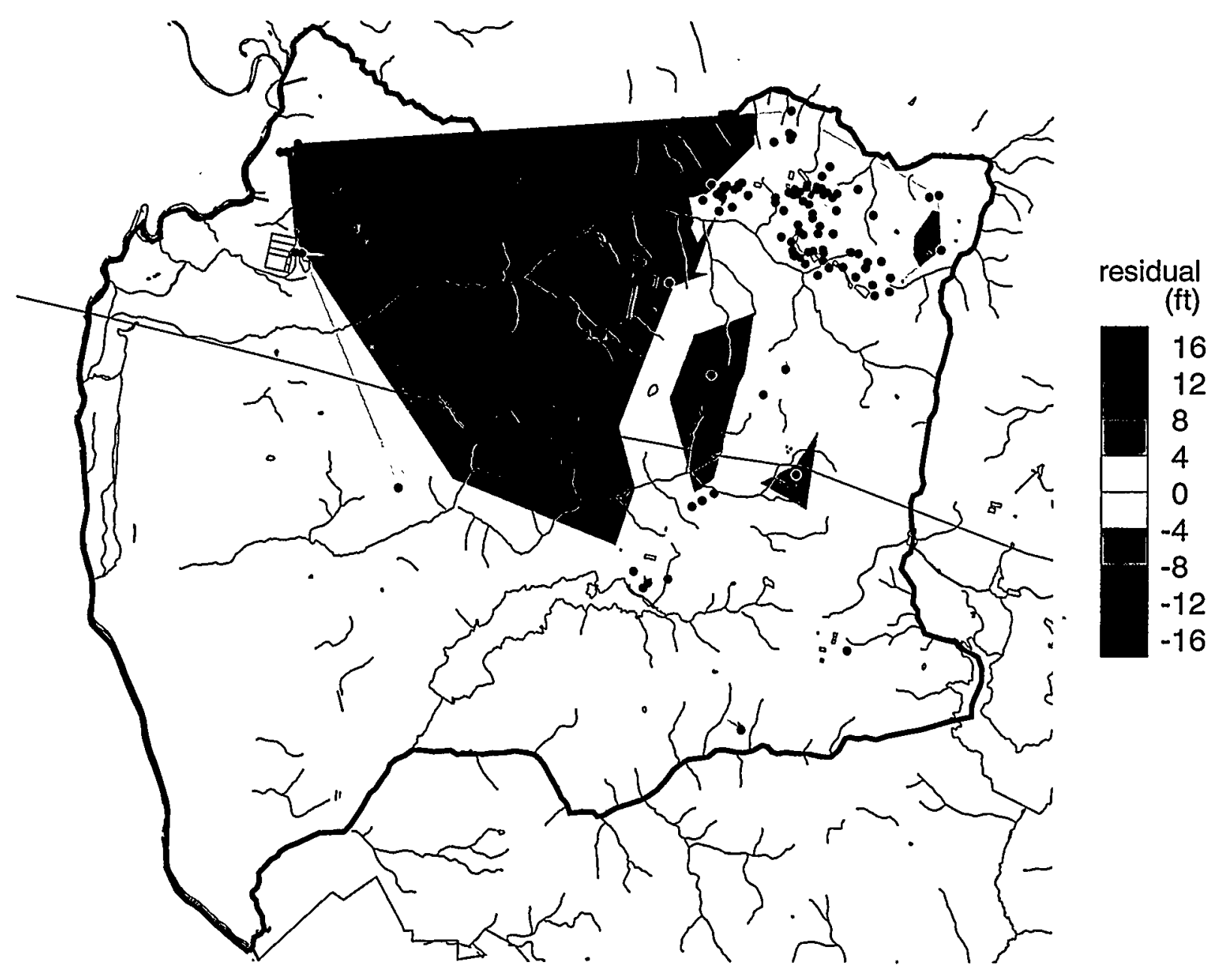

Figure H-2-2. (uncertainty case 2; compare to Figure 4-2) 

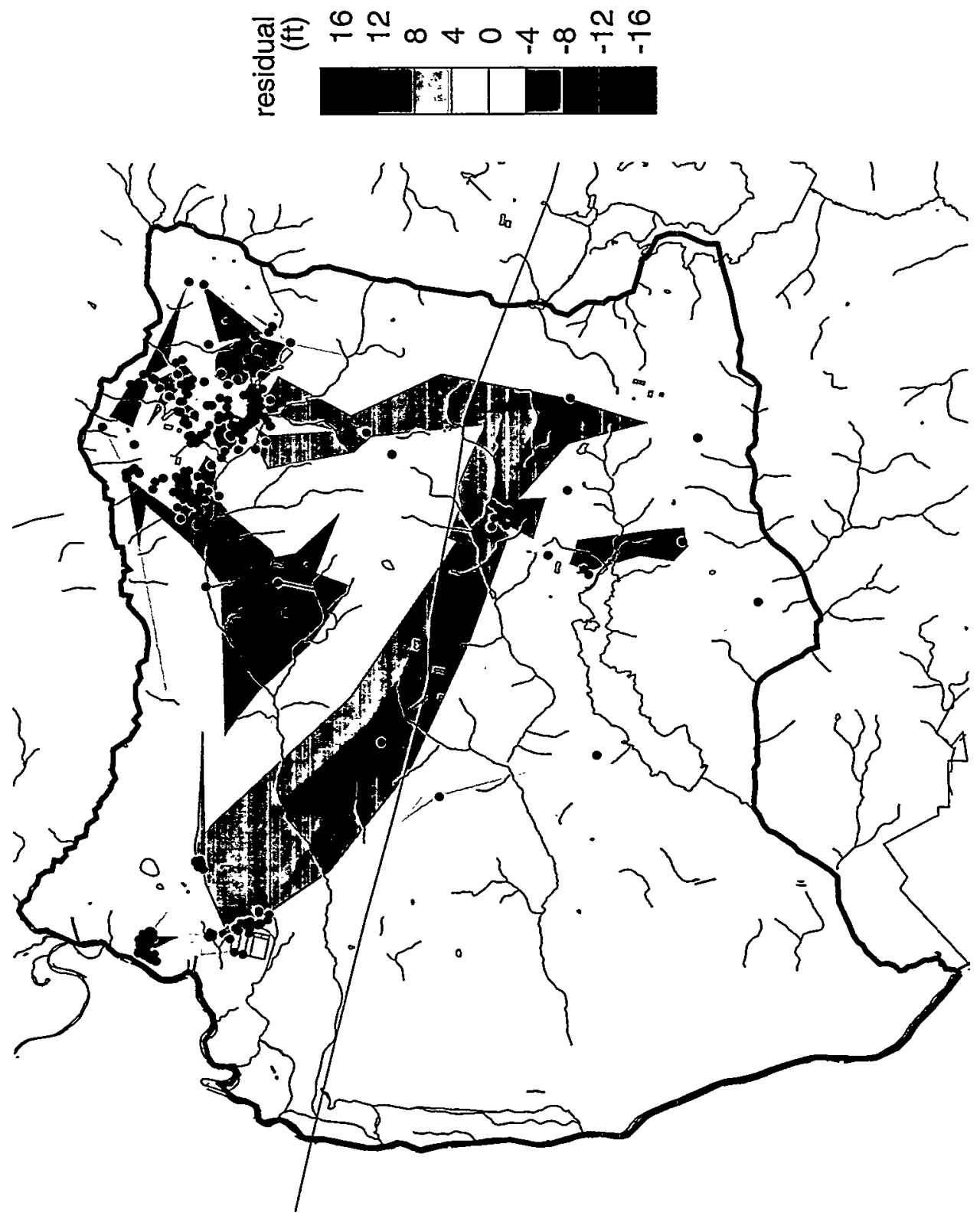


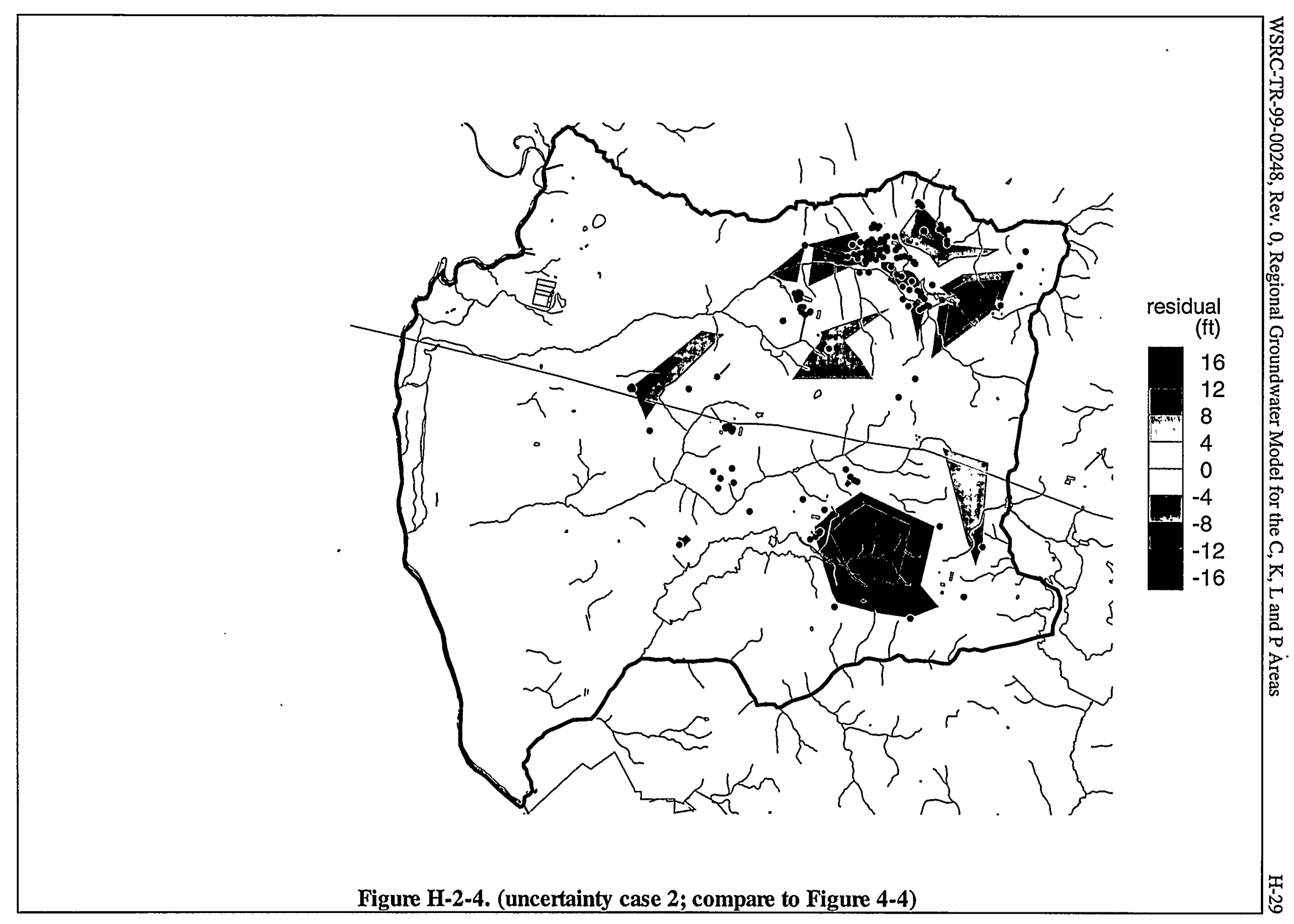




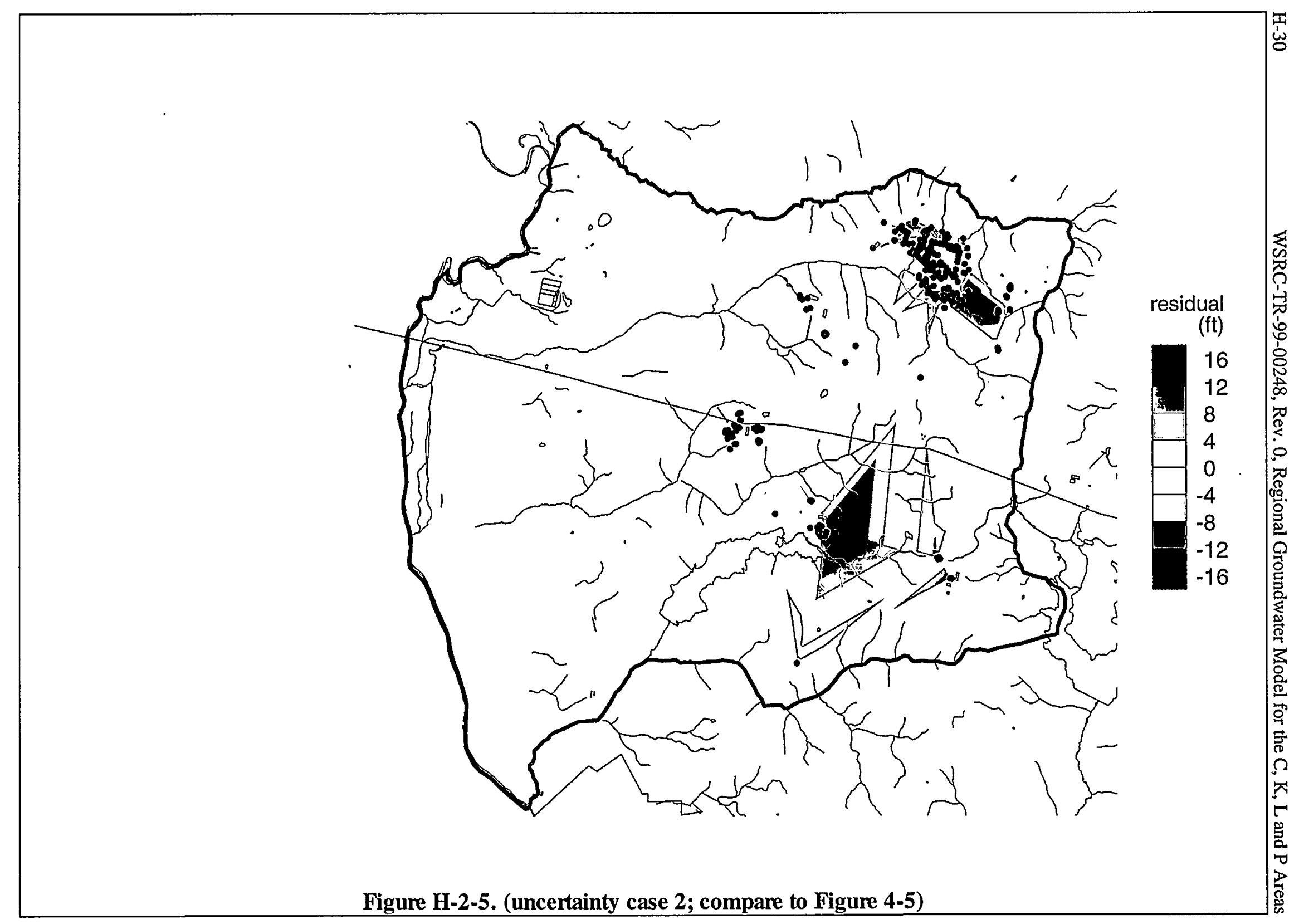




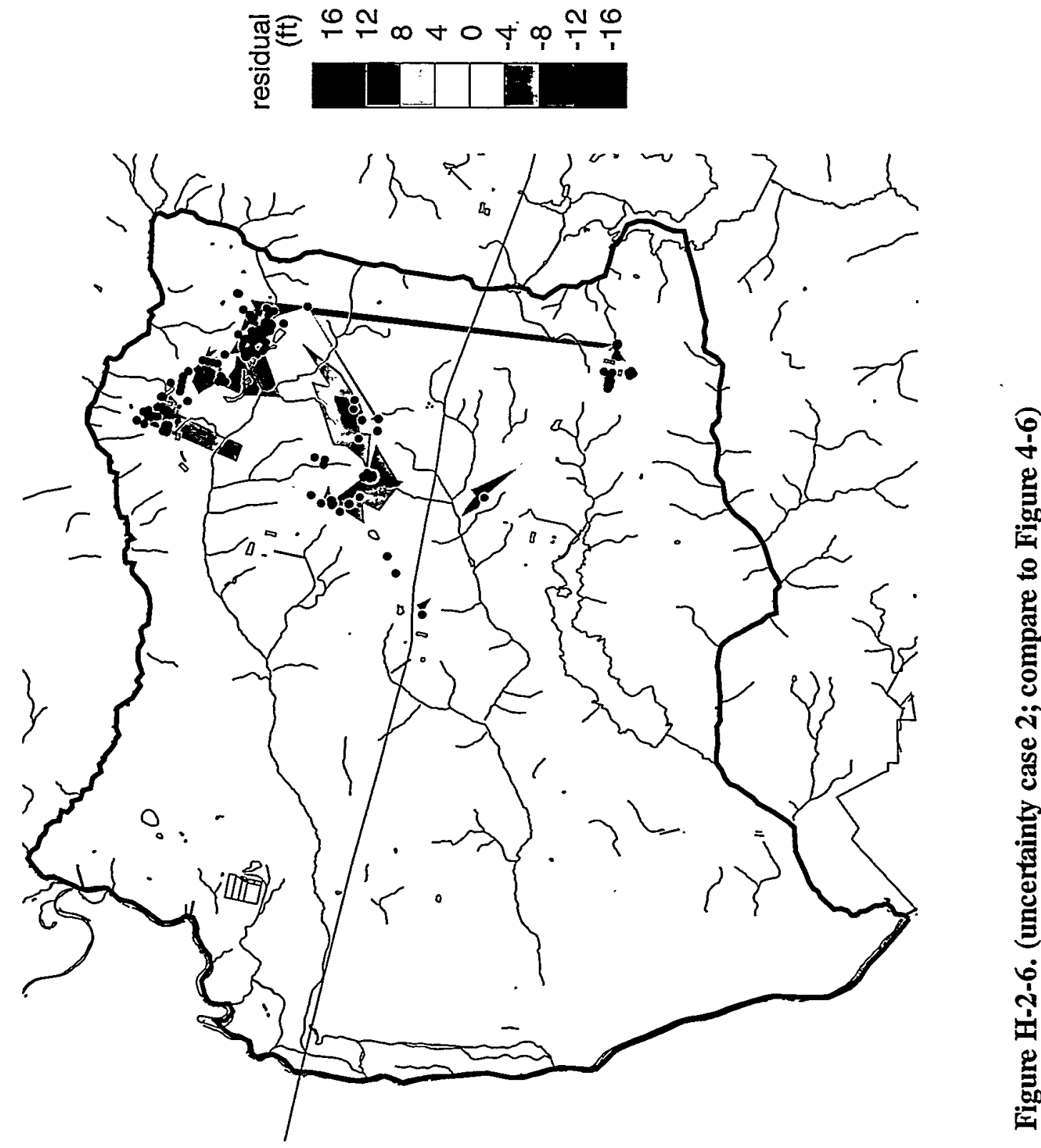




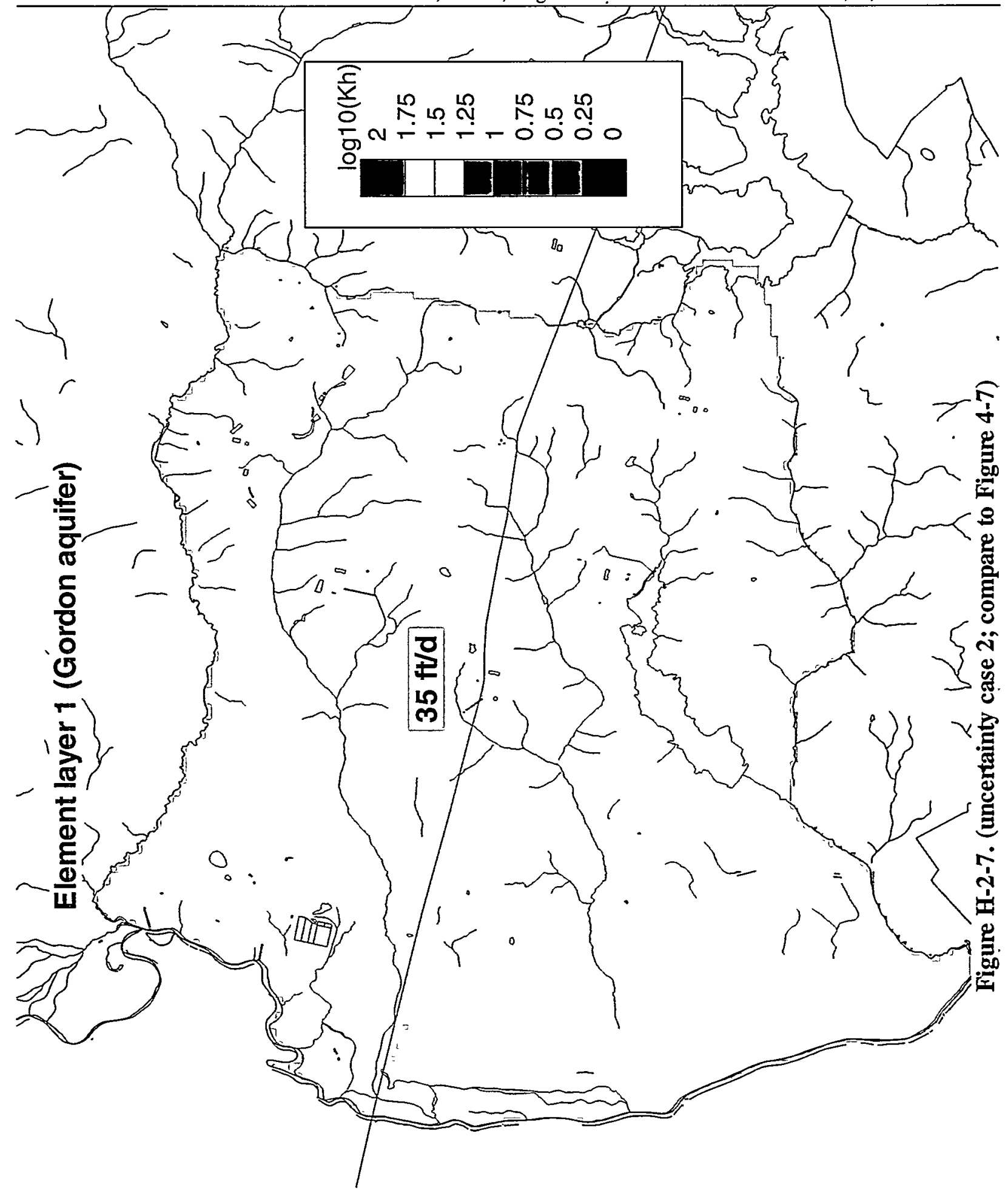




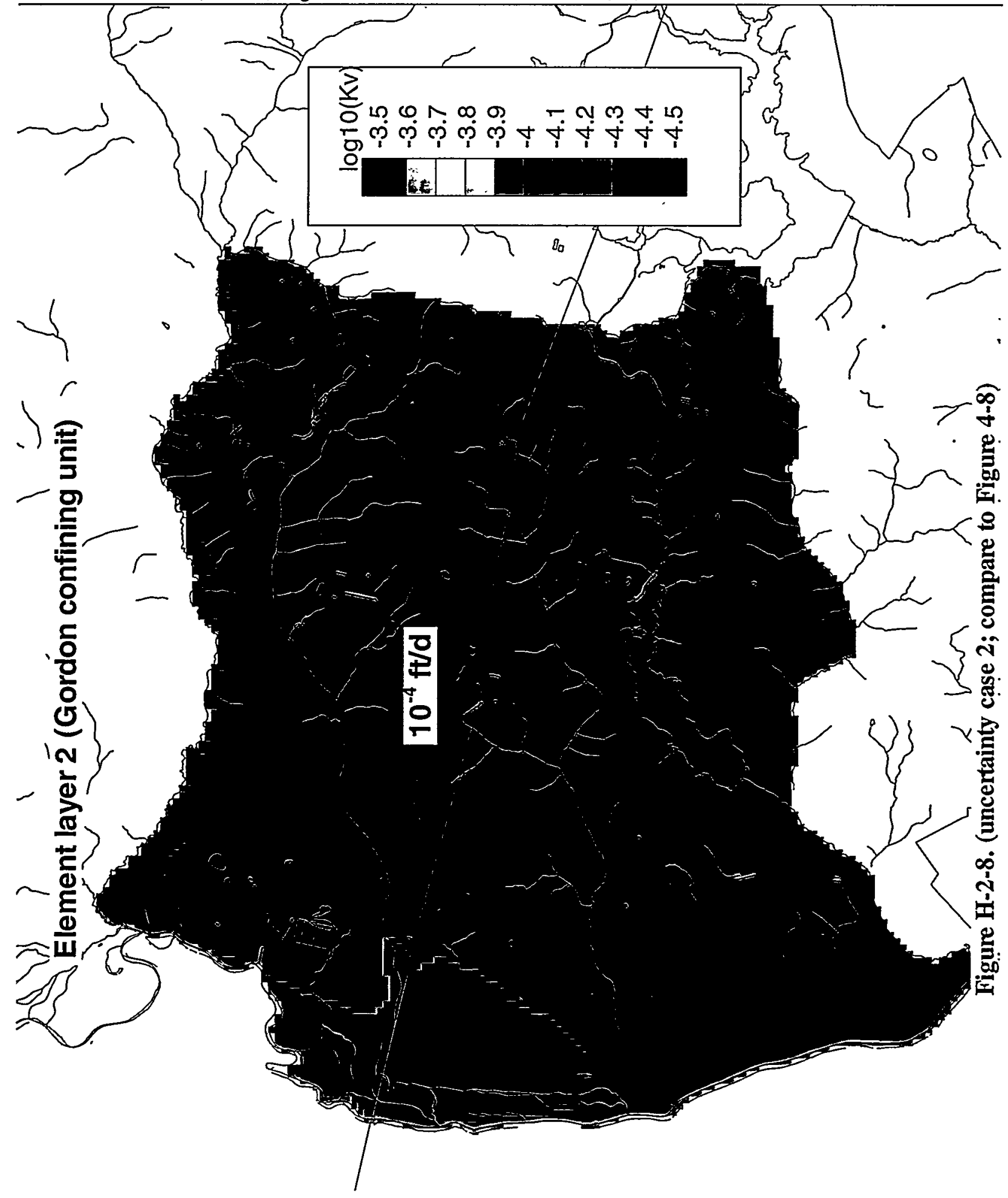




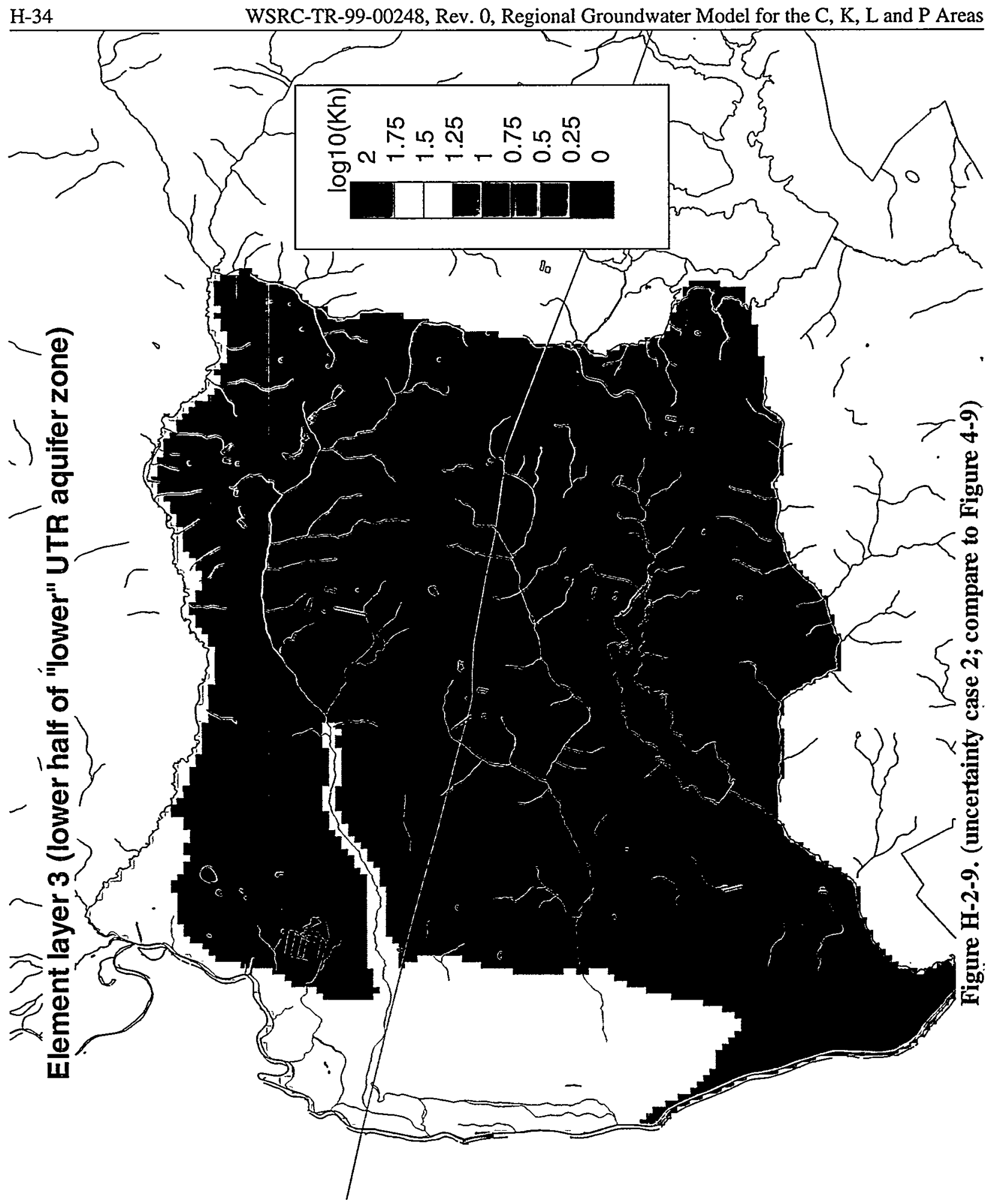




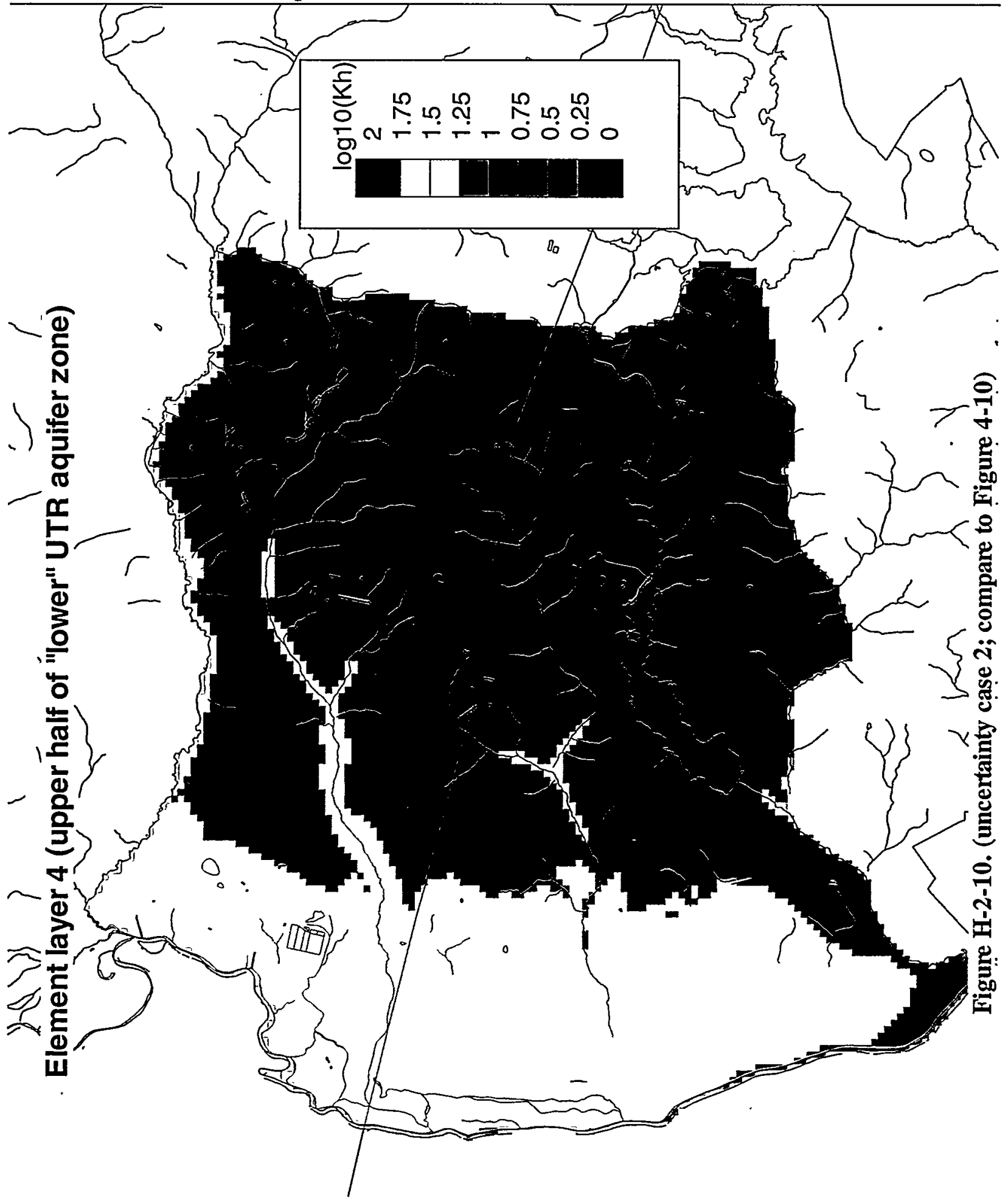




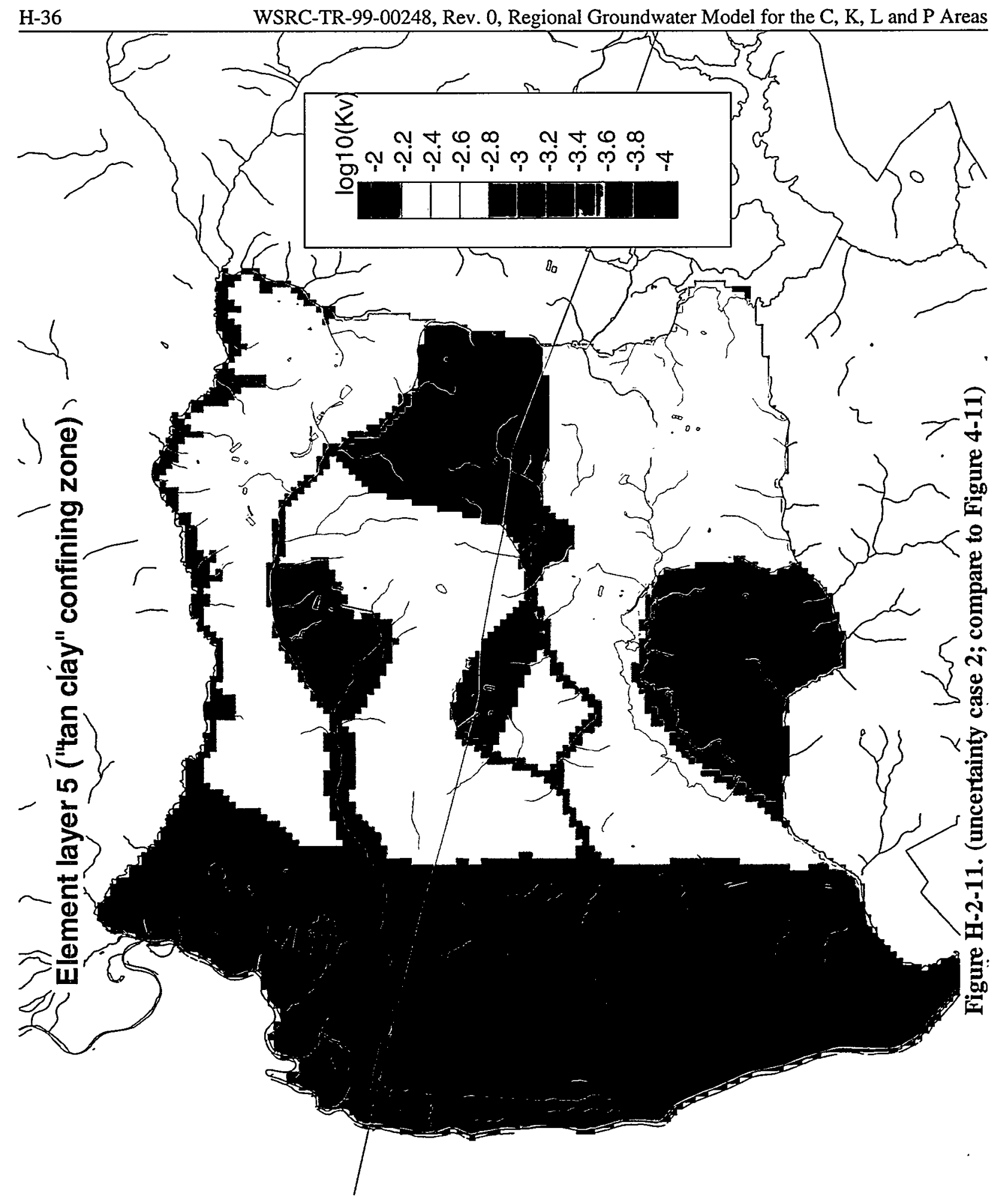




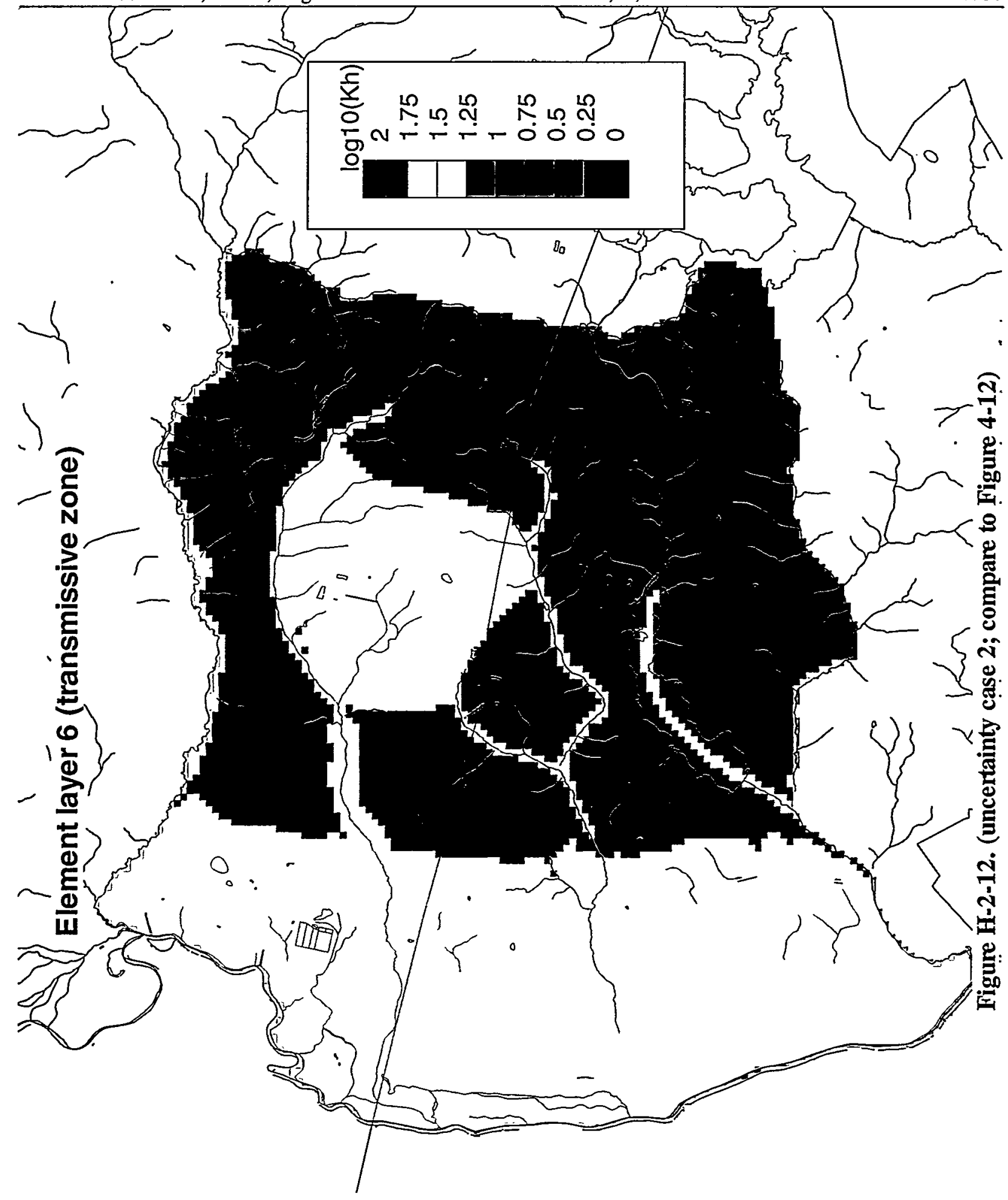




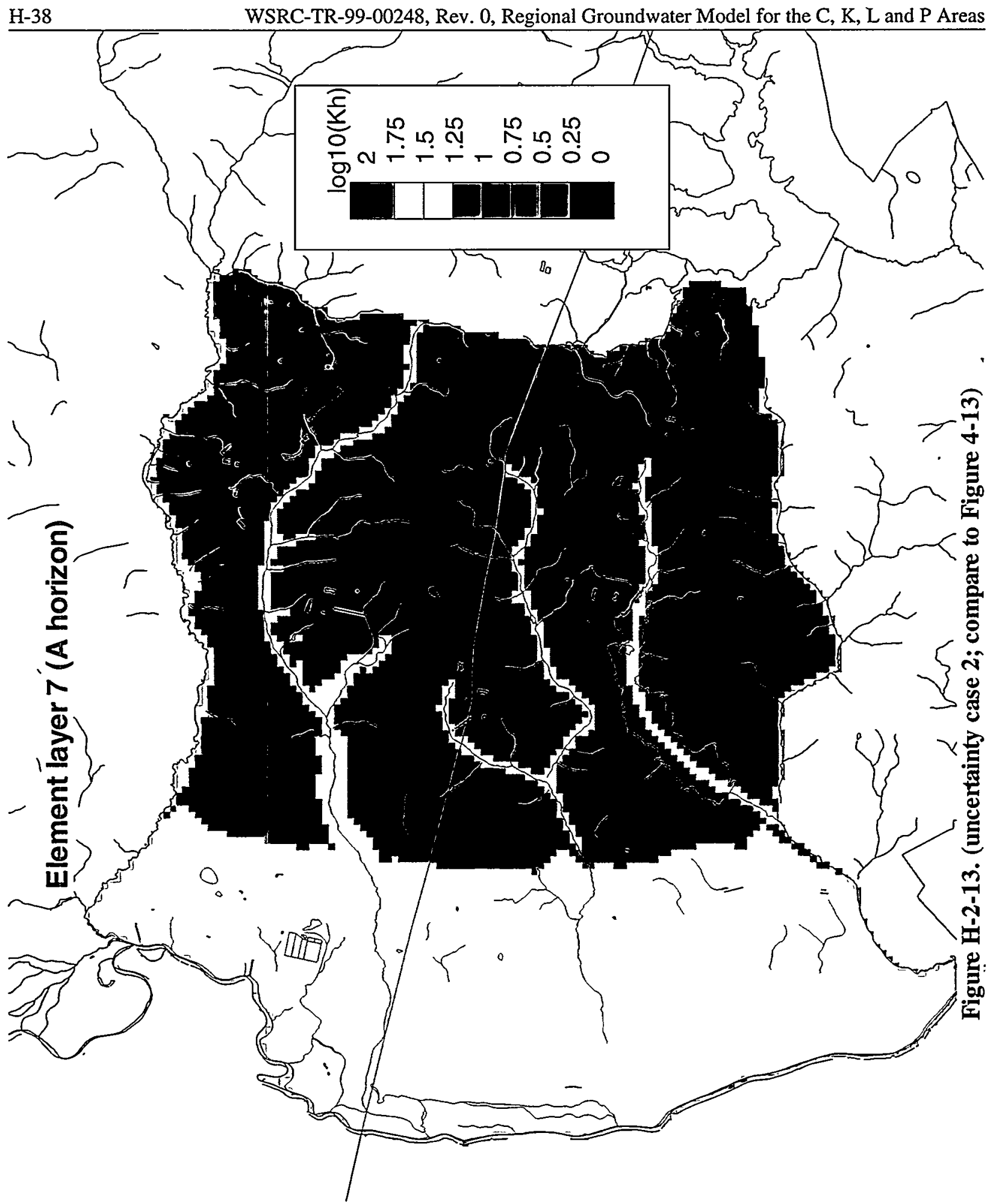




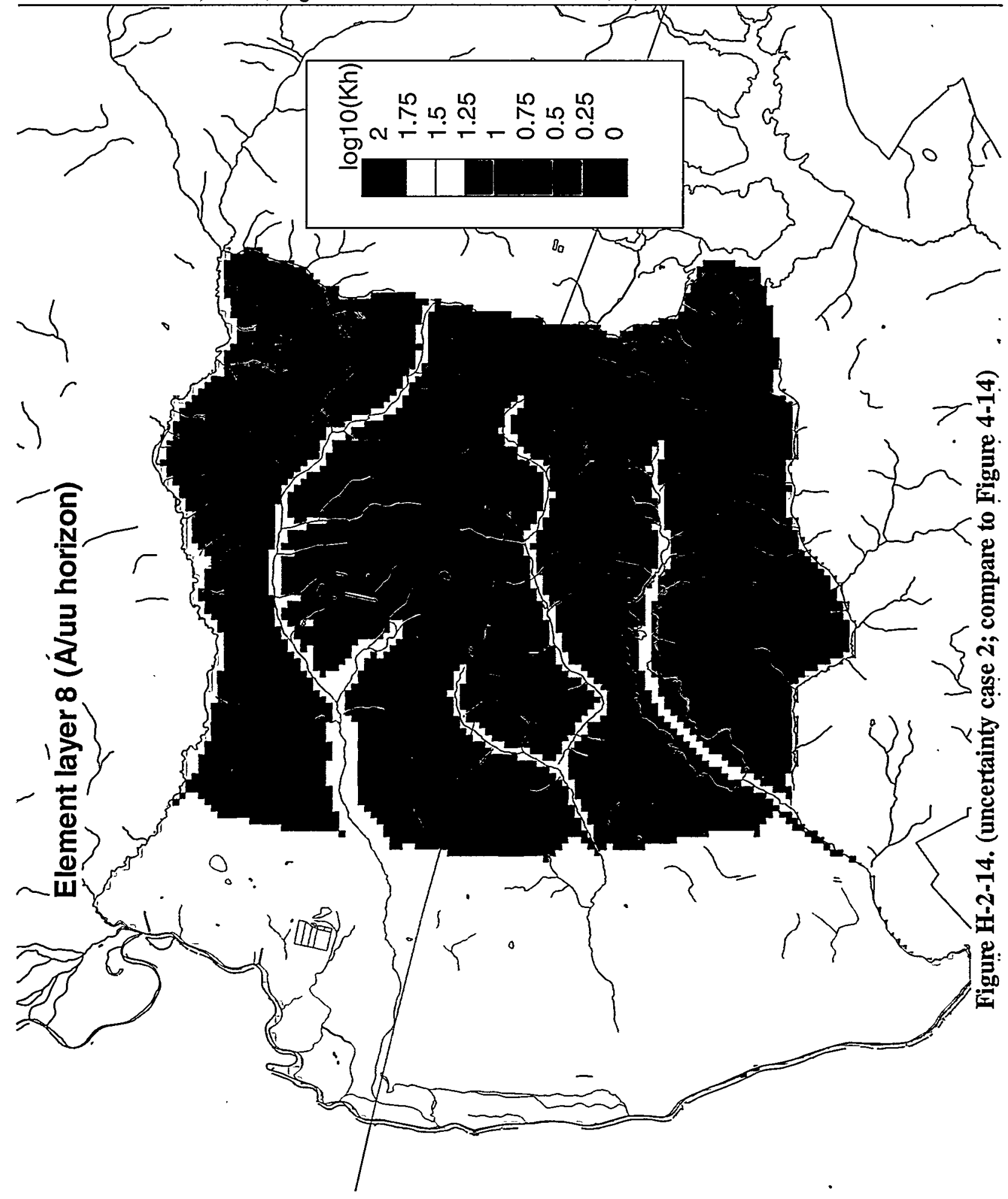




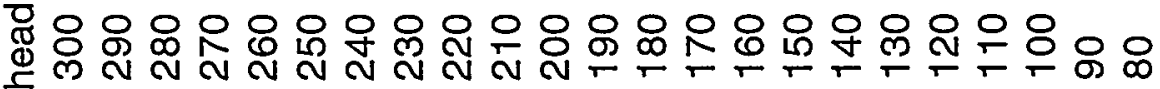

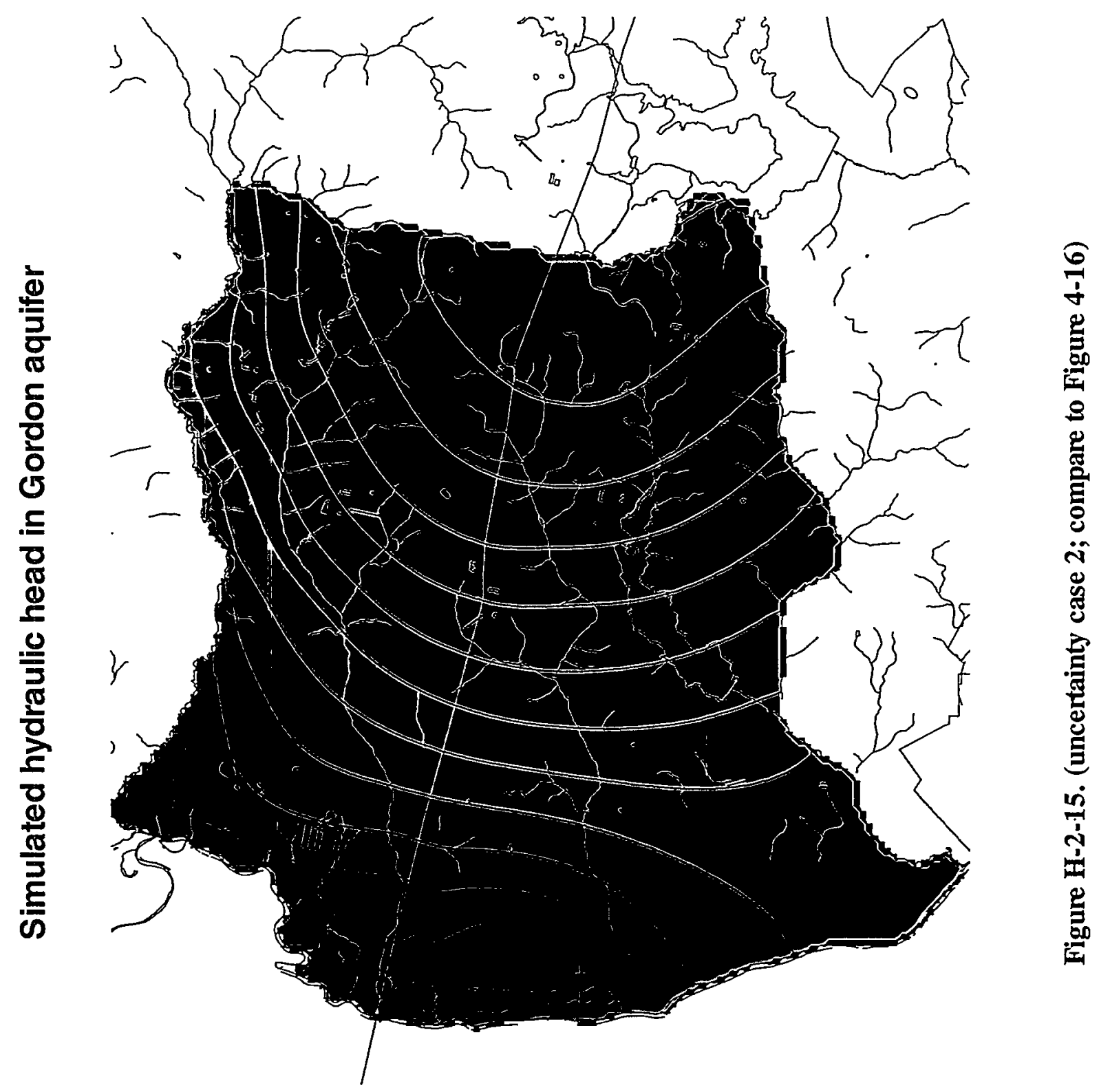




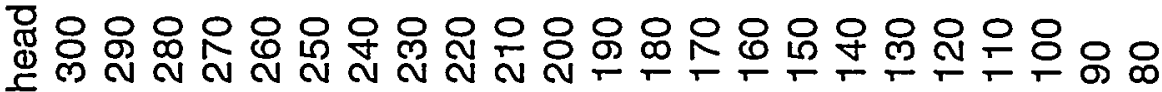
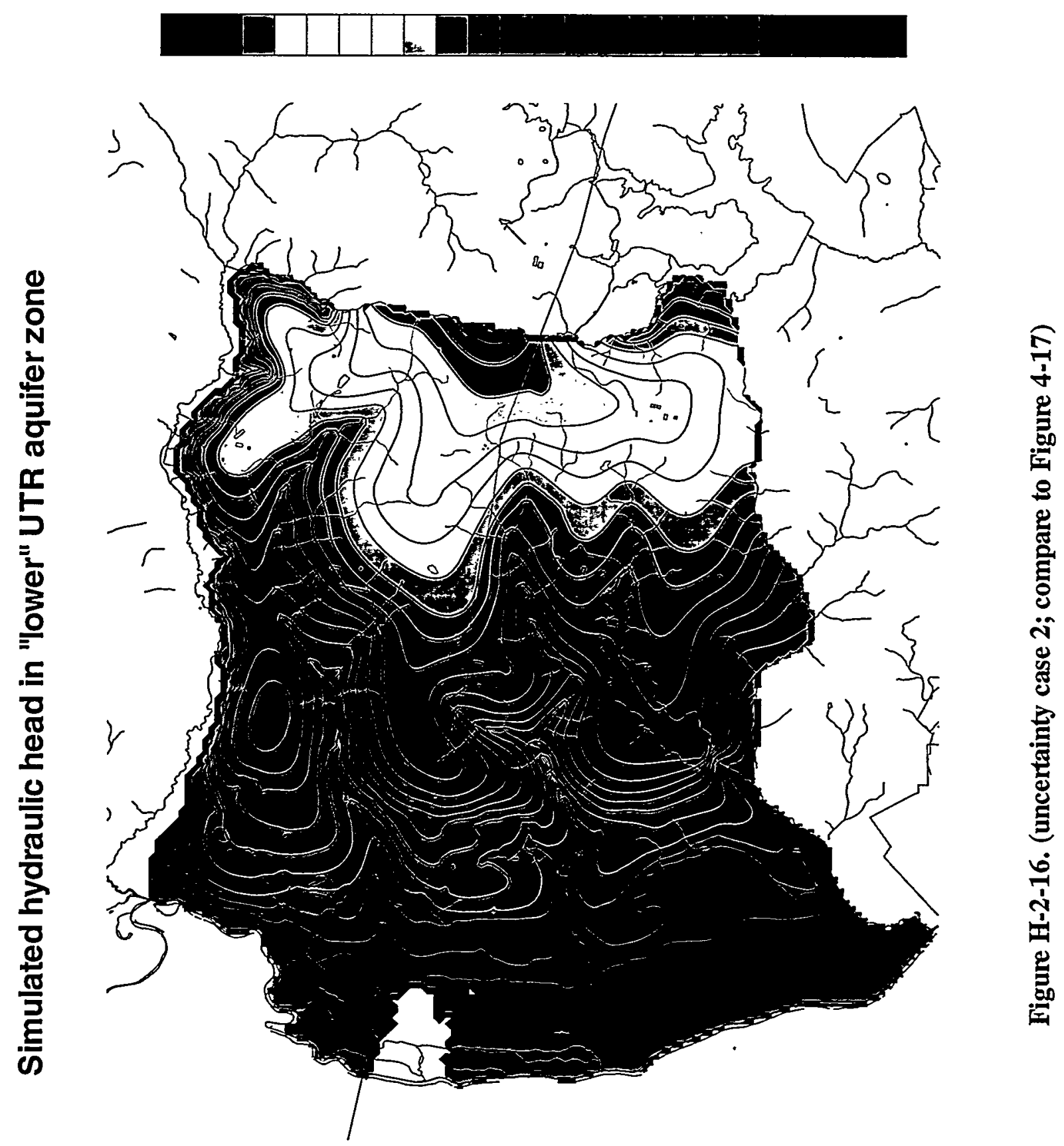


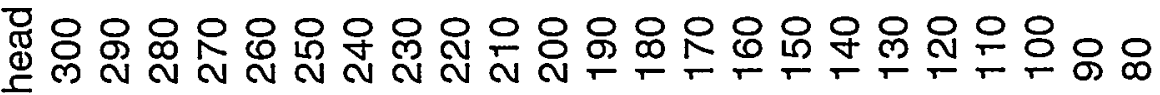

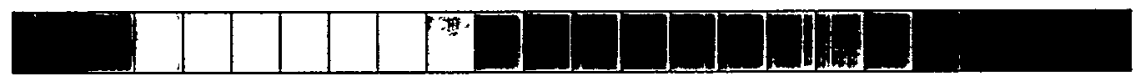

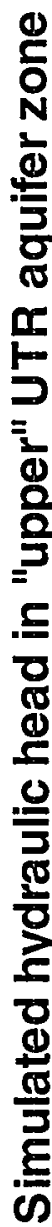
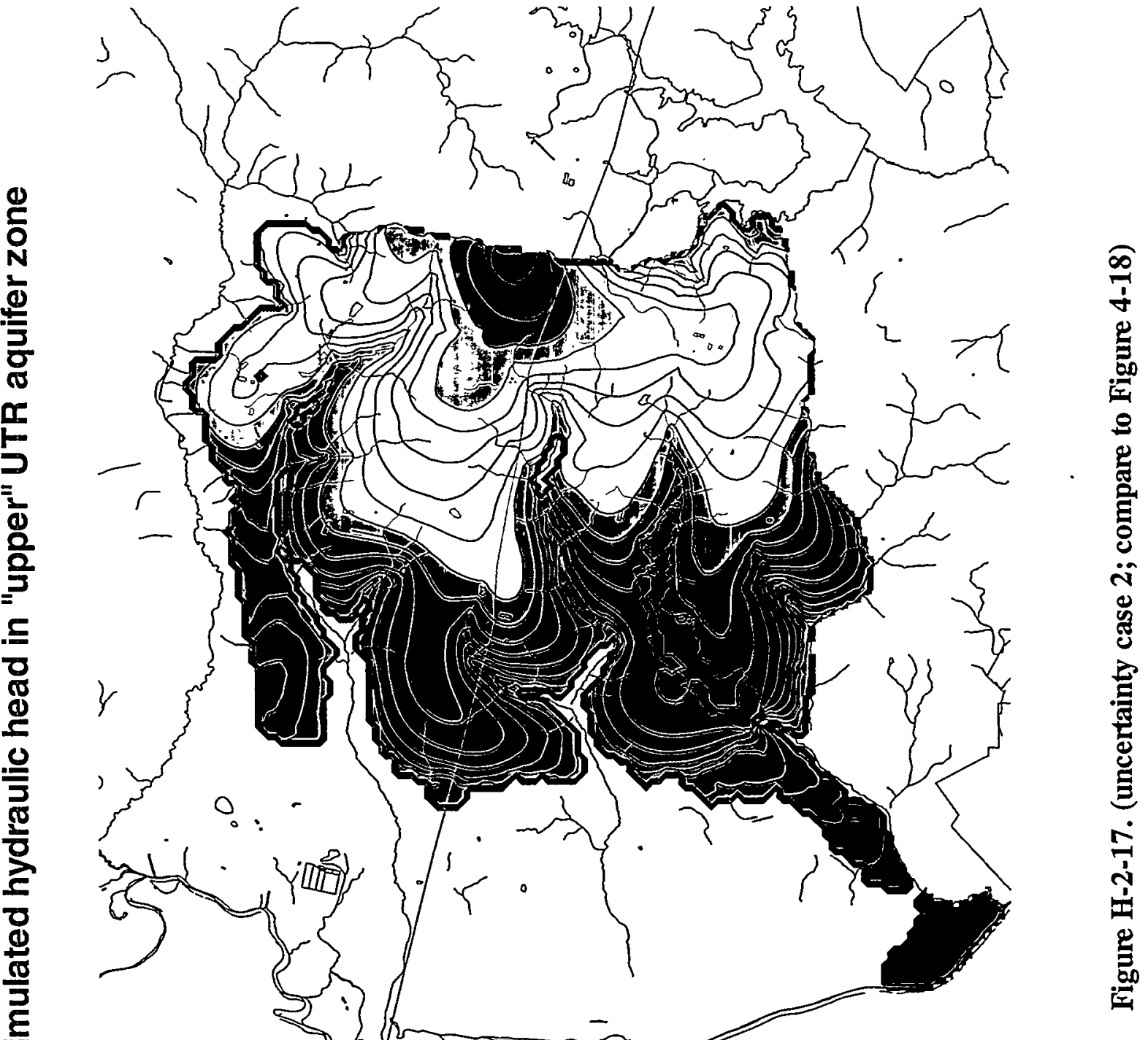


\section{Simulated hydraulic head in aquifer zone containing water table}

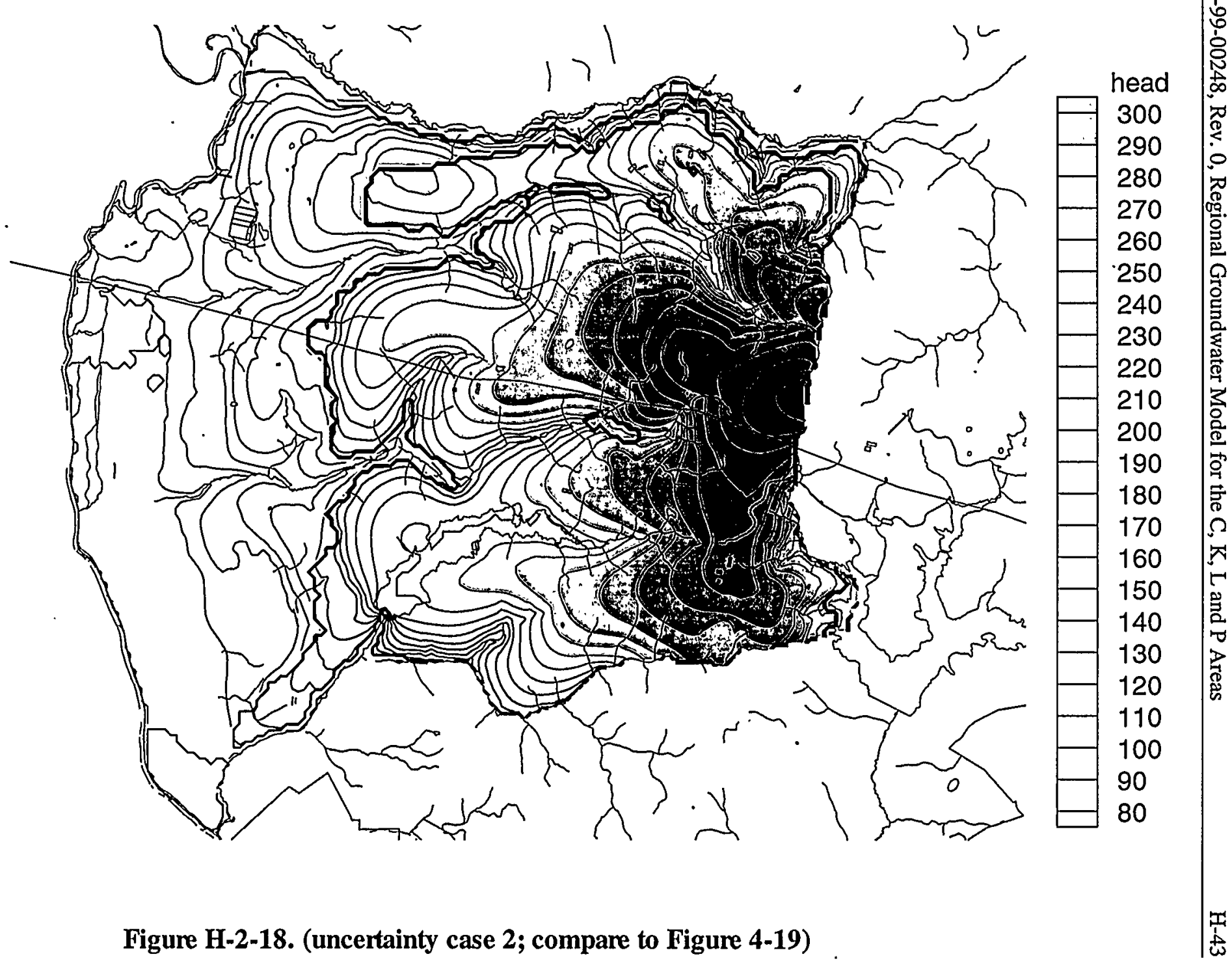




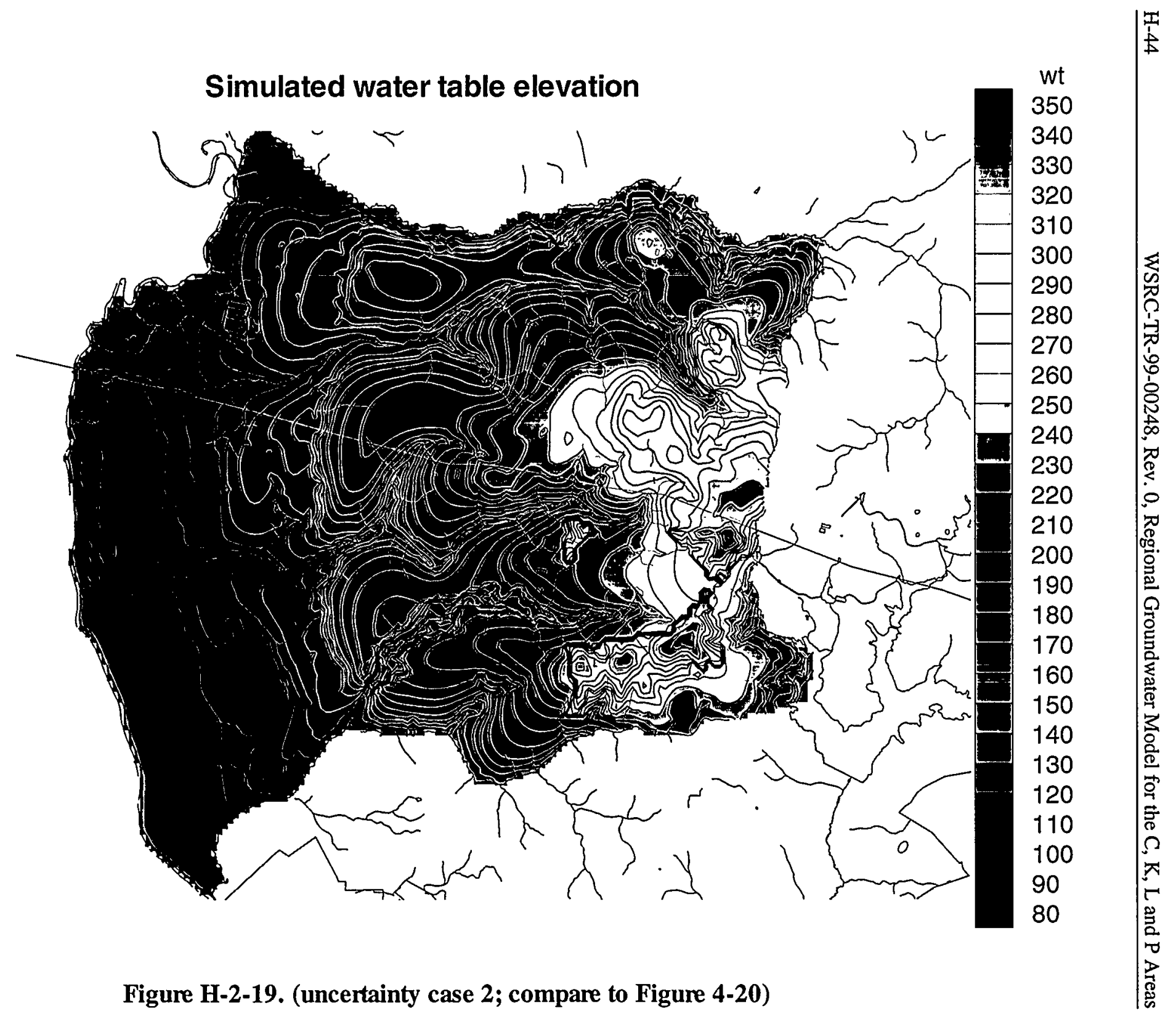




\section{Simulated groundwater recharge (discharge)}

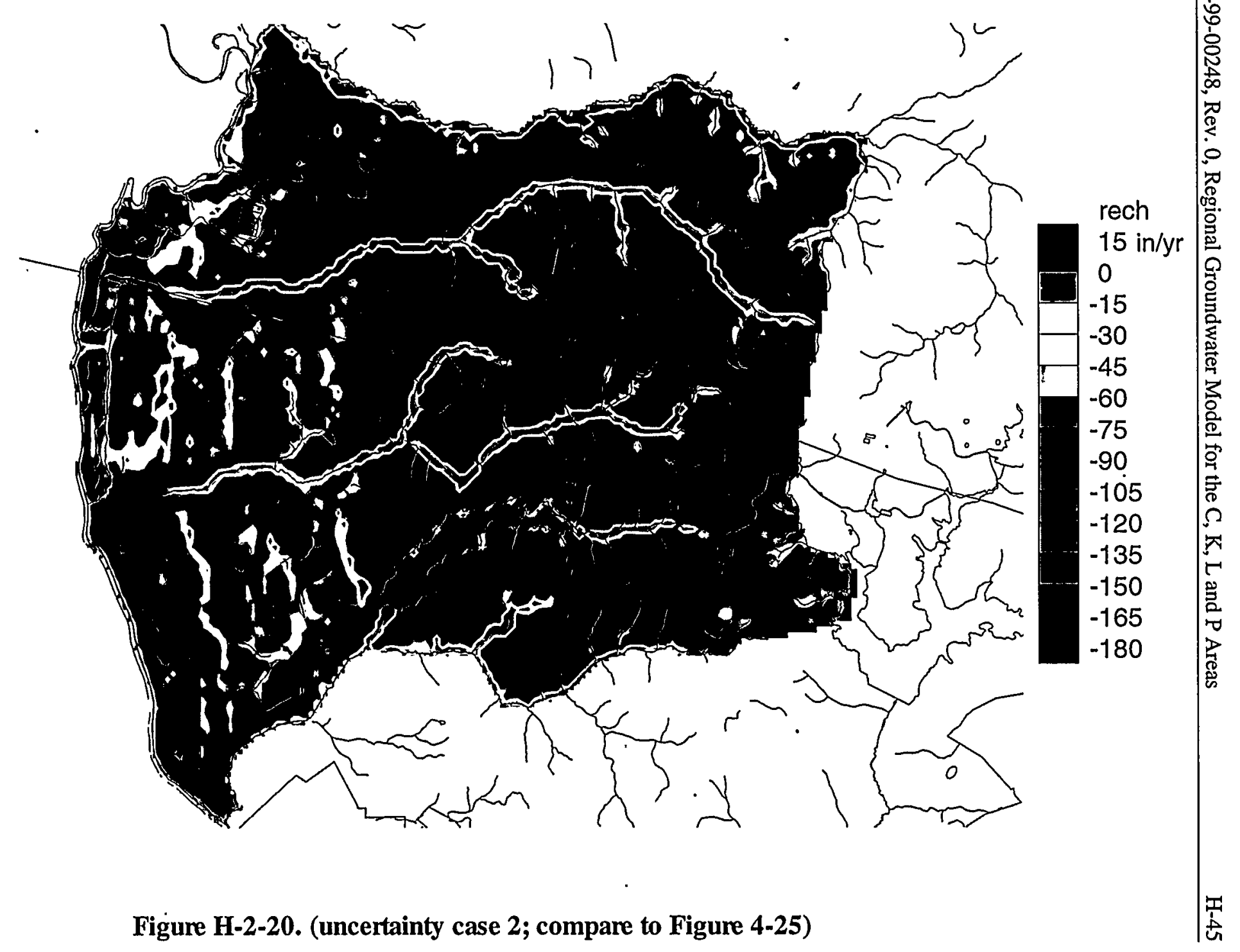




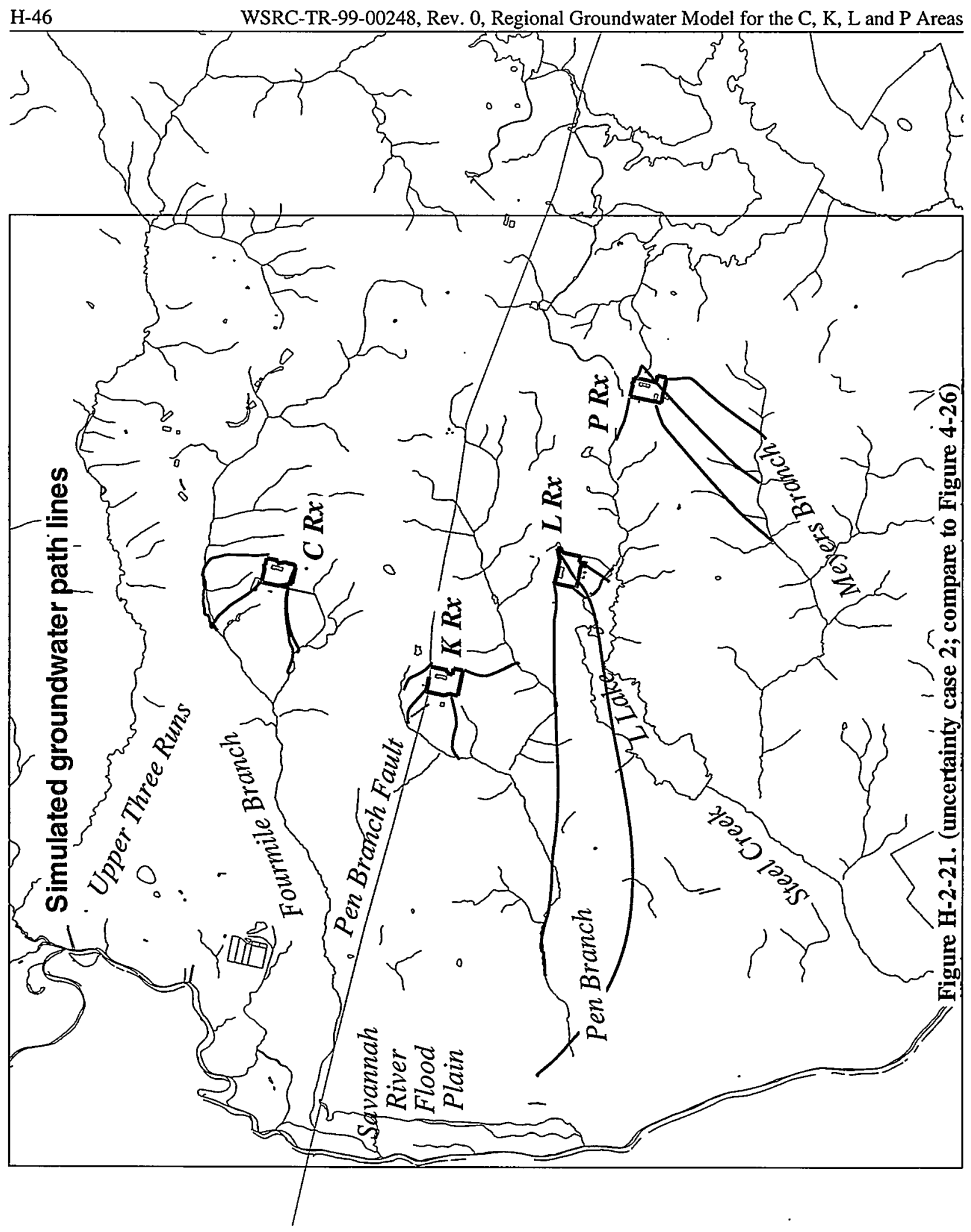




\section{Simulation results for uncertainty case 3}

Uncertainty case 3 involves an increase in Gordon confining unit vertical conductivity by a factor of 5 to $5 \times 10^{-4} \mathrm{ft} /$ day (Table 4-4). Summary calibration results are provided in Table 4-5. This appendix presents detailed simulation results for uncertainty case 3 for comparison to the nominal results shown in figures in the main text. The correspondence between figures for the nominal and uncertainty case 3 is as follows:

\begin{tabular}{|l|l|l|}
\hline Plot type & Nominal case & Uncertainty case 3 \\
\hline Head residual summary & Figure 4-1 & Figure H-3-1 \\
\hline Head residuals in Gordon aquifer & Figure 4-2 & Figure H-3-2 \\
\hline Head residuals in "lower" UTRA & Figure 4-3 & Figure H-3-3 \\
\hline Head residuals in transmissive zone & Figure 4-4 & Figure H-3-4 \\
\hline Head residuals in AA horizon & Figure 4-5 & Figure H-3-5 \\
\hline Head residuals in A/uu horizons & Figure 4-6 & Figure H-3-6 \\
\hline Kh in element layer 1 & Figure 4-7 & Figure H-3-7 \\
\hline Kv in element layer 2 & Figure 4-8 & Figure H-3-8 \\
\hline Kh in element layer 3 & Figure 4-9 & Figure H-3-9 \\
\hline Kh in element layer 4 & Figure 4-10 & Figure H-3-10 \\
\hline Kv in element layer 5 & Figure 4-11 & Figure H-3-11 \\
\hline Kh in element layer 6 & Figure 4-12 & Figure H-3-12 \\
\hline Kh in element layer 7 & Figure 4-13 & Figure H-3-13 \\
\hline Kh in element layer 8 & Figure 4-14 & Figure H-3-14 \\
\hline Gordon aquifer head & Figure 4-16 & Figure H-3-15 \\
\hline "Lower" UTRA head & Figure 4-17 & Figure H-3-16 \\
\hline "Upper" UTRA head & Figure 4-18 & Figure H-3-17 \\
\hline Head in aquifer containing water table & Figure 4-19 & Figure H-3-18 \\
\hline Water table & Figure 4-20 & Figure H-3-19 \\
\hline Recharge/discharge & Figure 4-25 & Figure H-3-20 \\
\hline Example particle tracing & Figure 4-26 & Figure H-3-21 \\
\hline
\end{tabular}



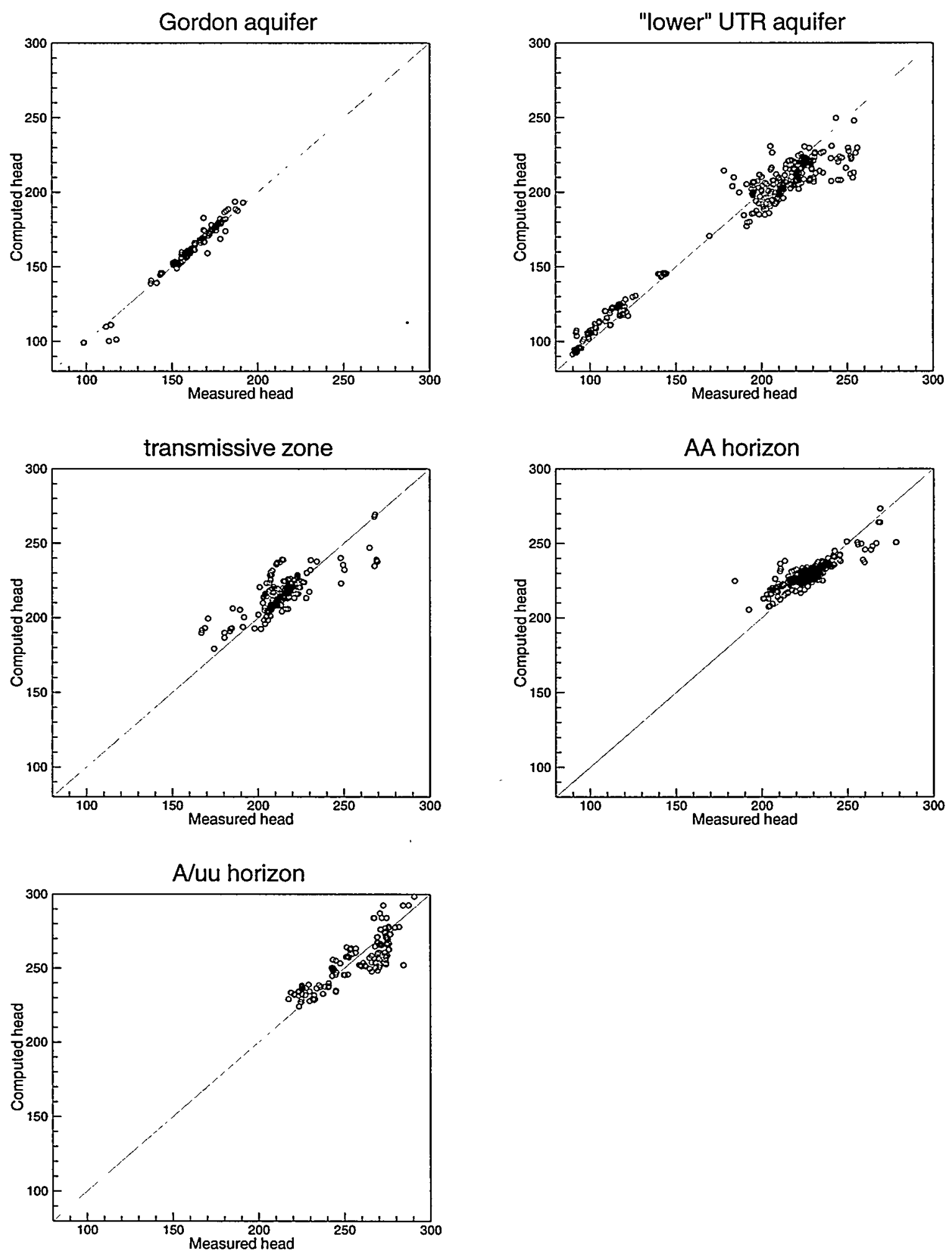

Figure H-3-1. (uncertainty case 3; compare to Figure 4-1) 


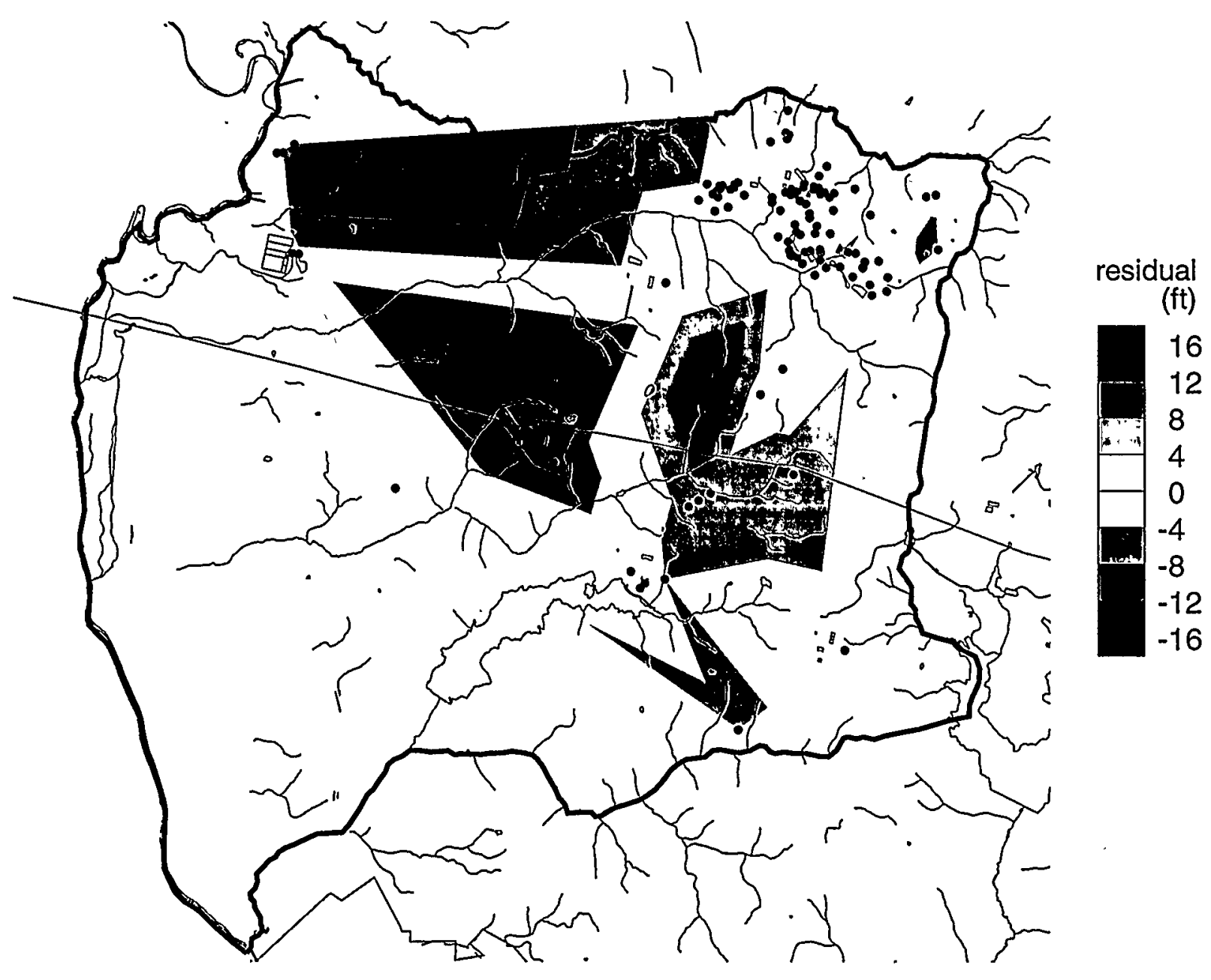

Figure H-3-2. (uncertainty case 3; compare to Figure 4-2) 

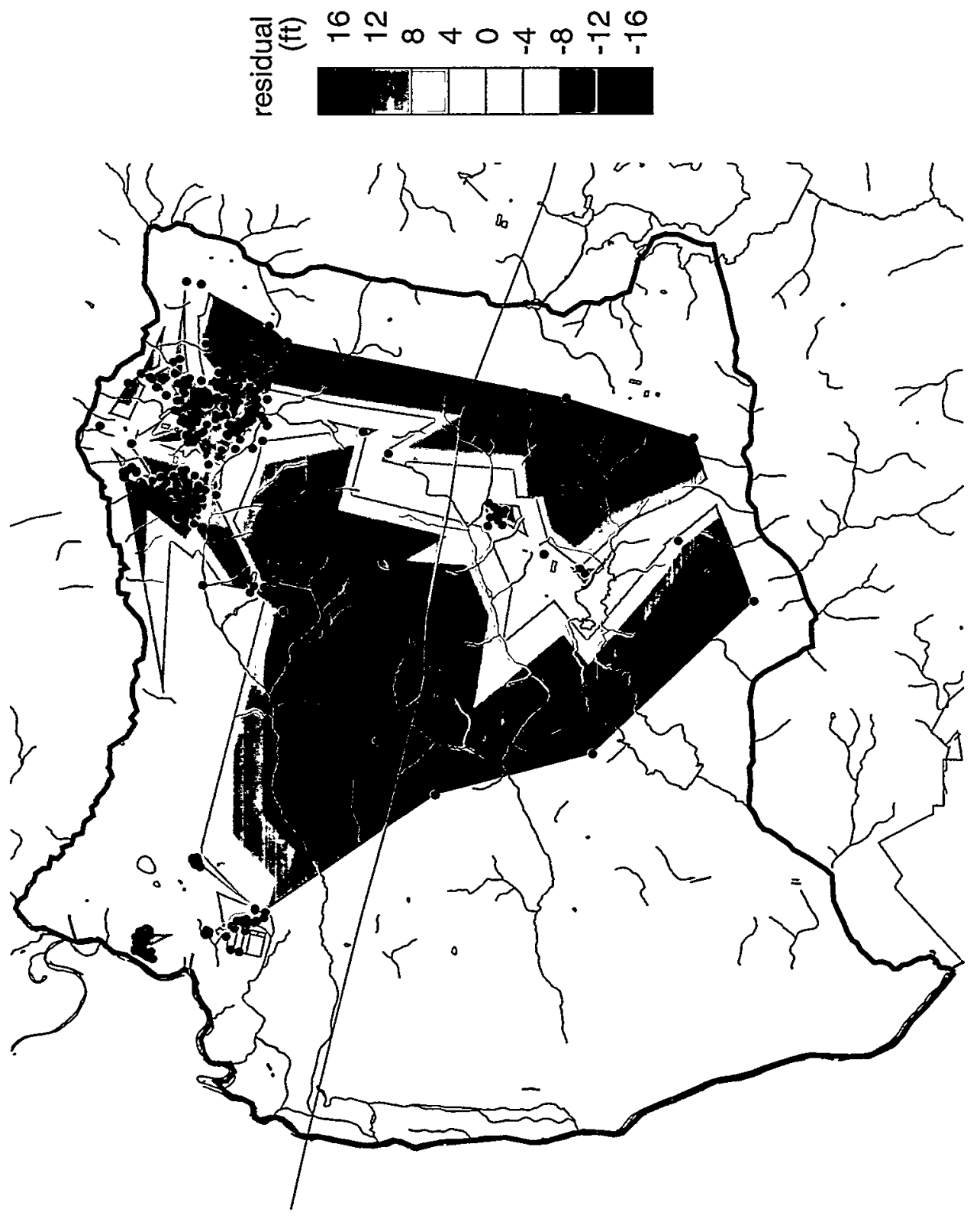

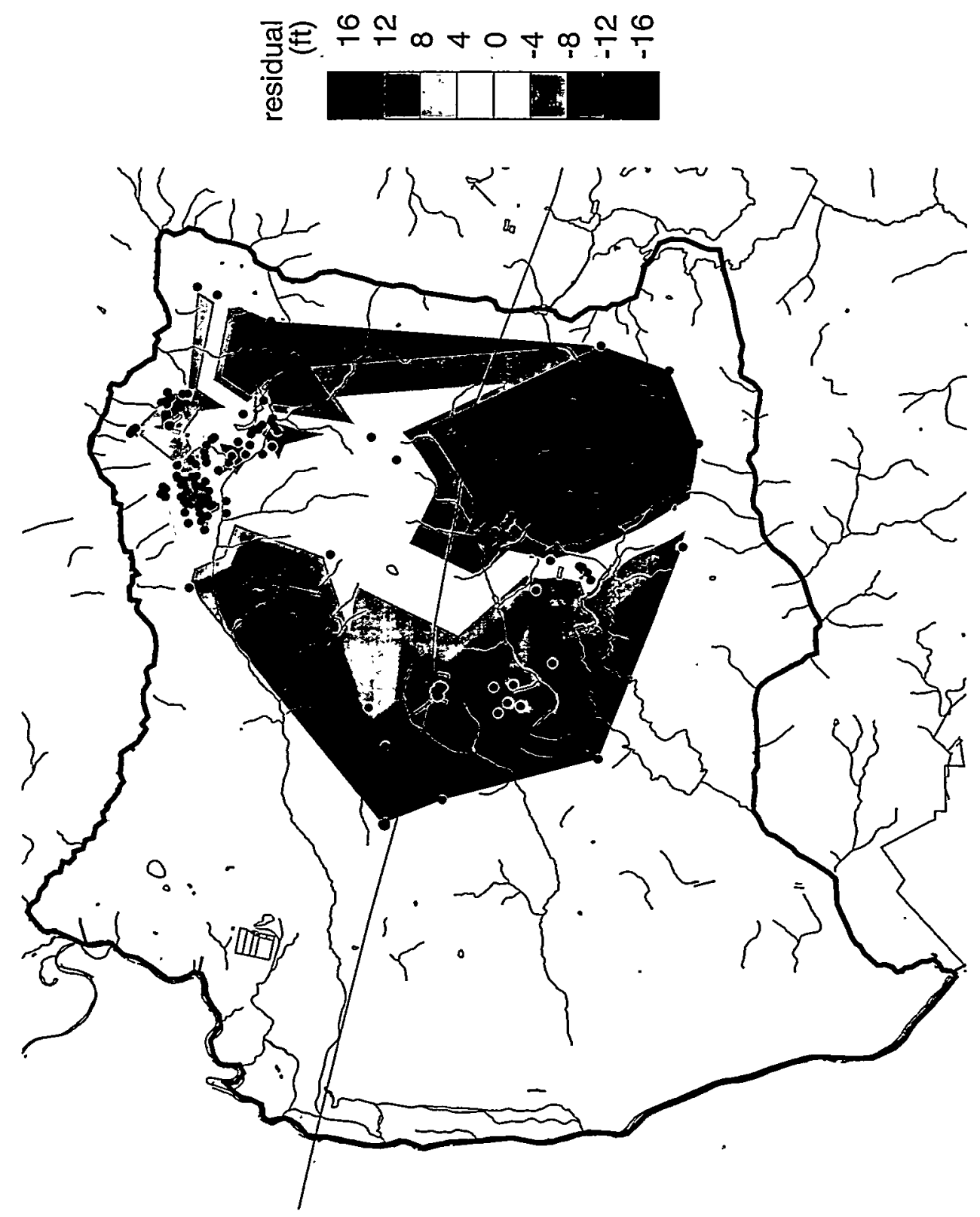


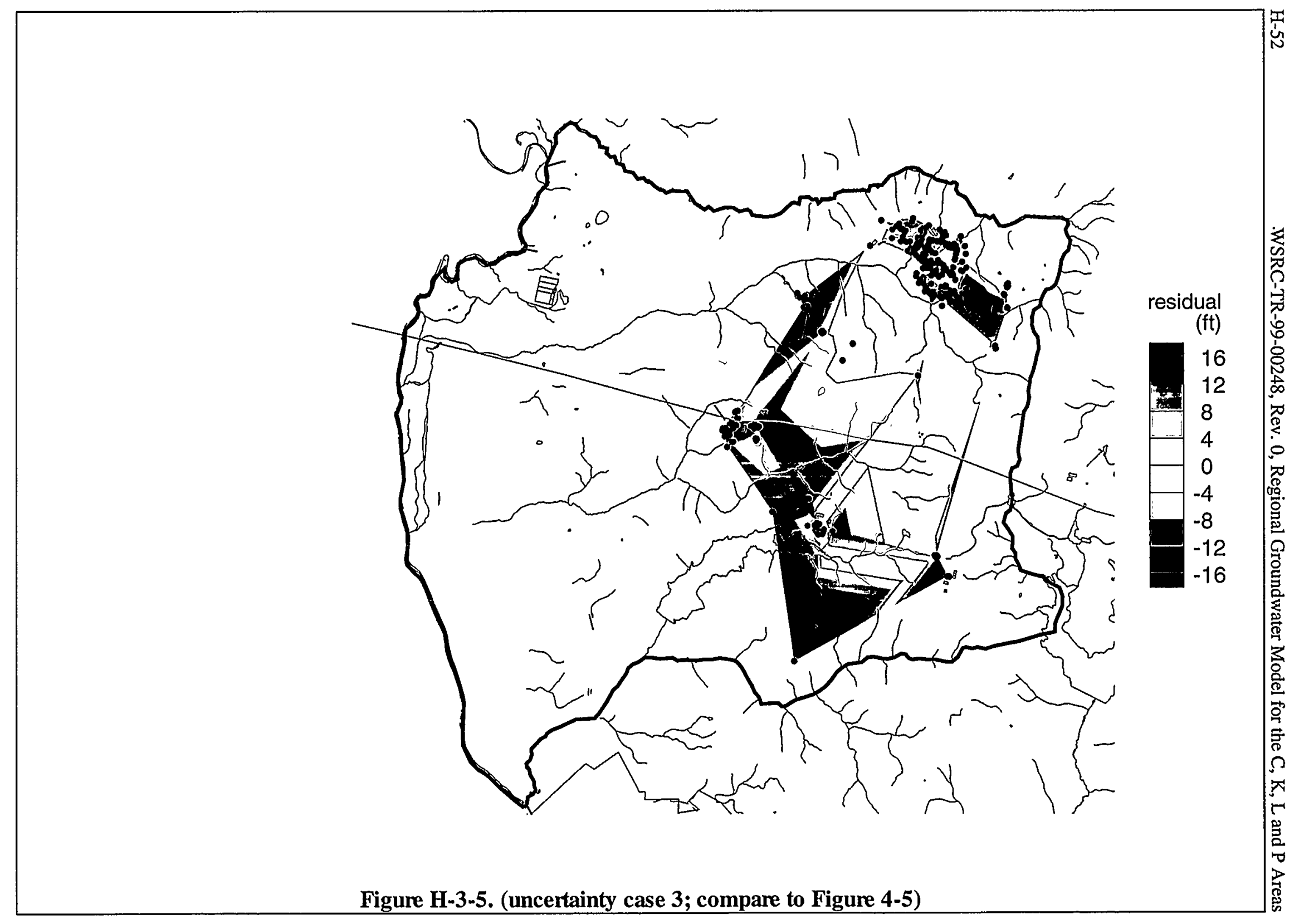




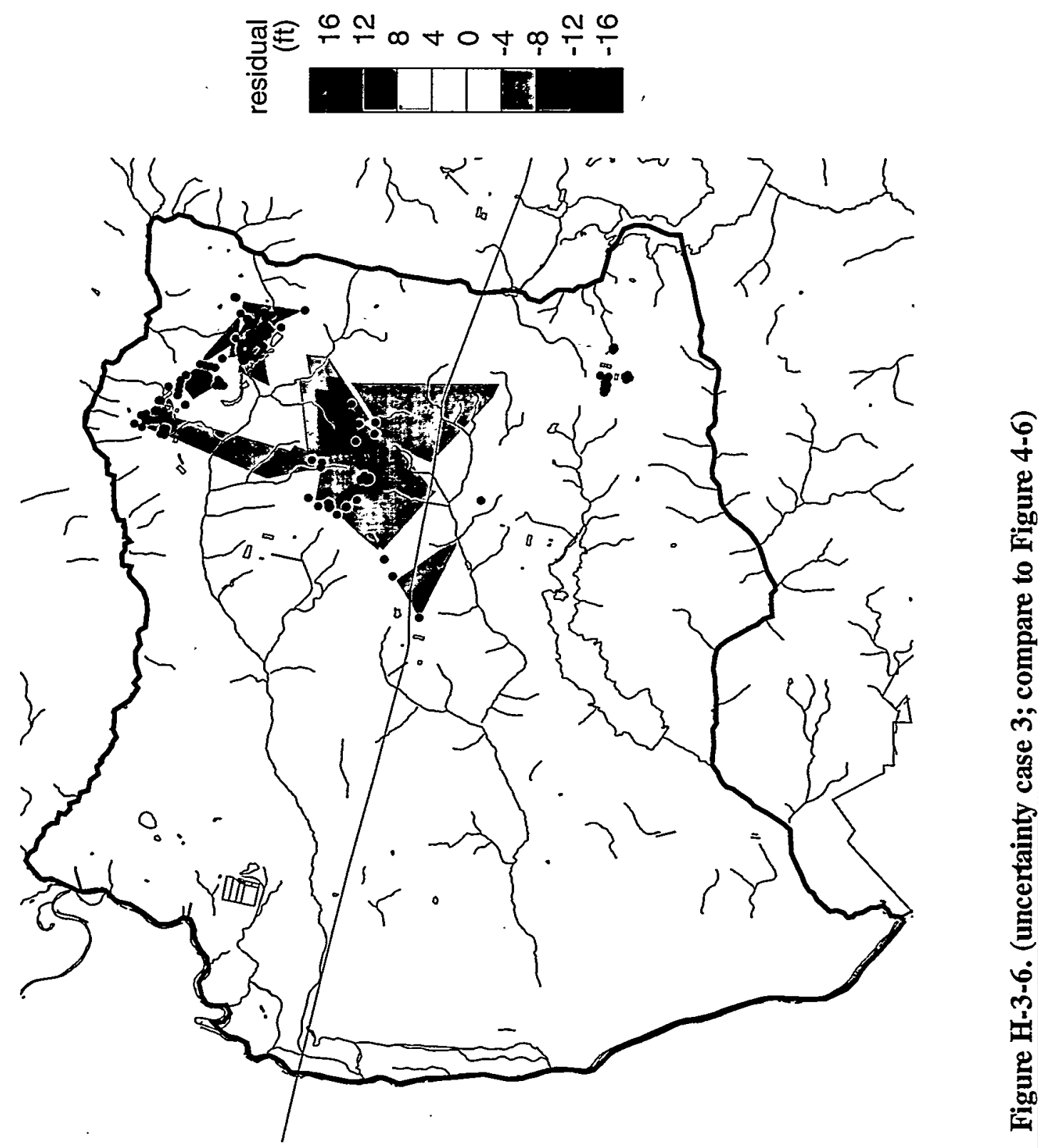




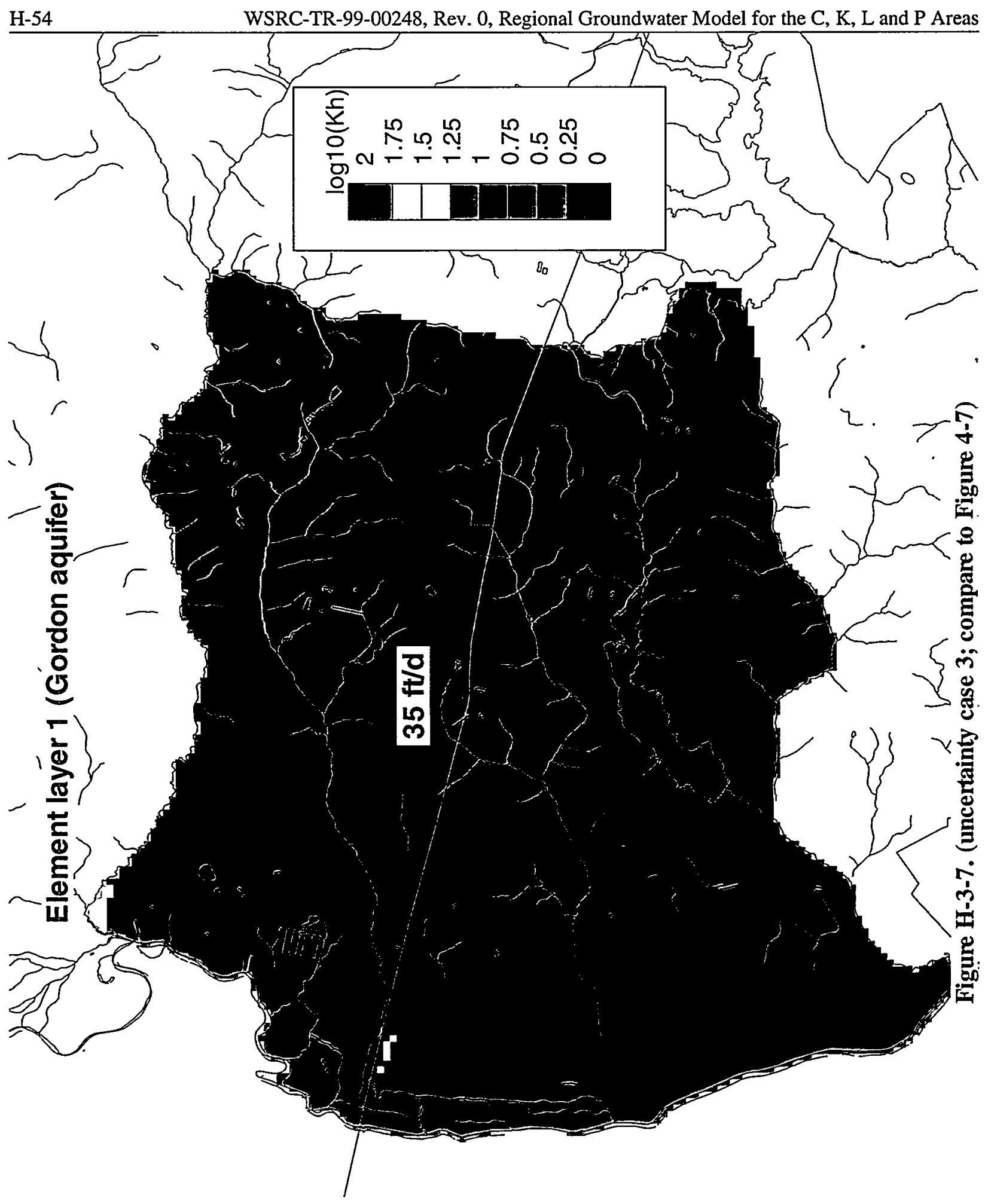




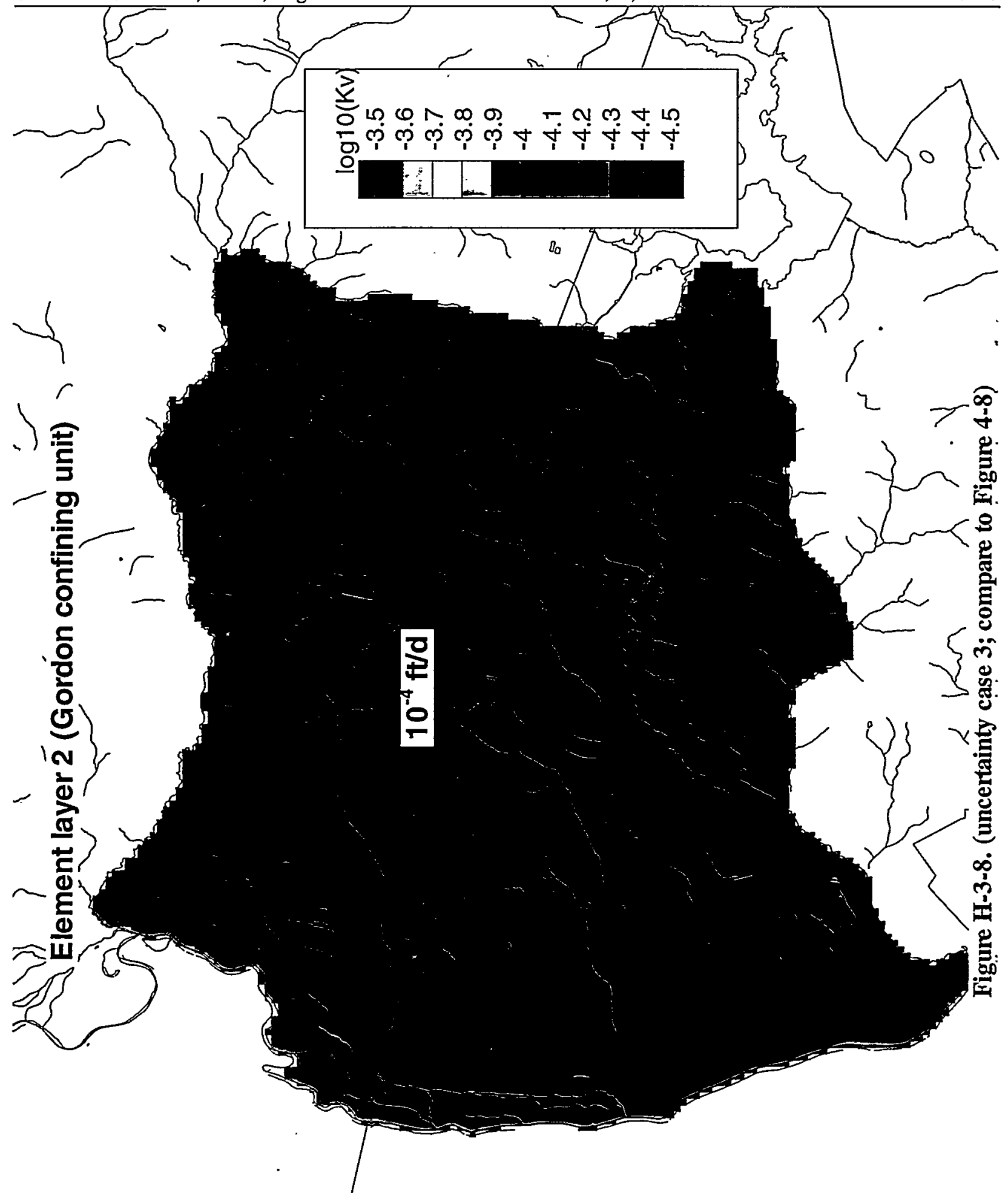




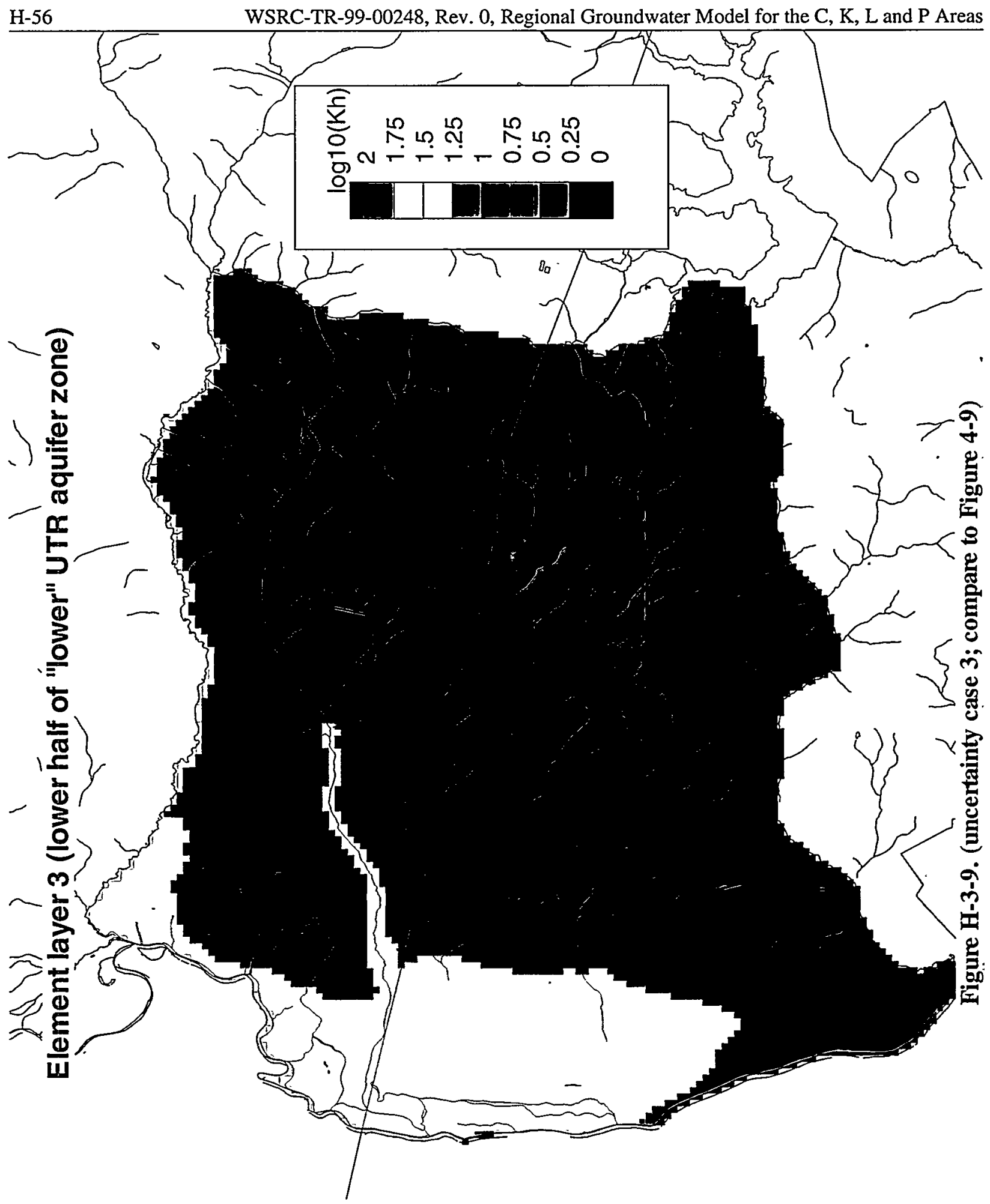




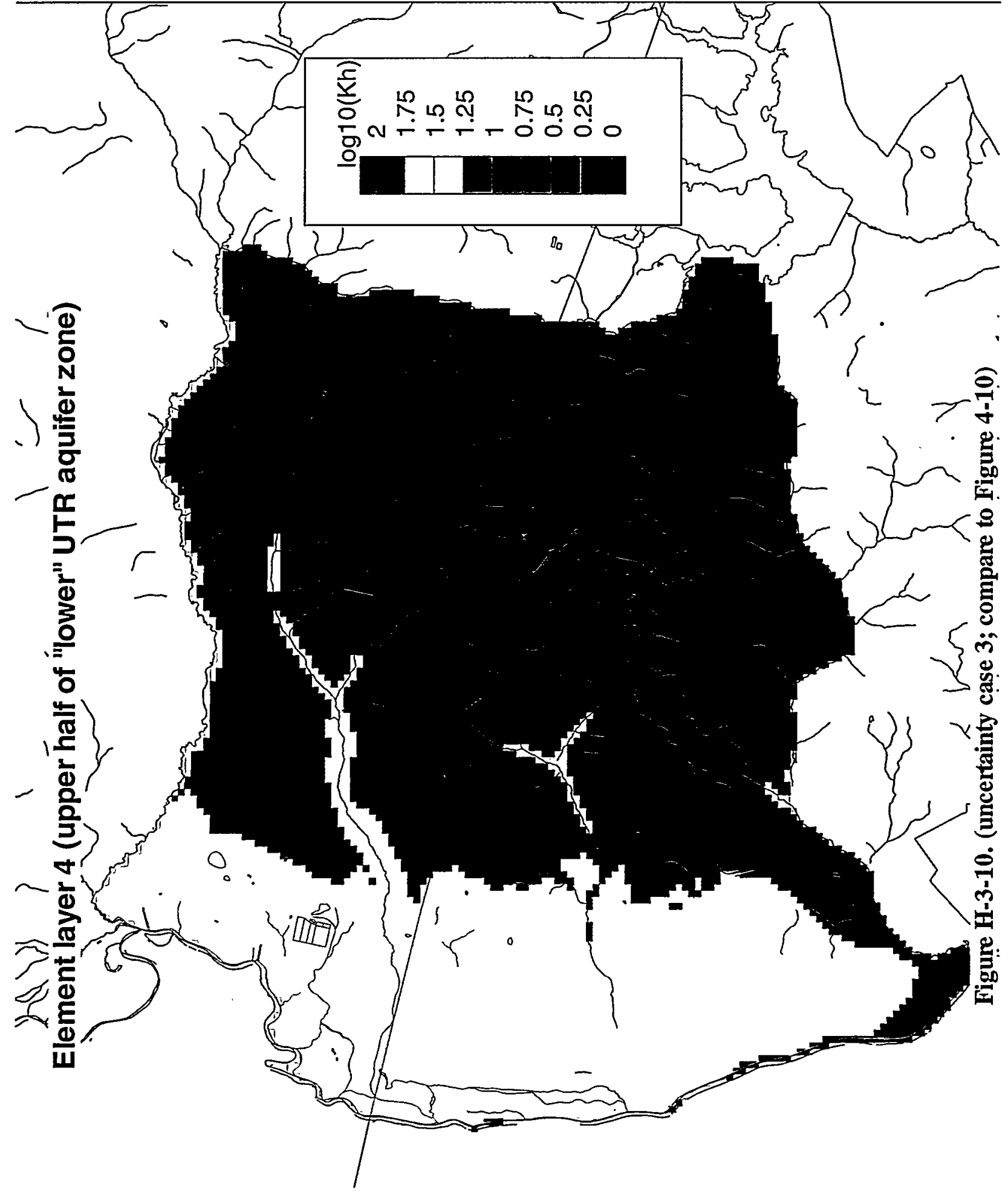




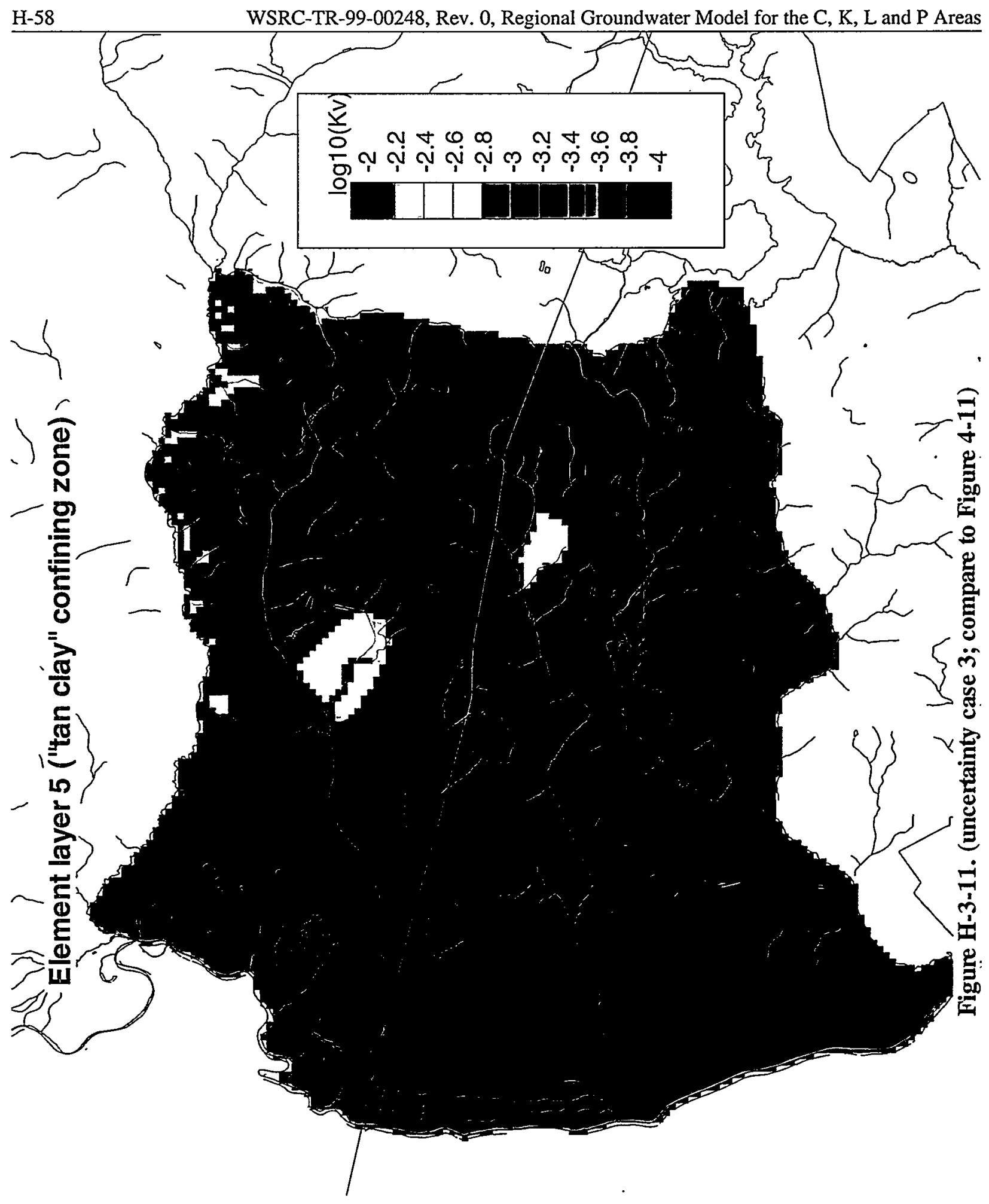




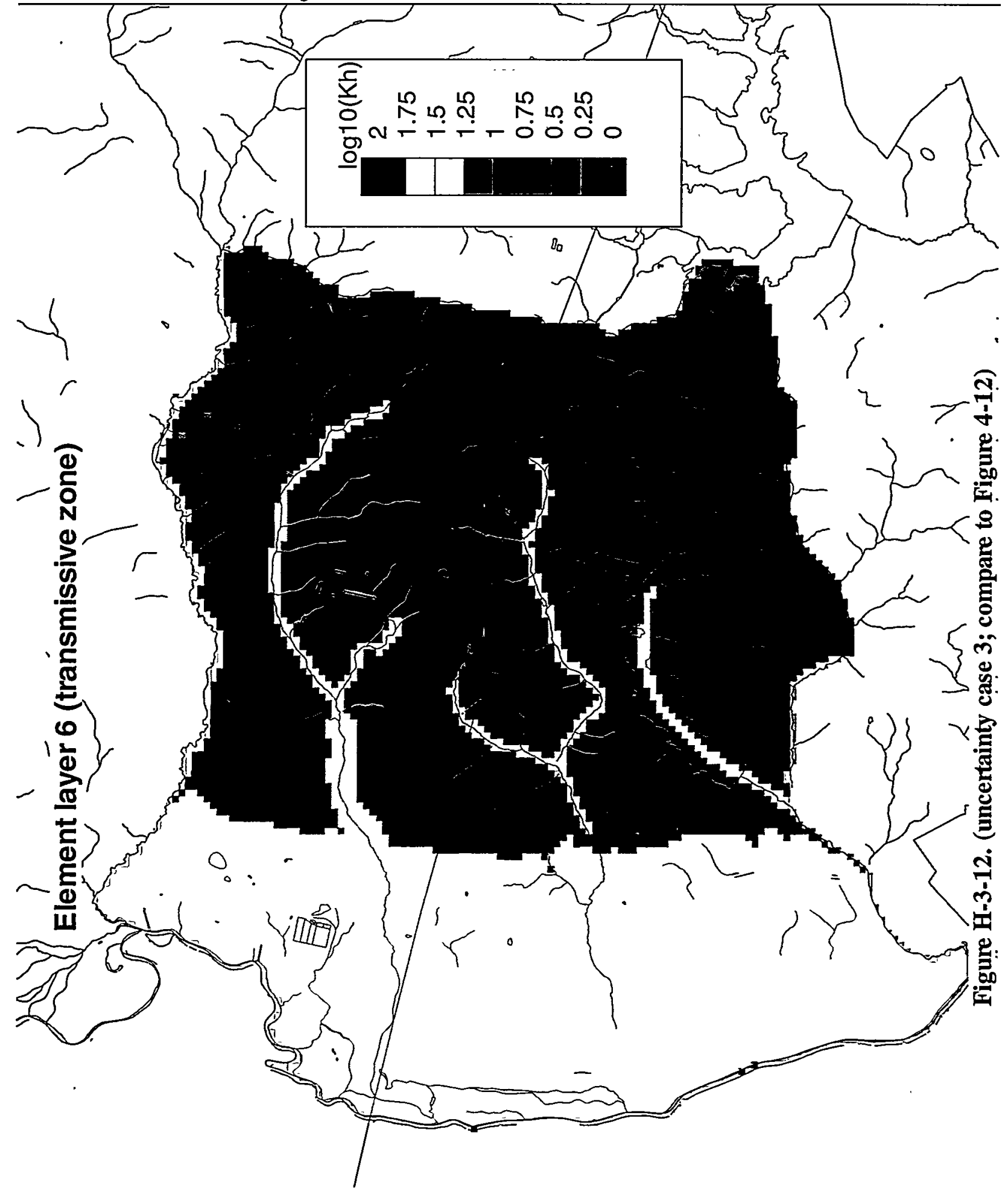




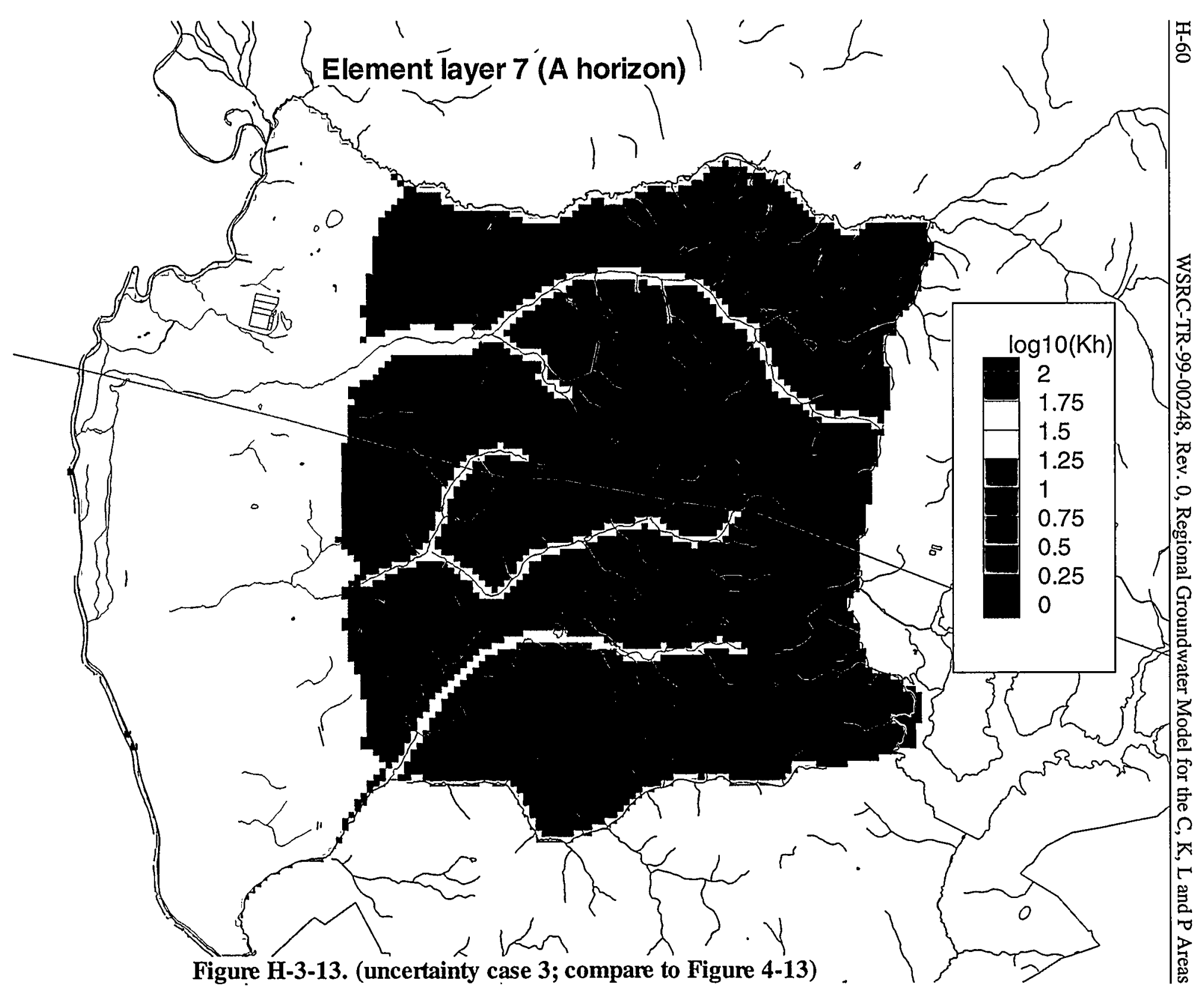




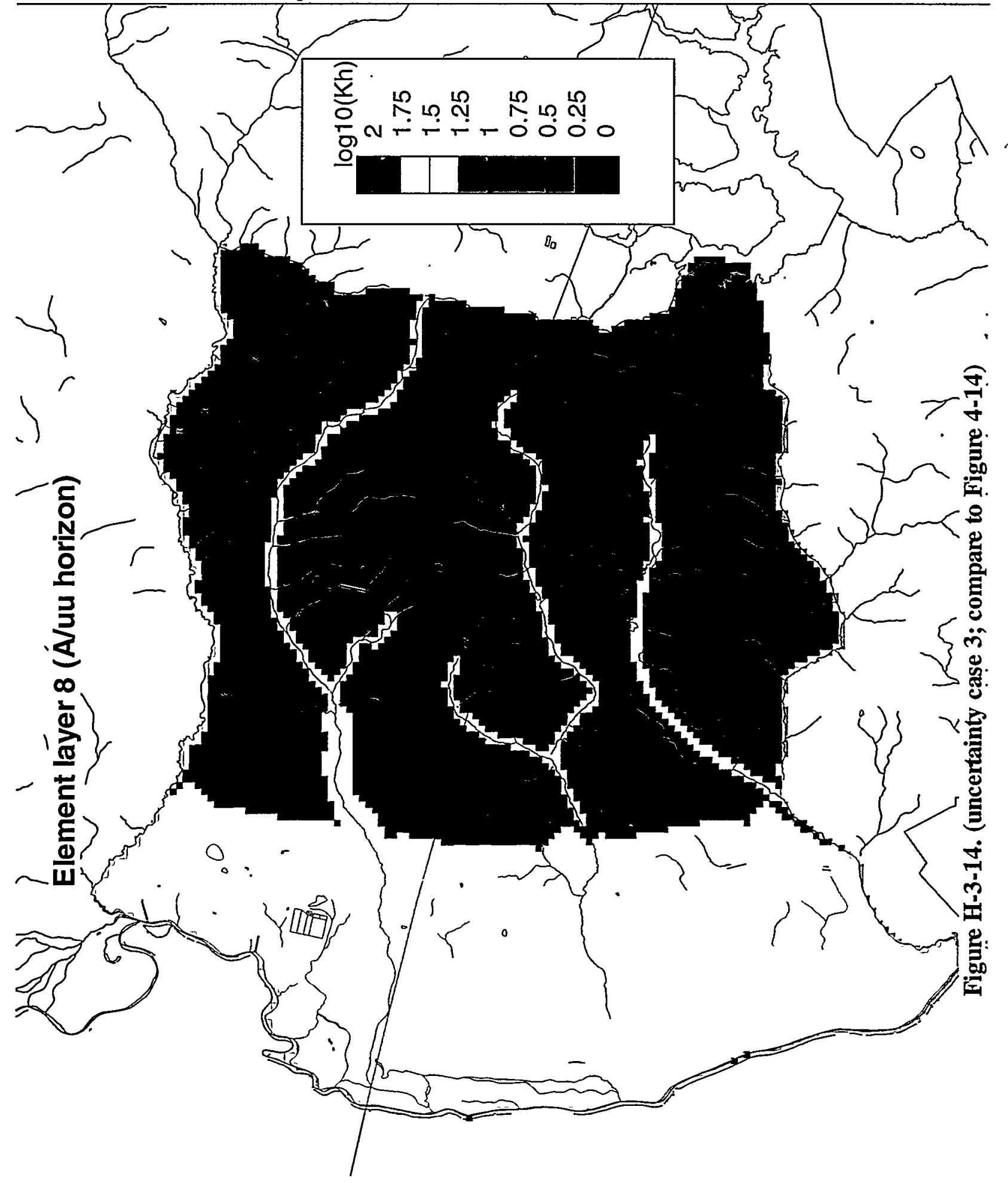




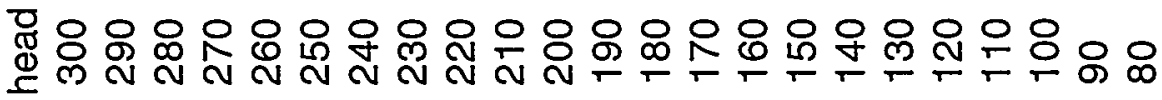

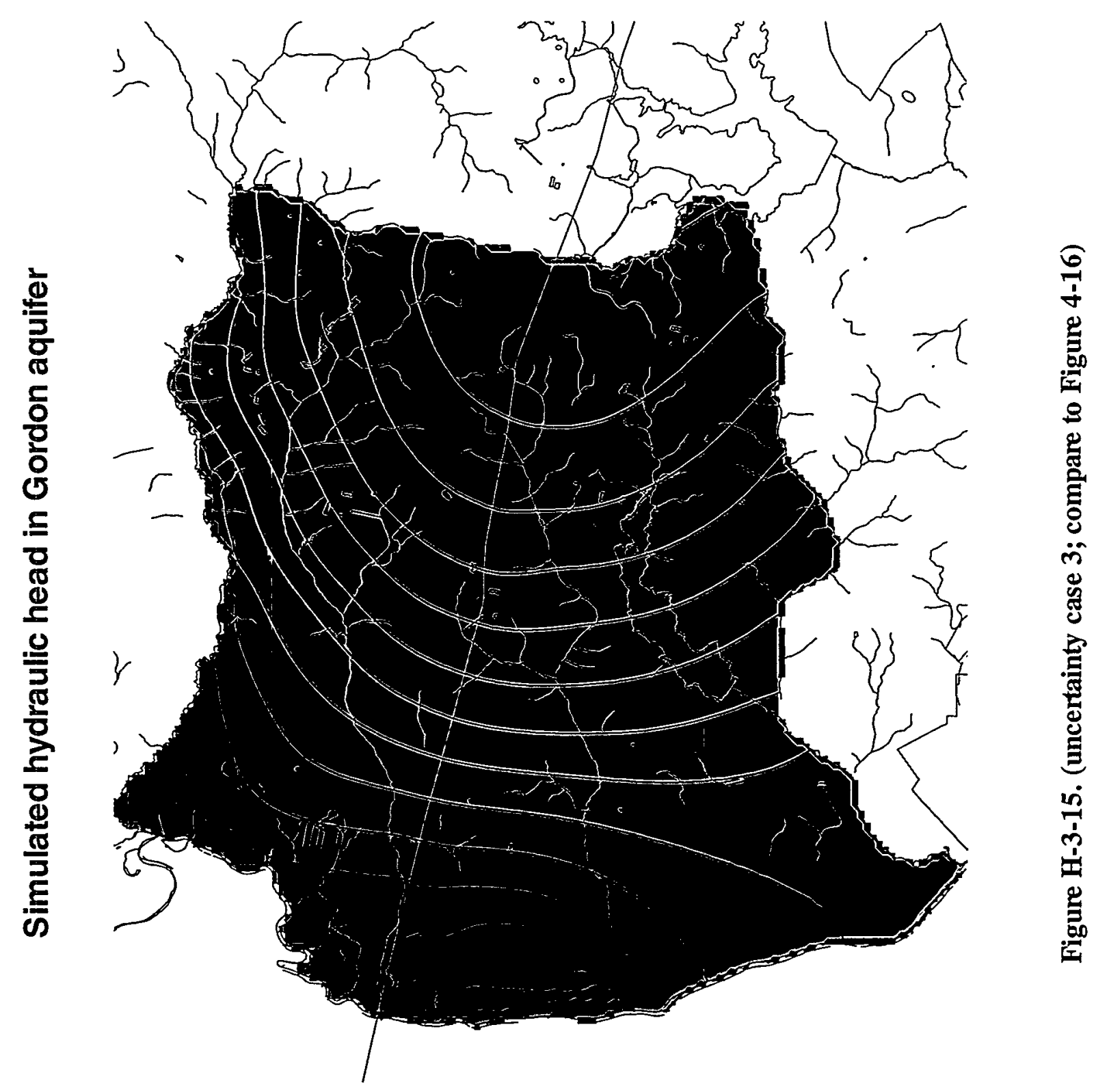




\section{Simulated hydraulic head in "lower" UTR aquifer zone}

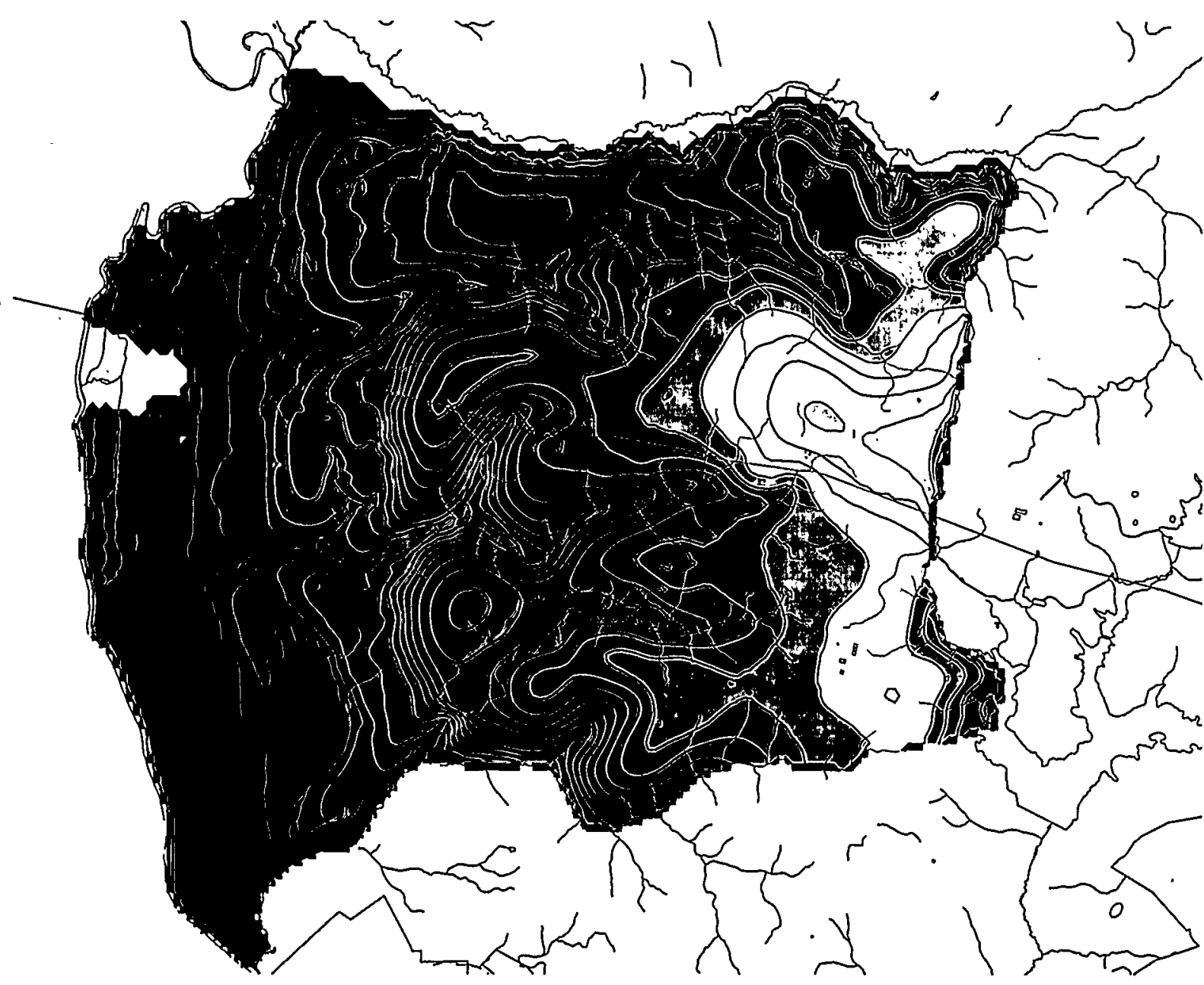

head 
Simulated hydraulic head in "upper" UTR aquifer zone

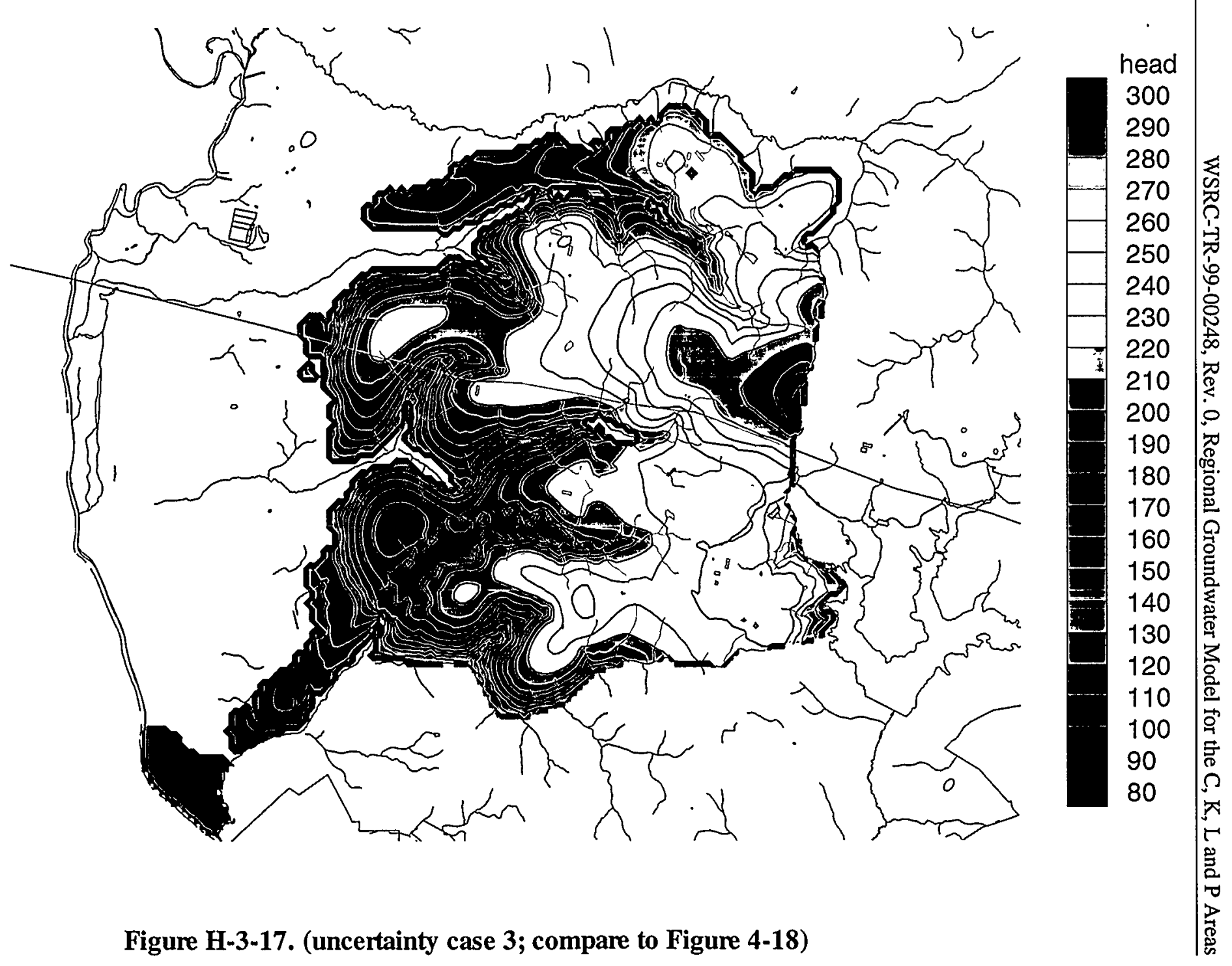




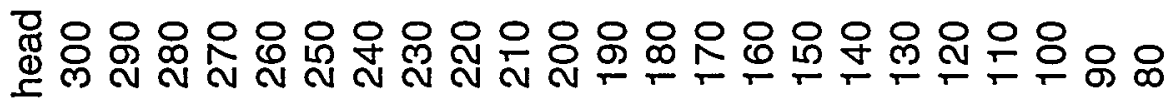
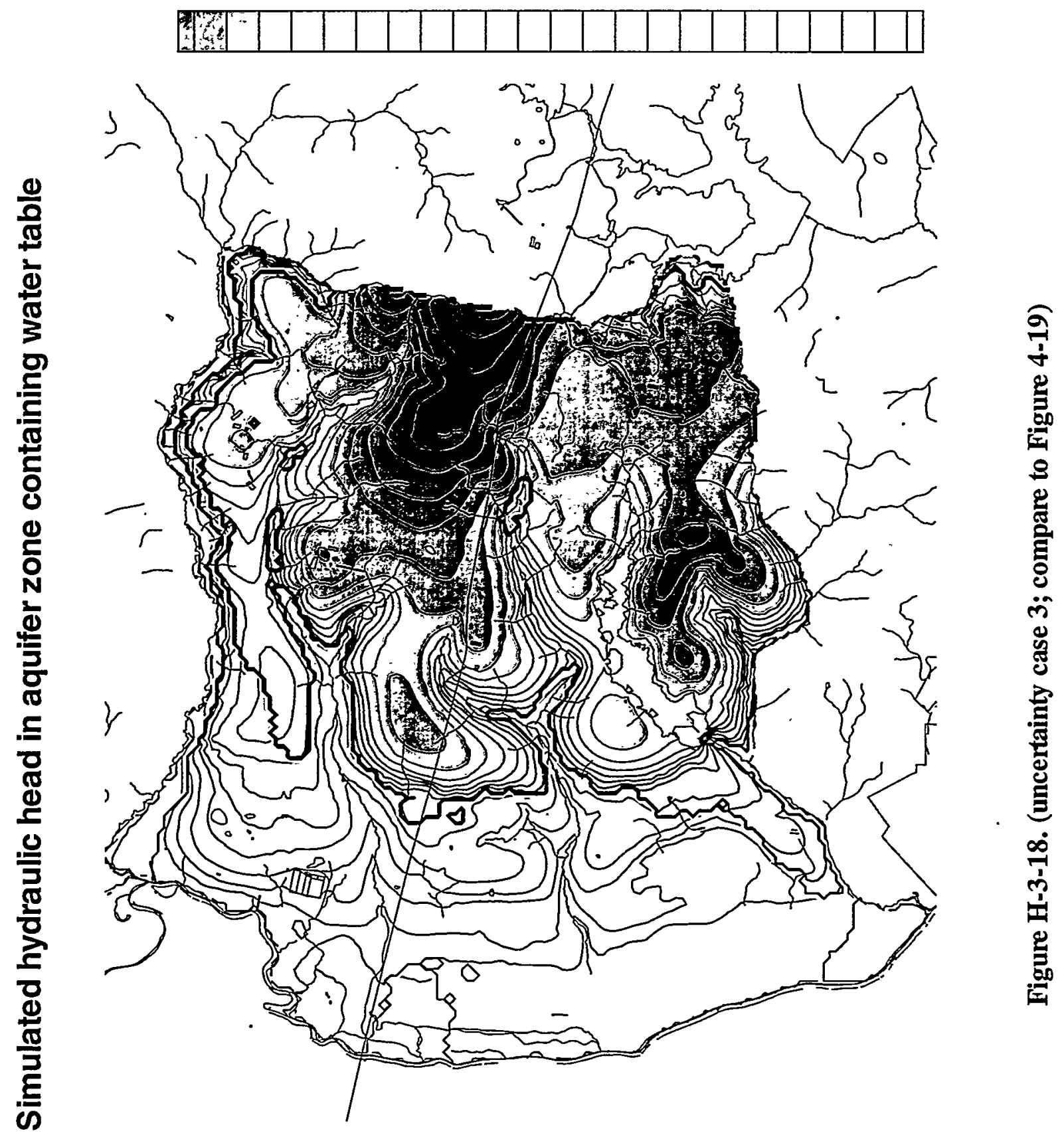


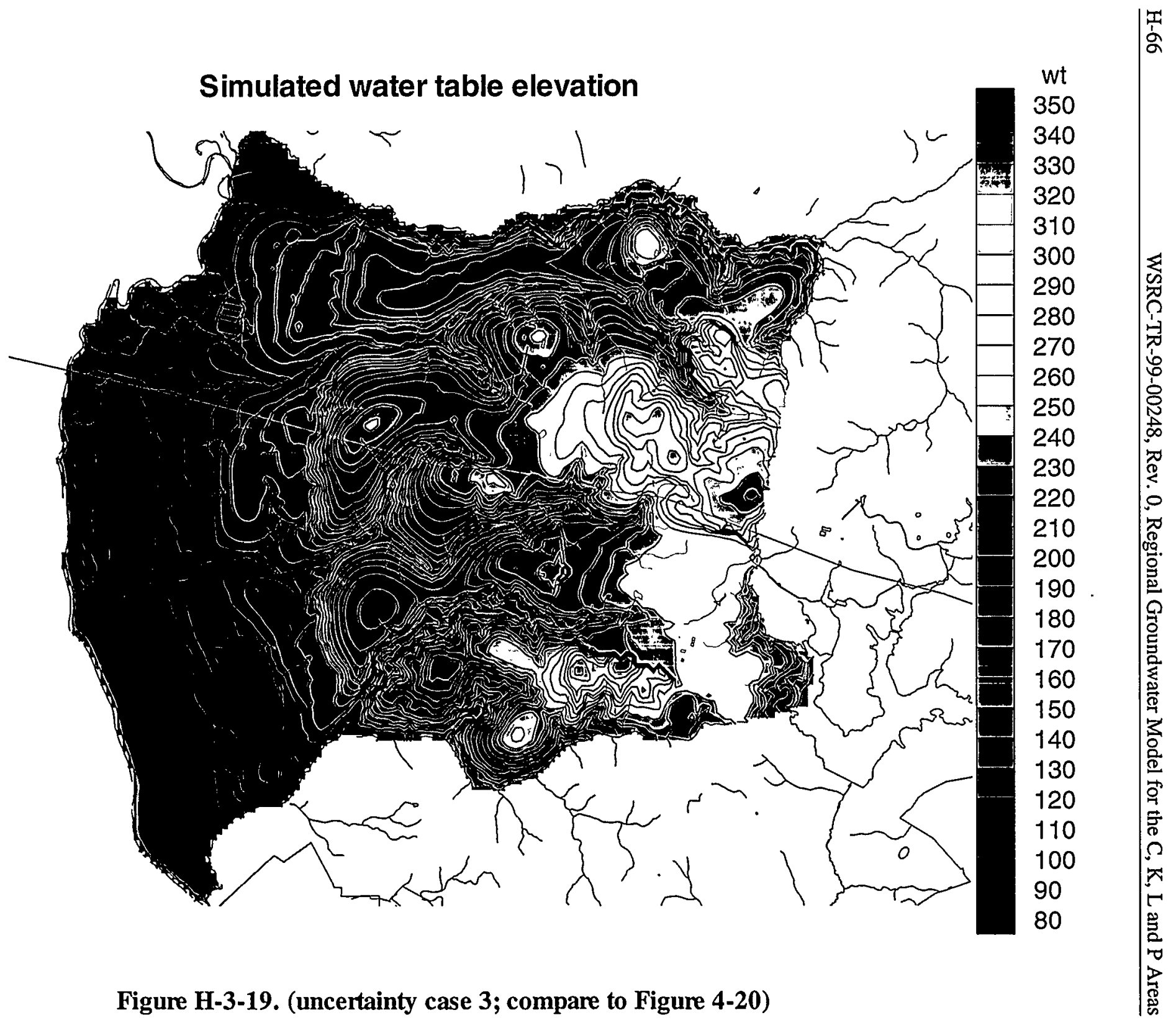




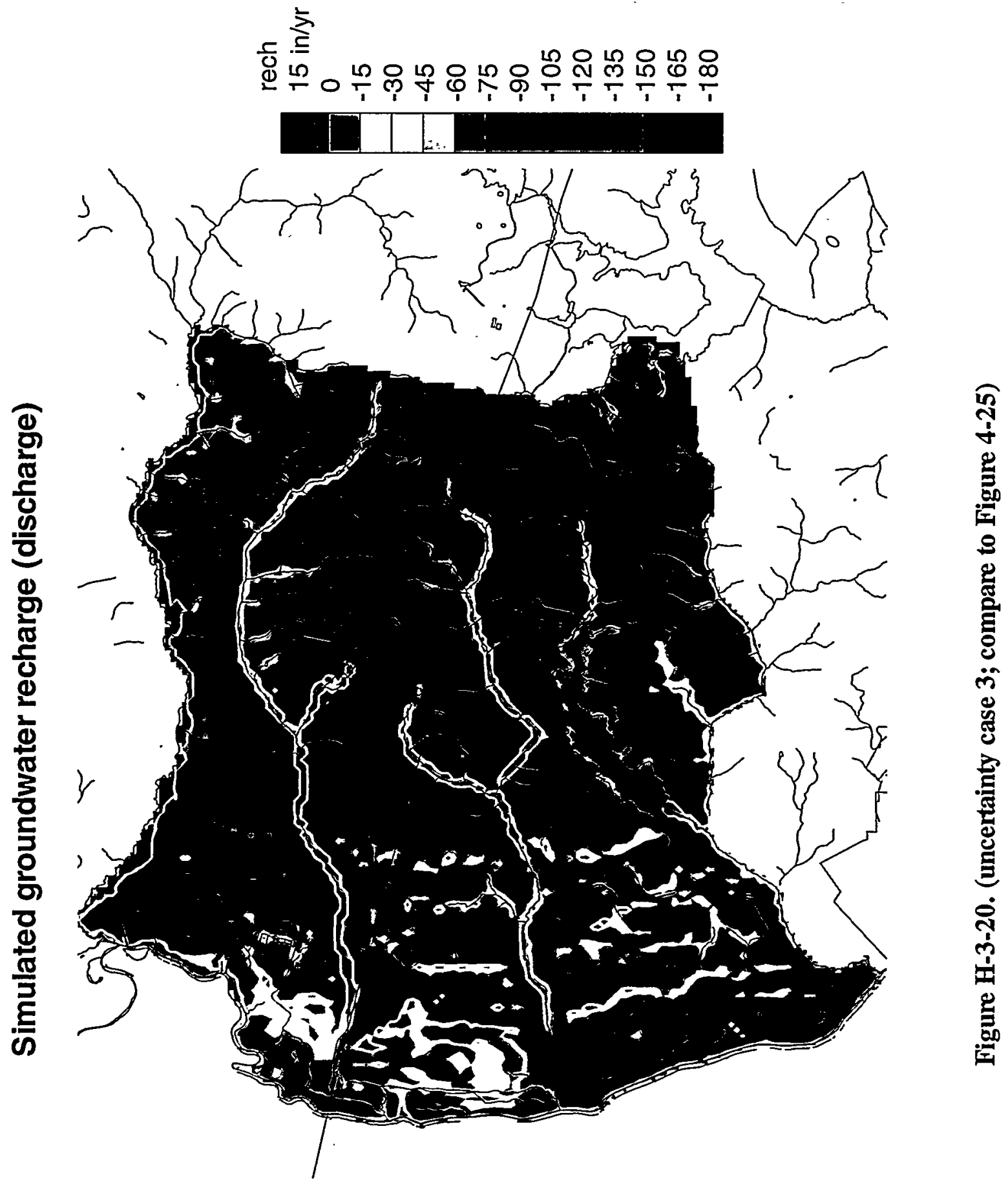




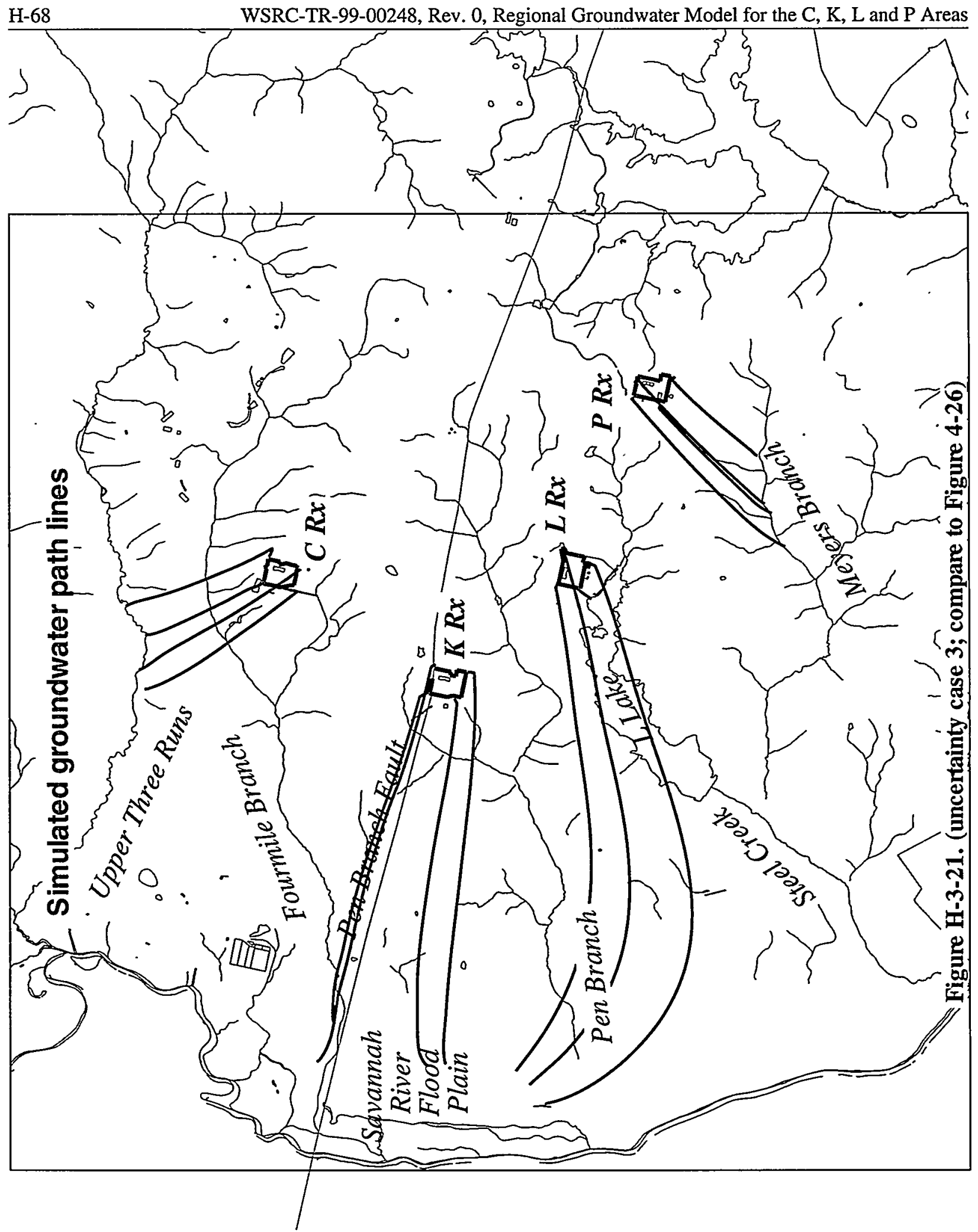




\section{Simulation results for uncertainty case 4}

Uncertainty case 4 involves a decrease in Gordon confining unit vertical conductivity by a factor of 5 to $2 \times 10^{-5} \mathrm{ft} /$ day (Table 4-4). Summary calibration results are provided in Table 4-5. This appendix presents detailed simulation results for uncertainty case 4 for comparison to the nominal results shown in figures in the main text. The correspondence between figures for the nominal and uncertainty case 4 is as follows:

\begin{tabular}{|l|l|l|}
\hline Plot type & Nominal case & Uncertainty case 4 \\
\hline Head residual summary & Figure 4-1 & Figure H-4-1 \\
\hline Head residuals in Gordon aquifer & Figure 4-2 & Figure H-4-2 \\
\hline Head residuals in "lower" UTRA & Figure 4-3 & Figure H-4-3 \\
\hline Head residuals in transmissive zone & Figure 4-4 & Figure H-4-4 \\
\hline Head residuals in AA horizon & Figure 4-5 & Figure H-4-5 \\
\hline Head residuals in A/uu horizons & Figure 4-6 & Figure H-4-6 \\
\hline Kh in element layer 1 & Figure 4-7 & Figure H-4-7 \\
\hline Kv in element layer 2 & Figure 4-8 & Figure H-4-8 \\
\hline Kh in element layer 3 & Figure 4-9 & Figure H-4-9 \\
\hline Kh in element layer 4 & Figure 4-10 & Figure H-4-10 \\
\hline Kv in element layer 5 & Figure 4-11 & Figure H-4-11 \\
\hline Kh in element layer 6 & Figure 4-12 & Figure H-4-12 \\
\hline Kh in element layer 7 & Figure 4-13 & Figure H-4-13 \\
\hline Kh in element layer 8 & Figure 4-14 & Figure H-4-14 \\
\hline Gordon aquifer head & Figure 4-16 & Figure H-4-15 \\
\hline "Lower" UTRA head & Figure 4-17 & Figure H-4-16 \\
\hline "Upper" UTRA head & Figure 4-18 & Figure H-4-17 \\
\hline Head in aquifer containing water table & Figure 4-19 & Figure H-4-18 \\
\hline Water table & Figure 4-20 & Figure H-4-19 \\
\hline Recharge/discharge & Figure 4-25 & Figure H-4-20 \\
\hline Example particle tracing & Figure 4-26 & Figure H-4-21 \\
\hline
\end{tabular}



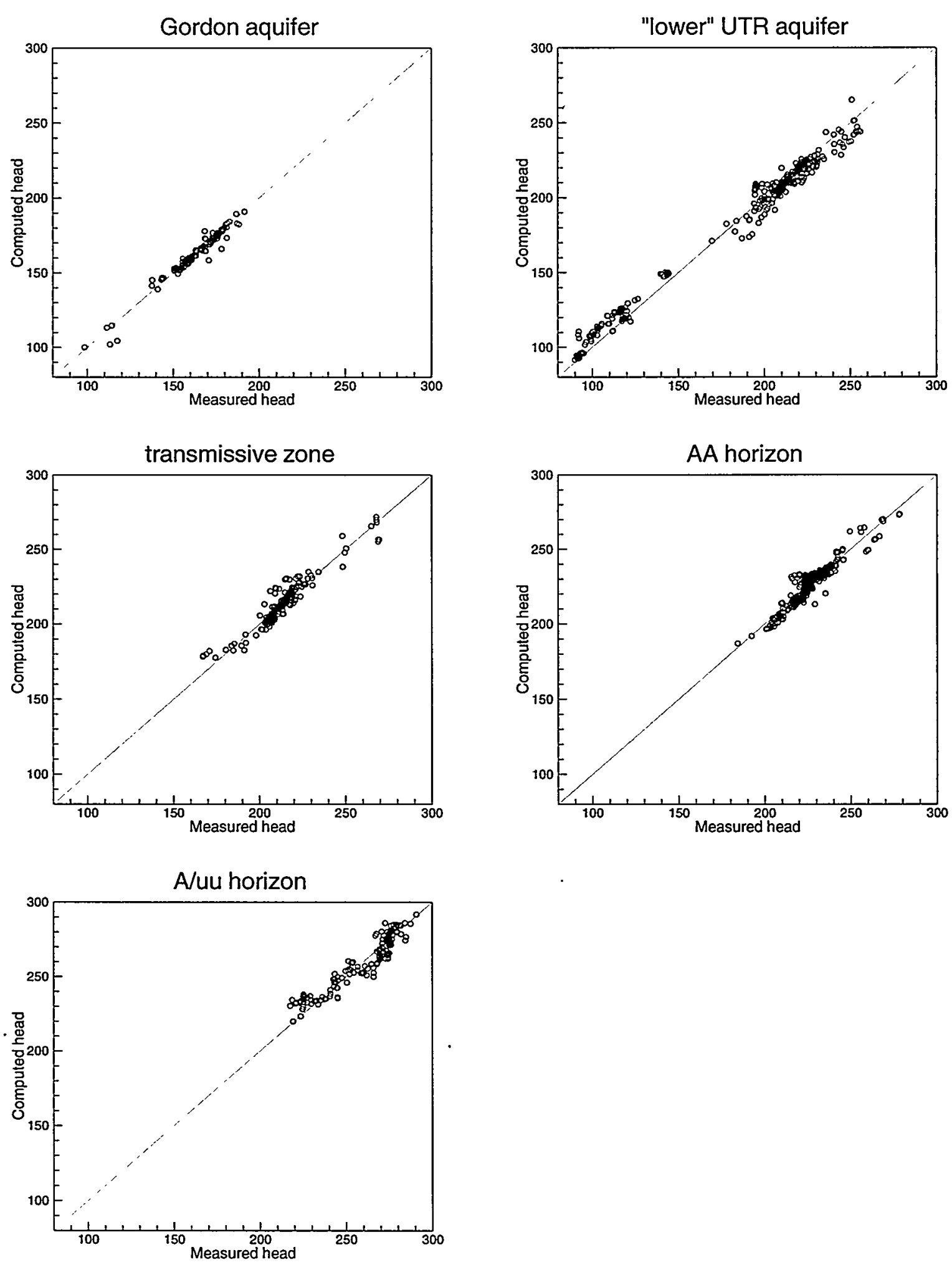

Figure H-4-1. (uncertainty case 4; compare to Figure 4-1) 

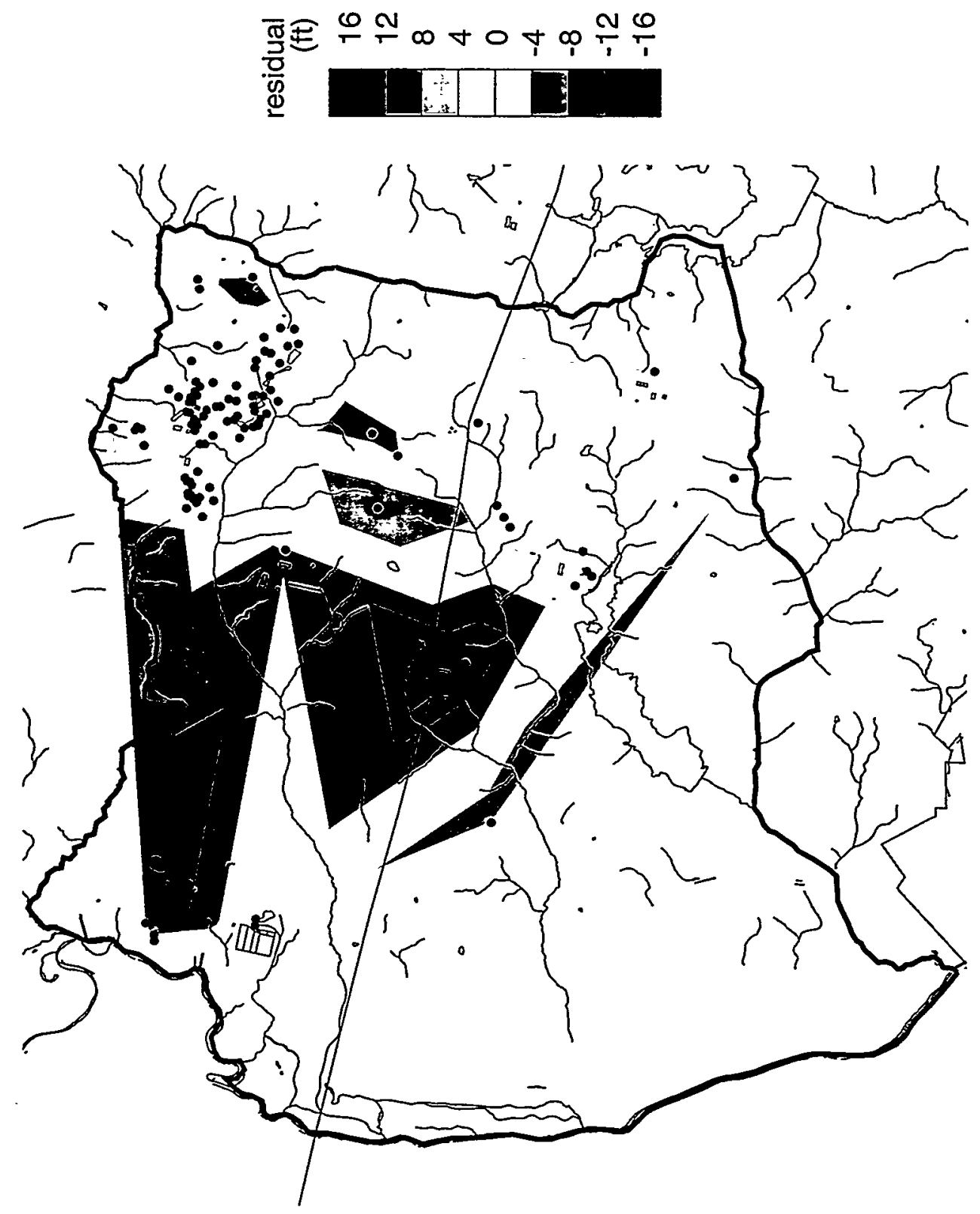


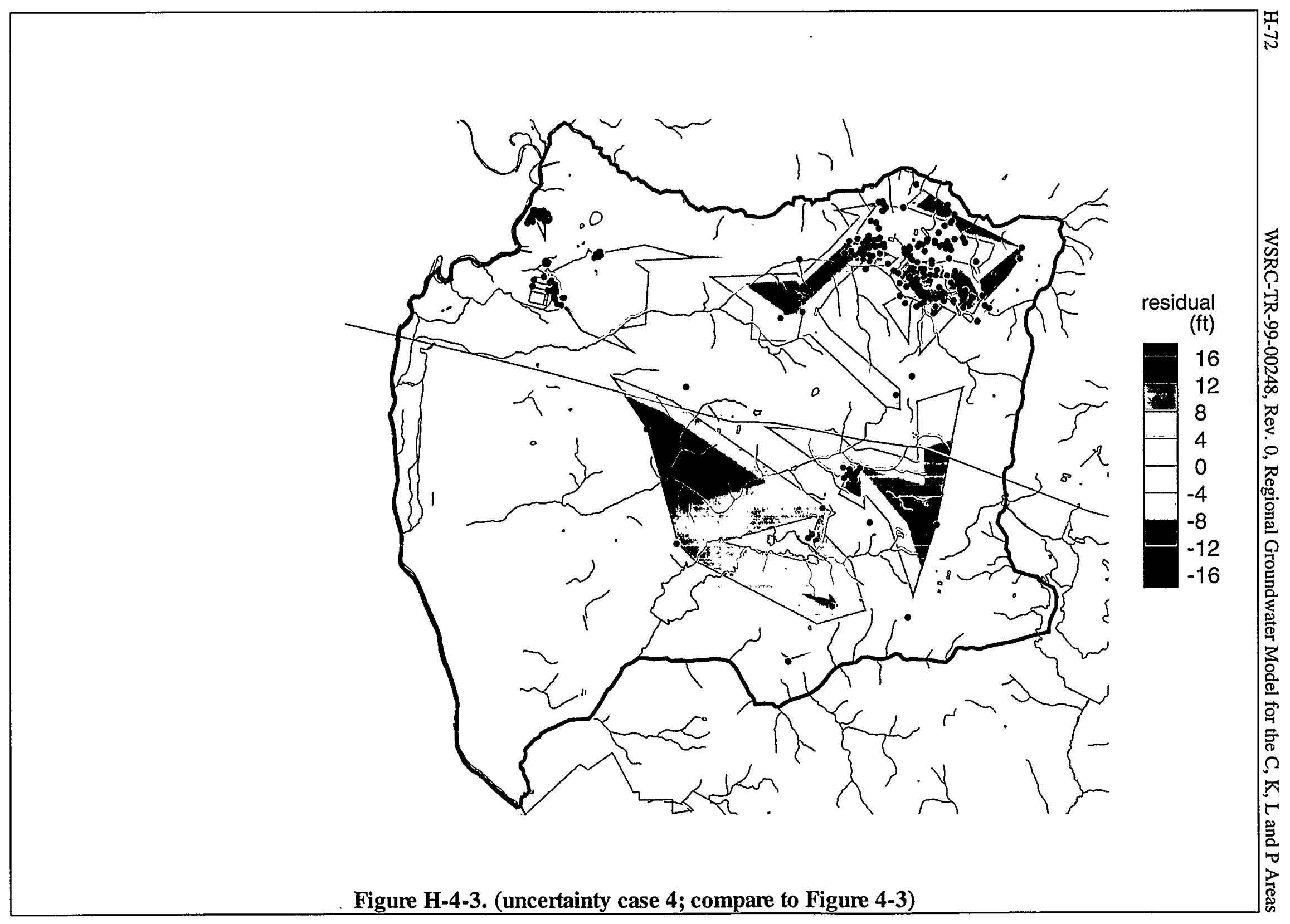



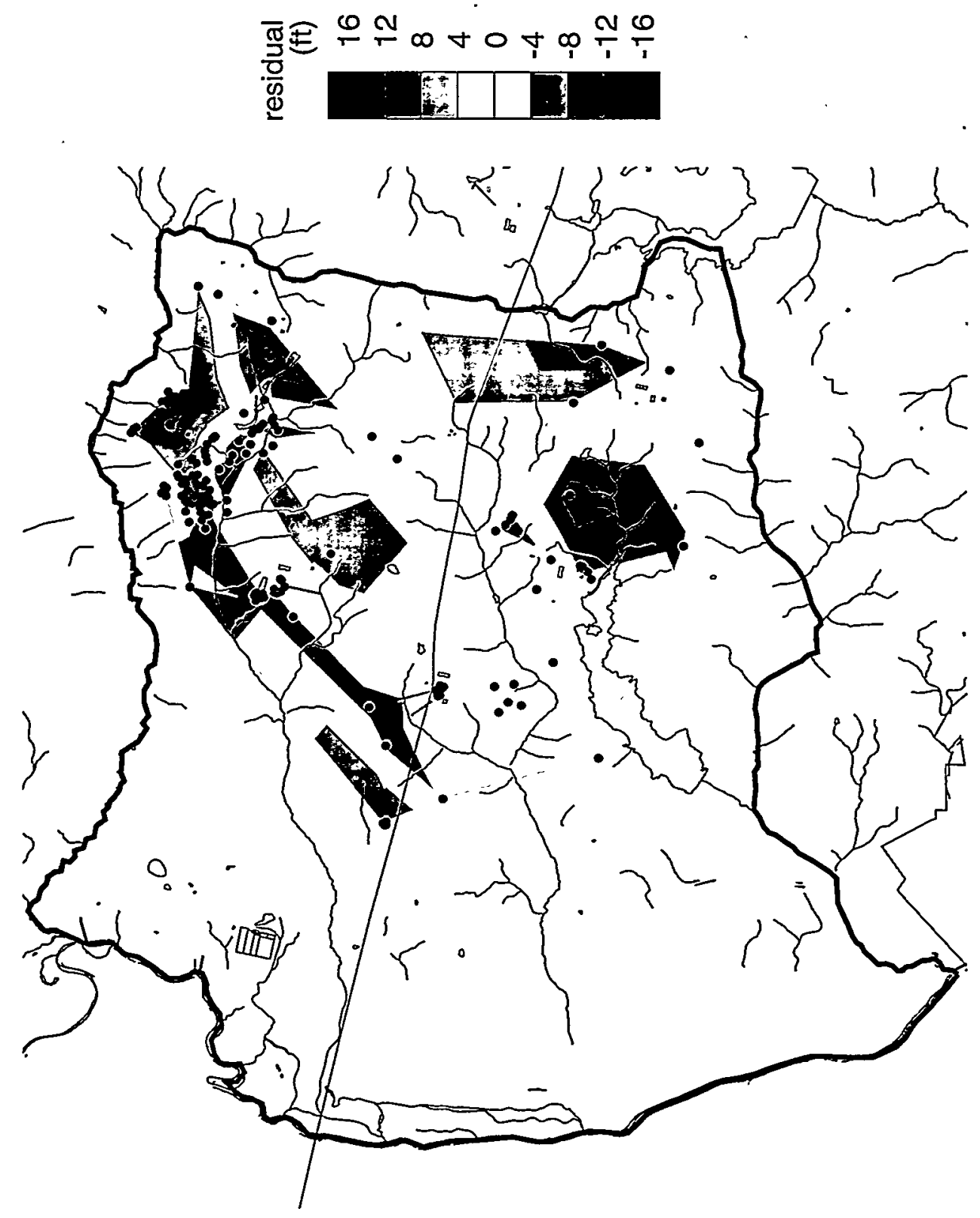

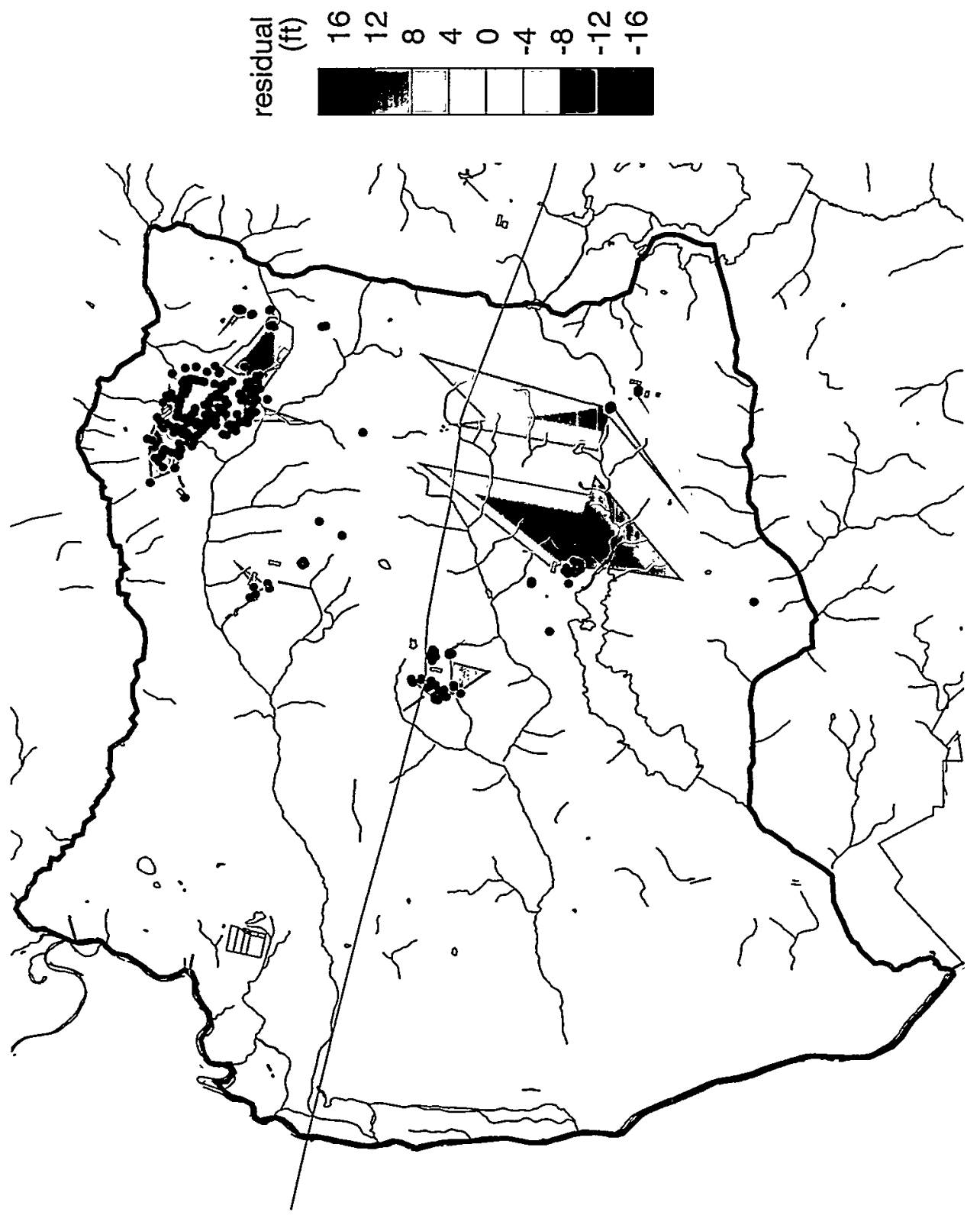


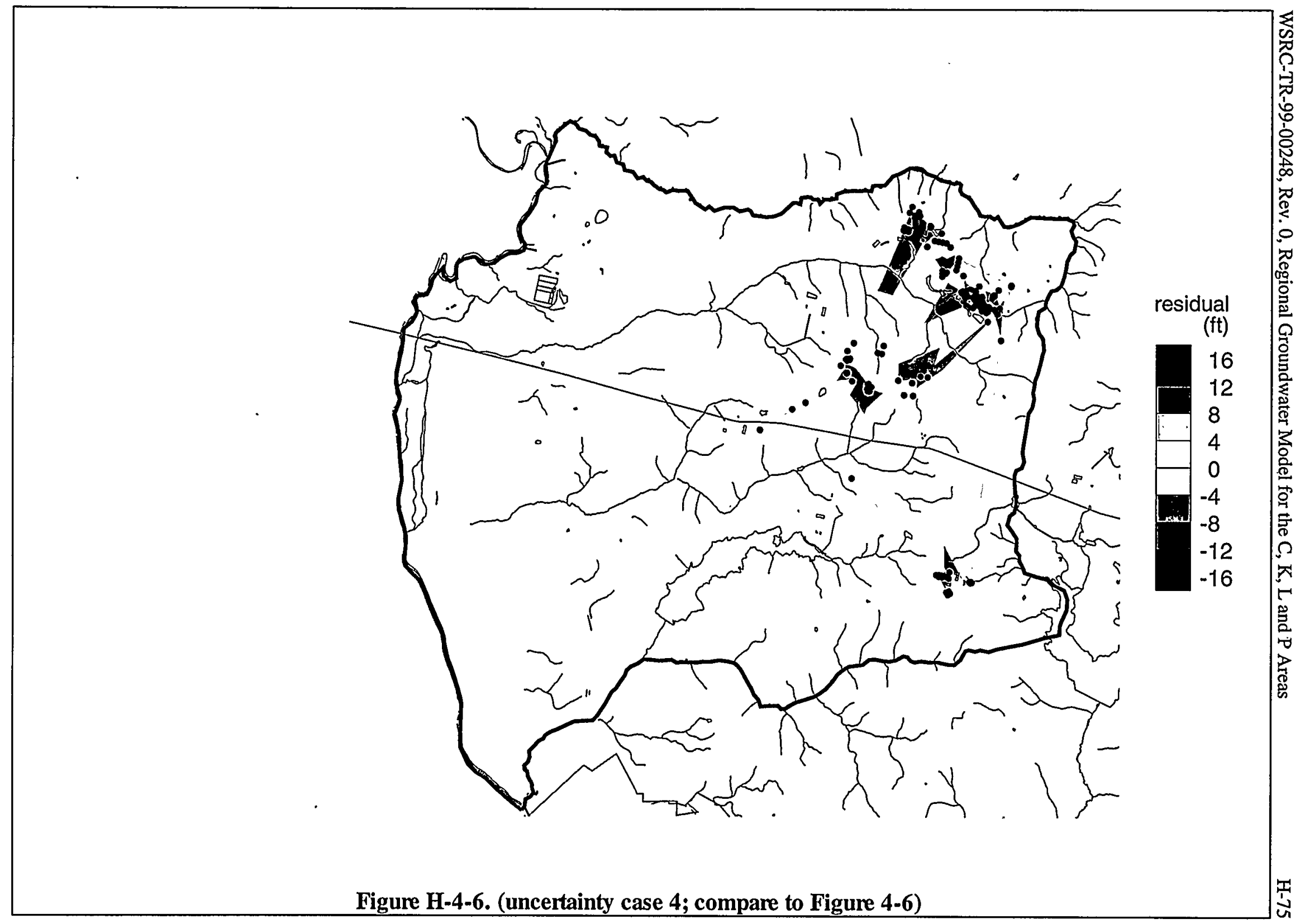




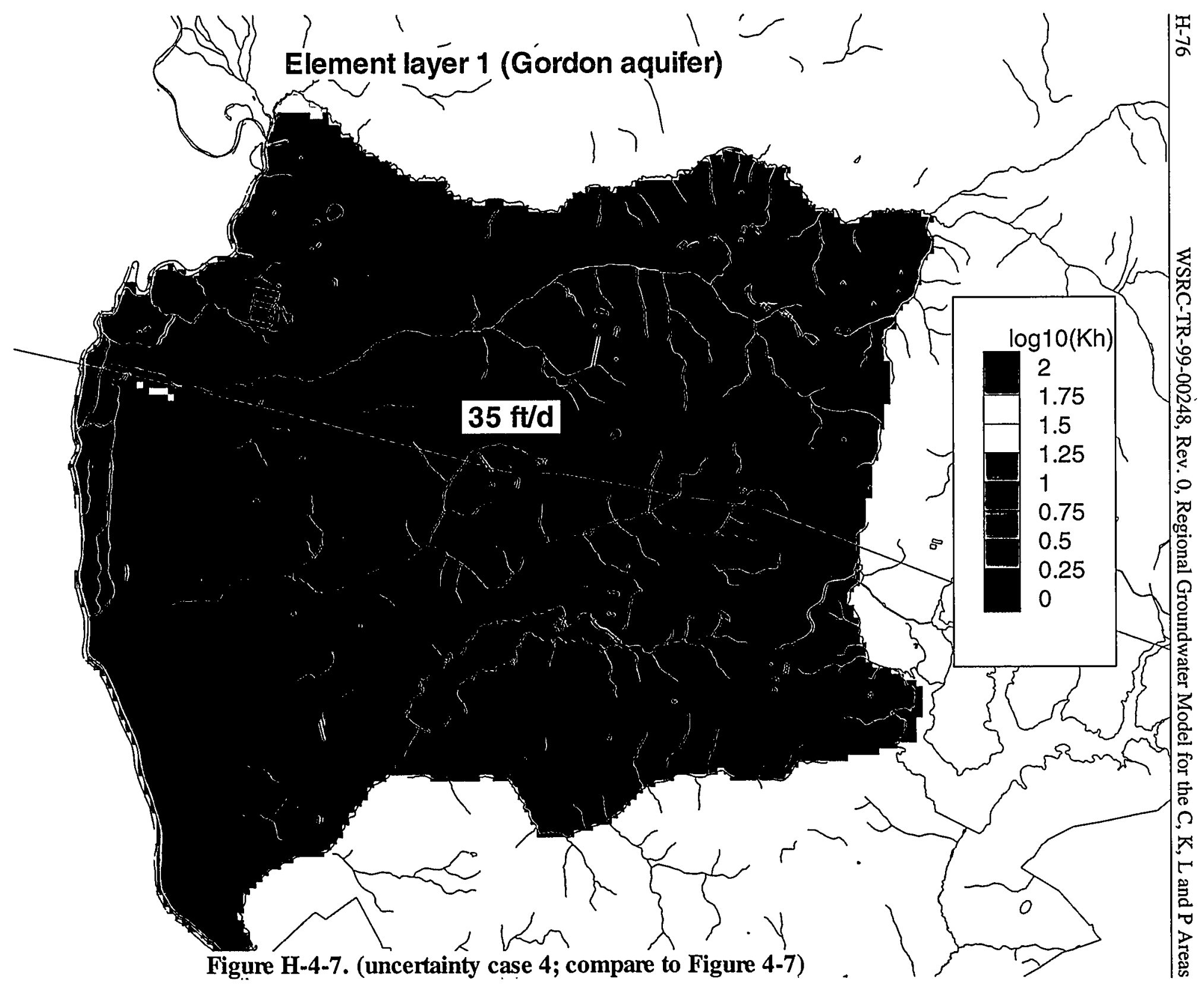




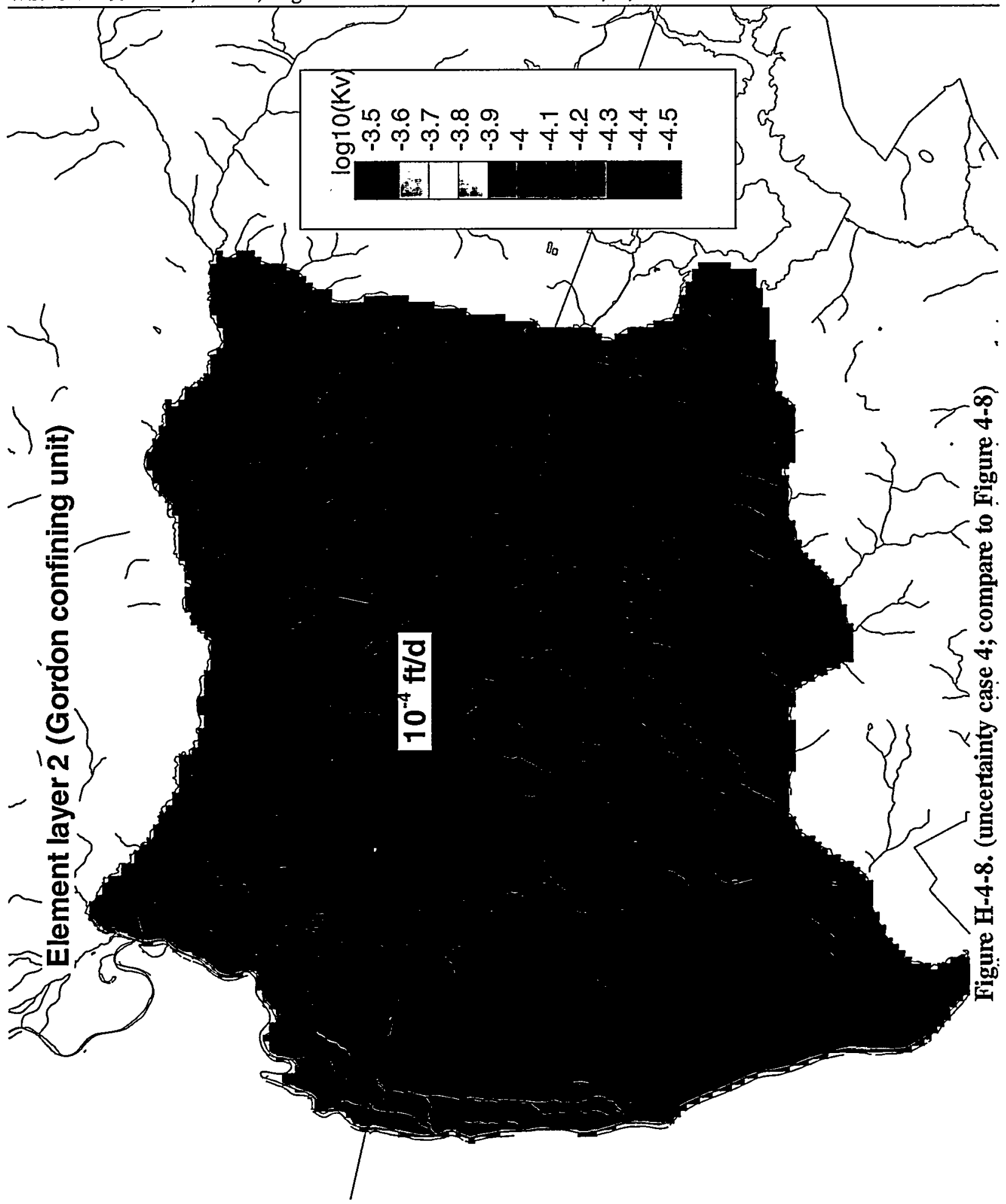




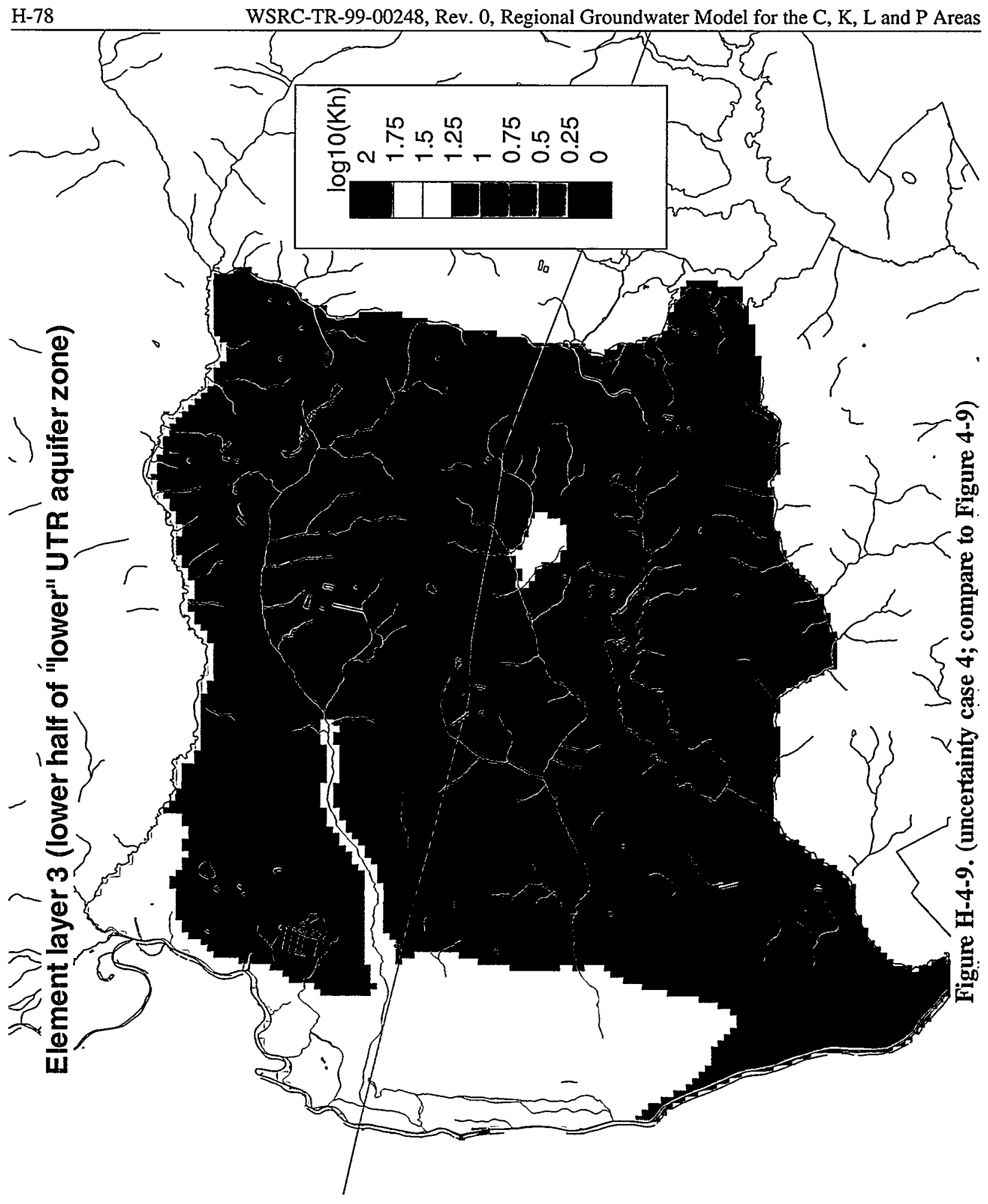




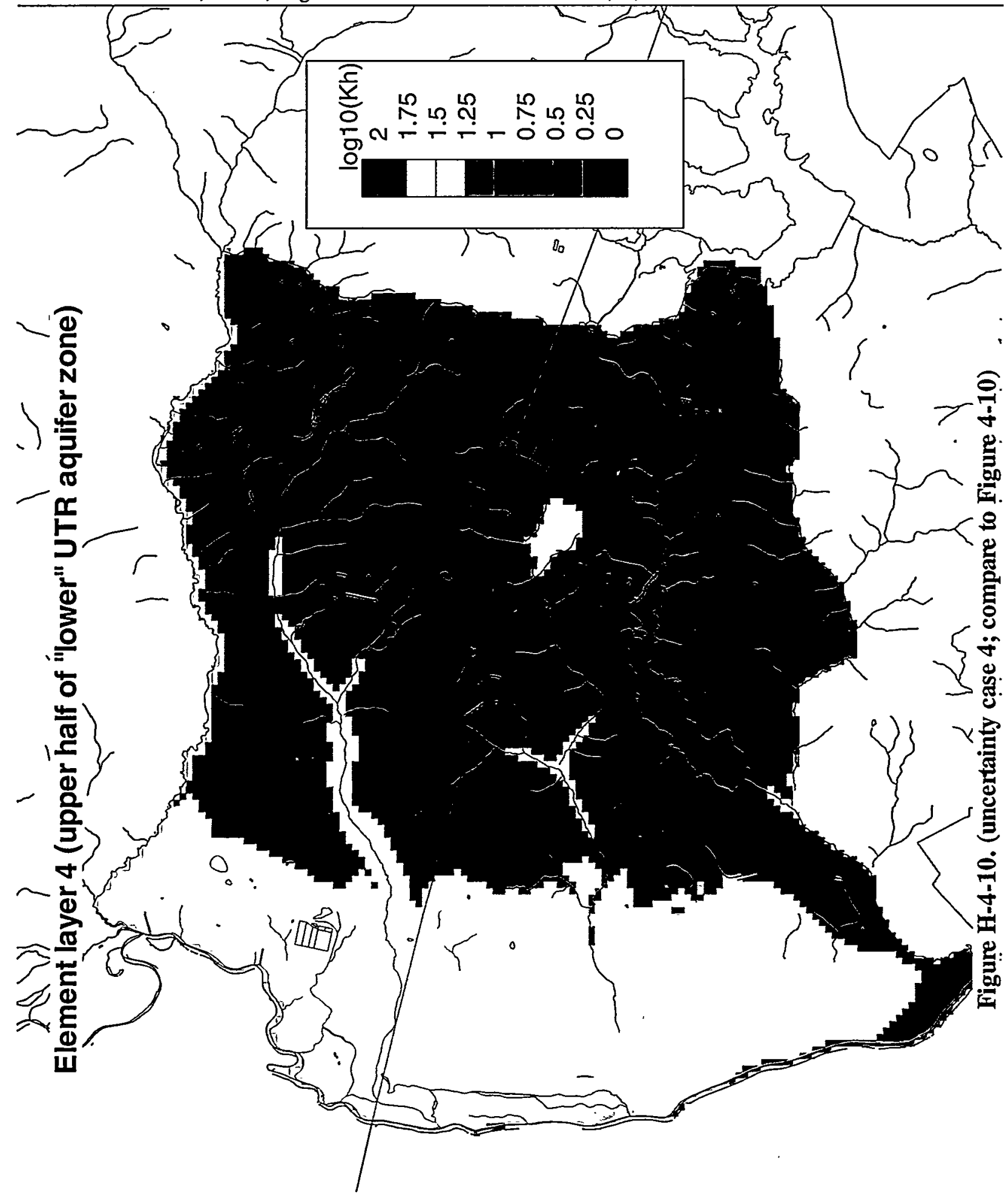




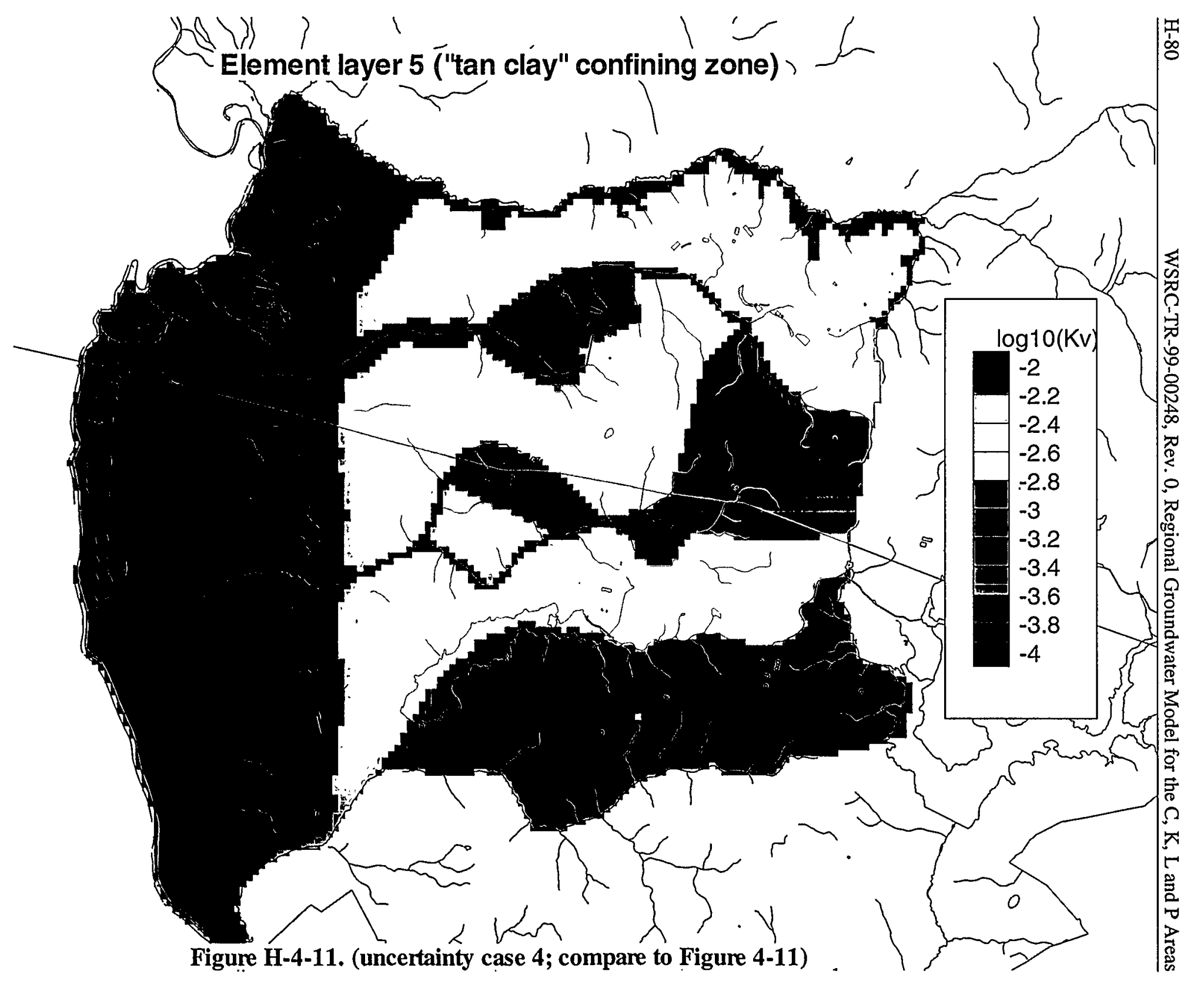




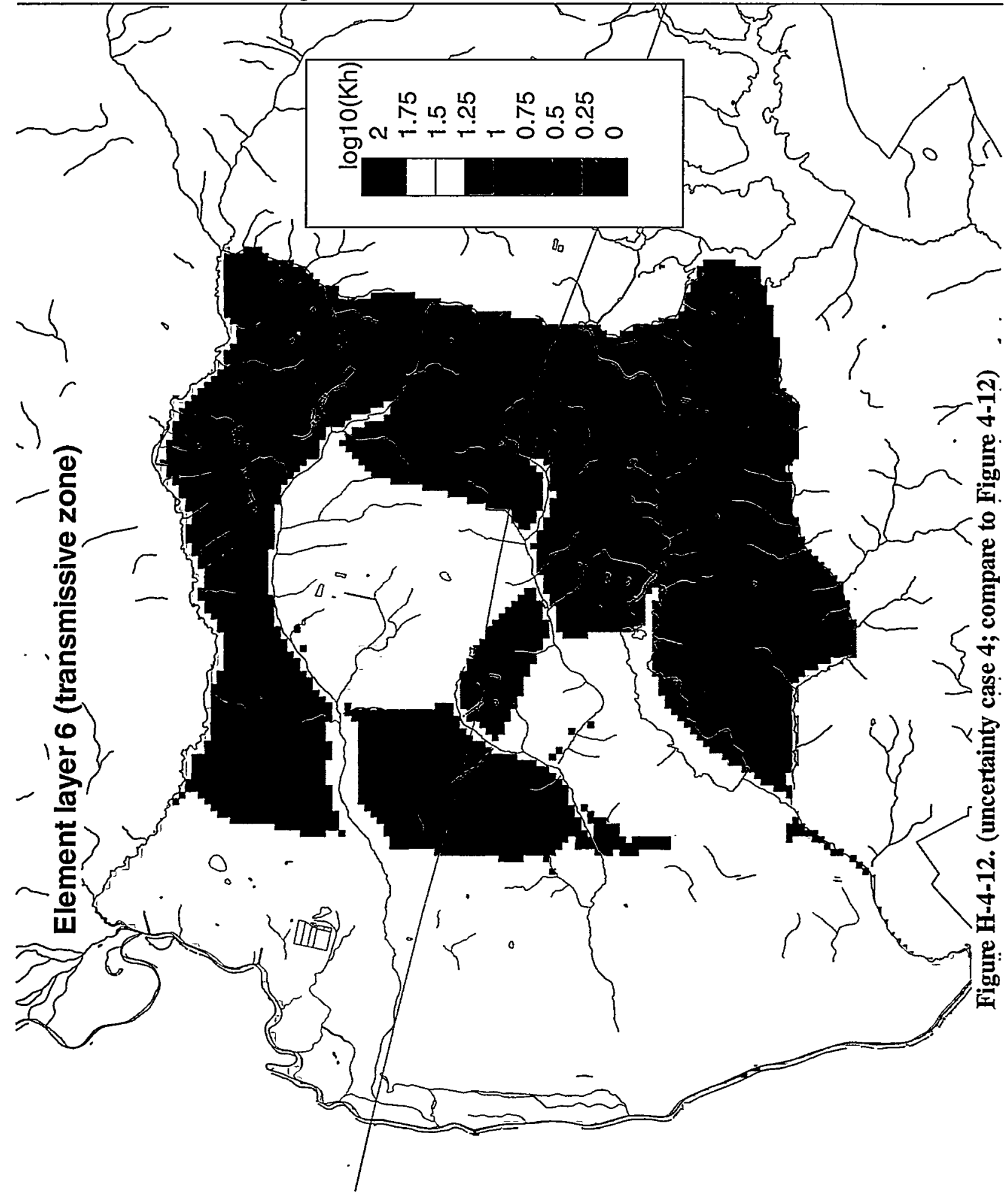




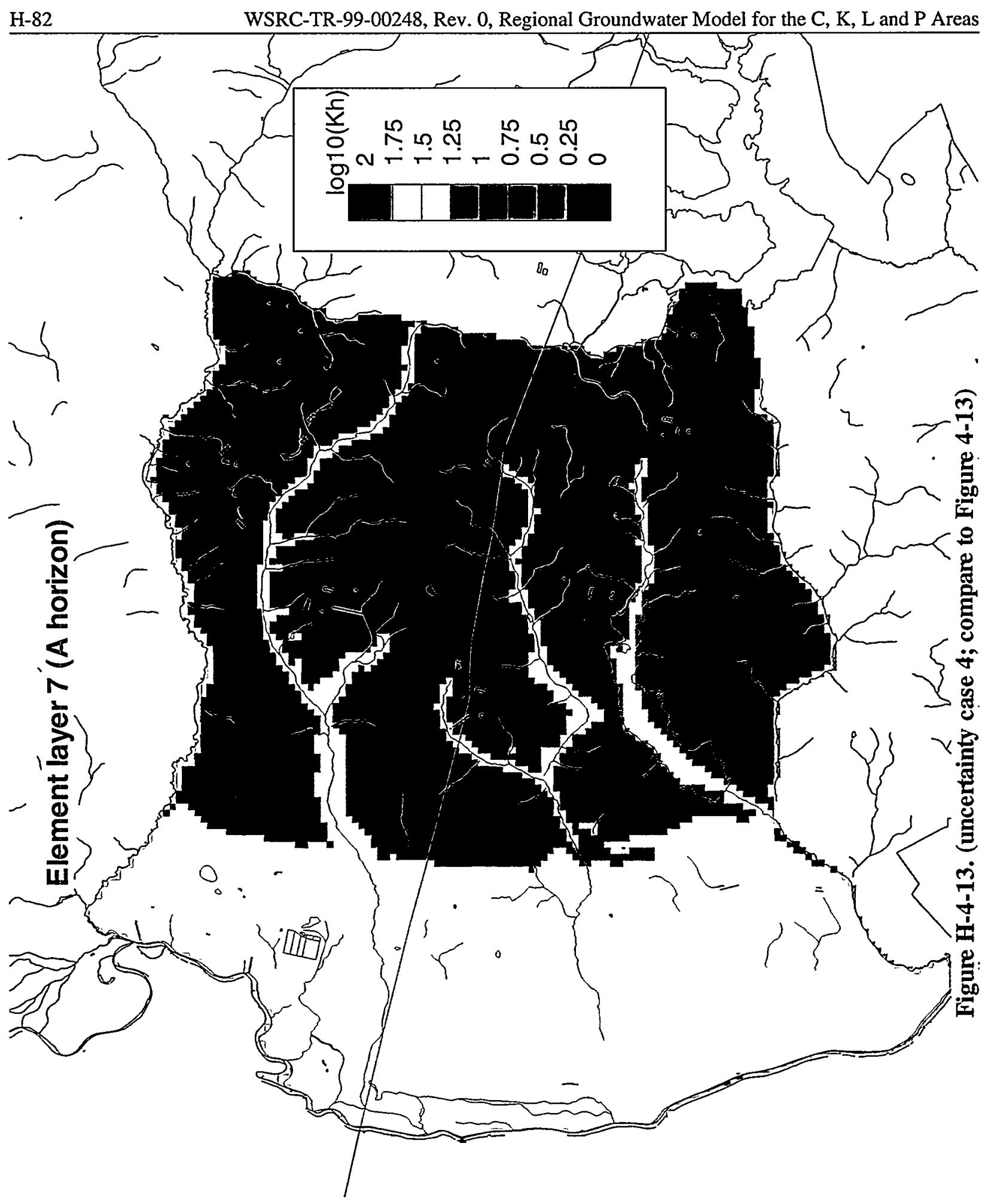




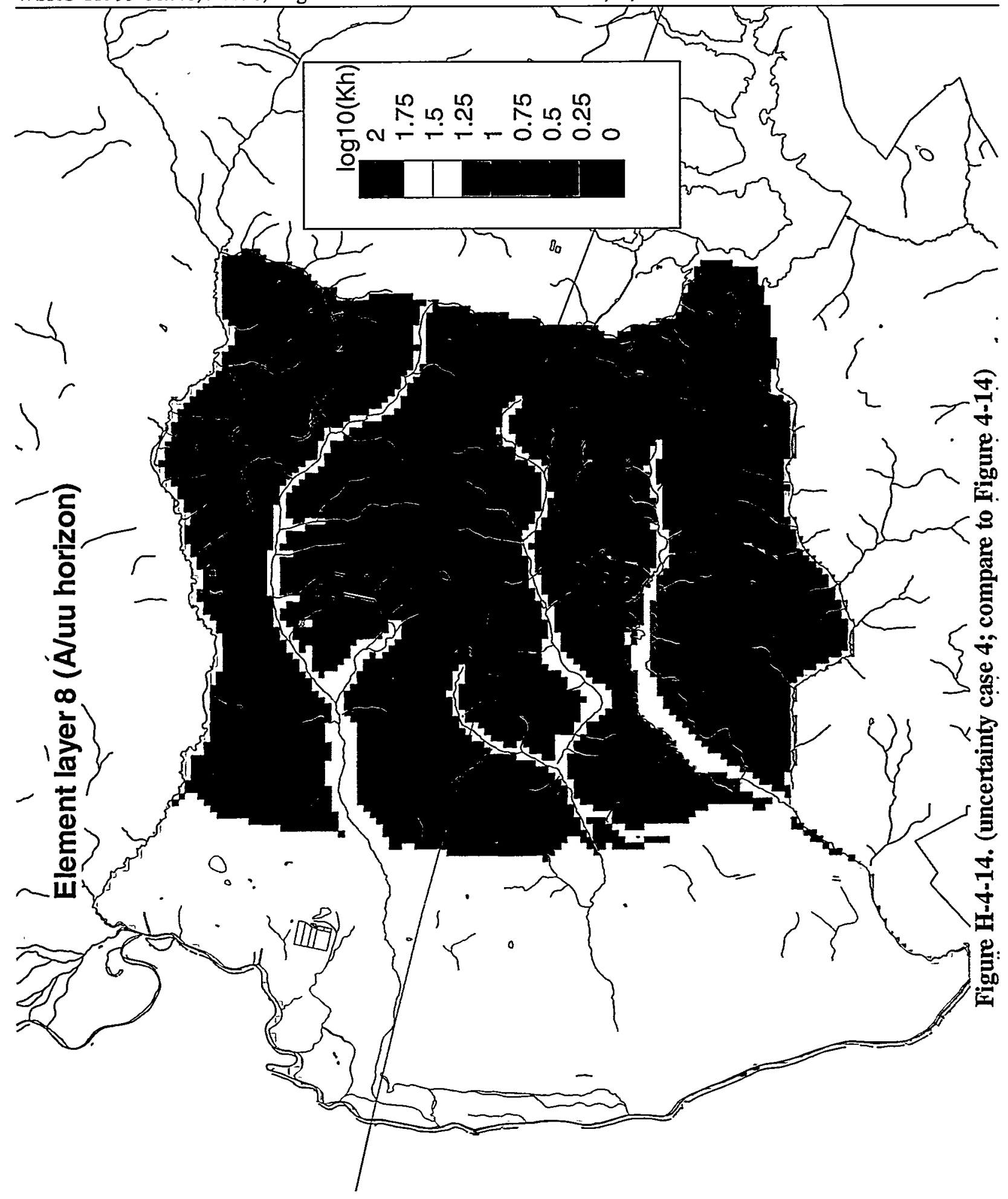




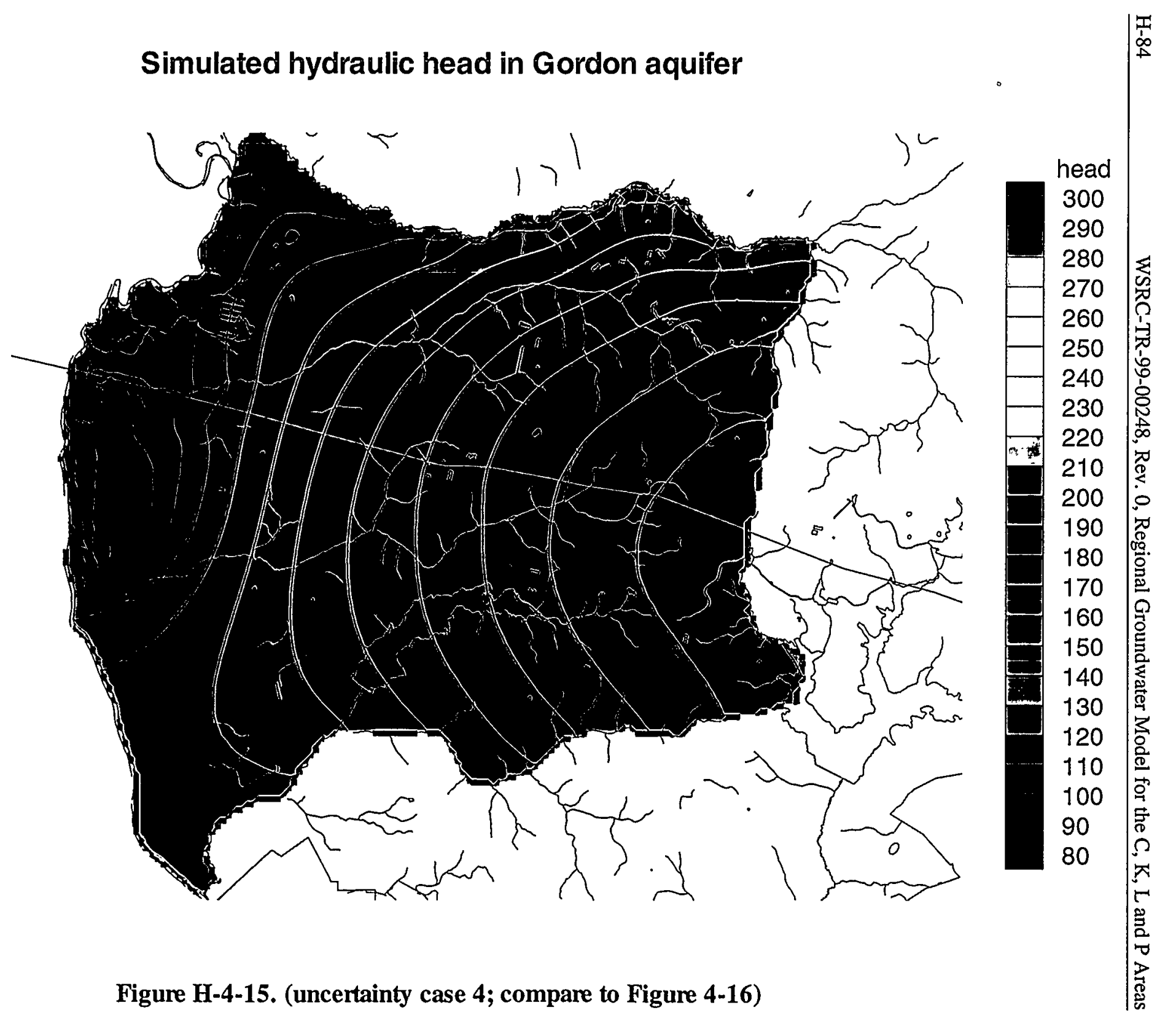


Simulated hydraulic head in "lower" UTR aquifer zone

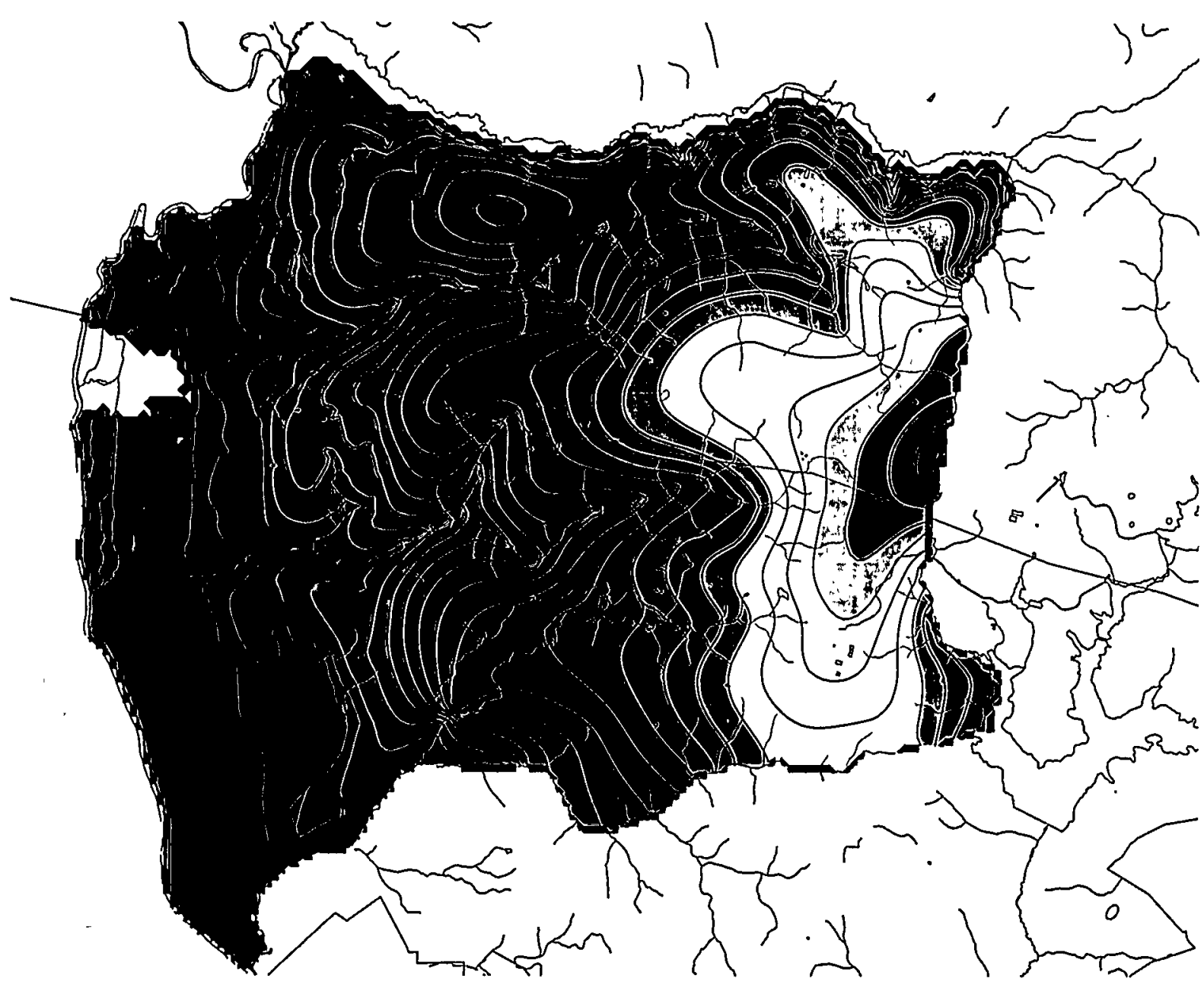

head 


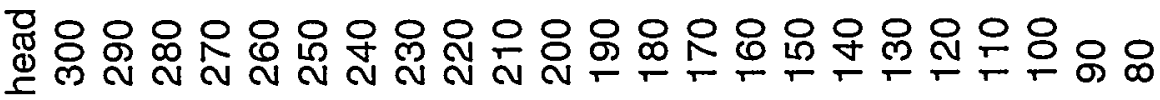
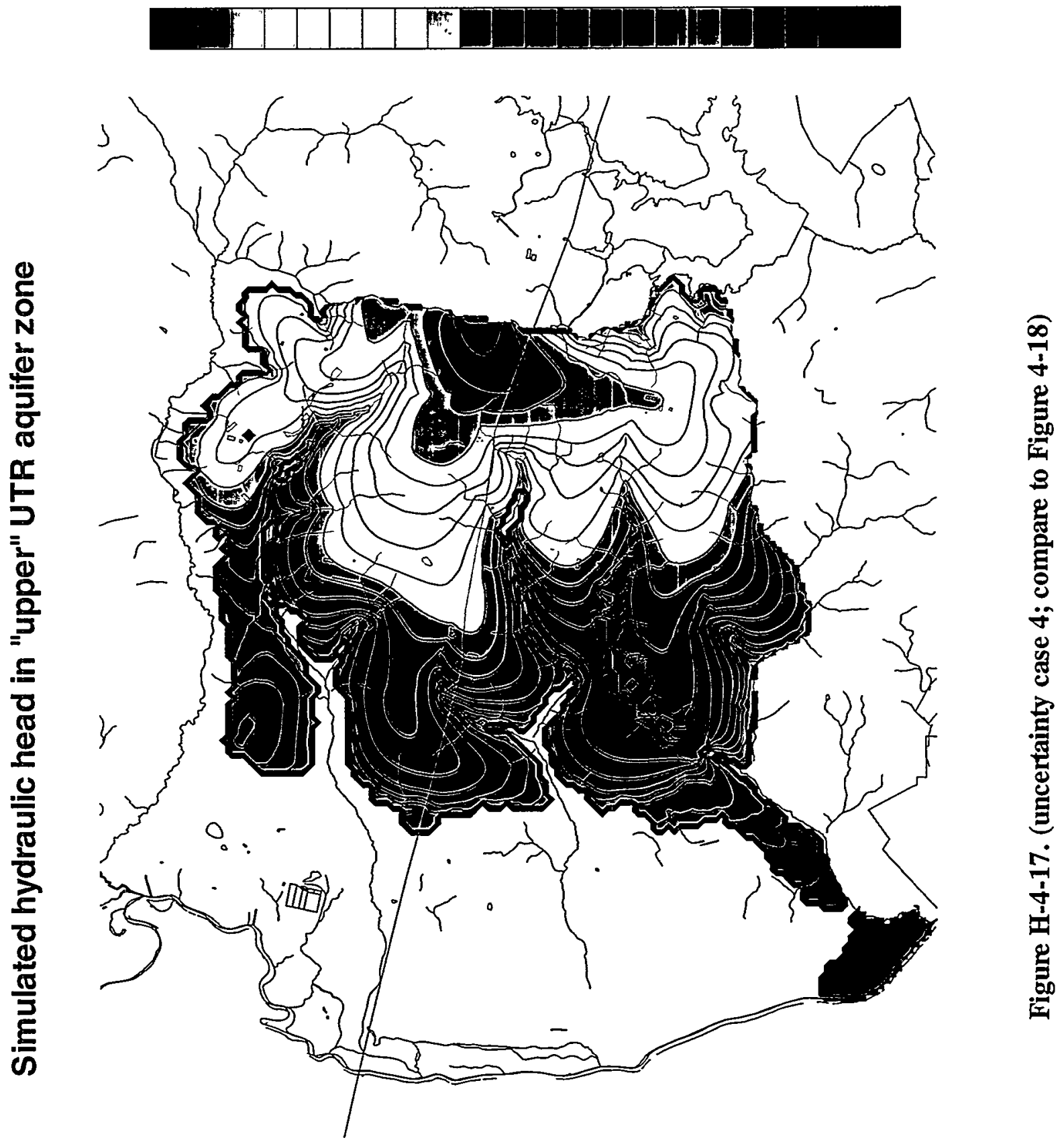


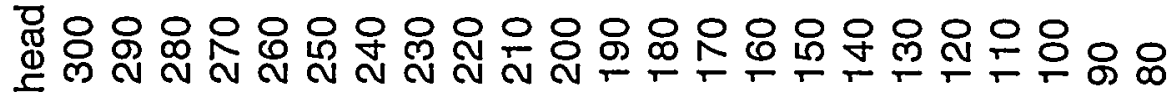

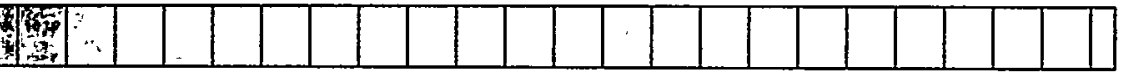

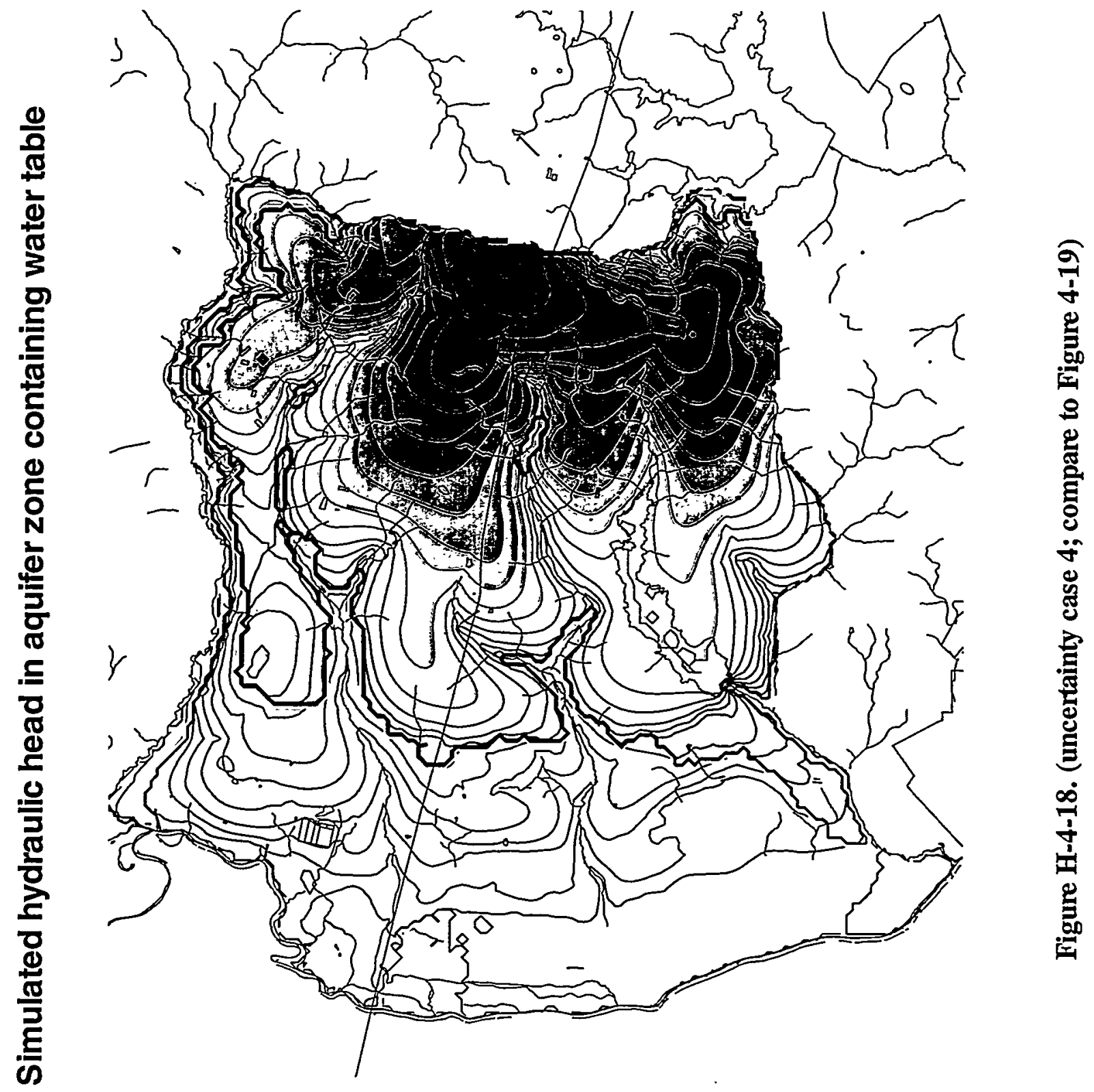




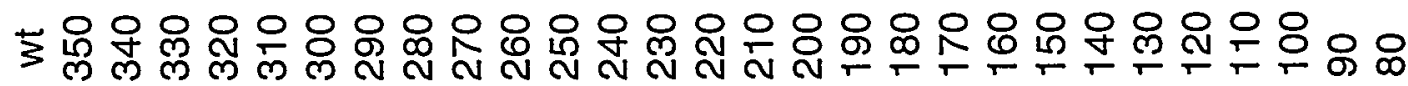

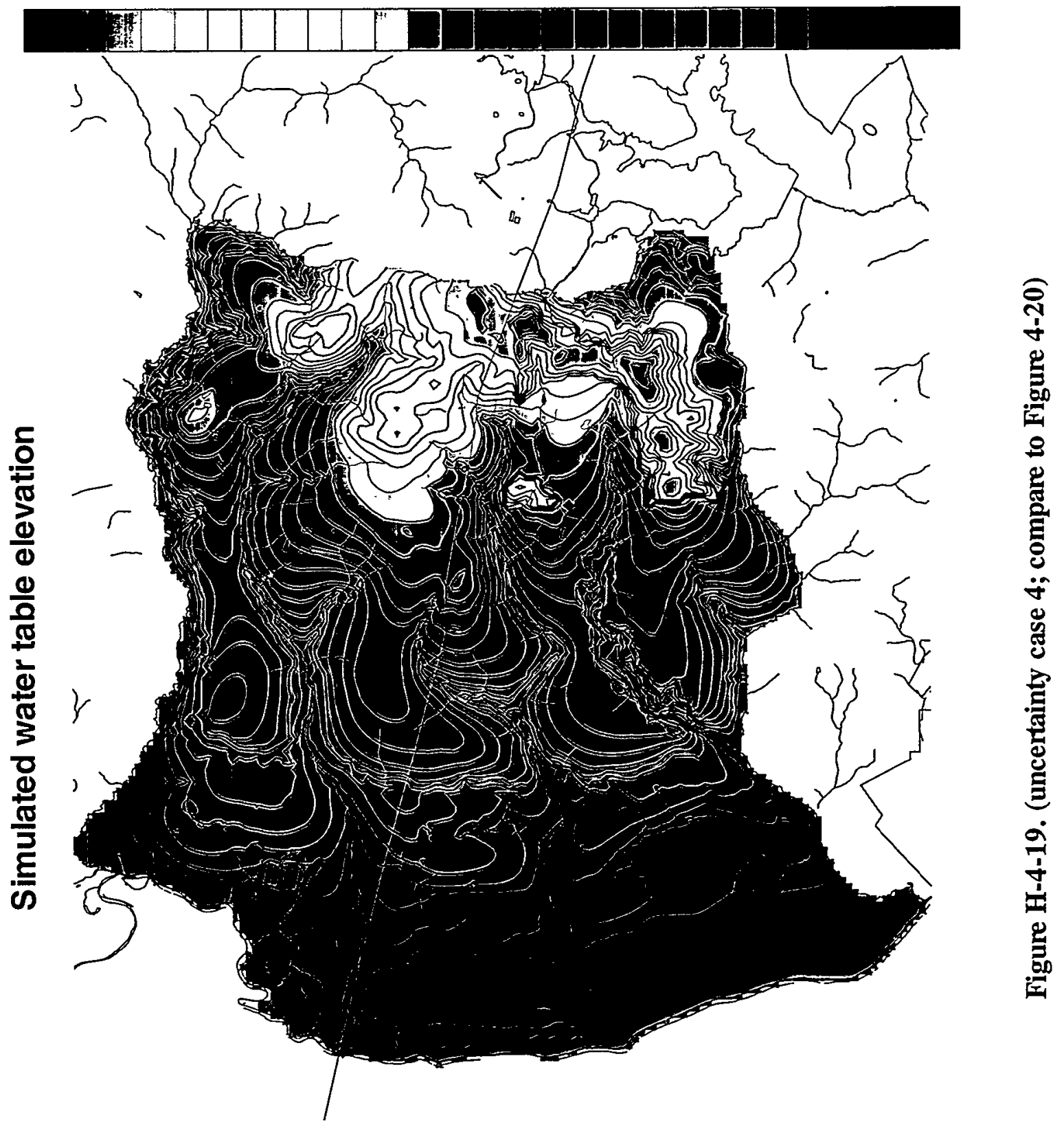



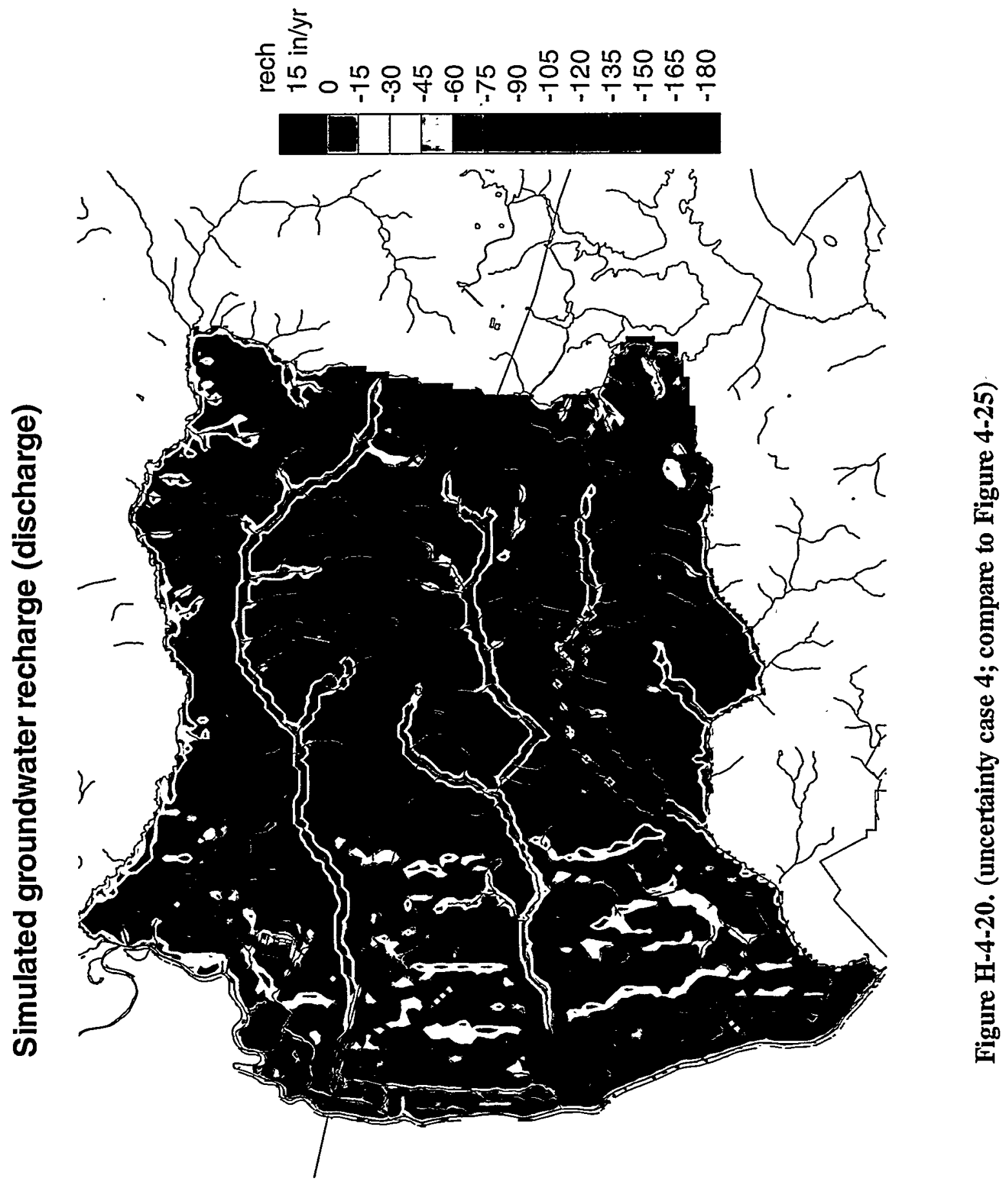


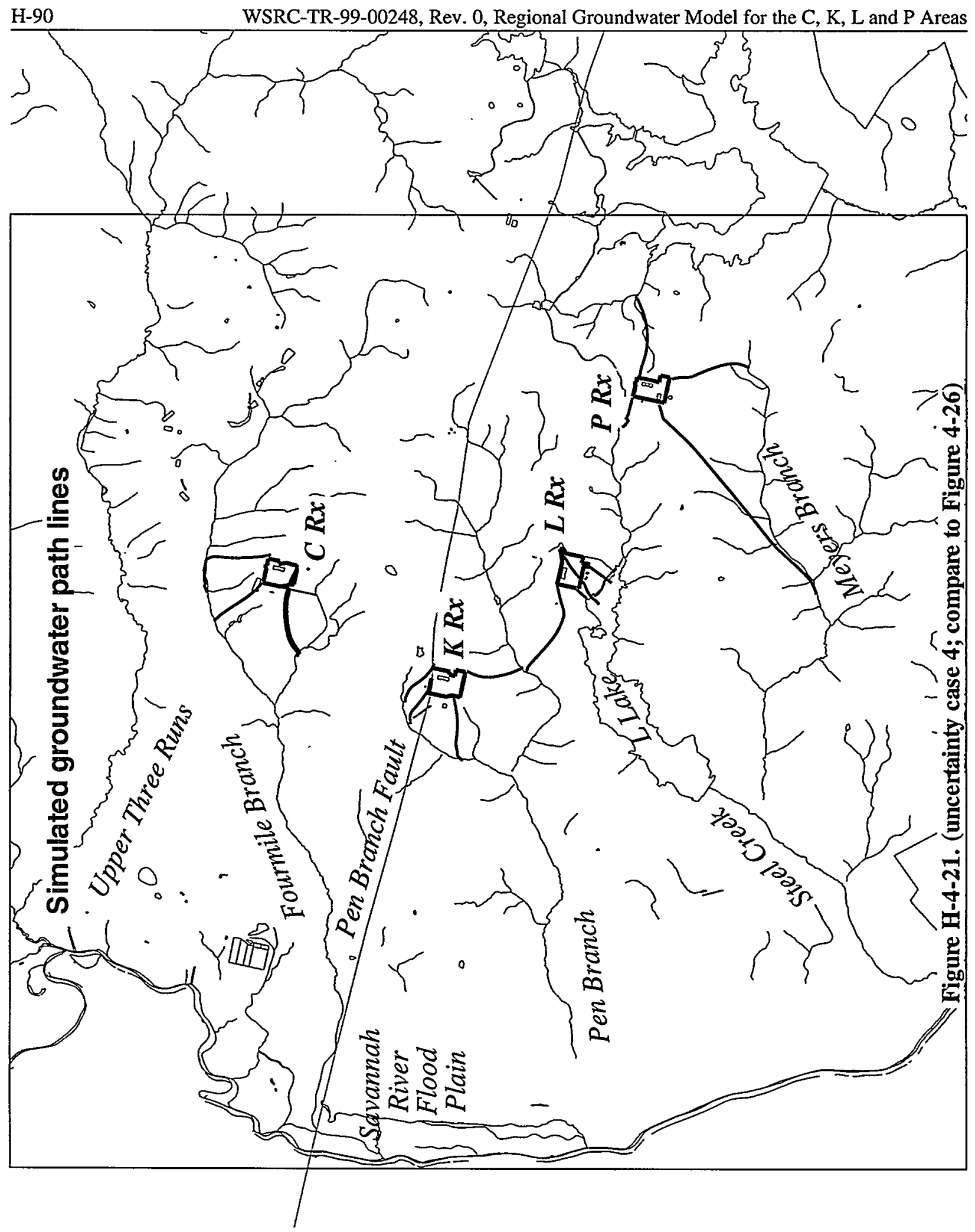

